SLAC-R-979

\title{
1994 SSRL Activity Report
}

\author{
By A. Bienenstock and H. Winick
}




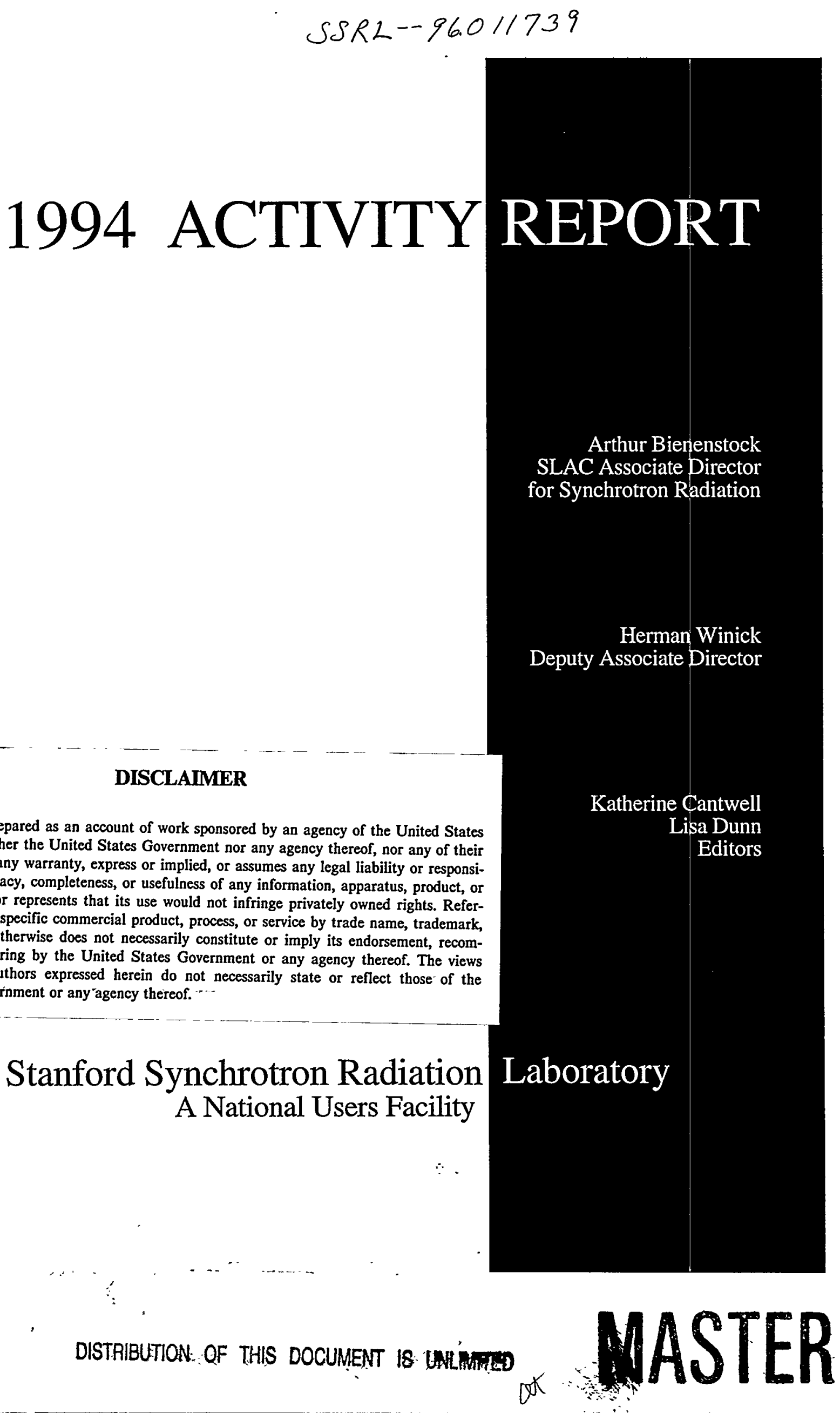

This report was prepared as an account of work sponsored by an agency of the United States Government. Neither the United States Government nor any agency thereof, nor any of their employees, makes any warranty, express or implied, or assumes any legal liability or responsibility for the accuracy, completeness, or usefulness of any information, apparatus, product, or process disclosed, or represents that its use would not infringe privately owned rights. Reference herein to any specific commercial product, process, or service by trade name, trademark, manufacturer, or otherwise does not necessarily constitute or imply its endorsement, recommendation, or favoring by the United States Government or any agency thereof. The views and opinions of authors expressed herein do not necessarily state or reflect those of the United States Government or any'agency thereof.

\section{Stanford Synchrotron Radiation}

Laboratory

Cantwel

a Dunn

Editors 


\section{DISCLAIMER}

Portions of this document may be illegible in electronic image products. Images are produced from the best available original document. 


\section{ABOUT THE STANFORD SYNCHROTRON RADIATION LABORATORY}

5

SRL, a division of the Stanford Linear Accelerator Center, is a national user facility which provides synchrotron radiation, a name given to x-rays or light produced by electrons circulating in a storage ring at nearly the speed of light. These extremely bright $\mathrm{x}$-rays are used for a large variety of scientific, medical and technological pursuits by over 900 researchers from more than 150 institutions. The techniques employed include $\mathrm{X}$-ray studies of atomic arrangements, ultraviolet studies of electronic states and several types of imaging. The facility, which provides 25 experimental stations on 21 beam ports as well as ancillary equipment, is used by researchers from industry, government laboratories and universities in many areas, including the fields of astronomy, biology, chemical engineering, chemistry, electrical engineering, environmental science, geology, materials science, medicine and physics.

The synchrotron radiation is produced by the $3.3 \mathrm{GeV}$ storage ring, SPEAR. SPEAR is a fully dedicated synchrotron radiation facility which has been operating for user experiments 6 to 7 months per year.

Additional information for prospective users can be obtained from SSRL by writing or calling Katherine Cantwell at SSRL, SLAC, MS 69, PO Box 4349, Stanford, CA 94309-0210 telephone (415) 926-3191, e-mail - k@slac.stanford.edu.

This report summarizes the activity at SSRL for the period January 1, 1994 to December 31, 1994.

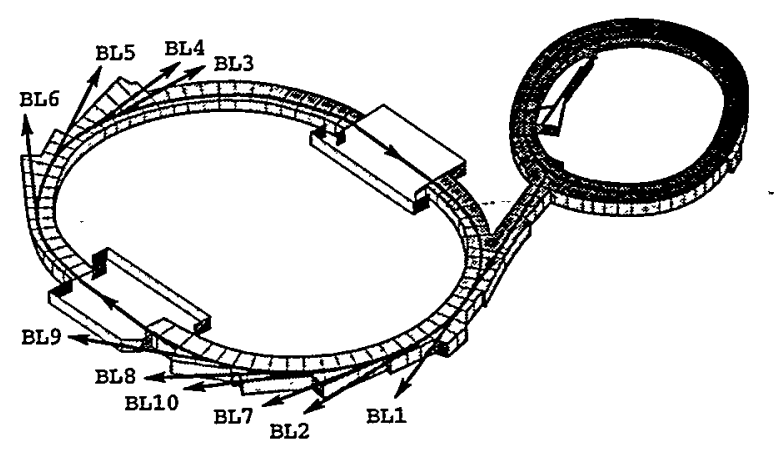

SSRL is funded by the Department of Energy, Office of Basic Energy Sciences. The Biotechnology Program is supported by the National Institutes of Health, Biomedical Research Technology Program, National Center for Research Resources. Further support is provided by the Department of Energy, Office of Health and Environmental Research. 


\section{CONTENTS}

\section{Introduction}

3 Laboratory Operations

Accelerator Operations

Accelerator Improvements

Beam and SPEAR Usage Tables

\section{Accelerator Physics Research and New Source Development}

Accelerator Physics Research

New Source Development

17 Experimental Facilities

Experimental Stations

Station Improvements

Instrumentation Development

Computers

Biotechnology Projects and Developments

Structural Molecular Biology Beam Line 9

Environmental Molecular Science Beam Line 11

\section{Workshops and Meetings}

21st Annual Users Meeting

Analytical Applications of Synchrotron Radiation Workshop Linear Coherent Light Source Workshops

35 SSRL Organization

SSRL Organization Chart

SSRL Experimental Stations - Responsible Personnel

SSRL Advisory Boards

\section{Experimental Progress Reports}

Indexes to Experimental Progress Reports

Materials Proposals

Biology Proposals

VUV Proposals

\section{Active Proposals}




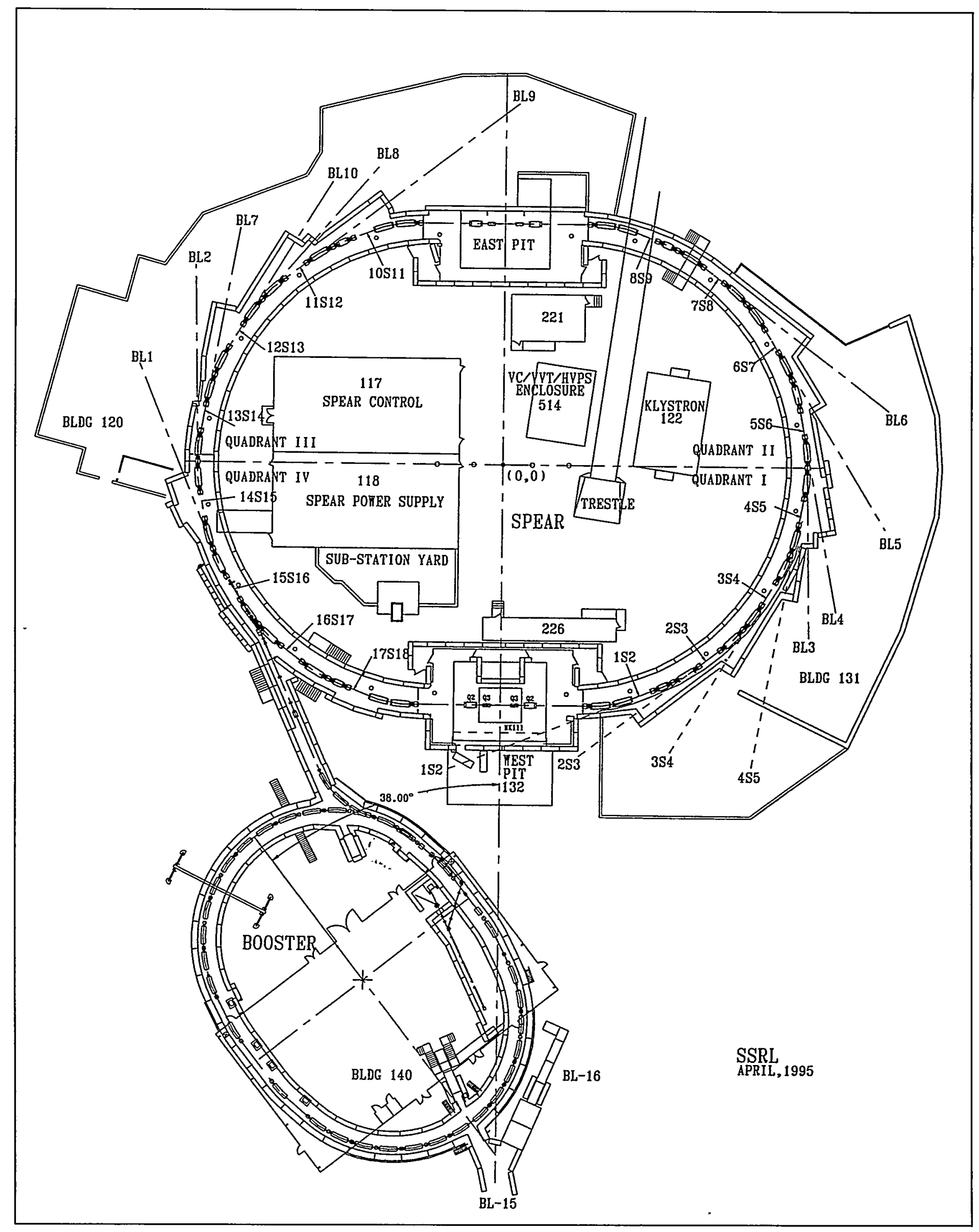




\section{INTRODUCTION}

1994, the third year of operation of SSRL as a fully dedicated, low-emittance, independent user facility was superb. The facility ran extremely well, delivering $89 \%$ of the scheduled user beam to 25 experimental stations during 6.5 months of user running. Over 600 users came from 167 institutions to participate in 343 experiments. Users from private industry were involved in $31 \%$ of these experiments.

The SPEAR accelerator ran very well with no major component failures and an unscheduled down time of only 2.9\%. In addition to this increased reliability, there was a significant improvement in the stability of the beam. The enhancements to the SPEAR orbit as part of a concerted three-year program were particularly noticeable to users. The standard deviation of beam movement (both planes) in the last part of the run was 80 microns, major progress toward the ultimate goal of 50-micron stability. This was a significant improvement from the previous year when the movement was 400 microns in the horizontal and 200 microns in the vertical.

A new accelerator Personal Protection System (PPS), built with full redundancy and providing protection from both radiation exposure and electrical hazards, was installed in 1994.

It is not possible to describe in this summary all of the scientific experimentation which was performed during the run. However, the flavor of current research projects and the many significant accomplishments can be realized by the following highlights.

- A multinational collaboration performed several experiments involving $x$-ray scattering from nuclear resonances. This resulted in the first observation of the nuclear resonance of Ta-181 and the measurement of its lifetime. In another experiment $\mathrm{Kr}-83$ was used as a gas sample, leading to the first observation of synchrotron-excited nuclear fluorescence in a gas.

- Studies related to nuclear waste remediation by groups from Los Alamos National Laboratory and Pacific Northwest Laboratories continued in 1994. A portion of the studies involved looking at the "sludge" from the Hanford Tanks. The group developed procedures for quantitative species analysis of solids using EXAFS measurements obtained with the synchrotron beam. For example, strontium in the tanks was shown to be incorporated substitutionally in calcium-containing minerals. Therefore, removal of strontium by waste remediation processes must be accomplished through removal of calcium.

- Diffraction data sets for a number of important protein crystals were obtained. A group from Harvard University collected a diffraction data set from crystals of yeast topoisomerase II. This protein is critical for correct DNA replication and cell division. A group from the University of Alabama collected data on influenza virus neuraminidase with aromatic inhibitors and feline calicivirus. The inhibitors of influenza virus neuraminidase have been licensed by BioCryst Pharmaceuticals, Inc., to be developed as a drug against flu. The structure solution of feline calicivirus will help in design of antiviral vaccines against this newly emerging virus. In another example, an SSRL staff scientist working with Anergen, Inc., obtained a data set of NB1, a protein present in normal breast cells but almost entirely absent in cancerous breast cells.

- During the past two years a collaboration consisting of groups from Hewlett Packard, Intel, Fisons Instruments and SSRL has been exploring the utility of synchrotron radiation for total reflection $\mathrm{x}$-ray fluorescence (TRXRF). This technique has emerged as a leading candidate for non-destructive measurement of silicon wafer surface cleanliness. Rotating-anode based TRXRF has been in use by many semiconductor chip manufacturers and has been sufficiently sensitive to levels of surface contamination until recently. Projections of the sensitivity needed for the near future indicate that these conventional facilities will not be adequate. Over the two years of the collaboration an increase in sensitivity to $3 \times 10^{8}$ atoms $/ \mathrm{cm}^{2}$ (compared to $5 \times 10^{9}$ atoms $/ \mathrm{cm}^{2}$ for the rotating anode based facility) has been achieved. SLAC internal CRADA funds have recently been awarded to improve the $\mathrm{Si}(\mathrm{Li})$ solid-state detector used for these experiments by reducing the parasitic signal in the solid-state detector due to transition-metal materials used in its construction.

- High-resolution angle-resolved photoemission experiments have continued to generate exciting new results from highly correlated and magnetic materials. More refined experiments from various high- $T_{c}$ samples continue to support earlier discovery at SSRL that the superconducting gap is very anisotropic and is suggestive of a novel $d$-wave pairing mechanism. (The work by Shen et al. is listed as one of the key 
experiments in a NEWS article in Science [Science 265, 862 (1994)]. An MIT-Stanford collaboration has successfully studied an insulating compound that is related to high- $T_{c}$ superconductors. This experiment unambiguously suggests the importance of antiferromagnetic order on the electronic structure of these materials. Finally, various other important novel materials such as $\mathrm{FeSi}, \mathrm{NiS}_{2-\mathrm{x}} \mathrm{Se}_{\mathrm{x}}$ have also been studied.

Several key advances in facility instrumentation occurred in 1994. A new elliptically polarizing undulator on SPEAR's Beam Line 5, the first device of its type, was commissioned. It is capable of producing intense soft $\mathrm{x}$-rays in the $500-1000 \mathrm{eV}$ energy range, corresponding to the L-edges of the first row transition elements. This undulator can be made to generate plane polarized $x$-rays in the vertical or horizontal directions, and left- or rightcircularly polarized radiation. It produces about $99 \%$ circular polarization. Changing the polarization does not affect SPEAR, so the experimenter may shift rapidly $(10 \mathrm{sec})$ between senses of polarization. In collaboration with LBL, a thin-film polarizer was built to characterize the $\mathrm{x}$-rays, the first such device in this energy range. The new undulator and polarizer will be used to study magnetic circular dichroism, Faraday rotation, and the Kerr magneto-optical effect in thin multilayers.

A new focusing mirror was installed in Station 1-5 ES2 at the end of the experimental run. This development will provide a 50 -fold increase in flux to the area detector end station used by the crystallography community. The small-angle scattering community will also see benefits from a development using synthetic Mo-C multilayers as the monochromator crystal on Station 4-2. These multilayers produce 10 times more flux on a spot size which is typically used for small-angle scattering studies.

1994 was the second year of the Structural Molecular Biology Beam Line construction project funded by the Office of Health and Environmental Research. As originally conceived, this beam line was to have three experimental stations designed for: (a) $x$-ray absorption spectroscopy, (b) protein crystallography and (c) smallangle scattering. On the advice of a number of outside review panels, the originally planned Small-Angle Scattering station will now be a Rotation Camera facility. This decision was driven by the high demand for the present rotation camera and the rapid expansion of the community which uses it.

The building extension to house the beam line and ancillary laboratories was completed. The wiggler magnet, an 8-period device with a peak field of 1.9 Tesla, was delivered in the spring of 1995 . This magnet is of a new design developed to achieve a wide fan of hard $x$-ray radiation over the three experimental stations. The wiggler vacuum chamber design and fabrication is being done at SLAC, the first such chamber to be designed and fabricated in-house. The design goal was to permit the smallest possible wiggler gap while preserving the necessary beam aperture in SPEAR. To achieve this the chamber must be extremely straight and have thin-walled pockets into which the wiggler poles fit.

A removable exit chamber crotch has been installed; a beryllium window module capable of withstanding the power from a high field wiggler at $200 \mathrm{~mA}$ has been designed and is being fabricated; and the beam line front end components have been fabricated and are being installed. A prototype photon beam position monitor and an improved electron beam monitor have been tested. The project is on track and should be ready for beam tests in early 1996.

Throughout 1994, the multi-institutional technical group (SLAC, LBL, UCLA, LLNL, University of Milan) has continued to meet monthly to pursue the study of the use of the last $1 / 3$ of the SLAC linac to drive a Linac Coherent Light Source (LCLS) - an X-ray laser capable of reaching very short wavelengths. A change in direction for the project took place following a workshop on Scientific Applications of Coherent X-rays held in February 1994. At this workshop several important applications of 1-2 \& radiation were presented, making use of the unique properties of the LCLS (ultra-high brightness and peak power, subpicosecond pulse length, full transverse coherence). The technical group has, therefore, shifted its emphasis away from the $30 \AA$ design that was previously considered and toward the design of an LCLS that could reach the 1-2 $\AA$ range. Such a source would provide about $10 \mathrm{GW}$ of peak coherent power in $100 \mathrm{fs}$ pulses, resulting in a peak brightness about 10 orders of magnitude higher than third-generation $\mathrm{x}$-ray synchrotron sources. At a $120 \mathrm{~Hz}$ repetition rate, the average brightness is about 3 orders of magnitude higher. 


\section{LABORATORY OPERATIONS}

\section{ACCELERATOR OPERATIONS}

During 30.5 weeks of operation in 1994, 89\% of the shifts scheduled for users were delivered. A combination of improved reliability and beam stability yielded far better data and, in some cases, allowed users to perform sensitive experiments that they had been unable to perform in the past. During the two experimental periods, which went from January 31August 17 and December 5-21, 1994, 314 experiments representing 207 proposals were scheduled. These experiments were from 165 spokespersons from 60 institutions. Approximately 600 scientists actually came to SSRL. Usable beam was delivered for 8019 8-hour user experimental shifts.

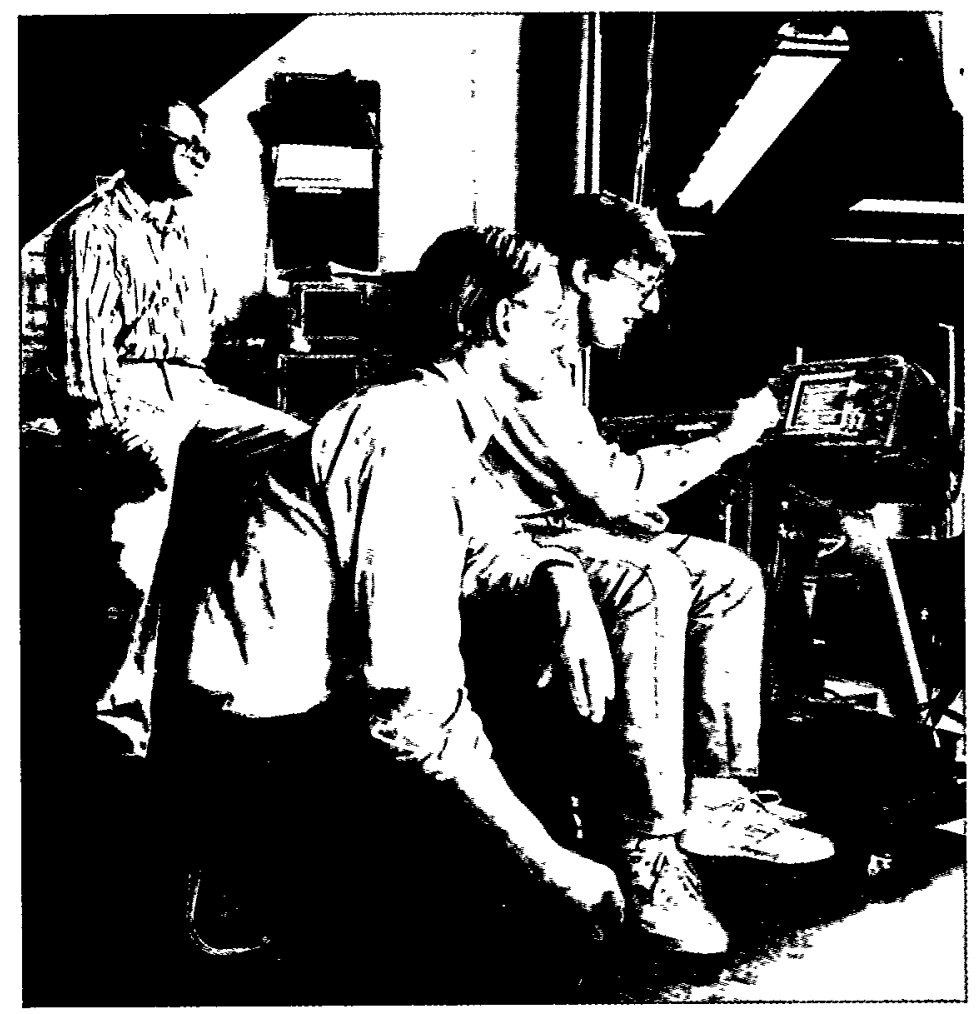

(From left) Gennadi Smirnov (Kurchatov Institute), Uwe Van Bürck (TU München) and Alfred Baron (Stanford University), members of a multinational collaboration which performed several experiments involving $x$-ray scattering from nuclear resonances during the August 1994 timing mode run.

Timing Mode. For a two and one half week period (August 1-17, 1994) SSRL ran in "timing mode" or four equally spaced electron bunches $190 \mathrm{~ns}$ apart. This mode allows experimenters to study the processes initiated by photons from each bunch. Normally SPEAR operated with a train of about 30 more closely spaced bunches. Due to its large circumference ( $235 \mathrm{~m}$ ), SPEAR is well suited to the timing mode of operation. The relatively high $\mathrm{rf}$ frequency also helps since this shortens the duration of each bunch.

\section{ACCELERATOR IMPROVEMENTS}

The SSRL accelerator group maintained its focus on improving the quality of the photon beams delivered to the users. Primary emphasis continued to be on the three-year orbit feedback program, which is scheduled to be completed in 1995. As described below, a major step was completed with the operation of a computerbased global orbit feedback system throughout the second half of the 1994 run. After initial tests a unified global/local version of the feedback program was introduced which enables simultaneous control of the global electron beam orbit and the local beam positioning at the photon beam position monitors (BPMs). This system also produced significant improvement in electron beam stability by reducing the diurnal motion of the beam as well as the fill-tofill movement.

Orbit Feedback. The new computer-based global orbit feedback system was successfully operated throughout the second half of the 1994 user run. At first, problems were encountered with orbit shifts that were apparently caused by changes in the ring circumference. Since this type of shift can be compensated for by adjusting the ring rf frequency, the feedback code was modified to feedback with the rf frequency as a servo parameter. Later, it was discovered that the beam position monitor signal processor was susceptible to current-dependent errors; a single large bunch was introduced into the fill pattern to ameliorate this effect. Further improvements included optimization of the number of eigenvectors used in the feedback algorithm, improvements in the technique used to measure response matrices, and digital filters to reject $\mathrm{BPM}$ noise. At this point, users were pleased with the improvement in orbit stability.

Later in the 1994 run, a unified global/local version of the feedback program was introduced which enabled simultaneous control of the global electron beam orbit and local beam positioning at the photon BPMs. At the top of each fill, the unified system was used to steer the photon beam lines and optimally position the remaining electron beam orbit. As a result, fill-tofill orbit motions were reduced. Work continues during 1995 to improve feedback system performance and extend the bandwidth by implementing dedicated digital signal processing components. 

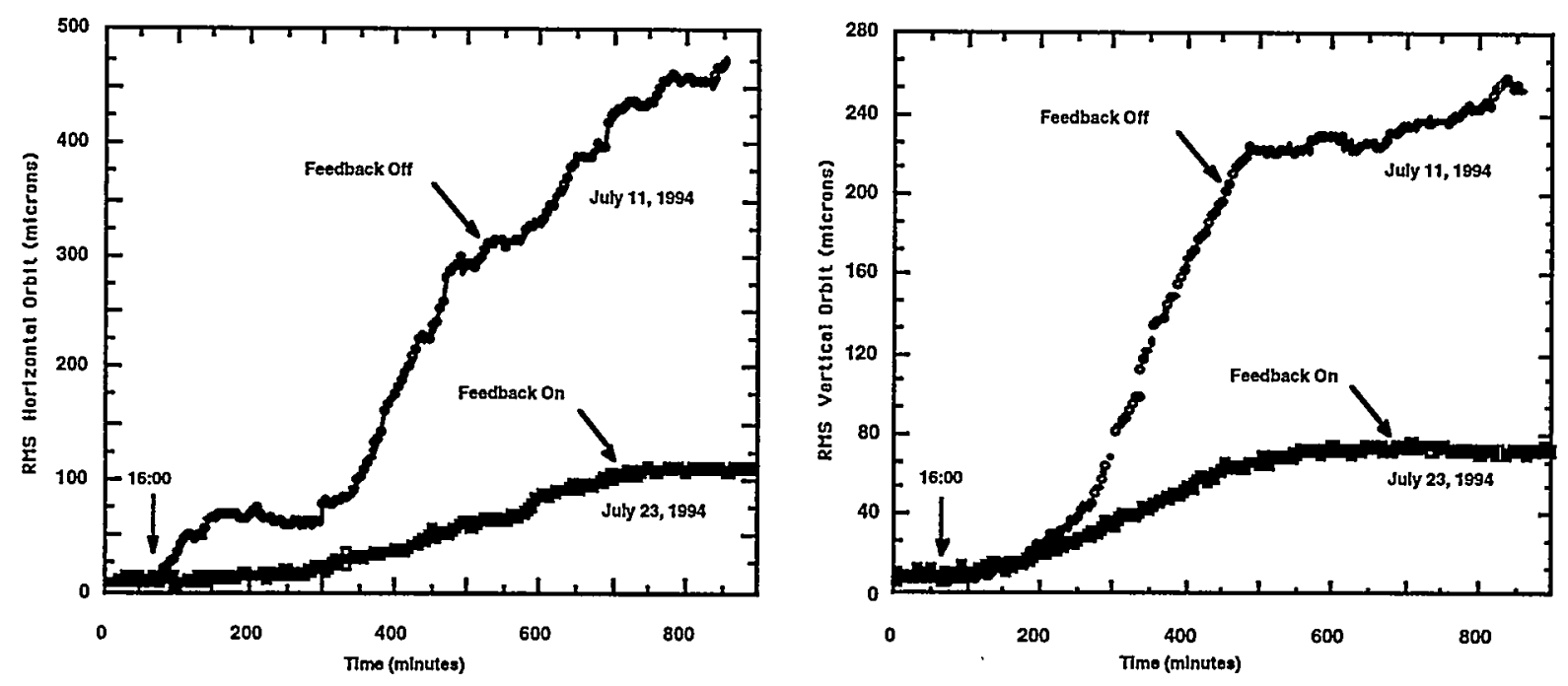

FIGURE 1. Performance of horizontal and vertical orbit feedback system over a 15-hour interval. Plots show rms orbit motion on two different days (starting at 16:00) with identical machine conditions. The upper line is with the feedback off and the lower line with feedback on.

Electron Beam Position Monitoring. Two new electron beam position monitors were installed in SPEAR during the 1994 shutdown, increasing the total number of monitors from 27 to 29 . The monitor design has been developed over the last two years, with the first prototype having been installed during the 1993 shutdown. The new design offers increased position sensitivity and lower beam impedance than most of the older BPM designs because of its optimized electrode placement, improved vacuum feedthroughs, and minimal vacuum chamber discontinuity.

The SPEAR BPM processing system presently measures a complete orbit in $\sim 1$ msec. By averaging 1200 orbits, orbits can be measured every few seconds with an rms reproducibility on the order of $10 \mu \mathrm{m}$. Temperature-dependent horizontal motion of the BPMs in the ring $(-50 \mu \mathrm{m} \max )$ and current dependent signal processing increase orbit monitoring error to thê order of $100 \mu \mathrm{m}$ over the course of 24 hours. A new processing system (that detects the $717 \mathrm{MHz}$ second harmonic of the ring if frequency with narrowband receivers) is being implemented during the 1995 user run. The new system is expected to substantially reduce the current dependent error and increase orbit acquisition speed by a factor of four. Braces will be installed on many BPMs to reduce their mechanical motion. The goal is to obtain orbit detection resolution and stability of $50 \mu \mathrm{m}$ or less with these changes.

Experimental Evaluation of BPM Alignment in SPEAR. Orbit control in the SPEAR storage ring is important to hold the location of photon beams constant for experimenters. Due to ground movements during the 22-year existence of
SPEAR, the location of the BPMs has shifted and, thus, limits the accuracy of orbit control and correction. An experimental method has been developed to determine BPM offsets from the equilibrium orbit by measuring and analyzing the orbit under different conditions. This allows researchers to subtract such offsets from orbit measurements and more efficiently correct the orbit.

Electron Beam Stability. Global orbit feedback produced great improvements in the electron beam stability during 1994. As one satisfied user remarked, "the beam stability this run was far better than ever before!" The main improvements came in two areas. First, the diurnal motion of the electron beam orbit was reduced during the course of each fill (see Figure 1). In addition, the feedback system was used to correct fill-to-fill orbit deviations. The net result is a more reliable and more stable photon beam for users.

SPEAR Lattice. An alternate lattice has been developed for SPEAR (NOQ3) with a similar emittance but improved stability compared to the presently used low-emittance lattice. It allows the removal of one, and the movement of another, of the low beta quadrupole families. This has the potential to create two long drift spaces of up to $18 \mathrm{~m}$, which could be utilized to accept special insertion devices, including long undulators.

The new lattice is being installed in a phased approach. In the initial phase, the Q3 magnets will be removed from the set of quadrupole families. If this is successful, a relocation of the Q2 magnets can be considered to generate the larger drift spaces. First attempts to inject into the NOQ3 lattice, carried out in 1994 without any hardware modifications, were 
unsuccessful due to aperture limitations. The vacuum chamber in the vicinity of the Q3 magnet, close to the symmetry point, had been designed with the low beta insertion in mind. The new lattice, with a larger beta-function at the symmetry points, requires a larger beam stay clear. For that reason, new vacuum chambers for the four Q3 magnets have been built and were installed during the fall shutdown of 1994. These chambers increase the available physical aperture in the area by more than $50 \%$.

Inserting a larger vacuum chamber in the existing Q3 magnets, rather than replacing the magnet with an even larger pipe, allows operation with the present low-emittance lattice while commissioning the new lattice during accelerator physics periods.

It is planned that the initial phase of the new lattice will go into operation during 1995.

Beam Loss Characterization in the Injector. A study was made to determine the amount and location of beam loss in the linac in the event of an "open chopper" failure mode. Normally, the chopper selects $3 \mathrm{~s}$-band bunches from the $1 \mu \mathrm{s}$ bunch train emitted at the rf gun. In the event that the chopper and the average current monitor interlocks fail, so as to pass the entire $1 \mu \mathrm{s}$ bunch train, beam loading in the linac would cause up to a $50 \%$ reduction in the beam energy at the end of the bunch train. Monte Carlo simulations indicate that about $30 \%$ of the beam (the low energy component) could be lost in the linac. The bulk of the beam would be lost after the B1 magnet in the linac-to-booster. Of the $\sim 50$ bunches that could enter the booster, it is estimated that 15 bunches would be captured, a factor of 3-5 higher than the normal operating condition. These results will be studied by Radiation Physics personnel to determine if additional shielding is required.

SPEAR Computer Control System. During 1994, SPEAR was successfully run on the newly commissioned upgrade of the computer control system. This upgrade consisted mainly of replacing the old CAMAC crate controllers with Ethernet-based controllers and new software. This upgrade facilitated the retirement of the old VAX 11/780 which had been used for running the SPEAR control system. That computer was replaced by a VAXstation 4000-90 which has considerably more capability.

A new Alpha computer was procured from Digital (3000-600 AXP) to which the SPEAR control system will begin to be moved in 1995. The VAXstation $4000-90$ will be moved to control the injector.

Software. The main improvement in accelerator control software came in the form of an $\mathrm{x}$-windows interface to replace the older graphics program. This changeover simplifies both accelerator diagnostics and accelerator control and reduces fill times. The corrector reduction program was streamlined to permit more emphasis on the fine tuning of beam lines during start-ups. Work was also done on the SPEAR history buffers and SPEARTALK to allow accelerator physicists to quickly isolate any operational problems that might arise. The R\&D program for accelerator modeling at SPEAR was used at the NSLS with very impressive results, including detection of coupling errors.

\section{Study of Energy Consumption of SPEAR} Magnets and Proposals for Conservation Measures. Supported by a $\$ 40,000$ grant from the DOE "In-House Energy Management" Department, a study of the energy use by SPEAR magnets was done. The SPEAR magnets were designed and built in 197071 , before the energy crisis and while by present-day standards energy costs were extremely low. Results from this study show that substantial cost savings could be realized if new coils were installed in a number of the magnets. Full implementation of the proposed energy conservation measures would result in reduced energy costs in excess of $\$ 400,000$ per year, amortizing the implementation costs in less than $3-5$ years.

\section{BEAM AND SPEAR USAGE TABLES}

The following tables and graph contain statistics on SPEAR running, experimental use and characteristics of SSRL stations.

For earlier information, consult previous Activity Reports. Table 1 and Figure 2 show the number of SPEAR shifts scheduled and delivered since dedicated running commenced in 1979. Table 2 shows the use of beam at SSRL for experimental purposes. Table 3 lists the characteristics of the 25 SSRL experimental stations. 
TABLE 1

\section{HOURS OF USER BEAM SCHEDULED AND DELIVERED}

\begin{tabular}{|c|c|c|c|}
\hline DEDICATED RUN & $\begin{array}{l}\text { SCHEDULED } \\
\text { HOURS for } \\
\text { USERS }\end{array}$ & $\begin{array}{l}\text { DELIVERED } \\
\text { HOURS for } \\
\text { USERS }\end{array}$ & $\begin{array}{l}\text { \% DELI } \\
\text { (Steered } \\
\text { to Beam }\end{array}$ \\
\hline $10 / 20-11 / 05 / 79$ & 152 & 95 & \\
\hline $12 / 03-12 / 21 / 79$ & 352 & 299 & \\
\hline $02 / 08-03 / 05 / 80$ & 472 & 366 & \\
\hline $04 / 16-05 / 19 / 80$ & 764 & 588 & \\
\hline $06 / 30-07 / 30 / 80$ & 726 & 320 & \\
\hline $09 / 29-10 / 14 / 80$ & 336 & 195 & \\
\hline $12 / 02-12 / 22 / 80$ & 440 & 309 & \\
\hline $01 / 26-03 / 03 / 81$ & 792 & 601 & \\
\hline $05 / 16-06 / 30 / 81$ & 988 & 727 & \\
\hline $11 / 18-12 / 21 / 81$ & 546 & 364 & \\
\hline $01 / 08-02 / 22 / 82$ & 748 & 613 & \\
\hline $03 / 09-04 / 26 / 82$ & 995 & 831 & \\
\hline 1 $/ 15-11 / 05 / 82$ & 473 & 316 & \\
\hline $27-02 / 22 / 83$ & 1050 & 826 & \\
\hline - $9-06 / 30 / 83$ & 1195 & 960 & \\
\hline $1 / 07-12 / 23 / 83$ & 857 & 663 & \\
\hline $03 / 21-04 / 30 / 84$ & 835 & 674 & \\
\hline $01 / 10-02 / 21 / 85$ & 905 & 607 & \\
\hline $03 / 15-07 / 22 / 85$ & 1502 & 1056 & \\
\hline $10 / 14-11 / 11 / 85$ & 416 & 204 & \\
\hline $04 / 11-06 / 30 / 86$ & 1550 & 1106 & \\
\hline $11 / 17-12 / 24 / 86$ & 752 & 527 & \\
\hline $01 / 02-02 / 07 / 87$ & 696 & 522 & \\
\hline $03 / 18-05 / 02 / 87$ & 1112 & 769 & \\
\hline $10 / 26-12 / 24 / 87$ & 1360 & 801 & \\
\hline $01 / 23-03 / 17 / 89$ & 1160 & 504 & \\
\hline $04 / 02-05 / 02 / 90$ & 720 & 536 & \\
\hline $05 / 27-09 / 18 / 91$ & 2304 & 1376 & \\
\hline $02 / 17-09 / 07 / 92$ & 4392 & 3385 & \\
\hline $01 / 11-07 / 26 / 93$ & 4207 & 3544 & \\
\hline $01 / 31-08 / 17 / 94$ & 4082 & 3650 & \\
\hline $12 / 05-12 / 21 / 94$ & 393 & 339 & \\
\hline
\end{tabular}

The history of dedicated time at SSRLSPEAR since its inception in 1979 is shown. From 1974-79 SSRL ran in a mode parasitic to the high energy physics program on SPEAR. From 1979-90 SPEAR was dedicated to synchrotron radiation for $50 \%$ of the operating time. In the fall of 1990 SPEAR became a dedicated synchrotron radiation source. The first full user run from the SSRL injector occurred in 1992.
SSRL's Accelerator Operations Group. (Standing, from left) Craig Haggart, Chris Hoover, Howard Page, Ed Guerra, Leif Johnson (sitting, from left) Pezhman Boussina, Thomas Nguyen and Glen Kerr.

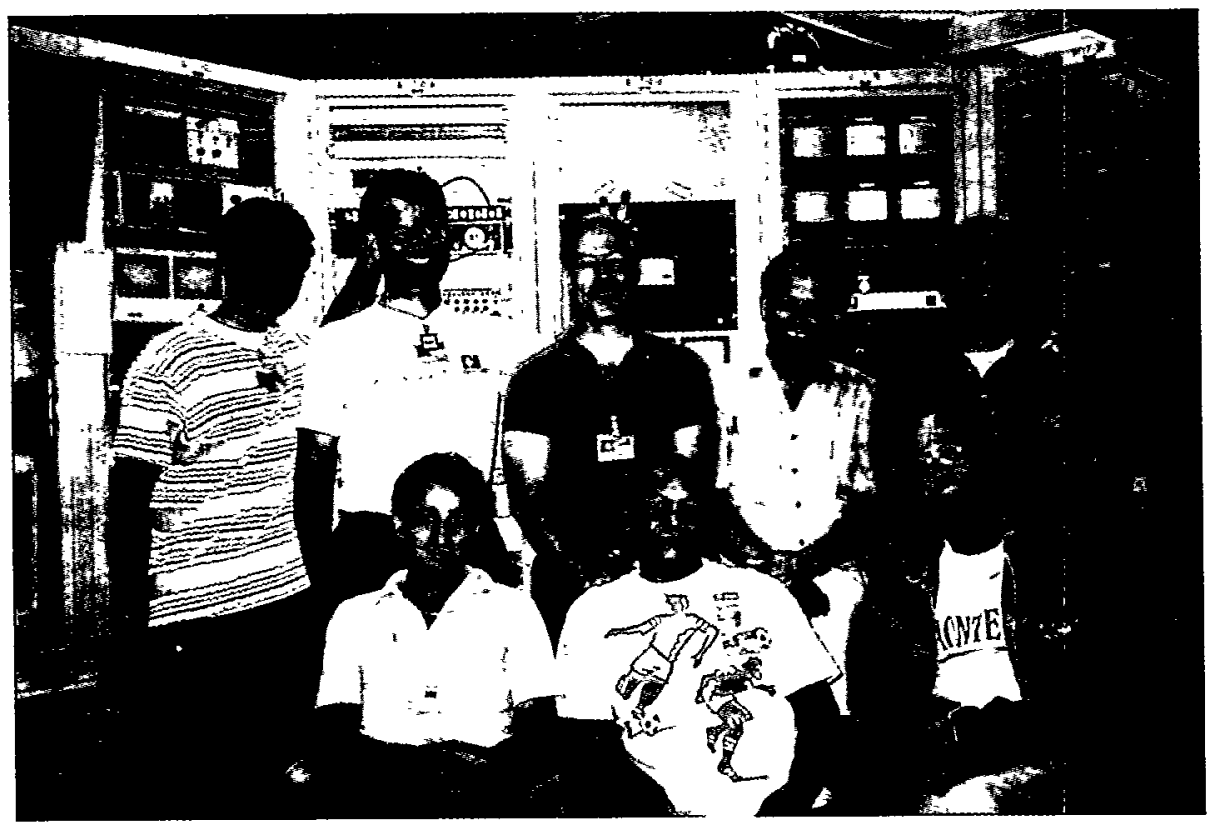




\section{SCHEDULED AND DELIVERED}

8-HOUR USER SHIFTS

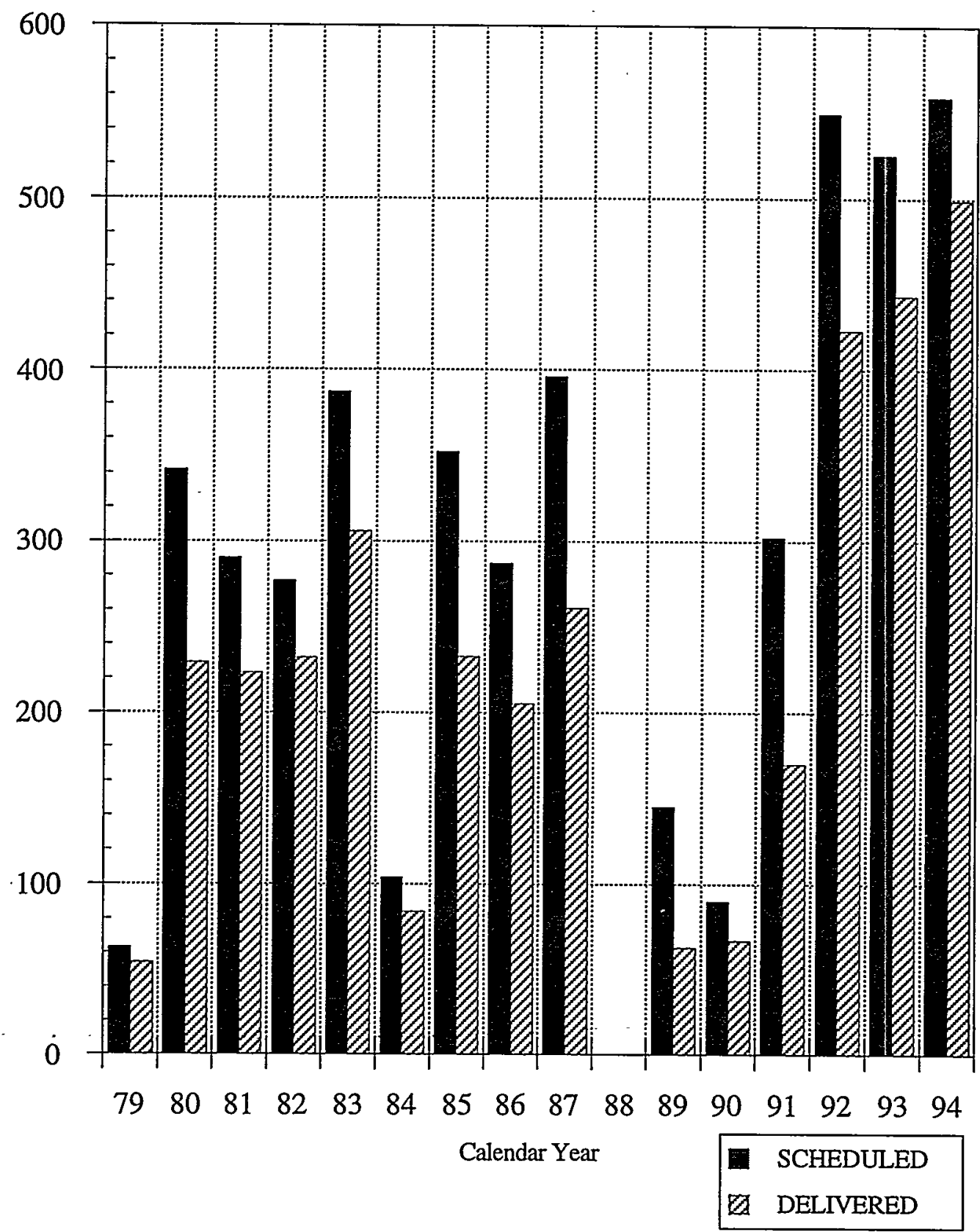

FIGURE 2. The graph above illustrates the number of SPEAR 8-hour shifts scheduled for user data taking each year and the percentage of those shifts actually delivered to users. 
TABLE 2

DELIVERED USER SHIFTS BY EXPERIMENTAL STATION January 31-August 17, 1994

\& December 5-21, 1994

\begin{tabular}{|c|c|c|}
\hline $\begin{array}{l}\text { EXPERIMENTAL } \\
\text { STATION }\end{array}$ & $\begin{array}{l}\text { SHIFTS FOR } \\
\text { EXPERIMENTAL } \\
\text { DATA TAKING }\end{array}$ & $\begin{array}{l}\text { SHIFTS FOR FACILITY } \\
\text { CHARACTERIZATTON }\end{array}$ \\
\hline $\begin{array}{l}\text { Beam Line } 1 \\
1-2 \\
1-4 \\
1-5\end{array}$ & $\begin{array}{l}228 \\
245 \\
121\end{array}$ & $\begin{array}{l}(41) \\
(94)\end{array}$ \\
\hline $\begin{array}{l}\text { Beam Line } 2 \\
2-1 \\
2-2 \\
2-3\end{array}$ & $\begin{array}{l}\text { Closed } \\
426 \\
389\end{array}$ & $\begin{array}{l}(52) \\
(34)\end{array}$ \\
\hline $\begin{array}{l}\text { Beam Line } 3 \\
3-1 \\
3-3 \\
3-4\end{array}$ & $\begin{array}{l}467 \\
318 \\
485\end{array}$ & (136) \\
\hline $\begin{array}{l}\text { Beam Line } 4 \\
4-1 \\
4-2 \\
4-3\end{array}$ & $\begin{array}{l}375 \\
394 \\
450\end{array}$ & $\begin{array}{l}(10) \\
(22)\end{array}$ \\
\hline Beam Line 5 & & $\begin{array}{l}\text { (Only one Station on BL5 can be } \\
\text { used at a time) }\end{array}$ \\
\hline $\begin{array}{l}5-2 \\
5-3\end{array}$ & $\begin{array}{r}32 \\
273\end{array}$ & $(36)$ \\
\hline $\begin{array}{l}\text { Beam Line } 6 \\
6-2\end{array}$ & 444 & (5) \\
\hline $\begin{array}{l}\text { Beam Line } 7 \\
7-1 \\
7-2 \\
7-3\end{array}$ & $\begin{array}{l}361 \\
452 \\
421\end{array}$ & $\begin{array}{l}(28) \\
(19) \\
(47)\end{array}$ \\
\hline $\begin{array}{l}\text { Beam Line } 8 \\
8-1 \\
8-2\end{array}$ & $\begin{array}{l}272 \\
398\end{array}$ & (39) \\
\hline $\begin{array}{l}\text { Beam Line } 10 \\
10-1 \\
10-2\end{array}$ & $\begin{array}{l}443 \\
439\end{array}$ & $\begin{array}{r}(5) \\
(18)\end{array}$ \\
\hline $\begin{array}{l}\text { TOTAL } \\
\text { Shifts Used } \\
\text { Proposals Run }\end{array}$ & $\begin{array}{r}8019 \\
207\end{array}$ & (includes facility characterization) \\
\hline
\end{tabular}

In 1994, 7433 8-hour shifts were actually used by experimenters for data taking on SSRL's 21 operating beam ports. A total of 586 station shifts was used by SSRL staff, PRT members and collaborators for characterization, upgrading and commissioning time. Additional shifts were used for check-outs and experimental set-up time between users. 
TABLE 3

\section{CHARACTERISTICS OF SSRL EXPERIMENTAL STATIONS}

SSRL presently has 25 experimental stations on 21 beam ports with 3 more stations under construction. Eleven of these stations are based on insertion devices while the remainder use bending magnet radiation.

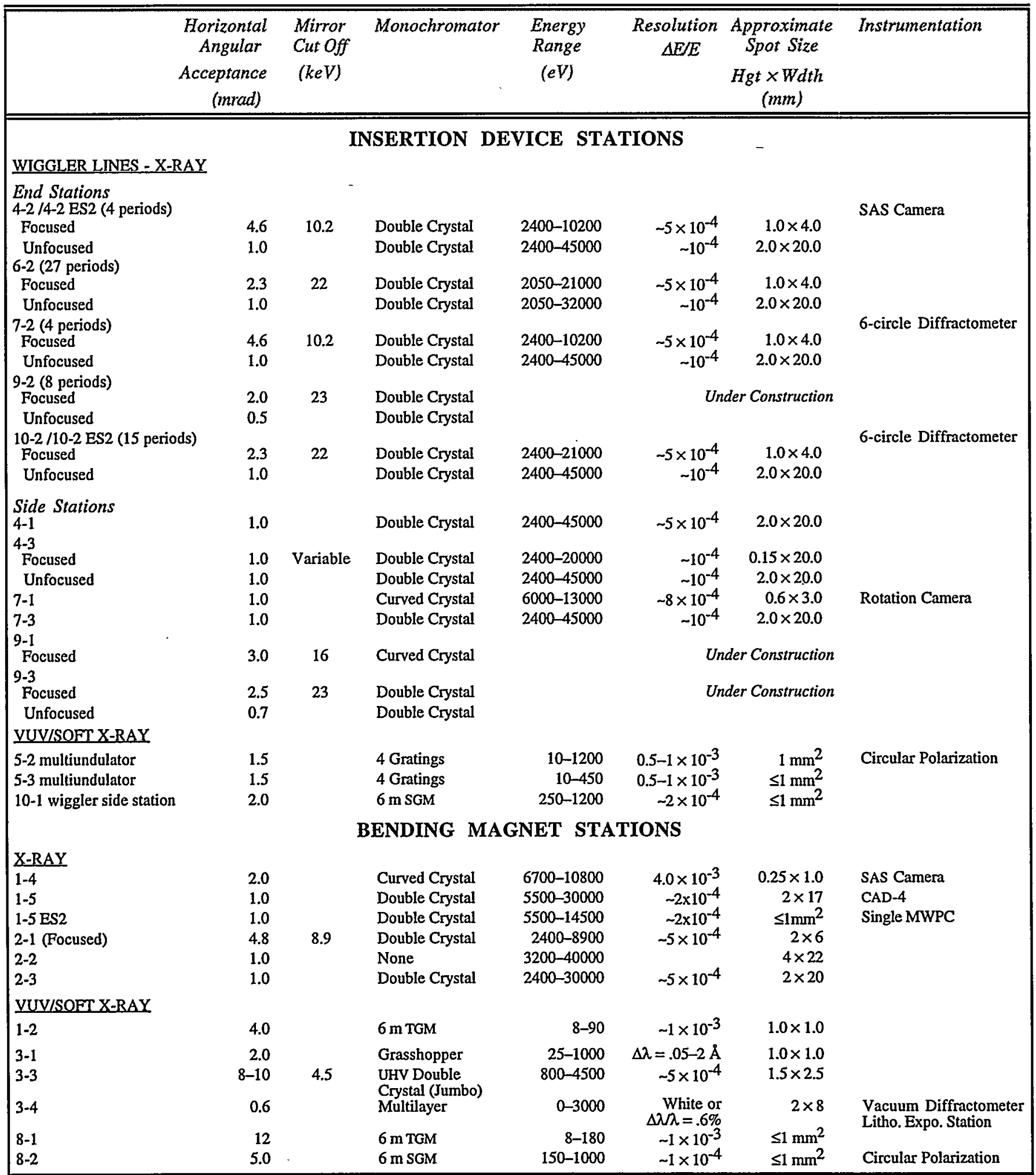




\section{ACCELERATOR PHYSICS RESEARCH AND NEW SOURCE DEVELOPMENT}

In addition to programs directly related to the improvement of the operation of SPEAR, research in long-term accelerator physics programs is carried out by the SSRL accelerator department in collaboration with colleagues at SLAC and other institutions. At the moment, the activity centers around the development of three new sources, the LCLS, FATE and a source to produce high-intensity electron pulses of subpicosecond duration (the SUNSHINE Experiment).

The training of graduate students in accelerator physics is also a key activity of the department.

\section{ACCELERATOR PHYSICS RESEARCH}

Phase-Space Studies. The development of a diagnostic phase-space monitor to enhance the lattice diagnostic capability in SPEAR continued in 1994. Progress has been made toward obtaining the longitudinal phase-space when the longitudinal bunch excitation and phase oscillation detection systems are integrated into the phase-space monitor assembly. The monitor has been used to examine the single-bunch phase-space decoherence characteristics as functions of total bunch current, horizontal displacement amplitude, octupole field perturbation, and horizontal chromaticity. In addition, an effort to investigate the coupling between the horizontal and longitudinal dimensions was initiated. Currently, work is underway to develop a means to excite the electron bunch vertically using the separation plates, and modifications are being made to enable low current study and tune-space mapping.

\section{NEW SOURCE DEVELOPMENT LINAC COHERENT LIGHT SOURCE (LCLS)}

During 1994 a group of accelerator scientists from SLAC, UCLA, LBL, LLNL, and the University of Milan continued a study of the use of the SLAC linac to drive short wavelength free electron lasers (FELs). The source, called a Linac Coherent Light Source (LCLS), is to achieve lasing in a single pass of a high peak current, low emittance electron beam through a long undulator by amplifying spontaneous emission. By avoiding the use of an optical cavity, which is difficult to implement at short wavelengths, the LCLS approach opens the way to achieving laser brightness and coherence at very short wavelengths.

With the present state of technology of if photocathode guns and precision undulators and with the understanding achieved on the Stanford Linear Collider in transporting, accelerating, and compressing low emittance electron beams, studies show that it is possible to produce about $10^{14}$ coherent photons in a subpicosecond pulse at wavelengths down to about $3 \mathrm{~mm}$ within a $0.1 \%$ bandwidth using 5-7 GeV electrons. The peak coherent power is about $10 \mathrm{GW}$. At a repetition rate of $120 \mathrm{~Hz}$, the average power is about $0.5 \mathrm{~W}$.

The expected average brightness of the LCLS is $2-3$ orders of magnitude higher than third-generation synchrotron light sources and the peak brightness is about 9 orders of magnitude higher. With improvements in photocathode rf gun technology and increased electron energy, it should be possible to extend LCLS operation to shorter wavelengths, perhaps as short as $0.1 \mathrm{~nm}$. By comparison, the shortest wavelength that has been reached in an FEL to date is $240 \mathrm{~nm}$. See Table 4 for parameters of several LCLS examples.

During 1994 the LCLS group continued its studies of relevant physical and scientific issues. A strong impetus toward developing LCLS technology down to 0.1 nm wavelengths was provided by a February 12,1994 Workshop on Scientific Applications of Coherent $\mathrm{X}$-rays. At this meeting, significant interest was evinced by the scientific community in a $0.1-0.2 \mathrm{~nm}$ source with LCLS properties for a number of important research applications. These include realtime studies of fast chemical reactions and phase transitions, real-time lattice dynamics studies using speckle interferometry, structural analysis, non-linear physics, and coherence-based interferometric and imaging techniques. With this stimulus, research has been directed toward the development and improved understanding of the physical theory and technology necessary to support SASE FEL operation at $0.1-0.2 \mathrm{~nm}$. To help plan, budget, and guide the group's R\&D efforts, work on the compilation of a comprehensive document describing and budgeting the required research through 1997 has been initiated.

Significant theoretical and experimental work was conducted in 1994 on the development of a new lowemittance photocathode of gun, optimized for mini- 
mizing the growth of transverse emittance following emission. The ultimate goal of this work, a joint BNL, LBL, SLAC, UCLA effort, is to produce a structure that can attain a normalized emittance of 1 $\mathrm{mm}-\mathrm{mrad}$ or lower with $1 \mathrm{nC}$ of change.

To support the further development of this gun, plans for constructing a photocathode rf gun linac test stand in the existing SSRL injector linac enclosure were initiated. To perform demonstration experiments on exponential gain growth at the $100-150 \mathrm{MeV}$ linac energies available at this location, a new SSRL project, the FEL Amplifier Test Experiment (FATE), has been created. This project, portions of which have already been technically assessed and budgeted, will entail the extension of the existing linac enclosure and the construction and installation of a 3-6 m undulator, together with the required transport and diagnostic (photon and electron) optics. (See next section on FATE)

TABLE 4

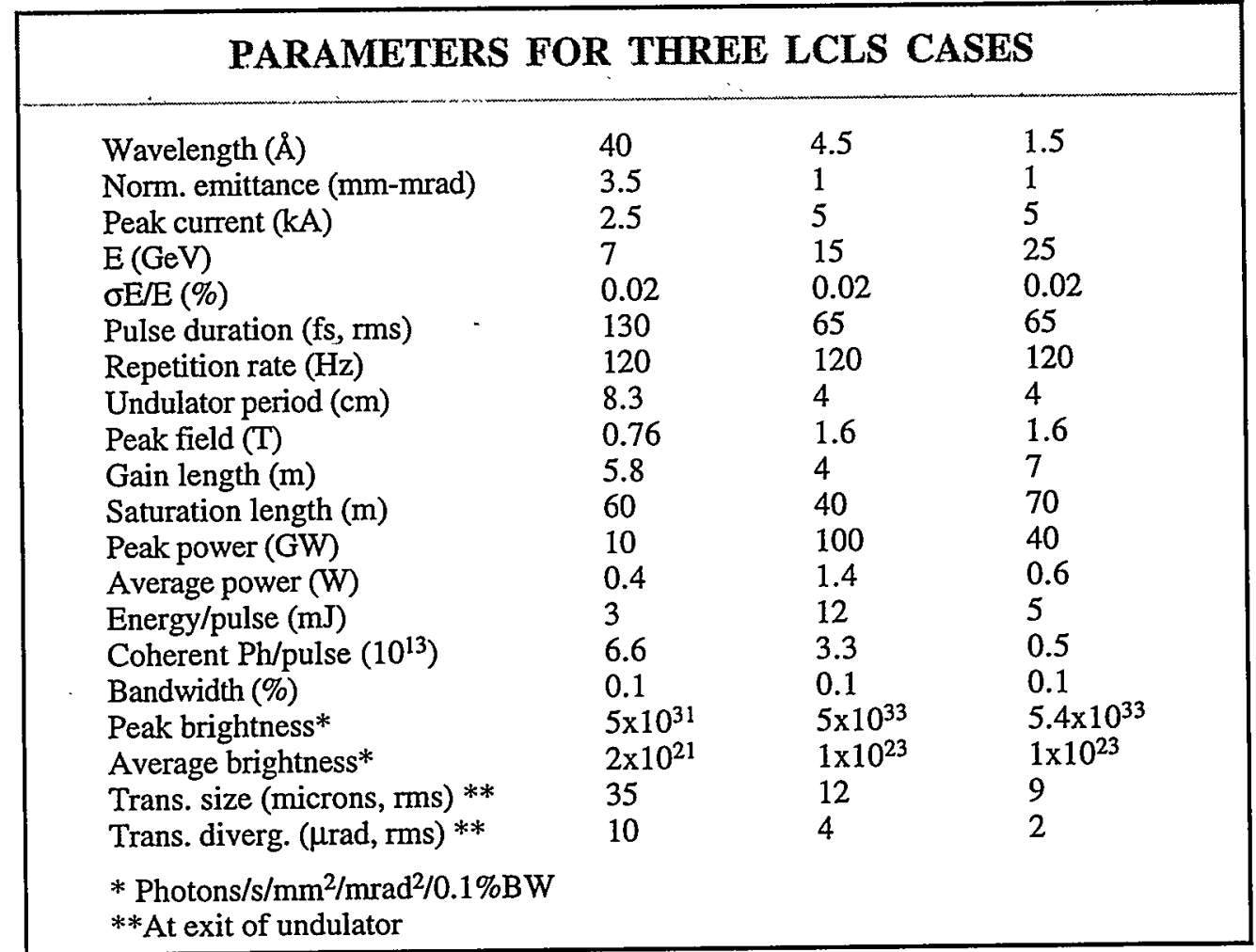

Approximate values of calculated performance parameters are given for three cases. The first column assumes conservative performance of an $r$ photocathode gun and compression scheme. In this column is included the results of a detailed study of the effects of undulator errors and misalignments, which reduces the output by about a factor of three. Error effects aren't included in the last two columns, which are based on improved guns and stronger compression schemes. In all cases, a third harmonic with about 1/30 the number of coherent photons/pulse would also be available.

The output photon beams could be synchronized with other sources to a precision of about 0.5 ps. This would make it possible to conduct pump-probe experiments with conventional lasers or SSRL synchrotron radiation beams from SPEAR. The beams are diffraction limited and hence have full transverse coherence. The longitudinal coherence length is given by the wavelength/bandwidth. Since the bandwidth is about 0.001 in all cases, the longitudinal coherence length is about 1000 times the wavelength.

Systematic research into candidate insertion device technologies for fabricating the $0.1 \mathrm{~nm}$ LCLS undulator continued during this period. A significant distinction between the LCLS device and earlier classes of undulators is that the LCLS device must be designed to provide a focusing field two or more orders of magnitude stronger than the natural focusing of a conventional structure. Three technologies are presently under study: 1) superconducting (SC), 2) hybrid/PM, and 3) pulsed-Cu. This work, performed in collaboration with staff at LBL, UCLA, Los Alamos, and the Kurchatov Institute, is aimed at identifying 
the shortest and least expensive structure of the necessary quality, as well as the basic limitations and advantages of the various technologies for attaining this goal.

The temporal, peak power, brightness, and spectralangular parameters of the LCLS are all critical to its beam line and end-use experimental designs. A graphical display of both the spontaneous and coherent (angle-integrated) photon flux distributions generated by the three LCLS cases of Table 4 is shown in Figure 3. The opening angle of the coherent FEL peaks $\left(\left[\left(1+K^{2}\right) / N_{l}\right]^{1 / 2} / \gamma_{l}\right.$ for the 1st harmonic) is at least a factor $\sqrt{N_{t}}$ smaller than that of the spontaneous background. For $K=6$ the total power in the spontaneous spectrum, which is seen to extend out to the vicinity of the 100th harmonic, can be comparable to or even greater than the power under the coherent peaks. The indicated spectral calculations have been used to begin exploring the design of optical instrumentation for the LCLS, such as mirrors, monochromators, and other elements for controlling its spectralangular, temporal, coherence, and polarization properties.

Theoretical FEL studies, continued through 1994, have focused on more comprehensive physical models of both SASE and seeded gain startup from noise. Studies of the effects of non-Gaussian bunch distributions, as well as more refined statistical models of startup noise, on gain growth have been initiated. These studies have been important in furthering understanding of the statistical properties of LCLS output radiation and do not invalidate the earlier projected performance parameters of the LCLS.

- LCLS PEAK AND AVERAGE PHOTON FLUX VS PHOTON ENERGY

$\left(N_{i}=\right.$ number of periods $(i=1,2,3) ; K=6$ for $i=1,2,3$; linac pulse frequency $120 \mathrm{~Hz}$ )



Homan Tatchyn - SSRL - 2/11/94

FIGURE 3. Energy-normalized spectral flux curves for three LCLS undulators. 


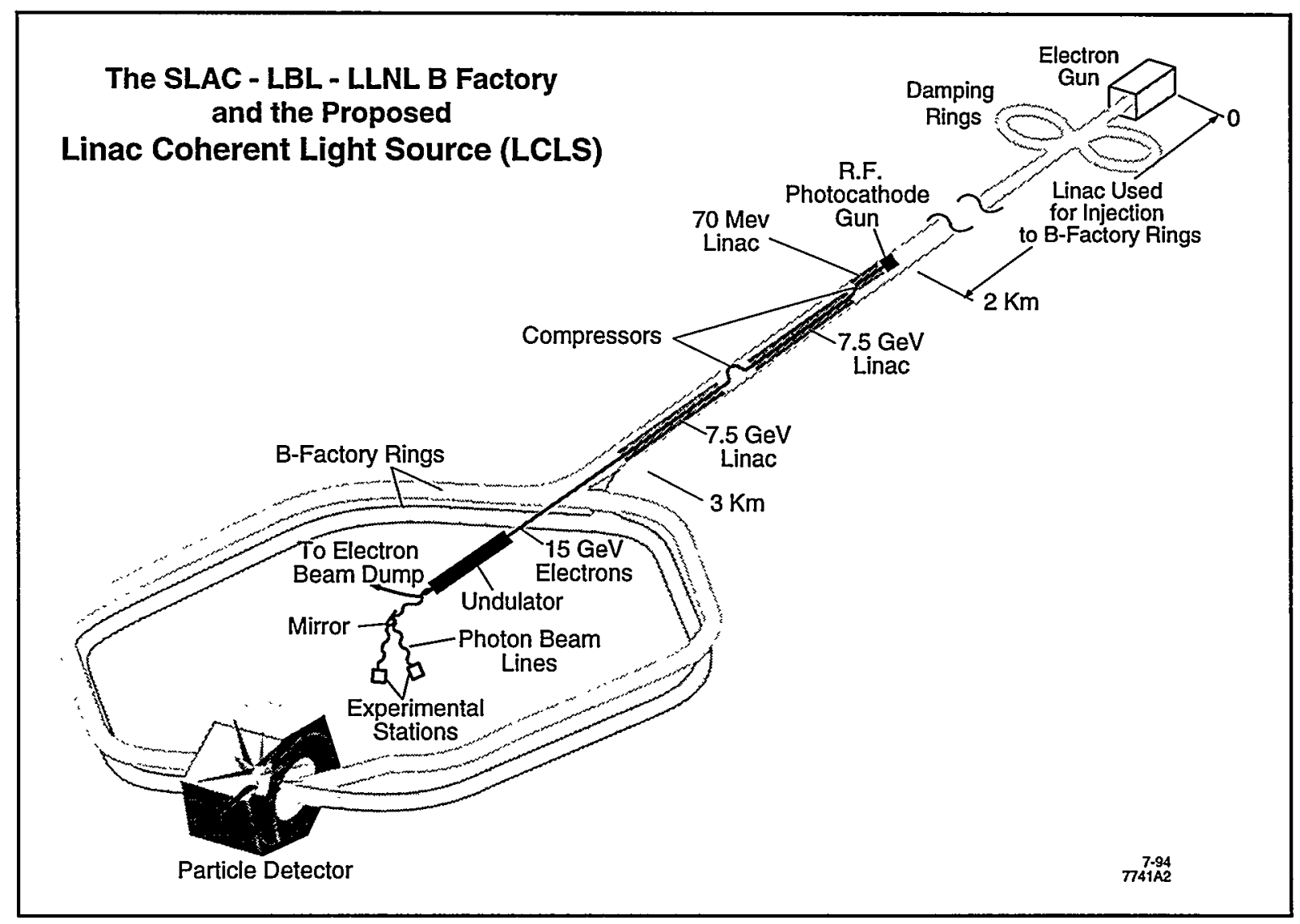

Layout showing SLAC linac serving the LCLS and B Factory

\section{THE FATE PROJECT}

In September 1994 it was recognized that the properties of the beam produced in the SSRL linac with alpha magnet compression are of sufficiently high quality to allow the performance of an FEL Amplifier Test Experiment (FATE) in the micrometer region. This experiment aims at providing the first experimental test and critical analysis of the so-called superradiant regime of a high-gain FEL, which is the radiation process underlying both short- and longbunch operation starting from noise.

The characteristics of the experiment are given in Table 5 for 1 and $10 \mu$ operation with undulator length ranging from 2.5 to $8 \mathrm{~m}$.

It turns out that the SSRL injector, based on a thermionic radio-frequency gun and an alpha magnet bunch compressor, provides an electron beam of quality good enough for a high gain single-pass operation in the $1 \mu$ region. This electron beam has already been produced on the Stanford campus with an apparatus identical to the SSRL injector.
TABLE 5

\begin{tabular}{|c|c|c|}
\hline$\lambda(\mu)$ & 1.06 & 10.6 \\
\hline$E(M e V)$ & 95 & 30 \\
\hline $\mathrm{I}_{\mathrm{p}}(\mathrm{A})$ & 250 & 160 \\
\hline$\varepsilon_{\mathrm{n}}\left(10^{-6} \mathrm{~m}-\mathrm{rad}\right)$ & $10 \pi$ & $10 \pi$ \\
\hline$\lambda_{w}(\mathrm{~cm})$ & 2.5 & 2.5 \\
\hline $\mathrm{L}_{w}(\mathrm{~m})$ & $2.5(8)$ & $2.5(8)$ \\
\hline $\mathrm{B}_{w}(\mathrm{~T})$ & 0.8 & 0.8 \\
\hline $\mathrm{L}_{b}(\mu)$ & 60 & 120 \\
\hline $\mathrm{L}_{\mathrm{s}}(\mu)$ & $106(320)$ & $1060(3200)$ \\
\hline $2 \pi \mathrm{L}_{\mathrm{c}}(\mu)$ & 92 & 336 \\
\hline$\rho$ & $5.8 .10^{-3}$ & $\cdots$ \\
\hline
\end{tabular}

Here $\mu$ is the radiation wavelength, $E$ is the electron beam energy, Ip is the peak current, $\varepsilon_{n}$ is the normalized emittance, $\mu w$ is the wiggler wavelength, $L_{w}$ is the total wiggler length and $B_{w}$ is the wiggler peak field. $L_{b}, L_{s}$ and $L_{c}$ are, respectively, the bunch length, the slippage length and the cooperation length. $\rho$ is the fundamental FEL parameter. The number of superradiant spikes (see later) is given by $L_{b} / 2 \pi L_{c}$. 
The FATE experiment will operate in the pure superradiant regime since the bunch length is smaller than the slippage length (see Table 5).

The physics of superradiance is new and unexplored. Theory predicts that a single radiation pulse, starting from noise, will develop with peak intensity scaling as the square of the electron current. From a fundamental viewpoint, in the superradiant regime the electrons do not radiate by stimulated emission but by "cooperative spontaneous emission". This is defined as a regime where the electrons radiate coherently because of self-bunching, but the strong slippage inhibits re-absorption and saturation since the electrons radiate almost in vacuum. The FATE experiment is in a position to demonstrate for the first time and to critically analyze this new and unexplored phenomenon in FEL physics.

The proposed experiment is still in evolution, but at this time an undulator up to about 6 meters long in one, two or three segments is envisioned. The use of a planar helical undulator is favored since it provides both horizontal and vertical focusing strong enough to prevent increase of the electron beam diameter. Other focusing strategies are also being investigated. Six meters is long enough to cause bunching of the electron beam followed by high-gain, superradiant emission of 1.06 micron light. This is the 'startup from noise' operational mode; it would generate enough radiation to be detectable but would not get to saturation. To generate enough output power to saturate, a startup from a 1.06 micron wavelength optical seed laser would be needed. This will be provided for in the design, but this capacity may not be built initially since analysis of superradiance and startup from noise are the primary project goals.

FATE has been modeled extensively using the GINGER code written by T. Fawley at LLNL. Assuming a 200 fsec, $10 \mathrm{~mm}$-mrad Gaussian unseeded electron pulse, a single superradiant output pulse would have power levels as shown:

$\begin{array}{llll}\text { Undulator } & \begin{array}{l}\text { B field } \\ (\mathrm{kG})\end{array} & \begin{array}{l}\text { Current } \\ \text { (Amp) }\end{array} & \begin{array}{l}\text { Power Output } \\ \text { (W) }\end{array} \\ \text { Helical } & 6.9 & 250 & 3.5 \times 10^{6} \\ \text { Helical } & 5.9 & 250 & 6 \times 10^{5} \\ \text { Linear } & 8.0 & 100 & 6 \\ \text { Linear } & 8.0 & 200 & 1300 \\ \text { Linear } & 8.0 & 250 & 9000\end{array}$

The lower performance of the linear undulator is due to the spread of the beam, which is not focused in the horizontal. The linear undulator examples show the strong dependence on peak electron current. The detection limit is estimated to be on the order of a picojoule, which corresponds to 5 watts for 200 fsec.

In summary, the FATE experiment has as its goals:

i) To demonstrate and critically analyze high-gain, single-pass FEL operation in the 1-micron wavelength region, using a thermionic gun and alpha magnet beam compression to obtain sufficient peak current, emittance, and energy spread.

ii) To demonstrate and critically analyze superradiant emission in the short bunch, strong slippage regime, which provides an indirect but fundamental test of short-wavelength, long-bunch operation starting from noise.

\section{SHORT-PERIOD UNDULATOR SOURCES FOR SPEAR}

During 1994, numerical studies were conducted to determine the potential performance of short-period, small-gap undulators operated at SPEAR energies of 3 and $3.5 \mathrm{GeV}$. Projecting $\mathrm{K}$ values smaller than 0.5 , the attainable photon energy (in $\mathrm{keV}$ ) is approximable by $E(G e V)^{2} / \lambda_{u}(\mathrm{~cm})$. Assuming a gap/period ratio smaller than 0.5 and a minimum attainable gap of 0.2 $\mathrm{cm}, 30 \mathrm{keV}$ photons could be generated with SPEAR running at $3.5 \mathrm{GeV}$. Since the length of the shortperiod device is limited to the order of the focusing beta (which in turn must be of the order of tens of centimeters to focus the electron beam through the small gap), its output can only be made comparable to a larger gap, longer period device by generating a substantial on-axis field amplitude. To this end, both SC and hybrid/PM technologies can be considered suitable for developing short-period insertion devices on SPEAR. Although the net flux generated by a short-period, small-gap device may be significantly smaller than that of a $2-\mathrm{m}$-long conventional wiggler, its in-band flux at the 1st harmonic peak, as well as its brightness, can be substantially larger, and its much smaller opening angle can be advantageous in designing $\mathrm{x}$-ray instrumentation with high throughput and efficiency. With suitable design, undulator structures or arrays capable of spanning the 10-36 $\mathrm{keV}$ range with acceptable efficiency could be developed. A suitable location for a short-period undulator station may be the long straight section in SPEAR's West Pit, provided an operating lattice with the requisite short beta can be activated.

\section{SUNSHINE EXPERIMENT}

High Intensity Coherent Far-Infrared Radiation from Subpicosecond Electron Bunches. The combination of a thermionic rf gun with an alpha magnet allows the production of highintensity electron pulses of subpicosecond duration. 
Such particle pulses can be transformed into equally short pulses of coherent radiation. The radiation spectrum is the Fourier transform of the electron distribution and, therefore, spans the far-infrared regime (FIR) between $10 \mu \mathrm{m}$ and about $1 \mathrm{~mm}$. Such a system has been assembled by SSRL accelerator physicists at the SUNSHINE facility (Stanford UNiversity SHort INtense Electron source) consisting of an if gun, an alpha magnet for bunch compression, and a 10-foot linear accelerator with associated supplies at the Hansen Experimental Physics Laboratory (HEPL) on the Stanford campus.

Experiments have begun and measurements of the coherent far-infrared radiation quantitatively confirm simulations of the electron beam dynamics in the rf gun. Radiation has been produced in the form of synchrotron radiation as well as transition radiation. The intensity in both processes exceeds the radiance available from conventional black body radiators by five to seven orders of magnitude. A permanent magnet undulator (a surplus undulator from the old PEP beam line) has also been installed at the SUNSHINE facility. The emission of coherent radiation in conjunction with the undulator field is expected to greatly enhance the brightness of the available FIR radiation as well as its collimation. The electron beam emittance is very small and, as a consequence, the FIR radiation has full spatial and temporal coherence properties. First measurements have been conducted confirming these theoretically expected radiation characteristics. At present, this is by far the brightest FIR radiation source in the world. These measurements will continue to fully characterize this radiation source, including measurements of the undulator tuning range, spectrum, and available radiation intensity as a function of photon energy, as well as the geometric distribution and collimation of the radiation.

Subpicosecond Bunch Length Measurement. A Michelson Fourier Transform Spectrometer has been assembled to measure the autocorrelation function of the FIR radiation emitted from ultra-short electron bunches. This autocorrelation function is a direct representation of the electron distribution in the bunch and can be used to measure extremely short subpicosecond bunches by optical interferometry where electronic means fail. Using this instrument, it was possible to achieve the shortest electron bunches ever produced. The rms bunch length was determined to be about $50 \mathrm{f}-\mathrm{sec}$ or $15 \mu \mathrm{m}$ long. A portable replication of the Michelson interferometer has been assembled for the Superconducting Accelerator (SCA) at Stanford to facilitate the optimization of its free electron lasers. Due to its portability and ease of use, it is expected that this method to determine and optimize for subpicosecond electron pulses will be a key diagnostics instrument in the development of appropriate beams for future linear colliders and drivers for free electron lasers.

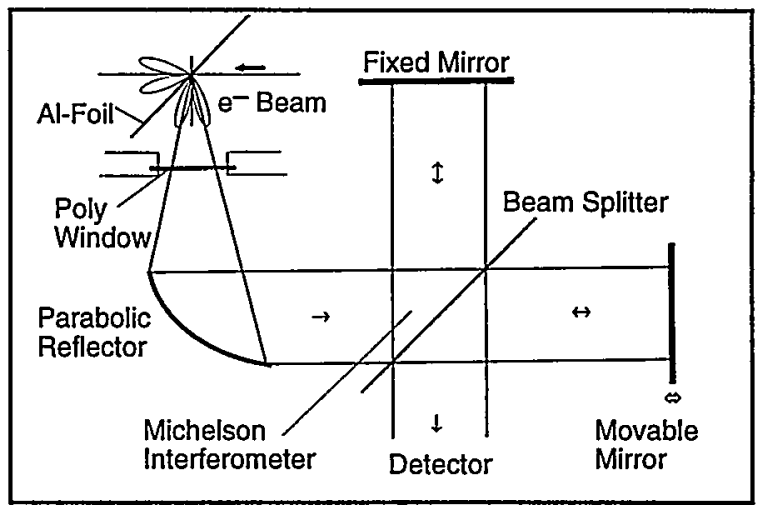

FIGURE 4. Transition radiation source and Michelson interferometer for bunch length measurement.

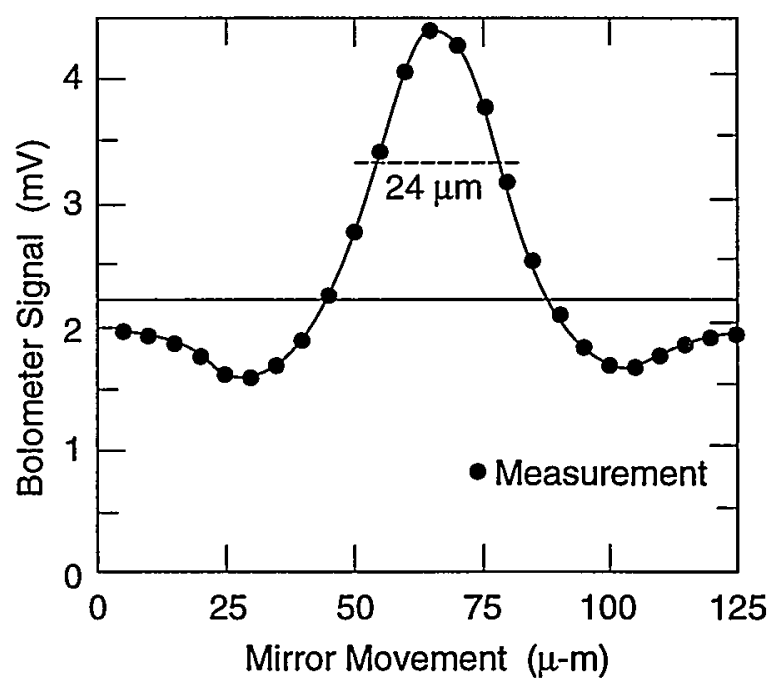

FIGURE 5. Main peak of the interferogram for the shortest electron bunch length obtained so far. The effective average bunch length in this case is $\sqrt{2 \pi \sigma_{z}}=36 \mu \mathrm{m}$ or $\sqrt{2 \pi \sigma_{t}}=\mathrm{fs}$.

Theoretical Model for Bunch Length Instability. During simulation studies of electron bunch generation in rf guns and bunch compression, an instability appeared which limits the degree of bunch compression for high electron intensities. This instability was unknown, and a theoretical model has been developed which qualitatively describes the features of the instability. In further studies, a quantitative agreement with actual parameters will be pursued as well as experimental verification of the instability. 


\section{EXPERIMENTAL FACILITIES}

\section{EXPERIMENTAL STATIONS}

The 25 experimental stations at SSRL are of two types: general facility stations and participating research team (PRT) stations. General user stations have been funded by various government agencies, principally the DOE, NIH, and NSF and are open to the user community on a competitive basis. In addition to experimental stations, there are facilities such as sample preparation laboratories, dark rooms, computer terminal rooms and a machine shop available to users.

SSRL presently has four PRT collaborations [EXXON (6-2), The National Laboratories/University of California (8-2, 10-2), IBM/Stanford University Center for Materials Science (10-1) and Stanford University (5-3)]. The standard PRT arrangement at SSRL is that each PRT institution receives one-third of the beam time. SSRL general users have access to the remainder of the time.

There are $16 \mathrm{x}$-ray stations located on 13 beam ports at SSRL. Two ports, with three stations, are PRT lines (wiggler lines 6 and 10); the others are SSRL facility stations. Of the facility lines, two (Beam Lines 4 and 7) have wigglers as sources for a total of seven stations and the other two (Beam Lines 1 and 2), serving six $x$-ray stations, have bending magnet sources.

There are currently nine VUV or soft $x$-ray stations at SSRL. Three of these facilities are PRT lines. The facilities being scheduled for users are: two TGMs (1$2,8-1$ ), one grasshopper monochromator (3-1), one in-vacuum double-crystal monochromator (3-3), two SGMs (8-2, 10-1), one lithography/optics line (3-4), and two stations on a multiundulator line (5), only one of which can be used at a time. Station 1-1, the "old grasshopper" was decommissioned in 1994.

\section{STATION IMPROVEMENTS}

Diffractometers Consolidated. In the past, x-ray diffractometers were maintained on Stations 6-2, 7-2 and 10-2. In an effort to improve the support for these complicated instruments, the decision was made (after consultation with users) to consolidate all of the X-ray diffraction experiments onto Station 10-2 and Station 7-2. Significant effort has been put into upgrading and optimizing the 10-2 and 7-2 diffractometers. The 10-2 diffractometer has been permanently installed and aligned in the 10-2 back hutch, and its associated motor driver hardware has been upgraded so that all motors have separate electronics. An eight-channel motor driver chassis has been added so that users can control additional motors that they may need for their experiments.

The venerable Station 7-2 diffractometer has been retired after 15 years of hard use. Built for a horizontal scattering geometry, it was retrofitted for a vertical scattering geometry for use at this then-new wiggler beam line. This instrument was a critical part of the scattering community during the early and mid ' 80 s prior to the completion of the NSLS. Many of the first 2-dimensional phase transition and grazing incidence scattering experiments were performed using it. It even has file marks on it as proof of the zealousness of SSRL's users. It has been replaced by a (nearly) new diffractometer which was purchased for use on the PEP $1 \mathrm{~B}$ beam line. The new instrument benefits from the many advances in design since the original diffractometer, and early reports indicate that it is much more accurate than its predecessor. With the new instrument, a total of 16 channels of motor electronics have been installed. The new instrument has a motorized alpha rotation, which should simplify certain alignment tasks.

Scattering experiments which received time on Station 6-2 in the past will be assigned time on $10-2$ or 7-2 instead.

Station 10-2 Upgrades. During the short summer shutdown, several improvements were made to Station $10-2$ by SSRL and the PRT staff. The assembly and installation of the Station 10-2 slit/mirror driver chassis were completed, a project that was actually begun the previous shutdown. The Compumotor drives which used to hang up so frequently are now gone. A new collimator system has also been designed and built for Station 10-2. The new system allows collimators ranging in size from 0.750 to $0.100 \mathrm{~mm}$ to be interchanged with no loss of alignment. After this initial success, even smaller collimators (down to $0.060 \mathrm{~mm}$ ) were built for the system. The smaller $\mathrm{X}$-ray beams are advantageous for diffraction experiments using laser-heated diamondanvil pressure cells. A new image plate holder for 20 $x 40 \mathrm{~cm}$ plates was also assembled and tested on Station 10-2. The holder can more accurately position and align the image plates normal to the incident beam. A hole in the center of the holder allows the transmitted beam to pass through the plate to a Bicron detector that can be used to more rapidly align high pressure cells to the beam.

Station 4-2 Mirror. Subtle mechanical problems have plagued the focusing mirror at Station 4-2 for two years, preventing proper focusing and delaying testing of a new Pt-coated silicon mirror installed in 
1993. During the 1994 shutdown the entire mirror tank was moved from SPEAR to a clean room where exhaustive mechanical and optical tests were performed. Though no obvious problems were discovered, when the mirror was reinstalled on SPEAR it produced an excellent focus.

Station 2-1 to be Redeveloped for Powder Diffraction. A diffractometer has been ordered and received to redevelop Station $2-1$ as a powder diffraction facility. This project is being implemented by SSRL in collaboration with a part-time visiting scientist. This bend magnet station has been mothballed for several years due to lack of staff to provide support.

Monochromator Improvements. The last user run saw the commissioning of Station 4-2 in white light mode (for SAXS). A double-pass Mo-C multilayer monochromator fabricated at the Center for $\mathrm{X}$-ray Optics at LBL was used on Station 10-2 for microcontamination experiments and then on Station 4-2 for SAXS. (For further details see the Biotechnology Developments Section below.) With a

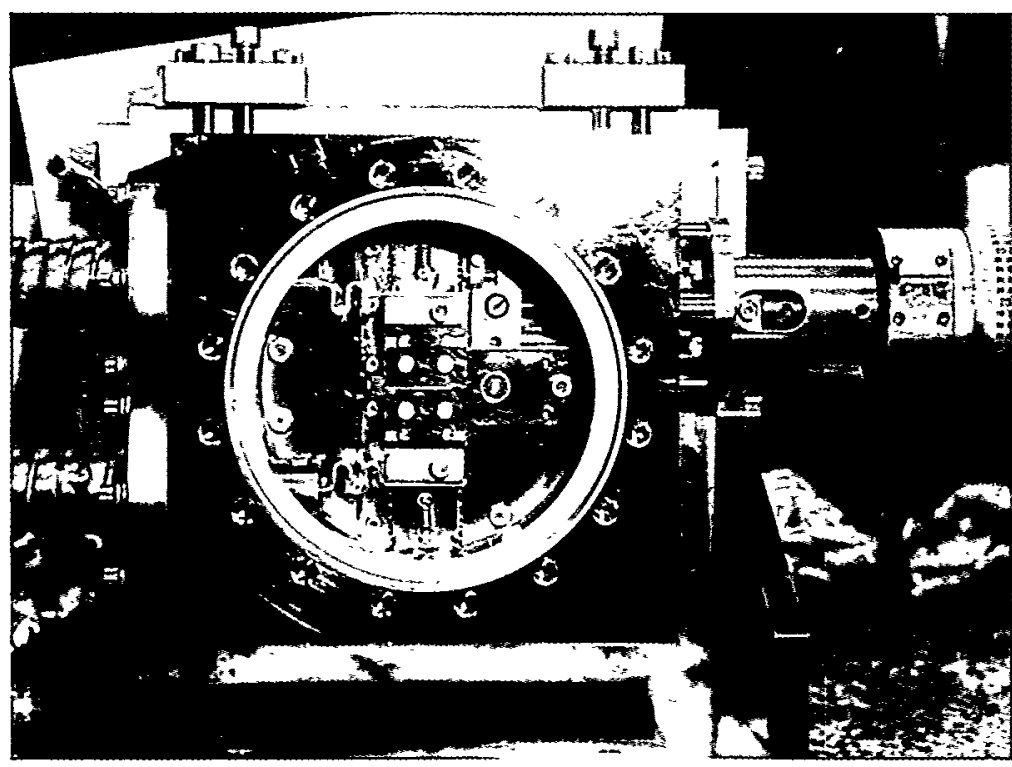

Station 10-1 SGM entrance slit assembly after modification to conductive cooling.

bandpass of approximately $3 \%$ it results in some two orders of magnitude more photons in the experimental hutch. On 10-2 this is $10^{13}$ photons per sec.

The Station 6-2 monochromator coarse motion was improved. A decision was made to remove the motor and gear reducer from the existing goniometer and use an inchworm piezoelectric to push a lever arm mounted to the goniometer platform. This turned out to be a fortunate direction to choose because late in the shutdown the vacuum group removed the cold trap for routine cleaning and observed heavy contamination, determined to be coming from the gear reducer. The inchworm piezoelectric has so far proven to be a very effective way of achieving parallelism between the two diffracting elements.

All of the monochromator motors were upgraded to the damped ferrofluid style. This design was first implemented on Station 7-3 and should reduce the vibration caused by motor steps. A 5-phase microstepping motor is also being tested on the Station 4-1 monochromator. For the first time, the monochromator viewport windows have been removed and baked to remove the color centers which, in some cases, had completely obscured observation of the photon beam.

\section{INSTRUMENTATION DEVELOPMENT}

\section{Performance of the Elliptically Polarizing}

Undulator. An Elliptically Polarizing Undulator (EPU) was installed on the SPEAR Beam Line 5 multiundulator mover in November 1994. The first tests of the EPU satisfied optimistic expectations. The EPU is the first device of its type; it is capable of producing plane polarized light in the vertical and horizontal and rightand left-circularly polarized light in the $500-1000 \mathrm{eV}$ range.

The EPU was developed to provide circularly polarized soft $\mathrm{x}$-rays for a variety of experiments on magnetic and biological materials that exhibit circular dichroism in $x$-ray absorption. These materials are currently of great research interest and frequently contain metals in the first row transition series that absorb at L-edges in the 500-1000 $\mathrm{eV}$ range.

Bending magnet sources of circularly polarized radiation have low flux, require separate optical paths for right- and left-circularly polarized radiation, and are not useful at very high polarization rates. To obtain a higher flux and polarization, various insertion devices have been designed, including several undulators. Of these approaches, the single helical undulator for simplicity and high flux was the most attractive. The result is the elliptically polarizing undulator (EPU). The EPU design was built on a magnet arrangement of Sasaki and the phase tunability 


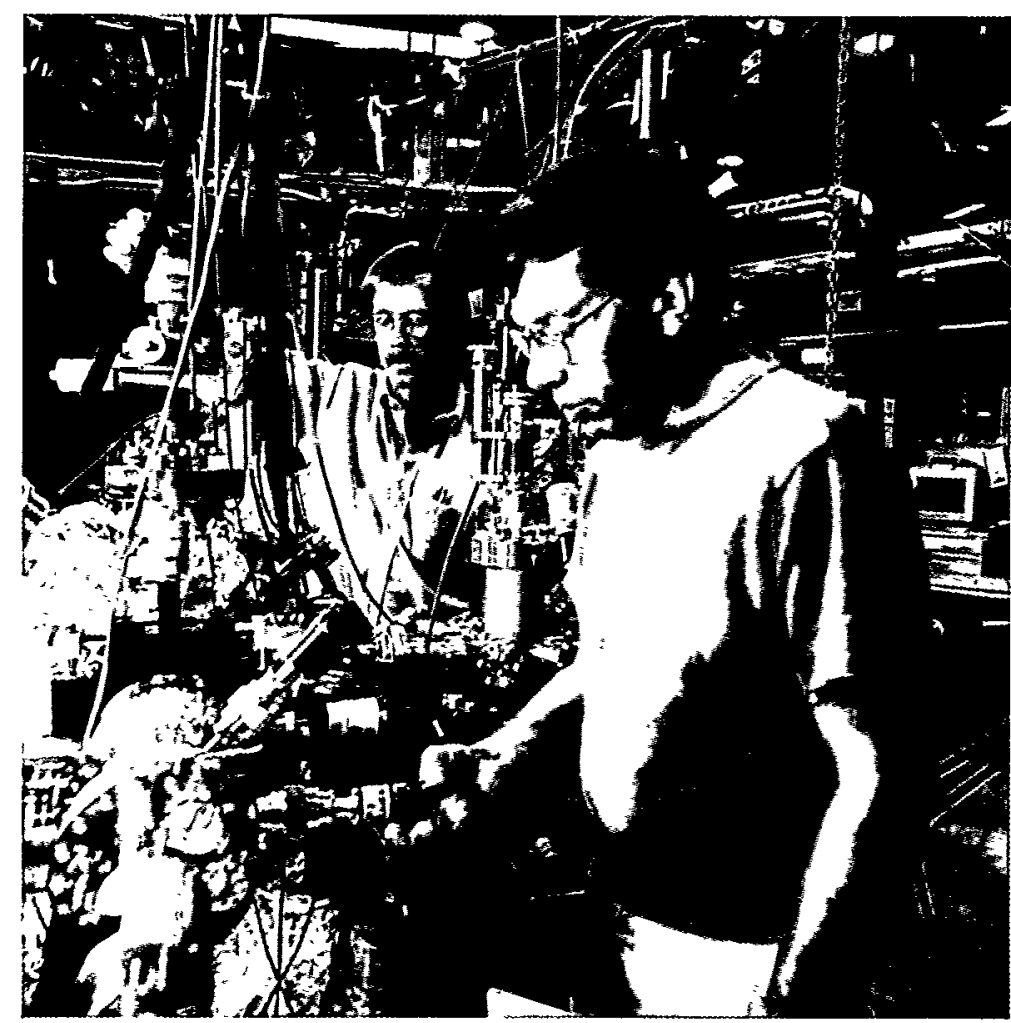

(From left) Jeff Kortright (LBL) and Roger Carr (SSRL) are shown setting up a soft $x$-ray polarimeter that was used to measure the polarization of light from the new SSRL elliptically polarizing undulator. This light was used to demonstrate an extremely large Faraday rotation through thin multilayers of iron and chromium. This rotation of the polarization is caused by a magnetic field and is the largest ever seen for any wavelength of light.

undulator. At the zero of motion, the magnetic field is horizontal, so polarization is vertical.

The EPU phase changes are transparent to SPEAR; neither row, jaw, pair nor uncoordinated phase motions have any noticeable effect on beam position monitors around the ring, and users at other beam lines see no effects. Therefore, it should be possible to move the EPU between right- and left-circular polarization modes at frequent intervals; the mechanical time required is about 5 seconds. The SPEAR operations group regularly allows independent control of the phase motions at the user endstation.

Because of SPEAR's large emittance (129 nm-rad horizontal, 3 nm-rad vertical), the width of the EPU fundamental is much larger than $1 / \mathrm{N}=1 / 26$, and a broad second harmonic peak has been noticed. The second harmonic peak was examined by polarimetry and found to be strongly polarized in the same sense as the fundamental. Beam position monitor measurements showed that the beam did not pass exactly along the EPU axis. The undulator

concept of the adjustable phase undulator. This design, shown in Figure 6, comprises two planes of magnets, one above and one below the storage ring's electron beampipe.

Each plane consists of two rows of pure NdFeB magnet blocks in the Halbach sinusoidal arrangement. Each of the rows is mounted on slides and can be moved longitudinally parallel to the electron beam by $\pm 1 / 2$ the undulator period length, $\lambda$.

The Beam Line 5 multiundulator mover can vary the gap between 30 and $200 \mathrm{~mm}$. The EPU has 26 periods; each period is $65 \mathrm{~mm}$ long and it generates $x$-rays in the $500-1000 \mathrm{eV}$ range when SPEAR is operated at $3 \mathrm{GeV}$. To select a state of circular polarization, the upper left and lower right rows of magnets are moved in one direction, while the other two rows are moved oppositely. With such a 'phase' movement of about $\pm 0.16 \lambda$, depending slightly on the gap, the magnetic field is a circular helix, so the $x$-rays are left or right circularly polarized. At $\pm 0.5 \lambda$ the magnetic field is vertical, and the $x$-rays are plane polarized horizontally, as in a conventional was moved transversely to minimize the energy of the fundamental, but even then, the beam moved at an angle to the axis both vertically and horizontally.

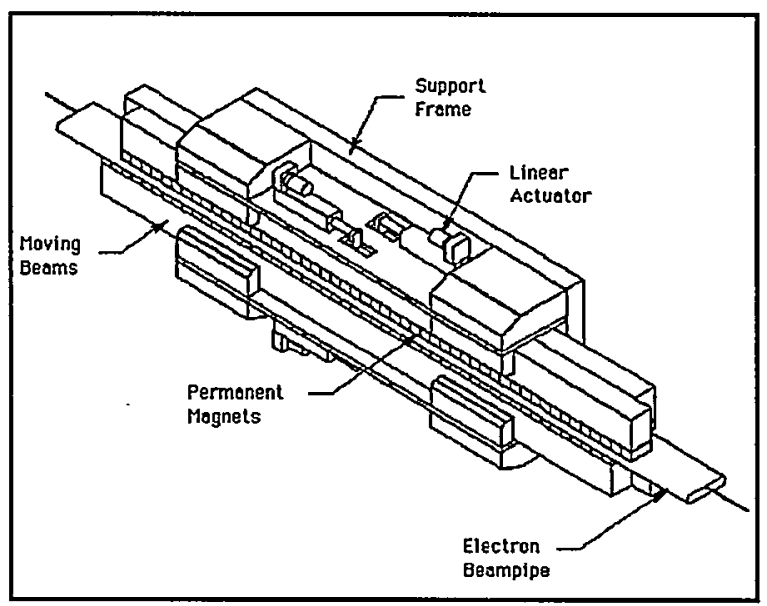

FIGURE 6. Schematic concept drawing of the EPU. The four rows of magnets are movable independently.

This movement degrades polarization only a little. 
FET shunts have since been put on the quadrupole magnets adjacent to the Beam Line 5 insertion region with the intention of being able to steer the beam more accurately by precisely locating the electron beam.

A simple multilayer polarimeter was used to characterize the polarization of EPU beams as the phase was varied. The polarimeter comprised a rotatable linear polarizer in the form of a multilayer reflector set at the Brewster incidence angle, $45^{\circ}$. A sputtered W/ $/ \mathrm{B}_{4} \mathrm{C}$ multilayer with $\mathrm{d}=12.43 \AA$ and 75 periods was used, yielding a Bragg peak at $708 \mathrm{eV}$. The extinction ratio $R_{s} / R_{p} \approx 3 \times 10^{4}$ is quite high, and even though $R_{s} \approx 0.01$, this reflector acts as an excellent linear polarizer at $700 \mathrm{eV}$ considering that roughly 1010 photons/second enter the polarimeter through its entrance pinhole. This is the highest photon energy at which multilayers have been used as polarizers to date, and the results demonstrate that multilayers can be useful for polarimetry at the 3-d transition metal L-edges.

A set of polarimetry scans for a variety of phase settings ranging from vertical linear to circular to horizontal linear polarization is shown in Figure 7. Each scan shows the intensity reflected by the polarizer as it rotates $360^{\circ}$ about the beam direction. Zero on the azimuthal angle scale corresponds to an upward vertical reflection. The scans show the expected polarization behavior ranging from predominantly vertical linear at phase $\approx 0$ to circular at phase $\approx 0.161$.

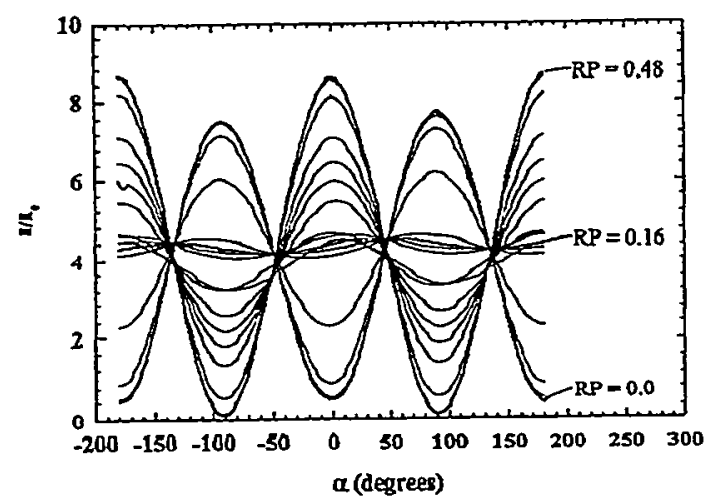

FIGURE 7. Polarimeter scans show strong dependence of polarization state on phase setting. Vertical linear polarization produces maxima at $0^{\circ}$ and $180^{\circ}$, while horizontal linear polarization produces maxima at $\pm 90^{\circ}$. Circular polarization produces the flattest scans.

The polarimetry data were analyzed to determine the Stokes parameters of the beam at each setting. Each scan was fit to obtain values for $S_{0} . S_{1}$, and $S_{2}$ which were normalized by $S_{0}$. Using just a polarizer, $S_{1}$, and $S_{2}$, but not $S_{3}$ can be determined because a distinction cannot be made between the circularly polarized and unpolarized fractions. To set an upper limit on possible unpolarized radiation, the phase value $(0.48 \lambda)$ yielding the highest degree of linear polarization $\mathrm{P}_{\mathrm{L}}=\left(\mathrm{S}_{1}{ }^{2}+\mathrm{S}_{2}{ }^{2}\right) / \mathrm{S}_{0}=0.98$ was identified with the assumption that all remaining intensity was unpolarized radiation. For calculations, the same degree of total polarization $\mathrm{P}=\left(\mathrm{S}_{1}{ }^{2}+\mathrm{S}_{2}{ }^{2}\right.$ $\left.+S_{3}{ }^{2}\right) / S_{0}=0.98$ was used for all phases. Then the value of $S_{3}$ representing circular polarization was calculated from this last expression with $S_{1}$ and $S_{2}$ measured and $\mathrm{P}$ assumed the same for each. When radiation does not come from the peak of the undulator fundamental, it has a linearly polarized and an unpolarized component. The unpolarized component comes from off-axis electrons and offaxis photons. These effects account for the observed unpolarized radiation.

The variation obtained in Stokes parameters with phase is shown in Figure $8 . S_{2}$ represents linear polarization at $\pm 45^{\circ}$ and is negligible for all phases. The largest amount of vertical linear polarization $S_{1}$ was -0.90 at phase $=0$, while the largest amount of horizontal linear polarization was 0.98 at phase $=$ $0.48 \lambda$. The circular polarization shows a broad peak near phase $=0.16 \lambda$. with maximum of $S_{3}=0.98$.

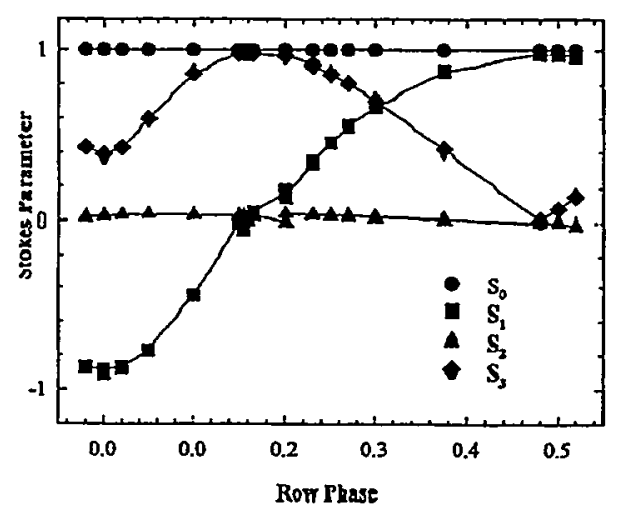

FIGURE 8. Stokes parameters as a function of phase setting, showing the inverse relationship between linear and circular polarization states.

To confirm that both senses of circular polarization are produced at the appropriate phase settings, magnetic circular dichroism data were collected from a $\mathrm{Fe} / \mathrm{Cr}$ multilayer. The multilayer consisted of 30 periods of nominally $30 \AA \mathrm{Fe}$ and $10 \AA \mathrm{Cr}$ deposited by magnetron sputtering onto a silicon wafer substrate. MCD data were obtained using total yield with the sample remnantly magnetized in-plane after magnetizing between the poles of a $1 \mathrm{kG}$ electromagnet and are shown in Figure 9. X-rays 
struck the multilayer at grazing incidence. The distinct $M C D$ signal at the $F e L_{I I}$ and $L_{\text {III }}$ edges shows the opposite helicity of circular polarization for phase $= \pm 0.16 \lambda$, which cannot be verified using the linear polarizer alone.

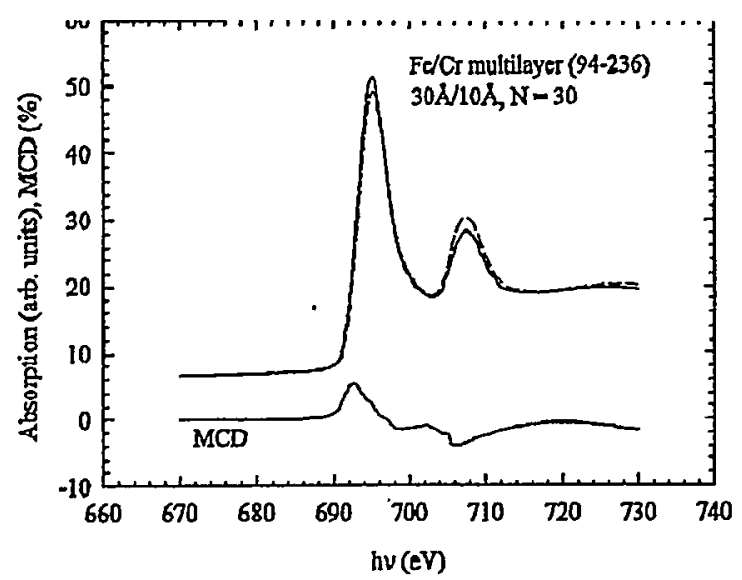

FIGURE 9. Magnetic circular dichroism is observed in a remnantly magnetized $\mathrm{Fe} / \mathrm{Cr}$ multilayer. The top two scans show the total yield spectra taken with $S_{3} \geq$ \pm 0.98 and the bottom curve is the $M C D$ signal, $\left(I_{+}-I_{-}\right) /\left(I_{+}+I_{-}\right)$.

In summary, the EPU was tested and shown to produce negligible interference with the SPEAR electron beam when its rows of magnets were moved. The phase motion was shown to produce linearly and circularly polarized light, as expected from theory. The degree of polarization was almost $100 \%$, in spite of electron beam missteering. The first use of multilayers as polarizers in this energy range has also been demonstrated. The EPU is expected to be a very useful source for magnetic circular dichroism studies.

\section{COMPUTERS}

The SSRL Computer \& Network Group has acquired a DEC model 3000/600 Alpha VMS-based computer system and has installed it on the SSRL network as node SSRL03 for data analysis and other numerical computations needed by staff or beam line users. Many of the computation intensive jobs have been moved from SSRL01 to SSRL03 which has increased the speed of computation by about 30 times.

Two mathematical packages, MATHEMATICA and MATLAB, have been installed on SSRL03. These packages are able to handle tasks involving matrices, numeric, symbolic and graphical calculations and each package incorporates a high-level programming language.
Ethernet hookups have been installed above each beam line to allow users to hook up their own computer to the network. New Ethernet controllers have been installed on beam line computers to allow the use of DECNET phase 5 in the near future.

New printing software (DCPS) has been installed on all the beam lines, bringing queue management up-todate. The operating systems on all the beam lines have been upgraded to version VMS5.5-2 with the exception of the area detector station on Beam Line 1. MicroVAX IIs have been upgraded to MicroVAX IIIs, which has increased the speed of interaction and computation. The memory for all the $x$-ray beam line computers has been increased from $16 \mathrm{MB}$ to $24 \mathrm{MB}$.

The User Support Database has been enhanced to help SSRL staff give users the support they need in a timely fashion. SPEAR-TALK is now available through the World Wide Web (http://wwwssrl.slac.stanford.edu/talk_display.html).

\section{BIOTECHNOLOGY PROJECTS AND DEVELOPMENTS}

The research and development projects in this area at SSRL continue to focus on developing and making available specialized facilities and instrumentation for research in structural molecular biology. These efforts are primarily supported through a Biomedical Research Technology Program Grant from NIH and through the DOE Office of Health and Environmental Research (OHER). During 1994 the NIH grant entered the fifth and last year of its then award cycle. In late 1993, a competitive renewal grant application was written and submitted to the NIH National Center for Research Resources. A site visit was held at SSRL in July 1994, the outcome of which was that the SSRL NIH Resource was funded for an additional 5-year period, through February 2000. The scientific and technological focus for both the NIH- and OHERfunded activities is toward applications of synchrotron radiation research in protein crystallography, small-angle x-ray scattering, and $x$-ray absorption spectroscopy. About $1 / 3$ of the experimental activity at SSRL typically falls into the "bio" category.

\section{PROTEIN CRYSTALLOGRAPHY}

The Rotation Camera Facility on Station 7-1. This station continues to be in very high overdemand. Several important structures were reported during 1994 using data collected at this station, including the complex between human growth hormone and the prolactin receptor, a 
most time-consuming tasks during the alignment of the Laue camera has been the precise manual alignment of the final $x$-ray collimator which has very small apertures. To overcome this problem, an automated collimator positioning mechanism was installed on the camera during the June 1994 Laue run.

In June 1994 an experiment was conducted that required the use of a class 4 laser that was brought to SSRL by collaborators. The necessary personnel protection systems and procedures that were required for this experiment to be accomplished safely were fabricated and installed, including a secondary laser containment door with safety interlocks, warning lights, and signs.

A suite of programs for processing Laue diffraction images has been installed on the DEC Alpha $3000 / 300$. The new program LAUEGEN, which incorporates several of the older pre-integration programs included in the package, has been installed and used to index Laue diffraction patterns, refine data collection parameters and generate predicted positions of diffraction spots. This program is Xwindow based (the graphics portion was written by J. Cambell at Daresbury) and greatly simplifies data processing.

\section{X-RAY ABSORPTION SPECTROSCOPY}

In the area of x-ray absorption spectroscopy, SSRL provides two 13-element Ge solid-state detector arrays and associated electronics, three liquid $\mathrm{He}$ cryostats for studies down to $4 \mathrm{~K}$, four remotely controlled optical alignment rails, four ionizationtype fluorescence detectors, and a large amount of peripheral equipment. All of this equipment is available for use on several experimental stations and much of it is thus of "ambulatory" type.

For the now permanently XAS-dedicated station 7-3, the major fraction of time $(85 \%)$ was scheduled for SMB research during the two 1994 runs. The permanent setup includes a motorized optical alignment rail, a remotely controlled in-hutch shutter system for protecting the sample from radiation during beam alignment, a liquid He cryostat, and a 13-element solid-state Ge detector array.

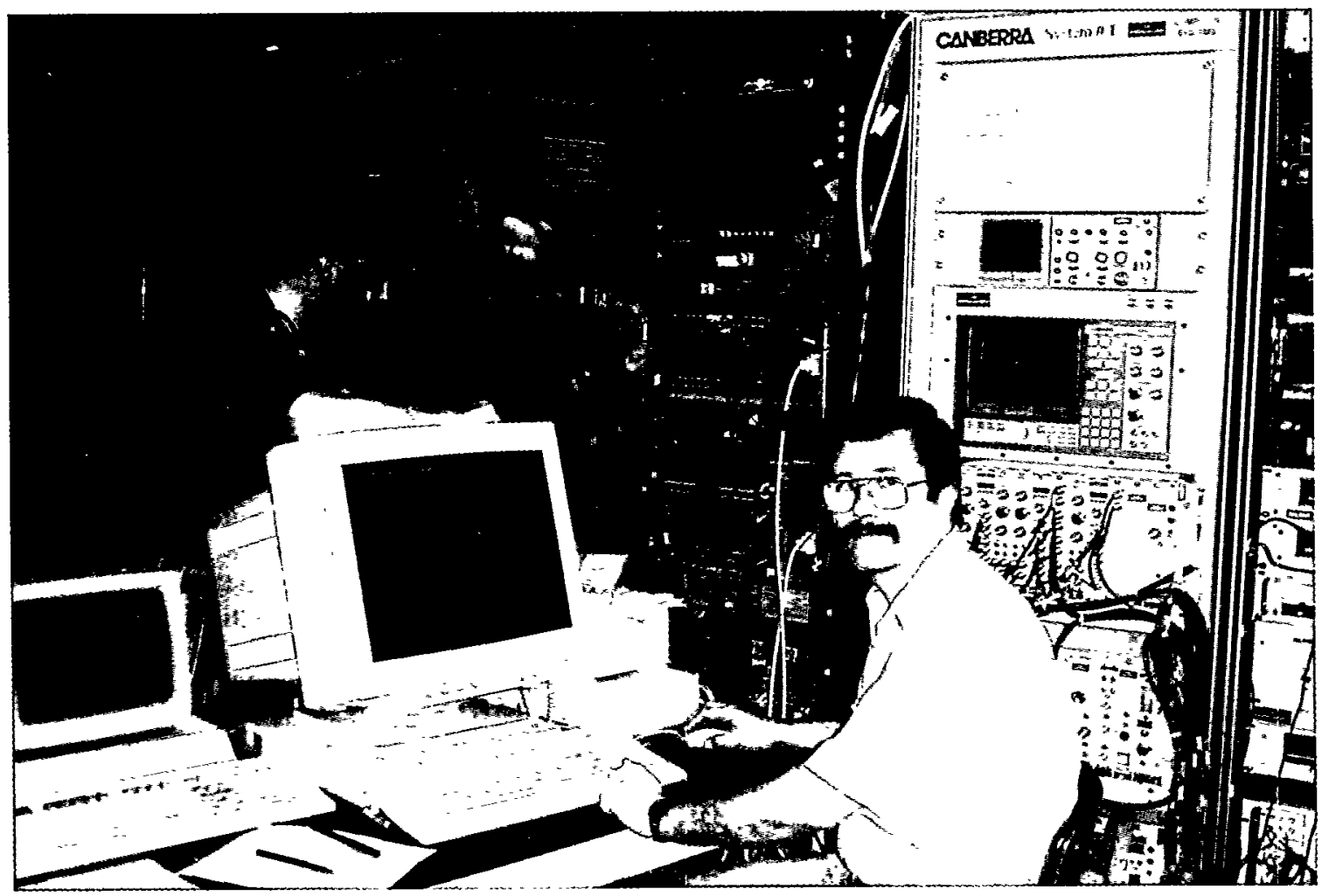

National User Facilities provide a unique opportunity for development of advanced instrumentation that can be ultimately shared among a large number of users in a very cost-effective manner. In the above photograph, scientists (from left) Bradley Patt, Jan Iwanczyk and Ryszard Szczebiot from the Xsirius Corporation in Camarillo are working at SSRL to test an $\mathrm{HgI}_{2}$ array detector for $x$-ray absorption spectroscopy. This work, supported by NIH research grants and SBIRs, is collaborative with scientists at SSRL. The advanced detector derived from this work will soon be made available to users studying problems ranging from dilute metallo-proteins to detection of trace impurities in contaminated soils. 
Array Detector Development. Development and improvement of advanced detectors for XAS continues to be of high priority. Significant progress was made during 1994 in the development of a large, next-generation multielement array detector (by Xsirius, Inc., in collaboration with SSRL). A 75element $\mathrm{HgI}_{2}$ detector system (based on three 25element modules, the prototype of which was tested at SSRL twice during 1993) was brought to SSRL in July 1994 for integration with SSRL-developed electronics control and data acquisition software. This was followed by testing of the detector system with increasingly high beam intensity and real data collection on a dilute metalloprotein. The $\mathrm{HgI}_{2}$-based system offers the advantage of non-cryogenically cooled detector elements that can be closely packed in large numbers giving a significant increase in total concentrate.

A major part of this detector effort has been the development of miniaturized, computer-controllable electronics. The advantages with this development are that it could be tailored to the characteristics of the $\mathrm{HgI}_{2}$ detector elements and it would drastically reduce the number of electronics modules needed (as 2-3 complete channels of amplifier, pile-up rejector, quad single channel analyzer, multiplexors could be housed on a single CAMAC board). For the 25element prototype, these electronics were implemented in hybridized technology. This approach had to be changed due to cost considerations, however, and for the 75-element system, the remaining electronics were realized using surface-mount technology, using the same inherent electronics design. Software for controlling the electronics from a VAXstation (setting gain levels, amplifier settings, SCA windows, etc.) was further developed in 1994 to allow for automatic calibration of the window setting and was implemented with a new type of CAMAC controller (see below). XAS data were collected with the 75-element detector on the dedicated XAS station 7-3 on a $\sim 1 \mathrm{mM}$ frozen solution of a Cu-cluster containing protein and a comparison made with data collected on a similar sample on a 13-element Ge detector array. Early indications are that the signal-to-noise ratio is improved using the $\mathrm{HgI}_{2}$ detector system. The complete 100-element system is now in final assembly and test at Xsirius, and the delivery date is at present targeted for spring of 1995.

Upgrade of the 13-element Ge Detector Systems. Planning for and investigations into how to incrementally improve the performance of the two existing 13-element $\mathrm{Ge}$ array detector systems have continued, and SSRL staff have been frequently in contact with the R\&D staff at the vendor, Canberra Instruments. In late spring 1994, it became clear that their development of a new low-noise, lowcapacitance pre-amplifier (based on Interfet technology) which when coupled with faster processing electronics appears capable of increasing throughput by a factor pf 3-10 (depending on the amplifier shaping time) had advanced to the point that an upgrade would be technically sound. Therefore in the early fall of 1994 one of the Ge systems was returned to Canberra for total rework (new elements, preamplifiers, amplifiers, cable harness, cryostat dewar, ion pump system, etc.). This system was delivered to SSRL in early 1995.

Software Development and Hardware Test of New CAMAC Controller for XAS and Other SSRL Stations. Both the implementation of a new VAXstation $4000 / 90$ as a replacement for the XAS station 7-3 VAXstation III and the test and use of the 100-element $\mathrm{HgI}_{2}$ detector array required that a replacement for the Q-bus interface used on the VAXstation III needed to be identified. A Kinetics Systems 3968 controller that uses Ethernet as a replacement for Q-bus and which physically replaces the DSP CC-LSI controllers typically used at SSRL was tested and found to match the necessary requirements. Software was furthermore developed to allow this controller to be used with other existing SSRL hardware and software. It was successfully used in the $\mathrm{HgI}_{2}$ tests, and the software is currently undergoing additional modifications and enhancements to enable final replacement on Station 7-3.

$X A S$ Data Collection Software Improvements. A new data collection package designed specifically for EXAFS was developed. The new software is built around low-level X-windows graphics and uses standard $C$ programming techniques. The window-driven software follows the outline and steps of an EXAFS experiment. Input from many of the users at SSRL was incorporated. The new software underwent initial testing and realtime debugging on one station during the first two months of the 1994 run. It was then implemented on all XAS stations at SSRL. It has been very well received by the user community and has become the standard, supported XAS software at SSRL. During the 1994 fall shutdown it was further enhanced, in particular in regard to plotting capabilities, and the code was also partly rewritten to increase the speed of execution. Simple post-processing (quick averaging, background and spline removal, simple Fourier transforms) was also added to enable "on-thefly" rough inspection of the data quality. A first version of a user manual was written and distributed to users. 


\section{SMALL-ANGLE X-RAY SCATTERING}

Two major technological developments of the SAXS facility in the 1994 run aimed at higher beam flux suitable for time-resolved studies were successfully implemented and tested: i) implemention of a pair of synthetic multilayers in the monochromator and ii) use of a white $\mathrm{x}$-ray beam.

Multilayer Monochromator. A matching pair of synthetic Mo-C multilayers, obtained from LBL, was installed in the Station 4-2 monochromator. Preliminary characterization revealed that the energy bandpass of the multilayer was approximately 200 $\mathrm{eV}$ (FWHM at $10.5 \mathrm{keV}$ ), which is considerably wider than that from a $\mathrm{Si}$ double-crystal monochromator. The pair of multilayers provided 10-20 times higher measured flux at $9.0 \mathrm{keV}$, depending on actual beam size used, than the $\mathrm{Si}(111)$ double-crystal monochromator in a comparable station configuration for solution small-angle scattering. The energy bandpass does not smear solution scattering patterns at angles corresponding up to $30 \AA$ in real space. A collagen fiber diffraction pattern recorded with the multilayer beam superimposes very well on the monochromatic data and showed very little broadening of the longitudinal diffraction peaks. This indicates that the wider energy bandpass of the multilayers does not worsen data quality even under the most stringent experimental conditions expected for biological small-angle scattering studies.

The multilayer monochromator considerably facilitates time-resolved studies. The higher flux also benefits static experiments, i.e., shorter data collection with more samples to run. The users of the 4-2 SAXS facility now have the choice of the $\mathrm{Si}$ crystal monochromator or the multilayer monochromator, depending on their needs for flux and energy bandpass.

Use of White X-ray Beam in Small-Angle Scattering. The second major technological development is the use of quasi-white beam, which is produced without a monochromator, in smallangle scattering. The main purpose of this development is to monitor fast structural events in protein folding, such as the formation of molten globule states, which often take place with a half life on the order of milliseconds. The use of a wide energy bandpass (a few hundred $\mathrm{eV}$ ) of the quasiwhite beam increases the total number of photons available to the experiments, much the same way as the use of multilayers does but with an even greater result. The actual energy profile is determined by the source characteristics of the synchrotron, transmis- sion spectra of the window/filter materials in the beam, and the mirror energy cutoff.

The first systematic white beam data collection of protein solution scattering was performed on Station 4-2 with a Pt-coated focusing mirror (to suppress higher harmonics; energy cutoff $\sim 11 \mathrm{keV}$ ) and aluminum filters, which cut off the lower side of the energy spectrum. The resulting energy bandpass with a $0.25-0.5 \mathrm{~mm}$ aluminum filter was approximately $2000 \mathrm{eV}$. The flux on the sample was too high to be effectively measured by an ionization chamber, and a scintillation counter (which monitors a small fraction of the beam scattered by a short air gap in front of sample) was incorporated in the camera system to measure incident white beam intensity. The count rate on the linear detector was more than two orders of magnitude higher, which is the regime where the detector begins to suffer significant dead time losses.

Proteins of different sizes were measured: lysozyme, calmodulin, bovine serum albumin, the trimeric form of the aspartate transcarbamylase (ATCase) catalytic subunit and ATCase holoenzyme. The values of radius of gyration measured with the quasi-white beam are very consistent when one of the values is taken as a known standard. The experiments showed that broad band "white light" gives satisfactory measurements of radius of gyration in practice, as was expected on theoretical grounds. Even some of the shape features could be extracted from the data taken at a rate two orders of magnitude faster than that possible with a monochromatic setup. This is believed to be the first practical test of this principle.

Characterization of the ANL 9-Element "Gold" CCD Detector and Acquisition of the Single-Element "Olive" CCD Detector. The 9-element CCD detector, primarily designed for protein crystallography by the group lead by $\mathrm{E}$. Westbrook and described above under "Protein Crystallography", was also tested/characterized in a small-angle x-ray scattering setup on Station 4-2. It appeared that the high spatial resolution and the twodimensional images produced by the detector offer advantages in small-angle $x$-ray scattering, although the read-out time in the current version was not fast enough for time-resolved studies. Due to the primary objectives of this particular detector development, the present level of dark current of the detector may not be low enough for small-angle scattering studies of protein solutions.

SSRL has recently obtained a single-element version of the CCD detector based on the same design principle. It will be primarily used at Station 1-4, the bending magnet small-angle scattering station 


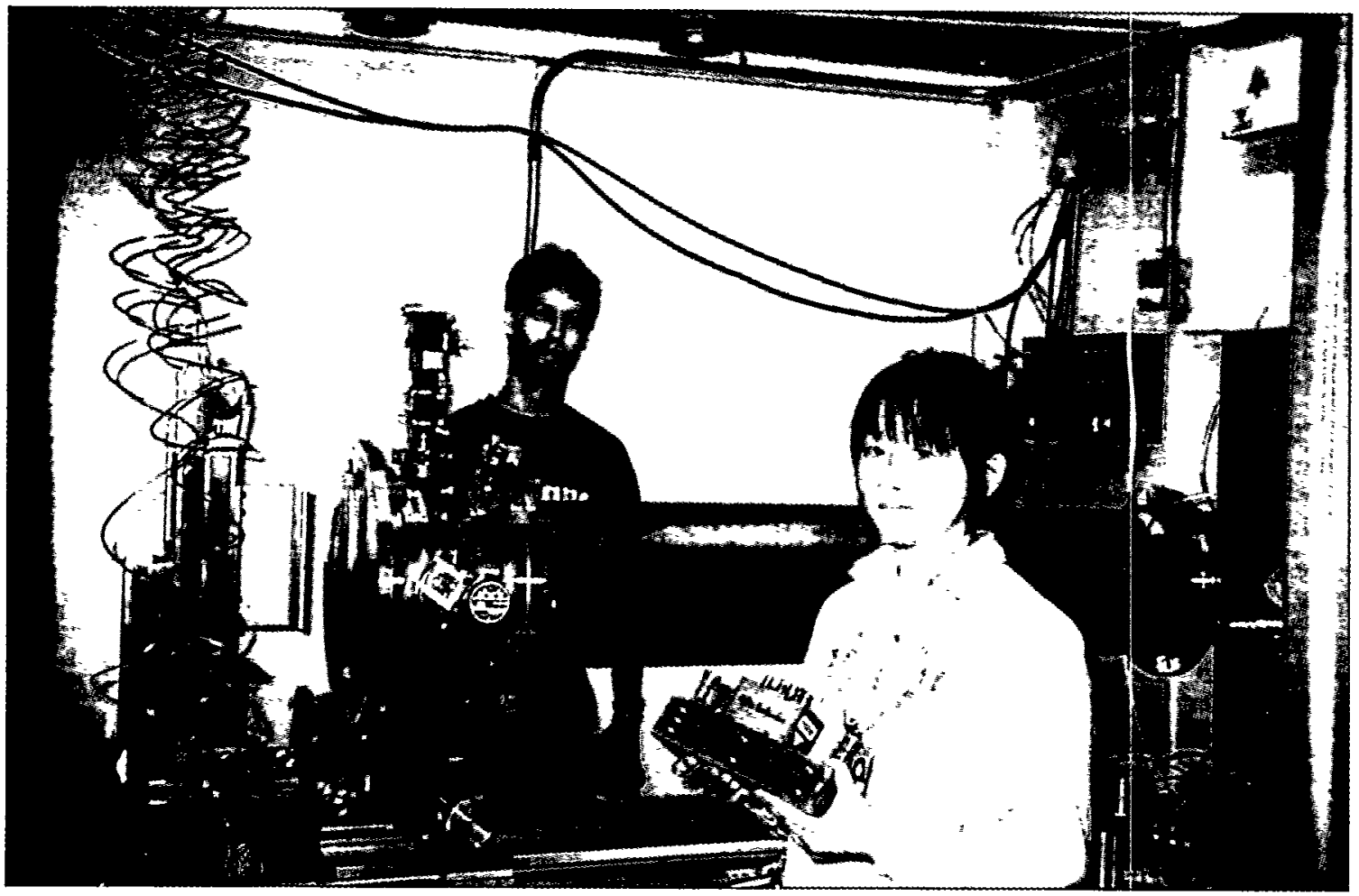

Graduate students Dan Segel and Lingling Chen (holding a linear position sensitive detector) at the SAS camera on Station 4-2.

whose users are mostly polymer scientists, but will be available on special request on other stations, including the SAXS facility on Station 4-2.

A beam profile monitor which combines a CCD digital camera and a phosphor screen was constructed. This monitor will be used when optimizing the focusing of the beam and when recording and diagnosing the size/divergence of the beam. The CCD digital camera, together with a zoom lens, can also be used to check operations of remotely controlled devices in the experimental hutch while the beam is still on.

Finally, adding to the existing data collection/analysis package at the SAXS facility, a powerful indirect Fourier transform data analysis program GNOM (developed by D. Svergun, EMBL-Hamburg) was installed and made available to the general users.

\section{BIOCHEMICAL SAMPLE PREPARATION LABORATORY}

The Building 120 extension that will accommodate Beam Line 9, user setup and workspace areas was completed in 1994. As part of this extension, two new OHER-funded sample preparation laboratories were built, one for users and a smaller one for the SSRL NIH Biotechnology staff. The user laboratory includes a walk-in coldroom, the second at SSRL.
These laboratories were made ready for use in late 1994 with final installation of laboratory furniture, fume hoods and purchase of basic laboratory equipment. Some redistribution of instrumentation between the two user labs (Bldgs. 131 and 120) also took place. Although some further equipment is still needed, the laboratories were ready for use by the December 1994 run.

\section{HIGH PERFORMANCE COMPUTING AND GRAPHICS FACILITIES}

With the completion of the Building 120 extension, rooms were provided upstairs for the DEC Alpha $3000 / 500$ workstations, including the 3-D graphics hardware which was acquired in 1993 via a DEC grant to SSRL and matching funds from the SSRL Biotechnology Resource. A separate room was built for the off-line BAS-2000 imaging plate scanner. These facilities were opened for users in the beginning of the December 1994 run.

\section{STRUCTURAL MOLECULAR BIOLOGY BEAM LINE 9}

Significant progress was made on Beam Line 9 during 1994. A SPEAR girder chamber of a new design with a removable crotch was successfully fabricated. During the summer/fall SPEAR shutdown period the 
exit chamber and the high power beam line front end components were installed. The modifications had no adverse affect on subsequent SPEAR operation.

The wiggler was not installed during the 1994 window due to delays in fabrication. The wiggler foundation, however, was installed and changes were made in SPEAR to ready it to receive the wiggler. In addition, a method was devised for opening the shielding for installation without having to remove the SPEAR roof. This should make the installation simpler during the 1995 summer shutdown.

The wiggler is now complete and has exceeded the specified 1.9 Tesla peak magnetic field. It delivers $2.04 \mathrm{~T}$ peak at the specified minimum gap of $21 \mathrm{~mm}$. This greatly enhances the hard $\mathrm{x}$-ray output of the beam line by raising the critical energy to $12.2 \mathrm{keV}$ at the nominal $3 \mathrm{GeV}$ SPEAR electron energy. The wiggler vacuum chamber, another design first for SSRL, was successfully fabricated and is ready for installation.

As a result of a continuing increase in the demand for beam time on SSRL's existing rotation camera station (7-1) and recommendations by a number of advisory and funding committees, SSRL made the decision in 1994 to build a second rotation camera facility on one of the Beam Line 9 side stations (2-1).

Originally, the three branch lines that were planned for this beam line were: i) an end station for protein crystallography (for MAD phasing studies, highresolution monochromatic data collection, and white radiation Laue diffraction); ii) a side station for XAS; and iii) a side station for SAXS. The decision to develop Station 9-1 for protein crystallography, rather than for SAXS, was influenced by the recent multilayer monochromator development that has significantly increased the beam flux on the existing semi-dedicated station (4-2) for SAXS. Plans have now been made to further enhance Station 4-2 for SAXS while developing the new branch line Station 9-1 for protein crystallography.

The new rotation camera facility is planned to be functionally very similar to the existing rotation camera station and will be of even higher $\mathrm{x}$-ray brightness. It will be run for extended periods at a fixed $\mathrm{x}$-ray wavelength of $0.98 \AA$, which is the Se K absorption edge. The feasibility of incorporating multiple monochromator crystals and other instrumentation to enable the occasional tuning of the facility to other $\mathrm{X}$-ray wavelengths in the range $0.8 \AA$ to $1.75 \AA$ is also being looked into. This would permit data collection (at a single $x$-ray wavelength) at absorption edges of a number of commonly occurring elements found in proteins and at the edges of many of the commonly used heavy atom derivatives. Although the $\mathrm{x}$-ray bandwidth of the beam line will be fairly broad, some degree of anomalous scattering will be possible. It is anticipated that the station will be equipped with a CCD detector system.

The optics configuration for Beam Line 9 is defined. Much of the hardware has been designed and is in fabrication. The first optical elements for $9-2 / 3$ are vertically collimating mirrors while the 9-1 mirror focuses vertically with $\sim 2: 1$ demagnification. The tight figure requirements for the collimating mirrors require that they be actively cooled. Three such mirrors are on order from the Rocketdyne Division of Rockwell International, with a promised November 1995 delivery. The Beam Line 9 project team is designing the holder/benders for these mirrors.

In order to make use of the high flux from the wiggler, it will be necessary to cool the first monochromator crystal in all of the branch lines. The Beam Line 9 team is working on a double-crystal monochromator design which will be capable of accepting either a pinpost crystal, presently being developed by Rocketdyne in collaboration with SSRL staff scientists, or possibly other cooling schemes. The design incorporates two pairs of crystals, either of which can be positioned in the beam without entering the monochromator enclosure. This should speed up the crystal change process and possibly permit changes without support staff intervention.

Refocusing mirrors will follow the monochromators in 9-2/3, producing a $\sim 1: 1$ vertical and $\sim 2: 1$ demagnified horizontal image of the source at the experiment. Station 9-1 will use a curved crystal monochromator to focus the $\mathrm{x}$-rays horizontally with 5:1 demagnification at the sample. Design of this monochromator is at an early stage.

Design and fabrication of the control system is progressing around EPICS, which is a client-server based data acquisition system jointly developed at a number of DOE laboratories. This system has been chosen for implementation on beam lines at the Advanced Photon Source. The program set is being ported to run on Alpha processors for Beam Line 9.

The EPICS system will control the optics and other hardware on Beam Line 9. One aim is to provide computer-assisted alignment and setup functions which should optimize the productivity of the beam line and minimize support staff workload.

Interfaces are being designed to allow existing data collection programs to use the facilities provided by the hardware, and new applications will be developed 
which will use the native EPICS facilities.

The present plan is to install the entire in-alcove transport system in the summer/fall 1995 SPEAR shutdown and to complete test and commissioning in the winter/spring SPEAR running cycle. Supports will be installed during the April 1995 shutdown.

\section{ENVIRONMENTAL MOLECULAR SCIENCE BEAM LINE 11}

The initial funding for a new beam line to enable the study of environmentally relevant materials was received in 1994. Studying such samples is very challenging because of the often very dilute nature of the contaminants and their presence in matrices that are often highly absorbing. This combination of sample characteristics typically results in low signalto-noise data or, in the most dilute cases (when the metal contaminant is at ppb or lower levels), little signal at all.

The overall goal of the Beam Line 11 project is to develop a state-of-the-art facility which enables signi ficantly improved sensitivity of these measurements. This will be enabled by improvements in beam line optics and instrumentation (in particular detectors) coupled with an extremely high x-ray flux to be obtained from a high-field (2 Tesla), multipole (2426) wiggler whose detailed design is currently underway. The engineering and design of Beam Line 11 will utilize experience and design of components from Beam Line 9. Eventually, an associated laboratory facility specifically for the study of dilute, hazardous environmental samples is being planned to be located adjacent to the beam line. This facility will, for example, permit the safe handling of materials containing actinides.

A management structure has been developed, including a user advisory group chaired by $\mathrm{N}$. Edelstein of LBL, for the project and a design group assembled to begin to define the optics for this new high-powered wiggler beam line for environmental molecular science.

The design of three SPEAR alcoves, one of which will be for Beam Line 11, and a 4440-square-foot extension to Building 131 is underway.

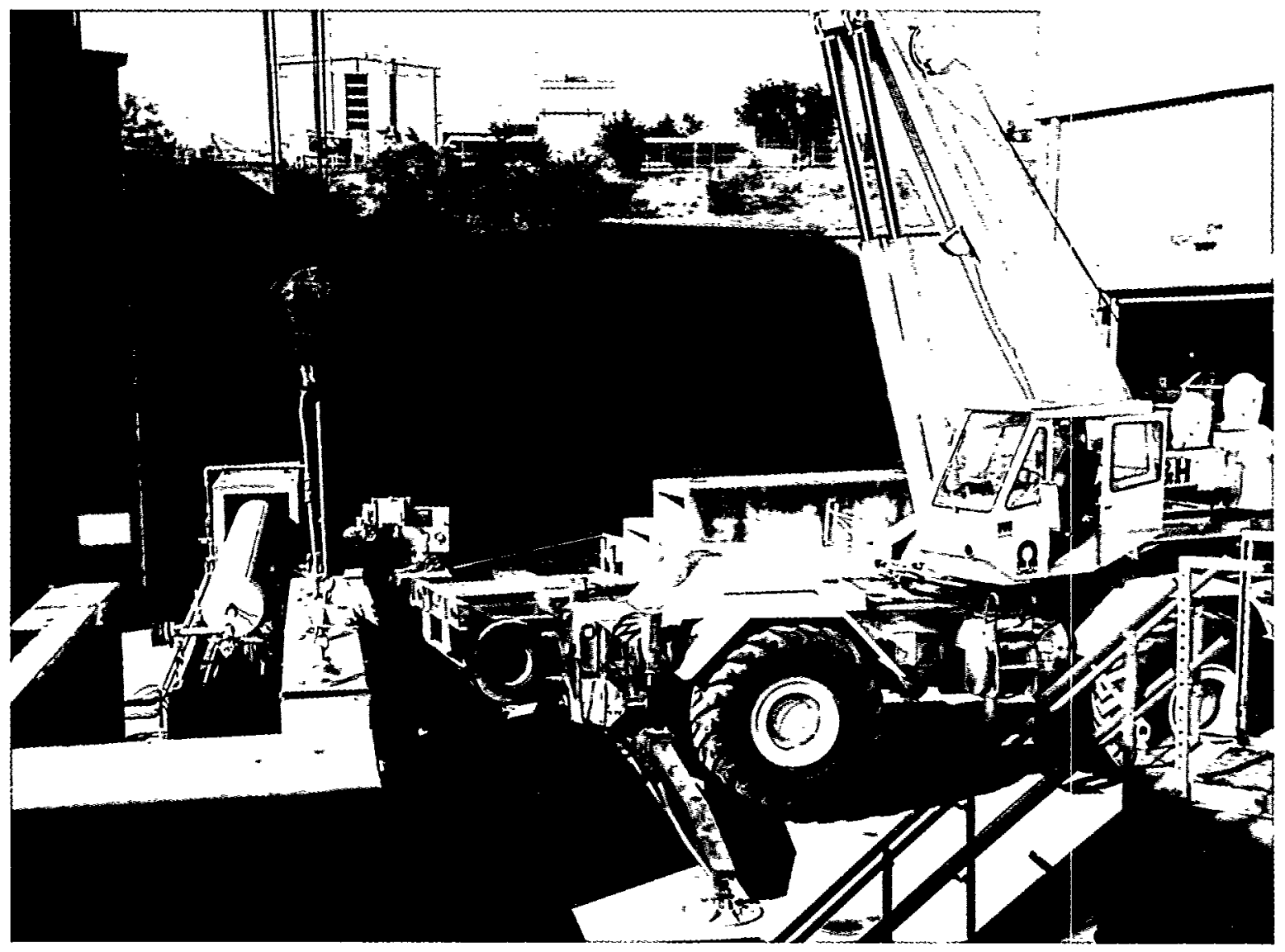

Removing shielding from around Beam Line 17 to facilitate the Building 131 extension which will house three alcoves for new beam lines. 


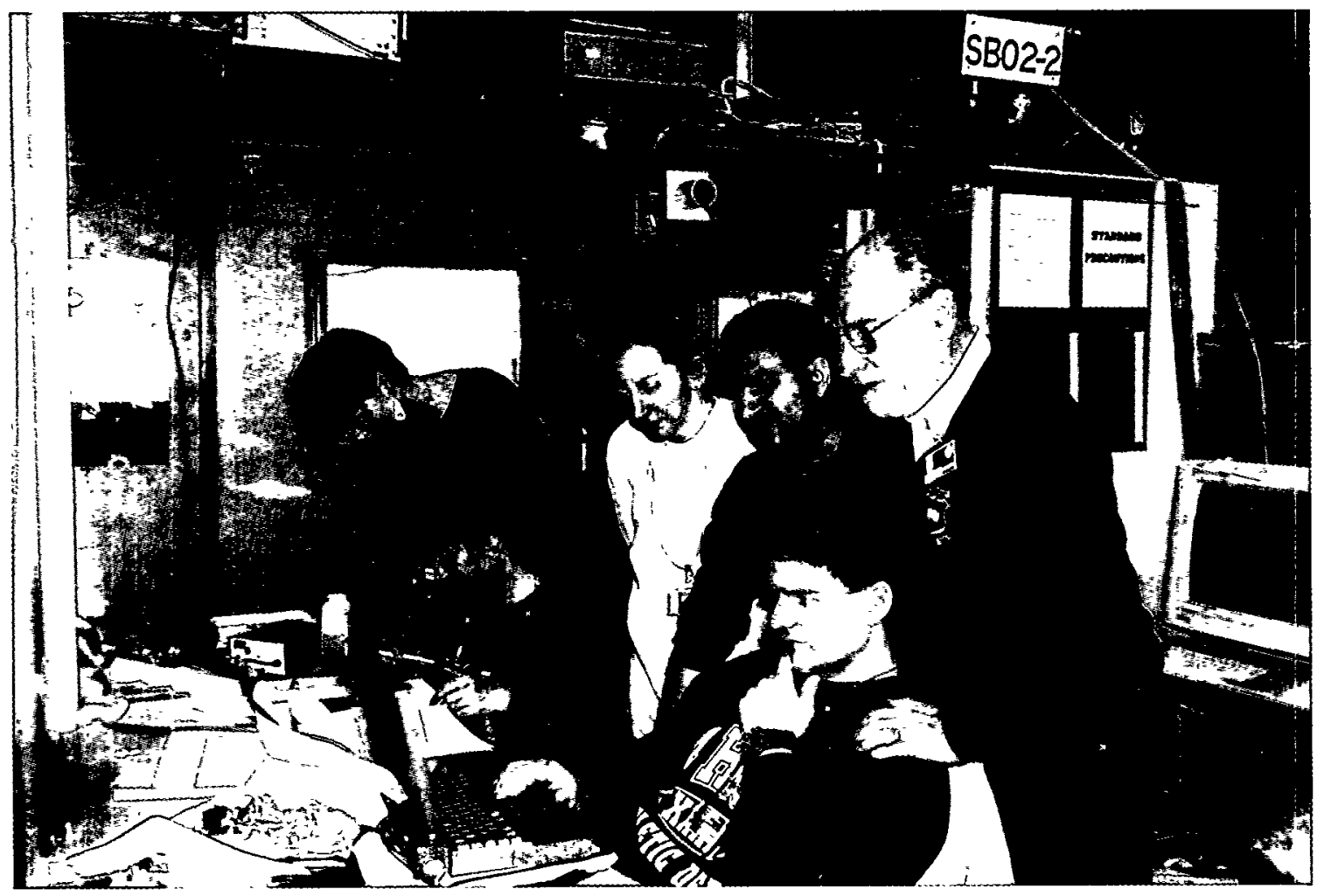

A user group from the University of Michigan working on Station 2-2. (Standing from left) Laraba Parfitt and Sandra G. Malhotra, grad students, Ashwani Malhotra, post-doc associate, and Professor John C. Bilello. (Sitting from left) Zhen Zeng and Oleh Karpenko, grad students.

(From left) Larry Cox and Steve Conradson shown taking data on Station 4-2 for the Los Alamos National Laboratory actinide experiment.

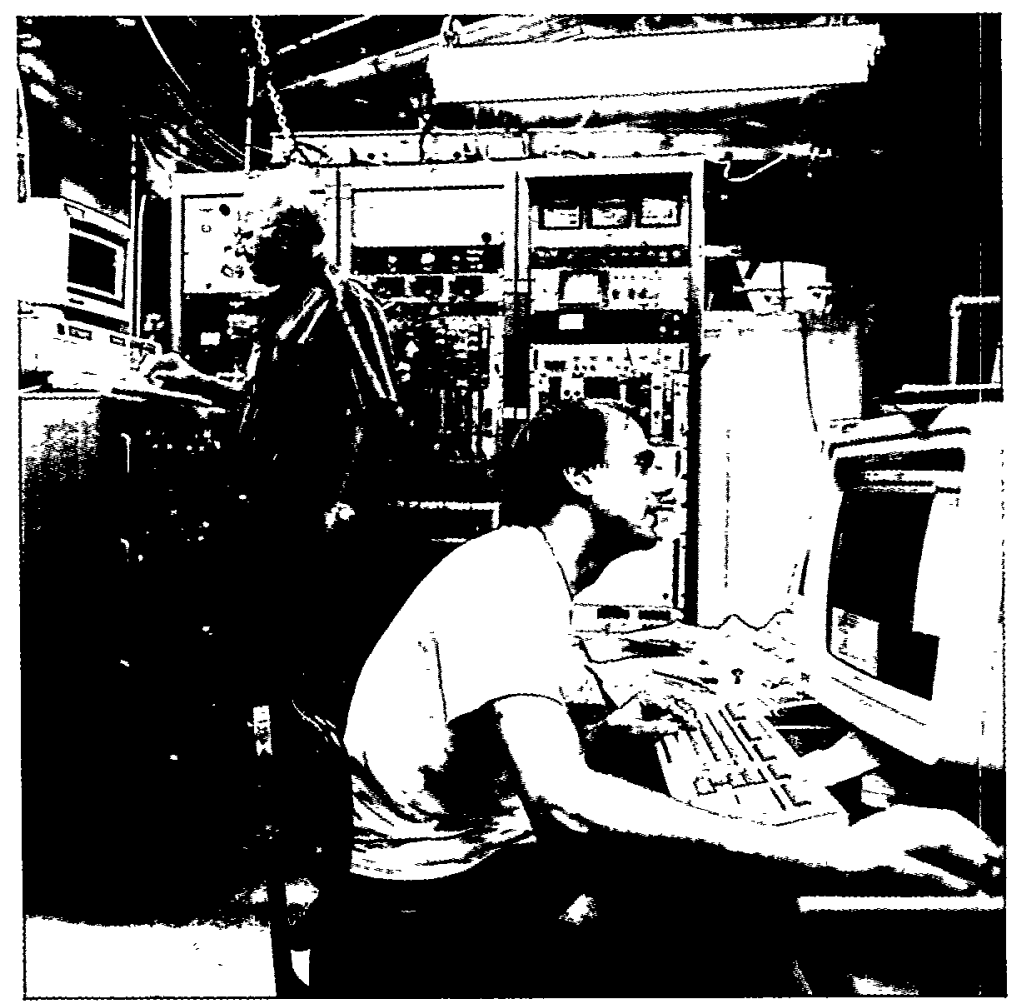




\section{WORKSHOPS AND MEETINGS}

\section{ST ANNUAL USERS MEETING}

The 21st Annual Stanford Synchrotron Radiation Laboratory Users Meeting saw many exciting new results presented by researchers from across the United States. The meeting, held October 17 and 18, 1994, was attended by 133 people. The program, put together by chairpersons L. Terminello ( $L L N L)$, I. Pickering (SSRL) and G. George (SSRL), was divided into six sessions. The four scientific sessions reflected strong areas of research at SSRL: Structural Molecular Biology; Environmental Science; General Materials; and Surface and Reduced Dimensional Studies. Two other sessions focused on facility developments, including the new structural molecular biology Beam Line 9, the new environmental molecular science Beam Line 11, the elliptically polarized undulator, and the possibility of an Angstrom free-electron laser.

The scientific sessions were particularly varied and included presentations on small-angle scattering, protein crystallography, several applications of $x$-ray absorption spectroscopy, anomalous dispersion, diffraction imaging, $x$-ray fluorescence trace element analysis, $\mathrm{x}$-ray magnetic circular dichroism, photoemission, standing wave studies, and nuclear resonance scattering.

The excellent operation of the SPEAR storage ring over the past year was highlighted in the accelerator physics presentation.

As always, the SSRL User Meeting had a vibrant poster session that showcased the efforts of many users of SSRL during the previous year. In particular, these poster sessions are an excellent forum for the many students who do their doctoral thesis research at SSRL. This year, the organizers inaugurated a prize for the best student poster, and the many outstanding contributions made the selection of a single prize winner very difficult. After much deliberation, the award was presented to M. Peterson of Stanford University who submitted a poster on the "Direct Evidence for Heterogeneous Redox at the Aqueous Chromium/Magnetite Interface".

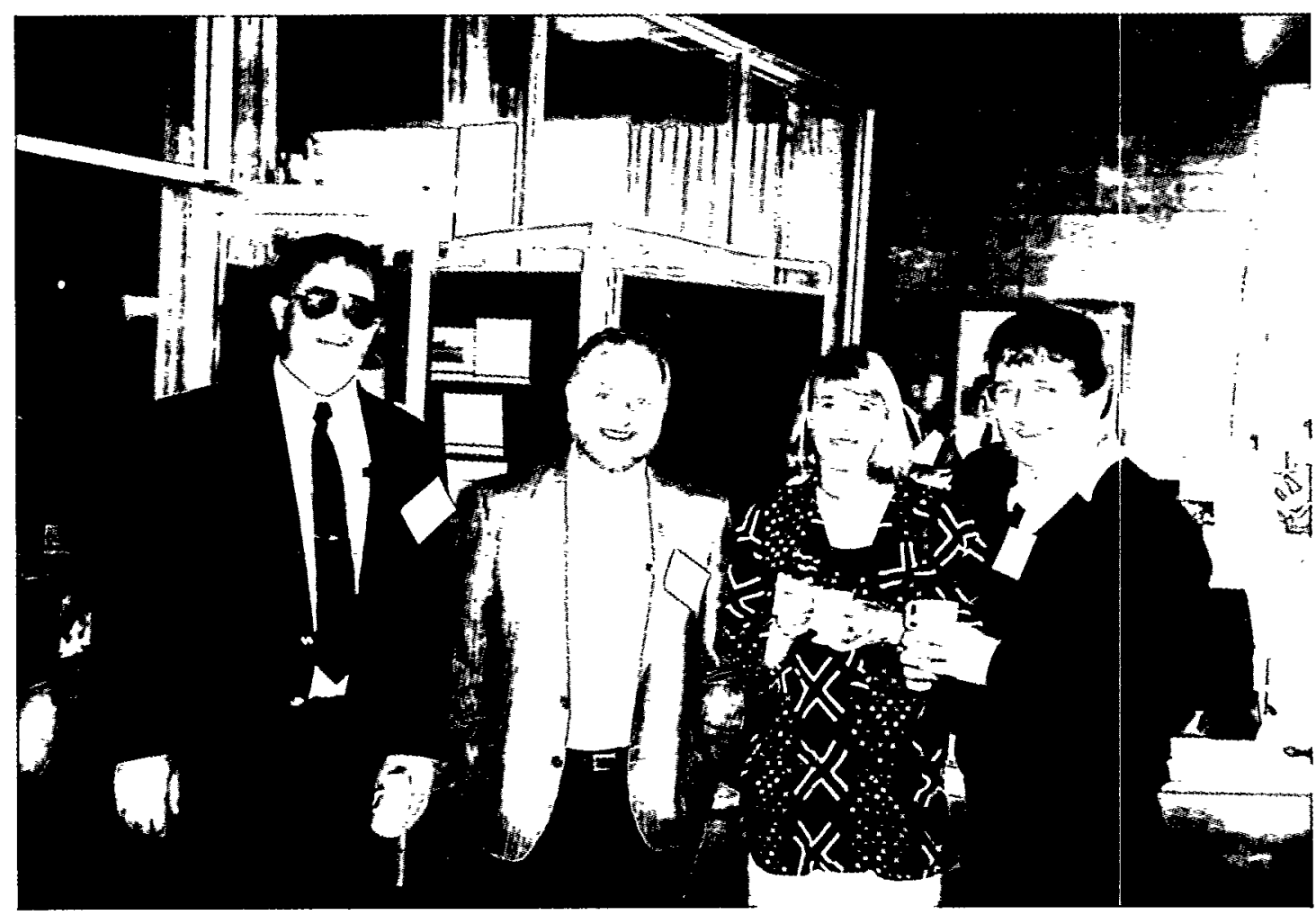

(From left) James Tobin (LLNL), Neville Smith (ALS), Katherine Cantwell (SSRL), and Paul Fuoss (AT\&T Bell Laboratories) - SSRL Users Meeting. 


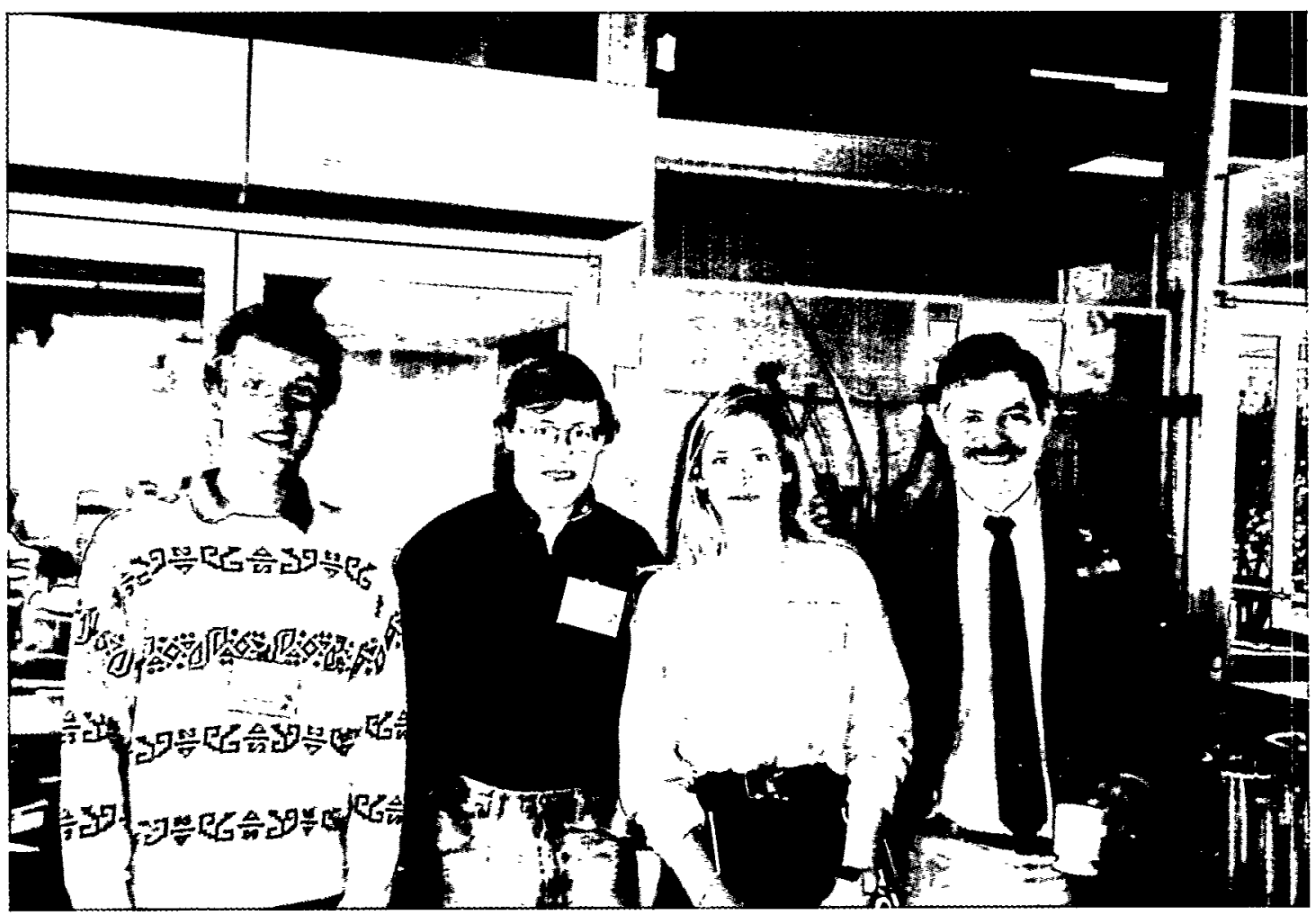

Members of SSRL Materials Research group: (from left) Alex Lessmann, postdoc, Alfred Baron and Anneli Munkholm, graduate students, and staff scientist Sean Brennan.

\section{ANALYTICAL APPLICATIONS OF SYNCHROTRON RADIATION}

The Stanford Synchrotron Radiation Laboratory (SSRL) and the Advanced Light Source (ALS) at Lawrence Berkeley Laboratory co-sponsored a "Workshop on Analytical Applications of Synchrotron Radiation: Environmental and Materials Sciences" that was held on October 19, 1994, the day between the SSRL and ALS annual users' meetings.

SSRL is building a new multipole wiggler beam line (Beam Line 11) for molecular-level analysis of chemical contaminants in diverse environments, and both SSRL and ALS plan to develop facilities for measurement of microcontamination of silicon wafers, eventually as a service to the semiconductor industry. The workshop thus served as an opportunity to inform the community about the status of these efforts, to review the capabilities of existing experimental techniques, to explore the scientific and technological opportunities in these areas, and to solicit input from the 120 persons in attendance on how to most effectively implement the proposed programs.
D. Sayers (North Carolina State University) set the stage for the day's activities with a presentation on the opportunities and challenges for commercial analytical services at synchrotron facilities. In the United States, government science policy encourages the facilities to demonstrate their relevance to industrial and societal needs, but it will be a challenge to develop the reliability and accessibility that commercial services require.

Molecular Environmental Science. G.E. Brown, Jr., (Stanford University) described waste cleanup, much of it involving radioactive materials, in the United States as a \$1-trillion job. The comparatively new field of molecular environmental science has an important role to play in this effort because remediation strategies depend on the ability to identify the elements present, often in low concentrations in complex, heterogeneous mixtures, and to determine their chemical states. It is also essential to track chemical reactions and transport processes involving contaminants and their environments, such as soils, sediments, surface and ground water, and artificial waste forms. 
X-ray absorption spectroscopy, particularly EXAFS (extended $x$-ray absorption fine-structure spectroscopy) and XANES ( $\mathrm{x}$-ray absorption near-edge spectroscopy), appears likely to be the workhorse synchrotron techniques for molecular environmental science. In support of this supposition, S. Conradson (Los Alamos National Laboratory) reviewed the results of EXAFS experiments on contaminated soils from the Fernald uranium-processing plant in Ohio.

When large numbers of EXAFS scans are needed, speed becomes an important factor. R. Frahm (HASYLAB) explained how the technique of QEXAFS (quickscanning EXAFS) could contribute. Frahm showed several examples of QEXAFS spectra obtained in times from a few seconds up to a minute. It has been possible at HASYLAB to reduce the scan time to the millisecond range with piezoelectric stages and undulator radiation.

The X-ray fluorescence microprobe is also likely to have important environmental applications. S. Sutton (University of Chicago) reviewed the performance of the microprobe on the X-26 Beam Line at the National Synchrotron Light Source and discussed the development of advanced detectors and focusing optics for spatially resolved (micro) XANES for chemicalstate mapping of contaminants, such as uranium and selenium.

Radioactive samples require special handling facilities and strict adherence to well-defined procedures in order to minimize hazards to personnel. P. Hoover (Los Alamos National Laboratory) described the trailer and hutch used at SSRL for EXAFS measurements of transuranic materials. I. Evans (SSRL) discussed engineering and administrative measures needed to ensure zero release of radioactivity. C. Donohue (Lawrence Berkeley Laboratory) reviewed the new radiation work authorization program at LBL.

The U.S. is not alone in having a radioactive waste problem. H. Nitsche (Forschungszentrum Rossendorf) (FZR) reported that in order to provide the basic information needed to address decontamination of environmental sites and assess potential waste repositories, the FZR is dedicating one of the three end stations on its proposed Collaborating Research Group bend-magnet beamline at ESRF to EXAFS of actinide materials.
Microcontamination of Semiconductors. As the minimum feature size of future integrated circuits shrinks below the 0.1-micrometer level, the detection of contaminants from the surface of silicon wafers becomes increasingly critical. A. Fischer-Colbrie (Hewlett Packard) reviewed experiments at SSRL using the technique of total $\mathrm{x}$-ray reflection fluorescence (TXRF) that lowered the detection sensitivity for a wide range of elements to about $3 \times 10^{8}$ per square centimeter. A large-area detector and other improvements could lower the limit further to about $5 \times 10^{7}$ per square centimeter.

Sematech, the collaborative research organization of the U.S. semiconductor industry, is studying the applicability of synchrotron-based TXRF for wafer screening prior to production runs. P. Pianetta (SSRL) reviewed plans at both SSRL and the ALS leading to dedicated facilities with advanced detectors and waferhandling capability. The two facilities have agreed to coordinate their operating schedules so that, at any time, one or the other is available to TXRF users. S. Brennan (SSRL) provided a technical overview of a side station on SSRL Beam Line 11 for TXRF.

Analysis of some kinds of wafer microcontamination can benefit from spatially resolved spectroscopy with chemical state sensitivity. T. Warwick $(A L S)$ described both scanning and full-field imaging spectromicroscopy end stations present and planned at ALS undulator Beamline 7.0, as well as a dedicated bendmagnet Beamline 7.3 that will be optimized for fullfield imaging with high spatial resolution and a circular polarization capability.

The day ended with two roundtable discussions, one focusing on Beam Line 11 and the other on the policy and procedural changes necessary to make the implementation and operation of analytical servicetype facilities a reality. A major problem described in this session was the wide gulf between the people doing synchrotron radiation research and the people in the field actually trying to solve the problems. Additionally, while the DOE is very supportive of technology transfer, there are a number of issues to be worked through to allow this type of routine service to become a reality at the national laboratories particularly if it were to be managed by outside companies. 


\section{SSRL ADVISORY BOARDS}

PROPOSAL REVIEW PANEL
The main task of the Proposal Review Panel (PRP) is the review and rating of scientific proposals to SSRL based largely on reports obtained from outside (non-panel) referees. The panel also advises SSRL on matters related to the user program. The PRP meets twice yearly. The panel met February 11, 1994 to rate the new proposals received in October 1993 and September 9-10, 1994, to review the May 1994 new proposals.

As of December 31, 1994, SSRL had received a total of 2336 full proposals of which 163 are presently active.

The panel members in 1994 were:

\begin{tabular}{l}
\hline \multicolumn{1}{|c|}{ Biology Subpanel } \\
\hline Stephen J. Lippard, MTT \\
Douglas C. Rees, Cal Tech \\
Jill Trewhella, LANL
\end{tabular}

\begin{tabular}{|l|}
\hline \multicolumn{1}{|c|}{ Materials Subpanel } \\
\hline Russell A. Chianelli, Exxon \\
Allan J. Jacobson, University of Houston \\
John Zachara, Pacific Northwest Laboratories \\
\hline
\end{tabular}

\begin{tabular}{|l|}
\hline \multicolumn{1}{|c|}{ VUV Subpanel } \\
\hline Warren Grobman, Motorola, (Chair) \\
Torgny Gustafsson, Rutgers University \\
Victor Henrich, Yale University \\
\hline
\end{tabular}

\section{SLAC SCIENTIFIC POLICY COMMITTEE}

The SLAC Scientific Policy Committee, which reports to the Stanford University President, reviews all aspects of the SLAC program and overviews SSRL's operation, development and plans for the future. The SPC met May 13-14 and September 30-October $1,1994$.

\section{During 1994, Scientific Policy Committee members were:}

Martin Blume, BNL

Marvin L. Cohen, UC Berkeley

Haim Harari, Weizmann Institute of Science

Wayne A. Hendrickson, Columbia University

Ernest M. Henley, University of Washington (Chair)

Robert Hollebeek, University of Pennsylvania

Ken Kikuchi, Japan Society for the Promotion of Science

David MacFarlane, McGill University

Piermarià J. Oddone, LBL

Stephen L. Olsen, University of Hawaii at Manoa

Robert D. Peccei, UC Los Angeles

Yves Petroff, ESRF

Kenneth C. Stanfield, Fermi Lab

Maury Tigner, Cornell University

Michael Witherell, UC Santa Barbara

Drs. Blume, Cohen, Hendrickson, and Petroff bring expertise in synchrotron radiation science to the committee. 
SSRL USERS ORGANIZATION

SSRL FACULTY
Members of the Executive Committee of the SSRLUO were elected following the 21st Annual SSRL Users Group Meeting as follows:

Frank G. Bridges, UC Santa Cruz

Katherine Cantwell (Secretary-SSRL Liaison)

Steven D. Conradson, LANL

Alice M. Fischer-Colbrie, Hewlett Packard, (Vice-Chair)

Jeffrey B. Kortright, LBL (Ex officio)

David B. McKay, Stanford University

David K. Shuh, LBL

David H. Templeton, LBL

Louis J. Terminello, LLNL (Chair)

Sarah H. Tolbert, UC Berkeley

Tami E. Westre, Stanford University

SSRL has been established as a department with an academic faculty of Stanford University. The SSRL faculty is augmented by term appointments from other Stanford University departments.

Arthur I. Bienenstock, SSRL, Applied Physics, Materials Science and Engineering

Gordon E. Brown, Jr.*, Earth Sciences

Sebastian Doniach*, Applied Physics (Faculty Chair)

Alice P. Gast*, Chemical Engineering

Keith O. Hodgson, Chemistry, SSRL

Piero A. Pianetta, Electrical Engineering, SSRL

R. Paul Phizackerley, SSRL

Z.-X. Shen, Applied Physics, SSRL

William E. Spicer*, Electrical Engineering, Materials Science and

Engineering

Helmut Wiedemann, SSRL, Applied Physics

Herman Winick, SSRL, Applied Physics

* Denotes Term Appointments

SSRL CONSULTING FACULTY

In 1993 the SLAC and SSRL faculty formalized the appointment of the following consulting faculty to the SSRL Department.

Robert Z. Bachrach, Applied Materials

George S. Brown, UC Santa Cruz

Stephen P. Cramer, UC Davis

Paul H. Fuoss, AT\&T Bell Laboratories

Klaus Halbach, LBL

Albert Hofmann, CERN

Stephen S. Laderman, Hewlett Packard

Claudio Pellegrini, UC Los Angeles

John J. Rehr, University of Washington

Thomas P. Russell, IBM

Joachim Stöhr, IBM

Joe Wong, LLNL 
X-ray absorption spectroscopy, particularly EXAFS (extended $x$-ray absorption fine-structure spectroscopy) and XANES (x-ray absorption near-edge spectroscopy), appears likely to be the workhorse synchrotron techniques for molecular environmental science. In support of this supposition, S. Conradson (Los Alamos National Laboratory) reviewed the results of EXAFS experiments on contaminated soils from the Fernald uranium-processing plant in Ohio.

When large numbers of EXAFS scans are needed, speed becomes an important factor. R. Frahm (HASYLAB) explained how the technique of QEXAFS (quickscanning EXAFS) could contribute. Frahm showed several examples of QEXAFS spectra obtained in times from a few seconds up to a minute. It has been possible at HASYLAB to reduce the scan time to the millisecond range with piezoelectric stages and undulator radiation.

The x-ray fluorescence microprobe is also likely to have important environmental applications. S. Sutton (University of Chicago) reviewed the performance of the microprobe on the X-26 Beam Line at the National Synchrotron Light Source and discussed the development of advanced detectors and focusing optics for spatially resolved (micro) XANES for chemicalstate mapping of contaminants, such as uranium and selenium.

Radioactive samples require special handling facilities and strict adherence to well-defined procedures in order to minimize hazards to personnel. P. Hoover (Los Alamos National Laboratory) described the trailer and hutch used at SSRL for EXAFS measurements of transuranic materials. I. Evans (SSRL) discussed engineering and administrative measures needed to ensure zero release of radioactivity. C. Donohue (Lawrence Berkeley Laboratory) reviewed the new radiation work authorization program at LBL.

The U.S. is not alone in having a radioactive waste problem. H. Nitsche (Forschungszentrum Rossendorf) (FZR) reported that in order to provide the basic information needed to address decontamination of environmental sites and assess potential waste repositories, the FZR is dedicating one of the three end stations on its proposed Collaborating Research Group bend-magnet beamline at ESRF to EXAFS of actinide materials.
Microcontamination of Semiconductors. As the minimum feature size of future integrated circuits shrinks below the 0.1-micrometer level, the detection of contaminants from the surface of silicon wafers becomes increasingly critical. A. Fischer-Colbrie (Hewlett Packard) reviewed experiments at SSRL using the technique of total $x$-ray reflection fluorescence (TXRF) that lowered the detection sensitivity for a wide range of elements to about $3 \times 10^{8}$ per square centimeter. A large-area detector and other improvements could lower the limit further to about $5 \times 10^{7}$ per square centimeter.

Sematech, the collaborative research organization of the U.S. semiconductor industry, is studying the applicability of synchrotron-based TXRF for wafer screening prior to production runs. P. Pianetta (SSRL) reviewed plans at both SSRL and the ALS leading to dedicated facilities with advanced detectors and waferhandling capability. The two facilities have agreed to coordinate their operating schedules so that, at any time, one or the other is available to TXRF users. S. Brennan (SSRL) provided a technical overview of a side station on SSRL Beam Line 11 for TXRF.

Analysis of some kinds of wafer microcontamination can benefit from spatially resolved spectroscopy with chemical state sensitivity. T. Warwick $(A L S)$ described both scanning and full-field imaging spectromicroscopy end stations present and planned at ALS undulator Beamline 7.0, as well as a dedicated bendmagnet Beamline 7.3 that will be optimized for fullfield imaging with high spatial resolution and a circular polarization capability.

The day ended with two roundtable discussions, one focusing on Beam Line 11 and the other on the policy and procedural changes necessary to make the implementation and operation of analytical servicetype facilities a reality. A major problem described in this session was the wide gulf between the people doing synchrotron radiation research and the people in the field actually trying to solve the problems. Additionally, while the DOE is very supportive of technology transfer, there are a number of issues to be worked through to allow this type of routine service to become a reality at the national laboratories particularly if it were to be managed by outside companies. 


\section{LINEAR COHERENT LIGHT SOURCE WORKSHOPS}

During 1994 two workshops were held focusing on the LCLS project. The first dealt with the scientific applications of such a source, while the second focused on developing possible collaborations to pursue the design and construction of an x-ray coherent light source.

\section{SCIENTIFIC APPLICATIONS OF COHERENT X-RAYS}

In parallel with a fierce snow storm on the East coast which prevented a number of invited speakers from arriving, a workshop on the Scientific Applications of Coherent X-rays was held on February 12, 1994. The charge to the workshop was to examine possible applications for a linac-based x-ray laser; identify critical issues and possible fundamental limits on the applications of such a source; identify variations in performance parameters that would extend possible uses; and consider the need for a pilot project as opposed to a user facility.

The initial impetus for this workshop came from technical analysis performed by a group of accelerator scientists from LBL, LLNL, SLAC, and UCLA. Stimulated by presentations and work done at earlier SLAC workshops, this group began to consider the possibility of using the SLAC linac to drive a shortwavelength Linac Coherent Light Source (LCLS), based on Self-Amplified Spontaneous Emission. This is a process by which very high coherent power can be achieved in an FEL in a single pass of a high brightness electron beam through a long undulator, without the need for an optical cavity. With the current state of technology, analysis shows that it should be possible now to construct an FEL that would operate at wavelengths down to about $30 \AA$, using electrons accelerated to about $7 \mathrm{GeV}$. With improvements in components and with the higher energies available at the SLAC linac, the operating wavelength could be reduced towards $1 \AA$. This workshop significantly changed the direction of the Linac Coherent Light Source (LCLS) project by moving the focus from a $20-40 \AA$ to a $1-4 \AA$ source. (For details of the LCLS performance see the description in the Accelerator Physics Research and New Source Development Chapter, including Figure 3.)
Discussions and presentations among the 63 attendees showed a strong interest by the scientific community in a source of $1-2 \AA$ coherent radiation for a number of important research applications. These include realtime studies of fast chemical reactions and phase transitions, real-time lattice dynamics studies using speckle interferometry, structural analysis, non-linear physics, and coherence-based interferometric and imaging techniques. The talks and discussion centered mostly on three characteristics of the LCLS radiation: extreme brightness, high transverse coherence, and timing reaching into the femtosecond regime. The use of linear polarization, circular polarization and wavelength tunability were also considered and are clearly seen as additional attractive characteristics of such a source. The high thermal power load was identified by the group as a technical problem which needs further consideration, though it was not thought to be unsolvable.

The workshop was organized by J. Arthur, G. Materlik, and $\mathrm{H}$. Winick. The proceedings are available as SLAC Report-437.

\section{JAPANESE-U.S. LCLS COLLABORATOR MEETING}

Fifty people, including scientists from Japan and Germany, came to SLAC May 27-28 for the first Japan-U.S. collaboration meeting on the project to develop a Linac Coherent Light Source (LCLS) using the SLAC linac. About one-third of the participants in the collaboration meeting were potential users of such a source; the others were accelerator physicists who were working on, or interested in, the technical challenges of creating such an x-ray laser. The Japanese delegation consisted of six scientists and was led by M. Ando of the Photon Factory at KEK.

J. Arthur, R. Tatchyn, and H. Winick of SSRL hosted the meeting along with G. Materlik, who was visiting SSRL from DESY.

The goals of the meeting were to: define additional work needed to set the range of LCLS parameters; review opportunities for $R \& D$ on technical aspects of the source at BNL, SLAC, and UCLA as well as other laboratories in the U.S. and Japan; describe experimental equipment and space needed; and recommend preliminary experiments that could be done at sources such as ESRF, APS and SPring-8. 


\section{SSRL ORGANIZATION}

SSRL is a division of the Stanford Linear Accelerator Center (SLAC). The internal SSRL organization consists of four departments: Accelerator Systems, Administrative Services, Experimental Systems, and Research. The Accelerator Systems department is responsible for the operation, maintenance and development of the booster and SPEAR. The Experimental Systems department is responsible for beam line management, beam line development, and user support. The Research department has responsibility for planning the short- and longterm in-house research of the Laboratory. The Safety Office and User Research Administration report directly to the Director of SSRL. A detailed organization chart appears on the next page.

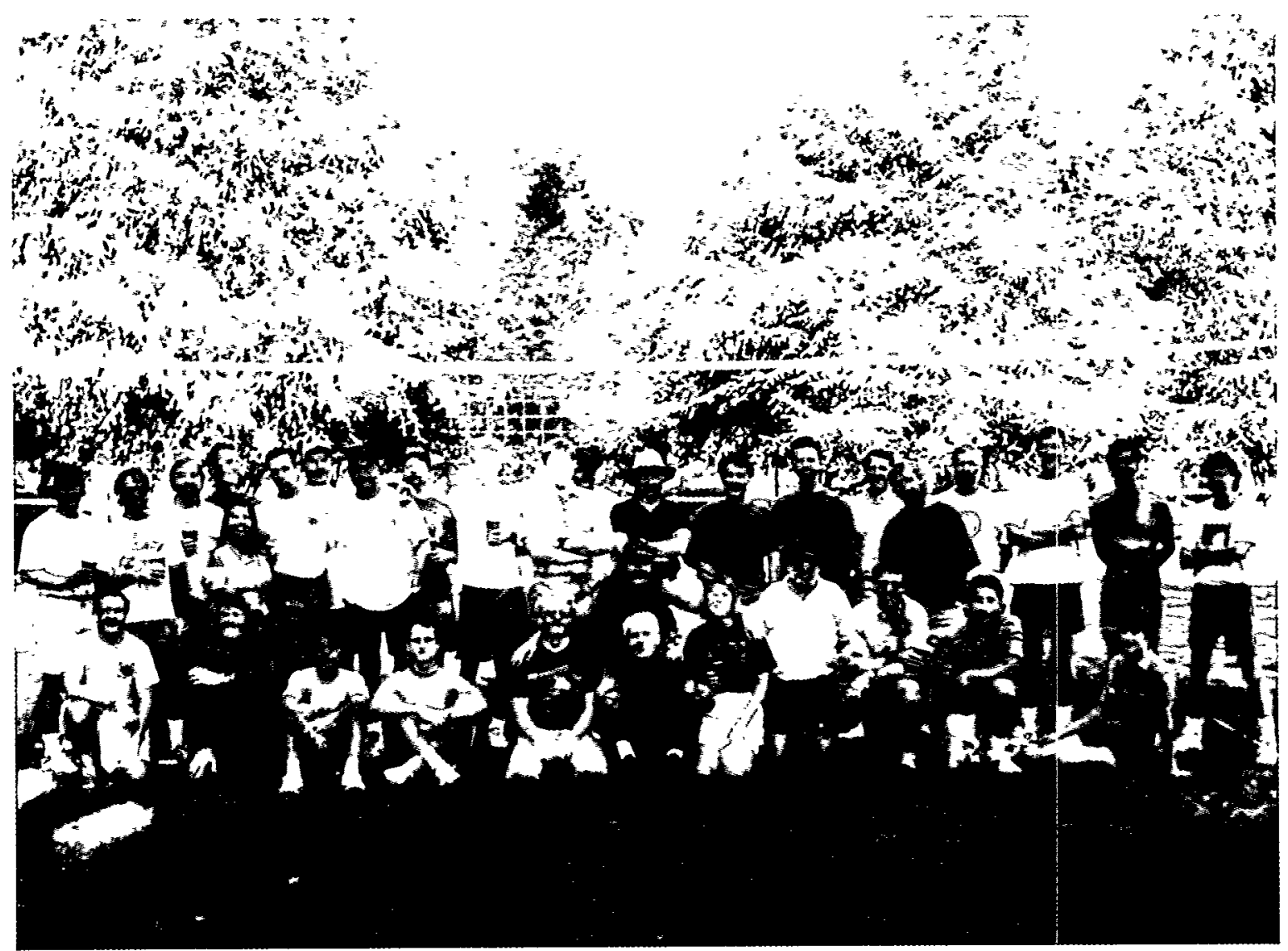

SSRL End-of-Run Party. (Back, from left) Chuck Troxel, Jr., Glen Kerr, Paul Phizackerley, Alex Lessmann, Pierre Capeder, Todd Slater, Bill Butler, Scott Jansson, Bart Johnson, Alan Swithenbank, Mike Nalls, Arthur Bienenstock, Ed Guerra, Chris Hoover, Tom Rabedeau, Rick Gabrielson, Piero Pianetta, Ian Evans, Jim Hayward, Mike Soltis, (front, from left) Pat Allen, Hal Tompkins, Joe Ormonde, Hiro Tsuruta, Jeff Moore, Jim Montgomery, Tom Hostetler, Katherine Cantwell, Herman Winick, Michael Baltay, Brian Choi, Sabine Bassen, (middle, from left) Courtenay Troxel, Michelle Steger 


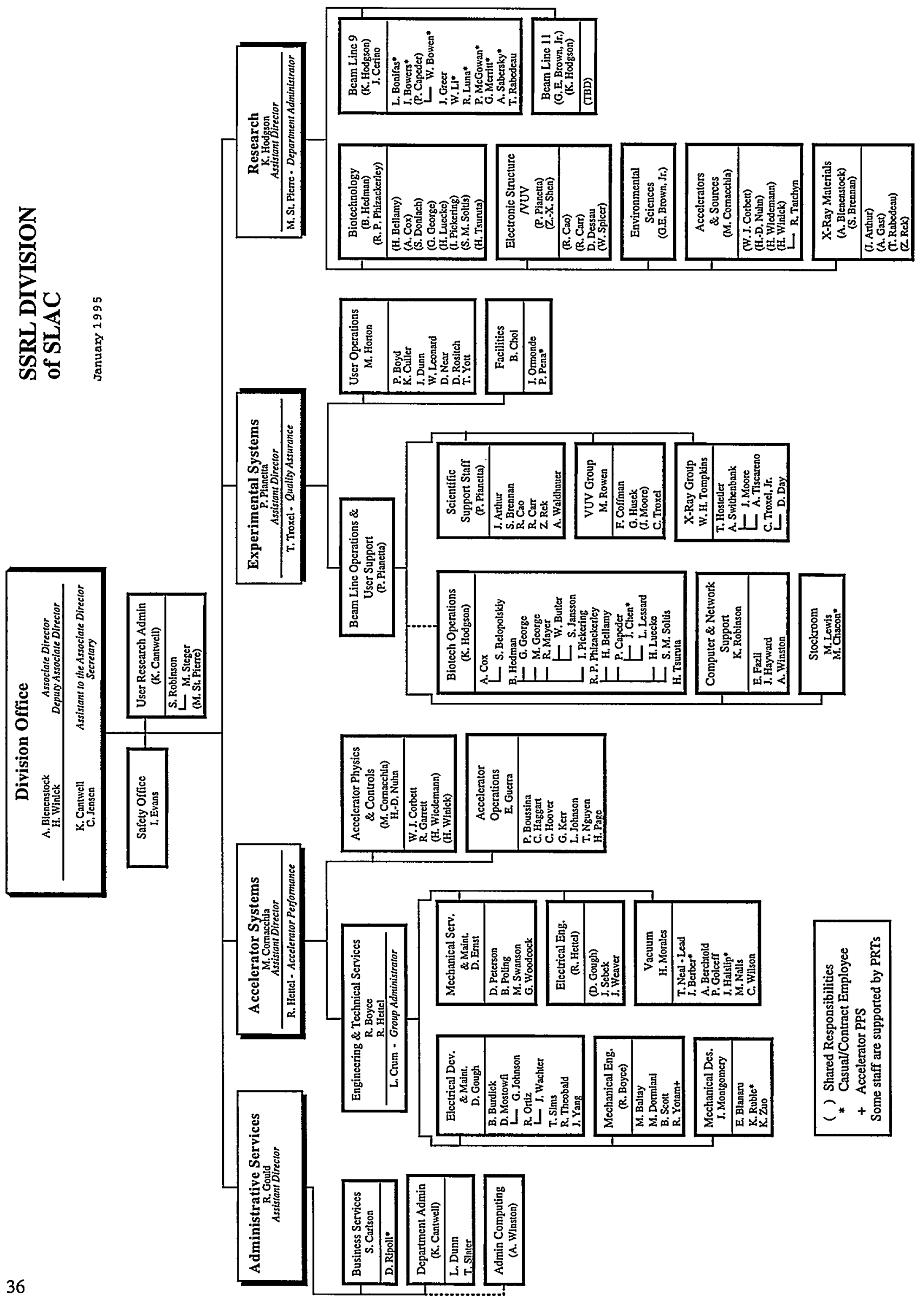




\begin{tabular}{|c|c|c|}
\hline \multicolumn{3}{|c|}{$\begin{array}{c}\text { SSRL EXPERIMENTAL STATIONS } \\
\text { RESPONSIBLE PERSONNEL }\end{array}$} \\
\hline \multicolumn{2}{|c|}{ BRANCH LINE } & RESPONSIBLE PERSON(S) \\
\hline $\begin{array}{l}1-2 \\
1-4 \\
1-5 \\
1-5 \text { ES2 }\end{array}$ & $\begin{array}{l}\text { TGM } \\
\text { SAS } \\
\text { Unfocused Bend Magnet } \\
\text { Protein Crystallography MAD Facility }\end{array}$ & $\begin{array}{l}\text { M. Rowen } \\
\text { H. Tompkins } \\
\text { H. Tompkins } \\
\text { P. Phizackerley, H. Bellamy }\end{array}$ \\
\hline $\begin{array}{l}2-1 \\
2-2 \\
2-3\end{array}$ & $\begin{array}{l}\text { Focused Bend Magnet } \\
\text { White Radiation } \\
\text { Unfocused Bend Magnet }\end{array}$ & $\begin{array}{l}\text { H. Tompkins } \\
\text { Z. Rek } \\
\text { H. Tompkins }\end{array}$ \\
\hline $\begin{array}{l}3-1 \\
3-3 \\
3-4\end{array}$ & $\begin{array}{l}\text { Grasshopper } \\
\text { Jumbo - Soft X-ray } \\
\text { Lithography }\end{array}$ & $\begin{array}{l}\text { F. Coffman } \\
\text { M. Rowen } \\
\text { P. Pianetta }\end{array}$ \\
\hline $\begin{array}{l}4-1 \\
4-2 \\
4-3\end{array}$ & $\begin{array}{l}\text { 8-Pole Wiggler } \\
\text { 8-Pole Wiggler } \\
\text { 8-Pole Wiggler }\end{array}$ & $\begin{array}{l}\text { H. Tompkins } \\
\text { H. Tompkins } \\
\text { H. Tompkins }\end{array}$ \\
\hline $5-1,-2,-3$ & Multiundulator & Z.-X. Shen, D. Dessau \\
\hline $6-2$ & 54-Pole Wiggler & J. Arthur, G. Via (EXXON) \\
\hline $\begin{array}{l}7-1 \\
7-2 \\
7-3\end{array}$ & $\begin{array}{l}\text { Rotation Camera for Protein Crystallography } \\
\text { 8-Pole Wiggler (6-circle Huber) } \\
\text { 8-Pole Wiggler }\end{array}$ & $\begin{array}{l}\text { P. Phizackerley, M. Soltis, H. Luecke } \\
\text { S. Brennan } \\
\text { H. Tompkins }\end{array}$ \\
\hline $\begin{array}{l}8-1 \\
8-2\end{array}$ & $\begin{array}{l}\text { TGM } \\
\text { SGM }\end{array}$ & $\begin{array}{l}\text { R. Cao } \\
\text { R. Cao, J. Tobin }(L L N L)\end{array}$ \\
\hline $10-1$ & SGM & M. Rowen, P. Pianetta, J. Stöhr (IBM) \\
\hline $10-2$ & 30-Pole Wiggler (6-circle Huber) & S. Brennan, J. Wong (LLNL), M. Nicol (UCLA) \\
\hline INS TRU & MENTATION/FACILITY & RESPONSIBLE PERSON(S) \\
\hline $\begin{array}{l}\text { PERKIN-E } \\
\text { CAD-4 DIF } \\
\text { COMPUTE } \\
\text { BIOTECH } \\
\text { BLDG. } 120 \\
\text { BLDG. } 120 \\
\text { BLDG. } 131 \\
\text { EXAFS CO } \\
\text { EXAFSEQ } \\
\text { FUIIMAC } \\
\text { SAXS CAN } \\
\text { SCATTERI } \\
\text { TOPOGRA } \\
\text { RAPID TU }\end{array}$ & $\begin{array}{l}\text { LMER CHAMBER } \\
\text { FRACTOMETER } \\
\text { R SYSTEMS, SOFTWARE and NETWORK } \\
\text { COMPUTER SYSTEMS and SOFTWARE } \\
\text { SAMPLE PREPARATIONLAB } \\
\text { BIOTECH SAMPLE PREPARATIONLAB } \\
\text { BIOTECH SAMPLE PREPARATION LAB } \\
\text { NSULTANTS } \\
\text { UTPMENT } \\
\text { ING PLATE SCANNER } \\
\text { IERA (BIOTECH) } \\
\text { NG CONSULTANTS } \\
\text { PHY EOUIPMENT } \\
\text { RNAROUND EXAFS FACILIIES }\end{array}$ & $\begin{array}{l}\text { R. Cao } \\
\text { M. Soltis } \\
\text { K. Robinson, J. Hayward, E. Fazli, A. Winston } \\
\text { S. Belopolskiy, M. George, H. Luecke } \\
\text { M. Rowen } \\
\text { R. Mayer, B. Hedman } \\
\text { R. Mayer, B. Hedman } \\
\text { G. George, B. Hedman, I. Pickering } \\
\text { R. Mayer, B. Hedman, G. George, I. Pickering } \\
\text { M. Soltis } \\
\text { H. Tsuruta } \\
\text { S. Brennan. J. Arthur } \\
\text { Z. Rek } \\
\text { I. Pickering, R. Mayer, B. Hedman }\end{array}$ \\
\hline
\end{tabular}




\section{SSRL ADVISORY BOARDS}

PROPOSAL REVIEW PANEL
The main task of the Proposal Review Panel (PRP) is the review and rating of scientific proposals to SSRL based largely on reports obtained from outside (non-panel) referees. The panel also advises SSRL on matters related to the user program. The PRP meets twice yearly. The panel met February 11, 1994 to rate the new proposals received in October 1993 and September 9-10, 1994, to review the May 1994 new proposals.

As of December 31, 1994, SSRL had received a total of 2336 full proposals of which 163 are presently active.

The panel members in 1994 were:

\begin{tabular}{|l|}
\hline Biology Subpanel \\
\hline Stephen J. Lippard, MTT \\
Douglas C. Rees, Cal Tech \\
Jill Trewhella, LANL
\end{tabular}

\begin{tabular}{|l|}
\hline \multicolumn{1}{|c|}{ Materials Subpanel } \\
\hline Russell A. Chianelli, Exxon \\
Allan J. Jacobson, University of Houston \\
John Zachara, Pacific Northwest Laboratories \\
\hline
\end{tabular}

\begin{tabular}{|l|}
\hline \multicolumn{1}{|c|}{ VUV Subpanel } \\
\hline Warren Grobman, Motorola, (Chair) \\
Torgny Gustafsson, Rutgers University \\
Victor Henrich, Yale University \\
\hline
\end{tabular}

\section{SLAC SCIENTIFIC POLICY COMMITTEE}

The SLAC Scientific Policy Committee, which reports to the Stanford University President, reviews all aspects of the SLAC program and overviews SSRL's operation, development and plans for the future. The SPC met May 13-14 and September 30-October $1,1994$.

\section{During 1994, Scientific Policy Committee members were:}

Martin Blume, BNL

Marvin L. Cohen, UC Berkeley

Haim Harari, Weizmann Institute of Science

Wayne A. Hendrickson, Columbia University

Ernest M. Henley, University of Washington (Chair)

Robert Hollebeek, University of Pennsylvania

Ken Kikuchi, Japan Society for the Promotion of Science

David MacFarlane, McGill University

Piermaria J. Oddone, LBL

Stephen L. Olsen, University of Hawaii at Manoa

Robert D. Peccei, UC Los Angeles

Yves Petroff, ESRF

Kenneth C. Stanfield, Fermi Lab

Maury Tigner, Cornell University

Michael Witherell, UC Santa Barbara

Drs. Blume, Cohen, Hendrickson, and Petroff bring expertise in synchrotron radiation science to the committee. 
SSRL USERS ORGANIZATION

SSRL FACULTY
Members of the Executive Committee of the SSRLUO were elected following the 21st Annual SSRL Users Group Meeting as follows:

Frank G. Bridges, UC Santa Cruz

Katherine Cantwell (Secretary-SSRL Liaison)

Steven D. Conradson, LANL

Alice M. Fischer-Colbrie, Hewlett Packard, (Vice-Chair)

Jeffrey B. Kortright, LBL (Ex officio)

David B. McKay, Stanford University

David K. Shuh, LBL

David H. Templeton, LBL

Louis J. Terminello, LLNL (Chair)

Sarah H. Tolbert, UC Bèrkeley

Tami E. Westre, Stanford University

SSRL has been established as a department with an academic faculty of Stanford University. The SSRL faculty is augmented by term appointments from other Stanford University departments.

Arthur I. Bienenstock, SSRL, Applied Physics, Materials Science and Engineering

Gordon E. Brown, Jr.*, Earth Sciences

Sebastian Doniach*, Applied Physics (Faculty Chair)

Alice P. Gast*, Chemical Engineering

Keith O. Hodgson, Chemistry, SSRL

Piero A. Pianetta, Electrical Engineering, SSRL

R. Paul Phizackerley, SSRL

Z.-X. Shen, Applied Physics, SSRL

William E. Spicer*, Electrical Engineering, Materials Science and

Engineering

Helmut Wiedemann, SSRL, Applied Physics

Herman Winick, SSRL, Applied Physics

* Denotes Term Appointments

\footnotetext{
Robert Z. Bachrach, Applied Materials

George S. Brown, UC Santa Cruz

Stephen P. Cramer, UC Davis

Paul H. Fuoss, AT\&T Bell Laboratories

Klaus Halbach, LBL

Albert Hofmann, CERN

Stephen S. Laderman, Hewlett Packard

Claudio Pellegrini, UC Los Angeles

John J. Rehr, University of Washington

Thomas P. Russell, IBM

Joachim Stöhr, IBM

Joe Wong, LLNL
} 


\section{EXPERIMENTAL PROGRESS REPORTS}

\section{MATERIALS PROPOSALS}

\begin{tabular}{|c|c|c|}
\hline \\
\hline & & Page \\
\hline $1 \mathrm{E} 12 \mathrm{M}$ & $\begin{array}{l}\text { Oxidation State of Sulfur in Soil Organic Matter and Iron Sulfide Oxidation } \\
\text { Products - S. Fendorf and M. Morra }\end{array}$ & 53 \\
\hline $1 \mathrm{E} 16 \mathrm{M}$ & $\begin{array}{l}\text { Trace Element Coordination in Natural Sediments at Ambient and Cryogenic } \\
\text { Temperatures -P.A. O'Day, S.A. Carroll, G.A. Waychunas and B. Phillips }\end{array}$ & 57 \\
\hline $\begin{array}{l}2138 \mathrm{Mp} / \\
9905 \mathrm{M}\end{array}$ & $\begin{array}{l}\text { Evidence of Negatively Correlated Displacements of the Axial } \mathrm{O}(2) \text { Atom and the } \\
\mathrm{Cu} \text { and Ba Atoms in } \mathrm{HgBa}_{2} \mathrm{CuO}_{4} \text { from XAFS - F. Bridges, C.H. Booth, E. Bauer, } \\
\text { J.B. Boyce, T. Claeson and C.H. Chu }\end{array}$ & 61 \\
\hline $\begin{array}{l}2138 \mathrm{Mp} / \\
9905 \mathrm{M}\end{array}$ & $\begin{array}{l}\text { XAFS Standards: A Comparison of Experiment and Theory -G.G. Li, F. Bridges } \\
\text { and C.H. Booth }\end{array}$ & 65 \\
\hline $2142 \mathrm{Mp}$ & $\begin{array}{l}\text { Polarized Dispersion for X-rays Scattered by an Aromatic Bromide } \\
\text {-D.H. Templeton and L.K. Templeton }\end{array}$ & 69 \\
\hline $2143 \mathrm{Mp}$ & $\begin{array}{l}\text { XAS Study of Cation Chemisorption at Oxide-Water Interfaces -M.L. Peterson, } \\
\text { J.R. Bargar, S.-F. Cheah, A.L. Foster, T. Tingle, T. Tokunaga, G.A. Parks and } \\
\text { G.E. Brown, Jr. }\end{array}$ & 70 \\
\hline $2153 \mathrm{Mp}$ & $\begin{array}{l}\text { Anomalous Small-Angle X-ray Scattering Studies of Amorphous Molybdenum- } \\
\text { Germanium Alloys - M.J. Regan and A. Bienenstock }\end{array}$ & 74 \\
\hline $2157 \mathrm{M}$ & $\begin{array}{l}\text { Sorption of Aqueous } \mathrm{Zn}^{2+} \text { on Ferrihydrite: Observation of the Onset of } \\
\text { Precipitation -G.A. Waychunas, C.C. Fuller and J.A. Davis }\end{array}$ & 78 \\
\hline $2165 \mathrm{Mp}$ & $\begin{array}{l}\text { Dynamic Study of the Monomer-Polymer Phase Transformation in Diacetylene } \\
\text { Bicrystals via White Beam Synchrotron X-ray Topography -J. Liao, D.C. Martin, } \\
\text { Z. Zeng, P.M. Wilson, Z.U. Rek and J.C. Bilello }\end{array}$ & 81 \\
\hline $2165 \mathrm{Mp}$ & $\begin{array}{l}\text { Strain Eigenvalues as a Function of Penetration Depth for Sputter Deposited Mo } \\
\text { Thin Films - S.G. Malhotra, Z.U. Rek, S.M. Yalisove and J.C. Bilello }\end{array}$ & 87 \\
\hline $2165 \mathrm{Mp}$ & $\begin{array}{l}\text { White Beam Transmission Laue Studies of Texture in Mo/W and Ta/W } \\
\text { Multiscalar Multilayer Composite Films - A.K. Malhotra, Z.U. Rek, S.M. Yalisove } \\
\text { and J.C. Bilello }\end{array}$ & 91 \\
\hline $2175 \mathrm{Mp}$ & $\begin{array}{l}\text { Determination of Chromium Oxidation States in Ash Samples Derived from Coal } \\
\text { Combustion - M. Najih, F.E. Huggins and G.P. Huffman }\end{array}$ & 95 \\
\hline $2175 \mathrm{Mp}$ & $\begin{array}{l}\text { XAFS Spectroscopic Investigation of the Behavior of Zinc During Coliquefaction } \\
\text { of Coal and Waste Lubricating Oil -F.E. Huggins, J. Zhao and G.P. Huffman }\end{array}$ & 98 \\
\hline $2182 \mathrm{Mp}$ & $\begin{array}{l}\text { In Situ X-ray Studies of OMVPE Growth -P.H. Fuoss, D.W. Kisker, } \\
\text { G.B. Stephenson and S. Brennan }\end{array}$ & 101 \\
\hline $2189 \mathrm{Mp}$ & $\begin{array}{l}\text { Seasonal Changes in Valence and Chemical Speciation of Bioaccumulated Mn in } \\
\text { Sago Pondweed - C.M. Lytle and F.W. Lytle }\end{array}$ & 106 \\
\hline $2193 \mathrm{Mp}$ & $\begin{array}{l}\text { XAFS Studies of Perovskite Related Structures at High Pressures -R. Ingalls, } \\
\text { F. Wang, S. Jones and B. Houser }\end{array}$ & 109 \\
\hline & & 41 \\
\hline
\end{tabular}


2194Mp Strain Distribution in Metallic Thin Films and Patterned Lines - R.P. Vinci,

E.M. Zielinski and J.C. Bravman

2197Mp Investigation of Heavily-Doped III-V and II-VI Semiconductors Using Fluorescent

115 EXAFS -K.M. Yu, W. Walukiewicz, C. Rossington, E. Bourret, J.M. Jaklevic and E.E. Haller

2200Mp Kinetic Studies of OMVPE Growth Using In Situ X-ray Scattering -D.W. Kisker, P.H. Fuoss, I. Kamiya, G.B. Stephenson, S. Brennan, L. Mantese and D.E. Aspnes

2208M Measurement of Chemical and Structural Anisotropy in Amorphous Tb-Fe Thin Films - T.C. Hufnagel, B.M. Clemens and S. Brennan

2210Mp Grazing-Incidence XAS Study of Cation Chemisorption at Single-Crystal Oxide Interfaces -S.N. Towle, J.R. Bargar, G.E. Brown, Jr, G.A. Parks and T.W. Barbee, Jr.

2212Mp Ti XAS of Crystalline Silicotitanates -M.L. Balmer, N.J. Hess and S.D. Conradson

2219Mp Cation Valence and Coordination in Cerium and Uranium Mixed Oxides

-M.R. Antonio and L. Soderholm

2224Mp Effect of Structure, Stress, Strain, and Alloying on the Hardness of

Fe(001)/Pt(001) Epitaxial Multilayers - B.J. Daniels, W.D. Nix and B.M. Clemens

2224Mp Structure and Magneto-optical Properties of Epitaxial PtMnSb(001) on 138 $\mathrm{W}(001) / \mathrm{MgO}(001)$-M.C. Kautzky and B.M. Clemens

2224Mp Surface Anisotropy and Structure of Fe(110)/MO(110) Bilayers - R.M. Osgood, B.M. Clemens, R.L. White and S. Brennan

2224Mp The Effect of Cr Doping on the Magnetoresistance and Saturation Field of Epitaxial $\mathrm{Fe}_{1-\mathrm{x}} \mathrm{Cr}_{\mathrm{x}}(001) / \mathrm{Cr}(001)$ Multilayers - B.J. Daniels and B.M. Clemens

2226Mp Sources of Strain in Rapidly Grown Crystals of $\mathrm{KH}_{2} \mathrm{PO}_{4}$-J.J. De Yoreo, Z.U. Rek, N.P. Zaitseva and B.W. Woods

2229Mp Mechanics of Advanced Composites Using X-ray Tomographic Microscopy

-T.M. Breunig, M.C. Nichols, J.H. Kinney and D.L. Haupt

2237Mp Studies of Actinides by X-ray Absorption Spectroscopy -P.G. Allen,

I. Almahamid, R.A. Andersen, G. Bernhard, J.C. Bryan, J.J. Bucher, A.K. Burrell,

D.L. Clark, N.M. Edelstein, S.A. Ekberg, J.W. Gohdes, E.A. Hudson, N. Kaltsoyannis, W.W. Lukens, W. Matz, H. Moll, M.P. Neu, H. Nitsche, P.D. Palmer, T. Reich,

K. Roberts, D.K. Shuh, C.D. Tait and B.D. Zwick

2242M Nd Substitution into the $\mathrm{Y}$ and $\mathrm{Ba}$ Layers of $\mathrm{Y}_{1} \mathrm{Ba}_{2} \mathrm{Cu}_{3} \mathbf{0}_{7}-\mathrm{J} . \mathrm{V}$. Acrivos, 160 C.M. Burch and K.K. Sing

2245Mp Reconstruction at Metal/Silicon Interfaces -C.A. Lucas and D. Loretto

162

2249Mp X-ray Micro-Tomography with a Diamond Anvil Cell - S. Weir and J. Akella

2252Mp Surface Structure Determination Using Anomalous X-ray Scattering:

Underpotential Deposition of Copper on Pt(111) - I.M. Tidswell, C.A. Lucas, N.M. Markovic and P.N. Ross 
2253M Structural Characterization of Organocuprate Complexes in Solution

-T.M. Barnhart, H. Huang and J.E. Penner-Hahn

2254M Structural Studies of Manganese Doped Bismuth Vanadate Ionic Conductors -A.J. Jacobson, Y.L. Yang, L. Qiu and I.J. Pickering

$2256 \mathrm{Mp} \quad \mathrm{X}$-ray Absorption Studies of Uranium Sorption on Mineral Substrates

-E.A. Hudson, L.J. Terminello, B.E. Viani, T. Reich, J.J. Bucher, D.K. Shuh and N.M. Edelstein

2261Mp Sorption and Coprecipitation of Arsenate and Chromate in Alkaline

Environments -S.C.B. Myneni, S.J. Traina, G.A. Waychunas and T.J. Logan

2264Mp High Resolution Synchrotron X-ray Diffraction Tomography of Polycrystalline

Samples -S.R. Stock, A. Guvenilir, D.P. Piotrowski and Z.U. Rek

2268Mp Correlation of XANES Features with the Scintillation Efficiencies of Ce Doped Alkaline Earth Lithium Silicate Glasses -D.L. Blanchard, Jr., D.S. Sunberg, R.A. Craig, M. Bliss and M.J. Weber

2269Mp XAS Study of Metal Ion Partitioning at Clay-Water Interfaces - C. Papelis, C.-C. Chen and K.F. Hayes

2276Mp Delta-Phase Stabilization and Effects of Aging on Microstructure of Plutonium Alloys -S. Conradson, L. Cox and N. Hess

2283Mp EXAFS Investigation of PtCu Carbon-Supported Catalysts -F.W. Lytle, R.M. Friedman and S.R. Bare

$2286 \mathrm{Mp} \quad$ On the Structure of the Solvated Mercury(I) Ion -I. Persson 204

2286Mp Structure of the Hydrated Gallium(III) Ion -M. Sandström and I. Persson 207

2298M Size-dependent Catalytic Activity of Supported Metal Clusters - Z. Xu, 208 F.-S. Xiao, S.K. Purnell, O. Alexeev, S. Kawi, S.E. Deutsch and B.C. Gates

2300M 1.05 Pr Substitution into the $\mathrm{Y}$ and $\mathrm{Ba}$ Layers of $\mathrm{Y}_{1} \mathrm{Ba}_{2} \mathrm{Cu}_{3} \mathrm{O}_{7}$-J.V. Acrivos, C.M. Burch, M. Rose, H.A. Blackstead and D.B. Pulling

8001M Short-Period AlAs/GaAs Superlattices Studied by X-ray Diffraction and Standing Waves -A. Lessmann, M. Schuster, S. Brennan, G. Materlik and H. Riechert

$8001 \mathrm{M}$ Wafer Cleaning Influence on the Roughness of the $\mathrm{Si}_{\mathrm{SiO}} \mathrm{I}_{2}$ Interface -A. Munkholm, S. Brennan and J.P. Goodbread

8010M Nuclear Scattering of Synchrotron Radiation by ${ }^{181} \mathrm{Ta}$-A.I. Chumakov, A.Q.R. Baron, J. Arthur, S.L. Ruby, G.S. Brown, G.V. Smirnov, U. van Bürck and G. Wortmann

$8013 \mathrm{M} X$-ray Absorption Spectroscopic Studies of the Superconductor $\mathrm{Ba}_{\mathbf{4}} \mathrm{BiPb}_{\mathbf{2}} \mathrm{TlO}_{12-\delta}$

-I.J. Pickering, G.N. George, T.E. Sutto and B.A. Averill

$8103 \mathrm{M}$ Characterization of $\mathrm{Si}^{-\mathrm{TaSi}_{2}}$ Composites for Use as Wide-band-pass Optical Element for Synchrotron Radiation -Z.U. Rek, S.R. Stock and M. Goorsky 
8103M Correlation of the Growth Parameters of YB 66 with Defect Structure and Crystal

Performance as Soft X-ray Monochromator -Z.U. Rek, T. Tanaka, M. Rowen and J. Wong

9104M Thermal Studies of Ceramics -E.D. Crozier, G.J. Soerensen, T. Tiedje and

T. Troczynski

9108M Structure in Rigid Polymers and in Nafion Membranes -P.S. Russo, J. Cheng, I. Negulescu and R.B. Moore

9113M Grazing Incidence XAS of Titania Sol-Gel Films - T. Hanley, R.F. Howe and I. Pickering

9127M Low-Energy X-ray Dosimetry Studies (7 to $17.5 \mathrm{keV}$ ) with Synchrotron Radiation -N.E. Ipe, H. Bellamy, J.R. Flood, K.R. Kase, P. Phizackerley, K.J. Velbeck and R. Tawil

9200M TXRF R\&D Studies - S. Laderman, A. Fischer-Colbrie, A. Waldhauer, S. Brennan, N. Takaura and P. Pianetta

9500M S K-edge XANES on Lubricant Additives -S.D. Cameron, R.S. Polizzotti and M. Sansone

9503M The Chemistry of Sulfur During Hydropyrolysis of Coals -M.L. Gorbaty, S.R. Kelemen, S.N. Vaughn, M. Sansone, P.J. Kwiatek and G.N. George

9902M Direct Observation of Phase Transformation in Fusion Welds - J. Wong, J.W. Elmer, M. Fröba, P.A. Waide and E.M. Larson

9912M Pressure Induced Structural Transformations in Silicon Nanocrystals: Surface and Shape Effect - S.H. Tolbert, A.B. Herhold, L.E. Brus and A.P. Alivisatos

9932M Synthesis and Characterization of Novel Materials at High Pressures and Temperatures by DAC X-ray Laser Heating Studies -C.-S. Yoo, J. Akella and M.F. Nicol 
2A14B Structural Studies of Type I DNA Topoisomerases -C. Lima, N. Lue, A. Sharma, V. Tokars and A. Mondragón

V. Tokars and A. Mondragón

2A27B Evaluation/Data Collection of Cytochrome Reductase Crystals -E.A. Berry, L.-S. Huang, V. Schulmeister and S.-H. Kim

2A50B X-ray Crystallographic Studies of the Hydroxylase Component of Methane Monooxygenase from Methylococcus capsulatus (Bath) -A.C. Rosenzweig,

2A53B Design of Influenza Virus Neuraminidase Inhibitors Based on Crystal Structure -M. Luo

2A54B Low Resolution Data Collection of Yeast RNA Polymerase II -P.R. David, D.A. Bushnell and R.D. Kornberg

2A59B Heat-labile Enterotoxin from $E$. coli (cholera family toxins) -E.A. Merritt,

F. van den Akker and W.G.J. Hol

2A60B Crystallographic Studies on Enzymes of the Pyruvate Dehydrogenase

2A61B X-ray Crystal Structure Determination of Complexes Between Chymotrypsin and

2A62B Structure of the Envelope Glycoprotein from Tick-borne Encephalitis Virus (TBE) - S.C. Harrison and F. Rey

2A74B Crystal Structure of a Type II DNA Topoisomerase -J.M. Berger, J.C. Wang and S.C. Harrison 
2A83B Structure of the Human Protective Protein Precursor Determined by X-ray Crystallography to 2.2 Å Resolution -G. Rudenko, E. Bonten, A. d'Azzo and W. Hol

2A87B A Structural Investigation of Familial Amyotrophic Lateral Sclerosis -S. Redford, M. Johnson, R. Hallewell, E. Getzoff and J. Tainer

2A90B Human Cu, Zn Superoxide Dismutase Turn Mutant and Permutant Structures to Study the Greek Key $\beta$-Barrel Fold -M.M. Thayer, H. Badkoobehi, M. Boissinot, R.A. Hallewell, J.A. Tainer and E.D. Getzoff

3A01B Structure of a 90kD Fragment of Yeast Topoisomerase II -J. Berger, J.C. Wang and S.C. Harrison

3A02B Structure Determination of Proteins Involved in Signal Transduction -U. Schulze-Gahmen, H.D. Jones, D.O. Morgan and S.-H. Kim

2129Bp Synchrotron Radiation Detector Development Program -W.K. Warburton,

B. Hubbard, Y. Kong and C. Zhou

2146Bp Ligand K-edge X-ray Absorption Spectroscopic Studies: Metal-Ligand Covalency

in a Series of Transition Metal Complexes - S.E. Shadle, K.R. Williams,

B. Hedman, K.O. Hodgson and E.I. Solomon

2167B Laue Studies of $\boldsymbol{R} b$. sphaeroides Photosynthetic Reaction Center and Other

Proteins - T. McPhillips, M.H.B. Stowell, M. Mas, M. Soltis, R.P. Phizackerley and D.C. Rees

2181Bp X-ray Absorption Spectroscopy Studies of Manganese Redox Enzymes -P.J. Riggs-Gelasco, T.L. Stemmler, J.E. Penner-Hahn, T.M. Sossong, D.E. Ash, R. Mei, C.F. Yocum and D. Ghanotakis

2184B Small-Angle X-ray Scattering Studies of Conformational Changes in E. coli Aspartate Transcarbamoylase -E.R. Johnson, H. Tsuruta, B.-B. Zhou, H. Kihara and H.K. Schachman

2187Bp Use of White Beam and Small Angle Scattering for the Study of Compact Intermediates -D. Segel, H. Tsuruta, L. Chen, D. Eliezer, S. Doniach and K.O. Hodgson

2190Bp/ Photosynthetic Water Oxidation: Structural Insights to the Catalytic Manganese Complex -J.C. Andrews, R. Cinco, H. Dau, M.J. Latimer, W. Liang, T.A. Roelofs, A. Rompel, K. Sauer, V.K. Yachandra and M.P. Klein

2192Bp Structural Characterization of the Active Site of a Manganese-Enzyme, Prolidase -P.J. Ellis, H.C. Freeman, B. Hedman, K.O. Hodgson, E.M. Proudfoot and L. Zhang

2227Bp

EXAFS Determination of a $2.5 \AA \mathrm{Cu}$-Cu Interaction at the $\mathrm{Cu}_{\mathrm{A}}$ Center of Oxidized and Reduced Cytochrome Oxidase from Bacillus subtilis -N.J. Bläkburn, J.S. Boswell and S. de Vries

2227Bp EXAFS Studies of the Copper Centers in Peptidylglycine $\alpha$-Hydroxylating Monooxygenase -N.J. Blackburn, J.S. Boswell, A.S. Quon, R.E. Mains and B.A. Eipper 
2227Bp Structural Investigations on the $\mathrm{Cu}_{\mathrm{B}}$ Center of the Quinol Oxidase from Bacillus subtilis and an Oxo-bridged Heterobimetallic Fe-Cu Model Compound: Multiple Scattering Determination of the Cu-O(H)-Fe Angle - N.J. Blackburn, J. Boswell, M. Wikström and K.D. Karlin

2240Bp Solution Small-Angle X-ray Scattering Studies of the Heat Shock Cognate Protein D.B. McKay

2241B EXAFS Studies of the Molybdenum-Iron Cluster from Nitrogenase - H.I. Liu, B. Hedman, K.O. Hodgson, L. Ma and B.K. Burgess

K $\beta$ Emission Spectroscopy of Iron and Nickel -C.R. Randall, A.E. Froeschner, M.M. Grush, J.A. Moore, X. Wang and S.P. Cramer

Site Selective and Spin-Polarized X-ray Fluorescence Spectroscopy -X. Wang, C.R. Randall and S.P. Cramer

2260Bp Determination of Iron-Ligand Bond Lengths in Horse Heart Met-Myoglobin, Deoxy-Myoglobin and Nitrosylmyoglobin Using Multiple-Scattering EXAFS -R.S. Armstrong, P.J. Ellis, H.C. Freeman, B. Hedman, K.O. Hodgson, P.A. Lay and A.M. Rich

2267B De Novo Design of Metalloprotein Metal Sites - G.R. Dieckmann, D.L. Tierney, J.E. Penner-Hahn, V.L. Pecoraro and W.F. DeGrado

Histone Variants H2A.X and H2B.0 Induce Unfolding of 195b.p. DNA from Nucleosomes -J.M. Gatewood, B.S. Imai, J. Siino, S. Krueger and P. Yau

SAXS Measurements on H4 Acetylated and Nonacetylated 195 b.p. Nucleosomes -B.S. Imai, J.M. Gatewood, J.S. Siino, Jr., S. Krueger, P.M. Yau, E.M. Bradbury and G. McConnell

8012B An X-ray Absorption Spectroscopic Study of Pyrococcus furiosus Rubredoxin —G.N. George, I.J. Pickering, R.C. Prince, M.W.W. Adams and Z.H. Zhou

8012B Constrained EXAFS Refinement of the Core Dimensions of the 3-Iron-Sulfur Ferredoxin from Pyrococcus furiosus - G.N. George, I.J. Pickering, R.C. Prince, M.W.W. Adams and Z.H. Zhou

8014B Preliminary.X-ray Crystallographic Analysis of Tritrichomoñas foetus Inosine-5'Monophosphate Dehydrogenase -F.G. Whitby, J. Huete-Perez, H. Luecke and C.C. Wang

8014B The Crystal Structure of Bovine $\gamma \mathrm{D}$-Crystallin at $1.65 \AA$ Resolution - D.K. Wilson, H. Luecke and F.A. Quiocho

8014B Very High Resolution Data for Phosphate-Binding Protein -H. Luecke and F.A. Quiocho

8054B Laue Diffraction Experiments on Phosphoglycerate Kinase - T. McPhillips, M. Stowell, D. Rees, S.M. Soltis and R.P. Phizackerley 
8110B Reductive Complexation of Vanadate by Tunichrome: An X-ray Absorption Spectroscopy Study -P. Frank, D. Ryan, K.B. Grant, K. Nakanishi and K.O. Hodgson

8110B Studies of Lysozyme Folding by Synchrotron Small-Angle X-ray Scattering -L. Chen, S. Doniach and K.O. Hodgson

8110B Vanadium in Blood Cells from Ascidia ceratodes: A Survey Using X-ray Absorption Spectroscopy -P. Frank, K. Kustin, W.E. Robinson, L. Linebaugh and K.O. Hodgson

8116B Angle Dependence of Multiple-Scattering Effects in EXAFS as Seen in Cytochrome Oxidase Analogues -H.H. Zhang, M.J. Scott, S.C. Lee, B. Hedman, R.H. Holm and K.O. Hodgson Using X-ray Absorption Spectroscopy - T.E. Westre, K.E. Loeb, J.M. Zaleski, B. Hedman, K.O. Hodgson and E.I. Solomon

9911B Electronic and Magnetic Properties of Iron Atoms in Nitrogenase: Fe L-edge Absorption Studies Using Linear and Circular Polarized Light - J. Chen, C.Y. Ralston, Z.-H. Zhou, M.W.W. Adams, B. Xia, J.L. Markely, B.A. Feinberg, R.C. Tittsworth, B.J. Hales and S.P. Cramer 


\begin{tabular}{|c|c|c|}
\hline & & Page \\
\hline $\begin{array}{l}2103 \mathrm{Vp} / \\
9700 \mathrm{~V}\end{array}$ & $\begin{array}{l}\text { Angle Resolved Photoemission Spectroscopy (ARPES) Study of MBE-grown } \\
\mathrm{Bi}_{2} \mathrm{Sr}_{2} \mathrm{CaCu}_{2} \mathrm{O}_{8-\delta} \text { Thin Films -D.S. Marshall, D.S. Dessau, D.M. King, C.-H. Park, } \\
\text { A.Y. Matsuura, Z.-X. Shen, W.E. Spicer, J.N. Eckstein and I. Bozovic }\end{array}$ & 367 \\
\hline $\begin{array}{l}2103 \mathrm{VP} / \\
9700 \mathrm{~V}\end{array}$ & $\begin{array}{l}\text { Development of a Spin-Polarized Inverse Photoemission System -S.L. Friedman, } \\
\text { P.J. White, Z.-X. Shen and J. Hurst }\end{array}$ & 370 \\
\hline $\begin{array}{l}2103 \mathrm{Vp} / \\
9700 \mathrm{~V}\end{array}$ & $\begin{array}{l}\text { Low Energy Excitations and Symmetry of the Order Parameter in High-T } \\
\text { Superconductors - D.S. Dessau, Z.-X. Shen, D.M. King, D.S. Marshall, A.G. Loeser, } \\
\text { C.H. Park, A. Matsuura, W.E. Spicer and A. Kapitulnik }\end{array}$ & 372 \\
\hline $\begin{array}{l}2103 \mathrm{Vp} / \\
9700 \mathrm{~V}\end{array}$ & $\begin{array}{l}\text { Photoemission Studies of High-T } T_{c} \text { Superconductors -D.M. King, Z.-X. Shen, } \\
\text { W.E. Spicer, D.S. Dessau and B.O. Wells }\end{array}$ & 375 \\
\hline $2145 \mathrm{~V}$ & $\begin{array}{l}\text { The Interaction of Propylene and Oxygen with } \mathrm{Cu}_{2} \mathrm{O}(111) \text { : A Study of } \\
\text { Heterogeneous Oxidation Catalysis - B. Reitz, P. Kennepohl and E.I. Solomon }\end{array}$ & 379 \\
\hline $2199 \mathrm{Vp}$ & $\begin{array}{l}\text { Angle Resolved Photoemission on Quasi-Low Dimensional Materials } \\
\text {-G.-H. Gweon, R. Claessen, F. Reinert, W.P. Ellis, J.W. Allen and Z.-X. Shen }\end{array}$ & 382 \\
\hline $2202 \mathrm{Vp}$ & $\begin{array}{l}\text { Test of XMCD Sum Rules with Total Electron Yield Measurement } \\
\text {-R. Nakajima, J. Stöhr, D. Weller and M. Samant }\end{array}$ & 386 \\
\hline $2213 \mathrm{Vp}$ & $\begin{array}{l}\text { Surface EXAFS and X-ray Standing Wave Study of the Cleaved CaO(100) and } \\
\text { MgO(100) Surfaces - T. Kendelewicz, P. Liu, W.B. Labiosa and G.E. Brown, Jr. }\end{array}$ & 388 \\
\hline $2233 \mathrm{~V}$ & $\begin{array}{l}\text { Correlation of Multiple Scattering Features in the K-edge XANES Spectra of Al } \\
\text { and Si to the Al-O-Si Bond Angle in Aluminosilicate Sodalites: An Empirical } \\
\text { Study -M. Fröba, J. Wong, P. Behrens, P. Sieger, M. Rowen, T. Tanaka, Z. Rek and } \\
\text { J. Felsche }\end{array}$ & 390 \\
\hline $2233 \mathrm{~V}$ & $\begin{array}{l}\text { SiK XANES Spectroscopic Investigations of Mesoporous M41S Silica Phases } \\
-\mathrm{M} \text {. Fröba, B. Behrens, J. Wong, C.H. Haggenmüller, G. van de Goor, M. Rowen, } \\
\text { T. Tanaka and W. Schwieger }\end{array}$ & 394 \\
\hline $2243 \mathrm{Vp}$ & $\begin{array}{l}\text { Magnetic Circular Dichroism in Soft X-ray Emission of Fe and Co Excited by } \\
\text { Monochromatic Circularly-Polarized X-rays -L.-C. Duda, J. Stöhr, M.G. Samant, } \\
\text { D.C. Mancini, N. Wassdahl, A. Nilsson, P. Kuiper and J. Nordgren }\end{array}$ & 398 \\
\hline $8058 \mathrm{~V}$ & $\begin{array}{l}\text { Surface Science Study of Negative Electron Affinity Formation on GaAs with Cs } \\
\text { and } \mathrm{NF}_{3}-\mathrm{R} \text {. Cao, H. Tang and P. Pianetta }\end{array}$ & 402 \\
\hline $8119 \mathrm{~V}$ & $\begin{array}{l}\text { Direct Observation of a Narrow Band Near the Gap Edge of FeSi -C.-H. Park, } \\
\text { Z.-X. Shen, A.G. Loeser, D.S. Dessau, D. Mandrus, A. Migliori, J. Sarrao and Z. Fisk }\end{array}$ & 405 \\
\hline $9053 \mathrm{~V}$ & $\begin{array}{l}\text { Imaging of Hydrated Biological Specimens with a New Type of Soft X-ray } \\
\text { Microscope }- \text { G. Hirsch }\end{array}$ & 409 \\
\hline $9105 \mathrm{~V}$ & $\begin{array}{l}\text { E Versus } \mathrm{k} \text { Relations and Many Body Effects in the Model Insulating Copper } \\
\text { Oxide } \mathrm{Sr}_{2} \mathrm{CuO}_{2} \mathrm{Cl}_{2}-\mathrm{B} \text {.O. Wells, Z.-X. Shen, A. Matsuura, D.M. King, } \\
\text { M.A. Kastner, M. Greven and R.J. Birgeneau }\end{array}$ & 412 \\
\hline & & 49 \\
\hline
\end{tabular}



M. Rice and R. Carr

9126V/ Characterization of Alkyl Monolayers on $\mathrm{Nh}_{4} \mathrm{~F}$ Etched si(111) - J. Terry,

9901V Linear and Circular Dichroism in Angle Resolved Fe 3p Photoemission

-E. Tamura, G.D. Waddill, J.G. Tobin and P.A. Sterne

9933V Magnetic X-ray Circular Dichroism in Nickel-Gold Multilayers -A.F. Jankowski, 427 G.D. Waddill and J.G. Tobin 
EXPERIMENTAL PROGRESS REPORTS INDEXED BY SPOKESPERSON

\begin{tabular}{|c|c|c|c|c|c|}
\hline SPOKESPERSON & PROPOSAL & PAGE & SPOKESPERSON & PROPOSAL & PAGE \\
\hline \multirow[t]{2}{*}{ Acrivos, J.V. } & $2242 \mathrm{M}$ & 160 & Klein, M.P. & $2190 \mathrm{Bp} / 2278 \mathrm{~B}$ & 308 \\
\hline & $2300 \mathrm{M}$ & 213 & Kortright, J.B. & $9121 \mathrm{~V} / 8115 \mathrm{~V}$ & 415 \\
\hline Alivisatos, A.P. & $9912 \mathrm{M}$ & 253 & Laderman, S.S. & $9200 \mathrm{M}$ & 241 \\
\hline Allen, J.W. & $2199 \mathrm{Vp}$ & 382 & Lay, P.A. & $2260 \mathrm{Bp}$ & 327 \\
\hline Antonio, M.R. & 2219Mp & 130 & Lippard, S.J. & $2 \mathrm{~A} 50 \mathrm{~B}$ & 265 \\
\hline Arthur, J. & $8010 \mathrm{M}$ & 221 & Lucas, C.A. & $2245 \mathrm{Mp}$ & 162 \\
\hline Berry, E.A. & 2A27B & 260 & Luecke, H. & $8014 \mathrm{~B}$ & $343,345,346$ \\
\hline Bilello, J.C. & $2165 \mathrm{Mp}$ & $81,87,91$ & Luo, M. & $2 \mathrm{~A} 53 \mathrm{~B}$ & 267,268 \\
\hline Blackburn, N.J. & $2227 \mathrm{Bp}$ & $312,313,314$ & Lytle, F.W. & $2189 \mathrm{Mp}$ & 106 \\
\hline Blanchard, D.L. & $2268 \mathrm{Mp}$ & 188 & & $2283 \mathrm{Mp}$ & 202 \\
\hline Boyce, J.B. & $2138 \mathrm{Mp} / 9905 \mathrm{M}$ & 61,65 & Marcovic, N.N. & $2252 \mathrm{Mp}$ & 168 \\
\hline Bravman, J.C. & $2194 \mathrm{Mp}$ & 111 & McKay, D.B. & $2 \mathrm{~A} 67 \mathrm{~B}$ & 276 \\
\hline Brennan, S. & $8001 \mathrm{M}$ & 215,218 & & $2240 \mathrm{Bp}$ & 316 \\
\hline Breunig, T.M. & $2229 \mathrm{Mp}$ & 152 & Mendelson, $\mathrm{R}$. & $2277 \mathrm{~B}$ & 336 \\
\hline \multirow[t]{2}{*}{ Brown, Jr, G.E. } & $2143 \mathrm{Mp}$ & 70 & Merritt, E.A. & $2 \mathrm{~A} 59 \mathrm{~B}$ & 270 \\
\hline & $2210 \mathrm{Mp}$ & 125 & Mondragon, A. & $2 \mathrm{~A} 14 \mathrm{~B}$ & 257 \\
\hline Burgess, B.K. & $2241 B$ & 317 & Nordgren, J.E. & $2243 \mathrm{Vp}$ & 398 \\
\hline $\mathrm{Cao}, \mathrm{R}$. & $8058 \mathrm{~V}$ & 402 & O'Day, P.A. & $1 \mathrm{E} 16 \mathrm{M}$ & 57 \\
\hline Clemens, B.M. & $2224 \mathrm{Mp}$ & $134,138,142,145$ & Penner-Hahn, J.E. & $2181 B p$ & 300 \\
\hline Conradson, S.D. & $2276 \mathrm{Mp}$ & 197 & & $2253 \mathrm{M}$ & 171 \\
\hline \multirow[t]{2}{*}{ Cramer, S.P. } & 2257Bp & 320,324 & & 2267B & 330 \\
\hline & $9911 \mathrm{~B}$ & 364 & Persson, I.N. & $2286 \mathrm{Mp}$ & 204,207 \\
\hline Crozier, E.D. & $9104 \mathrm{M}$ & 232 & Pickering, I.J. & $8013 \mathrm{M}$ & 224 \\
\hline David, P.R. & 2A54B & 269 & Qiu, X. & $2 \mathrm{~A} 72 \mathrm{~B}$ & 277 \\
\hline de Vos, A.M. & $2 \mathrm{~A} 61 \mathrm{~B}$ & 274 & Rayment, I. & 2A79B & 281 \\
\hline DeYoreo, J.J. & $2226 \mathrm{Mp}$ & 149 & Redford, S.M. & 2A87B & 287 \\
\hline Doniach, S. & $2187 \mathrm{Bp}$ & 306 & Rees, D.C. & 2A47B & 263 \\
\hline Fendorf, S.E. & $1 \mathrm{E} 12 \mathrm{M}$ & 53 & & 2167B & 299 \\
\hline Forest, K.T. & $2 A 29 B$ & 262 & Regan, M.J. & $2153 \mathrm{Mp}$ & 74 \\
\hline Freeman, H.C. & 2192Bp & 310 & Rek, Z.U. & $8103 \mathrm{M}$ & 227,229 \\
\hline Fuoss, P.H. & $2182 \mathrm{Mp}$ & 101 & Rodgers, D.W. & $2 \mathrm{~A} 74 \mathrm{~B}$ & 279 \\
\hline Gates, B.C. & $2298 \mathrm{M}$ & 208 & Rudeńko, G. & $2 A 83 B$ & 285 \\
\hline Gatewood, J.M. & 2273Bp & 333,334 & Russo, P.S. & $9108 \mathrm{M}$ & 234 \\
\hline George, G.N. & $8012 B$ & 337,339 & Shen, Z.-X. & $2103 \mathrm{Vp} / 9700 \mathrm{~V}$ & $367,370,372,375$ \\
\hline Gorbaty, M.L. & $9503 \mathrm{M}$ & 245 & & $8119 \mathrm{~V}$ & 405 \\
\hline Hackert, M.L. & $2 A 82 B$ & 282 & Shuh, D.K. & $2237 \mathrm{Mp}$ & 156 \\
\hline \multirow[t]{2}{*}{ Harrison, S.C. } & $2 \mathrm{~A} 62 \mathrm{~B}$ & 275 & Solomon, E.I. & $2145 V^{2}$ & 379 \\
\hline & $3 \mathrm{A01B}$ & 289 & Soltis, S.M. & $8054 \mathrm{~B}$ & 347 \\
\hline Hayes, K.F. & $2269 \mathrm{Mp}$ & 192 & Stock, S.R. & $2264 \mathrm{Mp}$ & 184 \\
\hline Hedman, B. & $8116 \mathrm{~B}$ & 357,360 & Stöhr, J. & $2202 \mathrm{Vp}$ & 386 \\
\hline Hendle, J. & $2 \mathrm{~A} 60 \mathrm{~B}$ & 272 & Templeton, D.H. & $2142 \mathrm{Mp}$ & 69 \\
\hline Hess, N.J. & $2212 \mathrm{Mp}$ & 129 & Terry, J.H. & $9126 \mathrm{~V} / 8104 \mathrm{~V}$ & 419 \\
\hline Hirsch, G. & $9053 V^{2}$ & 409 & Thayer, M.M. & $2 \mathrm{~A} 90 \mathrm{~B}$ & 288 \\
\hline \multirow[t]{2}{*}{ Hodgson, K.O. } & $2146 \mathrm{Bp}$ & 296 & Tobin, J.G. & $9901 \mathrm{~V}$ & 423 \\
\hline & $8110 \mathrm{~B}$ & $350,352,355$ & Traina, S.J. & $2261 \mathrm{Mp}$ & 180 \\
\hline Howe, R.F. & $9113 \mathrm{M}$ & 236 & Tsuruta, $\mathrm{H}$. & $8059 \mathrm{~B}$ & 348 \\
\hline Hudson, E.A. & $2256 \mathrm{Mp}$ & 177 & Varughese, K.I. & $2 A 77 B$ & 280 \\
\hline Huffman, G.P. & $2175 \mathrm{Mp}$ & 95,98 & Via, G.H. & $9500 \mathrm{M}$ & 244 \\
\hline Hufnagel, T.C. & $2208 \mathrm{M}$ & 122 & Warburton, W.K. & $2129 \mathrm{Bp}$ & 293 \\
\hline Ingalls, R.L. & $2193 \mathrm{Mp}$ & 109 & Waychunas, G.A. & $2157 \mathrm{M}$ & 78 \\
\hline Ipe, N.E. & $9127 \mathrm{M}$ & 239 & Weir, S.T. & $2249 \mathrm{Mp}$ & 165 \\
\hline Jacobson, A.J. & $2254 \mathrm{M}$ & 174 & Wells, B.O. & $9105 \mathrm{~V}$ & 412 \\
\hline Jankowski, A.F. & $9933 \mathrm{~V}$ & 427 & Wong, J. & $2233 \mathrm{~V}$ & 390,394 \\
\hline Johnson, E.R. & $2184 B$ & 304 & & $9902 \mathrm{M}$ & 248 \\
\hline Kendelewicz, T. & $2213 \mathrm{Vp}$ & 388 & Yoo, C.-S. & $9932 \mathrm{M}$ & 256 \\
\hline Kim, S.-H. & $3 \mathrm{~A} 02 \mathrm{~B}$ & 290 & Yu, K.M. & 2197Mp & 115 \\
\hline Kisker, D.W. & $2200 \mathrm{Mp}$ & 118 & & & \\
\hline
\end{tabular}




\title{
OXIDATION STATE OF SULFUR IN SOIL ORGANIC MATTER AND IRON SULFIDE OXIDATION PRODUCTS
}

\author{
Scott Fendorf and Matt Morra \\ Soil Science Division, University of Idaho. Moscow, ID 83844-2339
}

\section{Introduction}

Sulfur (S) is tremendously important in both agriculture and environmental quality. It is an integral part of protein and is therefore an essential element for life as we know it. Fundamental reactions in the biogeochemical S cycle are responsible for maintaining a constant supply of this element in biologically available forms. From the opposite perspective, $S$ has been the focus of intense interest because of its negative role in environmental quality. Oxidation of reduced forms of inorganic $S$, such

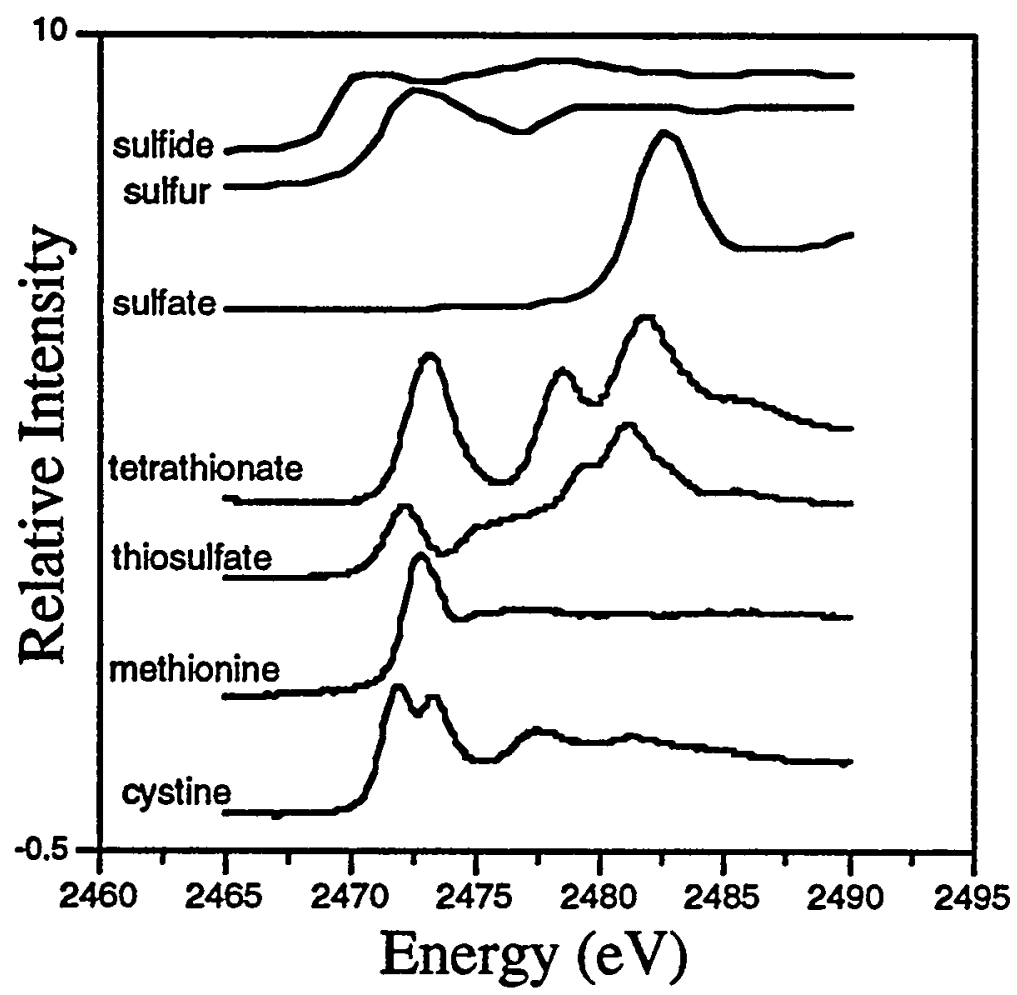

$\mathrm{kg} \mathrm{S} \mathrm{yr}^{-1}$ to the ocean in runoff (Stevenson, 1986) and contribute $5 \times 10^{9} \mathrm{~kg} \mathrm{~S} \mathrm{yr}^{-1}$ to the atmosphere (Brown, 1982). The internal S cycle facilitates mineralization of organic $S$ and redox changes of $S$ from +6 to -2 . The largest proportion of $S$ in most noncalcareous surface soil horizons is in organic forms. Organic soil $S$ is thought to consist of carbon-bonded $S$ in the form of amino acids and sulfonates, and esterbonded sulfates.

Our knowledge of the $S$ cycle in soil and the various compounds involved is severely limited by the relatively crude techniques used in measurement and speciation. Only indirect and destructive methods involving differential reduction of the various organic $S$ compounds to $\mathrm{H}_{2} \mathrm{~S}$ have been used to speciate soil $S$ (Freney, 1967; Tabatabai, 1982). Similar indirect methods to speciate organic $S$ in humic and fulvic acids have been reported (Keer et al., 1990; Vannier and Guillet, 1994). Pyrolytic methods for $S$ determination in humic substances and geologic materials (van Loon, 1993; Calkins et al., 1992), likewise provide only indirect evidence for precursor $S$ species.

$X$-ray absorption near edge structure (XANES) spectroscopy provides a means to directly determine $S$ oxidation states. This technique has been used to determine $S$ oxidation states in petroleum and coal Figure 1. X-ray absorption near edge structure (XANES) spectra of $S$ standards.

as iron sulfide, can lead to highly acidic environments that diminish water quality. Atmospheric deposition of acid forming $S$ compounds also contributes to the acidification of surface waters, but equally important is the potential acceleration of cation leaching in soils (Cronan et al., 1978; Turner et al., 1985).

The soil environment plays a key role in the global S cycle (Germida et al., 1992). Sulfur transformations within the soil environment result in the transport of $208: 10^{12}$ materials (George and Gorbaty, 1989; Gorbaty et al., 1990; Huffman et al., 1991; Waldo et al., 1991), biological systems (Frank et al., 1987; Frank et al., 1994), and marine sediments (Vairavmurthy et al., 1994). We studied S species in soils using XANES spectroscopy to help clarify the chemical and structural state of inorganic and organic $S$ in soils, increasing our understanding of the biogeochemical cycling of $S$ in the environment. 


\section{Soil Sulfur}

In a temperate soil more than $95 \%$ of the total soil sulfur is in the organic form (Tabatabai, 1982; Freney, 1967). Organic S can be further fractionated into that reduced to $\mathrm{H}_{2} \mathrm{~S}$ by hydriodic acid. Hydriodic acid reducible $S$ is mainly considered to exist in soils as ester bonded sulfate, although the identity of a portion of the $S$ recovered through this treatment is uncertain (Stevenson, 1982). Soil S not released by hydriodic acid is

assumed to be carbon-bonded $S$ (Stevenson, 1982). Raney nickel is used as the reductant to produce $\mathrm{H}_{2} \mathrm{~S}$ from carbon-bonded $\mathrm{S}$ existing in the form or amino acids. Some of the carbonbonded $S$ not released by Raney nickel apparently exists in the form of sulfonates (Autry and Fitzgerald, 1990; Strickland et al., 1986). Hydriodic acid reducible $S$ is thought to represent approximately $56 \%$ of the total organic $S$ fraction while the balance is carbon-bonded $S$ (Freney, 1967).

Acid hydrolysates of soil and extracted soil organic matter have yielded organic $S$ compounds identified as cystine, cysteic acid, methionine, methionine sulfoxide, and

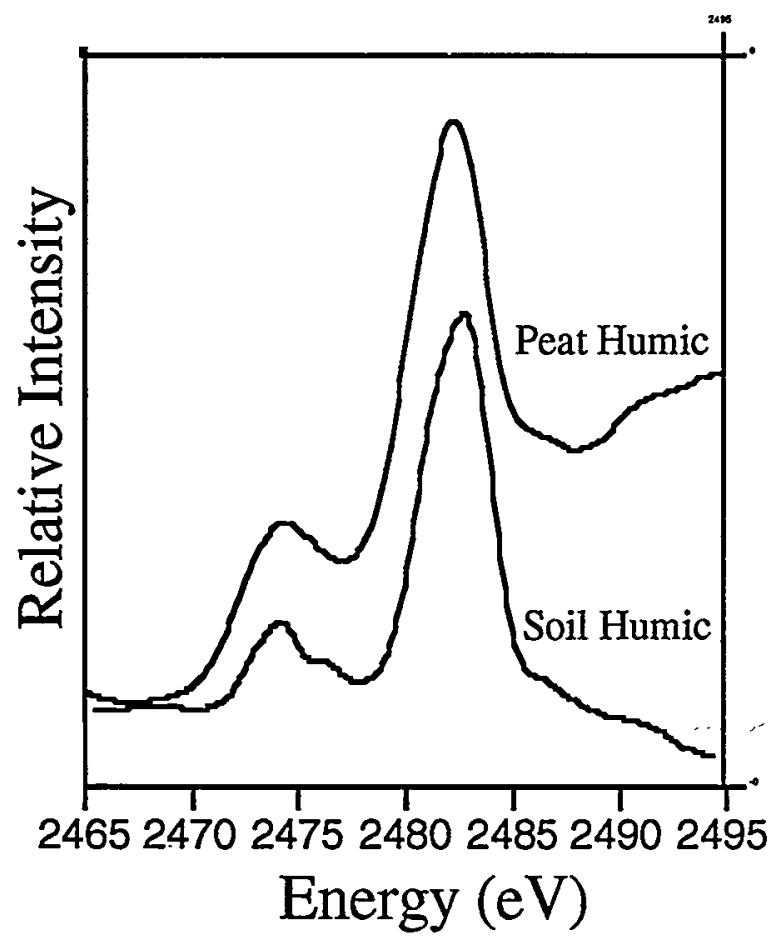

Figure 2. White-line positions in the XANES spectra of sulfur from humic materials indicates oxidation states of $+6.0(2482.2-2482.5 \mathrm{eV})$ and less than $+1.0(2474 \mathrm{eV})$. methionine sulfone (Freney and Stevenson, 1966). Hydrolysates of Scottish mineral soils and peats have shown that cysteine and methionine comprise from 19 to $31 \%$ of the carbon-bonded $S$ in mineral soils and $11 \%$ in peats (Scott et al., 1981). Analysis of selected Australian soils produced an estimate equal to $46 \%$ of the carbon-bonded $S$ (Freney et al., 1972). The ratio of cysteine to methionine was 2:1 for the Scottish mineral soils and 3:1 for the peat soils (Scott et al., 1981). The ratio for the Australian soils was approximately 1.6:1 (Freney et al., 1972).

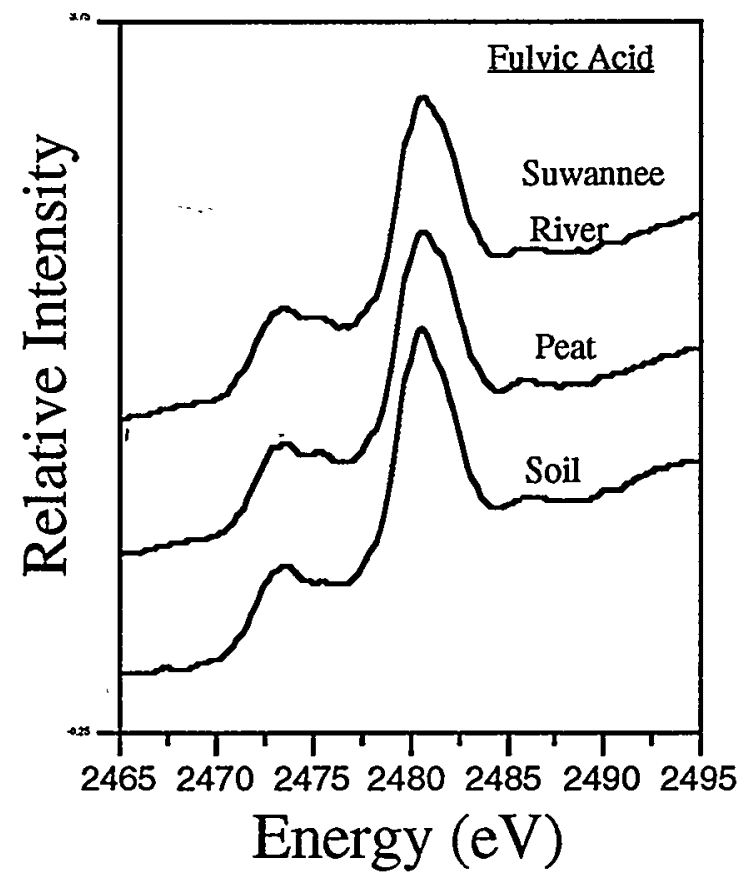

Figure 3. In fulvic acids, the white-line positions of the $S$ XANES spectra indicate oxidation $S$ species with oxidation states of +5.0 $(2480.4 \mathrm{eV})$ and between 0 and +2.0 (2473-2475 eV).

\section{Sulfur Speciation with XANES Spectroscopy}

The speciation of $S$ in soil solids has previously been studied using severely invasive techniques. Due to artifacts induced by these procedures, the actual state of $S$ within soils remains unclear. X-ray absorption near edge structure (XANES) spectroscopy circumvents many of these limitations, and offers information that is somewhat unique among the currently available spectroscopies. A useful linear relationship between the edge or white-line energy and oxidation state occurs for $S$ 
compounds (Frank et al., 1987; George and Gorbaty, 1989; Huffman et al., 1991; Waldo et al., 1991; Vairavamurthy et al., 1993, 1994). In fact, the intensity and energy dependence of white-line features has allowed for a quantitative determination of $S$ species in complex matrices such as heavy petroleums, petroleum source rock, sediments, and coal (George and Gorbaty, 1989; Waldo et al., 1991; Huffman et al., 1991; Vairavamurthy et al., 1994).

We used organic and inorganic $S$ compounds of known oxidation states to develop a relationship between the white-line energy position and $S$ oxidation state (Fig. 1). It is important to realize that the "apparent" oxidation states of organic S-containing compounds such as cysteine and methionine are not necessarily integers. For example, we confirmed oxidation states of +0.4 to +0.6 previously reported for cysteine, cystine, and methionine (Frank et al., 1987; Waldo et al., 1991).

Humic materials obtained from the International Humic Substances Society were placed directly into the sample holder of the spectrometer without any additional treatment. All spectra from the humic materials produced white-line peaks indicating the presence of $S$ in multiple oxidation states. Soil and peat humic acids produced white-lines indicative of $S$ in oxidation states of +6.0 and less than +1.0 (Fig. 2). Fulvic acids produced white-lines indicative of $S$ in oxidation states of +5.0 and species between 0 and +2.0 (Fig. 3). Leonardite humic acid produced white-lines suggesting the presence of $S$ in oxidation states of $+6.0,+5.0$, and at least two additional species ranging from 0 to +2.0 (Fig. 4). Suwannee River humic acid was dominated by $S$ in the +6.0 oxidation state, but also showed species with oxidation states ranging from 0 to +2.0 (Fig. 4).

We concluded that both the aquatic and soil humic acids contained $S$ in the +6.0 oxidation state; indicating the presence of esterbonded sulfates. In contrast, fulvic acids contained $S$ predominately in the +5.0 oxidation state. Species with this oxidation state likely exist as sulfonic acids. However, reduced forms of $S$ are also present in both humic and fulvic acids. Reduced $S$ in soil and peat humic acids with oxidation states between 0 and +1.0 probably represents amino acid $S$. Deconvolution techniques are necessary to further speciate and quantify $S$ in humic materials.

The valence states of $S$ in two soils were also investigated in preliminary studies using XANES spectroscopy. Dried clay-size fractions $(<2 \mu \mathrm{m})$ separated from the two soils as previously described (Morra et al., 1991a,b) were placed in the spectrometer without further alteration. Sulfur oxidation states were determined using the white-line positions observed in the respective soils. The predominant form of $S$ in both the Latahco and Morton soils had white-line peaks at energies near that of $\mathrm{SO}_{4}{ }^{2-}$ (data not shown). The presence of reduced $S$ species was indicated, but their low concentrations restricted further speciation.

The results obtained with XANES spectroscopy are valuable for definitively identifying sulfur compounds within soil materials. This information is needed for determining sulfur cycling within the environment.

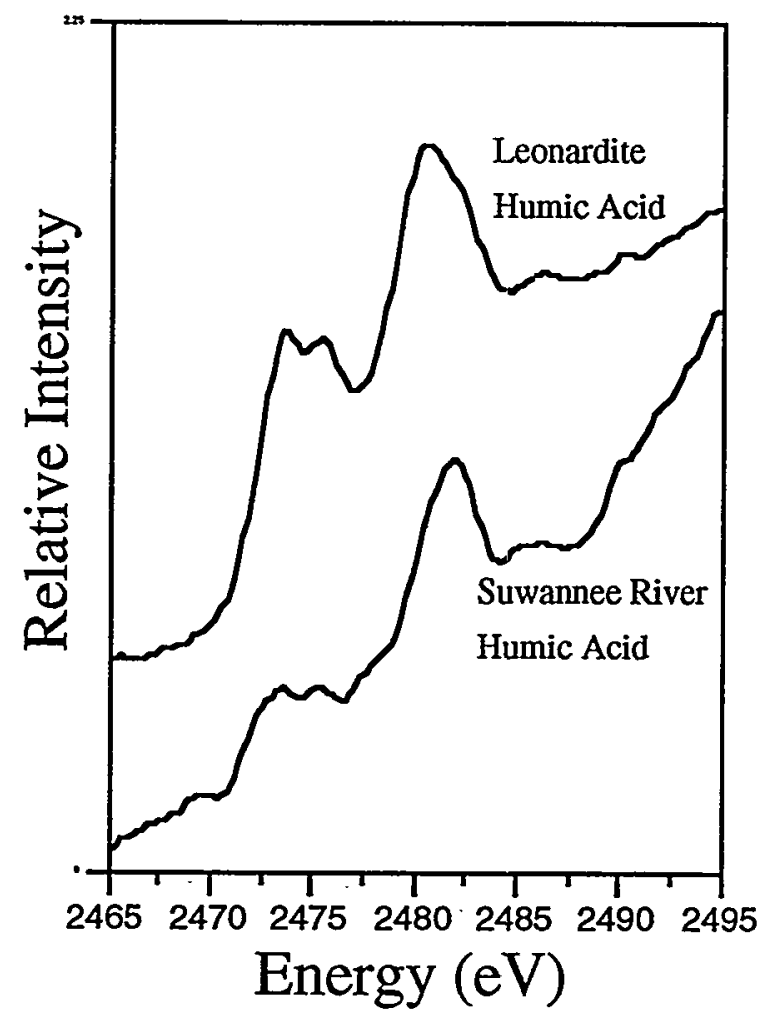

Figure 4. The energy position of the white-lines in Leonardite humic acid reveals oxidation states of $+6.0,+5.0$, and at least two additional species ranging from 0 to +2.0 . The spectra indicate that for Suwannee River humic acid an oxidized species of +6.0 is present in addition to species ranging between 0 and +2.0 . 


\section{References}

Autry, A.R., and J.W. Fitzgerald. 1990. Biol. Fertil. Soils 10: 50-56.

Brown, K.A. 1982. Environ. Pollut. 3:47-80.

Calkins, W.H., R.J. Torres-Ordonez, and B. Jung. 1992. Energy Fuels 6:411-413.

Cronan, C.S. W.A. Reiners, R.C. Reynolds, Jr. and G.E. Lang. 1978. Science 200: 309-311.

Frank, P., B. Hedman, R. M. K. Carlson, T.A. Tyson, A.L. Roe, and K.O. Hodgson. 1987. Biochemistry 26: 4975-4979.

Frank, P., B. Hedman, R. M. K. Carlson, and K.O. Hodgson. 1994. Inorg. Chem. Inorg. Chem. 33: 3794.

Freney, J.R. 1967. In A.D. McLaren and G.H. Peterson (ed.) Soil biochemistry. Marcel Dekker, New York. p. 229-259.

Freney, J.R., and F.J. Stevenson. 1966. Soil Sci. 101:307-316.

Freney, J.R., F.J. Stevenson, and A.H. Beavers. 1972. Soil Sci. 114:468-476.

George, G.N., and M.L Gorbaty. 1989. J. Am. Chem. Soc. 111: 3182-3186.

Germida, J. J., Wainwright, M. and Gupta, V. V. S. R. 1992. in Soil Biochemistry, vol. 7 (eds. Stotzky, G \& Bollag, J. M.) pp. 153 (Marcel Dekker, NY)

Gorbaty, M. L., G.N. George, and S.R. Kelemen. 1990. Fuel 69: 945-949.

Huffman, G.P., Mitra, S., Huggins, F. E., Shah, N., Vaidya, S. and Lu, F. 1991. Energy Fuels 5: 574-581.

Keer, J. I., R.G. McLaren, and R.S. Swift. 1990. Soil Biol. Biochem. 22: 97-104.

Morra, M. J., Blank, R. R., Freeborn, L. L. and Shafii, B. 1991a. Soil Sci. 152: 294-303.

Morra, M. J., Hall, M. H. and L. L. Freebom. 1991b. Soil Sci. Soc. Am. J. 55: 288-291.

Scott, N.M., W. Bick, and H.A. Anderson. 1981. J. Sci. Food Agric. 32:21-24.

Stevenson, F.J. 1982. Humus Chemistry. John Wiley \& Sons, New York.

Stevenson, F.J. 1986. Cycles of soil. John Wiley \& Sons, New York.

Strickland, T.C., J.W. Fitzgerald, and W.T. Swank. 1986. Can. J. For. Res. 16: 549553.

Tabatabai, M. A. 1982. In A.L. Page, R.H. Miller, and D. Keeney (Eds.) Methods of Soil Analysis, Part 2. ASA, SSSA, Madison, WI. pp. 501-538.

Turner, R.S., A.H. Johnson, and D. Wang. 1985. J. Environ. Qual. 14: 314-323.

Vairavamurthy, A., B. Manowitz, G. W. Luther, III, and Y. Jeon. 1993. Geochim. Cosmochim. Acta 57: 1619-1623.
Vairavamurthy, A., W. Zhou, T. Eglinton, and B. Manowitz. 1994. Geochim. Cosmochim. Acta 58: $4681-4687$.

van Loon, W.M.G.M., J.J. Boon, and B. de Groot. 1993. Environ. Sci. Technol. 27: 2387-2396.

Vannier, C., and B. Guillet. 1994. Soil Biol. Biochem. 26: 149-151.

Waldo, G. S., R.M.K. Carlson, J.M. Moldowan, K.E. Peters, and J.E. Penner-Hahn. 1991. Geochim. Cosmochim. Acta 55, 801-814. 
Trace Element Coordination in Natural Sediments at Ambient and Cryogenic Temperatures

\author{
Peggy A. O'Day ${ }^{1}$, Susan A. Carroll ${ }^{2}$, Glenn A. Waychunas ${ }^{3}$, and Brian Phillips ${ }^{4}$ \\ ${ }^{1}$ Dept. of Geology, Arizona State University, ${ }^{2} \mathrm{~L}-219$ Earth Sciences Division, Lawrence \\ Livermore National Laboratory, ${ }^{3}$ Center for Materials Research, Stanford University, and \\ ${ }^{4}$ Dept. of Chemical Engineering and Material Science, UC Davis
}

\section{Abstract}

The molecular coordination of trace levels of $\mathrm{Zn}$ (1-2 wt. \%) in natural sediments from an acid mine drainage and of $\mathrm{V}, \mathrm{Cr}$, and $\mathrm{Mn}$ (10-300 ppm) from weathered hydrothermal soils was examined by synchrotron X-ray absorption spectroscopy (XAS) at ambient and cryogenic $(\approx 10 \mathrm{~K})$ temperatures. XAFS analyses of mine drainage samples show that, with increasing distance from the mine source, $\mathrm{Zn}$ coordination changes from that of a sulfide phase in its host mineral, sphalerite ( $\mathrm{ZnS})$, to coordination indicative of hydroxide phases. In samples with silica, carbonate, and Fe-hydroxide phases (3-54 wt.\% $\left.\mathrm{Fe}(\mathrm{OH})_{3}\right)$, the local atomic $\mathrm{Zn}$ environment in hydroxide phases varies with the total amount of bulk iron hydroxide, but $\mathrm{Zn}$ is not incorporated into carbonate or silica phases. Analysis of pre-edge and $\mathrm{K}$-edge spectral features of very low concentrations of $\mathrm{V}, \mathrm{Cr}$, and $\mathrm{Mn}$ in kaolinite-rich soils from hydrothermal areas indicates reduced valence states for these ions $\left(\mathrm{V}^{3+}, 4+, \mathrm{Cr}^{3+}, \mathrm{Mn}^{2+}\right)$. These soils, now present at oxidizing surface conditions, were derived from extreme alteration of volcanic rocks. The XAS data suggests trapping of metal ions under paleoreducing conditions during clay formation. This work demonstrates the utility of XAS for identifying the coordination sites of elements of environmental interest at low concentrations in untreated natural materials containing complex mixtures of mineral phases.

\section{Introduction}

Hazard assessment and remediation of toxic metal contamination in natural soils and sediments is often difficult because little is known about how metals are incorporated into solid phases. Metals may be mobilized or retarded depending upon their mode of incorporation in the solid. The application of XAS to determining local metal ion bonding in natural soils and sediments is complicated by the presence of multiple solid phases, amorphous and disordered phases, low concentrations of metal ions, and multiple metal ion absorbers. In this study, we examined the feasibility of using XAFS and XANES to obtain structural information about the local atomic coordination of trace metals in crystalline and amorphous phases in untreated, complex natural sediments. We analyzed low-temperature XAFS spectra of $\mathrm{Zn}$ in sediments from an acid mine drainage area and ambient-temperature XANES spectra of $\mathrm{V}, \mathrm{Cr}$, and $\mathrm{Mn}$ in weathered hydrothermal soils [1].

\section{Experimental}

Zinc-bearing sediments ( $1-2 \mathrm{wt} . \% \mathrm{Zn}$ ) were collected from the U.S. tri-state mining district (KS, $\mathrm{MO}, \mathrm{OK})$. Clay-rich soils containing 10-300 npm V, $\mathrm{Cr}$, and $\mathrm{Mn}$ were collected from hydrothermal vent areas in central Iceland. Absorption spectra for dried, untreated solid soils and sediments were collected at SSRL on beam line II-3 at cryogenic temperature $(\approx 10 \mathrm{~K})$ and on beam line IV-1 at ambient temperature ( $3 \mathrm{GeV}, 40-90 \mathrm{~mA}$ ). Lightly crushed, undiluted samples were loaded into $1 \mathrm{~mm}$ thick teflon or cardboard sample holders and sealed with Mylar film. Zinc K-edge and EXAFS spectra (9400 - 10,700 eV; $\mathrm{k} \approx 3$ to $12-15 \AA^{-1}$ ) were collected at low $\mathrm{T}$ using an unfocused $\mathrm{Si}(220)$ monochromator crystal and a StermHeald-type fluorescence detector. Vanadium $(5465 \mathrm{eV})$, $\mathrm{Cr}(5990 \mathrm{eV})$, and $\mathrm{Mn}(6540 \mathrm{eV})$ absorption edges were collected at ambient temperature using an unfocused $\mathrm{Si}(111)$ monochromator crystal and a SternHeald-type fluorescence detector. Solid model 
compounds were diluted with inert $\mathrm{B}(\mathrm{OH})_{3}$ to produce $\approx 30 \%$ transmission of the incoming beam. Both transmission (using $\mathrm{N}_{2}$ - and Ar-filled ion chambers) and fluorescence absorption spectra were collected for model compounds. Energy calibration was obtained by simultaneous collection of a metal foil spectrum for the element of interest. Harmonic rejection was achieved by detuning the incoming beam by $30-50 \%$ of maximum intensity. Depending on absorber concentration, 3-16 scans were collected and averaged for each sample. Data reduction and analysis was done with the EXAFSPAK programs (G. George, SSRL). For Zn XAFS analysis, reference phase-shift and amplitude functions were generated using FEFF6 [2] based on atomic clusters of known crystal structures $\left(\mathrm{Zn} \mathrm{E} \mathrm{E}_{0}=9670 \mathrm{cV}\right.$ ). Theoretical FEFF functions were fit to experimental XAFS data for $\mathrm{Zn}$ model compounds to determine values for $\sigma^{2}$ and $S_{0}^{2}$. FEFF6 was used for XANES analysis of $\mathrm{V}, \mathrm{Cr}$, and $M n$ edges and $V$ and $M n$ reference compounds.

\section{Results}

Zn-Bearing Samples: Analyses of XAFS spectra of $\mathrm{Zn}$ unknown sediment samples, precipitated Zn-hydroxides, and known model compounds show that $\mathrm{Zn}$ does not form pure, known carbonatc or hydroxide phases in the acid mine samples, nor does it appear to sorb to quartz surfaces (Fig. 1). In sediments near the mine source, $\mathrm{Zn}$ is present primarily in sphalerite $(\mathrm{ZnS})$ as indicated by $\mathrm{S}$ and $\mathrm{Zn}$ backscatterers at 2.32 and $3.83 \AA$, respectively. With increasing distance from the source, $\mathrm{Zn}$ tetrahedral coordination by oxygen $(R=1.97-2.00 \AA$ ) is apparent in the spectra along with second-neighbor backscattering from $\mathrm{Fe}$ and/or $\mathrm{Zn}$ at distances shorter than $\mathrm{Zn}-\mathrm{Zn}$ backscattering observed in $\mathrm{ZnS}$ (Fig. 2). Due to similarities in the phase-shift and amplitude backscattering functions of second-neighbor $\mathrm{Zn}$ and $\mathrm{Fe}$, we cannot yet determine whether the backscattering at $\approx 3.3-3.6 \AA$ is from $\mathrm{Fe}, \mathrm{Zn}$, or a mixture of both $\mathrm{Fe}$ and $\mathrm{Zn}$. The position and intensity of spectral features attributed to second-neighbor $\mathrm{Zn}$ and/or Fe change with bulk $\mathrm{Fe}(\mathrm{OH})_{3}$ concentration in different samples.

Analyses of these sediments by XRD indicatcs that the primary crystalline phases are quart\%

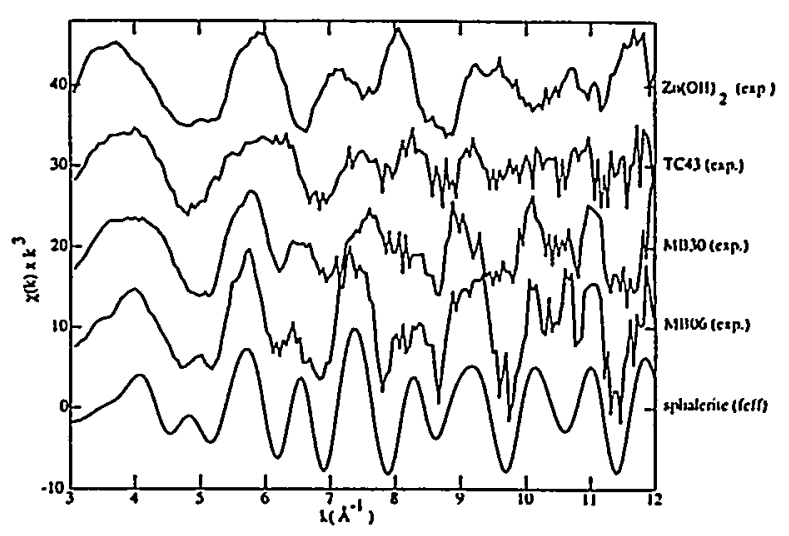

Fig. 1. Normalized XAFS spectra of $\mathrm{Zn}$ acid-mine sediments (MB06, MB30, TC43) compared to spectra of precipitated $\mathrm{Zn}$-hydroxide $\left(\mathrm{Zn}(\mathrm{OH})_{2}\right)$ and sphalerite (ZnS; theoretical spectra calculated with FEFF).
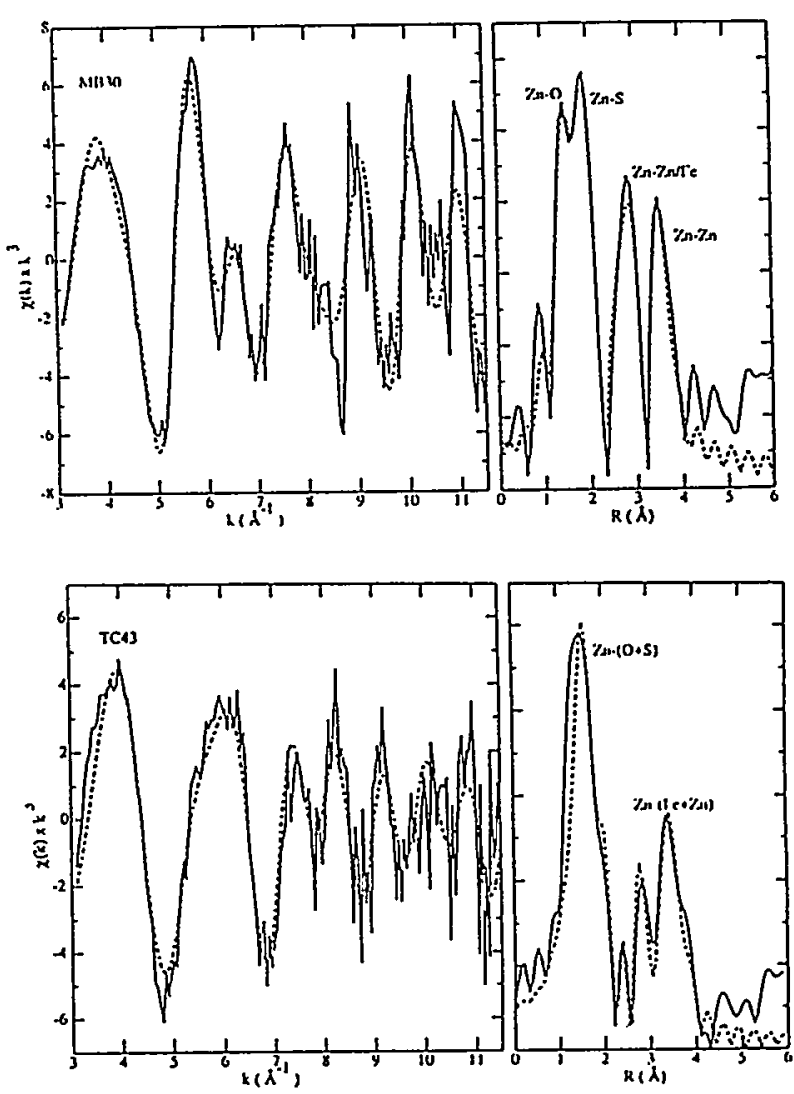

Fig. 2. Best fits (dashed lines) to experimental spectra (solid lincs) for sample MB30 (top) and sample TC43 (bottom). Fits indicatc $\mathrm{O}$ and $\mathrm{S}$ first-ncighbor backscatterers and $\mathrm{Zn}$ and/or Fe second-neighbor backscatterers, suggesting a mixture of $\mathrm{Zn}$ coordination environments. 
$\left(\alpha-\mathrm{SiO}_{2}\right)$, calcite $\left(\mathrm{CaCO}_{3}\right)$ or dolomite $\left(\mathrm{CaMg}\left(\mathrm{CO}_{3}\right)_{2}\right)$, goethite $(\alpha-\mathrm{FeOOH})$, and sphalerite $(\mathrm{ZnS})$. In addition, sample TC43 contains a large percentage of amorphous Fe-hydroxide [3, 4]. Calculation of the thermodynamic saturation state of waters in contact with the sediments indicates near- or over-saturation with respect to calcite and quartz, and undersaturation with respect to pure $\mathrm{Zn}$-carbonate, $\mathrm{Zn}$ hydroxide, and $\mathrm{Zn}$-sulfate phases $[3,4]$. There is no evidence in the XAFS spectra, however, for $\mathrm{Zn}$ coordination in carbonate phases or association with $\mathrm{Si}$ phases. The coexisting waters are also highly oversaturated with respect to crystalline Fe-oxide (goethite, hematite) and amorphous Fe-hydroxide $\left(\mathrm{Fe}(\mathrm{OH})_{3}(\mathrm{am})\right)$ phases. The XAFS spectra support the hypothesis that $\mathrm{Zn}$, as it weathers from the host ore $(\mathrm{ZnS})$, is scavenged primarily by Fe-oxide and/or -hydroxide phases, although we cannot yet determine whether the Fe phase is crystalline or amorphous, or a solid-solution of $\mathrm{Zn}$ and $\mathrm{Fe} .[1,3]$.

Hydrothermal Soils: Analysis of the XANES spectra of $\mathrm{V}-, \mathrm{Cr}-$, and $\mathrm{Mn}$-bearing hydrothermal clays indicates that these metals are present in reduced oxidation states $\left(\mathrm{V}^{3+}, 4+, \mathrm{Cr}^{3+}, \mathrm{Mn}^{2+}\right)$ (Fig. 3).

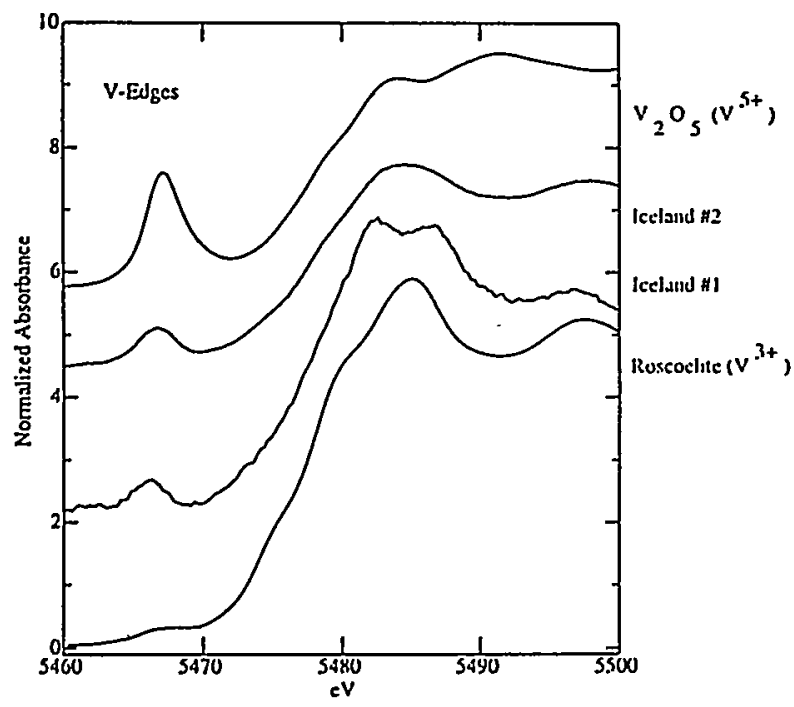

Fig. 3. V K-edge spectra of Iceland soil samples compared to $\mathrm{V}_{2} \mathrm{O}_{5}\left(\mathrm{~V}^{5+}\right)$ and roscoelite $\left(\mathrm{KAIV}_{2} \mathrm{SiO}_{10}(\mathrm{OH})_{2} ; \mathrm{V}^{3+}\right.$ ) model compounds. Preliminary modeling results suggest mixed $\mathrm{V}$ oxidation states (probably $3+$ and $4+$ ) in the soils.
Analysis of EXAFS spectra was not possible for $\mathrm{V}$ and $\mathrm{Cr}$ due the very low concentrations of these metals and interference in the EXAFS region from $\mathrm{Cr}$ and $\mathrm{Mn}$ $\mathrm{K}$-edges and $\mathrm{L}$ II,II -edges of trace concentrations of $\mathrm{Ba}$. Analyses of Mn-EXAFS would have been possible except for very large monochromator glitches in the Mn-EXAFS region. Comparison of the soil sample spectra to that of reference oxide compounds shows no large pre-edge features that would indicate oxidized cations. Edge features are similar to those observed in oxide model compounds with reduced cations $[5,6]$. These soils were derived from extreme alteration of volcanic rocks and are now present at the surface under very oxidizing conditions. The XAS data suggests trapping of metal ions under paleo-reducing conditions during clay formation. The oxidation state and coordination of $\mathrm{V}, \mathrm{Cr}$, and $\mathrm{Mn}$ in these clays serve as indicators of changes in groundwater redox chemistry during soil formation. Further studies of the coordination of these ions in or on clays may provide information about long-term metal ion mobility.

\section{Conclusion}

$\mathrm{X}$-ray absorption spectroscopy can provide valuable information on the local atomic coordination of environmentally-sensitive trace elements in natural soils and sediments at low concentrations. In $\mathrm{Zn}$-bearing mine drainage sediments, $\mathrm{Zn}$ liberated by weathering of $\mathrm{ZnS}$ is associated primarily with $\mathrm{Fe}$-oxides and/or hydroxide phases rather than carbonate and silica phases as might have been predicted from thermodynamic analyses of coexisting waters. $\mathrm{Zn}$ coordination is sensitive to the bulk $\mathrm{Fe}(\mathrm{OH})_{3}$ concentration in the sediment. In hydrothermallyaltered clay-rich soils, reduced metals are apparently trapped in clays during their formation under reducing conditions and strongly retained despite subsequent weathering under highly oxidizing conditions. We find that accurate determination of metal coordination in multicomponent spils and sediments from XAFS requires analysis of a variety of known experimental reference compounds as well as quantitative fitting with theoretical reference functions from FEFF. Data collection at low $T(\approx 10 \mathrm{~K})$ greatly enhanced spectral resolution, especially for atomic backscattering beyond the first coordination shell. 
Acknowledgments

We thank J. J. Rehr for access to and help with FEFF6. PAO acknowledges support from the U.S. National Science Foundation Earth Sciences post-doctoral fellowship program. Financial support was provided by the Institute of Geophysics and Planetary Physics (IGPP) at LLNL.

[1] O'Day, P. A. et al., Physica B (1994) in press.

[2] J. J. Rehr et al., Phys. Rev. Let. 69 (1992) 3397.

[3] Carroll, S. A. et al., Geol. Soc. Amer. Annual Meeting (abstr.), Seattle (1994).

[4] Piechowski, M. and Carroll, S. A., 207th American Chemical Society National Meeting (abstr.), San Diego (1994).

[5] J. Wong et al., Phys. Rev. B 30 (1984) 5596.

[6] J. Garcia et al., J. Physique 47 C8 (1986) 49. 


\title{
Evidence of negatively correlated displacements of the axial $\mathrm{O}(2)$ atom and the $\mathrm{Cu}$ and $\mathrm{Ba}$ atoms in $\mathrm{HgBa}_{2} \mathrm{CuO}_{4}$ from XAFS
}

\author{
F. Bridges, ${ }^{(1)}$ C. H. Booth, ${ }^{(1)}$ E. Bauer, ${ }^{(1)}$ J. B. Boyce, ${ }^{(2)}$ T. Claeson, ${ }^{(3)}$ and C. H. $\mathrm{Chu}^{(4)}$ \\ (1) Department of Physics, University of California Santa Cruz, Santa Cruz, CA 95064 \\ ${ }^{(2)}$ Xerox Palo Alto Research Center (PARC), Palo Alto, CA 94304 \\ ${ }^{(3)}$ Physics Department, Chalmers University of Technology, S-41296 Gothenberg, Sweden \\ ${ }^{(4)}$ Texas Center for Superconductivity, University of Houston, Houston, Texas 77204
}

\section{INTRODUCTION}

Since the first high $T_{c}$ materials were discovered [1], a major question has remained unanswered what is the mechanism for the formation of Cooper pairs? The recently discovered $\mathrm{Hg}$ series of high $T_{c}$ compounds [2] provide yet another opportunity to address this question. The $\mathrm{Hg}$ series of high $T_{c}$ materials have the highest $T_{c}$ to date $(164 \mathrm{~K}$ for the three $\mathrm{CuO}_{2}$ layer material under hydrostatic pressure [3]). More importantly, they also have the largest change in $T_{c}$ with pressure [3] and consequently may be more sensitive to local structural distortions than most or all other superconductors. In addition, the one $\mathrm{CuO}_{2}$ layer compound, $\mathrm{Hg}$ $1201,\left(\mathrm{HgBa}_{2} \mathrm{CuO}_{4}\right)$ is a relatively simple structure (See Fig. 1 for definitions of sites); it has no chains as in the YBCO family and, although the structure is very similar to the $\mathrm{Tl}$ series, there is very little $\mathrm{O}$ in the $\mathrm{Hg}$ layer. Thus if superconductivity involves the $\mathrm{O}$ states it must occur within the $\mathrm{Cu}$ and/or $\mathrm{Ba}$ layers. Conventional wisdom assigns superconductivity to the $\mathrm{CuO}_{2}$ plane while the $\mathrm{BaO}$ and to a lesser extent the $\mathrm{Hg}$ layer provides a charge carrier reservoir.

Over the last five years there has been increasing evidence that structural distortions play an important role, and consequently models for superconductivity that involve the heavy-metal oxide layer (these include the polaronic models, ferro- or antiferroelectric coupling and charge transfer models) have received increased attention (for a review, see Ref. [4]). In this report we present XAFS measurements that can be viewed as evidence for a negative correlation in the displacements of the $\mathrm{Cu}$ and axial $\mathrm{O}(2)$ atoms, as well as for the $\mathrm{Ba}$ and $\mathrm{O}(2)$ atoms. These results can therefore be interpreted in terms of a local, quasistatic, antiferroelectric (or ferroelectric) order, or alternatively, in terms of a dynamic process in which the atoms are moving together or apart in a correlated manner. The large number of reports of small structural anomalies that occur near or at $T_{c}$ (often in the width of the atomic positions) $[5-7]$ or with oxygen concentration $[8,9]$ are suggestive that these anomalies, including the negative correlation we have observed, arise from a lattice coupling to the superconducting pairs and hence are dynamic. Such a coupling is not immediately obvious from diffraction measurements because diffraction necessarily only measures average positions and broadening factors with respect to the lattice. Other measurements which are more sensitive to correlated displacements, such as ion channeling and XAFS, are far more likely to find evidence of the anticorrelations suggested by the above models.

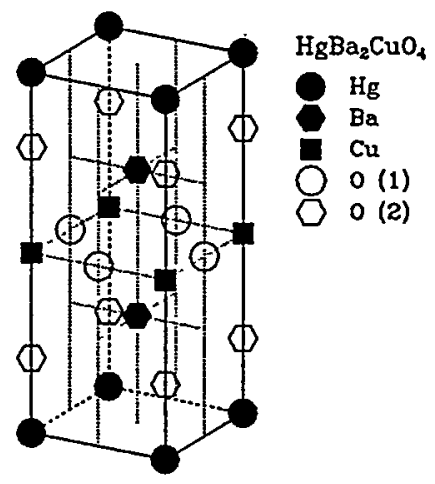

FIG. 1. Structure of $\mathrm{HgBa}_{2} \mathrm{CuO}_{4}$ ( $\mathrm{Hg}$ 1201).

\section{THE EXPERIMENT}

Transmission $\mathrm{x}$-ray absorption data were collected at $80 \mathrm{~K}$ for the $\mathrm{Cu}$ and $\mathrm{Ba} K$-edges and the $\mathrm{Hg} L_{3}$-edge on $\mathrm{Hg} 1201$ samples using $\mathrm{Si}(220)$ monochromator crystals at SSRL. Measurements were obtained for two powder samples with $T_{c}$ 's of $96 \mathrm{~K}$ (optimal $\mathrm{O}$ concentration) and $45 \mathrm{~K}$ (slightly $\mathrm{O}$ depleted). The XAFS sample preparation, data reduction and analysis procedures have been described in detail in other papers [10-12]. We note that for the $\mathrm{Ba}$ and $\mathrm{Hg}$ edges, there is significant structure in the background and we have used our iterative method [11] to remove it. Diffraction measurements indicated the sample was essentially sin- 
gle phase. Futher details of the diffraction study for this material will be provided in Ref. [13].

The Fourier transformed XAFS data and fits at the $\mathrm{Hg} L_{3}$-edge and the $\mathrm{Cu}$ and $\mathrm{Ba} \mathrm{K}$-edges for the $T_{c}=96 \mathrm{~K}$ sample are shown in Fig. 2. The $T_{c}=$ $45 \mathrm{~K}$ data and fits are similar. For the $\mathrm{Hg}$ edge the first peak corresponds to two $O$ neighbors while the multi-peak in the 3-4 $\AA$ range is the sum of the $\mathrm{Hg}-\mathrm{Ba}$ and $\mathrm{Hg}-\mathrm{Hg}$ contributions. Simple FEFF [14] simulations, using typical Debye-Waller factors and assuming little distortion, indicate that the amplitude of the $\mathrm{Hg}-\mathrm{Ba} / \mathrm{Hg}-\mathrm{Hg}$ peak is too small. This means that there is some disorder in one or both of these peaks. We have tried several models for this distortion, but have no yet found a model that accurately describes the data; including $7 \%$ oxygen at the $\mathrm{O}(3)$ defect site in the $\mathrm{Hg}$ layer as suggested by diffraction [2] does not appreciably change this result.

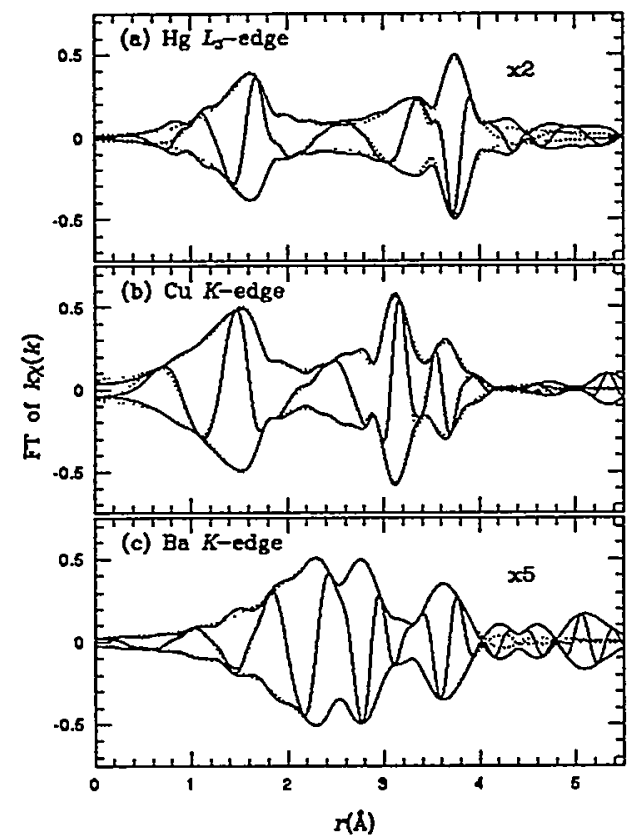

FIG. 2. Fourier transforms (FT) of $k \chi(k)$ from $\mathrm{Hg}$ $1201\left(T_{c}=96 \mathrm{~K}\right)$ at various edges. The top panel (a) is $\mathrm{Hg} L_{3}$-edge data (solid) and fit (dotted), both transformed from 3.5 to $16.6 \AA^{-1}$. The middle panel (b) is $\mathrm{Cu} K$-edge data (solid) and fit (dotted), both transformed from 3.5 to $12.5 \AA^{-1}$. The bottom panel (c) is $\mathrm{Ba} K$-edge data (solid) and fit (dotted), both transformed from 3.0 to $16.5 \AA^{-1}$. All transform windows are Gaussian broadened by $0.3 \AA^{-1}$.

For the $\mathrm{Cu}$ edge, the first peak corresponds to the four planar $O(1)$ atoms while the multi-peak from the 3-4 $\AA$ region arises from the four $\mathrm{Ba}$ and four planar $\mathrm{Cu}$ neighbors. The positions and amplitudes of these peaks are consistent with the structure obtained from diffraction studies on other samples $[2,15,16]$. However there should also be another $O$ peak in the XAFS spectra, near $2.2 \AA$ from the two axial $O(2)$ atoms. This peak is not obvious in the spectra shown in Fig. 2, which can be fit quite well without this peak. To explain its absence when several diffraction studies $[2,15,16]$ as well as our $\mathrm{Hg}$ $L_{3}$-edge data show this $\mathrm{O}(2)$ site to be fully occupied, requires a very broad $\mathrm{Cu}-\mathrm{O}(2)$ peak. A constrained fit requiring two $\mathrm{O}(2)$ neighbors, yields a very large Debye-Waller parameter (Table I).

The Ba K-edge data are similar to the $\mathrm{Cu} \mathrm{K}$ edge data in that the $\mathrm{Ba}-\mathrm{O}(1)$ peak and the $\mathrm{Ba}$ $\mathrm{Cu}$ peaks are consistent with the diffraction data while the $\mathrm{Ba}-\mathrm{Hg}$ is slightly broadened. However, the $\mathrm{Ba}-\mathrm{O}(2)$ peak with four $\mathrm{O}(2)$ neighbors at $\sim 2.8$ $\AA$ also appears to be missing. Again, a constrained fit yields a large Debye-Waller factor.

As mentioned in Sec. I, XAFS measurements of the broadening factors are fundamentally different than corresponding diffraction measurements. Diffraction measures the mean-square deviation of a given site from its average site with respect to the crystal. However, in XAFS we measure the broadening of a bond consisting of atoms $a$ and $b$ :

$$
\sigma_{a b}=\sqrt{\left\langle\left(r_{a}-r_{b}\right)^{2}\right\rangle},
$$

thus XAFS measures a correlated Debye-Waller factor. A comparison of the XAFS Debye-Waller parameters with the uncorrelated ones obtained in diffraction [13] for similar samples and measurement temperatures allows a measure of the degree of correlation of the displacements of the neighboring atoms. For atoms $a$ and $b$, diffraction gives the distributions for the positions of the two atoms independently (Debye-Waller parameters $(\sqrt{U})$ are $\sigma_{a}$ and $\sigma_{b}$ ), while XAFS measurements determine the broadening of the pair distance $\sigma_{a b}$. If we assume that the XAFS measurements of $\sigma_{a b}$ are absolute, we can define a correlation parameter $\phi$ by

$$
\sigma_{a b}^{2}=\sigma_{a}^{2}+\sigma_{b}^{2}-\sigma_{a} \sigma_{b} \phi
$$

If $\phi$ is near +1 we have a correlated width, such as we expect for well ordered materials at low temperatures; if $\phi$ is zero we have the uncorrelated result which often applies to more distant neighbors. However if there is anti-correlation, as for small offcenter displacements in an antiferroelectric system, then $\phi$ would be negative. The data in Table I clearly show that the $\mathrm{Cu}-\mathrm{O}(2)$ and $\mathrm{Ba}-\mathrm{O}(2)$ pairs are negatively correlated while the $\mathrm{Hg}-\mathrm{O}(2)$ pair is positively correlated. Together, these results suggest that the displacements of the $\mathrm{Cu}$ and $\mathrm{Hg}$ atoms may also be correlated. 
Measurements of $\phi$ should have fairly large systematic errors. For instance, the parameters $\sigma_{a b}$ must be determined absolutely; consequently, the standards used to fit the data must have well known Debye-Waller factors. We have recently shown that fits to the XAFS of model compounds using FEFF calculations of the XAFS function give values of $\sigma_{a b}$ that agree with the correlated-Debye model (the method used by the program) generally within $10 \%$ [12]. Another concern is that the samples used in the diffraction experiment are different than the ones used in the XAFS experiment. This is potentially a much more serious problem, since measurements of the width parameters by diffraction vary over a large range (in some cases by factors of $100 \%$ !). However, most of this variation is probably due to different fabrication techniques, etc. The values of $\sigma_{a}$ and $\sigma_{b}$ that we used to calculate $\phi$ were obtained from samples made with the same procedures and in the same laboratory as the samples used in the XAFS experiments. We therefore feel that there may be an absolute difference between the samples of $\lesssim 20 \%$ in the broadening factors. We have tried to reflect such systematic errors in Table I.

\section{DISCUSSION}

As noted above, we cannot determine from XAFS whether the displacements are static or dynamic, as XAFS is a very fast probe and the lattice is essentially frozen for times as short as the fluorescence lifetime. If the distortions were static one might expect well-defined off-center displacements as found in ferroelectrics; this would lead to a split nearest neighbor peak. However fits to a split peak rather than a broadened peak did not improve the fit. This means that either the magnitudes of the static displacements are random or that the atoms are moving, driven dynamically by some local interaction. We assume the latter. Then a charge hopping mechanism provides a simple model to explain both of the observed negative correlations, and may also provide the coupling for the formation of Cooper pairs. The $\mathrm{Hg}-\mathrm{O}(2)$ peak is narrow, which may be evidence that one of the electrons is tightly bound in the $\mathrm{Hg}-\mathrm{O}(2)$ bond. However, the second electron is shared between the $\mathrm{Cu}$ and the $\mathrm{Ba}$ atoms and may be weakly bound. If so it could hop off, forming a hole on the $\mathrm{O}(2)$ ion. This would reduce the attraction between the $\mathrm{O}(2)$ ion and the $\mathrm{Ba}$ and $\mathrm{Cu}$ ion neighbors. and these atom pairs would separate slightly. When the electron hops back onto the $O(2)$ ion the full attraction would be restored and the ions would move together. This would result in local negatively correlated displacements.

How can this hopping produce a coupling mechanism? Consider a hole moving in the $\mathrm{CuO}_{2}$ plane that approaches an electron on an $\mathrm{O}(2)$ site. The presence of the hole (say in the neighboring unit cell) induces the electron to hop into the $\mathrm{CuO}_{2}$ layer, either onto the $\mathrm{Cu}$ ion or into another hole in the plane. This negative charge will attract another hole in much the same way as a negative polarization of the lattice would in the usual phonon coupling model. Thus an electron hopping back and forth between the $\mathrm{O}(2)$ atom and a localized hole state in the $\mathrm{CuO}_{2}$ plane (which may extend for several unit cells and may determine the coherence length) would provide a coupling mechanism. To produce a high $T_{c}$ the hopping rate would have to be high; the average hopping frequency would take the place of the maximum phonon frequency in phonon coupling. How this process would couple to the lattice depends on two different times - the time spent on the $O(2)$ atom and the time spent in the $\mathrm{CuO}_{2}$ plane. To have a maximum effect in displaceing the atoms these two hopping times should be comparable and not too much faster than the highest optical phonon frequencies. If one of the hopping times is very short the electron will spend most of the time in one state and the effect on the lattice will be small. Also, if both hopping times are very short then the lattice will not have time to respond.

The coupling between the hopping electron and the lattice will also produce an isotope effect. If the decrease in $T_{c}$ were the result of a slower average hopping frequency then the coupling to the lattice would increase, and the displacements would change. This might explain the observed correlation between a strong isotope effect for low $T_{c}$ and a small one at high $T_{c}$. This model might also provide a basis for understanding the larger coherence length observed in the Pr substituted material. When the electron hops into the plane, it may be localized on the $\mathrm{Cu}$ atoms or jump into existing holes. However it is likely that the electron moves into a superposition of states that extends for a few unit cells. In the Pr substituted material our recent work indicates that there are additional localized states in the $\mathrm{CuO}_{2}$ plane in the $\mathrm{Pr}-\mathrm{O}$ bonds [8]. If this leads to a superposition state that is more spread out it might increase the coherence length.

\section{CONCLUSION}

We have collected and analyzed XAFS data on $\mathrm{HgBa}_{2} \mathrm{CuO}_{4}$ at $\mathrm{Hg}, \mathrm{Cu}$, and $\mathrm{Ba}$ edges. The bond length fits agree reasonably well with diffraction 
studies. Correlated Debye-Waller factors compare well with diffraction measurements, indicating that most near-neighbor bonds involve correlated motions of their atomic constituents, with the exception of the $\mathrm{Cu}-\mathrm{O}(2)$ and the $\mathrm{Ba}-\mathrm{O}(2)$ bonds. These latter bonds must involve anti-correlated motions to adequately describe the data. Such anticorrelation has been observed in other systems (notably the $\mathrm{Tl}$ compounds) and is further evidence of an antiferroelectric-like distortion occuring in a high $T_{c}$ superconductor. The nature of this distortion prompted us to outline a simple electronhopping model that might explain both the distortion and a possible pairing mechanism.
[1] J. G. Bednorz and K. A. Müller, Z. Phys. B 64, 189 (1986).

[2] S. N. Putilin et. al., Nature 362, 226 (1993).

[3] L. Gao et al., Phys. Rev. B 50, 4260 (1994).

[4] T. Egami, Ferroelectrics 130, 15 (1992).

[5] R. P. Sharma, L. E. Rehn, P. M. Baldo, and J. Z. Liu, Phys. Rev. Lett. 62, 2869 (1989).

[6] J. M. de Leon, S. D. Conradson, I. Batistic, and A. R. Bishop, Phys. Rev. Lett. 65, 1675 (1990).

[7] H. Kimura et al., (1993).

[8] C. H. Booth et al., Phys. Rev. B 49, 3432 (1994).

[9] G. G. Li et al., Phys. Rev. B (1995), in press.

[10] T. M. Hayes and J. B. Boyce, in Solid State Physics, edited by H. Ehrenreich, F. Seitz, and D. Turnbull (Academic, New York, 1982), Vol. 37, p. 173.

[11] F. Bridges, C. H. Booth, and G. G. Li, Physica B , in press.

[12] G. G. Li, F. Bridges, and C. H. Booth, submitted to Phys. Rev. B .

[13] Q. Huang, J. W. Lynn, Q. Xiong, and C. W. Chu (un. published).

[14] J. J. Rehr, R. C. Albers, and S. I. Zabinsky, Phys. Rev. Lett. 69, 3397 (1992).

[15] O. Chmaissem et al., Physica C 212, 259 (1993).

[16] J. L. Wagner et al., Physica C 210, 447 (1993).

TABLE I. Fit results for $\sigma$ and $R$ from XAFS at the $\mathrm{Hg} L_{3}$-edge, and the $\mathrm{Cu}$ and $\mathrm{Ba} K$-edges of $\mathrm{HgBa}_{2} \mathrm{CuO}_{4}$. $\mathrm{A}$ single value of the amplitude reduction factor $S_{0}^{2}$ was used for the $\mathrm{Ba}$ and $\mathrm{Cu}$ edges. The $\mathrm{Hg}$ edge data required a separate reduction factor for the first peak. $\phi$ was calculated via Eq. 2 using diffraction data from Ref. [13]. Results listed without an affixed error was held constant in the fit based on the diffraction result.

\begin{tabular}{|c|c|c|c|c|c|c|}
\hline \multirow[b]{2}{*}{ Bond } & \multicolumn{2}{|c|}{$\sigma(\AA)$} & \multicolumn{2}{|c|}{$\phi$} & \multicolumn{2}{|c|}{$R$} \\
\hline & $T_{c}=96 \mathrm{~K}$ & $T_{c}=45 \mathrm{~K}$ & $T_{c}=96 \mathrm{~K}$ & $T_{c}=45 \mathrm{~K}$ & $T_{c}=96 \mathrm{~K}$ & $T_{\mathrm{c}}=45 \mathrm{~K}$ \\
\hline $\mathrm{Hg}-\mathrm{O}(2)$ & $0.034(1)$ & $0.034(1)$ & $0.9(2)$ & $0.9(2)$ & $1.98(1)$ & $1.98(1)$ \\
\hline $\mathrm{Hg}-\mathrm{Hg}$ & $0.09(2)$ & $0.09(4)$ & $0.6(2)$ & $0.3(3)$ & $3.83(4)$ & $3.82(4)$ \\
\hline $\mathrm{Hg}-\mathrm{Ba}$ & $0.08(1)$ & $0.07(2)$ & $0.6(2)$ & $0.5(2)$ & $3.97(4)$ & $3.95(4)$ \\
\hline $\mathrm{Cu}-\mathrm{O}(1)$ & $0.047(10)$ & $0.045(10)$ & $0.9(2)$ & $0.8(2)$ & $1.93(1)$ & $1.94(1)$ \\
\hline $\mathrm{Cu}-\mathrm{O}(2)$ & $0.21(4)$ & $0.19(5)$ & $\lesssim-1.0(3)$ & $\lesssim-1.0(3)$ & $2.76(4)$ & $2.85(4)$ \\
\hline $\mathrm{Cu}-\mathrm{Ba}$ & $0.064(3)$ & $0.065(4)$ & $0.5(2)$ & $0.5(2)$ & $3.34(2)$ & $3.33(2)$ \\
\hline $\mathrm{Cu}-\mathrm{Cu}$ & $0.055(6)$ & $0.063(10)$ & $0.4(2)$ & $0.1(2)$ & $3.88(2)$ & $3.91(2)$ \\
\hline $\mathrm{Cu}-\mathrm{Hg}$ & $0.069(2)$ & $0.074(10)$ & $0.8(2)$ & $0.3(2)$ & $4.76(4)$ & $4.76(4)$ \\
\hline $\mathrm{Ba}-\mathrm{O}(1)$ & $0.075(1)$ & $0.065(7)$ & $0.5(2)$ & $0.6(2)$ & $2.77(1)$ & $2.73(1)$ \\
\hline $\mathrm{Ba}-\mathrm{O}(2)$ & $0.17(4)$ & $0.19(5)$ & $-0.9(3)$ & $\lesssim-1.0(3)$ & 2.87 & 2.87 \\
\hline $\mathrm{Ba}-\mathrm{Cu}$ & $0.069(5)$ & $0.070(5)$ & $0.4(2)$ & $0.4(2)$ & $3.34(1)$ & $3.30(1)$ \\
\hline $\mathrm{Ba}-\mathrm{Ba}$ & $0.062(4)$ & $0.075(6)$ & $0.6(2)$ & $0.4(2)$ & $3.91(4)$ & $3.89(4)$ \\
\hline $\mathrm{Ba}-\mathrm{Hg}$ & $0.120(25)$ & $0.10(1)$ & $0.05(20)$ & $0.06(20)$ & $3.94(4)$ & $3.94(4)$ \\
\hline
\end{tabular}




\title{
XAFS Standards: A Comparison of Experiment and Theory
}

\author{
G. G. Li, F. Bridges, and C. H. Booth \\ Physics Department, University of California, Santa Cruz, CA 95064
}

The reliability of the structural parameters obtained from XAFS spectra strongly depends on the quality of the standards used in the data analyses. Recently, the quality and availability of the FEFF code $[1,2]$ has allowed for the easy and accurate theoretical calculations of standards, which may then be used in fitting procedures. Although some comparisons of these calculations to actual data have been performed, no published work has compared the calculations to the data from a large number of compounds that might serve as a sample of the periodic table. We have therefore compared about 30 isolated pair standards, extracted from experimental data, with those generated by the FEFF5 code. The absorbing atoms range from $\mathrm{Ni}(Z=28)$ to $\mathrm{Pb}(Z=82)$ and the backscattering atoms from $\mathrm{O}(Z=8)$ to $\mathrm{Au}(Z=79)$. We followed standard data collection and fitting procedures $[3,4]$. In particular, each embedded-atom background absorption function $\mu_{o}$ was determined using our iterative technique [4]. This technique helped us remove the adverse effect of multi-electron excitations and the possible influence of any atomic-XAFS [5]. The influence of the energy resolution on XAFS, and correlations in the fitting parameters were also included.

Theoretical standards were calculated using $\mathrm{x}$ ray diffraction data (corrected for any thermal expansion) and Debye temperatures for the correlated Debye model used in FEFF. In general, good agreement between the FEFF calculations and the XAFS data is achieved, with typical errors of $0.005 \AA$ for nearest-neighbor distances, $R$ (Table I). In cases where the shape of the backscattering amplitude function was very similar to the data, the effect of the correlation between the amplitude reduction factors $S_{o \text { eff }}^{2}$ and the broadening factors $\sigma$ was greatly reduced (eg. $\mathrm{Cu}$ and $\mathrm{Ni}$ foils) (Table II). Estimated experimental errors for the amplitudes are $<10 \%$, and $\sim 5-6 \%$ in many cases. $S_{o \text { eff }}^{2}$ varied across the periodic table by about $20 \%$, well outside the error for most of the fits(Fig. 1). $E_{o}$ was also calculated (Table II).

Significant differences are sometimes found in the backscattering amplitude, $F(k, R)$, at low values of the photoelectron wave vector $k\left(<7 \AA^{-1}\right)$, especially for heavy backscattering atoms such as $\mathrm{Au}$ and $\mathrm{Pt}$. In such cases the relative change with $Z$ in the phase and amplitude functions that FEFF gen- erates, is still quite accurate. Therefore we demonstrate that an experimental standard shifted from one atom-pair to another provides a better fit to the data than obtained using the theoretical standard (Fig. 3).

TABLE I. Comparisons of the structural parameters for $\mathrm{Ni}, \mathrm{Au}, \mathrm{NiO}$, and $\mathrm{Cu}_{2} \mathrm{O}$ obtained from diffraction (Diff) and XAFS studies. $R$ (in $\AA$ ) is the distance to the backscattering atoms, $N$ is the number of neighboring atoms at that distance, and $\sigma$ (in $10^{-2} \AA^{-1}$ ) is the Debye-Waller factor for the corresponding pair.

\begin{tabular}{|c|c|c|c|c|c|}
\hline & Shell & 1st & 2nd & 3rd & 4th \\
\hline \multicolumn{6}{|c|}{$\mathrm{Ni}\left(\mathrm{T}=83 \mathrm{~K}, S_{o e f f}^{2}=0.82, \Delta t \mu\left(\mathrm{E}_{o}\right)=2.7\right)$} \\
\hline \multirow[t]{2}{*}{$R$} & Diff & 2.4863 & 3.5161 & 4.306 .3 & 4.9725 \\
\hline & XAFS & 2.482 & 3.512 & 4.312 & 4.976 \\
\hline \multirow[t]{2}{*}{$N$} & Diff & 12.0 & 6.0 & 24.0 & 12.0 \\
\hline & XAFS & 12.0 & 5.4 & 24.9 & 15.3 \\
\hline \multirow[t]{3}{*}{$\sigma$} & Debye $^{a}$ (FEFF5) & 5.04 & 5.47 & 5.45 & 5.42 \\
\hline & XAFS & 4.8 & 5.6 & 6.1 & 6.6 \\
\hline & $\mathrm{Au}(\mathrm{T}=77 \mathrm{~K}, \mathrm{~s}$ & $;_{o e f f}^{2}=0$. & $36, \Delta t \mu($ & $\left.\mathrm{E}_{o}\right)=1.1$ & \\
\hline \multirow[t]{3}{*}{$R$} & Diff & 2.8757 & 4.0669 & 4.9809 & 5.7515 \\
\hline & XAFS & 2.877 & 4.065 & 4.983 & 5.753 \\
\hline & $\mathrm{XAFS}^{b}$ & 2.876 & 4.070 & 4.987 & 5.757 \\
\hline \multirow[t]{2}{*}{$N$} & Diff & 12.0 & 6.0 & 24.0 & 12.0 \\
\hline & XAFS & 12.0 & 6.6 & 24.9 & 13.1 \\
\hline \multirow[t]{3}{*}{$\sigma$} & Debye $^{c}$ (FEFF5) & 5.59 & 6.16 & 6.21 & 6.25 \\
\hline & XAFS & 4.7 & 6.2 & 6.2 & 6.4 \\
\hline & \multicolumn{5}{|c|}{$\mathrm{NiO}\left(\mathrm{T}=83 \mathrm{~K}, S_{o e f f}^{2}=1.04, \Delta t \mu\left(\mathrm{E}_{o}\right)=1.1\right)$} \\
\hline \multirow[t]{2}{*}{$R$} & Diff & 2.0852 & 2.949 & & \\
\hline & XAFS & 2.090 & 2.951 & & \\
\hline \multirow[t]{2}{*}{$N$} & Diff & 6.0 & 12.0 & & \\
\hline & XAFS & 6.0 & 12.6 & & \\
\hline \multirow[t]{3}{*}{$\sigma$} & Debye $^{d}$ (FEFF5) & 6.78 & 4.79 & & \\
\hline & XAFS & 7.2 & 6.3 & & \\
\hline & \multicolumn{5}{|c|}{$\mathrm{Cu}_{2} \mathrm{O}\left(\mathrm{T}=83 \mathrm{~K}, S_{o e f f}^{2}=0.69, \Delta t \mu\left(\mathrm{E}_{o}\right)=0.6\right)$} \\
\hline \multirow[t]{2}{*}{$R$} & Diff & 1.8472 & 3.0165 & 3.5372 & \\
\hline & XAFS & 1.832 & 3.015 & 3.534 & \\
\hline \multirow[t]{3}{*}{$N$} & Diff & 2.0 & 12.0 & 6.0 & \\
\hline & XAFS & 1.9 & 12.3 & 6.3 & \\
\hline & XAFS & 3.5 & 8.4 & 6.2 & \\
\hline
\end{tabular}

${ }^{a} \Theta_{D}=450 \mathrm{~K}$, Ref. [6]. ${ }^{b}$ Phase-shift $=-0.25$ radian.

${ }^{c} \Theta_{D}=165 \mathrm{~K}$, Ref. [6]. ${ }^{d} \Theta_{\mathcal{D}}=560 \mathrm{~K}$, Ref. [7]. 


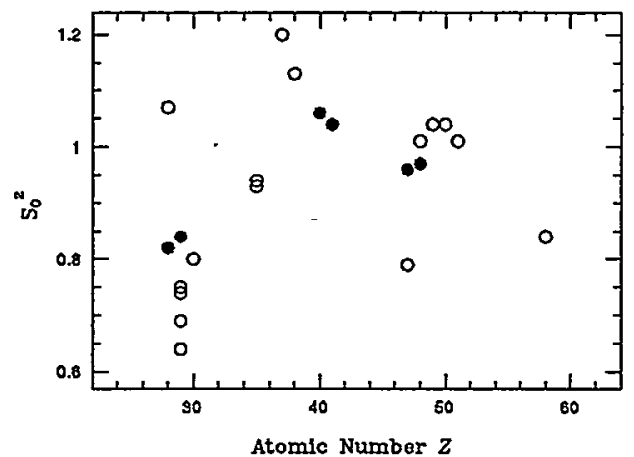

FIG. 1. Variation of $S_{\text {oeff }}^{2}$ as a function of $Z$ for the $K$ edge experimental standards. Note that for some atoms there is a large difference in $S_{o \text { eff }}^{2}$ for different crystal environments. The solid circles are for the pairs of metal atoms which are neighbors in the periodic table.

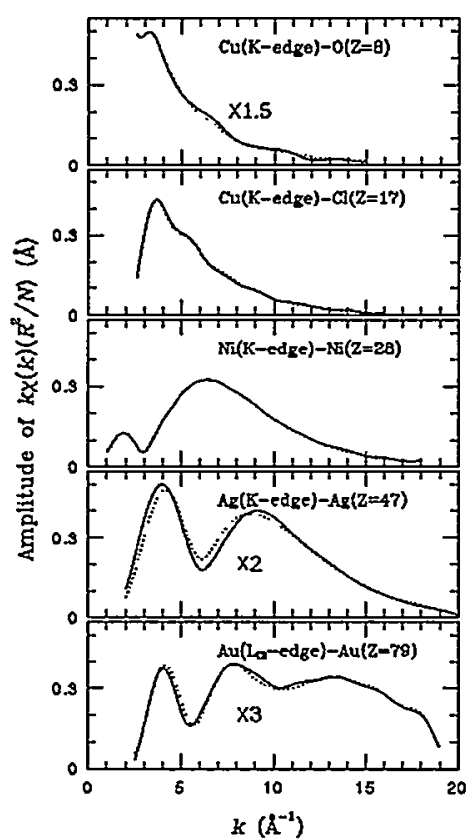

FIG. 2. Amplitude functions, $k \chi(k)\left(R^{2} / N\right), \mathrm{Cu}-\mathrm{Cl}$ (in $\mathrm{CuCl}$ ), $\mathrm{Ni}-\mathrm{Ni}$ (in $\mathrm{Ni}$ ), $\mathrm{Ag}-\mathrm{Ag}$ (in $\mathrm{Ag}$ ), and $\mathrm{Au}-\mathrm{Au}$ (in $\mathrm{Au}$ ) pairs. The solid lines are the experimental data and the dotted lines the fits to the data using the FEFF standards. The amplitudes are increased by the indicated factors for the $\mathrm{Cu}-\mathrm{O}, \mathrm{Ag}-\mathrm{Ag}$, and $\mathrm{Au}-\mathrm{Au}$ pairs, to put all data on the same vertical scale.

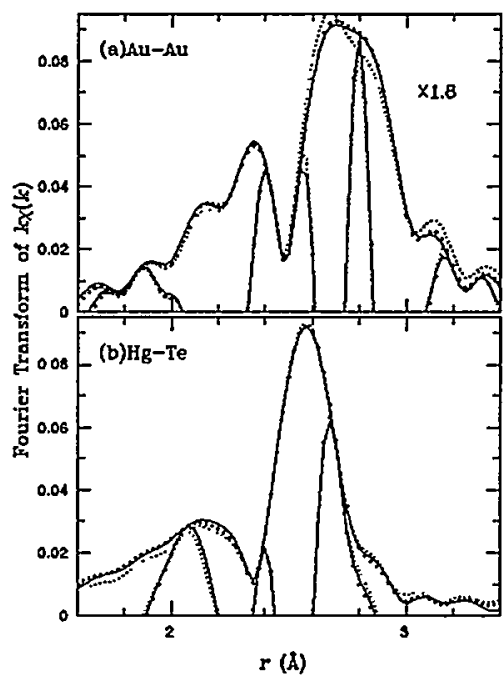

FIG. 3. Fourier transform of $k \chi(k)$ for (a) the $\mathrm{Au}-\mathrm{Au}$ pair in $\mathrm{Au}$ and (b) the $\mathrm{Hg}$-Te pair in HgTe. Solid lines, the experimental data; dotted lines, the fits to the experimental data using the FEFF standards; bold dots, the fits to the experimental data using the shifted experimental (a) Pt-Pt and (b) In-Sb pair standards, respectively. The transform range is from 3.0 to $18.5 \AA^{-1}$ for Au-Au and 2.8 to $19 \AA^{-1}$ for Hg-Te, Gaussian rounded by $0.3 \AA^{-1}$. The magnitude of the transform for $A u-A u$ is increased by a factor of 1.8 to be on the same scale.

In conclusion, we have found the quality of the FEFF standards to be very good and acceptable for fitting standard compounds in most cases. In a few cases, the backscattering amplitude function clearly has the wrong shape. If an experimental standard is not available, shifting a standard of another material with a similar composition is an excellent alternative.

The work is supported in part by NSF grant DMR-92-05204.

[1] J. Mustre de Leon, J. J. Rehr, and S. I. Zabinsky, Phys. Rev. B 44, 4146 (1991).

[2] J.J. Rehr, S.I. Zabinsky and R.C. Albers, Phys. Rev. Let. 69, 3397 (1992).

[3] T. M. Hayes and J. B. Boyce, in Solid State Physics, edited by H. Ehrenreich, F. Seitz, and D. Turnbull (Academic, New York, 1982), Vol. 37, p. 173.

[4] F. Bridges, C. Booth, and G. G. Li, Physica B, to be published. 
[5] J. J. Rehr, C. H. Booth, F. Bridges, and S. I. Zabinsky, Phys. Rev. B 49, 12347 (1994).

[6] G. T. Furukawa, T. B. Douglas, and N. Pearlman, in American Institute of Physics Handbook, edited by D. E. Gray (McGraw-Hill, New York, 1972), p. 4-115.

[7] Landolt-Börnstein: Numerical Data and Functional Relationships in Science and Technology, Group III, Vol. 17, part g, edited by K. H. Hellwege and O. Madelung (Springer-Verlag, Berlin, 1984).

[8] Landolt-Börnstein: Numerical Data and Functional Relationships in Science and Technology (Springer-Verlag, Berlin, 1982), Vol. 17, pt. b.

[9] Calculated from Wyckoff [16] with thermal expansion corrections.

[10] Calculated from FEFF5 with the correlated Debye model. The Debye temperatures are from Ref. [6].

[11] T. Suzuki, J. Phys. Soc. Jpn. 15, 2018 (1960).

[12] G. K. White and J. G. Collins, Proc. R. Soc. Lond. A 333, 327 (9973).

[13] Extrapolated using the data for $28-284^{\circ} \mathrm{C}$ from Ref. [18].

[14] Ref. [19]. The lattice constant of $\mathrm{Nb}_{3} \mathrm{Sb}$ is corrected using data for $\mathrm{Nb}_{3} \mathrm{Sn}$.

[15] Calculated from Wyckoff [16] and corrected by the thermal expansion, $\epsilon=\left(L_{80}-L_{298}\right) / L_{298}=0.0013$, from NiO. [7]

[16] R. W. G. Wyckoff, Crystal Structures, Vol. 1 (New York, Interscience Publishers LTD., 1960).

[17] R. K. Kirby, T. A. Hahn, and B. D. Rothrock, in American Institute of Physics Handbook, edited by D. E. Gray (McGraw-Hill, New York, 1972), p. 4119.

[18] Landolt-Börnstein: Numerical Data and Functional Relationships in Science and Technology (Springer-Verlag, Berlin, 1973), Vol. 7, pt. a.

[19] Landolt-Börnstein: Numerical Data and Functional Relationships in Science and Technology (Springer-Verlag, Berlin, 1971), Vol. 6. 
TABLE II. Comparisons of experimental and theoretical XAFS standards. $R_{d}$ is the distance to the nearest neighbor given by diffraction measurements. $\Delta \mu\left(E_{o}\right) t$ is the edge height in the experimental data. The fitting parameters obtained using the theoretical XAFS standards (FEFF5) are given; $\sigma$ is the Debye-Waller factor; $\Delta R$ is the shift in distance relative to $R_{d} ; \Delta E_{o}$ is the shift of the absorption edge relative to the experimentally defined one; $S_{o_{e f f}}^{2}$ is the amplitude correction factor for the theoretical XAFS standard. The values of $S_{o \text { ef } f}^{2}$ in parentheses are obtained with $\sigma$ fixed at the indicated theoretical value. Variation ranges are estimated using different $k$-weighting $\left(k \chi(k), k^{2} \chi(k)\right.$, and $\left.k^{3} \chi(k)\right)$ in the fits.

\begin{tabular}{|c|c|c|c|c|c|c|c|c|}
\hline Edge & Compound & $R_{d}(\AA)$ & $\sigma\left(10^{-2} \AA\right)$ & $\Delta R\left(10^{-2} \AA\right)$ & $\Delta E_{o}(\mathrm{eV})$ & $S_{o e f f}^{2}$ & $\Delta \mu\left(E_{o}\right) t$ & Crystal \\
\hline \multirow[t]{3}{*}{$\mathrm{Ni}(Z=28) \mathrm{K}$} & $\mathrm{NiO}(83 \mathrm{~K})$ & $2.085^{8}$ & $6.33 \pm 0.47$ & $-0.1 \pm 0.1$ & $-2.4 \pm 0.1$ & $1.04 \pm 0.04$ & 1.1 & $\overline{\mathrm{Si}(400)}$ \\
\hline & $\mathrm{Ni}(83 \mathrm{~K})$ & $2.486^{9}$ & $4.83 \pm 0.01$ & $-0.4 \pm 0.1$ & $-0.5 \pm 0.2$ & $0.82 \pm 0.00$ & 2.7 & $\mathrm{Si}(220)$ \\
\hline & & & $(5.04)^{10}$ & & & $(0.85 \pm 0.01)$ & & \\
\hline \multirow[t]{7}{*}{$\mathrm{Cu}(Z=29) \mathrm{K}$} & $\mathrm{CuO}(83 \mathrm{~K})$ & 1.955 & $4.87 \pm 0.12$ & $0.0 \pm 0.4$ & $-6.1 \pm 0.5$ & $0.64 \pm 0.01$ & 1.6 & $\operatorname{Si}(111)$ \\
\hline & $\mathrm{Cu}_{2} \mathrm{O}(83 \mathrm{~K})$ & $1.847^{11}$ & $3.95 \pm 0.39$ & $-0.8 \pm 0.7$ & $-6.8 \pm 1.0$ & $0.69 \pm 0.02$ & 0.6 & $\mathrm{Si}(111)$ \\
\hline & $\mathrm{CuCl}(4.2 \mathrm{~K})$ & $2.342^{13}$ & $6.15 \pm 0.26$ & $0.0 \pm 0.2$ & $-5.2 \pm 0.3$ & $0.74 \pm 0.02$ & 1.4 & $\mathrm{Si}(111)$ \\
\hline & $\mathrm{Cu}(83 \mathrm{~K})$ & $2.548^{11}$ & $5.56 \pm 0.01$ & $-0.4 \pm 0.2$ & $0.2 \pm 0.3$ & $0.84 \pm 0.01$ & 3.0 & $\mathrm{Si}(220)$ \\
\hline & & & $(5.75)^{9}$ & & & $(0.89 \pm 0.01)$ & & \\
\hline & $\mathrm{CuBr}(83 \mathrm{~K})$ & $2.458^{8}$ & $6.45 \pm 0.10$ & $-0.5 \pm 0.4$ & $-2.5 \pm 0.7$ & $0.84 \pm 0.02$ & 1.0 & $\mathrm{Si}(220)$ \\
\hline & $\mathrm{CuI}(4.2 \mathrm{~K})$ & $2.611^{8}$ & $4.61 \pm 0.11$ & $-0.9 \pm 0.0$ & $-2.5 \pm 0.1$ & $0.75 \pm 0.01$ & 0.8 & $\operatorname{Si}(111)$ \\
\hline $\mathrm{Zn}(Z=30) \mathrm{K}$ & $\mathrm{ZnTe}(83 \mathrm{~K})$ & $2.637^{8}$ & $4.50 \pm 0.07$ & $0.0 \pm 0.3$ & $-4.4 \pm 0.7$ & $0.80 \pm 0.02$ & 0.7 & $\mathrm{Si}(220)$ \\
\hline \multirow[t]{2}{*}{$\mathrm{Br}(Z=35) \mathrm{K}$} & $\mathrm{CuBr}(83 \mathrm{~K})$ & $2.458^{8}$ & $6.43 \pm 0.02$ & $-0.9 \pm 0.0$ & $-3.1 \pm 0.2$ & $0.93 \pm 0.03$ & 0.6 & $\mathrm{Si}(220)$ \\
\hline & $\mathrm{RbBr}(83 \mathrm{~K})$ & $3.405^{12}$ & $8.76 \pm 0.09$ & $0.9 \pm 0.7$ & $-0.5 \pm 1.0$ & $0.94 \pm 0.02$ & 0.8 & $\mathrm{Si}(220)$ \\
\hline $\mathrm{Rb}(Z=37) \mathrm{K}$ & $\operatorname{RbBr}(83 \mathrm{~K})$ & $3.405^{12}$ & $9.12 \pm 0.18$ & $1.6 \pm 0.5$ & $-3.5 \pm 0.7$ & $1.2 \pm 0.1$ & 0.8 & $\mathrm{Si}(220)$ \\
\hline $\operatorname{Sr}(Z=38) \mathrm{K}$ & $\mathrm{SrF}_{2}(4.2 \mathrm{~K})$ & $2.499^{13}$ & $6.70 \pm 0.54$ & $-1.0 \pm 0.6$ & $-0.8 \pm 0.6$ & $1.13 \pm 0.07$ & 0.6 & $\operatorname{Si}(111)$ \\
\hline \multirow[t]{2}{*}{$\mathrm{Zr}(Z=40) \mathrm{K}$} & $\alpha-\operatorname{Zr}(83 \mathrm{~K})$ & $3.202^{11}$ & $5.63 \pm 0.18$ & $2.0 \pm 0.1$ & $-1.3 \pm 0.1$ & $1.06 \pm 0.05$ & 0.7 & $\operatorname{Si}(220)$ \\
\hline & & & $(5.26)^{10}$ & & & $(0.94 \pm 0.01)$ & & \\
\hline \multirow[t]{2}{*}{$\mathrm{Nb}(Z=41) \mathrm{K}$} & $\mathrm{Nb}(83 \mathrm{~K})$ & $2.854^{11}$ & $5.24 \pm 0.04$ & $0.0 \pm 0.1$ & $0.1 \pm 0.2$ & $1.04 \pm 0.01$ & 0.7 & $\mathrm{Si}(220)$ \\
\hline & & & $(5.47)^{10}$ & & & $(1.10 \pm 0.02)$ & & \\
\hline \multirow[t]{3}{*}{$\operatorname{Ag}(Z=47) \mathrm{K}$} & $\operatorname{Ag}(83 \mathrm{~K})$ & $2.879^{11}$ & $5.73 \pm 0.05$ & $-0.3 \pm 0.3$ & $-3.6 \pm 0.9$ & $0.96 \pm 0.02$ & 1.7 & $\mathrm{Si}(220)$ \\
\hline & & & $(6.00)^{10}$ & & & $(1.07 \pm 0.03)$ & & \\
\hline & $\beta-\operatorname{AgI}(83 \mathrm{~K})$ & $2.806^{8}$ & $5.74 \pm 0.13$ & $0.3 \pm 0.5$ & $-4.3 \pm 1.2$ & $0.79 \pm 0.03$ & 1.7 & $\mathrm{Si}(220)$ \\
\hline \multirow[t]{3}{*}{$\mathrm{Cd}(Z=48) \mathrm{K}$} & $\mathrm{Cd}(83 \mathrm{~K})$ & $2.969^{9}$ & $6.17 \pm 0.05$ & $0.6 \pm 0.1$ & $5.0 \pm 0.2$ & $0.97 \pm 0.02$ & 1.1 & $\mathrm{Si}(220)$ \\
\hline & & & $(6.11)^{10}$ & & & $(0.96 \pm 0.01)$ & & \\
\hline & CdTe (83 K) & $2.804^{8}$ & $4.72 \pm 0.09$ & $1.0 \pm 0.2$ & $-12.6 \pm 0.3$ & $1.01 \pm 0.03$ & 1.0 & $\mathrm{Si}(220)$ \\
\hline $\operatorname{In}(Z=49) \mathrm{K}$ & $\operatorname{InSb}(83 \mathrm{~K})$ & $2.803^{8}$ & $4.36 \pm 0.03$ & $-0.1 \pm 0.2$ & $-6.4 \pm 0.6$ & $1.04 \pm 0.01$ & 1.7 & $\mathrm{Si}(220)$ \\
\hline $\operatorname{Sn}(Z=50) \mathrm{K}$ & $\mathrm{Nb}_{3} \mathrm{Sn}(83 \mathrm{~K})$ & $2.954^{14}$ & $4.95 \pm 0.05$ & $0.2 \pm 0.0$ & $-4.8 \pm 0.1$ & $1.04 \pm 0.01$ & 0.8 & $\operatorname{Si}(220)$ \\
\hline $\mathrm{Sb}(Z=51) \mathrm{K}$ & $\mathrm{Nb}_{3} \mathrm{Sb}(83 \mathrm{~K})$ & $2.939^{14}$ & $4.74 \pm 0.12$ & $0.2 \pm 0.3$ & $-8.5 \pm 0.9$ & $1.01 \pm 0.03$ & 0.4 & $\operatorname{Si}(220)$ \\
\hline $\mathrm{Ce}(Z=58) \mathrm{K}$ & $\mathrm{CeO}_{2}(83 \mathrm{~K})$ & $2.340^{15}$ & $5.21 \pm 0.14$ & $0.2 \pm 0.1$ & $3.6 \pm 0.1$ & $0.84 \pm 0.02$ & 0.8 & $\mathrm{Si}(400)$ \\
\hline \multirow[t]{2}{*}{$\operatorname{Pt}(Z=78) \mathrm{L}_{I I I}$} & Pt $(83 \mathrm{~K})$ & $2.77^{9}$ & $3.78 \pm 0.11$ & $-0.4 \pm 0.1$ & $-10.9 \pm 0.5$ & $0.88 \pm 0.03$ & 1.1 & $\mathrm{Si}(220)$ \\
\hline & & & $(4.23)^{10}$ & & & $(1.01 \pm 0.04)$ & & \\
\hline \multirow[t]{2}{*}{$\operatorname{Au}(Z=79) \mathrm{L}_{I I I}$} & $\mathrm{Au}(77 \mathrm{~K})$ & $2.876^{9}$ & $4.93 \pm 0.07$ & $-0.5 \pm 0.2$ & $-7.6 \pm 0.7$ & $0.86 \pm 0.02$ & 1.1 & $\mathrm{Si}(220)$ \\
\hline & & & $(5.59)^{10}$ & & & $(1.11 \pm 0.01)$ & & \\
\hline $\mathrm{Hg}(Z=80) \mathrm{L}_{I I I}$ & HgTe (83 K) & $2.7955^{8}$ & $4.87 \pm 0.08$ & $-0.3 \pm 0.0$ & $-3.8 \pm 0.0$ & $0.75 \pm 0.02$ & 0.5 & $\mathrm{Si}(220)$ \\
\hline $\mathrm{Pb}(Z=82) \mathrm{L}_{I I I}$ & $\beta-\mathrm{PbO}_{2}(83 \mathrm{~K})$ & $2.161^{15}$ & $5.13 \pm 0.11$ & $-0.3 \pm 0.2$ & $-1.4 \pm 0.4$ & $0.73 \pm 0.01$ & 0.6 & $\mathrm{Si}(220)$ \\
\hline
\end{tabular}




\title{
Polarized Dispersion for X-rays Scattered by an Aromatic Bromide
}

\author{
David H. Templeton and Lieselotte K. Templeton \\ Department of Chemistry, University of California, Berkeley, CA 94720
}

Large changes of amplitude and phase occur in the scattering of $\mathrm{X}$-rays by crystals as the wavelength is changed near an absorption edge. This dispersion is the basis of the multiwavelength anomalous diffraction (MAD) method, an important technique for solving macromolecular structures with the help of synchrotron radiation [1]. This radiation is highly polarized. Similar changes of scattering occur in many materials if the direction of polarization is changed. This polarized dispersion is very sensitive to chemical state and molecular geometry. It can enhance the power of the MAD method, but also makes it more complicated [2]. It also permits phases to be determined in various other ways $[3,4]$.

Bromine is a good element to use in the MAD method because its $K$ edge is at a convenient wavelength $(0.92 \AA)$ and because it can be incorporated in a macromolecule at definite positions, for example substituted for hydrogen on aromatic rings. As a test case for this chemical state we used crystals of a bromophenyl derivative of homocubanecarboxamide $\left(\mathrm{C}_{16} \mathrm{H}_{14} \mathrm{BrNO}\right)$ [5]. Dispersion tensors for two independent $\mathrm{Br}$ atoms were derived from intensities measured in diffraction experiments. They are uniaxial within experimental error with the unique principal

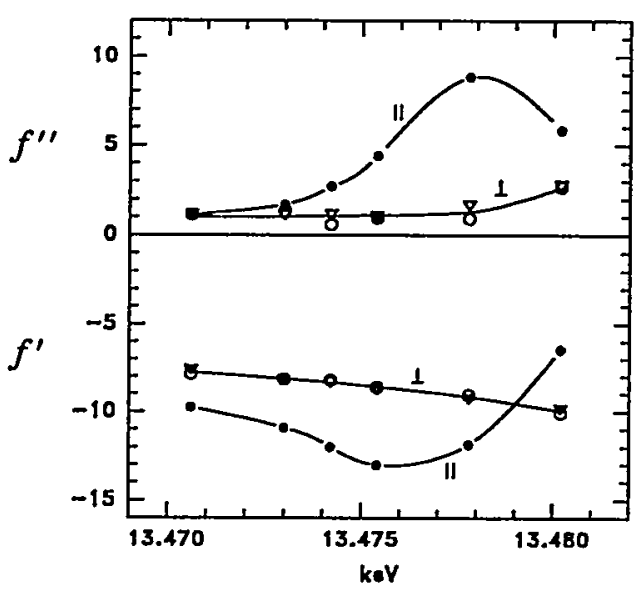

Figure 1: Principal values from diffraction data. Filled circles, polarization parallel with bond; open circles, in benzene plane; triangles, out of plane. value aligned with the respective $\mathrm{Br}-\mathrm{C}$ bond direction. Average principal values are plotted in Figure 1. Figure 2 shows principal values derived from absorption spectra measured by flourescence technique with a single crystal specimen. Here the anisotropy is underestimated somewhat because of saturation of the spectra. A strong resonance with a $\sigma$ antibonding orbital makes the amplitudes change with polarization direction by amounts that are quite comparable with the changes with wavelength. Exploitation of both effects promises to be more powerful than the use of either by itself.

1. W.A. Hendrickson, Science 254, 51 (1991).

2. E. Fanchon and W.A. Hendrickson, Acta Cryst. A 46, 809 (1990).

3. D.H. Templeton and L.K. Templeton, Acta Cryst. $A$ 43, 573 (1987); 47, 414 (1991); 48, 746 (1992).

4. A. Kirfel and A. Petcov, Z. Kristallogr. 195, 1 (1991); Acta Cryst. A. 48, 247 (1992).

5. W.D. Dauben and D.L. Whalen, Tetrahedron Lett. 1966, 3743.

6. R.C. Pettersen, $\mathrm{PhD}$ Thesis, Univ. of California, Berkeley (1966).

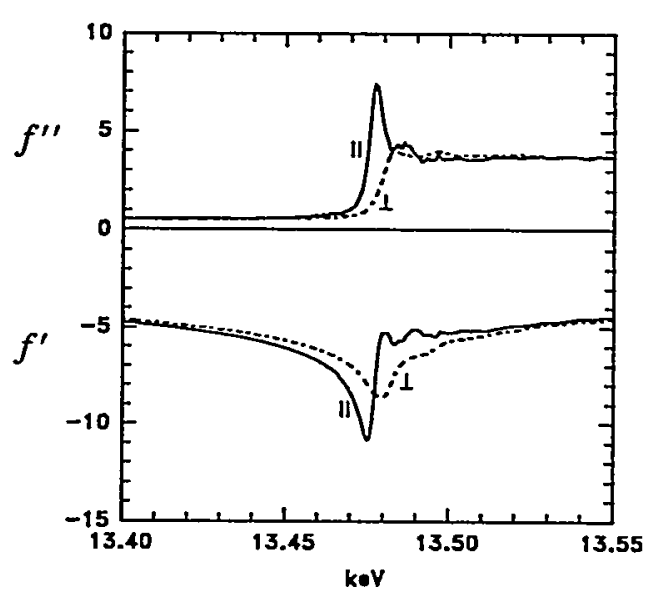

Figure 2: Principal values derived from absorption spectra. 
Proposal 2143Mp

\title{
XAS STUDY OF CATION CHEMISORPTION AT OXIDE-WATER INTERFACES
}

\author{
Maria L. Peterson ${ }^{1}$, John R. Bargar ${ }^{1}$, Sing-Foong Cheah ${ }^{1}$, Andrea L. Foster ${ }^{1}$, \\ Tracy Tingle ${ }^{2}$, Tetsu Tokunaga ${ }^{3}$, George A. Parks', and Gordon E. Brown, Jr. 1,4 \\ (1) Geological and Environmental Sciences, Stanford University, Stanford, CA 94305-2115 \\ (2) Center for Materials Research, Stanford University, Stanford, CA $94305-4045$ \\ (3) Lawrence Berkeley Laboratory, Earth Sciences Division, MS 50-E, Berkeley, CA 94720 \\ (4) Stanford Synchrotron Radiation Laboratory, Stanford, CA 94304
}

\section{INTRODUCTION}

The modes by which aqueous contaminants are removed from solution by sorption to mineral surfaces or by sequestration in solids are pertinent to environmental remediation and predictive modeling of contaminant uptake and transport. X-ray absorption spectroscopy is uniquely suited to provide local structural information (number, type, and distance of ligands coordinating a particular type of atom) as well as oxidation state of contaminant ions associated with crystalline, poorly crystalline, or amorphous compounds often found in soils, sediments, and mine tailings. Such information is critical to understanding the mode of sorption of environmental contaminants by solid surfaces and, potentially, predicting the fate of contaminants in groundwaters. We have studied the uptake of several ions $(\mathrm{Cr}, \mathrm{Cu}, \mathrm{As}, \mathrm{Se}$, and $\mathrm{Pb}$ ) on high surface area mineral substrates (magnetite, maghemite, hematite, goethite, $\alpha$-alumina, kaolinite, silica) and natural soils. In addition, we have analyzed model compounds of known structure and composition to (1) directly compare model compound and sorption EXAFS spectra, (2) extract phase and amplitude functions from theoretically calculated structures for modeling sorption complexes of unknown structure, and (3) ascertain how experimental model-derived phase and amplitude functions compare with $a b$ initio FEFF ${ }^{[1]}$ derived phase and amplitude functions.

\section{EXPERIMENTAL METHODS}

Sorption experiments are conducted under conditions of controlled $\mathrm{pH}$ and ionic strength to maximize uptake of ions from solution onto the oxide surface. Reactions are done under dry nitrogen to minimize carbonato complex formation (e.g., $\mathrm{PbCO}_{3}$ whose precipitation would prevent direct sorption of $\mathrm{Pb}$ on iron oxide) and to eliminate dissolved or gaseous oxygen as an oxidant in redox studies. Light is excluded from the system when photoredox might be expected to occur, as in the case of $\mathrm{Cr}$ sorption on iron oxides. Solids are characterized by BET for surface area analysis, XPS for chemical characterization, and XRD for structural characterization and phase identification. After reaction, the mineral/solution mixture is centrifuged and the supernatant decanted for analysis by atomic absorption spectroscopy. The solid centrifugate is mounted as a still-wet paste into Teflon sample holdèrs used for XAS studies, and 25 mil Mylar tape is used to seal the paste in the holder to minimize sample drying.

SSRL beamlines used include II-3, IV-3, and VI2. We used both the Ge array and Lytle detectors for fluorescent $x$-ray detection, and worked with a liquid He cryostat for some of the samples.

\section{RESULTS}

\section{I. $C r(I I I)$ and $C r(V I)$ Sorption on Iron Oxide Minerals}

The reduction of aqueous $\mathrm{Cr}$ (VI) to $\mathrm{Cr}$ (III) at the surface of magnetite $\left(\mathrm{Fe}^{2+} \mathrm{Fe}^{3+}{ }_{2} \mathrm{O}_{4}\right)$ occurs via heterogeneous electron transfer. $\mathrm{Cr}(\mathrm{VI})_{\mathrm{aq}}$ reacted with freshly-synthesized magnetite at surface coverages of $1-4.5 \mu \mathrm{mol} / \mathrm{m}^{2}$ is entirely reduced to $\mathrm{Cr}$ (III), as evidenced by the $\mathrm{Cr} \mathrm{K}$-edge position and by the absence of a $1 \mathrm{~s} \rightarrow 3 \mathrm{~d}$ pre-edge peak. XAS spectra of $\operatorname{Cr}(\mathrm{VI})_{\mathrm{aq}}$ reacted with progressively oxidized magnetite surfaces, however, show increasing pre-edge peak areas indicating the presence of some $\mathrm{Cr}$ (VI) at the mineral surface, hence a reduction in the $\mathrm{Cr}$ reducing capacity of oxidized (maghemite-coated) magnetite grains. As expected, $\mathrm{Cr}(\mathrm{VI})$ sorbed to a ferric oxide, synthetic maghemite $\left(\gamma-\mathrm{Fe}^{3+}{ }_{2} \mathrm{O}_{3}\right)$, is not significantly reduced (see Fig. 1). EXAFS fitting parameters were derived from FEFF (v. 6.01a) models of $\mathrm{Cr}$ (III)- and $\mathrm{Cr}(\mathrm{VI})$-containing compounds. Fits to the data suggest that (1) $\mathrm{Cr}(\mathrm{VI})$ is reduced to $\mathrm{Cr}(\mathrm{III})$ at the surface of fresh magnetite and adsorbed innerspherically with two shells of neighbor atoms detectable $\left(N_{\text {oxygen }}=6, R=1.98 \AA\right.$ and $N_{F e}$ or $C_{F}=1-3$, $\mathrm{R}=2.97 \AA$ ) providing direct, in-situ XAS evidence for heterogeneous redox; (2) $\mathrm{Cr}$ (VI) on maghemite is outer-spherically adsorbed with only firsi-neighbor oxygen atoms detectable $(\mathrm{N}=4, \mathrm{R}=1.62 \AA)$; and (3) oxidation of the magnetite surface to maghemite inhibits electron transfer so that $\mathrm{Cr}(\mathrm{VI})$ is only partially reduced to $\mathrm{Cr}$ (III) at the oxidized magnetite surface. Two shells of oxygen fit the surface-oxidized 

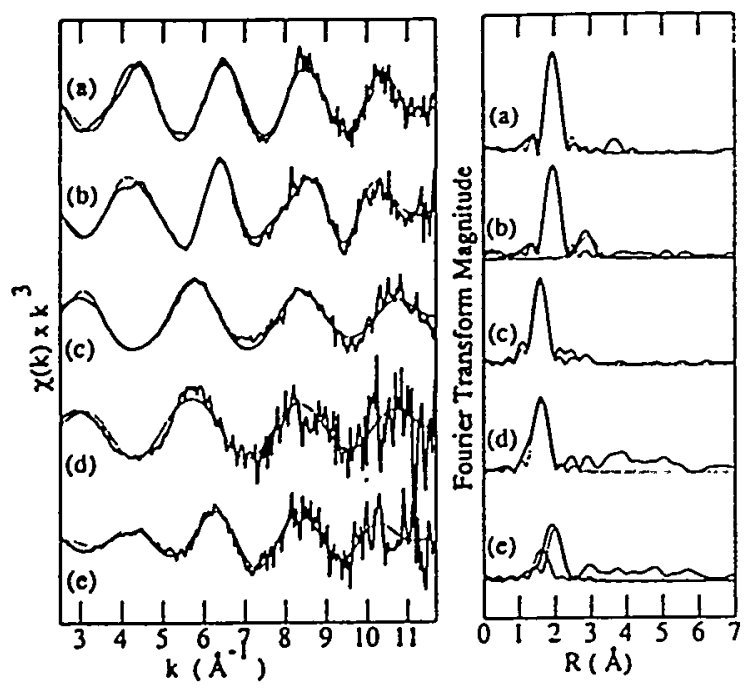

Figure 1. Cr K EXAFS and phase-corrected Fourier transforms showing experimental data (solid lines) and FEFF fits (dotted lines) of (a) $\mathrm{Cr}$ (III) model $\mathrm{CrCl}_{3} \cdot 6 \mathrm{H}_{2} \mathrm{O}$ (aq), (b) reduced $\mathrm{Cr}$ (III) on fresh magnetite, (c) $\mathrm{Cr}$ (VI) model $\mathrm{Na}_{2} \mathrm{CrO}_{4}$ (aq) (d) $\mathrm{Cr}$ (VI) on maghemite, and (e) partially reduced $\mathrm{Cr}$ in both +3 and +6 valence states on surfaceoxidized magnetite. Note the shift in oxygen distances from $2.0 \AA$ for $\mathrm{Cr}(\mathrm{III})$ to $1.6 \AA$ for $\mathrm{Cr}(\mathrm{VI})$.

magnetite data, with distances of $1.62 \AA$ (consistent

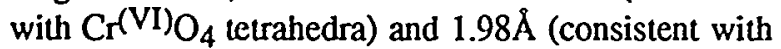
$\mathrm{Cr}^{(\mathrm{III})} \mathrm{O}_{6}$ octahedra) (Fig. 1).

We also collected XAS spectra of several $\mathrm{Cr}$ model compounds at both room temperature and $10 \mathrm{~K}$ using a liquid He cryostat. Distances derived from FEFF fits to the data were virtually identical for the two temperatures, but the Debye-Waller factors were 30-50\% lower for low temperature compounds due to the decrease in thermal atomic vibration. When we compared experimental model-derived phase and amplitude fits to FEFF-derived fits, we found that FEFF gives statistically better fits to the experimental data, in part because there is no noise in the theoretically-derived FEFF models.

\section{As and Se in Soils and Mine Tailings}

As part of an ongoing study of the speciation of toxic and redox-sensitive elements in natural materials, we collected approximately 20 XANES spectra of Se and $A s$ in natural soils and model compounds during our March 94 time on BL IV-3 and our June 1994 beamtime on VI-2. In addition, XAS spectra for three sets of undisturbed and acid-digested As-bearing mine tailings (6 samples) and As model compounds were collected in June 1994 on BL VI-2 (with $\mathrm{M}_{0} \mathrm{Pt}$ focusing mirror). The acid digestion process was carried out to simulate steps in human digestion that might result in the release and absorption of As from tailings particles in the human gastrointestinal tract. During the March beamtime, there were problems with the $\mathrm{Ge}$ array detector (11 of 13 channels unusable), and the poor resolution of BL VI-2 inhibited collection of high quality XANES spectra (needed for quantitative analysis) in June. However, collection of EXAFS spectra for As in mine tailings was successful on BL VI-2.

All tailings samples (sample names RM and VAN) have a major peak in the EXAFS Fourier transform $\Leftrightarrow 1.4 \AA$, uncorrected for phase shift) corresponding with that of $\mathrm{AsO}_{4}$ (Fig. 2). Although the $R M / R M D$ samples ("D" refers to digested sample) are predominantly quartz $\left(\mathrm{SiO}_{2}\right)$ and illite (a poorly ordered aluminosilicate clay), $N(>2)$ and $R(\approx 2.9$ $3.3 \AA$ ) values and the prevalence of mixed $Z$ and/or mixed $R$ second shells suggest that As is mainly coprecipitated with poorly ordered Fe hydroxides, and a minor component is sorbed onto illite. Singleelement X-ray maps generated from electron microprobe analysis of the RM/RMD samples support this interpretation, as regions of high $\mathrm{Fe}$ fluorescence also show high As fluorescence. The VAN/VAND samples have Fourier transform peaks at $=2.1 \AA$, too close to represent binding of $\mathrm{AsO}_{4}$ on or in a mineral. This distance is constent with As-S correlations. Electron microprobe analyses of As in VAN/VAND samples reveal that $A s K \alpha \quad x$-ray fluorescence is highest when small grains of iron sulfides are probed.
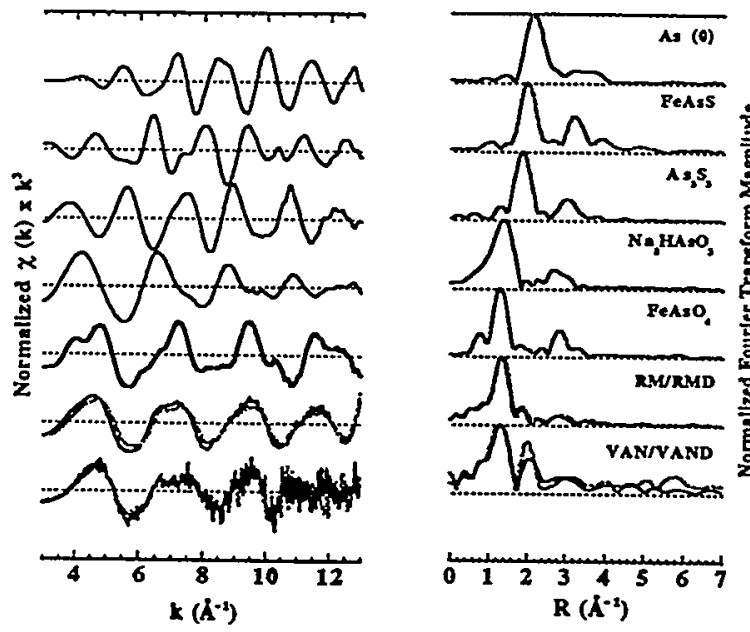

Figure 2. Arsenic K EXAFS of acid-digested and undisturbed mine tailings and model compounds. Unperturbed samples (RM and VAN; solid lines) and aciddigested samples (RMD and VAND; dotted lines) are overlain for comparison; there is little difference between the two. Samples are not identical to any of the model compounds, although the phase of oscillations and the position of the first peak of the Fourier transform most resembles $\mathrm{FeAsO}_{4}$. Note the strong peak at $2.1 \AA$ in the VAN/VAND samples, which may be attributable to $S$. 
Therefore, the VAN/VAND samples are most likely a mixture of reduced and oxidized As, although XANES analysis is required for testing of this hypothesis.

\section{Se(IV) Sorption on Kaolinite}

Preliminary XAS data for Se(IV) sorbed on kaolinite $\left(\mathrm{Al}_{2} \mathrm{Si}_{2} \mathrm{O}_{5}(\mathrm{OH})_{4}\right)$ and for model compounds were collected on $\mathrm{BL}$ VI-2 (with a $\mathrm{Pt}$ focusing mirror) in June, 1994 under ambient conditions, in fuorescence mode, and with the Ge array detector (Fig. 3). Model compound data were collected in transmission. Phase shift and amplitude functions of $\mathrm{Se}-\mathrm{O}$ and $\mathrm{Se}-\mathrm{Mg}$ were extracted from FEFF-calcutated EXAFS of $\mathrm{MgSeO}_{3} \cdot 6 \mathrm{H}_{2} \mathrm{O}$ because a suitable model compound with both $\mathrm{Se}$ (IV) and $\mathrm{Al}$ was not available at the time of data collection. The sensitivity of EXAFS $(\mathrm{Z}+1-2)$ is such that $\mathrm{Mg}$ phase and amplitude functions may serve as a surrogate for Al phase and amplitude functions, since $\mathrm{Mg}$ and $\mathrm{Al}$ are both in octahedral sites in these minerals. $\mathrm{Al}$ and $\mathrm{Si}$, both present in kaolinite, cannot be resolved on the basis of their phase and amplitude functions alone for the same reason.

Preliminary fitting of the sorption samples spectra indicates the presence of $3.2-3.8 \mathrm{O}$ atoms at $1.70 \AA$ (phase corrected for $\mathrm{O}$ ) in agreement $\mathrm{Se}(\mathrm{IV})-\mathrm{O}$ distances calculated in other EXAFS studies. In addition, the "high coverage" spectrum is best fit to 2.7 $\mathrm{Al}$ or $\mathrm{Si}$ at $3.60 \AA$, and the "low coverage" spectrum to $1.3 \mathrm{Al}$ or $\mathrm{Si}$ at $3.21 \AA$ (phase corrected for $\mathrm{Mg}$ ). It would appear that with increasing surface coverage, bidentate binding of Se becomes predominant, although data reduction and interpretation for these samples is still in progress.

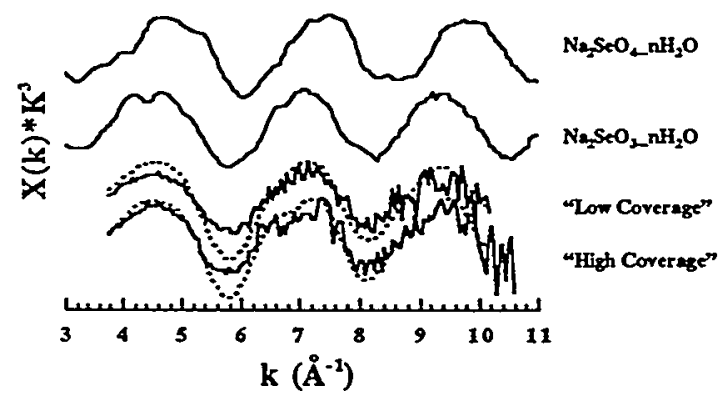

Figure 3. EXAFS spectra of $\mathrm{Se}$ (VI) and Se(IV) models (top two lines) and Se(IV) sorbed on kaolinite. Qualitative comparison of samples with model compounds demonstrates that samples are indeed $\mathrm{Se}(\mathrm{IV})$, since the spectral oscillations are in phase with $\mathrm{Se}$ (IV) model $\mathrm{Na}_{2} \mathrm{SeO}_{3} \cdot \mathrm{nH}_{2} \mathrm{O}$, and not with $\mathrm{Se}(\mathrm{VI})$ model $\mathrm{Na}_{2} \mathrm{SeO}_{4} \cdot \mathrm{nH}_{2} \mathrm{O}$. Dotted lines overlaying sample spectra are preliminary fits, in which $\mathrm{Se}$ is coordinated by approximately $3 \mathrm{O}$ at $1.70 \AA$ and $1-2 \mathrm{Al}$ or $\mathrm{Si}$ at $3.21-3.60 \AA$.

\section{Lead(II) Sorption on Al-and Fe-Oxides}

Lead is another metal of environmental importance due to its widespread occurrence as a contaminant of soils and waters Knowledge of the molecular composition and geometry of chemisorbed lead(II) is of critical importance in constructing thermodynamic models and rate laws for reactions at mineral surfaces which control lead mobility in natural environments. Lead $\mathrm{L}_{\mathrm{II}}$-edge spectra were collected for lead model compounds, and lead(II) sorbed on Aland Fe-oxides and hydroxides on beamlines II-3, IV-3 and VI-2. Results were of mixed success. Highquality spectra of lead(II)/alumina $\left(\alpha-\mathrm{Al}_{2} \mathrm{O}_{3}\right)$ at submonolayer and monolayer adsorption densities were obtained at room temperature and $10 \mathrm{~K}$ on BL IV-3 in March 1994. Combined with data from 1993 SSRL experiments, results indicate that lead(II) chemisorbs on alumina in a bridging or bidentate geometry (i.e. it forms two chemical bonds to oxide or hydroxide ions in the alumina surface). At monolayer adsorption density, lead ions link through hydroxo bridges to form surface oligomers. Lead(II) coordination number decreases from 6 in water to 3 on the surface, and average $\mathrm{Pb}-\mathrm{O}$ bond length shortens from $2.61 \AA$ to $2.25 \AA$. The preferred binding site was clearly shown to be edges of $\mathrm{AlO}_{6}$ octahedra exposed on the surface. This contradicts previous macroscopic solution studies [2-3], which suggested that lead(II) chemisorbs in an atop geometry on alumina.

Spectra for lead(II) sorbed on hematite $\left(\alpha-\mathrm{Fe}_{2} \mathrm{O}_{3}\right)$ and goethite $(\alpha-\mathrm{FeOOH})$ were collected on BL VI-2 in June 1994 using $\mathrm{Si}(111)$ monochromator crystals, and the toroidally bent $\mathrm{Pt}$ focusing mirror. Flux was approximately 100 times greater than on BL IV-3. Results were disappointing; beamline noise ranged from 1 to $3 \%$ (compared to $0.3 \%$ on BL IV-3) and completely offset the enhanced $S / N$ from increased flux. In spite of this, analyzable data were collected. Preliminary data reduction suggests that lead(II) chemisorbs to edges of $\mathrm{FeO}_{6}$ octahedra on hematite and goethite surfaces, but does not form surface oligomers as on alumina.

\section{Copper Model Compound Study}

We have collected transmission mode data for a series of crystalline inorganic copper model compounds containing $\mathrm{Cu}$ in different chemical environments and oxidation states, and two crystalline copper compounds with the copper atom coordinated to the organic ligand 2,2'-bipyridine (bipy). We have chosen these compounds to discem the contribution of different ligands and geometrical environments on the XANES and EXAFS features of $\mathrm{Cu}$ compounds and to help analyze data of $\mathrm{Cu}$ (II) adsorbing on silica and alumina surface in the presence and absence of bipy. 
Phase and amplitude parameters generated from FEFF were used to fit the data and lead to the following conclusions: (1) the main oscillation is due to 1st shell oxygens at about 1.9 to $2.0 \AA$, (2) oxygens that are farther away, at about 2.2 to $2.6 \AA$ due to Jahn Teller distortions, have large Debye-Waller factors and contribute insignificantly to the EXAFS features, and (3) multiple scattering from $N$ and $C$ in bipy contribute significantly to the EXAFS features.

\section{CONCLUSIONS}

XAS is uniquely suited for non-destructive analysis and characterization of heavy metal environmental contaminants sorbed to minerals, sediments, and soils. The information derived from fitting of XAS data, i.e. the oxidation state of sorbed $\mathrm{Cr}, \mathrm{As}$, and $\mathrm{Se}$, and the configuration of sorbed complexes of $\mathrm{Cr}$ on iron oxides, Se and As on natural mine tailings and kaolinite, $\mathrm{Pb}$ on iron oxides, and $\mathrm{Cu}$ on silica and alumina is of great importance for realistic chemical transport and surface complexation modeling. Model compound study has shown that (1) direct comparison of sorption comlexes with models can indicate the dominant oxidation state of a sorption complex at a solid surface, and (2) FEFF-derived phase and amplitude functions give statistically better fits to experimental data than do model-derived phase and amplitude functions.

\section{ACKNOWLEDGMENTS}

We gratefully acknowledge the SSRL staff for their support, in particular Hal Tompkins for his ongoing improvements of the BL IV-3 harmonics rejection mirror. Financial support for this work was provided through NSF grant EAR-9105105, DOE grant DE-EG03-93ER14347-A000, and graduate fellowships from the Corning Foundation and the National Science Foundation.

\section{REFERENCES}

[1] Rehr, J.J., Mustre de Leon, J., Zabinsky, S.I., Albers, R.C. J. Am. Chem. Soc. 1991, 113, 5135-5140.

[2] Davis J.A. III and Leckie J.O. J. Colloid Interf. Sci. 1978,67, 90-107.

[3] Hohl H. and Stumm W. J. Colloid Interf. Sci. 1976, 55, 281-288. 
Proposal 2153Mp

\title{
ANOMALOUS SMALL-ANGLE X-RAY SCATTERING STUDIES OF AMORPHOUS MOLYBDENUM-GERMANIUM ALLOYS
}

\author{
M.J. Regan* and A. Bienenstock \\ Stanford Synchrotron Radiation Laboratory, P.O. Box 4349, Stanford, CA 94309
}

\section{Introduction}

For the past several years, this group has been involved in a study of composition fluctuations in sputtered amorphous $M_{x} \mathrm{Ge}_{1-x}(M=\mathrm{Fe}, \mathrm{Mo}, \mathrm{W})$ and $\mathrm{Fe}_{x} \mathrm{Si}_{1-x}$ alloys using anomalous small-angle $x$-ray scattering.[1] As a result of these efforts, it is now clear that the metal/insulator transition $(0.1<x<0.3)$ in these and similar alloys is not an attribute of homogeneous material. Rather, we observe significant chemical inhomogeneity that exists on a very fine-size scale $(1-3 \mathrm{~nm})$ throughout a wide composition range $(x<0.3)$, is quite anisotropic, and depends on the growth conditions.[2]

The microstructure can be determined by utilizing the cylindrical correlation function or suitable particle models[3]. Both methods suggest a physical picture in which wellcorrelated inhomogeneities are formed at the surface during film growth, with little correlation between those formed in one layer and those formed after $2 \mathrm{~nm}$ of material have been deposited. These results indicate that composition fluctuations in the growth direction play a pivotal role in preventing simple columnar structure growth or structures that evolve systematically as the film grows. As a consequence, electron tunneling and hopping phenomena are probably strongly influenced by the resulting microstructure, which also leads to unique percolation paths for the metallic phase.

The method we use to study these issues is small-angle $x$-ray scattering, which arises from variations in the scattering density on size scales that are large (typically $10 \AA-1000 \AA$ ) compared to atomic dimensions. At $x$-ray energies far removed from any absorption edges in the material, the scattering density is simply the electron density. For energies near an absorption edge, however, the anomalous scattering factors define an effective electron density. By varying the anomalous scattering factors with changes in the $x$-ray energy, the effective electron density is altered, leading to species-specific information which allows one to differentiate between voids and chemical inhomogeneities, among others [4].

In the past year, we have undertaken further studies of the amorphous $\mathrm{Mo}_{x} \mathrm{Ge}_{1-x}$ alloys, since, in addition to the well-known metal/insulator transition near 10 at.\% Mo, there is a normal/superconducting transition near 13.5 at.\%
Mo.[5] Characterization of Mo-containing alloys with anomalous small-angle $x$-ray scattering (ASAXS) is extremely difficult with hard $x$-rays (Mo $K$ edge at $20 \mathrm{keV}$ ), and the purpose of this brief report is to highlight the methods we have used to measure the ASAXS with hard $x$-rays.

\section{Experiment}

As mentioned in previous Annual Reports to SSRL (1991-1993), amorphous films were prepared by magnetron sputtering of elemental targets onto Si wafers attached to a rapidly rotating substrate table. The Si substrates were then removed and the films ( $-6-9$ microns thick) rendered free-standing after immersion in a warm $\mathrm{KOH}$ bath.

ASAXS data were collected on SSRL beam line 4-2 at several energies near the the Ge $K$ edge (11.1 keV) and the Mo $K$ edge $(20.0 \mathrm{keV})$. $B y$ varying the $x$-ray energy from -400 to $-10 \mathrm{eV}$ below, for example, the Mo edge, the real part of the Mo anomalous scattering factor becomes increasingly negative, while the imaginary component remains small and slowly varying. The atomic scattering factor can be altered by $-12-15 \%$ for these elements with this method, leading to variable contrast.

To obtain ASAXS data at the Mo and Ge edges, a Xe/CO $\mathrm{CO}_{2}$ gas mixture was used in the position sensitive proportional counter to improve the detector efficiency over the usual $\mathrm{Ar} / \mathrm{CO}_{2}$ efficiency. With a $70 \% \mathrm{Ar} / \mathrm{balance} \mathrm{CO}_{2}$ mixture, the $1 / \mathrm{e}$ absorption length is $77 \mathrm{~cm}$ at 20 $\mathrm{keV}$, which leads to very poor detector efficiency (conversion depth is typically only $6 \mathrm{~mm}$ for these detectors). In order to record the Mo edge coherent scattering and perform ASAXS, however, the $80 \% \mathrm{Xe} /$ balance $\mathrm{CO}_{2}$ counter gas that gave an absorption length of $8.2 \mathrm{~cm}$ at the Mo edge was used.

This was still not sufficient, however, to carry out ASAXS since the large fraction of Ge in the samples swamped the detector with fluorescence $\left(\mathrm{K} \alpha_{1}=9886, \mathrm{~K} \alpha_{2}=9855 \mathrm{eV}\right.$, etc.). These lower energy photons are distributed over $4 \pi$ solid angle and are detected as a constant background superimposed on the coherent scattering. With the fluorescence emitted at lower energies and, hence, preferentially absorbed in the limited detector depth (absorption length is $1.5 \mathrm{~cm}$ for the $\mathrm{Xe}$ mixture at $10 \mathrm{keV}$ ), the count rates are so 
large that space-charge effects and dead time corrections become appreciable. Although the detector energy resolution is approximately $20 \%$ and the fluorescence can be discriminated, the beam flux must be reduced in any case to avoid the high count rates, and the resulting coherent scattering signal is poor.

To avoid this problem, a large, sputtered $\mathrm{Zn}$ filter [which has an absorption edge between the fluorescent radiation and the coherent signal $(\mathrm{Zn}$ $K$ edge at $\sim 9.7 \mathrm{keV}$ )] was placed over the linear detector, downstream of the beam stop, so the $\mathrm{Ge}$ fluorescence could be preferentially absorbed without excitation of $\mathrm{Zn}$ fluorescence by the direct beam. In previous studies, fluorescence from $\mathrm{Fe}$ at $\mathrm{X}$-ray energies near the Ge edge in the Fe-Ge samples was not so great as to warrant the use of an absorbing filter and was removed with the background subtraction method outlined. This is due to the small $\mathrm{Fe}$ concentrations studied and the low fluorescence yield of the lighter elements.

In addition, ASAXS data were collected with the scattering vector both parallel to the film surface plane and at oblique angles to it. These data have been discussed in previous SSRL Annual Reports and will not be mentioned this year. We are in the process of further analyzing these data in terms of particle-particle correlation functions, more commonly known as radial distribution functions for isotropic systems.

\section{Data Analysis}

The spectra were: (a) corrected for background scattering, (b) normalized to incident intensity, sample absorption and thickness, and (c) placed on an absolute scale with polyethylene. There remained, however, the problem of isolating the sample's coherent scattering, the differential cross-section per sample volume $d \sigma d \Omega$, which is superimposed on the angular-independent incoherent scattering, $\Re(E)$, to yield the total scattering, $d \sigma(k, E) / d \Omega+\Re(E)$.

- The incoherent scattering consists of fluorescence and resonant Raman scattering (Compton scattering is negligible at small angles). The $\Re(E)$ in these data were subtracted in the following way. For a particular sample and oblique transmission angle, the coherent scattering at sufficiently large $k$ was assumed to follow a power law, so that the total scattering can be written as

$$
\frac{d \sigma(k, E)}{d \Omega}+\Re(E)=A / k^{\alpha}+B .
$$

The $B$ was then removed from each scan, rendering $d \sigma(k, E) / d \Omega$. We tried to determine $A, \alpha$ and $B$ using a non-linear least-squares routine to fit the observed scattering. The results were inconclusive, however, since the relevant $k$ range that can be fit is less than a decade--limited by the large interparticle interference at small $k$ and the poor signal at very large $k$ and is extremely sensitive to errors.

Maret et al.[6] used the Porod scattering power-law for sharp interfaces, $\alpha=4$, to fit their data. Schmidt et al.[7] have shown that the SAXS at large $k$ can decay at a rate greater than $\alpha=4$ and as great as $\alpha=6$ ("fuzzy" boundary model). We did not find it possible to determine $\alpha$ uniquely over the range 4-5. Since that was the case, the coherent scattering was extracted using two different values of $\alpha, 4$ and 5 , to test the sensitivity of physical results to the choice of $\alpha$. See Regan [1] for a futher discussion of background subtraction and power law scattering.

\section{Results}

Figure 1 illustrates the recorded scattering at normal incidence (i.e. $k$ lies in the sample surface plane) for several $\mathrm{Mo}_{x} \mathrm{Ge}_{1-x}$ alloys collected at an $\mathrm{X}$-ray energy of $10.8 \mathrm{keV}$ with the $\mathrm{Xe} / \mathrm{CO}_{2}$ gas filled detector. The 6.5 and 12.4 at.\% Mo samples exhibit the greatest magnitude of scattering, with it less intense for the 16.5 at.\% Mo sample, while little scattering is recorded for 23.6 at.\% Mo.

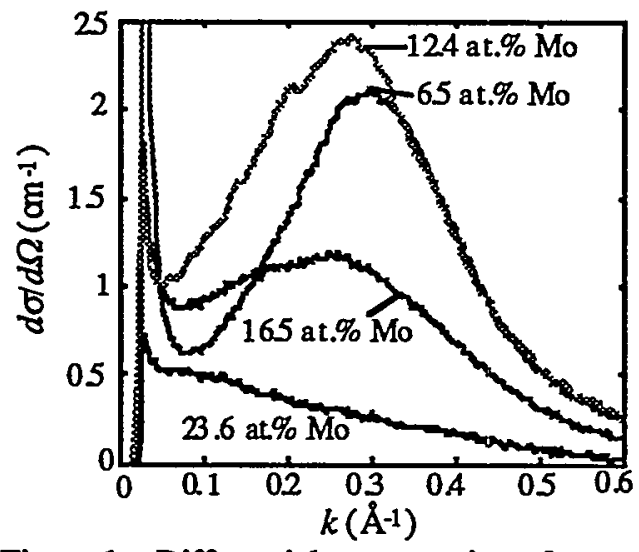

Figure 1. Differential cross-section of scattered radiation as a function of composition for several $\mathrm{Mo}_{x} \mathrm{Ge}_{1-x}$ alloys. Samples held at normal. incidence, with the scattering vector in the sample surface plane.

The scattering that is recorded for 23.6 at.\% Mo is extremely weak and is of a different kind, at small $k$, with only a small scattering maximum resolvable. We have observed this sample's scattering pattern during different data collection periods as well as during a single collection period but with different camera lengths. It is thus likely that the small SAXS reported for this sample indicates that the volume fraction of the chemical inhomogeneity is decreasing rapidly and becomes essentially zero at -25 at.\% Mo. Attempts with anomalous scattering to verify that this is, in fact, the case, have not been successful. Differences in the extremely small SAXS signal 
at energies near the $\mathrm{Ge}$ and Mo edges lead to such poor signal-to-noise ratios that prevent a systematic study at this composition with anomalous scattering.

The oblique transmission results ( $k$ along different film directions) along with the interpretation of the SAXS maxima have been reported in previous Annual Reports, but the anomalous scattering results at the Mo edge have not. This has to do with the difficulty we encountered in recording the data in previous years, and the fact that these results were obtained only in late 1993 and 1994.

For samples with $6.5,12.4$, and 16.5 at.\% Mo, only slight if any changes in scattering are observed as the incident photon energy is increased beneath the Ge absorption edge while there are noticeable differences for similar changes beneath the Mo edge. This observation has been made previously by Rice et al.[4] for amorphous $\mathrm{Fe}-\mathrm{Ge}$ and W-Ge alloys at the Fe, W, and $G e$ edges. These authors show that a zero variation of the intensity at the Ge edge implies that the $\mathrm{Ge}$ is distributed homogeneously, with the change at the metal edge associated with an inhomogeneous distribution of metal atoms.

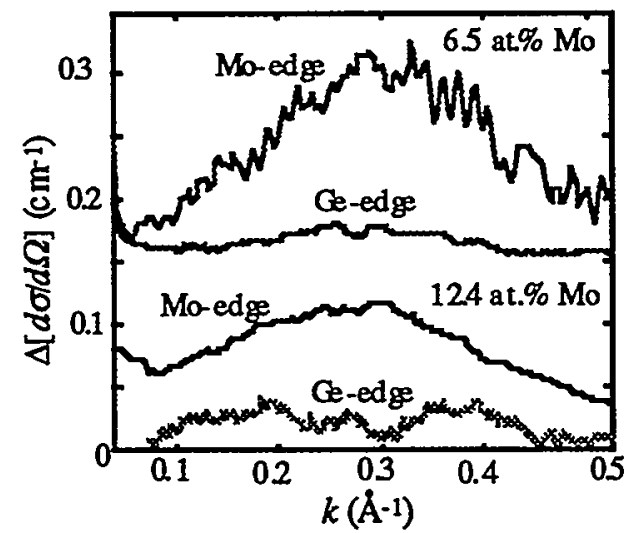

Figure 2. Absolute changes in SAXS at the Mo and $\mathrm{Ge}$ edges, scaled by the relative change in $f(E)$ at each edge. At the Ge edge, $E_{1}=-300$ and $E_{2}=-10 \mathrm{eV}$ below the edge, and $E_{1}=-400$ and $E_{2}=-10 \mathrm{eV}$ below the Mo edge.

Figure 2 shows the changes in $d \sigma / d \Omega$ for two samples at the $\mathrm{Ge}$ and Mo edges scaled by the relative change in the anomalous scattering factors. That is, we plot

$$
\frac{d \sigma\left(k, E_{1}\right) / d \Omega-d \sigma\left(k, E_{2}\right) / d \Omega}{\left|f_{v}\left(E_{1}\right)-f_{v}\left(E_{2}\right)\right|}
$$

for $v=M o$ and $G e, E_{1}=-300$ and $E_{2}=-10 \mathrm{eV}$ below the $\mathrm{Ge}$ edge, and $E_{1}=-400$ and $E_{2}=-10 \mathrm{eV}$ below the Mo edge. Similar results have also been obtained for the 16.5 at.\% Mo film. The relative change at the Mo edge is approximately a factor of 5-10 greater than that observed at the Ge edge. An $11 \%$ change in $\mathrm{f}_{\mathrm{Mo}_{0}}(E)$ causes an approximate $30 \%$ change of the main scattering peak, whereas the $16 \%$ change in $\mathrm{f}_{\mathrm{Ge}}(E)$ leads to a mere $6 \%$ intensity change.

The large change at the Mo edge is evidence that the Mo atoms are not distributed homogeneously. As with the Fe-Ge and W-Ge materials in the $x<0.3$ region, the $\mathrm{Ge}$ appears relatively homogeneously distributed whereas the Mo is inhomogeneously distributed. PSFs have not been obtained from these data. The poor Mo edge SNR introduces large uncertainties in the PSFs, if either the standard Keating[8] or Munro[9] approaches are used.

Although Mo edge data were collected for samples only at normal incidence, due to the long measurement times needed to perform ASAXS from such weak scattering and poor detector efficiency with hard $x$-rays, it is expected that the anomalous scattering results are similar at all oblique angles. Consistent with this view, the Ge edge data were collected for these samples at the oblique angles and little, if any, change of intensity with $f_{G e}(E)$ is observed.

\section{Conclusions}

We have observed anomalous small-angle $\mathrm{x}$ ray scattering at the Mo and Ge $K$ edges of amorphous Mo $\mathrm{Ge}_{1-x}$ films. Technically, we have shown that ASAXS is possible with hard $\mathrm{X}$ rays, even in the presence of significant fluorescence from other elements. The result is particularly satisfying since we now have definitive evidence that there is a true chemical inhomogeneity in the Mo-Ge alloys as was observed for the Fe-Ge and W-Ge systems[4]. The results indicate that the Mo atoms are distributed inhomogeneously, within a homogeneous distribution of $\mathrm{Ge}$, leading to a general model of phase separation into amorphous Ge and a Mo-Ge intermetallic with a Ge number density similar to that for $\mathrm{a}-\mathrm{Ge}$.

Analysis of the data in terms of specific models and correlation functions have been reported elsewhere[2,3], indicating, among others, that the Mo-Ge intermetallic is $\mathrm{MoGe}_{3}$, but we are still in the process of analyzing the oblique transmission data collected in 1994. These results should help in better understanding the microstructure, in particular the structural characteristics near the percolation limit and the superconducting transition.

\section{Acknowledgements}

The research was performed at and supported by the Stanford Synchrotron Radiation Laboratory, which is funded by the Department of Energy through the Office of Basic Energy Science and the NIH, Biotechnology Resource Program, Division of Research Resources. 


\section{References}

* Present Address: Division of Applied Sciences, Harvard University, Cambridge, MA 02138

[1] M. Rice, Ph.D. Thesis, Stanford University, 1993. M.J. Regan, Ph.D. Thesis, Stanford University, 1993.

[2] M.J. Regan, M. Rice, M.B. Fernandez van Raap, and A. Bienenstock, Phys. Rev. Lett. 73, 1118 (1994).

[3] M.J. Regan and A. Bienenstock, Phys. Rev. B (accepted for publication); J. Non-Cryst. Sol. (accepted for publication).
[4] M. Rice, S. Wakatsuki, and A. Bienenstock, J. Appl. Cryst. 24, 598 (1991).

[5] See S. Yoshizumi, T.H. Geballe, M. Kunchur, and W.L. McLean, Phys. Rev. B 37, 7094 (1988) and references therein.

[6] M. Maret, J.P. Simon, B. Boucher, R. Tourbot, and O. Lyon, J. Phys. Condens. Matter 4, 9709 (1992).

[7] P.W. Schmidt, D. Avnir, D. Levy, A. Hohr, M. Steiner, and A. Roll, J. Chem. Phys. 94, 1474 (1991).

[8] R.G. Munro, Phys. Rev. B 25, 5037 (1982).

[9] D.T. Keating, J. Appl. Phys. 34, 923 (1963). 


\title{
Sorption of Aqueous $\mathrm{Zn}^{2+}$ on Ferrihydrite: Observation of the Onset of Precipitation.
}

\author{
Glenn A. Waychunas ${ }^{1}$, Christopher C. Fuller ${ }^{2}$ and James A. Davis ${ }^{2}$ \\ ${ }^{1}$ Center for Materials Research, Stanford University, Stanford, CA $94305-4045$ \\ ${ }^{2}$ United States Geological Survey, Water Resources Division, Menlo Park, CA 94025
}

\section{Introduction}

As part of our continuing studies on the sorption of toxic metallic and anionic complexes on hydrous iron oxides we have investigated the local structure near $\mathrm{Zn}^{2+}$ complexes on ferrihydrite.

Ferrihydrite is a poorly crystalline to amorphous hydrous iron oxide with a formula near $5 \mathrm{Fe}_{2} \mathrm{O}_{3} \cdot \mathrm{9H}_{2} \mathrm{O}$. It is found abundantly in natural waters, sediments and soils, and its small particle size and high surface area make it an important sorbent for metals and anionic complexes [1].

$\mathrm{Zn}$ contamination in soils and waters is produced from both industrial processing and mining operations. Often the $\mathrm{Zn}$ occurs with other heavy metals like Cd and $\mathrm{Pb}$. $\mathrm{Zn}$ crystal chemistry is especially complex, e.g. carbonates, oxides and hydroxides may have zinc in tetrahedral, 5-coordinated and 6-coordinated (by oxygen or hydroxyls) sites in a variety of structural motifs. For example, the zinc mineral stable at normal surface conditions is hydrozincite, a zinc carbonate-hydroxide. Hydrozincite has a layer structure with sheets containing both octahedrally and tetrahedrally-coordinated $\mathrm{Zn}$ polyhedra [2].

$\mathrm{Zn}$ sorption on ferrihydrite is additionally interesting because of the form of the adsorption isotherm that has been observed [3]. The sorption obeys a Langmuir (linear) dependence with bulk composition up to sorption densities of about 0.003 $(\mathrm{Zn} / \mathrm{Fe})$. At larger sorption densities the dependence becomes non-linear, suggesting a change in sorption character or precipitation, but this happens at much lower bulk $\mathrm{Zn}$ concentrations than where $\mathrm{Zn}(\mathrm{OH})_{2}$ is expected to form. Thus we have prepared sorption samples with sorption densities from 0.003 to 0.150 $(\mathrm{Zn} / \mathrm{Fe})$, plus an additional sample with sufficient $\mathrm{Zn}$ such that precipitation of $\mathrm{Zn}(\mathrm{OH})_{2}$ is assured. All of these samples, model compounds of both $\mathrm{Zn}$ and $\mathrm{Fe}$, and model aqueous solutions were examined by XAS.

\section{Experimental Details}

Ferrihydrite samples were prepared in two distinct forms: precipitated in the presence of aqueous $\mathrm{Zn}^{2+}$ (coprecipitated samples, CPT); and precipitated separately then exposed to aqueous $\mathrm{Zn}^{2+}$ solutions (adsorbed samples, ADS). The $\mathrm{pH}$ range of preparations was 6-7. Complete details are available in our past publications $[1,4]$.

$\mathrm{Zn}$ and $\mathrm{Fe} \mathrm{K}$-edge EXAFS and XANES spectra were collected on beam lines 4-1 and 4-3 using Si (111) monochromator crystals. Detection was carried out in fluorescence and transmission modes simultaneously. Franklinite $\left(\mathrm{ZnFe}_{2} \mathrm{O}_{4}\right.$, tetrahedral $\mathrm{Zn}$, octahedral $\left.\mathrm{Fe}^{3+}\right)$, zincite $(\mathrm{ZnO}$, tetrahedral $\mathrm{Zn})$, smithsonite $\left(\mathrm{ZnCO}_{3}\right.$, octahedral $\left.\mathrm{Zn}\right)$, willemite $\left(\mathrm{Zn}_{2} \mathrm{SiO}_{4}\right.$, tetrahedral $\left.\mathrm{Zn}\right)$ and hematite $\left(\alpha-\mathrm{Fe}_{2} \mathrm{O}_{3}\right.$, octahedral $\mathrm{Fe}^{3+}$ ) standards were used to acquire empirical phase and amplitude functions for EXAFS analysis. Calculations of these functions was also done using Feff 6.01. Fe and $\mathrm{Zn}$ metal foils were used for energy calibration. Analysis was performed with the EXAFSPAK routines available at SSRL.

\section{Results}

The most surprising result was the change in $\mathrm{Zn}$ coordination noted between sorbed aqueous $\mathrm{Zn}^{2+}$ and aqueous $\mathrm{Zn}^{2+}$. We obtained $\mathrm{Zn}-\mathrm{O}$ distances of 2.11(2) $\AA$ for all solutions measured, with coordination numbers of 6.5(1). This was in agreement with work done several years ago at SSRL [5]. However, all sorbed $\mathrm{Zn}^{2+}$ had $\mathrm{Zn}-\mathrm{O}$ distances of $1.99(2) \AA$ with coordination numbers of 4(1). A comparison of $\mathrm{Zn}-\mathrm{O}$ bond lengths in our samples and in well-known structures is shown in Fig. 1.

Besides the coordination change, EXAFS structure functions of sorbed $\mathrm{Zn}^{2+}$ on ferrihydrite all show small second-shell peaks indicative of a metal second coordination environment. These peaks varied in amplitude with zinc sorption density, having largest amplitude for the lowest densities and vice versa for the range of $\mathrm{Zn} / \mathrm{Fe}$ of $0.003-0.150$ (Fig. 2). These second shells can be fitted by $\mathrm{Zn}$-Fe contributions at a distance of about $3.45(5) \AA$, with about $2 \mathrm{Fe}$ second neighbors at the lowest sorption densities. This distance and coordination number is consistent with a bidentate tetrahedral $\mathrm{Zn}^{2+}$ complex sharing the oxygen 


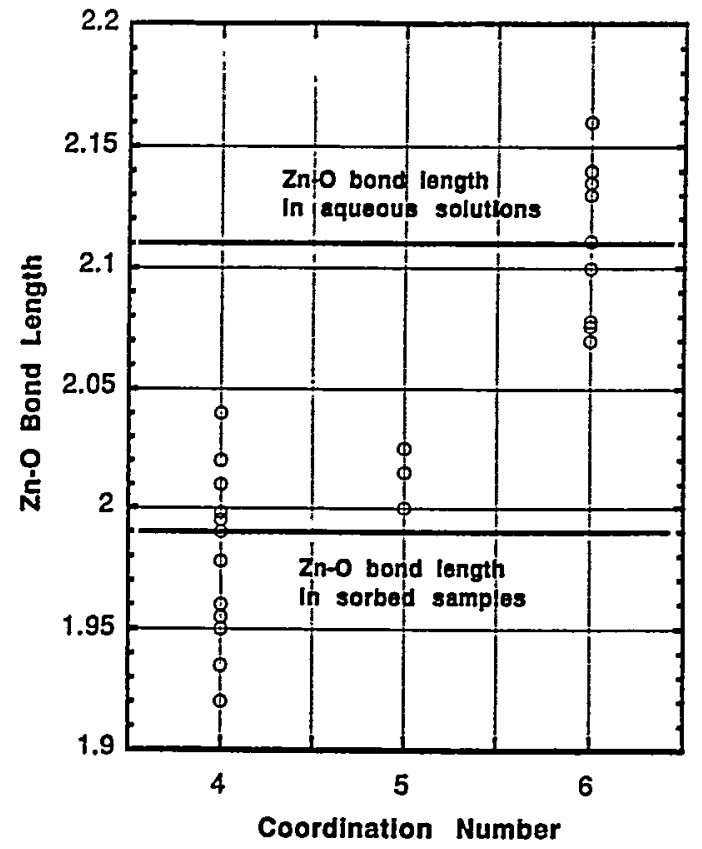

Figure 1: $\mathrm{Zn}-\mathrm{O}, \mathrm{OH}$ bond lengths in various model compounds having 4,5 or 6 -coordinated $\mathrm{Zn}^{2+}$ (open circles). Observed $\mathrm{Zn}-(\mathrm{O}, \mathrm{OH})$ bond lengths in this study (solid horizontal lines).

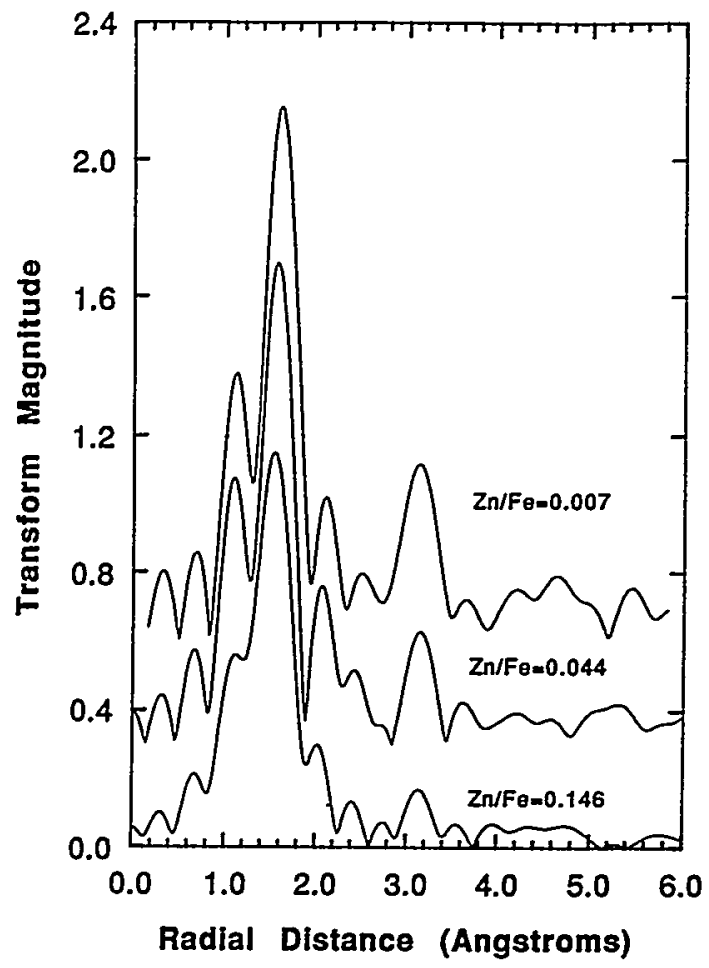

Figure 2: Comparison of $\mathrm{Zn}$ EXAFS radial structure functions for CPT ferrihydrite sorption samples with varying $\mathrm{Zn} / \mathrm{Fe}$ bulk ratios (sorption densities). apices of adjacent $\mathrm{Fe}^{3+}$ oxyhydroxide octahedra. This is a quite different result than observed for $\mathrm{Zn}^{2+}$ sorption on various commercial inorganic materials in more alkaline solutions [6]. At the higher sorption densities the number of neighbors is difficult to determine accurately due to the weak amplitudes. However, the amount of static disorder appears to increase markedly with sorption density up to 0.150 .

In the sample of ferrihydrite prepared with exposure to more concentrated $\mathrm{Zn}^{2+}$ aqueous solutions, the second shell in the structure function was markedly different from that observed for the lower sorption density samples. Two well-resolved contributions are seen at radial distances distinct from the single $\mathrm{Zn}$ $(\mathrm{Zn}, \mathrm{Fe})$ peak seen in samples with $\mathrm{Zn} / \mathrm{Fe}$ of 0.003 0.150 . These features resemble those observed in the structure function of amorphous precipitated $\mathrm{Zn}(\mathrm{OH})_{2}$ (Fig. 3), but with reduced amplitude.

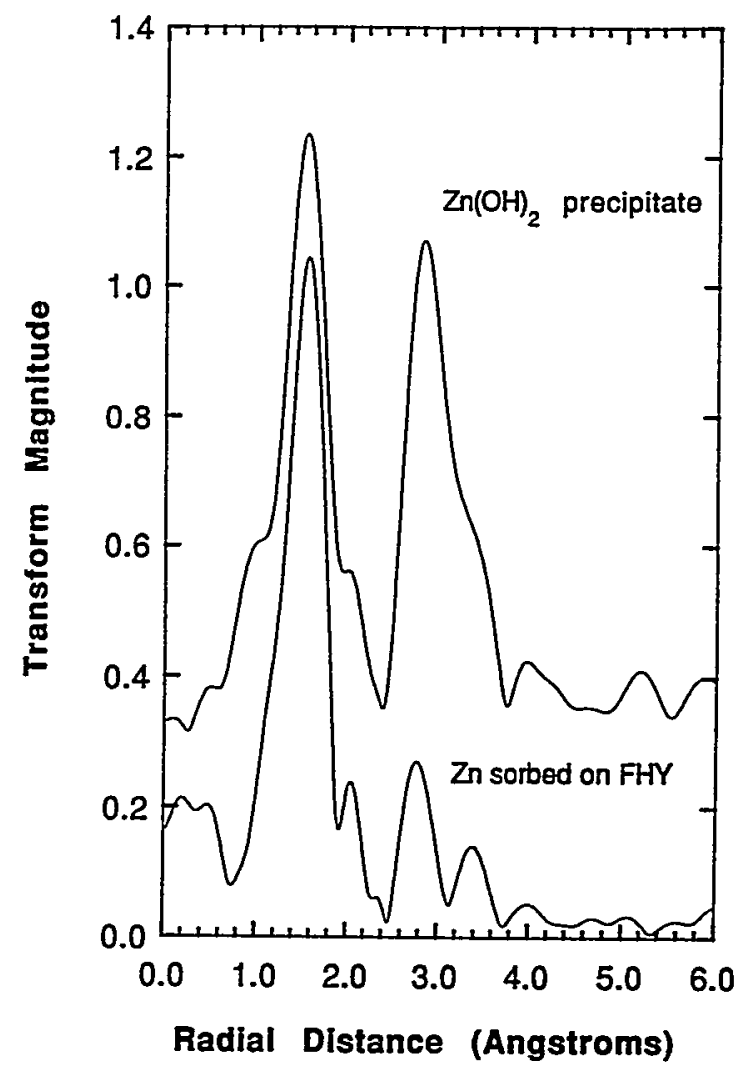

Figure 3: Comparison of $\mathrm{Zn}$ EXAFS radial structure functions from: (top) Zn EXAFS radial structure function of synthetic amorphous $\mathrm{Zn}(\mathrm{OH})_{2}$ precipitate. (bottom) ferrihydrite sample with sorbed $\mathrm{Zn}^{2+}$ exposed to additional more concentrated $\mathrm{Zn}^{2+}$ aqueous solution.

\section{Discussion}

The evolution of the amplitude in the second shell contribution of the sorbed $\mathrm{Zn}^{2+}$, (which we attribute 
to $\mathrm{Fe}^{3+}$ next nearest neighbors) suggests some combination of increasing static disorder as the $\mathrm{Zn}^{2+}$ sorption density increases. This could arise from a number of mechanisms, for example: (1) As the sorption density increases there is a tendency for surface $\mathrm{Zn}-\mathrm{O}-\mathrm{Zn}$ bonds to form, producing a mixture of both $\mathrm{Zn}-\mathrm{Fe}$ and $\mathrm{Zn}-\mathrm{Zn}$ contributions in the second shell of the structure function. $\mathrm{Zn}$ and $\mathrm{Fe}$ backscattering phase and amplitude are not so different so as to cancel one another's contributions, and thus lower the observed peak amplitude. However, the linkages are connecting the initially sorbed zinc tetrahedra to additional zinc tetrahedra away from the surface, and with iron octahedra in the surface. These polyhedra will produce metal-metal distances unlikely to be equal, and thus the EXAFS phases from the contributions may be more disparate causing destructive interference as well as increased static disorder. (2) As the ferrihydrite surface becomes saturated with bidentate $\mathrm{Zn}(\mathrm{O}, \mathrm{OH})_{4}$ units, all additional zinc may form clusters or clots of amorphous zinc hydroxide. Hence, the average $\mathrm{Zn}^{2+}$ retains tetrahedral coordination, but the second shell peak is reduced by the low order in the amorphous precipitate. (3) The zinc complexes may react with the ferrihydrite at higher sorption densities to produce a $\mathrm{Zn}$-Fe precipitate with high structural disorder.

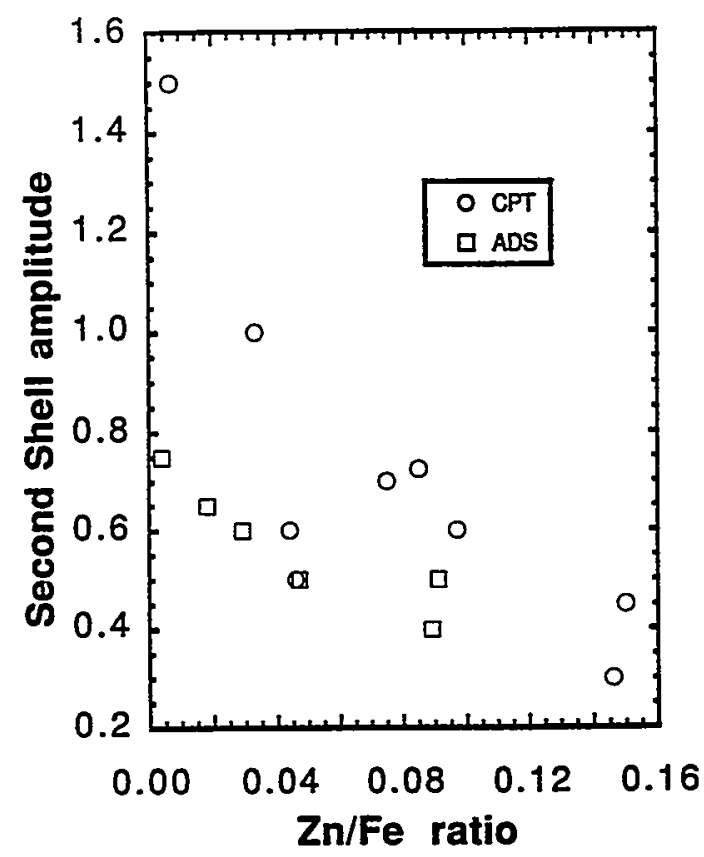

Figure 4: Second shell RSF Zn-(Zn,Fe) peak amplitude for CPT and ADS samples as a function of bulk $\mathrm{Zn} / \mathrm{Fe}$ ratio.

From the results on the high $\mathrm{Zn}$ sample where precipitation is expected, we see that even a small amount of precipitate has a different structure function contribution than does $\mathrm{Zn}$ on ferrihydrite at the lower sorption densities. This suggests that over the range of $\mathrm{Zn} / \mathrm{Fe}$ of $0.003-0.150$ we are observing the change of sorption behavior from isolated bidentate attachment of tetrahedral $\mathrm{Zn}(\mathrm{O}, \mathrm{OH}) 4$ units, to the progressive beginnings of multiple mixed cation linkages, but distinct from the simple formation of the amorphous precipitate. Model calculations of the EXAFS from $\mathrm{Zn}^{2+}$ substituted in the ferrihydrite structure, are also clearly distinctive from the observations. Hence "solid solution" or mixed precipitate formation in the normal sense cannot be occurring.

The only differences observed between the ADS and CPT samples over the sorption densities examined is in the second shell structure function peak amplitude change with sorption density. The CPT samples have larger second shell peak amplitudes than the ADS samples for all sorption densities, though the differences decrease with increasing density (Fig. 4). As we expect the CPT samples to provide the greatest numbers of surface attachment sites for the aqueous zinc complexes $[1,4]$, the actual sorption density (i.e. $\mathrm{Zn}$ complexes per surface attachment site) at a given $\mathrm{Zn}^{2+}$ sorption concentration ought to be lower in the CPT samples. Hence, the CPT ferrihydrite particle surfaces at low bulk $\mathrm{Zn}^{2+}$ are more likely than the ADS samples with equivalent adsorbed zinc to have isolated bidentate sorbed $\mathrm{Zn}(\mathrm{O}, \mathrm{OH})_{4}$ complexes, and thus, the largest second shell amplitudes for this single geometry.

We are currently further examining these various structural possibilities with both molecular and EXAFS simulations.

This work was supported through the Materials Research, Science and Engineering Center Program of the NSF-DMR, and by the USGS Water Resources division.

1. G.A. Waychunas, B. A. Rea, C.C. Fuller and J.A. Davis, Geochim. Cosmochim. Acta 57, 2251 (1993).

2. S. Ghose, Acta Cryst. 17, 1051 (1964).

3. D.A. Dzombak and F. M. M. Morel, J. Colloid Interface Sci. 112, 588 (1986).

4. C. C. Fuller, J.A. Davis and G.A. Waychunas, Geochim. Cosmochim. Acta 57, 2271 (1993).

5. D. L. Parkhurst, M.S. Thesis, Geology Department, Stanford University (1985).

6. F. Wegmüller, J. Colloid Interface Sci. 116, 312 (1987). 


\title{
Dynamic Study of the Monomer-Polymer Phase Transformation in Diacetylene Bicrystals via White Beam Synchrotron X-ray Topography
}

\author{
J. Liao, D. C. Martin, Z. Zeng, P. M. Wilson, Z. U. Rek*, and J. C. Bilello \\ Department of Materials Science and Engineering, \\ The University of Michigan, Ann Arbor, MI 48109-2136 \\ *Stanford Synchrotron Radiation Laboratory, Stanford University, Stanford, CA 94309
}

\section{INTRODUCTION}

Grain boundaries in organic polymer solids are expected to play roles in determining properties as they do in metallic and ceramic materials. In order to understand the impact of grain boundary defects on properties of conjugated, highly ordered crystalline polymers, we have selected poly(diacetylenes) to develop schemes to isolate grain boundaries and study their influences on the mechanical and electrical properties. Diacetylene polymer bicrystals are obtained via solid state polymerization of precursor diacetylene monomer bicrystals. The boundary strength of diacetylene polymer bicrystals has been found depending on initial grain boundary geometric conditions and polymerization methods, and essentially, depending on microstructural evolution at grain boundaries during solid state polymerization. To understand the evolving microstructural changes at the grain boundaries, we have dynamically studied the monomer-polymer phase transformation in constructed diacetylene bicrystals with small angle grain boundaries by real-time White Beam Synchrotron X-ray Topography (WBSXT). Our primary goal for this study is to establish conditions to obtain useful WBSXT patterns and examine beam sensitivity of polymerizable diacetylene crystals. We have been in an effort to explore the utility of WBSXT to characterize the microstructure and microstructural evolution of diacetylene polymer bicrystals.

\section{EXPERIMENTAL}

DCHD and pTS diacetylene have been chosen for the present study due to its high resistance to radiation damage, well-defined crystalline structure, and interesting mechanical and electrical properties. The construction and characterization of DCHD diacetylene polymer bicrystals with small angle grain boundaries will be discussed elsewhere (1). In this report, we mainly focus on the WBSXT studies.
We use the transmission Laue method in this experiment. When a perfect single crystal is illuminated by a white X-ray beam, a number of lattice planes (hkl) pick up their proper wavelength to meet the Bragg's Law. Due to the low divergence and high intensity of the synchrotron radiation, each spot on the transmission Laue pattern becomes a highresolution topograph that carries the information about defects and their distribution in the crystalline samples. Like most of organic crystalline polymers, poly(diacetylenes) are sensitive to $\mathrm{X}$-ray radiation. But the high flux and energy of synchrotron X-ray beam, in another hand, makes it possible to shorten exposure time and avoid $\mathrm{X}$-ray radiation damage. Using the synchrotron X-ray source at the Stanford Synchrotron Radiation Laboratory ( $3 \mathrm{GeV}$ and $50-85 \mathrm{~mA}$ ), we are able to induce and simultaneously capture the whole monomerpolymer phase transformation in pTS and DCHD diacetylenes at an exposure time base of 0.5 seconds. The films used in these studies are Polaroid 57 and Kodak SR-5.

\section{RESULTS AND DISCUSSIONS}

(1). The Monomer-polymer Phase Transformation in Diacetylene Single Crystals

The microstructure in diacetylene polymer crystals has been imaged previously by conventional monochromatic X-ray topography (2-4). The diacetylene monomer-polymer phase transformation has been studied by WBSXT (5).

The critical factor in imaging the diacetylene monomer-polymer phase transformation is the control of the amount of dose accepted by samples (6). To minimize the $\mathrm{X}$-ray dose rate on the samples, care needs to be taken to establish conditions of obtaining WBSXT patterns with high sensitive films. The previous WBSXT work done by M. Dudley et al(5) on the pTS diacetylene monomer-polymer phase transformation have captured, from what we 
observed, only part of the phase transformation. At SSRL, we were able to record a transmission WBSXT Laue pattern within 0.5 seconds. This allowed us observe the whole process of the diacetylene monomer-polymer phase transformation. In contrast to Dudley's report, there is no visible changes observed on the first patterns taken at the beginning of the phase transformation. What we found is that in pTS crystals the majority of the phase transformation completes within first 10-20 seconds under beam conditions of $3 \mathrm{GeV}$ and $60-85 \mathrm{~mA}$, which was about the time required to record one single transmission WBSXT Laue pattern in Dudley's work.

Fig. 1 is a series of topographs obtained from a pTS single crystal sample showing the microstructural evolution during the monomerpolymer phase transformation induced by $\mathrm{X}$-ray. Subgrain boundaries can be seen on the topographic images in Fig. 1a. The topographic image smear in Fig. 1 evidently results from the crystal lattice bending during the phase transformation, indicating the existence of internal strain inside the intermediates. The subgrain boundary image contrast fades away at the final stage of the phase transformation, which means each subgrain Orients to achieve orientation perfection. This topographic observation is coincident with Laue diffraction observation. We have seen diffraction streaking, initiated at the beginning of the phase transformation, also fades away at the final stage of the phase transformation. This type of diffraction streaking has also been observed during the phase transformation of DCHD diacetylene crystals, but it does not fade away after completing the phase transformation. Diffraction streaking corresponds to 2-D disordering or planar defects in crystal lattice. Therefore, both pTS and DCHD diacetylene monomers undergo a defect-mediated phase transformation into diacetylene polymers.

(2). Characterization of Grain Boundary Geometry in DCHD Diacetylene Monomer Bicrystals

One of our research objectives is to construct diacetylene bicrystals with controlled grain boundary structures. We have developed schemes to control grain boundaries in precursor diacetylene monomer bicrystals. But how accurately can we control it? To answer this crucial question, we need to find a method to characterize the grain boundary geometry in the constructed bulk diacetylene monomer bicrystals.
Grain boundary geometry can be characterized by measuring the amount of grain-boundary-image separation on WBSXT Laue diffraction patterns obtained from bicrystal samples (7). But obtaining WBSXT diffraction patterns from monomer bicrystals are even more difficult than from polymer bicrystals, because the minimum $\mathrm{X}$-ray dose acceptable for diacetylene monomer crystals is expected to be a few order smaller than that for polymer crystals (6). During this SSRL run, we have successfully obtained WBSXT Laue diffraction patterns from DCHD diacetylene monomer bicrystals. Fig. 2 is a series of WBSXT Laue diffraction patterns obtained from a DCHD diacetylene bicrystal with a " $0^{\circ}$ tilt grain boundary". By measuring the image separation, we characterize the bicrystal having only a $0.575^{\circ}$ tilt component. The construction accuracy for this bicrystal is thus less than $1^{\circ}$.

(3). Strain Evolution Near Grain Boundaries of Diacetylene Bicrystals During the Monomerpolymer Phase Transformation

As mentioned before, the boundary strength of diacetylene polymer bicrystals essentially depends on the microstructural evolution at the grain boundaries. It is obvious that the microstructural evolution can be studied by some high resolution techniques such as TEM, AFM, and WBSXT. TEM study risks the possibility of introducing extra defects and extra misorientation during sample preparation and lacks of information about bulk phenomena. AFM study does not carry information about crystallography. WBSXT turns out to be an ideal high resolution technique providing spatial and crystallography information about defect structures in nondestructive, bulk samples.

At SSRL, we have mainly focused on constructed DCHD diacetylene bicrystals with small angle grain boundaries. Due to space limitation for this report, we take Fig. 2 as a typical example of these studies. Other results will be discussed in detail elsewhere (1). Fig. 2 dynamically shows the monomer-polymer phase transformation of a DCHD diacetylene bicrystal with " 00 tilt grain boundary". Fig. $2 \mathrm{a}$ is a nonstreaked, monomer bicrystal pattern. Starting at Fig. $2 b$, topographic diffraction spots start to streak. The streaking continues to develop through Fig. 2d but does not disappear at the final stage of the phase transformation as revealed by previous TEM studies in DCHD diacetylene thin film samples. Fig. 3 is the magnified WBSXT topographs showing development of strain field in the vicinity of this " $0^{\circ}$ tilt grain 
boundary". Fig. 3a are a pair of the grainboundary-images without distortion obtained at the beginning of the phase transformation. As the bicrystal accepts more X-ray photons, localized strain field develops in the vicinity of the grain boundary as shown in Fig. 3b-3c. However, no severe grain-boundary-image smearing has been seen in a naturally-grown pTS tilt bicrystal with about $20^{\circ}$ tilting in polymer chains. This might be due to that the conjugated, covalent polymer chains continue through the " 00 tilt grain boundary" during the phase transformation which leads to lattice distortion near the grain boundary interface, while none, or at least, no enough polymer chains continue through the large angle tilt grain boundary interface in the pTS bicrystal. In fact, the pTS bicrystal was de-bonded at the grain boundary after the phase transformation.

\section{CONCLUSIONS}

1. WBSXT has proven to be a useful method to study the dynamic process of the diacetylene monomer-polymer phase transformation by providing both morphological and crystallographic information.

2. At SSRL, we have successfully captured the whole phase transformation by recording transmission WBSXT Laue patterns every 0.5 seconds.

3. We have also characterized the grain boundary geometry of constructed diacetylene monomer bicrystals and obtained information about the accuracy of the bicrystal construction.

4. There is strain development near small angle grain boundary defects in DCHD diacetylene bicrystals, but no severe strain development in a naturally-grown pTS bicrystal with large angle tilt grain boundary.

\section{ACKNOWLEDGMENT}

This work is supported by the College of Engineering of the University of Michigan, the National Science Foundation (NSF) through grant DMR-9024876 and DOE, and, in part, synchrtron studies at SSRL by DOE. D. C. Martin also thanks the NSF for a National Young Investigator award.

\section{REFERENCES}

1. J. Liao, Ph. D. Dissertation, 1995.
2. J. M. Schultz, J. Mater. Sci., 12, 1977, 2258-2266.

3. D. Bloor, D. K. Bowen, S. T. Davies, K. J. Roberts, and J. N., Sherwood, J. Mater. Sci. Lett., 1, 1982, 150-152.

4. P. Rosemeier, R. E. Green, and R. H. Baughmann, J. Appl. Phys., 52 (12), 1981, 7129-7135.

5. M. Dudley, J. N. Sherwood, D. J. Ando, and D. Bloor, Mol. Crys. Liq. Cryst., 93, 1983, 223-237.

6. J. Liao and D. C. Martin, Science, 260, 1993, 1489-1491.

7. Z. Zeng, Z. Rek, and J. C. Bilello, Mat. Res. Soc. Symp. Proc., 307, 1993. 

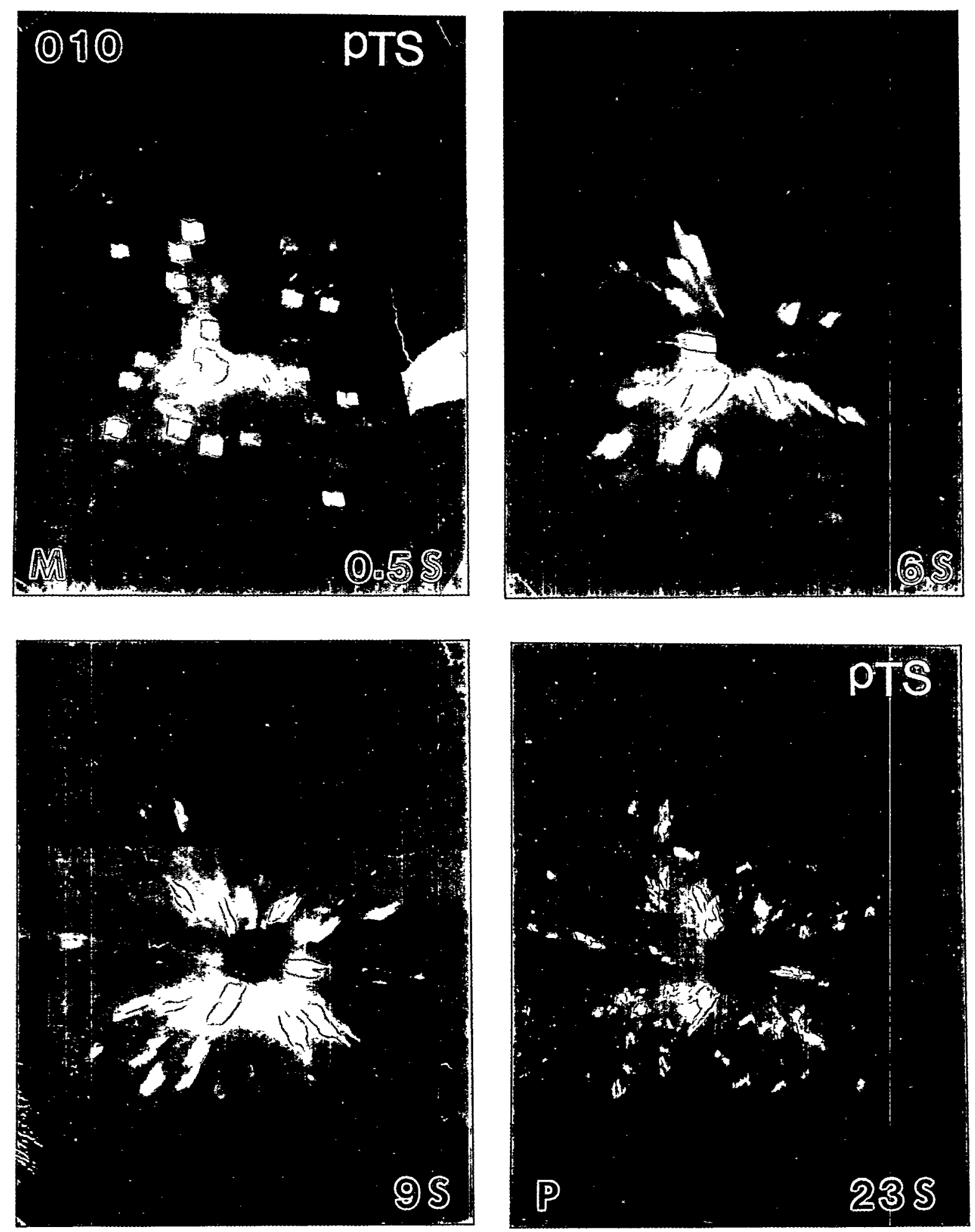

Fig. 1. A series of WBSXT topographs obtained from a pTS single crystal sample showing the initiation and development of diffraction streaking during the monomerpolymer phase transformation. Subgrain boundaries can be observed within the topographs as shown in Fig. 1a. The subgrain boundary image contrast fades away at the final stage of the phase transformation. 

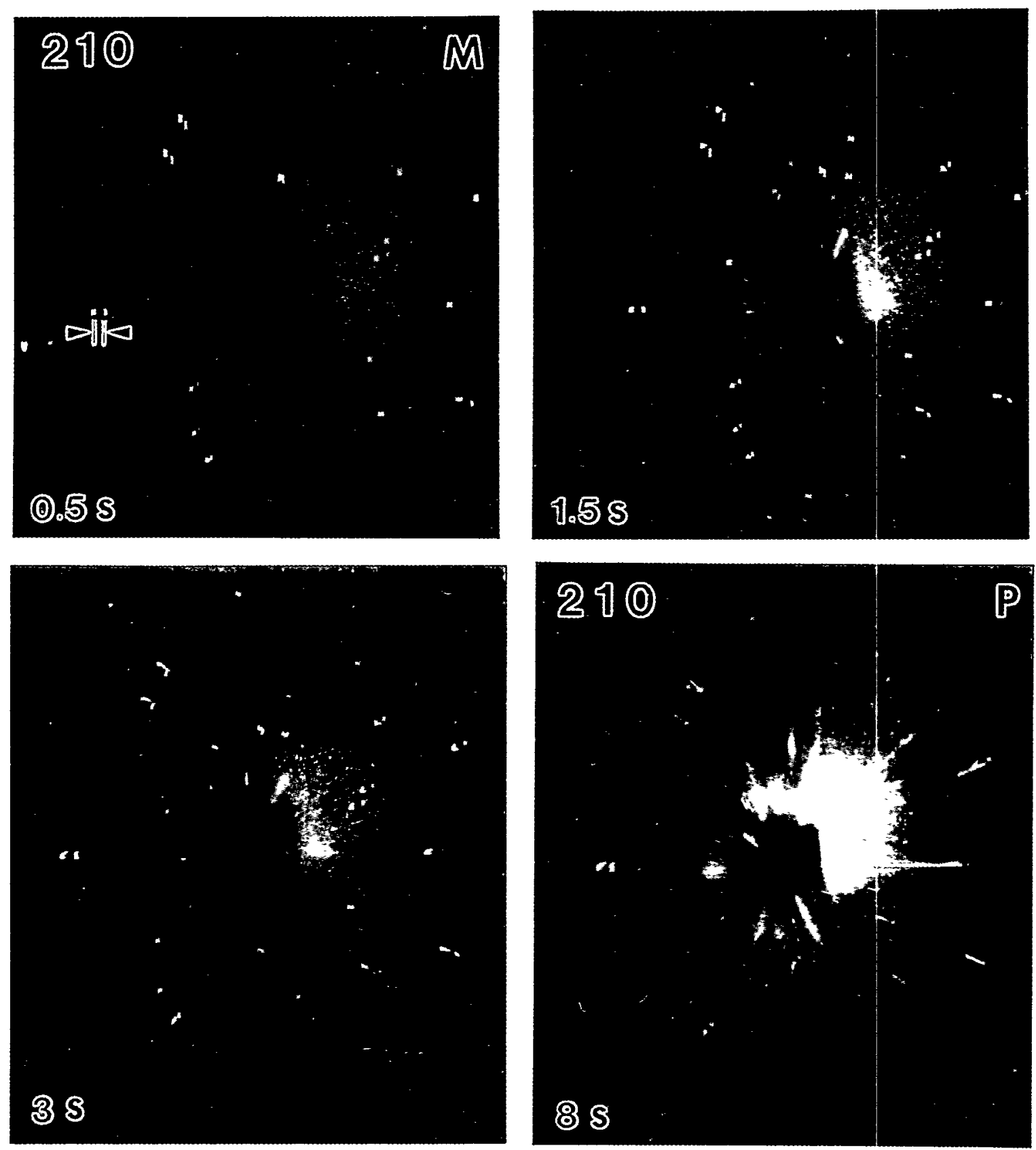

Fig. 2. A series of WBSXT Laue diffraction patterns obtained from a DCHD diacetylene bicrystal with a "00" tilt grain boundary. By measuring the image separation, we characterize the monomer bicrystal having only a $0.575^{\circ}$ tilt component. The construction accuracy for this bicrystal is thus less than $1^{0}$. 

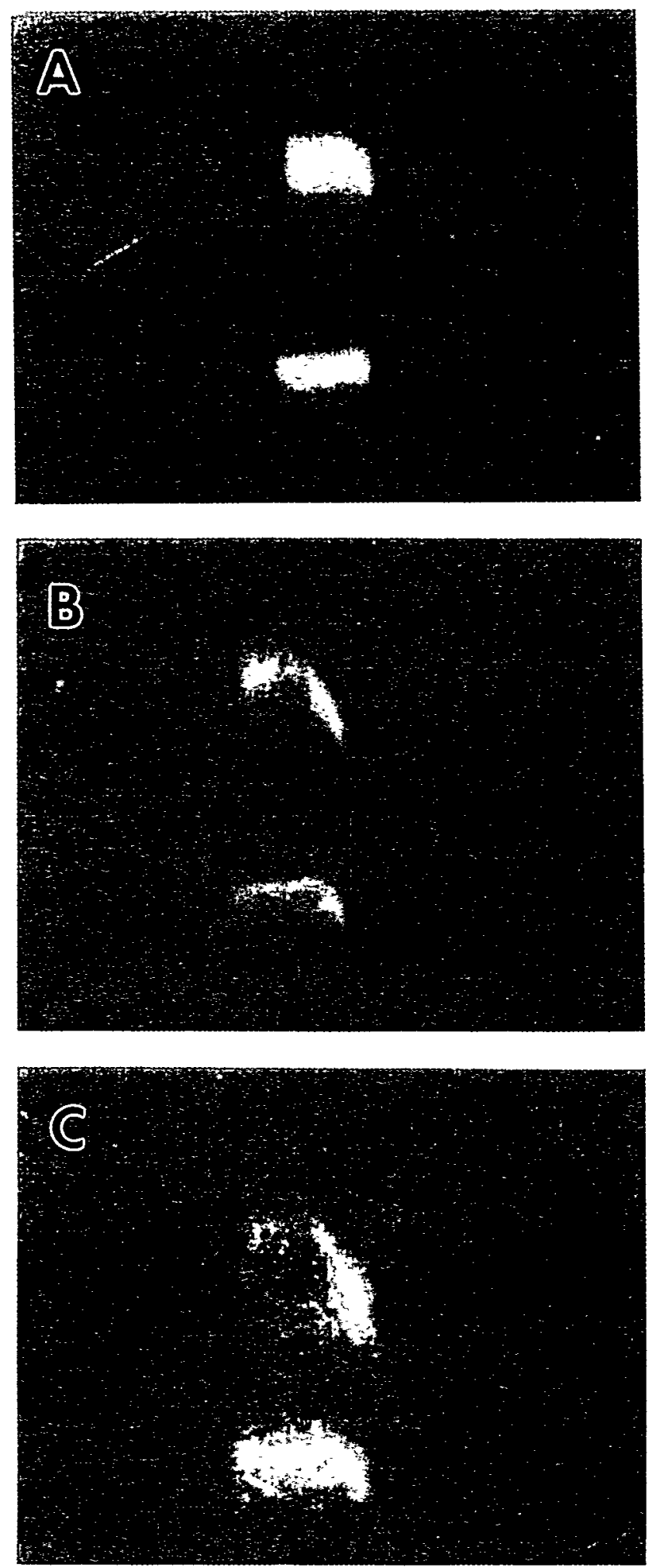

Fig. 3. The magnified WBSXT topographs showing there is strain field developed in the vicinity of this " 0 " tilt grain boundary. Fig. 3a are a pair of grain-boundary-images without distortion obtained at the beginning of the phase transformation. As the bicrystal accepts more and more X-ray photons, localized strain field is observed developing in the vicinity of the grain boundary as shown in Fig. $3 \mathrm{~b}$ and $3 \mathrm{c}$. 


\title{
STRAIN EIGENVALUES AS A FUNCTION OF PENETRATION DEPTH FOR SPUTTER DEPOSITED MO THIN FILMS
}

\author{
S.G. Malhotra, Z.U. Rek*, S.M. Yalisove, and J.C. Bilello \\ Univ. of Michigan, Dept. of Materials Science and Eng., Ann Arbor, MI 48109-2136 \\ *Stanford Synchrotron Radiation Laboratory, Stanford, CA 94305
}

\section{INTRODUCTION}

The development of residual strains during thin film processing is inevitable and can compromise the integrity of the thin film structure. Therefore, it is important to be able to quantify the amount of residual strains and stresses so that reliable thin film structures can be produced. An understanding of the depth variation of strains (and hence stresses) in thin films would be helpful because their microstructure and strain state may vary significantly throughout the thickness of the film.[1,2] A study of the depth variation is possible with grazing incidence $x$-ray scattering (GIXS) because it utilizes conventional Bragg diffraction and the phenomenon of total extemal reflection.[3] This combination allows the $x$-rays to selectively penetrate only the thin film and not the substrate so that the signal-tonoise ratio for the film is greatly improved.

In recent studies of strain in polycrystalline thin films using GIXS, the in-plane strain was determined as a function of depth in several crystallographic directions by an analysis of diffraction peak positions.[4-6] The authors did not identify an in-plane strain gradient and concluded that a gradient did not exist in the films. In another recent study, the authors identified an average in-plane and out-of-plane strain for epitaxial films with a range of film thicknesses. [7] In contrast to the above work, the goal of this study was to determine both inplane and out-of-plane strains in approx. $500 \AA$ and $1000 \AA$ Mo films at a variety of $x$-ray penetration depths. These strains were then used for the calculation of the strain eigenvalues as a function of penetration depth.

\section{EXPERIMENTAL PROCEDURES}

Molybdenum films with thicknesses of $500 \AA \pm$ $50 \AA$ and $1000 \AA \pm 100 \AA$ (determined by Rutherford Backscattering[8]) were deposited from a $99.95 \%$ pure Mo target onto $3^{n}$ diameter $\mathrm{Si}$ (100) wafers by direct current planar magnetron sputtering. The wafers were in the "as-received" condition, with a native oxide coating. The sputtering occurred without significant heating of the substrates. The sputtering power was 308 watts ( $-83 \mathrm{~A} /$ minute deposition rate) and the chamber was pumped to a base pressure of at least $5 \times 10^{-6}$ Torr. The chamber was then backfilled with Ar and maintained at a pressure of approx. $10 \mathrm{~m}$ Torr. The samples were mounted face-down 5 inches above the sputter source in a horizontal carousel which rotated at $20 \mathrm{rpm}$. The target was presputtered onto the shutter for at least 1 minute to prevent oxides or contaminants from being sputtered on to the wafers.

The GIXS experiments were conducted under standard conditions ( $3 \mathrm{GeV}$ and $60 \mathrm{~mA}$ at fill) on the eight-pole focused wiggler station BL 7-2 at SSRL. A Si (111) double-crystal monochromator was used to select the incident $x$ ray wavelength of $1.24 \AA(10 \mathrm{keV})$ from the continuous spectrum. The horizontal and vertical divergence of the beam on BL 7-2 is $3 \mathrm{mrad}$ and $0.2 \mathrm{mrad}$, respectively. Slits $1 \mathrm{~mm} \times 1 \mathrm{~mm}$ were used for the incoming beam and $1 \mathrm{mrad}$ Soller slits were used for the diffracted beam to limit vertical divergence. The diffracted beam was detected with a scintillation counter. The samples were mounted on an automated Hüber 5020 four-circle goniometer and a beam-line computer was dedicated to control the goniometer motions, the shutter, and the photon counting. The experiments were conducted in the "dose" mode by putting a scintillation counter in the path of the incident beam because the current in the synchrotron ring decreased linearly with time.

Two different scattering geometries were used to collect the data: symmetric and highly asymmetric GIXS, as illustrated in Figure 1.[6] Note that the strains obtained from symmetric GIXS will be referred to as in-plane strains and the strains obtained from highly asymmetric GIXS will be referred to as out-of-plane strains. For each sample, the $\{110\},\{200\},\{211\}$, $\{310\},\{222\},\{321\}$, and $\{400\}$ diffraction peaks were collected in the symmetric GIXS geometry and the $\{110\},\{200\}$, and $\{211\}$ peaks were collected in the highly asymmetric GIXS 
geometry at four penetration depths from the froe surface. The penetration depths were varied by changing the angle of the incoming radiation near the critical angle for total external reflection.[3] The penetration depths for the $500 \AA$ film include $140 \AA, 220 \AA, 440 \AA$, and $500 \AA$; the penetration depths for the $1000 \AA$ film include $220 \AA, 530 \AA, 750 \AA$, and $1000 \AA$. The upper limit for the error in the penetration depths was $\pm 100 \AA$, except very near the critical angle, where the uncertainty was $\pm 300 \AA$.

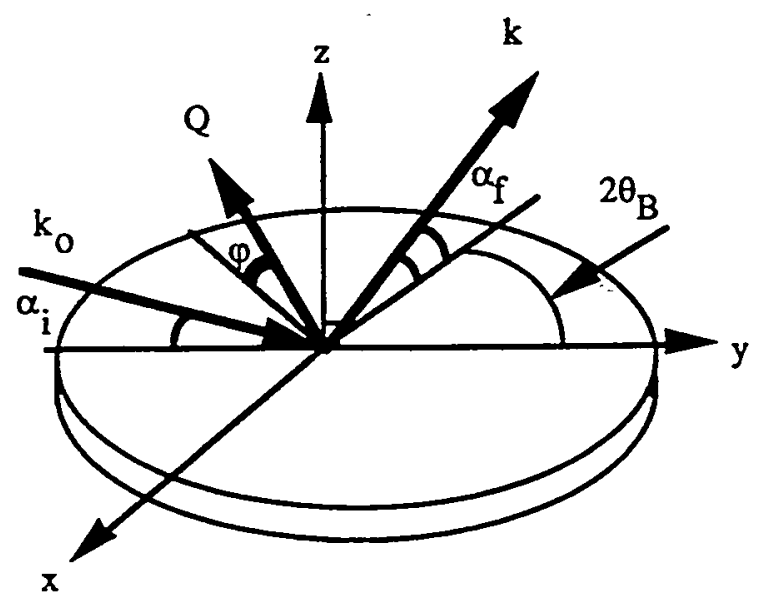

(a)

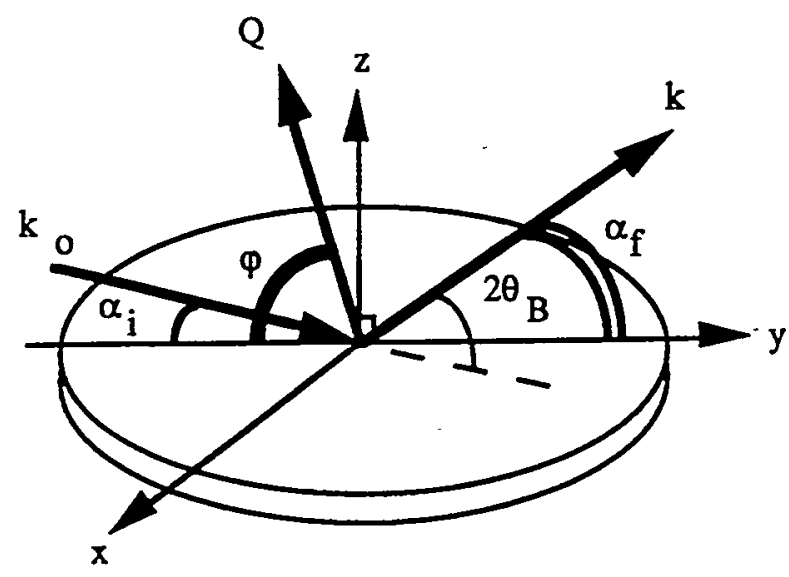

(b)

Figure 1. Schematic of (a) symmetric and (b) highly asymmetric GIXS geometries. In symmetric GIXS the scattering planes are nearly perpendicular to the sample surface, and in highly

\section{RESULTS AND DISCUSSION}

The diffraction data was fit with a Voigt or Gaussian function to determine the location of each peak, $2 \theta$, which was then used to determine the interplanar spacings for each $\{\mathrm{hkl}$ \} family of planes, dhkl, from Bragg's Law. The strain, $\varepsilon_{h k l}$ $=\left(d_{h k l}-d_{0}\right) / d_{0}$, was calculated with $d_{0}$ equal to the equilibrium interplanar spacings from a sintered Mo polycrystalline standard.

The integrated in-plane and out-of-plane strains as a function of penetration depth for the $1000 \AA$ film are shown in Figure 2. The in-plane strain vs. penetration depth data shown in Figure 2(a) does not indicate a significant strain gradient. This is the same trend previously observed for inplane strains in $\mathrm{Al}[4,9]$ and $\mathrm{Al}-2 \% \mathrm{Cu}[6]$ films. However, an out-of-plane strain gradient was identified as shown in Figure 2(b). This would agree with investigations that have identified a gross strain gradient in $M o$ and other thin films.[10-12] These same trends were observed for the $500 \AA$ Mo film as well.
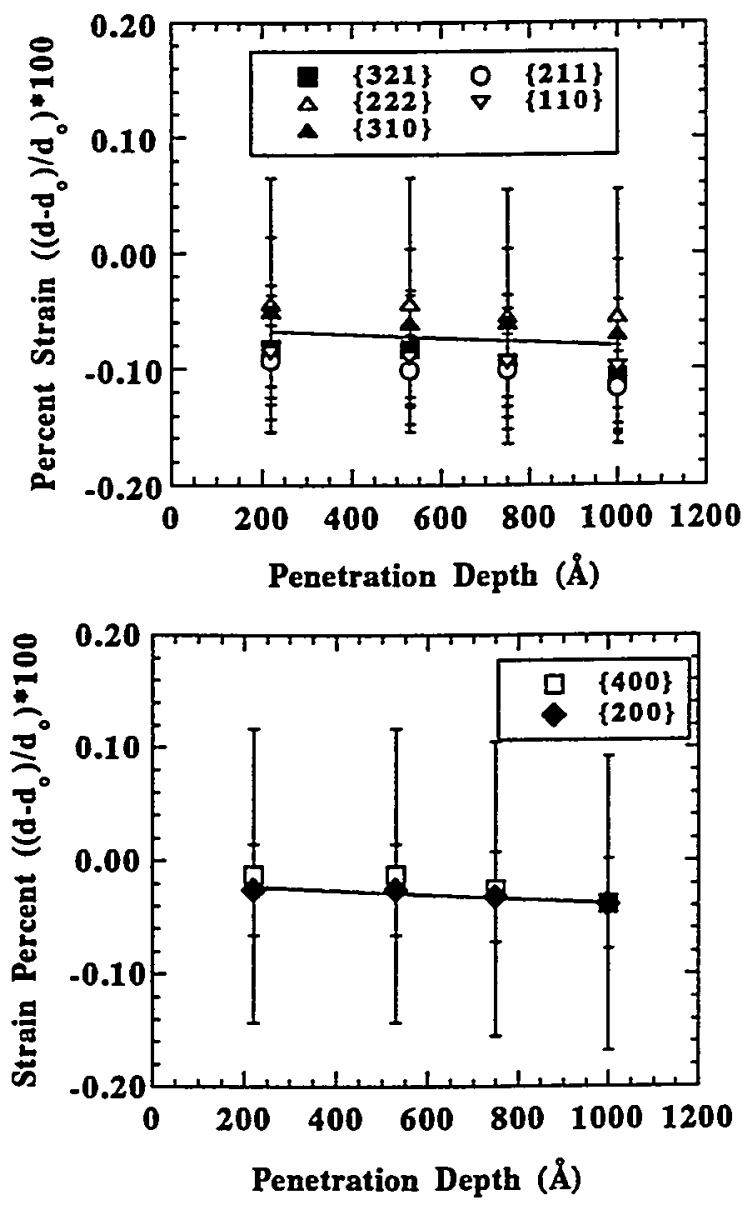

(a) 


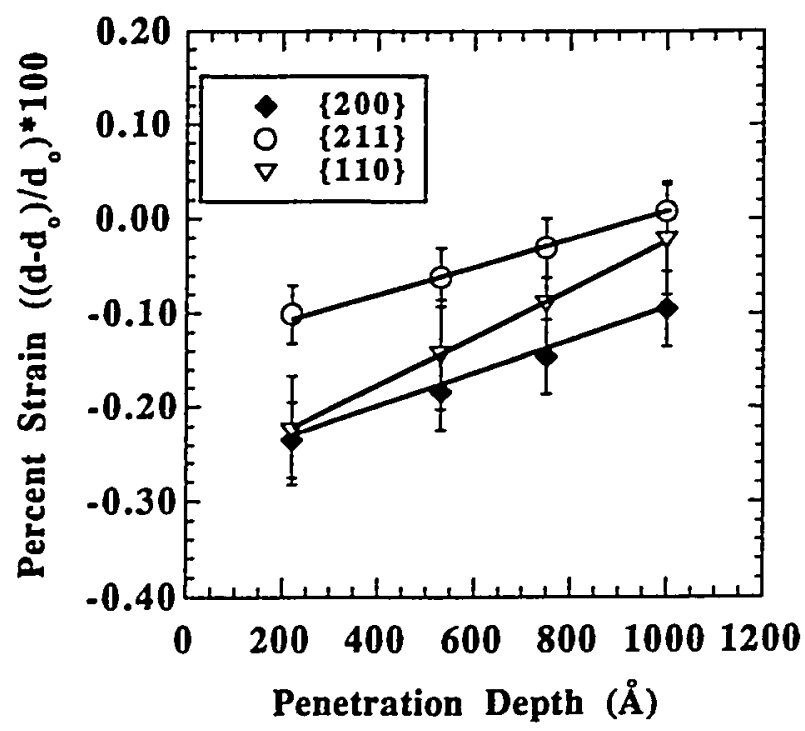

(b)

Figure 2. Plot of (a) in-plane and (b) out-ofplane strains for $1000 \AA$ Mo.

The most significant error in the strain calculations arose from the scatter in the diffraction data because associated with each curve fit is an uncertainty in the peak centroid, or peak $2 \theta$ value. The uncertainty in the peak centroids was determined from an RMS error analysis of the raw data. This error propagated from $2 \theta$, to $\theta$, and then to the $\sin \theta$ term in Bragg's Law. For a given error in $\theta$, the error which propagates through $\sin \theta$ is minimized as $\theta$ approaches $90^{\circ}$, thus the ideal Bragg angle is close to $180^{\circ}$. The error which propagated from $\sin \theta$ to the $d$ spacings via Bragg's Law and then to the strains was calculated using the common error propagation formula.[13] The error bars in Figures 2 and 3 reflect this random error.

The strain tensors for each depth of penetration were calculated using the least squares methodology developed by Imura, Weissmann, and Slade for each film.[14] The ten in-plane and out-of-plane strain displacement vectors were used to solve for the six unknowns in the strain tensor. The strain eigenvalues were then calculated for each symmetric strain tensor using the classical approach outlined in Nye, for example.[15] To check the validity of this technique, the strain eigenvalues were converted to stress eigenvalues using Hooke's Law.[16] The average stress eigenvalues for each entire film thickness were then compared to the average stresses for the same films determined by a substrate curvature method, double crystal diffraction topography (DCDT).[8,17] The agreement between this new approach and the curvature method was within the same order of magnitude. In addition to average strain (stress) information, this new approach yields three dimensional information as a function of $x$-ray depth of penetration. For example, the strain eigenvalues as a function of x-ray depth of penetration for the $500 \AA$ and $1000 \AA$ film are shown in Figure 3 below. Work is presently underway to resolve the strain eigenvalues shown below onto a laboratory axis frame with components parallel and normal to the sample surface.

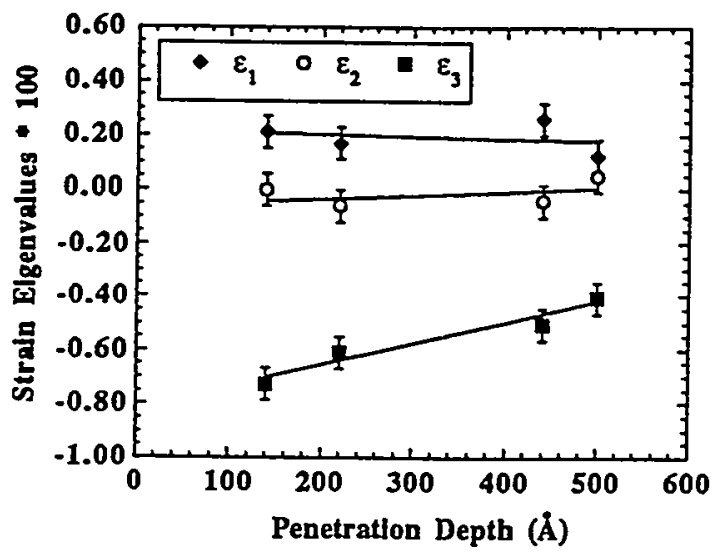

(a)

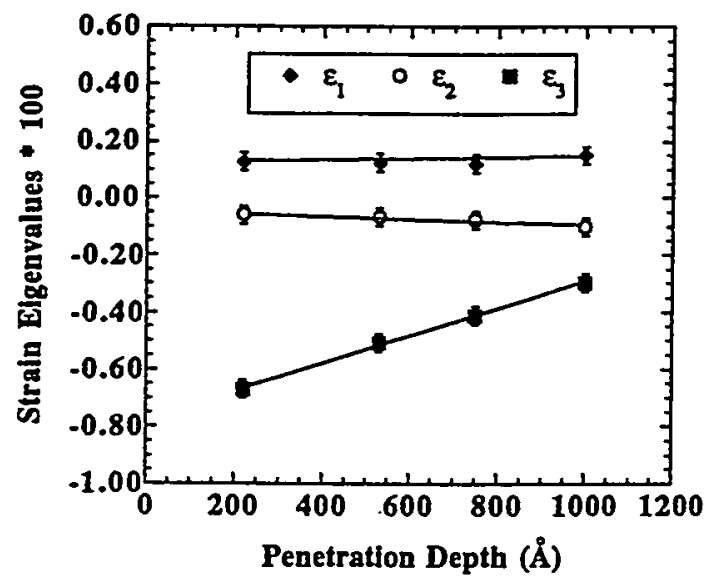

(b)

Figure 3. Strain eigenvalues vs. penetration depth for (a) $500 \AA$ Mo and (b) $1000 \AA$ Mo 
The error in the strain eigenvalues in Figure 3 are of the same order as determined for the strains in Figure 2. From Figure 3 it appears that the strain eigenvalues towards each film surface become more compressive and anisotropic in nature. Work is presently underway to determine the mechanisms behind this behavior.

\section{CONCLUSIONS}

A new method for determining the variation in three dimensional strain (and stress) eigenvalues with $x$-ray penetration depth was described. The stresses for the entire $500 \AA$ and $1000 \AA$ Mo film thicknesses determined from this new method were in good agreement with the average stresses determined by a substrate curvature technique. The following were also shown:

- Method reveals detailed information about strain variation as a function of penetration depth with high depth resolution

- There did appear to be an out-of-plane strain gradient in both films.

- Three dimensional strain (stress) tensors have been determined with GIXS.

\section{ACKNOWLEDGMENTS}

This work was supported by the USARO and ARPA under contract DAAL03-91-0235. The synchrotron work was conducted at the Stanford Synchrotron Radiation Laboratory, funded by the DOE, Office of BES. The RBS experiments were conducted at the Michigan Ion Beam Laboratory. The authors would like to thank S. Brennan for heipful discussions. One of us (SGM) would also like to thank Dr. A.K. Malhotra for his valuable input.

\section{REFERENCES}

1. B.A. Movchan and A.V. Demchishin, Fizika Metall. 28, 83 (1969).

2. J.A. Thornton, Ann. Rev. Mater. Sci. 7, 239 (1977).

3. H. Dosch, Phys. Rev. B. 35, 2137 (1987).

4. R. Venkatraman, P.R. Besser, J.C. Bravman,
5. P.R. Besser, S. Brennan, J.C. Bravman, J. Mater. Res. 9, 13 (1994).

6. C.J. Shute and J.B. Cohen, J. Appl. Phys. 70, 2104 (1991).

7. L.J. Martinez-Miranda, J.J. Santiago-Aviles, W.R. Graham, P.A. Heiney, M.P. Siegal, and J. Mater. Res. 9, 1434 (1994).

8. L.J. Parfith, private communication.

9. M.F. Doerner and S. Brennan, J. Appl. Phys. 63, 126, (1988).

10. R.M. Fisher, J.Z. Duan, and A.G. Fox, in Thin Films: Stresses and Mechanical Properties, (Mat. Res. Symp. Proc. 130, Pittsburgh, PA, 1989) p. 249.

11. R.W. Hoffman, Surf. and Int Analy. 3,62 (1981).

12. T.J. Vink, M.A.J. Somers, J.L.C. Daams, and A.G. Dirks, J. Appl. Phys. 70, 4301 (1991).

13. Y. Beers, Introduction to the Theory of Error, 3rd ed. (Addison-Wesley Publishing Company, 1962), p. 28.

14. T. Imura, S. Weissmann, and J.J. Slade, Jr., Acta. Cryst. 15, 786 (1962).

15. J.F. Nye, Physical Properties of Crystals (Oxford University Press, 1985), p. 41.

16. G.E. Deiter, Mechanical Metallurgy (McGraw-Hill Book Company, Inc., 1961), p.39.

17. J. Tao, L.H. Lee, and J.C. Bilello, J. Elect. Mat. 20, 819 (1991). 


\section{WHITE BEAM TRANSMISSION LAUE STUDIES OF TEXTURE IN Mo/W AND Ta/W MULTISCALAR MULTILAYER COMPOSITE FILMS \\ A.K. Malhotra, Z.U. Rek *, S.M. Yalisove, \& J.C. Bilello \\ Department of Materials Science and Engineering, The University of Michigan, Ann Arbor, MI 48109-2136 \\ * Stanford Synchrotron Radiation Laboratory, Stanford, CA 94309}

\section{INTRODUCTION}

The transmission Laue technique using white beam synchrotron $\mathrm{x}$-ray radiation has been recently used for determining preferred orientations for 60 $\mu \mathrm{m}$ thick films.[1,2] Using this technique, average texture information through the entire thickness of the films is revealed on the order of several hours.[1,2]. In contrast, using a laboratory $\mathrm{x}$-ray source, the texture information is limited to a rather thin region of the specimen determined by the corresponding extinction depth. For example, with a $\mathrm{Cu} \mathrm{K} \alpha$ source, less than 3 $\mu \mathrm{m}$ of a Mo film is penetrated.

In this work, metallic multiscalar multilayer composite $\left(\mathrm{M}^{3} \mathrm{C}\right.$ ) films of $\mathrm{Mo} / \mathrm{W}$ and $\mathrm{Ta} / \mathrm{W}$ with thickness of $45 \mu \mathrm{m}$ and 22 $\mu \mathrm{m}$, respectively, were studied via the transmission Laue technique using whitebeam synchrotron radiation.

Both $\mathrm{Mo} / \mathrm{W}$ and $\mathrm{Ta} / \mathrm{W}$ films have prospective applications as high temperature coatings. Therefore, their microstructural stability after exposure to high temperatures is of interest. In the present work, preliminary results on the effect of thermal exposure on the texture characteristics of a $\mathrm{Mo} / \mathrm{W}$ film are presented and compared to that of an as-deposited Mo/W film.

\section{EXPERIMENTAL}

Metallic multiscalar multilayer composite $\left(\mathrm{M}^{3} \mathrm{C}\right)$ films of $\mathrm{Mo} / \mathrm{W}$ and $\mathrm{Ta} / \mathrm{W}$ were magnetron sputter deposited to thickness of $45 \mu \mathrm{m}$ and $22 \mu \mathrm{m}$, respectively, onto as-received Si substrates. A schematic of the multiscalar multilayer composites is shown in Fig. 1. The configuration consisted of alternating layers of hard stacks and tough phases. The hard stacks themselves were made of 29 alternating layers of $40 \AA$ thick Mo (for $\mathrm{Mo} / \mathrm{W}$ ) or $\mathrm{Ta}$ (for $\mathrm{Ta} / \mathrm{W}$ ) and $\mathrm{W}$ layers.
The tough phases were much thicker layers (microns thick) of Mo (for Mo/W) or Ta (for Ta/W). Two sets of Ta/W films were studied. Both had similar hard stack configuration and a total thickness of $22 \mu \mathrm{m}$, but differed in their tough phase thickness $(3$ and $6 \mu \mathrm{m})$. A more detailed description of the processing parameters for these films is provided elsewhere [3]. Although these films were deposited onto Si substrates, the analysis was performed on free standing films which were obtained by simply peeling them off the $\mathrm{Si}$.

In order to study the effect of thermal exposure, a free standing $\mathrm{Mo} / \mathrm{W}$ $\mathrm{M}^{3} \mathrm{C}$ film with a total thickness of $45 \mu \mathrm{m}$ and a tough phase thickness of $5 \mu \mathrm{m}$ was heat treated in vacuum (better than $1 \times 10^{-6}$ Torr) at $900^{\circ} \mathrm{C}$ for 8 hours followed by furnace cooling.

Transmission Laue patterns were recorded on Kodak SR-5 x-ray photographic films at beamline II-2 of Stanford Synchrotron Research Laboratory (SSRL). The incident beam, after exiting from a beryllium window was narrowed by a $1 \mathrm{~mm}$ diameter aperture. The distance between the exit window and specimen was kept constant at $\sim 250 \mathrm{~mm}$. The sample to film distance (SFD) was varied between 74 and $96 \mathrm{~mm}$ for the $\mathrm{Mo} / \mathrm{W}$ and $\mathrm{Ta} / \mathrm{W}$ films, respectively. Transmission Laue patterns were taken unfiltered and using $\mathrm{Cu}, \mathrm{Nb}$, $\mathrm{Zr}$, and Pd prefilters. The $\mathrm{x}$-ray energy in the high and low energy regions of the spectrum was eliminated by absorption from a combination of atmosphere, air gap, and applied pre-filters. The x-ray beam that was incident upon the specimen had a reduced wavelength range of $0.65 \AA-0.95 \AA$. This spectrum was nearly uniformly attenuated, but otherwise unaffected, by absorption from the specimens, because $\mathrm{K}$ edge of all the components $(0.62 \AA$ for Mo, 
$0.184 \AA$ for $\mathrm{Ta}$, and $0.178 \AA$ for $\mathrm{W}$ ) in the films lie outside its wavelength range.

\section{RESULTS AND DISCUSSION}

Transmission Laue patterns of the heat treated $\mathrm{Mo} / \mathrm{W}$ films revealed two notable features: a series of rings and a lobe pattern, as shown in Fig. 2a. A preferred direction of alignment in the planes perpendicular to the incident beam is indicated by the presence of lobes. The angular spread of the lobes is a measure of the misorientation of the planes of a family in various grains. The radial spread of these lobes on the other hand, is a measure of the incident beam's energy range. The presently observed Laue pattern for the heat treated sample with a two fold symmetry shown by the lobes is very similar to that of an as-deposited Mo/W film under similar orientation conditions (Fig. 2b) which was studied previously. A comparison of the measured ring radii and d-spacings for the two patterns with the calculated d-spacings of the planes allowed for a BCC Mo sample is shown in Table I. As is seen from Fig. 2 and Table I the as-deposited and heattreated $\mathrm{Mo} / \mathrm{W}$ films show quite similar transmission Laue patterns under similar orientation conditions. The as-deposited Mo/W films with processing parameters similar to the Mo/W film studied presently were shown to grow with a (110) type outof-plane and in-plane preferred orientation characteristics [2]. The results on the heattreated Mo/W films indicate that the (110) out-of-plane and in-plane texture is present even after the thermal exposure. Therefore, from a qualitative examination, the preferred orientation characteristics of the Mo/W films were unchanged after thermal exposure of the films.

Transmission Laue patterns of the two Ta/W films studied revealed a series of rings as is illustrated by an example in Fig. 3. Patterns taken using unfiltered and prefiltered incident radiation showed the same number of rings for both films. Prefiltering only resulted in attenuation of the intensity for the rings. A comparison of the measured ring radii and d-spacings with the standard equilibrium values showed that all of the allowed planes in the diffraction patterns were present, thus indicating a random orientation of grains in the direction of observation (see Table II). This observation is in contrast to that for Mo/W films for which a (110) type in-plane and out-of-plane growth texture was observed $[1,2]$. The results of a microstructural comparison of the two $\mathrm{M}^{3} \mathrm{C}$ films (Mo/W and $\mathrm{Ta} / \mathrm{W}$ ) are consistent with the Laue patterns and are presented elsewhere [4]. The microstructure of Mo/W films show grains which are elongated and aligned parallel to each other in the plane of growth along $\langle 110\rangle$ directions. However, Ta/W films show grains randomly oriented with respect to each other in the plane of growth. Such a contrast in the orientation characteristics of the Mo/W and Ta/W films is very interesting because exactly similar processing parameters were used for the two films and the structures of bulk Mo and $\mathrm{Ta}$ are very similar. Future work in this area will involve determining the mechanism giving rise to such a difference in the orientation characteristics of these two films.

\section{CONCLUSIONS AND FUTURE WORK}

From this work-in-progress it can be concluded that the preferred orientation characteristics of $\mathrm{Mo} / \mathrm{W}$ films remain unaffected by thermal exposure up to $900^{\circ} \mathrm{C}$ for 9 hours. However, a more detailed study using the transmission Laue technique, in addition to using other characterization techniques, is being performed which would provide a quantitative comparison of the heat-treated and non heat-treated Mo/W films. In addition, similar studies are planned for the $\mathrm{Ta} / \mathrm{W}$ films.

\section{REFERENCES}

1. M. Vill, Z.U. Rek, J. Tao, S.M. Yalisove, and J.C. Bilello, SSRL Activity Report, 1993.

2. M. Vill, Z.U. Rek, S.G. Malhotra, S.M. Yalisove, and J.C. Bilello, SSRL Activity Report, 1994.

3. D.P. Adams, M. Vill, J. Tao, J.C. Bilello, and S.M. Yalisove, J. Appl. Phys. 74 (2), 1993, 1015-1021.

4. A.K. Malhotra, S.M. Yalisove, and J. C. Bilello, work in progress. 


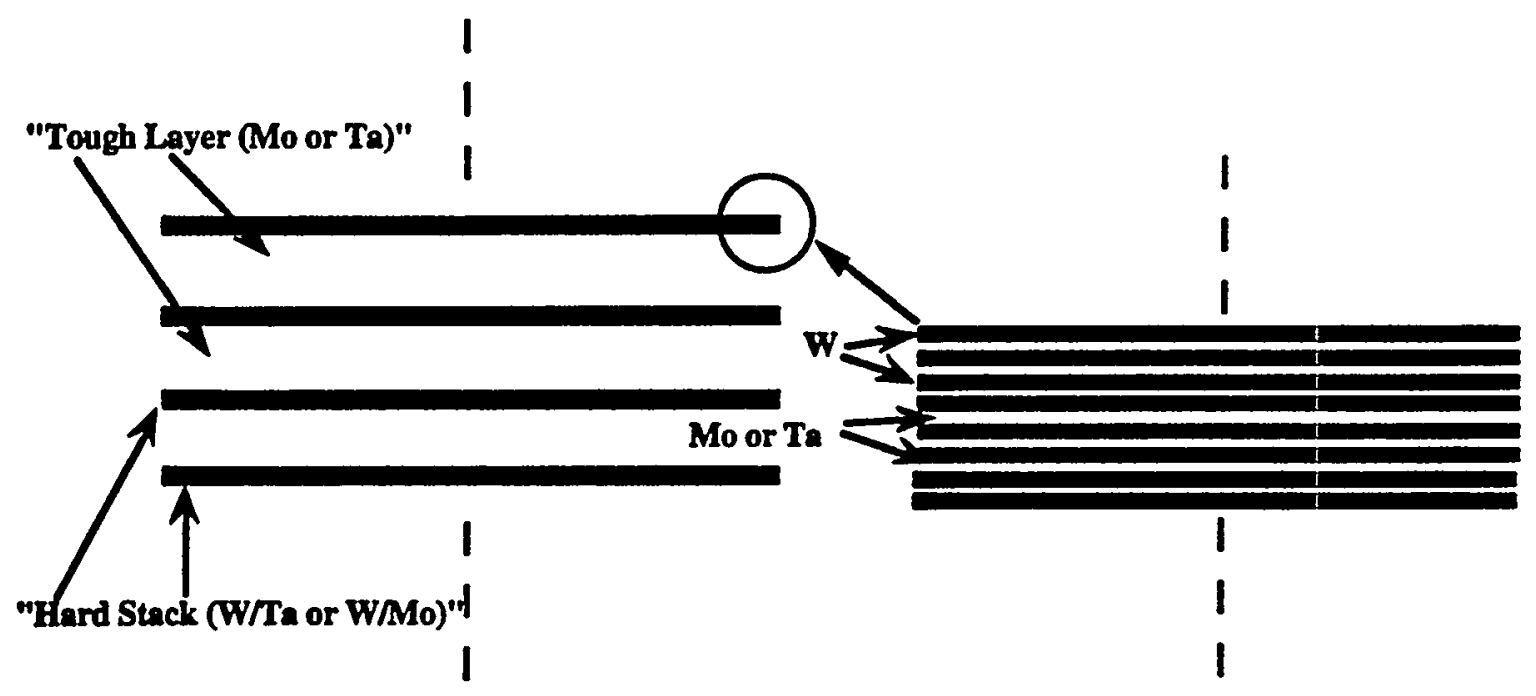

Figure 1 - Schematic of the multiscalar multilayer composite design.
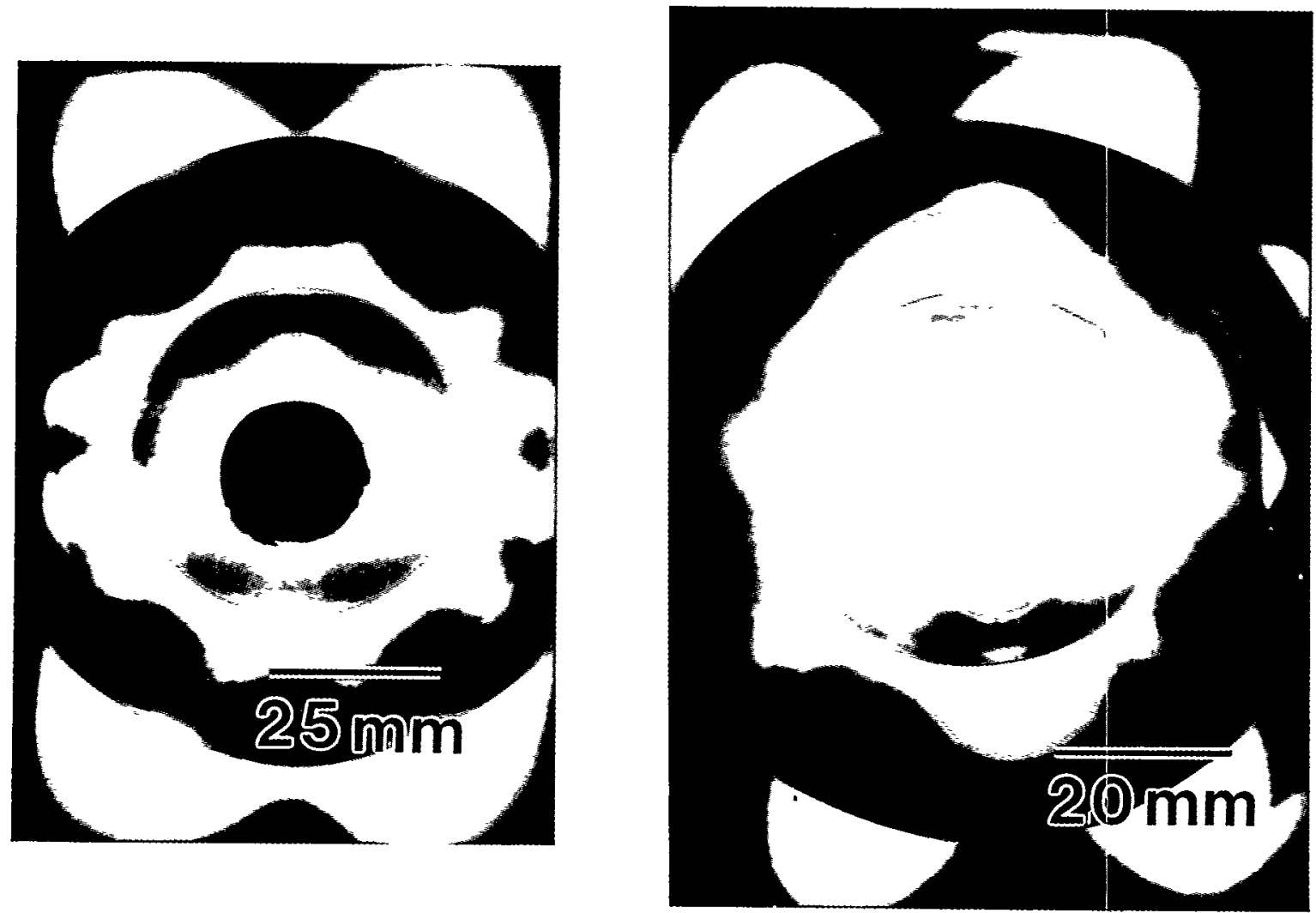

Figure 2 - Transmission Laue pattern obtained for a $45 \mu \mathrm{m}$ thick Mo/W sample with $5 \mu \mathrm{m}$ Mo tough layer (a) in the as-deposited condition and (b)after the heat-treatment. 
Table I - Measured ring radii and d-spacings for the transmission Laue patterns shown in Fig. 2 and calculated d-spacings for Mo using equilibrium lattice parameter.

\begin{tabular}{ccccccc}
\hline \hline \multicolumn{3}{c}{ Measured from the diffraction pattern } & \multicolumn{2}{c}{ Calculated Values } \\
\cline { 1 - 5 } & \multicolumn{3}{c}{ As-deposited } & \multicolumn{2}{c}{ Heat-Treated } & \\
\hline \hline Debye Ring \# & $\mathrm{R}(\mathrm{mm})$ & $\mathrm{d}_{\text {meas }}(\mathrm{A})$ & $\mathrm{R}(\mathrm{mm})$ & $\mathrm{d}_{\text {mens }}(\mathrm{A})$ & hkl & $\mathrm{d}_{\text {cal }}(\mathrm{A})$ \\
\hline \hline 1 & 24.0 & 2.205 & 22.5 & 2.342 & 110 & 2.225 \\
2 & 35.5 & 1.553 & 33.0 & 1.654 & 200 & 1.573 \\
3 & 45.0 & 1.277 & 43.0 & 1.324 & 211 & 1.285 \\
4 & 55.0 & 1.096 & 51.0 & 1.159 & 220 & 1.113 \\
5 & 64.5 & 0.982 & 60.0 & 1.031 & 310 & 0.995 \\
6 & 74.5 & 0.896 & & & 222 & 0.908 \\
7 & 85.0 & 0.831 & & & 321 & 0.841 \\
\hline \hline
\end{tabular}

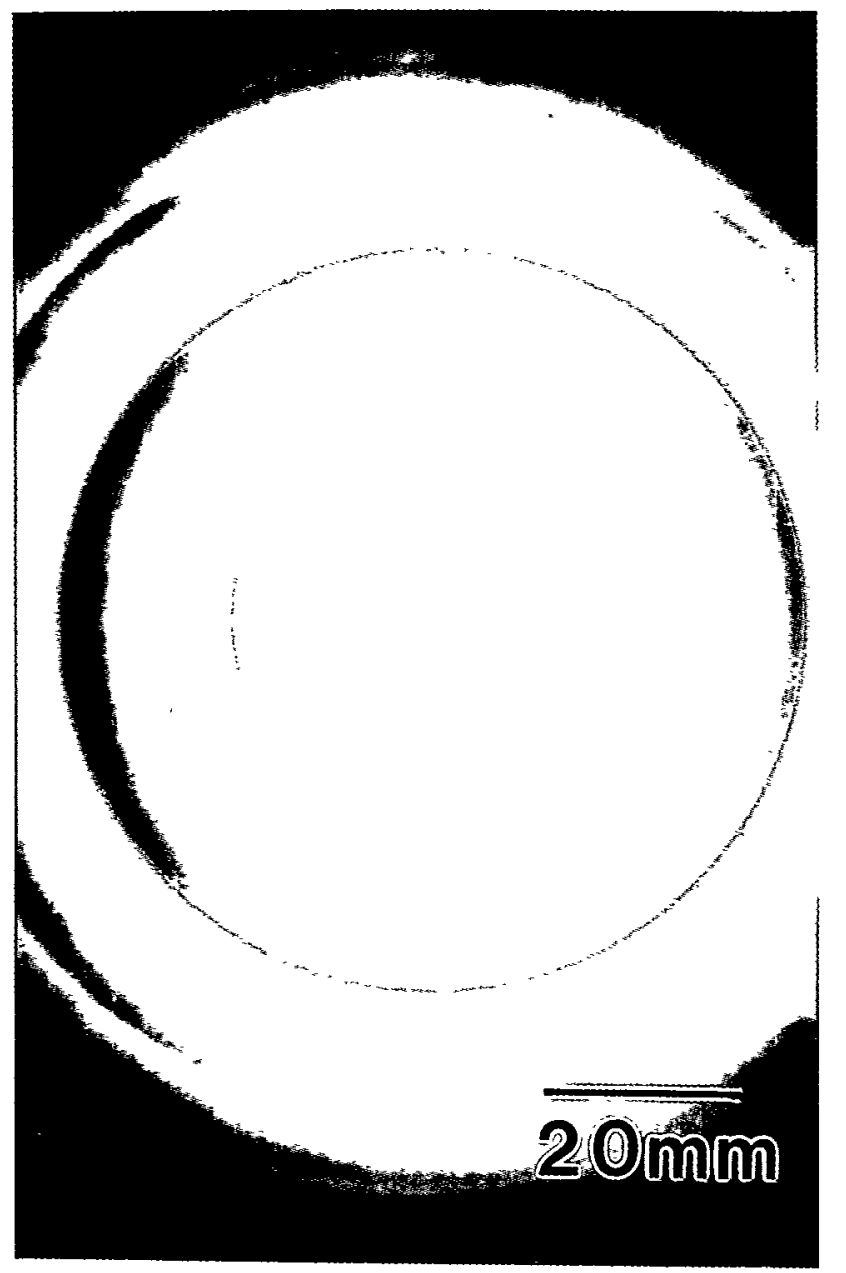

Table II - Measured ring radii and dspacings for the transmission Laue pattern shown in Fig. 3 and calculated d-spacings for $\mathrm{BCC} \mathrm{Ta}$ using equilibrium lattice parameter.

\begin{tabular}{cc}
\hline \hline Measured from the diffraction \\
pattern
\end{tabular}$\quad \begin{gathered}\text { Calculated } \\
\text { Values }\end{gathered}$

\begin{tabular}{lllll}
\hline $\begin{array}{l}\text { Debye } \\
\text { Ring }\end{array}$ & $\mathrm{R}(\mathrm{mm})$ & $\mathrm{d}_{\text {meas }}(\mathrm{A})$ & $\mathrm{hkl}$ & $\mathrm{d}_{\text {cal }}(\mathrm{A})$ \\
\hline \hline 1 & 21.0 & 2.369 & 110 & 2.335 \\
2 & 31.0 & 1.637 & 200 & 1.651 \\
3 & 38.0 & 1.359 & 211 & 1.348 \\
4 & 45.25 & 1.165 & 220 & 1.168 \\
5 & 54.0 & 1.005 & 310 & 1.044 \\
6 & 58.0 & 0.949 & 222 & 0.953 \\
7 & 63.5 & 0.884 & 321 & 0.883 \\
\hline
\end{tabular}

Figure 3 - Transmission Laue pattern for a $22 \mu \mathrm{m}$ thick $\mathrm{Ta} / \mathrm{W} \mathrm{M}^{3} \mathrm{C}$ film using a Pd filter. 


\title{
Determination of Chromium Oxidation States in Ash Samples Derived from Coal Combustion
}

\author{
M. Najih, F. E. Huggins, and G. P. Huffman \\ 341 Bowman Hall, University of Kentucky, Lexington, KY 40506-0059
}

One trace element listed in the 1990 Amendments to the Clean Air Act [1] that is of great concern in coal combustion for electrical power generation is chromium. Despite the facts that this element exists in most coals at the $10-20 \mathrm{ppm}$ level, and only as little as $1 \%$ of the chromium may actually escape to the atmosphere, the possible presence of the $\mathrm{Cr}(\mathrm{VI})$ oxidation state in such emissions and other waste materials (bottom ash, flyash, etc.) is a significant concern to health issues arising from coal combustion because of the toxicity and carcinogenic properties of this oxidation state [2]. In contrast, the other common oxidation state of chromium, $\mathrm{Cr}$ (III), at such concentration levels is of no significant concern to human health.

There are a number of sophisticated speciation methods for determining the oxidation states of trace chromium in solution [3-5]. However, in order to ensure that the solid materials from coal combustion completely dissolve would involve strong acid dissolution, during which the oxidation state of $\mathrm{Cr}$ and other multivalent elements can be altered. This source of uncertainty can be eliminated by determining the oxidation state of chromium directly on the solid materials. In this work, we have developed a determinative method for the $\mathrm{Cr}(\mathrm{VI}) /[\mathrm{Cr}(\mathrm{VI})+\mathrm{Cr}(\mathrm{III})]$ ratio in solid materials based on the Cr pre-edge peak in XAFS spectra. This method is then used to assess the significance of the presence of the $\mathrm{Cr}(\mathrm{VI})$ oxidation state in coal combustion products.

\section{EXPERIMENTAL}

Calibration samples were prepared from mixtures of potassium chromate $\left(\mathrm{K}_{2} \mathrm{CrO}_{4}\right)$ and $\mathrm{K}-\mathrm{Cr}$ alum sulfate $\left(\mathrm{KCr}\left(\mathrm{SO}_{4}\right)_{2} \cdot 12 \mathrm{H}_{2} \mathrm{O}\right)$. The total chromium content was diluted to $4 \mathrm{wt} \%$ by mixing the chromium compounds in various ratios from $0 \% \mathrm{Cr}(\mathrm{VI})$ to $100 \% \mathrm{Cr}(\mathrm{VI})$ in measured amounts of boric acid $\left(\mathrm{HBO}_{3}\right)$.
XAFS experiments were conducted at SSRL on the wiggler beam-line, IV-3, and absorption data were collected in fluorescent mode using a Lytle detector for the calibration samples and a 13-element, Ge detector [6], for the combustion ash samples, in which the $\mathrm{Cr}$ contents were usually less than 150 ppm. A $6 \mu$ vanadium filter was used with the $\mathrm{Ge}$ detector to enhance the signal-to-noise ratio.

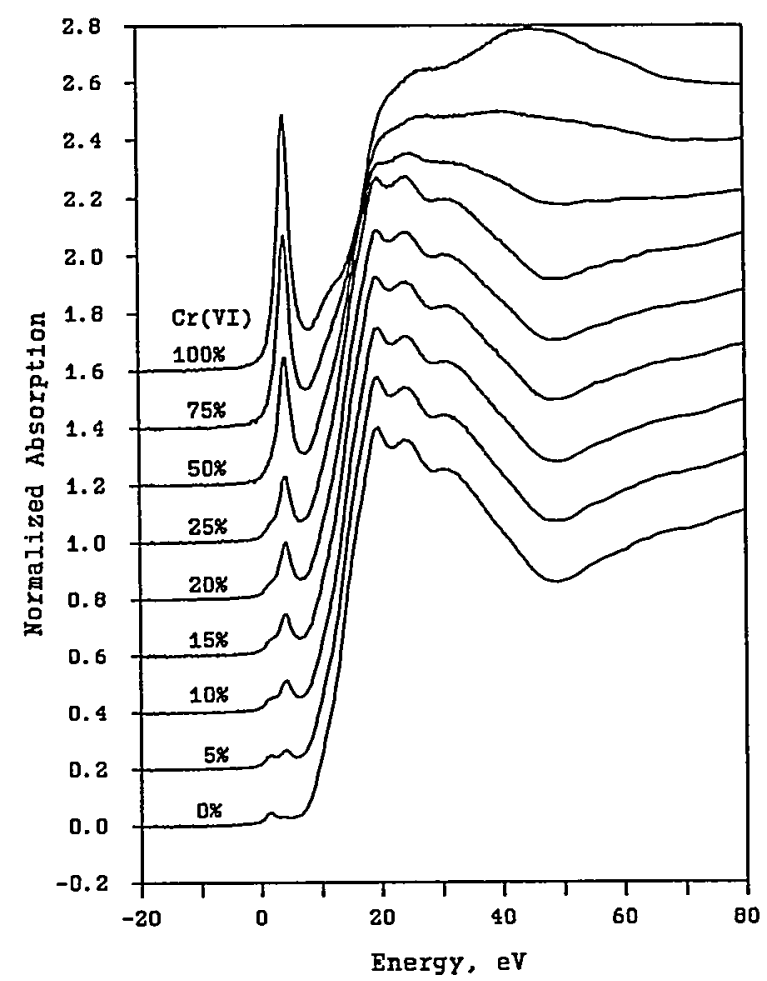

Figure 1: $\mathrm{Cr}$ XANES Calibration Spectra.

\section{RESULTS AND DISCUSSION}

(a) Calibration Data: The suite of Cr XANES calibration spectra are shown in Figure 1. It is clear that the pre-edge peak is the spectral feature that is most sensitive to the variation in $\mathrm{Cr}$ oxidation state. It is 
also the easiest feature to quantify in the XAFS spectra. Determinations of the height and intensity (area) of the pre-edge peak normalized to the edge step were made as a function of the $\mathrm{Cr}(\mathrm{VI})$ content in order to construct possible calibration curves. These calibration curves are shown in Figure 2.

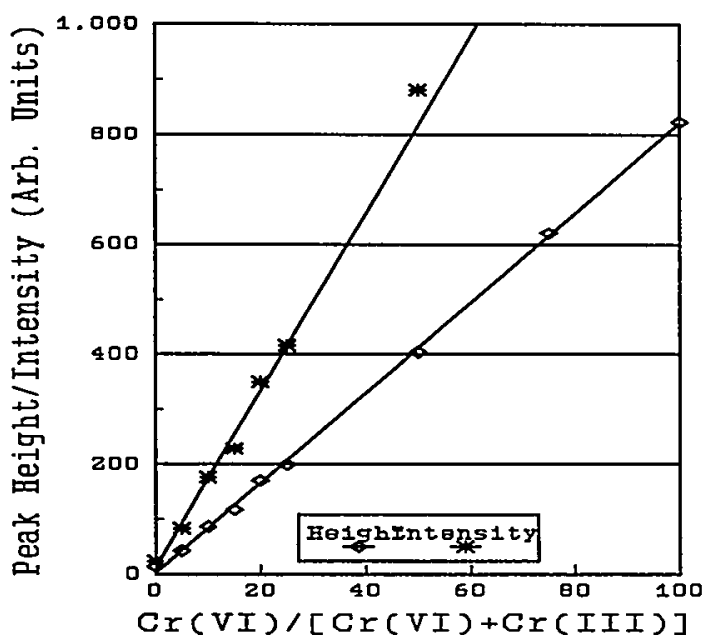

Figure 2: Calibration curves derived from Fig. 1.

It should be noted that the pre-edge feature in the $\mathrm{Cr}$ (III) alum consists of two very weak peaks at about 1.5 and $4.0 \mathrm{eV}$, whereas the $\mathrm{Cr}(\mathrm{VI})$ pre-edge feature consists of one major peak at about $4.0 \mathrm{eV}$. The peak at $4.0 \mathrm{eV}$ only was used to construct the calibration curves shown in Figure 2.

(b) $\mathrm{Cr}$ (VI) in Combustion Products: A variety of combustion products have been examined in the course of this investigation. These have varied from actual waste products (fly-ash, bottom ash) from commercial power plants to experimental samples obtained from bench-scale combustion apparatus. Chromium XANES spectra of a selection of these materials are shown in Figure 3. As can be seen from this figure, the pre-edge peaks in the Cr XANES spectra are uniformly weak and consist of two components. Such peaks were subjected to least-squares fitting to derive peak parameters for the separate components (Figure 4). The Cr(VI) contents of these ash samples were then estimated using the generated calibration data. It was found that in all samples the $\mathrm{Cr}(\mathrm{VI})$ content was less than $5 \%$ of the total chromium in these samples.

It is likely that such determinations represent an upper limit for $\mathrm{Cr}(\mathrm{VI})$ because of the choice of the $\mathrm{Cr}$ (III) calibration standard. Owing to the isolated and symmetric nature of the $\mathrm{Cr}$ octahedra in the $\mathrm{K}$ -
Cr-alum structure, there is little or no intensity enhancement of the $\mathrm{Cr}$ (III) pre-edge peaks due to deviation of the $\mathrm{Cr}$ site from perfect octahedral symmetry. Such enhancement has been demonstrated for structure-symmetry systematics reported for Ti(IV) octahedra in oxides and silicates [7]. However, we note that the low-energy $\mathrm{Cr}$ (III) pre-edge peak is generally of equal or higher intensity than the high energy pre-peak in these ash spectra. This observation would appear to indicate that the $\mathrm{Cr}(\mathrm{VI})$ oxidation state in these ash materials is negligible.

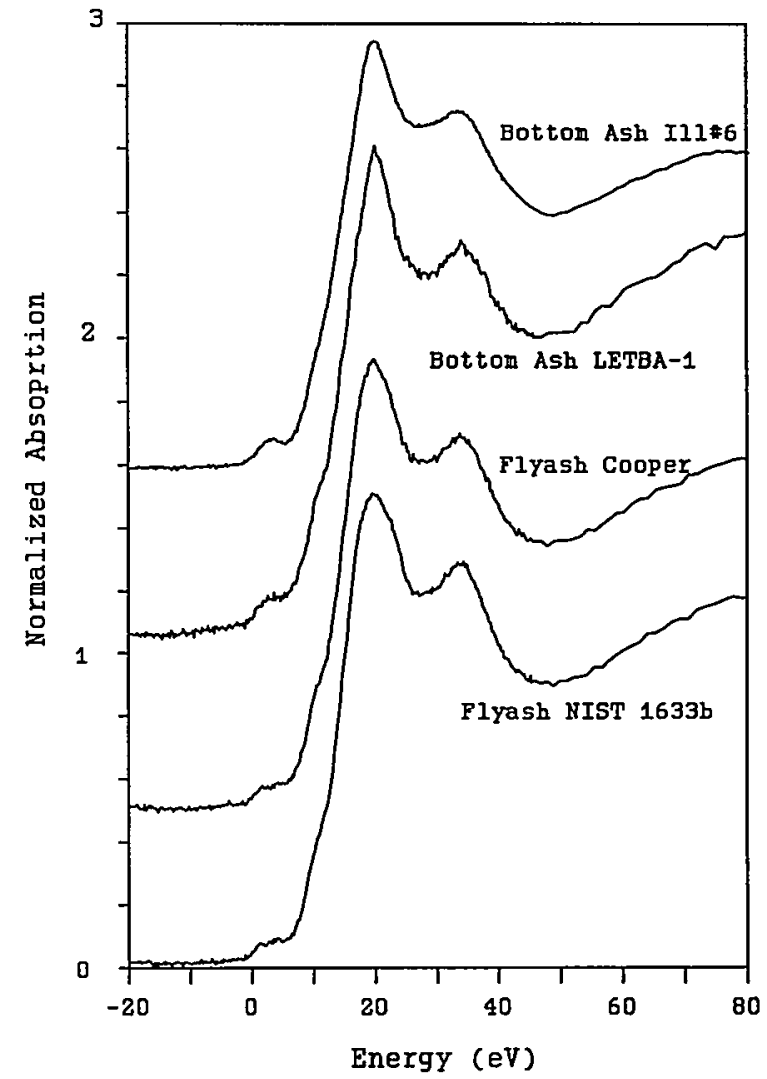

Figure 3: Cr XANES spectra of selected bottom ash and flyash samples from coal combustion.

\section{CONCLUSIONS}

Least-squares fitting of the pre-edge peaks in Cr XANES spectra has been used to construct a calibration curve from which $\mathrm{Cr}(\mathrm{VI}) / \mathrm{Cr}$ (III) ratios can be estimated for solid combustion products. For the most precise work, especially at very low levels of $\mathrm{Cr}(\mathrm{VI})$ in such systems, additional work needs to be done in order to take into account the variation of the pre-edge peaks' intensities with different $\mathrm{Cr}$ (III) structures of varying degrees of octahedral site distortion. The $\mathrm{Cr}(\mathrm{VI})$ contents of the ash samples derived from coal combustion that we have so far 
examined by Cr XANES spectroscopy do not exceed $5 \%$ of the total chromium.

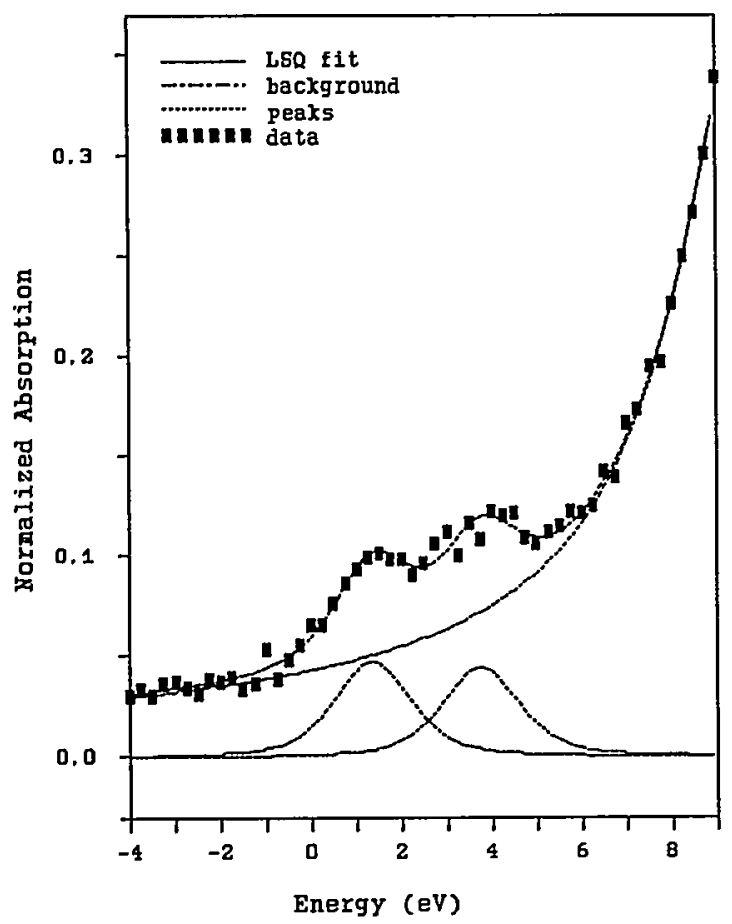

Figure 4: Least-squares fit on pre-edge peaks for NIST Flyash sample 1633b.

\section{ACKNOWLEDGEMENTS}

Research into the speciation of "air-toxic" trace elements in coal and ash has been supported by the Office of Exploratory Research, Electric Power Research Institute, Palo Alto, CA. We also acknowledge the SSRL Biotechnology group for their assistance in this work and the U.S. Department of Energy for its support of the synchrotron facilities at SSRL.

1. United States Public Law 101-549, Nov. 15, 1990, U.S. Government Printing Office, Washington DC, 1990, $314 \mathrm{pp}$.

2. Baruthio, F., Biol. Trace Elem. Research, $\underline{32}$ 145-153, (1992).

3. Horvath, Zs., et al., Talanta, 41, 1165-1168 (1994).

4. de Beer, H., and Coetzee, P. P., S.-Afr. Tydskr. Chem., 41(4), 152-156, (1988).

5. Elleouet, C., et al., Analyt. Chim. Acta., 257, 301-308, (1992).

6. Cramer, S. P., et al., Nucl. Instr. Meth., A266, 586, (1988).

7. Waychunas, G. A., Amer. Mineral., $\underline{72}, 89-101$, (1987). 
Proposal 2175Mp

\title{
XAFS Spectroscopic Investigation of the Behavior of Zinc during Coliquefaction of Coal and Waste Lubricating Oil
}

\author{
F. E. Huggins, J. Zhao, and G. P. Huffman \\ 341 Bowman Hall, University of Kentucky, Lexington, KY 40506-0059
}

Coliquefaction of waste carbonaceous materials, such as plastics, rubber, and waste oil, with coal is being examined as a possible strategy to derive a useful liquid fuel that could be used as a feed-stock for conventional petroleum cracking or similar processes [1]. The use of hydrogen-rich waste materials in liquefaction with hydrogen-poor coal should enhance both the yield and quality of desirable liquid products. Moreover, this process also results in a solid residue, derived in large part from the ashforming elements in coal, that can act as an effective internal filter for unwanted components from both the waste material and the coal. For example, much of the sulfur and ash present in coal reports to the solid residue, and it is anticipated that most inorganic elements, whether derived from the coal or from the waste material, should also migrate into this solid residue.

Of great environmental importance in coliquefaction of carbonaceous waste materials and coal is the behavior and mobilization of specific trace and minor elements and their compounds that have been identified by the U.S. Environmental Protection Agency as potentially detrimental to the Nation's air and water quality if they were to be released to the environment in significant concentrations. Such species may derive from either the waste material or the coal or both components in the coliquefaction process. To follow the mobilization and segregation (partitioning) reactions of critical elements during coliquefaction, it is necessary to utilize an elementspecific technique such as XAFS spectroscopy to determine the speciation of the element in these complex materials.

Generally, the ash-forming elements, including most of the elements of greatest environmental concern, exist in coal in much higher concentrations than they do in waste lubricating oil [2]. However, zinc and sometimes phosphorus have concentrations that are significantly greater in the waste oil than in the coal because of special liquid additives, such as zinc dialkyldithiophosphate (ZDDP), zinc dialkyldithiocarbamate, etc., that are included in the oil formulation as antioxidant/antiwear agents. For the sample of waste oil used in this study, both zinc and phosphorus were shown by ICP to be present in the oil at about the $800 \mathrm{ppm}$ level, suggesting that ZDDP had been added to the oil at about the $0.50 \mathrm{wt} \%$ level. The presence of metals such as zinc in the oil is an anathema to other methods of recycling and reprocessing of waste lubricating oil.

In this report, XAFS spectroscopy is used to examine the structure and behavior of zinc in the coliquefaction of coal and waste lubricating oil. It was anticipated that the zinc and other undesirable elements would end up in the solid residue, rather than the oil, by means of reaction of the zinc with sulfur derived from the oil and coal.

\section{EXPERIMENTAL}

Coliquefaction experiments: Mixtures of a low sulfur, low ash bituminous coal (Blind Canyon seam, UT) and the waste lubricating (15W40) oil were subjected to liquefaction tests at $400-450^{\circ} \mathrm{C}, 1250 \mathrm{psi}_{2}$ (cold), for $60 \mathrm{~min}$, in tubing bombs at Auburn University, under the supervision of Prof. A. R. Tarrer. In these experiments, the oil and residue samples are defined as the fractions of the product that are soluble and insoluble in hexane, respectively. In all experiments, the solid residue was separated from the liquid product and used for the XAFS experiments.

XAFS Experiments: Zinc K-edge XAFS spectroscopy was carried out at beam-line IV-3 at SSRL on the waste oil and on residues obtained from the coliquefaction tests. A Lytle detector was used for all 
zinc XAFS measurements; however, some concentrated samples and various zinc standards were also measured in absorption geometry.

\section{RESULTS AND DISCUSSION}

The $\mathrm{Zn}$ XANES spectrum of the waste oil is compared to that of zinc dialkyldithiophosphate (ZDDP) in Figure 1. There is clearly a close correspondence between the two spectra indicating that the zinc remains in that form during use, despite exposure to temperatures as high as $150^{\circ} \mathrm{C}$ in internal combustion engines. However, the $\mathrm{Zn}$ XANES spectrum of the coliquefaction residue sample shown in Figure 1 is clearly different from that of the waste oil and much more closely resembles that of zinc sulfide (ZnS), also shown in Figure 1.

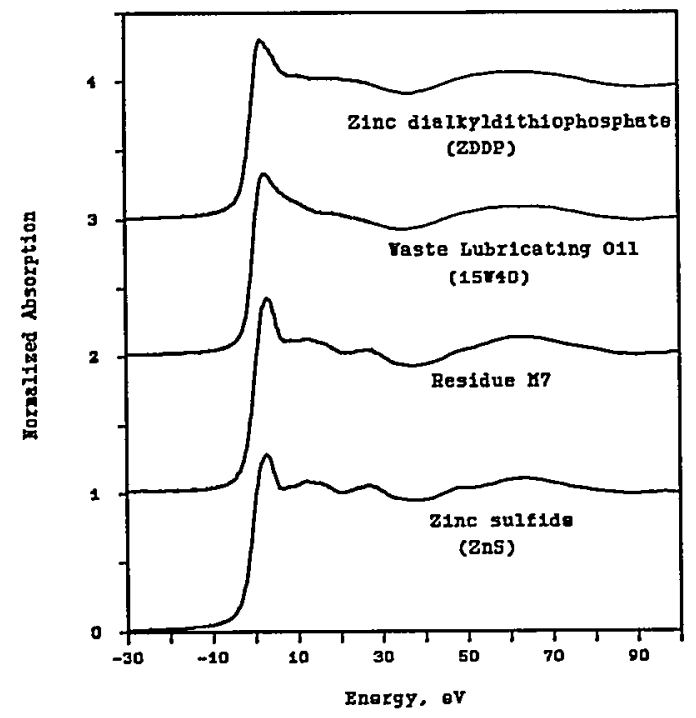

Figure 1: Zn XANES spectra of waste lubricating oil and a coliquefaction residue compared with those for two zinc standard compounds.

Such findings are also confirmed by analysis of the EXAFS regions of the XAFS spectra, as can be seen from the comparison of the Fourier transforms shown in Figure 2. In addition, the structural data obtained from a quantitative analysis of the FT peaks of ZDDP provides constraints on the local zinc environment in ZDDP and leads to the fundamental structural unit shown in Figure 3, in which the zinc in the liquid organothiophosphate ester is surrounded by about four sulfur atoms at a distance of $2.35 \AA$ and about one phosphorus atom at about $2.9 \AA$. Such a structural unit is inconsistent with a monomeric structure for ZDDP (in which the phosphorus coordination must equal 2), but is consistent with a polymeric structure involving two or more molecules of
ZDDP. This result is in agreement with similar findings based on ${ }^{31} \mathrm{P}$ NMR studies of ZDDP and related materials [3].

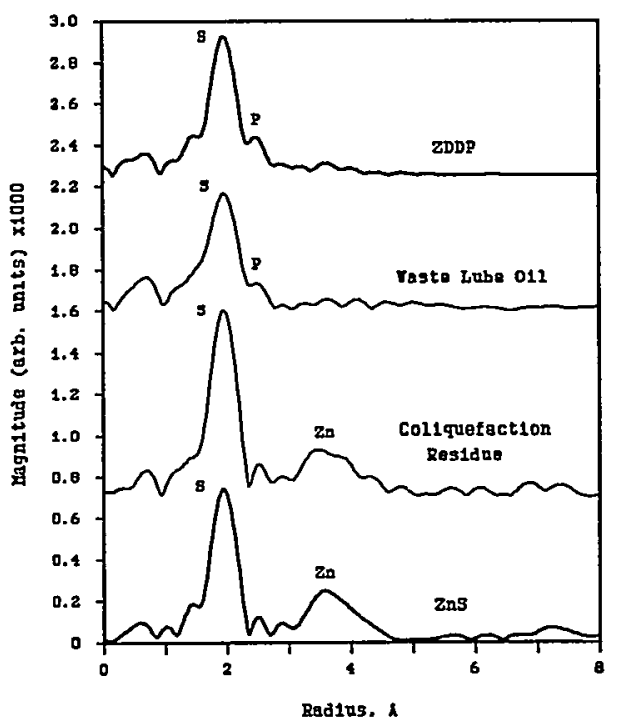

Figure 2: Fourier transforms of $Z n$ EXAFS regions of ZDDP, waste oil, coliquefaction residue, and zinc sulfide.

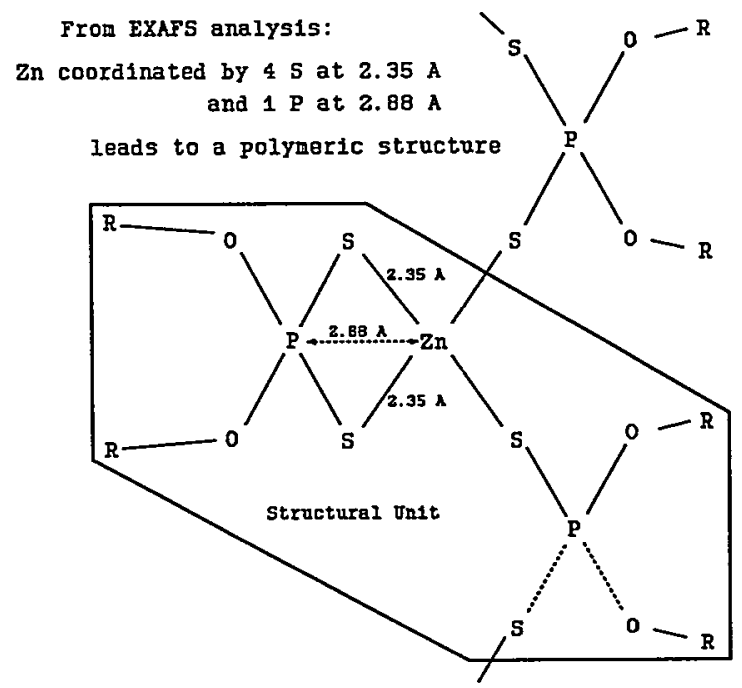

Figure 3: Structural unit derived from analysis of EXAFS spectral region from zinc in $Z D D P$.

\section{CONCLUSIONS}

These results show that during coliquefaction of coal and waste oil the zinc present in waste lubricating oil in the form of an organothiophosphate ester will report to the solid residue in the form of a zinc sulfide. As discussed elsewhere [1,2], this partitioning occurs reasonably quantitatively with much of the 
zinc $(70-100 \%)$ reporting to the residue. From the nature of the coliquefaction process involving coal, it is anticipated that most of the environmentally sensitive elements in carbonaceous materials will exhibit similar behavior.

\section{ACKNOWLEDGEMENTS}

We are grateful to Prof. A. R. Tarrer, Auburn University, for his donation of samples and analysis for these studies, and to Dr. M. Farcasiu, Pittsburgh Energy Technology Center, for samples of the zinc antioxidant agents and general discussion of this problem. Research into the partitioning of environmentally sensitive minor and trace elements in coal and waste carbonaceous materials during co- liquefaction is being supported by the Consortium for Fossil Fuel Liquefaction Science Research Program, administered by the U.S. DoE through Contract No.DE-FC22-93PC93053. We also acknowledge the SSRL Biotechnology group for their assistance in this work and the U.S. Department of Energy for its support of the synchrotron facilities at SSRL.

1. Consortium for Fossil Fuel Liquefaction Science Technical Progress Report, U.S. DoE Contract No. DE-FC22-93PC93053, for the period May 1, 1993 to April 30, 1994.

2. Huggins, F. E., submitted to J. Environmental Science and Health, September, 1994.

3. Harrison, P. G., and Kikabhai, T., J. Chem. Soc. (Dalton Trans.), 807-814, (1987). 


\title{
IN SITU X-RAY STUDIES OF OMVPE GROWTH
}

\author{
P.H. Fuoss ${ }^{a}$, D.W. Kisker ${ }^{\text {b }}$, G.B. Stephenson ${ }^{\text {b }}$, S. Brennan ${ }^{\text {c }}$ \\ ${ }^{2}$ AT\&T Bell Laboratories, Murray Hill, NJ 07974 \\ bIBM Research Division, Yorktown Heights, NY 10598 \\ 'Stanford Synchrotron Radiation Laboratory, Stanford, CA 94309
}

This report summarizes our recent use of x-ray measurements to determine the atomic mechanisms of organometallic vapor phase epitaxy (OMVPE). Detailed information about OMVPE processes has been difficult to obtain because of the high pressure, chemically reactive environment present during growth. X-ray scattering measurements have been uniquely productive in determining the structure of surfaces during OMVPE growth. For example, we have studied surface smoothness, step ordering, growth modes, growth rates and surface reconstructions. In addition, $x$-ray spectroscopy measurements have revealed much about the complicated gas phase behavior in the OMVPE reactor. From these scattering and spectroscopy measurements, a more complete model of OMVPE growth is being developed.

The following will discuss some of our new results. First, we will show results profiling the concentration of the arsenic precursor as a function of height above the substrate. Next, growth modes and growth rates are determined by observing $\approx 1 \mathrm{~Hz}$ oscillations of the $\mathrm{x}$ ray intensity from the (11l) crystal truncation rod near the 110 position. A careful analysis of these growth oscillations suggests that use of trimethylgallium (TMG) as a precursor leads to the adsorption of an organic group on the surface while use of triethylgallium does not. We believe that the desorption of this methyl group is the rate-limiting step in growth of GaAs using TMG.

\section{Experimental Background}

The experiments were performed using a purposebuilt OMVPE reactors and a diffractometer in which high-quality semiconductor films can be grown while simultaneously allowing the diffraction of $x$-rays from the surface[1]. This year we have constructed a new OMVPE reactor which has an improved response time and provisions for optical access. This system has been described in more detail elsewhere.
The substrates used for these measurements were $\mathrm{GaAs}(001)$ wafers $25 \mathrm{~mm}$ in diameter and $1 \mathrm{~mm}$ thick. For various experiments the wafers were misoriented by $0.25^{\circ}$ along the (111) $\mathrm{A}, 0.25^{\circ}$ along the (111) B, or were nominally singular $\left(<0.05^{\circ}\right.$ in a random direction). After mounting in the reactor, the sample was annealed at $600^{\circ} \mathrm{C}$ in flowing tertiarybutylarsine (TBAs) and $\mathrm{H}_{2}$ until the surface was smooth as indicated by a sharp and intense 110 CTR. Typical partial pressures were 70 Torr of $\mathrm{H}_{2}$ and 100 mTorr of TBAs. While under a continuous flow of TBAs, growth was started and stopped by switching either trimethylgallium (TMG) or triethylgallium (TEG) between the reactor and vent line.

For the $\mathrm{x}$-ray spectroscopy experiments, the $\mathrm{x}$-ray fluorescence was monitored with a high-count rate intrinsic Ge detector. This detector was positioned along the polarization of the synchrotron radiation to minimize scattering from the system and to maximize the signal to background. In order to profile the reactor, the entire growth system was translated. The spatial resolution was defined by the incident $x$-ray slits and was $\approx 100 \mu$.

\section{X-ray Spectroscopy Measurements}

A number of important growth characteristics of an OMVPE reactor are strongly influenced by the gas dynamics of the reactor. For example, growth uniformity is often controlled by flow patterns in the reactor, the temperature distribution controls the pyrolysis of the precursors, and switching of gas flows may limit the production of sharp interfaces and doping levels. Even though these parameters are crucial to the design of a successful reactor, they are difficult to control and even harder to predict. While much effort is being expended on improving modeling of reactor designs and performance, those efforts are limited by the availability of accurate data on existing designs. Data that would be useful include composition and temperature profiles of the gas phase, response of the reactor to transients and accurate measurements of growth rate 
for a wide range of conditions. X-ray absorption spectroscopy can be used to directly measure compositions in the gas phase.

We have made substantial progress on each of these three issues. Figure 1 shows the $\mathrm{AsK}_{\alpha}$ fluorescence from the gas phase $1 \mathrm{~mm}$ above the substrate as a flow of TBAs is switched alternately from the reactor to a bypass line. The total flow through the reactor nozzle is $1.8 \mathrm{slpm}$, the reactor pressure is 60 Torr and the TBAs partial pressure is 0.32 Torr. Looking at the inset, which is an average of 10 switching cycles, the reactor response time is approximately 1 second.

Figure 2 shows the behavior of this signal as the reactor is moved vertically past the stationary synchrotron beam. The fluorescence rate, and hence the number density in the gas phase, is relatively constant at room temperature. At high temperature, the fluorescence rate falls off smoothly as the distance above the substrate is decreased. Since this fluorescence rate is a measure of the number density in the gas phase, it should increase smoothly above the surface simply because the gas density at constant pressure drops with increasing temperature.

Also shown in Figure 2 are computational fluid dynamics (CFD) calculations of the concentration gradients in our reactor under these conditions[2]. Clearly there is excellent agreement between the CFD predictions. and our data. These calculations confirm that the

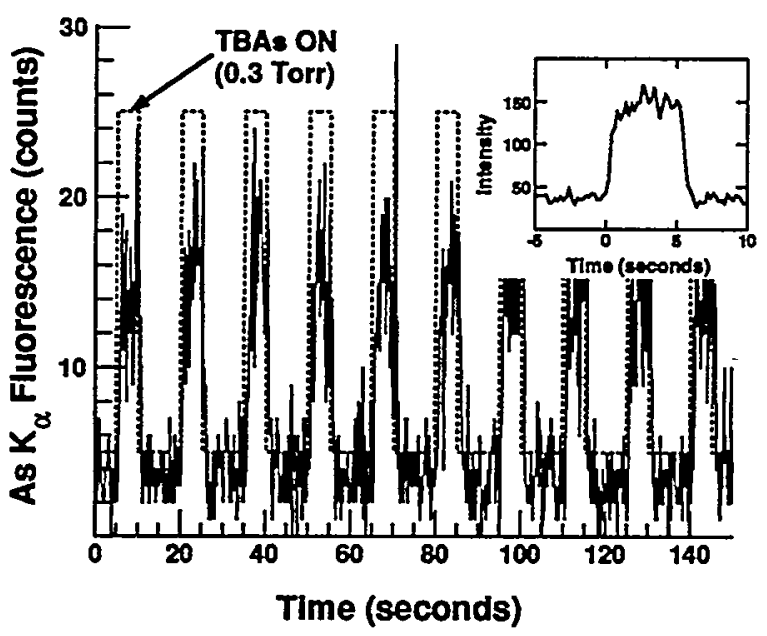

Figure 1: AsK $\mathrm{K}_{\alpha}$ fluorescence from the gas phase while switching the TBAs supply from the reactor to a bypass line. dominate effect is heating of the gas stream. However, an interesting and unexpected result is the slight downturn in the concentration near the substrate for the room temperature data. This turns out to be due to a pinching effect from the purge flow used to eliminate window deposition.

\section{X-Ray Scattering Measurements}

Figure 3 shows the intensity of the 110 and the $\overline{1} 10 \mathrm{re}$ flections as a function of time during two identical pulses of GaAs homoepitaxial growth. Considering the 110 data, the strong oscillations in the scattered $x$ ray intensity are obvious. These oscillations arise for the following reason. The GaAs 110 reflection is bulk forbidden because, as the $x$-rays penetrate into the bulk, successive $\mathrm{Ga}$ and As bilayers scatter exactly out of phase. However, if attenuation and extinction are included, the scattered intensity is given by[3]:

$$
I \propto \sum_{j=0}^{\infty} \Gamma\left[e^{-\mu \Delta}-e^{-\mu \Delta(j+1 / 2)}\right]
$$

which has the limits:

$$
\begin{array}{ll}
I \propto \Gamma^{2} & \text { for large } \mu \Delta \\
I \propto(\Gamma / 2)^{2} & \text { for small } \mu \Delta
\end{array}
$$

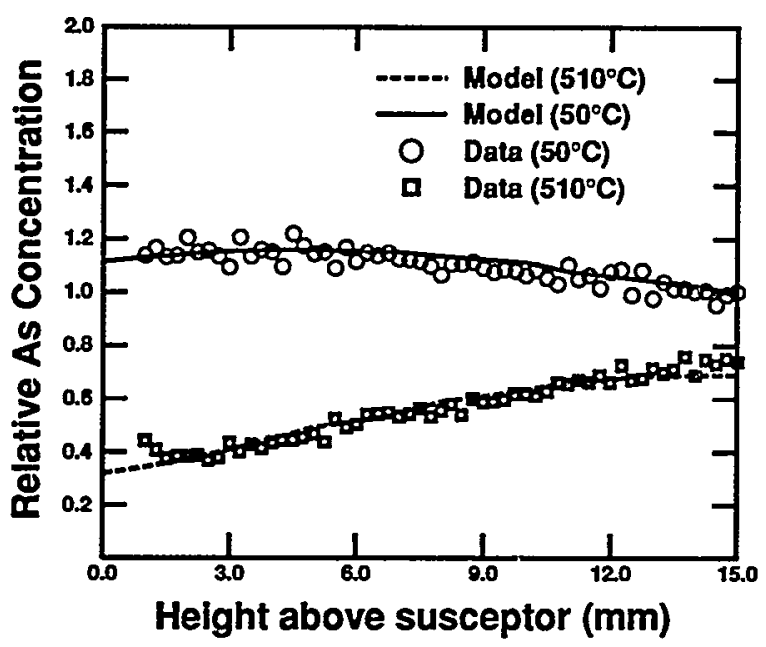

Figure 2: $\mathrm{AsK}_{\alpha}$ fluorescence from the gas phase as a function of height above the GaAs substrate. Note that the dependence at high temperature is much stronger than that at room temperature. This can largely be explained by temperature gradients in the gas phase. 


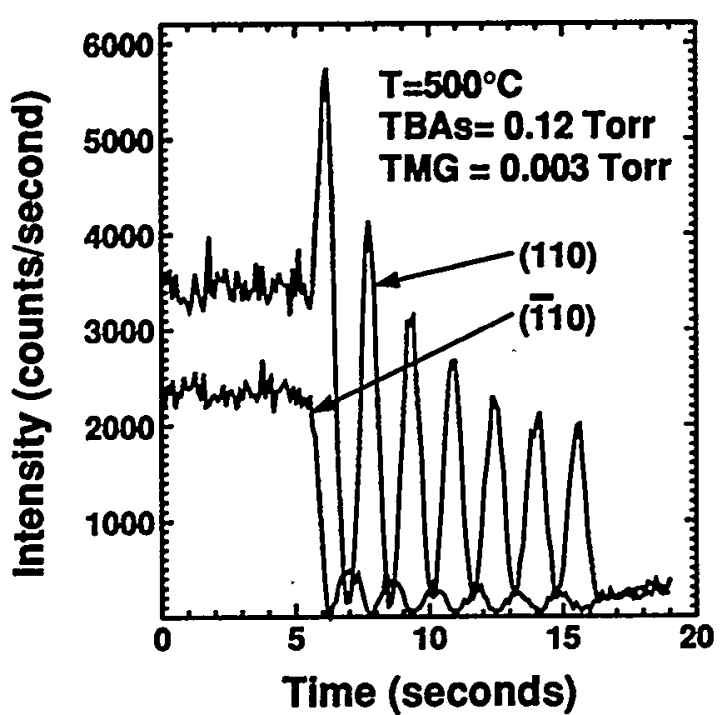

Figure 3: The CTR intensity oscillations for the (110) and (i10)directions from a GaAs (001) surface during growth at $500^{\circ} \mathrm{Cusing}$ TBAs and TMG as the organometallic precursors.

where $\Gamma$ is the scattering per $\mathrm{Ga} /$ As bilayer, $\mu$ is the linear absorption coefficient, $\Delta$ is the thickness of the atomic bilayer and the sum runs over all of the layers in the sample. Thus, if the sample is atomically flat over the coherence length of the $x$-rays, there will be scattering at the 110 position which is equivalent to about one monolayer. This accounts for the strong scattering seen before growth and as each bilayer is completed. However, as the coverage of small islands on the surface approaches one half monolayer, the positive and negative phases in Eqn. (1) will cancel out and the scattering will vanish. Thus, we see strong oscillations of the $\mathrm{x}$-ray signal at the $\mathbf{1 1 0}$ during layerby-layer growth.

It is possible to use these growth oscillations in much the same fashion as RHEED oscillations in MBE growth. For example, Figure 4 shows the growth rate as a function of TMG partial pressure for two different temperatures. While it was thought that the growth rate was proportional to the TMG partial pressure at these temperatures, these data clearly show that the growth rate is kinetically limited; probably by the decomposition of the TMG species on the surface.

The nature of the growth process using TMG can be further explored by examining the behavior of the CTR's during growth in more detail. In Figure 3, the behavior of the (110) and (110) CTR's during growth

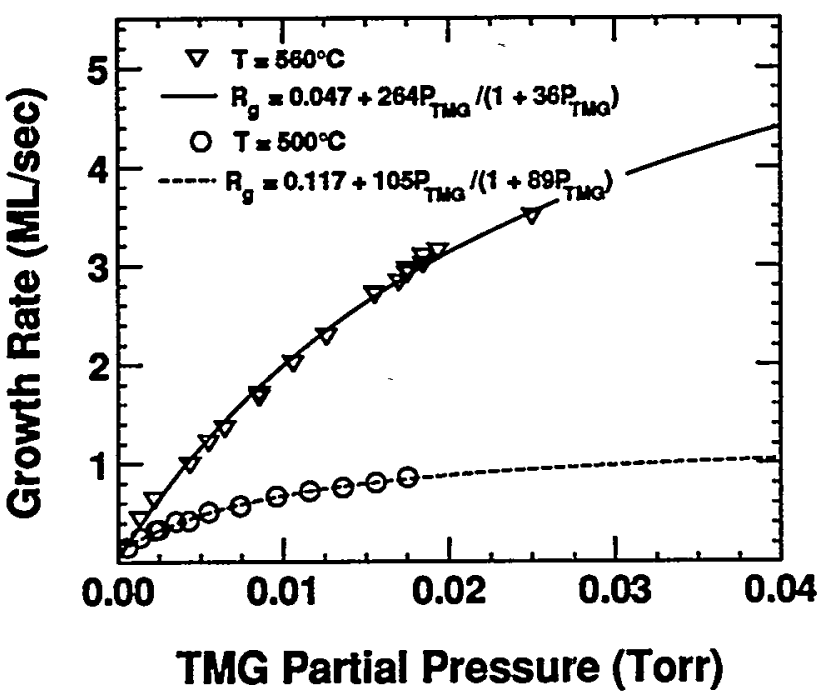

Figure 4: Growth rate as a function of TMG partial pressure for two different growth temperatures. The agreement with the LangmuirHinshelwood form (fits) shows that growth is limited by surface chemical reactions.

at $500^{\circ} \mathrm{C}$ are compared. Several differences between the two azimuths are clear in Figure 3: 1) the oscillations are much stronger at the (110) position, 2) there is a strong initial peak at the (110) and 3) the weak oscillations at the (110) are out of phase with the (110) oscillations. It is interesting to note that none of this behavior is observed during growth using TEG, suggesting that the chemical processes associated with TMG are leading to this behavior.

Our preliminary analysis leads to a model where decomposition of a methylgallium species on the surface is the rate-limiting step. To see this, first consider a simple model in which the surface reconstruction is not a function of island morphology during growth. The intensities of the (110) and $(\overline{1} 10)$ reflections can be written as:

$$
I_{110} \propto\left\{\left[\frac{1}{2}\left(f_{A s}-f_{G z}\right)+f_{\text {surfacc }}\right][1-2 \theta]\right\}^{2}(E Q 2)
$$

and

$$
I_{I 10} \propto\left\{\left[\frac{1}{2}\left(f_{A s}+f_{G z}\right)-f_{s u r f a c t}\right][1-2 \theta]\right\}^{2}(E Q 3)
$$

where $f_{\text {surface }}$ is the scattering factor of the atoms occupying the sites at the surface (which are Ga sites in this model), and $\Theta$ is the fraction of surface covered by bilayer-height islands (which runs from 0 to 1 as each bi- 
layer is grown). The initial state under a flow of TBAs has a partial second layer of As, giving $f_{\text {surface }} \approx 5 / 8$ and $\Theta=0$. Neglecting the small difference between the scattering factors of arsenic $\left(f_{A s}\right)$ and gallium $\left(f_{G_{a}}\right)$, the intensities before growth are:

$$
I_{110} \propto\left\{\frac{5}{8} f_{A s}\right\}^{2}
$$

and

$$
\mathrm{I}_{\mathrm{I} 10} \propto\left\{\frac{3}{8} \mathrm{f}_{\mathrm{As}}\right\}^{2}
$$

This result, which arises because of the particular stacking of the zinc blende structure, qualitatively explains the difference between the initial intensities of the (110) and (110) reflections in Figure 3.

To understand the behavior of the data in Figure 3 as growth is initiated, we have developed a model shown in Figure 5. The initial starting surface, looking at (1) in Figure 5, has a partially completed extra layer of arsenic with a coverage of $\approx 5 / 8$ 's adsorbed on what would normally be gallium sites [4]. We postulate that TMG is only partially decomposed at this temperature and that, at the beginning of growth a methylgallium species either displaces this adsorbed arsenic layer or completes the layer. In either case, looking at (2) in Figure 5 , the methylgallium species scatters $x$-rays more strongly than either arsenic or gallium and enhances the scattering at the (110) position. Similarly, along the (110) direction, the methylgallium species reduces the $x$-ray scattering by enhancing $f_{\text {surface }}$.

After the surface is saturated with methylgallium, the rate-limiting step for further growth is the desorption of the methyl radicals. As the organic desorbs, sites become available for arsenic and more methylgallium to attach. Such isolated atoms then coalesce into bilayerheight islands. As the island coverage $\theta$ increases, the intensity at the (110) position decreases to zero and then increases, due to the final factor in equations (23 ). Each bilayer produces one oscillation in the intensity.

If the new islands formed on the surface were immediately terminated with the methylgallium species, the intensity of the (110) CTR would oscillate weakly around zero but in-phase with the (110) CTR. There are many possible sources for the weak, out-of-phase
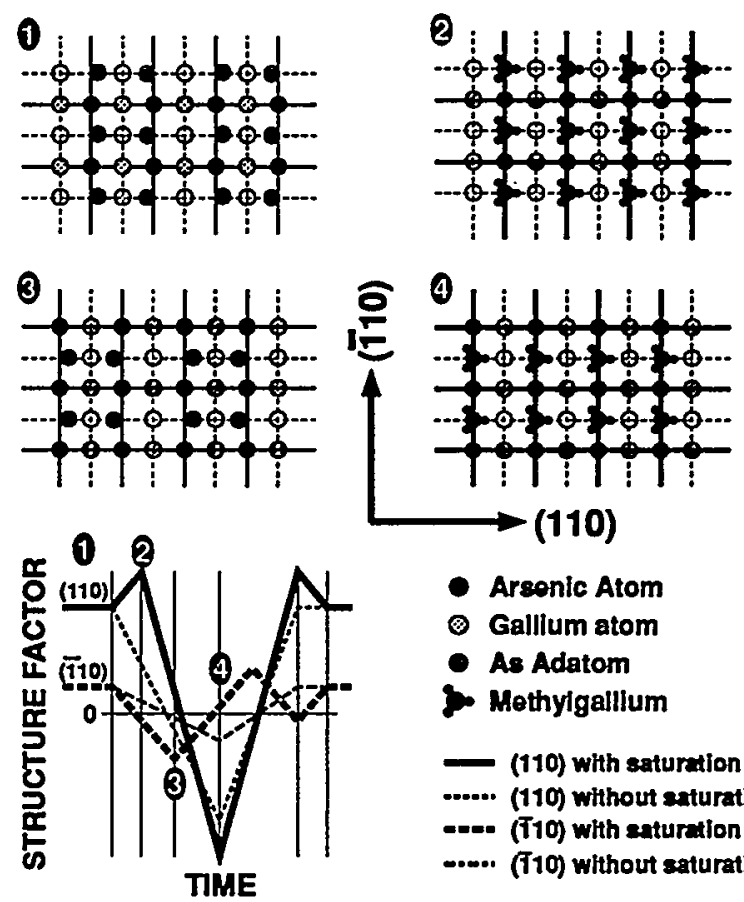

- Arsonic Atom

- Galllum atom

- As Adatom

iv Methyigallium

(110) with saturation

..... (110) without saturation

-es. (i10) with saturation

-.... (T10) without saturation

Figure 5: A schematic picture of the postulated growth process. Before the TMG source is turned on (1), the surface has an extra, partially filled layer of arsenic. When growth is started (2), this layer is replaced with a layer of partially decomposed TMG. This layer then reacts with arsenic and forms islands with a partially filled layer of arsenic (3). These islands are slowly covered with methylgallium and coalesce to form a complete GaAs bilayer (4). At the conclusion of the growth cycle (when the TMG is turned off), the surface slowly recovers to the starting termination. In the real space plan views, the solid lines represent planes of heavy electron density while the dashed lines represent planes of low electron density.

(110) scattering shown in Figure 3. For example, the surface of the islands could be saturated with arsenic more rapidly than with the methylgallium species (because of the much higher partial pressure of TBAs), or the edge of the islands might have a different structure than the center, resulting in an average surface structure that oscillates as the island morphology oscillates. After the TMG source is turned off, the surface gradually returns to its pre-growth state.

This model is consistent with the non-linear growth rate dependence shown in Figure 4 and is supported by the greatly enhanced growth rate, at constant TMG flow, as the TBAs partial pressure is increased. Thus, 
we believe these results are strong evidence for saturation of the surface with a methylgallium compound during growth with TMG. It should be emphasized that similar effects are not seen under similar conditions using TEG. In that case there is no upward spike at the onset of growth, the growth rate is linear with TEG partial pressure and strong, in-phase oscillations are seen at both the (110) and (i10). These differences are consistent with the much different decomposition mechanisms of TMG and TEG.

\section{Summary and Conclusions}

In summary, we continue to use $x$-ray spectroscopy and scattering techniques to study the surface of a film growing in the chemically harsh, high pressure and high temperature environment of organometallic vapor phase epitaxy (OMVPE). The use of $x$-ray intensity oscillations to study growth rates and growth modes (in much the same way as RHEED analysis has been used in UHV techniques) has been used to study growth processes. In a simple example, we showed that the growth was kinetically limited by the decomposition of the TMG at $500^{\circ} \mathrm{C}$ and $560^{\circ} \mathrm{C}$.

Finally, it is well established that $\mathrm{x}$-ray diffraction techniques can be used to understand the surface structures present during growth[4-8]. In a significant difference from the MBE case where growth occurs on a $p(2 \times 4)$ reconstruction, we typically find either a $p(1 \times 2)$ or a $c(4 \times 4)$ reconstruction at lower growth temperatures and a lattice gas of As dimers terminating the surface at higher temperatures. We find little evidence that the presence of a well-defined reconstruction has any effect on growth kinetics and, in fact, see no change in the growth rate as we cross the well-defined phase transition boundary between the ordered phases and the As dimer lattice gas. However, the nature of these reconstructions is thought to control the shape of islands during growth and to influence the morphology of device structures during epitaxial regrowth[9]; and we are currently exploring the dependency of island size and shape as a function of reconstruction and V/ III ratio. While not discussed here, $x$-ray diffuse scattering associated with these intensity oscillations can be used to probe correlations on the growing surface. Diffusion coefficients and activation energies can be extracted from these correlations and show that OMVPE growth is substantially different than MBE or MOMBE growth [10].

\section{REFERENCES}

${ }^{1}$ S. Brennan, P.H. Fuoss, J.L. Kahn, and D.W. Kisker, Nucl. Instrum. Methods Phys. Res., Sec. A, 291, 86(1990).

${ }^{2}$ D.W. Kisker, T. Merchant, P.H. Fuoss, T.J. Mountziaris and K.F. Jensen, to be published.

${ }^{3}$ P.H. Fuoss, K.S. Liang and P. Eisenberger, Synchrotron Radiation Research: Advances in Surface Science, R.Z. Bachrach, Editor, Plenum Publishing Company, 388(1990)

${ }^{4}$ A. Payne, P.H. Fuoss, D.W. Kisker, G.B. Stephenson, S. Brennan, Phys. Rev. B, 49, 14427(1994).

${ }^{5}$ FJ. Lamelas, P.H. Fuoss, D.W. Kisker, G.B. Stephenson, P. Imperatori and S. Brennan, Phys. Rev. B, 49, 1957(1994).

${ }^{6}$ FJ. Lamelas, P.H. Fuoss, P. Imperatori, D.W. Kisker, G.B. Stephenson and S. Brennan, Appl. Phys. Letters, 61, 1034(1992)

${ }^{7}$ D.W. Kisker, P.H. Fuoss, S. Brennan, G. Renaud, K.L. Tokuda and J.L. Kahn, J. of Crystal Growth, 101,42(1990)

${ }^{8}$ D.W. Kisker, G.B. Stephenson, P.H. Fuoss, FJ. Lamelas, S. Brennan and P. Imperatori, J. of Crystal Growth, 124, 1(1992).

${ }^{9} \mathrm{H}$. Asai, J. of Crystal Growth, 80, 425(1987).

${ }^{10}$ D.W. Kisker, G.B. Stephenson, P.H. Fuoss, and S. Brennan, to be published in J. of Crystal Growth, (1994). 


\title{
Seasonal Changes in Valence and Chemical Speciation of Bioaccumulated Mn in Sago Pondweed
}

\author{
C. Mel Lytle, Brigham Young University, Provo, UT 84602 \\ Farrel W. Lytle, The EXAFS Company, Pioche, NV 89043
}

\begin{abstract}
Methylcyclopentadieny manganese tricarbonyl (MMT) is often added to modern gasoline as an antiknock compound to increase the octane rating necessary for modern high-compression engines. We have published a report describing Mn accumulation in soils and plants alongside highways.(1) The accumulation was greatest nearest the highway and in aquatic plants the dry leaf concentrations reached $14,000 \mathrm{ppm}$ in Ranunculus aquatilis and $5000 \mathrm{ppm}$ in Potamogeton pectinatus (sago pondweed). In this report we discuss the speciation of the $\mathrm{Mn}$ accumulating in $P$. pectinatus, a known bioaccumulator of $\mathrm{Mn}$ and other metals (2), over a growing season. A more detailed paper has been submitted for publication.(3) Although necessarily present at a much lower concentration, the bioaccumulated $\mathrm{Mn}$ discussed here was not similar in either concentration or chemical structure to the $\mathrm{Mn}$ in the $\mathrm{O}_{2}$-evolving complex of the Photosystem Apparatus. See Guiles et al. (4) and Goodin et al. (5) for discussions and spectra.
\end{abstract}

Whole plants were collected monthly (JuneJanuary, 1991-2) in replicate from waterways within the lower Provo River drainage, Utah County, Utah. Leaf tissue was separated from root tissue for element and XAS analysis. Clean samples were triply rinsed and dried at $70^{\circ} \mathrm{C}$. Dry tissue samples were ground, digested in $\mathrm{HNO}_{3}, \mathrm{HClO}_{4}$, diluted appropriately and analyzed by Atomic Absorption analysis. Sediment and water samples were obtained from the same locations and intervals as plant samples. The element analyses are collected in Table 1. Pressed pellets of the plant tissue (the speciation of the root tissue is not discussed here) were analyzed by the fluorescent X-ray absorption spectroscopy technique at SSRL. Beam Line 4-3 was set up with $S i(220)$ crystals and a $1 \mathrm{~mm}$ monochromator entrance slit which produced a beam of $\sim 1 \mathrm{eV}$ band width at the $\mathrm{Mn} \mathrm{K}$ edge. Replicate scans were accumulated to obtain an acceptable signal to noise ratio. A series of reference $\mathrm{Mn}$ oxides and solutions was also measured.

In the following we describe our results in terms of finger print comparisons to the reference spectra although a more detailed analysis is being attempted. In Fig. 1 the XANES spectra of a series of Mn oxide reference compounds are shown. Also shown in later figures are spectra from a $0.1 \mathrm{M}, \mathrm{Mn}^{2+}\left(\mathrm{H}_{2} \mathrm{O}\right)_{6}$ solution. There are three qualities of these spectra which we will use in our comparisons. First, the $\sim 1$ $\mathrm{eV}$ positive shift of the main absorption rise with each unit increase in valence, second, the general appearance of the first large peak at the top of the absorption edge and, third, the position and amplitude of the $3 \mathrm{~d}$ prepeaks before the edge. Together they are sensitive indicators of the valence and site symmetry of the $\mathrm{Mn}$ atoms.

Differences were found in the XANES of $\mathrm{Mn}$ in $\mathrm{P}$. pectinatus plant tissue between June, October and January as shown in Fig. 2. Bioaccumulated Mn changed in average valence from $\mathrm{Mn}^{2+}$ in June to mixed $\mathrm{Mn}^{2}+\mathrm{Mn}^{3}+$ in October and back toward $\mathrm{Mn} 2+$ in January. Also note the concentration changes in Table 1. The June leaf tissue was similar to the reference $\mathrm{Mn}^{2+}\left(\mathrm{H}_{2} \mathrm{O}\right)_{6}$ in all three respects (discussed above) as well as in the Fourier transforms shown if Fig. 3. The structure of the $0.1 \mathrm{M} \mathrm{Mn}^{2}+\left(\mathrm{H}_{2} \mathrm{O}\right)_{6}$ solution was solved by fitting with FEFF 3 (6) parameters and found that $\mathrm{N}_{1}=6$ and $\mathrm{R}_{1}=2.15 \pm 0.02$ $\AA$. The Fourier transforms of Fig. 3 are particularly indicative that $\mathrm{Mn}$ in the June plant tissue is coordinated by water since even the more distant peaks agree in position. By October the $\mathrm{Mn}$ valence had changed with an average valence near than of $\mathrm{Mn}_{3} \mathrm{O}_{4}$. $\mathrm{Mn}_{3} \mathrm{O}_{4}$ has the spinel structure with mixed valence (average $=\mathrm{Mn}^{2.67+}$ ) and is more accurately written $\left(\mathrm{Mn}^{2+}\right)\left(\mathrm{Mn}^{3+}\right)_{2} \mathrm{O}_{4}$ where $\mathrm{Mn}^{2+}$ is in tetrahedral, four-coordinated sites, $\mathrm{Mn}-\mathrm{O}=1.86 \AA$ and the $\mathrm{Mn}^{3+}$ is in octahedral, six-coordinated sites, $\mathrm{Mn}-\mathrm{O}=2.03 \AA$ (7). The $\mathrm{Mn}^{2+}$ in tetrahedral coordination causes the larger pre-edge peak as discussed by Kutzler et al. (8). It is evident that at no time during its growing season does $P$. pectinatus leaf tissue have the larger pre-peak of $\mathrm{Mn}_{3} \mathrm{O}_{4}$. This proves that the bioaccumulated $\mathrm{Mn}$ was always in a coordination with inversion symmetry; probably octahedral. - In October there was a strong 2 nd neighbor peak similar to that in $\mathrm{MnO}$. In the $\mathrm{NaCl}$ structure of $\mathrm{MnO}$ this peak is due to 2 nd neighbor $\mathrm{Mn}$ atoms. The similarity in position and amplitude suggests that this peak is also $\mathrm{Mn}$ in $\mathrm{P}$. pectinatus. By January the valence had shifted back toward $\mathrm{Mn}^{2+}$, probably by selective leaching of the $\mathrm{Mn}^{3+}$. Note in Table 1 that the concentration had decreased to $1 / 5$ of the maximum October concentration by January. 
Table 1. Manganese content and valence in $P$. pectinatus leaf and root tissues from June to January. Mn content as ppm \pm S. E., no. samples $=3$.

Sample

June

August

September

October

December

January

Water

Soil
Concentration, ppm

Leaf

$122 \pm 6 \quad 81 \pm 17$

$1744 \pm 102 \quad 1248 \pm 89$

$3861 \pm 117 \quad 3640 \pm 302$

$4990 \pm 48 \quad 3072 \pm 221$

$2130 \pm 65 \quad 2953 \pm 177$

$1002 \pm 890 \quad 891 \pm 33$

0.001
$13 \pm 2$ DTPA extract

Horiguchi and Iwakiri (9) have found that a peroxidase-malale dehydrogenase system oxidized $\mathrm{Mn}^{2+}$ in root cells. 'This suggests that June-October Mn compound formation in plant tissue is also an A'TP-dependent biological mechanism and not due to a physical process (such as the leached condition in January) caused by the atmosphere or aquatic environment. As the concentration of $\mathrm{Mn}$ increases along side the nation's highways and becomes ubiquitous in our cities, the biological signilicance of its presence must be examined. Also, the appearance of $\mathrm{Mn}^{3+}$ in bioaccumulating plants may have serious biological signilicance since $\mathrm{Mn}^{3+}$ is more toxic than $\mathrm{Mn}^{2+}$ to animals that may ingest them.(10)

\section{Relerences}

1. C. M. Lytle, C. Z. McKinnon and B. N. Smith, Naturwissenschaften 8 1, 509 (1994).

2. H. A. Kantrud, Sago Pondweed: A Literature Review, U. S. Fish and Wildlife Service, Resource Publication 1.76 (1990) and references therein.

3. C. M. Lytle, F. W. Lytle and B. N. Smith, J. Envir. Qual., Submitted, (1995).

4. R. D. Guiles, V. K. Yachandra, A. E. McDermott, J. L. Cole, S. L. Dexheimer, R. D. Britt, K. Sauer and M. P. Klein, J. Amer. Chem. Soc. 29, 471 (1990) and ibid. p. 486.

5. D. B. Goodin, V. K. Yachandra, R. D. Britt, K. Sauer and M. P. Klein, Biochim. Biophys. Acta. 767, 209 (1984).

6. J. J. Rehr, J. Mustre de Leon, S. I. Zabinsky and R. Albers, J. Amer. Chem. Soc. 113, 5135 (1991).

7. R. W. G. Wyckoff, Crystal Structures, 2nd Edition, John Wiley and Sons, New York, NY (1963). 8. F. W. Kutzler, C. R. Natoli, D. K. Misemer, S. Doniach and K. O. Hodgson, J. Chem. Phys. 73, 3274 (1980).

Valence

$2^{+}$

Mixed $2^{+}$and $3^{+}$

$2^{+}$

9. T. Horiguchi and Y. Iwakiri, Abst. 1982 Meeting Jpn. Soc. Soil Sci. Plan Nutr., p. 59.

10. J. Komura, M. Sakamoto, Linviron. Res. 57, 34 (1992).
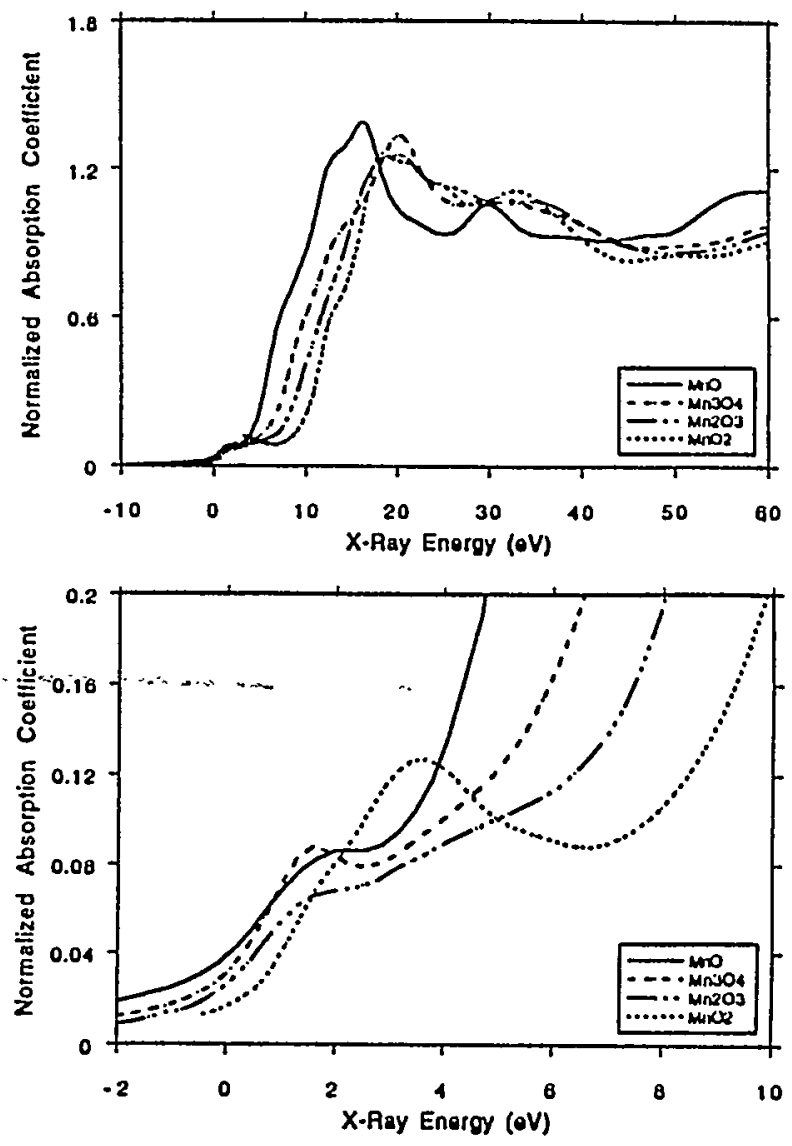

Fig. 1 XANES, top, and pre-edge, bollom, spectra of $\mathrm{Mn}$ oxide reference compounds. 

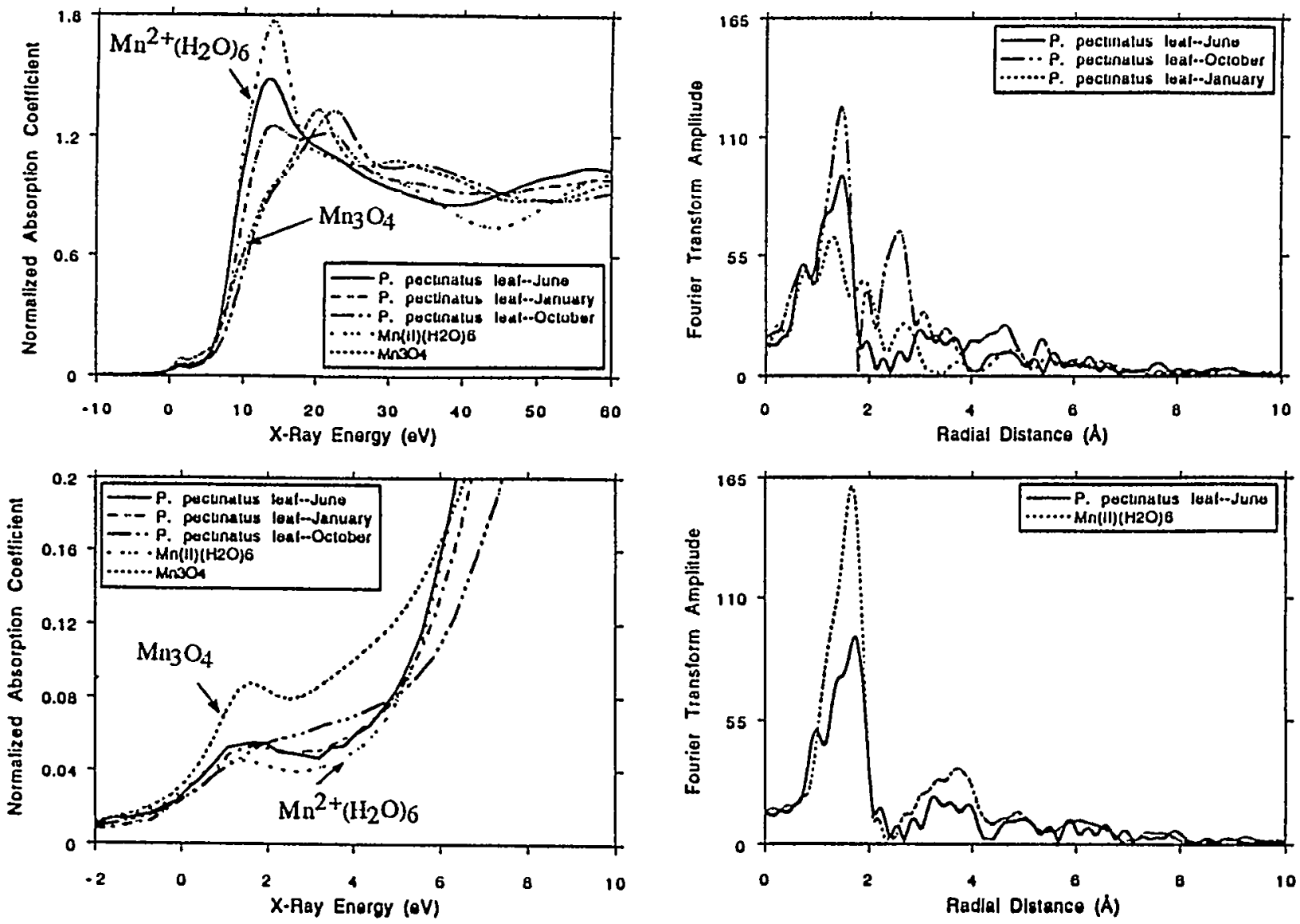

Fig. 2. Top, comparison of the June, October, January P. p. XANES spectra with that of $0.1 \mathrm{M}$ $\mathrm{Mn}^{2+}\left(\mathrm{H}_{2} \mathrm{O}_{6}\right.$ and the mixed valence $\mathrm{Mn}_{3} \mathrm{O}_{4}$. The June spectrum is an excellent match to that of $\mathrm{Mn}^{2+}\left(\mathrm{H}_{2} \mathrm{O}_{6}\right.$. By October the valence of $\mathrm{P}$. $\mathrm{p}$. is much like $\mathrm{Mn}_{3} \mathrm{O}_{4}$ and by January the valence has shifted nearly back to $\mathrm{Mn} \mathrm{2t}$. In the pre-edge spectra, bottom, note that at no time during the year did the P. p. spectra show the increased intensity characteristic of a tetrahedral or other noninversion symmetry site.

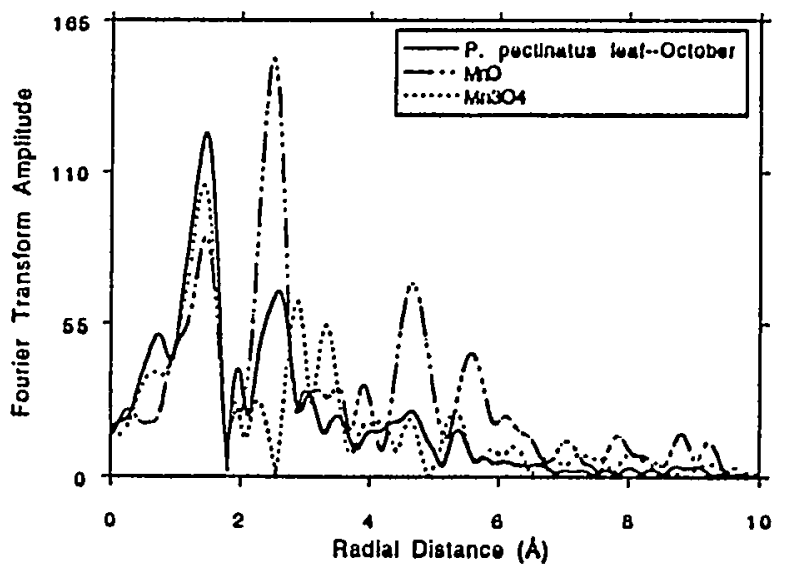

Fig. 3 Top, comparison of structural changes shown by their Fourier transforms during the year in P. p. Middle, the overlay of P. p. June with $\mathrm{Mn}^{2+}\left(\mathrm{H}_{2} \mathrm{O}_{6}\right.$ is a good match even for the higher coordination shells. Bottom, in October the P. p. Fourier transform has a large 2nd neighbor peak similar in position to that in $\mathrm{MnO}$. 


\title{
XAFS STUDIES of PEROVSKITE RELATED STRUCTURES AT HIGH PRESSURES
}

\author{
R. Ingalls, F. Wang and S. Jones \\ Department of Physics, University of Washington, Seattle, WA 98195 \\ B. Houser \\ Department of Physics, Eastern Washington University, Cheney, WA 99004
}

During this report period we have continued to study the XAFS of materials undergoing pressure induced phase transitions, using methods described earlier. ${ }^{1}$

We obtained further measurements on the material $\mathrm{ReO}_{3}$ which undergoes a rotational (buckling) structural transition, sometimes referred to as a "compressibility collapse," at $0.5 \mathrm{GPa}$. With the aid of state of the art FEFF programs, ${ }^{2}$ the data was analyzed to give very accurate values for the rotation angles of the $\mathrm{ReO}_{6}$ octahedra as a function of pressure. In the future this material is expected to be a very precise XAFS pressure calilbrant for the 0 - 2 Gpa pressure range.

A major advance in our capabilities was the development of a low temperature, liquid nitrogen, cryostat to contain our pressure cell. It was used to to study $\mathrm{ReO}_{3}$ along with $\mathrm{RbCl}$ which also has a structural phase transition ca. $0.5 \mathrm{GPa}$, in this case from the $\mathrm{B} 1$, or $\mathrm{NaCl}$ structure, to the $\mathrm{B} 2$, or $\mathrm{CsCl}$ structure. In the case of $\mathrm{ReO}_{3}$, the data should help to remove the thermal disorder from the rotational disorder at the transition.

The low temperature dramatically enhanced the EXAFS of RbCl. We found that it also caused the B1 - B2 transition to be more extended in pressure showing evidence of a mixed phase region. Figure 1 shows this effect where low temperature first modifies the EXAFS of the B1 phase, this is enhanced below the transition and even more so above the transition, while the XANES continue to evolve from one phase to the other.

Several techniques have been used to investigate the pressure induced amorphization, including $\mathrm{x}$ ray diffraction, Mössbauer spectroscopy, and Raman scattering. The amorphization has been determined by the complete loss of the diffraction

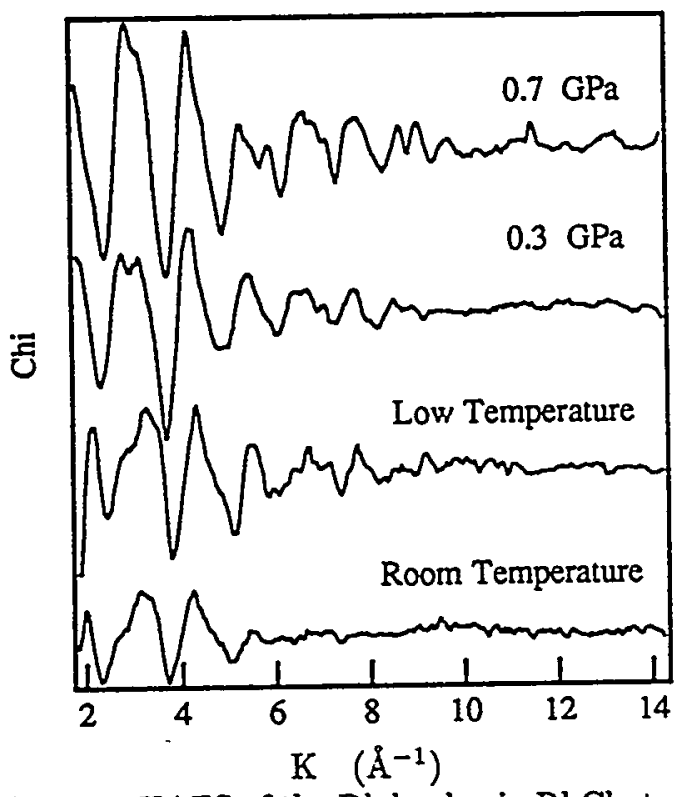

Figure 1. XAFS of the $\mathrm{Rb}$ k-edge in $\mathrm{RbCl}$ at room and liquid nitrogen temperature as a function of pressure showing the $\mathrm{B} 1-\mathrm{B} 2$ phase transition that occurs ca. 0.5 GPa.

pattern, and its accompanying information at the same time. The usefulness of Raman scattering technique for structural studies of the amorphized state is impaired, because metallization turns the sample opaque. XAFS, on the other hand, is a very good local probe, that can study both crystalline and amorphous states.

Tin iodide $\left(\mathrm{SnI}_{4}\right)$ undergoes a phase transition between approximately $8 \mathrm{GPa}$ and $17 \mathrm{GPa}$. The transformation is simultaneously from crystalline to amorphous and from insulating to metallic. ${ }^{3}$. Sintered boron carbide anvils ${ }^{1}$ were used to abtain pressures to above $19 \mathrm{GPa}$. In this first run, a pressure calibrant was not used, rather the sample 
pressure was estimated from the applied pressure. XAFS spectra of the $k$ edges of $S n$ and I were measured at different pressures. For the $\mathrm{Sn}$ edge, the amorphization process is clearly observed in Figure 2, where the very strong EXAFS oscillations are greatly diminished above the transition due to the lack of long range order in the amorphous phase. After release of pressure the ordered phase is again obtained. It is noted that the $29 \mathrm{keV}$ edge of $\mathrm{Sn}$ and $33 \mathrm{keV}$ edge of I were the highest edges so far studied at high pressures. Analysis of these results is in progress.

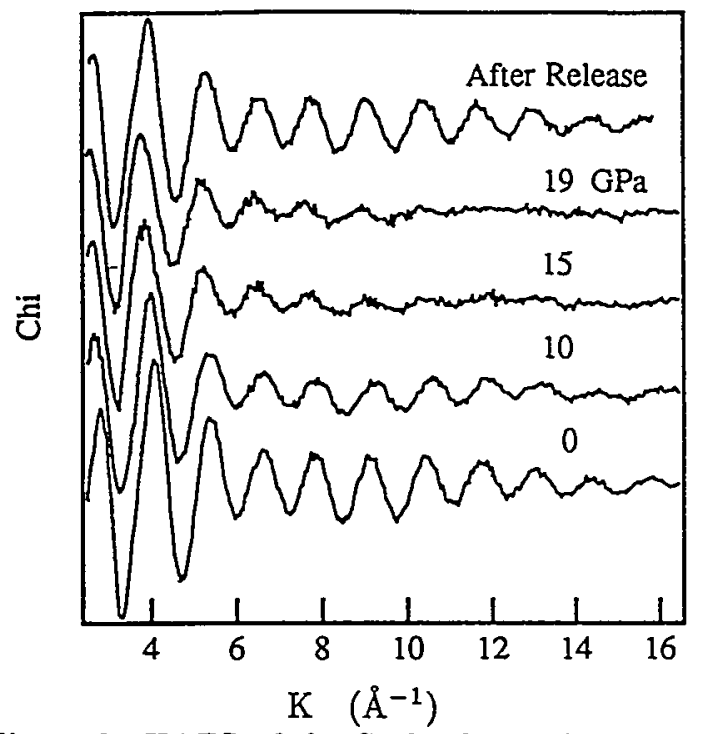

Figure 2. XAFS of the $\mathrm{Sn} \mathrm{k-edge} \mathrm{in} \mathrm{SnO}_{4}$, illustrating the loss of atomic order above the pressure induced crystalline to amorphous structural transition.

Another recent study was the pressure induced Wurtzite to $\mathrm{BI}$ transition in $\mathrm{ZnO}$ which occurs at approximately $10.5 \mathrm{GPa}$. Figure 3 shows both the change in XANES and EXAFS as one proceeds through the transition. Analysis of these results is in progress.

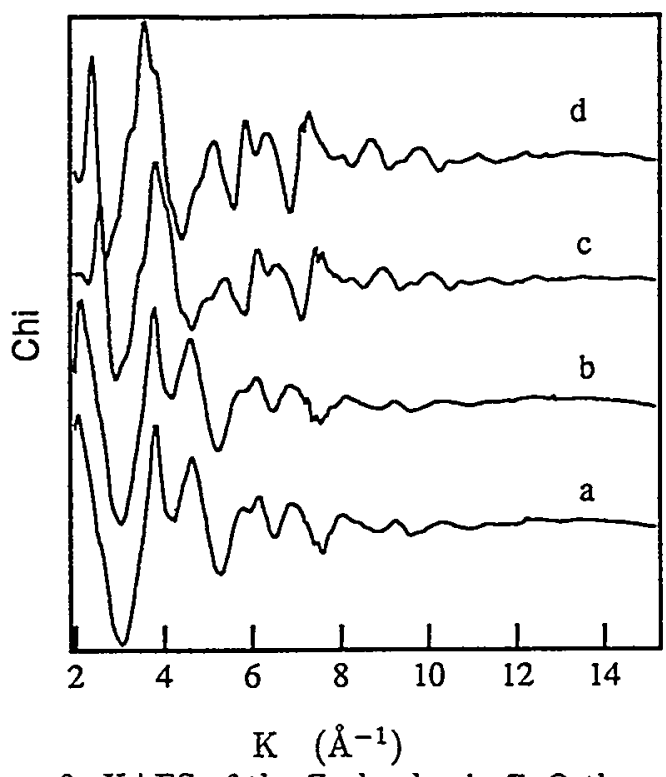

Figure 3. XAFS of the $\mathrm{Zn}$ k-edge in $\mathrm{ZnO}$ through the $10.5 \mathrm{GPa}$ Wurtzite to $\mathrm{B} 1$ structural phase transition: curves a to $d$ are in order of increasing pressure with $a$ and $b$ below the transition, and $c$ and $\mathrm{d}$, above.

\section{ACKNOWLEDGEMENTS}

This research is supported in part by the Depratment of Energy, Grant No. DE-FG06-84ER45163, through the office of Basic Energy Sciences.

\section{REFERENCES}

${ }^{1}$ R. Ingalls et al., J. Appl. Phys. 51, 3158 (1980)

${ }^{2}$ J.J. Rehr and R.C. Albers, Phys. Rev. B41,8139 (1990)

${ }^{3}$ Moshe P. Pasternak et al., Phys. Rev. Lett. 72, 2736 (1994) 
Proposal 2194Mp

\title{
Strain Distributions in Metallic Thin Films and Patterned Lines
}

\author{
Richard P. Vinci, Eden M. Zielinski, and John C. Bravman \\ Department of Materials Science and Engineering, Stanford University, Stanford CA 94305-2205
}

\section{BACKGROUND}

While aspects of the mechanical behavior of metallic thin films have been widely investigated, general relationships between processing, microstructure, and properties remain largely undetermined. Aluminum and copper films have been the foci of our work because aluminum is the most commonly used material for interconnect lines in integrated circuits while copper is considered a likely successor as reliability and performance demands continue to increase. While many similarities exist between these metals, significant differences have been observed and must be explained.

Following are selected recent results, showing measurements of strain in grains of different orientation in aluminum and copper thin films. It is difficult to produce an aluminum or copper film with a perfect preferred grain orientation, and a large random component or secondary texture component often exists [1]. The difference in stress state between grains of different orientation in films of mixed texture may preferentially influence the growth of certain grains [2], resulting in a microstructure detrimental to electromigration resistance in aluminum interconnects $[3,4]$. Furthermore, hillock growth in aluminum films has also been attributed to stress differences in a film of imperfect texture [4]. Thus, it is important to understand the stress states not only of grains corresponding to the primary texture of a film, but also of randomly oriented grains or grains corresponding to a secondary texture.

Several models have been proposed which explain observed high thin film flow stresses in terms of the stress required for dislocation glide in the constraining presence of substrate and surface layer interfaces. One such model, developed by Nix [5], predicts an orientational dependence of flow stress in a single crystal film. It is common to add to this a grain size strengthening term, often in the form of the Hall-Petch relation [6], which is not orientation dependent. Thompson has also derived an expression in which the grain size effect does have an orientational dependence [7]. For an FCC material, both models predict that a film of (111) orientation (normal to the film plane) will have a larger yield stress than (100) or (110) films.

To extend the Nix model to a polycrystalline thin film, it is generally presumed that the flow stress in a grain with a particular orientation can be described by the single crystal model. This is based on two assumptions: first, each grain is assumed to be columnar and rigidly attached to the substrate, limiting the forces imposed by adjacent grains, and second, the dislocation flow processes are assumed to occur solely in an individual grain and therefore will not induce flow in neighboring grains.

This model has several implications for microstructural evolution in thin films of imperfect texture. If grains with different orientations support different stresses, a local stress gradient will be established. Hillocking may arise from a mechanodiffusional mass flux from (111) grains to grains of a different orientation. Differences in the strain states between grains of different orientations, and the resulting differences in strain energy density, may also provide a large driving force for preferential grain growth. In both cases, the effect of film thickness on flow stress must also be taken into consideration.

Strain determination by $\mathrm{x}$-ray measurements of interplanar spacing as a function of $\sin ^{2} \psi$ (where $\psi$ is the angle between the film normal and the direction of interest) is a commonly used technique. The unstrained lattice parameter $\left(\mathrm{d}_{0}\right)$ for a film can be found at a value of $\psi$ which is a function of the particular material and microstructure. Knowledge of the unstrained lattice parameter and the lattice parameter in an arbitrary direction defined by $\psi$ (and by $\phi$, the angle in the plane of the film) allows calculation of the strain in that direction. Once the strain at several values of $\psi$ has been determined, the strain in any arbitrary direction may be calculated.

If the unstrained lattice parameter has been determined by the $\sin ^{2} \psi$ method for a particular film, it is possible to directly determine the normal strain of grains of a particular orientation from a single additional measurement of interplanar spacing. In practice, since the strain is calculated from one measurement, rather than from a fit to a series of measurements, this approach requires great instrumental accuracy, and error is likely to be greater than with the $\sin ^{2} \psi$ method. The low equatorial beam divergence of the synchrotron source coupled with the use of Soller slits perpendicular to the plane of the diffractometer dramatically reduces error in the measurement normally encountered in a standard diffractometer. Axial divergence error, however, must be identified by extrapolation techniques and corrected prior to strain calculation. Still, this approach guarantees that there is no contribution to the measurement from misoriented grains, a significant concern when attempting to determine the strain state 
of grains not corresponding to the primary texture when using the $\sin ^{2} \psi$ method [8].

Once the strain in the direction normal to the film is determined for grains of a certain orientation, then it is also possible to calculate the strain and stress in those grains in the plane of the film by applying appropriate assumptions of mechanical behavior.

\section{RESULTS}

\section{Single Crystal Aluminum Films}

The biaxial stress-temperature behavior of a quasiepitaxial (111) aluminum single crystal film on (111) silicon and a (110) aluminum bicrystal film on (100) silicon was determined using the $\sin ^{2} \psi$ technique. Measurements were taken while heating to $400^{\circ} \mathrm{C}$ and cooling to room temperature. Large differences in yield stress were apparent, with the (111) single crystal yield stress exceeded that of the (110) bicrystal by approximately $150 \mathrm{MPa}$ during heating, demonstrating that the basic principal behind the yield models is valid.

\section{Polycrystalline Aluminum Film}

An aluminum sample, $1.2 \mu \mathrm{m}$ thick, was sputter deposited at room temperature on a (100) silicon substrate. The texture, determined with $x$-rays, was weakly (111) with a small (110) component. As a result, (200) and (220) out-of-plane lattice spacings were measured to determine strain throughout a thermal cycle.

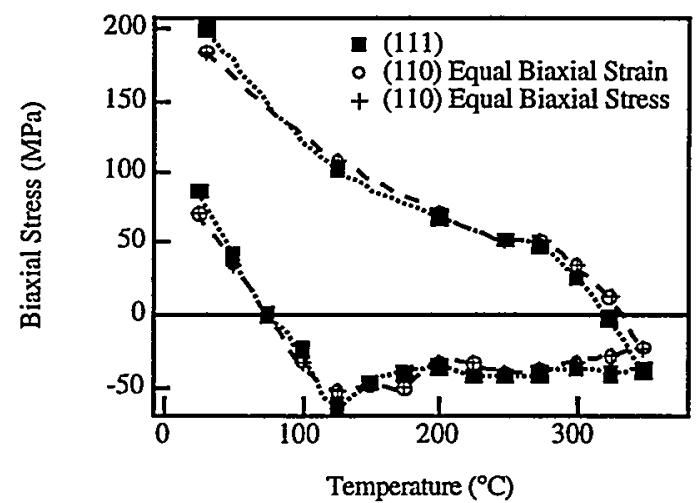

Figure 1. Biaxial stress in (110) and (111) oriented Al grains during thermal cycling.

Figure 1 shows the biaxial stress-temperature behavior for both (111) and (110) oriented grains. Although there are some small differences in the stress of the two orientations in the compressive regime, these are within the error of the measurement. The yield stresses predicted by the Nix model, ignoring grain size strengthening effects, differ by approximately $60 \mathrm{MPa}$. The expected difference is even greater using the Thompson model, which includes an orientational dependence in the grain size strengthening mechanism. The predicted differences are within the sensitivity of the technique but are not observed.

Hillocking was observed by optical and atomic force microscopy after the thermal cycle, and is manifested as a small yield drop in the compressive regime. The magnitude of the yield drop is consistant with that due to hillocking reported elsewhere [9]. The low stress supported by the film at these temperatures means that orientationally dependent differences in stress should be small. Since this is the regime in which hillocks are thought to occur, it is difficult to directly evaluate the validity of the hillocking model with this data.

Cross-sectional TEM revealed that the asdeposited film was only partially columnar, with columnar regions separated occasionally by equiaxed regions. In addition, plan-view TEM revealed a relatively small grain size of $0.4 \mu \mathrm{m}$ in a $0.9 \mu \mathrm{m}$ thick film. We previously measured the thickness dependent strengthening term in films where the grain size approximately equaled the film thickness. The different microstructure of this film may weaken the thickness dependent strengthening, making the orientational differences smaller and therefore harder to detect. That said, this film does demonstrate that the stress state associated with a perfectly columnar microstructure is not necessary for hillocking to occur.

\section{Polycrystalline Copper Films}

Copper films $0.2,0.4,0.8$, and $1.2 \mu \mathrm{m}$ thick were sputter deposited at $250^{\circ} \mathrm{C}$ on oxidized (100) silicon wafers with a tantalum barrier layer. Median grain sizes determined after deposition were $0.20,0.37,0.71$, and $0.72 \mu \mathrm{m}$, in increasing order of film thickness. $\mathrm{X}$ ray texture scans before and after thermal cycling showed that the films were strongly (111) oriented, but with small (100) and random components, and no indications of abnormal grain growth. Cross-sectional TEM revealed a fully columnar microstructure.

Measurements were taken as each sample was heated and cooled in vacuum. Several reflections, both symmetric and off-axis, were measured at each temperature step. Symmetric scans were taken of (222) and (200) planes whose normals were parallel to the film normal. Off-axis scans were made of (331) planes over a wide range of $\psi$ angles (selected assuming (111) grain orientation). While significant differences in the hysteresis of the stress-temperature behavior existed between the samples of different thickness, the basic orientation behaviors were similar. Thus, only the trends in the $1.2 \mu \mathrm{m}$ film are presented in the following discussion. 


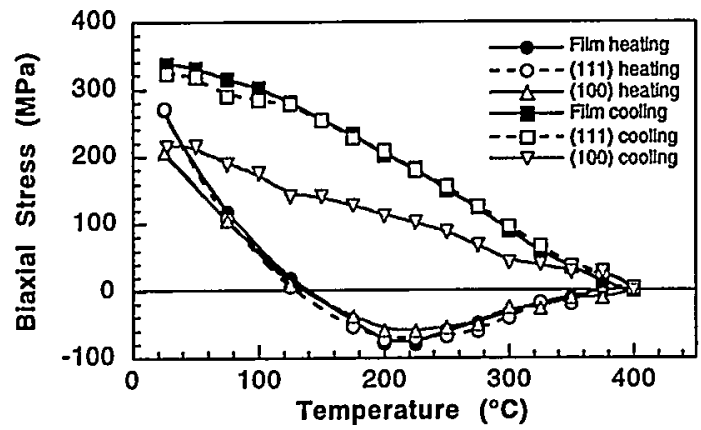

Figure 2. Biaxial stress in a $1.2 \mu \mathrm{m}$ thick bare $\mathrm{Cu}$ film.

Biaxial stresses derived from direct $x$-ray measurements of (111) and (100) grain populations are plotted in Figure 2, along with biaxial stresses determined for the entire film from off-axis measurements of (111) grains. The agreement between the direct (111) measurements and the off-axis measurements is excellent. While the initial room temperature stresses in the two orientations are different, the grains follow an isostress behavior during much of the heating leg of the cycle. The initial strain (not plotted) in the (100) grains is large compared to the (111) grains, contrary to the simple isostrain expectation. Throughout the thermal cycle, the biaxial strain in the $(100)$ grains exceeds that in the (111) grains except at temperatures above $300^{\circ} \mathrm{C}$, where relaxation is sufficiently rapid to eliminate the strain in both orientations. As in the aluminum sample, there is no obvious difference in the yield stress of the two orientations. This may imply that the small grain boundary displacement assumption is invalid, leading to an appearance of yielding in the stronger orientation. Upon cooling, a stress difference between the orientations is present once again. The room temperature stress in the (111) grains increases as a result of the cycle, while the stress in the (100) grains is relatively unchanged. This behavior is consistent throughout the samples, suggesting that room temperature relaxation occurred primarily in the (111) grains prior to measurement or that an additional strengthening mechanism is at work in the (111) grains.

In Figure 3, the final room temperature stresses of both grain populations are plotted as a function of 1 thickness, with linear fits applied to the data. The room temperature flow stresses calculated using the Nix and Thompson models are also plotted in Figure 3. Both models for (111) oriented grains, including grain boundary strengthening, underestimate the magnitude of the stresses but match reasonably well in slope. The offset between the predicted flow stresses and the measured flow stresses is suggestive of another strengthening mechanism at work, perhaps in the form of obstacles to dislocation glide resulting from numerous twin boundaries or work hardening. The scatter of the (100) data is large, so it is not certain that a linear fit is most appropriate. Unlike the (111) stresses, the room temperature stresses of the (100) grains show no strong dependence on thickness, contrary to the predictions of the dislocation glide models. This has the effect of greatly increasing the stress differences between the orientations as film thickness decreases.

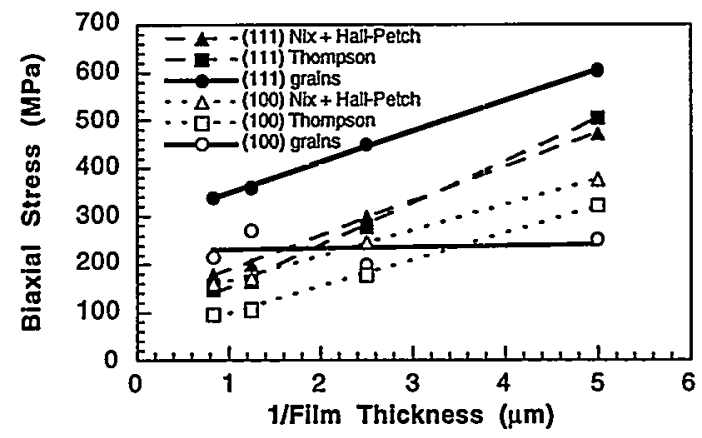

Figure 3. Room temperature flow stresses in grains of (111) and (100) orientation in films of different thicknesses.

Based on these results, it is inappropriate to apply simple assumptions to the yield behavior of individual grains within a bare polycrystalline copper film. Dislocation glide models are only appropriate for the majority grain orientation population in these textured films, since the surrounding grains are likely to be of the same orientation and will therefore provide a homogeneous local environment. Nonetheless, a difference in low temperature stress level between grains of (111) and (100) orientation does exist, and, in fact, the difference increases with decreasing film thickness even more rapidly than predicted. The preceding analysis was conducted with the assumption that obstacle controlled dislocation glide is the primary relaxation mechanism acting in the copper films. The behavior of the minority grains also may deviate from expectations in part because of other relaxation mechanisms active in these films [8].

\section{Qrientation Dependent Grain Growth in Copper}

Two $\mathrm{Cu}$ samples, $1.2 \mu \mathrm{m}$ thick, were sputter deposited, one at room temperature, the other at $250^{\circ} \mathrm{C}$. Texture scans identified almost random orientation for the room temperature deposited film and strong (111) texture for the $250^{\circ} \mathrm{C}$ film. As before, (200) and (222) normal lattice spacings were measured to determine strain.

Biaxial stress in the random film, which exhibited abnormal grain growth, is plotted in Figure 4. This graph is a composite of measurements from several identical samples from a single wafer; strain of both orientations was measured from a different sample at each temperature. This technique was chosen over the 
standard method of heating a single sample and taking measurements at each temperature step in an attempt to directly measure the strain energy driving force force as a function of annealing temperature. However, some grain growth occurred during thermal cycling to the measurement temperature for all temperatures above $125^{\circ} \mathrm{C}$, as monitored by (200) intensity.

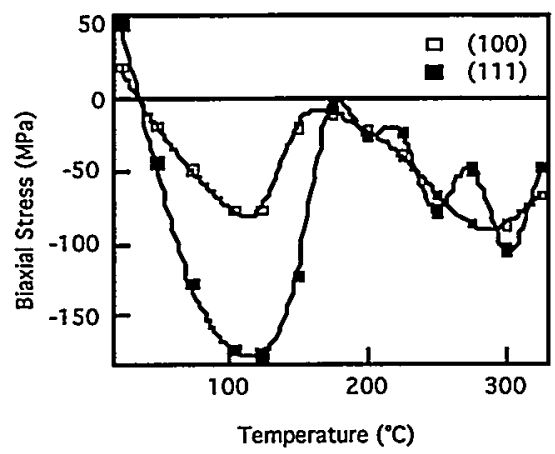

Figure 4. Biaxial stress from several different identical samples. Abnormal grain growth of the (100) orientation was observed above $125^{\circ} \mathrm{C}$.

From room temperature to $125^{\circ} \mathrm{C}$, both orientations supported identical biaxial strains. Due to the large difference in biaxial modulus, this manifests itself as a large difference in biaxial stress. Since the strain energy density for both of these orientations is given by $\sigma \varepsilon$, the difference in biaxial stress directly determines the driving force for growth of (100) grains. At 100 and $125^{\circ} \mathrm{C}$, significant deviation from linearity shows that some of the thermal strain is accommodated by a plastic flow mechanism. Although grain growth is known to relieve compressive stresses, the (200) and (222) peak intensities did not appreciably change at these two temperatures, indicating that this is not the primary relaxation mechanism. This allows for the calculation of the maximum strain energy driving force for this film, since plasticity determines the maximum achievable strain energy difference. At $125^{\circ} \mathrm{C}$, the driving force is equal to $68 \times 10^{3} \mathrm{~J} / \mathrm{m}^{3}$, which is far less than the 700 $\times 10^{3} \mathrm{~J} / \mathrm{m}^{3}$ predicted by applying the $\mathrm{Nix}$ or Thompson models [2]. Above $125^{\circ} \mathrm{C}$, a factor of 100 increase in the (200) peak intensity indicated that abnormal grain growth occurred and therefore that the measurement did not represent the driving force for (100) growth, but-rather merely measured the residual strain after growth.

The biaxial stress in a single textured sample, which did not exhibit any abnormal (100) growth, is shown in Figure 5. Contrary to the assumption that the thermal strain is applied equally to grains of each orientation, the $(100)$ and the $(111)$ orientations supported significantly different strains but very similar stresses. It is not strictly true that the grains are following isostress averaging, since the measured ratio of the slopes in the elastic region of a strain versus temperature plot is not equal to the inverse of the ratio of the biaxial moduli. Nearly isostress behavior, however, does explain why (100) abnormal grain growth is absent. Throughout the cycle, the large strain of the (100) orientation relative to that of the (111) orientation means that the (111) grains will have the lower strain energy density, favoring (111) growth.

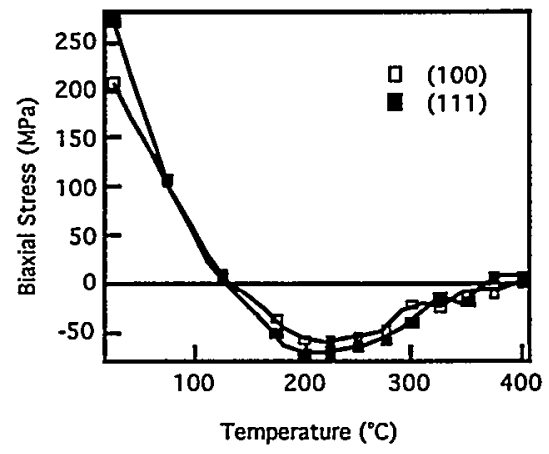

Figure 5. Biaxial stress in a $\mathrm{Cu}$ film in which the abnormal growth of (100) grains was suppressed.

Plan-view TEM revealed in-plane grain sizes of 0.11 and $0.98 \mu \mathrm{m}$ for the random and (111) textured films, respectively. Perhaps more importantly, crosssectional TEM demonstrated that the film in which isostrain behavior was observed was only partially columnar while the isostress film was fully columnar. In both films, individual grains must both incorporate the applied biaxial strain and respond to local force balance requirements. Grains in a non-columnar film are less constrained locally and therefore may on average be able to support an isostrain state; in a columnar film, it is possible that the local force balance is the deciding factor. Further work is required for clarification.

This work was performed at SSRL, which is supported by the Department of Energy.

\section{REFERENCES}

1. D. P. Tracy, D. B. Knorr, J. of Elec. Mater. 22, 611 (1993)

2. E. M. Zielinski, R. P. Vinci, J. C. Bravman, J. Appl. Phys. 76, 4516 (1994).

3. C. V. Thompson, Scripta Met. 28, 167 (1993).

4. J. Sanchez, E. Arzt, Scripta Met. 27, 285 (1992).

5. W.D. Nix, Met. Trans. A 20A, 2217 (1989).

6. A. J. Griffin, F. R. Brotzen, C. Dunn, Scripta Met. 20, 1271 (1986).

7. C. V. Thompson, J. of Mat. Res. 8, 237 (1993).

8. R. P. Vinci, E. M. Zielinski, J. C. Bravman, submitted to Thin Solid Films, (1994).

9. R. Venkatraman, J. C. Bravman, J. of Mat. Res. 7, 2040 (1992). 


\title{
INVESTIGATION OF HEAVILY-DOPED III-V AND II-VI SEMICONDUCTORS USING FLUORESCENT EXAFS
}

\author{
Kin Man Yu, Wladyslaw Walukiewicz, Carolyn Rossington, \\ E. Bourret, J. M. Jaklevic, and E. E. Haller \\ Lawrence Berkeley Laboratory
}

\section{INTRODUCTION}

Ge, a group IV element is, in principle, an amphoteric dopant in III-V compound semiconductors, in particular GaAs and $\mathrm{InP}^{1-4}$. Using ion channeling and electrical measurement, Kringh $\varnothing \mathrm{j}^{3}$ showed that $\mathrm{Ge}$ occupies both In and $\mathrm{P}$ sites in InP. In our previous report we have discussed the substitutionality of $\mathrm{Ge}$ in InP at lower Ge concentration $\left(-1 \times 10^{20} \mathrm{~cm}^{-3}\right)$ in which we oberved $\mathrm{Ge}-\mathrm{P}$ and $\mathrm{Ge}-\mathrm{In}$ bonding environments for $\mathrm{Ge}$ implanted in InP by extended $x$-ray fine structure (EXAFS). As a result, $\mathrm{Ge}$ can be used as an n-type dopant in InP but its doping efficiency is low due to the presence of compensating Gep acceptors. We report in here the fluorescent EXAFS measurements we performed in the past year to investigate the behavior of $\mathrm{Ge}$ in heavily $\mathrm{Ge}$ implanted InP and $\mathrm{AlSb}$ both in the as-implanted state and after annealing. In the as-implanted sample it is expected that the Ge atoms reside in an amorphous InP (and AlSb) layer. Therefore the Ge substitutionality cannot be studied by ion channeling and electrical methods which rely on the monocrystalline nature of the host crytal.

\section{EXPERIMENT}

Ge ions were implanted into single crystal InP wafer at $60 \mathrm{keV}, 110 \mathrm{keV}$ and $180 \mathrm{keV}$ to doses of $1 \times 10^{15} / \mathrm{cm}^{2}, 1.5 \times 10^{15} / \mathrm{cm}^{2}$ and $2.4 \times 10^{15} / \mathrm{cm}^{2}$, respectively. The purpose of the multiple energy implantation is to produce $\mathrm{a} \sim 2000 \AA \mathrm{InP}$ layer with uniform Ge concentration of $\sim 4 \times 10^{20} / \mathrm{cm}^{3}$. The InP substrates were held at both room temperature (RT) and liquid nitrogen temperature (LNT). Rapid thermal annealing (RTA) were performed at $950^{\circ} \mathrm{C}$ for $5 \mathrm{sec}$.

Large grain bulk AlSb crystals were implanted with Ge at $180 \mathrm{keV}$ to a dose of $5 \times 10^{15} / \mathrm{cm}^{2}$. The AlSb samples were kept at room temperature during implantation. After implantation the sample was rapid thermal annealed (RTA) at $750^{\circ} \mathrm{C}$ for $5 \mathrm{sec}$.

The local atomic environments of the Ge in InP and AlSb were studied using fluorescence EXAFS measurements performed at room temperature at beamline 4-3 at SSRL.

\section{RESULTS AND DISCUSSION}

\section{Ge implanted InP}

In this study, the Ge concentration is $-4 \times 10^{20} / \mathrm{cm}^{3}$ which is expected to be above the solubility limit of $\mathrm{Ge}$ in InP. Fig. 1 shows the isolated $k^{3}$-weighted Ge K-edge EXAFS of the RT implanted InP as-implanted and after RTA, where $k$ is the photoelectron wave number. The Fourier transform (FT) magnitudes, $|\mathrm{F}(\mathrm{r})|$ of the EXAFS of these samples are shown in Fig. 2. Notice that the EXAFS spectrum of the as-implanted sample has a much reduced amplitude indicating that the $\mathrm{Ge}$ atoms in the sample have reduced pair correlation with the host In and $\mathrm{P}$ atoms. This can be explained by the heavy damage created by the Ge implantation in the as-implanted state. In fact, ion channeling measurement showed that an amorphous layer on the surface (up to $\sim 2000 \AA$ ) was created by the $\mathrm{Ge}$ implantation. The EXAFS spectrum of the RTA sample shows a much higher amplitude revealing that the Ge atoms have high correlation in the crystal after annealing.

Two peaks are resolved in the as-implanted sample in Fig. 2 indicating that the Ge atoms have two different bonding environments. In contrast, only a single broad peak is observed for the RTA sample. Multi-parameter least square fitting of the filtered FT data showed that in the as-implanted sample the $\mathrm{Ge}$ atoms substitute both the In and the $\mathrm{P}$ sites in roughly the same proportion. After RTA, the single peak shown in Fig. 2 arises from the Ge$\mathrm{Ge}$ bonding configuration indicating $\mathrm{Ge}$ precipitation at this high concentration. Analysis of the filtered FT data in Fig. 2 reveals that as much as $70 \%$ of the implanted $\mathrm{Ge}$ atoms formed $\mathrm{Ge}-\mathrm{Ge}$ bonding after RTA.

$\mathrm{PIXE} /$ channeling results show that the nonsubstitutional Ge, probably in the form of $\mathrm{Ge}$ precipitates present in InP after RTA amount to $\sim 65 \%$. This is consistent with the EXAFS measurements. From the EXAFS and PIXE 


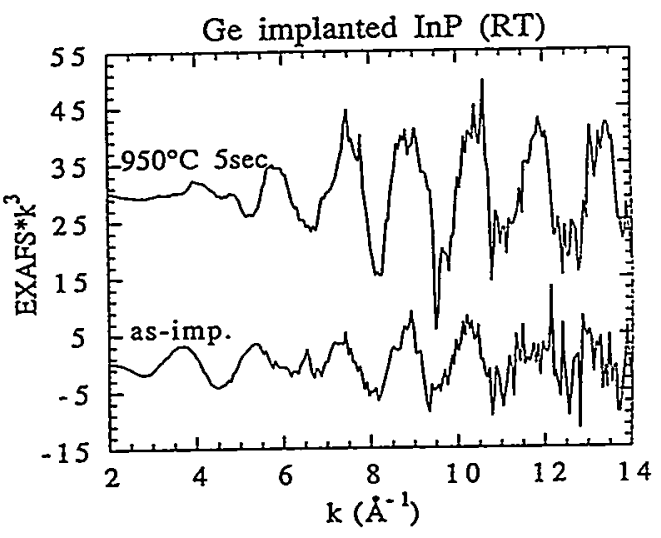

Fig. 1 The isolated EXAFS oscillations at the Ge Kedge weighed by $\mathrm{k}^{3}$ from the $\mathrm{Ge}$ implanted InP samples as-implanted and after RTA.

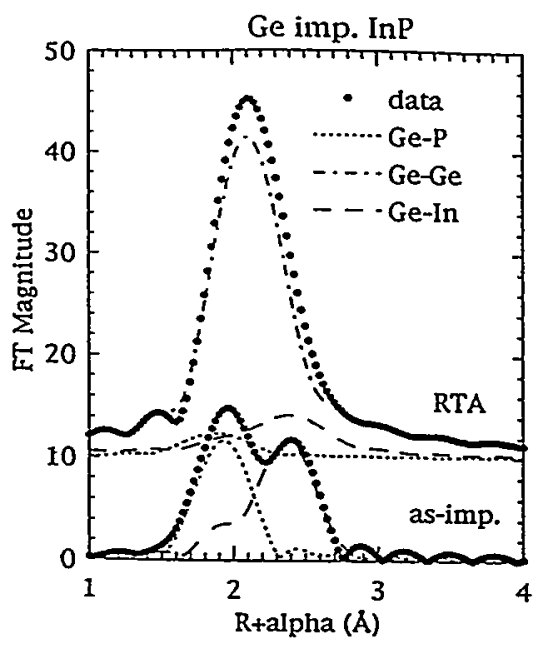

Fig. 2 Fourier transform magnitudes of the EXAFS oscillations from the RT implanted and RTA samples shown in Fig. 1. The dashed curves show the FT of the best-fit data.

measurement we determined that the solubility limit of $\mathrm{Ge}$ in InP is $\sim 1.2 \times 10^{20} / \mathrm{cm}^{3}$. Hall measurements on the RTA sample show that the sample is n-type with sheet carrier concentration $3.4 \times 10^{14} \mathrm{~cm}^{-2}(\sim 8 \%$ activation).

\section{Ge implanted AlSb}

Figure 3 shows the isolated $k^{3}$-weighted EXAFS spectra of the Ge implanted AISb sample asimplanted and after RTA. Solid phase epitaxial regrowth of implant induced amorphous layers in GaSb and AlAs has been shown to occur by RTA at $750^{\circ} \mathrm{C}$ by Pearton et. al.$^{5}$ Our EXAFS results

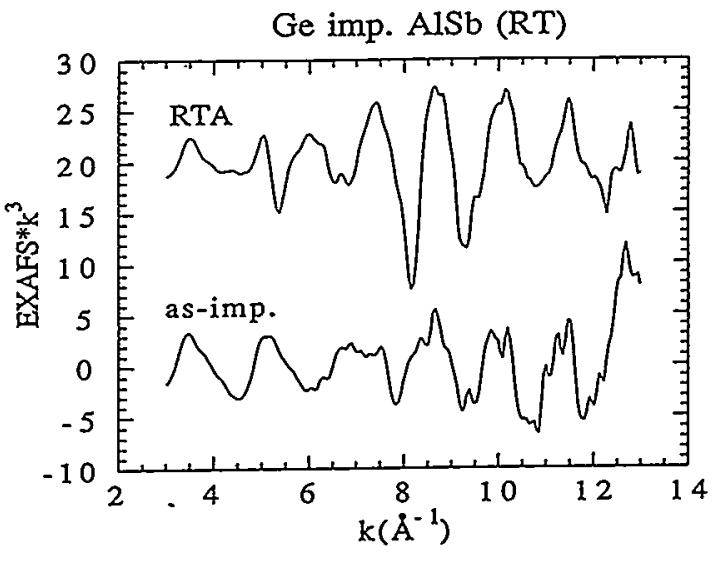

Fig. 3 The EXAFS oscillations at the Ge K-edge weighed by $k^{3}$ as a function of photoelectron wave number $k$ from Ge implanted AlSb samples asimplanted and after RTA.

in Fig. 3 suggest that $750^{\circ} \mathrm{C}$ RTA in fact also induces recrytallization of the Ge implantation induced amorphous layer in AlSb. However, the degree of residual damage in the layer has not been measured in the present study.

Fig. 4 shows the filtered Fourier transform (FT) magnitude of the $k^{3}$-weighted EXAFS spectra of the as-implanted and RTA AlSb samples. The small FT magnitude of the as-implanted sample is a result of the small EXAFS amplitude (Fig. 3 ) due to $\mathrm{Ge}$ atoms in the amorphous layer. The FT magnitude of EXAFS from the as-implanted sample shows two major peaks at distances 2.1 and $2.4 \AA$. After phase shift corrections, these two peaks correspond to nearest neighbor distances of $\mathrm{Ge}-\mathrm{Al}$ at $2.52 \AA$ (RGe$\mathrm{Al})$ and $\mathrm{Ge}-\mathrm{Sb}$ at $2.66 \AA\left(\mathrm{R}_{\mathrm{Ge}} \mathrm{Sb}\right)$. This result indicates that in the as-implanted state, although the $\mathrm{Ge}$ atoms are in an amorphous AlSb layer, they reside in two specific positions, one surrounded by $\mathrm{Al}$ and the other by Sb atoms, similar to the case of InP. However, the average coordination number of the $\mathrm{Ge}$ atoms is about 3 instead of 4 for Ge substituting a host atom in the crystalline state. The dotted and dashed lines Fig. 4 show the FT of individual contribution of the best-fitted data.

The filtered FT magnitude of the RTA sample displayed in Fig. 4 shows a significant difference from that of the as-implanted sample. Most notable is the disappearance of the double peak feature in the as-implanted sample. The single peak in Fig. 4 for the RTA sample is asymmetrically skewed to the low $\mathrm{R}$ side. Moreover, the FT magnitude (and also the EXAFS amplitude) is much larger indicating that the crystallinity of the AlSb has been restored. Fitting of the filtered back-transformed data reveals a two peak structure corresponding to $\mathrm{Ge}-\mathrm{Al}$ and $\mathrm{Ge}-\mathrm{Sb}$ bondings. The measured $\mathrm{R}_{\mathrm{Ge}-\mathrm{Al}}$ and $\mathrm{R}_{\mathrm{Ge}} \mathrm{Sb}$ in the RTA samples are $2.46 \AA$ and $2.66 \AA$, respectively. 


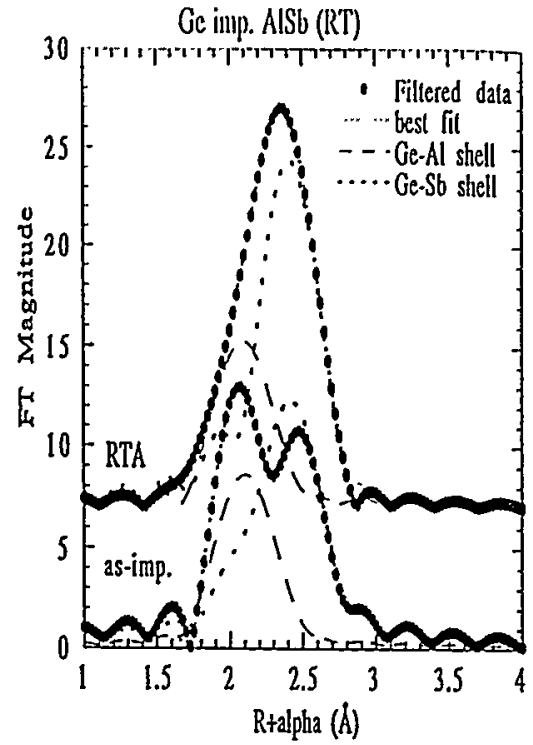

Fig.4 Fourier transform magnitudes of the EXAFS in Fig. 3.

Using the sum of the tetrahedral covalent radii of the elements involved compiled by Van Vechten and Philips $s^{6}$ as the natural bond lengths, we calculated the theoretical bond lengths $\mathrm{Ge}-\mathrm{Al}$ and $\mathrm{Ge}-\mathrm{Sb}$ in $\mathrm{AlSb}$ to be $2.50 \AA$ and $2.64 \AA$, respectively. Our experimental results in both the as-implanted and RTA samples agree well with these theoretical predictions.

Unlike the as-implanted sample where the fractions of $\mathrm{Ge}$ atoms surrounded by $\mathrm{Al}$ and $\mathrm{Sb}$ are approximately equal, the $\mathrm{Ge}-\mathrm{Sb}$ bonding dominates in the RTA sample. An estimate from the EXAFS data reveals that the $\mathrm{Ge}-\mathrm{Sb}$ bonding or $\mathrm{Ge}$ substituting the $\mathrm{Al}\left(\mathrm{Ge}_{\mathrm{Al}}\right)$ in the RTA sample is $\sim 80 \%$. This can be understood by considering the local strains due to $\mathrm{Ge}$ substitution in AlSb. The substitution of $\mathrm{Ge}$ in $\mathrm{Al}$ and $\mathrm{Sb}$ positions in AlSb involve shortening of the bond length by 0.02 and $0.16 \AA$, respectively. While the distortion due to $\mathrm{Ge}_{\mathrm{Al}}$ substitution is small, the lattice distortion due to GeSb substitution is significant. In the as-implanted sample, such local strain can be relaxed due to the reduced coordination number in the amorphous state. After RTA, as the $\mathrm{AlSb}$ recrystallizes the $\mathrm{Ge}_{\mathrm{Al}}$ substitution, which introduces negligible lattice distortion becomes energetically more favorable than the GeSb substitution.

In an earlier report on Ge implantation in InP, we found that $\mathrm{Ge}$ atoms occupy both the In and $\mathrm{P}$ sites after $\mathrm{RTA}^{4}$. The ratio of $\left[\mathrm{Ge}_{\mathrm{In}}\right]:[\mathrm{Gep}]$ is 0.7:0.3 which is slightly less than the $\left[\mathrm{Ge}_{\mathrm{Al}}\right]:\left[\mathrm{Ge}_{\mathrm{Sb}}\right]$ ratio of $0.8: 0.2$ we found in the present work. This can also be explained by the fact that the $\mathrm{GeAl}$ substitution in AISb introduces much less lattice distortion $\left(\triangle \mathrm{R} / \mathrm{R}_{\mathrm{Al}-\mathrm{Sb}}=0.008\right)$ than the GeIn substitution of a $\mathrm{Ge}$ into an In position in InP

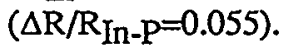

\section{SUMMARY}

We have studied two systems of heavily doped III-V semiconductors using the EXAFS technique. $\mathrm{Ge}$ is found to be in two definite configurations, GeIII and Ge-V, in the as-implanted amorphous materials in both InP and AlSb. We have directly observed the amphoteric substitutionality and precipitation of $\mathrm{Ge}$ in high dose $\mathrm{Ge}$ implanted InP. The Ge-In and Ge-P bond lengths formed by $\mathrm{Ge}$ substitution in the $P$ and In sublattices are very similar to the theoretically predicted values. The solubility limit of $\mathrm{Ge}$ in InP is estimated to be $\sim 1.2 \times 10^{20} \mathrm{~cm}^{-3}$.

The EXAFS results on the AISb samples indicate that $\mathrm{Ge}$ preferentially occupies the $\mathrm{Al}$ site after annealing. Therefore we expect that Ge in AlSb act as a donor species. The high $\left[\mathrm{Ge}_{\mathrm{Al}}\right]:\left[\mathrm{Ge}_{\mathrm{Sb}}\right]$ ratio suggests that n-type doping with $\mathrm{Ge}$ can be very efficient. However, compensation and complexing of $\mathrm{Ge}_{\mathrm{Al}}$ donors by other implantation related defects, such as vacancies can reduce the n-type conductivity of AlSb:Ge.

\section{ACKNOWLEGMENT}

We would like to thank the staff at the Stanford Synchrotron Radiation Laboratory (SSRL) for their assistance in performing the EXAFS experiments. This work was supported in part by the Laboratory Directed Research and Development Program and by the Director, Office of Energy Research, Office of Basic Energy Sciences, Materials Science Division of the U. S. Department of Energy under Contract No. DE-AC03-76SF00098. SSRL is supported by the Office of Basic Energy Sciences of the US Department of Energy.

\section{REFERENCES}

1. S. J. Pearton, W. S. Hobson, and C. R. Abernathy, Mat. Res. Symp. Proc. 147, 261 (1989).

2 J. P. Donnelly and G. A. Ferrante, Solid. State Electron. 23, 1151 (1980).

3 P. Kringhoj, J. Appl. Phys. 7 1, 1748 (1992).

4 Kin Man Yu, A. J. Moll, W. Walukiewicz, N. Derhacobian, and C. Rossington, Appl. Phys. Lett. 6 4, 1643 (1994).

5. S. J. Pearton, A. R. Von Neida, J. M. Brown, K. T. Short, L. J. Oster, and U. K. Chakrabarti, J. Appl. Phys. 64, 629 (1988).

6. J. A. Van Vechten and J. C. Philips, Phys. Rev B 2, 2160 (1970). 
Proposal 2200Mp

\title{
KINETIC STUDIES OF OMVPE GROWTH USING IN SITU X-RAY SCATTERING
}

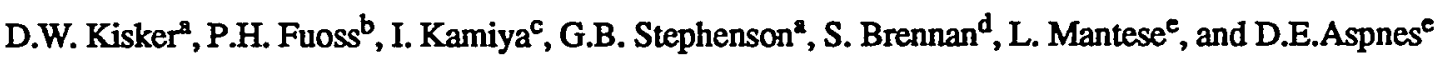 \\ ${ }^{2}$ IBM Research Division, Yorktown Heights, NY 10598 \\ bAT\&T Bell Laboratories, Murray Hill, NJ 07974 \\ 'JRDC and Imperial College, London, UK

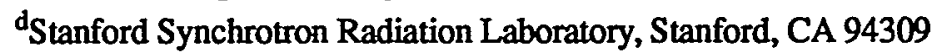 \\ 'North Carolina State University, Raleigh, NC 27695
}

Chemical vapor deposition (CVD) represents a complex interplay between chemistry and surface structure, making detailed understanding difficult to achieve. As a step towards this goal, we have performed the first simultaneous measurement of surface chemistry and atomic order using reflectance difference spectroscopy ${ }^{1}$ (RDS) and grazing-incidence $x$-ray scattering ${ }^{2}$ (GIXS), respectively, during homoepitaxial growth of GaAs(001) surface.

The specific growth process we have studied is organometallic vapor phase epitaxy (OMVPE). During OMVPE growth, reactant gases are introduced into the reactor, flow past a heated substrate, are decomposed and growth occurs. To grow GaAs, we have used tertiarybutylarsine (TBAs) as the arsenic source and either trimethylgallium (TMG) or triethylgallium (TEG) as the gallium source. While this growth process is conceptually simple, little is known about where the reactants decompose, what the surface structures are before and during growth, and how growth actually proceeds. Our research at SSRL is aimed at answering questions such as those.

The principle $t$ ool we have used to study these reactions is GIXS. Since OMVPE occurs at relatively high total pressure $(\approx 70 \mathrm{Torr})$ and the atmosphere in the reactor is both at high temperature and very reactive; normal surface science probes cannot be used. However, GIXS can be used to non-invasively probe the surface structures present before, during and after growth. Previously, we have shown that one can do time-resolved $x$-ray measurements and determine growth modes, growth rates and surface order. ${ }^{3}$

While GIXS is extremely powerful, it has two major shortcomings for this work. First, GIXS cannot directly measure the electronic structure of the surface and, second, the techniques and approaches cannot be moved to a factory because they rely on the very bright synchro- tron $x$-ray sources. To solve these problems, we have been investigating other, primarily optical, approaches to in situ monitoring and control. A promising technique is reflectance difference spectroscopy (RDS). RDS is an optical (usually 1.5-5.5 eV) spectroscopy where the difference in reflectivity along the principle directions of the substrate are measured. For example, for $\mathrm{GaAs}(001)$ surface, we measure the difference in the normal optical reflectivity with the polarization vector along the [1110] and [110] directions. Since these two reflectivities are identical in the bulk, the difference arises primarily from surface anisotropies. While these differences are measurable, they are also small, $\Delta r / r \approx 0.003$. Thus, the specialized equipment shown in Figure 1 has been developed to measure these small differences. ${ }^{4}$

The RDS spectrometer shown in Figure 1 was constructed at North Carolina State University. It uses the photoelastic-modulator approach, where the incident light is modulated at a $50 \mathrm{kHz}$ rate to produce first and second harmonic components that are proportional to the imaginary and real parts, respectively, of the relative anisotropy $\Delta r / r=2\left(r_{110}-r_{110}\right)\left(r_{110}+r_{110}\right)$ or $\Delta r / r=\Delta r / r+i \Delta \theta$. Here, $r_{110}$ and $r_{110}$ are the complex reflectances for light linearly polarized along the $[\overline{1} 10]$ and $[110]$, respectively. With phase sensitive detection at a time constant of 0.1 seconds, our sensitivity is of the order of 0.01 monolayer. The usable spectral range is 1.5 to $5.5 \mathrm{eV}$. Both $\Delta \mathrm{r} / \mathrm{r}$ and $\Delta \theta$ were recorded.

Also shown in Figure 1 are the $x$-ray diffractometer and the OMVPE reactor. The $x$-ray diffractometer is a Z-axis configuration which has been described elsewhere along with the gas handling system for the reactor. ${ }^{5}$ The OMVPE reactor is a new design which has been optimized for 1) fast gas switching, 2) higher 


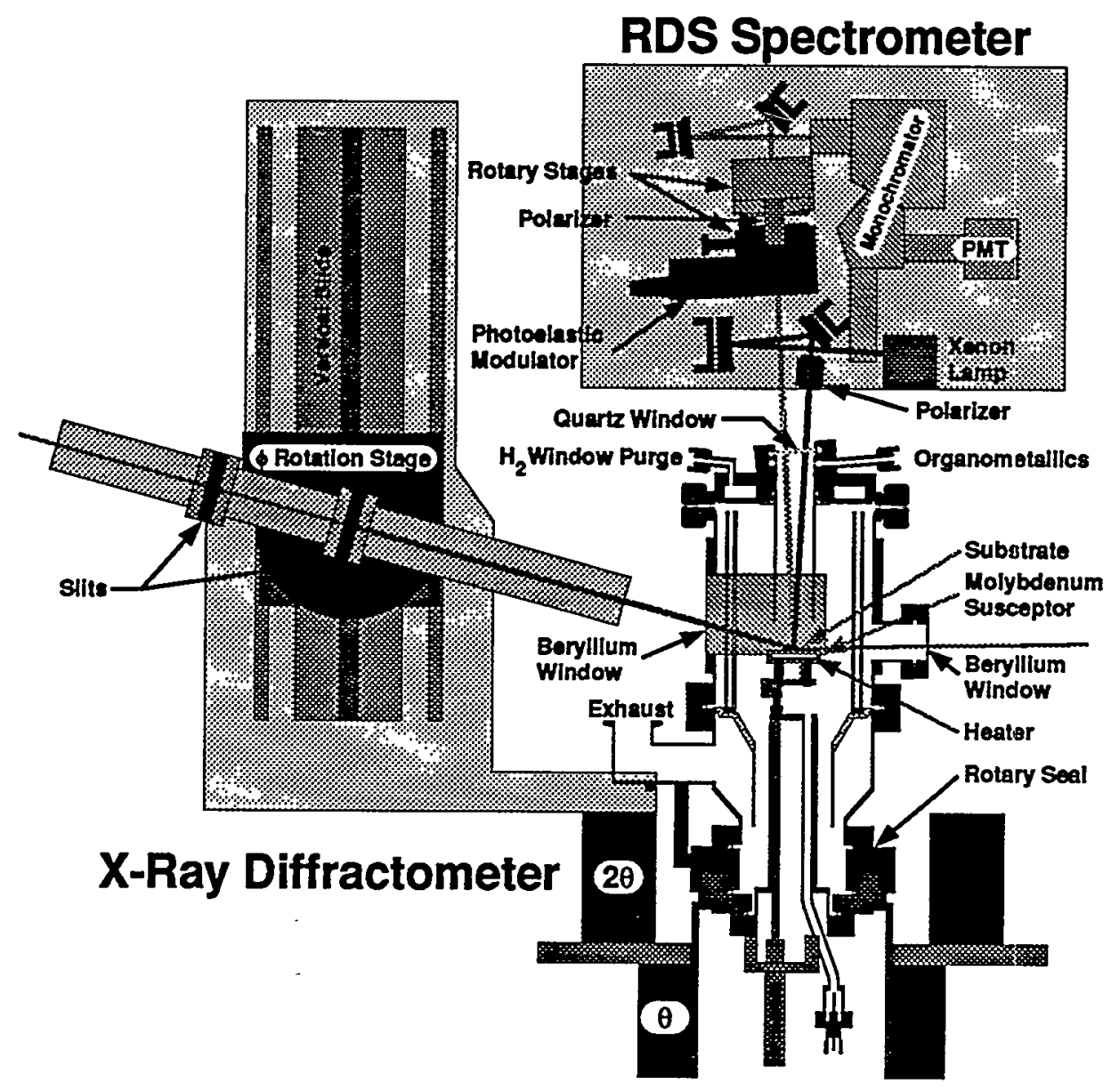

Figure 1: A schematic drawing of the new OMVPE system showing the redesigned reactor, the RDS spectrometer and the $\mathrm{x}$-ray diffractometer

temperature growth and 3) optical access. In addition, it uses a much simpler and cheaper Be window which is directly clamped to the stainless steel reactor housing and sealed with a viton o-ring. This reactor was constructed this year, primarily for the optical experiments, and has grown excellent GaAs films.

While RDS is a powerful approach to measuring surface optical properties, interpreting those signals is difficult. There is not a quantitative theory for the surface-induced optical anisotropy because it represents a small difference between large numbers. So the main approach to data interpretation in RDS analysis has been to establish a catalog of spectra which are referenced to structures determined, for example, by RHEED analysis in a UHV system. It is not obvious that a catalog of spectra determined under UHV conditions will be transferable to OMVPE conditions; testing this hypothesis was one of our major goals. In addition, while RDS oscillations have been observed during growth and have been interpreted as coming from layer-by-layer growth, direct evidence for that correlation is sparse. Thus, we were interested in testing whether RDS oscillations were correlated with the deposition of each atomic bilayer and whether the presence or absence of RDS oscillations meant that layer-by-layer growth either was or was not occurring, respectively.

Thus, using these complementary probes, we have performed a systematic investigation of OMVPE as a function of temperature, growth rate, V-III ratio, and precursor species. In the remainder of this report we will describe our main results including 1 ) the catalog of $R D$ signals from an OMVPE environment is similar but not identical to that from MBE, 2) RDS oscilla- 


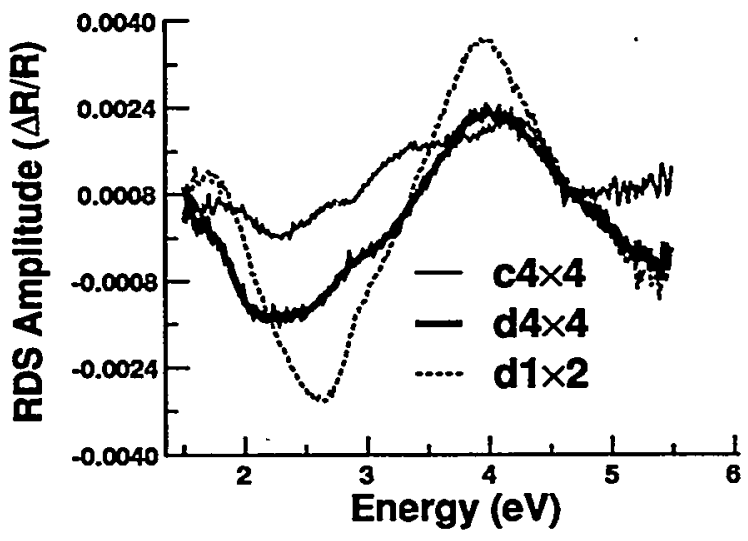

Figure 2: RDS spectra from several different $\mathrm{GaAs}$ (001) surface reconstructions.

tions are unambiguously connected to layer-by-layer growth but 3 ) the disappearance of RD oscillations with increasing temperature does not mean that stepflow growth has been achieved.

Figure 2 shows typical RDS spectra obtained from our OMVPE reactor before growth is started. The analogy between these patterns and those found in UHV with arsenic stabilization is very good. However, we find that these patterns do not uniquely define a structure. For example, the curve label $c(4 \times 4)$ has been observed for both the $c(4 \times 4)$ reconstruction and a reconstruction which is has a scattering pattern similar to that of the $c(4 \times 4)$ but with a significant tetragonal distortion.

We've also demonstrated that RDS spectra are not par-

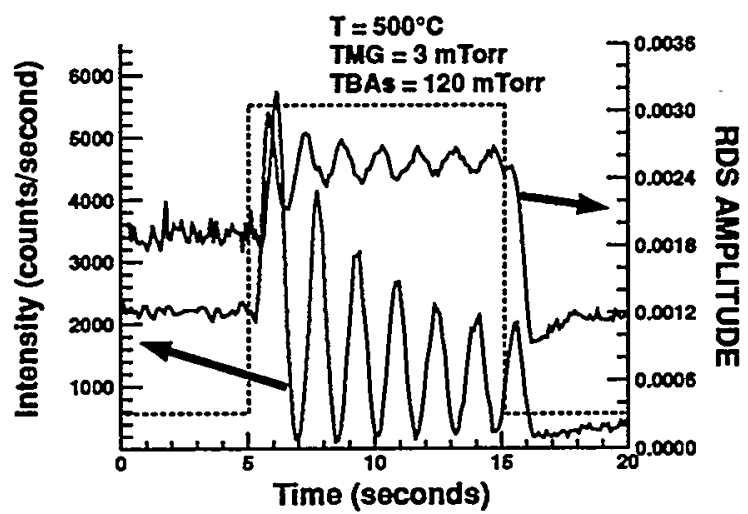

Figure 4: X-ray and RDS oscillations during layer-bylayer homoepitaxial growth of GaAs.

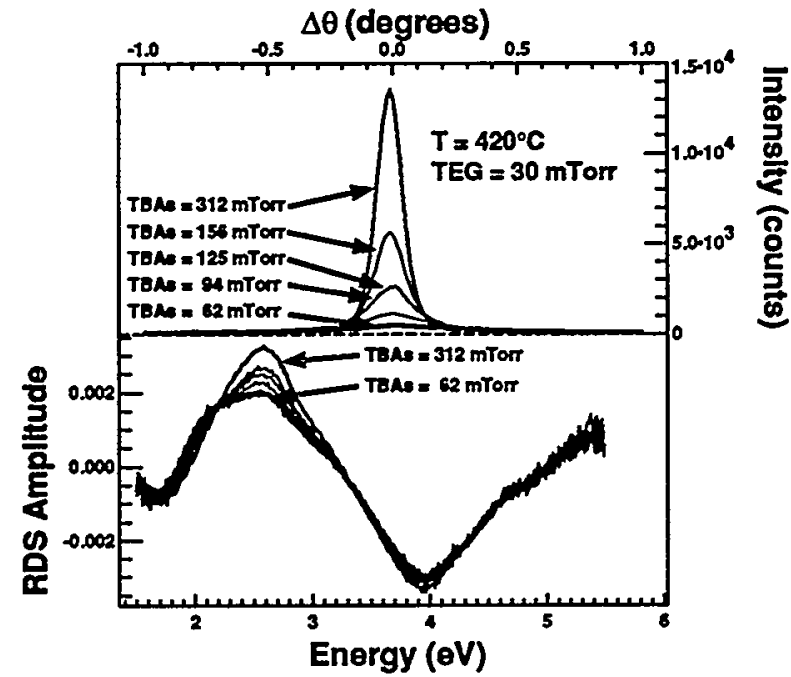

Figure 3: X-ray and RDS spectra from a GaAs (001) surface as an order-disorder transition is induced by changing the TBAs partial pressure.

ticularly sensitive to long range order. Figure 3 shows the RDS spectra from tetragonal phase along with one of the principle $\mathrm{x}$-ray diffraction peaks. As the TBAs partial pressure is changed, the tetragonal phase goes through an order-disorder transition while the RDS spectra changes by a moderate amount. This result would be expected from the relatively local nature of the RDS information.

The final important point is oscillations in the RDS

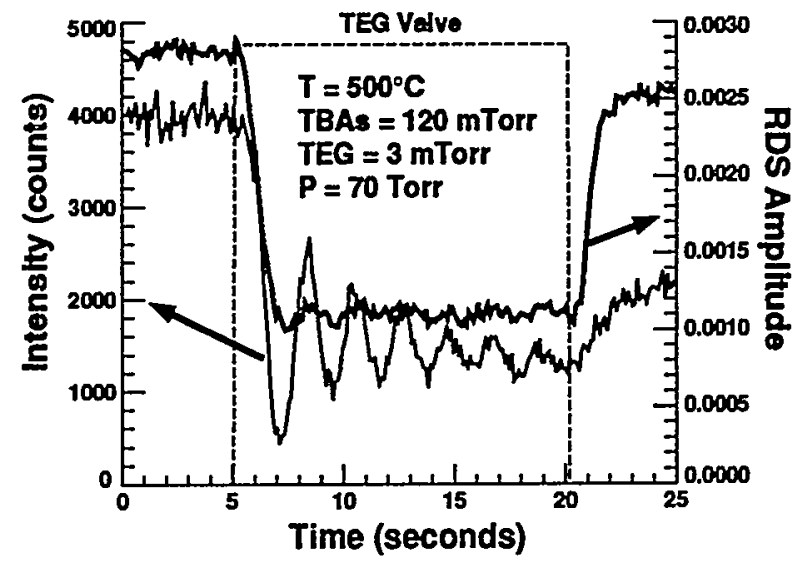

Figure 5: The $x$-ray oscillations show that layer-bylayer growth is occurring yet the RDS signal shows no oscillations 
signal during growth directly correspond to deposition of individual atomic layers. Figure 4 shows the (110) CTR intensity during layer-by-layer growth along with the $2.6 \mathrm{eV}$ RDS signal. Clearly there is a direct correspondence between the two signals. Since we know that each $\mathrm{x}$-ray oscillation corresponds to the deposition of one atomic bilayer, the RDS signal is also sensitive to layer-by-layer growth. However, as seen in Figure 5, the lack of RDS oscillations does not mean that layer-by-layer growth is not occurring. In this figure, there is a dip in the RDS amplitude but no significant oscillations while the $x$-ray signal clearly shows layer-by-layer deposition occurring.

In summary, we've developed equipment and methodology to perform simultaneous $x$-ray scattering and reflection-difference spectroscopy measurements. This capability has been applied to the homoepitaxial growth of GaAs using tertiarybutylarsine and either triethylgallium or trimethylgallium as the reactants. We find that RDS is sensitive to changes in the local order on the surface but is not sensitive to subtle changes in long-range order. We also find that periodic oscillations in the RDS signal can be correlated to a layer-by-layer growth mode.

\section{REFERENCES}

[1] I. Kamiya, DE. Aspnes, H. Tanaka, L.T. Florez, J.P. Harbison and R. Bhat, Phys. Rev. Lett., 68, 627(1992).

[2] P.H. Fuoss and S. Brennan, Annual Rev. of Mater. Sci., 20, 365(1990) and references therein.

[3] P.H. Fuoss, D.W. Kisker, FJ. Lamelas, G.B. Stephenson, P. Imperatori and S. Brennan, Phys. Rev. Lett., 69, 2791(1992).

[4] D.E. Aspnes, J.P. Harbison, A.A. Studna and L.T. Florez, J. Vac. Sci. Technol. A6, 1327(1988).

[5] S. Brennan, P.H. Fuoss, J.L. Kahn and D.W. Kisker, Nucl. Instrum. and Methods Phys. Res. Sect. A, 291, 86(1990). 
Proposal 2208M

\title{
MEASUREMENT OF CHEMICAL AND STRUCTURAL ANISOTROPY IN AMORPHOUS Tb-Fe THIN FILMS
}

\author{
T.C. Hufnagel and B.M. Clemens \\ Department of Materials Science and Engineering \\ Stanford University, Stanford, CA 94305-2205 \\ S. Brennan \\ Stanford Synchrotron Radiation Laboratory \\ Bin 69, P.O. Box 4349, Stanford, CA 94309
}

\section{INTRODUCTION}

One of the most significant developments in information storage technology in the past five years has been the introduction of magneto-optic media and drives for high density, erasable storage of information. The current generation of magneto-optic media is based on amorphous rare earth-transition metal alloy thin films. These alloys have a number of properties which make them ideal for magneto-optic recording applications, including a large perpendicular magnetic anisotropy, large Kerr rotation, low noise, and high coercivity at room temperature.

Although it has been known for more than twenty years that some amorphous rare earthtransition metal thin film materials (especially $\mathrm{Tb}-\mathrm{Fe}$ and $\mathrm{Gd}-\mathrm{Co}$ ) display perpendicular magnetic anisotropy, the origins of this anisotropy are still the subject of debate. A variety of theoretical explanations have been proposed, almost all of which postulate the existence of some corresponding structural anisotropy (even in the case of Tb-based alloys, in which single ion anisotropy can be important ${ }^{3-3}$. Until recently, however, there had been no experimental observations of such an anisotropy.

Recently, two groups have reported observations of structural anisotropy in rare earth-transition metal thin films. The first report of a structural anisotropy was from a Tb-Fe-Co thin film, where the investigators used symmetric reflection and grazing-incidence $x$-ray scattering to obtain interference functions for the out-of-plane and inplane directions, respectively ${ }^{4}$. They inferred from a shift in the positions of the first two interference peaks the presence of bond orientation anisotropy (BOA) in their sample. This type of anisotropy is characterized by a contraction of bonds in the plane of the film, and a lengthening of bonds in the out-of-plane direction. The anisotropy is anelastic, that is, it persists even after the removal of residual stresses. It has been postulated that BOA can arise due to compressive stresses and local heating which occur during sputter deposition. It is important to note that $\mathrm{BOA}$ is a purely structural anisotropy; no chemical short-range ordering is involved.

A second group employed polarization dependent extended $x$-ray absorption fine structure (EXAFS) to examine $\mathrm{Tb}-\mathrm{Fe}$ thin films ${ }^{5}$. By modeling the data with a computer simulation of the EXAFS, they reported a preference for unlike nearneighbor pairs in a direction perpendicular to the film plane. This type of anisotropy, which involves a chemical short-range ordering, is known as pair ordering anisotropy (POA). In contrast to the $\mathrm{x}$-ray scattering results of the first group, they reported no change in the distance of the first near-neighbor shell between the in-plane and out-of-plane directions.

The present experiment has been undertaken in an attempt to clarify the situation, and determine whether a structural or chemical anisotropy (or both) underlies the magnetic anisotropy in amorphous $\mathrm{Tb}-\mathrm{Fe}$ thin films. We made $\mathrm{X}$-ray scattering measurements in both symmetric reflection and symmetric transmission modes at several $x$-ray energies to determine both the outof-plane and in-plane structure of these materials, including the presence of chemical ordering (if any).

\section{EXPERIMENT}

One micron thick $\mathrm{Tb}_{.25} \mathrm{Fe}_{.75}$ films were deposited by $\mathrm{DC}$ magnetron cosputtering from elemental targets. The substrates were $12 \mathrm{~mm} \mathrm{x} 3 \mathrm{~mm} \mathrm{x}$ $3000 \AA$ A thick amorphous SiN films, prepared by depositing a $\mathrm{SiN}$ film via LPCVD onto a $\mathrm{Si}$ wafer, followed by patterning and etching the $\mathrm{Si}$ to produce SiN 'windows'. These substrates are ideal for $\mathrm{x}$-ray scattering measurements on amorphous thin films, because they contribute very little to the overall scattering. 
Scattering data were collected on beamline 7-2 in both symmetric transmission (to obtain in-plane structural information) and symmetric reflection (for out-of-plane information) modes. Several different $x$-ray energies were chosen for different experiments. The beamline was operated in focused mode for data collection at 10-12 keV; the high intensity of this beamline allows for rapid collection of data of high statistical quality in this energy range. Additional scans were taken near the $\mathrm{Fe}-\mathrm{K}$ and $\mathrm{Tb}-\mathrm{L}_{\mathrm{III}}$ absorption edges for anomalous dispersion scattering experiments, to allow determination of chemical short-range ordering. The beamline was also operated in unfocused mode at $21.5 \mathrm{keV}$ and $30 \mathrm{keV}$ to allow data to be taken at larger magnitudes of the scattering vector, which is important both for normalizing the scattering data and for improving the resolution of the nearest-neighbor shell (due to the reduction of termination effects in the Fourier analysis).

The magnetic anisotropy of the samples was measured with a torque magnetometer. The as-deposited samples showed a large perpendicular magnetic anisotropy energy of $7 \times 10^{6} \mathrm{erg} / \mathrm{cc}$. After annealing for one hour at 200 degrees Celsius, the magnetic anisotropy energy dropped to $2 \times 10^{6} \mathrm{erg} / \mathrm{cc}$. The saturation magnetization (measured by vibrating-sample magnetometer) did not change upon annealing.

\section{RESULTS}

Reduced radial distribution functions (RRDFs) for both the in-plane and out-of-plane directions for an as-deposited film are shown in Figure 1. The presence of a structural anisotropy, although small, is clearly shown by the differences in the RRDFs in the two directions. The coordination number is slightly larger (about one-half average atom) in the in-plane direction, while there is a slight shift of the in-plane nearest neighbors to larger distances (by $0.02 \AA$ ). The approximate distances of $\mathrm{Fe}-\mathrm{Fe}, \mathrm{Fe}-\mathrm{Tb}$, and $\mathrm{Tb}-\mathrm{Tb}$ near-neighbors (based on metallic radii) are shown. It is apparent that there is a slight preference for $\mathrm{Fe}-\mathrm{Fe}$ and $\mathrm{Tb}-\mathrm{Tb}$ near-neighbors in-plane, and for $\mathrm{Fe}$-Tb pairs out-of-plane.

After annealing, the magnetic anisotropy is reduced and we see a corresponding reduction in the structural anisotropy. Figure 2 shows RRDFs for the same film after annealing. Qualitatively, we see that the anisotropy is reduced; the near-neighbor peaks are now much more similar in size and position. The coordination number of the first near-neighbor shell is now the same in the two directions, although the spacing is still larger in the in-plane direction.

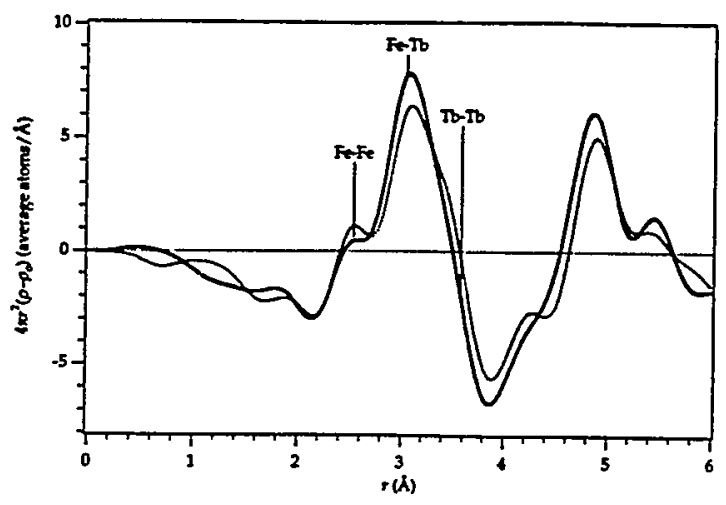

Figure 1. In-plane (dashed line) and out-of-plane (solid line) RRDFs for an amorphous Tb-Fe film as-deposited.

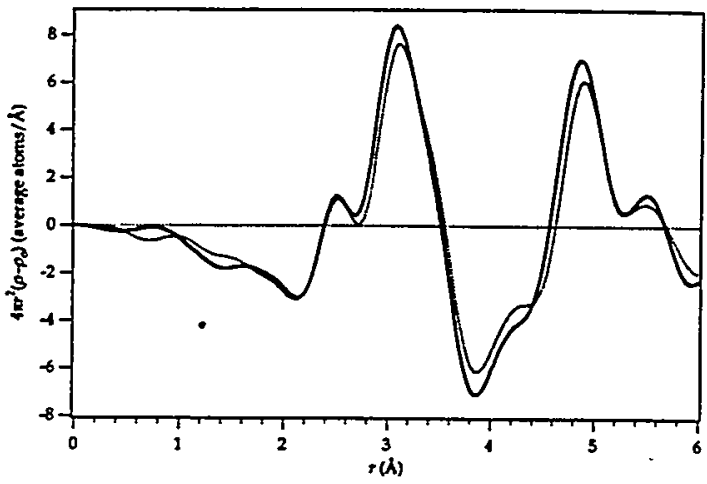

Figure 2. In-plane and out-of-plane RRDFs for an amorphous $\mathrm{Tb}-\mathrm{Fe}$ film after annealing.

The effects of annealing can be seen more clearly by considering the in-plane and out-of-plane directions separately. Figure 3 shows the RRDFs for the out-of-plane direction before and after annealing. The peaks in the RRDF are sharper, indicating a more ordered structure. The coordination number of the first shell increases by about one-half average atom (primarily $\mathrm{Fe}-\mathrm{Fe}$ and $\mathrm{Fe}-\mathrm{Tb}$ pairs) with annealing, but there is no change in the near-neighbor spacing.

In the in-plane direction (Figure 4), the changes are somewhat different. There is no change in the overall coordination number or near-neighbor spacing, but there is a definite increase in the 


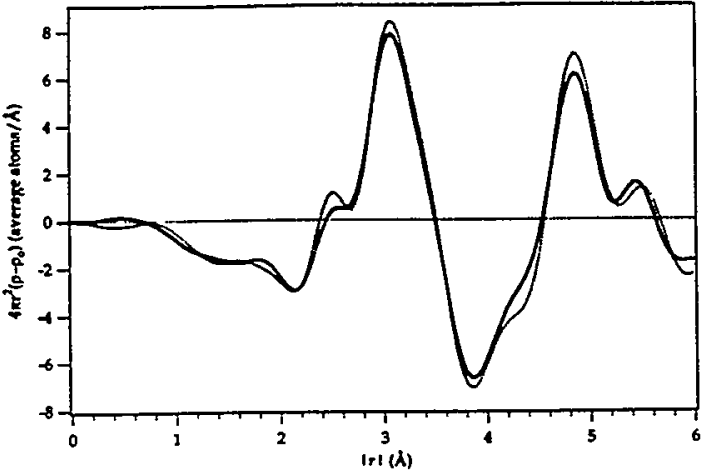

Figure 3. Out-of-plane RRDFs for an amorphous $\mathrm{Tb}-\mathrm{Fe}$ film before (solid line) and after (dashed line) annealing.

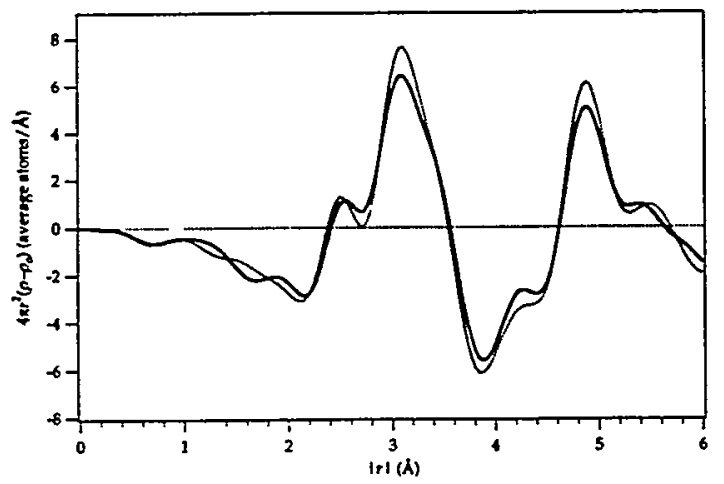

Figure 4. In-plane RRDFs for an amorphous Tb-Fe film before (solid line) and after (dashed line) annealing.

number of $\mathrm{Fe}-\mathrm{Tb}$ pair correlations, with a corresponding decrease in the number of $\mathrm{Fe}-\mathrm{Fe}$ pairs.

Our results indicate that anisotropic pair-ordering does occur in amorphous Fe-Tb thin films, with a preference for Fe-Tb pairs to align in the out-of-plane direction. The effect of annealing is to increase the chemical ordering by increasing the overall number of $\mathrm{Fe}-\mathrm{Tb}$ near-neighbor pairs. This increase is larger in the in-plane direction than the out-of-plane direction, thus reducing the net anisotropy in pair ordering.

\section{CONCLUSIONS}

We have employed $x$-ray scattering in transmission and reflection modes to observe a structural anisotropy in amorphous $\mathrm{Tb}-\mathrm{Fe}$ thin films. We see a preference for unlike near-neighbor pairs to align in the out-of--plane direction. There is also a slightly larger coordination number in the plane of the film. Upon annealing, the structural anisotropy of the films is reduced, primarily by an increase in the number of unlike near-neighbors in the in-plane direction (while the number of unlike near-neighbors in-plane decreases). There is also an increase in the overall coordination number in the out-of-plane direction. The net effect is to make the distribution of Fe-Tb near-neighbor pairs more isotropic. This structural change is responsible for the reduction in magnetic anisotropy upon annealing.

\section{ACKNOWLEDGEMENTS}

This research is supported by Kobe Steel USA through their Applied Electronics Center. The authors gratefully acknowledge useful discussions with A. Bienenstock, V. G. Harris and E. Marinero; and are indebted to R. Serimaa for MATLAB macros used in the data analysis.

\section{REFERENCES}

1. T. Mizoguchi and C.S. Cargill. J. Appl. Phys. 50, 3570 (1979).

2. H. Fu, M. Mansuripur, and P. Meystre. Phys. Rev. Lett. 66, 1086 (1991).

3. F. Hellman and E.M. Gyorgy. Phys. Rev. Lett. 68, 1391 (1992).

4. X. Yan, M. Hirscher, T. Egami, and E. Marinero. Phys. Rev. B 43, 9300 (1991).

5. V.G. Harris, K.D. Aylesworth, B.N. Das, W.T. Elam, and N.C. Koon. Phys. Rev. Lett. 69, 1939 (1992). 


\title{
Grazing-Incidence XAS Study of Cation Chemisorption at Single-Crystal Oxide Interfaces
}

\author{
STEVEN N. TOWLE*,**, JOHN R. BARGAR ${ }^{* *}$, GORDON E. BROWN, JR ${ }^{* *}$, GEORGE A. PARKS ${ }^{* *}$, AND \\ TROY W. BARBEE, JR.*** \\ "Department of Materials Science and Engineering, Stanford University, Stanford, CA 94305 \\ *Department of Geological and Environmental Science, Stanford University, Stanford, CA 94305-2115 \\ Lawrence Livermore National Laboratory, Livermore CA
}

This work to be published in Material Research Society Proceedings Volume 357

\section{INTRODUCTION}

The structural chemistry of inorganic adsorption complexes at oxide-water interfaces is a crucial issue in a number of processes of scientific and technological interest, such as geochemical modeling 1 and catalyst preparation ${ }^{2}$. However, little work has been done to define the geometry of complexes and adsorption sites of metal ions at these interfaces, mostly because few methods can provide this information. During the 1994 SSRL run, we collected data which show that $x-$ ray absorption fine structure (XAFS) spectroscopy can be of considerable aid in investigating such complexes on single crystal surfaces, and that in favorable cases a unique adsorption site can be determined.

\section{EXPERIMENTAL}

The substrates used in this study consisted of (0001) and (0112) $\alpha-\mathrm{Al}_{2} \mathrm{O}_{3}$ wafers, $(110)$ and $(001)$ $\mathrm{TiO}_{2}$ (rutile) wafers, and ultra-polished $\mathrm{SiO}_{2}$ glass blocks. These substrates were cleaned and thoroughly characterized (by XPS, profilometry, and AFM) before being equilibrated at $20^{\circ} \mathrm{C}$ with solutions of $\mathrm{Co}$ (II) or $\mathrm{Pb}$ (II) at selected concentrations and $\mathrm{pH}$ values. The samples were dried by blowing down the meniscus with a jet of clean $\mathrm{N}_{2}$ as they were removed from the solution - a method that is, in our view, likely to minimize desorption of weakly bound complexes. The XPS data and the edge position in the XAFS spectra confirmed that the $\mathrm{Co}$ (II) and $\mathrm{Pb}$ (II) were neither oxidized nor reduced.

Data were collected either in situ under a thin water film (see, for example, Melroy et al. ${ }^{3}$ for details of this experimental geometry), or ex situ. The in situ experiments were conducted as follows: the substrates were pre-equilibrated with the solution, as described above, then dried and loaded into the solution cell, after which the cell was filled with the reserved supernatant and reequilibrated before withdrawing most of the solution in order to collect the data. Since the Co Kedge $x$-rays (7.7-8.3 keV) are less energetic than those of the $\mathrm{Pb}$ LIII-edge(13-13.5 keV), they are less penetrating and also the the critical angle for total external reflection is greater. As a consequence, it was impossible to get the water film thin enough for a significant fraction of the Co $x$-rays to go through it, so these samples were collected ex situ. The ex situ experiments were conducted both in air and in $\mathrm{H}_{2} \mathrm{O}$-saturated $\mathrm{N}_{2}$. This difference did not affect the XAFS data.
All data were collected at SSRL during the 1994 run, on BL 6-2 from April 27-May 10 and on BL 4-3 from May 17-31. Data were collected in fluorescence mode using a Canberra 13-element $\mathrm{Ge}$ energy dispersive detector. The energy dispersion allowed us to eliminate Bragg scattered peaks from the substrate; also, in cases where Bragg scattering did affect the data, the unaffected channels could still be analyzed. X-ray optics on beam line 6-2 consisted of a toroidally bent $\mathrm{Pt}$ focusing mirror and a double crystal Si (111) monochromator, along with associated slits and windows. Unfortunately, the data we collected on this beamline were marred by mechanical noise from the monochromator. At about 3\%, this source of noise overwhelmed the statistical noise level and in fact resulted in the data from the BL 6-2 run being scarcely superior to that from BL 4-3. On BL 4-3, a Si (220) monochromator and a downstream flat $\mathrm{Pt}$ mirror were used. The incident angle on the sample ranged from $0.10^{\circ}$ to $0.20^{\circ}$ (the critical angle for total external reflection for $8 \mathrm{keV} x$-rays from $\alpha-\mathrm{Al}_{2} \mathrm{O}_{3}$ is $0.22^{\circ}$, for $13.5 \mathrm{keV} x$-rays is $0.13^{\circ}$ ), and was set by using it as parameter to optimize the signal-to-noise ratio. The energy resolution on BL $6-2$ and BL $4-3$ at $8 \mathrm{keV}$ is about $4 \mathrm{eV}$ and $2 \mathrm{eV}$, respectively.

For the Co samples, separate spectra were collected with the sample in various orientations with respect to the electric field polarization vector of the synchrotron beam. For the $\alpha-\mathrm{Al}_{2} \mathrm{O}_{3}(0112)$ samples, the surface net has rectangular symmetry, so there are two distinguishable directions with the polarization parallel to the surface. Since the surface oxygen atoms form zigzag rows (see Figure $2 b$ ), the spectra were collected with the polarization parallel to these rows, perpendicular to them, and normal to the surface. The $\alpha-\mathrm{Al}_{2} \mathrm{O}_{3}$ (0001) face, on the other hand, has hexagonal symmetry; thus, the sensitivity of the polarization dependence is not adequate to distinguish different directions on this surface. Consequently, only two spectra were collected on this sample, with the polarization vector parallel to the surface and normal to it. Only one orientation was collected on each crystal for the $\mathrm{Pb}$ samples, since the angular dependence of the electric field polarization is much weaker for an $\mathrm{L}_{\mathrm{II}}$ edge $\mathrm{e}^{4}$.

Data were reduced using the EXAFSPAK programs ${ }^{5}$. The final fitting was done with the raw data, using theoretical phase and amplitude models generated with the FEFF 6 code ${ }^{6}$. 


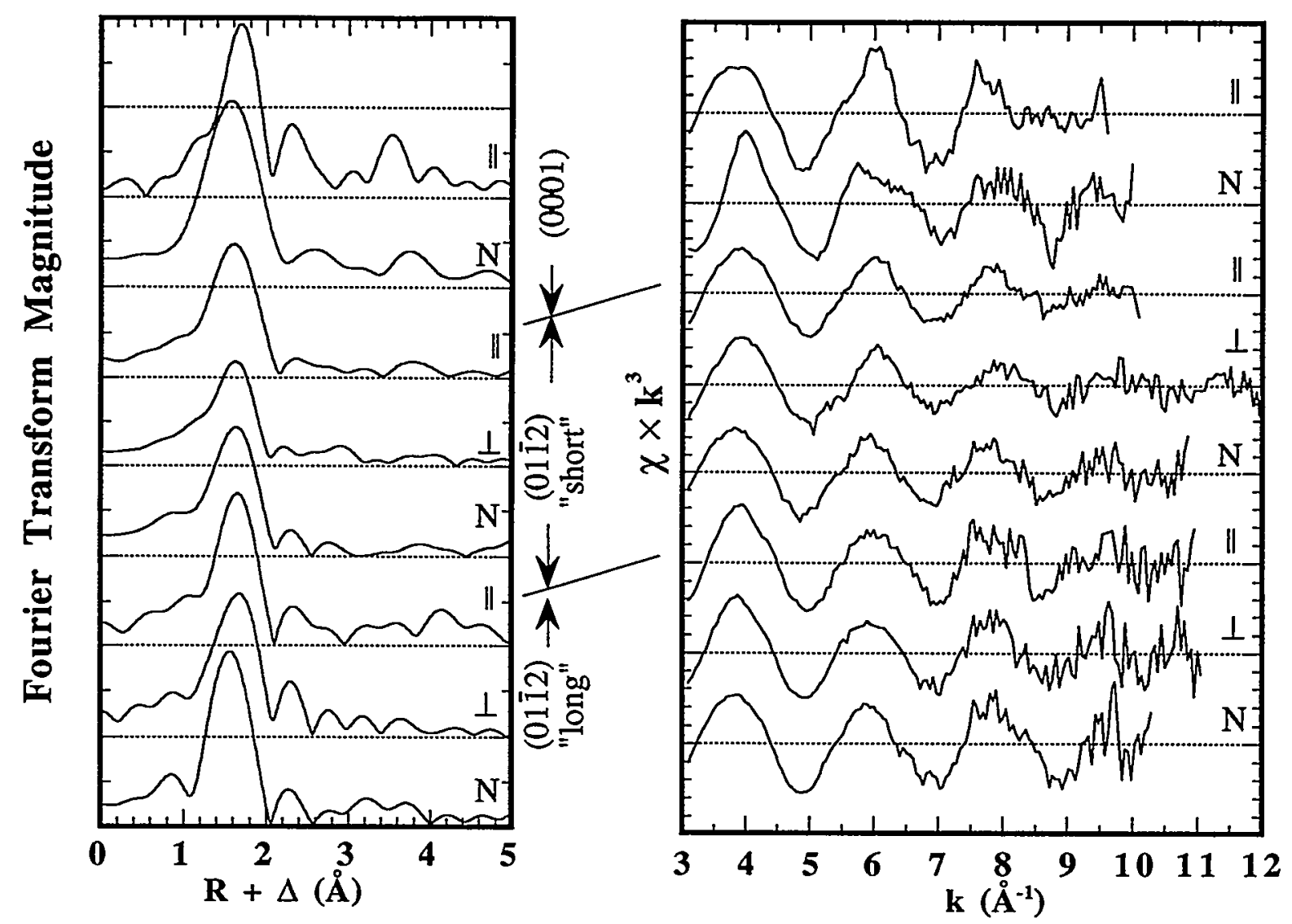

Figure 1. EXAFS spectra and Fourier transforms (FT). The top two spectra and FT"s are from the $\alpha-\mathrm{Al}_{2} \mathrm{O}_{3}$ (0001) sample, the next three from the (01ī2) sample where the solution had a short pre-equilibration, and bottom from the $\alpha-\mathrm{Al}_{2} \mathrm{O}_{3}(01 \overline{1} 2)$ sample with a long pre-equilibration. The FT's are not corrected for phase shift $(\Delta)$.

\section{Co(II) ADSORPTION RESULTS}

The background-subtracted, normalized, deglitched EXAFS spectra for the $\mathrm{Co} / \alpha-\mathrm{Al}_{2} \mathrm{O}_{3}$ samples, along with their Fourier transforms (FT's) (not corrected for phase shift), are presented in Figure 1. The ranges of data shown are the same as those used in the quantitative analysis. Results of least-squares fitting of the spectra are summarized in Table 1. In all fits, the significance of each of the fitted parameters was tested and found to be significant at the $99.5 \%$ confidence level using the student $t$ test. XPS results showed that surface coverages were $3.0 \mu \mathrm{mole} / \mathrm{m}^{2}$ on the $\alpha-\mathrm{Al}_{2} \mathrm{O}_{3}$ (0001) and (0112) "long" samples and $1.4 \mu \mathrm{mole} / \mathrm{m}^{2}$ on the $\alpha-\mathrm{Al}_{2} \mathrm{O}_{3}(0112)$ "short" sample.

The first neighbor shell is, in all cases, due to backscattering from a near perfect octahedron of oxygens. The measured number of neighbors is proportional to the cosine of the angle between the electric field vector and the axis linking the absorber and the backscatter axis ${ }^{4}$. Hence, a regular octahedron in any orienta-

\begin{tabular}{|c|c|c|c|c|}
\hline Sample & 1st shell & $\begin{array}{c}\text { edge-shared } \\
\text { 2nd neighbors }\end{array}$ & $\begin{array}{l}\text { comer-shared } \\
\text { 2nd neighbors }\end{array}$ & $\begin{array}{l}\text { more distant } \\
\text { shells }\end{array}$ \\
\hline$(0001)$ II & $5.60 @ 2.05 \AA(0.008)$ & $1.3 \mathrm{Al} @ 2.73 \AA$ & $7.6 \mathrm{Al} @ 4.09 \AA$ & \\
\hline$(0001) \mathrm{N}$ & $6.50 @ 2.08 \AA(0.008)$ & $1.8 \mathrm{Al} @ 2.89 \AA$ & 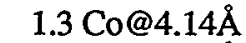 & \\
\hline (0112) "S" II & $5.60 @ 2.05 \AA(0.010)$ & & $0.4 \mathrm{Co@4.10 \AA}$ & \\
\hline (0112) "S" 1 & $5.30 @ 2.04 \AA(0.010)$ & & $2.0 \mathrm{Al} @ 3.36 \AA$ & \\
\hline (0112) "S"N & $5.50 @ 2.08 \AA(0.009)$ & & & \\
\hline (011̄2) "L" II & $6.10 @ 2.06 \AA(0.009)$ & $0.6 \mathrm{Al} @ 2.77 \AA$ & $3.8 \mathrm{Al} @ 4.14 \AA$ & 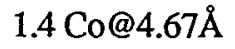 \\
\hline (0112) "L" & $5.90 @ 2.07 \AA(0.008)$ & $1.0 \mathrm{Al} @ 2.74 \AA$ & & \\
\hline (0112) "L" N & $5.30 @ 2.07 \AA(0.006)$ & $0.4 \mathrm{Al} @ 2.74 \AA$ & $\begin{array}{r}3.5 \mathrm{Al} @ 3.66 \AA \\
+1.0 \mathrm{Co} @ 4.10 \AA \\
\end{array}$ & \\
\hline
\end{tabular}




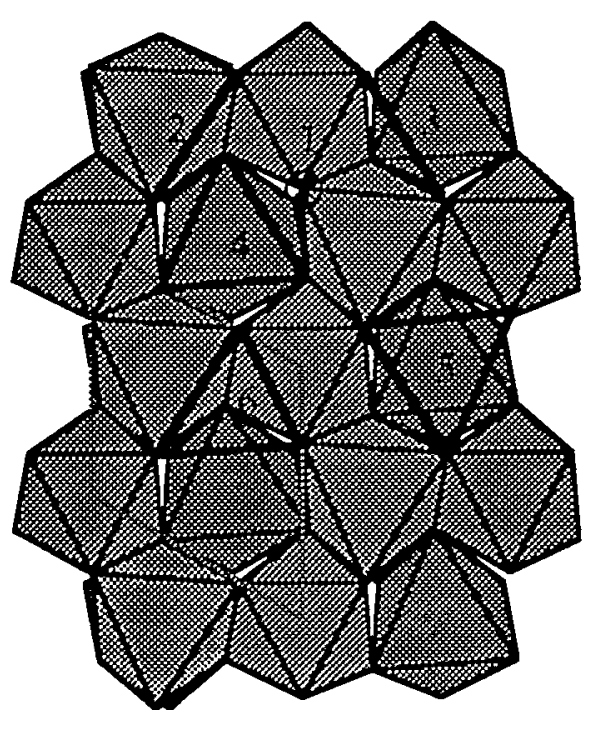

(a)

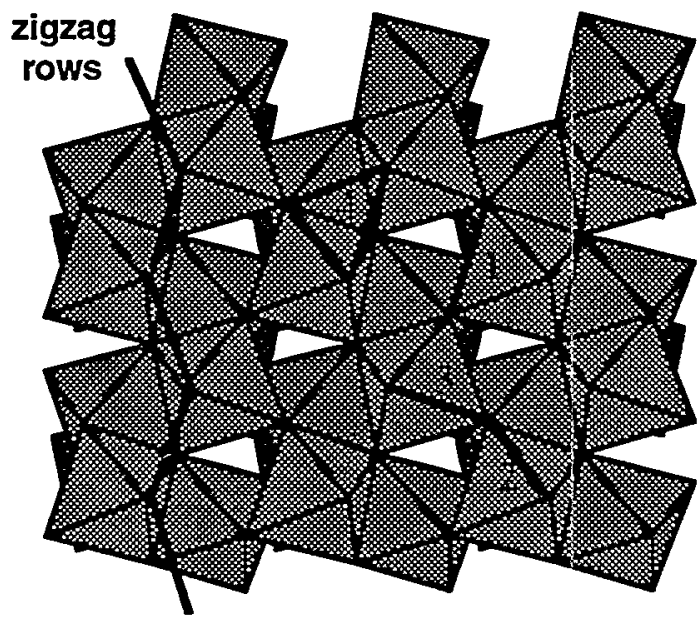

(b)

Figure 2. Structural drawings of the oxygen terminated (0001) (a) and (01ī2) (b) surfaces of $\alpha-\mathrm{Al}_{2} \mathrm{O}_{3}$. Possible adsorption sites are marked with a heavy line for polydentate sites, or with $\otimes$ for a monodentate site; the light line in (a) describes the surface unit cell; the heavier line in (b) shows the zigzag row of $\mathrm{O}$ atoms. The XAFS data indicate that Co adsorption occurs predominantly on site 6 on the (0001) surface and on site 4 on the (0112) surface.

tion yields 6 neighbors.

All second and higher shells are identified as being due $\mathrm{Al}$ or Co atoms. As the backscattering of $\mathrm{Co}$ and $\mathrm{Al}$ is almost exactly out of phase, it is virtually impossible to confuse the two, though in cases with overlapping shells there will be destructive interference with a consequent reduction in the fitted coordination number. This reason, plus the fact that the Debye-Waller factors of the higher shells are not known, causes uncertainty in the second neighbor numbers for the more distant shells. In all fits, the Debye-Waller factor for shells beyond the first was set to 0.004 , a value that works well for similar $\mathrm{Co}$ (II) compounds? ${ }^{7}$. However, the uncertainty in the second-neighbor numbers does not prevent identification of the sorption site, at least in favorable cases.

The raw data reveal that there are significant differences in the measured local structures between different crystallographic orientations and also among different polarization directions on the same face. From this observation alone, it is clear that Co must be bound to the surface in a position that has characteristic structure, i.e., it is specifically adsorbed. More detailed conclusions, though, require that the relevant surfaces be examined site by site, comparing the second neighbor $\mathrm{Al}$ distances from different possible surface complex geometries to those observed in the spectra. In all analyses of adsorption sites, the surfaces have been considered to be oxygen terminated. This termination is not expected for a vacuum-cleaved crystal; however, under water it seems likely that the oxygen lattice sites would be occupied by either hydroxyl ions or adsorbed water molecules.
A problem that complicates analysis of adsorption sites is the possibility that surfaces may relax, i.e., that atoms in the vicinity of a surface may not lie on their bulk lattice positions. This effect is likely to be particularly strong in $\alpha-\mathrm{Al}_{2} \mathrm{O}_{3}$ because the structure consists of linked pairs of highly disorted face-shared octahedra. Since the distortion is caused by cationcation repulsion, missing cations at the $\alpha-\mathrm{Al}_{2} \mathrm{O}_{3}$ surface will likely reduce the disortion. In most cases, though, departures from bulk positions need to be large to affect the analysis of adsorption sites.

The $\alpha-\mathrm{Al}_{2} \mathrm{O}_{3}(0001)$ surface consists of interlinked six-member rings of edge-sharing $\mathrm{AlO}_{6}$ octahedra, each with a triangular face oriented upward, as illustrated in Figure 2a. All of the surface oxygens are equivalent, thus there is only one monodentate site (site 1). Of the three bidentate sites, two are likely to be quite similar due to surface relaxation, hence only edge-sharing (site 2) and bridging (site 3 ) sites are considered. There are three possible tridentate sites: sharing a face (site 4), above an octahedral interstice (site 5), and above a tetrahedral interstice (site 6). Two other sites were also considered, though they are not shown: within the octahedral cavity (site 7) and within the lattice at a bulk Al position (site 8).

Sites 7 and 8 and the tridentate face-shared position (site 4) can be rejected because they require that in the normal orientation an Al backscatterer be present at the characteristic distance for face-shared octahedra, $\approx 2.5-2.6 \AA$. No peak is seen at this distance in the FT, despite the fact that it would have to be quite large since the $\mathrm{Al}$ atom would be almost exactly lined up with the polarization vector. The closest second- 
neighbor $\mathrm{Al}$ distance observed, $\approx 2.75 \AA$, requires edgesharing. For this reason all the sites that only have corner-shared octahedra $(1,3$, and 5) can be eliminated. The sorption geometry can be further constrained by noting the large $A l$ peak at $4.1 \AA$ in Figure 1 (the peak at $3.6 \AA$ before phase shift correction in the FT for (0001) parallel orientation). Since the Co-O distance is $\approx 2.1 \AA$ and the longest $\mathrm{Al}-\mathrm{O}$ distance is $\approx 2.0 \AA$, this peak must be due to a linear arrangement of $\mathrm{Co}, \mathrm{O}$, and $\mathrm{Al}$ atoms. Such a linear arrangement would also explain why the peak is so large, since linear multiple scattering is known to greatly increase the amplitude of backscattering ${ }^{13}$. Of all the sites considered, only the tridentate, edge-shared site (site 6) has such an arrangement. Thus, this site must predominate in the chemisorption of $\mathrm{Co}$ (II) on $\alpha-\mathrm{Al}_{2} \mathrm{O}_{3}(0001)$.

The spectra from the "long" sample allow a similar site analysis to be done on the $\alpha-\mathrm{Al}_{2} \mathrm{O}_{3}(01 \overline{1} 2)$ surface. In these spectra the most diagnostic feature is the $\mathrm{Al}$ peak at $2.77 \AA(2.3 \AA$ in the $F T$ uncorrected for phase shift, in figure 1) which is seen in all of the orientations. As before, a shell at this distance requires that the Co be linked to the surface in an edge-shared manner. Hence, in this analysis, only the edge-shared sites will be considered. One bidentate site is at the bottom of the zigzag trough (site 1). There are, in addition, two sites that stretch from the trough to ridge (sites 2 and 3). The only other edge-shared site is tridentate, with two ridgetop oxygens and one trough-bottom oxygen (site 4). The two other diagnostic Al peaks, at $4.14 \AA$ in the parallel-to-the-rows spectrum and at $3.66 \AA$ in the normal spectrum, are much weaker and hence allow less certain conclusions, though they are still significant at the $99.5 \%$ confidence level. These peaks correspond to $\mathrm{Al}$ distances for the tridentate site, though in this case considerable surface relaxation $(\approx 0.2 \AA)$ has to be invoked to make them match exactly. Another argument in favor of this site is its similarity to the Co sorption site on (0001) - both lie above tetrahedral interstices in the $\alpha-\mathrm{Al}_{2} \mathrm{O}_{3}$ lattice. The exact site is not as definite on (0112) as on (0001).

Spectra were also collected in three different orientations for $\mathrm{Co} / \mathrm{TiO}_{2}(110)$ and two on $\mathrm{TiO}_{2}(001)$. As with $\alpha-\mathrm{Al}_{2} \mathrm{O}_{3}(0001)$, the in-plane symmetry of the $\mathrm{TiO}_{2}(001)$ surface is too high to distinguish separate orientations parallel to the surface. These data are still being analyzed.

Also during the 1994 run, we started a project to investigate Co(II) sorption on fused silica. This substrate material lacks some of the advantages of single crystal substrates, such as being able to investigate different crystallographic orientations. However, it has the advantages of not producing Bragg diffraction peaks, and being available in ultrapolished form. The exceptionally low roughness improves the signal-tonoise and the surface sensitivity.

A dry sample of Co(II) adsorbed on a polished fused silica surface $(4 \times 10 \mathrm{~cm})$ at a coverage of approximately $0.75 \mathrm{~mole} / \mathrm{m}^{2}$ was analyzed in two orientations. Twenty five spectra were collected with the electric vector perpendicular to the sample surface and eighteen spectra were collected with the electric vector horizontal to the sample surface. As predicted by macroscopic modeling of uptake curves, no secondnearest neighbors were observed in either of the orientations. The molecular model will be tested during the SSRL winter 1995 run under conditions where Co(II) clustering is predicted and Co nearest neighbors should be observed.

\section{Pb(II) ADSORPTION RESULTS}

Lead LIII-edge XAFS spectra were collected from dry samples of $\mathrm{Pb}$ (II) sorbed on $\alpha-\mathrm{Al}_{2} \mathrm{O}_{3}(0001)$ and (0112) single crystal wafers. The $\mathrm{Pb}(\mathrm{II}) / \alpha-\mathrm{Al}_{2} \mathrm{O}_{3}$ (0112) sample was also run in situ (i.e., under a film of aqueous solution). To our knowledge, this is the first in situ, glancing incidence XAFS experiment performed at the oxide-water interface. Spectra were noisy (due to monochromator vibrations on BL 6-2, or low flux on BL 4-3) but analyzable to $k \leq 8 \AA^{-1}$, which allowed the first coordination shell to be characterized.

Results of numerical analysis of these spectra are intriguing. The wet and dry (0112) samples have virtually identical lead environments: 3.5 oxygen first neighbors at $2.30 \pm 0.03 \AA$. This is similar to $\mathrm{Pb}$ (II) chemisorbed on high surface area powders of $\alpha-\mathrm{Al}_{2} \mathrm{O}_{3}$. In contrast, $\mathrm{Pb}$ (II) sorbed on the $(0001)$ orientation has a much longer $\mathrm{Pb}-\mathrm{O}$ bond ( $\mathrm{RPb}$ $\mathrm{O}=2.53 \AA$ ), which is similar to $R_{P b}-\mathrm{O}$ observed in aqueous solution. This strongly suggests that $\mathrm{Pb}(\mathrm{II})$ is adsorbed on the (0001) surface in an outer-sphere mode (i.e., $\mathrm{Pb}(\mathrm{II})$ is weakly sorbed via electrostatic attraction and/or hydrogen bonding), as opposed to chemisorption on (0112). Hence, the structure of the oxide surface has a very important role in determining the molecular geometry and mode of $\mathrm{Pb}(\mathrm{II})$ sorption. Also, the effect of drying the samples was shown to be unimportant.

We express our gratitude to Per Persson (Umea University, Sweden), to Hal Tompkins and the rest of the SSRL staff, and to the DOE, which supported this work through grant DE-FG03-93ER1437A000.

${ }^{1}$ G. Sposito, The Surface Chemistry of Soils (Oxford University Press, New York, 1984).

${ }^{2}$ B. C. Gates, Catalytic Chemistry (John Wiley \& Sons, New York, 1992).

${ }^{3}$ O.R. Melroy, M.G. Samant, G.L. Borges, and J.G. Gordon, II, Langmuir 4, 728-732 (1988).

${ }_{4}^{4}$ P.H. Citrin, Phys. Rev. B 31 (2), 700-721 (1985).

${ }^{5}$ G.N. George, EXAFSPAK (SSRL, Stanford, 1993).

$6_{\mathrm{J}}$. J. Rehr, J. Mustre de Leon, S. I. Zabinsky et al., J. Amer. Chem. Soc. 113, 5135-5140 (1991).

7 P.A. O'Day, J.J. Rehr, S.I. Zabinsky and G.E. Brown, Jr., J. Amer. Chem. Soc. 116, 2938-2948 (1994). 


\title{
Ti XAS of Crystalline Silicotitanates
}

\author{
Mari Lou Balmer and Nancy J Hess \\ Pacific Northwest Laboratory, Richland WA \\ and \\ Steven D Conradson \\ Los Alamos National Laboratory, Los Alamos NM
}

Crystalline Silicotitanates (CSTs) have been proposed as ion exchange materials for the removal of $\mathrm{Cs}$ and $\mathrm{Sr}$ from high level waste streams produced during the reprocessing of fuel rods. $\mathrm{Cs}$ and $\mathrm{Sr}$ are released from the loaded exchange materials by elution and incorporated into solid waste forms. Another strategy is to directly incorporate the $\mathrm{Cs}$ loaded ion exchange materials into a solid waste form, for example, borosilicate glass logs, by melting. Recent studies at PNL have demonstrated that a formulation of CST can be directly transformed to a crystalline material isostructural with pollucite $\left(\mathrm{CsAlSi}_{2} \mathrm{O}_{6}\right)$ at temperatures well below those required for formation of borosilicate glass. In addition, Ti-pollucite, $\mathrm{CsTiSi}_{2} \mathrm{O}_{6}$, has leach rates that lower than that for the borosilicate glass, generates lower volumes of high level waste, and due to the low processing temperatures, the Cs losses due to volatilization during processing are extremely low.

Ti XANES and EXAFS were performed to determine how $\mathrm{Ti}$ is incorporated into the pollucite structure. If $\mathrm{Ti}$ substitutes for $\mathrm{Al}^{3+}$, the Ti would occupy tetrahedral sites which is rare for $\mathrm{Ti}$, and $\mathrm{Ti}$ would have to be in the trivalent oxidation state which requires strongly reducing conditions during synthesis.

Figure 1 compares the Ti XANES region of Ti-pollucite to a titanium standard compound, anatase with $\mathrm{Ti}$ in octahedral coordination, and a commercial sodium silicotitanate zeolite, ETS-4 with Ti occupying a highly distorted octahedral site. Ti-pollucite exhibits a single pre-edge feature approximately $2 \mathrm{eV}$ lower than a similar feature on ETS-4. The amplitude of the this feature and its position relative to the $\mathrm{Ti} \mathrm{K}$-edge suggests either a distorted pentagonal or tetrahedral site. Figure 2 overlays the Fourier transforms of the EXAFS from $k$ $=2.5$ to $9.5 \AA^{-1}$. Comparison of the first shell amplitudes suggests that Ti-pollucite has fewer oxygen nearest neighbors than anatase ETS-4. Initial fitting results using FEFF6 indicate that Ti-pollucite is coordinated by 4 oxygens at $1.9 \AA$ whereas ETS- 4 has 5 oxygens at $1.96 \AA$ and anatase has 6 oxygens at $1.94 \AA$. A. multi-probe spectroscopic study is in progress to verify these results.

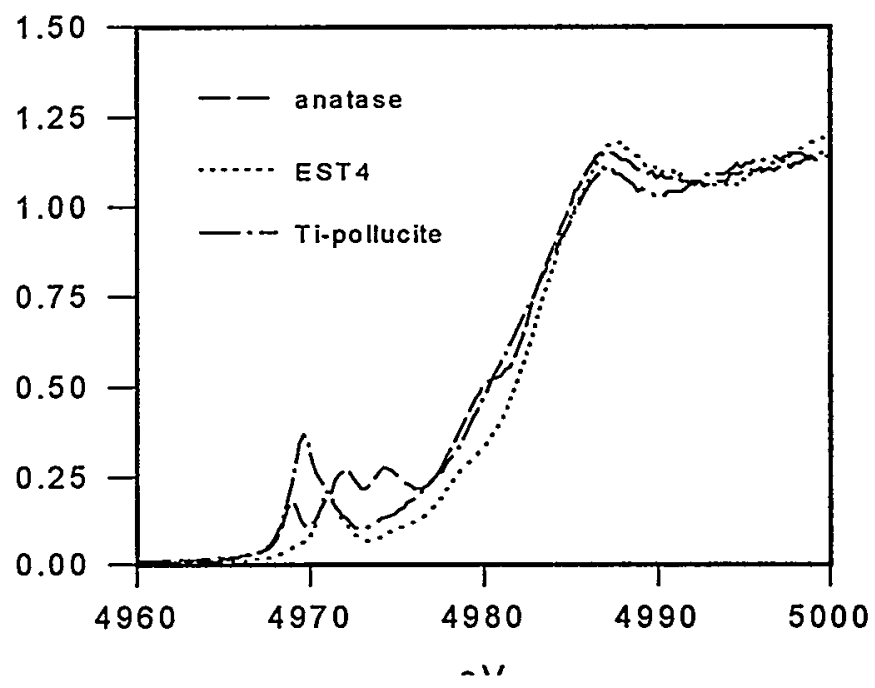

Figure 1. Ti XANES

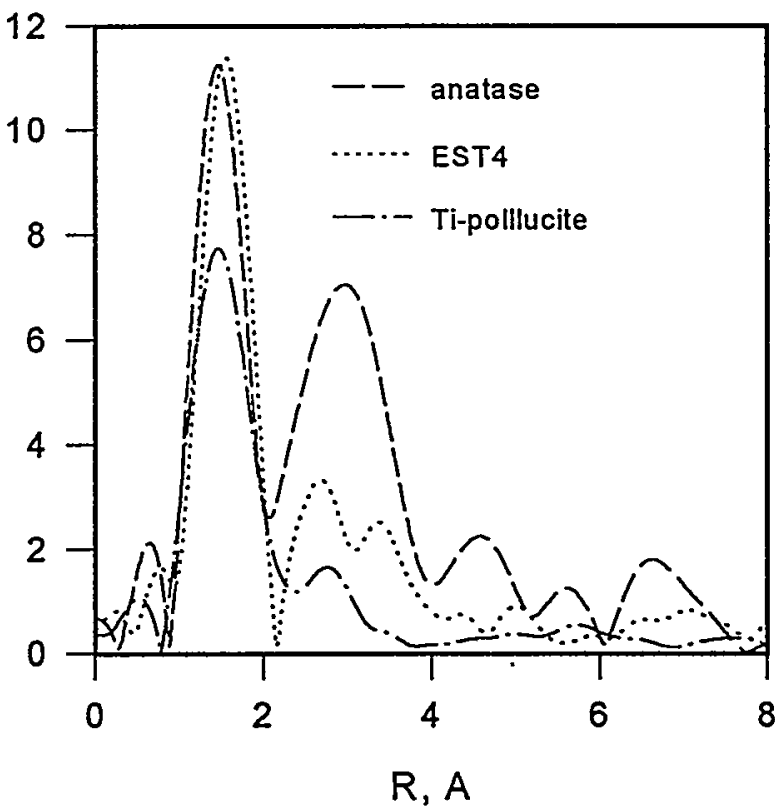

Figure 2. Ti EXAFS 


\title{
Cation Valence And Coordination In Cerium And Uranium Mixed Oxides
}

\author{
Mark R. Antonio and L. Soderholm
}

\section{Chemistry Division, Argonne National Laboratory, Argonne, IL 60439}

Metal oxides containing f-element and transition metal cations may exhibit unusual physical properties, particularly if the combination of cations is such that the chemical potentials for their valence orbitals are similar. Both dynamic electron delocalization and static electron localization can result in unique electronic properties, which may be modified by chemical, thermal, or optical means. This is particularly true when light lanthanide or actinide ions with two (or more) stable valence states (e.g., CeIII,IV and UIV,VI) are combined in an oxide with a transition-metal ion with at least two stable valence states (e.g., MoIV,V,VI and WIV,V,VI). Compounds with this combination of elements may exhibit valence instabilities if the energy barrier to the electronic charge transfer between the rare earth and transition metal cations is small. The focus of this study is on the cation valence distribution and coordination environments in two mixed oxides of composition $\mathrm{Ce}_{2} \mathrm{MO}_{6}$ for $\mathrm{M}=\mathrm{Mo}$ and $\mathrm{U}$.

$\mathrm{Ce}_{2} \mathrm{MoO}_{6}$ and $\mathrm{Ce}_{2} \mathrm{UO}_{6}$ were prepared as black and blue powders, respectively. It has not been possible to grow suitable crystals for single-crystal Xray diffraction (XRD) analyses to determine the crystal and molecular structures of $\mathrm{Ce}_{2} \mathrm{MO}_{6}$. The powder XRD patterns have been reported to be consistent with the fluorite structure, $F_{\mathrm{m} 3 \mathrm{~m}}$ space group.[1,2] The $F_{\mathrm{m} 3 \mathrm{~m}}$ lattice requires that each cation (Ce, Mo, and $U$ ) be disordered on the same site and, therefore, that each has the same coordination environment. In light of the different cation valence distributions that can be envisioned for $\mathrm{Ce}_{2} \mathrm{MO}_{6}$, i.e.,

$$
\begin{aligned}
& \mathrm{Ce}_{2} \mathrm{II}_{\mathrm{MVI}_{6}} \text { or } \mathrm{Ce} \mathrm{III}_{\mathrm{Ce}} \mathrm{IV}_{\mathrm{MVO}} \text { or } \\
& \mathrm{Ce}_{2}^{\mathrm{IV}} \mathrm{M}^{\mathrm{IV}} \mathrm{O}_{6}
\end{aligned}
$$

the structure and properties of ceriummolybdenum "black" and cerium-uranium "blue" may be of prospective technological importance for oxidation catalysis. This view follows from the fact that a bismuth molybdate of the same empirical formula, $\mathrm{Bi}_{2} \mathrm{MoO}_{6}$, and with a fluorite-related structure is well-known for its catalytic activity in the selective oxidation and ammoxidation of olefins.[3]

Through use of $\mathrm{K}$ - and L-edge Mo XAFS as well as Ce and U L-edge XAFS and M-edge (U) XANES, we have elucidated the cation valence distributions and coordination environments in $\mathrm{Ce}_{2} \mathrm{MO}_{6}$. XAFS data were obtained by transmission and electron-yield methods on SSRL beam lines 4-1 and 4-3 using $1 \mathrm{~mm}$ premonochromator slits and $\mathrm{Si}<111>$ and $\mathrm{Si}<220>$ crystals, respectively. The Oxford CF1208 cryostat was provided through the SSRL Biotechnology Program.

It is immediately apparent from the Ce $\mathrm{L}_{3}$-edge XANES for $\mathrm{Ce}_{2} \mathrm{MoO}_{6}$ and $\mathrm{Ce}_{2} \mathrm{UO}_{6}$ (Figure 1 ) that the cerium valences are not the same. The single edge

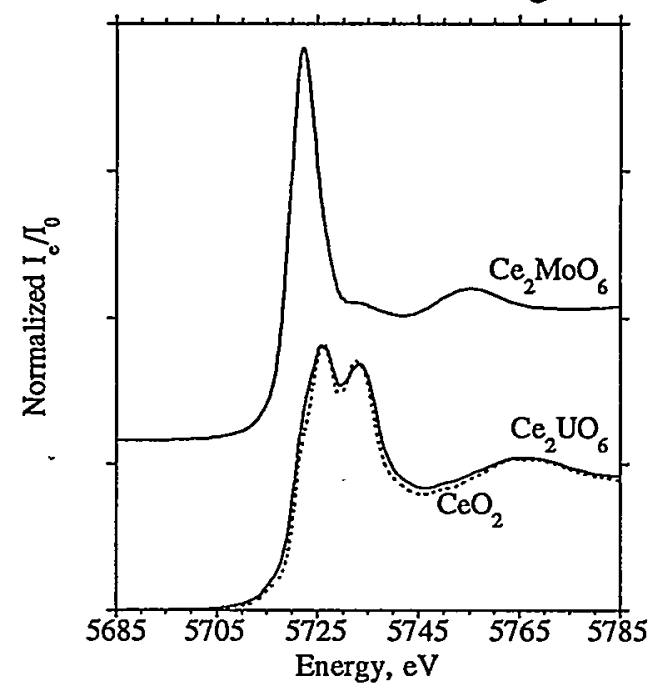

Figure 1. Ce L3-edge, electron-yield XANES obtained at $295^{\circ} \mathrm{K}$ for $\mathrm{Ce}_{2}^{\mathrm{III}} \mathrm{MoO}_{6}$, $\mathrm{Ce}_{2}^{\mathrm{IV}} \mathrm{UO}_{6}$, and $\mathrm{Ce}^{\mathrm{IV}} \mathrm{O}_{2}$ (dashed line). 
resonance observed for ceriummolybdenum black is indicative of trivalent $\mathrm{Ce}$, whereas the double edge resonance for cerium-uranium blue is indicative of quadrivalent Ce.[4] Despite the limited resolution due to the onset of the ${\mathrm{Ce} \mathrm{L}_{2}-}^{-}$ edge, the different valence distributions are also manifest in the EXAFS as different coordination environments. The $\mathrm{k}^{2} \chi(\mathrm{k})$ EXAFS and the corresponding Fourier transform data for $\mathrm{Ce}_{2} \mathrm{MoO}_{6}, \mathrm{Ce}_{2} \mathrm{UO}_{6}$, and $\mathrm{CeO}_{2}$ are shown in Figures 2 and 3. Here too, it is clear that the oxygen

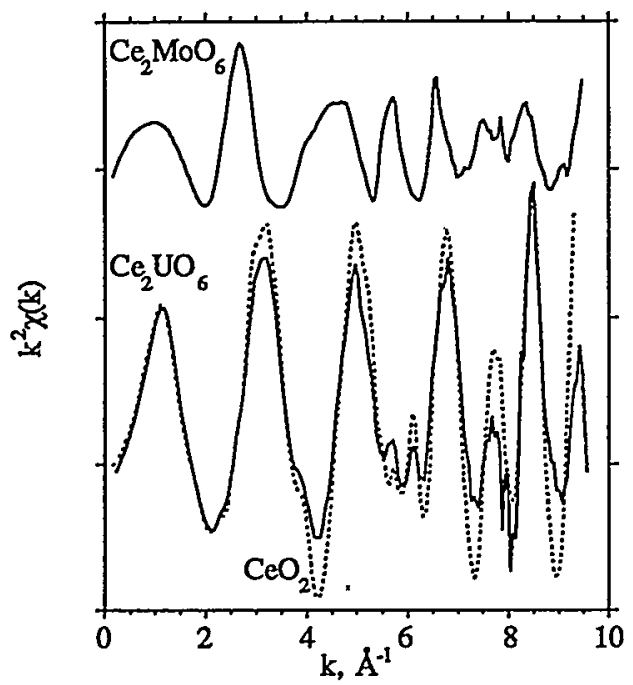

Figure 2. $\mathrm{Ce} \mathrm{L}_{3}$-edge, electron-yield, $\mathrm{k}^{2} \chi(\mathrm{k})$ EXAFS $\left(295^{\circ} \mathrm{K}\right.$ ) for $\mathrm{Ce}_{2}^{\mathrm{II}} \mathrm{MoO}_{6}, \mathrm{Ce}_{2}^{\mathrm{IV}} \mathrm{UO}_{6}$, and $\mathrm{Ce}^{\mathrm{IV}_{2}} \mathrm{O}_{2}$ (dashed line).

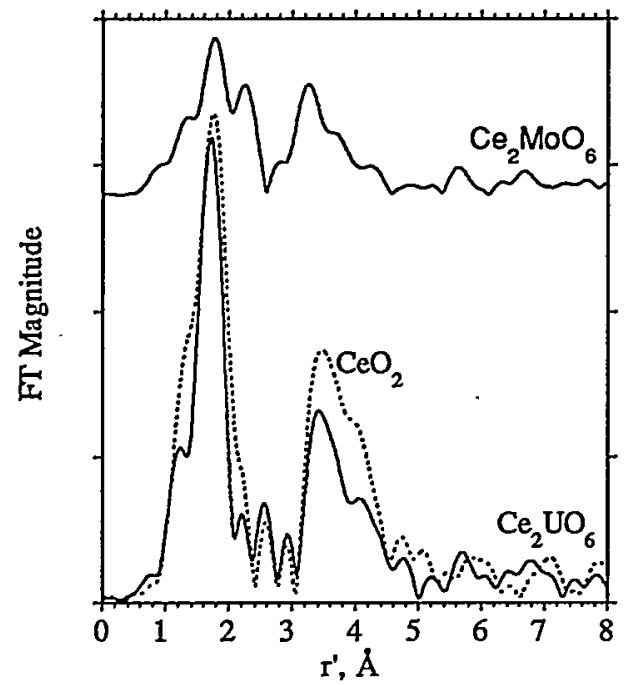

Figure 3. Fourier transforms of the $k^{2} \chi(k)$ EXAFS of Figure 2. coordination about $\mathrm{Ce}$ III in $\mathrm{Ce}_{2} \mathrm{MoO}_{6}$ (4 at 2.43(3) and 4 at 2.70(3) $\AA$ ) is different from the oxygen coordination about $\mathrm{Ce}^{\mathrm{IV}}$ in $\mathrm{Ce}_{2} \mathrm{UO}_{6}$ (8 at 2.30(3) $\AA$ ). The $\mathrm{Ce}$ coordination in $\mathrm{Ce}_{2} \mathrm{UO}_{6}$ is identical to that for $\mathrm{CeO}_{2}$, which has the fluorite $\left(\mathrm{F}_{\mathrm{m} 3 \mathrm{~m}}\right)$ structure.

By use of both $\mathrm{K}$ - and L-edge XANES (see Figures 4 and 5), we have determined that molybdenum is hexavalent in $\mathrm{Ce}_{2} \mathrm{MoO}_{6}$. Similarly, through use of

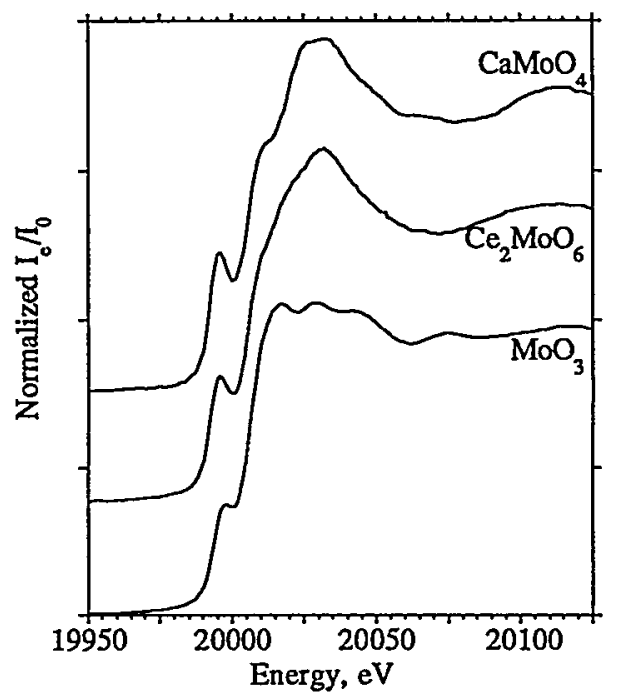

Figure 4. Mo K-edge, electron-yield XANES $\left(295^{\circ} \mathrm{K}\right)$ for $\mathrm{Ce}_{2} \mathrm{MoVIO}_{6}$ and hexavalent Mo model compounds $\mathrm{CaMo} \mathrm{VIO}_{4}$ and $\mathrm{MoVIO}_{3}$.

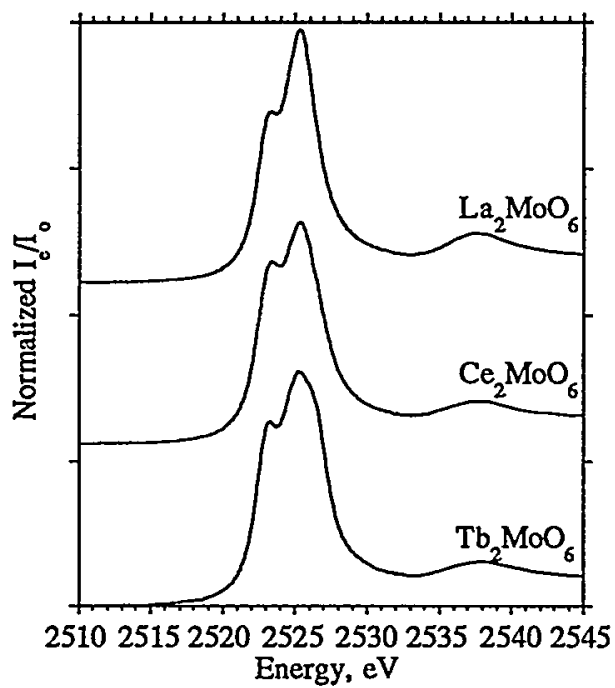

Figure 5. Mo $\mathrm{L}_{3}$-edge, electron-yield XANES for $\mathrm{Ce}_{2} \mathrm{MoV} \mathrm{O}_{6}$ and hexavalent $\mathrm{Mo}$ model compounds $\mathrm{La}_{2} \mathrm{MoVI}{ }^{\mathrm{VI}}$ and $\mathrm{Tb}_{2} \mathrm{MoVIO}_{6}$. 
both L- and M-edge XANES (see Figure 6 top and bottom), we have determined that uranium is quadrivalent in $\mathrm{Ce}_{2} \mathrm{UO}_{6}$. In view of the difference between the ionic radii of MoVI and UIV (ca. $0.3 \AA$ ), it is not surprising to find different coordination
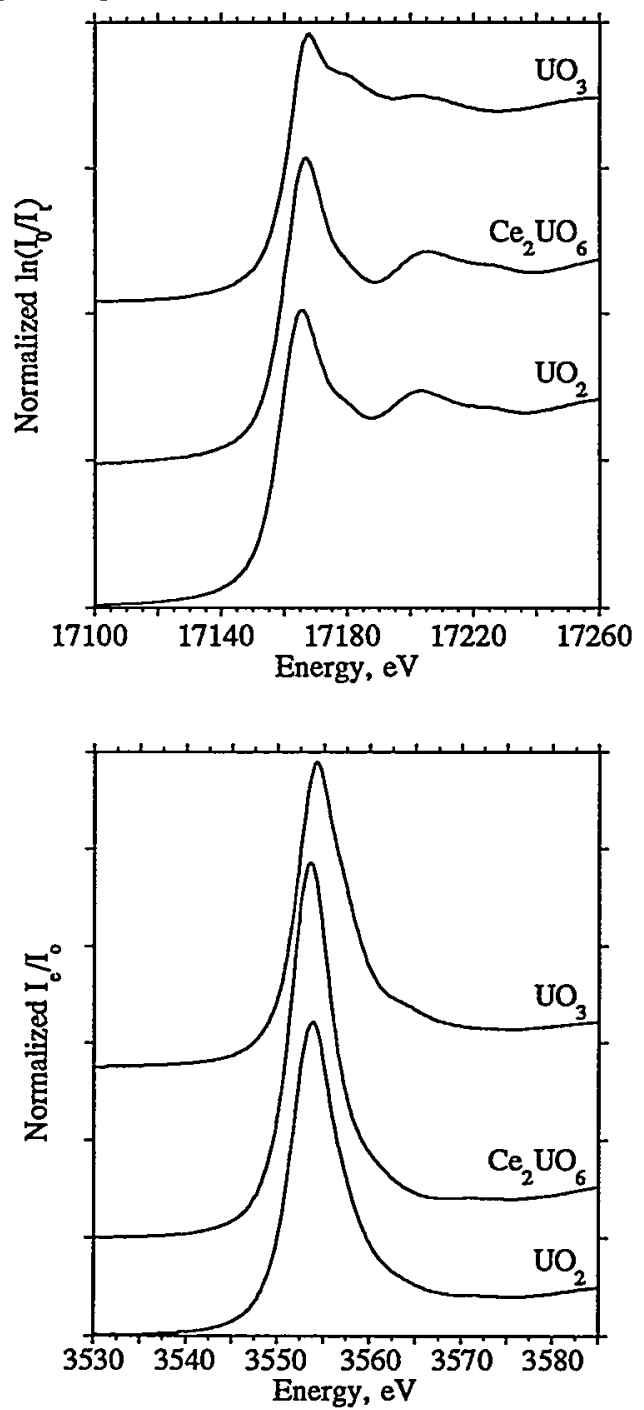

Figure 6. (TOP) $\mathrm{U}^{2}$-edge, transmission XANES $\left(10^{\circ} \mathrm{K}\right)$ and (BOTTOM) U M M- $^{-}$ edge, electron-yield XANES $\left(295^{\circ} \mathrm{K}\right)$ for $\mathrm{Ce}_{2} \mathrm{UO}_{6}$ and model compounds $\mathrm{UVI} \mathrm{O}_{3}$ and $\mathrm{UNO}_{2}$.

environments about Mo and $\mathrm{U}$. The Mo Kedge and $\mathrm{U} \mathrm{L}_{3}$-edge $\mathrm{k}^{2} \chi(\mathrm{k})$ EXAFS and the corresponding Fourier transform data are shown in Figures 7 and 8. Molybdenum(VI) is coordinated to $4 \mathrm{O}$ atoms at $1.80(2) \AA$, whereas uranium(IV) is coordinated to $8 \mathrm{O}$ atoms at 2.30(2) $\AA$.

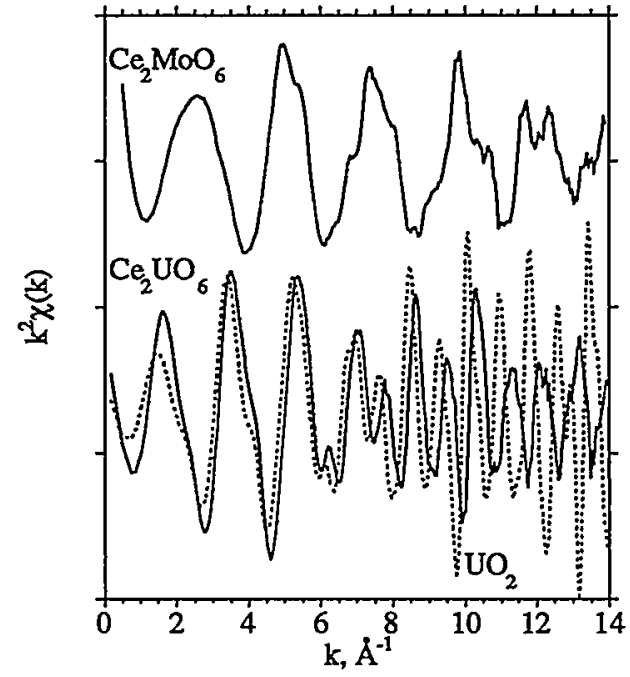

Figure 7. Transmission $\mathrm{k}^{2} \chi(\mathrm{k})$ EXAFS $\left(10^{\circ} \mathrm{K}\right)$ from the $\mathrm{Mo} \mathrm{K}$-edge of $\mathrm{Ce}_{2}^{\mathrm{III}} \mathrm{MoVI}_{6}$

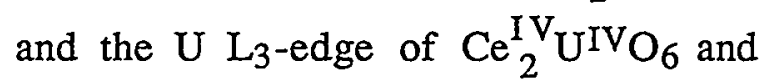
$\mathrm{U}^{\mathrm{IV}} \mathrm{O}_{2}$ (dashed line).

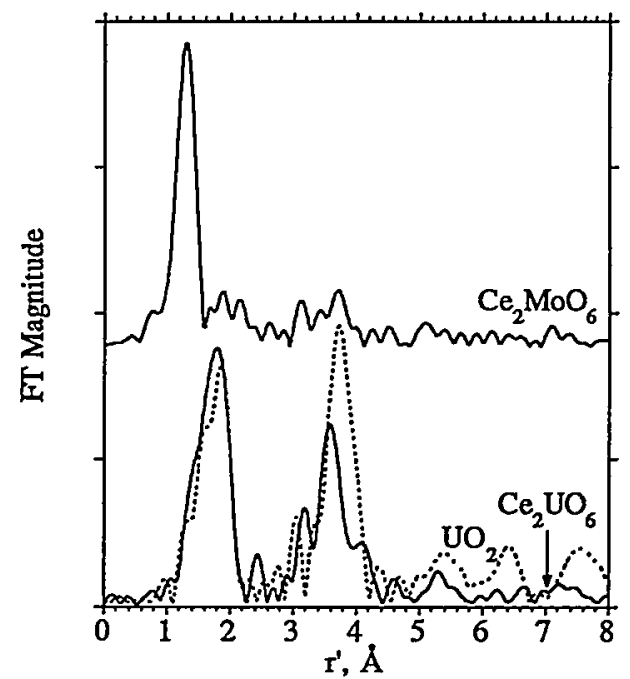

Figure 8. Fourier transforms of the $\mathrm{k}^{2} \chi(\mathrm{k})$ EXAFS shown in Figure 7.

In summary, for $\mathrm{Ce}_{2} \mathrm{UO}_{6}$, both $\mathrm{Ce}$ and $U$ have the same valence (IV) and the same eight-fold oxygen coordination. These XAFS results are consistent with the available powder XRD data that indicate the $\mathrm{F}_{\mathrm{m} 3 \mathrm{~m}}$ space group for the cerium-uranium blue.[1] In view of the fact that $\mathrm{CeO}_{2}$ and $\mathrm{UO}_{2}$ are isostructural with only a small difference in their lattice constants, the EXAFS metrical results and the XANESdetermined valences are not surprising. This stands in stark contrast to the situation for 
$\mathrm{Ce}_{2} \mathrm{MoO}_{6}$, which is much different from that for $\mathrm{Ce}_{2} \mathrm{UO}_{6}$. In $\mathrm{Ce}_{2} \mathrm{MoO}_{6}$, cerium is trivalent with a $\mathrm{Ce}^{\mathrm{III}_{-} \mathrm{O}_{8}}$ coordination environment, whereas molybdenum is hexavalent with a $\mathrm{MoVI}_{-} \mathrm{O}_{4}$ coordination environment. The XAFS results for $\mathrm{Ce}_{2} \mathrm{MoO}_{6}$ are inconsistent with the reported space group $\left(\mathrm{F}_{\mathrm{m} 3 \mathrm{~m}}\right)$ - one (or more) of the structure studies is in error. To resolve this discrepancy, powder neutron diffraction and electron diffraction studies of $\mathrm{Ce}_{2} \mathrm{MoO}_{6}$ are underway.

This work was supported by the U.S. DOE, Basic Energy SciencesChemical Sciences, under contract No. W-31-109-ENG-38.

1. A. Magneli, L. Kihlborg, Acta Chemica Scand. 5, 578 (1951).

2. L.H. Brixner, A.W. Sleight, M.S. Licis, J. Solid State Chem. 5, 186 (1972).

3. D.J. Buttrey, T. Vogt, U. Wildgruber, W.R. Robinson, J. Solid State Chem. 111, 118 (1994)

4. M.R. Antonio, J.S. Xue, L. Soderholm, J. Alloys \& Cmpds. 207/208, 444 (1994). 


\section{EFFECT OF STRUCTURE, STRESS, STRAIN, AND ALLOYING ON THE HARDNESS OF Fe(001)/Pt(001) EPITAXIAL MULTILAYERS \\ B.J. Daniels, W.D. Nix, and B.M. Clemens \\ Department of Materials Science and Engineering, Stanford University}

\section{Introduction}

Metal multilayer thin films are currently of interest for many materials applications in part because the mechanical properties of these structures differ significantly from those of the bulk materials. Although researchers have reported the observation of a hardness enhancement in these films at small $\Lambda$ [1-5], an adequate explanation of this behavior over the entire range of $\Lambda$ is still lacking. The hardness enhancement in the large $\Lambda$ regime has been explained through the use of a Hall-Petch-like model which treats one half of the bilayer spacing as the grain size $[2,5]$. For materials in which there is a significant shear modulus discontinuity the image dislocation model may explain the higher hardness $[2,3]$. Another proposed strengthening mechanism requires a structural discontinuity between layers (e.g. FCC/BCC). This cannot be the dominant mechanism, however, as significant hardness enhancements are seen in the $\mathrm{Fe}(001) / \mathrm{Cr}(001)$ system in which there is no structural barrier $[1,2]$.

In this study we investigate three other mechanisms for strengthening in metal multilayer systems. Strain relaxation by generation of misfit dislocations at the $\mathrm{Fe} / \mathrm{Pt}$ interface could result in enhanced hardness. The motion of a dislocation would be impeded as its stress field interacts with the stress field of the misfit dislocation network. Another phenomenon which would result in enhanced hardness is a large compressive film stress. This compressive stress constrains the film under and around the indenter, resulting in an increased yield strength. A third method for strengthening in multilayer structures is solid solution strengthening or precipitation hardening. This could result from intermixing or diffusion upon film deposition. In this study we use the $\sin ^{2} \psi$ x-ray diffraction technique to investigate the role of misfit dislocation density, compressive film stress, and solid solution strengthening in the observed hardness enhancement.

\section{Experimental}

Epitaxial $\mathrm{Fe}(001) / \mathrm{Pt}(001)$ multilayers with $\Lambda=25$, 44,76 , and $121 \AA$ were examined in this study.
Details of the sample fabrication, characterization, and testing are discussed elsewhere $[1,2]$, however the main results will be summarized here. Samples were fabricated by dc magnetron sputtering onto single crystal $\mathrm{MgO}(001)$ substrates. A seed layer of $5 \AA \mathrm{Fe}+200 \AA \mathrm{Pt}$ was deposited at $360^{\circ} \mathrm{C}$ to promote the epitaxial growth of the superlattice. Equal thicknesses of each component were deposited sequentially at $50-60^{\circ} \mathrm{C}$ to form multilayers. Although $\Lambda$ was varied from sample to sample, the total thickness of the multilayer was held constant at $1 \mu \mathrm{m}$. The bilayer spacing and the epitaxial orientation for these films was then determined by $\mathrm{x}$-ray diffraction (XRD) while the mechanical properties were characterized via nanoindentation.

High-accuracy measurements of the strain in the films were made using asymmetric and grazing incidence $x$-ray scattering (GIXS) at the Stanford Synchrotron Radiation Laboratory (SSRL). An Xray wavelength of $1.2087 \AA$ was used in these experiments. The standard $\sin ^{2} \psi$ analysis $[6,7]$ (where $\psi$ is the angle between the normal of the diffracting planes and the film normal) was used to determine the in-plane strain, out-of-plane strain, and the unstrained lattice parameter as a function of $\Lambda$ for both $\mathrm{Fe}$ and Pt. Specifically we investigated the $\mathrm{Fe}\{211\}$ and $\mathrm{Pt}\{420\}$ peaks. By using peaks from the same family (i.e. the same value of $2 \theta$ ) error due to diffractometer alignment is minimized. These particular families of peaks were chosen because the diffraction condition for each is satisfied for multiple values of $\psi$ and the diffracted peaks are far from those of the substrate and the other film component.

\section{Results and Discussion}

Nanoindentation results for the $\mathrm{Fe}(001) / \mathrm{Pt}(001)$ system were reported previously $[1,2]$. Figure 1 shows that the hardness of these multilayers is enhanced over the rule of mixtures value by a factor of approximately 2.5 for a broad range of $\Lambda$. The four samples examined in this study (filled circles) lie within this region of constant hardness enhancement. The decrease in hardness at large $\Lambda$ can be explained by a Hall-Petch-like effect $[2,5]$, while the hardness decrease at small $\Lambda$ is presumed to 


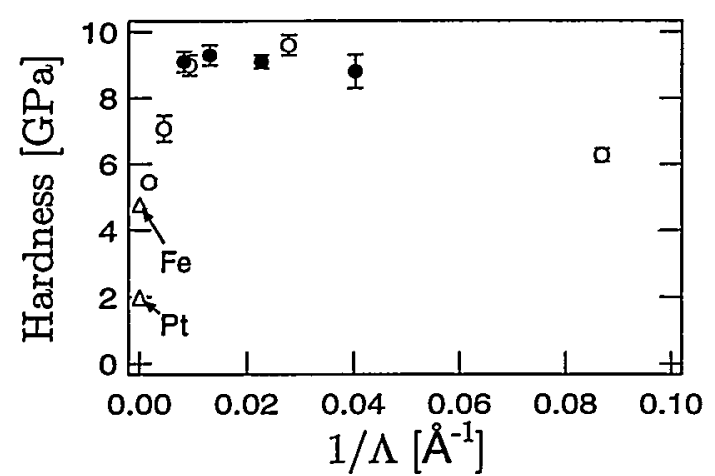

Figure 1: Hardness vs $1 / \Lambda$ for $\mathrm{Fe}(001) / \mathrm{Pt}(001)$ multilayer films. The filled circles are the samples used in this experiment. Indentation depths are nominally $150 \mathrm{~nm}$.

be the result of alloying.

Asymmetric XRD data were used to determine the epitaxial orientation of each of the four films. For $44 \AA \leq \Lambda \leq 121 \AA$ the $\mathrm{Fe}$ and Pt films are rotated relative to one another by $45^{\circ}$ around the [001], i.e. the Bain orientation. For the $\Lambda=25 \AA$ sample the Fe layer adopts the FCC structure. In this case the lattice parameter of the Fe is such that it grows with no relative rotation around the [001] with respect to the Pt, i.e. cube-on-cube epitaxy. In this configuration there is no structural barrier to dislocation motion. This is in agreement with the results for the $\mathrm{Fe}(001) / \mathrm{Cr}(001)$ system in which the structural barrier to dislocation motion between layers was shown to be a minor strengthening effect $[1,2]$.

The $\sin ^{2} \psi$ technique was used to determine the strain and extent of alloying in each film. The governing equation for $d$ vs $\sin ^{2} \psi$ analysis in the case of the biaxial strain present in (001) epitaxial films is:

$$
\begin{aligned}
\epsilon(\psi)= & \frac{d(\psi)-d_{0}}{d_{0}}=\frac{\sigma}{\left(c_{11}-c_{12}\right)\left(c_{11}+2 c_{12}\right)} \mathrm{x} \\
& {\left[-2 c_{12}+\left(c_{11}+2 c_{12}\right) \sin ^{2} \psi\right] }
\end{aligned}
$$

where $\epsilon(\psi)$ and $d(\psi)$ are the strain and the measured plane spacing as a function of $\psi, d_{0}$ is the unstrained plane spacing, $\sigma$ is the equi-biaxial stress, and the $c_{i j}$ are the elements of the elastic stiffness matrix. This yields an equation for $d(\psi)$ which is a linear function of $\sin ^{2} \psi$. In order to determine the value of $\sin ^{2} \psi$ which corresponds to the unstrained d-spacing, $d_{0}$, we use Equation 1 for the case where $d(\psi)=d_{0}$ to obtain:

$$
\left.\sin ^{2} \psi\right|_{d(\psi)=d_{0}}=\frac{2 c_{12}}{c_{11}+2 c_{12}}
$$

By using the elastic constants for each material and a linear fit to $d$ vs $\sin ^{2} \psi$ data, the unstrained lattice parameter, in-plane strain, and out-of-plane strain can be obtained.

A plot of the in-plane and out-of-plane strains obtained from $d$ vs $\sin ^{2} \psi$ fits is shown in Figure 2(a). Both the in-plane and out-of-plane strains are relatively small and roughly constant in the Pt layers of each of the four films tested. The strains in the $\mathrm{Fe}$ layers, however, are quite large at $\Lambda=44 \AA$ and relax toward zero as $\Lambda$ increases. The Fe in-plane compressive strain of nearly $2.5 \%$ for $\Lambda=44 \AA$ lies between the theoretical coherency strains of $-3.3 \%$ for $\mathrm{Fe}$ on rigid $\mathrm{Pt}$ and $-1.8 \%$ for a free-standing multilayer. The large amount of strain relaxation in the Fe layers is accompanied by a large increase in the misfit dislocation density at the Fe/Pt interface. Since the hardness is independent of $\Lambda$ in this region, it is unlikely that the stress fields of the misfit dislocations are responsible for the hardness enhancement observed in these films.

In-plane strains can be converted to in-plane stresses via the biaxial modulus for each component in the following manner [8]:

$$
\sigma=\left(c_{11}+c_{12}-\frac{2 c_{12}^{2}}{c_{11}}\right) \epsilon=\mathrm{M}_{(001)} \epsilon
$$

where $\epsilon$ is the equi-biaxial in-plane strain the $c_{i j}$. The in-plane stress data, shown in Figure 2(b), indicates that there is a constant compressive stress in the Pt layers of these films. The in-plane stress in the Fe layers is quite large at $\Lambda=44 \AA$ and relaxes toward zero for larger $\Lambda$. Although the constraints imposed by large in-plane compressive stresses could result in enhanced hardness, the stress in the Fe layer varies dramatically with no observed change in the hardness of the film. One could argue that the smaller, but constant, compressive stress in the $\mathrm{Pt}$ layer could contribute to the hardness enhancement if deformation were to occur preferentially in the $\mathrm{Pt}$ layer, however the magnitude of this stress makes it unlikely that this is the dominant effect.

Figure 3 shows the unstrained lattice parameters, $a_{0}=d_{0} \sqrt{h^{2}+k^{2}+l^{2}}$, as determined from $d$ vs $\sin ^{2} \psi$ fits. The unstrained lattice parameter of the $\mathrm{Pt}$ is constant at approximately the bulk value for all four films. The Fe unstrained lattice parameter, however, increases as $\Lambda$ decreases. This 

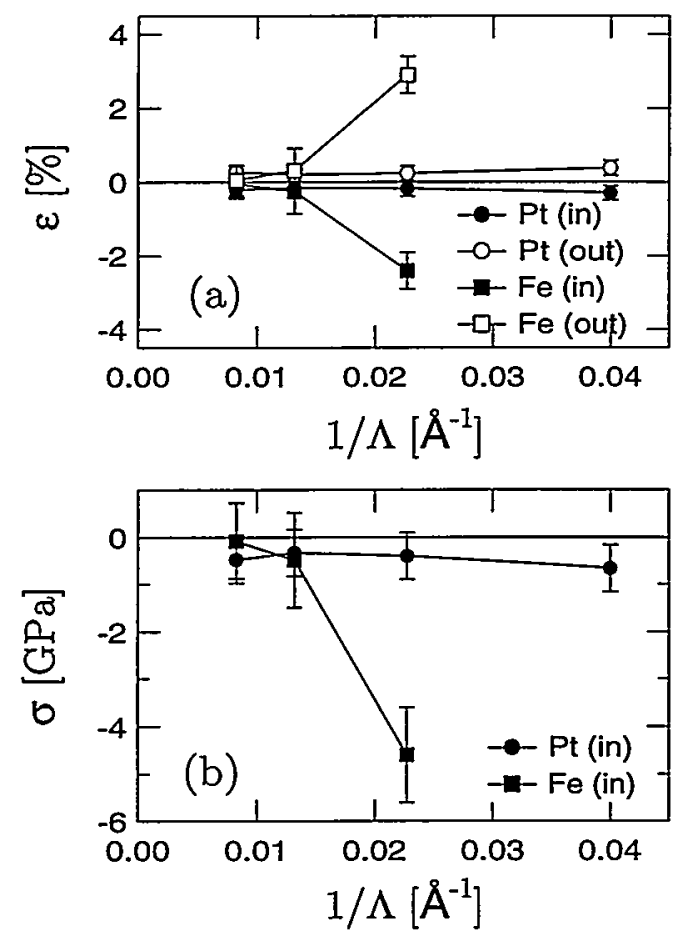

Figure 2: (a) Strain and (b) total stress in each layer vs $1 / \Lambda$ as determined by $\sin ^{2} \psi$ analysis.

is assumed to be due to Pt intermixing and diffusion upon deposition. One possible explanation for the difference between layers is that reflected $\mathrm{Ar}$ ions have a larger energy during $\mathrm{Pt}$ deposition and tend to knock $\mathrm{Pt}$ atoms into the Fe layer. Extrapolating a linear fit to the data shows that $a_{0}$ approches the bulk value for $\mathrm{Fe}$ in the infinite $\Lambda$ limit. The extent of alloying in each layer can be determined by using the size factors for metallic solid solutions which have been tabulated by King [9]. Although data was not available in the literature for the atomic volume of $\mathrm{Pt}$ in $\mathrm{Fe}$, a reasonable estimate of the $\mathrm{Pt}$ concentration can still be obtained. Since Pt and Pd are both FCC noble metals, differ in atomic volume by only $2.5 \%$, and are completely miscible in each other, their alloying behavior with $\mathrm{Fe}$ would be expected to be similar. Thus the data for $\mathrm{Pd}$ in $\mathrm{Fe}$ can be used as an approximation. Using this analysis we find that the concentration of $\mathrm{Fe}$ in $\mathrm{Pt}$ is negligible, while the concentration of $\mathrm{Pt}$ in $\mathrm{Fe}$ varies from approximately 11 at.\% for $\Lambda=44 \AA$ to 5 at.\% for $\Lambda=121 \AA$. Most systems which exhibit solution solution strengthening behavior show a strong dependence of the yield strength on the impurity con-
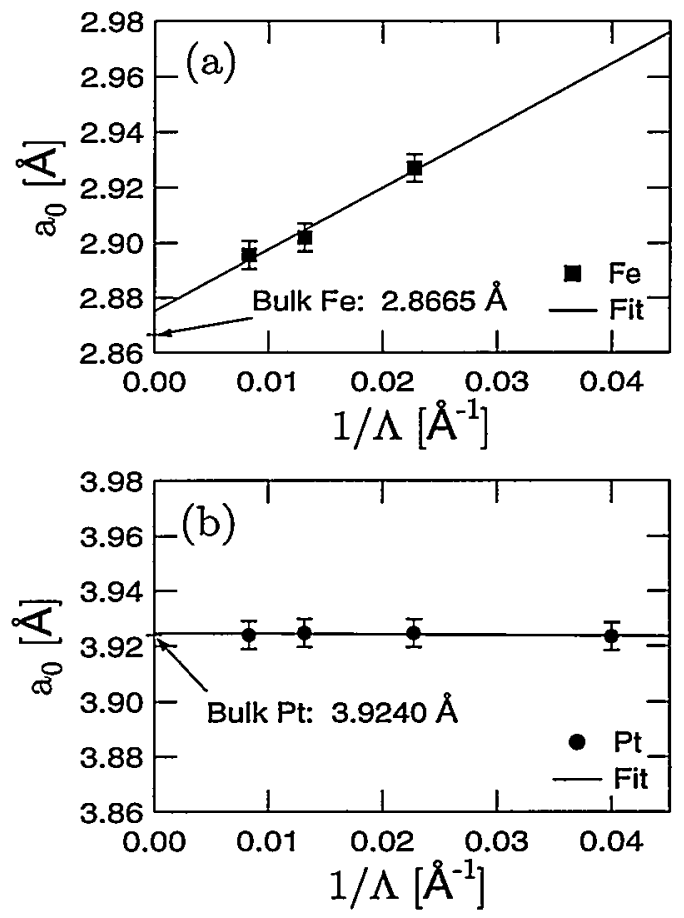

Figure 3: Unstrained lattice parameter, $a_{0}$, of (a) Fe and (b) Pt vs $1 / \Lambda$. Solid lines are least-squares fits to the data.

centration. Since the hardness remains constant in the regime where the alloy content varies by 6 at.\%, it seems unlikely that solid solution strengthening is responsible for the enhanced hardness.

\section{Conclusions}

We have investigated the possible origins of enhanced hardness in epitaxial $\mathrm{Fe}(001) / \mathrm{Pt}(001)$ multilayers. The hardness was enhanced by a factor of 2.5 over the rule of mixtures value for $25 \AA \leq \Lambda \leq 121 \AA$. The Fe in the $\Lambda=25 \AA$ film adopts the FCC structure, verifying that the contribution of a structural discontinuity to the hardness enhancement is small. Values for the in-plane strain, out-of-plane strain, and unstrained lattice parameter were obtained as a function of $\Lambda$ through the use of standard $d$ vs $\sin ^{2} \psi$ analysis. The large strain relaxation $(2.4 \%)$ in the $\mathrm{Fe}$ layers made it unlikely that strengthening was the result of dislocation interactions with the stress fields of misfit dislocations. The in-plane stresses were calculated from elastic constants and showed that the Fe layer was highly compressed at $\Lambda=44 \AA$ and relaxed at 
larger $\Lambda$. The stress in the $\mathrm{Pt}$ layer was constant, smaller, and compressive. This would seem to indicate that compressive film stress is not a dominant factor in the enhanced hardness. The unstrained lattice parameter of the $\mathrm{Fe}$ also varied dramatically with $\Lambda$, indicating a significant amount of $\mathrm{Pt}$ alloying. This behavior makes it unlikely that solid solution strengthening or precipitate hardening is responsible for the constant hardness enhancement over this region of $\Lambda$. Although in this investigation we have enhanced our understanding of the structure-property relationships in the $\mathrm{Fe} / \mathrm{Pt}$ system, we conclude that structural discontinuities, misfit dislocation density, compressive film stress, and solid solution or precipitate strengthening are not dominant factors in the large hardness enhancement observed in this system.

\section{Acknowledgements}

The authors would like to acknowledge helpful discussions with G.R. English and the assistance of T.C. Hufnagel, R.M. Osgood, M.C. Kautzky, J. Xu, S.A. Newell, C.T. Wang, T. Ueda, D.T. Carpenter, and K.P. Fahey in the acquisition of synchrotron $\mathrm{x}$-ray scattering data. Funding for this investigation was provided by NSF under contract DMR-9100271. SSRL is funded by the U.S. Department of Energy (DOE), Office of Basic Energy Sciences.

\section{References}

[1] B.J. Daniels, W.D. Nix, and B.M. Clemens, Thin Solid Films 253, 218 (1994).

[2] B.J. Daniels, W.D. Nix, and B.M. Clemens, Mater. Res. Soc. Symp. Proc. 343, 549 (1994).

[3] M.F. Doerner, Ph.D. Dissertation (Stanford University, 1987).

[4] G.R. English, G.F. Simenson, B.M. Clemens, and W.D. Nix, Mater. Res. Soc. Symp. Proc. 356 (1995).

[5] T. Foecke and D.S. Lashmore, Script. Met. et Mat. 27, 651 (1992).

[6] J.A. Bain, L.J. Chyung, S. Brennan, and B.M. Clemens, Phys. Rev. B 44, 1184 (1991).

[7] I.C. Noyan and J.B. Cohen, Residual Stress, Measurement by Diffraction and Interpretation (Springer-Verlag, New York, 1987).
[8] W.D. Nix, Met. Trans. A 20A, 2217 (1989).

[9] H.W. King, J. Mater. Sci. 1, 79 (1966). 


\title{
Structure and Magneto-optical Properties of Epitaxial PtMnSb(001) on $\mathrm{W}(001) / \mathrm{MgO}(001)$
}

\author{
M.C. Kautzky and B.M. Clemens
}

Dept. of Materials Science and Engineering, Stanford University, Stanford, CA 94305-2205

The Heusler alloy PtMnSb has attracted attention due to its unique magnetic, magneto-optic, and transport properties, including the theoretical prediction and limited experimental observation of half-metallic ferromagnetism $[1,2]$, and the presence of an intrinsic Kerr rotation which exceeds $1^{\circ}$ at red wavelengths [3]. While progress has been made in controlling these properties through microstructural development, more detailed studies of the structure-property relationships in this system are necessary before they can be exploited. Such studies could benefit from the fabrication of high quality epitaxial thin films and multilayers.

One area which could potentially benefit from epitaxial growth is the development of PtMnSb as a magneto-optical recording medium. The unusually high Kerr rotation in this material is attractive for achieving a large readback signal, but to date $\mathrm{PtMnSb}$ thin films have lacked the strong perpendicular magnetic anisotropy required for magneto-optical data storage. Various attempts have been made to overcome this problem [4-9], but none have been successful in producing a combination of perpendicular magnetization and large Kerr rotation. In this paper we report our attempt to induce perpendicular magnetization through inverse magnetostriction during growth of strained epitaxial PtMnSb layers. We show that high quality epitaxial films can be produced by sputter deposition, but that the strain levels we observe in them are insufficient to produce perpendicular anisotropy. We expect, however, that the development of a successful epitaxial growth technique will now lead to an improved understanding and application of PtMnSb's properties.

The choice to use epitaxial strain to achieve perpendicular magnetization is a result of magnetic energy considerations. Calculations of the strain necessary to overcome shape anisotropy in $\mathrm{PtMnSb}$ indicate that the most favorable case is for an (001)-oriented film, which requires a tensile strain of $\approx 2 \%$. The search for a substrate with a positive mismatch of this magnitude and the 138

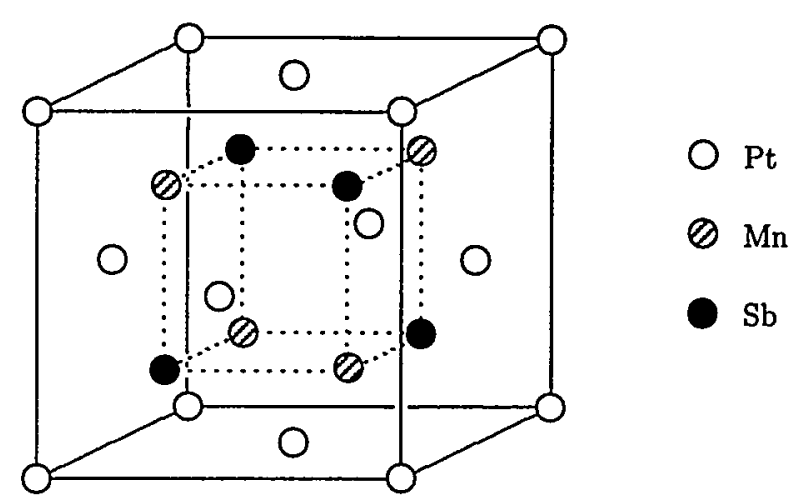

Figure 1: $\mathrm{Cl}_{b}$-type crystal structure of $\mathrm{PtMnSb}$. The unstrained, stoichiometric lattice parameter is $6.20 \AA[10]$.

same fourfold in-plane symmetry as $\mathrm{PtMnSb}(001)$ (fig. 1) led to the choice of W (bcc, $\mathrm{a}_{o}=3.1653 \AA$ ) as the best candidate. Comparing the atomic arrangements of $\{001\}$ planes in both materials, we predict that the PtMnSb film will minimize its misfit by adopting a cube-on-cube epitaxial relationship with no relative rotation between the film and seed layer (PtMnSb[100] $\| \mathrm{W}[100])$. In this orientation, for which $a_{P t M n S b} \approx 2 a_{W}$, the theoretical misfit strain required for coherent growth of $\mathrm{PtMnSb}$ is 2.1\%. In the results below, we describe the growth of (001)-oriented PtMnSb on W(001) seed layers in the predicted orientation, and the magneto-optical properties that result.

Although a systematic study of these films is in progress, the following discussion will be restricted to a single sample deposited in a dc magnetron sputtering system equipped with a load lock and four confocally-aimed sputter guns at a source-to-sample distance of 6 inches. In the experiment, a $300 \AA \mathrm{PtMnSb}$ layer was deposited onto a $1000 \AA$ epitaxial, (001)-oriented $W$ seed layer on a polished (001)-oriented $\mathrm{MgO}$ substrate. The substrate was etched for $2 \mathrm{~min}$. in a solution of $\mathrm{NH}_{4} \mathrm{OH}: \mathrm{H}_{2} \mathrm{O}_{2}: \mathrm{H}_{2} \mathrm{O}$ (1:1:100 volumetric) 
and blown dry with nitrogen just before introduction into the load lock. The room temperature base pressure of the system was $5 \times 10^{-9}$ torr. The substrate was first annealed for $2 \mathrm{hrs}$. at the deposition temperature, nominally $500^{\circ} \mathrm{C}$, until a pressure of $6 \times 10^{-8}$ torr was achieved. (Substrate temperature was measured by a thermocouple calibrated earlier by optical pyrometry.) The $\mathrm{W}$ seed layer was then sputtered in $3 \mathrm{mT} \mathrm{Ar}$ at a rate of $0.94 \AA / \mathrm{s}$. Immediately following deposition of the seed layer, the PtMnSb layer was grown at the same temperature and pressure by cosputtering from elemental targets, at a total rate of $\approx 1.2 \AA / \mathrm{s}$. During deposition of both layers, the substrate was rotated to insure compositional uniformity.

Structural characterization of the film was done using symmetric and asymmetric $x$-ray diffraction, and grazing-incidence $x$-ray scatterirg (GIXS). Measurements were made at the Stanford Synchrotron Radiation Laboratory (beamline 7-2) on a four-circle diffractometer running in focused mode at a beam energy of $10,000 \mathrm{eV}$. Additional symmetric diffraction was done using $\mathrm{Cu} \mathrm{K} \alpha$ radiation on a powder diffractometer. All peaks were fit to Voigt profiles for extraction of peak parameters.

Figure 2 shows a full symmetric scan of the sample. The absence of any underlayer or film reflections besides $W(002)$ and $\mathrm{PtMnSb}(004)$ indicates that both have a single (001) out-of-plane orientation. The inset of figure 2 next shows asymmetric spectra from $\phi$ scans of the W\{011\} and $\mathrm{PtMnSb}\{111\}$ peaks. In both cases, the presence of four peaks at $90^{\circ}$ intervals in $\phi$ is consistent with a four-fold in-plane symmetry, indicating that both the film and underlayer are epitaxial. In addition, the $45^{\circ}$ offset in $\phi$ between these two different families of planes indicates that the $\mathrm{PtMnSb}$ grows on the $\mathrm{W}$ with no rotation, as. predicted. Finally, while no asymmetric peaks for $\mathrm{MgO}$ are shown, we have confirmed the presence of a $45^{\circ}$ relative rotation between the $W$ underlayer and $\mathrm{MgO}$ substrate in this growth orientation (W[100] || $\mathrm{MgO}[110]$ ), as reported previously by others [11].

The crystalline quality of the film and underlayer was measured by rocking curves taken through both the symmetric and in-plane peaks. The W(002) rocking curve FWHM is $0.72^{\circ}$, indicating high quality growth on the $\mathrm{MgO}$ substrate. The PtMnSb (004) peak has a slightly larger width

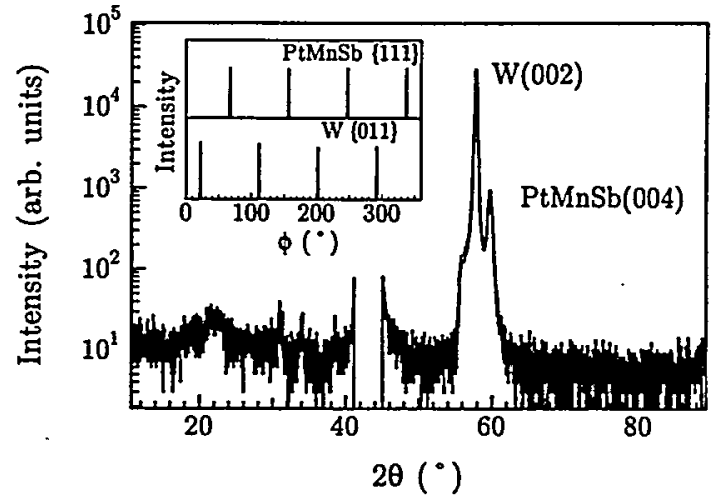

Figure 2: Symmetric $x$-ray diffraction scan showing an (001) out-of-plane orientation for the W underlayer and PtMnSb film. The missing peak at $42^{\circ}$ is from the $\mathrm{MgO}$ substrate. The inset $\phi$ scan confirms unrotated epitaxial growth of $\mathrm{PtMnSb}$ on W.

of $0.89^{\circ}$, which would suggest the defect structure of the growing PtMnSb film is largely controlled by that of the underlayer. Surprisingly, though, the opposite behavior is observed for the in-plane peaks, for which the $\mathrm{PtMnSb}(400)$ rocking curve width $\left(0.71^{\circ}\right)$ is actually smaller than the $W(200)$ width $\left(0.83^{\circ}\right)$. The atomic processes which would cause such a reduction in the in-plane mosaic spread are not clear, and a better understanding of the type and distribution of defects present is needed to explain this behavior.

An analysis of the strain state in the film was done using asymmetric diffraction in conjunction with the $\sin ^{2} \psi$ technique. Details of this technique have been presented elsewhere [12]. We chose to use the PtMnSb $\{531\}$ family of planes for this analysis since there are three members at inclinations below $\psi=90^{\circ}$, the scattered intensity from them is sufficiently large, and because they are well separated in reciprocal space from any underlayer or substrate peaks. A plot of the experimentally measured values of $d_{\psi}^{531}$ vs. $\sin ^{2} \psi$, and the resulting least squares fit, is shown in figure 3. A direct solution from this fit for values of the in-plane strain $\left(\epsilon_{1}\right)$, out-of-plane strain $\left(\epsilon_{3}\right)$, and unstrained lattice parameter $\left(d_{o}\right)$ is not possible, since the elastic stiffnesses $C_{11}$ and $C_{12}$ are not independently known for $\mathrm{PtMnSb}$. What can be determined, though, is the range of values that these variables take for a given range of $\frac{C_{12}}{C_{11}}$. Based on a survey of this ratio 


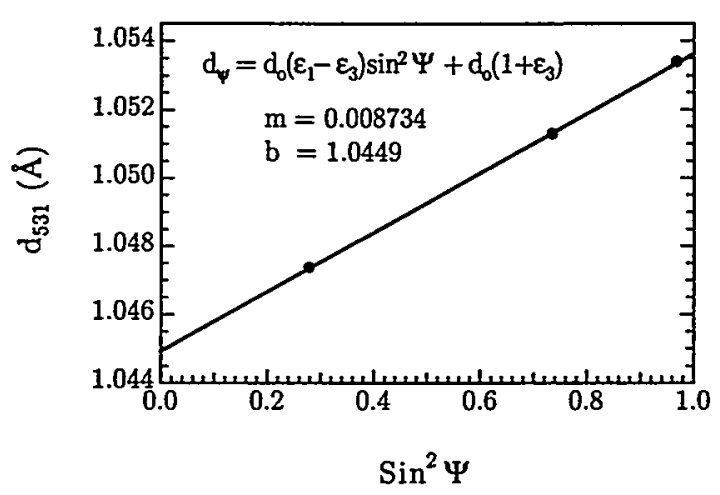

Figure 3: d-spacing of $\mathrm{PtMnSb}\{531\}$ planes as a function of $\sin ^{2} \psi$. The positive slope of tie fit denotes in-plane tension.

at room temperature for fcc metals $(\mathrm{Pd}=0.78$, $\left.\mathrm{Cu}=0.73, \mathrm{Pt}_{t}=0.72, \mathrm{Ni}=0.64\right)[13,14]$ and fccbased intermetallic compounds $\left(\mathrm{Cu}_{2} \mathrm{MnAl}=0.72\right.$, $\mathrm{Ni}_{3} \mathrm{Al}=0.67, \mathrm{Ni}_{2} \mathrm{MnGa}=0.41$ ) $[15,16]$, we believe $0.3-0.8$ to be a sufficiently large interval to include the probable value of $\frac{C_{12}}{C_{11}}$ for PtMnSb. The corresponding range of $d_{o}^{531}$ is then $1.0482-1.0503 \AA$, resulting in an estimated in-plane tensile strain of $0.31-0.52 \%$. By way of comparison, if we instead assume a completely stoichiometric film, and use the bulk d-spacing of $1.048 \AA$, a strain of $0.53 \%$ results.

The strain in the underlayer was measured using the $\mathrm{W}\{200\}$ in- and out-of-plane reflections directly. The observed d-spacings for the (002) and (200) peaks were $1.593 \AA$ and $1.575 \AA$ respectively. Using these and the bulk values for $C_{11}$ and $C_{12}[13], d_{o}^{200}$ is calculated to be $1.585 \AA$, which is slightly larger than the bulk value of $1.583 \AA$. Calculation of $\epsilon_{1}$ indicates that a small, compressive in-plane strain $(-0.51 \%)$ is present in the underlayer, which is consistent with nearly full accommodation of the $-5.9 \%$ misfit strain between $W$ and $\mathrm{MgO}$. The magnitude of the observed strain is similar to levels reported by others for singlelayer $\mathrm{W}$ films on $\mathrm{MgO}$ [11].

Polar magneto-optic Kerr effect measurements of the film were made at room temperature on a Kerr looper equipped with a monochromator and halogen lamp. The angle of incidence was $15^{\circ}$ from the sample normal. Values of the saturation rotation were taken to be half of the total difference in rotation at forward and reverse fields of $20 \mathrm{kOe}$ applied perpendicular to the film

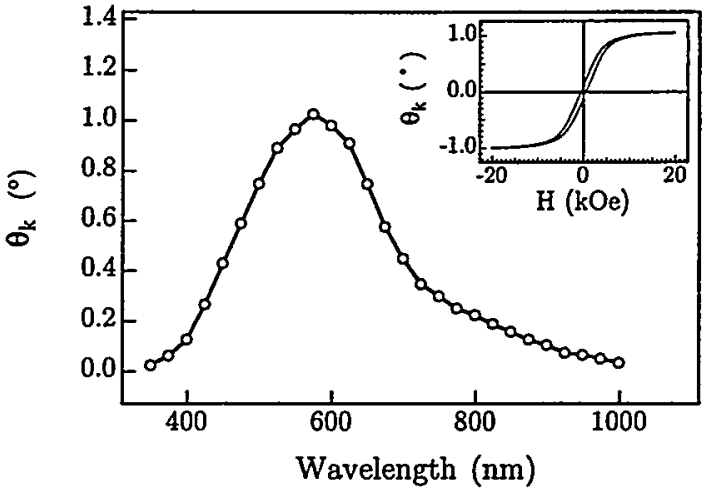

Figure 4: Room temperature saturation rotation as a function of wavelength. Inset shows a polar Kerr loop taken at $575 \mathrm{~nm}$.

surface. Figure 4 shows the MOKE spectrum in the range $350-1000 \mathrm{~nm}$. The rotation peaks strongly near $575 \mathrm{~nm}$, with a maximum value of $1.02^{\circ}$. This peak occurs at a substantially lower wavelength than those reported for polycrystalline PtMnSb spectra, which typically have maxima near $700 \mathrm{~nm}[6,17]$. We attribute this enhancement at lower wavelengths to the presence of the $\mathrm{W}$ underlayer, since such effects are routinely observed in metallic bilayers of magneto-optic and reflector materials [18-20]. In addition, the magnitude of the Kerr rotation, while smaller than those reported previously for polycrystalline $\mathrm{PtMnSb}$ films at higher wavelengths, is nonetheless approximately two and a half times larger than the rotations of rare-earth/transition metal alloys [21] and $30 \%$ larger than that of epitaxial ordered FePt [22] at this wavelength. This large rotation, however, does not persist down into the blue region due to the sharp dropoff below $500 \mathrm{~nm}$. Finally, the inset of figure 4 shows a room-temperature polar Kerr hysteresis loop taken at $575 \mathrm{~nm}$. The easy axis of magnetization lies in the plane of the film, resulting in a hard loop. This is consistent with a level of strain which is insufficient to induce perpendicular magnetization through inverse magnetostriction. The remanence rotation and coercive field are estimated to be $\approx 0.1^{\circ}$ and $\approx 500$ Oe respectively.

In conclusion, we have grown high-quality, epitaxial (001)-oriented PtMnSb on epitaxial $\mathrm{W}(001) / \mathrm{MgO}(001)$ by de magnetron cosputtering. The PtMnSb was found to grow unrotated on the $\mathrm{W}$ seed layer, accompanied by a modest in-plane 
tensile strain. The magnitude of this strain was insufficient to overcome shape anisotropy and induce perpendicular magnetization. From a structural standpoint, we suggest that this may still be possible through use of thinner single layers, or through a strained multilayer structure. Alternatively, it is also hoped that the successful growth of epitaxial PtMnSb films will lead to the development of structures with useful properties that depend less sensitively on strain.

The authors would like to thank R. Victora, R. White, E. Marinero, B. Daniels, K. Fahey, T. Hufnagel, R. Osgood, J. Xu, D. Carpenter, S. Newell, and C. Wang for helpful discussions and experimental assistance. This work was supported by the NSIC/DARPA UHDR Program. SSRL is funded by the U.S. Department of Energy, Office of Basic Energy Sciences.

\section{References}

[1] H. Ebert and G. Schütz. J. of Appl. Phys., 69:4627-9, 1991.

[2] R. DeGroot. Europhys. News, 23:146-148, 1992.

[3] P.G. van Engen, K.H.J. Buschow, and R. Jongebreur. Appl. Phys. Lett., 42:202, 1983.

[4] N. Hayashi, K. Morii, T. Matsui, and Y. Nakayama. Mat. Sci. Eng., B10:209-15, 1991.

[5] S. Hatta, T. Shiono, H. Adachi, and K. Wasa. Jap. J. of Appl. Phys., 26:L2082, 1987.

[6] Y. Hosoki, N. Mikami, H. Noda, T. Koyanagi, and K. Matsubara. IEEE Trans. Mag. Jpn., 6:855-61, 1991.

[7] T. Matsui, N. Iketani, K. Morii, and Y. Nakayama. Mat. Sci. Eng., B8:L5-8, 1991.

[8] T. Matsui, N. Iketani, K. Morii, and Y. Nakayama. Mat. Sci. Eng., B14:95-100, 1992.

[9] N. Sugimoto, T. Inukai, M. Matsuoka, and K. Ono. Jap. J. of Appl. Phys., 28:1139-40, 1989.

[10] K. Watanabe. J. Phys. Soc. Jpn., 28:302-7, 1970.

[11] H. Kimura, I. Petrov, F. Adibi, and J.E. Greene. J. Crystal Growth, 123:344-56, 1992.
[12] I.C. Noyan and J.B. Cohen. Residual Stress: Measurement by Diffraction and Interpretation. Springer-Verlag, New York, 1987.

[13] G. Simmons and H. Wong. Single Crystal Elastic Constants and Calculated Aggregate Properties: A Handbook. M.I.T. Press, Cambridge, 1971.

[14] J.F. Nye. Physical Properties of Crystals. Oxford University Press, Oxford, 1957.

[15] A. Vasil'ev, V. Kokorin, Y. Savchenko, and V. Chernenko. Sov. Phys. - JETP, 71:803-5, 1990.

[16] H. Landolt and R. Börnstein. Zahlenwerte und Funktionen aus Naturwissenschaft und Technik. Neue Serie, volume III/11. SpringerVerlag, Berlin, 1979.

[17] K. Takanashi, H. Fujimori, J. Watanabe, M. Shoji, and A. Nagai. IEEE Trans. Mag. Jpn., $5: 348-54,1990$.

[18] W. Reim and D. Weller. Appl. Phys. Lett., 53:2453-4, 1988.

[19] K. Song, M. Naoe, and H. Ito. J. of Appl. Phys., 70:6395-7, 1991.

[20] J. Cai, M. Lu, H. Zhai, Y. Miao, H. Wang, Y. $\mathrm{Xu}$, and H. Huang. J. of Appl. Phys., 70:5045$8,1991$.

[21] M. Kaneko, Y. Sabi, I. Ichimura, and S. Hashimoto. IEEE Trans. Mag., 29:3766-71, 1993.

[22] B.M. Lairson and B.M. Clemens. Appl. Phys. Lett., 63:1438-40, 1993. 


\section{SURFACE ANISOTROPY AND STRUCTURE OF FE(110)/MO(110) BIILAYERS \\ R.M. Osgood III, B.M. Clemens, R.L. White, S. Brennant \\ Department of Materials Science and Engineering,Stanford University \\ †Stanford Synchrotron Radiation Laboratory}

Magnetic anisotropy, the tendency for the magnetization of a ferromagnet to lie along a certain axis, is relevant to data storage technology. A highly anisotropic material can theoretically support a large density of information. Among the materials systems that promise a high storage density are thin films, whose properties are strongly affected by what happens at the interfaces of the magnetic thin film. It is equally important to understand the contribution of the strain state of the film to the total magnetic anisotropy.

Surface magnetic anisotropy (hereafter termed 'surface anisotropy') has its origin in the broken symmetry of the surface of the magnetic layer, whether that surface be bordering on vacuum or on a different material. It is typically inversely proportional to the thickness of the layer. Our goal was to compare the surface anisotropy of an epitaxial $\mathrm{Fe}(110) / \mathrm{Mo}(110)$ bilayer. Since the magnetization is confined to lie in the plane of the sample, we are interested only in the in-plane component of the anisotropy energy density, $K_{\|}$. It is composed of magnetocrystalline and magnetoelastic components ( $K_{1}$ and $k_{\epsilon}^{\|}$, respectively) and the surface anisotropy. The latter can be found by using torque magnetometry to measure $K_{\|}$and subtracting $K_{1}$ (a constant) and $k_{\epsilon}^{\|}$[1]. The magnetoelastic component $k_{\epsilon}^{\|}$can be calculated if the strain state of the film is known.

Samples were synthesized in a UHV chamber with base pressure of $1.0 \times 10^{-8}$ torr by $\mathrm{DC}$ magnetron sputtering. Single crystal (1120)-oriented $\mathrm{Al}_{2} \mathrm{O}_{3}$ substrates were cleaned with solvents and heated to $700^{\circ} \mathrm{C}$ for deposition of a 600 monolayer (henceforth ' $m$ l') Mo (110) underlayer. Previous work in [2] has shown that the Mo grows epitaxially on the $\mathrm{Al}_{2} \mathrm{O}_{3}$ with the $[\overline{1} 11]$ direction parallel to the [0001] axis of the $\mathrm{Al}_{2} \mathrm{O}_{3}$. The $\mathrm{Fe}$ layer was deposited at room temperature and capped with an insulating, transparent $100 \AA$ layer of SiC. Thicknesses were determined by calibration runs and rate monitors.

Five samples were deposited with a $600 \mathrm{ml}(=$ $1335 \AA$ ) thick, (110)-oriented Mo underlayer, a 100 $\AA$ ) thick $\mathrm{SiC}$ capping layer, and Fe layer thicknesses of $9,12,25,50$, and $400 \mathrm{ml}$ (the thickest Fe (110) sample had no SiC cap). Grazing inci- dence, symmetric, and asymmetric X-ray diffraction measurements were performed at the Stanford Synchrotron Radiation Laboratory (SSRL) Beamline $10-2$ with photon energy of $10 \mathrm{keV}$. These measurements allowed the complete determination of the stress and strain state as well as the unstrained lattice parameter of the $\mathrm{Fe}$ constituent of the bilayer. Torque magnetometry was used to determine the total in-plane uniaxial anisotropy $\left(K_{\|}\right)$. A small biaxial anisotropy was also present in the samples; its magnitude was zero to within our error bars, whose height is approximately equal to the fourthorder contribution from the crystalline anisotropy $\left(0.36 \times 10^{6} \mathrm{ergs} / \mathrm{cm}^{3}\right)$.

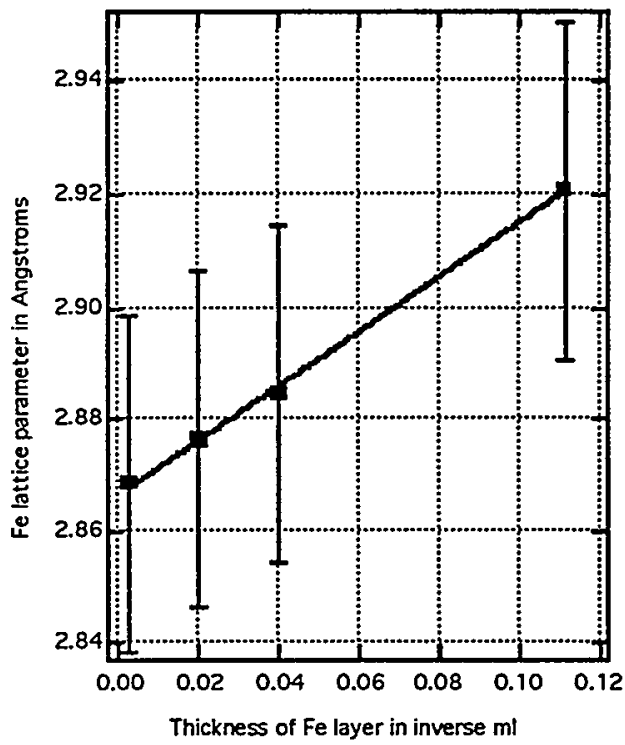

Figure 1: Fe lattice parameter as function of the inverse thickness of the Fe bilayer constituent.

The epitaxy of both the Mo underlayer and the bilayer was determined by laboratory X-ray sources and verified at SSRL. Rocking curves of the $\mathrm{Fe}$ constituent were typically $5^{\circ}$ wide. Grazing incidence and asymmetric $x$-ray scattering were used to observe three $\{121\}$ type reflections for these BCC structures. These three reflections are due to planes which are crystallographically identical, but oriented at different angles relative to the sample surface and in-plane axes, and therefore experience different strains. Least squares fitting was used to determine the values of the of in-plane stress (assumed unequal biaxial) and unstrained lattice pa- 
rameter which gave the best match to the observed $d$-spacings.

The change of the average $\mathrm{Fe}$ lattice parameter $a^{F e}$ as a function of the inverse thickness of the $\mathrm{Fe}$ layer is displayed in Fig. 1. The lattice parameter for the $12 \mathrm{ml}$ sample was not deducible from the data because of an error in data retrieval; we assume that it falls at the same value as that predicted by the fit to the other data points. Note that the fit to the data extrapolates to the Fe bulk lattice parameter $\left(a_{0}^{F c}=2.8664 \AA\right)$ at infinite thickness as it should, and also that the average lattice parameter scales linearly with the inverse thickness of the Fe layer, extrapolating to $3.36 \AA$ at $1 \mathrm{ml}$ coverage. This indicate that we may separate the average $\mathrm{Fe}$ lattice parameter into a bulk and surface contribution as indicated below:

$$
a^{F e}=\frac{a_{0}^{F e}(n-1) \mathrm{ml}+3.36 \AA 2 \times 1 \mathrm{ml}}{n \mathrm{ml}}
$$

where $n$ is the number of monolayers in the Fe bilayer constituent.

We can rewrite this equation to show explicitly the bulk and surface contributions to $a_{F e}$ :

$$
a^{F e}=a_{0}^{F e}+\left(3.36 \AA-a_{0}^{F e}\right) \frac{1 \mathrm{ml}}{n \mathrm{ml}} .
$$

We find this data consistent with a picture where the interdiffusion at the $\mathrm{Mo} / \mathrm{Fe}$ interface has resulted in a Vegard's law swelling of the Fe lattice due to incorporation of the larger Mo atoms. This is consistent with trends observed in [3].

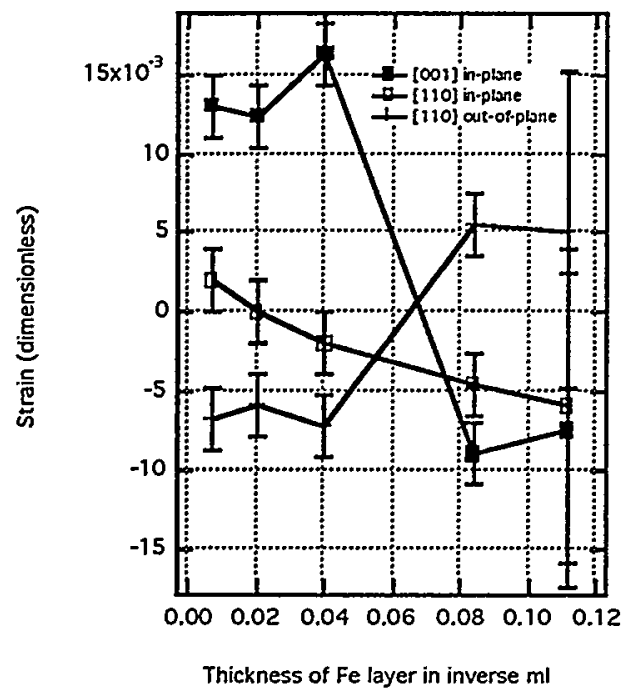

Figure 2: Strain state of the Fe bilayer constituent.
The strain in the Fe bilayer constituent is plotted in Fig. 2. The strain in the (001) direction is larger in magnitude, and becomes suprisingly negative (compressive) at small thicknesses. Similar behavior has been observed in $\mathrm{Fe}(100)$ films on $\mathrm{MgO}$ (100) [4], where the Fe film initially grows in an islanded fashion and then coalesces to form a continuous film under tension stress from the $\mathrm{MgO}$ substrate. Such an effect was not observed in an Fe (110) film grown epitaxially on Mo (110) in [2].

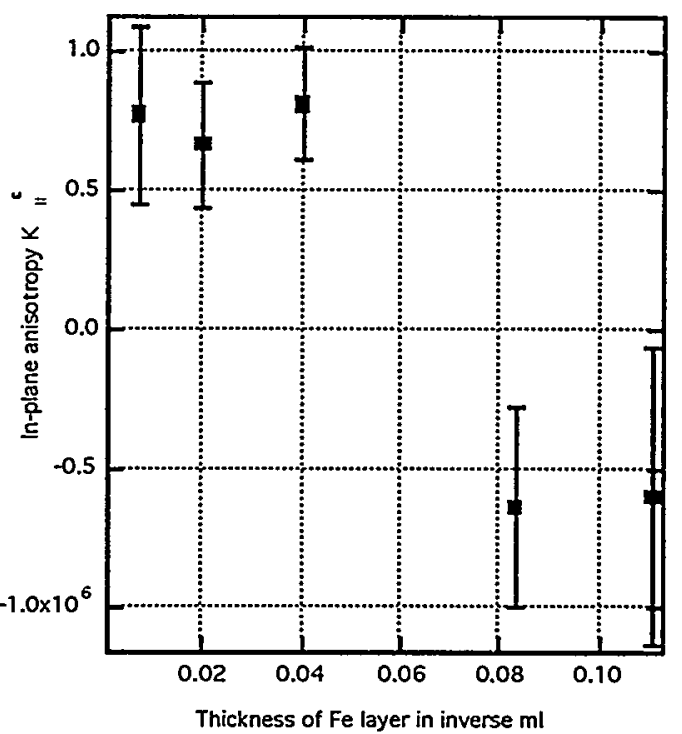

Figure 3: Magnetoelastic contribution to the magnetic anisotropy.

The magnetoelastic contribution to the magnetic anisotropy $\left(k_{\epsilon}^{\|}\right)$was calculated from the strain data and is displayed in Fig. 3. Positive $k_{\epsilon}^{\|}$prefers the (001) orientation of the magnetization. Subtracting this from $K_{\|}$gives the surface anisotropy, which is displayed in Fig. 4. Except for the thinnest samples, whose strain state is not completely well understood, the surface anisotropy is zero to within our error bars, in contrast to [1], where the surface anisotropy of the 'atomically smooth' surface of Fe (110) on W (110) prefers the (110) orientation of the magnetization. This is consistent with disorder at the interfaces; both interdiffusion and island formation [5] can lower the anisotropy of a surface.

Epitaxial, sputter-deposited $\mathrm{Fe}(110) / \mathrm{Mo}(110)$ bilayers with varying Fe thicknesses were prepared to study the surface anisotropy of the Fe bilayer constituent. X-ray diffraction was used to determine the unstrained lattice parameter and strain state of the Fe film. The unstrained lattice parameter of the $\mathrm{Fe}$ constituent increased linearly with the inverse thickness of the Fe layer, which would be expected from a Vegard's law swelling of the Fe lattice due to 
incorporation of the larger Mo atoms at the interface. The strain state of the Fe constituent shows evidence of structural imperfection, consistent with the surface anisotropy being zero within our error bars (except for the thinnest sample, which is unexpectedly in a state of compressive in-plane strain).

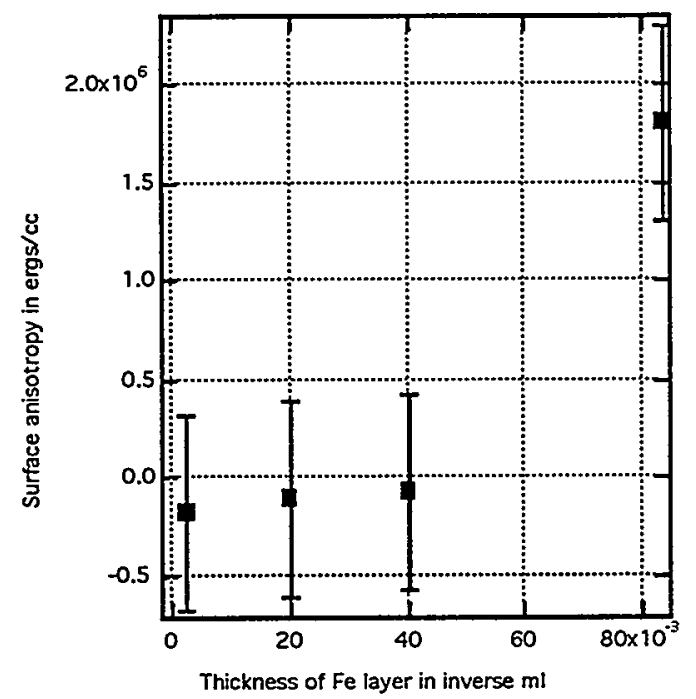

Figure 4: Surface anisotropy of the Fe bilayer constituent.

The authors would like to acknowledge funding for this project from NSF under contract No. 2DHZ452. One of us (RMO) acknowledges helpful discussions with and assistance from B. J. Daniels, C.-T. Wang, S. A. Newell, M. C. Kautzky, and J. $\mathrm{Xu}$.

1. H.J. Elmers and U. Gradmann, App. Phys. A, 51,255 (1990).

2. B.M. Clemens, R.M. Osgood, A.P. Payne, B.M. Lairson, S. Brennan, R.L. White, and W.D. Nix, J. Mag. Mag. Materials, 121, 37 (1993).

3. R.M. Osgood III, B.M. Clemens and R.L. White, Mechanisms of Thin Film Evolution, Materials Research Society (1994).

4. H.A. Atwater, E. Chason, M.H. Grabow, and M.G. Lagally, Evolution of Surface and Thin FIlm Microstructure, 280, 441 (1993)

5. M. Albrecht, T. Furubayashi, M. Przybylski, J. Korecki, and U. Gradmann, J. Mag. Mag. Materials, 113, 207 (1992). 


\section{THE EFFECT OF Cr DOPING ON THE MAGNETORESISTANCE AND SATURATION FIELD OF EPITAXIAL $\mathrm{Fe}_{1-x} \mathrm{Cr}_{x}(001) / \mathrm{Cr}(001)$ MULTILAYERS B.J. Daniels and B.M. Clemens Department of Materials Science and Engineering, Stanford University}

\section{Introduction}

Recent studies of "giant" magnetoresistance (GMR) in $\mathrm{Fe} / \mathrm{Cr}$ multilayers have revealed both large room temperature magnetoresistances in epitaxial multilayers [1-5] and increases in the magnetoresistance with $\mathrm{Cr}$ doping of the $\mathrm{Fe}$ layer in polycrystalline [6-9] and epitaxial [10] multilayers. Currently the GMR effect is believed to be due to spin-dependent scattering of electrons in these antiferromagnetically coupled multilayers. This preferential scattering has been postulated to occur both at interfaces and impurity sites throughout the layers, although the relative importance of each is still under debate $[9,11-18]$. Since the GMR is defined to be $\Delta \rho / \rho_{\text {sat }}$, one can increase the GMR by either increasing the spin-dependent scattering $(\Delta \rho)$ or decreasing the spin-independent scattering $\left(\rho_{\text {sat }}\right)$. Progress has been made toward reducing the spin-independent scattering via the fabrication of films with a greater degree of crystalline perfection [4], however methods for increasing the spin-dependent scattering are still lacking. Another practical consideration for these materials is the percent change in resistivity with field, which we will refer to as the sensitivity, $S$. This parameter, which can be defined as the slope of the GMR vs. H curve, depends not only on scattering, but also on the strength of the antiferromagnetic coupling. In this work we expand upon the approach of Petroff et al. [10] and report a technique to increase the spin-dependent scattering and sensitivity in $\mathrm{Fe} / \mathrm{Cr}$ multilayers. Specifcally we investigate the effect of $\mathrm{Cr}$ doping of the Fe layer on the GMR and saturation field of epitaxial $\mathrm{Fe}_{1-x} \mathrm{Cr}_{x}(001) / \mathrm{Cr}(001)$ superlattices.

\section{Experimental and Results}

Samples were deposited in a UHV sputter deposition chamber [19] equipped with three shuttered, 2-in. planar dc magnetron sputtering guns confocally aimed at a vertically-mounted substrate, 6 in. away. Organically cleaned, polished $1 \mathrm{~cm} \times 1 \mathrm{~cm}$ single crystal $\mathrm{MgO}(001)$ substrates were placed in the chamber which was then evacuated to a base pressure of $1 \times 10^{-9}$ Torr. All films were sput- tered in $3 \mathrm{mTorr}$ of Ar at a rate of approximately $1 \AA /$ s. Alloy layers were deposited by cosputtering from elemental targets. Deposition rates were determined by an oscillating quartz crystal rate monitor and corrected to account for the tooling factor for each gun and target. A $100 \AA \mathrm{Cr}$ seed layer was deposited at $600^{\circ} \mathrm{C}$, as measured by infrared optical pyrometry, to promote epitaxial growth of the multilayer $[4,5,20,21]$. Multilayers consisting of 50 bilayers of $14 \AA \mathrm{Fe}_{1-x} \mathrm{Cr}_{x} / 8 \AA \mathrm{Cr}$ where $\mathrm{x}=$ $0,0.1,0.2,0.3,0.4$, and 0.5 were then deposited sequentially at $180^{\circ} \mathrm{C}$.

Sample structure was determined by $\mathrm{x}$-ray diffraction (XRD). Symmetric scans taken with a powder diffractometer using $\mathrm{CuK} \alpha$ radiation and equipped with an exit beam monochromator showed only (002) and (004) film peaks. Asymmetric XRD was used to ascertain if this were due to strong texture or epitaxy in these multilayers. Phi scans were obtained with a three-circle diffractometer using $\mathrm{CuK} \alpha$ radiation. A phi scan of a $100 \AA \mathrm{Cr} /\left[14 \AA \mathrm{Fe}_{0.8} \mathrm{Cr}_{0.2} / 8 \AA \mathrm{Cr}\right]_{50}$ film is shown in Fig. 1(a). For the $\mathrm{Fe}(001) / \mathrm{Cr}(001)$ system only one combined peak can be distinguished for each $\mathrm{Fe}\{110\}$ and $\mathrm{Cr}\{110\}$ reflection since these materials differ in lattice parameter by only $0.7 \%$ and are not rotated relative to one another. The observed fourfold symmetry in $\phi$ indicates that these films grow in the cube-on-cube epitaxial orientation with the [001] normal to the sample surface $[4,5,20,21]$. These peaks have a full width at half maximum (FWHM) of less than $1^{\circ}$, indicating excellent crystalline quality. Peaks due to the $\mathrm{MgO}$ substrate can be seen $45^{\circ}$ from each film peak, indicating that both $\mathrm{Fe}$ and $\mathrm{Cr}$ grow with the Bain epitaxial orientation on $\mathrm{MgO}(001)[4,5,20-23]$. The diffraction spectra for films with different $\mathrm{Cr}$ concentrations were nearly identical to that shown in Fig. 1(a).

Low-angle superlattice data were obtained at the Stanford Synchrotron Radiation Laboratory (SSRL) using an x-ray wavelength of $1.5405 \AA$. Figure 1(b) shows a symmetric scan (upper curve) for a $100 \AA \mathrm{Cr} /[14 \AA \mathrm{Fe} / 8 \AA \mathrm{Cr}]_{50}$ film. The first order superlattice line can be seen at $2 \theta \approx 4.23^{\circ}$. Finite thickness oscillations are also clearly resolved in this figure, indicating the high quality 
of these epitaxial multilayers. The lower curve in this figure is an offset-Bragg scan with $\omega=0.1^{\circ}$. Although two orders of superlattice lines can be distinguished, the finite thickness oscillations can no longer be seen since the sample has been rocked off the symmetric $\left(\omega=0^{\circ}\right)$ spine in reciprocal space. The presence of these peaks in the diffusely scattered intensity, however, implies that the roughness in these multilayers is correlated $[24,25]$. A model proposed by Hood et al. [18] links correlated roughness to the large values of GMR observed in this system. The bilayer spacing, $\Lambda$, was determined to be $20.6 \AA$ from a fit to the superlattice peak positions. This is within $7 \%$ of the value calculated from rate monitor rates and is a constant offset for all samples measured. The inset of Fig. 1(b) shows a rocking curve through the first order superlattice peak at $2 \theta=4.23^{\circ}$. The broad peak in the non-specular intensity implies that the lateral length scale of the correlated roughness is small. Symmetric and offset-Bragg scans for multilayers with 10 and 20 at.\% $\mathrm{Cr}$ yielded similar results for both the calculated bilayer spacing and diffusely scattered intensity. However, the 10 at.\% Cr sample exhibited an extra small-angle diffraction peak at a position corresponding to a structural periodicity of about double that of the nominal bilayer spacing. The origin of this peak is unclear, but it may be evidence of a structural defect in this sample.

Magnetotransport measurements were made at the Francis Bitter National Magnet Laboratory using a $2 \frac{3}{16}$ in. diameter warm bore superconducting magnet. Sample temperature during these measurements was $15-20^{\circ} \mathrm{C}$. The field was swept from -1 to $30 \mathrm{kG}$ with a sweep rate of approximately $100 \mathrm{G} / \mathrm{s}$ while a standard four-point probe was used to determine the resistance of the sample. Resistivity, $\rho$, was obtained using the relationship: $\rho=\alpha t V / I$, where $\alpha$ is a correction factor related to the lateral size of the film, $t$ is the thickness of the film (both the multilayer and the Cr seed layer, $V$ is the voltage drop across the inner probes, and $I$ is the current forced between the outer probes. A correction factor of 4 [ideal $=\pi / \ln (2)=4.53$ ] was used for our probe spacing-to-film width ratio of approximately 8 [26].

Figure 2(a) shows magnetoresistance as a function of field for six different $100 \AA$ $\mathrm{Cr} /\left[14 \AA \mathrm{Fe}_{1-x} \mathrm{Cr}_{x} / 8 \AA \mathrm{Cr}\right]_{50}$ films with $\mathrm{H} \| \mathrm{I}$ \| [110]. GMR versus $H$ curves were also obtained for both $\mathrm{H} \| I \perp$ [100] and $\mathrm{H} \perp \mathrm{I} \|$ [110], however these showed no differences from the curves in Fig. 2(a). For alloys from 0 to 20 at.\% $\mathrm{Cr}$ the mag- netoresistance remains constant at approximately $31 \%$ before decreasing for larger $\mathrm{Cr}$ concentrations. This behavior can be interpreted in terms of the spin-independent scattering, manifested in the saturation resistivity $\left(\rho_{s a t}\right)$, and the spin-dependent scattering, shown by the change in resistivity $(\Delta \rho)$. These data are plotted in Fig. 2(b) and show that $\Delta \rho$ (the spin-dependent scattering) actually increases from 10.0 to $11.2 \mu \Omega-\mathrm{cm}$ for a 10 at.\% $\mathrm{Cr}$ alloy and to $10.5 \mu \Omega-\mathrm{cm}$ for a 20 at.\% Cr alloy before decreasing at higher $\mathrm{Cr}$ concentrations. This increase in spin-dependent scattering offsets an increase in $\rho_{\text {sat }}$ to keep the GMR constant over this range. We believe that the increase in the spin-dependent scattering is a result of preferential scattering from $\mathrm{Cr}$ impurities throughout the Fe layer, as was reported by Rensing et al. [9], although the functional form of this variation is still unclear. The spin-independent scattering $\left(\rho_{\text {sat }}\right)$ increases with increasing $\mathrm{Cr}$ content due to the introduction of more scattering centers in the $\mathrm{Fe}$ layers. This is in agreement with the classical theory of impurity scattering in metals. Although the value of $\rho_{\text {sat }}$ appears to be anomalously high for the film with 10 at.\% $\mathrm{Cr}$, this effect was also reported by Rensing et al. for a 10 at.\% $\mathrm{Cr}$ polycrystalline $\mathrm{Fe} / \mathrm{Cr}$ film [9]. This could be due to the structural difference noted earlier, but the origin of this increase in $\rho_{\text {sat }}$ is still unclear and further investigation is warranted.

At this point it is interesting to compare the effects of alloying the Fe layer in our epitaxial multilayers with the effects seen by Rensing et al. [9] in polycrystalline multilayers. The field-induced change in resistivity, $\Delta \rho$, is larger in the epitaxial films, with a maximum in $\Delta \rho$ of $11.2 \mu \Omega-\mathrm{cm}$ for 10 at. $\% \mathrm{Cr}$ as compared with $5.6 \mu \Omega$-cm for 20 at. $\%$ in the polycrystalline films. The larger value of $\Delta \rho$ in the epitaxial multilayers can be attributed to the longer electron mean free path which allows electrons to traverse the $\mathrm{Cr}$ layer without encountering a spin-flip scattering event. The maximum change in $\Delta \rho$ due to $\mathrm{Cr}$ alloying is larger in the polycrystalline system $(2.7 \mu \Omega$-cm for 20 at.\% $\mathrm{Cr})$ than in the epitaxial system $(1.2 \mu \Omega$-cm for 10 at.\% $\mathrm{Cr})$. We speculate that segregation of the $\mathrm{Cr}$ to grain boundaries in the polycrystalline films could result in a larger increase in the spin-dependent scattering than that observed in epitaxial films. The saturation resistivity in all of the epitaxial multilayers is lower than the lowest saturation resistivity observed in polycrystalline multilayers. This is presumably due to the absence of grain boundaries and other structural imperfections in the epitaxial 

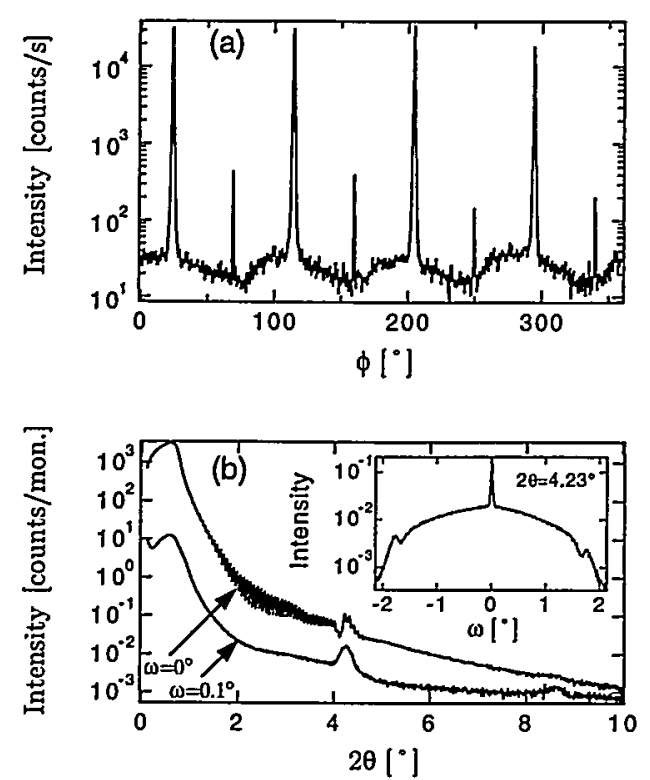

Figure 1: (a) XRD phi scan for a $100 \AA$ $\mathrm{Cr} /\left[14 \AA \mathrm{Fe}_{0.8} \mathrm{Cr}_{0.2} / 8 \AA \mathrm{Cr}\right]_{50}$ multilayer. The four small peaks $45^{\circ}$ from each film peak are due to the substrate. (b) Symmetric $\left(\omega=0^{\circ}\right)$ and offsetBragg $\left(\omega=0.1^{\circ}\right)$ low-angle XRD scans for a $100 \AA$ $\mathrm{Cr} /[14 \AA \mathrm{Fe} / 8 \AA \mathrm{Cr}]_{50}$ film. The inset shows a rocking curve through the first order superlattice peak.
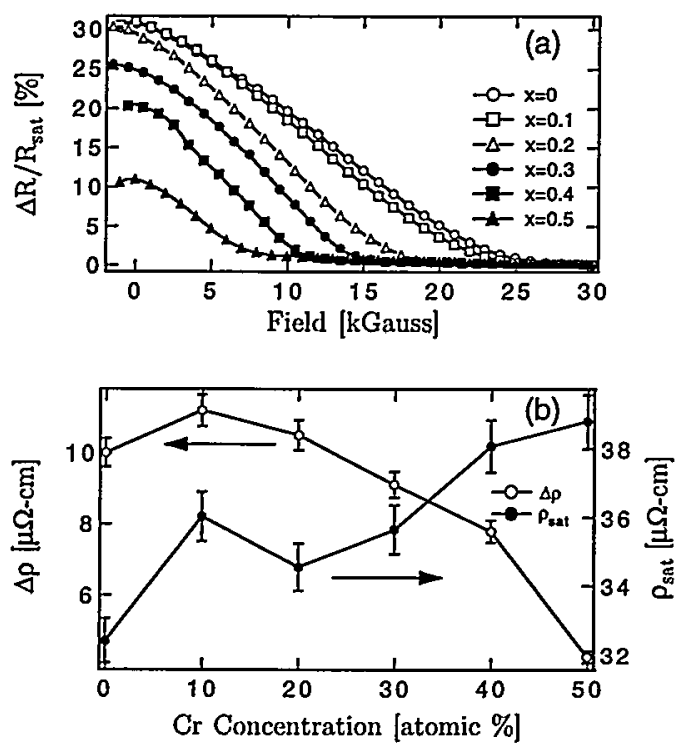

Figure 2: (a) $\Delta \mathrm{R} / \mathrm{R}_{\text {sat }}$ vs applied field and (b) $\Delta \rho$ and $\rho_{\text {sat }}$ vs $\mathrm{Cr}$ concentration for $100 \AA \mathrm{Cr} /\left[14 \AA \mathrm{Fe}_{1-x} \mathrm{Cr}_{x} / 8 \AA \mathrm{Cr}\right]_{50}$ multilayers. samples.

Another effect of alloying the magnetic layer is that the field required to saturate these films decreases linearly with increasing $\mathrm{Cr}$ concentration. This is the result of a decrease in the antiferromagnetic coupling strength with larger $\mathrm{Cr}$ concentrations and is consistent with the moment reduction observed in polycrystalline films [9]. By assuming the GMR versus $H$ curves are linear from zero field to saturation, we can calculate the effect of alloying on the sensitivity, $S$, of these films $\left[S=\partial(G M R) / \partial H \approx G M R / H_{\text {sat }}\right]$. These data, summarized in Table $I$, reveal a sensitivity increase of over $50 \%$ for alloy compositions of $20-40$ at.\% $\mathrm{Cr}$ when compared to the $\mathrm{x}=0$ film.

Table I: Values of GMR, $\mathrm{H}_{\text {sat }}$, sensitivity $(S)$, and change in sensitivity $(\Delta S)$ for six different $100 \AA \mathrm{Cr} /\left[14 \AA \mathrm{Fe}_{1-x} \mathrm{Cr}_{x} / 8 \AA \mathrm{Cr}\right]_{50}$ films.

\begin{tabular}{|c|c|c|c|c|}
\hline $\mathrm{Cr}[\%]$ & GMR $[\%]$ & $H_{\text {sat }}[k G]$ & $S[\% / k G]$ & $\Delta S[\%]$ \\
\hline 0 & 30.8 & 28 & 1.10 & 0 \\
\hline 10 & 31.0 & 26 & 1.19 & 8.2 \\
\hline 20 & 30.4 & 18 & 1.69 & 53.6 \\
\hline 30 & 25.5 & 15.5 & 1.65 & 50.0 \\
\hline 40 & 20.4 & 12 & 1.70 & 54.5 \\
\hline 50 & 10.9 & 8 & 1.36 & 23.6 \\
\hline
\end{tabular}

\section{Conclusions}

In conclusion, we have examined the structure and magnetotransport properties of six $\mathrm{Fe}_{1-x} \mathrm{Cr}_{x}(001) / \mathrm{Cr}(001)$ multilayers sputter-deposited onto $\mathrm{MgO}(001)$ substrates. Films with a nominal composition of $100 \AA \mathrm{Cr} /\left[14 \AA \mathrm{Fe}_{1-x} \mathrm{Cr}_{x} /\right.$ $8 \AA \mathrm{Cr}]_{50}$ where $0 \leq \mathrm{x} \leq 0.5$ were studied. XRD indicated that all films are high quality epitaxial multilayers. The room temperature magnetoresistance is constant at $31 \%$ for $\mathrm{x} \leq 0.2$ and decreases for larger $\mathrm{Cr}$ concentrations. An increase in the spin-independent scattering $\left(\rho_{\text {sat }}\right)$ with increasing $\mathrm{Cr}$ concentration is responsible for this decrease in the magnetoresistance. The spin-dependent scattering $(\Delta \rho)$ increases for alloys with 10 and 20 at.\% $\mathrm{Cr}$, due to preferential scattering at impurity sites throughout the $\mathrm{Fe}$ layer. This increase in $\Delta \rho$ is less than that observed for polycrystalline multilayers but offsets the increase in $\rho_{\text {sat }}$ to keep the GMR constant for $x \leq 0.2$. For larger concentrations of $\mathrm{Cr}$, the spin-dependent scattering decreases. The magnetic field required to saturate these multilayers decreases linearly with increasing $\mathrm{Cr}$ concentration due to a decrease in the strength of the antiferromagnetic coupling. By alloying the $\mathrm{Fe}$ layer with $20-40$ at.\% $\mathrm{Cr}$, the slope of the GMR versus $\mathrm{H}$ curve can be increased, resulting in an increase 
in the sensitivity of these films of over $50 \%$. It is expected that this technique can be applied to other multilayer systems, resulting in larger values for both the magnetoresistance and the sensitivity.

\section{Acknowledgements}

The authors are greatly indebted to the staff of the Francis Bitter National Magnet Laboratory, in particular Larry Rubin and Thang Vu, for assisting in the magnetotransport measurements of these films and to M.C. Kautzky for helpful discussions regarding this manuscript. SSRL is funded by the U.S. Department of Energy, Office of Basic Energy Sciences. The authors would also like to acknowledge the support of NSF Grant No. DMR9022248 and DMR-9100271.

\section{References}

[1] M.N. Baibich, J.M. Broto, A. Fert, F. Nguyen Van Dau, F. Petroff, P. Etienne, G. Creuzet, A. Friederich, and J. Chazelas, Phys. Rev. Lett. 61, 2472 (1988).

[2] A. Barthelemy, M.N. Baibich, J.M. Broto, R. Cabanel, G. Creuzet, P. Etienne, A. Fert, A. Friederich, S. Lequien, and F. Nguyen Van Dau, Mater. Res. Soc. Symp. Proc. 151, 43 (1989).

[3] A. Barthelemy, A. Fert, M.N. Baibich, S. Hadjoudj, F. Petroff, P. Etienne, R. Cabanel, S. Lequien, F. Nguyen Van Dau, and G. Creuzet, J. of Appl. Phys. 67, 5908 (1990).

[4] E.E. Fullerton, M.J. Conover, J.E. Mattson, C.H. Sowers, and S.D. Bader, Appl. Phys. Lett. 63, 1699 (1993).

[5] E.E. Fullerton, M.J. Conover, J.E. Mattson, C.H. Sowers, and S.D. Bader, Phys. Rev. B 48, 15755 (1993).

[6] L.H. Chen, T.H. Tiefel, S. Jin, R.B. Van Dover, E.M. Gyorgy, and R.M. Fleming, Appl. Phys. Lett. 63, 1279 (1993).

[7] N.M. Rensing and B.M. Clemens, J. Appl. Phys. in press (1995).

[8] N.M. Rensing, Ph.D. Dissertation, (Stanford University, 1994).

[9] N.M. Rensing, B.M. Clemens, and D.L. Williamson, submitted to J. Appl. Phys.
[10] F. Petroff, A. Barthelemy, A. Hamzic, A. Fert, P. Etienne, S. Lequien, and G. Creuzet, $J$. Magn. Magn. Mater. 93, 95 (1991).

[11] R.E. Camley and J. Barnás, Phys. Rev. Lett. 63, 664 (1989).

[12] M. Johnson, Phys. Rev. Lett. 67, 3594 (1991).

[13] P. Baumgart, B.A. Gurney, D.R. Wilhoit, T. Nguyen, B. Dieny, and V.S. Speriosu, J. Appl. Phys. 69, 4792 (1991).

[14] B.L. Johnson and R.E. Camley, Phys. Rev. B 44, 9997 (1991).

[15] B.A. Gurney, P. Baumgart, D.R. Wilhoit, B. Dieny, and V.S. Speriosu, J. Appl. Phys. 70, 5867 (1991).

[16] R.Q. Hood and L.M. Falicov, Phys. Rev. B 46,8287 (1992).

[17] S. Zhang, P.M. Levy, and A. Fert, Phys. Rev. $B$ 45, 8689 (1992).

[18] R.Q. Hood, L.M. Falicov, and D.R. Penn, Phys. Rev. B 49, 368 (1994).

[19] A.P. Payne, S. Brennan, and B.M. Clemens, Rev. Sci. Instr. 63, 1147 (1992).

[20] B.J. Daniels, W.D. Nix, and B.M. Clemens, Mater. Res. Soc. Symp. Proc. 343, 549 (1994).

[21] B.J. Daniels, W.D. Nix, and B.M. Clemens, Thin Solid Films 253, 215 (1994).

[22] B.M. Lairson, A.P. Payne, S. Brennan, N.M. Rensing, B.J. Daniels, and B.M. Clemens, Mater. Res. Soc. Symp. Proc. 280, 441 (1993).

[23] A.P. Payne, B.M. Lairson, S. Brennan, B.J. Daniels, N.M. Rensing, and B.M. Clemens, Phys. Rev. B 47, 16064 (1993).

[24] D.E. Savage, J. Kleiner, N. Schimke, Y.-H. Phang, T. Jankowski, J. Jacobs, R. Kariotis, and M.G. Lagally, J. Appl. Phys. 69, 1411 (1991).

[25] A.P. Payne and B.M. Clemens, Phys. Rev. B 47,2289 (1993).

[26] W.F. Beadle, J.C.C. Tsai, and R.D. Plummer, Eds., Quick Reference Manual for Semiconductor Engineers (Wiley, New York, 1985). 
Sources of strain in rapidly grown crystals of $\mathrm{KH}_{2} \mathrm{PO}_{4}$

James J. De Yoreo, Zofia U. Rek*, Natalia P. Zaitseva and Bruce W. Woods

Lawrence Livermore National Laboratory, Livermore, CA 94550

*Stanford Synchrotron Research Laboratory, Stanford, CA 94309

Due to their interesting electrical and optical properties, structural phase transitions, and ease of crystallization, $\mathrm{KH}_{2} \mathrm{PO}_{4}(\mathrm{KDP})$ and its isomorphs have been the subject of a wide variety of investigations for over 40 years 1,2 . Today, KDP and its deuterated analog, $\mathrm{KD}_{2} \mathrm{PO}_{4}$ (DKDP), are widely used to control the parameters of laser light such as pulse length, polarization and frequency through the first and second order electro-optic effects 3,4 .

Efficient operation of electro-optic devices such as Pockels cells and frequency converters requires crystals with a high degree of perfection. In particular, internal strains in the crystals generate spatial variations in the refractive index tensor through the stress-optic effect. 5 While these phenomena are a minor issue for the small crystals typically used in laboratory research applications, the effect of strain is the limiting factor on performance in applications requiring large aperture crystals such as inertial confinement fusion ${ }^{6}$ and high average power laser systems ${ }^{7}$. De Yoreo et al. ${ }^{8}$ analyzed the effect of internal stresses on the refractive index tensor and quantitatively related the magnitude of the stresses to experimentally determined variations in the transmitted wave front and beam polarization in KDP and DKDP crystals grown by conventional techniques. Recently, Zaitseva et al. ${ }^{9}$ described a method for growing bulk single-crystals of KDP from solutions at high supersaturation which produces growth rates of ten to fifty times those obtained with conventional methods. The purpose of this paper is to describe the results of X-ray topographic studies on KDP crystals grown by this technique. We show that strain in these crystals is caused primarily by three sources: dislocations, variations in composition between adjacent growth sectors of the crystal and variations in composition between adjacent sectors of vicinal growth hillocks within a single growth sector of the crystal. We find that the compositional variations cause variations in the refractive index and induce distortion of the transmitted wave front while large groups of dislocations are responsible for strain induced birefringence which leads to beam depolarization.

Single crystal boules of KDP grown at high

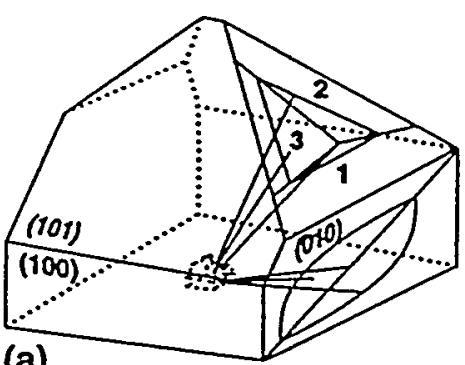

(a)

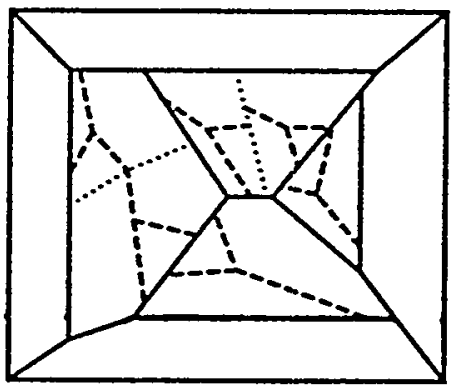

(b)

Figure 1: (a) Illustration of the growth habit of a single crystal boule of KDP showing the location of the seed, dislocations and the geometry of vicinal growth hiilocks. (b) Location of sector boundaries in an plate of KDP cut perpendicular to the $\{001\}$ axis.

supersaturation advance on both the $\{101\}$ (pyramidal) and $\{100\}$ (prismatic) facets of the crystal leading to a pyramidal crystal habit as shown in Figure 1a. Advance of the crystal face on both sets of facets occurs on steps generated at vicinal growth hillocks formed by dislocations emanating either from the seed (see Figure 1a) or from foreign inclusions incorporated during growth. As Figure $1 \mathrm{~b}$ shows, the vicinal hillocks on the $\{101\}$ face have an asymmetric triangular pyramidal geometry. The sectors of the vicinal hillock with the shallowest (sector 3) and steepest (sector 1) slopes generate steps oriented along the pyramid-pyramid and pyramidprism boundaries respectively. The crystals used for this study were $1 \mathrm{~cm}$ thick plates oriented with the normal to the plate along the $<001>$ axis and were cut from the full cross section of single crystal boules. In general, such crystals contain eight 
different growth sectors corresponding to the eight $\{101\}$ and $\{100\}$ directions. Within the individual $\{101\}$ sectors, lie sub-boundaries corresponding to the division between the three sectors of the vicinal hillocks (vicinal sector boundaries) as well as the boundaries between adjacent vicinal hillocks (intervicinal boundaries).

Figure 2a shows a composite of white beam $\mathrm{X}$ ray topographs of a $8.8 \times 7.6 \mathrm{~cm} \mathrm{KDP} \mathrm{crystal} \mathrm{cut} \mathrm{from}$ the central portion of a boule grown at $5 \mathrm{~mm} /$ day along the $<001>$ axis. The $\{101\}-\{101\}$ boundaries are faintly visible and there is pronounced contrast between the $\{101\}$ and the $\{100\}$ sectors. This contrast is also seen in the transmitted wave front profile in Figure $2 b$, showing that this contrast is correlated with a variation in the optic index of refraction of the crystal, a reflection of its composition. The results support those of

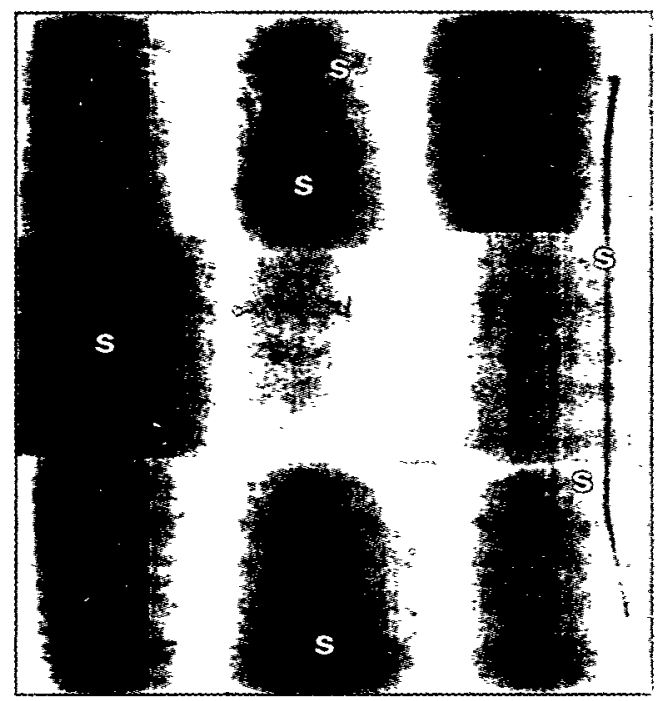

(a)

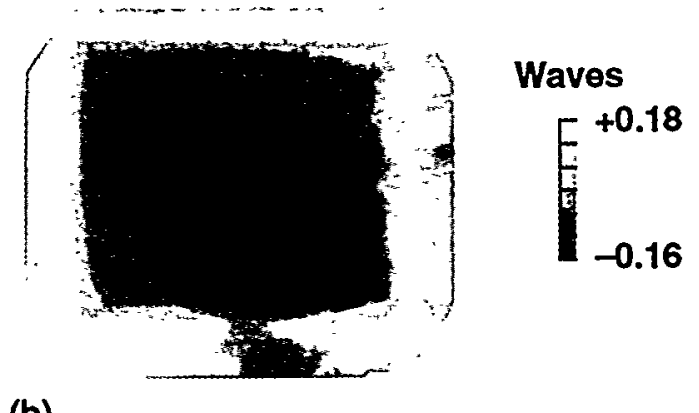

(b)

Figure 2: (a) Composite white beam X-ray topograph and (b) transmitted wave front profile of an (001) plate of KDP with dimensions $8.8 \times 7.6 \times 1.0 \mathrm{~cm}^{3}$. Scrystal sector boundaries. previous investigators 10 which showed that impurities are preferentially incorporated on the $\{100\}$ faces of KDP.

Figure $3 \mathrm{a}$ shows a composite of X-ray topographs of a portion of a $11.5 \times 10.0 \mathrm{~cm} \mathrm{KDP}$ crystal cut from the upper portion of a boule grown at $13 \mathrm{~mm} /$ day along $<001\rangle$. There are three main features to this topograph: pyramid-pyramid sector boundaries (S), numerous groups of dislocations (D) and a set of domain-like structures with rectilinear boundaries which correspond to vicinal sector boundaries $(\mathrm{V})$ and intervicinal boundaries $(\mathrm{I})$. The shallowest sector exhibits the highest contrast relative to the other two sectors indicating that its
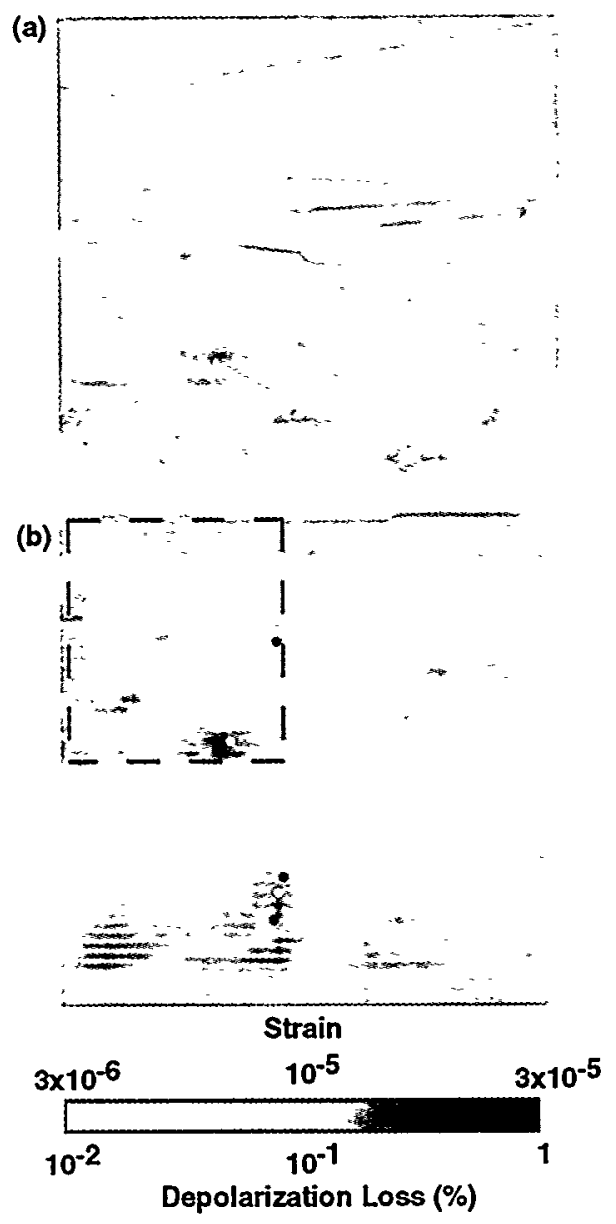

Figure 3: (a) Composite white beam X-ray topograph and (b) depolarization profile of an (001) plate of KDP with dimensions $11.5 \times 10.0 \times 1.0 \mathrm{~cm}^{3}$. The dashed line in (b) gives the location of the topograph. S- crystal sector boundaries, V- vicinal sector boundaries, I- intervicinal boundaries, D- strong dislocation bunches, and $\mathbf{T}$ - tops of growth hillocks. 
lattice parameters are the most dissimilar. Smolskii 11 suggested that the contrast was due to variations in impurity content caused by differences in the segregation coefficient for the three step directions. The effect of these defects on the birefringence of the crystal is seen in the depolarization profile given in Figure $3 \mathrm{~b}$. While the sector boundaries are clearly visible in this profile, only the groups of dislocations with the strongest contrast cause significant levels of beam depolarization.

The effect of vicinal sectorality on the optic index of refraction is illustrated in Figure 4 which shows a composite of white beam X-ray topographs of a crystal cut from the upper portion of an
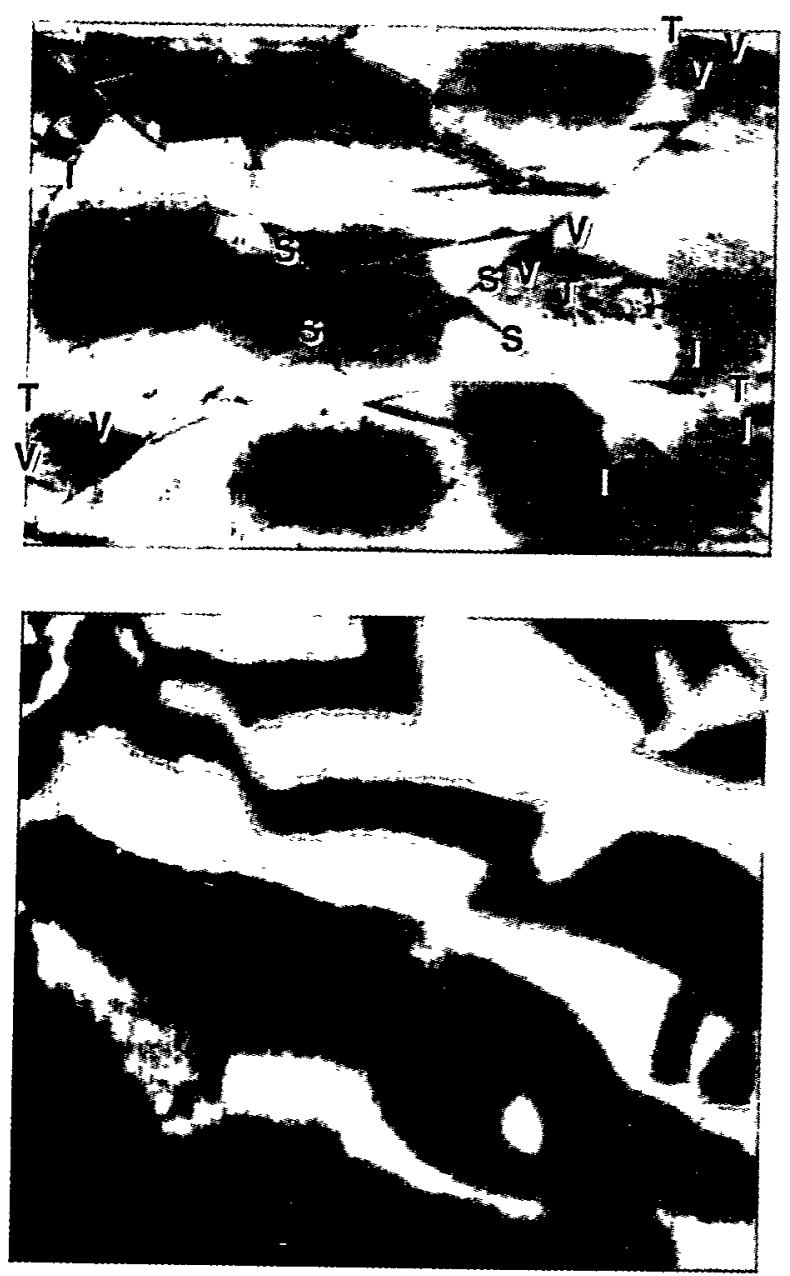

(b)

Figure 4: (a) Composite white beam X-ray topograph and (b) static fringe interferogram of an (001) KDP plate with dimensions $8.5 \times 7.5 \times 1 \mathrm{~cm}^{3}$. In the absence of bulk index inhomogeneities, the fringes would be straight and parallel. Symbols are as in Figure 3.
$8.5 \times 7.5 \mathrm{~cm}$ boule grown at $30 \mathrm{~mm} /$ day and the corresponding static fringe interferogram. As Figure 4a shows, vicinal sectorality is strongly pronounced in this sample. Comparison of Figure $4 a$ and $b$ shows that the breaks and distortions in the fringes coincide with the locations of vicinal and intervicinal sector boundaries. These results demonstrate that vicinal sectorality is strongly correlated with variations in optic index of refraction and supports the hypothesis that the contrast in the topographs is caused by differences in impurity content between adjacent vicinal sectors.

The results presented here show that optical distortion from KDP crystals can be related to defects visible with $\mathrm{X}$-ray topography. Strong bundles of dislocations cause high levels of strain induced birefringence while differences in composition between adjacent sectors of the crystal as well as vicinal hillocks on the faces of the growing crystal generate variations in the optic index of refraction.

This work was performed under the auspices of the Division of Material Sciences, US Department of Energy and Lawrence Livermore National Laboratory under Contract No. W-7405-ENG48.

1. For a review on crystal growth see: Rashkovich, L.N., KDP-Family Single Crystals (Adam Higer, New York, 1991).

2. For a review on KDP structure, properties and applications see:Ferroelectrics, 71 and $\mathbf{7 2 ,}$ (1987).

3. Zernicke, F. and Midwinter, J.E., Applied Nonlinear Optics (John Wiley \& Sons, New York, 1973).

4. Eimerl, D., Ferroelectrics, 72, 95 (1987).

5. Nye, J. F., Physical properties of crystals, ch. 13 (Oxford University Press, New York, 1985).

6. J.J. De Yoreo, and B.W. Woods, in: Inorganic crystals for optics, electro-optics and frequency conversion, Proc. Int. Soc. Optic. Engin., SPIE,1561, P.F. Bordui, Ed. (SPIE, Bellingham, WA ,1991, p.50).

7. Eimerl, D., IEEE Journal of Quantum Electronics, 23, 2238 (1987).

8. De Yoreo, J.J. and Woods, B.W., J. App. Phys. 73, 7780 (1993).

9. N.P. Zaitseva, I.L. Smol'skii and L.N. Rashkovich, Kristallografiya 36, 113 (1991).

10. Belouet, C., Dunia, E. and Petroff, J.F., J. Cryst. Growth, 29, 109 (1975).

11. I.L. Smol'skii, A.A. Chernov, Yu. G. Kuznetsov, V.F. Parvov, and V.N. Pozhanskii, Sov. Phys. Crystallogr. 30, 971 (1985). 
Proposal 2229Mp

\title{
Mechanics of Advanced Composites Using X-ray Tomographic Microscopy
}

\author{
Thomas M. Breunig, and Monte C. Nichols \\ Sandia National Laboratories, Livermore, CA 94551-0969 \\ John H. Kinney, and David L. Haupt \\ Lawrence Livermore National Laboratory, Livermore, CA 94551
}

\section{INTRODUCTION}

The macromechanical responses of composite materials exhibit a complex dependence on the microstructure present, the processing method used to develop the microstructure and the way damage is initiated and accumulated during deformation. Unlike monolithic materials, damage in composite microstructures frequently initiates internally and the associated changes in local properties may not significantly change a composite's macroscopic properties. Until recently, the only way to threedimensionally analyze the microstructure of an opaque material was with time consuming serial sectioning, a destructive technique that is acknowledged to be artifact prone [1] and requires that applied loads be removed before examination. X-ray computed tomography is uniquely suited for monitoring damage accumulation since the incremental changes in internal microstructure can be examined within a single sample at multiple loads during the course of an experiment $[2,3]$. Since for most composites, the size scale of the damage features of interest is less than $10 \mu \mathrm{m}$, high resolution X-ray tomographic microscope (XTM) is ideally suited to provide the three-dimensional resolution and sensitivity required [4].

We have designed a servo-mechanical load frame for use with the XTM which allows imaging of samples under load. The XTM load frame (XTM-LF) is capable of generating tensile or compressive forces up to 15.6 $\mathrm{kN}$. The test specimen is rotated through $360^{\circ}$, without induced bending or torque, by synchronously rotating the grips on precision bearings. With this load frame it is possible to image internal damage development during the application of a monotonic or low frequency cyclic load. This is accomplished by interrupting the test and maintaining a fixed load (or displacement) during the XTM data collection procedure.

\section{EXPERIMIENTS}

The materials examined included three man-made composites, and three natural biological composites. An example of both composite material types will be presented. The man-made composite system is a ceramic matrix composite with carbon fibers in a silicon carbide matrix. The biological composite is a section of trabecular bone from the proximal tibia of a sheep. These initial experiments were conducted in compression.

XTM data was collected prior to the application of load (a slight preload was used to hold sample in place) to provide an initial material condition for comparison purposes. The compression test were performed in displacement control with the specimen mounted between a fixed and a spherically mounted compression platen. During the deformation process and XTM data collection, both the applied load and displacement were continuously monitored. The deformation tests were interrupted at pre-set displacement levels or after critical deformation response events. During XTM data collection scans, the deformation conditions were maintained in displacement control. The volume of material examined was based on the region of critical interest for modeling deformation damage development.

\section{RESULTS}

A carbon fiber/SiC matrix ceramic matrix composite material system was examined. The material was fabricated by duPont during 1990. A balanced, plain-weave, fabric of T-300 PAN carbon fibers was layed-up with a $0 \% 190^{\circ}$ orientation in the sample examined. The panel was densified using diffusive chemical vapor infiltration (CVI). The mechanical compression test specimen was cut parallel to the cloth weave from a $3.62 \mathrm{~mm}$ thick panel. The specimen geometry used was straight sided with a width of $3.26 \mathrm{~mm}$ and a length of $9 \mathrm{~mm}$. The major fiber direction was parallel to the load axis (specimen 
length). This specimen was large enough to contain multiple unit cells.

Figure 1 shows the engineering stress-strain plot of the data collected. XTM data collection is indicated by the circled regions on the plot. XTM data was also collected prior to the application of load. For this material system data was collected over the entire test specimen. The total time required for XTM data collection at each load was approximately four hours. During that time the deformation conditions were maintained in displacement control. The amount of load drop in four hours was less than two percent. The XTM data collection displacements were selected to determine the undeformed microstructure, linear elastic response, microstructural transformations associated with the change in stiffness, and final fracture. The moduli measured from this test specimen were 11.4 $\mathrm{GPa}$ and $20 \mathrm{GPa}$. The modulus changed at $120 \mathrm{MPa}$ $(1,416 \mathrm{~N})$ and $1.08 \%$ strain with final fracture occurring at $383 \mathrm{MPa}(4,520 \mathrm{~N})$ and $2.47 \%$ strain (222 $\mu \mathrm{m}$ displacement). These mechanical properties results are in good agreement with the preliminary data from duPont [9].

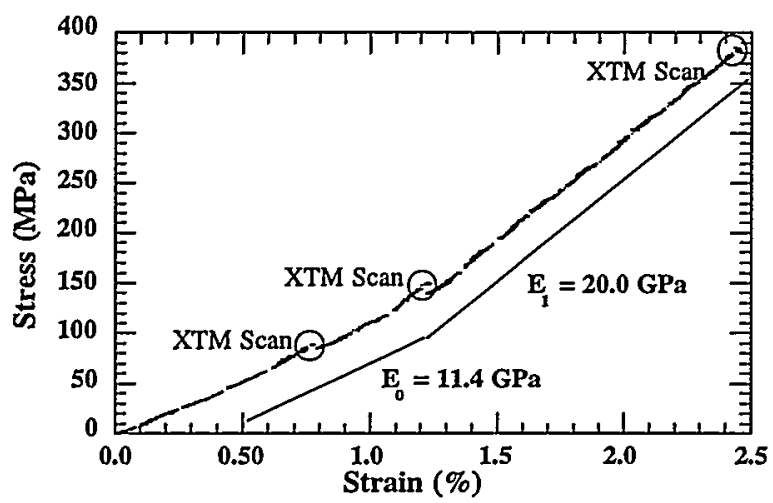

Figure 1. Engineering stress-strain plot for a carbon fiber/SiC matrix composite. XTM data was collected before deformation and at the deformation levels indicated by the circles on the plot. The first change in modulus occurred at $120 \mathrm{MPa}$ and $1.08 \%$ strain with final fracture at $383 \mathrm{MPa}$ and $2.47 \%$ strain.

A representative vertical section parallel to the fiber plies of this material is shown in Figure 2a. The distinctive over-under pattern of the woven fiber tows can be clearly identified. Figure $2 b$ is a vertical section normal to the fiber plies in the test specimen at failure. Figure $2 \mathrm{c}$ is a cross-section of the test specimen at failure. The white and light colored regions are the $\mathrm{SiC}$ deposited by the CVI process, the dark gray regions are the carbon fibers and black represents air.

The incomplete consolidation characteristic of diffusional CVI can be seen clearly in these Figures.
The thick deposits of the matrix material on the surface of the sample blocks the gas paths to the interior of the sample and stops the consolidation process. The result is large voids between the fiber tows in each cloth and between cloth layers. The SiC coating on the individual fiber tows can be identified. The balanced plain-weave of the T-300 PAN fiber based fabric is apparent in the section parallel to the fiber plies. The over and under character of the weave can be readily identified and characterized. From these images and the measured linear attenuation coefficients, it has been determined that only small quantities of $\mathrm{SiC}$ were deposited inside the carbon fiber tows.

The cause for the change in modulus observed in Figure 1 is not readily apparent from direct observation of the microstructures in Figures 2. The total structural strain displacement difference between the images collected before and after the modulus change was less than $0.5 \%$ strain, or $45 \mu \mathrm{m}$ displacement. When the numeric data are more closely examined, several changes can be detected. Between the initial structure and the linearly deformed structure there was a decrease in the total number of low density voxels associated with the pore structure. After the specimen changed in modulus there was an increase in the number of low density pore voxels. The total number of low density pore voxels after the modulus change remained smaller than detected in the initial microstructure. These results indicate that there was some type of failure that allowed some strain accommodation. The most probable event to cause this type of response would be cracking of the $\mathrm{SiC}$ deposited on the carbon fiber bundles. The resulting increase in structural modulus associated with the matrix cracking indicates that the deformation structure is distributing the load more evenly over all of the fiber plies in the structure.

The final test specimen failure was graceful with the load dropping slowly as the ply delaminations and fiber tow kinking occurred. The displacements caused by the failure event were not constrained to the crossply orientation. The in-ply displacements were most notable at the samples surface where fiber tows appears to be turned at an angle and protrude from the side of the specimen. The final failure process also caused the $\mathrm{SiC}$ coating on a large number of fiber tows to crack. In the center of Figure 2c, the $\mathrm{SiC}$ deposit between two fiber tows have failed and the fiber tows have remained intact. There are two major delaminations present in the final structure. These delaminations are contained in the inner fiber plies where there has not been as much $\mathrm{SiC}$ deposited on the fiber tows. The failure in the outer plies where there was more $\mathrm{SiC}$ deposited on the fiber tows appears to be more shear in 
nature. The failure modes observed are shear, fibermatrix delamination and kinking of fiber bundles.

The load-displacement records for the trabecular bone samples is shown in Figure 3. The displacement rate used for these tests is considered to be very slow when compared to normal physiological processes. The first deformation step was continuous with a total of $2 \%$ strain introduced into the sample. The load increased to $135 \mathrm{~N}$ and relaxed to $45 \mathrm{~N}$. After completion of the second XTM data collection scan, the sample was deformed additional $2 \%$ at the same strain rate used for the first deformation sequence. As the load increased on the sample, the deformation response appears to be following the initial loaddisplacement trace. A dotted line is included in Figure 3 to indicate the continuous response trace. The load displacement record in Figure 3 is interesting because it appears that when the loading process was continued after the second XTM scan, the load increased from the steady state value back to the initial response trace. The dotted line in Figure 3 is an estimate of the continuous load-displacement trace.

The general trend of the stress and strain data are consistent with observations of Carter and Hayes [5] for samples containing marrow and deformed with a strain rate of $0.01 \mathrm{~s}^{-1}$. The compressive stress was taken to be the maximum stress achieved prior to pore collapse and the modulus was taken to be the slope of the stress-strain curve in the most linear portion of the pre-yield loading region. Using these definitions, the modulus from the sheep trabecular bone sample is 82 $\mathrm{MPa}$ and the compressive strength is between $1.4 \mathrm{MPa}$ and 1.7 $\mathrm{MPa}$. The compressive strength and modulus measured on the sheep bone sample is within the range reported in the literature [5].

The trabecular bone sample shown in Figure 4 is $15 \mathrm{~mm}$ long and $8 \mathrm{~mm}$ in diameter. The region examined with XTM was $8 \mathrm{~mm}$ in height and covered the region containing the physis (growth plate). Figure $4 \mathrm{a}$ is a tomographic slice through the trabecular bone above the physis. From this image the general trabecular microstructure is apparent. At the perimeter of the sample there are a series of bone fragments embedded into the marrow space. They were formed and embedded by the machining process used to fabricate the test specimen. The presence of these bone chips at the samples surface in the marrow space does not have an effect on the mechanical properties measured. A representative vertical section through the data showing the physis is shown in Figure $4 \mathrm{~b}$. The vertical section location is indicated in Figure 4a by two dashed lines and a solid line. The goal of examining this type of samples is to determine how displacements and strains are transferred across the growth plate. An extension of the direct observations will be to measure the changes in stereologic and topologic properties of the structure. If the mean spacing of the trabeculi changes as a function of load, will the changes be the same for the regions above and below the growth plate? Will the growth plate deform or change orientation because of the deformation? The microstructural changes associated with the deformation states examined should be helpful in explaining why the stresses and strains change in the manner observed.

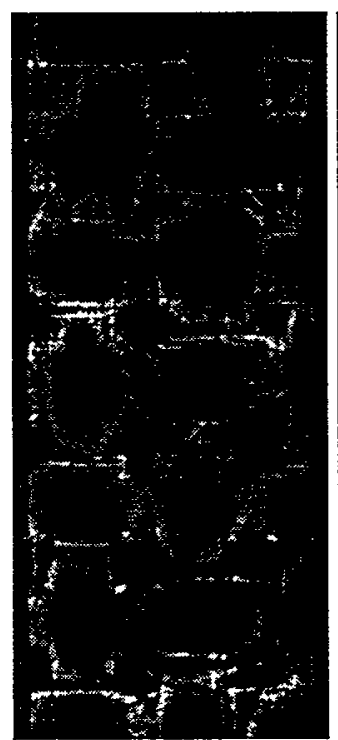

a)

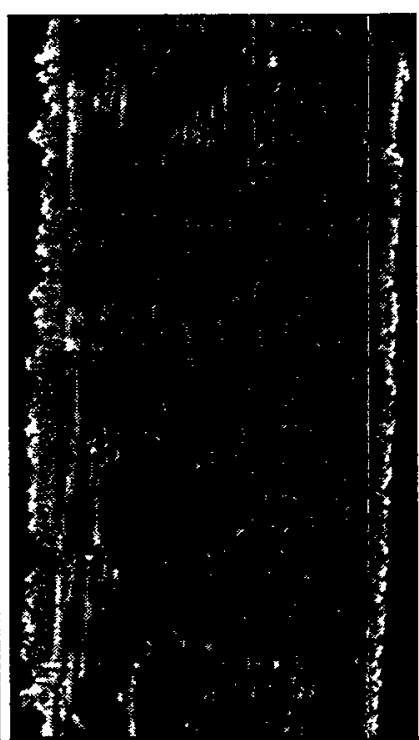

b)

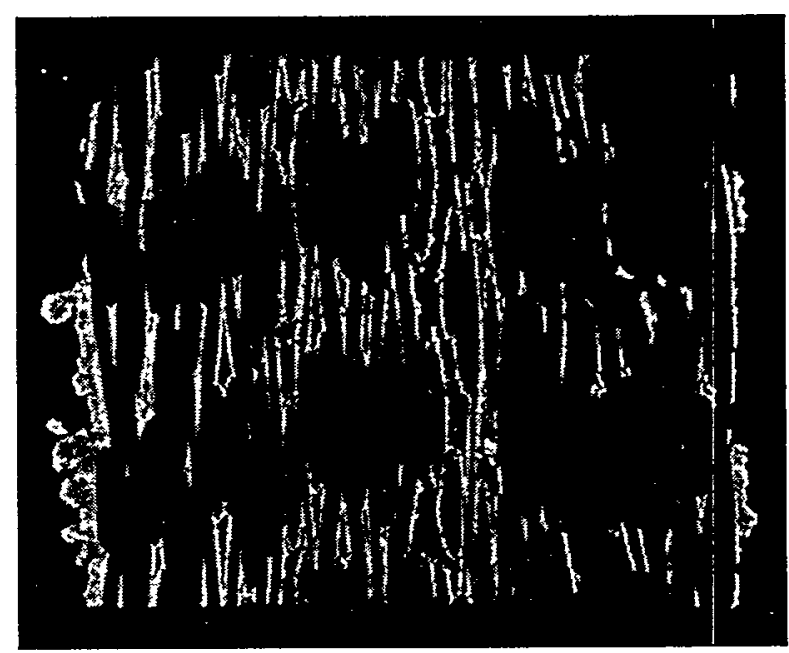

c)

Figure 2. XTM sections from a carbon fiber/SiC matrix composite under load. a) Vertical slice parallel to the plies showing the weave of the carbon fiber bundles. b) Vertical section normal to the fiber plies at failure. c) Cross-sectional slice through the failed sample. Failure is at the interface between the SiC matrix and the fiber bundles. (2a and $2 \mathrm{~b}-18 \mu \mathrm{m}$ spatial resolution. $2 \mathrm{c}$ ) $5 \mu \mathrm{m}$ spatial resolution.) 


\section{SUMMARY}

During the first year of this proposal we have tested the XTM-LF's mechanical stability and have verified that the rotation synchronization precision was adequate for high resolution XTM data collection. The material systems examined were intended to represent a variety of synthetic and biologic composite microstructures. The mechanical properties measured with the XTM-LF during imaging are in excellent agreement with previously published results.

\section{REFERENCES}

1. E.R. Weibel, "Measuring Through the Microscope: Development and Evolution of Stereological Methods," J. Microscopy, Vol. 155, Pt 3, 1989, pp. 393-403.

2. J.H. Kinney, T.M. Breunig, T.L. Starr, D. Haupt, M.C. Nichols, S.R. Stock, M.D. Butts, and R.A. Saroyan, "X-ray Tomographic Study of Chemical Vapor Infiltration Processing of Ceramic Composites", Science, Vol. 260, 7May, 1993, pp.789-792.

3. T.M. Breunig, S.R. Stock, S.D. Antolovich, J.H. Kinney, W.N. Massey, and M.C. Nichols, "A Framework for Relating Macroscopic Measures and Physical Processes of Crack Closure Illustrated by a Study of Aluminum-Lithium Alloy 2090," Fracture Mechanics: Twenty-Second Symposium (Volume I), ASTM STP 1131, H.A. Ernst, A. Saxena, and D.L. McDowell, Eds., American Society for Testing and Materials, Philadelphia, 1992, pp. 749-761.

4. J.H. Kinney, and M.C. Nichols, "X-ray Tomographic Microscopy (XTM) Using Synchrotron Radiation," Ann. Rev. Mater. Sci. Vol. 22, 1992, pp. 121-152.

5. D.R. Carter and W.C. Hayes, "The Compressive Behavior of Bone as a Two-Phase Porous Structure, J. Bone and Joint Surgery, Vol. 59-A, 1977, pp. 954962.

\section{ACKNOWLEDGMENTS}

This work was supported under the auspices of the U.S. DOE by Sandia National Laboratories under contract DE-AC04-76DP00789 and by the Lawrence Livermore National Laboratory under contract W7405-Eng-48; XTM experiments were performed at the Stanford Synchrotron Radiation Laboratory, which is supported by the U.S. DOE, Office of Basic Energy Sciences.

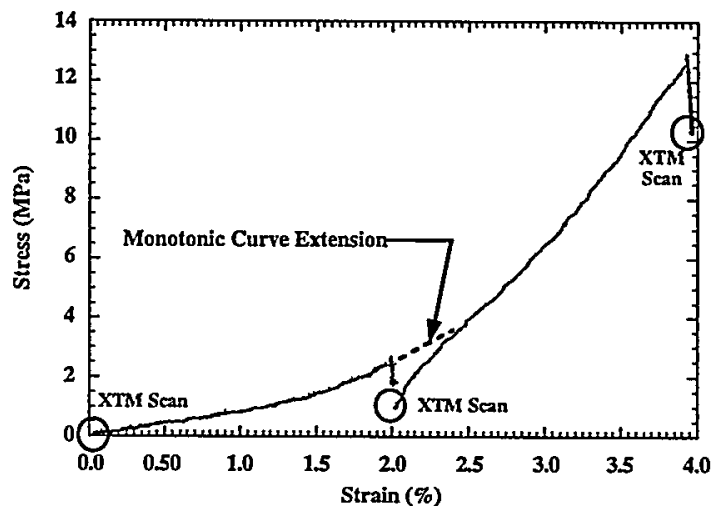

Figure 3. Engineering stress-strain plot for trabecular bone from a sheep's proximal tibia. XTM data was collected before deformation and at the deformation levels indicated by the circles on the plot.

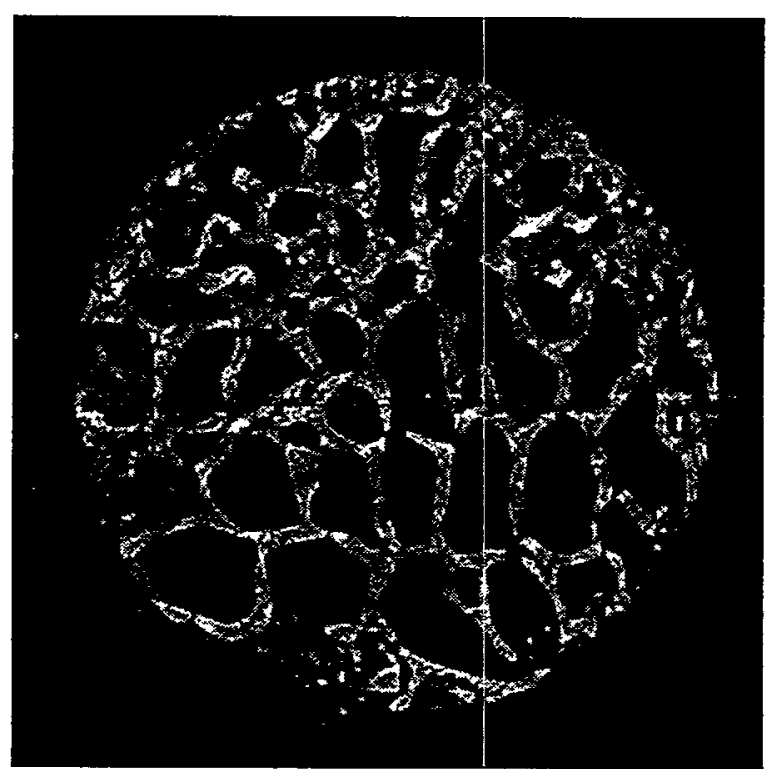

a)

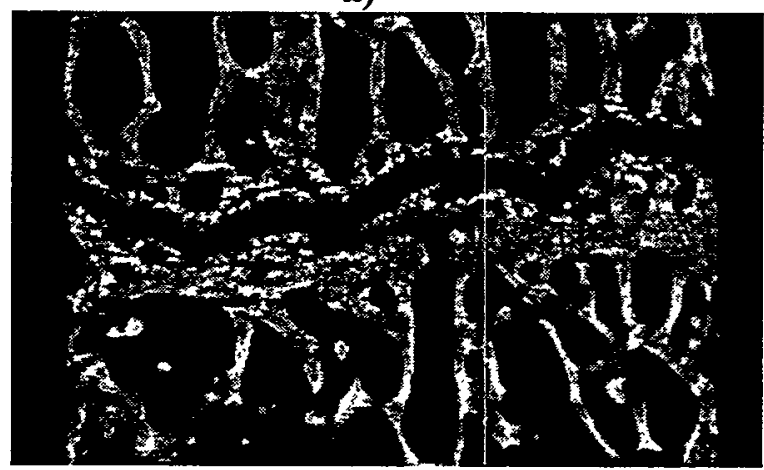

b)

Figure 4. Tomographic images of the trabecular bone structure from the proximal tibia of a sheep. The sample displacement is being held at $2 \%$ strain in these images. The cross-section image in a) shows some bone chips from the fabrication process along the circumference. The location of the vertical section shown in b) is indicated by the solid and dashed lines in a). 


\section{Studies of Actinides by X-ray Absorption Spectroscopy}

P. G. Allenn, ${ }^{1,6}$ I. Almahamid, ${ }^{2}$ R. A. Andersen, ${ }^{1}$ G. Bernhard, ${ }^{3}$ J. C. Bryan, ${ }^{4}$ J. J. Bucher, ${ }^{1}$ A. K. Burrell, ${ }^{4} D_{1}$ L. Clark, 5 N. M. Edelstein, S. A. Ekberg, J. W. Gohdes, E. A. Hudson, N. Kaltsoyannis, W. W. Lukens, W. Matz, ${ }^{5}$ H. Moll, ${ }^{4}$ M. P. Neu, ${ }^{4}$ H. Nitsche, ${ }^{4}$ P. D. Palmer, ${ }^{4}$ T. Reich, ${ }^{3}$ K. Roberts, ${ }^{2}$ D. K. Shuh, C. D. Tait, and B. D. Zwick

${ }^{1}$ Chemical Sciences Division, Lawrence Berkeley Laboratory, Berkeley, USA

${ }_{3}^{2}$ Earth Sciences Division, Lawrence Berkeley Laboratory, Berkeley, USA

${ }^{3}$ Research Center Rossendorf (FZR), Institute of Radiochemistry, Germany

${ }_{5}^{4}$ Chemical Sciences \& Technology Div., Los Alamos National Laboratory, Los Alamos, NM USA

5 Research Center Rossendorf (FZR), Institute of Ion Beam Physics \& Materials Research, Germany

${ }^{6}$ Glenn T. Seaborg Institute, Lawrence Livermore National Laboratory, Livermore, CA USA

\section{INTRODUCTION}

Synchrotron radiation investigations of radioactive and hazardous materials systems by XAS are particularly valuable. XAS is amenable to both aqueous and low concentration samples. Samples can be safely contained and examined in-situ. Thus, $\mathrm{XAS}$ is an ideal technique for the investigation of a range of fundamental issues concerning actinide materials, fission products, separation science, and topics in environmental remediation. XANES has been utilized to investigate electronic structure and oxidation states, whereas EXAFS has been used for the determination of coordination numbers and distances of a chosen atom to its nearest-neighbors.

The XAS measurements were performed on wiggler branch lines on Beamline IV utilizing $\mathrm{Si}(220)$ crystals detuned $50 \%$. The spectra were collected at the U LIII and Tc K edges at $\sim 17.2$ and $\sim 21.0 \mathrm{keV}$, respectively. Spectra were collected simultaneously with reference materials utilizing a four pixel Ge detector, a Lytle detector, and ion chambers. The data analysis was accomplished with the EXAFSPAK software.

\section{EXAFS OF URANYL CARBONATE COMPLEXES IN NEAR-NEUTRAL AQUEOUS SOLUTION}

Carbonate and bicarbonate anions are found in significant concentrations in natural waters and are exceptionally strong complexing agents for actinide ions. Depending on factors such as $\mathrm{pH}$, ionic strength, and the concentration of carbonate anion, several different complex actinide ions can exist in rapid equilibria with one another and with the uncomplexed or hydrolyzed actinide cation. The uranyl carbonate system is by far the most extensively studied of all actinide carbonate systems. The composition and structure of the tris-carbonato complex of formula $\mathrm{UO}_{2}\left(\mathrm{CO}_{3}\right)_{3}{ }^{4-\cdot}$ is wellestablished, ${ }^{2}$ and a trimeric species of composition $\left[\mathrm{UO}_{2}\left(\mathrm{CO}_{3}\right)_{2}\right]_{3}{ }^{6-}$ has been reported. ${ }^{3}$ This trimer is strongly stabilized in high ionic strength solutions and is thought to be responsible for the very high solubility of $\mathrm{UO}_{2} \mathrm{CO}_{3}(\mathrm{~s})$ in carbonate solutions, and thus important in aquatic transfer of uranyl ions.

${ }^{13} \mathrm{C}$ and ${ }^{17} \mathrm{O} \mathrm{NMR}$, and Raman spectroscopies were used to monitor the fractions of $\left[\mathrm{UO}_{2}\left(\mathrm{CO}_{3}\right)_{3}\right]^{4-}$ and $\left[\left(\mathrm{UO}_{2}\right)_{3}\left(\mathrm{CO}_{3}\right)_{6}\right]^{6-}(1)$ in aqueous carbonate solutions as a function of $\mathrm{pH}$, ionic strength, carbonate concentration, uranium concentration, and temperature.

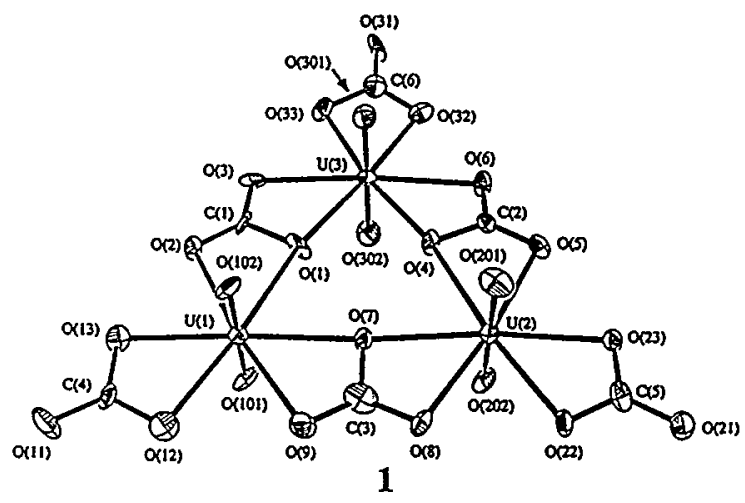

Fig. 1. The solid state structure of the $\left[\left(\mathrm{UO}_{2}\right)_{3}\left(\mathrm{CO}_{3}\right)_{6}\right]^{6-}$ anion.

Multinuclear NMR and Raman data are consistent with the formation of 1 . EXAFS data from solid $\left[\mathrm{C}\left(\mathrm{NH}_{2}\right)_{3}\right]_{6}\left[\left(\mathrm{UO}_{2}\right)_{3}\left(\mathrm{CO}_{3}\right)_{6}\right]$ and a solution of 1 suggest that the same uranium species is present in both the solid and solution. Fourier Transforms (FT) of the EXAFS spectra of both solid and solution revealed five well-resolved peaks 
corresponding to nearly identical near-neighbor distances for the solid and solution of 1 (Fig. 2).

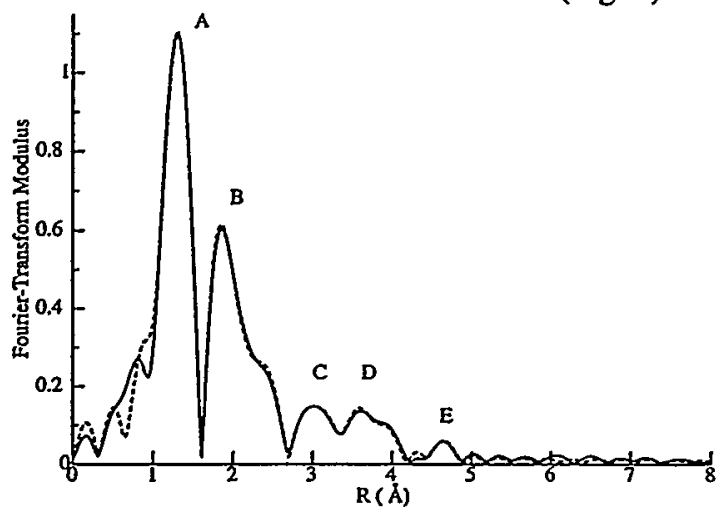

Fig. 2. The uncorrected EXAFS FT of $\left[\left(\mathrm{UO}_{2}\right)_{3}\left(\mathrm{CO}_{3}\right)_{6}\right]^{6-}$ in solution. The solid line is the experimental data and the dashed line a best fit.

Fitting of these peaks yields $\mathrm{U}-\mathrm{O}$ (uranyl) $=$ $1.79, \mathrm{U}-\mathrm{O}$ (carbonate) $=2.45, \mathrm{U}-\mathrm{C}=2.90, \mathrm{U}-\mathrm{O}$ (terminal carbonate) $=4.16$, and U--U $=4.91 \AA$ for the solid, and similar distances for the solution sample. The peak at $4.75 \AA$ in both FT (uncorrected for phase shift) corresponds to the U--U interaction at $4.91 \AA$, a conclusion which is supported by the absence of this peak in the FT of the crystalline monomeric $\mathrm{K}_{4}\left[\left(\mathrm{UO}_{2}\right)\left(\mathrm{CO}_{3}\right)_{3}\right]$. Multiple scattering along the uranyl vector plays a significant role in the EXAFS of all three systems. The EXAFS data are consistent with the trimeric uranyl carbonate species indicated by NMR.

Table I. Comparison of X-ray and EXAFS Metrical Parameters (in $\AA$ ).

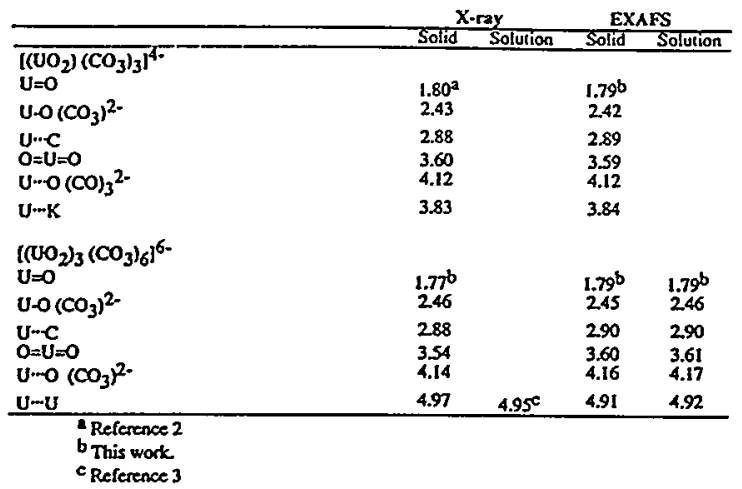

The solid state molecular structure of $\left[\mathrm{C}\left(\mathrm{NH}_{2}\right)_{3}\right]_{6}\left[\left(\mathrm{UO}_{2}\right)_{3}\left(\mathrm{CO}_{3}\right)_{6}\right]^{\bullet} 6.5 \mathrm{H}_{2} \mathrm{O}$ contains a planar $\mathrm{D}_{3 \mathrm{~h}}$ trimetallic $\left(\mathrm{UO}_{2}\right)_{3}\left(\mathrm{CO}_{3}\right)^{6-}$ anion (Fig. 1), the structure that Åberg et. al originally proposed for the trimeric solution species. Particularly significant is the average nonbonding $U--U$ distance of $4.97 \AA$ which compares to the $4.91 \AA$ distance seen in the EXAFS. A comparison of the bond distances obtained by EXAFS and a later X-ray structure analysis is given in Table 1.

\section{CHARACTERIZATION OF HYDROUS URANYL SILICATE BY EXAFS ANALYSIS}

The local atomic arrangement of uranium in $\left(\mathrm{UO}_{2}\right)_{2} \mathrm{SiO}_{4} \cdot 2 \mathrm{H}_{2} \mathrm{O}$ has been investigated by EXAFS and compared to the structural parameters obtained by $\mathrm{X}$-ray Diffraction fine structure analysis (XRD). This comparison illustrates the possibilities and limitations of EXAFS applied to a system containing $\mathrm{U}, \mathrm{O}$, and $\mathrm{Si}$. Results of these studies are pre-requisite to the EXAFS investigations of the speciation of $\mathrm{UO}_{2}{ }^{2+}$ sorbed on Si-containing minerals in the environment. The hydrous uranyl silicate can be used as a reference for the EXAFS data analysis of similar but non-crystalline samples. The details of the synthesis and XRD analysis of the sample used in this study are given in Ref. 4.

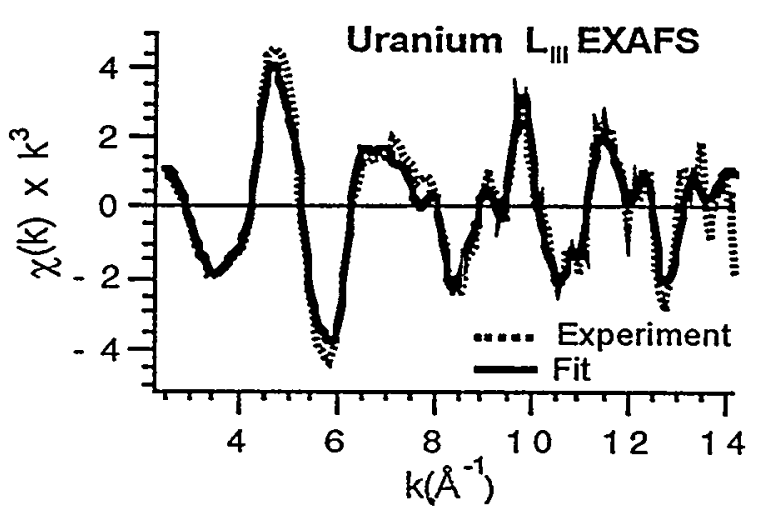

Fig. 3. EXAFS of $\left(\mathrm{UO}_{2}\right)_{2} \mathrm{SiO}_{4} \cdot 2 \mathrm{H}_{2} \mathrm{O}$.

The uranium atom in $\left(\mathrm{UO}_{2}\right)_{2} \mathrm{SiO}_{4} \cdot 2 \mathrm{H}_{2} \mathrm{O}$ is surrounded by two axial oxygen atoms, $\mathrm{O}_{1}$, and five oxygen atoms in the equatorial plane, $\mathrm{O}_{2}$ and $\mathrm{O}_{3}$. The atoms $\mathrm{O}_{2}$ are part of $\mathrm{SiO}_{4}$-tetrahedra bonded to uranium. $\mathrm{O}_{3}$ is an oxygen atom of the crystal water. Figs. 3-4 show the unsmoothed experimental EXAFS curve of $\left(\mathrm{UO}_{2}\right)_{2} \mathrm{SiO}_{4} \cdot 2 \mathrm{H}_{2} \mathrm{O}$ and its FT, respectively. The main peaks centered at $1.8,2.4$, 3.1, and $3.9 \AA$ in the FT correspond to the U-O $\mathrm{O}_{1}, \mathrm{U}-$ $\mathrm{O}_{2,3}, \mathrm{U}-\mathrm{Si}$, and $\mathrm{U}-\mathrm{U}$ absorber-backscatterer pairs, respectively.

In fitting the EXAFS absorber-backscatterer distances, $R_{i}$, and the mean square deviation in $R_{i}$, $\sigma_{i}$, were varied; the coordination number, $N_{i}$, were held constant at those obtained from XRD analysis. Scattering amplitudes and scattering phases were calculated with the code FEFF 5 in the single- 
scattering approximation. 5 The results of the fit are given in Table 2. All features of the experimental

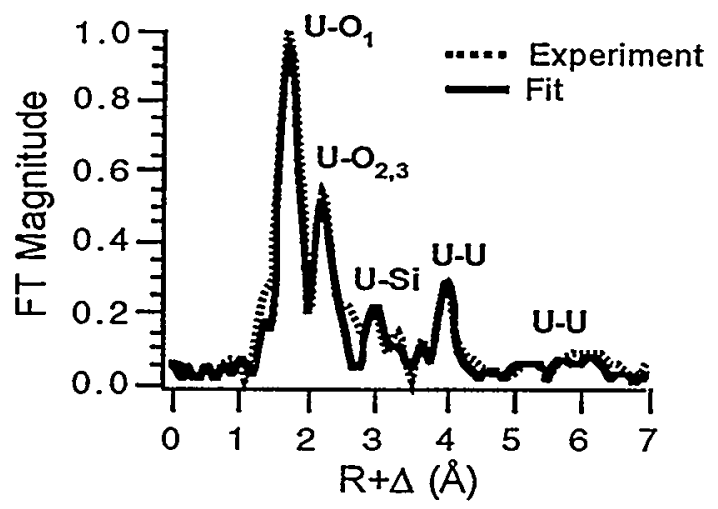

Fig. 4. FT magnitude over the range $2.5-14.2 \AA^{-1}$.

EXAFS curve are reproduced well by the fit (Figs. 34). The backscatterers $\mathrm{Si}$ and $\mathrm{O}$ at $\mathrm{R}_{\mathrm{i}}>3.5 \AA$ (coordination shells 4,6 , and 8 ) contribute less than $1 \%$ to the EXAFS. Structural parameters for these light elements in higher coordination shells cannot be determined without a priori knowledge of their presence. It is interesting that in this well-ordered structure, $U$ scatterers can be determined up to $\mathrm{R}=5.8 \AA$ at room temperature. It was impossible to obtain a good fit by including only lighter elements such as $\mathrm{Si}$ or $\mathrm{O}$ in the 5 th coordination shell, instead of $U$. The values of $\sigma_{i}$ given in Table 2 correlate well with the bond distance distribution of the elements determined by XRD.

For example, the 5 equatorial atoms $\mathrm{O}_{2}$ (2.31, $2.40 \AA$ ) and $\mathrm{O}_{3}(2.34 \AA$ ) cannot be resolved by EXAFS and appear in the FT as a single peak at $2.36 \AA$. These exhibit a larger $\sigma_{i}$ than for the first $O_{1}$ shell. Based upon the agreement of the $R_{i}$ values determined by EXAFS and XRD, the theoretical scattering amplitudes and scattering phases calculated by FEFF can be used for an accurate $R_{\mathbf{i}}$ determination. This is especially important when experimental scattering amplitudes and scattering phases are unavailable.

Table. 2. Summary of EXAFS and XRD results on uranyl silicate.

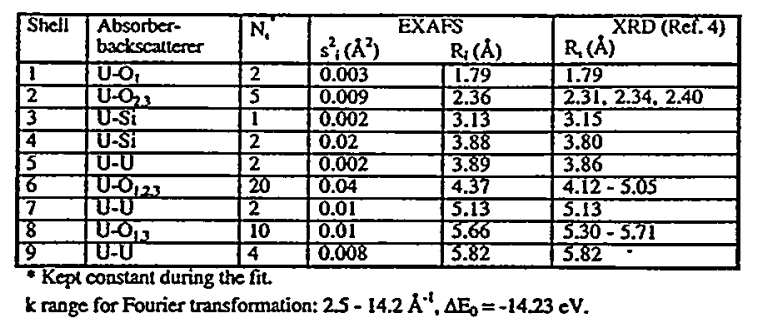

\section{ELECTRONIC AND STRUCTURAL INVESTIGATIONS OF TECHNETIUM COMPOUNDS BY XAS}

${ }^{99} \mathrm{Tc}$ is a by-product of nuclear fission, and its significant half-life $\left(t / / 2=2.13 \times 10^{5}\right.$ years) necessitates its consideration in the long-term disposal of nuclear waste. The principal Tccontaining component of nuclear wastes is $\mathrm{TcO}_{4}^{-}$, and the propensity of $\mathrm{TcO}_{4}^{-}$to migrate in the geosphere has led to research aimed at the facile conversion of pertechnetate into other, less mobile compounds of ${ }^{99} \mathrm{Tc}$. An XANES evaluation of the efficacy of a process designed to reduce $\mathrm{TcO}_{4}^{-}$to $\mathrm{TcO}_{2}$ in wasteform cement was recently reported.

XANES has been used to establish the chemical shifts of the technetium $\mathrm{K}$ edge in a wide range of Tc compounds, as a function of the formal oxidation states $0,+2,+4,+5$ and +7 . The shifts in the edge positions relative to $\mathrm{NH}_{4} \mathrm{TcO}_{4}$ are shown in Fig. 5. Clearly, there is a strong correlation between formal oxidation state and $\mathrm{K}$ edge position. An increase in formal oxidation state implies an increase in effective charge. $\mathrm{X}$-ray absorption edge energies are governed mainly by the effective charge on the absorbing atom or ion such that edges shift progressively toward higher energies with the formal oxidation state of the absorbing atom. ${ }^{\top}$ Of the eight systems studied, six display an almost linear relationship; $\mathrm{Tc}_{2}(\mathrm{CO})_{10}$ and $\mathrm{Tc}(\mathrm{ArN})_{3} \mathrm{I}$ show more significant differences.

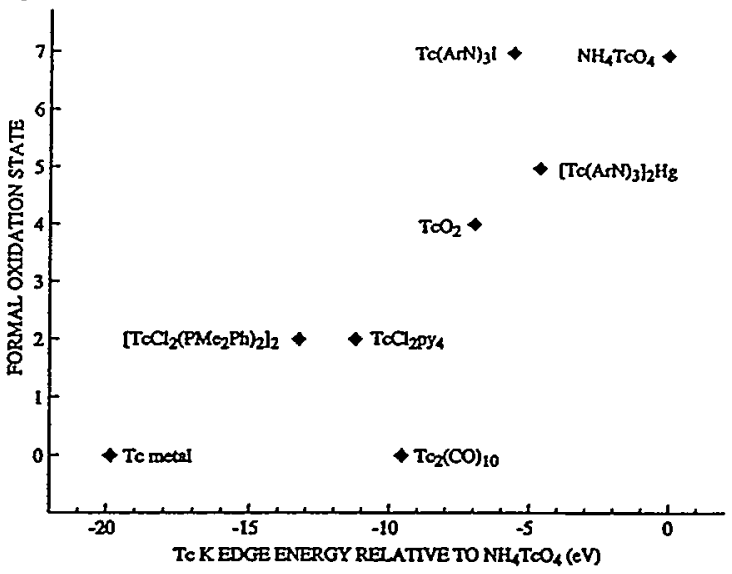

Fig. 5. Chemical shifts of the Tc $\mathrm{K}$ edge versus formal oxidation state for a range of $\mathrm{Tc}$ materials $\left[\mathrm{TcCl}_{2} \mathrm{py}_{4}\left(\mathrm{py}=\mathrm{C}_{5} \mathrm{H}_{5} \mathrm{~N}\right)\right.$ and $\mathrm{Tc}(\mathrm{ArN})_{3} \mathrm{I}(\mathrm{ArN}=$ 2,6- $\left.\left.\mathrm{Pr}_{2}{ }_{2} \mathrm{C}_{6} \mathrm{H}_{3} \mathrm{~N}\right)\right]$.

$\left[\mathrm{TcCl}_{2}\left[\left(\mathrm{PMe}_{2} \mathrm{Ph}\right)_{2}\right]_{2}\right.$ and $\mathrm{TcCl}_{2} \mathrm{Py}_{4}$ contain $\mathrm{Tc}$ as $\mathrm{Tc}(\mathrm{II})$, and their edge positions are 13.2 and $\mathbf{1 1 . 2}$ $\mathrm{eV}$ lower than $\mathrm{NH}_{4} \mathrm{TCO}_{4}$, respectively. That 
$\left[\mathrm{TcCl}_{2}\left[(\mathrm{PMe} 2 \mathrm{Ph})_{2}\right]_{2}\right.$ has a $2.0 \mathrm{eV}$ greater shift than $\mathrm{TcCl}_{2} \mathrm{py}_{4}$ is a result of the stronger $\sigma$-donor and weaker $\pi$-acceptor nature of $\mathrm{PMe}_{2} \mathrm{Ph}$ versus $\mathrm{C}_{5} \mathrm{H}_{5} \mathrm{~N}$. Both $\mathrm{Tc}(\mathrm{ArN})_{3} \mathrm{I}$ and $\mathrm{NH}_{4} \mathrm{TcO}_{4}$ contain $\mathrm{Tc}$ in a formal oxidation state of +7 , but $\operatorname{Tc}(\operatorname{ArN}) 3 \mathrm{I}$ has a Tc $\mathrm{K}$ edge energy which is $5.6 \mathrm{eV}$ lower than $\mathrm{NH}_{4} \mathrm{TcO}_{4}$. This reflects the greater electron withdrawing properties of the oxygens, thereby inducing a greater effective charge on the Tc compared to the ArN/I combination. The magnitude of the difference is somewhat surprising, as the Tc edge of $\operatorname{Tc}(\operatorname{ArN})_{3} I$ has an edge is further from $\mathrm{NH}_{4} \mathrm{TcO}_{4}$ than the $\mathrm{Tc}(\mathrm{V})$ in $\left[\mathrm{Tc}(\operatorname{ArN})_{3}\right]_{2} \mathrm{Hg}$.

The most significant deviation from the formal oxidation state/K edge energy relationship is that of $\mathrm{Tc}_{2}(\mathrm{CO})_{10}$. Its edge position is closer to $\mathrm{NH}_{4} \mathrm{TcO}_{4}$ than either $\mathrm{TcCl}_{2} \mathrm{py} 4$ or $\left[\mathrm{TcCl}_{2}\left[\left(\mathrm{PMe}_{2} \mathrm{Ph}\right)_{2}\right] 2\right.$. This results from the electron withdrawing ability of the carbonyl unit, and indicates that $\pi$ backbonding from metal to ligand dominates ligand to metal $\sigma$ donation. These results are entirely consistent with previous $\mathrm{XPS}_{8-9}$ measurements on $\mathrm{Cr}(\mathrm{CO}) 6$ and $\mathrm{Mn}_{2}(\mathrm{CO})_{10}$, $^{8-9}$ which show the binding energy of the metal $2 \mathrm{p} 3 / 2$ atomic orbital to be close to that in $\mathrm{MO}_{2}$ and well-removed from the value obtained in the pure element. The compound $\mathrm{TcO}_{2}$ is shown by EXAFS to possess a distorted rutile structure with a closest Tc-Tc distance of $2.61 \AA$ and is consistent with the Goodenough 10 model for bonding in transition metal dioxides.

\section{MMPLEMENTATION OF A MONOLITHIC Ge FLUORESCENCE DETECTOR ARRAY}

The continued development of fluorescence detection techniques is essential for improved investigations of environmentally-relevant systems. Much of the chemistry and physics of environmental concern occurs at extremely low concentration and/or in matrices that necessitate fluorescence detection.

The quad-pixel fluorescence detector system consists of a monolithic high-purity $4.0 \mathrm{~cm}^{2} \mathrm{Ge}$ crystal that is divided electrically into four $1.0 \mathrm{~cm}^{2}$ pixel elements solely by careful placement of the signal contacts. The monolith, in comparison to standard multi-element arrays, suffers no dead volume loss. The configuration of the detector system utilizes four channels of commercially available pulse detection electronics and programs running on a personal computer. The detector preamplifiers are small and allow for positioning of the detector close to the sample.

The performance of the quad-pixel detector system has been tested at LBL with sealed sources $\left({ }^{55} \mathrm{Fe},{ }^{238} \mathrm{Pu}\right.$, and $\left.{ }^{241} \mathrm{Am}\right)$ and has been utilized for XAS measurements on the Beamline IV. The charge collection process for an event that occurs in one pixel is only associated with signal from that pixel and the individual pixels are identical for all practical purposes. These measurements show that the overall performance characteristics of the quad-pixel detector, in terms of throughput and resolution, is comparable to that of existing multi-element detectors.

The performance of this detector in its current configuration, represents a significant improvement over existing multi-element Ge detector systems and is considerably less expensive. Enhancements to improve both the resolution and the maximum linear count rate of the existing detector are planned.

\section{REFERENCES}

1. I. Grenthe, R. J. Lemire, A. B. Muller, C. Nguyen-Trung, and H. Wanner, Chemical Thermodynamics of Uranium; OECD-NEA, Paris, (1991).

2. R. Graziani, G. Bombieri, and E. Forsellini, J. Chem. Soc., Dalton Trans. 19, 2059 (1972).

3. M. Åberg, D. Ferri, J. Glaser, and I. Grenthe, Inorg. Chem. 22, 3981 (1983).

4. H. Moll, G. Bernhard, and W. Matz, 1994 Annual Report, FZR, Germany.

5. J. J. Rehr, R. C. Albers, and S. I. Zabinsky, Phys. Rev. Lett. 69, 3397 (1992).

6. D. K. Shuh, N. Kaltsoyannis, J. J. Bucher, N. M. Edelstein, S. B. Clark, H. Nitsche, T. Reich, E. A. Hudson, I. Almahamid, et. al, Mat. Res. Soc. Symp. Proc., xxx (1994).

7. Agarwal, B. K. in X-Ray Spectroscopy, Second ed. Springer-Verlag: Berlin, (1991).

8. S. Pignataro, A. Foffani, and G. Distefano, Chem. Phys. Lett. 20, 351 (1973).

9. D. F. Van der Vondel, L. .F. Wuyts, G. P. Van der Kelen, and L. Bevernage, J. Electr. Spectr. 10, 389 (1977).

10. J. B. Goodenough, Progr. Solid State Chem. 5, 145 (1971).

\section{ACKNOWLEDGMENTS}

The XAS measurements were performed at the SSRL which is operated by the US Department of Energy, Office of Basic Energy Sciences, Division of Chemical Sciences. This work was supported by the Director, Office of Energy Research, Office of Basic Energy Sciences, Chemical Sciences Division of the US Department of Energy under Contract No. DE-AC03-76SF00098. 


\title{
Nd substitution into the $\mathrm{Y}$ and $\mathrm{Ba}$ layers of $\mathrm{Y}_{1} \mathrm{Ba}_{2} \mathrm{Cu}_{3} \mathrm{O}_{7}$
}

\author{
J.V. Acrivos, C.M. Burch San José State University, San José CA 95192-0101 \\ and
}

K.K. Sing, IRC for Superconductivity, UK and Morris Res. Inc., Berkeley, CA 94704

\begin{abstract}
The Nd L3 XAS reveal the progressive substitution of $\mathrm{Nd}$ in the $\mathrm{Y}$ and $\mathrm{Ba}$ layers by the shell occupation. The data is compared to ESR spectra line widths versus substitution.
\end{abstract}

\section{Introduction:}

Lower than 1:1 substitution of $\mathrm{Nd}$ for $\mathrm{Y}$ in the regular 123 goes both in the $\mathrm{Y}$ and in the $\mathrm{Ba}$ layers. $\mathrm{A}$ quantitative knowledge of the relative $\mathrm{Y} / \mathrm{Ba}$ substitution is important for the developing theories of superconductivity. ESR spectra give evidence on the defects introduced by $\mathrm{Nd}^{3+},{ }_{9 / 2} \mathrm{I}_{9 / 2}$ into the $\mathrm{Ba}$ layer, ${ }^{1}$ and XRD gives a pseudo $\mathrm{D}_{4 \mathrm{~h}}{ }^{1}$ symmetry for the $D_{2 h}{ }^{1}$ compounds due to $\mathbf{a}, \mathbf{b}$ axes twining, introduced into the $\mathrm{CuO}$ chain layer of the 123 structure by the defects in the $\mathrm{Ba}$ layer. The ESR spectra are similar, but not identical, to $\mathrm{Cu}^{2+}$ defects in plain 123 but broaden as substitution increases. Nd-Nd spin-spin interactions are always present:

$\mathrm{d}_{\mathrm{Na}(\mathrm{Y})-\mathrm{Nd}(\mathrm{Ba})}=3.76 \AA<\mathrm{a}, \mathrm{b}=3.85,3.91 \AA^{2}$ ESR line widths' increase with $\mathrm{Nd}$ substitution above 1:1 indicates that the relaxation path must include the proximity of $\mathrm{Nd}^{3+}, 4 \mathrm{I}_{9 / 2}$ to $\mathrm{Cu}^{2+}$ defects, introduced by the charge inbalance in the $\mathrm{Ba}$ layer. The EXAFS analysis was undertaken in order to ascertain how the substitution changes the FT radial function vs $\mathrm{Nd}$ substitution above 1:1.

\section{Experimental:}

The compounds were prepared at Morris Laboratories and the ESR spectra measured at SJSU; the XAS $(4-77 \mathrm{~K})$ were measured at the SSRL, Station II-3.

\section{Discussion:}

The data analysis is still on-going using EXAFSPAK. $^{3}$ The $\mathrm{d}_{\mathrm{Nd}-A}$ distances (Table I) are known from excellent XRD data,

$\mathrm{d}_{\mathrm{Nd}(\mathrm{Y})-\mathrm{Cu}(2)} \cong \mathrm{d}_{\mathrm{Nd}(\mathrm{Ba})-\mathrm{Cu}(2)}=3.33 ; 6.45 ;$

$\mathrm{d}_{\mathrm{Nd}(\mathrm{Ba})-\mathrm{Cu}(1)}=3.46 ; 6.52 ; \mathrm{d}_{\mathrm{Nd}(\mathrm{Y})-\mathrm{Cu}(1)}=6.48$

$\mathrm{d}_{\mathrm{Nd}(\mathrm{Y})-\mathrm{Nd}(\mathrm{Y})}=\mathrm{d}_{\mathrm{Nd}(\mathrm{Ba})-\mathrm{Br}(\mathrm{Ba})}=3.85,391,5.49$;

$\mathrm{d}_{\mathrm{Na}(\mathrm{Y})-\mathrm{Ba}}=\mathrm{d}_{\mathrm{Nd}(\mathrm{Ba}) \mathrm{Nd}(\mathrm{Y})}=3.76 ; 5.75 ; 6.65$

$\mathrm{d}_{\mathrm{Nd}(Y)-O(1,2,3)}=2.53-2.55 ; 4.62-4.66 ; 5.04$

6.18-6.47;

$\mathrm{d}_{\mathrm{N}(\mathrm{Ba})-\mathrm{O}(1,23)}=2.78-2.88 ; 4.65-4.84 ; 5.73-$ $5.76 ; 6.19-6.62 \AA$

The respective Point Groups about $\mathrm{Y}\left(\mathrm{D}_{2 \mathrm{~h}}\right)$ and $\mathrm{Ba}\left(\mathrm{C}_{2 \mathrm{v}}\right)$ are made evident by the spread $\Delta \mathrm{d}_{\mathrm{Nd}-A}: \Delta \mathrm{d}_{\mathrm{Nd}(Y)-O(1,2,3)}=0.02 \AA$ $\Delta \mathrm{d}_{\mathrm{Nd}(\mathrm{Ba})-\mathrm{O}(1,2,3)}=0.1 \AA$. The first shell radial FT near $R+\Delta=2.5 \AA$ shows three peaks within $0.2 \AA$; the highest relative amplitudes are for $\mathrm{R}=2.78,2.88 \AA$; they increase with $\mathrm{x}$ in Figure 1, suggesting a preference for $\mathrm{Nd}$ substitution in the $\mathrm{Ba}$ layer. The amplitudes centered near $R+\Delta$ $=3.3$ (highest) and $6.5 \AA$ show relative amplitude loss near $\mathrm{R}=\mathrm{a}, \mathrm{b}$ with increasing $\mathrm{x}$ in the Ba layer; $\left(\mathrm{d}_{\mathrm{Na}(\mathrm{Y})-\mathrm{Cu}(2)} \cong\right.$ $\mathrm{d}_{\mathrm{N}(\mathrm{Ba})-\mathrm{Cu}(2)}=3.33,6.46 \AA$ and, in-plane $\mathrm{d}$ $\cong \mathrm{a}, \mathrm{b}, .$.$) ; suggesting that out of plane$ multiple scattering paths increase with $x$. The valence deduced from the edge shifts remain constant.

Conclusions:

The EXAFS together with XRD data on Nd substituted 123 compounds points to 
greater substitution of $\mathrm{Nd}$ in the $\mathrm{Ba}$ layer than indicated by the stoichiometry. The charge inbalance in the $\mathrm{Ba}$ layer causes the increase in the ESR line widths.

\section{Acknowledgements:}

Work at SJSU supported by NSF Grants DMR 9307387 and INT 9312176 . KKS wishes to thank Dr. W.M. Morris for support in the preparation of the componds. The work at SSRL was aided by DOE, Drs. G. George and I. Pickering and, Mr. R. Mayer.

\section{References:}

1. J.V. Acrivos, L. Chen, C. Burch, P. Metcalf, J.M. Honig, R.S. Liu and K.K. Singh, Phys. Rev. B 50, 13710 (1994)

2. (a) J.M. de Lón, E.A. Stern, D.E. Sayers, E.A. Stern, D.E. Sayers, . Ma and J. Rehr, ed., "XAFS V", 1988 p.433-491; (b) D.M. Ginsburg, a Physical Properties of High $\mathbf{T}_{\mathbf{c}}$ Superconductors, In $^{\boldsymbol{m}}$ World Scientific, p. 150, 1990

3. G. Georege and I. Pickering, "EXAFSPAK", 1994

Table I: Closest Shell Distances: $\mathrm{d}_{\mathrm{NadA}}$. $\mathrm{D}^{1} \mathrm{zh}_{\mathrm{f}} \mathrm{a}, \mathrm{b}, \mathrm{c}(\AA)=3.91,3.85,11.74,{ }^{2}$ in different unit cells (hk $\ell$ ) about the origin unit cell $(000)$ containing the absorber:
(hkl): (000)
(100)
(110)

(200)

\begin{tabular}{|c|c|c|c|c|c|}
\hline & $A / N d$ & & - & $\operatorname{Vd}(\mathbf{Y})$ & - \\
\hline 4 & $\mathbf{Y}$ & $\underline{3.85}$ & 3.91 & 5.49 & \\
\hline 3 & $\mathbf{O}(3)$ & 2.53 & 4.66 & 4.66 & 8.22 \\
\hline & $\mathbf{O}(3)$ & 2.55 & 2.55 & 4.62 & 6.0 \\
\hline 3 & $\mathrm{Cu}(2)$ & $\underline{3.33}$ & 3.33 & $\underline{3.33}$ & \\
\hline & $\mathbf{B a}^{*}$ & 3.76 & 5.42 & $\underline{6.65}$ & \\
\hline & $\mathbf{O}(2)$ & 5.04 & 5.04 & 5.04 & \\
\hline & $\mathrm{Cu}$ & 6.48 & 6.48 & 6.48 & 8.5 \\
\hline & $\mathbf{O ( 1 )}$ & 6.18 & 7.31 & 7.31 & 9.9 \\
\hline-1 & $O(2)$ & 8.00 & 8.00 & 8.00 & 9.7 \\
\hline & $\mathbf{B a}$ & $\underline{7.98}$ & 8.89 & 9.69 & 11. \\
\hline-3 & $\mathrm{Cu}(2)$ & 10.24 & 10.24 & 10.24 & 11.63 \\
\hline-3 & $O(3)$ & 10.28 & 10.28 & 10.98 & 11.6 \\
\hline-3 & $O(3)$ & 10.28 & 11.00 & 11.00 & 12.91 \\
\hline & $Y$ & 11.74 & 12.37 & 12.96 & \\
\hline
\end{tabular}
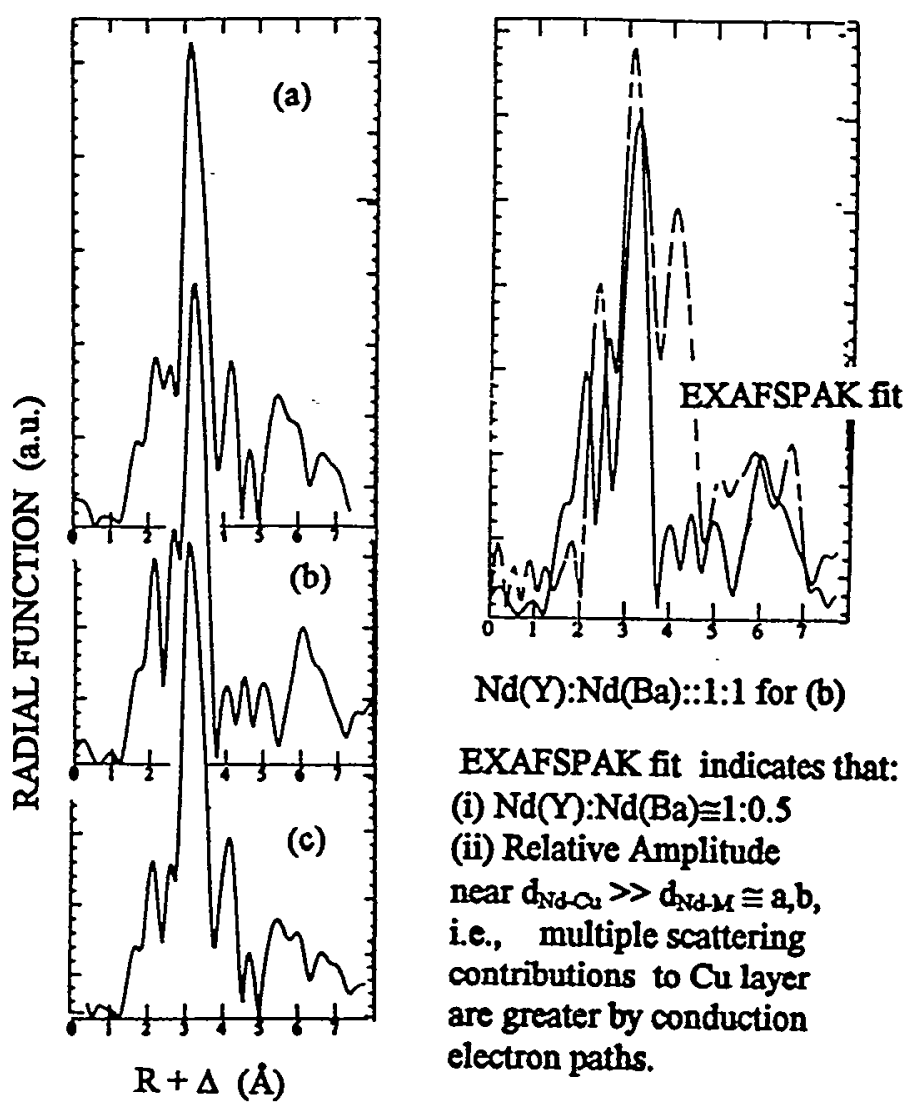

$\mathrm{Nd}(\mathrm{Y}): \mathrm{Nd}(\mathrm{Ba}):: 1: 1$ for (b)

EXAFSPAK fit indicates that:

(i) $\mathrm{Nd}(\mathrm{Y}): \mathrm{Nd}(\mathrm{Ba}) \cong 1: 0.5$

(ii) Relative Amplitude

near $\mathrm{d}_{\mathrm{N} \alpha \mathrm{O}_{3}} \gg \mathrm{d}_{\mathrm{x} \alpha-\mu \mathrm{s}} \cong \mathrm{a}, \mathrm{b}$, i.e., multiple scattering contributions to $\mathrm{Cu}$ layer are greater by conduction electron paths.

Fig.1: $\mathrm{Nd}\left(\mathrm{O}_{2} \mathrm{Cu}\right)_{2}\left(\mathrm{Nd}_{\mathbf{x}} \mathrm{Ba}_{1-\mathrm{x}}\right)_{2}\left(\mathrm{O}_{2}\right) \mathrm{CuO}$ Radial Distribution Function EXAFS FT (a) $x=0.05$. (b) $x=0.15$. (c) $x=0.25$.

The closest $d_{N d-O}(\AA)$ are shown bold. The closest $d_{\text {Nd-Metal }}(\AA)$ are underlined. Not all shells are evaluated.

\begin{tabular}{|c|c|c|c|c|c|}
\hline & (000) & 100) & (110) & (200) & (001) \\
\hline & & & $\mathbf{N d}(\mathbf{B} \mathbf{2})$ & & $>$ \\
\hline 11.74 & 3.76 & 5.75 & 6.65 & 8.68 & 15.50 \\
\hline 10.28 & 2.86 & 4.84 & 4.84 & 8.33 & 13.99 \\
\hline 10.28 & 2.88 & 2.88 & 4.81 & 6.23 & 13.99 \\
\hline 10.24 & 3.33 & 3.33 & 3.33 & 6.45 & 13.89 \\
\hline 7.98 & 0.00 & 3.91 & $\underline{5.49}$ & 7.82 & 11.74 \\
\hline 8.00 & 2.78 & 2.78 & 2.78 & 6.19 & 11.60 \\
\hline & & & & 6.52 & 10.01 \\
\hline 6.18 & 2.86 & 4.84 & $\overline{4.84}$ & 8.33 & 9.82 \\
\hline 04 & 4.65 & 4.65 & 4.65 & 7.23 & 8.44 \\
\hline 3.76 & 4.23 & 5.76 & 6.93 & 8.89 & 7.51 \\
\hline 3.33 & & 6.69 & & 8.68 & \\
\hline 2.55 & 6.63 & 6.63 & 7.67 & 8.64 & 5.74 \\
\hline 2.53 & 6.63 & 7.69 & 7.69 & 10.25 & 5.73 \\
\hline 0.00 & 7.98 & 8.89 & 9.69 & 11.18 & 3.76 \\
\hline
\end{tabular}




\title{
Reconstructions at Metal/Silicon Interfaces
}

\author{
C. A. Lucas and D. Loretto
}

\begin{abstract}
Materials Sciences Division, Lawrence Berkeley Laboratory, University of California, Berkeley, CA 94720.
\end{abstract}

The study of metal/semiconductor interfaces has grown rapidly in the past decade and a number of prototypical systems have emerged. In particular, interest has focused on the atomic structure at the interface and its influence on the electronic properties, characterised by the Schottky barrier height (SBH) $[1,2]$. In many cases, buried interface reconstructions have been observed and these are conceptually similar to the reconstructions observed at clean surfaces in ultra high vacuum (UHV). However, due to the buried nature of the interface reconstruction, standard surface probes cannot be used for structural studies. Such studies require techniques that employ highly penetrating beams, such as $x$-ray diffraction and transmission electron microscopy (TEM). In our program we have used both of these techniques to study a number of different metal-semiconductor reconstructions in an effort to understand both the interface structure and its influence on the subsequent metal epitaxial growth. Some highlights are presented below.

\section{(i) $\mathrm{CoSi} / \mathrm{Si}(001)$.}

The CoSi2/Si(001) interface exhibits a two-domain (2x1) reconstruction which has the same symmetry as the $(2 \times 1)$ reconstruction observed at the $\mathrm{Si}(001)$ and $\mathrm{Ge}(001)$ surfaces [3]. The $\mathrm{CoSi}_{2} / \mathrm{Si}-(2 \times 1)$ reconstruction was originally observed by TEM and a model involving $\mathrm{Si}$ dimers at the interface was proposed [4]. A conflicting model of compositional modulation was proposed in a separate TEM study [5] and has recently been supported by a high resolution $Z$ contrast imaging experiment [6].

The samples we studied were prepared by molecular beam epitaxy (MBE) at AT\&T Bell Laboratories [7]. $\mathrm{X}$-ray diffraction measurements were performed on beamline 7-2 at SSRL using focussed radiation collimated by a Si(111) monochromator. Scattering from the $(2 \times 1)$ reconstuction occurs at half-integer positions in the Si reciprocal lattice. A representative rocking scan through one of these peaks $(1 / 2,0,0.15)$ is shown in Figure 1(a). In total we measured 248
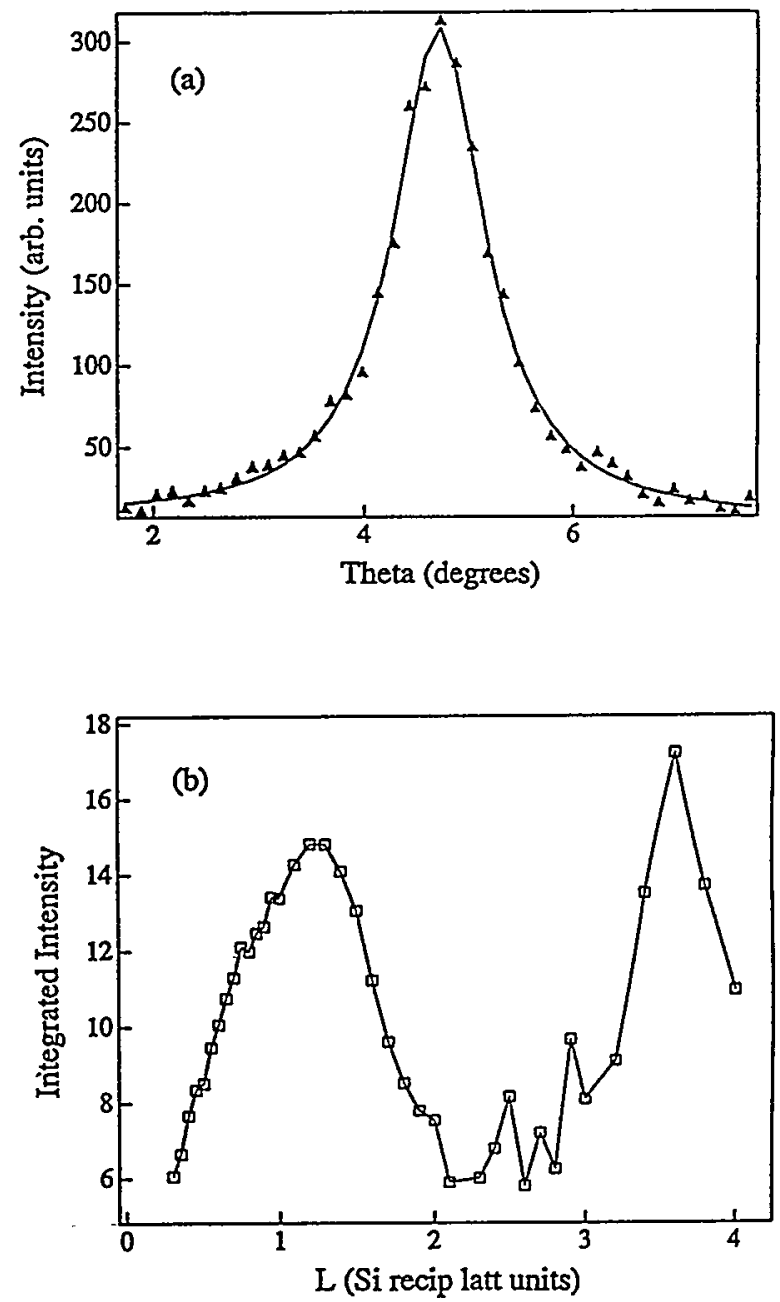

Figure 1. (a) A rocking scan through the $(1 / 2,0$, 0.15 ) superlattice Bragg reflection for $\mathrm{CoSi}_{2} / \mathrm{Si}(001)$. The solid line is a calculated Lorentzian lineshape. (b) The $(1 / 2,0, L)$ surface rod showing the oscillatory behavior. 
independent structure factors, specific to the $(2 \times 1)$ phase, combining in-plane scans (i.e. zero surface normal momentum transfer) and out-of-plane scans (i.e. along the surface normal) for 6 of the in-plane reflections. The out-of-plane measurements (or 'surface rods') show an oscillatory behavior as a function of perpendicular momentum transfer, L. Figure 1(b) shows the $(1 / 2,0, L)$ rod as an example. This oscillatory behavior is similar to results from the clean $\mathrm{Si}(001)$ and $\mathrm{Ge}(001)-2 \times 1$ surfaces and implies relaxation away from bulk atomic positions over at least 6 monolayers at the interface [8].

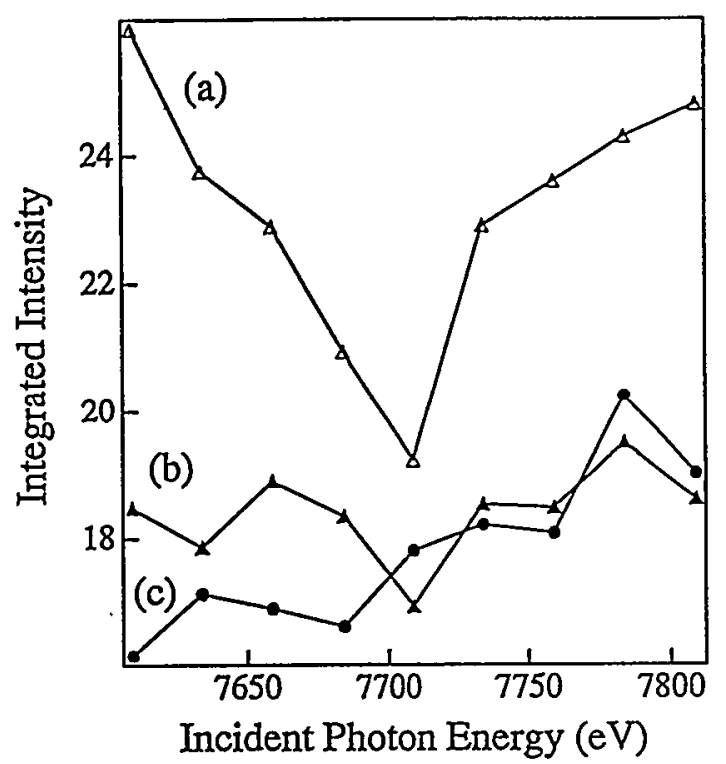

Figure 2. The energy dependence of some $\mathrm{CoSi}_{2} / \mathrm{Si}(001)$ data. (a) At $(2,0,0.15)$ on a bulk Si CTR, (b) at $(1 / 2,0,0.15)$ and (c) at $(1 / 2,1,0.15)$. See text for explanation.

In order to resolve the controversy over the nature of the reconstruction; i.e compositional modulation or Si dimers, we extended our $x$-ray study by performing diffraction measurements using incident $x$-ray energies close to the Co $\mathrm{K}$ adsorption edge (7709 eV). We measured the scattered intensity at a number of $(2 \times 1)$ superlattice Bragg reflections as the $x$-ray energy was scanned across the Co edge. Representative results are shown in Figure 2. The $(2,0,0.15)$ position lies on a bulk Si crystal truncation rod (CTR) where the scattering is given by the sum of the scattering from the $\mathrm{Si}$ substrate and the epitaxial, lattice matched $\mathrm{CoSi}_{2}$ film (the thickness was $\sim 30 \AA$ as measured by specular reflectivity through the Si(004) Bragg reflection). As expected, the scattering is reduced at the
Co edge due to the anaomalous corrections to the Co atomic form factor. In contrast, the scattering at the (2x1) Bragg reflections is unchanged (this lack of effect was observed at several in-plane peaks and along 3 surface rods). Two in-plane reflections are shown in Figure 2. These results indicate that there is no Co (or missing Co atom) in the $(2 \times 1)$ unit cell. A detailed analysis of the structure factor data, in order to derive a structural model, is currently in progress.

\section{(ii) $\mathrm{Pb} / \mathrm{Si}$.}

The mutual solubilities of $\mathrm{Pb}$ and $\mathrm{Si}$ are negligible and thus the $\mathrm{Pb} / \mathrm{Si}$ is an ideal system for the study of metal layer growth. As such, the early stages of interface formation have been studied by a variety of techniques. For $\mathrm{Pb}$ deposition on $\mathrm{Si}(111)$ at room temperature the $(7 \times 7)$ reconstruction, characteristic of the clean surface, is modified but retains the $(7 \times 7)$ symmetry [9]. This is a metastable phase which irreversibly transforms to an incommensurate $(\sqrt{3} \times \sqrt{3})$ structure upon heating. Further deposition of $\mathrm{Pb}$ on the monolayer structures leads to (111) surface-normaloriented island growth and these islands eventually join up to form a continuous film [10]. The SBH was found to be different for the two interfaces indicating the importance of the atomic arrangement at the interface $[2,10]$. In contrast to the $\mathrm{Pb} / \mathrm{Si}(111)$ system the $\mathrm{Pb} / \mathrm{Si}(001)$ system has received little attention [11].

We have performed a systematic study of the $\mathrm{Pb} / \mathrm{Si}$ interface and thin film structure. All samples were prepared by MBE in a UHV system with a base pressure of $1 \times 10^{-11}$ torr. The Si substrate was cooled to below room temperature after cleaning and $\mathrm{Pb}$ films of thickness $\sim 200-300 \AA$ were grown in each case. The films were capped with amorphous Si before removal from the vacuum. The unifomity of the $\mathrm{Pb}$ films was checked by plan view TEM. In some cases $\sim 2$ monolayers of $\mathrm{Ge}$ were deposited on the hot $\mathrm{Si}$ substrate before cooling for $\mathrm{Pb}$ deposition.

The diffraction pattern for $\mathrm{Pb} / \mathrm{Si}(001)$ exhibits a two-domain $(2 \times 1)$ reconstruction at the interface (analogous to the clean $\mathrm{Si}$ surface). The $\mathrm{Pb}$ film on top of this reconstruction is oriented with its (111) direction along the Si(001) surface normal. Due to the different lattice constants of the $\mathrm{Si}$ substrate and the $\mathrm{Pb}$ film, the scattering from each is separated in reciprocal space. We have measured a number of $(2 \times 1)$ superlattice Bragg reflections and surface rods in order to derive a structural model. The oscillation period in the surface rods indicate that the reconstruction is 2-3 monolayers deep and the intensities are consistent with a Pb-decorated Si reconstruction. The in-plane orientation of the (111) surface normal oriented $\mathrm{Pb}$ film was measured by large angle rocking scans at momentum transfer $Q=12001 \mathrm{~Pb}$. The result is shown in Figure 3(a) and indicates an interesting case of rotational epitaxy. The rotational domains and the 
$(2 \times 1)$ interface reconstruction are stable up to the $\mathrm{Pb}$ bulk melting point. At this point there is a sharp melting transition which causes the $\mathrm{Pb}$ to unwet the $\mathrm{Si}$ substrate and form islands. Even at temperatures $\sim 50^{\circ}$ above the melting point we find that the $(2 \times 1) \mathrm{Pb}$ interface phase remains below the liquid $\mathrm{Pb}$ droplets.

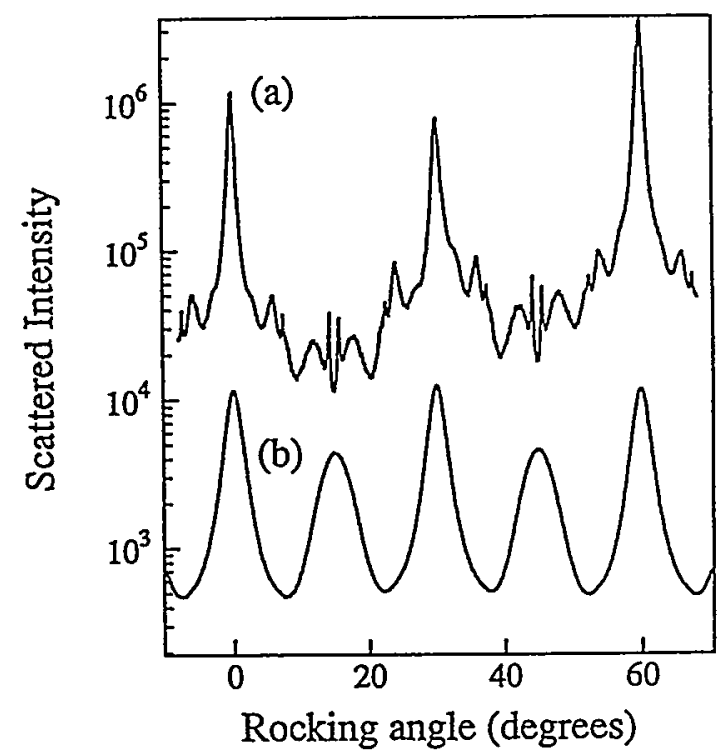

Figure 3. Extended rocking scans at $Q=\left|\begin{array}{llll}2 & 0 & 0\end{array}\right|$ $\mathrm{Pb}$. (a) for $\mathrm{Pb} / \mathrm{Si}(001)$ and (b) $\mathrm{Pb} / \mathrm{Ge} / \mathrm{Si}(001)$. In each case the $S i<1 \quad 10>$ direction is at $\phi=0$. The data in (b) is divided by 100 for reasons of clarity.

Deposition of 2 monolayers of $\mathrm{Ge}$ on the clean $\mathrm{Si}$ (2x1) surface results in a similar $(2 \times 1)$ reconstruction (observed by RHEED and as studied by several other authors [12]). Deposition of $\mathrm{Pb}$ onto this surface also results in (111) oriented $\mathrm{Pb}$ film and a two-domain ( $2 \times 1)$ interface reconstruction. The $L$ dependence of the surface rods and the intensity distribution of the inplane peaks indicate that the reconstruction differs from the the structure found at the $\mathrm{Pb} / \mathrm{Si}$ interface. This difference has a dramatic effect on the in-plane orientation of the $\mathrm{Pb}(111)$ film. Figure $3(\mathrm{~b})$ shows a rocking scan equivalent to the one in Figure 3(a). The epitaxial orientation is drastically changed, even

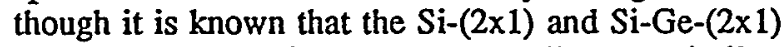
surface reconstructions are structurally very similar. Clearly the strain and chemical composition at the interface plays an important role in the subsequent epitaxial growth.

The results presented in this report are at an early stage of analysis but are chosen to highlight some of the experimental findings. We have also studied the buried phase transition that occurs when the metastable
$\mathrm{Si}(111)-(7 \times 7) / \mathrm{Pb}$ interface is heated and an equivalent structural transition that occurs at the $\mathrm{Pb} / \mathrm{Ge}$ (5x5)/Si(111) interface. Again we observe different $\mathrm{Pb}(111)$ orientational epitaxy for the $\mathrm{Si}$ and $\mathrm{Si} / \mathrm{Ge}$ samples.

We would like to thank the staff and user administration at SSRL for their hospitality and Sean Brennan for his excellent support of beamline 7-2. This work was supported by the Director, Office of Basic Energy Sciences, Materials Sciences Division of the U.S. Department of Energy under Contract No. ACO3-76SF00098.

\section{References}

[1] R. T. Tung, Phys. Rev. Lett., 52, 461 (1984).

[2] D. R. Heslinga et al., Phys. Rev. Lett., 64, 1589 (1990).

[3] see for example, C. A. Lucas et al., Phys. Rev. B, 47, 10375 (1993).

[4] D. Loretto, J. M. Gibson and S. M. Yalisove, Phys. Rev. Lett., 63, 298 (1989).

[5] C. W. T. Bulle-Lieuwma, A. F. de Jong and D. E. Vandenhoudt, Phil. Mag., 64, 255 (1991).

[6] M. F. Chisholm et al., Appl. Phys. Lett., 64, 3608 (1994).

[7] the samples were kindly provided by $R$. T. Tung.

[8] see for example, R. Rossmann et al., Surf. Sci., 279, 199 (1992).

[9] F. Grey et al., J. Phys. (Paris) 50, 7181 (1989).

[10] H. H. Weitering, D. R. Heslinga and T. Hibma, Phys. Rev. B, 45, 5991 (1992).

[11] a recent summary can be found in L. Li et al., Phys. Rev. B, 50, 10834 (1994).

[12] H. J. Gossmann, L. C. Feldman and W. M. Gibson, Surf. Sci., 155, 413 (1984). 


\title{
X-Ray Micro-Tomography with a Diamond Anvil Cell
}

\author{
S. Weir and J. Akella \\ Lawrence Livermore Laboratory, Livermore, CA 94550
}

The properties of materials under ultra-high pressures is a fascinating field of study. Using diamond anvil cells (DAC's), pressures of several Mbars are now possible. Such pressures are high enough to dramatically affect the physical properties of most materials.

To study the high pressure properties, a wide variety of experimental techniques are now in use including $x$-ray diffraction, optical reflectivity, electrical conductivity, and Raman scattering. For crystalline materials, $\mathrm{x}$-ray diffraction is a valuable technique because it yields the pressure-vs.-volume equation-of-state (EOS), which is useful in the understanding and exploration of high pressure phases. In the case of amorphous and fluid materials, however, there is currently no general technique for determining pressure-vs.-volume equations-of-state to high pressures in a diamond anvil cell. This is unfortunate because the availability of a general technique for determining the EOS's of fluids and amorphous materials would be very valuable in the study of a number of high pressure phenomena such as pressure-induced crystalline-to-amorphous phase transitions as well as the study of high-pressure materials above their melt lines. Such a technique would also be valuable in the study of the EOS's of complex, multi-component systems such as highpressure detonation products. Our project has been to develop an experimental technique for determining the equations-of-state of fluids and amorphous materials to pressures of several hundred kbars in a diamond anvil cell.

\section{Technique}

The approach which we are exploring for obtaining EOS's of fluids and amorphous materials is a direct volumetric imaging approach using $x$-rays to determine the dimensional changes in the tiny highpressure sample as it undergoes compression. This approach is similar in concept to an optical vol- umetric imaging technique which was successfully used to measure the EOS of hydrogen to several hundred kbars in a DAC 1 .

Although ambient-pressure 3-D x-ray imaging axial tomography utilizing $x$-ray beams has been explored by a number of researchers $2,3,4,5$, it has not been applied to the in situ study of highpressure DAC samples. The application of conventional axial tomography to diamond anvil cells is problematic because the technique requires $360^{\circ}$ access to the sample, a requirement incompatible with the large amount of mechanical support material needed around the sample. As a result, we are exploring an alternative volumetric imaging approach which is made possible by the fact that the geometry of the sample is constrained by the diamond anvils.

A schematic diagram of the apparatus is shown in Figure 1. In order to perform accurate volumetric measurements of the high-pressure diamond anvil cell samples (typically disk-shaped with diameters in the range of 75-150 $\mu \mathrm{m}$, and thicknesses in the range of $20-50 \mu \mathrm{m}$ ), we are developing a scanning tomographic technique using a micro-collimated beam (Fig. 1). Due to constraints on the sample geometry, this technique only requires two scan directions, one performed normal to the plane of the sample, and one parallel to the sample plane.

The micro-collimator forms a highly collimated $\mathrm{x}$-ray beam $(10 \mu \mathrm{mx} 10 \mu \mathrm{m})$ which is rasterscanned across the face of the sample while the $x$-ray absorption is simultaneously recorded as a function of position. A second scan perpendicular to the axes of the diamond anvils yields information on the sample thickness. In our experiments, a monochromatic beam of $15 \mathrm{keV}$ was used since the total mean free path through steel is $\approx 25 \mu \mathrm{m}$, which is comparable to typical gasket thicknesses of 10-100 $\mu \mathrm{m}$. 


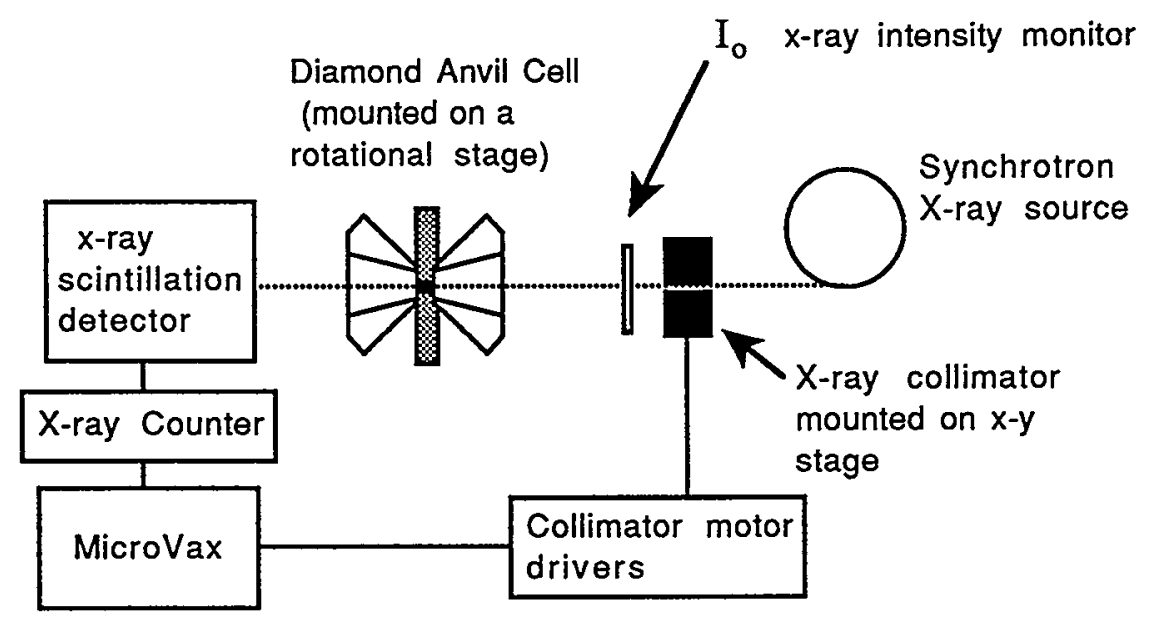

Figure 1. Schematic of the proposed high-pressure x-ray micro-tomography apparatus. Micro-collimator scans would be made both parallel and perpendicular to the plane of the high-pressure sample disk, while monitoring both the incident $\left(\mathrm{I}_{\mathrm{o}}\right)$ and transmitted intensities.

In our first experimental run, a sulfur sample with an initial diameter of $150 \mu \mathrm{m}$ and an initial thickness of $110 \mu \mathrm{m}$ was studied. A few grains of ruby $(\approx 5 \mu \mathrm{m}$ in size) were also added so that the pressure could be measured by the ruby fluorescence method. Such small grains were used to ensure that their combined volume was negligible compared to that of the sample. For each pressure, the $10 \mu \mathrm{m} \times 10 \mu \mathrm{m} \times$-ray collimator was raster-scanned across the face of the sample by $2 \mu \mathrm{m}$ increments, resulting in an image such as that shown in Figure 2. These raster scanned images were then processed by a deconvolution algorithm to correct them for the finite-sized collimated beam. In principle, the spatial resolution of the images can approach the step size of $2 \mu \mathrm{m}$ after undergoing deconvolution, although inevitable statistical noise will tend to decrease the resolution. We are in the process of studying various deconvolution algorithms in order to obtain the best images.

The thickness of the disk-shaped sample is also measured at each pressure. To do this, the DAC is rotated by $90^{\circ}$ with respect to the beam and a transverse $\mathrm{x}$-ray absorption scan is performed across the gasket. In order to obtain an unobstructed view of the portion of the gasket sandwiched between the diamond anvils, a special steel gasket was prepared in which two sides of the gasket were laser-cut away.
A profile of the sample thickness at one pressure is shown in Figure 3.

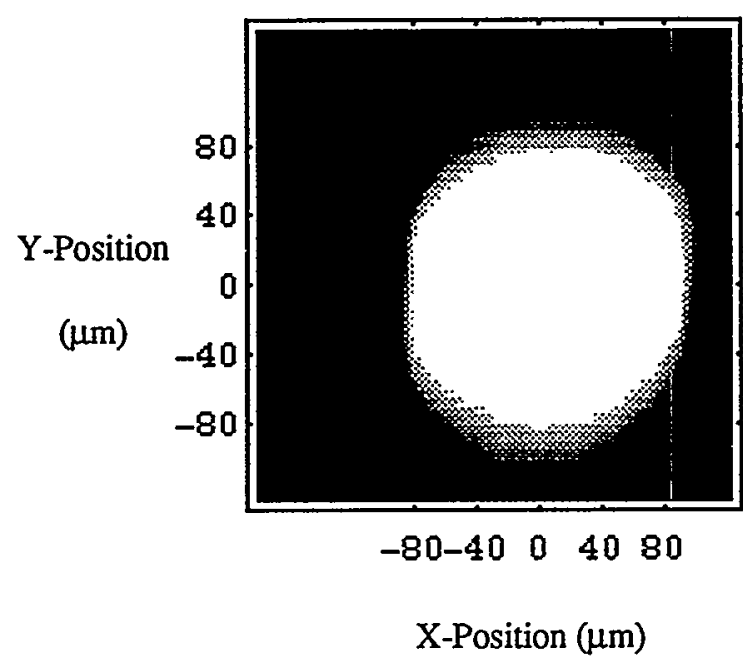

Figure 2. X-ray image of high-pressure sulfur DAC sample at a pressure of $20 \mathrm{kbars}$. Microcollimator steps of $2 \mu \mathrm{m}$ were used to raster-scan the image. 


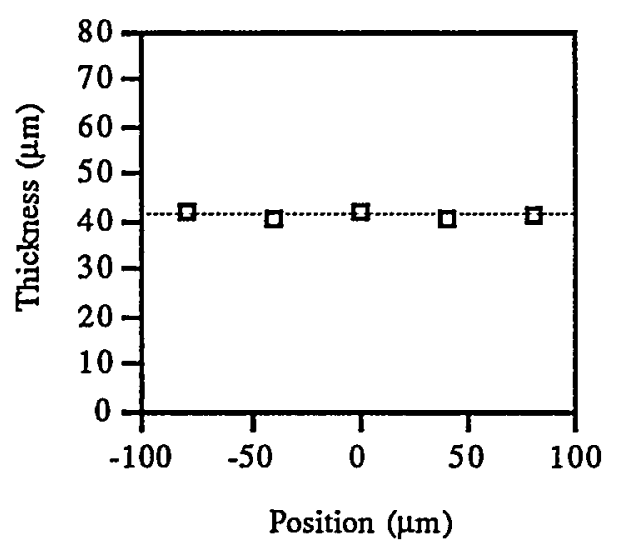

Figure 3. Thickness profile of sulfur sample at a pressure of $20 \mathrm{kbars}$. Negligible elastic "cupping" deformation was observed at this pressure.

\section{Conclusions}

In the experimental runs performed thus far at SSRL, the feasibility of the micro-tomographic volumetric imaging technique has been demonstrated. Clear, high-resolution images were obtained of both the face of the high-pressure sample and its thickness at several pressures. Our efforts are now focused on improving image quality still further by means of smaller micro-collimators and better deconvolution algorithms. In addition, we are working on improving the alignment procedures for the micro-collimator and the diamond anvil cell, so that available experimental time may be more efficiently used for data collection. Future experiments are expected to focus on the pressureinduced crystalline-to-amorphous transition in sulfur ${ }^{6}$ at $25 \mathrm{GPa}$ and on the equations-of-states of various high-pressure detonation products.

\section{Acknowledgments}

This work was performed under the auspices of the U.S. Department of Energy by Lawrence Livermore National Laboratory under Contract W7405-ENG-48. We thank the staff at SSRL for their excellent support.

1 J. van Straaten and I.F. Silvera, Phys. Rev. B 37, 1989 (1988).

2 P. Spanne and M.L. Rivers, Nucl. Instrum. Meth. Phys. Res. B24/25, 1063 (1987).
3 J.H. Kinney and M.C. Nichols, Annu. Rev. Mater. Sci. 22, 121 (1992).

4 J.H. Kinney, R.A. Saroyan, W.N. Massey, M.C. Nichols, U. Bonse, and R. Nusshardt, Review of Progress in Nondestructive Evaluation 10A, 427 (1991).

5 J.H. Kinney, T.M. Breunig, T.L. Starr, D. Haupt, M.C. Nichols, S.R. Stock, M.D. Butts, and R.A. Saroyan, Science 260, 789 (1993).

${ }^{6}$ H. Luo and A.L. Ruoff, Phys. Rev. B 48, 569 (1993). 
Proposal 2252Mp

\title{
Surface Structure Determination using Anomalous X-ray Scattering: Underpotential Deposition of Copper on $\mathrm{Pt}(111)$
}

\author{
I. M. Tidswell, C. A. Lucas, N. M. Marković and P. N. Ross \\ Materials Sciences Division, Lawrence Berkeley Laboratory, University of California, Berkeley, \\ CA 94720.
}

In-situ $x$-ray scattering offers a unique opportunity to obtain detailed information on the structure of the electrode/electrolyte interface $[1,2,3]$. In particular, measurements of the $x$-ray crystal truncation rod (CTR) are sensitive to the surface normal structure and the atomic density of any adsorbed layers at the electrode surface. Grazing incidence $x$-ray scattering measurements can be used to solve the in-plane structure of an adsorbed layer, although a precise structural solution can be difficult if the adsorbed layer consists of more than one species. In this report, we describe some $x$-ray scattering measurements of the copper underpotential deposition (UPD) layer on $\mathrm{Pt}(111)$ in the presence of chloride. By using "anomalous scattering", i.e. the dependence of the $\mathrm{Cu}$ atomic $\mathrm{x}$-ray cross section on the $\mathrm{x}$-ray photon energy near to a $\mathrm{Cu}$ adsorption edge, we are able to determine the adsorbate structure with a high degree of confidence. These measurements have been described in detail in a recent paper [4].

From electrochemical studies it is clear that electrosorption of $\mathrm{Cu}$ onto platinum surfaces (and other surfaces) is very sensitive to the anions present in solution $[5,6]$. Focusing on the Pt(111) surface, in perchloric acid solutions (with essentially no anion adsorbates) cyclic voltammetry (CV) shows that the $\mathrm{Cu}$ UPD process is very slow and kinetically controlled. The addition of a small amount of either $\mathrm{Cl}$ or $\mathrm{Br}$ anions results in a fast, diffusion limited, two step process for both deposition and desorption [5]. For ease of discussion we shall divide the potential behavior into three regions, I, II and III, shown on the $\mathrm{CV}$ in Figure 1(a) (the CV was measured in the $\mathrm{x}$-ray cell in an electrolyte consisting of $0.1 \mathrm{M} \mathrm{HClO}_{4}$ $0.001 \mathrm{M} \mathrm{NaCl}$ and $10^{-3} \mathrm{M} \mathrm{Cu}^{2+}$ ). X-ray measurements were made on beamline 7-2 at SSRL (the (111) face is indexed to a hexagonal lattice described in reference 7). As the potential was swept at approximately $1 \mathrm{mV} / \mathrm{s}$, the $x$-ray scattering intensity, measured at the
(0 11 1.5) non-specular CTR position, shifted between three constant levels (Figure 1(b)). These three regions of constant $\mathrm{x}$-ray scattering are consistent with the presence of three different interface structures. In all three regions we searched for in-plane scattering peaks which would be associated with an ordered interface superstructure. No such peaks were found in the potential region where no $\mathrm{Cu}$ is absorbed, or in the region where a full $\mathrm{Cu}$ monolayer is adsorbed. In the middle region (i.e., in between the two UPD peaks) four symmetry independent peaks were observed at $(0$, $0.765),(0.765,0.765),(0,1.53)$ and $(0.765,1.53)$. These positions are shown in figure 2 (top). The changes in the scattered intensity at $(0.765$, $0.765,0.1$, Figure $1(\mathrm{c})$, clearly shows that the structure responsible for this peak is potential dependent and present only in the central region. The location of these peaks indicates that the adsorbed species form a hexagonal superstructure which is incommensurate with the underlying platinum lattice; the nearest commensurate unit cell is $(4 \times 4)$, as was seen by LEED. The insets in Figure 2(a) and 2(b) show two rocking scans ( $\phi$ scans) through the $(0,1$, $0.1)$ and $(0.765,0.765,0.1)$ positions. Both peaks have a lorentzian lineshape with a half width at half maximum (HWHM) of $\sim 0.005 \AA$ in (b), corresponding to domain size of $200 \AA$. This is somewhat smaller than the estimated terrace size, $1000 \AA$, obtained from the width of the $(0,1)$ peak.

The relative intensities of the four in-plane peaks can be used to derive a structural model for the adsorbed layer. Additional information, however, can first be obtained by utilizing "anomalous scattering" methods, i.e. measuring the peak intensities as the incident photon energy is tuned close to the $\mathrm{Cu} \mathrm{K}$ adsorption edge $(8979 \mathrm{eV})$ [8]. In general, the intensities of the relatively strong $(0.765,0.765)$ and $(0,1.530)$ peaks were weaker close to the $\mathrm{Cu}$ edge, whereas the relatively weak $(0,0.765)$ and $(0.765,1.530)$ peaks 


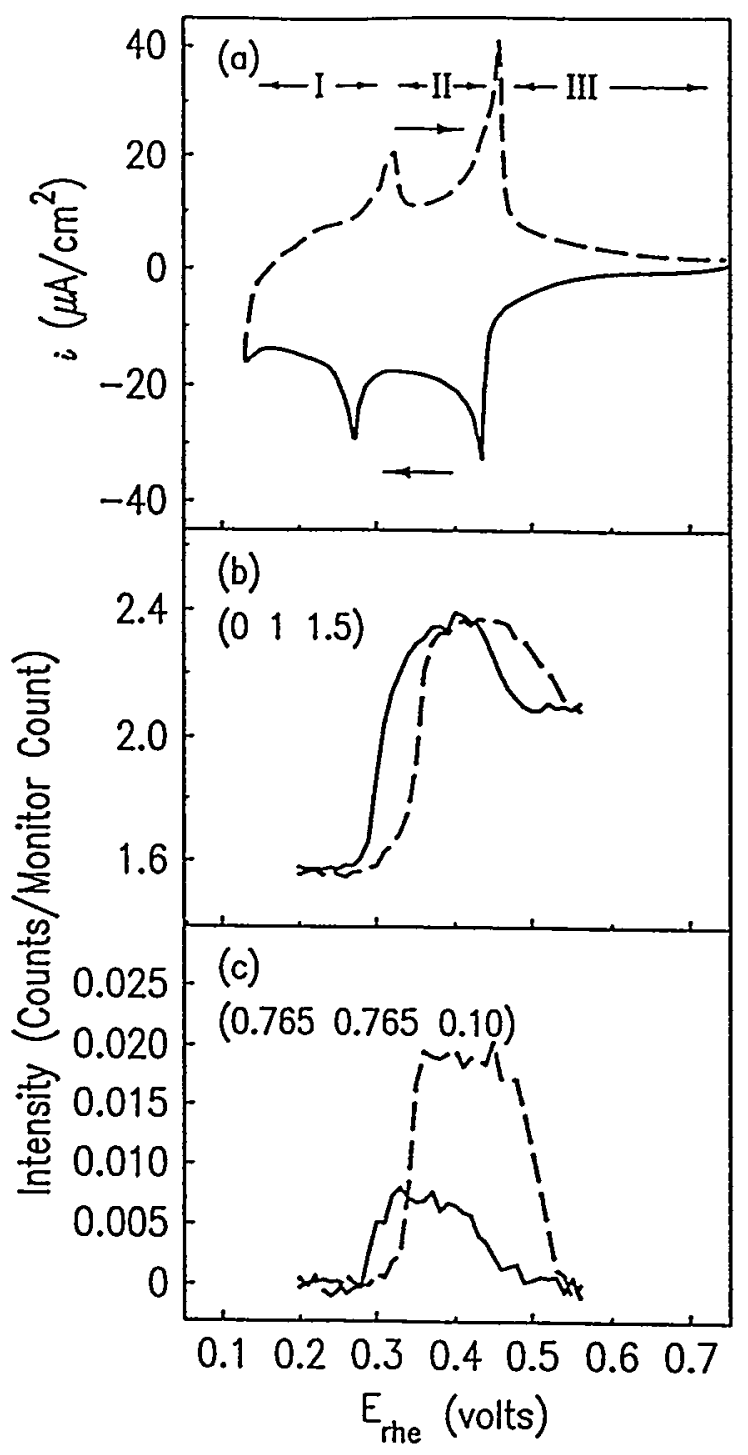

Figure 1. (a) $\mathrm{CV}$ of the $\mathrm{Pt}(111)$ crystal measured in the $x$-ray electrochemical cell with the polymer film expanded. (b) Changes in the $x$-ray intensity measured at the $\left(\begin{array}{lll}0 & 1 & 1.5\end{array}\right)$ CTR position. (c) Changes in the $x-$ ray intensity at the $(0.765,0.765,0.1)$ peak. In all cases the solid line is the negative going (adsorbing) sweep and the dashed line is the positive going (stripping) sweep. The numerals I to III designate the three potential regions described in the text.

were stronger close to the $\mathrm{Cu}$ edge. Without further analysis (for details see reference 4 ), this result indicates that the adsorbed superstructure does not consist of only $\mathrm{Cu}$ atoms (which would result in a reduction in intensity of all the in-plane peaks near the $\mathrm{Cu}$ edge) but must also include $\mathrm{Cl}$. The simplest real space structure consistent with the data has a hexagonal unit cell with a lattice spacing $3.63 \AA$. The unit cell contains one $\mathrm{Cu}$ and one $\mathrm{Cl}$ atom arranged in two hexagonal layers one of $\mathrm{Cu}$ and one of $\mathrm{Cl}$, each with a coverage of $0.585 \mathrm{ML}$. Good agreement with the data was obtained with $\mathrm{Cl}$ atoms in the three-fold hollow sites of the $\mathrm{Cu}$ layer. The result of optimizing the model, by allowing the Debye Waller disorder terms for the two atoms to vary, is shown in Figure 2 by the left hand side quadrants for each $\mathrm{X}$-ray energy at the four measured positions.
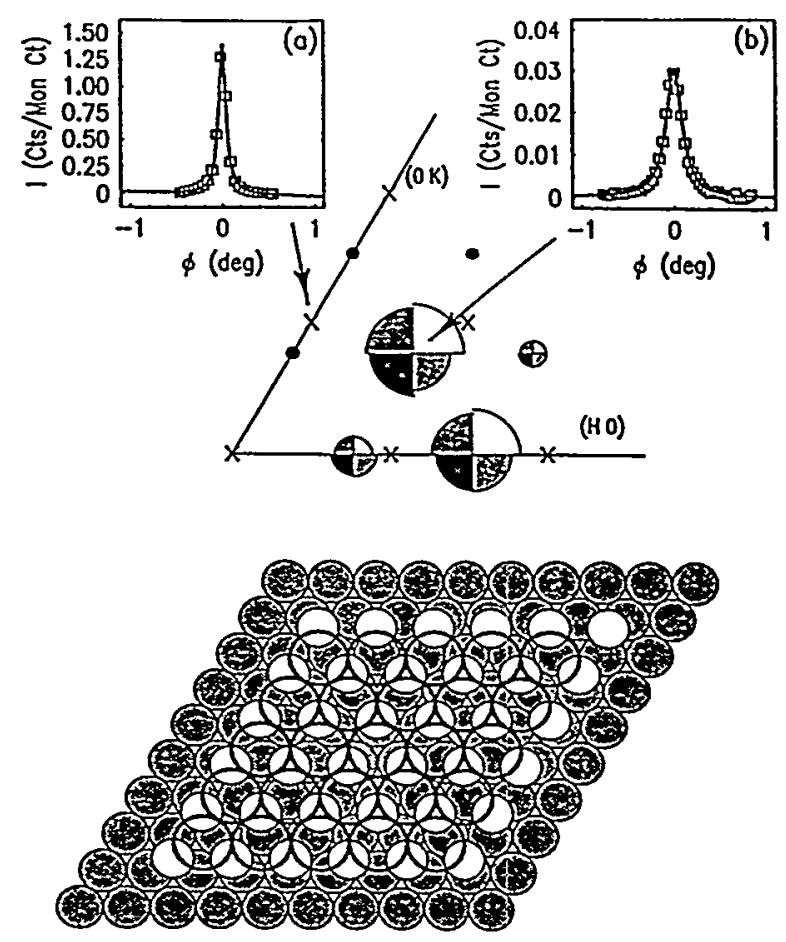

Figure 2. The top figure shows a representation of the in-plane scattering in reciprocal space in the potential region between the two UPD peaks in Figure 1(a). The locations of CTR's are shown by crosses and the superstructure peaks by circles. The lower figure shows the proposed structure of the adsorbed layer. The $\mathrm{Pt}$ substrate is shown in grey, while $\mathrm{Cu}$ atoms are represented in white. The top layer of $\mathrm{Cl}$ atoms are drawn in outline to show the position relative to the underlying $\mathrm{Cu}$ and $\mathrm{Pt}$ atoms. The insets (a) and (b) are two $\phi$ scans through the $(0,1,0.1)$ and $(0.765,0.765,0.1)$ positions respectively (the solid lines are lorentzian fits).

While there are some discrepancies in the absolute intensities (which could be reduced by allowing the position of the $\mathrm{Cl}$ atom to vary), it is clear that the size of the change of scattering intensity is quite well described by this structure. No other physically reasonable structure was able to describe the data, although we cannot rule out a more complex model. The $\mathrm{Pt}-\mathrm{Cu}$ and $\mathrm{Cu}-\mathrm{Cl}$ surface normal spacings 
of $2.2 \pm 0.1 \AA$ and $1.2 \pm 0.3 \AA$ respectively were derived from the out-of-plane intensity dependence of the in-plane peaks and from the specular CTR (the results are not shown here). It is interesting to note that our proposed structure of the adsorbed bilayer is very similar to the (111) plane of $\mathrm{Cu}(\mathrm{I}) \mathrm{Cl}$.

We would like to thank Lee Johnson for technical assistance and acknowledge very useful discussions with Tom Rabedeau, Sean Brennan and Mike Toney. This work was supported by the Director, Office of Energy Research, Office of Basic Energy Science, Materials Sciences Division of the U.S. Department of Energy (USDOE) under contract number DE-AC0376 SF00098.

\section{References.}

[1] O. R. Melroy, M. F. Toney, G. L. Borges, M. G. Samant, J. B. Kortright, P. N Ross, and L. Blum, Phys. Rev. B, 38, 10962 (1988).

[2] J. Wang, B. M. Ocko, A. Davenport, and H. Isaacs, Phys. Rev. B, 46, 10321 (1992).

[3] I. M. Tidswell, N. M. Markovic and P. N. Ross, Phys. Rev. Lett., 71, 1601 (1993).

[4] I. M. Tidswell, C. A. Lucas, N. M. Markovic and P. N. Ross, Phys. Rev. B, in press (1995).

[5] N. M. Marković and P. N. Ross, Langmuir 9, 580 (1993).

[6] R. Michaelis, M. S. Zei, R. S. Zhai and D. M. Kolb, J. Electroanal. Chem. 339, 299 (1992).

[7] A. R. Sandy et al., Phys. Rev. B 43, 4667 (1991).

[8] S. Brennan and P. L. Cowan, Rev. Sci. Instrum. 63, 850 (1992). 


\title{
STRUCTURAL CHARACTERIZATION OF ORGANOCUPRATE COMPLEXES IN SOLUTION
}

\author{
Terence M. Barnhart, Hui Huang, and James E. Penner-Hahn \\ Department of Chemistry, University of Michigan, Ann Arbor, MI 48109-1055
}

\section{INTRODUCTION}

Organocopper reagents $\left(\mathrm{R}_{2} \mathrm{Cu}^{-}\right)$are used extensively for $\mathrm{C}-\mathrm{C}$ bond formation reactions in organic synthesis [1]. However, little is known of their solution structure, and the data that are available are often equivocal. In the present report, we describe recent results in two areas of organocuprate structural chemistry: Characterization of the structure of "higher-order" cuprates and determination of the structure of the cuprate reagents prepared from transmetellation of $\mathrm{CuCN}$ and Grignard reagents.

\section{"Higher-Order" Cuprates.}

Solutions of $\mathrm{R}_{2} \mathrm{Cu}^{-}$are made from addition of $2 \mathrm{RLi}$ to slurries of either $\mathrm{CuI}$ or $\mathrm{CuCN}$ to form clear solutions. In the latter case, the resulting solutions are referred to as "higher order" cuprates, which have been suggested to contain a three-coordinate $\mathrm{Cu}(\mathrm{I})$ center in which both $\mathrm{R}$ groups and the $\mathrm{CN}^{-}$are directly coordinated. Recently, this structural proposal has been questioned. NMR [2-3] and EXAFS [4] spectra have both been interpreted as indicating that cyanide is not linearly coordinated to the $\mathrm{Cu}$ in " $\mathrm{R}_{2} \mathrm{Cu}(\mathrm{CN}) \mathrm{Li}{ }_{2}$ ". The "higher-order" cuprate proposal was originally formulated in terms of a linearly coordinated cyanide ligand [1]. However, it has recently been suggested that the cyanide may, instead, be $\pi$-coordinated to the $\mathrm{Cu}$ center [5].

We have used EXAFS and XANES to show that $\mathrm{Cu}$ is unequivocally two coordinate in the so-called "higher-order" cuprate formed from CuCN+2RLi. The XANES spectra for the dimethyl cuprates prepared from $\mathrm{CuCN}$ or from Cul are compared in Fig. 1. It is clear that the presence or absence of $\mathrm{CN}^{-}$does not cause any detectable change in the Cu XANES. Similarly, the EXAFS $\mathrm{Cu}-\mathrm{C}$ bond lengths are identical for both species. The average $\mathrm{Cu}-\mathrm{C}$ bond length $(1.95 \AA)$ is indicative of a two- rather than a three-coordinate $\mathrm{Cu}$ site. The absence of any change in either XANES or EXAFS when $\mathrm{I}^{-}$is changed for $\mathrm{CN}^{-}$suggests that the cyanide is not bound directly to the $\mathrm{Cu}$ in this species.

When cyanide is coordinated linearly to $\mathrm{Cu}$, two shells of scatterers $(\mathrm{Cu}-\mathrm{C}$ and $\mathrm{Cu} \cdot \cdots \mathrm{N}$ ) should be apparent in the EXAFS spectrum as a result of the intense multiple scattering of the linear cyanide ligand. This is seen, for example, in preparations of $\mathrm{BuCuCN}^{-}$, which contains a two coordiante $\mathrm{Cu}(\mathrm{I})$ with a $\sigma$-bound cyanide ligand. Furthermore, the $1 \mathrm{~s} \rightarrow 4 \mathrm{p}$ transition in the XANES region should decrease in intensity on going from a linear two coordinate geometry to a three coordinate structure. Finally, EXAFS spectrum should show an increase in the bond distance, and apparent number of first shell scatters on addition of a third $\pi$-bound ligand.

In order to test the sensitivity of XAS to the formation of a three coordinate $\pi$-complex, we have measured XANES and EXAFS spectra for the $\pi$ complex formed by addition of $\mathrm{Me}_{2} \mathrm{CuLi}$ (from Cul) to Methyl-trans-cinnamate ester. This has previously been shown by NMR to form an authentic three-coordinate $\mathrm{Cu}(\mathrm{I})$ complex containing a $\pi$-bonded enoate. The XANES spectrum of this solution shows a markedly decreased $1 \mathrm{~s} \rightarrow 4 \mathrm{p}$ transition consistent with the formation of a three-coordinate complex. Similarly, the $\mathrm{Cu}-\mathrm{C}$ bond length increases to $1.99 \AA$ and the apparent first shell coordination number increases from two to four. All of these observations are consistent with formation of a $\pi$-bound enolate, as previously shown by NMR.

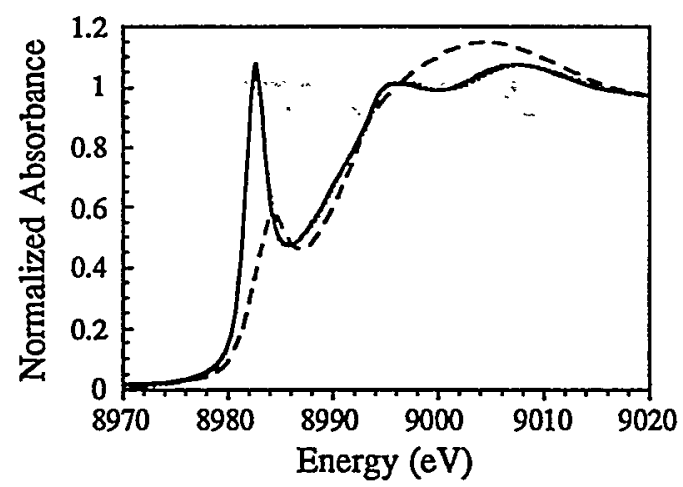

Fig. 1. XANES spectra for "higher-order" cuprates prepared from CuCN (solid) and CuI (dots). For comparison, the XANES spectrum of the dimethylcuprateenolate $\pi$ complex is also shown (dashed line). 
This demonstrates the sensitivity of EXAFS and XANES to the presence of a $\pi$-complex and thus strongly suggests that there is no significant $\pi$-complexation between $\mathrm{Cu}$ and $\mathrm{CN}^{-}$in the "higher-order" cuprates.

These observations are inconsistent with direct coordination of cyanide ligand in either a $\pi$ or a $\sigma$ mode in "higher order" cuprate solutions. These observations comprise the first unequivocal study of the copper coordination sphere of "higher order" cuprate complexes in solution.

\section{Grignard Cuprates.}

In addition to being prepared using alkyl lithium reagents, cuprate reagents can also be prepared using alkyl Grignard starting materials. The Grignard cuprate reagents are important because they are easier to prepare than lithium cuprates, because they have greater stability, and because they show different reactivity patterns thus allowing access to different products. We have used EXAFS to determine the structure of the cuprate reagents generated from $\mathrm{MeMgCl}+\mathrm{CuCN}$ or $\mathrm{CuI}$ and to compare their structures with the corresponding cuprates formed from RLi.

The normalized XANES spectra of $\mathrm{CuCN} \cdot 2 \mathrm{LiCl}$ and $\mathrm{CuCN} \cdot 2 \mathrm{LiCl}+\mathrm{nMeMgCl}(\mathrm{n}=1)$ are shown in Figure 2. For comparison, XANES for $\mathrm{CuCN} \cdot 2 \mathrm{LiCl}+\mathrm{nMeLi}(\mathrm{n}=1,2)$ are also shown. There is a continuum resonance at ca. $8995 \mathrm{eV}$ due to the presence of linear cyanide ligands. The intensity of this peak decreases on going from $\mathrm{CuCN} \cdot 2 \mathrm{LiCl}$ to $\mathrm{CuCN} \cdot 2 \mathrm{LiCl}+\mathrm{RLi}$ to $\mathrm{CuCN} \cdot 2 \mathrm{LiCl}+2 \mathrm{RLi}$, consistent with the displacement of successive cyanide ligands. A similar change is observed on addition of $\mathrm{MeMgCl}$ to $\mathrm{CuCN} \cdot 2 \mathrm{LiCl}$. In this case however, the spectra for samples containing 2 or 3 equivalents of $\mathrm{MeMgCl}$ are identical to that seen for 1 equivalent of $\mathrm{MeMgCl}$. This suggests that cyanide displacement is not complete in this case. Although the spectra for $\mathrm{CuCN}+\mathrm{RLi}$ and $\mathrm{CuCN}+\mathrm{RMgCl}$ are very similar, they are not identical. In particular, the cuprate prepared from $\mathrm{RMgCl}$ has a weak transition at $8986 \mathrm{eV}$ which is absent in other cuprate spectra.

The Fourier transforms of the EXAFS spectra are shown in Fig. 3. As with the edge spectra, the FTs for samples having 2 or 3 equivalents of $\mathrm{MeMgCl}$ are identical to that for $\mathrm{CuCN}+\mathrm{RMgCl}$. As noted previously [5] , addition of 1 equivalent of $\mathrm{RLi}$ causes loss of ca. one-half of the $\mathrm{Cu}$ -

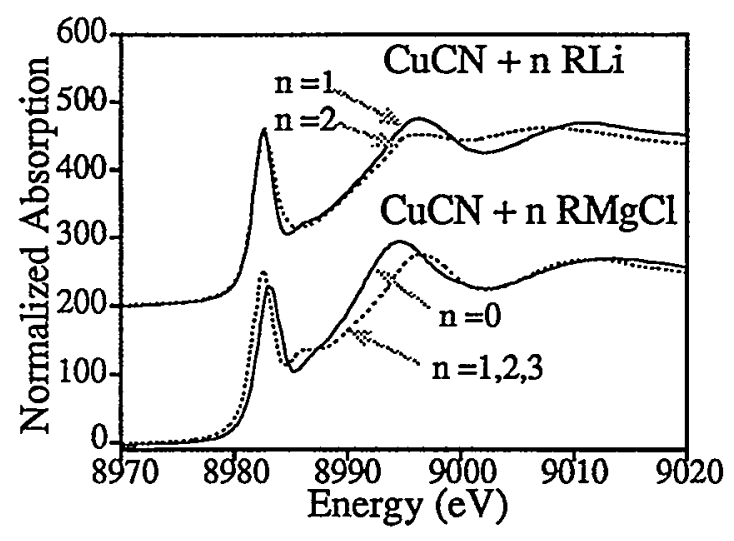

Figure 2. XANES spectra for mono and dimethyl cuprates prepared using $\mathrm{MeLi}$ and $\mathrm{MeMgCl}$.

$\mathrm{CN}$ coordination, while addition of a second equivalent of $\mathrm{RLi}$ causes complete displacement of the cyanide ligation. Addition of the first equivalent of $\mathrm{RMgCl}$ similarly causes loss of ca. one-half of the cyanide ligands (see Fig. 3, bottom). In contrast, even a three-fold excess of $\mathrm{RMgCl}$ is not sufficient to displace the remaining cyanide. This is confirmed by $I R$ measurements (not shown). Even more striking is the difference in the FTs for MeCuCNLi and $\mathrm{MeCuCNMgCl}$. For the Grignard cuprate, there is a small feature at $R+\alpha=4.5 \AA$ that is not present in the FT of the lithium cuprate. This peak is not due to residual $\mathrm{Cu} \cdots \mathrm{Cu}$ EXAFS, as indicated by the fact that it does not disappear on addition of excess $\mathrm{RMgCl}$ and by the fact that the apparent $\mathrm{Cu} \cdots \mathrm{X}$ distance is significantly longer than in $\mathrm{CuCN}-2 \mathrm{LiCl}$. This peak can be modeled as a $\mathrm{Cu}-\mathrm{Mg}$ interaction at 5.02-5.05 $\AA$, although other interpretations cannot be ruled out at this

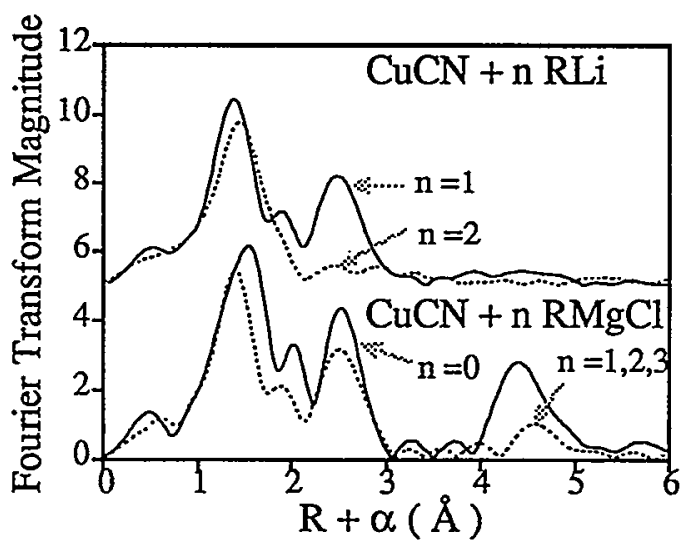

Figure 3. FTs of EXAFS data for mono and dimethyl cuprates prepared from $\mathrm{MeLi}$ and $\mathrm{MeMgCl}$. 
time. In other regards, the curve fitting resuls for $\mathrm{CuCN}+\mathrm{MeMgCl}$ are identical with those for the lithium cuprate.

These data demonstrate that there are two principal difference between the $\mathrm{Li}$ and the Grignard cuprates. Most importantly, $\mathrm{RMgCl}$ does not appear able to carry out a transmetallation reaction with $\mathrm{RCuCN}$. Only the first transmetallation reaction:

$\mathrm{RMgCl}+[-\mathrm{Cu}(\mathrm{CN})-]_{\mathrm{n}} \rightarrow \mathrm{RCuCN}^{-}+\mathrm{MgCl}^{+}$

appears to take place. Secondly, there appears to be a scatterer coordinated to the $\mathrm{N}$ of the $\mathrm{CN}$ in the $\mathrm{CuCN}+\mathrm{RMgCl}$ adduct. A likely candidate for this scatterer is $\mathrm{Mg}^{2+}$, which may play an important role in controlling the reactivity of the cuprate reagent (6).

In contrarast with the $\mathrm{CuCN}$ system, the cuprate reagents generated from $\mathrm{CuI}+\mathrm{MeMgCl}$ are indistinguishable by EXAFS or XANES from the reagents prepared using MeLi. This illustrates the important role played by $\mathrm{CN}^{-}$of the cuprate reagent.

\section{REFERENCES}

1. For reviews of cyanocuprate chemistry, see: a) Lishutz, B. H.; Sengupta, S.; Org. React. 1992, 41, 135. b) Lipshutz, B. H. Synlett $1990,3,119$.

2. a) Bertz, S. H. J. Am. Chem. Soc. 1991, 113,5470 and references therein.

3. a) Snyder, J. P.; Spangler, D. P.; Behling, J. R.; Rossiter, B. E. J. Org. Chem. 1994, 59,2665 and references therein.

4. Stemmler, T.; Penner-Hahn, J. E.; Knochel, P. J. Am. Chem. Soc. 1993, 59, 348.

5. Lipshutz, B. H. and James, B. J. Org. Chem. 1994, 59, 7585.

6. Tseng, C.C.; Paisley, S.D.; Goering, H.L. J. Org. Chem. 1986, 51, 2884-91.

\section{ACKNOWLEDGEMENTS}

This work has been supported in part by grants from the A.P. Sloan Foundation (to JEPH) and the Sokol Foundation (to TMB). Spectra were measured in part at SSRL, which is supported by the US DOE and by the NIH, Biotechnology Resource Program, Division of Research Resources. 
Proposal 2254M

Structural Studies of Manganese Doped Bismuth Vanadate Ionic Conductors

Allan J. Jacobson, Yuemei L. Yang, and Longbao Qiu

Department of Chemistry, University of Houston, Houston, TX 77204-5641

Ingrid J. Pickering

Stanford Synchrotron Radiation Laboratory, Stanford CA 94309-0210.

Very high oxide ion conductivity has recently been reported in a series of compounds derived from $\mathrm{Bi}_{2} \mathrm{O}_{2} \mathrm{VO}_{3} .5$ by partial substitution of vanadium by other transition metals [1]. The parent structure is the first member of the Aurivillius structural series which consists of layers of composition $\mathrm{Bi}_{2} \mathrm{O}_{2}$ that alternate with perovskite layers of ideal composition $A_{n-1} B_{n} O_{3 n+1}$. In the bismuth vanadate case, $n=1$ and the presence of oxygen vacancies (ㅁ) gives a single layer of square pyramids $\mathrm{VO}_{3.5}{ }^{-} 0.5$. In reality the structure is much more complex. At low temperatures, ordering of the oxygen vacancies results in a very large supercell, the structure of which is not known in detail. On heating the room temperature $\alpha$ phase transforms first to $\beta$ and finally to a simple tetragonal structure $(\gamma)$ that has the ideal symmetry of the $n=1$ Aurivillius structure $(14 / \mathrm{mmm})$. The successive phase transitions are believed to be associated with disordering of the oxygen vacancies within the perovskite layers [2], though the supporting evidence for this is limited. What is clear is that the ionic conductivity follows the phase transitions. The ionic conductivity in the $\gamma$ phase is unusually high and comparable to the best previously known examples of oxide anion conductors.

Following the initial work on pure $\mathrm{Bi}_{2} \mathrm{O}_{2} \mathrm{VO}_{3.5}$, several groups have shown that the $\gamma$ structure can be stabilized at lower temperature by partial substitution of vanadium with another transition metal [3-5]. All of the first row transition elements stabilize the $\gamma$ structure over some range of composition and temperature, substitution with copper being particularly effective. Our specific interest is in the synthesis of stabilized $\gamma$ phases with both high ionic and electronic conductivity for application in mixed electron ion conducting membranes. For this reason, we have concentrated on the mixed conducting manganese doped phases. To date we have established by diffraction and thermal analysis studies that the $\gamma$ phase can be stabilized in the compound $\mathrm{Bi}_{2} \mathrm{O}_{2} \mathrm{~V}_{1-x} \mathrm{Mn}_{x} \mathrm{O}_{3.5-\delta}$ for $0.1 \leq x \leq 0.25$. We have determined the synthesis conditions to obtain reproducible single phase materials in this composition range and cell constants and average structures by fitting the powder X-ray diffraction data. We have measured the total conductivities as a function of temperaturc by ac impedance techniques. The data confirm the high conductivity at temperatures below $500^{\circ} \mathrm{C}$. Results are shown in Figure 1 for one representative

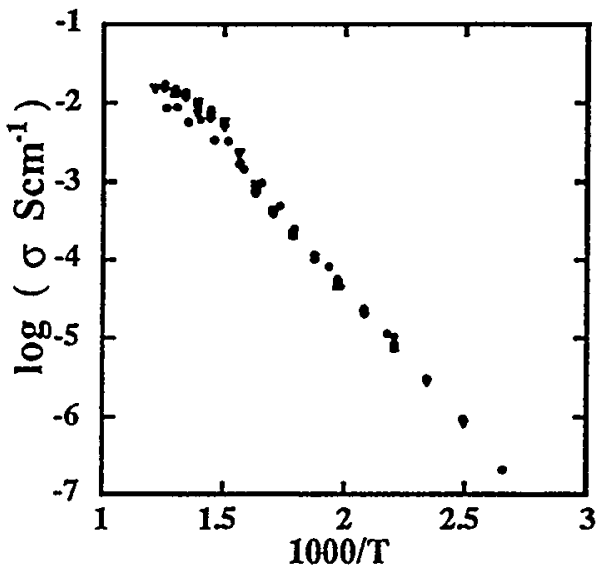

Figure 1 Temperature dependence of the conductivity of $\mathrm{Bi}_{2} \mathrm{~V}_{0.8} \mathrm{Mn}_{0.2} \mathrm{O}_{3.5} \delta$.

composition $(x=0.20)$. The high value of the total conductivity below $500^{\circ} \mathrm{C}(1000 / \mathrm{T}=1.3)$ is remarkable and suggests that the materials are excellent candidates for oxygen transport materials. The transport properties are being investigated by other techniques in order to separate the ionic and electronic contributions to the total conductivity. An understanding of the transport mechanism requires more detailed information about the local structure and electronic changes that occur on doping. We have made a first series of XAS measurements at the $\mathrm{Mn}$ and $\mathrm{V} \mathrm{K}$ edges and also at the $\mathrm{Bi} \mathrm{L}_{\mathrm{LI}}$ edge for a series of $\mathrm{Bi}_{2} \mathrm{O}_{2} \mathrm{~V}_{1-\mathrm{x}} \mathrm{Mn}_{\mathrm{X}} \mathrm{O}_{3.5-\delta}$ compositions to begin to address these issues. The preliminary results are described in this report.

XAS experiments have been performed on powder samples of $\mathrm{Bi}_{2} \mathrm{O}_{2} \mathrm{~V}_{1-\mathrm{x}} \mathrm{Mn}_{\mathrm{x}} \mathrm{O}_{3.5-\delta} \delta$ with $\mathrm{x}=$ 
$0,0.05,0.10,0.15,0.20$ and 0.25 . The samples were prepared by two procedures. In one method, samples were annnealed at increasing temperatures up to a maximum value of $890^{\circ} \mathrm{C}(\mathrm{x}=0,0.05,0.15)$. In the second procedure, the samples were melted and then ground and annealed at $890^{\circ} \mathrm{C}(x=0.1,0.15$, $0.2,0.25$ ). Samples with $x=0.15$ prepared by both trechniques were compared. XAS measurements were carried out at beamline 2-3 at SSRL, using a $\mathrm{Si}(220)$ double crystal monochromator and an upstream vertical aperture of $1 \mathrm{~mm}$. Finely ground powders were packed into a specimen holder $20 \times 4$ $\mathrm{mm}^{2}$ in area and $0.5 \mathrm{~mm}$ thick. Samples were held at $10 \mathrm{~K}$ in an SSRL Biotechnology Laboratory flowing liquid helium cryostat, and measurements were made in fluorescence for the $\mathrm{Mn}$ and $\mathrm{V} \mathrm{K}$-edges using an SSRL Biotech. 13-element germanium detector. Fluorescence measurements were required due to the presence of the highly absorbing bismuth in the sample.

The EXAFS data were analyzed using the program feff-5 of Rehr and coworkers. (6) The fitted EXAFS data and the corresponding Fourier transforms are shown for both the $\mathrm{V}$ and $\mathrm{Mn} \mathrm{K}$ edges in Figures 2 and 3 for one composition $(x=0.2)$. The data for other compositions prepared by the same technique are closely similar. The results indicate that the the vanadium and the manganese coordination environments are very different. Previous
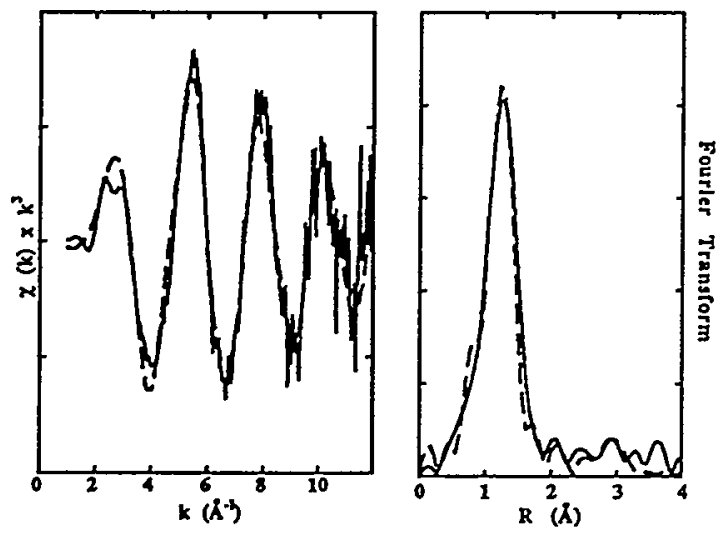

Figure 2: $\mathrm{V} \mathrm{K}$ edge EXAFS and Fourier Transform data for $\mathrm{Bi}_{2} \mathrm{~V}_{0.8} \mathrm{Mn}_{0.2} \mathrm{O}_{3.5-\delta}$

$\mathrm{X}$-ray and neutron diffraction studies of the manganese and other metal substituted phases have assumed that the dopant replaces $V$ in the lattice and adopts a similar coordination but on a local level this is clearly not the case. In particular, little evidence for well defined next nearest neighbor distances is apparent in the vanadium K edge EXAFS, suggesting a high degree of disorder. A preliminary fit to the data indicates $4 \mathrm{~V}-\mathrm{O}$ distances at $1.70 \AA$. This $\mathrm{V}-\mathrm{O}$ bond distance is close to that expected for four coordinate $V(V)$. No strong evidence is observed for the presence of a vanadium atom with the square pyramidal coordination by oxygen atoms assumed in the analysis of diffraction data (expected distances 1.V-O at $1.6 \AA$ and $4 \mathrm{~V}-\mathrm{O}$ at $2.0 \AA$ from neutron data).

In contrast to the vanadium EXAFS, the corresponding $\mathrm{K}$ edge data for Mn (Figure 3) contain better developed peaks in the Fourier transformed data at distances beyond the first nearest neighbor shell indicative of a more regular coordination environment. Analysis of the EXAFS data and the
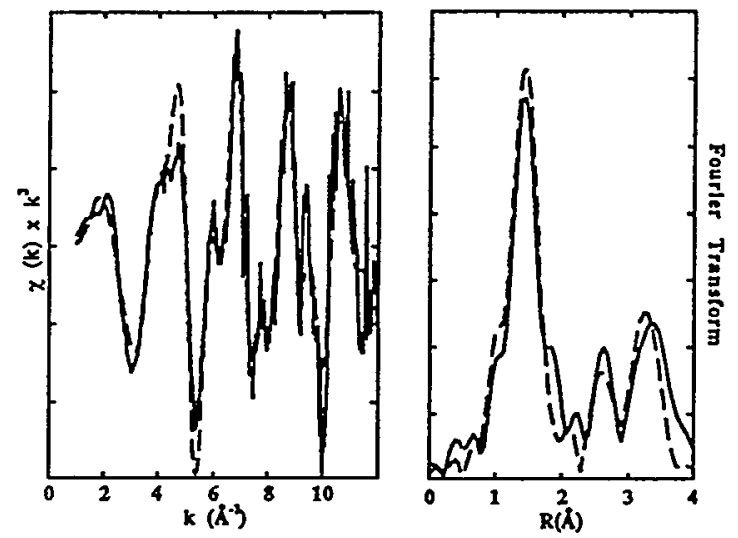

Figure 3: Mn K edge EXAFS and Fourier Transform data for $\mathrm{Bi}_{2} \mathrm{~V}_{0.8} \mathrm{Mn}_{0.2} \mathrm{O}_{3.5-\delta}$

XANES data below suggest that the $\mathrm{Mn}$ is octahedrally coordinated. Some preliminary EXAFS data at the $\mathrm{Cu}$ edge in $\mathrm{Bi}_{2} \mathrm{O}_{2} \mathrm{~V}_{0.9} \mathrm{Cu}_{0.1} \mathrm{O}_{3.5-\delta}$ also indicate some degree of order for the copper coordination. [7] No $\mathrm{V} \mathrm{K}$ edge EXAFS data have been previously reported.

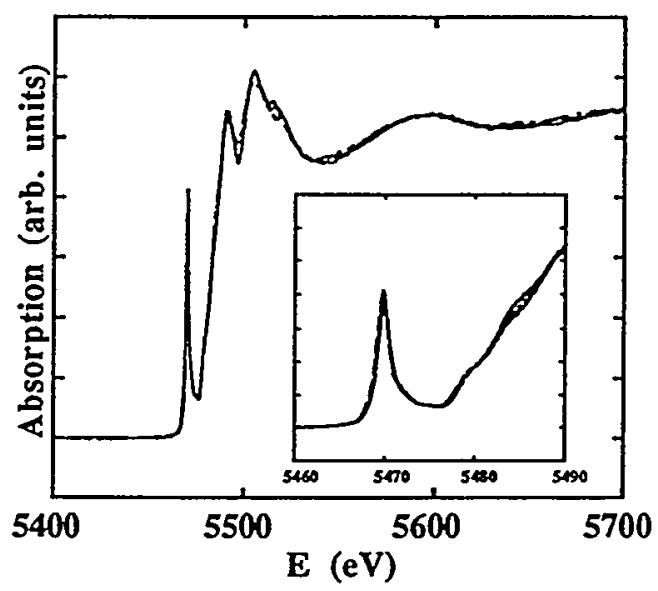

Figure 4: $\mathrm{V} X A N E S$ data for $\mathrm{Bi}_{2} \mathrm{~V}_{1-\mathrm{x}} \mathrm{Mn}_{\mathrm{x}} \mathrm{O}_{3.5-\delta}$ with $x=0$ to 0.25 in increments of 0.05 . 
The $\mathrm{V}$ and $\mathrm{Mn} \mathrm{K}$ edge XANES data are shown in Figure 4 and 5 for all of the compositions. The vanadium data show a very intense is to $3 \mathrm{~d}$ transition consistent with a non-centrosymmetric vanadium site. The variations with composition are small for $0.1 \leq x \leq 0.25$ but systematic and are different from the spectra of $x=0$ and 0.05 . The latter compositions are structurally distinct and do not form part of the tetragonal solid solution series. The corresponding data at the Mn edge show a very weak and splitd is to $3 \mathrm{~d}$ transition characteristic of centrosymmetric probably octahedral geometry. A pre edge feature at close to $6550 \mathrm{eV}$ is also apparent in the data. This feature is most pronounced for the $x=$ 0.20 and 0.25 compositions (see inset in Figure 5) for reasons that are not yet understood.

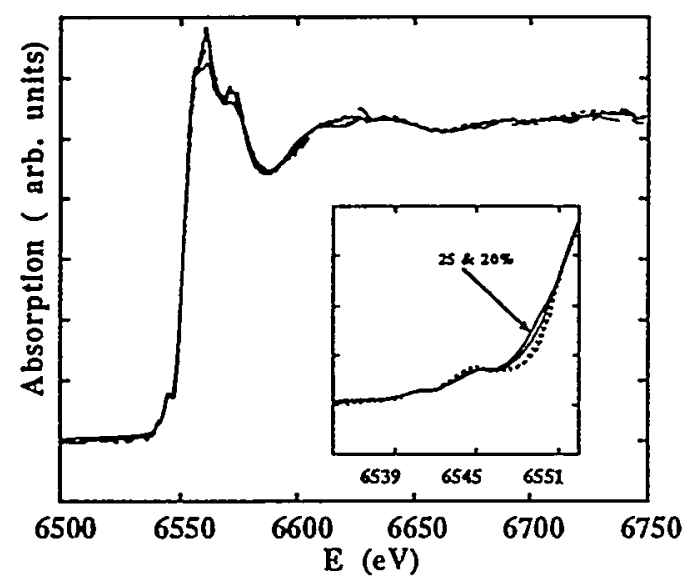

Figure 5: Mn XANES data for $\mathrm{Bi}_{2} \mathrm{~V}_{1-\mathrm{x}} \mathrm{Mn}_{\mathrm{x}} \mathrm{O}_{3.5-\delta}$ with $x=0.05$ to 0.25 in increments of 0.05 .

A comparison of the Mn EXAFS data for two samples with $x=0.15$ that were prepared by two different synthesis procedures showed distinct differences in the outer shell coordination. This is an important result that may resolve some previous anomalies in the diffraction data. Further XAS studies of samples as a function of annealing conditions will be correlated with diffraction data and conductivity measurements in future studies.

The work was supported by the Department of Energy through the Office of Basic Energy Sciences and Los Alamos National Laboratories and The Robert A. Welch Foundation.

[1] F. Abraham, M. F. Debreuille-Gresse, G. Maircsse and G. Nowogrocki, Solid State Ionics 28-30 (1988) 529.

[2] F. Abraham, J. C. Boivin, G. Mairesse and G.
Nowogrocki, Solid Statc Ionics 40/41 (1990) 934.

[3] J. B. Goodenough, A. Manthiram, M. Paranthaman, and Y. S. Zhen, Mater. Sci and Eng. B12 (1992) 357.

[4] V. Sharma, A. Shukla and J. Gopalakrishnan, Solid State Ionics 58 (1992) 359.

[5] R. Essalim, B. Tanouti, J. Bonnet and J. Reau, Mater. Lett. 13 (1992) 382.

[6] J.J. Rehr, J. Mustre de Leon, S.I. Zabinsky, and R.C. Albers, J. Am. Chem. Soc. 113, 5135 (1991)

[7] A. J. Franklin, A. V. Chadwick and J. W. Couves, Solid State Ionics, 70/71, 215 (1994). 


\title{
X-Ray Absorption Studies Of Uranium Sorption On Mineral Substrates
}

\author{
Eric A. Hudson, Louis J. Terminello, and Brian E. Viani \\ Lawrence Livermore National Laboratory, Livermore, CA 94551 \\ Tobias Reich \\ Forschungszentrum Rossendorf e. V., Institut für Radiochemie, \\ Postfach 5105 19, D-01314 Dresden, Germany \\ Jerome J. Bucher, David K. Shuh, and Norman M. Edelstein \\ Lawrence Berkeley Laboratory, Berkeley, CA 94720
}

Aqueous transport of actinides in the geosphere can be significantly retarded by sorption on mineral surfaces. Despite the relevance of this effect to environmental remediation efforts and nuclear waste repository design, there is only limited direct knoledge about the microscopic nature of the actinidemineral interactions. X-ray Absorption Spectroscopy (XAS) is one of the few techniques which can study the in-situ local environment of elements present in low concentrations on a complex solid matrix. In these experiments, described in greater detail elsewhere, ${ }^{1}$ XAS was used to characterize uranium in contact with two forms of vermiculite, an expansible layer silicate.

Vermiculite particles are characterized by negatively-charged Mg-Al-silicate layers, with chargecountering cations residing between the layers. In the natural vermiculite, these interlayer regions are hydrated and thus accessible to aqueous-phase cations, permitting cation exchange. Some vermiculite was treated to obtain a collapsed phase, thereby limiting access to the interior cation exchange sites. Results of our Extnded X-ray Absorption Fine Structure (EXAFS) measurements for uranium bound to these two types of vermiculite indicate that the uranyl ion has a more distorted equatorial shell when bound to the collapsed form. This suggests that uranyl adopts a more symetric local structure within the interlayer regions of vermiculite than on the external particle surfaces. Results also indicate that, for both types of vermiculite, the bound uranium is monomeric, i.e. there is no evidence of surface precipitates, oligomers, or polymers.

Natural vermiculite was ground in a shatter box to $<45 \mu \mathrm{m}$. The collapsed form of vermiculite was produced by exchange of the interlayer cation, which is predominantly $\mathrm{Mg}^{2+}$ in the original mineral, for the $\mathrm{K}^{+}$cation. $\mathrm{XRD}$ analysis revealed differences in the basal-plane layer (001) spacings for the two forms of saturated vermiculite as follows: (1) $\mathrm{Mg}$ vermiculite (i.e. the natural form) showed a sharp peak at $14.44 \AA$, indicating the presence of two layers of water in the interlayer region, (2) K-vermiculite showed a broad peak centered $\sim 10.3 \AA$, indicating collapse of the layers to a non-hydrated mica-like structure, and a very small $\sim 14.4 \AA$ peak, indicating that only a trace amount of uncollapsed vermiculite remained. These spacings are similar to published values for relatively high-charge vermiculite. ${ }^{2}$

To obtain samples containing uranium bound to the mineral substrates, the solids were exposed to aqueous uranyl solutions. Calculations based upon established thermodynamic constants ${ }^{3}$ predict that, for the conditions used, the uranyl $\left(\mathrm{UO}_{2}{ }^{2+}\right)$ ion exists predominantly in solution as the fully hydrated species $\mathrm{UO}_{2}\left(\mathrm{H}_{2} \mathrm{O}\right) 5^{2+}$. After exposure, solids were separated by centrifugation and the resulting wet pastes were double-sealed in 2-mil polyethylene bags for XAS analysis. A solution of $0.2 \mathrm{M}$ uranyl chloride in $0.5 \mathrm{M} \mathrm{HCl}$, referred to here as $\mathrm{UO}_{2}{ }^{2+}$ (aq.), was also prepared for XAS analysis.

Uranium $L_{3}$-edge EXAFS was measured in both transmission and fluorescence modes at SSRL beamline IV-1 using a Si(111) double-crystal monochromator. X-ray fluorescence from the sample was measured using a single-element, four-pixel germanium detector, gated to the $L_{\alpha}$ fluorescence lines of U. EXAFS results were modeled by leastsquares fitting using scattering amplitudes and phase shifts calculated for each pair of atoms by the FEFF V code. 4 Distinct amplitudes and phase shifts were obtained for the axial oxygens of the uranyl species and the surrounding equatorial oxygens, and were used separately for these two shells in the fits. 


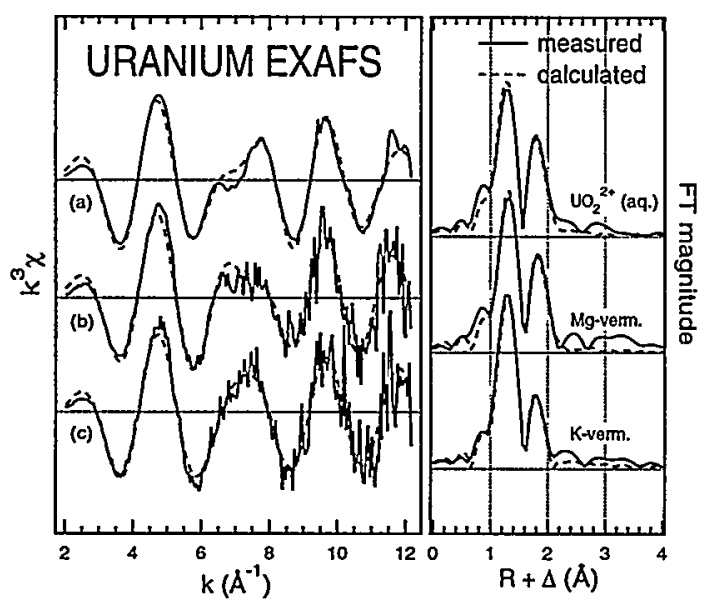

Figure 1: Uranium $L_{3}$ EXAFS results. Measured (solid lines) and calculated (dashed lines) chi curves (left) and the corresponding Fourier transforms (right) are compared for: (a) hydrated uranyl ion in aqueous solution; (b) uranium bound to $\mathrm{Mg}$-vermiculite; and (c) uranium bound to K-vermiculite.

Table I: Results of the EXAFS analysis. Derived U-O bond distances, R, and Debye-Waller factors, $\sigma^{2}$, are tabulated. Bond-distance uncertainties, as determined from the least-squares fit, are $<0.002 \AA$ (axial) and $<0.008 \AA$ (equatorial).

\begin{tabular}{llll}
\hline \hline Sample & Shell & $\mathrm{R}(\AA)$ & $\sigma^{2}\left(\AA^{2}\right)$ \\
\hline $\mathrm{UO}_{2}{ }^{2+}$ (aq.) & axial & 1.776 & 0.00250 \\
& equatorial & 2.411 & 0.00826 \\
$\mathrm{UO}_{2}{ }^{2+}$-- & axial & 1.798 & 0.00236 \\
on $\mathrm{Mg}$-verm. & equatorial & 2.391 & 0.00787 \\
$\mathrm{UO}_{2}{ }^{2+--}$ & axial & 1.784 & 0.00181 \\
on K-verm. & equatorial & 2.389 & 0.01267 \\
\hline \hline
\end{tabular}

Figure 1 compares measured and calculated EXAFS for several uranium"samples. In the calculation, the uranyl local structure was modeled by two shells of oxygen atoms, representing the axial and equatorial ligands. Coordination numbers were fixed at 2 and 5, respectively, based upon previously reported values for the hydrated uranyl species. 5,6 Bond distances and Debye-Waller factors were allowed to vary for each shell, yielding the numerical results listed in Table I.
The experimental Fourier Transforms (FT) in Fig. 1 have little magnitude for $R+\Delta>2.5 \AA$, indicating the absence of an ordered shell of uranium atoms outside the uranyl moiety. This is evidence that the uranyl sorption is monomeric, i.e. the uranyl does not bind to the minerals as a uranium-bearing phase, such as a surface precipitate, oligomer, or polymer. Surface complexes can be classified as outer- or innersphere, depending on the presence or absence of an intervening water molecule between the sorbate and the substrate. The absence of strong, distant scatterers in the FT's is consistent with sorption as an outer-sphere complex, but inner-shell sorption to $\mathrm{Al}$ or Si cannot be ruled out.

The most striking feature of the experimental FT's is the variation of the equatorial peak at $\sim 1.8 \AA$, which is much less intense for $\mathrm{U} / \mathrm{K}$-vermiculite. This difference is reflected in the numerical fit results by a smaller Debye-Waller (DW) factor for U/Mgvermiculite than obtained for $\mathrm{U} / \mathrm{K}$-vermiculite. In principle, a decreased FT peak intensity could also arise from a reduction in coordination number, with little change in the DW factor. However the difference observed here would require an unreasonably large change of $>1.5$ in the coordination number. The assumption of a constant cordination number for the equatorial shell is also supported by the very small variation in the derived equatorial bond distances. Therefore the difference in derived DW factors is judged to be significant.

The DW factor determined for the hydrated uranyl species is very close to that of U/Mg-vermiculite, indicating that the latter sample has a similarlyordered local environment. The larger DW factor for $\mathrm{U} / \mathrm{K}$-vermiculite reflects greater disorder, most likely static disorder resulting from a mixture ofdifferent equatorial bonding distances. These variations could arise from a range of bond distances within the equatorial shell of a single species, or from a mixture of species with different bond distances in the equatorial shell. The former explanaton seems more likely in this case, upon examination of the DW factors derived for the axial oxygens. If the U/Kvermiculite disorder were caused by a mixture of species with differing equatorial bond distances, the axial bond distance would also assume arange of values, due to the roughly inverse relationship between axial and equatorial bond lengths. ${ }^{7}$ Such a variation in axial bond length would lead to an increased DW factor for the axial oxygens, as compared to U/Mg-vermiculite or $\mathrm{UO}_{2}{ }^{2+}$ (aq.). In 
fact, the opposite effect is observed--the derived axial $\mathrm{DW}$ factor is less for $\mathrm{U} / \mathrm{K}$-vermiculite. This result is more consistent, therefore, with a larger range of equatorial bond distances within a single species which possesses, at the most, two discrete axial bond distances. The uranyl ion is known to exhibit such distortion of the equatorial shell for a number of different complexes in the solid state. 6,8

In summary, EXAFS results indicate that the local uranyl structure is modified upon changing from $\mathrm{U} / \mathrm{Mg}$-vermiculite to U/K-vermiculite. The average equatorial bond distance remains constant, but the individual bonds assume a wider range of values, which is reflected by the larger derived equatorial DW factor.

These results can be interpreted by considering structural properties of the two forms of vermiculite. $\mathrm{Mg}$-vermiculite is known to have two layers of partially ordered water in the interlayer region. The interlayer $\mathrm{Mg}^{2+}$ cation occupies a highly symmetric outer-sphere site, with six equidistant waters of hydration. ${ }^{9}$ EXAFS results indicate that the uranyl ion has a relatively uniform set of equatorial bond distances, indicating that it also occupies a fairly symmetric site in the interlayer region. The similarity of the EXAFS to that measured for the hydrated urany ion in solution, and the absence of strong scatterers outside the uranyl moiety, suggests that the uranyl ion is bound to $\mathrm{Mg}$-vermiculite as an outer-sphere complex.

Final interpretation of the EXAFS results for the $\mathrm{U} / \mathrm{K}$-vermiculite sample depends upon the results of pending laboratory analysis. However the following picture is consistent with our present understanding. For the collapsed $\mathrm{K}$-vermiculite, the interlayer region is no longer hydrated and $\mathrm{K}^{+}$occupies the interlayer cation-exchange sites. ${ }^{10}$ This leaves only the external surfaces of the vermiculite particles accessible to the aqueous phase. At the relatively low $\mathrm{pH}$ used here, specific adsorption at amphoteric sites on the edge surfaces is not expected, so the uranium most likely populates cation exchange sites on the external basal plane surfaces. For these sites, the ordering influence of an opposing basal plane surface is absent, and the structural order which characterizes the interlayer region is therefore reduced. EXAFS results indicate that the uranyl ion adopts a mor distorted equatorial shell in this case. This suggests the interpretation that uranyl ions in the interlayer region are influenced by both basal plane layers to produce a fairly symmetric species, while uranyl ions on external basal plane surfaces experience less balanced forces, leading to a reduced symmetry. Further work is in progress to test the assumption that uranyl does not intercalate into the $\mathrm{K}$-vermiculite.

This work was supported in part by the Division of Materials Science, Office of Basic Energy Science, and performed under the auspices of the U.S. Department of Energy by Lawrence Livermore National Laboratory under contract No. W-7405ENG-48. This work was also supported by the Director, Office of Energy Research, Office of Basic Energy Science, Chemical Sciences Division of the U.S. Department of Energy under contract No. DEAC03-76SF00098.

1 E. A. Hudson, L. J. Terminello, B. E. Viani, T. Reich, J. J. Bucher, D. K. Shuh, and N. M. Edelstein, to be published in Applications of Synchrotron Radiation Techniques to Materials Science, Materials Research Society Symposium Proceedings (MRS,Pittsburgh, 1995).

2. G.W. Brindley and G. Brown, Crystal Structures of Clay Minerals and their X-ray Identification (Mineralogical Society, London, 1980), p.204.

3. Chemical Thermodynamics of Uranium, edited by $\mathrm{H}$. Wanner and I. Forest (North-Holland, New York, 1992) p. 107 and p. 195.

4. J. J. Rehr, J. Mustre de Leon, S. I. Zabinsky, and R. C. Albers, J. Am. Chem. Soc. 113, 5135 (1991).

5. P. Charpin, A. Dejean, G. Folcher, P.Rigny, and P. Navaza, J. de Chim. Phys. 82 (1985); C. Görller-Walrand and W. Colen, Chem. Phys. Lett. 93, 82 (1982).

6. N. W. Alcock and S. Esperas, J. Chem. Soc. Dalton Tran. 1977, 893.

7. W. H. Zachariasen, Acta Cryst. 7, 795 (1954).

8. M. J. Bannister and J. C. Taylor, Acta Cryst. B 26, 1775 (1970); J. C. Taylor, Acta Cryst. B 27, 1088 (1971); B. O. Loopstra, J. C. Taylor, and A. B. Waugh, J. Solid State Chem. 20, 9 (1977).

9. J. F. Alcover, L. Gatineau, and J. Méring, Clays and Clay Minerals 21, 131 (1973)

10. G. Sposito, Surface Chemistry of Soils, (Oxford University Press, New York, 1984), p.15. 


\title{
Sorption and Coprecipitation of Arsenate and Chromate in Alkaline Environments
}

\author{
Satish C.B. Myneni, Samuel J. Traina, Glenn A. Waychunas* and Terry J. Logan \\ Environmental Sciences Graduate Program, The Ohio State University, Columbus, OH 43210 \\ *Center for Materials Research, Stanford University, Stanford, CA 94305
}

The Clean Air Act of 1990 mandated a significant reduction of $\mathrm{SO}_{2}$ emissions. As a result the coal-burning power plants have to retrofit their flue gas units with $\mathrm{CaO}$-scrubbing systems. The absorbent $\mathrm{CaO}$ reacts with the $\mathrm{S}$ present in flue gases and forms calcium sulfites and other sulfosalts. In addition to $S$, other volatile elements such as As, Se and B also partition into the calcareous phases. Thus introduction of these scrubbing systems will result in the generation of large quantities of alkaline materials with high concentrations of toxic elements. These wastes are commonly recognized as flue-gas desulphurization wastes. The disposal of these waste materials in natural landfills may result in the release of these toxic elements into the natural waters, and thus is of major environmental concern. The geochemistry of these elements in the environment is determined by the sorption and coprecipitation reactions that can occur during weathering of these alkaline waste materials. Ettringite is one of the dominant secondary minerals of the weathering product and is found to control the chemistry of several trace elements present in these wastes.

Ettringite is a calcium aluminum hydroxy sulfate with several molecules of crystalline water. Its occurrence is not limited to the flue-gas desulphurization wastes, but also commonly encountered in alkaline flyashes, cements and cement based waste solidification byproducts. The name "ettringite" is used to identify a mineral family with a general composition, $\mathrm{X}_{6} \mathrm{Y}_{2}(\mathrm{Z})_{3}(\mathrm{OH})_{12} .26 \mathrm{H}_{2} \mathrm{O}$ (where $\mathrm{X}=\mathrm{Ca}^{2+}, \mathrm{Mg}^{2+}, \mathrm{Sr}^{2+}, \mathrm{Ba}^{2+}, \mathrm{Na}^{+}, \mathrm{K}^{+} ; \mathrm{Y}=\mathrm{Al}^{3+}, \mathrm{Fe}^{3+}$, $\mathrm{Cr}^{3+}, \mathrm{Si}^{4+}, \mathrm{Mn}^{4+}$; and $\mathrm{Z}=$ oxyanions such as $\mathrm{SO}_{4}{ }^{2-}$, $\mathrm{AsO}_{4}{ }^{3-}$ ) and also the sulfate endmember of this family. Several minerals of ettringite family are reported to occur in alkaline waste materials and have been shown to exhibit high partition constants for both cations and oxyanions in their stability range. However, oxyanion substitution is relatively favored over cationic sorption because of this mineral's structural compatibility for oxyanion incorporation
(1). The peculiar structure of ettringite, and its high stability in alkaline environment also makes it a useful tool to probe oxyanion interactions with mineral surfaces. The main objective of this research is to understand the geochemistry of As and $\mathrm{Cr}$; and to identify the mode of interactions that their oxyanions ( $\mathrm{AsO}_{4}{ }^{3-}$ and $\mathrm{CrO}_{4}{ }^{2-}$ respectively) exhibit with the ettringite surfaces in alkaline environments.

Structure of Ettringite and Surface Complexes of $\mathrm{AsO}_{4}{ }^{3-}$ and $\mathrm{CrO}_{4}{ }^{2-}$

The structure of ettringite (Fig. 1) consists of columns of $\left\{\mathrm{Ca}_{6}\left[\mathrm{Al}(\mathrm{OH})_{6}\right]_{2} 24 \mathrm{H}_{2} \mathrm{O}\right\}^{6+}$, and the intercolumn space is occupied by $\mathrm{SO}_{4}{ }^{2-}$ with water molecules between the oxyanion (as an outer-sphere complex) and the columns (2). The oxyanion holds the columns together by electrostatic interactions. Thus the structure of this mineral allows an oxyanion to form both the inner sphere (IS), and outer sphere (OS) complexes. Depending on the nature of the oxyanion, it can form IS, and OS complexes with $\mathrm{Al}$, or $\mathrm{Ca}$ atoms exposed on the surface of ettringite. Although the channels are apt to form OS complexes (since water molecules surround the columns), channel sites may also exhibit IS complexes (by replacing water and directly complexing with the $\mathrm{Ca}$ polyhedra). In this case the oxyanion of the channels may not interact with $\mathrm{Al}$ directly, since it is present farthest inside the columns. The solubility of the oxyanion in these alkaline wastes depends on how it interacts with $\mathrm{Ca}$, and $\mathrm{Al}$ in ettringite. Previous studies have shown that $\mathrm{AsO}_{4}{ }^{3-}$ always form IS complexes and $\mathrm{CrO}_{4}{ }^{2-}$ forms $\mathrm{OS}$ complexes with $\mathrm{Fe}$ and Al-hydroxide surfaces. However, little is known about their interactions at high $\mathrm{pH}$, and with calcareous phases. 


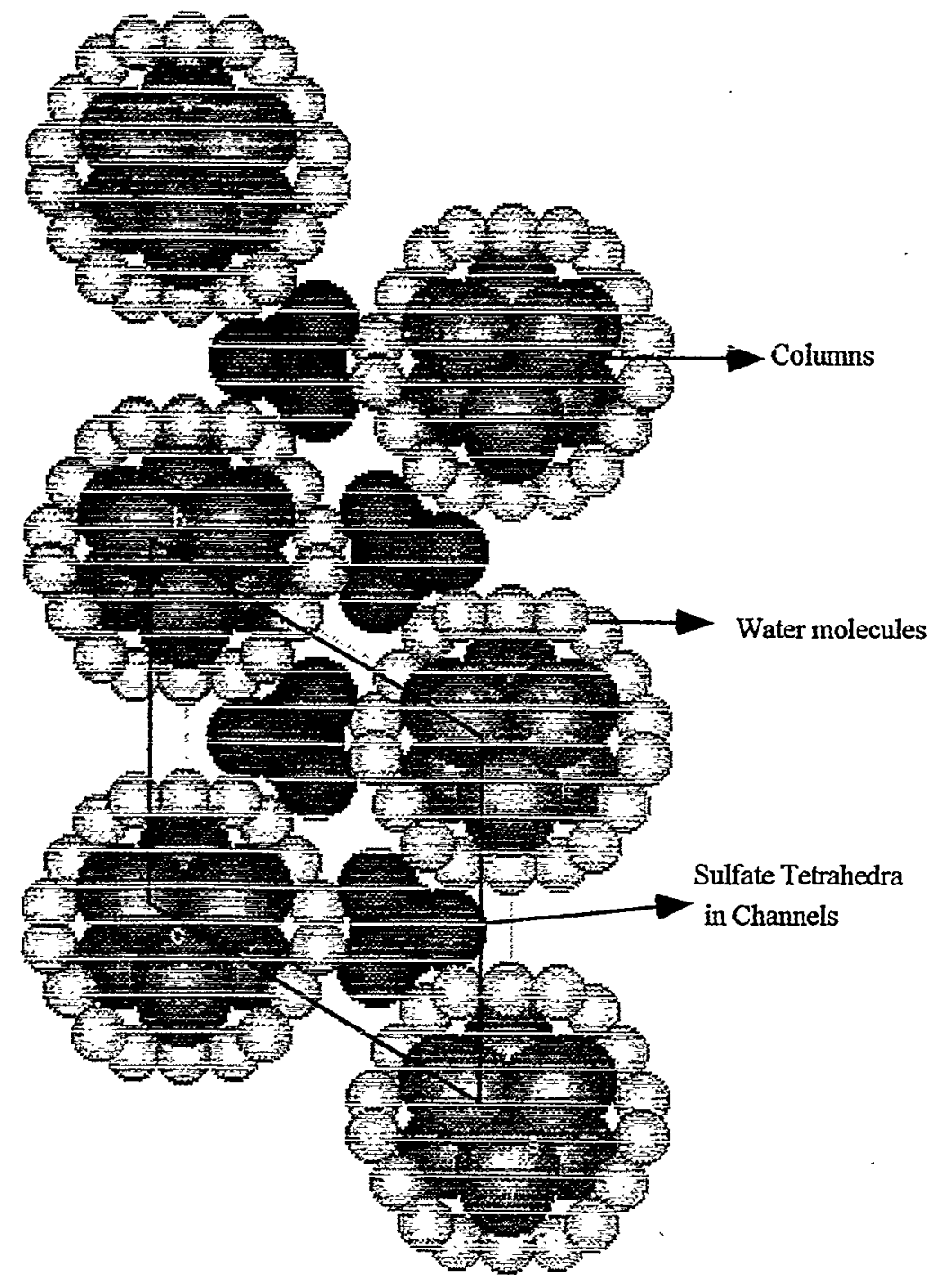

Fig. 1 Structure of ettringite (001) 


\section{Materials and Methods}

Adsorption and coprecipitation experiments were conducted for $\mathrm{AsO}_{4}{ }^{3-}$, and $\mathrm{CrO}_{4}{ }^{2-}$ in ettringite separately. Synthetic ettringite was used in all adsorption experiments and ettringite was allowed to precipitate in the presence of $\mathrm{AsO}_{4}{ }^{3-}$, and $\mathrm{CrO}_{4}{ }^{2-}$ during coprecipitation experiments. The initial concentrations of the oxyanions in reacting solutions ranged from $4 \mu \mathrm{M}$ to $20 \mathrm{mM}$. The final (equilibrium) concentrations of different ions were measured using ICP and AA spectrometers. The solid phase concentrations of the oxyanions were estimated from mass balance calculations. The mineralogy of the precipitated solids was identified using X-ray diffraction (XRD), and the molecular environment of the sorbed oxyanion was probed with the help of infrared (IR), and Raman spectroscopic techniques. The same solids were also studied using X-ray absorption spectroscopy (XAS) to obtain information on the type of surface complexes, and the coordination environment.

The XAS measurements were conducted at SSRL in the months of April, August and December 1994 on beam lines IV-1 and IV-3 using Si(111) monochromator crystals. The EXAFS spectra were collected for As and $\mathrm{Cr} \mathrm{K}$-edges at 298 , and $10 \mathrm{~K}$ (using a helium cryostat). The concentrated samples, and the model compounds were diluted with boron nitride. For EXAFS data collection the samples were mounted between mylar windows attached to plastic or Al-sample holders. Both transmission and fluorescence (Lytle Detector) spectra were collected for all the samples. The number of scans for a sample ranged from 4 to 30 with a duration of $25 \mathrm{~min}$ to $1 \mathrm{hr}$ for each scan, depending on the concentration of the absorbing atom in the samples. The scans were averaged and analyzed using EXAFSPAK. Details of this type of EXAFS data analysis has been described by Teo (4).

\section{Results and Discussion}

Mass balance from the sorption experiments of $\mathrm{AsO}_{4}{ }^{3-}$, and $\mathrm{CrO}_{4}{ }^{2-}$ suggest that these oxyanions can replace $\mathrm{SO}_{4}{ }^{2-}$ from the channels of ettringite. Of these two oxyanions, $\mathrm{AsO}_{4}{ }^{3-}$ was preferred for the channel sites, and coprecipitation resulted in higher uptake than the adsorption experiments. XRD studies of the reacted solids suggest that the crystal structure of ettringite was not affected at low concentrations of the substituted oxyanion. However, new solid phases (not reported in ICDD manual) formed at high sorption concentrations and high oxyanion-to- $\mathrm{SO}_{4}{ }^{2-}$ ratios. The vibrational spectra of the sorbed and coprecipitated samples were different, and they showed distinct changes in the molecular environment of the sorbed oxyanion with the increase in concentration. These studies also gave evidence for the presence of different types of surface complexes at different concentrations of adsorbate. XAS studies of these samples were helpful in identifying the type of complexes the oxyanions form with this mineral. The data reduction of the collected EXAFS spectra is in progress, and the preliminary results of $\mathrm{AsO}_{4}{ }^{3-}$ sorption is presented here.

EXAFS spectra were collected for $\mathrm{AsO}_{4}{ }^{3-}$ sorbed samples that has As as low as $2 \mathrm{mmol} \mathrm{Kg}^{-1}$. The data is noisy for very dilute samples, and the data collection at $10 \mathrm{~K}$ significantly improved the quality of these spectra. Radial structure functions for adsorbed arsenate are shown in Figure 2. At very low concentrations of sorbed $\mathrm{AsO}_{4}{ }^{3-}$ (As $=2 \mathrm{mmol} \mathrm{Kg}{ }^{-1}$ ), $\mathrm{AsO}_{4}{ }^{3-}$ formed bidentate complexes. The theoretical

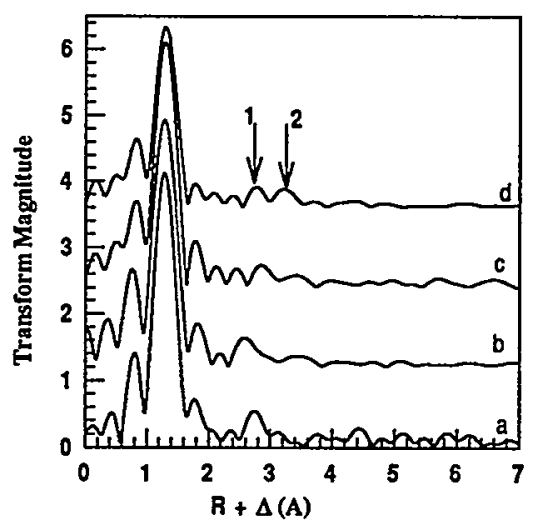

Fig. 2 Adsorption of $\mathrm{AsO}_{4}{ }^{3-}$ in ettringite. The concentration of As decreases in the order $d>c>b>a$. The first peak $(R \sim 1.25)$ in the fourier transform corresponds to the first shell $O$ atoms. Arrow-1 (R 2.7) corresponds to the bidentate complexes, and arrow-2 (R 3.1) represents monodentate complexes of $\mathrm{AsO}_{4}{ }^{3-}$ with $\mathrm{Ca}$ or $\mathrm{Al}$ (in case of Fig. 2a). 
phase and amplitude functions (calculated using FEFF601) for the second shell at 2.8A (not corrected for phase) suggest that the second nearest neighbor is $\mathrm{Al}$ rather than $\mathrm{Ca}$. This suggests that $\mathrm{AsO}_{4}{ }^{3-}$ is probably binding to the external surfaces of the mineral. However, with the increase in adsorbate concentration, $\mathrm{AsO}_{4}{ }^{3-}$ tend to form both mono-, and bidentate complexes with $\mathrm{Ca}$ in the channels of ettringite. The macroscopic observation of these adsorption experiments showed that the number of moles of $\mathrm{SO}_{4}{ }^{2-}$ released from ettringite is similar to the number of moles of sorbed $\mathrm{AsO}_{4}{ }^{3-}$, suggesting that $\mathrm{AsO}_{4}{ }^{3-}$ is present in the channels of this mineral at high coverages. The $\mathrm{pH}$ of the initial solution has greater effect on the type of complexes that $\mathrm{AsO}_{4}{ }^{3-}$ formed with ettringite surfaces. When $\mathrm{pH}$ is maintained at 11.8 for all adsorbate concentrations (10 to $700 \mathrm{mmol} \mathrm{Kg}^{-1}$ ) $\mathrm{AsO}_{4}{ }^{3 \cdot}$ formed both mono-, and bidentate complexes with $\mathrm{Ca}$. In addition, high $\mathrm{pH}$ stabilized the structure of ettringite even at high adsorbate concentrations.

On the contrary, the coprecipitated samples showed completely different behavior (Fig. 3), and the structure of ettringite was preserved upto very high concentrations of the adsorbate. At low adsorbate concentrations $\mathrm{AsO}_{4}{ }^{3-}$ formed only monodentate

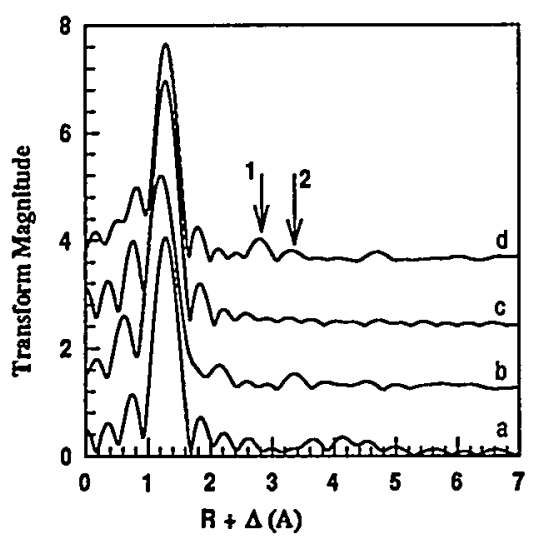

Fig. 3 Coprecipitation of $\mathrm{AsO}_{4}{ }^{3-}$ in ettringite. The concentration of the oxyanion decreases in the order $d>c>b>a$. Arrows 1 , and 2 represent bi-, and mono-dentate complexes of $\mathrm{AsO}_{4}{ }^{3-}$ respectively. complexes. However, both mono-and bidentate complexes were present at high adsorbate concentrations. When the molar ratios of $\mathrm{AsO}_{4}{ }^{3-}$ to $\mathrm{SO}_{4}{ }^{2-}$ were close to 0.2 , no significant back-scattering from the second or higher shells was observed (Fig. 3c). This may suggest that $\mathrm{AsO}_{4}{ }^{3-}$ is present as an OS complex in the channels of ettringite. Although large thermal disorder from low atomic number, large radius cations such as $\mathrm{Ca}$ may significantly reduce the backscattering amplitude (3) for the second shell, our data collection at $10 \mathrm{~K}$ did not show any improvement in the Debye-Waller factor $\left(\sigma^{2}\right)$. Relatively low $\sigma^{2}$ $(0.005$ to 0.009$)$ for the second and third shell fits of calcium arsenate model compounds (rauenthalite, and pharmacolite) also suggest that there is typically significant backscattering from the $\mathrm{Ca}$ atom. Combining this information with the vibrational spectroscopic data, it may be possible to establish that $\mathrm{AsO}_{4}{ }^{3-}$ forms $\mathrm{OS}$ complexes in the channels of ettringite. Ionic strength dependence desorption experiments $(I=0.05$ to $1.0 \mathrm{M} \mathrm{NaClO} 4)$ were conducted to further identify the nature of these OS complexes. Desorption of coprecipitated $\mathrm{AsO}_{4}{ }^{3-}$ (outerspherically bound $\mathrm{AsO}_{4}{ }_{4}^{3}$ ) from As-ettringite did not show any changes in the coordination environment of coprecipitated arsenic with changes in ionic strength. In addition, there is no significant release of $\mathrm{AsO}_{4}{ }^{3-}$ from these samples. This may also suggest that $\mathrm{AsO}_{4}{ }^{3-}$ present in the channels can not be displaced easily irrespective of the type of complexes it forms.

The chemistry of these surface complexes of $\mathrm{AsO}_{4}{ }^{3-}$, and $\mathrm{CrO}_{4}{ }^{2-}$ will help us to understand the behavior of $\mathrm{As}$, and $\mathrm{Cr}$ and their redox behavior and kinetics in alkaline environments.

This work was supported by Ohio Coal Development Office, Electric Power Research Institute, DOE, and U.S Geological Survey.

1. Kumarathasan P., McCarthy G.J., Hassett D.J., and Hassett D.F.P., (1990), Mat. Res. Soc. Symp. Proc., V.178, 83 .

2. Moore A.E., and Taylor H.F.W., 1968, Nature, V.218, 1085.

3. O'Day P.A., Rehr J.J., Zabinsky S.I, and Brown Jr. G.E., 1994, J. Am. Chem. Soc, 116, 2938.

4. Teo B.K., 1986, "EXAFS: Basic Principles and Data Analysis", Spr. Ver., 349pp 


\title{
HIGH RESOLUTION SYNCHROTRON X-RAY DIFFRACTION TOMOGRAPHY OF POLYCRYSTALLINE SAMPLES
}

\author{
S.R. Stock ${ }^{1}, A$. Guvenilir' ${ }^{1}$ D.P. Piotrowski ${ }^{1}$ and Z.U. Rek ${ }^{2}$ \\ 'School of Mat. Sci. \& Eng., Georgia Inst. Technology, Atlanta, GA \\ ${ }^{2}$ SSRL
}

The macroscopic response of polycrystalline materials to loading depends on both the spatial distribution of strain and the variation of microtexture on the scale of $100 \mu \mathrm{m}$. In understanding of fatigue crack growth, nondestructive measurements are needed if one is to understand the influence of the three-dimensionally varying microtexture on evolution of strains at the crack tip and on determination of fatigue crack path. Approaches are summarized for high resolution polychromatic $\mathrm{x}$-ray diffraction tomography of polycrystalline samples. Preliminary results are reported on the two extremes of samples which could be encountered: large-grained samples in which the diffraction images of individual grains can be resolved and small-grained samples in which the grains' images form an essentially continuous, although nonuniform, distribution of diffracted intensity.

In Al-Li 2090, fatigue crack growth rates are unusually low compared to other $\mathrm{Al}$ alloys. This is related to a characteristic macrotexture in the center of plates which produces a rough, asperity dominated crack face and significant crack deflection [1]. A result of the roughness is a phenomenon known as crack closure, i.e., premature contact of the crack faces during the unloading portion of the fatigue cycle which leads to a reduced driving "force" for crack propagation and lower crack propagation rates [2]. The prominent macrotexture in $\mathrm{Al}-\mathrm{Li} 2090$ has been correlated with the geometry of asperities on fatigue crack faces [1], but the role of microtexture on the path of fatigue cracks through the solid has not been investigated.

$\mathrm{X}$-ray polychromatic diffraction tomography can be performed in transmission using microbeams and involves the following. For ease of discussion of the technique, it will be assumed that the sample is in the form of a relatively thin plane, and the $x$-ray microbeam is incident normal to the plate and to the two-dimensional detector.

Mapping in the plane of the sample involves an $x-y$ translation of the beam. All grains within the column $(x, y)$ illuminated by the beam will diffract different photon energies in different directions, and a two-dimensional detector measures the angular distribution of diffracted intensity $I_{D}\left(r_{D}, \phi_{D}\right)$, where $r_{D}$ and $\phi_{D}$ are polar coordinates of the detector pixels and $r_{D}$ is measured from the incident beam (see Fig. 1 and 2). For position ( $x, y)$, the depth $\mathrm{z}$ from which volume element $\mathrm{dV}$ diffracts is determined by ray tracing: varying the sample-detector distance changes $I_{D}\left(x_{D}, y_{D}\right)$ as shown in Fig. 1.

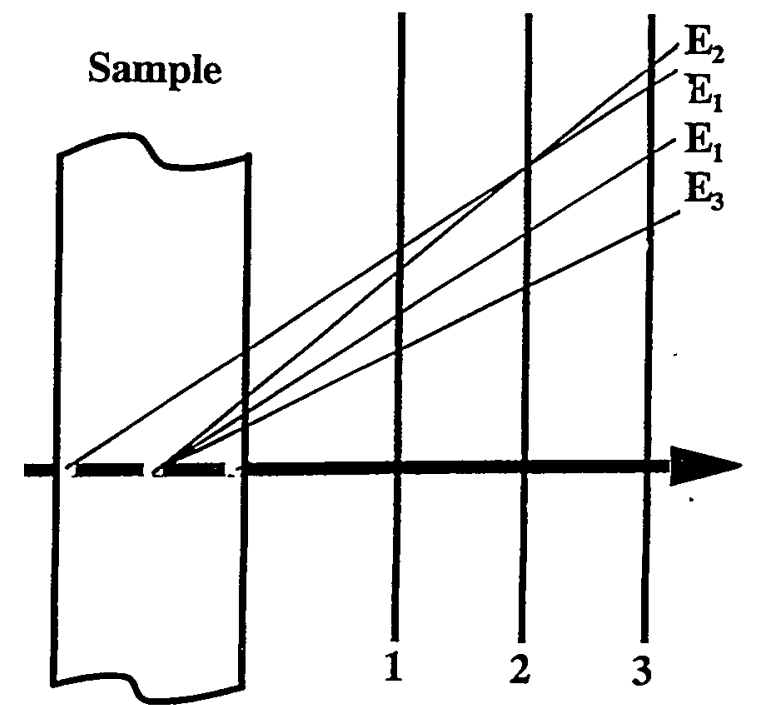

Figure 1. Depth of $d V$ from diffraction of polychromatic $\mathrm{x}$-rays. Different detector positions (labeled 1-3) allow diffraction of $x$-rays with energy $E_{1}$ from near-surface $d V$ to be separated from diffraction of energies $\left(E_{2}<E_{1}\right.$ or $\left.E_{3}>E_{1}\right)$ by $d V$ deeper within the sample. Diffracted intensity from interior $\mathrm{dV}$ can appear closer or farther from the incident beam than that of entrance surface $d V$ despite diffracting lower energy photons. 
The pattern of diffracted intensity is recorded with and without a filter possessing an absorption edge in the wavelength range of interest for the sample being studied: the conflicting requirements of high transmissivity for rapid data collection and rapidly decreasing $\mathrm{x}$-ray flux for higher photon energies must be carefully considered for each combination of material and sample thickness. With a filter in the beam, a large change in contrast appears between images of grains diffracting $x$-rays with wavelengths on either side of the absorption edge [3]. This allows one to differentiate, for example, between diffraction from $\{111\}$ by $1.00 \AA$ $x$-rays and $\{200\}$ by $0.87 \dot{A} x$-rays. If an energy sensitive, high spatial resolution, two-dimensional detector were available, a filter would be unnecessary.

Strain within a grain producing a particular diffraction spot or within the grains producing a particular portion of the diffraction pattern is determined using a post-sample analyzer crystal (see Fig. 6). Normally strain is measured with a monochromatic beam, and diffracted intensity is measured as a function of sample rotation. This is undesirable for diffraction tomography because the volume irradiated will change with rotation. In the present approach the sample's orientation is unchanged, and the analyzer rotated to measure the peak profile; this is equivalent to scanning the energies in the diffracted beam in order to measure the mosaicity associated with the microstrain. Using imaging plates, one can very quickly position the analyzer crystal to intercept a particular diffraction spot or area of high density of diffracted intensity. Only two axes of translation are required to position the two-circle rotator carrying the analyzer and detector. Rotation of the sample about the incident beam direction is used to align the diffraction plane normals of the analyzer crystal and $\mathrm{dV}$ so that diffraction profile is not artificially broadened.

One sample (large-grained) was fabricated by cleaving approximately $1 \mathrm{~mm} \times 1 \mathrm{~mm}$ pieces of a Si wafer, by randomly orienting the pieces of $\mathrm{Si}$ in a 5 $\mathrm{mm}$ diameter capillary tube (a standard thin-walled, low absorption glass tube for powder diffraction) and by fixing the pieces in place with lacquer. The small-grained sample was a compact tension sample machined from the center $2 \mathrm{~mm}$ of a $12.5 \mathrm{~mm}$ thick plate of Al-Li 2090 T8E41 [4].

The diffraction experiments were performed with bending magnet radiation on Beam Line 2-2.
The primary two-dimensional detector for diffraction tomography was Fuji HR-IIIN Imaging Plates $(20 \mathrm{~cm}$ $x 25 \mathrm{~cm}$ dimensions), although Kodak SR-5 film (13 $\mathrm{cm} \mathrm{x} 18 \mathrm{~cm}$ dimensions) was used to record the data shown below for the polycrystalline Si sample and Polaroid P57 film was used during setup. Typical exposures for diffraction from $2 \mathrm{~mm}$ thick $\mathrm{Al}-\mathrm{Li}$ 2090 were about $7 \times 10^{2} \mathrm{~mA} \cdot \mathrm{sec}$ for Type 57 film, $1 \times 10^{4} \mathrm{~mA} \cdot \mathrm{sec}$ for SR-5 film and $2 \times 10^{1} \mathrm{~mA} \cdot \mathrm{sec}$ for the image plate. Subsequent to exposure the films were developed and the image plates were read with $100 \mu \mathrm{m}$ spatial resolution and 1024 levels of contrast in a Fuji BAS-2000 Imaging Plate Scanner. The SR5 film was digitized using a Molecular Dynamics microdensitometer, and the pixel size and contrast levels were $100 \mu \mathrm{m}$ and 1024 respectively, for the resulting digital image.

Large areas were scanned with a $1.0 \mathrm{~mm}$ diameter collimator while a $0.1 \mathrm{~mm}$ diameter collimator was used for small fields of view. The $\mathrm{Si}$ polycrystal data discussed below was taken with the $1 \mathrm{~mm}$ diameter collimator while both collimators were used with the Al-Li 2090 sample. In some exposures of the Al- $\mathrm{Li}$ sample a $75 \mu \mathrm{m}$ thick Mo filter was placed between the storage ring and the collimator in order to identify where on the diffraction pattern grains were diffracting radiation at the Mo K-edge $(\lambda=0.620 \AA)$. In all cases, the imaging plate or film was centered on the transmitted beam.

Microtexture within Al-Li 2090 varies significantly from position to position as the imaging plate data in Fig. 2 shows. The lighter pixels in Fig. 2 represent higher diffracted intensity, the patterns were collected with a $1 \mathrm{~mm}$ collimator from positions $2 \mathrm{~mm}$ apart, and " $\mathrm{A}$ " and " $\mathrm{B}$ " indicate the changing microtexture revealed with the beam normal to the sample surface.

Results of ray tracing for three "grains" of the polycrystalline sample are shown in Fig. 3. The data was recorded on SR-5 film with a $1 \mathrm{~mm}$ collimator and subsequently digitized. Each diffraction spot is an $\mathrm{x}$-ray diffraction topograph of a piece of $\mathrm{Si}$ and maps the spatial distribution of the piece's diffracting power. A particular feature in each grain's images was used to extrapolate to the origin of the diffracted beam within the sample. As it is difficult to measure sample-detector separation directly with precision better than about $2 \mathrm{~mm}$, a detector reference position is defined and the detector is positioned by hand on an optical rail relative to the reference with precision 

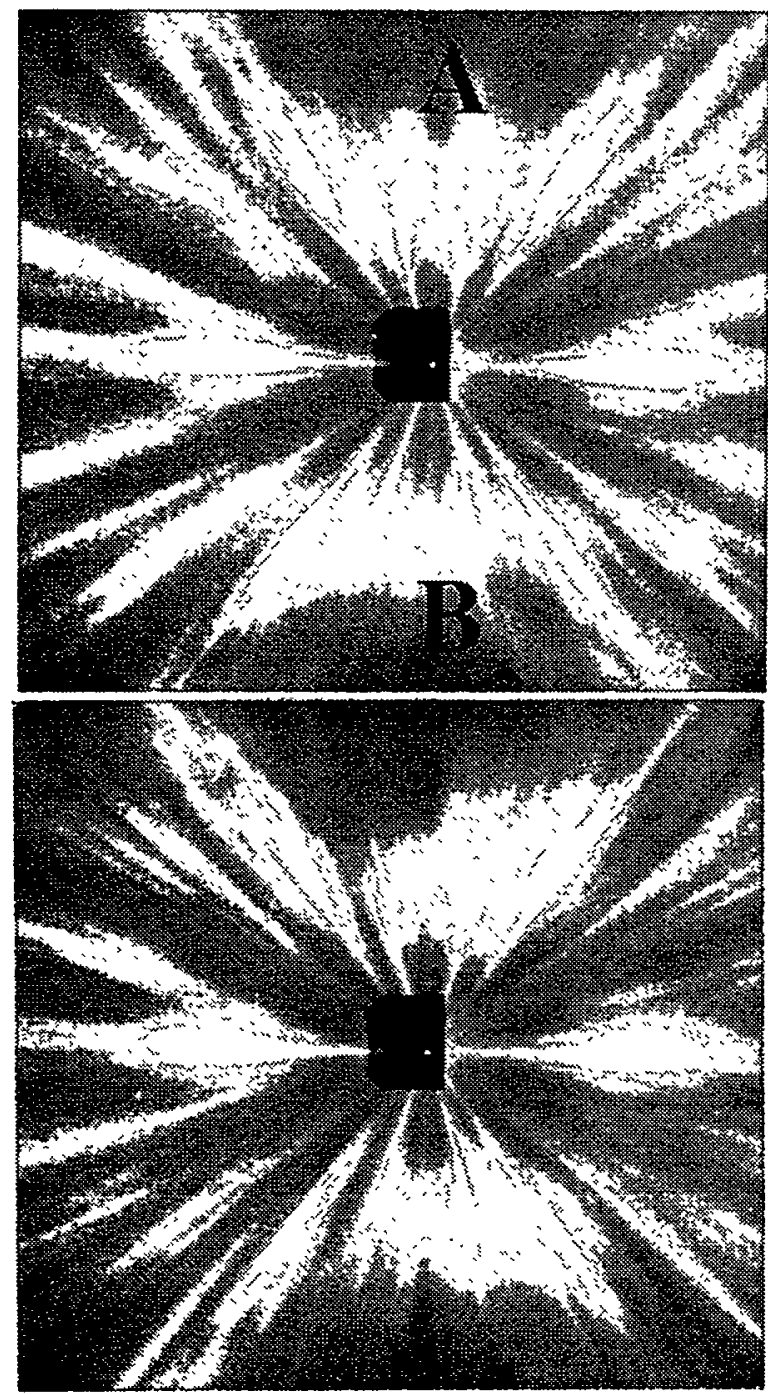

Figure 2. Microtexture from positions $2 \mathrm{~mm}$ apart (1 $\mathrm{mm}$ collimator, imaging plate).

of about $0.25 \mathrm{~mm}$. Least squares fitting of $r_{D}$ as a function of detector position is used to determine where within the sample the diffracted beam originated. Seven or more detector positions are used, and the standard deviation of the grain positions within the sample (i.e., the horizontal axis in Fig. 3) are 0.2 to $0.3 \mathrm{~mm}$ for this data.

The imaging plate data in Fig. 4 shows the difference in the distribution of diffracted intensity with and without the Mo filter (the left and right images, respectively). The positions diffracting the wavelength of the Mo K-edge show the expected change in intensity, and the numerals $1-4 b$ label the edge positions for five different hkl. The calculated values of the diffraction angle are compared to those
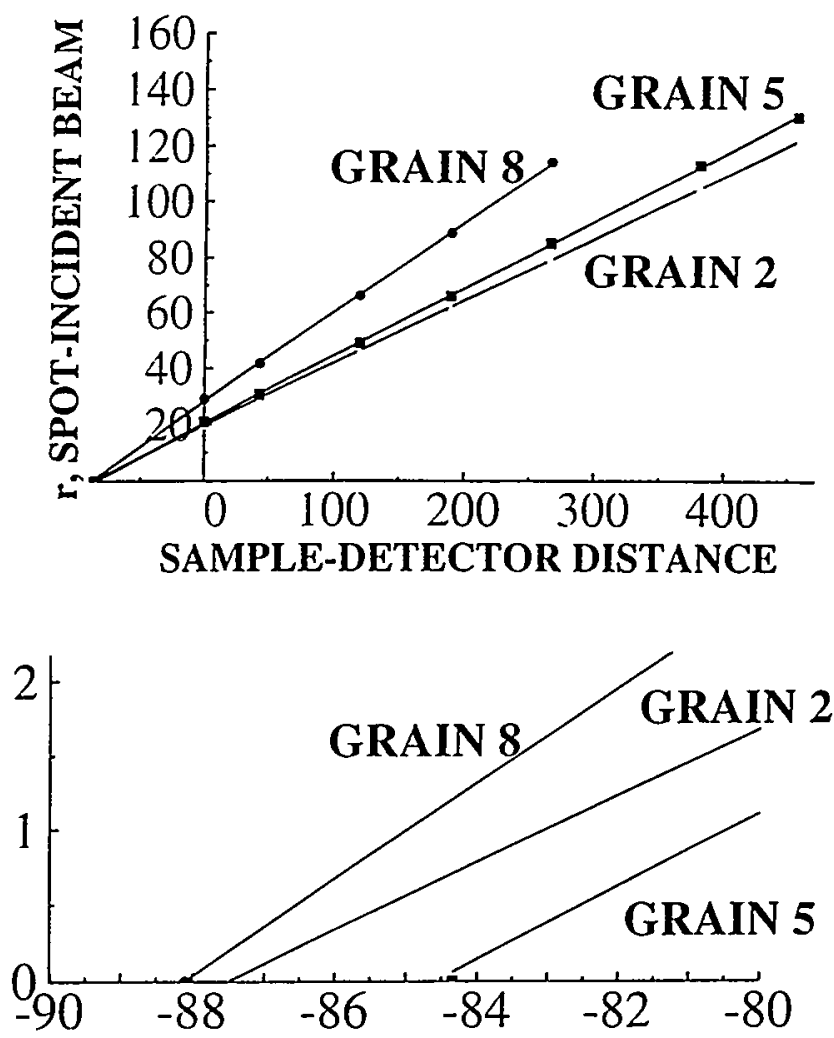

Figure 3. Separation $r_{D}$ between diffraction spots and incident beam vs. detector position (both axes are in $\mathrm{mm}$ ). The lower plot enlarges the far-left portion of upper graph.

expected for the Al reflections (Fig. 5), and the agreement is excellent. There is a small systematic difference between the two sets of angles, but this is due to a slight inaccuracy in the estimate of the detector-sample separation which was physically measured and not determined by regression as were the positions of the Si grains 2, 5 and 8 (Fig. 3).

The diffraction profiles of several grains within the large-grained $\mathrm{Si}$ sample and from an $\mathrm{Al}$ single crystal have been measured in the transmission geometry using an analyzer after the sample (Fig. 6). The $\mathrm{Si} 111$ reflection from a $\mathrm{TaSi}_{2} / \mathrm{Si}$ eutectic composite crystal was used as the analyzer for the $200 \mathrm{Al}$ reflection shown in Fig. 6 for intensity diffracted from the analyzer crystal as a function of rotation of the analyzer crystal. A $1 \mathrm{~mm}$ diameter collimator was used, and the counting time was $1 \mathrm{sec}$ per data point. Background can reduced by improved shielding, and recording such patterns with collimator diameters of $100 \mu \mathrm{m}$ should not prove too difficult. 


\section{ACKNOWLEDGMENTS}

This research was supported by the Office of Naval Research (Grants N00014-89-J-1708 and N00014-94-1-0306). We thank Dr. E. Morgan of the Department of Pharmacology, Emory University for use of the microdensitometer.

\section{REFERENCES}

1. G.R. Yoder, P.S. Pao, M.A. Imam and L.A. Cooley, in Proceedings of the Fifth International Aluminum-Lithium Conference, Sanders and Starke, eds., (Mat. \& Comp. Eng. Publ., Birmingham, UK, 1989) p. 1033.

2. R.O. Ritchie, in Fatigue Thresholds, Backland, Blom and Beevers, eds. (Eng. Adv. Ser., Warley, UK, 1981) p. 503.
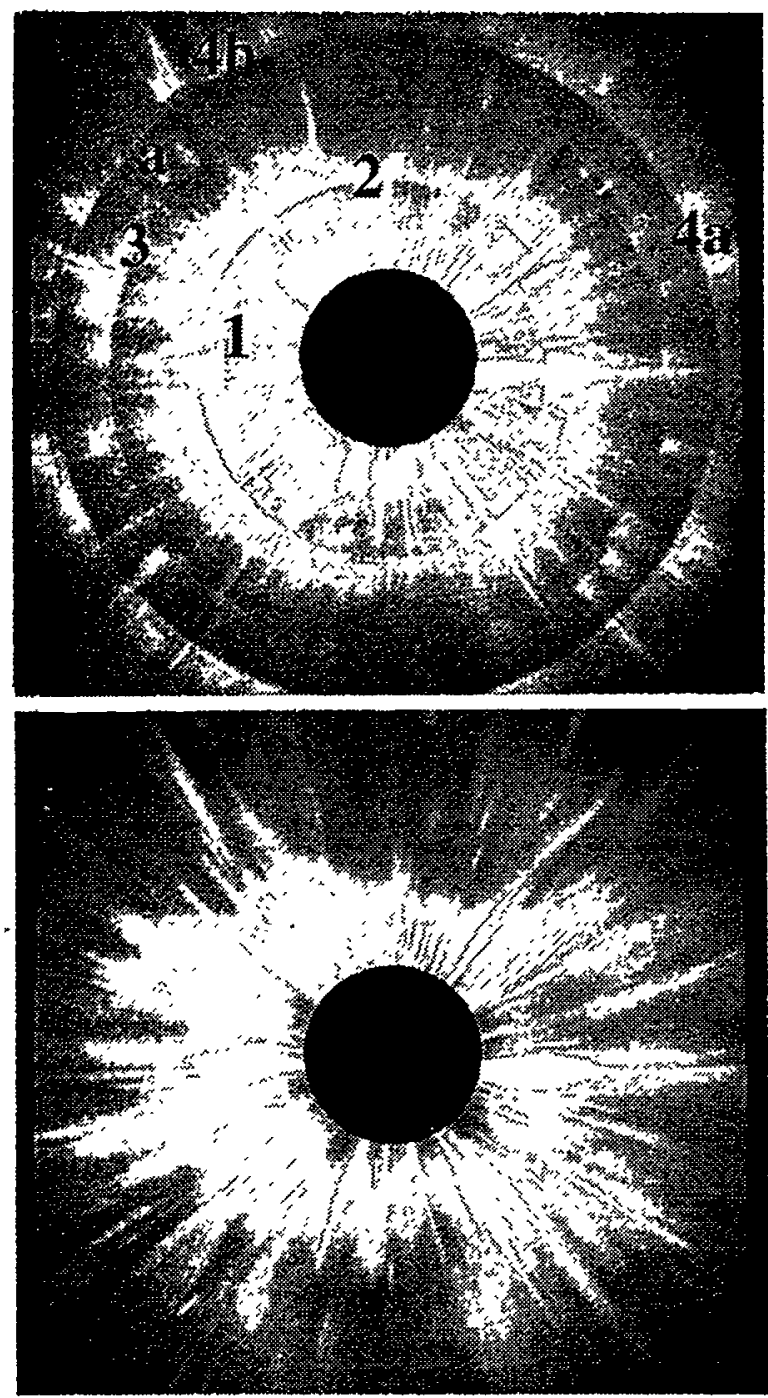

Figure 4. Diffracted intensity with and without Mo filter (top and bottom images, respectively).
3. S.R. Stock, Z.U. Rek, Y.H. Chung, P.C. Huang and B.M. Ditchek, J. Appl. Phys. 73, 1 (1993).

4. A. Guvenilir, S.R. Stock, M.D. Barker and R.A. Betz, in Aluminum Alloys: Their Physical and Mechanical Properties, Volume II, Proceedings of the Fourth International Conference, (Georgia Institute of Technology, Atlanta, 1994) p. 413.

$2 \Theta^{\circ} \quad 2 \Theta^{\circ}$

Feature $\underline{\mathrm{hkl}}$ Observed Expected

\begin{tabular}{cccc}
\hline 1 & & & \\
\hline 1 & 111 & 15.8 & 15.24 \\
2 & 200 & 17.9 & 17.61 \\
3 & 220 & 25.3 & 25.01 \\
$4 \mathrm{a}$ & 311 & 29.8 & 29.41 \\
$4 \mathrm{~b}$ & 222 & 31.2 & 30.86 \\
& 400 & & 35.75 \\
5 & 311 & 39.7 & 39.00 \\
& or 420 & & 40.04 \\
\hline
\end{tabular}

Figure 5. Expected and calculated diffraction angles of six hkl for the wavelength of the Mo K-edge for the images shown in Fig. 4.
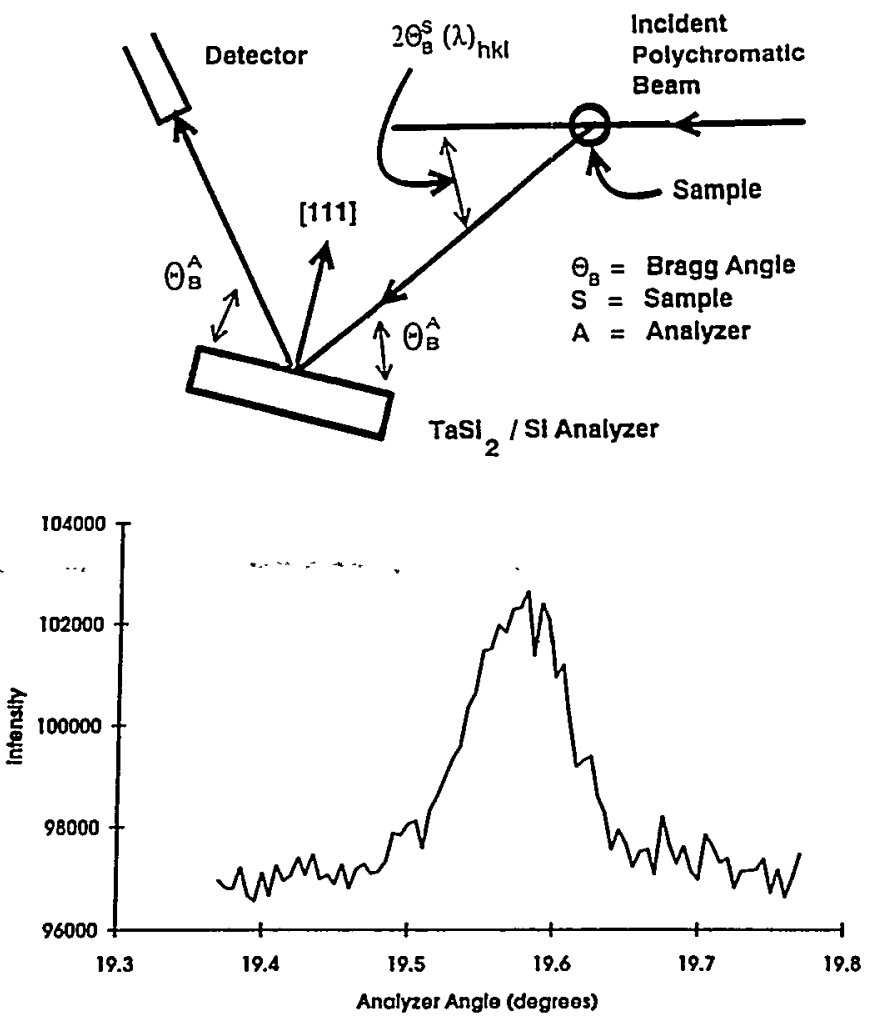

Figure 6. Geometry (top) for recording the Al 200 diffraction peak profile as a function of analyzer crystal rotation (bottom). 


\title{
CORRELATION of XANES FEATURES with the SCINTILLATION EFFICIENCIES of Ce DOPED ALKALINE EARTH LITHIUM SILICATE GLASSES
}

\author{
David L. Blanchard Jr., Debra S. Sunberg, Richard A. Craig, Mary Bliss \\ Pacific Northwest Laboratory, Box 999, Richland, WA 99352 \\ Marvin J Weber \\ Livermore National Laboratory, Livermore, CA 94551
}

Cerium-activated, lithium-silicate glasses are widely used as thermal neutron detectors because of their versatility, robustness and low cost. As previously observed, 1 the glasses $20 \mathrm{Li}_{2} \mathrm{O} \cdot 15 \mathrm{MO} \cdot 64.4 \mathrm{SiO}_{2} \cdot 0.6 \mathrm{Ce}_{2} \mathrm{O}_{3}$ (where $\mathrm{M}$ is $\mathrm{Mg}, \mathrm{Ca}, \mathrm{Sr}$, or $\mathrm{Ba}$ ) exhibit scintillation efficiencies that vary by more than a factor of 2.5 with the alkaline earth (Fig. 1). Measurements of the Ce $\mathrm{L}_{\mathrm{III}} \mathrm{x}$-ray absorption edge in the $\mathrm{Mg}, \mathrm{Ca}$ and $\mathrm{Sr}$ glasses display a feature near the absorption edge that is suggestive of the presence of $\mathrm{Ce}^{4+}$. The area of this peak is, in fact, correlated with the scintillation efficiency of the glass. The amount of $\mathrm{Ce} 4+$ indicated by the intensity of this feature is, however, too high to be a permanent population. We suspect that the feature is a transient phenomenon related to creation of $\mathrm{Ce}^{4+}$ and trapped electrons due to photoionization by the $\mathrm{x}$-ray beam.

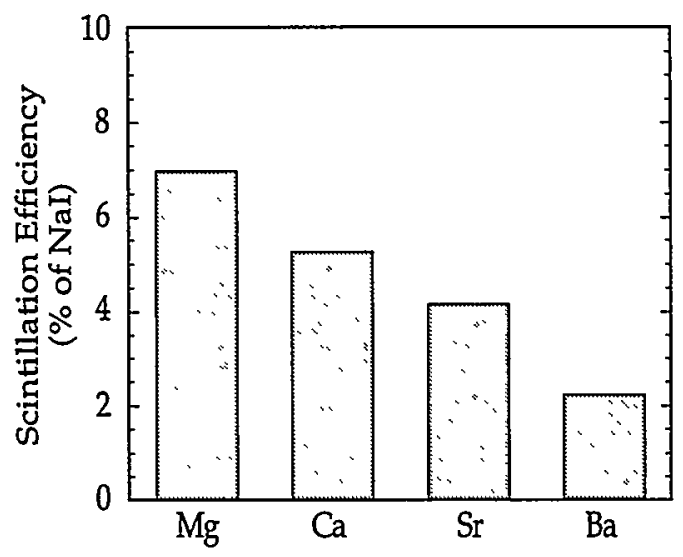

Figure 1. Scintillation efficiency of alkaline earth glasses as a percentage of that of $\mathrm{NaI}$.
In this report, $\mathrm{Ce}$ (III) indicates the valence state and $\mathrm{Ce}^{3+}$ indicates an actual ion or ions. $\mathrm{Ce}(\mathrm{IV})$ and $\mathrm{Ce}^{4+}$ have the corresponding meanings. The glass containing $15 \mathrm{~mol} \%$ $\mathrm{MgO}$ will be referred to as the $\mathrm{Mg}$ glass, and glasses with other alkaline earths will be referred to similarly.

Although it is theoretically possible for $\mathrm{Ce}^{4+}$ to be a scintillation center, this ion absorbs light strongly over a range of frequencies that includes the $\mathrm{Ce}^{3+}$ fluorescence. Therefore the presence of $\mathrm{Ce}^{4+}$ is believed to reduce the scintillation efficiency of a glass by absorbing scintillation light from $\mathrm{Ce}^{3+}$. The observation that the glasses are clear and colorless is consistent with the lack of evidence for $\mathrm{Ce}^{4+}$.

The $\mathrm{x}$-ray absorption near edge structure (XANES) for Ce(III) and Ce(IV) compounds is quite different, suggesting a method of detecting the presence of $\mathrm{Ce}^{4+}$ ions in the glass. Although $\mathrm{Ce}^{4+}$ was not detected in previous experiments by fluorometry, frontface fluorescence or UV-Vis spectroscopy, its concentration is expected to be extremely low (less than $10 \%$ of the total $\mathrm{Ce}$, or less than $0.06 \mathrm{~mol} \%$ in the glass). The difference in the XANES for the two ions is sufficient to provide a detection limit for $\mathrm{Ce}^{4+}$ of at least $1 \%$ of the total $\mathrm{Ce}$.

The gamma-ray-induced scintillation efficiency for the series of glasses is shown in Figure 1 as a percentage of that for crystalline $\mathrm{NaI}$. As observed previously 1 , the amount of light produced varies significantly (greater than a factor of 2.5) as the alkaline earth is varied.

$\mathrm{X}$-ray absorption spectra of the $\mathrm{Mg}, \mathrm{Ca}$ and $\mathrm{Sr}$ glasses and $\mathrm{Ce}$ reference compounds were 
recorded at room temperature. (Analyses of the $\mathrm{L}_{\mathrm{III}}$ edge of $\mathrm{Ce}$ in the $\mathrm{Ba}$ glass was complicated by the presence of the $\mathrm{Ba}_{\mathrm{II}}$ edge at approximately $5625 \mathrm{eV}$, and is not included here.) The X-ray absorption structure near the Ce LIII edge is shown in Fig 2 for Ce(III) acetate $\left[\mathrm{Ce}\left(\mathrm{C}_{2} \mathrm{H}_{3} \mathrm{O}_{2}\right)_{3}\right], \mathrm{Ce}$ (IV) hydroxide $\left[\mathrm{Ce}(\mathrm{OH})_{4}\right]$, and for the series of glasses. The difference between the $\mathrm{Ce}$ (III) and $\mathrm{Ce}$ (IV) edges is quite striking, and has been observed for $\mathrm{CeF}_{3}$ and $\mathrm{CeF}_{4}$ as well.2

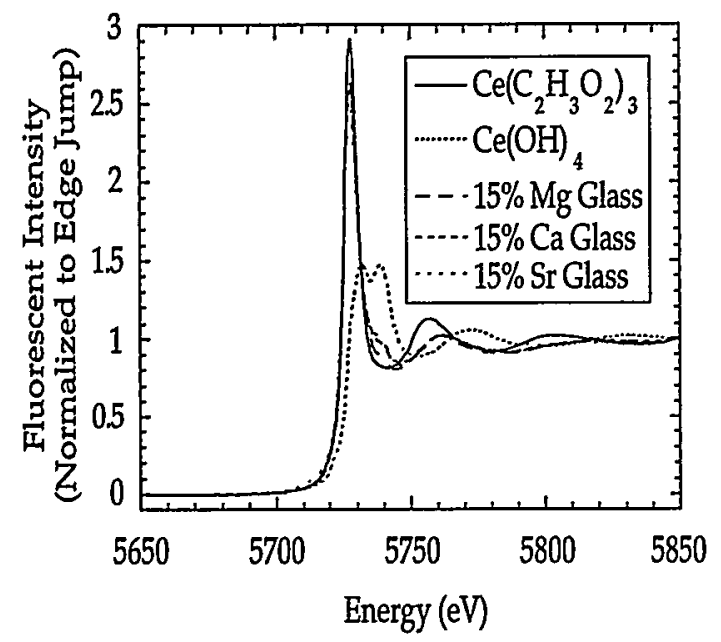

Figure 2. Ce $L_{\text {III }} X A N E S$ for the series of alkaline earth glasses and for two reference compounds.

The Ce(III) spectrum displays a single large white line due to excitation of an electron from the $2 p_{3 / 2}$ core level to empty $5 \mathrm{~d}$ bands. The Ce(IV) XANES shows two strong absorption peaks. A theoretical treatment that includes multiple scattering effects and many body final states suggests that these are $2 p$ to $5 d$ transitions with two different final state configurations, $4 \mathrm{f}^{0}$ and $4 \mathrm{f} 1 \mathrm{~L}$ (the latter designates hybridization of the $\mathrm{Ce} 4 \mathrm{f}$ orbitals with $\mathrm{O} 2 \mathrm{p}$ ligand orbitals). 3 Such hybridization of cation and anion wavefunctions has been shown to be significant in insulating $\mathrm{Ce}$ (IV) compounds, which have been the subject of much investigation and discussion.2-10 The Ce $\mathrm{L}_{\text {III }}$ XANES for the glass samples indicate that they contain predominantly $\mathrm{Ce}^{3+}$. This may be inferred from the presence of a single white line in the glass spectra and from the proximity of the glass spectra edges to the $\mathrm{Ce}$ (III) reference edge. However, the alignment of the shoulder at $5739 \mathrm{eV}$ in the glass spectra with the second major peak in the Ce(IV) reference suggests the presence of $\mathrm{Ce}^{4+}$.

To estimate the amount of $\mathrm{Ce}^{4+}$ present in the glasses, the spectra were normalized to the step edge and then fit using a combination of Voigt peaks and a cumulative step function. A Voigt peak, the convolution of Lorentzian and Gaussian lineshapes, accounts for both the natural lineshape (Lorentzian) and the detector function (Gaussian). The method of Puerta and Martin 11 was used to approximate the Voigt peaks. Transitions to the continuum were represented by a cumulative Gaussian step function. The widths of the step function and the Gaussian components of the pseudoVoigt peaks were set to the same value but allowed to vary together to reflect a consistent instrument response. Best fits were found for a Gaussian width of $1.80 \pm 0.03 \mathrm{eV}$, consistent with the observed resolution of approximately $0.5 \mathrm{eV}$. The Lorentzian widths of the pseudo-Voigt peaks were allowed to vary to reflect differences in the natural (lifetime) linewidths.

The Ce(II) standard best fit is shown in Fig. 3. Solid squares indicate data points, and the solid line through them is the fit. The Ce(IV) standard fit is shown in Fig. 4. The four features corresponding to the four Voigt peaks have been observed previously and reproduced by various calculations. $3-5$ The peak at $5739 \mathrm{eV}$ is believed to cause the shoulder in the glass spectra.

The peak fit for the Ca glass is shown in Fig 5. Peak positions and half widths found for the Ce(III) standard were used as starting parameters to fit the Ce near edge structure of the glasses, with the addition of a peak to fit the shoulder. Two of the three largest Ce(IV) peaks are very close in energy position $(0.1$ $\mathrm{eV}$ and $0.5 \mathrm{eV}$ for the peaks at $5727.5 \mathrm{eV}$ and $5730.1 \mathrm{eV}$, respectively) to peaks present in the $\mathrm{Ce}$ (III) edge. For this reason it was assumed that variation in the intensity and width of these peaks would be sufficient to account for the $\mathrm{Ce}^{4+}$ peaks present in the 


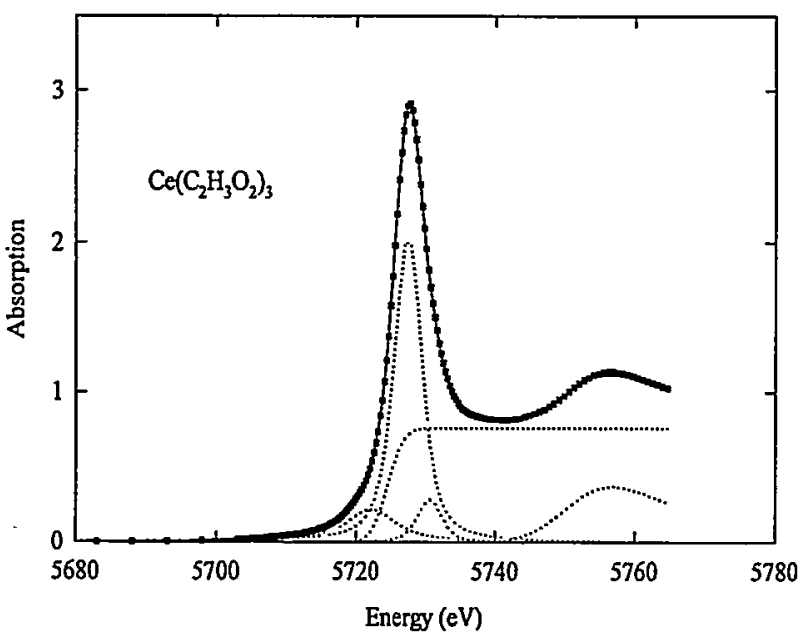

Figure 3. Peak fits to the $\mathrm{Ce} \mathrm{L}_{\mathrm{II}}$ near edge structure of the $\mathrm{Ce}^{3+}$ reference, $\mathrm{Ce}\left(\mathrm{C}_{2} \mathrm{H}_{3} \mathrm{O}_{2}\right)_{3}$.

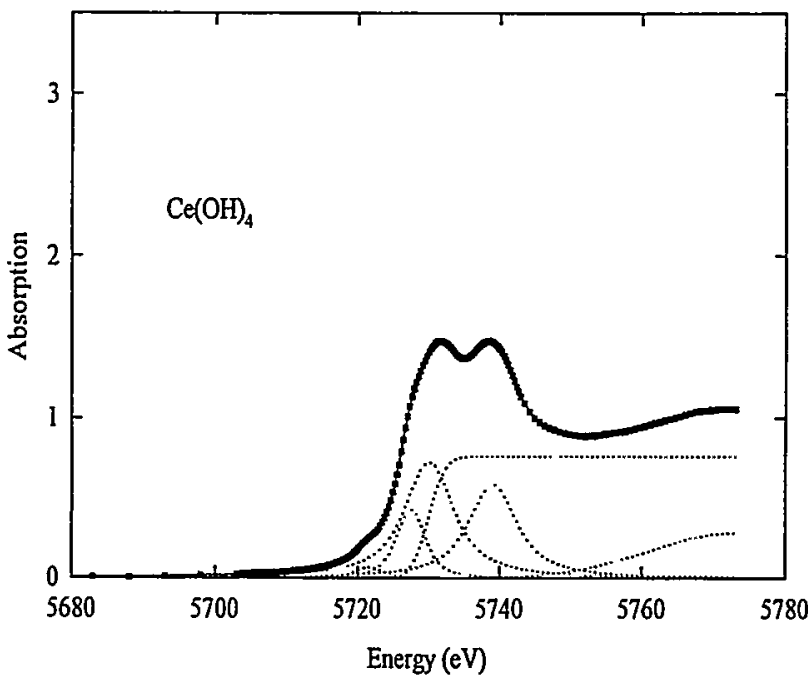

Figure 4. Peak fit to the Ce $\mathrm{L}_{\text {III }}$ near edge structure of the $\mathrm{Ce}^{4+}$ reference, $\mathrm{Ce}(\mathrm{OH})_{4}$.

glasses other than that explicitly added at 5739 $\mathrm{eV}$. In fact, good fits were obtained with fairly small shifts (less than $1.8 \mathrm{eV}$ ) in the positions of all the peaks relative to the corresponding peaks in $\mathrm{Ce}$ (III) and $\mathrm{Ce}$ (IV). Significant differences in the intensities and values of the full width at half maximum were observed. These differences are due in part to the presence of peaks from both $\mathrm{Ce}^{3+}$ and $\mathrm{Ce}^{4+}$, and to inhomogeneous broadening of the peaks that results from the variety of $\mathrm{Ce}$ sites present in the glass. This fitting

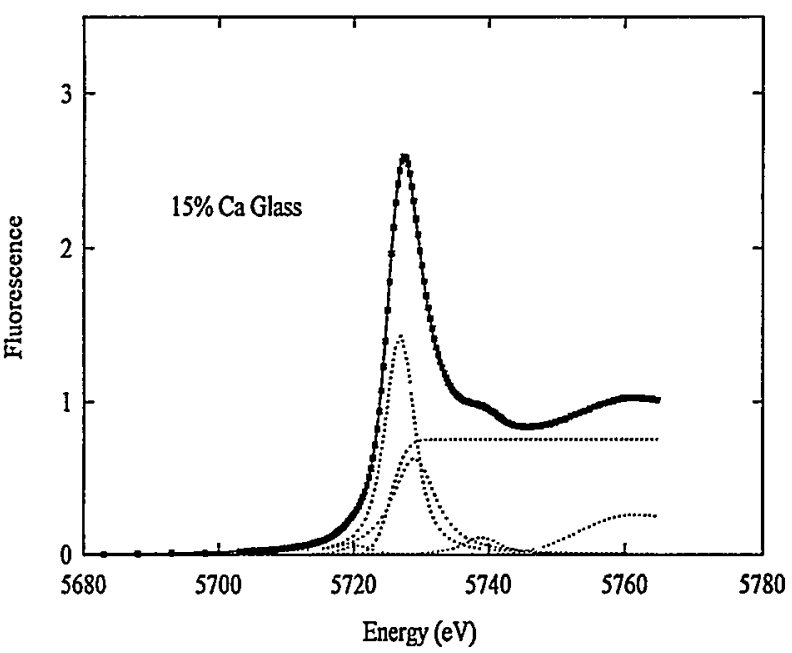

Figure 5. Peak fit to the Ce $\mathrm{L}_{\text {III }}$ near edge structure of the $\mathrm{Ca}$ glass.

procedure, though not rigorous, is sufficient to extract relative changes in the $\mathrm{Ce}^{4+}$ concentration in the glasses.

The peak that accounts for the shoulder in the glass spectra was numerically integrated, and the resulting area was plotted versus the scintillation efficiency (Fig. 6). They are clearly correlated. Normalizing these peak areas by the corresponding peak area found for the $C(I V)$ reference should give an estimate of the concentration of $\mathrm{Ce} 4+$ in the samples. However, the estimates using this method are unreasonably high (up to $19 \%$ of the total Ce for the Sr glass), as discussed below. The line through the data is a best fit assuming the scintillation efficiency varies as $1 /\left[\mathrm{Ce}^{4+}\right]$. This is an approximation of the behavior expected assuming $\mathrm{Ce}^{4+}$ absorbs the scintillation output of the $\mathrm{Ce}^{3+}$.

The high concentration of $\mathrm{Ce}^{4+}$ determined by the peak fitting method cannot represent a permanent population. Ishii et al. 12 have shown that the $\mathrm{Ce}^{4+}$ ion in silicate glasses produces a broad absorption peak centered about $275 \mathrm{~nm}$. Depending on the matrix, the Ce $4+$ absorption tail in the visible is 1 to $10 \%$ of the maximum absorption value. This would imply, for the glass compositions used here, absorption coefficient should be on the order of 3-30 $\mathrm{cm}^{-1}$; in this case the samples would be grossly colored. They are not; the 


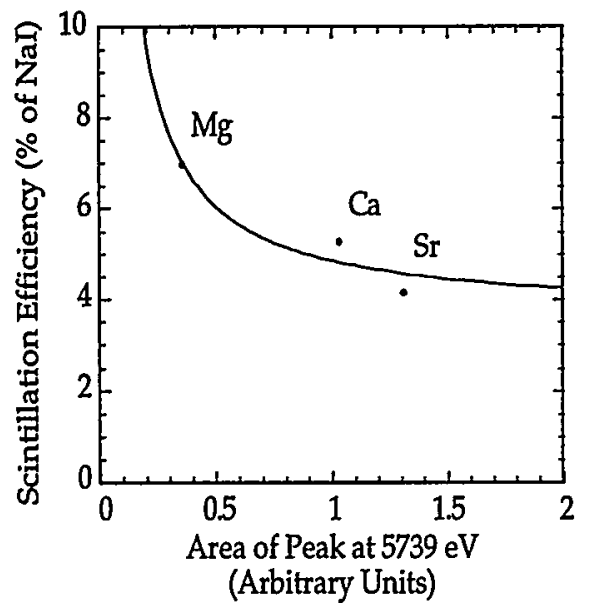

Figure 6. Plot of the $5739 \mathrm{eV}$ peak area vs scintillation efficiency for the glasses.

samples are water-clear. The use of other estimation methods, such as changes in the $\mathrm{Ce}^{3+}$ white line, give somewhat lower values for the $\mathrm{Ce}^{4+}$ concentration, but are still unreasonably high. It is evident that the feature identified as $\mathrm{Ce}^{4+}$ cannot be a permanent population of $\mathrm{Ce}^{4+}$ in the samples.

A possible explanation is that the feature is indeed $\mathrm{Ce}^{4+}$, but is a transient phenomenon related to beam effects. Weber et al.19 have performed UV-stimulated low-temperature thermoluminescence on the sample series studied here. They found a glow peak at approximately $20^{\circ} \mathrm{C}$; the strength of this glow peak increased in going from $\mathrm{Mg}$ to $\mathrm{Ba}$. It is plausible, and consistent with Weber et al., that the $\mathrm{Ce}^{4+}$ found in this study is a result of photoionization of $\mathrm{Ce}^{3+}$ by the $\mathrm{x}$-ray beam. If this is occurring, an equilibrium population of $\mathrm{Ce}^{4+}$ and trapped electrons in the $20^{\circ} \mathrm{C}$ traps could be established. On removal from the beam, the traps will quickly depopulate (with the emission of thermoluminescence), because the sample is at or near $20^{\circ} \mathrm{C}$, and restore the $\mathrm{Ce}^{4+}$ to $\mathrm{Ce}^{3+}$. This explanation could be tested simply by performing the beam measurements at higher or lower temperatures. Such experiments are planned.

A correlation was observed between the scintillation efficiency of a series of $\mathrm{Ce}$ doped lithium silicate glasses and the intensity of a feature in the Ce LIII XANES of the glasses.
This feature appears to indicate the presence of $\mathrm{Ce}^{4+}$ due to its location. However, an estimate of the concentration of $\mathrm{Ce}^{4+}$ in the glasses by peak area comparison to a $\mathrm{Ce}$ (IV) compound results in an unrealistically high value. We suspect that the feature is a transient phenomenon related to creation of $\mathrm{Ce}^{4+}$ and trapped electrons due to photoionization by the $\mathrm{x}$-ray beam. The correlation indicates that the presence of $\mathrm{Mg}$ is more effective at preventing creation of such a species than are $\mathrm{Ca}$ and $\mathrm{Sr}$.

This work was supported, in part, by the Department of Energy, NN-20. Pacific Northwest Laboratory is operated by Battelle Memorial Institute for the U. S. Department of Energy under Contract DE-AC06-76RLO 1830. The authors wish to thank FW Lytle, DL Brewe, and SM Heald for useful and stimulating discussions.

1. M. Bliss et al., MRS Symposium Proceedings, 348, 195 (1994).

8. G. Kaindl et al., Phys. Rev. Lett. 58, 606 (1987).

9. A.V. Soldatov et al., Phys. Rev. B, 50 5074 (1994).

10. L.D. Finkelstein et al., Materials Letters 14, 115 (1992).

11. A. Bianconi et al., Phys Rev B 35, 806 (1987).

12. H. Dexpert et al, Phys Rev B, 36, 1750 (1987).

13. R.D. Parks et al., Phys Rev B, 28, 3556 (1983).

14. A. Fujimori, Phys Rev B 28, 4489 (1983).

15. E Wuilloud et al., Phys Rev Lett 53, 202 (1984).

16. F Mirabelli et al., Phys Rev B 46, 10012 (1992).

17. J. Puerta and P. Martin, Appl. Optics 20, 3923 (1981).

18. Y. Ishii et al., J. Am. Ceram. Soc.70, 72 (1987).

19. M. J. Weber, et al., "Scintillators and Applications: Cerium-DopedMaterials", Proceedings of EURODIM94 (Seventh Europhysical Conference on Defects in Materials), Lyon, France, July 5-8, 1994. 


\title{
XAS Study of Metal Ion Partitioning at Clay-Water Interfaces
}

\author{
Charalambos Papelis ${ }^{1}$, Chia-Chen Chen ${ }^{2}$, and Kim F. Hayes ${ }^{2}$ \\ ${ }^{1}$ Desert Research Institute, Water Resources Center, University System of Nevada \\ P.O. Box 19040, Las Vegas, NV 89132-0040
}

${ }^{2}$ Environmental and Water Resources Engineering, Department of Civil and Environmental Engineering,
The University of Michigan, Ann Arbor, MI 48109-2125

\section{Introduction}

Metal cations are thought to sorb to smectite clays either at permanent-charge sites on the interlayer basal planes or at surface-hydroxyl sites at the clay edge where the crystal structure is interrupted (1-4). Although most of the surface area and cation exchange capacity in smectites is found in the interlayer region, recent studies suggest that the less-abundant sites on the external surface may be even more important, depending on solution conditions (4). To date, direct confirmation of the structure of sorbed metal ion complexes on smectites is lacking. This is partly because most spectroscopic methods cannot distinguish the number and type of nearest neighbors surrounding a sorbing atom or distinguish among different sorption complexes. X-ray absorption spectroscopy (XAS) can be used to determine the average number, type, and interatomic distance between a sorbed metal cation and its nearest neighbors in situ.

For this study two smectites have been selected, montmorillonite and hectorite, to distinguish between $\mathrm{Co}$ (II) and $\mathrm{Sr}$ (II) surface complexes formed with permanent-charge sites in.the interlayer region and surface complexes on external surface-hydroxyl sites. These two metal cations differ in their relative affinities for surface hydroxylated mineral surfaces. Co(II), a relatively strongly binding ion, has been shown to form inner-sphere complexes on oxide and clay surfaces and polynuclear species at surface coverages above $5-10 \%(5-7)$. Sr(II), a weakly binding ion, only forms outer-sphere complexes and mononuclear species, even at high surface coverages on oxide minerals (Hayes, unpublished XAS data). The hypotheses of the different coordination environments expected for the two metal cations and the two types of sites are being tested by collecting XAFS spectra at the $\mathrm{Co}$ and $\mathrm{Sr} \mathrm{K}$-edge energies. The results from this study will be used to improve current surface complexation models by imposing constraints on reaction stoichiometry.

\section{Experimental}

The adsorbents, Arizona Ca-montmorillonite (Saz-1) and California Hectorite (SHCa-1), were obtained from the Clay Minerals Society (University of Missouri, Columbia, MO), pretreated according to Kunze (8) and converted to the $\mathrm{Na}$ form by reacting with $4 \mathrm{~N}$ sodium chloride. Relative metal ion fractional uptake was determined by comparing the concentration in the supernatant to the concentration of a control (sample without adsorbent).

XAS Data Analysis. X-ray absorption spectra were collected at the Co K-edge $(7,709.5 \mathrm{eV})$ and the Sr K-edge $(16,105 \mathrm{eV})$ at the Stanford Synchrotron Radiation Laboratory (SSRL) using wiggler beam line 4-3 with $\mathrm{Si}(220)$ monochromators, beam energy $3.0 \mathrm{GeV}$, and beam current ranging from 30 to $100 \mathrm{~mA}$. Spectra were collected in transmission mode for the solid reference compounds and in fluorescence mode for sorption samples using a SternHeald type detector ( $(9)$ or a 13-element Ge array detector. To improve the signal-to-noise ratio, by minimizing thermal disorder, data were collected at liquid helium temperature (approximately $10 \mathrm{~K}$ ) using a liquid helium cryostat.

Spectra were averaged and background was subtracted by fitting a straight line in the pre-edge region and a cubic, three segment spline in the Extended X-ray Absorption Fine Structure (EXAFS) region. The background-subtracted spectra were normalized using tabulated McMaster atomic absorption fall-off coefficients $(10)$ followed by conversion from energy to frequency space. The background-subtracted, 
normalized, $\mathbf{k}^{3}$-weighted EXAFS spectra were Fourier transformed to obtain radial structure functions (rsf). Individual peaks in the Fourier transform were backtransformed to produce filtered EXAFS and to isolate specific contributions from individual shells in the EXAFS. The number and identity of the backscattering atoms were then determined by fitting the filtered EXAFS (with nonlinear, least-squares curve-fitting techniques) using phase and amplitude parameters derived from crystalline "reference" compounds with known structure or ab initio calculations using a curved wave formalism (11).

\section{Results and Discussion}

\section{(1) Co Sorption on Montmorillonite}

$\mathrm{Co}$ (II) sorption experiments at different $\mathrm{Na}$ ion concentrations, total $\mathrm{Co}$ (II) concentrations, and $\mathrm{pH}$, were conducted to selectively promote $\mathrm{C}$ (II) sorption at either permanent-charge or surface-hydroxyl sites. XAS data were collected at various conditions of $\mathrm{pH}$ and $\mathrm{Na}$ ion concentration and total $\mathrm{Co}$ (II) concentration

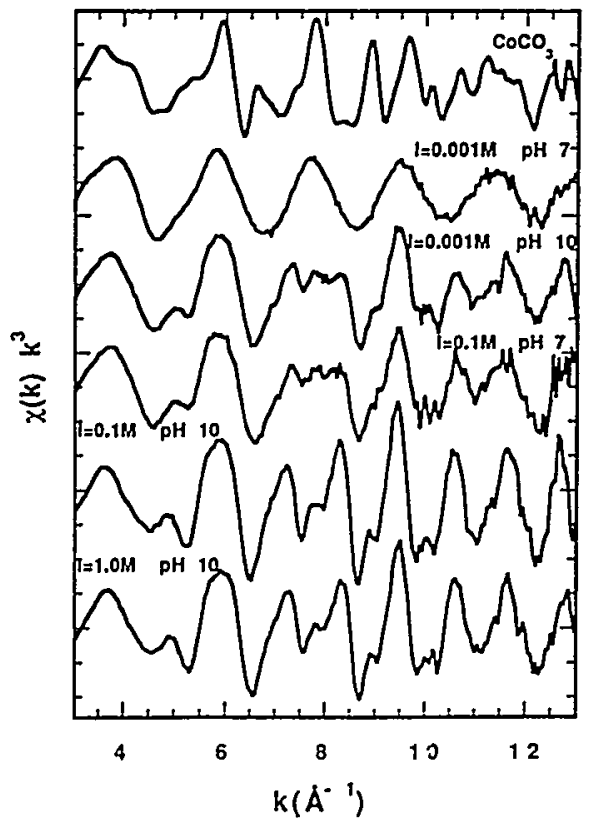

Figure 1: Co EXAFS spectra for one model compound, $\mathrm{CoCO}_{3}$ and $\mathrm{Co}$ (II) sorption samples on montmorillonite. Only the second spectrum, corresponding to $\mathrm{Co}$ (II) sorbed on montmorillonite at $\mathrm{pH} 7$ and $0.001 \mathrm{M} \mathrm{Na}$ ion concentration, shows a single frequency, suggesting a single oxygen shell around the central Co atom. The "beat patter" present in every other sorption sample suggests additional shells surrounding the central Co atom. of $1 \times 10^{-4} \mathrm{M}$. At a low $\mathrm{Na}$ ion concentration $(0.001$ $\mathrm{M})$ and $\mathrm{pH} 7, \mathrm{Na}$ is not expected to be able to displace Co(II) from permanent-charge sites. Under these conditions, $\mathrm{Co}$ (II) surface complexes with permanentcharge sites are expected. At higher $\mathrm{pH}$, however, because of increased affinity of $\mathrm{Co}$ (II) for surfacehydroxyl sites, formation of $\mathrm{Co}(\mathrm{II})$ complexes on surface-hydroxyl sites is anticipated. The backgroundsubtracted, normalized, $\mathrm{k}^{3}$-weighted Extended X-ray Absorption Fine Structure (EXAFS) spectra for a reference ("model") compound ( $\mathrm{CoCO}_{3}$, sphaerocobaltite) and 5 sorption samples (Figure 1) and the radial structure functions (rsf) resulting from Fourier transform of the EXAFS spectra (Figure 2) are consistent with these expectations. Peaks in the rsf correspond to shells of backscatterers at a certain distance (uncorrected for phase shift) from the absorber.

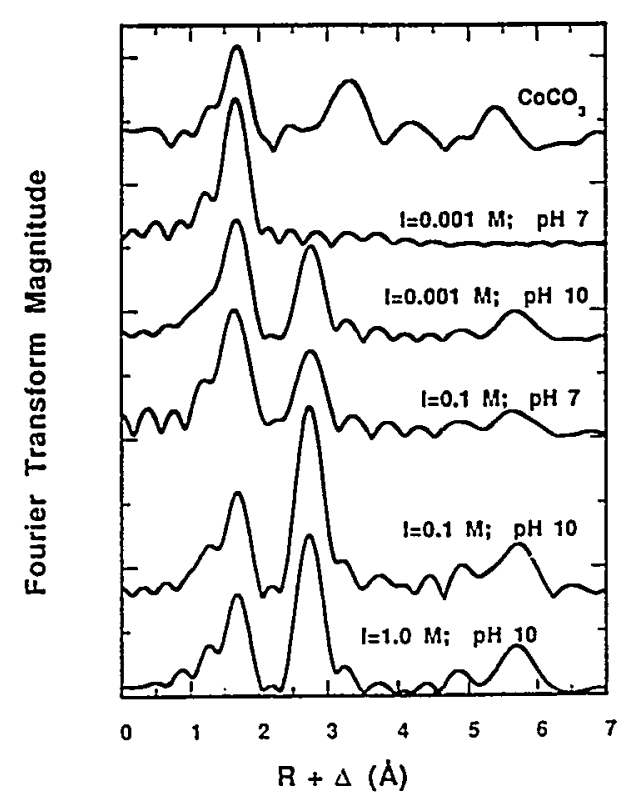

Figure 2: Radial structure functions produced by Fourier-transforming of the EXAFS spectra shown in Figure 1. The first peak corresponds to a shell of oxygens surrounding the central Co atom, at approximately the same distance for all samples. Only in the sorption sample corresponding to $\mathrm{pH} 7$ and $0.001 \mathrm{M}$ $\mathrm{Na}$ ion concentration is only one shell evident.

At the lowest $\mathrm{Na}$ ion concentration $(0.001 \mathrm{M})$ and $\mathrm{pH} 7$, only one peak is evident in the rsf, indicating a Co atom coordinated by 6 oxygens in an outer-sphere complex with permanent-charge sites. 
The Co-O distance and $\mathrm{CN}$ determined, $2.09 \pm 0.02 \AA$ and $6.6 \pm 20 \%$, respectively are consistent with the coordination environment of $\mathrm{Co}$ in aqueous solutions (12). At pH 10, two additional peaks in the rsf at approximately 2.75 and $5.75 \AA$ (uncorrected for phase shift) appear, corresponding to Co-Co shells and the formation of polynuclear species or a surface precipitate. The appearance of $\mathrm{Co}-\mathrm{Co}$ shells (2nd and 4th Co coordination shell at 2.75 and $5.75 \AA$, respectively, uncorrected for phase shift) at high coverage is consistent with past work of Co sorption on aluminas and clays which indicates the onset of multinuclear complex formation on external sites at coverages greater than $5-10 \%(5,7,12)$. The coordination environment in these polynuclear-species/surface-precipitates (CoCo distance $3.13 \pm 0.02 \AA, \mathrm{CN} 4.0 \pm 20 \%$ ) is consistent with the reported structure of polynuclear species of $\mathrm{Co}$ on $\gamma-\mathrm{Al}_{2} \mathrm{O}_{3}$ and kaolinite $(5,6,12)$. In addition, also in agreement with these previous studies, the $\mathrm{Co}-\mathrm{Co}$ distance $(3.12-3.13 \pm 0.02 \AA)$ is shorter than in crystalline $\mathrm{Co}(\mathrm{OH})_{2}(3.173 \AA)$, suggesting that the structure of the surface precipitate formed is modified to better match the structure of the underlying substrate.

At higher $\mathrm{Na}$ ion concentrations $(0.1 \mathrm{M}), \mathrm{Na}$ ions can exclude $\mathrm{Co}$ (II) ions from permanent-charge sites at all $\mathrm{pH}$ values. Therefore, at high $\mathrm{Na}$ concentration, it was expected that $\mathrm{Co}$ (II) would bond predominantly to external surface-hydroxyl sites. The appearance of the two peaks near 2.75 and $5.75 \AA$ in the rsf of the $0.1 \mathrm{M} \mathrm{Na}, \mathrm{pH} 7$ sample (Figure 2) confirms that multinuclear complexes with surfacehydroxyl groups are forming for this sample. The comparison of XAS results for the two $\mathrm{pH} 7$-samples (at two $\mathrm{Na}$ ion concentrations, 0.001 and $0.1 \mathrm{M}$ ) has significant implications. Although, based on macroscopic experiments, Co fractional uptake is similar in the two samples, the Co coordination environment is substantially different (absence vs. presence of secondneighbor $\mathrm{Co}$ ), suggesting that at lower $\mathrm{Na}$ ion concentration Co sorbs at permanent-charge sites, whereas at the higher $\mathrm{Na}$ concentration $\mathrm{Co}$ complexes at external surface-hydroxyl sites are formed.

At still higher $\mathrm{pH}(10)$ and $\mathrm{Na}$ ion concentration $(0.1$ or $1.0 \mathrm{M})$, as more $\mathrm{Co}$ (II) sorbs to the surface-hydroxyl sites, the amplitude of the second and fourth shell peaks ( $\mathrm{Co}-\mathrm{Co}$ ) increases (Figure 2), compared to the sample at $\mathrm{pH} 7$. The increased amplitudes are consistent with growing Co polymers or a more ordered surface precipitate on the surfacehydroxyl sites as surface coverage increases. Note also the increased $\mathrm{CN}$ in the second and fourth coordination shells of the $\mathrm{pH} 10$ sample at 0.1 (or 1.0) $\mathrm{M} \mathrm{Na}$ concentration, compared to the $\mathrm{pH} 10$ sample at the lower Na concentration $(0.001 \mathrm{M})$. Although $\mathrm{Co}$ is quantitatively removed (near 100\%) from solution under both experimental conditions, the spectroscopic results suggest a lower surface-hydroxyl surface coverage at the lower $\mathrm{Na}$ concentration. Because changes in the $\mathrm{Na}$ concentration are not expected to effect the amount or coordination environment of Co sorbed exclusively to surface-hydroxyl sites, the likely explanation for the lower $\mathrm{CN}$ at the lower $\mathrm{Na}$ concentration is that some of the $\mathrm{Co}$ is also sorbed, as an outer-sphere complex, in the interlayer region.

\section{(2) Sr(II) Sorption on Montmorillonite and Hectorite}

$\mathrm{Sr}$ (II) sorption experiments were conducted at different $\mathrm{Na}$ ion concentrations with montmorillonite and hectorite. XAS data were collected at various conditions of $\mathrm{pH}$ and $\mathrm{Na}$ ion concentration and total

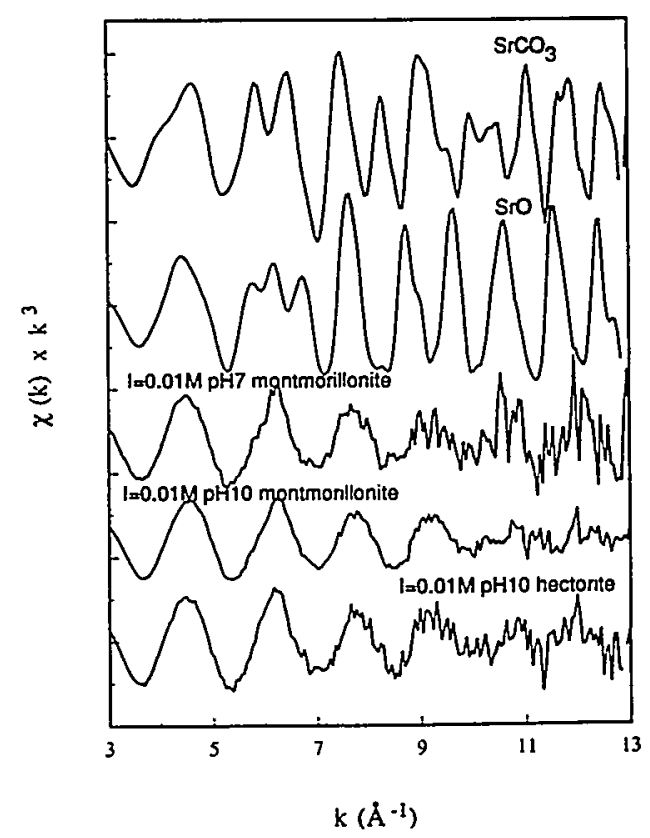

Figure 3: Normalized, background-subtracted, $\mathrm{k}^{3}$ weighted Sr EXAFS spectra for two model compounds, $\mathrm{SrCO}_{3}$ and $\mathrm{SrO}$, and $\mathrm{Sr}$ (II) sorption samples on montmorillonite and hectorite. No beat patterns are evident in any of the sorption samples, suggesting a single $\mathrm{O}$ shell around the central $\mathrm{Sr}$ atom. 
Sr(II) concentration of $1 \times 10^{-4} \mathrm{M}$. The backgroundsubtracted, normalized, $\mathrm{k}^{3}$-weighted Extended X-ray Absorption Fine Structure (EXAFS) spectra for two model compounds, $\mathrm{SrCO}_{3}$ and $\mathrm{SrO}$, and 3 sorption samples and the radial structure functions (rsf) resulting from Fourier transform of the EXAFS spectra are shown in Figures 3 and 4, respectively. No beat patterns are evident in any of the sorption samples, regardless of experimental conditions. Although the amplitude (and quality) of the EXAFS spectra are severely reduced beyond $\mathrm{k}$ approximately $8 \AA^{-1}$ (Figure $3)$, because of the high disorder in the coordination environment of aqueous $\mathrm{Sr}$, it is believed that the presence of second-neighbor $\mathrm{Sr}$ would be obvious in the earlier part of the spectrum (at $\mathrm{k}$ values smaller than $8-9 \AA^{-1}$, compare the EXAFS spectra of the model compounds).

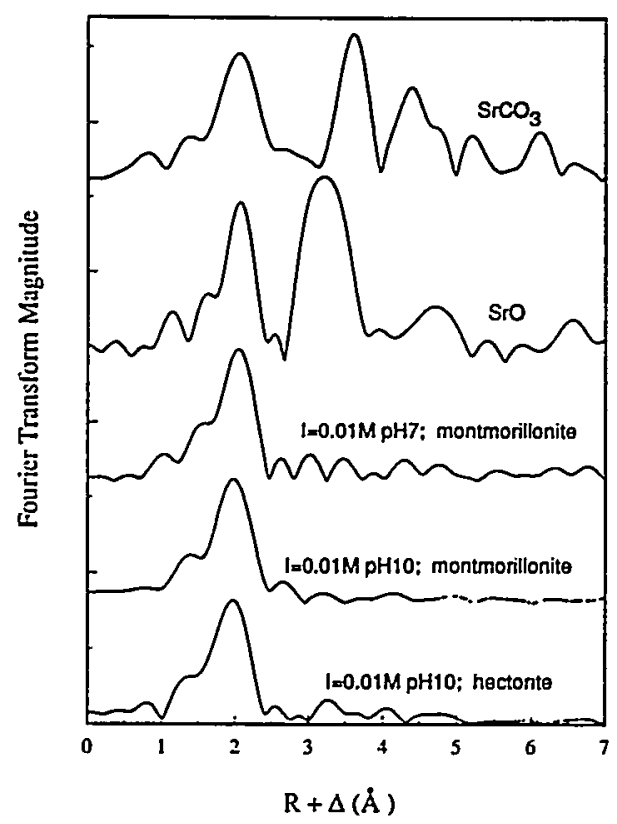

Figure 4: Radial structure functions produced by Fourier-transforming of the EXAFS spectra shown in Figure 3. The first peak corresponds to a shell of oxygens surrounding the central $\mathrm{Sr}$ atom, at approximately the same distance for all samples. Only one shell is evident in all sorption samples.

The absence of any evidence of a second shell is consistent with the formation of outer-sphere complexes, regardless of experimental conditions and adsorbate. It is also believed that the formation of inner-sphere complexes would result in decreased disorder in the $\mathrm{Sr}$ coordination environment, thereby allowing the detection of additional shells beyond the first. Sr $(\mathrm{Z}=38)$ is a strong enough backscatterer to allow detection of second-neighbor Sr. This is evident from the spectra of the $\mathrm{Sr}$ model compounds in Figures 3 and 4. The absence of second-neighbor $\mathrm{Sr}$, even at $\mathrm{pH} 10$, on both clay minerals, is consistent with previous studies suggesting absence of $\mathrm{Sr}$ multinuclear species and formation of outer-sphere complexes, even at relatively high surface coverages (Hayes, unpublished data). In addition, at higher $\mathrm{Na}$ ion concentration $(0.1 \mathrm{M})$, the decreased Sr fractional uptake resulted in unusable spectra, consistent with formation of weak outer-sphere complexes, even under conditions promoting sorption on extemal surface-hydroxyl sites. The results for $\mathrm{Sr}$ are, therefore, in contrast to the results for $\mathrm{Co}$, where the formation of multinuclear species/surface precipitates on surface-hydroxyl sites was evident under similar surface coverages.

\section{Conclusions}

The significant implication of these results for metal ion sorption on smectites is that for strongly binding cations like $\mathrm{Co}$ (II) both types of sites (permanent charge and surface hydroxyl) are operative over a wide range of $\mathrm{pH}$ at low ionic strength. These findings provide the first direct evidence that, even at intermediate and high $\mathrm{pH}$, interlayer sites can be important for sorption of these types of metal ions. This is in direct contrast to the recent speculation, based on ionic strength dependence studies, that only surface-hydroxyl sites are important at $\mathrm{pH}$ values greater than 6.5 (13). In addition, these studies are consistent with expected differences in the sorption behavior of the two cations studied, and with only the formation of $\mathrm{Sr}$ mononuclear outer-sphere complexes on smectites, regardless of experimental conditions.

\section{Acknowledgments}

Financial support was provided by the National Science Foundation and the Procter and Gamble Company. Graham George (SSRL) is gratefully acknowledged for providing the EXAFS data reduction program. We thank Lynn E. Kaz for assistance with XAS data collection. The facilities and staff of the Stanford Synchrotron Radiation Laboratory are much appreciated. 


\section{References}

(1) Peigneur, P.; Maes, A.; Cremers, A. Clays Clay Miner. 1975, 23, 71-75.

(2) Inskeep, W. P.; Baham, J. Soil Sci. Soc. Am. J. 1983, 47, 660-665.

(3) Fletcher, P.; Sposito, G. Clays Clay Miner. 1989, 24, 375-391.

(4) Zachara, J. M.; Smith, S. C.; Resch, C. T.; Cowan, C. E. Soil Sci. Soc. Am. J. 1992, 56, 1074-1084.

(5) O'Day, P. A.; Brown, G. E., Jr.; Parks, G. A. J. Colloid Interface Sci. 1994, 165, 269-289.

(6) O'Day, P. A.; Parks, G. A.; Brown, G. E., Jr. Clays \& Clay Minerals 1994, 42, 337-355.

(7) Katz, L. E.; Hayes, K. F. J. Colloid Interface Sci. 1995, in press.

(8) Kunze, G. W.; Dixon, J. B. In Methods of Soil Analysis. Part 1: Physical and Mineralogical Methods, 2nd ed.; A. Klute Ed.; Soil Science Society of America: Madison, WI, 1986; pp 91100.

(9) Lytle, F. W.; Greegor, R. B.; Sandstrom, D. R.; Marques, E. C.; Wong, J.; Spiro, C. L.; Huffman, G. P., et al. Nucl. Instr. and Meth. $1984,226,542-548$.

(10) McMaster, W. H.; Del Grande, N. K.; Mallett, J. H.; Hubbell, J. H. Compilation of $x$-ray cross-sections III; UCRL-50174; US Atom. Ener. Comm., 1969.

(11) McKale, A. G.; Veal, B. W.; Paulikas, A. P.; Chan, S. K.; Knapp, G. S. J. Am. Chem. Soc. $1988,110,3763-3768$.

(12) Chisholm-Brause, C. J.; O'Day, P. A.; Brown, G. E., Jr.; Parks, G. A. Nature 1990, 348, 528-531.

(13) Cowan, C. E.; Zachara, J. M.; Smith, S. C.; Resch, C. T. Soil Sci. Soc. Am. J. 1992, 56, 1084-1094. 
Proposal 227\%p

\title{
Delta-Phase Stabilization and Effects of Aging on Microstructure of Plutonium Alloys
}

\author{
Steven Conradson and Lawrence Cox, \\ Materials Science and Technology and Nuclear Materials Technology Divisions, Los Alamos National \\ Laboratory, Los Alamos, NM 87545; \\ and Nancy Hess, \\ Materials Science Department, Battelle Pacific Northwest Laboratory, Richland, WA
}

\begin{abstract}
Objectives. Plutonium demonstrates and readily converts between a large number of phases in the solid state. One means of addressing the technological problems involved in fabricating plutonium shapes to reasonable tolerances, resulting from the density variations among the various phases of plutonium accessed by casting, forging, and machining, was addressed by stabilizing it in the (putativlely fcc) delta phase. This was most commonly accomplished by alloying it with gallium in the $0.5-2.0 \mathrm{wt}-\%$ range. The long-term stability of these and other phases and alloys of plutonium, however, is unknown, but, as a radioactive element with a relatively short half life, they possess intrinsically the potential means for promoting conversions among the different phases.

As nuclear weapons are retired from the arsenals of the United States and Russia and close to 200 metric tons of plutonium "pits" are placed in interim storage until the means of their final disposition is determined, these stability issues are critical in ensuring that no unexpected incidents occur that could result in the accidental release during storage or subsequent disposal. The objective of this project is thus to characterize the microstructure of various newly prepared and aged plutonium alloys, investigating the mechanism(s) of phase stabilization and conversion and the long term stability of these materials with respect to structural instabilities induced by aging, e.g., phase transitions and other transformations to structural variants that may possess different densities and mechanical properties from the original materials. This information will be essential in establishing an effective, science-based approach to the storage and disposition of the surplus plutonium.
\end{abstract}

Approach. Several metals in addition to gallium are also delta-phase stabilizers, and the different sizes and electronic character of these as well as the complexity of the structures of various plutonium phases have made it difficult to assess the specific mechanism(s) of stabilization and phase transitions in plutonium alloys. Advances in structural determination techniques and theoretical methods over the 10-20 years since the last measurements of plutonium structures were made make it likely that readdressing the plutonium phase problem at this time will significantly enhance our understanding of and predictive abilities with respect to these phenomena. As stated above, such information is now even more critical because of the retirement of the majority of the existing nuclear weapons and the resulting need to ensure the integrity of the stored plutonium prior to its destruction or conversion to a long term storage form. It will also therefore be beneficial to analyze the microstructure of aged materials to establish the existence and mechanisms of structural transformations as the helium and americium concentrations and the amount of energy deposited in the material increase with time.

Over the two years of this project, it has involved $\mathrm{X}$-ray Absorption Fine Structure (XAFS) spectroscopy experiments on plutonium and plutonium alloys. In contrast to diffraction methods, which are sensitive to order on the scale of tens to hundreds of $\AA$ and thus determine the average positions of the atoms in a crystal over this same length scale, XAFS determines the average local structure around à selected element in a sample on a scale of 1-5 $\AA$ and does not require the assignment of a certain symmetry which imposes constraints on the final structure. XAFS is thus the technique of choice for identifying deviations from the average, symmetry-based structure of crystalline materials that may be critical in determining various macroscopic properties of interest as well as for providing information on the role of trace elements. High resolution (utilizing synchrotron radiation), powder $x$ ray diffraction measurements are currently planned as a complement to the XAFS experiments.

To date, XAFS spectra have been obtained at 80 $\mathrm{K}$ at both the $\mathrm{Pu} \mathrm{L}_{3}$ and $\mathrm{Ga} \mathrm{K}$ edges for freshly prepared $0.5,1.0$, and $2.0 \mathrm{wt}-\%$ gallium-plutonium, at both the $\mathrm{Pu} \mathrm{L} 3$ and $\mathrm{Ce} \mathrm{K}$ edges for 0.2 and $6.0 \mathrm{wt}-\%$ cerium-plutonium, at both the $\mathrm{Pu} \mathrm{L}_{3}$ and $\mathrm{In} \mathrm{K}$ edges for $0.5,1.5$, and $3.0 \mathrm{wt}-\%$ plutonium-indium, and at both the $\mathrm{Pu} \mathrm{L}_{3}$ and $\mathrm{Ga} \mathrm{K}$ edges for a sample of 8-year old $0.7 \mathrm{wt}-\%$ gallium $/ 0.5 \mathrm{wt} \%$-americium-plutonium and for samples of $\mathrm{ca} 25$-year old $1.0 \mathrm{wt}-\%$ gallium from two independent samples that have been in storage at LANL. We also have some additional data on alpha plutonium and temperature dependence on certain of these samples.

Summary of results on delta-stabilized materials. The alloys with gallium and indium and the higher concentration of cerium have been previously identified by XRD as the fcc "delta" phase. In contrast to these assignments based on similarities in the average, long-range order that result in similar diffraction patterns, the only common structural element observed by the XAFS measurements is the presence of an average plutonium-plutonium bond length of $3.3 \AA$ that accounts for all or most of the first shell of near neighbors around the plutonium. For the purposes of this study, therefore; the term "delta" will be used to describe phases possessing this moiety. In rigorously fcc metals, e.g., copper and platinum, the atoms in the more distant shells are distributed only very narrowly around the positions corresponding to a perfect fcc lattice and are observed in these positions by XAFS. Only certain of the plutonium alloys examined are apparently sufficiently well ordered with respect to the 
extended structure of the plutonium atoms - the specific locations of the plutonium atoms about their average locations over a minimum unit cell - to exhibit the structural features at the positions associated with such a well defined fcc lattice as determined by the first shell bond length. Those that do will be described as " $\mathrm{fcc}$ " in addition to being delta phase.

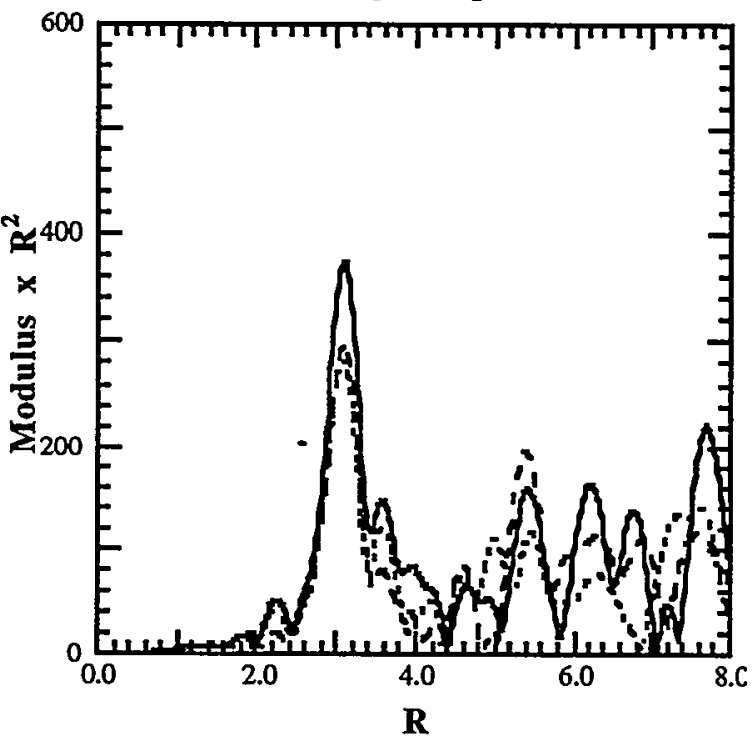

Figure 1. Fourier transform moduli of $\mathrm{Pu}_{3} \mathrm{~L}_{3}$ EXAFS of Pu-In alloy, with In concentrations of 0.5 (solid), 1.5 (dashed), and 3.0 (dotted) wt-\% In.

Table 1. Curve-fitting results for Pu EXAFS of Pu-In alloys.

\begin{tabular}{|c|c|c|c|c|c|}
\hline \multicolumn{2}{|l|}{} & Pu-fcc & $\begin{array}{c}\mathbf{0 . 5} \\
\text { wt\% In }\end{array}$ & $\begin{array}{c}\text { 1.5 } \\
\text { wt\% In }\end{array}$ & $\begin{array}{c}\text { 3.0 } \\
\text { wt\% In }\end{array}$ \\
\hline 1st & r & 3.281 & 3.28 & 3.28 & 3.28 \\
& $\sigma$ & $\#=12$ & 0.04 & 0.05 & 0.05 \\
& sf & & 0.78 & 0.73 & 0.74 \\
\hline 2nd & r & 4.640 & & 4.68 & \\
& $\sigma$ & $\#=6$ & & 0.05 & \\
& sf & & & 0.37 & \\
\hline 3rd & r & 5.683 & 5.69 & 5.68 & 5.72 \\
& $\sigma$ & $\#=24$ & 0.09 & 0.06 & 0.05 \\
& sf & & 0.88 & 0.70 & 0.86 \\
\hline 4th & r & 6.562 & 6.54 & 6.57 & 6.60 \\
& $\sigma$ & $\#=12$ & 0.08 & 0.07 & 0.09 \\
& sf & & 0.88 & 0.70 & 0.86 \\
\hline 5th & r & 7.336 & & & \\
& $\sigma$ & $\#=24$ & & & \\
\hline
\end{tabular}

Fits of $\mathrm{Pu} \mathrm{L}_{3}$ edge over $\mathrm{k}$-range $=3.9$ to $13.63, \mathrm{k}^{3}$

The local structure around the plutonium in the alloys with indium is delta and fcc for all three samples spanning the range of $0.5-3.0 \mathrm{wt}-\%$ indium. XRD also finds the same consistency in structure. In addition, although some variation in the order for the first shell as indicated in the amplitude of the EXAFS is observed (Figure 1), these amplitudes are nevertheless uniformly high (Table 1 ), indicative of a relatively well ordered structure. The distances are close to those expected in the fcc lattice, with a slight expansion consistent with the somewhat larger size of indium relative to plutonium. The inability to locate the second shell is not uncommon in these structures because of the small number of atoms and correspondingly minor to the overall EXAFS. The same is true for the identification of the fifth shell, its contribution is damped by the long distance and complicated by the overlap with many multiple scattering contributions at that distance.

The local structure around the plutonium in the alloys with $0.5,1.0$, and $2.0 \mathrm{wt}-\%$ gallium is delta, but not fcc, for these three samples, i.e the nearest neighbor $\mathrm{Pu}-\mathrm{Pu}$ distance is near $3.3 \AA$, but most other structural attributes do not correspond with the fcc lattice. A high degree of disorder is reflected in the diminished amplitude of even the first shell. (Figure 2) In addition, substantial variability occurs for the other aspects of the structure. The $2.0 \mathrm{wt}-\%$ alloy shows significant features beyond the first shell, which do not correspond to the fcc lattice. Curve-fits (Table 2) indicate a substantial contribution from the second shell, but at a significantly longer distance than expected. The $1.0 \mathrm{wt}-\%$ alloy exhibits virtually no extended order; no significant features are observed beyond the first shell. Curve-fits show contributions at or near the correct distances, but the amplitude is greatly diminished The $0.5 \mathrm{wt}-\%$ alloy demonstrates the most substantial departure from a regular structure. Even the first shell is split into three different distances, with the additional two longer than the $3.3 \AA$ delta moiety. This more complicated packing arrangement may account for the high density of this alloy, although the structure of this particular sample may have been altered by the sample being cooled to $80 \mathrm{~K}$ prior to any measurements and may therefore not be representative of the actual, high density phase. However, no conversion to the alpha phase with its much shorter first shell distance is observed.

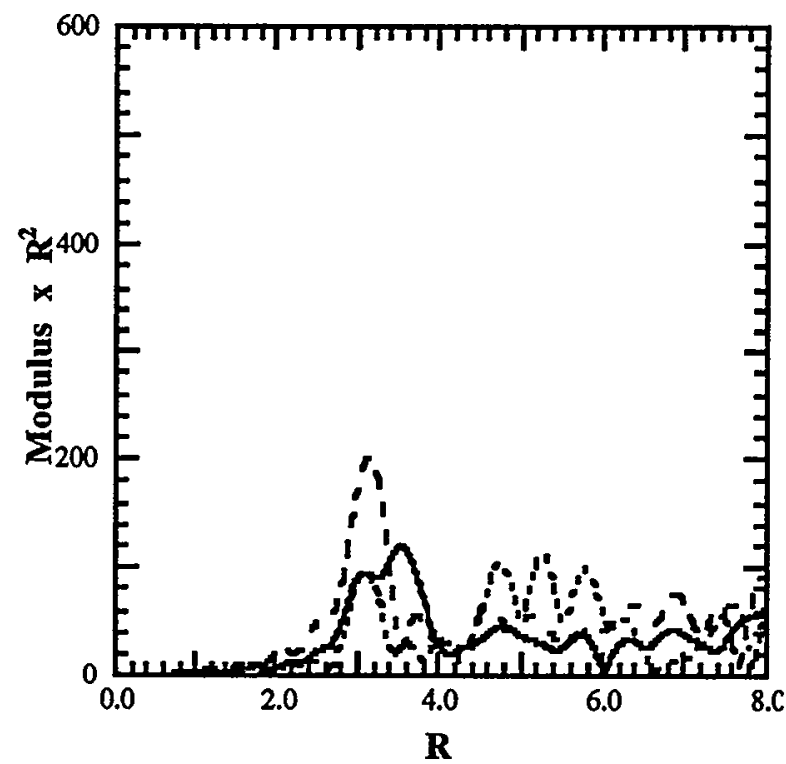

Figure 2 Fourier transform moduli of Pu L3 EXAFS of Pu-Ga alloy, with In concentrations of 0.5 (solid), 1.0 (dashed), and 2.0 (dotted) wt- $\% \mathrm{Ga}$ 
Table 2. Curve-fitting results for $\mathrm{Pu}$ EXAFS of $\mathrm{Pu}-\mathrm{Ga}$ alloys.

\begin{tabular}{|c|c|c|c|c|c|}
\hline \multicolumn{2}{|c|}{ shell } & Pu-fcc & $\begin{array}{c}\mathbf{0 . 5} \\
\text { wt\% Ga }\end{array}$ & $\begin{array}{c}1.0 \\
\text { wt\% Ga }\end{array}$ & $\begin{array}{c}\mathbf{2 . 0} \\
\text { wt\% Ga }\end{array}$ \\
\hline 1st & r & 3.281 & 3.27 & 3.29 & 3.28 \\
& $\sigma$ & $\#=12$ & 0.00 & 0.05 & 0.11 \\
& sf & & 0.70 & 0.68 & 1.25 \\
& n & & 3.67 & & \\
\hline 2nd & r & 4.640 & 3.47 & 4.64 & 4.76 \\
& $\sigma$ & $\#=6$ & 0.06 & 0.01 & 0.06 \\
& sf & & 0.70 & 0.18 & 0.73 \\
& n & & 4.44 & & \\
\hline 3rd & r & 5.683 & 3.87 & 5.63 & 5.64 \\
& $\sigma$ & $\#=24$ & 0.00 & 0.00 & 0.00 \\
& sf & & 0.70 & 0.09 & 0.10 \\
& n & & 1.90 & & \\
\hline 4th & r & 6.562 & & & \\
& $\sigma$ & $\#=12$ & & & \\
\hline 5th & r & 7.336 & & & \\
& $\sigma$ & $\#=24$ & & & \\
\hline
\end{tabular}

$0.5 \mathrm{wt} \%$, fits of $\mathrm{Pu} \mathrm{L}_{3}$ edge over $\mathrm{k}$-range $=2.8$ to 12.6 , $\mathrm{k}^{3}$

$1.0,2.0 \mathrm{wt} \%$, fits of $\mathrm{Pu}_{3}$ over $\mathrm{k}$-range $=3.9$ to 11.6 , $\mathbf{k}^{3}$

The behavior of the local structure around the alloying metal is the inverse of that found for the plutonium. The indium-plutonium bond lengths range from 3.23 to $3.28 \AA$, with the shortest distance and greatest deviation from the $3.28 \AA$ plutoniumplutonium bond length associated with the lowest indium concentration. The more distant shells are very close to the fcc distances for the two lower concentrations, but substantially different for the $\mathbf{3 . 0}$ wt-\% sample.

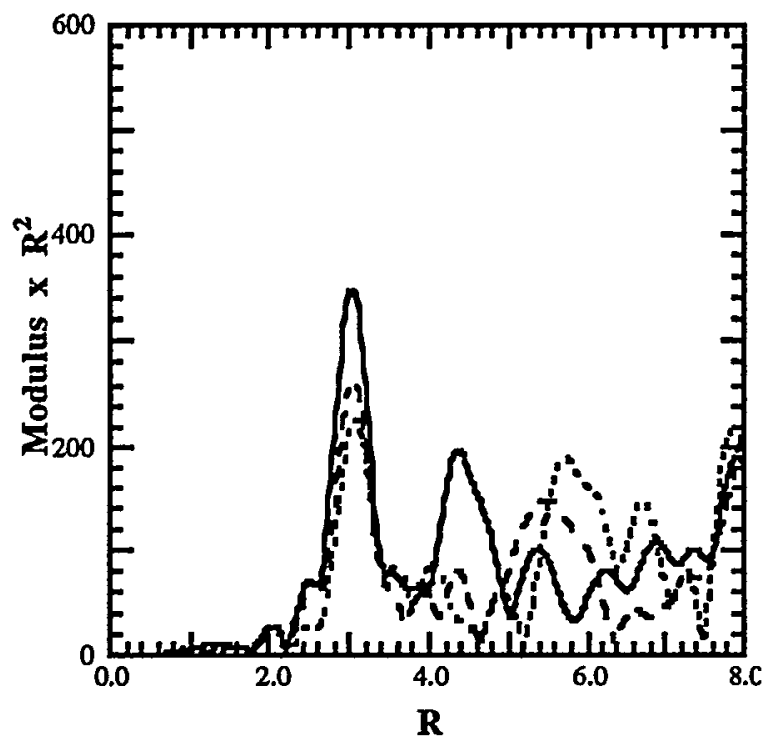

Figure 3. Fourier transform moduli of In K EXAFS of Pu-In alloy, with In concentrations of 0.5 (solid), 1.5 (dashed), and 3.0 (dotted) wt-\% In.
Table 3 Curve-fitting results for In EXAFS of Pu-In alloys.

\begin{tabular}{|c|c|c|c|c|c|}
\hline \multicolumn{2}{|l|}{ shell } & Pu-fcc & $\begin{array}{c}\mathbf{0 . 5} \\
\text { wt\% In }\end{array}$ & $\begin{array}{c}1.5 \\
\text { wt\% In }\end{array}$ & $\begin{array}{c}\text { 3.0 } \\
\text { wt\% In }\end{array}$ \\
\hline 1st & r & 3.281 & 3.23 & 3.25 & 3.28 \\
& $\sigma$ & $\#=12$ & 0.06 & 0.06 & 0.08 \\
& sf & & 1.09 & 0.95 & 1.27 \\
\hline 2nd & r & 4.640 & 4.60 & 4.63 & 4.46 \\
& $\sigma$ & $\#=6$ & 0.07 & 0.12 & 0.04 \\
& sf & & 1.59 & 1.50 & 0.30 \\
\hline 3rd & r & 5.683 & 5.69 & 5.67 & 5.78 \\
& $\sigma$ & $\#=24$ & 0.12 & 0.00 & 0.07 \\
& sf & & 1.30 & 0.18 & 0.29 \\
\hline 4th & r & 6.562 & & 6.44 & 6.44 \\
& $\sigma$ & $\#=12$ & & 0.09 & 0.10 \\
& sf & & & 1.32 & 3.27 \\
\hline 5th & r & 7.336 & & 7.41 & 7.16 \\
& $\sigma$ & $\#=24$ & & 0.07 & 0.00 \\
& sf & & & 0.36 & 0.13 \\
\hline
\end{tabular}

Fits of In Kedge over $\mathrm{k}$-range $=2.3$ to 12 for $0.5, \mathrm{k}^{3}$

Fits of In Kedge over k-range $=2.3$ to 12 for 1.5 and 3 , $\mathbf{k}^{3}$

The local structure around the gallium is, in contrast to that of the plutonium in these materials, quite independent of gallium concentration, exhibiting very well ordered shells of near neighbors with a 0.13 $\AA$ contraction of the gallium-plutonium bond relative to the $3.27 \AA$ plutonium-plutonium bond length in these materials, which relaxes to a distance near that characteristic of the plutonium lattice by the fourth shell. This decrease in the length of the galliumplutonium bond relative to the plutonium-plutonium pair is consistent with the smaller size of gallium, and may also explain some of the considerable disorder found for the structure around the plutonium atoms in these alloys. Because the average distance between the plutonium atoms of the first shell must equal the gallium-plutonium bond length there must be a large number of longer distances to balance out the required shorter ones, giving a wide distribution. However, the difference in bond length is so great that contributions from multiple shells would be expected in the EXAFS. These are not observed. It is also of interest that at the highest gallium concentration, the average spacing between gallium atoms should be equivalent to the fifth shell separation. The average plutoniumplutonium bond length from the EXAFS is significantly shorter than that derived from the unit cell determined by XRD. 


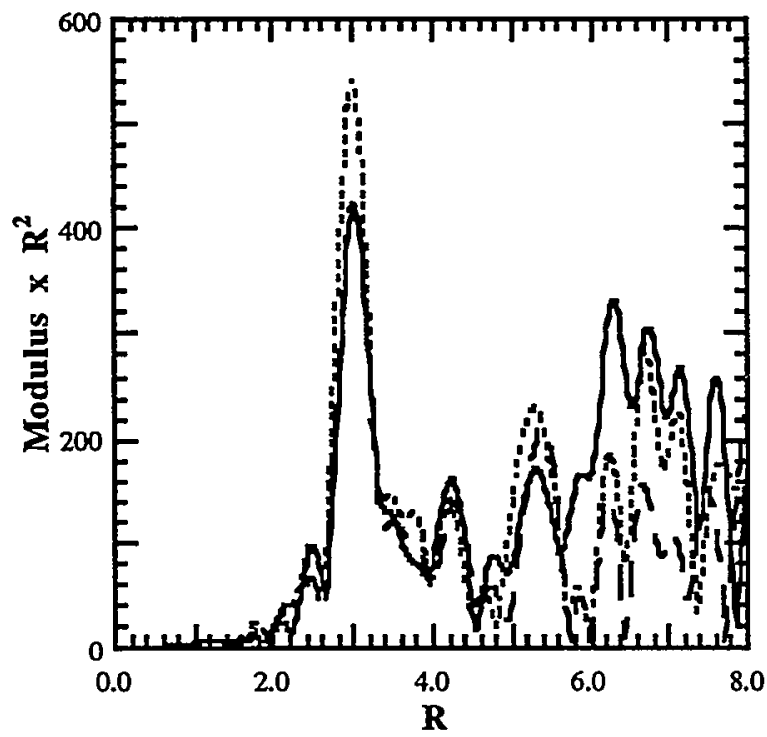

Figure 4 Fourier transform moduli of Ga K EXAFS of Pu-Ga alloy, with Ga concentrations of 0.5 (solid), 1.0 (dashed), and 2.0 (dotted) wt-\% In.

Table 4. Curve-fitting results for Ga EXAFS of $\mathrm{Pu}-$ Ga alloys.

\begin{tabular}{|c|c|c|c|c|c|}
\hline \multicolumn{2}{|c|}{ shell } & Pu-fce & $\begin{array}{c}\mathbf{0 . 5} \\
\text { wt\% Ga }\end{array}$ & $\begin{array}{c}\mathbf{1 . 0} \\
\text { wt\% Ga }\end{array}$ & $\begin{array}{c}\mathbf{2 . 0} \\
\text { wt\% Ga }\end{array}$ \\
\hline 1st & r & 3.281 & 3.15 & 3.15 & 3.14 \\
& $\sigma$ & $\#=12$ & 0.05 & 0.06 & 0.06 \\
& sf & & 0.67 & 0.86 & 0.99 \\
\hline 2nd & r & 4.640 & 4.56 & 4.58 & 4.55 \\
& $\sigma$ & $\#=6$ & 0.01 & 0.09 & 0.10 \\
& sf & & 0.35 & 0.89 & 1.20 \\
\hline 3rd & r & 5.683 & 5.61 & 5.64 & 5.60 \\
& $\sigma$ & $\#=24$ & 0.05 & 0.07 & 0.06 \\
& sf & & 0.14 & 0.30 & 0.30 \\
\hline 4th & r & 6.562 & & & \\
& $\sigma$ & $\#=12$ & & & \\
\hline 5th & r & 7.336 & & & \\
& $\sigma$ & $\#=24$ & & & \\
\hline
\end{tabular}

Fits of $\mathrm{Ga}$ Kedge over k-range $=2.3$ to $12.70, \mathrm{k}^{3}$

In summary, the mechanism of delta-phase stabilization is apparently complex. The opposite behavior resulting from the addition of gallium and indium, the counterintuitive concentration dependence, with the lowest alloying agent concentrations demonstrating the greatest deviation from the fcc lattice, and the fact that the ordering of the lattice is greatest around the element with the greatest difference in size from the plutonium host, suggest that there may be electronic as well as lattice mismatch factors involved and that there may be more than one mechanism. In addition, it is difficult to interpret the discrepancy between the average bond lengths expected for the fcc lattice and that actually found. What is apparent is that, in the gallium-based alloy, although the gallium is in a much different site than found for the pure metal, it nevetheless seems to determine the structure, whereas the plutonium is forced to comform to the arrangement of atoms preferred by the gallium. In the indium-based alloys, the plutonium demonstrates the structural consistency resulting from either less distortion of the lattice or a plutonium network which is relatively stronger than the distortion induced by the trace metal.

In addition, the average plutonium structure for the 0.5 wt-\% gallium alloy is extremely anomalous. It is the only material that exhibits a distinct set of distances in the first shell. None of these correspond to the alpha structure, they are equal or longer to the 3.3 $\AA$ delta plutonium-plutonium bond length. These results are consistent with the other anomalies characteristic of these low gallium wt-\% alloys and suggest a microstructural basis for the unusual macroscopic behaviors.

Local structure in aged materials. In contrast to the high degree of disorder exhibited by the freshly prepared materials of identical, $1.0 \mathrm{wt}-\%$ gallium concentration, the amplitudes of the features in the plutonium EXAFS of the aged samples are much larger, with distinct peaks corresponding to structural components. In addition, these transforms strongly resemble those found for the indium-containing materials (Figure 5), which demonstrate the features associated with the shells of an fcc structure. Specific and apparently reproducible changes from the atomic-level local structure of newly prepared gallium containing materials have thus apparently occurred in these two, independent, aged samples of $1 \mathrm{wt} \%$ gallium deltastabilized plutonium. Curve-fitting results (Table 5) corroborate this finding, with substantial amplitude found at the expected distances for most of the shells out to the fifth. The structure of these samples are thus not only "delta" but also "fcc," although the starting materials were (presumably) not. The structures are also quite similar to that observed in a $0.7 \mathrm{wt}-\%$ gallium/ 0.5 wt-\% americium sample which was approximately eight years in age (not shown).

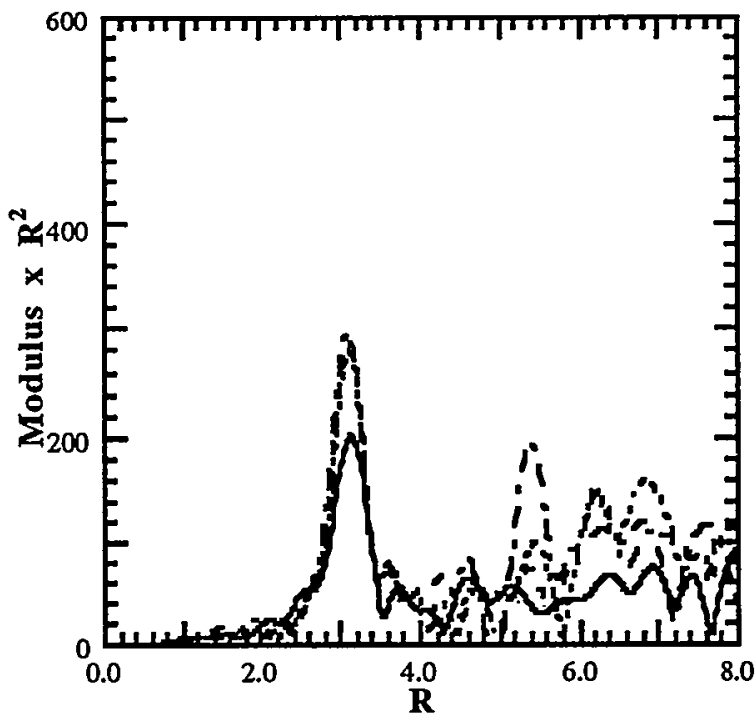

Figure 5 Fourier transform moduli of $\mathrm{Pu}_{3}$ EXAFS of Pu-Ga alloy, $1.0 \mathrm{wt}-\% \mathrm{Ga}$, freshly prepared (solid aged sample B (dashed), aged sample L (dotted), and $\mathrm{Pu}-$ In alloy, $1.5 \mathrm{wt}-\%$ In (dash-dot). 
Table 5. Curve-fitting results for Pu EXAFS of PuGa, 1.0wt-\%, aged samples B and L.

\begin{tabular}{|c|c|c|c|c|}
\hline \multicolumn{2}{|l|}{ shell } & Pu-fcc & $\begin{array}{c}\text { aged } \\
\text { B }\end{array}$ & $\begin{array}{c}\text { aged } \\
\text { L }\end{array}$ \\
\hline 1st & r & 3.281 & 3.27 & 3.27 \\
& $\sigma$ & $\#=12$ & 0.06 & 0.06 \\
& sf & & 0.81 & 0.97 \\
\hline 2nd & r & 4.640 & & \\
& $\sigma$ & $\#=6$ & & \\
& sf & & & \\
\hline 3rd & r & 5.683 & 5.61 & 5.69 \\
& $\sigma$ & $\#=24$ & 0.00 & 0.10 \\
& sf & & 0.05 & 0.57 \\
\hline 4th & r & 6.562 & 6.54 & 6.53 \\
& $\sigma$ & $\#=12$ & 0.00 & 0.05 \\
& sf & & 0.35 & 0.57 \\
\hline 5th & r & 7.336 & 7.35 & 7.30 \\
& $\sigma$ & $\#=24$ & 0.00 & 0.00 \\
& sf & & 0.20 & 0.18 \\
\hline
\end{tabular}

Fits of $\mathrm{Pu} \mathrm{L}_{3}$ edge over k-range $=3.9$ to $13.6, \mathrm{k}^{3}$

Although aging resulted in a significant modification of the local structure around the plutonium, it apparently had little or no effect on the local structure around the gallium (Figure and Table 6). The first three shells are easily located, at the same distances and with close to the same high amplitudes as in the freshly prepared sample. The mechanism by which the local structures around the two elements are decoupled in this way is not known.

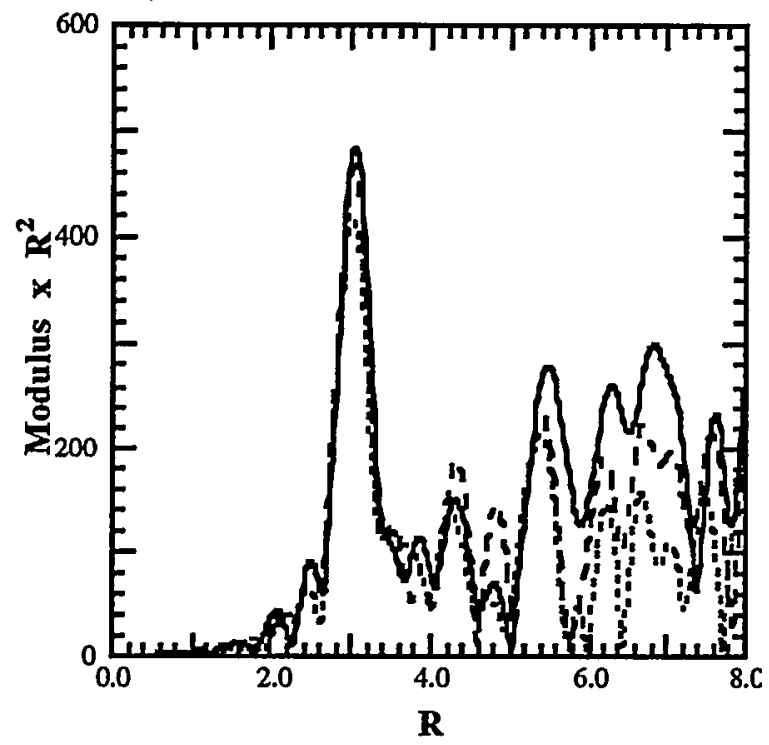

Figure 6 Fourier transform moduli of Ga K EXAFS of Pu-Ga alloy, $1.0 \mathrm{wt}-\% \mathrm{Ga}$, freshly prepared (solid), aged sample B (dashed), and aged sample L (dotted)
Table 6. Curve-fitting results for Ga EXAFS of Pu$\mathrm{Ga}, 1.0 \mathrm{wt}-\%$, aged samples $\mathrm{B}$ and $\mathrm{L}$.

\begin{tabular}{|c|c|c|c|c|}
\hline \multicolumn{2}{|l|}{ shell } & Pu-fcc & $\begin{array}{c}\text { aged } \\
\text { B }\end{array}$ & $\begin{array}{c}\text { aged } \\
\text { L }\end{array}$ \\
\hline 1st & r & 3.281 & 3.15 & 3.15 \\
& $\sigma$ & $\#=12$ & 0.07 & 0.07 \\
& sf & & 1.00 & 1.00 \\
\hline 2nd & $\mathrm{r}$ & 4.640 & 4.59 & 4.56 \\
& $\sigma$ & $\#=6$ & 0.07 & 0.02 \\
& sf & & 0.74 & 0.29 \\
\hline 3rd & r & 5.683 & 5.66 & 5.62 \\
& $\sigma$ & $\#=24$ & 0.09 & 0.00 \\
& sf & & 0.53 & 0.12 \\
\hline 4th & r & 6.562 & & \\
& $\sigma$ & $\#=12$ & & \\
\hline 5th & r & 7.336 & & \\
& $\sigma$ & $\#=24$ & & \\
\hline
\end{tabular}

Fits of Ga Kedge over k-range $=2.3$ to $12.70, \mathrm{k}^{3}$

Summay and significance. Although previous XRD measurements on plutonium alloys would predict that the structures and the phases of all of the samples reported on here would be identical, these XAFS results indicate the opposite. The only characteristic shared by all of these samples is a plutonium-plutonium bond length of $3.8 \AA$. Substantial variability occurs in the extended structure as function of the trace element added, its concentration, the element probed, and the history of the sample. Nevertheless, analogous to XRD, similarities in the spectra suggest the division of these materials into the categories described above, the fcc structure or phase and its delta subset. The relationship between these XAFS structures or phases and those determined by XRD is under investigation.

Transformations or transitions between these structural classes are evidently induced by aging. The relative effects on this process of the buildup of helium bubbles, the increase in the concentration of decay products, and the deposition of energy in the lattice are not known. Although the transition from delta to fcc has apparently not resulted in the changes in the gross dimensions of the bulk material that are typical of phase transitions and observed for the XRD-identified phase transitions in plutonium, such modifications of the atomic structure can result in differences in, e.g., the elasticity of the material and other macroscopic phenomena such as its susceptibility to crack initiation and propagation. Insofar as the atomic structure of plutonium changes with age, other critical, macroscopic properties of plutonium may also be agedependent. Further studies are under way to resolve these issues. 


\title{
EXAFS Investigation of PtCu Carbon-Supported Catalysts
}

\author{
Farrel W. Lytle, The EXAFS Company, Pioche, NV 89043 \\ Robert M. Friedman, Monsanto Chemical Co., St. Louis, MO 63167 \\ Simon R. Bare, Dow Chemical Co., Midland, MI 48674
}

In earlier investigations of silica supported PtCu catalysts prepared by solution impregnation of the support, calcination and $\mathrm{H}_{2}$ reduction Via et al.(1) found mixed coordination around both the $\mathrm{Pt}$ and $\mathrm{Cu}$ atoms in a $1 \mathrm{wt} \% \mathrm{Pt}$ plus $\mathrm{Cu}$ catalyst with a $1: 1$ atomic ratio. In the 1 st coordination shell of $\mathrm{Pt}$ the ratio of $\mathrm{Pt}: \mathrm{Cu}$ was $80 \%: 20 \%$ with bond distances of $\mathrm{Pt}-\mathrm{Pt}=2.71 \AA$ and $\mathrm{Pt}-\mathrm{Cu}=2.66 \AA$. This implies a strong tendency for like atoms to cluster within the catalyst particles. We have investigated a series of carbon supported $\mathrm{Pt}$ catalysts prepared by solution impregnation of the support followed by $\mathrm{H}_{2}$ reduction but at different loadings and an atomic ratio, $\mathrm{Pt}: \mathrm{Cu}$ of 1:5.5. The compositions of the catalysts are given in Table 1.

The catalysts were examined on BL 4-1 with Si(111) crystals and $1 \mathrm{~mm}$ monochromator entrance slits. Catalysts were treated in situ in our catalyst cell. The presence of $\mathrm{Cu}$ in the catalyst created a particular difficulty when measuring the $\mathrm{Pt} L$ edges in fluorescence since the $\mathrm{Cu}$ fluorescence was overwhelming. In addition to the $\mathrm{Zn}$ filter normally used to reduce scattered radiation at the $\mathrm{Pt}_{2} \mathrm{~L}_{3}$ edge we added a $\mathrm{Co}$ filter to reduce the $\mathrm{Cu}$ fluorescence. Although this also reduced the Pt signal the 16-fold background reduction resulted in a 5-fold signal to noise ratio increase as measured by the relative edge jumps. The catalysts were reduced in flowing $\mathrm{H}_{2}$ at $200 \mathrm{C}$ and cooled in flowing He to prepare the catalyst surfaces before the EXAFS measurements. The data (not shown) were of excellent quality with EXAFS to $\mathrm{K}=16$. They were fitted using single-scattering, curved wave parameters calculated from FEFF 3 (2) with excellent fits as shown in Fig. 2. In Fig. 1 the $\mathrm{X}(\mathrm{K}) \mathrm{K}^{3}$ vs $\mathrm{K}$ plot of one of the catalysts is compared with that of a $5 \% \mathrm{Pt}$ on carbon catalyst. Although the data for the lower concentration catalyst were noisier the results were essentially identical as shown by the fitted parameters in Table 1. Note the high amplitude of the function in the 6-8 $\AA^{-1}$ region which is characteristic of $\mathrm{Cu}$ backscattering.

The interesting result for the carbon supported bimetallic catalyst which differs from the 1:1 silica supported catalyst reported by Via et al. (1) is the large number of $\mathrm{Cu}$ atoms in the 1st coordination shell of $\mathrm{Pt}$ (Table 1). Here the relative population of the 1st coordination shell of $\mathrm{Pt}$ is $\mathrm{Pt}: \mathrm{Cu} 19 \%: 81 \%$, just the opposite of Via's catalysts. We believe this simply reflects the effect of the higher proportion of $\mathrm{Cu}$ in the impregnating solutions. Evidently this is sufficient to overcome the like atom clustering effect, i. e. all the metal atoms become more equivalent. Note that there was little difference between the two carbon supported bimetallic catalysts as a result of metal loading. Our bond distances within the PtCu clusters are appreciably shorter than those reported in Ref. 1 and probably reflect the difference in average composition of the catalyst clusters, i. e. Via's were primarily $\mathrm{Pt}$, ours are primarily $\mathrm{Cu}$.

\section{References}

1. G. H. Via, K. F. Drake, G. Meitzner, F. W. Lytle and J. H. Sinfelt, Catalysis Letters 5, 25 (1990).

2. J. J. Rehr, J. Mustre de Leon, S. I. Zabinsky and R. C. Albers, J. Am. Chem. Soc. 113, 5135 (1991).

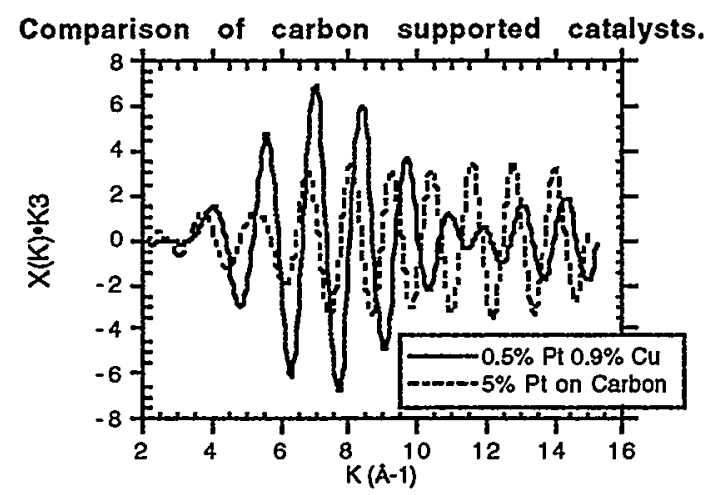

Fig. 1 Comparison of the $\mathrm{Pt}_{3} \mathrm{X}(\mathrm{K}) \mathrm{K}^{3}$ 1st shell EXAFS functions of a $\mathrm{Pt}$ on carbon catayst and a $\mathrm{PtCu}$ on carbon catalyst. In the $\mathrm{PtCu}$ catalyst note the large amplitude in the 6-8 $\AA^{-1}$ region which is characteristic of $\mathrm{Cu}$ neighbors.. 
Table 1. Description of carbon supported catalysts and $P t L_{3} X(K) K^{3}$ fitted structural parameters.

Sample Disp. $\begin{array}{lllllllll}\mathrm{N}_{\mathrm{Pl}} & \mathrm{R}_{\mathrm{Pl}} & \Delta \sigma^{2} \mathrm{Pl}_{\mathrm{l}} & \mathrm{N}_{\mathrm{Cu}} & \mathrm{R}_{\mathrm{Cu}} & \Delta \sigma^{2} \mathrm{Cu}_{\mathrm{l}} & \mathrm{E}_{\mathrm{o}} & \text { S. D. }\end{array}$

$\begin{array}{lllllll}5 \% \mathrm{Pt} & 0.5 & 5.0 & 2.75 & 0.0067 & 6.8 & 0.25\end{array}$

$\begin{array}{llllllllll}0.5 \mathrm{Pt} 0.9 \mathrm{Cu} & 0.2 & 1.4 & 2.69 & 0.0046 & 6.1 & 2.57 & 0.0110 & 6.0 & 0.25\end{array}$

$\begin{array}{llllllllll}0.1 \mathrm{Pt} 0.18 \mathrm{Cu} & 0.6 & 1.1 & 2.69 & 0.0045 & 7.1 & 2.62 & 0.0099 & 8.1 & 0.86\end{array}$

The Dispersions were measured by $C O$ chemisorption. $\Delta \sigma^{2}$ is relative to the calculated FEFF 3 parameters where $\sigma^{2}$ was set to 0.0. The Standard Deviation is that calculated from the variance between the data and the fitted curve.

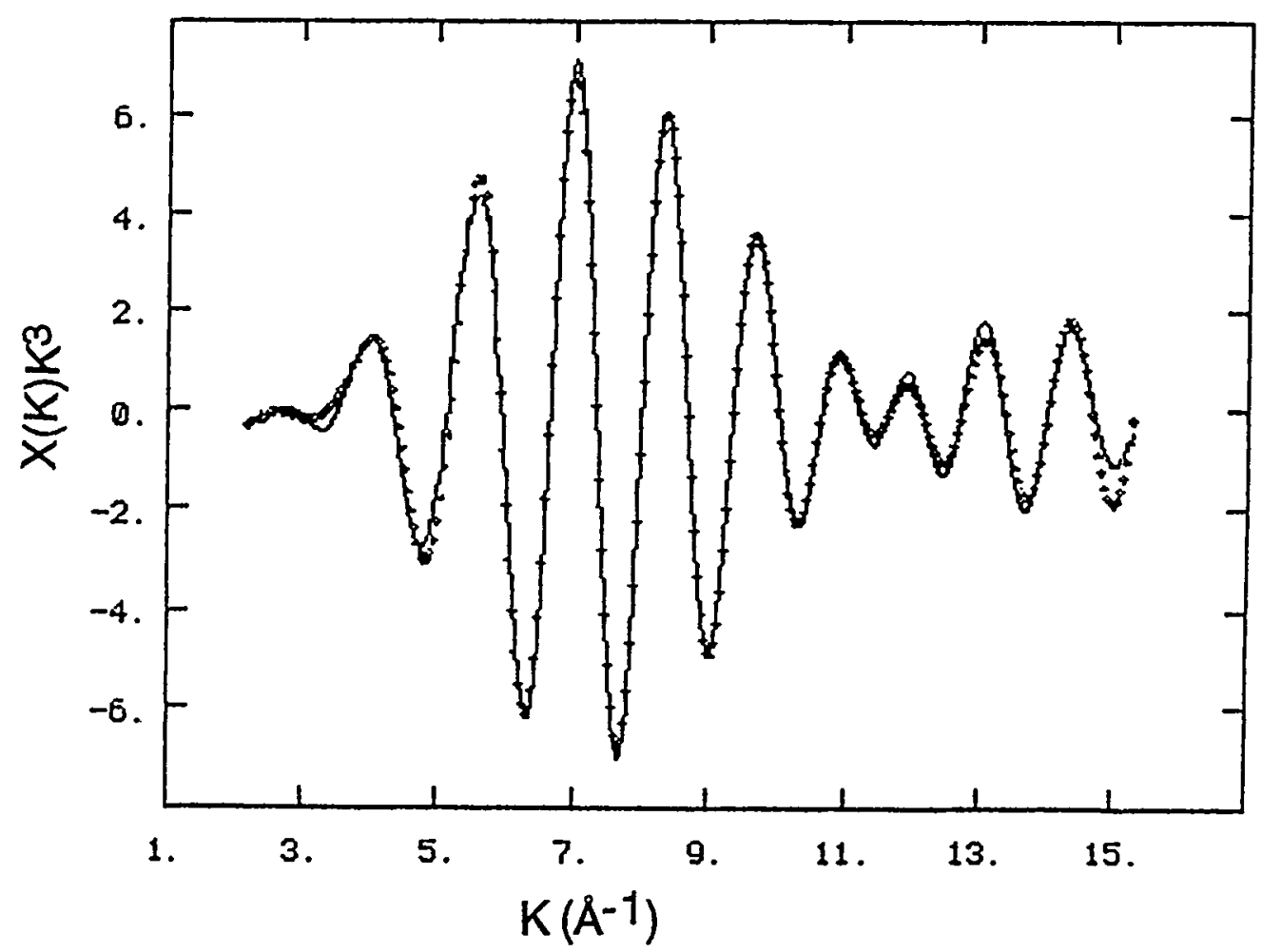

Fig. 2 Comparison of $\mathrm{Pt} \mathrm{L}_{3}$ EXAFS data (solid line) to the $X(\mathrm{~K}) \mathrm{K}^{3}$ fit for $0.5 \% \mathrm{Pt}$ $0.9 \% \mathrm{Cu}$ on carbon catalyst. The fitting parameters were calculated using FEFF 3 (2) and the results are listed in Table 1. 
Proposal 2286Mp

\title{
ON THE STRUCTURE OF THE SOLVATED MERCURY(I) ION
}

\author{
Ingmar Persson \\ Department of Chemistry, Swedish University of Agricultural Sciences, P.O.Box 7015, S- \\ 75007 Uppsala, Sweden.
}

\section{INTRODUCTION}

The stability of the mercury $(\mathrm{I})$ ion, $\mathrm{Hg}_{2}{ }^{2+}$, is very much depending on the coordinating properties of the solvent. ${ }^{1,2}$ The mercury(I) ion disproportionates into metllic mercury and mercury(II), $\mathrm{Hg}_{2}{ }^{2+} \rightleftharpoons \mathrm{Hg}^{0}+\mathrm{Hg}^{2+}$, in solvents with strong electron-pair donor properties as liquid ammonia and $\mathrm{N}, \mathrm{N}$-dimethylthioformamide, $\left(\mathrm{CH}_{3}\right)_{2} \mathrm{NCHS}$, while it is fairly stable in weak donor solvents as water, methanol, dimethyl sulfoxide and acetonitrile and it is partly disproportionated in pyridine. ${ }^{2}$ The disproportionation constant of the mercury(I) ion, $K_{\mathrm{D}}=\left[\mathrm{Hg}^{2+}\right]$ $\left[\mathrm{Hg}_{2}{ }^{2+}\right]^{-1}$, and the standard electrode potentials of the $\mathrm{Hg}^{0} / \mathrm{Hg}_{2}{ }^{2+}, \mathrm{Hg}^{0} / \mathrm{Hg}^{2+}$ and $\mathrm{Hg}_{2}{ }^{2+} / \mathrm{Hg}^{2+}$ couples versus NHE in water have been determined in a number of solvents with varying electron-pair donor properties, ${ }^{2}$ see Table 1 . The structure of the solvated mercury(I) ion water, methanol, dimethyl sulfoxide, acetonitrile and pyridine has been determined by means of XAFS methods. The obtained $\mathrm{Hg}-\mathrm{Hg}$ bond distance in the $\mathrm{Hg}_{2}{ }^{2+}$ ion has been correlated with thermodynamic properties of the mercury(I) ion in these solvents.

\section{EXPERTMENTAL}

XAFS Measurements. Mercury $\mathrm{L}_{\text {III }}$ edge $\mathrm{X}$-ray absorption data were collected in transmission mode at ambient temperature at the Stanford Synchrotron Radiation Laboratory (SSRL), Stanford University, U.S.A., under dedicated conditions: $3.0 \mathrm{GeV}$, maximum current 100 $\mathrm{mA}$ using wiggler beamline 4-1. A Si(111) double monochromator was detuned to $50 \%$ of the maximum intensity in order to reduce higher order harmonics. EXAFS spectra of an aqueous solution of mercury(I) perchlorate was recorded in order to check that the $\mathrm{Hg}-\mathrm{Hg}$ and $\mathrm{Hg}-\mathrm{O}$ bond distances found in a large angle $\mathrm{X}$-ray scattering study ${ }^{3}$ was in agreement with distances obtained from theoretical calculations using ab initio calculated parameters from FEFF. ${ }^{4}$ The solutions were kept in cells with thin glass windows $(<35$ $\mu \mathrm{m})$ and Viton spacers $(1 \mathrm{~mm})$. All studied solutions contain only one complex except the pyridine solution where also $\mathrm{Hg}^{2+}$ ions are present due a disproportionation reaction, see above. The mercury concentration of the solutions, $\mathrm{ca}$. 0.5 $M$, gives an absorption change of about one logarithmic unit over the $\mathrm{L}_{\mathrm{II}}$ edge for a $1 \mathrm{~mm}$ path. The solids were diluted with $\mathrm{BN}$ to give a similar absorption.

Data Treatment. The treatment of EXAFS data and refinement of model parameters have been performed by means of the EXAFSPAK program package. ${ }^{5}$ Calculations of theoretical parameters for fitting the experimental data have been made by means of the program package FEFF $6{ }^{4}$

\section{RESULTS}

The $\mathrm{Hg}-\mathrm{Hg}$ bond distance increases as expected with increasing solvating ability of the solvent. The $\mathrm{Hg}-\mathrm{Hg}$ bond distance is furthermore proportional to the logarithmated disproportionation constant of the mercury $(I)$ ion, see Table 1 and Fig. 1.

The mean square deviation of the $\mathrm{Hg}-\mathrm{Hg}$ bond distance is very small, 0.0017-0.0034, and it increases with increasing solvation except for the value obtained for water. The $\mathrm{Hg}-\mathrm{Hg}$ interaction dominates very strongly the total EXAFS and the contribution from the Hg-solvent interactions is only observed below $k=6 \AA^{-1}$. The determination of the Hg-solvate distances and the number of solvent molecules coordinated to each mercury atom is therefore unususally unaccurate, see Table 2. The XANES region of the solvated mercury(I) ions indicates that the hydrate, and methanol and dimethyl sulfoxide solvates have the same configuration around mercury, while the acetonitrile and pyridine solvates seem to have a different configuration, see Fig. 2 and Table 2. 
Table 1. $\mathrm{Hg}-\mathrm{Hg}$ bond distance, $\mathrm{d} / \AA$, standard electrode potential of the couples $\mathrm{Hg}_{2}{ }^{2+} / \mathrm{Hg}(\mathrm{l})$ and $\mathrm{Hg}_{2} \mathrm{X}_{2} / \mathrm{Hg}(\mathrm{l}), E^{\mathrm{O}} / \mathrm{V}$, disproportionation constant of mercury $(\mathrm{I}), K_{\mathrm{D}}=\left[\mathrm{Hg}^{2+}\right]\left[\mathrm{Hg}_{2}{ }^{2+}\right]^{-1}, \mathrm{Hg}_{2}{ }^{2+} \rightleftharpoons \mathrm{Hg}^{2+}+$ $\mathrm{Hg}(\mathrm{l}), v(\mathrm{Hg}-\mathrm{Hg})$ stretching frequency/cm $\mathrm{cm}^{-1}$, of solvated mercury(I) ions aqueous, methanol, dimethyl sulfoxide, acetonitrile and pyridine solution and of mercury(I) halides in aqueous solution.

\begin{tabular}{lllll}
\hline & $d$ & $E^{\mathrm{o}}$ & $K_{\mathrm{D}}$ & $v(\mathrm{Hg}-\mathrm{Hg})$ \\
\hline $\mathrm{Hg}_{2}{ }^{2+}$ /acetonitrile & 2.515 & $+1.019^{\mathrm{d}}$ & $-2.67^{\mathrm{d}}$ & 161 \\
$\mathrm{Hg}_{2}{ }^{2+} /$ methanol & 2.523 & $+1.091^{\mathrm{d}}$ & $-2.38^{\mathrm{d}}$ & 160 \\
$\mathrm{Hg}_{2}{ }^{2+} /$ water & $2.528^{\mathrm{a}}$ & $+0.7973^{\mathrm{b}}$ & $-1.99^{\mathrm{c}}$ & 171.5 \\
$\mathrm{Hg}_{2}{ }^{2+} /$ dimethyl sulfoxide & 2.537 & $+0.452^{\mathrm{d}}$ & $-1.40^{\mathrm{d}}$ & 160.5 \\
$\mathrm{Hg}_{2}{ }^{2+}$ /pyridine & 2.558 & $+0.418^{\mathrm{d}}$ & $+0.76^{\mathrm{d}}$ &
\end{tabular}

\begin{tabular}{llll}
$\mathrm{Hg}_{2} \mathrm{~F}_{2}$ & $2.51^{\mathrm{e}}$ & & 185.9 \\
$\mathrm{Hg}_{2} \mathrm{Cl}_{2}$ & $2.53^{\mathrm{e}}$ & $+0.26808^{\mathrm{b}}$ & 166.5 \\
$\mathrm{Hg}_{2} \mathrm{Br}_{2}$ & $2.58^{\mathrm{e}}$ & $+0.13923^{\mathrm{b}}$ & 132.2 \\
$\mathrm{Hg}_{3} \mathrm{I}_{7}$ & $2.69^{\mathrm{e}}$ & $-0.0405^{\mathrm{b}}$ & 112.5 \\
\hline
\end{tabular}

${ }^{\mathrm{a}}$ The $\mathrm{Hg}-\mathrm{Hg}$ bond distance in aqueous $0.56-2.80 \mathrm{M} \mathrm{Hg}_{2}\left(\mathrm{ClO}_{4}\right)_{2}$ solutions was determined to $2.524(5) \AA$ by means of large angle X-ray scattering technique, ref. 3. ${ }^{b}$ Ref. $6 .{ }^{c}$ Ref. 7. ${ }^{d}$ Ref. $2 .{ }^{e}$ Ref. 8.

Table 2. Bond distance, $d / \AA$, mean square deviation, $\sigma^{2} / \AA^{2}$, and the number solvent molecules coordinated per mercury atom, $n$. Estimated error of refined parameters are given within parenthesis.

\begin{tabular}{lccccc}
\hline Complex & $r(\mathrm{Hg}-\mathrm{Hg})$ & $\sigma^{2}(\mathrm{Tl}-\mathrm{X})$ & $d(\mathrm{Hg}-\mathrm{O} / \mathrm{N})$ & $\sigma^{2}(\mathrm{Hg}-\mathrm{O} / \mathrm{N})$ & $n(\mathrm{Hg}-\mathrm{O} / \mathrm{N})$ \\
\hline Acetonitrile & $2.515(1)$ & $0.0017(1)$ & $2.14(1)$ & $0.0017(2)$ & 1 \\
Methanol & $2.523(1)$ & $0.0025(1)$ & $2.25(3)$ & $0.033(1)$ & 3 \\
Water & $2.528(2)$ & $0.0034(1)$ & $2.21(2)$ & $0.022(1)$ & 3 \\
Dimethyl sulfoxide & $2.537(1)$ & $0.0021(1)$ & $2.24(1)$ & $0.027(2)$ & 3 \\
Pyridine & $2.558(1)$ & $0.0024(2)$ & $2.24(2)$ & $0.0051(7)$ & 2 \\
\hline
\end{tabular}
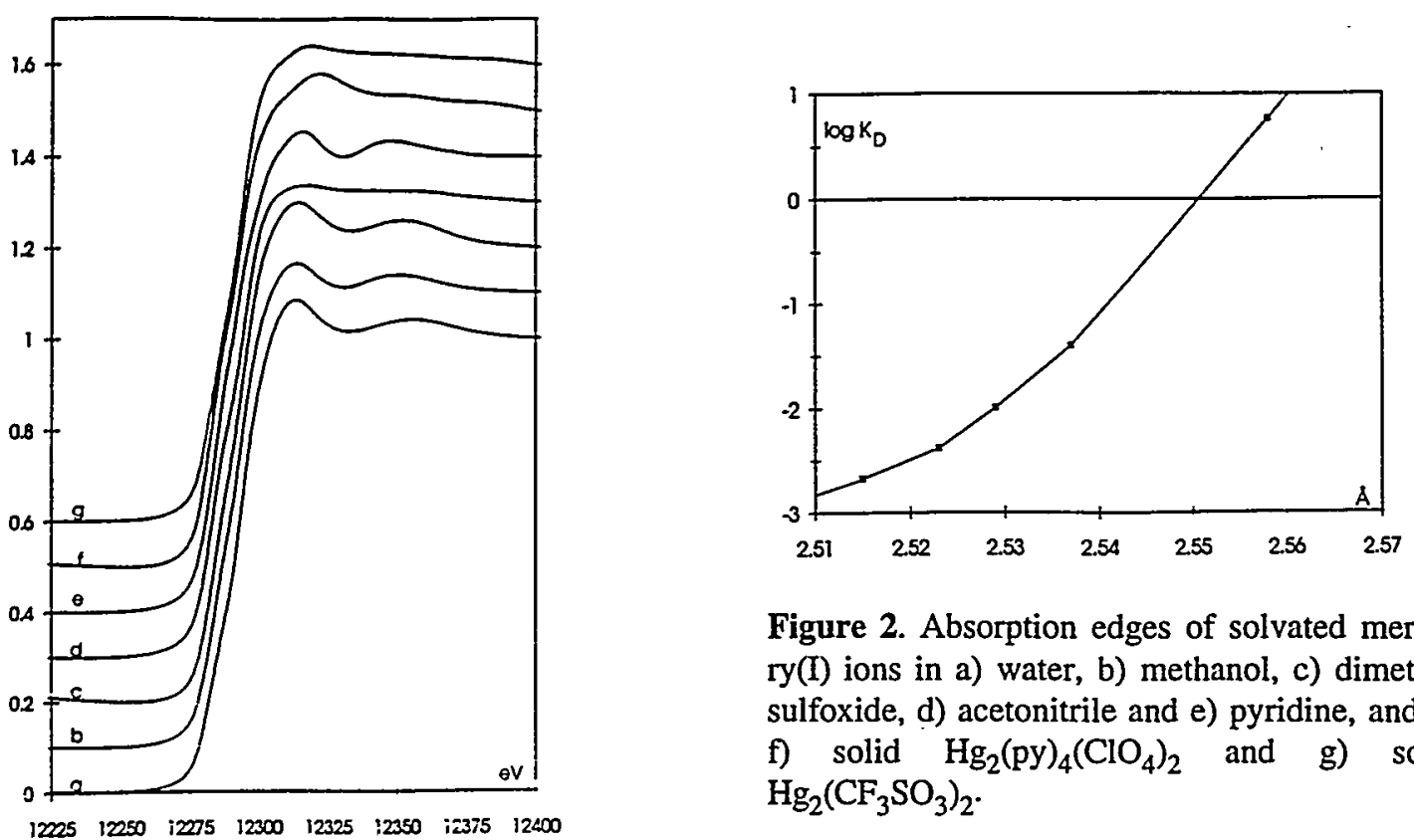

Figure 2. Absorption edges of solvated mercury(I) ions in a) water, b) methanol, c) dimethyl sulfoxide, d) acetonitrile and e) pyridine, and of f) solid $\mathrm{Hg}_{2}(\mathrm{py})_{4}\left(\mathrm{ClO}_{4}\right)_{2}$ and g) solid $\mathrm{Hg}_{2}\left(\mathrm{CF}_{3} \mathrm{SO}_{3}\right)_{2}$.

Figure 1. Correlation of of $\mathrm{Hg}-\mathrm{Hg}$ bond distance in solvated mercury(I) ions and the logarithmated disproportionation constant of mercury(I), $\log K_{\mathrm{D}}$ 


\section{ACKNOWLEDGMENTS}

The continuing support of the Swedish Natural Science Research Council is gratefully acknowledged. The EXAFS measurements were performed at the Stanford Synchrotron Radiation Laboaratory (SSRL), supported by the Department of Basic Energy Science, Division of Chemical/Material Science, and the National Institutes of Health, Biomedical Resource Technology Program. I am Drs Graham N. George and Ingrid J. Pickering for allowing me to use the EXAFSPAK software.

\section{REFERENCES}

1. P. Schwerdtfeger, P.D.W. Boyd, S. Brienne, J.S. McFeaters, M. Dolg, M.-S. Liao and W.H.E. Schwarz, Inorg. Chim. Acta 213, 233 (1993)

2. M. Chaudhry, K.C. Dash, E. KamienskaPiotrowicz, Y. Kinjo and I. Persson, J. Chem. Soc., Faraday Trans. 90, 2235 (1994) and references therein

3. J.J. Rehr, R.C. Albers and S.I. Zabinsky, Phys. Rev. Lett. 69, 3397 (1992).
J.J.Rehr, J. Mustre de Leon, S.I. Zabinsky and R.C. Albers, J. Am. Chem. Soc. 113, 5135 (1991)

J. Mustre de Leon, J.J. Rehr, S.I. Zabinsky and R.C. Albers, Phys. Rev. B 44, 4146 (1991)

4. G.N. George and I.J. Pickering, "EXAFSPAK - A Suit of Computer Programs for Analysis of X-ray Absorption Spectra", SSRL, 1993.

5. G. Johansson, Acta Chem. Scand. 20 (1966) 553.

6. Handbook of Chemistry and Physics, CRC Press, Boca Raton, Fl. 1992, pp. 8-17 - 8-27

7. L.G. Sillén and A.E. Martell, Stability Constants of Metal Ion Complexes, The Chemical Society, London, 1964, 1971.

E. Högfeldt, Stability Constants of Metal Ion Complexes, IUPAC Data Series, No. 21, Pergamon Press, Oxford 1982.

8. F.A. Cotton and G. Wilkinson, Advanced Inorganic Chemistry, 3rd Ed., John Wiley \& Sons, New York 1980, p. 594. 


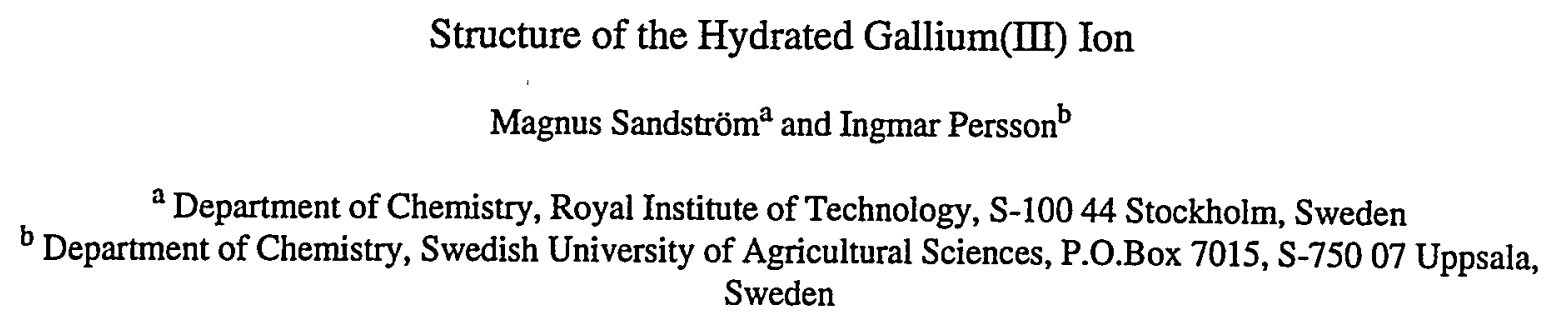

The structure of the hydrated gallium(III) has been determined in aqueous solution. The solid alum salt $\mathrm{CsGa}\left(\mathrm{SO}_{4}\right)_{2} \cdot 12 \mathrm{H}_{2} \mathrm{O}$, with a reported $\mathrm{Ga}$ $\mathrm{O}$ bond distance of $1.944 \AA$, not corrected for thermal motion [1], has been used as model compound. The surrounding of the gallium(III) ion aqueous solution and in the alun compound is identical, see Figs. 1 and 2.

$\mathrm{Ab}$ initio calculated EXAFS parameters from FEFF, v. 6.01 [2], have been used in the refinements. The refinement calculations gave for the solid alum salt $\mathrm{Ga}-\mathrm{O} d=1.956(1) \AA, \sigma^{2}=0.0038(1)$ $\AA, n=6(\mathrm{fix})$ and $E_{0}=-12.5(4) \mathrm{eV}$. The $\mathrm{Ga}-\mathrm{O}$ bond distance is as expected somewhat longer than the $\mathrm{Ga}-\mathrm{O}$ bond distance in the crystallographic study. A correction for thermal motion to make the distance between the mean positions of the $\mathrm{Ga}$ and $\mathrm{O}$ atoms in the crystal structure comparable with the measured EXAFS distance can explain the observed difference. Refinements of the aqueous solution gave for for $\mathrm{Ga}-\mathrm{O} d=1.957(1) \AA, \sigma^{2}=0.0041(1)$ $\AA, n=6$ (fix) and $E_{0}=-12.5$ (fix) $\mathrm{eV}$. No significant contributions were observed from a second sphere of hydration with $\mathrm{Ga} \cdots \mathrm{O}$ distances expected at about $3.9 \AA$.

This work was supported by the Swedish Natural Science Research Council.

1. J.K. Beattie, S.P. Best, B.W.Skelton and A.H. White, J. Chem.Soc., Dalton Trans. 2105 (1981).

2. S.I. Zabinsky, J.J. Rehr, A. Ankudinov and R.C. Albers, FEFF 6 - Ab initio Multiple-Scattering X-ray Absorption Fine Structure and Xray Absorption Near Edge Structure Code, University of Washington.

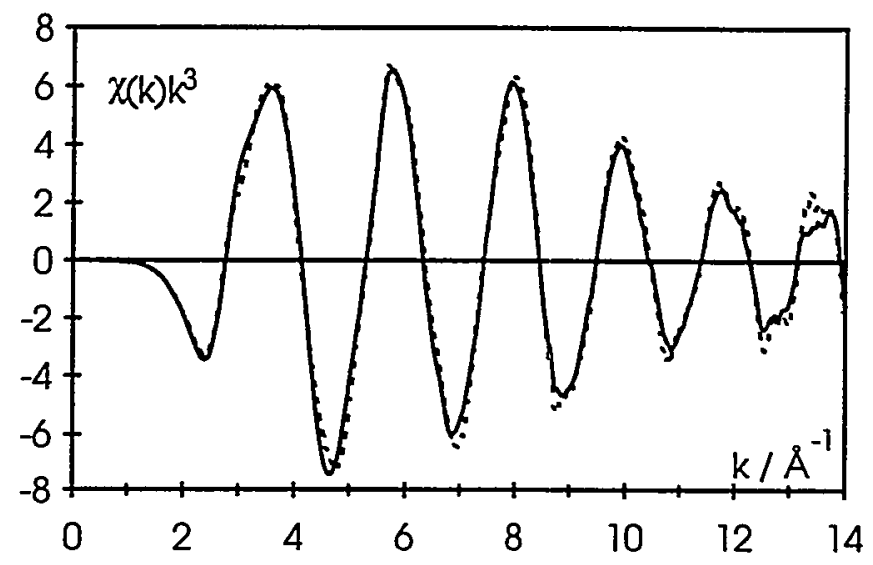

Figure 1. Comparison of EXAFS data for the hydrated gallium(III) ion in aqueous solution (solid line) and solid $\mathrm{CsGa}\left(\mathrm{SO}_{4}\right)_{2} \cdot 12 \mathrm{H}_{2} \mathrm{O}$ (dashed line).

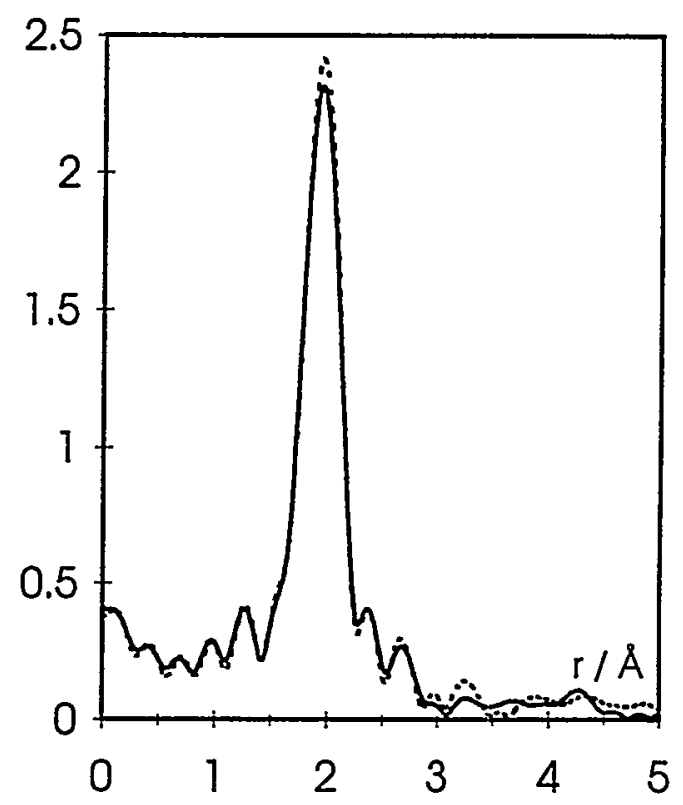

Figure 2. Fourier transforms of the hydrated gallium(III) ion in aqueous solution (solid line) and solid $\mathrm{CsGa}\left(\mathrm{SO}_{4}\right)_{2} \cdot 12 \mathrm{H}_{2} \mathrm{O}$ (dashed line); phase correction has been performed 
Proposal 2298M

\title{
Size-dependent Catalytic Activity of Supported Metal Clusters
}

\author{
Z. Xu, F.-S. Xiao, S. K. Purnell, O. Alexeev, S. Kawi, S. E. Deutsch and B. C. Gates \\ Department of Chemical Engineering and Materials Science, University of California, \\ Davis, CA 95616
}

Because catalysis by metals is a surface phenomenon, technological catalysts contain minute metal particles with a large fraction of the atoms exposed. The particles are dispersed on porous high-area metal oxide supports. Because the particles are nonuniform, it has not been possible to determine exact relationships between catalytic activity and particle size for most reactions. However, hydrocarbon hydrogenations and other reactions are structureinsensitive, proceeding at approximately the same rates on variously sized particles of a metal, provided they are metallic (larger than about $1 \mathrm{~nm}$ ). It is unknown how small metal particles may become before their catalytic properties change significantly. Here we show that the catalytic activities of $\mathrm{Ir}_{4}$ and $\mathrm{Ir}_{6}$ clusters on supports are markedly less than those of metallic iridium particles. Thus the concept of structure insensitivity is restricted to reactions on metallic surfaces and does not extend to small clusters. The clusters are quasi-molecular and catalytically unique.

The fascination with molecular metal clusters motivated many investigations of their catalytic properties, but few examples of cluster catalysis and no industrial applications have emerged. A decisive limitation is the lack of stability of most ligand-stabilized clusters. However, metal clusters dispersed on solid supports have been thought to exist among the complex mixtures of structures in industrial supported metal catalysts, and recently clusters of, on average, 5-6 $\mathrm{Pt}$ atoms in the pores of zeolite LTL have been identified by EXAFS spectroscopy and applied industrially as catalysts for selective reforming of naphtha to give aromatics. Thus the metal clusters of most importance as catalysts are robust supported clusters and not molecular clusters.
We report the catalytic activities of $\mathrm{Ir}_{4}$ and $\mathrm{Ir}_{6}$ clusters on porous metal oxide supports for structure-insensitive hydrocarbon hydrogenation reactions. Precursors of the supported catalysts were prepared by adsorption of $\left[\mathrm{Ir}_{4}(\mathrm{CO})_{12}\right]$ on supports, $\mathrm{MgO}$ and $\gamma-\mathrm{Al}_{2} \mathrm{O}_{3}$, and by formation of $\left[\mathrm{Ir}_{6}(\mathrm{CO})_{16}\right]$ in supercages of the molecularsieve zeolite $\mathrm{NaY}$ by carbonylation of sorbed $\left[\operatorname{Ir}(\mathrm{CO})_{2}\right.$ (acac)]. The resultant metal carbonyl clusters, $\left[\mathrm{HIr}_{4}(\mathrm{CO})_{11}\right]^{-}$and $\left[\mathrm{Ir}_{6}(\mathrm{CO})_{15}\right]^{2-}$ on $\mathrm{MgO}, \quad\left[\mathrm{Ir}_{4}(\mathrm{CO})_{12}\right]$ on $\gamma-\mathrm{Al}_{2} \mathrm{O}_{3}$, and $\left[\mathrm{Ir}_{6}(\mathrm{CO})_{16}\right]$ in zeolite $\mathrm{NaY}$, were treated in $\mathrm{He}$ at $300^{\circ} \mathrm{C}$, leading to complete decarbonylation, as shown by IR and EXAFS spectra.

EXAFS spectroscopy shows that the firstshell Ir-Ir coordination numbers charac-terizing the fresh and used $\mathrm{MgO}$-supported catalysts made from $\left[\mathrm{Ir}_{4}(\mathrm{CO})_{12}\right]$ are indistin-guishable from 3 , the value for a tetrahedron, as in $\left[\mathrm{Ir}_{4}(\mathrm{CO})_{12}\right]$ (Table 1). The decarbonylated clusters retained this metal frame. EXAFS shows that the decarbonylated $\operatorname{Ir}_{6}$ clusters had metal frames indistinguishable from the octahedra of the precursor hexairidium carbonyls, indicated by the coordination number of approximately 4 (Table 1).

The decarbonylated Ir clusters are markedly less active than the metallic aggregates on $\mathrm{MgO}$ (Table 2). $\mathrm{Ir}_{6}$ clusters are several times less active than $\mathrm{Ir}_{4}$ clusters. The effect of the support on the activities of the decarbonylated clusters is small (Table 2).

Pretreatment in $\mathrm{H}_{2}$ at $300{ }^{\circ} \mathrm{C}$ did not lead to a significant change in the metal framework structures of $\mathrm{MgO}$-supported Ir4 and Ir6, as shown by EXAFS, but it did lead to a decrease in their activities for toluene hydrogenation (Table 2). The results suggest that hydrogen 
bonded strongly to the clusters and inhibited catalysis. The clusters chemisorb several times less hydrogen per exposed atom than supported Ir particles, and these adsorb even less than transition metal clusters in the gas phase. Temperature-programmed desorption of chemisorbed hydrogen from the supported clusters indicates that some hydrogen is much more tightly bound than hydrogen on metallic particles.

However, the effect of hydrogen illustrated by these data is not the same as that observed for $\mathrm{Ir}_{6}$ in NaY zeolite. The hydrogen pretreatment led to a two fold increase in activity of this catalyst without a change in the metal framework structure. The data suggest that the different supports lead to different effects of hydrogen treatment. Additional evidence of support effects is shown by the data for Ir 4 supported on $\gamma-\mathrm{Al}_{2} \mathrm{O}_{3}$; following treatment in $\mathrm{H}_{2}$ at $573 \mathrm{~K}$, the catalytic activity (per total Ir atom) increased ten-fold (Table 2). The increase is explained at least in part by the EXAFS result for the used catalyst showing that the Ir had aggregated to give clusters of roughly 20 atoms each, on average (Table 1). Thus the support significantly affects the stability of the clusters, whereas it affects their intrinsic catalytic activity relatively little.

The low catalytic activity of $\mathrm{Ir}_{4}$ relative to that of aggregated (metallic) Ir correlates with the capacities of these structures for chemisorption of $\mathrm{H}_{2}$. Although the clusters catalyze at least two of the same reactions as metallic Ir, their catalytic character is different.

The clusters are metal-like but not metallic. The concept of structure insensitivity is limited to catalysis by metallic surfaces. The supported clusters are thus regarded as quasi molecular, with the support providing part of a ligand shell, which affects the activity as ligands affect the activities of molecular metal cluster catalysts.

The $\mathrm{MgO}$-supported $\mathrm{Ir}_{4}$ and $\mathrm{Ir}_{6}$ clusters are stable catalysts, as shown by the lack of significant changes in their activities in steadystate operation in a flow reactor for days and by EXAFS results indicating retention of the metal framework structures during catalysis (Table 1). Thus supported metal cluster catalysts are robust enough for practical application. The size dependence of the catalytic properties of the supported clusters is consistent with the observations of unique reactivities of sizeselected gas-phase metal clusters. The results suggest that it may be fruitful to search for reactions for which supported metal clusters have catalytic properties superior to those of conventional supported metals.

We thank M. A. Vannice for providing a catalyst sample and the National Science Foundation for support. EXAFS experiments were done at the National Synchrotron Light Source at Brookhaven National Laboratory (Beamline X-11A) and the Stanford Synchrotron Radiation Laboratory. 
Table 1. EXAFS results characterizing fresh and used supported metal cluster catalysts

\begin{tabular}{|c|c|c|c|c|c|c|c|}
\hline $\begin{array}{l}\text { sample } \\
\text { number }\end{array}$ & $\begin{array}{l}\text { catalyst } \\
\text { precursor }\end{array}$ & support & treatment/catalysis $^{a}$ & $\begin{array}{l}N,^{b} \\
\text { fresh } \\
\text { catalyst }\end{array}$ & $\begin{array}{l}R,{ }^{c} \AA \\
\text { fresh } \\
\text { catalyst }\end{array}$ & $\begin{array}{l}N,^{b} \\
\text { used } \\
\text { catalyst }\end{array}$ & $\begin{array}{l}R,{ }^{c} \AA, \\
\text { used } \\
\text { catalyst }\end{array}$ \\
\hline 1 & {$\left[\mathrm{HI}_{4}(\mathrm{CO})_{11}\right]^{-}$} & $\mathrm{MgO}$ & none & 3.2 & 2.71 & & \\
\hline 2 & {$\left[\mathrm{Ir}_{4}(\mathrm{CO})_{12}\right]$} & $\gamma-\mathrm{Al}_{2} \mathrm{O}_{3}$ & $\begin{array}{l}\text { decarbonylation and } \\
\text { catalysis of toluene } \\
\text { hydrogenation }\end{array}$ & 2.9 & 2.69 & 3.2 & 2.68 \\
\hline 3 & {$\left[\mathrm{HIr}_{4}(\mathrm{CO})_{11}\right]^{-}$} & $\mathrm{MgO}$ & $\begin{array}{l}\text { decarbonylation and } \\
\text { catalysis of toluene } \\
\text { hydrogenation }\end{array}$ & 3.2 & 2.71 & 2.8 & 2.69 \\
\hline 4 & {$\left[\operatorname{Ir}_{6}(\mathrm{CO})_{16}\right]$} & $\begin{array}{l}\mathrm{NaY} \\
\text { zeolite }\end{array}$ & $\begin{array}{l}\text { decarbonylation and } \\
\text { catalysis of toluene } \\
\text { hydrogenation }\end{array}$ & & & 3.9 & 2.70 \\
\hline 5 & {$\left[\operatorname{Ir}_{6}(\mathrm{CO})_{15}\right]^{2-}$} & $\mathrm{MgO}$ & $\begin{array}{l}\text { decarbonylation and } \\
\text { catalysis of toluene } \\
\text { hydrogenation }\end{array}$ & & & 4.1 & 2.69 \\
\hline 6 & {$\left[\mathrm{HIr}_{4}(\mathrm{CO})_{11}\right]^{-}$} & $\mathrm{MgO}$ & $\begin{array}{l}\text { decarbonylation, } \\
\text { treatment in } \mathrm{H}_{2} \text { at } \\
300^{\circ} \mathrm{C} \text {, and catalysis } \\
\text { of toluene } \\
\text { hydrogenation }\end{array}$ & 3.1 & 2.70 & 3.1 & 2.70 \\
\hline 7 & {$\left[\operatorname{Ir}_{6}(\mathrm{CO})_{15]^{2-}}\right.$} & $\mathrm{MgO}$ & $\begin{array}{l}\text { decarbonylation, } \\
\text { treatment in } \mathrm{H}_{2} \text { at } \\
300^{\circ} \mathrm{C} \text {, and catalysis } \\
\text { of toluene } \\
\text { hydrogenation }\end{array}$ & 3.8 & 2.69 & 3.7 & 2.70 \\
\hline
\end{tabular}

Notes: $N$ is coordination number, $R$ the average absorber-backscatterer distance, $\Delta \sigma^{2}$ the Debye-Waller factor, and $\Delta E_{0}$ the inner potential correction. Typical experimental errors in $N$ and $R$ are approximately $\pm 20 \%$ and $\pm 2 \%$, respectively. 
Table 2. Catalytic activities of supported metal clusters and supported metallic particles

\begin{tabular}{|c|c|c|c|c|c|}
\hline $\begin{array}{l}\text { sample } \\
\text { number }^{a}\end{array}$ & $\begin{array}{l}\text { catalyst } \\
\text { modeled as }\end{array}$ & support & $\begin{array}{l}\text { catalytic } \\
\text { reaction }\end{array}$ & $\begin{array}{l}\mathrm{H}_{2} \text { treatment } \\
\text { temp. of catalyst, }{ }^{\circ} \mathrm{C}\end{array}$ & $10^{3} \times$ TOF $^{c} \mathrm{~s}^{-1}$ \\
\hline 1 & {$\left[\mathrm{HIr}_{4}(\mathrm{CO})_{11}\right]^{-}$} & $\mathrm{MgO}$ & $\begin{array}{l}\text { toluene } \\
\text { hydrogenation }\end{array}$ & no treatment & 0.00 \\
\hline 2 & $\mathrm{Ir}_{4}$ & $\gamma-\mathrm{Al}_{2} \mathrm{O}_{3}$ & $\begin{array}{l}\text { toluene } \\
\text { hydrogenation }\end{array}$ & no $\mathrm{H}_{2}$ treatment & 0.94 \\
\hline 3 & $\mathrm{Ir}_{4}$ & $\mathrm{MgO}$ & $\begin{array}{l}\text { toluene } \\
\text { hydrogenation }\end{array}$ & no $H_{\text {, treatment }}$ & 0.63 \\
\hline 4 & $\mathrm{Ir}_{6}$ & NaY zeolite & $\begin{array}{l}\text { toluene } \\
\text { hydrogenation }\end{array}$ & no $\mathrm{H}_{3}$ treatment & 0.25 \\
\hline 5 & $\mathrm{Ir}_{6}$ & $\mathrm{MgO}$ & $\begin{array}{l}\text { toluene } \\
\text { hydrogenation }\end{array}$ & no $\mathrm{H}_{2}$ treatment & 0.23 \\
\hline 6 & $\mathrm{Ir}_{4}$ & $\mathrm{MgO}$ & $\begin{array}{l}\text { toluene } \\
\text { hydrogenation }\end{array}$ & 300 & 0.17 \\
\hline 7 & $\mathrm{Ir}_{6}$ & $\mathrm{MgO}$ & $\begin{array}{l}\text { toluene } \\
\text { hydrogenation }\end{array}$ & 300 & 0.03 \\
\hline 8 & $\begin{array}{l}\text { avg } 20 \text { atom } \\
\text { aggregates }\end{array}$ & $\gamma-\mathrm{Al}_{2} \mathrm{O}_{3} \ldots$ & $\begin{array}{l}\text { toluene } \\
\text { hydrogenation }\end{array}$ & 300 & $9.9^{d}$ \\
\hline 9 & Ir6 & NaY zeolite & $\begin{array}{l}\text { toluene } \\
\text { hydrogenation }\end{array}$ & 300 & 0.52 \\
\hline 10 & $\mathrm{Ir}_{4}$ & $\mathrm{MgO}$ & $\begin{array}{l}\text { cyclohexene } \\
\text { hydrogenation }\end{array}$ & 300 & 18 \\
\hline 14 & Ir crystallites & $\mathrm{MgO}$ & $\begin{array}{l}\text { toluene } \\
\text { hydrogenation }\end{array}$ & 500 & 2.0 \\
\hline
\end{tabular}

$a_{\text {Sample numbers match those of Table } 1 .}$ 


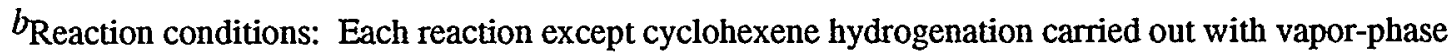
reactants in a once-through plug flow reactor operated at atmospheric pressure. Cyclohexene hydrogenation carried out with liquid-phase cyclohexene in $n$-hexane solvent saturated with $\mathrm{H}_{2}$ flowing through the stirred reactor, which was held at atmospheric pressure.

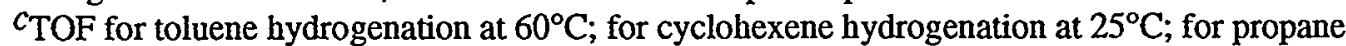
hydrogenolysis at $200^{\circ} \mathrm{C}$ with a $\mathrm{H}_{2} /$ hydrocarbon molar ratio of 2.5 ; for $n$-butane hydrogenolysis at $227^{\circ} \mathrm{C}$ with a $n$-butane $/ \mathrm{H}_{2} / \mathrm{He}$ molar ratio of $1 / 10 / 9$.

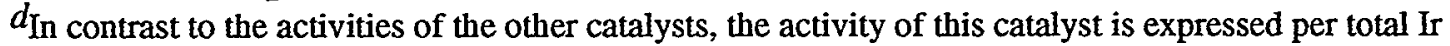
atom to facilitate the comparison with the results for sample 2. The comparison shows that the activity, even per total Ir atom, increased as a result of aggregation of the Ir caused by pretreatment of the sample in hydrogen. 


\title{
1.05 Pr substitution into the $\mathrm{Y}$ and $\mathrm{Ba}$ layers of $\mathrm{Y}_{1} \mathrm{Ba}_{2} \mathrm{Cu}_{3} \mathrm{O}_{7}$
}

\author{
J.V. Acrivos, C.M. Burch and M. Rose, SJSU, CA 95192-0101 \\ and \\ H.A. Blackstead and D.B. Pulling, University of Notre Dame, IN 46556
}

\begin{abstract}
The $\mathrm{Ba}$ and Pr L-edges' XAS reveal changes in the conduction band vs temperature $\mathrm{T}$. This is the first time report of an increase in the amplitude of both the $\mathrm{Ba}$ and $\mathrm{Pr} \mathrm{L}$ edges' white peaks (by more than double) as $\mathrm{T}$ decreases from 77 to $4.1 \mathrm{~K}$, indicating an increase in the transition probability as $\mathrm{T}$ decreases. This result is important because it provides a measure of the changes in the Fermi level vs $T$ in the cuprate superdconductors which can be used to test the various theories. There is considerable amount of disorder indicated in both the $\mathrm{Ba}$ and $\mathrm{Pr}$ EXAFS (by comparison with $\mathrm{Y}_{1} \mathrm{Ba}_{2} \mathrm{Cu}_{3} \mathrm{O}_{7}$ ) as if substitution of $\mathrm{Pr}$ in the Ba layer is greater than the stoichiometric substitution of $\mathrm{x}=(1.05-1) / 2$. The EXAFS FT radial distribution function is compared to ESR data.
\end{abstract}

\section{Introduction:}

The white peaks near the $L$ edges reveal the transition probability to conduction states near the Fermi level ( $p \rightarrow$ d symmetry). ${ }^{1}$ The amplitude of these peaks relative to the EXAFS $\mu_{0}$ have been correlated previously to the metal conductivity for polymorphs of layered dichalcogenides $\mathrm{TaS}_{2}$ and $\mathrm{TaSe}_{2 ;}{ }^{1}$, suggesting that similar measurements are of utmost importance for ascertaining the mechanism of superconductivity in the cuprates.

ESR measurements indicate that substitution of $\operatorname{Pr}$ for $Y$ in the regular 123 usually adds some of it into the $\mathrm{Ba}$ layer, $\mathrm{Pr}^{+3}$ is a non-Kramer's ion and the only ESR absorption expected is from defect $\mathrm{Cu}^{+2}$, introduced by the substitution of $\mathrm{Ba}^{+2}$ by $\mathrm{Pr}^{+3}$.3. Here in addition to the $\mathrm{Cu}^{+2}$ ion impurity ESR near $g \cong 2.1$, an absorption showing nuclear hyperfine structure $(g=2.06, I$ $=5 / 2$ ) has been observed, suggesting a large presence of $\mathrm{Pr}^{+2}$ (similar to that detected by ESR in $\mathrm{BaF}_{2}$ ). For some years the disorder in the $\mathrm{Y} / \mathrm{Ba} / \mathrm{Cu}$ layers of 123 has been known. ${ }^{4}$ If substitution into the $\mathrm{Ba}$ layer stabilizes a valence of +2 , the edge should give evidence of mixed valence.

The XAS analysis in this work was undertaken in order to ascertain the changes in these compounds produced by $T$ and $\operatorname{Pr}$ substitution.

\section{Experimental:}

The compound was prepared at the University of NotreDame;ESR spectra were measured at SJSU.

Discussion:
The data analysis is still on-going using EXAFSPAK ${ }^{5}$ The distances $d_{\text {Pr-A }}$ obtained from XRD data, ${ }^{4}$ are:

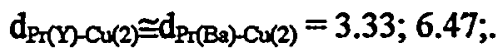

$d_{\text {Na(Ba })-C_{u}(1)}=3.47 ; 6.54 ; d_{\text {Na(P)-Cu(1) }}=6.48$

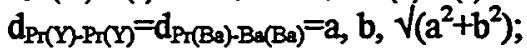

$d_{P(Y)-B a}=d_{P(B a)-N d(Y)}=3.75 ; 5.74 ; 6.66$

$d_{P r}(Y)-O(1,2,3)=2.53-2.56 ; 4.63-4.67 ; 5.04$

$6.17,6.69$;

$\mathrm{d}_{\mathrm{Pr}(\mathrm{Ba})-0(1,2,3)}=2.79-2.88 ; 4.65-4.85 ; 5.73-$

$5.74 ; 6.21,6.62 \AA$

The respective Point Groups about $Y\left(D_{2 h}\right)$ and $\mathrm{Ba}\left(\mathrm{C}_{2 \mathrm{r}}\right)$ are made evident by the spread $\Delta \mathrm{d}_{\mathrm{P}-\mathrm{A}}$ :

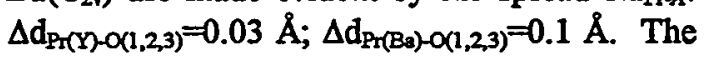
EXAFS radial FT for the Ba L2 edge near $R+\Delta$ $=2.5 \AA$ shows more than one peak for $x=0.025$ in the $\mathrm{Ba}$ layer, the peak intensity near $\mathrm{R}=\mathrm{a}, \mathrm{b}$ is strong, indicating uniform backscattering but the Pr L2 edge EXAFS FT amplitudes centered near $R+\Delta=3$ to 4 (highest) and 5 to $6 \AA$ show relative amplitude loss near $R=a, b$ indicating that out of plane multiple scattering paths follow conduction paths as in ref. 3.

Figure 1 shows the most important fact deduced from this work, the temperature changes of the amplitude of the L2 edge white peak. These peaks depend on the shape of the Fermi level vs T. Now the theories of superconductivity may be tested by this new phenomenon, reported for the first time here. The L2 edge was chosen because the Pr L3 edge almost coincides with the $\mathrm{Ba} \mathrm{Ll}$ edge. This is cumbersome for the EXAFS analysis but, we are searching for new phenomena of constructive interference by multiple paths when the edges 
of different elements, directly bonded, are almost degenerate.

Conclusions:

The XANES vs $\mathrm{T}$ point to changes in the Fermi level for the first time in cuprate superconductors. The EXAFS together with XRD data on Pr substituted 123 compounds points to greater substitution of $\mathrm{Pr}$ in the $\mathrm{Ba}$ layer than indicated by the stoichiometry.

Acknowledgments:

Work at SJSU supported by NSF Grants DMR 9307387 and INT 9312176; at SSRL by DOE, Mr. R. Mayer, Drs. G. George and I. Pickering.
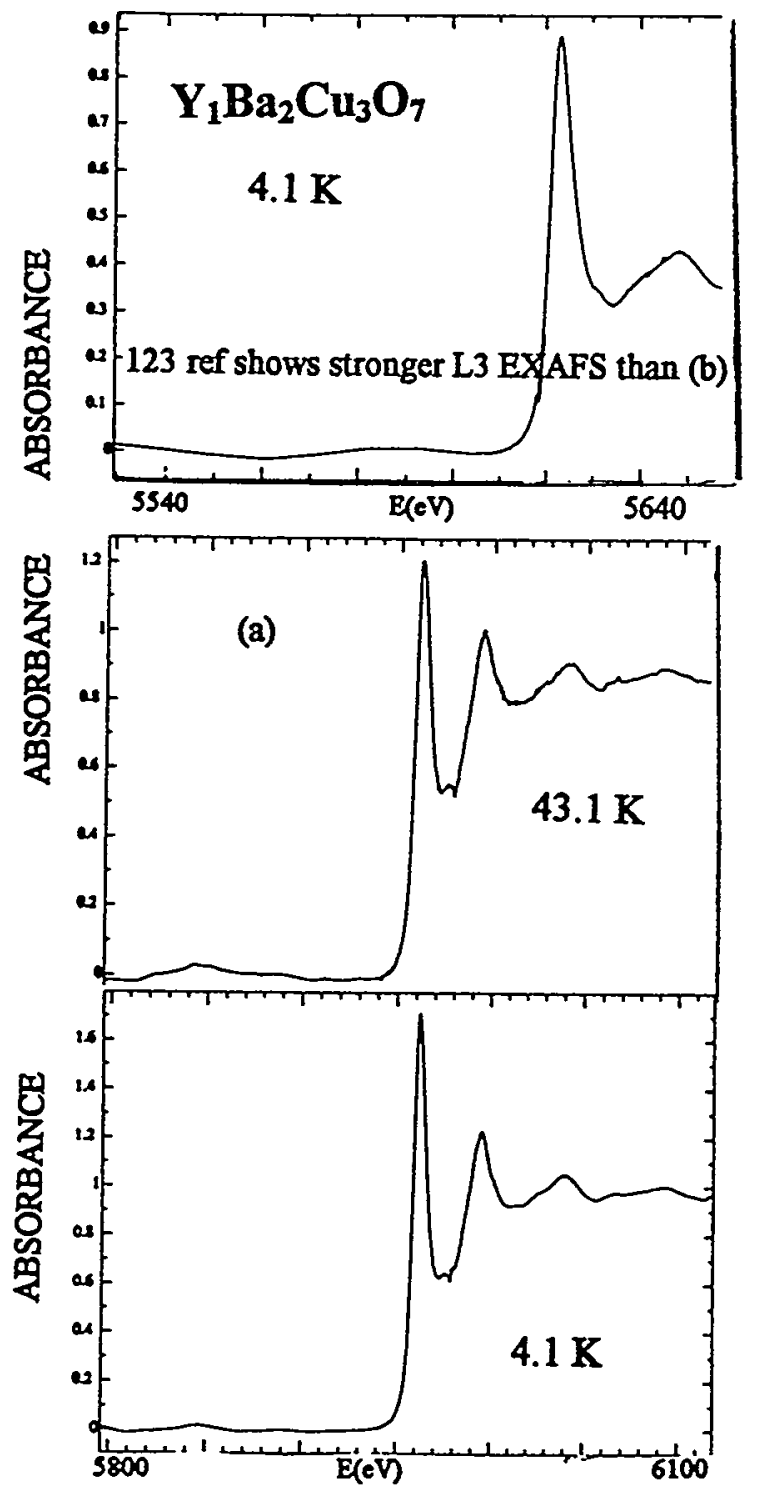

Figure 1: $\mathrm{Pr}_{1.05} \mathrm{Ba}_{1.95} \mathrm{Cu}_{3} \mathrm{O}_{7, s} \mathrm{~L}$ Edge white peak: (a) Pr L3, Ba L1 edges. (b) Ba L2 edgevs T.
References:

1. J.V. Acrivos, S.S.P.Parkin, J.R.Reynolds, J. Code and E. Marseglia, J. Phys. C. 14, L349 (1981)

2. J.V. Acrivos, L. Chen, C. Burch, P. Metcalf, J.M. Honig, R.S. Liu and K.K. Singh, Phys. Rev. B 50, 13710 (1994).

3. J.V. Acrivos, C.M. Burch and K.K. Singh, SSRL Report on Proposal 2242 (1995)

4. (a) J.M. de León, E.A. Stern, D.E. Sayers, E.A. Stern, D.E. Sayers, . Ma and J. Rehr, ed., "XAFS V", 1988 p.433-491; (b)D.M. Ginsburg,

* Phys. Prop. of Hight $\mathrm{T}_{\mathrm{c}}$ Superconductors, I" World Scientific, p. 150, 1990

5. G. Georege and I. Pickering, "EXAFSPAK", 1994

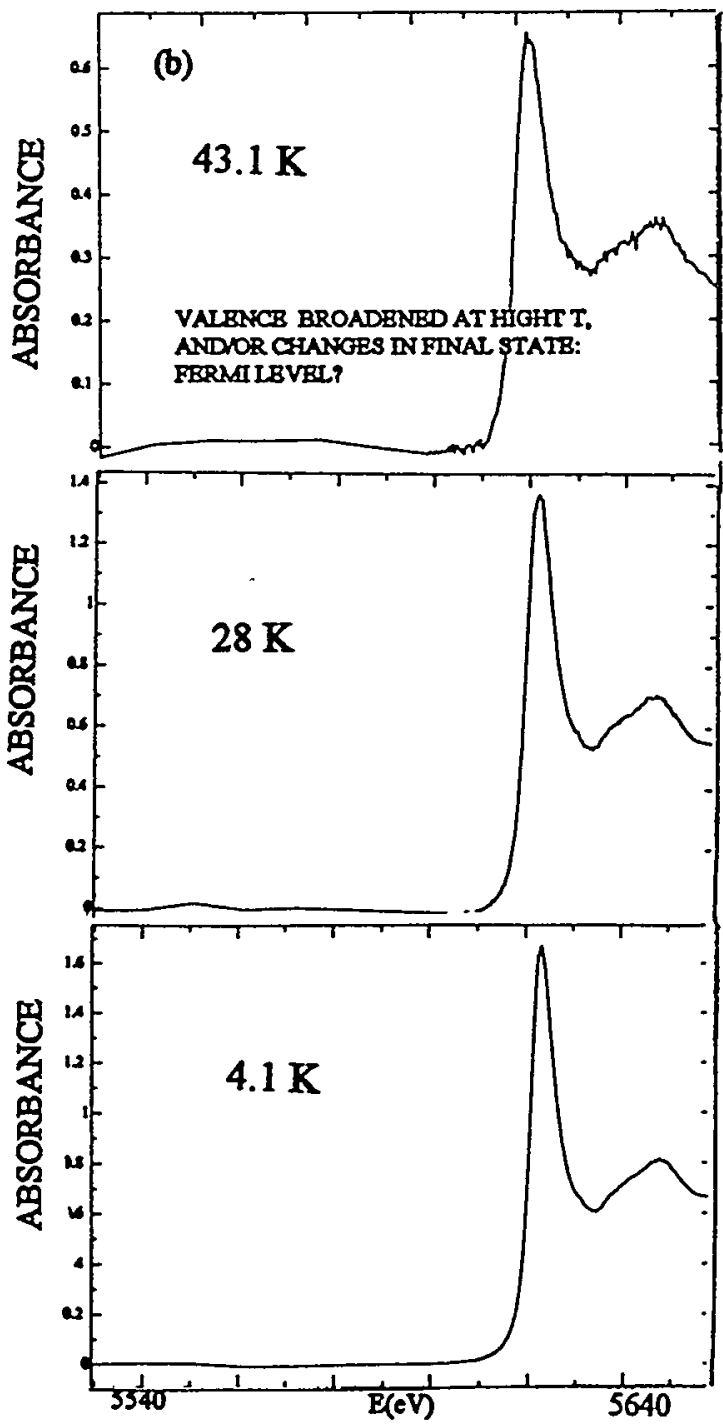




\title{
Short-Period AlAs/GaAs Superlattices Studied by $\mathrm{X}$-ray Diffraction and Standing Waves
}

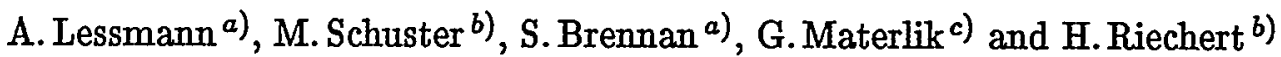 \\ a) Stanford Synchrotron Radiation Laboratory, Stanford, CA 94306 \\ b) Siemens AG, ZFE BT MR 3, D-81739 Munich, Germany \\ c) Hamburger Synchrotronstrahlungslabor HASYLAB, D-22607 Hamburg, Germany
}

\section{Introduction}

In x-ray diffraction (XRD) a measurement of the reflectivity $R=\left|D_{H}\right|^{2} /\left|D_{0}\right|^{2}$, given from the amplitudes $D_{0}$ and $D_{H}$ of incident and reflected $x$-ray wave, is the usual way to study structural parameters of heteroepitaxial layers and superlattices. An extension of this method offers the simultaneous registration of inelastic channels, as it is done in the $\mathrm{x}$-ray standing wave (XSW) method [1]. By recording the $x$-ray fluorescence or the Auger- or photoelectron yield from the sample, it is possible to measure the normalized intensity $\left|\left(D_{0}+D_{H}\right)\right|^{2} /\left|D_{0}\right|^{2}$ of the wavefield at the atomic positions. From the calculable phase between $D_{0}$ and $D_{H}$ the position of the selected atoms in the unit cell can then be determined. We performed XRD/XSW measurements using, among other heterostructures, AlAs/GaAs short-period superlattices (SL) on $\mathrm{GaAs}(001)$ to study the possible applications. The samples had several hundred periods and typical bilayer thicknesses of $3 \mathrm{~nm}$.

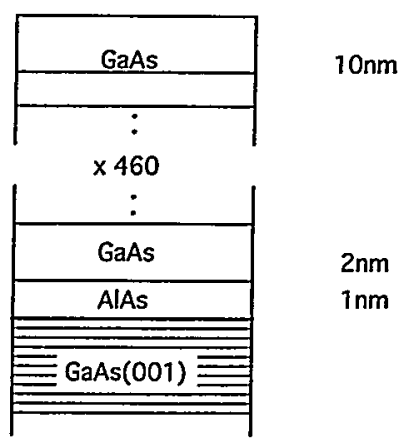

Fig. 1: Schematic sideview of one of the SL samples.

\section{Experimental}

Experiments were carried out at beamlines 2-2 and 7-2. For the XSW measurements at 22 monochromatization of the white beam was done at $5300 \mathrm{eV}$ with a double crystal $\mathrm{Ge}(004)$ monochromator in $(t,-)$ arrangement [2]. A symmetric 1st and asymmetric 2nd reflection was used to collimate the incoming $x$-rays with an asymmetry parameter $b \simeq-8$. For GaAs(004) sample reflections a nearly nondispersive arrangement is achieved, because of the almost identical lattice constants of $\mathrm{Ge}$ and GaAs.

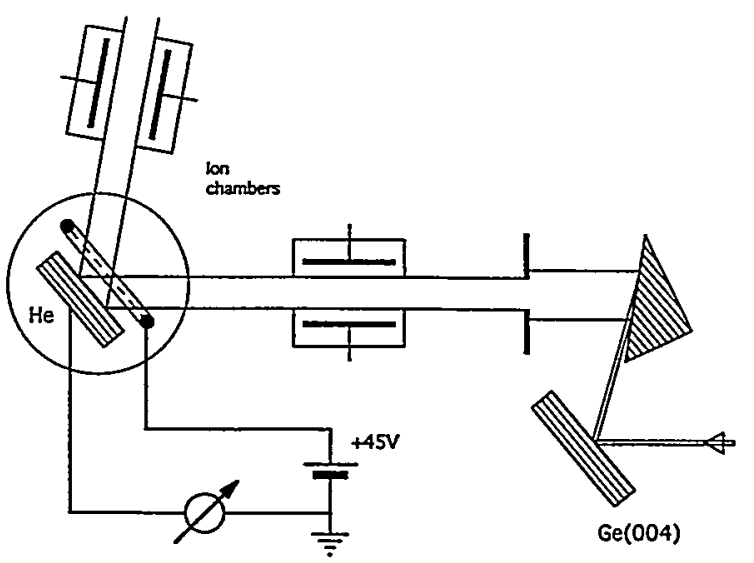

Fig. 2: Experimental setup for the XSW measurements of the photocurrent at beamline 2-2. The $\mathrm{Si}(\mathrm{Li})$ detector, which was used for detection of fluorescence radiation, is not shown.

Samples were mounted on a Huber- 410 circle motorized by a Compumotor microstepping motor. The $\mathrm{x}$-ray fluorescence was detected with a thin-window $\mathrm{Si}(\mathrm{Li})$ detector and the photoelectron yield was registered via the photocurrent flowing through a current amplifier to the sample. In the photocurrent experiments the samples were contacted and placed into small kapton housings flushed with He. For acceleration of the emerging photoelectrons a ring anode with a potential of $45 \mathrm{~V}$ with respect to the sample was placed around the illuminated area. Typical currents were several $10^{-10} \mathrm{~A}$. 
At beamline 7-2 a focussed beam, monochromatized by a pair of symmetric Si(111) reflections, was employed. The samples were mounted on the 4-circle diffractometer and incident and diffracted intensity were monitored with scintillation counters. Energy resolution and angular divergence of the incoming beam can be estimated to $8 \times 10^{-4}$ and $0.03^{\circ}$, respectively. Registration of the electron yield was done under rough vacuum conditions by a current amplifier attached to the floating sample.

\section{Results}

In its lower part Fig. 3 shows the GaAs(004) substrate reflection and at a smaller diffraction angle the 0th order SL reflection. The average lattice mismatch is $\Delta d / d=8.2 \times 10^{-4}$. Due to the nearly nondispersive arrangement between monochromator and sample reflection these data exhibit very good resolution over a range of angles in the vicinity of the GaAs(004) reflection. In the upper part the normalized photocurrent signal is shown. Here the thickness oscillations from the overall SL thickness of $1310 \mathrm{~nm}$ show a larger contrast and the XSW induced modulation shows pronounced differences between substrate and 0th order SL reflection.
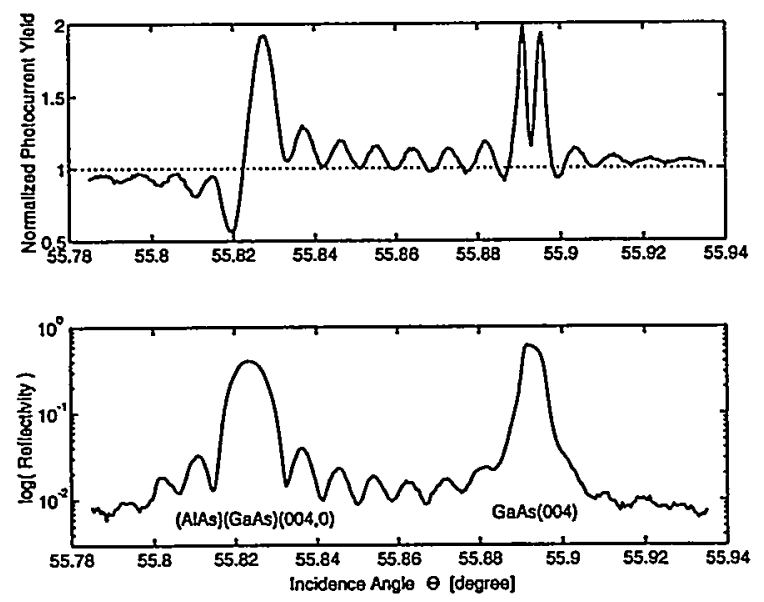

Fig. 3: Reflectivity and electron yield around the $\mathrm{GaAs}(004)$ substrate reflection and around the corresponding $(004,0)$ superlattice satellite.

Most of the observed effects can be explained by the periodicity of the $x$-ray wavefield, primarily given by wavelength and incidence angle $\Theta$ and the small information depth of the elec- tron signal. In the case of the SL reflection the wavefield has a modulation length that is close to the average lattice parameter of the SL and therefore most of the atoms are located coherently with respect to it. This reflection can be interpreted as a Bragg reflection from the average unit cell of one SL period, overlapping with thickness fringes from the overall SL thickness.

On the other side the (004) substrate reflection creates a wavefield with a slightly compressed periodicity, because the pure GaAs has a smaller lattice constant than the SL. Only at specific depths in the SL the wavefield is in phase with the atomic planes. From the difference in diffraction angle this moiré or beating effect can be calculated: After a distance of $\sim 170 \mathrm{~nm}$ the wavefield is again in phase with the atomic planes. As the electrons contributing to the photocurrent are only emerging from the uppermost region of the sample, their information depth is significantly smaller than this length.

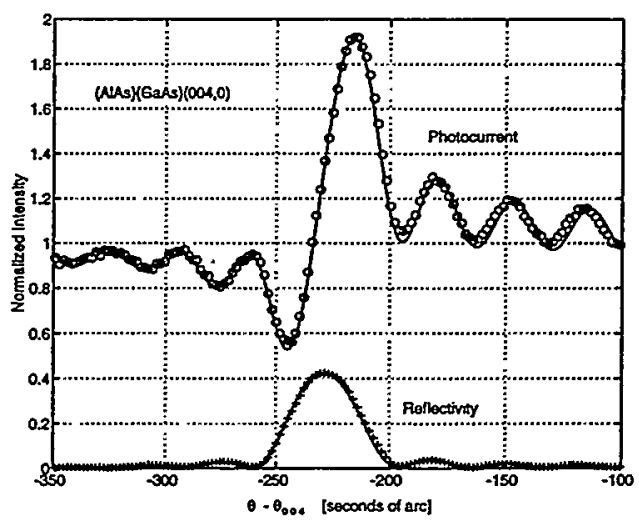

Fig. 4: Theoretical XSW fit to reflectivity and electron yield at the $(004,0) \mathrm{SL}$ satellite. The calculation was based on the Takagi-Taupin formalism.

A detailed interpretation of the data requires a dynamical calculation of the wave amplitudes in the SL and a fit to the data. The applied theory was based on a numerical solution of the Takagi-Taupin equations for each individual layer and recursion of this process from the substrate up to the surface [3]. Fig. 4 shows the results of a fit to reflectivity and electron signal, revealing the high epitaxial quality of the sample. The fraction of coherently ordered atoms was found to be $74 \%$. 
The very intense, focused wiggler radiation at beamline 7-2 made it possible to study also weak SL satellite reflections. In Fig. 5 the result of a $(\Theta-2 \Theta)$ scan performed at a photon energy of $8050 \mathrm{eV}$ is shown.

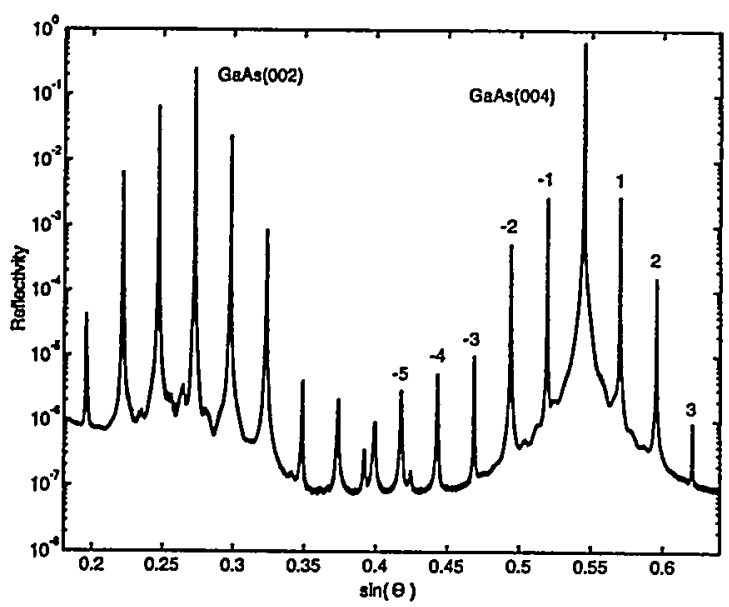

Fig. 5: Diffracted intensity in a $(\Theta-2 \Theta)$ scan from the (002) to the (004) reflection, taken at wiggler endstation 7-2. Numbers $n$ indicate the order of the $(004, n)$ superlattice satellite.

An evaluation of the satellite spacings in reciprocal space gives a SL period length of $3.03 \mathrm{~nm}$. Note that the satellite groups belonging to the $\mathrm{GaAs}(002)$ and $\mathrm{GaAs}(004)$ reflection are not overlapping, but are independent from each other and do not coincide in diffraction angle. This is due to an incommensurable ratio between the average SL period length and the thickness of the whole SL structure [4]. Possible reasons are varying bond lengths at the interfaces between the AlAs and GaAs layers, or an interdiffusion process where $\mathrm{Al}$ and $\mathrm{Ga}$ atoms change their positions.
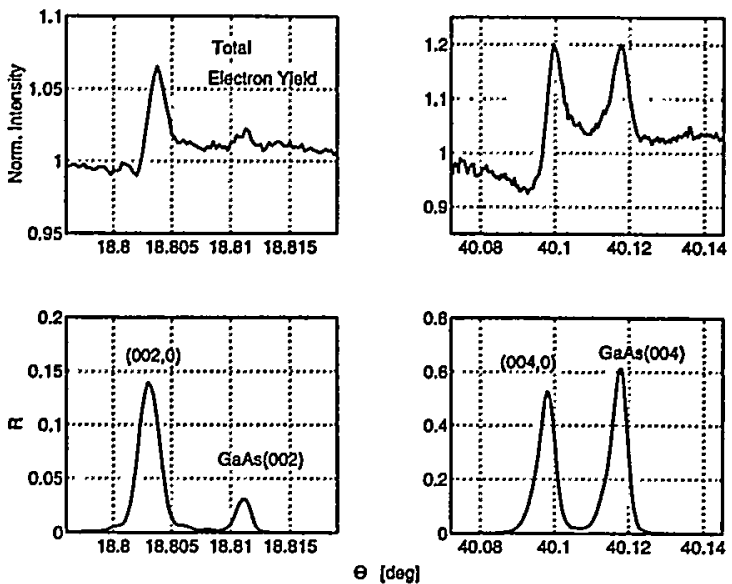

Fig. 6: XSW scans at the (002) and (004) reflection.
The results for a simultaneous registration of the total electron yield around the $\mathrm{GaAs}(002)$ and $\mathrm{GaAs}(004)$ reflection are shown in Fig. 6. In comparison to the XSW data in Fig. 3 , the data on the left side shows a reduced resolution, because of the dispersive monochromatorsample arrangement and the additional divergence of the focused beam. Nevertheless, the phase contrast is still large enough to extrac$t$ information about the structural parameters in the SL. The GaAs(002) diffraction plane spacing matches the $\mathrm{Si}(111)$ spacing from the monochromator much better, resulting in notable thickness fringes and strong modulations of the electron yield.

\section{Conclusion}

XSW measurements as well as the simultaneous registration of inelastic channels during $\mathrm{x}$ ray diffraction experiments is a valuable tool in the study of superlattices and heterostructures. The described photoyield detection setup can be easily implemented in existing diffraction instruments, giving additional information related to the phase of the x-ray wave. Obtained results were in good agreement with dynamical calculations for the depth and angle dependent wavefield based on the Takagi-Taupin theory. Around the $(004,0)$ satellite the wavefield was found to be aligned to the average lattice parameter $\left\langle d_{\perp}\right\rangle$ in the SL stack, enabling the study of the atomic arrangement in the AlAs and GaAs layers.

\section{Acknowledgments}

SSRL is funded by the Department of Energy, Office of Basic Energy Sciences. Two of us (M. S. and G. M. ) are grateful to the Volkswagen Stiftung for sponsoring their studies through a grant to Stanford University.

\section{References}

[1] B. W. Batterman, H. Cole, Rev. Mod. Phys. 36(1964)681

[2] A. Lessmann, M. Schuster, S. Brennan, G. Materlik, H. Riechert, in Proc.5th Int.Conf. on Synchrotron Radiation Instrumentation SRI'94

[3] W.J. Bartels, J. Hornstra, D.W. Lobeek, Acta Cryst. A 42(1986)539

[4] M.Schuster, A. Lessmann, A. Munkholm, S. Brennan, G. Materlik, H. Riechert, in Proc. 2nd European Symposium on X-ray Topography and High Resolution Diffraction 


\title{
Wafer cleaning influence on the roughness of the $\mathrm{Si} / \mathrm{SiO}_{2}$ interface
}

\author{
A. Munkholm and S. Brennan \\ Stanford Synchrotron Radiation Laboratory, Stanford, CA 94309 \\ Jon P. Goodbread \\ Hewlett-Packard Co., Palo Alto, CA 94301
}

\section{Introduction}

As device sizes decrease, the importance of interfacial roughness on the quality of gate oxides increases. With future generations of gate oxide thicknesses expected to be $35-50 \AA$, both surface and interfacial roughness can dramatically effect device performance. Although the top surface roughness can be measured using probes such as scanning tunneling microscopy (STM) or atomic force microscopy (AFM), in order to measure the roughness of a buried interface, either the oxide layer must be stripped off or a probe must be used which can penetrate through the oxide layer.

Although specular reflectivity has long been used to measure surface and interfacial roughness, for materials whose index of refraction differ only slightly the deconvolution of the roughnesses from the two interfaces is problematic. Over the past decade $x$-ray scattering techniques have been developed to study the truncation of bulk order in crystalline materials. ${ }^{1,2}$ By measuring only the termination of bulk order, the roughness of the $\mathrm{Si} / \mathrm{SiO}_{2}$ interface can be determined independently. The measurement is performed by studying the decay of $x$-ray scattered intensity away from a bulk diffraction peak in the direction of the surface. This fall-off in intensity is known as a Crystal Truncation Rod, or CTR. Many of the early studies of CTRs involved clean, reconstructed surfaces where the goal was to determine the rearrangement of the top layers of the bulk crystal. Recently, groups have started looking at interfacial roughness with CTRs, especially related to the technologically important $\mathrm{Si} / \mathrm{SiO}_{2}$ interface. ${ }^{3-5}$

In this study we have investigated the influence of different cleaning procedures on interfacial roughness using synchrotron radiation to perform CTR-scattering. In particular, we have looked at silicon(001) both before and after the growth of a $1000 \AA$ thermal oxide.

\section{Theory}

Crystal truncation rods (CTRs) are intensity streaks in reciprocal space perpendicular to the surface which arise due to the abrupt change in electron density from the termination of the bulk. The phenomenon can be thought of as a relaxation of the diffraction condition in the direction perpendicular to the surface, so that spots of intensity turn into rods. Because the diffracted intensity in reciprocal space is the fourier transform of the electron density in real space, the transformation of an infinite crystal (and the correspondingly tiny points in reciprocal space) into a semi-infinite crystal results in the points smearing out in the direction of the surface. The more abrupt the termination of the crystalline solid, the more fourier components in reciprocal space are needed to represent that transition, and the stronger the intensity of the CTRs. In contrast, a very rough interface can be represented by only a few fourier components and the scattered intensity decays more quickly in reciprocal space. This was also shown by the early describers of dynamical scattering theory, which predicts that the decrease in scattered intensity towards the surface is inversely proportional to the square of the scattering vector perpendicular to the surface $\left(q_{\perp}\right)$, if the surface is perfectly flat.

Following Robinson's formulation of the kinematic theory for the decay of bulk crystalline scattering, ${ }^{2}$ we use a layer summation method for the structure factor which is summed up for each atomic layer of the crystal. The diamond cubic lattice consists of bilayers perpendicular to the (001) direction with a basis of (surface units):

$$
G_{h k l}=1+e^{2 \pi i\left(\frac{h}{2}+\frac{l}{4}\right)}
$$

The bilayer translates into the above bilayer by the phase factor: 

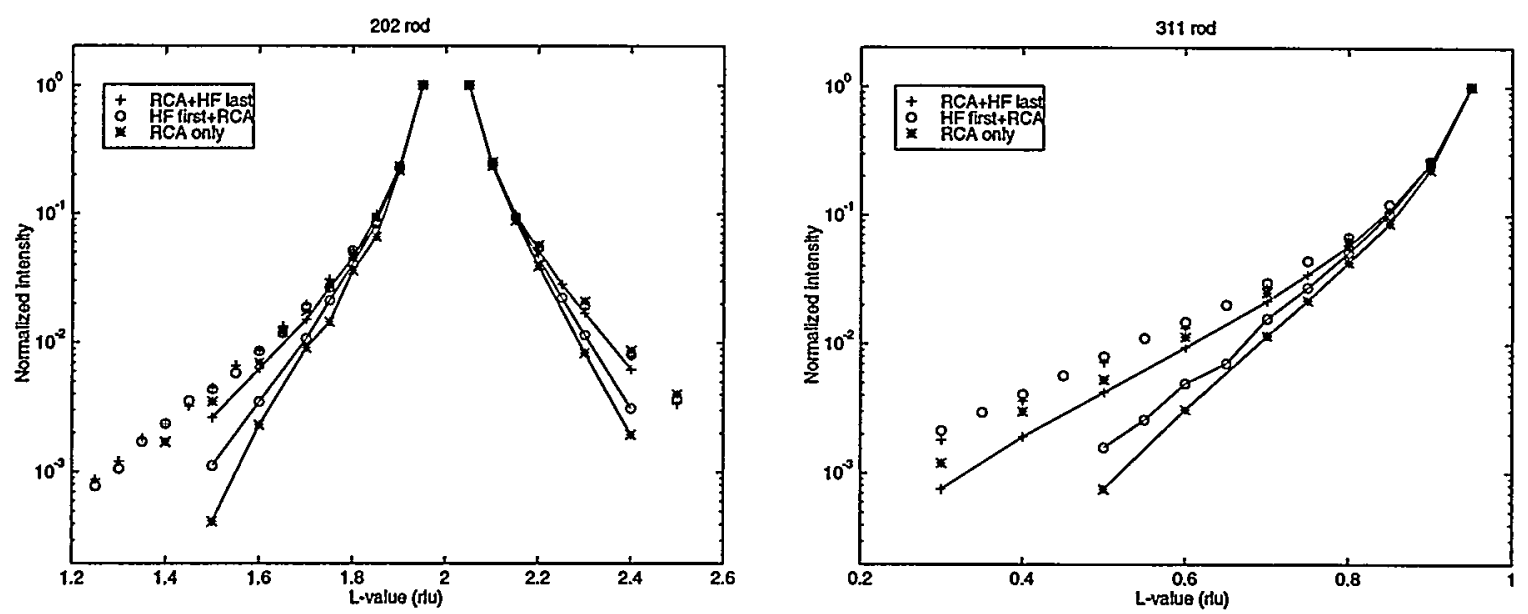

Figure 1: Crystal truncation rod (CTR) intensities around the 202 and 311 Bragg reflections

$$
\psi=2 \pi\left(\frac{h}{2}+\frac{k}{2}+\frac{l}{2}\right)
$$

The structure factor of the bulk is consequently given by adding the structure factor of the bilayers up over all the bilayers present in the crystal.

$$
\begin{aligned}
F= & \sum_{j=1}^{\infty} G_{h k l} e^{i \psi j}+\sum_{j=0}^{-\infty} \beta^{-2 j+1} e^{i \psi j} \\
& +\sum_{j=0}^{-\infty} \beta^{-2 j} e^{2 \pi i\left(\frac{h}{2}+\frac{l}{4}\right)} e^{i \psi j} \\
= & \frac{G_{h k l} e^{i \psi}}{1-e^{i \psi}}+\frac{\beta+e^{2 \pi i\left(\frac{h}{2}+\frac{l}{4}\right)}}{1-\beta^{2} e^{-i \psi}}
\end{aligned}
$$

where the roughness parameter $\beta$ is a measure of the partial occupancy of the top atomic layers. The layers for which $j>0$ correspond to the bulk with an atomic occupancy of 1 . For each adjacent layer of atoms the occupancy goes as $\beta^{-j}$. The roughness parameter can be related to rms roughness $\sigma$ by:

$$
\sigma_{r m s}=\frac{\sqrt{\beta}}{1-\beta} d_{\perp}
$$

where $d_{\perp}$ represents the atomic distance perpendicular to the surface.

\section{Experimental}

The data was collected on Beam line 6-2 at Stanford Synchrotron Radiation Laboratory (SSRL) using a focused beam. The photon energy passed by the
Si(111) double crystal monochromater was $10 \mathrm{keV}$. The wafers were mounted on a vacuum chuck and kept in a helium environment to reduce air scattering.

The scattered beam slits were defined using the procedure of Specht and Walker. ${ }^{6}$ This setup has a large in-plane slit opening, which allows the entire diffracted beam to be collected by the detector simultaneously, so that the integrated intensity is directly measured. The 4-circle diffractometer was operated in the $\omega=0$ mode and the sample surface normal was aligned with the phi axis, which results in the incident and exit beam angles being equal. Rod intensities were obtained by scanning $\theta$, which results in a trapezoidal rocking curve with the flat top of the curve resulting from diffraction of the entire rocking curve simultaneously. Thus the integrated intensity is the difference between that top and the background. The resolution along the rod is defined by the size of the scattered beam slits in the-direction perpendicular to the scattering plane. This resolution also depends on the relationship of the rod to the horizontal plane, which changes as the rod is scanned. For these measurements the resolution varied between 0.01-0.03 reciprocal lattice units (rlu). The data were corrected for the background, the Lorenz-factor, the change in atomic form factor as a function of the magnitude of the scattering vector, the area of the sample illuminated by the beam and the resolution function of the rod.

The samples used are all well oriented silicon (001) wafers and consist of three sets of two wafers, a native and a $1000 \AA$ thermal oxide wafer both 


\begin{tabular}{l|cc|cc|cc|} 
& \multicolumn{2}{|c|}{ RCA only } & \multicolumn{2}{c|}{ HF first+RCA } & \multicolumn{2}{c|}{ RCA+HF last } \\
& native & thermal & native & thermal & native & thermal \\
\hline 202 low & 6.55 & 2.75 & 4.73 & 2.39 & 2.99 & 2.38 \\
202 high & 5.75 & 2.26 & 4.30 & 2.41 & 2.74 & 2.48 \\
311 low & 5.38 & 2.37 & 4.16 & 1.76 & 3.02 & 1.92 \\
\hline
\end{tabular}

Table 1: rms roughness in $\AA$.

cleaned by the same methods. They are: an RCA clean only; HF dip followed by an RCA clean; and an RCA clean followed by HF dip. The RCA clean is a two step process performed at $70^{\circ} \mathrm{C}$ in which the wafers first are exposed to an $\mathrm{SCl}\left(\mathrm{H}_{2} \mathrm{O}, \mathrm{H}_{2} \mathrm{O}_{2}\right.$ and $\mathrm{NH}_{4} \mathrm{OH}$ in the ratio 8:1:1) followed by an $\mathrm{SC} 2$ clean $\left(\mathrm{H}_{2} \mathrm{O}, \mathrm{H}_{2} \mathrm{O}_{2}\right.$ and $\mathrm{HCl}$ in the ratio 8:1:1). The $\mathrm{HF}$ clean was processed at $25^{\circ} \mathrm{C}$ with a water to HF ratio of 60:1. Although there are several rods which can be measured, we focused on three, above and below the 202 reflection and below the 311 reflection.

\section{Results}

The crystal truncation rod intensities from the 202 and 311 reflections are shown in figure 1 . The rod intensity data from the samples containing a native oxide are connected with lines and the cleaning prpcedure is specific to the symbol. For the 202 rod significant differences are observed amongst the roughnesses of the native oxides; the thermal oxides are all smoother than the native oxides. Amongst the thermal oxides the wafer cleaned by RCA only is the roughest, consistant with its position as the roughest of the native oxides. Although the roughness of the two native oxides cleaned with HF are significantly different, the thermal oxides show very little difference.

Extracting consistent quantitative information from these curves has so far been problematic. Using currently available theories of CTR scattering we have fit these data, with the results tabulated in table 1. As can be seen there, the results for any one of the rods show a consistent trend, but the roughness extracted from different rods for a single sample show a wide variability.

We are currently studying this problem with a goal of improving the theory to explain these differences. Amongst the issues we will be exploring is the effect of miscut, the possibility of a surface reconstruction, and the effect of possible ordering within the amorphous oxide layer.

\section{Conclusions}

We have observed differences in the CTR-scattering for different rods from the same samples, which can not be explained by the current theories. As a result, quantitative results are not reliable. Qualitatively, however, the relative roughness of different samples can be determined by comparing the rod intensities of the same rods for different samples.

There is clear evidence of a correlation between the roughness of the $\mathrm{Si} / \mathrm{SiO}_{2}$ interface and the different wafer cleaning procedures, and that the growth of a thermal oxide reduces the initial roughness significantly.

\section{Acknowledgments}

This work was performed at and supported by SSRL, which is supported by the Department of Energy through the Office of Basic Energy Sciences.

\section{References}

[1] S.R. Andrews and R.A. Cowley. J. Phys. C: Solid State Phys. 18 (1985).

[2] I.K. Robinson. Phys. Rev. B. 33 (6), 15 March 1986.

[3] M.-T. Tang, K.W. Evans-Letterodt, G.S. Higashi, and T. Boone. Appl. Phys. Lett. 62 (24), 14 June 1993.

[4] M.-T. Tang, K.W. Evans-Letterodt, M.L. Green, D. Brasen, K. Krisch, L. Manchanda, G.S. Higashi, and T. Boone. Appl. Phys. Lett. 64 (6), 7 February 1994.

[5] M.L. Green, D. Brasen, K.W. EvansLetterodt, L.C. Feldman, K. Krisch, W. Lennard, H.-T. Tang, L. Manchanda and M.T. Tang. Appl. Phys. Lett. 65 (7), 15 August 1994.

[6] E.D. Specht and F.J. Walker. J. Appl. Crystallography. 26 (2), 1 April 1993. 


\title{
Nuclear Scattering of Synchrotron Radiation by $181 \mathrm{Ta}$
}

\author{
A. I. Chumakov ${ }^{1}$, A. Q. R. Baron ${ }^{2}$, J. Arthur ${ }^{2}$, S. L. Ruby ${ }^{2}$, G. S. Brown ${ }^{3}$, G. V. Smirnov ${ }^{4}$, U. van Bürck ${ }^{5}$, \\ and G. Wortmann ${ }^{6}$
}

(1) European Synchrotron Radiation Facility, BP 220 - F38043 Grenoble Cedex, France

(2) Stanford Synchrotron Radiation Laboratory, Stanford Linear Accelerator Center, Stanford CA 94309 USA

(3) Dept. of Physics, Univ. of California at Santa Cruz, Santa Cruz, CA 95064 USA

(4) Russian Research Center "Kurchatov Institute", Moscow 123182 Russia

(5) Department of Physics, Technical University of Munich, D-85747 Garching, Germany

(6) Department of Physics, University of Paderborn, D-33095 Paderborn, Germany.

The recent rapid development of hyperfine nuclear spectroscopy using synchrotron radiation [1] indicates that this method will become a standard technique in the near future. The broadband, pulsed, well-collimated, and highly polarized synchrotron radiation is very different in character from that of a conventional radioactive source, and offers distinct advantages for hyperfine spectroscopy experiments. However, only a few isotopes have been used in synchrotron-based experiments to date: ${ }^{57} \mathrm{Fe}[1]$, ${ }^{169} \mathrm{Tm}[2],{ }^{119} \mathrm{Sn}[3,4]$ and ${ }^{83} \mathrm{Kr}[5,6]$.

An attractive target is the $6.2 \mathrm{keV}$ nuclear E1 transition in ${ }^{181} \mathrm{Ta}$. The long lifetime of this nuclear level $\left(\tau_{0}=8.73 \mu \mathrm{s}\right.$ [7]) and the complementary narrow energy width $\left(\Gamma_{0}=7.5 \times 10^{-11} \mathrm{eV}\right.$, corresponding to a Doppler shift of $3.6 \mu \mathrm{m} / \mathrm{s}$ velocity), together with a very large nuclear moment, makes this resonance a very sensitive probe of hyperfine interactions. In the field of Mössbauer spectroscopy this resonance gives more than an order of magnitude higher energy resolution than the $14.4 \mathrm{keV}$ resonance of ${ }^{57} \mathrm{Fe}\left(\tau_{0}=141\right.$ ns). This has been shown in many experimental studies, including high-resolution measurements of the ${ }^{181} \mathrm{Ta}$ isomer shift in metallic systems and compounds [8], studies of hydrogen diffusion in Ta metal [9] and temperature-induced changes in the electronic structure of ferroelectric $\mathrm{LiTaO}_{3}$ [10], and observation of the double NMR Mössbauer resonance [11].

However, this high sensitivity causes severe technical difficulties in the preparation of good radioactive sources for ${ }^{181} \mathrm{Ta}$ Mössbauer analysis. The source lines are considerably broadened by crystal imperfections or by interstitial impurities such as oxygen or hydrogen $[12,13]$. These difficulties are reflected in the fact that the narrowest experimental linewidth observed so far in ${ }^{181} \mathrm{Ta}$ Mössbauer spectroscopy is about $15 \Gamma_{0}$ [13]. Another disadvantage of the radioactive sources is the low flux of $\gamma$-radiation due to the high internal conversion coefficient ( $\alpha=70.5$
$[14,15])$ and the large photoelectric absorption. In addition, the resonant $6.2 \mathrm{keV}$ radiation is strongly shadowed by the intense $L \alpha, \beta$ fluorescence $x$-radiation of Ta.

By contrast, the synchrotron radiation source provides almost perfect experimental conditions. The short pulse of synchrotron radiation allows high resolution time domain spectroscopy. The timing experiment also eliminates the problems arising from mechanical vibrations [16] and geometrical broadening. The method is practically free of any background radiation in the time window excluding the synchrotron radiation pulses.

The experiment was performed at the 27-period wiggler beamline 6-2 at the Stanford Synchrotron Radiation Laboratory (SSRL). The storage ring was run in timing mode with 4 electron bunches in the ring, providing pulsed radiation with a $195 \mathrm{~ns}$ period. During 12 hours a special single bunch mode with 780 ns between pulses was used for the studies of the time distribution of the ${ }^{181} \mathrm{Ta}$ nuclear decay.

A synchrotron radiation beam of about $3.6 \mathrm{eV}$ bandwidth at an energy of $6.2 \mathrm{keV}$ was prepared by a cooled $\mathrm{Si}(111)$ monochromator. Further reduction of the bandwidth down to about $83 \mathrm{meV}$ was achieved by a $\mathrm{Si}(333)$ symmetric-reflection channel-cut monochromator $\left(\theta_{B}=70.8 \mathrm{deg}\right)$. After passing through the ${ }^{181}$ Ta sample the $x$-rays were counted by an EG\&G avalanche photodiode (APD) timing detector [17]. The sample and high-resolution optics were contained in a helium-filled box to minimize flight-path absorption of the $6.2 \mathrm{keV}$ radiation.

A nuclear forward scattering geometry was used [18], in which radiation is transmitted straight through the sample into the detector. The resonant sample is excited by short pulses of synchrotron radiation, and emits radiation coherently into the forward direction after a delay determined by the lifetime of the nuclear excited state. The prompt flux on the detector, due to non-resonant transmission of the synchrotron pulses, 
was about $1.5 \times 10^{7}$ photons per second (as measured by a calibrated ion chamber). The APD signal was gated off during the prompt pulse, so that only delayed events were counted.

The resonant ${ }^{181} \mathrm{Ta}$ target was a $99.996 \%$ pure Ta foil of $15 \mathrm{~mm}$ diameter and $3.8 \mu \mathrm{m}$ thickness. This sample had been previously studied by conventional Mössbauer spectroscopy [13] and revealed an extremely narrow linewidth (measured as a convolution of the source and absorber lines) of about $57 \pm 1 \mu \mathrm{m} / \mathrm{s}\left(15.8 \pm 0.3 \Gamma_{0}\right)$. For the present experiment the sample was tilted at an angle of about 30 degrees, so its effective thickness along the beam path was about $7.5 \mu \mathrm{m}$.

A major technical challenge for the experiment arose from the large uncertainty in the value of the ${ }^{181} \mathrm{Ta}$ resonance energy. The error range derived from the Mössbauer literature was about $\pm 20 \mathrm{eV}$, around a central value $6238 \mathrm{eV}$ [14]. Recently, a somewhat more precise value for the resonance energy, $6224 \pm 5$ $\mathrm{eV}$, was found from measurements of Auger electron spectra of Ta compounds [19].

In the search for the nuclear resonance the total delayed count rate in the time interval 40-90 ns after the prompt flash was measured as a function of the incident $\mathrm{x}$-ray beam energy. A resonance peak was found having a maximum delayed count rate of 0.23 cps above a background of $0.013 \mathrm{cps}$. The observed width of the peak, $83 \pm 12 \mathrm{meV}$ (FWHM), was in good agreement with the calculated value $(87 \mathrm{meV})$ of the energy bandwidth of the $\mathrm{Si}(333)$ monochromator.

For the determination of the ${ }^{181} \mathrm{Ta}$ resonance energy we used the Nd $\mathrm{L}_{\mathrm{III}}$ absorption edge. The Si(111) monochromator was calibrated using the edge position (6209.5 eV [20]) for the $\mathrm{NdF}_{3}$ powder absorber. The absolute energy of the ${ }^{181} \mathrm{Ta}$ resonance was found to be $6214 \pm 2 \mathrm{eV}$ (the error is dominated by the uncertainty in the published data on the Nd LIII edge energy). It was possible to make a much more precise relative measurement of the resonance energy with respect to the pronounced absorption maximum (white line) of the absorber. The energy of the ${ }^{181} \mathrm{Ta}$ resonance was determined to be $1130 \pm 80 \mathrm{meV}$ above the maximum absorption point of the $\mathrm{NdF}_{3}$ absorber. This measurement provides a simple procedure for finding the resonance in further studies.

The time distribution of the nuclear forward scattering from the Ta foil was measured during a special 12-hour period of single bunch operation of the synchrotron light source. The time distribution is shown in Figure 1. The experimental data may be approximately described by an exponential with characteristic (1/e) decay time of $530 \pm 80 \mathrm{~ns}$.

The experimental data were fit using the dynamical theory of nuclear resonant scattering [21]. For an E1 nuclear transition with some inhomogeneous broadening, the response function $R(E)$ (the energy-dependent amplitude for nuclear scattering) takes the form $[22,23]$ :

$$
R(E)=\exp \left\{\frac{-\frac{i T \Gamma_{0}}{4}(1+2 i \xi)}{E-E_{0}+\frac{i \Gamma}{2}}\right\}
$$

where $T=\left(\sigma_{0} f_{\mathrm{LM}} \mathrm{nz}\right)=44$ is the effective resonant thickness of the sample; $\sigma_{0}=1.1 \times 10^{-18} \mathrm{~cm}^{2}$ is the resonance cross-section; $\mathrm{f}_{\mathrm{LM}}=0.96$ is the LambMössbauer factor; $\mathrm{n}=0.55 \times 10^{23} \mathrm{~cm}^{-3}$ is the density of resonant nuclei; $\mathrm{z}$ is the thickness of the sample; $\left(\mathrm{E}-\mathrm{E}_{0}\right)$ is the deviation from the resonance energy; $\Gamma$ is the inhomogeneously broadened width of the resonance; and $\xi=-0.16$ [24] is a parameter which accounts for an interference between electronic scattering and nuclear scattering for the E1 transition of ${ }^{181} \mathrm{Ta}[22,23]$. The fit used a Fourier transform of the response function. The overlap of the decays resulting from consecutive prompt pulses was taken into account. The best fit (solid line in Fig. 1) was obtained with the width of the nuclear resonance $\Gamma=6 \pm 2 \Gamma_{0}$, corresponding to $22 \pm 7 \mu \mathrm{m} / \mathrm{s}$.

The ability to determine directly the width of the nuclear resonance in a sample is one of the advantages of synchrotron nuclear spectroscopy. In standard Mössbauer spectroscopy the measured linewidth always involves a convolution with the linewidth of the radioactive source. On the contrary, the value obtained here for the inhomogeneous broadening of the nuclear transition, $\Gamma=6 \pm 2 \Gamma_{0}$, is a characteristic of the sample, determined only be the chemical environment of the ${ }^{181}$ Ta nuclei.

The $x$-ray beam created by nuclear scattering in the Ta sample (the delayed, coherent forward scattered radiation), had a bandwidth of about $4.5 \times 10^{-10} \mathrm{eV}$ This qualifies as the most monochromatic beam of synchrotron radiation ever prepared $\left(\Delta \mathrm{E} / \mathrm{E}=7 \times 10^{-14}\right)$. The longitudinal coherence length of such radiation is about $1 \mathrm{~km}$.

This demonstration of the feasibility of time resolved synchrotron radiation hyperfine nuclear spectroscopy using ${ }^{181} \mathrm{Ta}$ promises future developments. The general advantages of this method are particularly apparent in the case of ${ }^{181} \mathrm{Ta}$ spectroscopy, where synchrotron radiation provides almost ideal instrumental resolution and therefore allows direct measurement of the resonance parameters of the sample alone. Thus the extreme narrowness of the ${ }^{181} \mathrm{Ta}$ resonance can in principle be fully exploited for the study of very weak hyperfine interactions. Finally, the pronounced interference between nuclear and electronic scattering in ${ }^{181} \mathrm{Ta}$, observed so far only in absorption, could stimulate new developments in the dynamical theory of resonant nuclear scattering. 


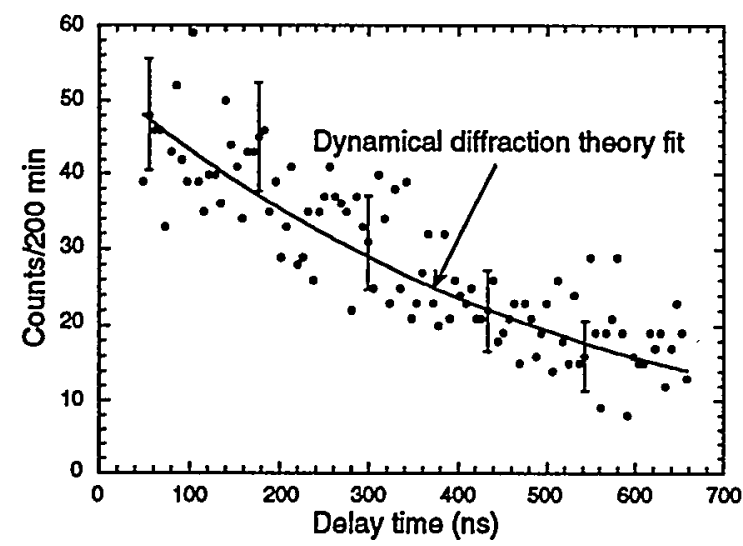

Figure 1. Time distribution of nuclear forward scattering of synchrotron radiation by the $7.5 \mu \mathrm{m}$ Ta foil. Experimental data are fit (solid line) by the dynamical theory of nuclear resonant scattering under the conditions of inhomogeneous broadening of the resonant line. A small background (2.7 counts per channel in the figure) was included in the fit. The width of the nuclear resonance is found to be $\Gamma=6 \pm 2$ $\Gamma_{0}$, corresponding to $22 \pm 7 \mu \mathrm{m} / \mathrm{s}$.

\section{Acknowledgments}

The authors would like to thank the SSRL staff for their tremendous support during the experiment, especially for providing a period of single bunch operation on very short notice. We thank Dr. A. Rogalev from ESRF for the loan of the $\mathrm{NdF}_{3}$ absorber, and Dr. V. I. Zhudov from the Russian Research Center Kurchatov Institute for his measurement of the resonant energy. Support for this research was provided by the US Department of Energy, office of Basic Energy Sciences, under contract DE-AAC03-76SF00515, by the European Synchrotron Radiation Facility, by the Russian Research Center "Kurchatov Institute", and by the German Bundesministerium für Forschung und Technologie under Contract 05 5WOAAI.

\section{References}

1. See the review by E. Gerdau and U. van Bürck, in Resonant Anomalous X-Ray Scattering -Theory and Applications. (Edited by G. Materlik, C. J. Sparks and K. Fisher, Elsevier, 1994), p. 589 and references therein.

2. W. Sturhahn, E. Gerdau, R. Hollatz, R. Rüffer, H. D. Rüter and W. Tolksdorf, Europhys. Lett. 14, 821 (1991).
3. E. E. Alp, T. M. Mooney, T. Toellner, W. Sturhahn, E. Withoff, R. Röhlsberger, and E. Gerdau, Phys. Rev. Lett. 70, 3351 (1993).

4. S. Kikuta, et al., presented at the International Conference on the Applications of the Mössbauer Effect, Vancouver 1993.

5. D. E. Johnson, D. P. Siddons, J. Z. Larese and J. B. Hastings, to be published.

6. A. Q. R. Baron, A. I. Chumakov, S. L. Ruby, J. Arthur, G. S. Brown, U. van Bürck and G. V. Smirnov, to be published.

7. D. Mouchel, A. Nylandsten Larsen, and H. H. Hansen, Z. Phys. A 300, 85 (1981).

8. G. Kaindl, D. Salomon and G. Wortmann, Phys. Rev. B 8, 1912 (1973).

9. A. Heidemann, G. Kaindl, D. Salomon, H. Wipf, and G. Wortmann, Phys. Rev. Lett. 36, 213 (1976).

10. M. Löhnert, G. Kaindl, G. Wortmann, and D. Salomon, Phys. Rev. Lett. 47, 194 (1981).

11. V. K. Voitovetskii, S. M. Cheremisin and S. V. Sazonov, Phys. Lett. A 83, 81 (1981).

12. C. Sauer, Z. Physik 222, 439 (1969).

13. V. A. Dornow, J. Binder, A. Heidemann, G. M. Kalvius, and G. Wortmann, Nucl. Instrum. and Meth. 163, 491 (1979).

14. R. B. Firestone, Nucl. Data Sheets 62, 101 (1991).

15. J. L. Campbell and B. Martin, Z. Physik A 277, 59 (1976).

16. Yu. V. Shvyd'ko, A. I. Chumakov, G. V. Smirnov, V. G. Kohn, T. Hertrich, U. van Bürck, E. Gerdau, H. D. Rüter, J. Metge and O. Leupold, Europhys. Lett. 22, 305 (1993).

17. A. Q. R. Baron, Nucl. Instrum. and Meth. A 352, 665 (1995).

18. J. B. Hastings, D. P. Siddons, U. van Bürck, R. Hollatz, and U. Bergmann, Phys. Rev. Lett. 66, 770 (1991).

19. V. I. Zhudov, private communication (July 1994).

20. J. A. Bearden, Rev. Mod. Phys. 39, 78 (1967). (The reported value has been re-evaluated by the authors using an original value in $\AA$-scale and the currently accepted wavelength-energy conversion factor).

21. Yu. Kagan, A. M. Afanas'ev, and V. G. Kohn, J. Phys. C: Solid State Phys. 12, 615 (1979).

22. G. T. Trammell and J. P. Hannon, Phys. Rev. 180, 337 (1969).

23. Yu. M. Kagan, A. M. Afanas'ev, and V. K. Voitovetskii, Pisma Zh. Eksp. Teor. Fiz. 9, 155 (1969) [JETP Lett. 9, 91 (1969)].

24. G. Kaindl and D. Salomon, Phys. Lett. B 32, 364 (1970). 


\title{
X-ray Absorption Spectroscopic studies of the Superconductor $\mathrm{Ba}_{4} \mathrm{BiPb}_{2} \mathrm{TIO}_{12-\delta}$
}

Ingrid J. Pickering and Graham N. George

Stanford Synchrotron Radiation Laboratory, PO Box 4349, Bin 69, Stanford CA 94309

\author{
Thomas E. Sutto and Bruce A. Averill \\ Department of Chemistry, University of Virginia, Charlottesville, Virginia 22901
}

The superconductor $\mathrm{Ba}_{4} \mathrm{BiPb}_{2} \mathrm{TlO}_{12-\delta}$ is a cubic perovskite with a maximum $T_{c}$ of $10.5 \mathrm{~K}$, and with superconducting properties that are extremely sensitive to the exact atomic composition [1]. The similarity in Xray form factors and neutron scattering cross sections of the component metals ( $\mathrm{Tl}, \mathrm{Pb}$, and $\mathrm{Bi})$ means that the structure of the system is very difficult to characterize using diffraction methods. We have therefore used X-ray Absorption Spectroscopy (XAS) to study the local structure of the metals in two forms of $\mathrm{Ba}_{4} \mathrm{BiPb}_{2} \mathrm{TlO}_{12-\delta}$, an oxygen-annealed, orthorhombic, superconducting form $(\delta=0.9)$ and an argon-annealed, tetragonal, non-superconducting form $(\delta=2.2)$.

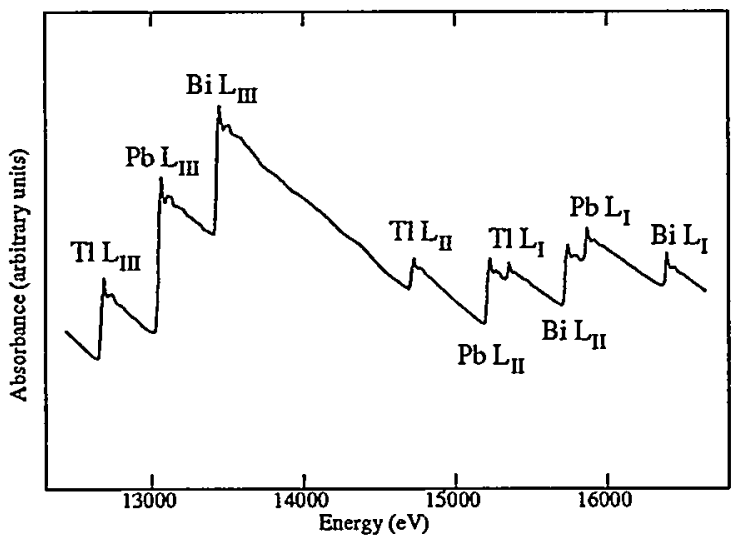

Figure 1: $X$-ray absorption spectrum of oxygenated $\mathrm{Ba}_{4} \mathrm{BiPb}_{2} \mathrm{TlO}_{12-\delta}$ at a temperature of about $4 \mathrm{~K}$.

XAS spectra were collected on beamline 7-3 using the SSRL Biotechnology group Oxford Instruments CF1204 liquid helium cryostat. XAS data were collected above and below $T_{c}(100 \mathrm{~K}$ and close to $4 \mathrm{~K}$, re- spectively). Figure 1 shows the $\mathrm{X}$-ray absorption spectrum of the superconducting oxygen-annealed form of $\mathrm{Ba}_{4} \mathrm{BiPb}_{2} \mathrm{TlO}_{12-\delta}$ between 12.4 and $16.7 \mathrm{keV}$, which encompasses a total of no less than nine absorption edges: the three L X-ray absorption edges of all three of the component metals. The dominant features of $\mathrm{X}$ ray absorption edge spectra are governed by $\Delta l= \pm 1$ electric dipole-allowed transitions. Thus, $\mathrm{L}_{\mathrm{I}}$ spectra are dominated by $2 s \rightarrow \mathrm{np}$ transitions, while $\mathrm{L}_{\mathrm{II}}$ and $\mathrm{L}_{\mathrm{III}}$ edges are dominated by $2 p \rightarrow$ nd transitions. The selection rules for $\mathrm{L}_{\mathrm{II}}$ and $\mathrm{L}_{\mathrm{III}}$ edges differ only with regard to electron spin effects. The $\mathrm{L}_{\mathrm{I}}$ and $\mathrm{L}_{\mathrm{TII}}$ near-edge spectra of all three metals are shown in Figures 2 and 3 , respectively. While little or no differences are observed above and below $T_{c}$, distinct differences are observed between the data from the oxygen-annealed superconducting form, and the argon-annealed nonsuperconducting form. It can be seen from figure 2 that the Tl $L_{\mathrm{I}}$ edge shifts to a lower energy (by about 2.8 $\mathrm{eV}$ ) in the argon treated sample, while the $\mathrm{Pb}$ showed no appreciable shift, and the $B i L_{I}$ edge shifted to higher energy (by about $1.4 \mathrm{eV}$ ). More subtle effects were observed with the $\mathrm{Bi} \mathrm{L}_{\mathrm{III}}$ edges and, while the $\mathrm{Pb}$ $\mathrm{L}_{\mathrm{III}}$ edges again showed no appreciable change (figure 3), quite pronounced differences appear at the $\mathrm{Tl} \mathrm{L}_{\text {III }}$ edge. Thus, the edges seem to suggest that thallium is more oxidized in the $\mathrm{O}_{2}$-treated sample (compared with the Ar-annealed), while lead is unchanged and 
bismuth may be slightly more oxidized in the reduced sample.

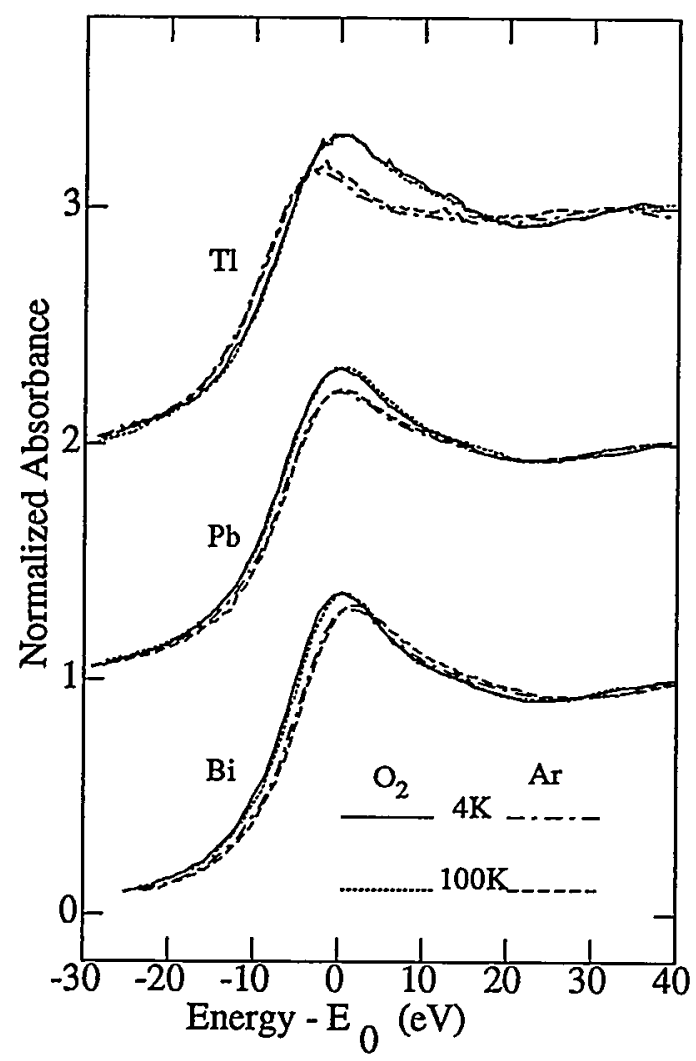

Figure 2: Comparison of $L_{r}$ edge $X$-ray absorption for $\mathrm{Ba}_{4} \mathrm{BiPb}_{2} \mathrm{TlO}_{12-\delta}$ annealed with oxygen and argon, $\mathrm{re}$ corded at temperatures above and below $T_{c}$.

We also examined the thallium, lead and bismuth $\mathrm{L}_{\mathrm{III}^{-}}$ edge EXAFS of $\mathrm{Ba}_{4} \mathrm{BiPb}_{2} \mathrm{TlO}_{12-\delta}$. Only subtle changes, attributable to the expected reduction in Debye-Waller factors, were observed above and below $T_{c}$ (not illustrated). Pronounced differences were observed between the EXAFS of the argon-annealed and the oxygen-annealed sample. Figure 4 compares the EXAFS Fourier transforms of the $\mathrm{L}_{\text {III }}$ EXAFS of the metals. In all cases, the EXAFS are dominated by first shell metal-oxygen interactions, with less intense transform peaks due to metal-metal contacts evident at longer distances. Significant differences are observed between the EXAFS of all three metals, which are particularly striking for the thallium EXAFS.

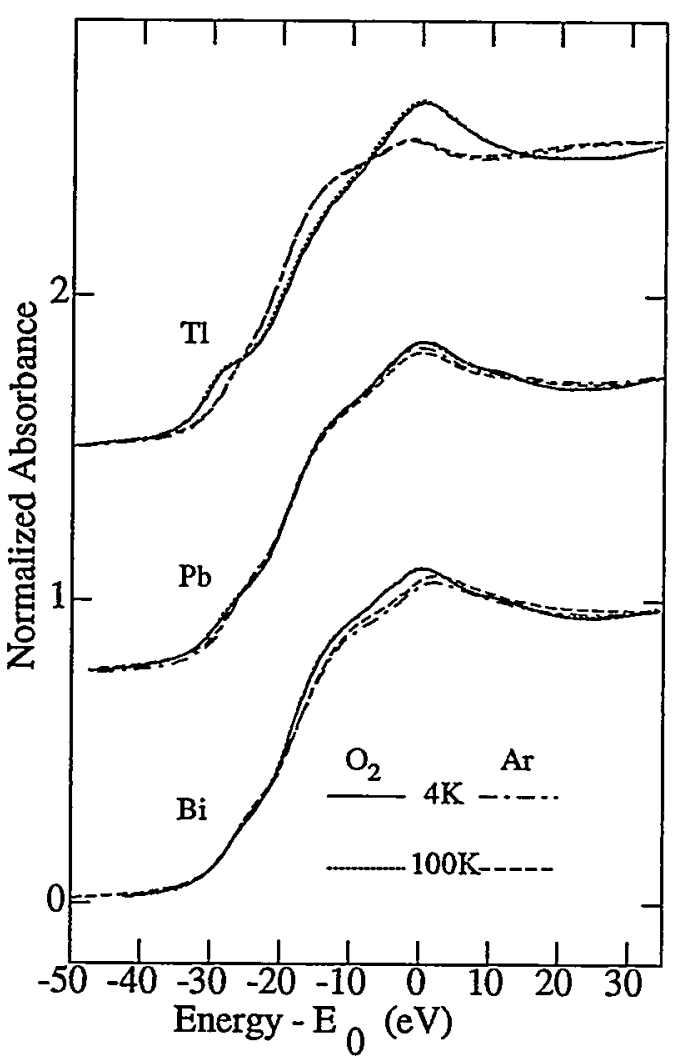

Figure 3: Comparison of $L_{I I r}$ edge $X$-ray absorption for $\mathrm{Ba}_{4} \mathrm{BiPb}_{2} \mathrm{TlO}_{12-\delta}$ annealed with oxygen and argon, recorded at temperatures above and below $T_{c}$

The superconducting form shows a well-defined first shell Tl-O backscattering, with a smaller backscattering at $\sim 3 \AA$. Striking differences are observed with the argon-annealed sample, with the EXAFS amplitude being very much decreased. This suggests a very highly disordered thallium environment in the argon-treated sample. Other, less marked, changes in the EXAFS of the other metals are visible (see figure 4). The bismuth EXAFS of the argontreated sample has slightly decreased amplitude throughout, and the lead EXAFS shows a marked decrease in the amplitude of the Fourier transform peak 
corresponding to $\sim 3.7 \AA$ metal-metal contacts, together with a more subtle decrease in amplitude of the oxygen peak.

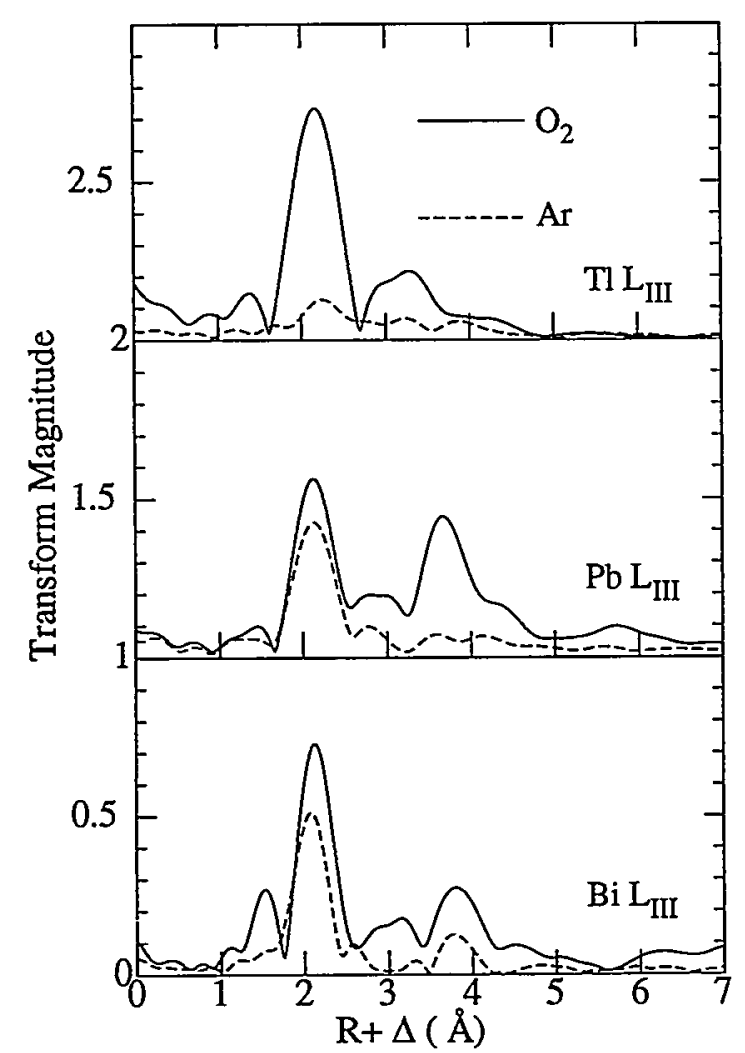

Figure 4. EXAFS Fourier transforms for thallium, lead and bismuth $L_{I I I}$ EXAFS. The $4 \mathrm{~K}$ data are compared for the superconducting oxygen-annealed (solid line) and the non-superconducting argon-annealed (broken line) samples.

Our results indicate that local environments of the metals in the non-superconducting form of $\mathrm{Ba}_{4} \mathrm{BiPb}_{2} \mathrm{TIO}_{12-\delta}$ are considerably more disordered than the metal environments in the superconducting form, and that this is particularly true for the thallium sites. Shifts in the absorption edges also indicate subtle changes in oxidation state.

\section{References:}

(1). Sutto, T.E.; Averill, B.A. Chem. Mater. 1992, 4, 1092-1096. 


\title{
Characterization of Si-TaSi ${ }_{2}$ Composites for Use as Wide-band-pass Optical Element for Synchrotron Radiation.
}

\author{
Z. U. Rek ${ }^{1}$, S. R. Stock ${ }^{2}$, M. Goorsky ${ }^{3}$
}

\author{
1 Stanford Synchrotron Radiation Laboratory \\ 2 Georgia Institute of Technology, Atlanta, GA \\ 3 University of California, Los Angeles
}

Crystal optics for synchrotron radiation typically employs perfect crystals of $\mathrm{Si}$ or Ge. In many cases, however, wide-band-pass monochromators, multilayers or even mosaic crystals could utilize the entire beam divergence and to increase the intensity available for an experiment [1]. There were a number of successful attempts to increase the band-pass of a perfect crystals by mechanical lapping of silicon wafers [2] or silicon annealing [3] to produce oxygen precipitation within the wafer.

A very promising material for broad-band optics seems to be the in situ composite $\mathrm{Si} / \mathrm{TaSi}_{2}$. Developed by GTE as a novel electronic material [4], the composite is obtained by directional solidification of semiconductor-metal eutectic mixtures in a Czochralski crystal growth system. With the addition of 5.5 wt\% of $\mathrm{Ta}$ to the charge, an array of $\mathrm{TaSi}_{2}$ rods aligned with the growth direction is produced in the single crystal Si matrix. The composites studied contain about $1.6 \times 10^{6}$ rods $/ \mathrm{cm}^{2}$ with an average rod diameter of $1.2 \mu \mathrm{m}$ and an average interrod spacing of $7.9 \mu \mathrm{m}$. Structural characterization of matrix's defect structure and of preferred orientation of the rods was previously performed using white beam $x$-ray diffraction and double-crystal diffraction [5].

The rocking curves taken with double-axis diffractometer for (111), (333) and (004) reflections and with $\mathrm{CuK} \alpha$ radiation gave very smooth peaks with FWHM about 20 times wider than those for perfect silicon crystals. Crystal reflectivity, however, decreased by a factor of four. Comparison of the results for a composite wafer and a perfect silicon crystal is presented in the followiong table. In order to check whether the large FWHM from the matrix of the composite was due to long range lattice curvature, rocking curves were recorded with different-sized irradiated area. Gradual change from $.4 \times 1 \mathrm{~mm}^{2}$ to $4 \times 12 \mathrm{~mm}^{2}$ resulted in $20 \%$ broadening of (333) rocking curve from 59 to 79 arc sec. Even with the very small beam size, FWHM remained very large, suggesting that the broadening is not due to long range curvature of the diffraction planes.

$$
\begin{array}{lll}
\text { Cuk } \alpha & \mathrm{Si}-\mathrm{TaSi}_{2} & \mathrm{Si}
\end{array}
$$

\begin{tabular}{|c|c|c|c|}
\hline Reflection & $\begin{array}{c}\text { FWHM } \\
\text { [arc sec] }\end{array}$ & $\begin{array}{c}\text { Peak } \\
\text { Reflectivity }\end{array}$ & $\begin{array}{c}\text { FWHM } \\
\text { [arc sec] }\end{array}$ \\
\hline (111) & 150 & $20 \%$ & 7.4 \\
\hline$(333)$ & 58 & $8 \%$ & 1.99 \\
\hline$(004)$ & 64.8 & $17 \%$ & 3.6 \\
\hline
\end{tabular}

Triple-axis diffractometry was employed to measure the reciprocal space maps around (111) and (333) reflections. An example, the (111) reciprocal space map, is presented in Fig.1. It shows the quite complex nature of deformation in the matrix of the composite: significant mosaic spread and compressive strain are identified. Despite the complex geometry and deformations in the matrix, $x$-ray transmission topography of wafers cut perpendicular to the crystal growth does not reveal non-uniformities, and, thus, these composites could be used as an optical element for imaging experiments. The (111) oriented wafer was utilized as a monochromator for radiography experiments. The images with composite monochromator were obtained 15-20 times faster than images of the same samples 
with a perfect silicon monochromator and with equal image quality [6]. The same (111) oriented $\mathrm{Si}-\mathrm{TaSi}_{2}$ crystal was used successfully as an analyzer crystal in high resolution synchrotron $x$-ray diffraction tomography of polycrystalline materials $[7,8]$.

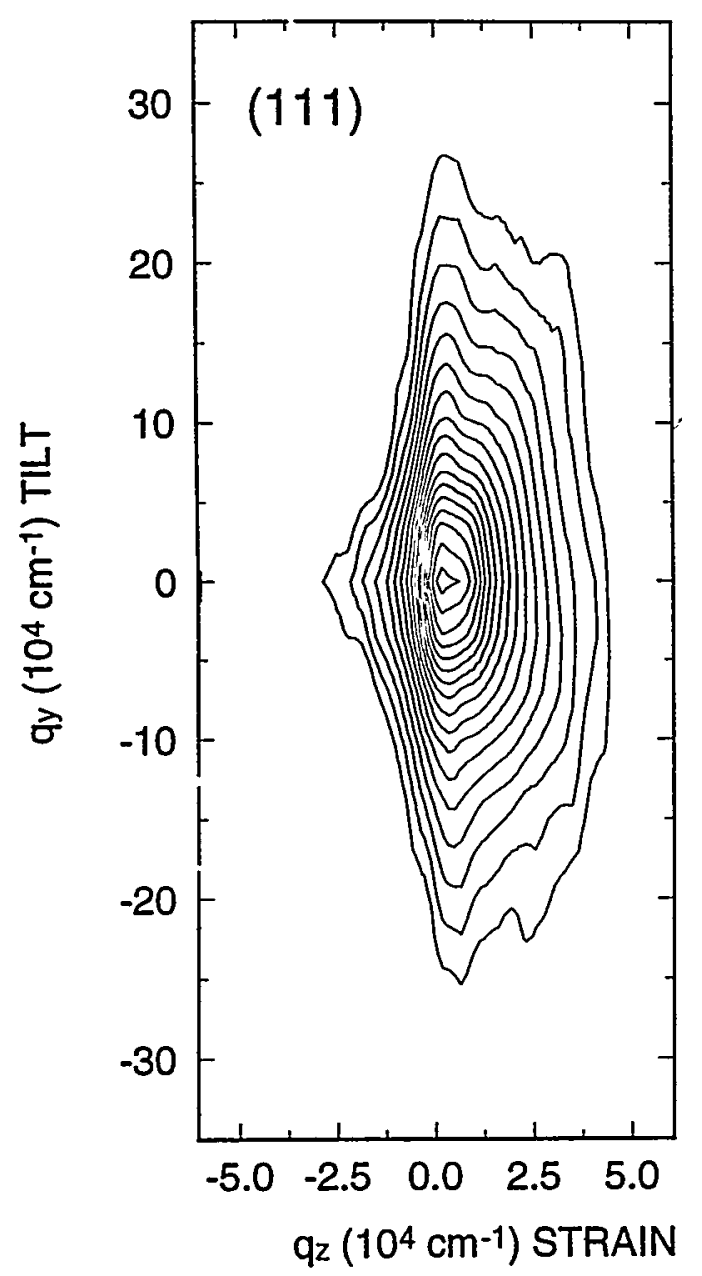

Figure 1

The (111) reciprocal space map of $\mathrm{Si}_{-} \mathrm{TaSi}_{2}$ composite wafer
Despite the geometrical complexity and the large fraction of material under significant strain, the $\mathrm{Si}^{-\mathrm{TaSi}_{2}}$ composites have far greater crystallographic perfection than many of bulk or layered semiconductors and seems to be very suitable for applications such as large area photodiodes and photovoltaic devices as well as very interesting material for synchrotron radiation optics. Further work on understanding the nature of deformation is in progress as is an extension of the study to three inch $\mathrm{Si} / \mathrm{TaSi}_{2}$ wafers.

\section{References}

1. A. Freund, SPIE, 1740, (1992).

2. H. Shiwaku, K. Hyodo, M. Ando, Jap. J. Appl. Phys., 30, 2065, (1991).

3. P. Zaumseil, U. Winter, S. Joksch, A. Freund, Rev. Sci. Instrum., 63, 907, (1992).

4. B.M. Ditchek, M. Levinson, Appl. Phys. Lett., 49, 1656, (1986).

5. S.R. Stock, Y. Chung, Z.U. Rek, B.M. Ditchek, J. Appl. Phys., 73, 1737, (1993).

6. Z.U. Rek, S. R. Stock, M. Goorsky, in preparation. 7. S.R. Stock, A. Guvenilir, D.P. Piotrowski, Z.U. Rek, ibid, experimental report, proposal 2264Mp. 8. S.R. Stock, A. Guvenilir, D.P. Piotrowski, to be published in Mat. Res. Proc., (1995). 


\title{
Correlation of the growth parameters of $\mathrm{YB}_{66}$ with defect structure and crystal performance as soft $x$-ray monochromator.
}

\author{
Z.U. Rek ${ }^{1}$, T. Tanaka ${ }^{2}$, M. Rowen ${ }^{1}$, Joe Wong ${ }^{3}$ \\ 1. Stanford Synchrotron Radiation Laboratory \\ 2. National Institute for Inorganic Materials, Japan \\ 3. Lawrence Livermore National Laboratory
}

$\mathrm{YB}_{66}$ crystals proved to be very good monochromators for soft $x$-ray energy range 1-2 keV [1,2]. Apart from the fact that the monochromators work efficiently already, the resolution and reflectivity are not yet optimal. Both of these parameters did not yet match the theoretical values. The crystal growth of this material is quite a complicated process. The single crystals of $\mathrm{YB}_{66}$ have been grown by indirect heating floating zone method [3]. In this method, a molten zone is heated by radiation from an inductively heated tungsten ring which is situated between the molten zone and a RF coil.

Crystals grown were systematically characterized using synchrotron radiation reflection and transmission $\mathrm{x}$-ray topography and double-crystal rocking curve measurements using $\mathrm{CuK} \alpha$ and several reflections, such as (400), (10 0 0), (12 00$),(1400)$ and (16 000$)$. They were finally tested as an actual monochromator on line
3-3. An appearance of grain structure and growthinduced defects $[4,5]$ as a function of crystal growth parameters and position on the crystal were mapped out. As a result, a number of changes in the crystal growth process were introduced in order to achieve higher crystal quality and reproducible growth. Some of them were reported earlier [4]. In particular, the downwards drive of the feed rod and a crystal during crystal growth combined with incongruent growth, where the composition $[\mathrm{B}] /[\mathrm{Y}]$ for molten zone and the growing crystal were different, gave higher quality crystals. The central part of the crystal was very uniform, (400) rocking curves, measured for $\mathrm{CuK} \alpha$ were ca 97 arc sec. The crystal \#6757, obtained at this stage of technology was used as monochromator for EXAFS measurements in Jumbo monochromator on beam line 3-3. The energy resolution of $0.3 \mathrm{eV}$ for $\mathrm{E}=1200,0.45 \mathrm{eV}$ for $\mathrm{E}=1500 \mathrm{eV}$ and $1 \mathrm{eV}$ for $\mathrm{E}=2000$ was obtained, fig. 1.

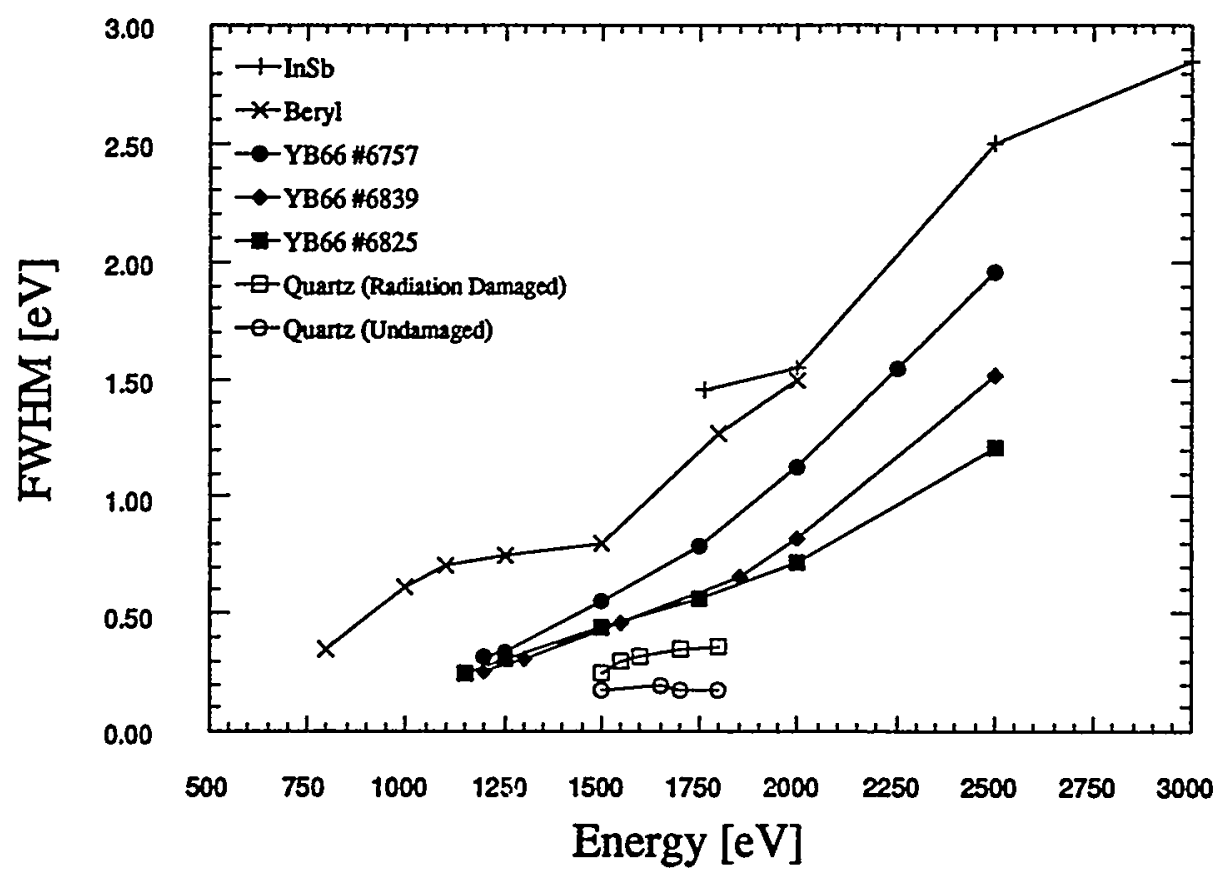

Figure 1. Comparison of rocking curve widths of $Y_{66}$, Beryl, Quartz and InSb. 


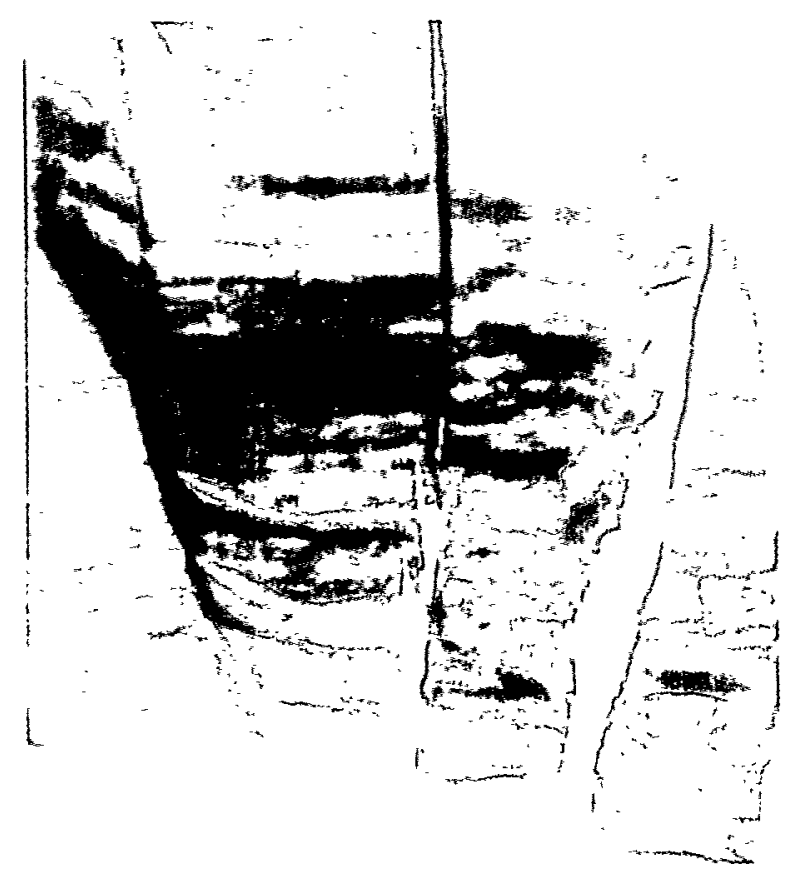

Figure 2. Reflection topograph of crystal \#6830, grown at high heating power.

Further improvements in crystal growth technology included sequentially: 1 . impurity addition, such as scandium to minimize number of grain boundaries and dislocation densities, 2. thorough studies of effect of heating power on crystal defect structure, 3 . change of the growth direction from [001] to [110] in order to solve a persisting faceting problem. A crystal grown with the addition of scandium, \#6825, was of high crystal quality, with a few grain boundaries located at the very side of crystal, $(400)$ rocking curve of the order of 85 arc sec and energy resolution better than for crystal \#6757, fig.1. The reflectivity, however, was low and fluorescence background high due to accidental contamination with iron during crystal growth. The effect of heating power has a crucial effect on a defect structure of grown crystals. This dependence was evaluated from white beam reflection and transmission topography. The crystal growth related defect structure differs dramatically for high and low heating power. In the first case, fig. 2, a large number of grain boundaries and lattice deformations were observed, with angular distribution of more than 1000 arc sec, dependent on the location in the crystal. The reason is a vibration of molten zone which may be induced by a strong convection in molten zone. As a contrast, the topograph of crystal \#6840, grown at lower heating power condition reveals less sub-grain boundary structure and higher crystal quality, with (400) rocking curve width of ca 80 arc sec, fig. 3. One of the important improvements seems to be growth in [110] direction. $\mathrm{YB}_{66}$ exhibits the strong tendency to faceting. The (100) plane shows a stronger faceting property than (110) and (210), respectively. In a growth parallel to the $[100]$ direction, the faceting is responsible for the subgrains boundaries mainly at the peripheral region of the crystal, only the central facet has a high quality. The growth along higher index axis and even about 10 degrees off towards [210] direction seems to help the problem of faceting. Several crystals were grown this way. The results are very good in the case of crystal \#6839 with only one grain boundary observed at the upper part of image, fig. 4. The (400) rocking curves width were 70-75 arc sec. Energy resolution obtained for this crystal was better than for the other two crystals, fig. 1. In the case of this crystal, the single dislocations were resolved in transmission topography for the first time.

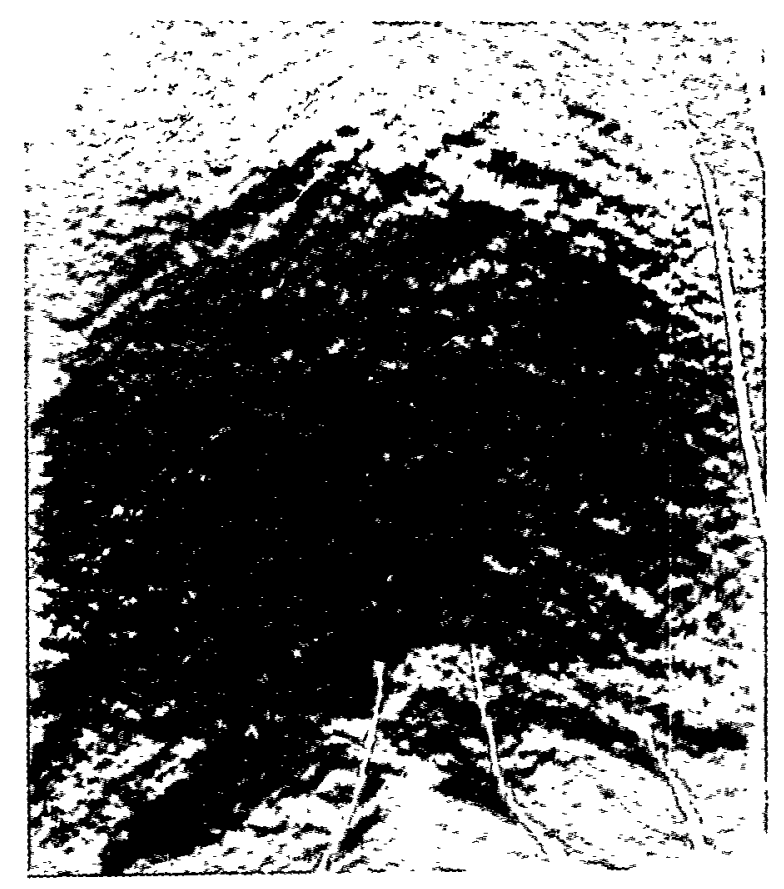

Figure 3. Reflection topograph of crystal \#6840 grown at low heating power

All recently obtained crystals are of high quality, but did not yet achieve the theoretical values for rocking curve width and reflectivity. The calculated value for (400) rocking curve, CuK $\alpha$ is of the order of $2 \operatorname{arcsec}$. 
In the measurements of higher order reflections, from $(1000)$ up to $(1600)$, the FWHM values of rocking curves are nearly constant. This indicates a very high mosaicity. Hard X-ray rocking curve measurements

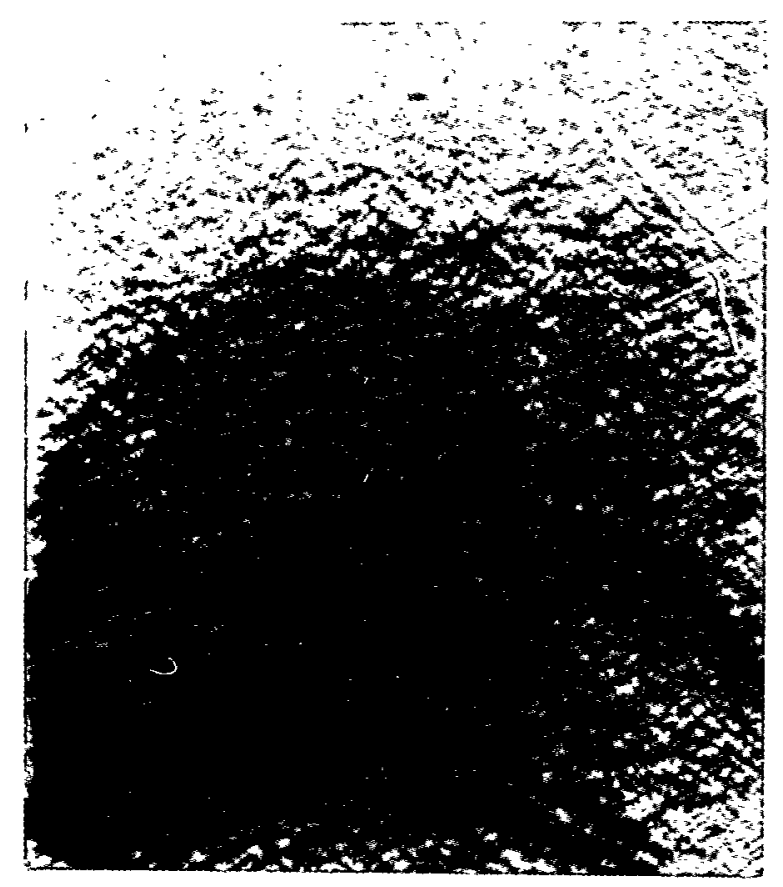

Figure 4. Reflection topograph of crystal \#6839 grown with [110] growth direction and low heating power. are more sensitive to observe this effect, because of the small Bragg angle and large area illuminated even with the small beam size. Data obtained with soft $x$ rays are much closer to theoretical, because the Bragg angle is very large and the beam sees only a small selected area. The detail analysis of these studies is in preparation [6]. Further planned improvements in crystal growth are expected to bring a big change in the reducing of crystal mosaicity.

\section{References}

1. J. Wong, G.N. George, IJ. Pickering, Z.U. Rek, M. Rowen, T. Tanaka, G.A. Via, B. DeVries, G.E. Brown, Solid St. Com., 92, 559, (1994).

2. M. Froba, J. Wong, M. Rowen, G.E. Brown, T. Tanaka, Z.U. Rek, Physica B,208 \& 209, 555, (1995) 3. T .Tanaka, S. Otani, Y. Ishizawa, J. Crystal Growth, 99, 994, (1990).

4. Z.U. Rek, J. Wong, T. Tanaka, Y. Kamimura, F. Schaffers, B. Muller, M. Krumrey, P. Muller, SPIE, 1740, 173, (1992).

5. T. Tanaka, Y. Ishizawa, J. Wong, Z.U. Rek, M. Rowen, F. Schaffers, B.R. Muller, Jpn. J. Appl. Phys., 10, 110, (1994).

6. Z.U. Rek, T. Tanaka, M. Rowen, J. Wong, in preparation. 


\author{
Thermal Studies of Ceramics \\ E. D. Crozier and G. J. Soerensen \\ Physics Department, Simon Fraser University \\ Burnaby, B. C., V5A 1S6, Canada \\ T. Tiedje ${ }^{+}$and T. Troczynski* \\ Department of Physics and Electrical Engineering ${ }^{+}$ \\ Department of Metals and Materials Engineering* \\ University of British Columbia, Vancouver, B. C., V6T 1Z1, Canada
}

Introduction

The 31-pole wiggler, beamline 10-2, SSRL, operating in the focussed, "white light" mode delivers 125 watts of $\mathrm{x}$-ray power to a $0.5 \times 5 \mathrm{~mm}$ area (power density $50 \mathrm{MW} / \mathrm{m}^{2}$ ). This is sufficient to create point defects and vacancies in irradiated materials and to achieve surface melting. In our initial experiments we have explored the feasibility of joining ceramics.

Recent technical breakthroughs in advanced ceramics cannot be fully exploited because of the lack of reliable joining technologies. ${ }^{1}$ Techniques investigated previously have involved: liquid state bonding through surface melting, or welding, of pure surfaces with laser or electron beams; deposition of a secondary phase at the interface (liquid eutectic phases, glassy phases, metallic brazes).

The high energy laser and electron beam techniques inconsistently produce good ceramic-toceramic joints. The crucial factors causing the inconsistency are the lack of control of microstructure, volume of solidifying material, and excessive thermal stresses. The key to success with the high energy beam techniques is the ability to achieve the melting point in a relatively thin (10-50 $\mu \mathrm{m})$ and uniform surface layer in a short time $(<10$ sec). This is difficult to accomplish if the energy is deposited only on the surface and dissipated only through poor thermal conduction (thermal conductivities of many ceramics of interest are in the range 1 to $30 \mathrm{~W} / \mathrm{mK}$ ).

$X$-rays offer the advantage of penetrating surfaces to a depth controlled by the $x$-ray energy and absorption properties of the material. The absorption can be controlled by suitable doping techniques.

The focussing on beamline $10-2$ is achieved with a Pt mirror, band pass $4 \mathrm{keV}$ to $20 \mathrm{keV}$. The absorption length of alumina is $22 \mu \mathrm{m}$ at the lower energy, $1160 \mu \mathrm{m}$ at the higher energy and averaged over the bandpass range is $415 \mu \mathrm{m}$. Thus the $\mathrm{x}$-ray energy is deposited throughout the subsurface region and does not rely entirely upon thermal conduction to transport the heat to the bulk of the join area.

The absorption length of a typical hightechnology zirconia ceramic for the same range is 45 $\mu \mathrm{m}$. Zirconia, $\mathrm{ZrO}_{2}$, is selected to show the advantage of doping. Zirconium has a K-absorption edge at $18 \mathrm{keV}$. The absorption length in $\mathrm{ZrO}_{2}$ is 4 $\mu \mathrm{m}$ at $4 \mathrm{keV}$, just below the K-edge is $132 \mu \mathrm{m}$ and just above it is $16 \mu \mathrm{m}$. It should be possible to vary the percentage of heavy elements to produce the appropriate absorption length in a ceramic.

\section{Preliminary Experiments}

In February, 1994 we did exploratory x-ray joining experiments on beamline 10-2, SSRL. A computer-controlled sample positioner was designed and constructed at Simon Fraser University to enable the remote positioning of samples in the $\mathrm{x}$-ray beam. In the experiments, two tubes or rods were rotated in the $x$-ray beam while under a longitudinal compression. To control the heating rate the rotation speed could be varied to give linear scan rates across the sample between $0.1 \mathrm{~mm} / \mathrm{s}$ and $300 \mathrm{~mm} / \mathrm{s}$.

Results

Silica tubes: a successful end-to-end or butt seal was made. The quality of the join region is revealed by inspection of the circular band on the right in Fig. 1. After the join was completed, the rotating silica tube was slowly raised through the $\mathrm{x}$ ray beam depositing silica on the cooler region and forming the white band on the top left in Fig. 1.

Sapphire rods: a butt seal was made but recrystallization occurred and the join was not mechanically strong.

Alumina and zirconia rods and tubes: we were unsuccessful in joining rods and tubes. The high power density $\mathrm{x}$-rays incident on the samples while at room temperature caused the rods or tubes to fracture. In an attempt to preheat the ceramics, they 
were gradually moved into the fluorescing air sheath around the $x$-ray beam and then eventually into the main $x$-ray beam. In this way the ceramics did not fracture immediately. In some samples it seemed

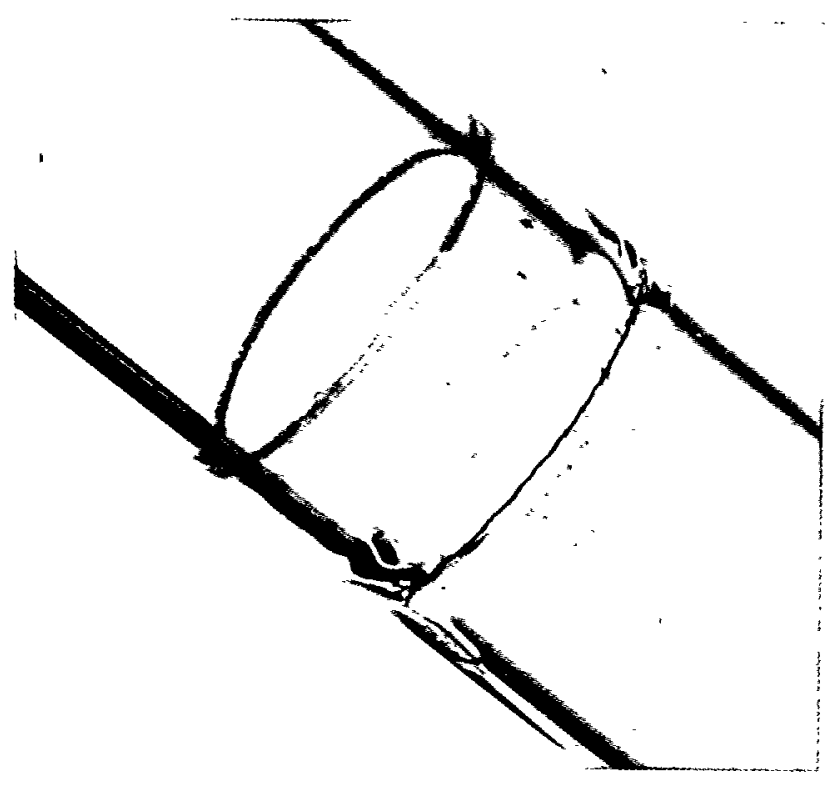

Figure 1: Silica tubes joined with focussed $x$-rays.

that a join may have been achieved but when removed from the sample holder there was no join.

\section{Conclusions}

We have successfully demonstrated that materials with high thermal conductivity and low thermal expansion coefficient can be joined using focussed $x$-rays available at SSRL. But ceramics with low thermal conductivity and/or larger expansion coefficients pose problems.

During our next run we will attempt to determine the conditions under which these ceramics can be joined consistently. Particular attention will be given to the effect of the irradiation on the ceramic microstructure.

The assistance of Hal Tompkins and Tom Hostetler of SSRL is much appreciated. This work was supported by a grant from the Natural Sciences and Engineering Research Council, Canada.

1 W. A. Zdaniewski, American Ceramic Society Bulletin, March, 1993. 


\section{Structure in Rigid Polymers and in Nafion Membranes}

Paul S. Russo, J. Cheng and I. Negulescu

Department of Chemistry, Louisiana State University, Baton Rouge, LA 70803

Robert B. Moore

Department of Polymer Science, University of Southern Mississippi, Hattiesburg, MS 39406

Beam station I-4 was used to conduct small angle X-ray scattering (SAXS) measurements on several polymer systems, discussed separately.

1. Fuzzy rod polypeptides containing an $\alpha-$ helical backbone and long, flexible sidechains exhibit a very complex melting transition to a liquid crystalline state, and are the longest, most rigid molecules known to form thermotropic liquid crystals. X-ray powder diffractometer measurements suggested that very long-range order could develop in well-annealed samples of poly $(\gamma$-stearyl- $\alpha, L$-glutamate), PSLG. Those measurements were at the limits of instrument resolution for our local diffractometer. Using the SAXS instrument at station I-4, a sharp, lowangle peak (at scattering vector $\mathrm{q} \approx 2 \times 10^{6} \mathrm{~cm}^{-1}$ corresponding to about $300 \AA$ ) was cleanly resolved, as shown in Figure 1. The meaning of this observation is not yet clear, but smectic ordering of these large rods is not ruled out. Additional studies were conducted in which the PSLG fuzzy rods were mixed with polyethylene.

2. PSLG fuzzy rods were covalently crosslinked with diamines and triamines. Different concentrations of crosslinker and polymer were used. After solvent exchange and critical point drying in $\mathrm{CO}_{2}$, lightweight aerogels containing fuzzy rods were obtained. The SAXS results so far (see Figure 2) are consistent with small pore sizes (60-170 $\AA$ ) and sharp interfaces (Porod regime slopes of -4 ). Production must still be developed to improve the consistency of these materials and to align the rodlike molecules, but these results are promising for the production of nanostructured polypeptide-based materials.

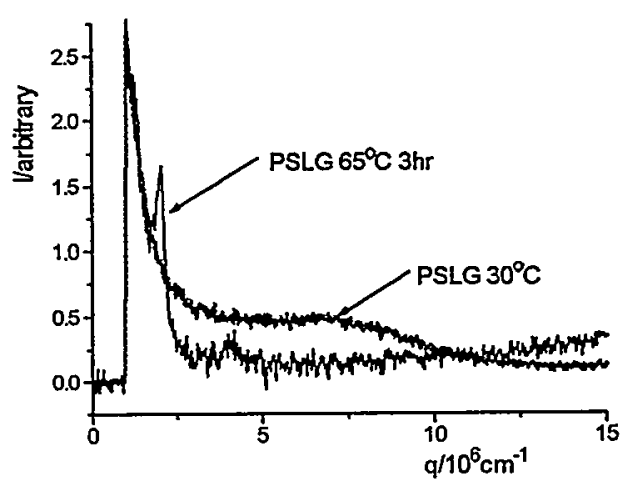

Figure 1. PSLG develops long-range order on annealing.

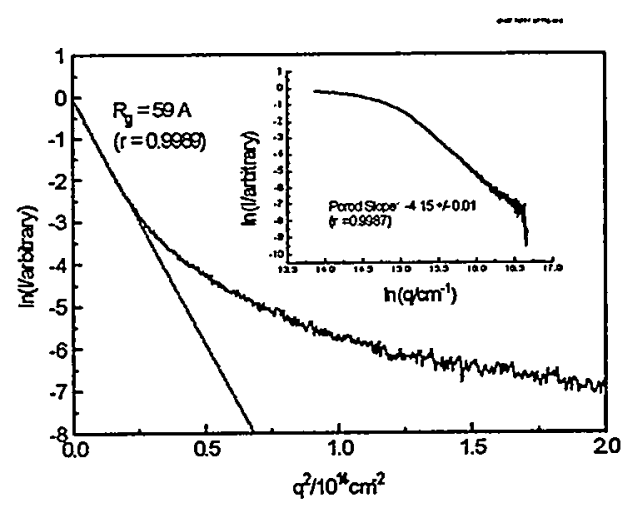

Figure 2. Guinier plot showing small correlation length and log-log plot (inset) for PSLG aerogel. 
3. Small angle $X$-ray scattering (SAXS) studies were carried out on $\mathrm{Na}^{+}$-form Nafion $(1100 \mathrm{EW}$ long side chain) and Dow (808 EW - short side chain) perfluorosulfonate ionomers. Samples of the PFSI membranes were heated to $330^{\circ} \mathrm{C}$, quenched to room temperature, and then annealed at $200^{\circ} \mathrm{C}$ for 6 and 24 hours. Figures 3 and 4 show intensity versus scattering vector plots of Nafion PFSI before and after thermal treatments. The prominent shoulder centered at about 0.4 reciprocal nanometers in the as received PFSI's results from inter-crystalline domain scattering and corresponds to a Bragg spacing of about $150 \AA$. The lack of a shoulder in the quenched samples implies that crystallinity can be eliminated or drastically diminished by thermally quenching the PFSI from above its crystalline melting temperature. The SAXS profiles of annealed samples show the profound effect of side chain length on the ability of the ionomers to recrystallize. Annealing, up to 24 hours, at $200^{\circ} \mathrm{C}$ has a negligible effect on recrystallization of the short side chain Dow ionomer while the longer side chain Nafion PFSI is able to recrystallize to a significant extent. It appears that the longer side chains allow the polymer backbone enough mobility to reorganize into crystalline domains.

This work is supported by the National Science Foundation, Polymers Program (DMR-9202434) and by Dow Chemical to PSR and by the National Science Foundation, Electric Power Research Institute (DMR-9211963) and the Air Force Office of Scientific Research (F49620-93-10189DEF) to RBM.
SAXS of $\mathrm{Na}^{+}$-form Nafion

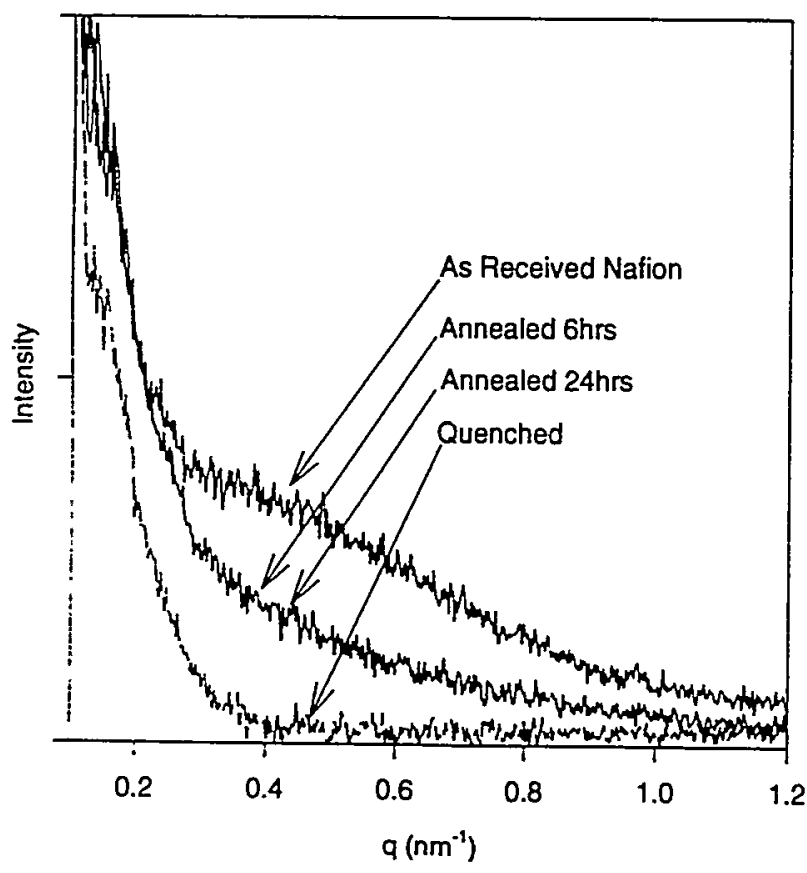

Figure 3. Nafion $\mathrm{Na}^{+}$-form SAXS plot. SAXS of $\mathrm{Na}^{+}$-form Dow

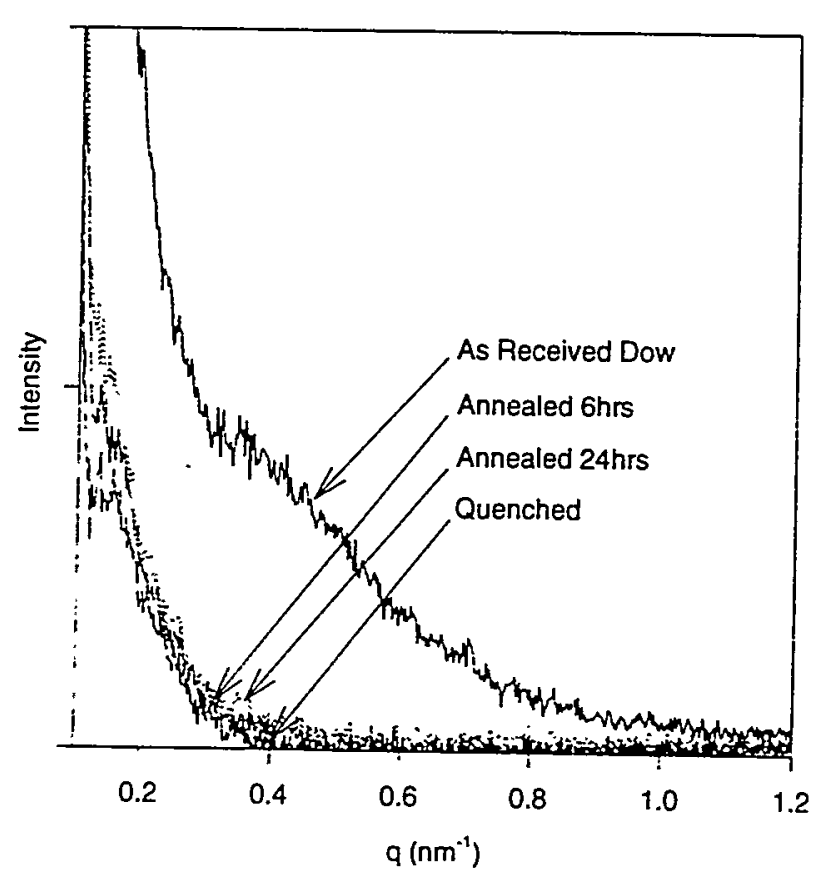

Figure 4. Nafion $\mathrm{Na}^{+}$-form SAXS plot (Dow). 


\title{
Grazing Incidence XAS of Titania Sol-Gel Films
}

\author{
Tracey Hanley and Russell F Howe \\ Department of Physical Chemistry, University of New South Wales, Sydney, Australia 2052 \\ Ingrid Pickering
}

Stanford Synchrotron Radiaition Laboratory, Stanford, CA 94309-0210

Titania thin films prepared by sol-gel methods have several important applications which exploit the semiconductor properties of titania; as photocatalysts in environmental remediation[1], in photovoltaic cells[2] and in photochromic windows[3], for example. The optical and photoelectronic properties of titania can be modified by doping with transition metal ions [4]. This project is concerned with optimizing the properties of doped titania sol-gel films for such applications. As part of that project, it is essential to know the structure of the films, the location of the dopant ions and the coordination environment in which they are found. The thin films made in this way are highly porous, and less than $100 \mathrm{~nm}$ thick. Conventional bulk characterization techniques therefore lack sensitivity when applied to these systems.

The geometry of total external reflection surface sensitizes the EXAFS and XANES techniques. Below the critical angle, X-ray absorption can be measured both in the relected beam or by fluorescence, and the region analyzed corresponds to a depth of ca $3 \mathrm{~nm}$. Depth profiling is possible in principle by varying the angle of incidence above the critical angle and measuring the fluorescence signal. Several studies of this type have been reported in recent literature [5-8]

This report describes results of initial feasibility studies carried out with titania films doped with iron and chromium using a grazing incidence instrument at SSRL. The purpose of these first experiments was to establish the feasibility of the method for such samples, and to determine the concentration range of dopant ions that could be usefully studied.

The samples investigated in this study were sol-gel coatings of doped and undoped titania of approximately $50 \mathrm{~mm}$ thickness. The doped sols

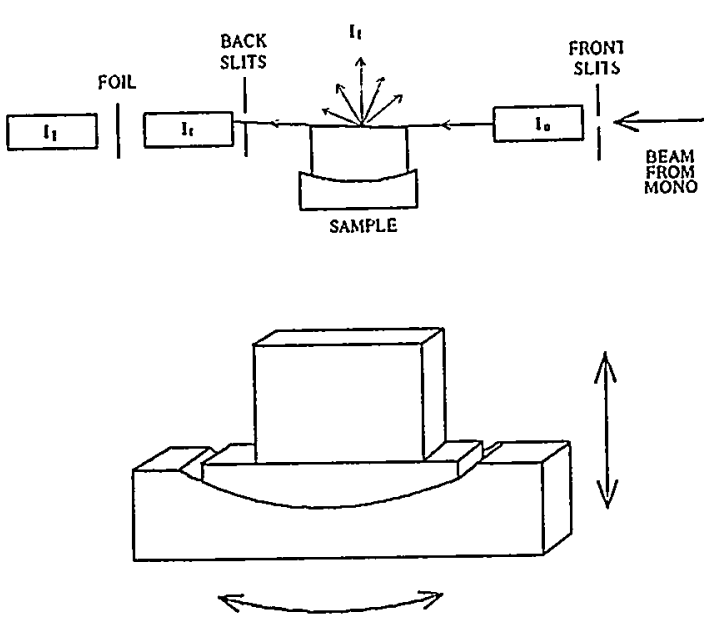

Figure 1: Schematic of grazing incidence experiment.

were produced by adding the appropriate metal salt to the peptising sol. The films were prepared by dip-coating quartz substrates into aqueous sols of titamia. The films were then left to air dry creating a green film. Some films were then fired at $100^{\circ} \mathrm{C}$ for 24 hours and then at $350^{\circ} \mathrm{C}$ for 24 hours to produce the $350^{\circ} \mathrm{C}$ fired films. A second set of films were fired further to $800^{\circ} \mathrm{C}$ for 4 hours to produce the $800^{\circ} \mathrm{C}$ fired films. Films investigated were $0.5 \%, 1 \%$ and $5 \%$ Fe doped titania, $1 \%$ and $5 \%$ $\mathrm{Cr}$ doped titania and undoped titania. Measurements were made of the EXAFS of the dopant $K$ edge and of the EXAFS and XANES of the Ti $K$ edge.

The grazing incidence experimental setup is illustrated in Figure 1. The samples were mounted on a motorised sample holder which had the ability to change the sample height, position and angle of the sample. The incident and reflected beams were monitored with ion chambers and the fluorescence 
signal with a 13 element Canberra solid state detector. The optimum angle for EXAFS was found by scanning the reflected and fluorescent intensities as a function of the angle of incidence, the angle corresponding to the maximum fluorescent yield was chosen (Figure 2). The sample is then fixed at this angle (just below the critical angle, typically $300 \mathrm{mdeg}$ ) and the EXAFS data is acquired in the usual manner.

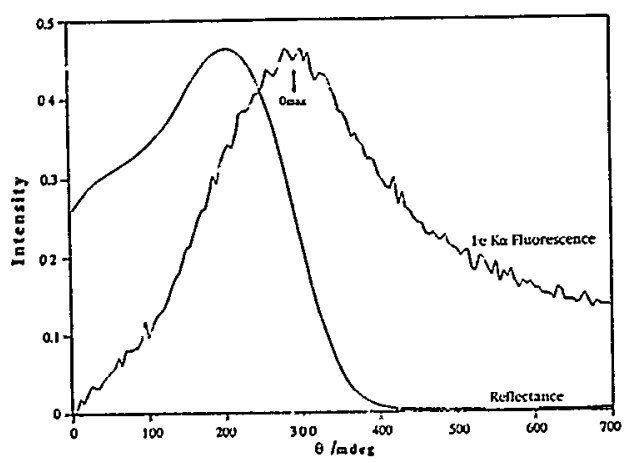

Figure 2: Reflected beam and fluorescence intensity as a function of angle for an Fe doped titania film.

The $\mathrm{Ti} \mathrm{K}$ edge measurements were acquired relatively quickly under these conditions. Freshly prepared films gave XANES spectra typical of anatase, and the Fourier transforms of the EXAFS are similar to published data for bulk anatase ( quantitative curve fitting analysis of the EXAFS is currently in progress). An unexpected result was that films fired to $800^{\circ} \mathrm{C}$ still gave a XANES spectrum of anatase. Bulk gels prepared under similar conditions can be completely transformed from anatase to rutile at $500-600^{\circ} \mathrm{C}$.

Measurement of the $\mathrm{Fe}$ or $\mathrm{Cr} \mathrm{K}$ edges required considerably longer accumulation times. The dopants were measured at levels of 5,1 and $0.5 \mathrm{wt}$ $\%$. Figure 3 shows examples of data measured from a $1 \% \mathrm{Fe}$ doped film (each spectrum typically measured for 5-6 hours).
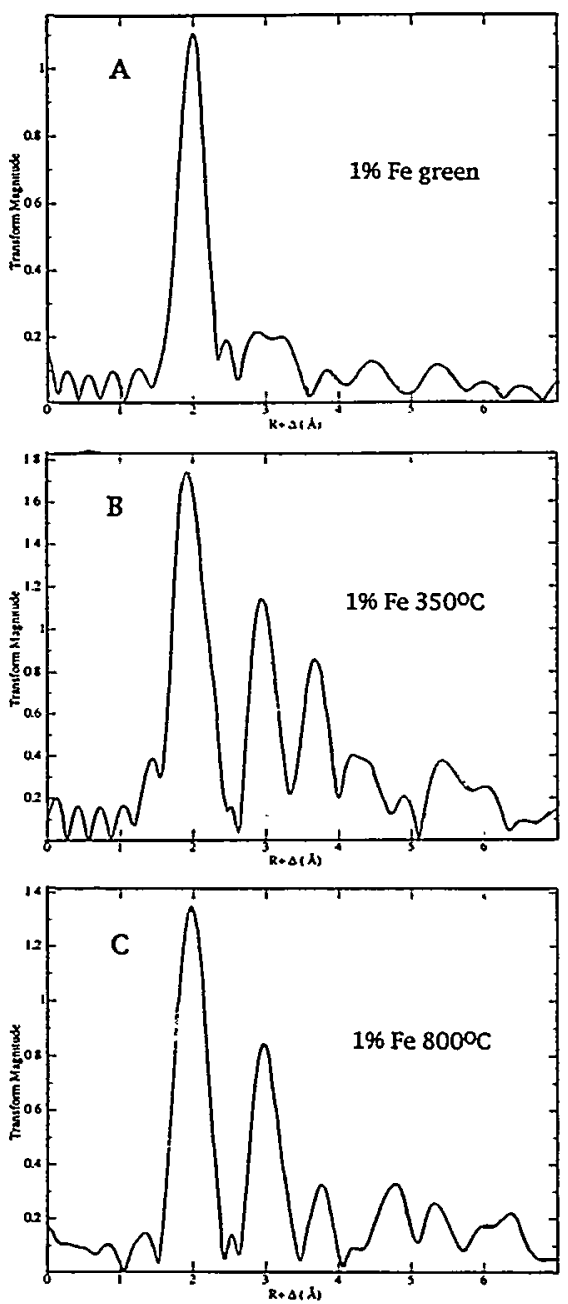

Figure 3: Fourier transforms of grazing incidence Fe K edge EXAFS from $1 \% \mathrm{Fe}$ doped titania films. A: as prepared; B: fired at $350 \mathrm{C}$; C: fired at $800 \mathrm{C}$.

The Fourier transform of the Fe K EXAFS from the freshly prepared film shows a single peak indicating $\mathrm{Fe}$ is adsorbed at the surface of the anatase nanocrystals ( the Fe doped sol is prepared by adding $\mathrm{Fe}^{3+}$ to the peptizing solution). After firing at $800^{\circ} \mathrm{C}$ however the Fe EXAFS develops second, third and possibly fourth coordination shell features in the Fourier transform. The resulting Fourier transform is closely similar to that of the Ti K EXAFS from the same sample. Quantitative curve fitting of the EXAFS is not yet complete, but the preliminary interpretation of the data is that on firing iron substitutes for $\mathrm{Ti}$ in the anatase lattice.

Figure 4 shows corresponding data for $5 \%$ and $0.5 \%$ doped titania films fired at $800 \mathrm{C}$, together with that for the Ti $K$ edge of anatase. These data 
indicate that complete substitution of $\mathrm{Fe}$ into in the anatase lattice does not occur at higher dopant levels.
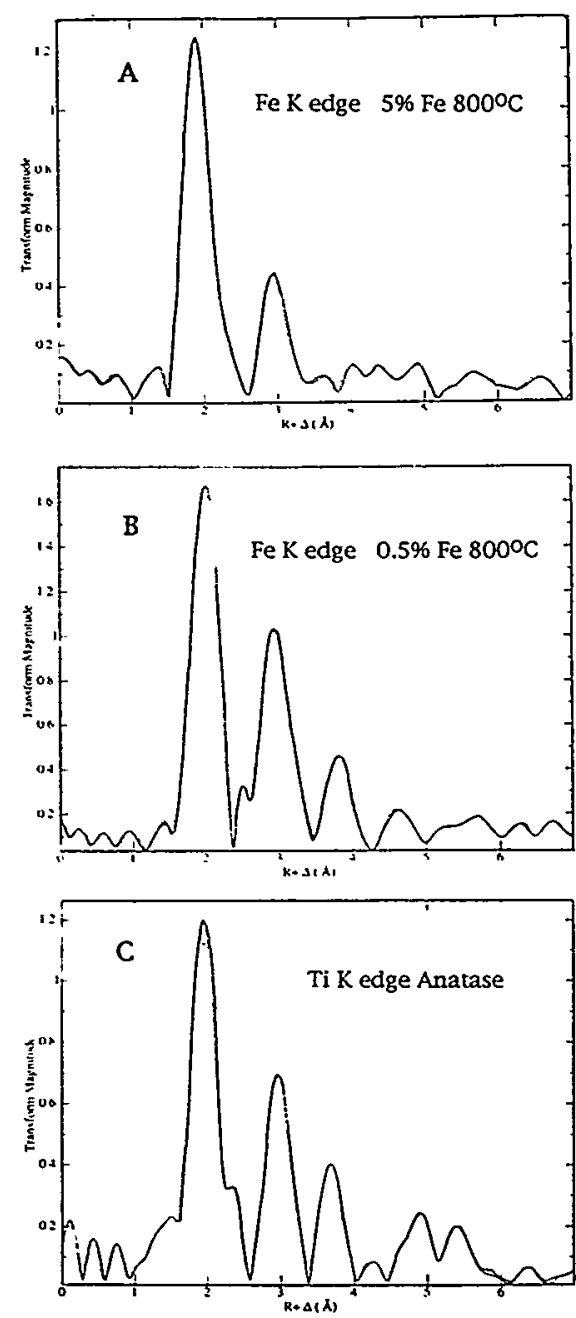

Figure 4: Fourier transforms of grazing incidence EXAFS measured from A: $5 \% \mathrm{Fe}$ doped titania film fired at $800 \mathrm{C} ; \mathrm{B}: 0.5 \% \mathrm{Fe}$ doped titania film fired at $800 \mathrm{C}$; C: pure titania film.

In the case of $\mathrm{Cr}$ doped films, XPS experiments previously undertaken at UNSW indicated that surface chromate species were present in freshly prepared films. This was confirmed from the grazing incidence XAS measurements. The intensity of the $\mathrm{Cr} \mathrm{K}$ edge decreased dramatically on firing the $\mathrm{Cr}$ doped films at successively higher temperatures; the corresponding changes in the $\mathrm{XANES}$ and EXAFS indicate a transition from adsorbed chromate to possibly $\mathrm{Cr}$ incorporated into the anatase. The loss of intensity would suggest diffusion of $\mathrm{Cr}$ away from the surface of the anatase, although depth profile measurements are needed to confirm this preliminary interpretation. Satisfactory data could not be measured from $\mathbf{C r}$ doped samples at less than $1 \%$ in the time available.

The conclusions reached from these preliminary measurements were that the grazing incidence technique is certainly appropriate for structural analysis of the doped titania films. Analysis at dopant levels of $1 \%$ or less ( the concentration range needed to ensure phase homogeneity) does however place extreme demands on signal to noise and hence X-ray flux.

RFH and TH acknowledge support from the Access to Major Research Facilities Program of the Australian Government. This project is part of a collaborative research project with the Australian Nuclear Science and Technology Organization.

1. M.A.Anderson et al., Chem.Tech. 1993(5), 21

2. B.O.Regan and M.Graetzel, Nature 353,257 (1991).

3. "Sol-Gel Optics", Society of Photo-optical Instrumentation Engineers, 1328 (1990)

4. R.F.Howe and M.Graetzel, J.Phys.Chem. 94,2566(1990).

5. S.M.Heald,E.Keller and E.A.Stern, Phys.Lett. 103A,155(1984).

6. S.Pizzini et al., Rev.Sci.Inst. 60,2525 (1989).

7. N.T.Barrett et al., J.Phys.D 22,542 (1989).

8. N.T.Barrett et al., Surface Science 227,337

(1990). 


\title{
Low-Energy X-Ray Dosimetry Studies (7 to $17.5 \mathrm{KeV}$ ) with Synchrotron Radiation ${ }^{*}$
}

\author{
N. E. Ipe, H. Bellamy, J. R. Flood, K. R. Kase, and P. Phizackerley \\ Stanford Linear Accelerator Center, Stanford University, Stanford, California 94309 \\ and \\ K. J. Velbeck and R. Tawil \\ HARSHAW/BICRON, 6753-I Cochran Road, Solon, Ohio 44139
}

Unique properties of synchrotron radiation, such as its high intensity, brightness, polarization, and broad spectral distribution (extending from $x$-ray to infrared wavelengths) make it an attractive light source for numerous experiments. As synchrotron radiation facilities are rapidly being built all over the world, they introduce the need for low-energy $\mathrm{x}$-ray dosemeters because of the potential radiation exposure to experimenters. However, they also provide a unique opportunity for low-energy $\mathrm{x}$-ray dosimetry studies because of the availability of monochromatic $\mathrm{x}$-ray beams. Results of such studies at the Stanford Synchrotron Radiation Laboratory are described. Lithium fluoride

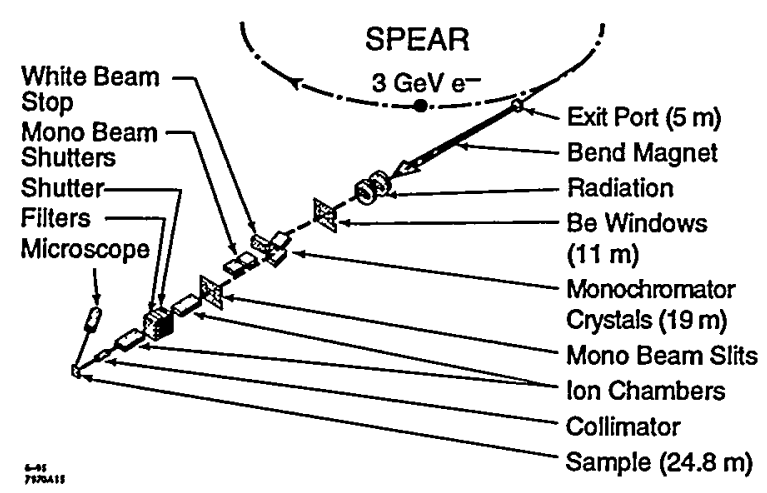

Fig. 1. Schematic of SSRL Beamline 1-5.
(TLD-100) TLDs of thicknesses varying from 0.015 to $0.08 \mathrm{~cm}$ were exposed free in air to monochromatic $\mathrm{x}$-rays, 7 to $17.5 \mathrm{keV}$. These exposures were monitored with ionization chambers. The response (nC/Gy) was found to increase with increasing TLD thickness and with increasing beam energy. A steeper increase in response with increasing energy was observed with the thicker TLDs; responses at 7 and $17.5 \mathrm{keV}$ were within a factor of 2.3 and 5.2 for the 0.015 and $0.08 \mathrm{~cm}$-thick TLDs, respectively. The effects of narrow (beam size smaller than the dosemeter) and broad (beam size larger than the dosemeter) beams on the response of the TLDs are also reported.

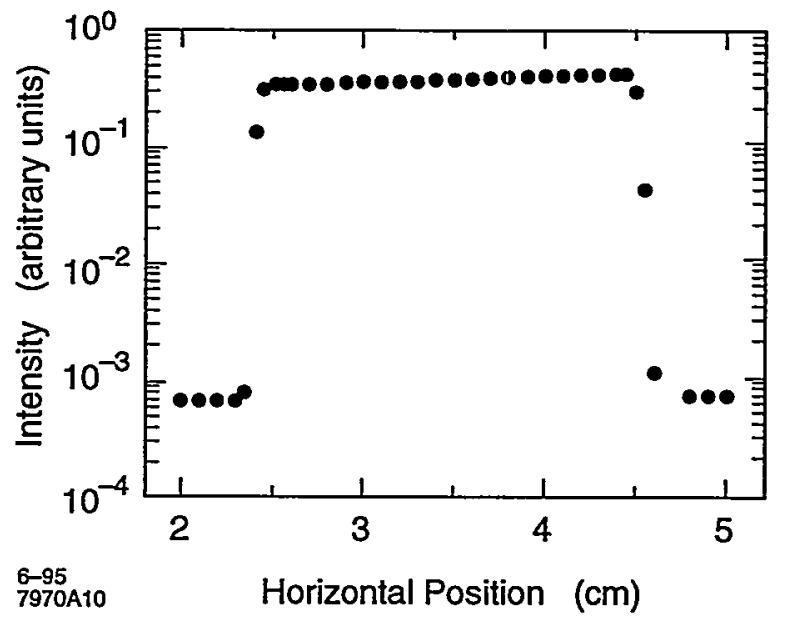

Fig. 2. Horizontal beam profile.

\footnotetext{
"Work supported by Department of Energy Contract DE-AC03-76SF00515.
} 


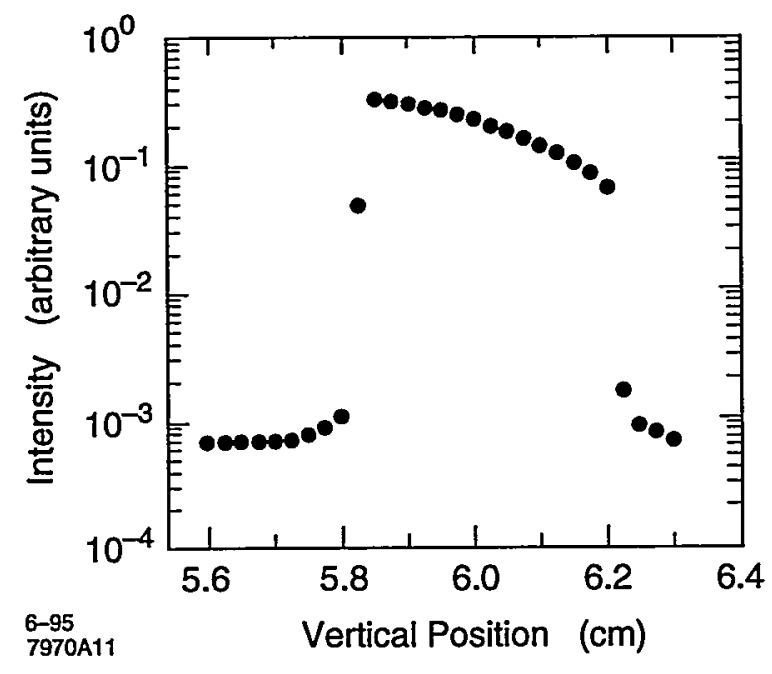

Fig. 3. Vertical beam profile.

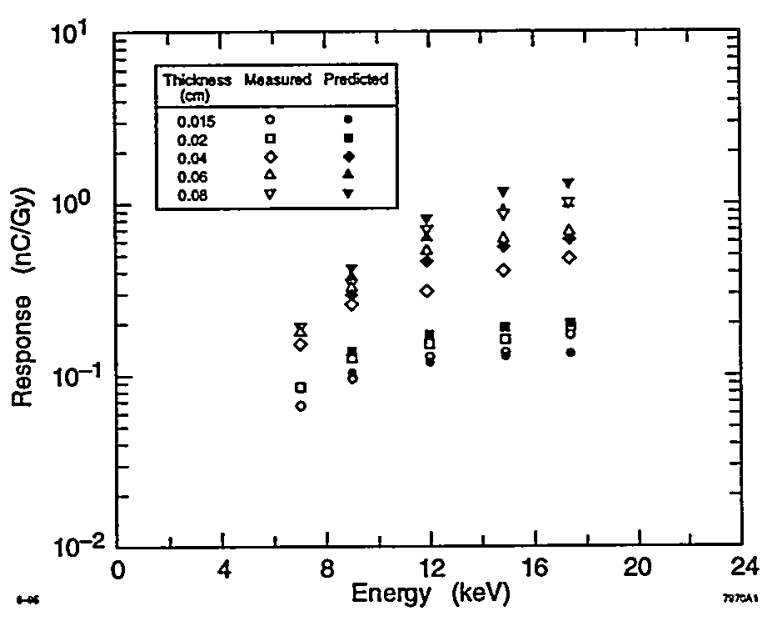

Fig. 4. Measured and predicted responses of $\mathrm{LiF}$ TLD-100 as a function of energy for various TLD thicknesses.

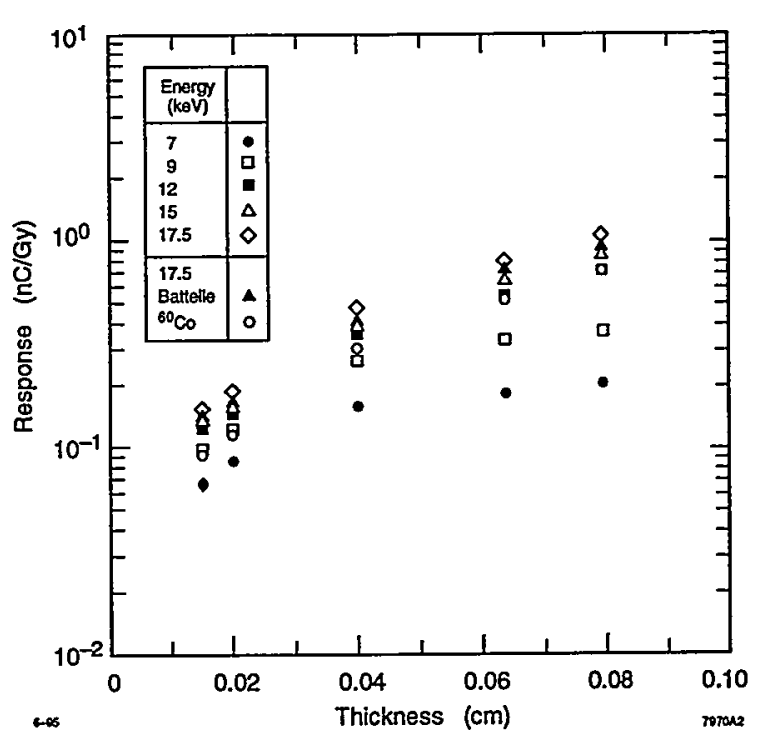

Fig. 5. Measured response of LiF TLD-100 as a function of thickness for various energies.

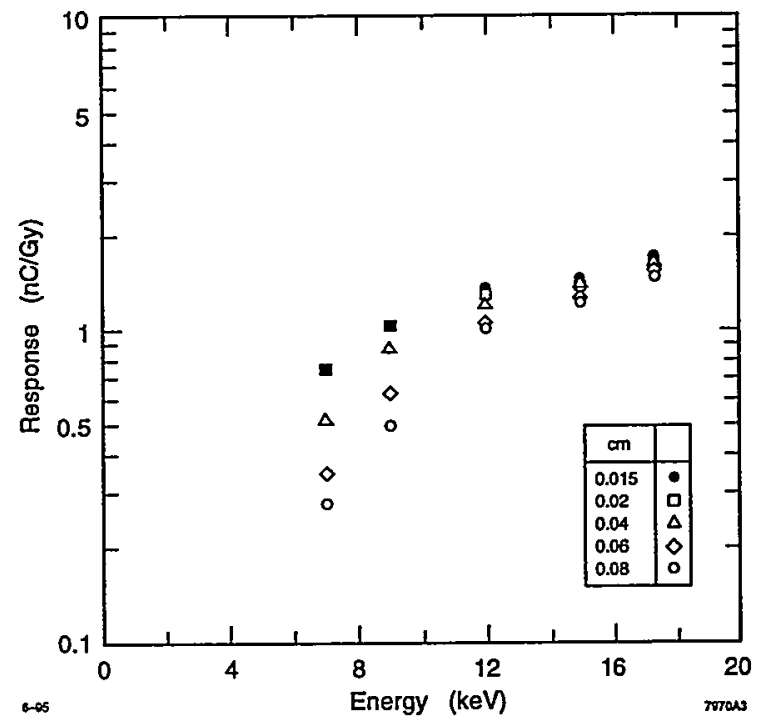

Fig. 6. Measured response of LiF TLD-100 relative to ${ }^{60} \mathrm{Co}$ as a function of energy for various TLD thicknesses. 


\title{
TXRF R \& D STUDIES
}

\author{
S. Laderman and A. Fischer-Colbrie \\ Hewlett-Packard Laboratories, Palo Alto, CA 94304 \\ A. Waldhauer, S. Brennan, N. Takaura and P. Pianetta \\ SSRL/SLAC, Stanford, CA 94309
}

This program covers research and development activities aimed at testing the possibility that synchrotron radiation sources might become a practical means of usefully extending total reflection $\mathrm{X}$-ray fluorescence analyses for mainstream application to the development, improvement, and maintenance of ULSI manufacturing processes. Total reflection X-ray fluorescence (TXRF) analyses using rotating anode sources are routinely used throughout the semiconductor industry. Nearly every major manufacturer of leading-edge VLSI circuits and many materials and equipment suppliers to these companies have one or more conventional TXRF installations in order to measure trace metal contamination on silicon wafer surfaces. Conventional TXRF equipment is typically reported to have a sensitivity to iron or nickel of nearly $5 \times 10^{9}$ atoms $/ \mathrm{cm}^{2}$ This high sensitivity to impurity contamination, efficient sample handling procedures, mapping and depth profiling capabilities, and the ease of reliably quantifying the data all contributed to the success of the method. However, current advanced wafer surface preparation methods can reproducibly create silicon surfaces that are free of transition metal impurities, such as iron, nickel and copper, to a level below the detection limit of widely available measurement methods, including conventional TXRF. Without more sensitive measurement methods, it is difficult to maintain, evaluate, and improve the processing technology. The motivation for the work reported here was this need to develop improved wafer surface analysis methods.

Prior work in this program established that with an appropriate configuration, the use of synchrotron radiation could improve TXRF detection limits to better than $3 \times 10^{8}$ atoms $/ \mathrm{cm}^{2}[1,2,3,4]$. This is more than an order of magnitude better sensitivity than is achieved with typical conventional equipment. Work continues to further improve the sensitivity and realize high sensitivities for all elements of interest, and to further test the concept by attempting prototypical applications.

This past year, SSRL's microcontamination program expanded its scope and accelerated its technical progress. Specifically, the program expanded

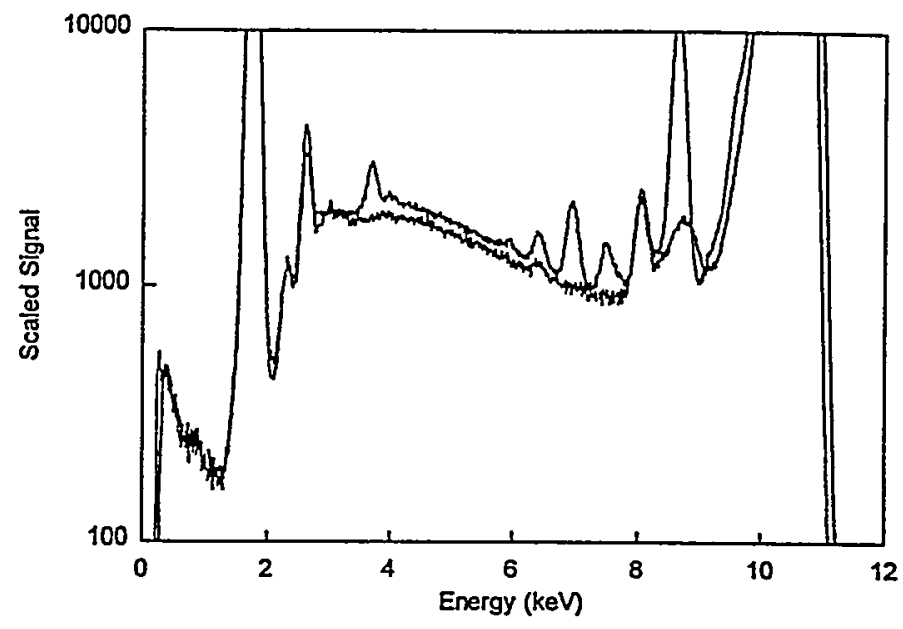

Fig. 1 Synchrotron radiation TXRF spectra from a very clean silicon wafer (upper) and from an as received wafer from a mainstream silicon wafer vendor (lower).
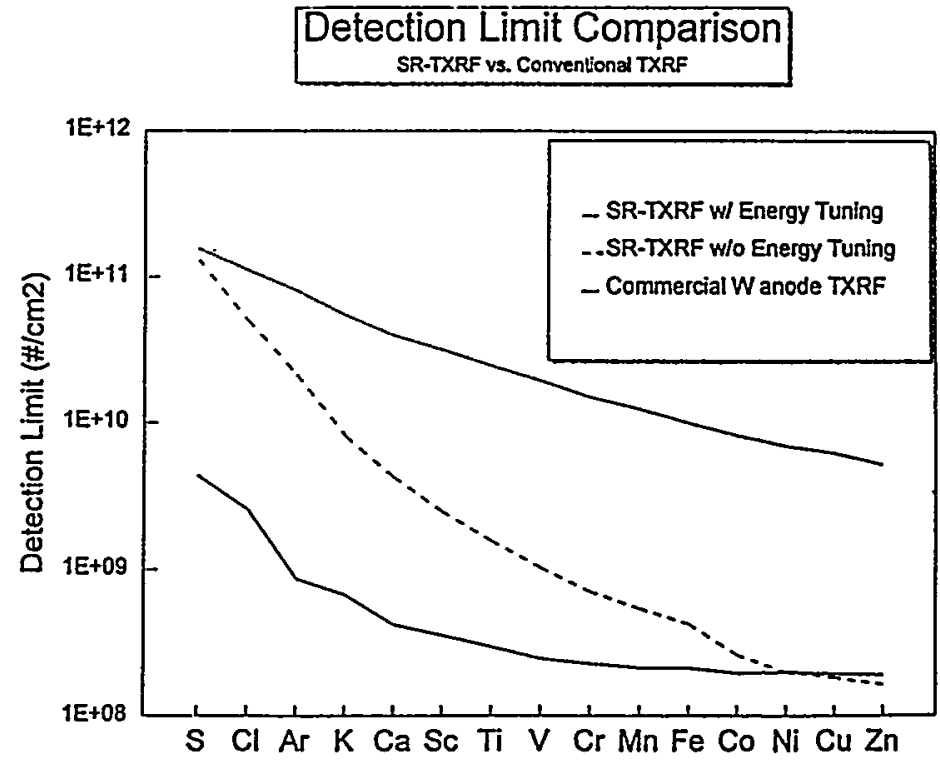

Fig. 2 Self-consistent detection limits for various elements using: conventional TXRF (dot-dash), synchrotron radiation using an incident $\mathrm{X}$-ray energy near $10 \mathrm{keV}$ (dash), and synchrotron radiation TXRF using an incident energy optimized for each element (solid). 
to include the formal involvement of seven U.S. semiconductor companies. In addition, the HewlettPackard Company and SSRL/SLAC signed a CRADA to jointly improve and test critical system components. Together, these activities continue SSRL's worldwide leadership role in this application, as evidenced by worldwide interest in the results and an expanding user base.

\section{Sematech Experiments}

Last August, a periodic meeting of the Sematech Analytical Lab Managers Working Group was convened in Santa Clara, CA, and devoted to prospective developments in $\mathrm{X}$-ray metrology methods for the semiconductor industry. In addition to Sematech member company representatives, staff members from SSRL, ALS, and DOE attended. Largely as a result of that meeting, the Sematech group committed to jointly exploring synchrotron radiation TXRF at SSRL through coordinated experimental investigations.

To date, experiments have been performed at SSRL by microcontamination analysis experts from Advanced Micro Devices (AMD), Digital Equipment Corporation (DEC), Hewlett-Packard Company (HP), International Business Machines (IBM), Intel, Motorola, and Texas Instruments (TI). Another round of experiments will be performed during June, 1995. In September, 1995, a joint report including data and conclusions will be issued. The data from one example of such experiments are shown in Figure 1. In Figure 1, synchrotron radiation TXRF spectra from a very clean wafer and from an "as-received" wafer from a mainstream wafer vendor are shown. The precision to which the clean wafer can be seen to be clean is more than an order of magnitude better with the synchrotron source than with conventional equipment. Interestingly, the as-received wafer is shown, by the synchrotron radiation TXRF spectra, to have measurable metallic impurities on the surface. With the exception of $\mathrm{Zn}$, these levels are well within the capabilities of SR TXRF but too low to be measured with conventional equipment. Similar prototypical applications have been and will soon be explored. After further analysis, self-consistent results from the various processes and fabrication facilities under test will be presented this year.

\section{SSRL/SLAC and HP CRADA}

Detection limits for various elements of interest to the semiconductor manufacturing community are shown in Figure 2 for the cases of conventional TXRF, synchrotron radiation TXRF at a fixed incident energy optimized for elements near $\mathrm{Ni}$ in atomic number, and synchrotron radiation TXRF optimized for each element in turn. We currently expect further improvements in the configuration to lead to even higher sensitivities in the case of synchrotron radiation TXRF, but little if any further improvement in the sensitivity of the conventional equipment. The curves displayed in Figure 2 have been directly derived from experimental data in the cases of conventional and single energy synchrotron radiation TXRF. Pending further analysis, the curve representing detection limits with the current apparatus and optimized for each element is calculated with the assumption that the background level will be fairly insensitive to changes in incident energy. Data testing this assumption are still being reviewed.

In order to achieve these detection limits for all the elements shown in Figure 2, detector improvements were required. The detector assembly needed to be reengineered to remove parasitic fluorescence peaks excited by X-rays scattered by the sample. The reengineering of the detector, its acquisition for the project, and its careful testing were the primary goals of the CRADA. Work to date has led to the removal of a parasitic Ni peaks and the diminishment of parasitic $\mathrm{Fe}$, and $\mathrm{Cu}$ peaks to the level of $5 \times 10^{8}$ and $2 \times 10^{9}$ atoms $/ \mathrm{cm}^{2}$ equivalent impurity levels, respectively. Furthermore, experimental apparatus improvements required to accurately test the detector changes were performed. These included the addition of efficient sample alignment algorithms and limited multipoint or mapping capability. Such improvements were leveraged to greatly benefit the Sematech member company trial runs described above. These improvements greatly increased the rate of technical learning over the past year.

\section{Future Prospects}

The results of the activities summarized here continue to attract worldwide attention. Invited and contributed presentations have been given or are committed to be given at numerous public scientific meetings. As awareness of the activities grows, inquiries continue to come in from staff at wafer vendors, semiconductor companies, and government research laboratories in the U.S. and abroad. The user base is therefore expected to further expand next year.

Further work is also planned to develop and test synchrotron radiation TXRF configurations with even higher sensitivities, to add greater wafer mapping capability, and to lay the groundwork for putting in place higher throughput apparatus capable of more contamination-free handling procedures. These steps are motivated by the desire to better utilize the capabilities of synchrotron radiation TXRF in practical cases. 
1. S.S. Laderman, Bull. Am. Phys. Soc., 39, 514 (1994).

2. P. Pianetta, N. Takaura, S. Brennan, W. Tompkins, S.S. Laderman, A. Fischer-Colbrie, A. Shimazaki, K. Miyazaki, M. Madden, D.C. Wherry and J.B. Kortright, Rev. Sci. Instrum., 66 (2), (1995).

3. A. Fischer-Colbrie, S.S. Laderman, S. Brennan, N. Takaura, P. Pianetta, A. Shimazaki, K. Miyazaki, J. Kortright and D.C. Wherry, Proceedings of the Second International Symposium on Ultra-clean Processing of Silicon Surfaces, ed. by M. Heyns, p. 57, Acco, Leuven/Amersfoort, 1994.

4. S.S. Laderman, A. Fischer-Colbrie, A. Shimakazi, K. Miyazaki, S. Brennan, N. Takaura, P. Pianetta and J.B. Kortright, Analytical Sciences (Japan), June, 1995. 


\section{S K-Edge XANES on Lubricant Additives}

\author{
S.D.CAMERON, R.S.POLIZZOTTI, \\ M.SANSONE \\ Exxon Research \& Engineering Co. \\ Annandale, NJ, 08801
}

X-ray Absorption Near-Edge Spectroscopy has provided the opportunity to measure changes in the bulk and surface states of the sulfur and phosphorous for two classic anti-wear/anti-friction lubricant additives, zinc dialkylthiophosphate $\{Z D D P\} \&$ molybdenum dithiocarbamate $\{\mathrm{Mo}$ DTC .

The figure below shows the sulfur XANES spectra taken from a $5 \mu \mathrm{m}$ mylar sheet with a 300 angstrom Fe film sputter deposited on the surface. The film grown in ZDDP (A) shows two distinct oxidation states. Oxidation of the sulfidic $\left\{\mathrm{R}^{-\mathrm{S}^{-}} \mathrm{M}^{+}\right\}$form to the sulfate $\left\{\mathrm{SO}_{4}{ }^{2-}\right\}$ is evident from the growth of the peak at $2479.8 \mathrm{eV}$. MoDTC (B), however, shows a heavily oxidized film with very little parent compound remaining in the film. Along with the S K-edge, a Mo L-edge appears with a distinctive pair of peaks at 2522.9 and $2525.4 \mathrm{eV}$. When equal weights of both compounds are added (C), a third S-state is observed from this binary mixture indicating a new coordination involving both the Mo-DTC and ZDDP in an aerobic environment.

\section{S K-edge XANES FROM Fe FILMS}

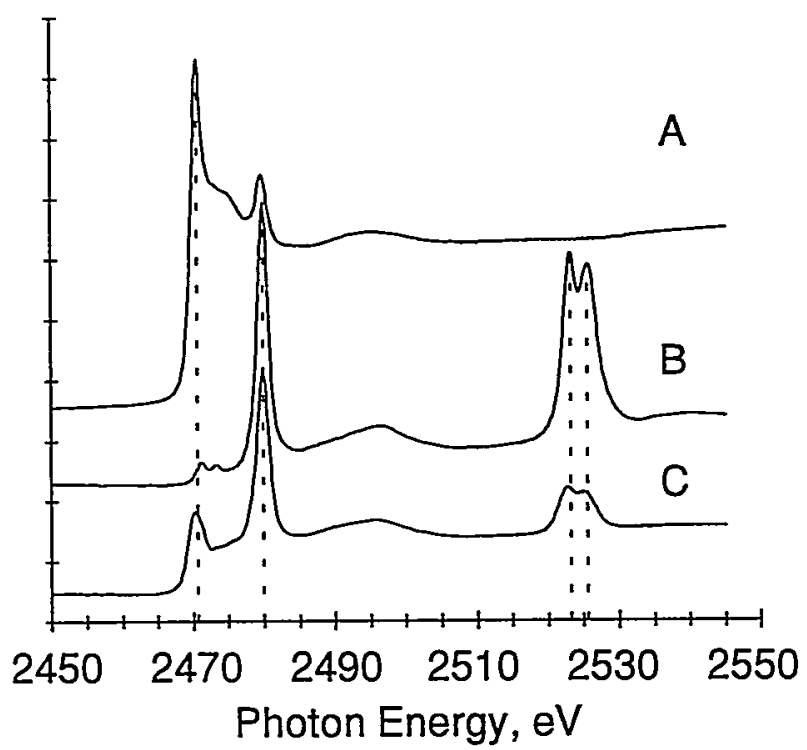

The figure below shows the S-XANES from the bulk solutions which created the previous films. These data show a great reduction in the amount of oxidation of the $S \&$ Mo species for these three solutions.

Time sequences taken a $1,3,5,8$ \& 16 hrs indicates no oxidation of the ZDDP sulfur $(A)$ at $150 \mathrm{C}$ in an air purged system. Mo-DTC (B), under the same conditions, shows only a slight oxidation feature at $2474 \mathrm{eV}$, consistent with the formation of a sulfone-like, $-\mathrm{SO}_{3}{ }^{-}$,sulfur species. The Mo Ledge shows an unresolved doublet structure from an untreated solution all the way to $16 \mathrm{hrs}$. at $150 \mathrm{C}$.

The S XANES for the binary solution (C), ZDDP \& Mo-DTC, showed a $0.6 \mathrm{eV}$ shift in the primary sulfidic sulfur form from 2470.3 to 2470.9 $\mathrm{eV}$ with no indication of any oxidized sulfur present in the bulk solution. The reduced intensity of the Mo L-edge is a result of the dilution in concentration by the addition of ZDDP by equal weight. The appearance of the unresolved doublet suggests the Mo in solution remains unoxidized after 16 hrs at $150 \mathrm{C}$ in an air sparged solution. These data compare the relative oxidation of both the surface adsorbed and bulk species. Clearly, the species adsorbed of the $\mathrm{Fe}$ film show a much higher oxidation than the solution components indicating a different mechanism for surface oxidation compared to the solution.

\section{S K-edge XANES FROM BULK SOLUTION}

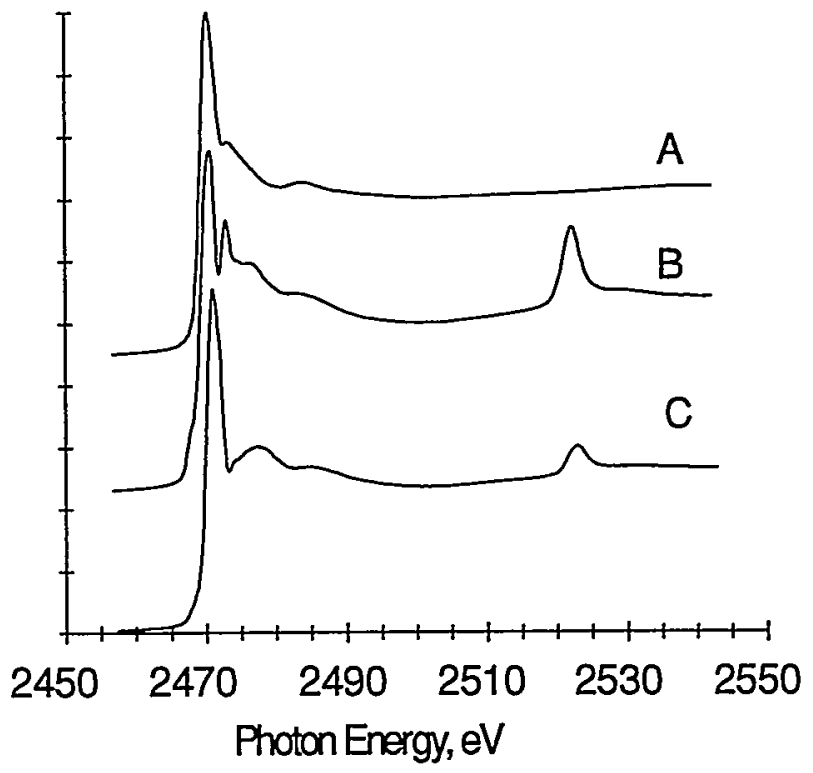




\title{
THE CHEMISTRY OF SULFUR DURING HYDROPYROLYSIS OF COALS
}

\author{
M. L. Gorbaty, S. R Kelemen, S. N. Vaughn, M. Sansone and P. J. Kwiatek \\ Exxon Research and Engineering Company, Annandale, NJ 08801 \\ G. N. George \\ Stanford Synchrotron Radiation Laboratory, Stanford, CA 94309
}

Sulfur K-edge X-Ray Absorption Near Edge Structure Spectroscopy (XANES) and sulfur $2 p$ X-Ray Photoelectron Spectroscopy have been used to identify organically bound sulfur forms in coals [1-3], and to follow the chemistry of organic sulfur during various treatments including mild oxidation [4], pyrolysis [57] and chemical reductions [8]. This paper focuses on the changes which take place with organically bound sulfur functionalities in coals of various ranks when they are subjected to hydropyrolysis conditions. Data from both $X$-Ray techniques and temperature programmed decomposition-mass spectrometry were used in tandem.

\section{Experimental Section}

Most coal samples used in this study were obtained from the Argonne Premium Coal Program [9]. The sample of Rasa coal was obtained from Dr. C. M. White of PETC. The procedures for obtaining and interpreting XPS and XANES spectra of coals have been reported and discussed previously $[1,2]$. XPS spectra were obtained on a Vacuum Generators (VG) ESCA lab system using $\mathrm{Mg} \mathrm{K}_{\alpha}$ nonmonochromatic radiation using a five channel detection system. XANES spectra were recorded at SSRL on beam line 6-2. Fluorescence spectra were recorded using a Stern-Heald-Lýlle detector. Hydropyrolysis char thermal reactivity data were obtained using a temperature programmed decomposition (TPD) apparatus, the design and use of which are described elsewhere $[5,6]$. Samples were heated under ultrahigh vacuum from room temperature to $750^{\circ} \mathrm{C}$ at a rate of $0.5^{\circ} \mathrm{C} / \mathrm{s}$, while the off gases were monitored by a mass spectrometer. Hydropyrolysis experiments were carried out in a closed reactor pressurized at room temperature to 70 atm with a $95 \%$ hydrogen - $5 \%$ helium gas mixture. Pyrolysis was done in helium, at $1 \mathrm{~atm}$ in a quartz lined reactor [5]. For pyrolysis experiments, the samples were heated at a linear heating rate of $0.5^{\circ} \mathrm{C} / \mathrm{s}$ to $400^{\circ} \mathrm{C}$ followed by holding at that temperature for 5 minutes. The reactor temperature program for hydropyrolysis was similar except that the maximum temperature was $427^{\circ} \mathrm{C}$ and the samples were held for 30 minutes under isothermal conditions. Samples were analyzed after the linear heatup and isothermal stages. These kinetic conditions favored the retention of coal liquid products in the pyrolysis and hydropyrolysis residues.

\section{Results and Discussion}

The hydropyrolysis residues were collected and examined by elemental analysis to determine sulfur loss, by XPS and XANES to determine the forms of bound sulfur remaining, and by TPD to determine the thermal reactivity of the residues. Table 1 shows the sulfur to carbon atomic ratios for

\section{Table 1}

\section{SULFUR/CARBON
ATOM RATIO $(x$ 100)}

$\begin{array}{lcc}\text { BASA } & \text { XPS } & \text { BULK } \\ \text { INITIAL } & 6.20 & 6.10 \\ \text { PYROLYSIS } & 4.70 & 5.30 \\ \text { HYDROPYROLYSIS } & 4.51 & 4.82 \\ \text { ILLINOIS H8 } & & \\ \text { INITIAL } & 1.25 & 2.32 \\ \text { PYROLYSIS } & 0.98 & 2.25 \\ \text { HYDROPYROLYSIS } & 0.98 & 1.58\end{array}$

samples of starting Rasa and Illinois No. 6 coals, as well as those for the residues from heating to $400^{\circ} \mathrm{C}$ under helium pyrolysis and hydropyrolysis after heating to $427^{\circ} \mathrm{C}$ as determined from bulk elemental analysis and from XPS by integration of the areas for sulfur and carbon [1]. The bulk and XPS elemental analyses are in good agreement for Rasa coal, which 
contains virtually no pyrite, and are not in agreement for Illinois No. 6. The discrepancy between the bulk elemental and XPS data for Illinois No. 6 coal is due to the fact that the surface concentration of pyrite and its oxidation products are different on this coal's surface relative to that in the bulk [10]. Both analyses indicate that more sulfur was lost during hydropyrolysis than pyrolysis under nitrogen.

Sulfur K-edge XANES absorption spectra of the hydropyrolysis residues of Illinois No. 6 coal are shown in Figure 1. Included in the figure are the

\section{Figure 1}

Illinois No. 6 Hydropyrolysis Chars
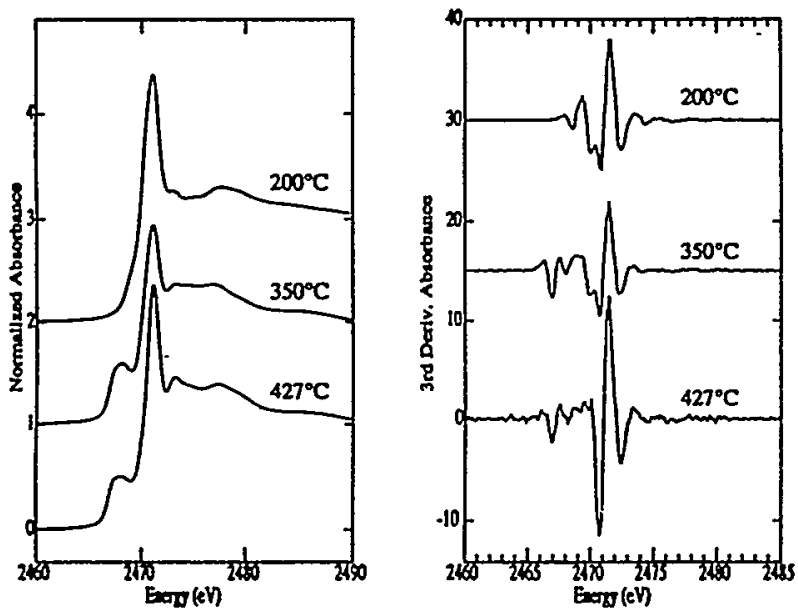

absorption spectra and their 3rd derivatives. The latter were analyzed to determine the aromatic and aliphatic sulfur components and the results of this analysis are shown in Table 2. The table shows the XANES results for aliphatic sulfur for the initial coals, hydropyrolysis residues obtained at 200,350

Table 2

\section{HOLEX ALJPHATIC SULFUR DETECTED IN CHARS} (XANES ANALYSIS)

\begin{tabular}{|c|c|c|c|c|c|}
\hline \multirow[b]{2}{*}{$\cos 1$} & \multirow[b]{2}{*}{ ORIGISAL } & \multicolumn{3}{|c|}{ HroROPYRacrsis } & \multirow{2}{*}{ 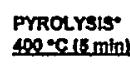 } \\
\hline & & & $100^{\circ}$ & $472 \cdot 6$ & \\
\hline mase & $\infty$ & 2 & 9 & 20 & - \\
\hline 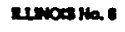 & m & 24 & zo & 18 & 5 \\
\hline $\operatorname{mroanx}$ & 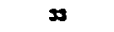 & $2 \pi$ & 2t & 12 & 11 \\
\hline
\end{tabular}

and $427^{\circ} \mathrm{C}$, and previously obtained data [5] on helium pyrolysis chars. The results indicate that the hydropyrolysis chars contain the same ratio of aliphatic to aromatic sulfur as the starting coals, and considerably more than that contained in the pyrolysis residues. XPS data on the same samples confirm these findings. In addition, the feature attributed to pyrite in the fresh Illinois No. 6 coal and its $200^{\circ} \mathrm{C}$ hydropyrolysis residue largely disappears in the $350^{\circ} \mathrm{C}$ residues and is replaced by a feature attributed to pyrrhotite.

The effect of temperature on the reactivity of bound sulfur on the hydropyrolysis residues was examined by TPD. Traces of the relative intensities of the $\mathrm{m} / \mathrm{e}=34$ mass spectra of the initial Rasa and Illinois No. 6 coals and their hydropyrolysis residues prepared at 200,350 and $427^{\circ} \mathrm{C}$ are plotted as a function of temperature in Figure 2. These were

Figure 2

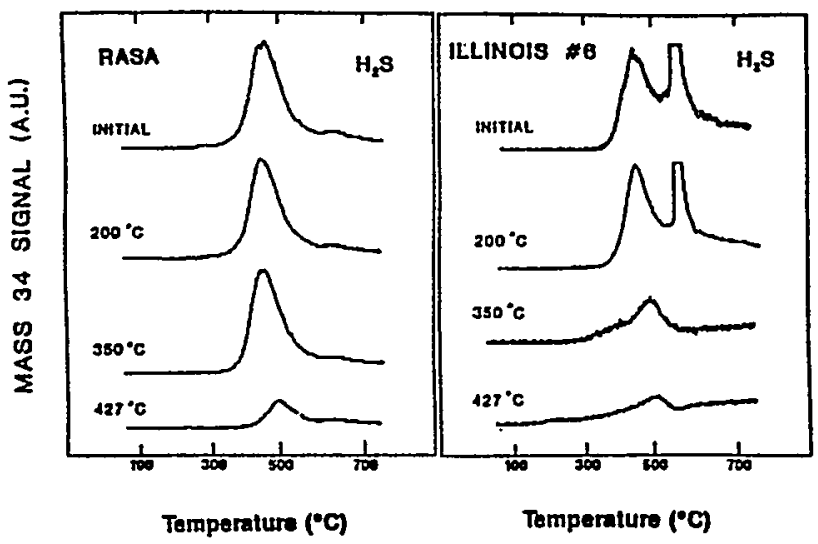

normalized so that the data for a given coal appear on the same relative intensity scale. Up to $350^{\circ} \mathrm{C}$, Rasa coal continues to evolve $\mathrm{H}_{2} \mathrm{~S}$, but at $427^{\circ} \mathrm{C}$ the amount released is considerably diminished. The TPD traces for fresh Illinois No. 6 coal and its $200^{\circ} \mathrm{C}$ hydropyrolysis residue show evolution of $\mathrm{H}_{2} \mathrm{~S}$ from both aliphatic sulfur and pyrite, but the total amount of $\mathrm{H}_{2} \mathrm{~S}$ released from the $350^{\circ} \mathrm{C}$ residue is diminished, and that from pyrite is virtually gone. There is even less $\mathrm{H}_{2} \mathrm{~S}$ evolution from the $427^{\circ} \mathrm{C}$ hydropyrolysis residue.

Taken together the data present an apparent discrepancy. It is known that on pyrolysis in an inert environment at $400^{\circ} \mathrm{C}$, aliphatic sulfur is lost from the coal as $\mathrm{H}_{2} \mathrm{~S}[5,6]$. Aromatic sulfur does not evolve at these temperatures [11]. Somewhat more sulfur is lost during hydropyrolysis than pyrolysis, but the differences are not great. TPD data indicate that most of the reactive sulfur in the hydropyrolysis residues 
had evolved at $427^{\circ} \mathrm{C}$. Nevertheless, XPS and XANES analyses indicate that the hydropyrolysis residues contain about as much aliphatic sulfur as the starting coals; the ratio of aliphatic to aromatic sulfur is about the same, even though significant amounts of $\mathrm{H}_{2} \mathrm{~S}$ evolved during the hydropyrolysis.

To account for these observations, we postulate that molecules containing aromatic sulfur forms are partially hydrogenated under hydropyrolysis conditions. To the XPS and XANES probes, the sulfur in the hydrogenated molecules appear as aliphatic sulfurs. However, during heating, carbon sulfur bonds are not cleaved; rather the molecules lose hydrogen to regain their aromaticity.

\section{Conclusions}

Unlike pyrolysis under inert gas, pyrite in coals is converted to pyrrhotite during hydropyrolysis, and significant quantities of aliphatic sulfur are detected in the residues. It is believed that these aliphatic sulfur forms result from partial hydrogenation of aromatic sulfur species. While these sulfur species appear to be aliphatic to the X-ray probes, they are not, and do not behave thermally as aliphatic species.

1. S. R. Kelemen et. al, Fuel 69, 939, 945(1990).

2. G. N. George et. al, Energy Fuels 5, 93(1991).

3. G. P. Huffman et. al, Energy Fuels 5, 574(1991).

4. M. L. Gorbaty et. al, Fuel 71, 1255(1992).

5. S. R. Kelemen et. al, Fuel 70, 396(1991).

6. S. R. Kelemen et. al, Fuel 72, 645(1993).

7. M. M. Taghiei et. al, Energy Fuels 6, 293(1992).

8. K. Chatterjee et. al, Energy Fuels 5, 773(1991).

9. K. Vorres Energy Fuels 4, 42(1990).

10. S. R. Kelemen et. al, Energy Fuels 5, 720(1991).

11. W. H. Calkins et. al, Energy Fuels 6, 411(1992). 


\title{
Direct Observation of Phase Transformation in Fusion Welds
}

\author{
Joe Wong 1 , J.W. Elmer ${ }^{1}$, M. Fröba ${ }^{1}$, P.A. Waide ${ }^{1}$ and E.M. Larson ${ }^{2}$ \\ ${ }^{1}$ Lawrence Livermore National Laboratory, University of California, P.O. Box 808, Livermore, CA 94551 , \\ ${ }^{2}$ Grand Canyon University, College of Science, 3300 W. Camelback Rd, Phoenix, AZ 85107
}

\begin{abstract}
We have developed a spatially resolved $\mathrm{x}$ ray diffraction (SRXRD) technique using synchrotron radiation and applied it successfully to map the phases present the heat affected zone, (HAZ), of fusion welds in-situ and in real time. For the case of a $1.9 \mathrm{~kW}$ arc weld in a commercially pure $\mathrm{Ti}$, which exhibits an allotropic transformation from a hcp $\alpha$-phase to a bcc $\beta$-phase at $\sim 922{ }^{\circ} \mathrm{C}$, the following results were obtained. (i) The width of the HAZ next to the liquid weld pool was found to be $3.33 \pm 0.33 \mathrm{~mm}$ as determined by the existence of the high temperature bcc phase in this zone. This experimental width corroborated well with that calculated from a scaled-up heat flow model. (ii) Concentration profiles derived from the SRXRD data revealed co-existence of both $\alpha$-and $\beta$-phases in the HAZ. These results represent the first direct observation of solid state transformation and mapping of phase boundaries in fusion welds, and provide the needed information for modelling the kinetics of phase transformation and microstructural evolution in allotropic and other more complex systems under steep thermal gradients and non-isothermal heating conditions.
\end{abstract}

During fusion welding, high intensity heat sources are used to create steep thermal gradients that rapidly heat and cool materials to and from their melting point. This rapid thermal cycling induces solid state phase transformations both on heating and on cooling, and causes melting and solidification in those parts of the weld where the liquidus temperature has been exceeded. Microstructural discontinuities exist at (or near) the location of each phase transformation isotherm. In general, two distinct microstructural regions form during the welding process: the fusion zone, $\mathrm{FZ}$, in which melting, solidification and solid state phase transformation have taken place, and the heat affected zone, $\mathrm{HAZ}$, in which only solid state phase transformations have taken place. In each zone, metastable microstructures may be created that can enhance or degrade the quality of the weld, depending on the materials and the application. Examples of materials where welding induced phase transformations play a significant role include allotropic elements such as pure titanium ( $\mathrm{hcp} \rightarrow \mathrm{bcc}$ ); two-phase alloys such as stainless steels $(\mathrm{fcc} \rightarrow \mathrm{bcc}$ ); martensitic alloys such as iron-based steel alloys (fcc $\rightarrow$ bct); and dispersion strengthened alloys. Most of the phase transformations of interest will involve deviations from equilibrium microstructures, resulting in partial transformations and/or the creation of metastable phases. The above types of phase transformations receive a considerable amount of attention in recent years in welding research development and application studies (1).

From a practical standpoint, solid state phase transformations play an important role in welding related problems such as sub-solidus cracking, cold cracking and distortion caused by residual stresses $(2,3)$. Solution to these problems will greatly be facilitated by the development of novel experimental methods for determining phase transformation behavior in the steep thermal gradients and at the high cooling rates that occur during welding. Theoretical methods will be particularly useful for predicting the such phase transformation behavior of electron beam welds, laser beam welds and high-speed arc welds. Currently there are only a handful of in-situ, real time studies of phase changes and chemical dynamics mostly carried out in high temperature reaction systems involving solid combustions (4-7). In welding processes in which steep thermal gradients exist in the materials, no direct method exists for investigating solid state phase transformations that take place. For example, conventional methods for studying general phase transformation behavior such as calorimetry (8), dilatometry (9), resistivity, Jominy end quench testing (10) and Gleeble testing (11) are all indirect. These techniques measure the response of the sample (changes in length, resisitivity, heat capacity or hardness) to the imposed thermal cycle, but do not in any way determine the phases that are present during the test. Moreover, these methods only provide data for low heating and cooling rates on the order of $\sim 1^{\circ} \mathrm{C} / \mathrm{s}$, which is much less than those of arc welds $\left(10-10^{3} \mathrm{C} / \mathrm{s}\right)$, and laser and electron beam welds $\left(10^{2}-10^{4} \mathrm{o} / \mathrm{s}\right)$. Also, no reliable models have been developed to predict the kinetics of phase transformation under the nonthermal cycling conditions that occur in the HAZ of welds. Combined thermal-mechanical numerical modelling is an area of active welding research for 
calculating residual stresses and weld distortion (1214). Such simulations required knowledge of microsturcutre evolution in the HAZ since the thermal and mechanical properties of the weld is a strong function of its microstructure.

We have used the high intensity provided by synchrotron radiation emitted from a multi-pole wiggler insertion device to produce a sub-millimeter probe and performed a series of spatially resolved $x$ ray diffraction (SRXRD) measurements to follow the phases and map their location in the HAZ of a titanium fusion weld. In pure titanium two phase transitions occur: an allotropic $\alpha(\mathrm{hcp}) \rightarrow \beta(\mathrm{bcc})$ transition in the solid state at $882{ }^{\circ} \mathrm{C}$, and melting at $1668{ }^{\circ} \mathrm{C}$. In Grade 4 titanium containing $\sim 0.38 \mathrm{wt}$ $\%$ oxygen, which was used in this work, the $\alpha \rightarrow \beta$ transition is elevated to $922^{\circ} \mathrm{C}(15)$. The SRXRD experiments were performed on the 31-pole wiggler beam line 10-2 (16) at SSRL with SPEAR (Stanford Positron-Electron Accumlation Ring) operating at an electron energy of $3.0 \mathrm{GeV}$ and injection current of $\sim 100 \mathrm{~mA}$.

A schematic of the SRXRD setup and welding apparatus is shown in Fig.1. The synchrotron beam emerging from the wiggler was focussed by a toroidal mirror, passed through a $1 \mathrm{~mm}$ entrance slit and monochromatized with a double Si(111) crystal. The monochromatic beam was then collimated with a Huber slit to render a sub-millimeter beam on the sample at an incident angle of $\sim 25^{\circ}$. An energy of $8.5 \mathrm{keV}(\lambda=1.45860 \AA)$ for the incident beam was chosen as an optimum energy to (a) maximize the penetration depth in $\mathrm{Ti}(-26 \mu \mathrm{m})$, which was sufficient to reduce the signal contribution from any thin oxide layer $(\sim 100 \AA)$ that might form during the welding process; (b) minimize the background contribution due to $\mathrm{Ti} \mathrm{K}$-fluorescence from the sample (Ti K-edge at $4966 \mathrm{eV}$ ) (17); (c) stay within the specified detection efficiency range $(<10 \mathrm{keV})$ of the silicon photodiodes and (d) maximize the range of $2 \theta$ to collect an adequate number of diffraction peaks for both hcp and bcc phases of $\mathrm{Ti}$ for phase identification. The diffracted beams were collected using a water-chilled 2048 element position sensitive photodiode array detector to cover a $2 \theta$ range of $\sim 30^{\circ}$. The detector and its associated ST1000 data collection software supplied by Princeton Instruments have been described elsewhere (18).

The temperature of the weld and its vicinity was measured using an IR scanning pyrometer

(Inframetrics Inc., Model 600) with a field of view covering the entire weld pool and HAZ. The camera was operated in a wideband mode in the range 3-12 $\mu \mathrm{m}$ to optimize sensitivity over a wide temperature range. A He-Ne laser and associated optics were used to align the IR camera in coincidence with the $x$-ray probe on the sample as shown in Fig. 1, and the infrared pyrometric images were recorded concurrently with the SRXRD measurements.

Gas tungsten arc welds were made on a cylindrical $\mathrm{Ti}$ bar, $10 \mathrm{~cm}$ in diameter during the SRXRD experiments using a $150 \mathrm{~A}$ power supply welding unit with a $4.6 \mathrm{~mm}$ diameter W-2\%Th electrode that was ground with a $60^{\circ}$ taper. The power was maintained constant at $1.9 \mathrm{~kW}$ for all welds and $\mathrm{He}$ was used as the welding and trailing gas. The welds were made at a rotational speed of $0.26 \mathrm{rpm}$ corresponding to a surface speed of 1.36 $\mathrm{mm} / \mathrm{s}$. These conditions yielded a $12-13 \mathrm{~mm}$ wide fusion zone on the surface of the Ti bar in good agreement with that calculated from a conductionbased heat flow model (19), which also estimated a width of a few $\mathrm{mm}$ for the HAZ. In order to spatially resolve the phases in the HAZ of the Ti fusion weld under these conditions, a $0.25 \mathrm{~mm} \times 0.5 \mathrm{~mm}$ beam spot was used for all experiments described in this paper. With this beam size, the photon flux at this energy was estimated to be $\sim 10^{10}$ photons/s at the sample plane and a $10 \mathrm{~s}$ integration time was found adequate to yield over 30,000 counts for the most intense reflection with a $S / N$ ratio of over 300 .

To map the phases in the HAZ region, the welding assembly was integrally mounted to an $x-y$ stage driven by stepper motors (not shown in Fig.1) with $10 \mu \mathrm{m}$ precision. The $x-y$ stage was used to manipulate the weld (welding torch and sample) with respect to the fixed $x$-ray beam in order to probe discrete regions about the weld. Movement in the $x$ direction (defined perpendicular to the welding direction) were performed by direct translation of the sample with respect to the X-ray beam. Movements in the y-direction, which is parallel to the welding direction, required translation on a geometric arc due to the curved surface of the cylindrical sample.

Results of a point-by-point phase mapping along the $x$-direction from the base Ti material into the HAZ are shown in Fig. 2. Inset A shows schematically the path taken by the sub-millimeter beam "scanning" sequentially in steps of $0.33 \mathrm{~mm}$ from a point $5 \mathrm{~mm}$ behind the center of the weld (+ mark in liquid pool) and $11 \mathrm{~mm}$ from the centerline of the weld. A diffraction pattem was recorded at each location with a $10 \mathrm{sec}$ integration time. A selected set of XRD patterns was plotted to show the critical phase changes along the path. Thus, beginning at $x=11 \mathrm{~mm}$, the diffraction pattern was that of the $h \mathrm{hp}$ $\mathrm{Ti}$ at some temperature below the $\alpha-\beta$ transition temperature. This pattern yielded the expected five low-angle reflections of hcp Ti calculated for the powder pattern (20). The high peak intensity of the 
observed (002) reflection indicates that the surface of the Ti cylinder is highly textured with the basal planes oriented at near-normal to the incident $x$-ray beam. Between $x=11 \mathrm{~mm}$ to $x=9.33 \mathrm{~mm}$, only the sharp hcp pattern was observed. As the beam was "scanned" deeper into the HAZ towards liquid pool, two notable changes in the SRXRD pattern took place concurrently. At $x=9 \mathrm{~mm}$, a shoulder

( $\downarrow$ in Fig. 2) on the low-angle side of this hcp (002) reflection emerged simultaneously with the appearance of the $b c c(110)$ reflection. Both of these features are shown clearly in Inset B. The bcc(110) reflection increased in intensity from $x=9 \mathrm{~mm}$ to $x=7$ $\mathrm{mm}$, then decreased from $\mathrm{x}=6.66 \mathrm{~mm}$ and persisted at $x=5.66 \mathrm{~mm}$. Also, from $x=9 \mathrm{~mm}$ inward, the shoulder feature on the low-angle side of the sharp hcp (002) reflection increased in intensity and evolved at $x=7.33 \mathrm{~mm}$ into a well defined peak, replacing the narrow reflection completely at $x=7 \mathrm{~mm}$. This is indicative of expansion of the hcp lattice along the caxis in a positive temperature gradient towards the liquid pool. Also at $x=7.33 \mathrm{~mm}$, low-angle shoulder features are also evident from the (101) and (102) reflections of the hcp phase. This is expected since both of these ( $h k l$ ) reflections have a non-zero $l$ value.

The relative fractions of the $\alpha$ - and $\beta$-phases in the HAZ may be estimated from the normalized peak intensities of the $(002)$ reflection of the hcp phase and the $(110)$ reflection of the bcc phase respectively. For each phase, normalization was performed with respect to the corresponding strongest peak in the series of SRXRD patterns recorded along the path indicated in Inset $A$ of Fig. 2. These intensity profiles are plotted in Fig. 3. By defining the region containing the bcc phase as a measure of the width of the HAZ, which is clearly seen from the intensity profile of the (110) reflection of the bcc phase, a value of $3.33 \pm 0.33$ $\mathrm{mm}$ may be deduced. This value was reproducible experimentally in a series of five SRXRD runs conducted in the same region with respect to the center of the weld pool using the same welding parameters. Moreover, the experimental value agrees well with a value of $\sim 3 \mathrm{~mm}$ calculated from a scaledup heat flow model under the same thermal input power (19). Although the spatial range of occurrence for the bcc phase correlates well with the calculated locations of the HAZ, the existence of minute amount of hcp phase and total absence of the bcc phase near the melt at $x=5 \mathrm{~mm}$ (see also Fig. 2), appear an anomaly, but reproducible from run to run. This may be due to local fluctuations in the position of the weld pool with respect to the $x$-ray probe arising from lateral instabilities in the dynamic weld pool. Also, scattering in the intensity profile of the $\alpha$-phase, particularly outside the HAZ in the range $x=11$ to $8 \mathrm{~mm}$, is largely due to strong texturing of the $\mathrm{Ti}$ base material discussed above. Thus, concentrations of phases from such intensity profiles are at best semi-quantitative.

The temperature profile along the same $x$-ray scanning path depicted in Fig. 2 Inset $A$ may be retrieved from the IR pyrometric images recorded synchronously with the SRXRD measurements. The measured pixel values were converted to temperature values using a 3-point calibration: $620^{\circ} \mathrm{C}$ at $\mathrm{x}=11$ $\mathrm{mm}$ and $1660^{\circ} \mathrm{C}$ (melting point of Ti) at $\sim 5 \mathrm{~mm}$ as derived from the heat flow model (19), and $922^{\circ} \mathrm{C}$ at $x=9 \mathrm{~mm}$ from the SRXRD data showing first appearance of the bcc phase. The IR thermal profile is plotted in Fig. 4 and shows general agreement with that calculated from the heat flow model to within $\pm 50 \mathrm{C}^{0}$. A major difference between the two temperature profiles lies in the region $x=5.5-7.0$ $\mathrm{mm}$, in which the IR profile shows a maximum feature at $x=6.5 \mathrm{~mm}$, whereas the calculated profile is monotonic with no maximum. The origin of this discrepancy remains to be elucidated.

Perhaps, a significant result of the present in-situ SRXRD measurements is the co-existence of the $\alpha$ and $\beta$-phase in the HAZ about the Ti fusion weld. This fact, not readily obtainable with either conventional structural techniques (because of their ex-situ and post-mortem nature) or simple heat flow calculations, must be taken into account in the kinetic modelling of phase transformation and microstructural evolution in allotropic systems under highly non-isothermal conditions. The present results are very encouraging and demonstrate experimental feasibility of our SRXRD technique to determine the phases and their boundaries in-situ and in real time during the welding process. With aid of further experimental refinements using a combination of more stabilized welds and smaller size probes from 3rd generation bright synchrotron sources such as those at ESRF (Grenoble) and APS (Argonne), we plan to obtain more extensive and finer spatiallyresolved phase mappings of fusion welds using large imaging plates (21). These data will then be used to develop a generalized model of phase transformation behavior in weld to predict microstructural evolution in both the heat-affected zone and fusion zone, and to verify the model for both allotropic phase transformations occurring in titanium and those occurring in two-phase stainless steel alloys that are of prime importance in a variety of core technologies.

Acknowledgments - This work was performed under the auspices of the U.S. DOE, LLNL, under Contract No. W-7405-ENG-48. MF is grateful to the Alexander von Humboldt Foundation, Germany, for a Feodor Lynen Research Fellowship. The synchrotron experiments were carried out at SSRL supported by DOE, Division of Chemical Sciences. 


\section{References and Notes}

1. International Trends in Welding Science and Technology, ed. S.A David and J.M. Vitek, ASM International, (1993).

2. B.K. Damkroger, G.R. Edwards and B.B. Rath, Welding Journal, 68, 290-s (1989).

3. M.C. Mcquire, M.L. Santella and B.K Damkroger, in The Science of Metal Joining, ed. by M. H., Cieslak, J. Perepezko, S. Kang and M.E. Glicksman, TMS (1992), p.41

4. Joe Wong, E.M. Larson, J.B. Holt, AP.A. Waide, B. Rupp, and R. Frahm, Science, 249, 1406 (1990)

5. R. Frahm, Joe Wong, J.B. Holt, E.M. Larson,

B. Rupp, and P.A. Waide, Phys. Rev. B46, 9205 (1992).

6. E.M. Larson, Joe Wong, J. B. Holt, P.A Waide, B. Rupp and L.J. Terminello, J. Mat. Res., 8, 1533 (1993).

7. R. Rupp, Joe Wong, J.B. Holt, and and P.A.

Waide, J. Alloys and Compounds, 209, 25 (1994).

8. W. Hemminger and G. Hohne, Calorimetry, Fundamentals and Practice, translated. by $Y$. Goldman, Verlag Chemie, Weinheim, (1984).

9. J. Valentich, Tube Type Dilatometer, Instrument Society of America, Research Triangle Park, NC (1981).

10. G. Krauss, Principles of Heat Treatment of Steel, ASM International, (1980).
11. C.D. Lundin, C.Y.P. Qiao, and C.H. Lee, Weldability of Matertials, ed. by R.A. Patterson and K.W. Mahin, ASM International, (1990), p.1 12. K.W. Mahin, W.S. Winters, J. Krafcik, T. Holden, R. Hobson and S. McEwen in Recent Trends in Welding Science and Technology, Ed. by S. A. David and J, M. Vitek, ASM International, (1990). p. 91.

13. J. Goldak, Ref. 12, p. 71

14. J. Goldak, M. Gu and L. Karlsson, in ASM Handbook, vol. 6, ASM International (1993), p. 1131.

15. M. Donachie Jr., Titanium: A Technical Guide, ASM International, (1988)

16. V. Karpenko, J. H. Kinney, S. Kulkarni, K. Neufeld, C. Poppe, K. Tirsell, Joe Wong, J. Cerino, T. Troxel, J. Yang, E. Hoyer, M. Green, D. Humphries, S. Marks, and D. Plate, Rev. Sci. Instrum., 60 1451, (1989)

17. A. Bearden and A.F. Bur, Rev. Mod. Phys. 39, 125 (1967).

18. E.M. Larson, P.A. Waide and Joe Wong, Rev. Sci. Instrum., 62, 53 (1991).

19. J.W. Elmer, Joe Wong, M. Fröba, P.A. Waide and E.M. Larson, (1995) to be published.

20. Calculated with the LAZY Program: Scientific

Software by PhysiSoft Corporation, DE (1994)

21. T. Hamaoka, Cell Technology, 9, 456, (1990)

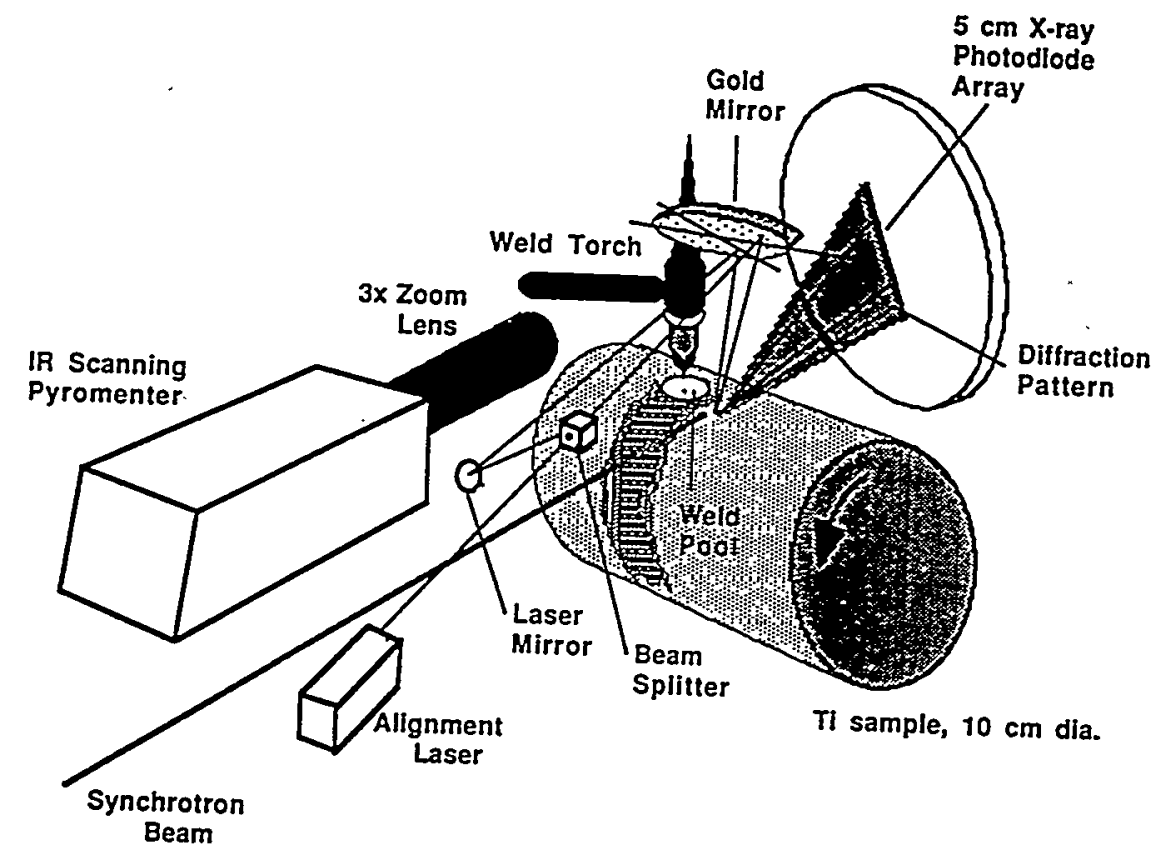

Fig. 1 Schematics of the SRXRD setup used for in-situ phase transformation and mapping in fusion welds. 


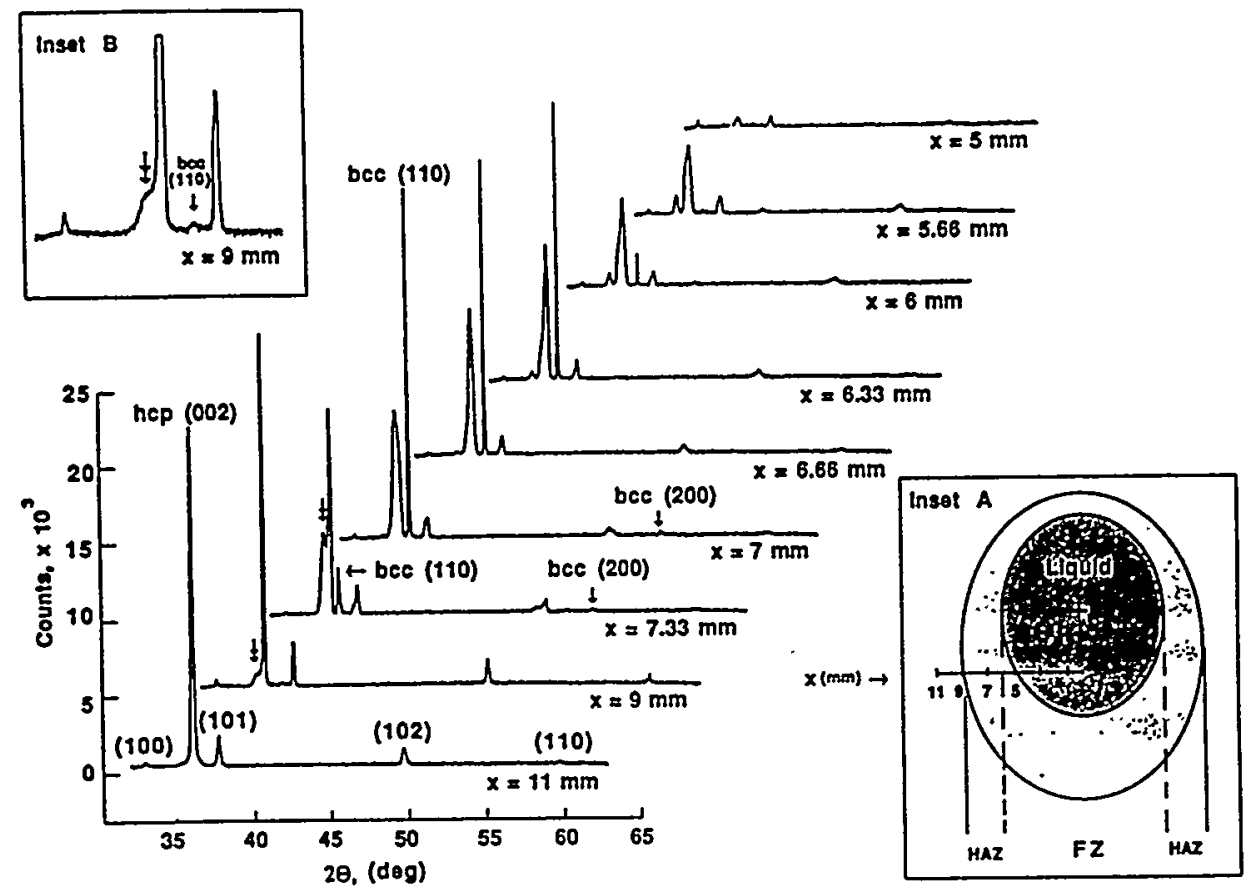

Fig. 2 Selected SRXRD diffraction patterns as a function of position in the HAZ of a Ti fusion weld. Inset A indicates schematically the path taken by the $x$-ray, $0.5 \mathrm{~mm} \times 0.25 \mathrm{~mm}$ in size, from the base material to the HAZ toward the liquid pool. The starting position at $x=11 \mathrm{~mm}$ is about $5 \mathrm{~mm}$ behind the center of the weld pool, which is denoted by the cross mark. Inset $B$ is an expanded SRXRD pattern at location $x=9 \mathrm{~mm}$, showing a discemible appearance of the bcc(110) reflection between the $h c p(002)$ and $h c p(101)$ reflections.
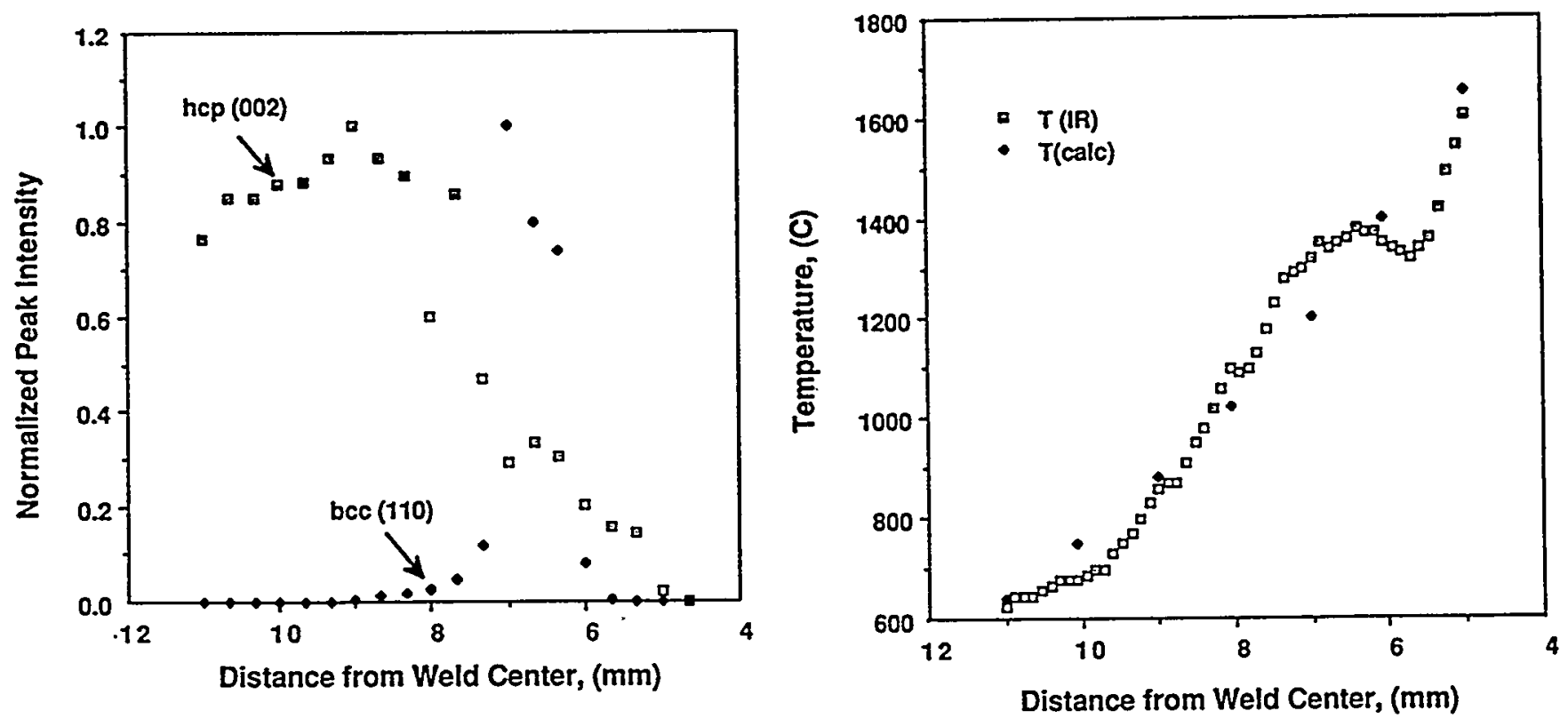

Fig. 3 Normalized peak intensity profiles for the hcp $\alpha-\operatorname{Ti}(002)$ and the bec $\beta$-Ti $(110)$ reflections showing the relative fraction of each phase as a function of distance from the weld center. Normalization was performed with respect to the corresponding strongest peak in the series of SRXRD patterns recorded along the path depecited in Fig. 2, Inset A.

Fig. 4. Temperature profile along the same path probed by the $\mathrm{x}$-ray beam in the $\mathrm{Ti}$ fusion weld as measured with an $\mathbb{R}$ scanning pyrometer. The crosses denote temperature values calculated with a conduction-based heat flow model (19). 


\title{
Pressure Induced Structural Transformations in Silicon Nanocrystals: Surface and Shape Effect
}

\author{
Sarah H. Tolbert, ${ }^{a}$ Amy B. Herhold, ${ }^{a}$ Louis E. Brus, ${ }^{b}$ and A. Paul Alivisatos ${ }^{a}$ \\ aDepartment of Chemistry, University of California, Berkeley, CA 94720, and \\ Materials Sciences Division, Lawrence Berkeley Laboratory
}

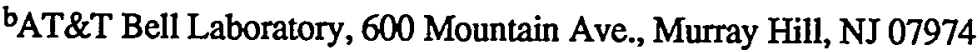

Introduction:

How does the intrinsic size of a material affect the stability of various possible solid phases of the system? In an attempt to answer this question, experiments have been carried out to study pressure induced structural transformations in $\mathrm{CdSe}$ nanocrystals. ${ }^{1}$ These experiments suggest that the phase stability in finite size is in large part controlled by the surface energy associated with any given phase of a nanocrystal. In addition, the experiments suggest that kinetic effects can play a major role in phase stability of finite systems, primarily through transition path induced changes in surface structure and nanocrystal shape. These results can be interpreted in terms of general rule for phase stability in nanocrystalline solids: Because of path induced changes in surface structure, solid-solid phase transition pressures will always be elevated in finite size in comparison to the analogous bulk system.

To address the generality of these finding, and to learn more about the effect of intrinsic size on solid state phase stability, experiments have recently been carried out to study on Silicon (Si) nanocrystals. These crystallite differ from the CdSe nanocrystals in a number of ways. In the first place, Si bonding in its various phases is covalent and metallic, in comparison to CdSe which is dominantly ionic. Si crystallites can also be made in a very wide range of sizes: $50-500$ $\AA$ in diameter. More importantly, the surface of these crystallites is very effectively passivated with $\mathrm{SiO}_{2}$. The $\mathrm{Si} / \mathrm{SiO}_{2}$ is a particularly low energy interface with almost no dangling bonds.

Experimental and Results:

Bulk Si is know to under go a solid-solid phase transition from the atmospheric pressure diamond structure (a semiconductor) to a high pressure $\beta$-Sn phase (metallic) at about $12 \mathrm{GPa}$. High pressure optical absorption experiments performed on $100 \AA$ Si nanocrystals, however show a very different result (figure 1). The large increase in optical density at the semiconductor to metal transition pressure is an indication of structural change. The upstroke transition is well above the bulk Si upstroke phase transition pressure. Upon release of pressure, a semiconducting state is recovered. This result is in contrast to the result for bulk $\mathrm{Si}$, where a metastable metallic phase (BC8) is recovered.

To understand this altered phase stability in nanocrystals of $\mathrm{Si}$, high pressure X-ray diffraction experiments have been carried out on bulk $\mathrm{Si}$ and 500 $\AA$ diameter Si nanocrystals. Experiments were performed at the Stanford Synchrotron Radiation Laboratory on wiggler beam line 10-2. Experiments utilized a Mao-Bell style diamond anvil cell with a diffraction slit. Focused, monochromatic, $0.62 \AA \mathrm{X}$ rays were collimated to a $0.1 \mathrm{~mm}$ diameter beam and aligned through the sample compartment of the high pressure cell. Diffracted X-rays were collected on Fuji image plates and read with $0.1 \mathrm{~mm}$ resolution. Scan times ranged from $30 \mathrm{~min}$. to 1.5 hours.

Bulk $\mathrm{Si}$ undergoes 3 solid-solid phase transitions between 11 and $16 \mathrm{GPa}$ (diamond to $\beta$-Sn to Imma to primitive hexagonal), with a fourth transition to a metastable phase upon release of pressure ( $\beta$-Sn to BC8). ${ }^{2}$ All of the structures other than the semiconducting diamond phase are metals, and so resonance Raman or optical techniques are not useful for studying these phases. Only diffraction can be used to fully sort out the high pressure phase behavior in Si nanocrystals.

Diffraction data, presented in figure 2, shows the complicated phase behavior displayed by $450 \AA$ diameter $\mathrm{Si}$ nanocrystals coated with $\mathrm{SiO}_{2}{ }^{3}$ Like CdSe nanocrystals, the phase transition pressure is much higher than that observed in bulk $\mathrm{Si}$, a particularly striking result given the large diameter of the nanocrystals in question. The system can, however, be converted from the diamond structure ${ }^{4}$ (1) to the primitive hexagonal structure (2). Whether this transition proceeds through the $\beta-S n$ and Imma phases is not clear from the data. Upon partial release 
of pressure, an Imma phase is observed (3) and a $\beta$-Sn phase (not shown). While the transition pressures are altered in the nanocrystal sample with respect to bulk $\mathrm{Si}$, all of these phases can be observed in bulk as well as nanocrystalline Si. Upon full release of pressure, however, instead of recovering a BC8 phase, ${ }^{5}$ the sample appears to form amorphous $\mathrm{Si}$ (4). This complicated phase behavior would be almost impossible to unravel with any technique other than diffraction.

\section{Discussion:}

During a solid-solid phase transformation, symmetry constrained atomic motion along a transition path necessitates a change in the shape of the unit cell of a solid. Because nanocrystals transform coherently over the entire crystallite (i.e., they do not fracture into multiple domains), this change in unit cell shape necessitates an overall change in the shape of each nanocrystal. For example, a spherical crystallite might transform to a prolate ellipsoid. While this shape change causes a small increase in surface area, a larger effect is found in the surface structure. Surface atoms are bonded to the interior, so the symmetry-allowed motions of the interior atoms determine the new surface structure in the high pressure phase. This creates a high pressure phase surface with a non-equilibrium number of edges, corners, and vacancies. In addition, the new surface structure is generally not optimized to take advantage of whatever surface passivation was present for the low pressure phase. The net result is an increase in surface energy after a solid-solid phase transformation. This increased surface energy destabilizes the newly formed high pressure phase, relative to the low pressure phase, and thus causes the observed elevation in phase transition pressure.

For a system with a low energy, completely passivated interface like the Si nanocrystals described above, the situation is quite dramatic. Not only does a change in shape create high index surfaces, it destroys the very low energy, $\mathrm{Si} / \mathrm{SiO}_{2}$ interface which is formed during high temperature synthesis. This $\mathrm{Si} / \mathrm{SiO}_{2}$ interface has almost no dangling bonds. It is impossible to preserve this stable structure as the atoms of the $\mathrm{Si}$, but not the $\mathrm{SiO}_{2}$, move along the transition path. The extremely large increase in surface energy is reflected in the substantial increases in phase transition pressure observed for $\mathrm{Si}$ nanocrystals as large as $450 \AA$ in diameter.

For the diamond to $\beta-S n$ to primitive hexagonal transition observed in Si nanocrystals, the low symmetry of all of the structures involved provides some valuable insights. In CdSe nanocrystals, the proposed changes in nanocrystal shape are very difficult to observe experimentally because the long and short axes of the nanocrystal are crystallographically degenerate. In an X-ray diffraction pattern, the only result of the shape change would be a slightly non-Gaussian line shape of the diffraction peaks. Unfortunately, background scattering and pressure inhomogeneity makes it impossible to determine whether these subtle shape effects are present in a diffraction experiment. In contrast, the long and short axes of primitive hexagonal $\mathrm{Si}$ nanocrystals should be crystallographically distinct. Differences in peak widths along various crystallographic orientations can thus be seen clearly.

Figure 3 shows high pressure X-ray diffraction data for approximately $450 \AA \mathrm{Si}$ nanocrystals in the primitive hexagonal phase. The (100) diffraction peak is clearly broader than the (001) diffraction peak. By assuming ellipsoidal nanocrystals, these diffraction peak widths can be converted to domain sizes. The ratio of the (100) to the (001) domain size calculated in this way is in reasonable agreement with that predicted by transition path induced changes in the shape of the unit cell. ${ }^{6}$ Both the (001) and the (100) domain sizes are, however, somewhat smaller than the nominal crystallite size ( $317 \AA$ and $221 \AA$ respectively, versus an approximate diamond structure domain size of 450 $\AA$ ). While this apparent decrease in domain size (peak broadening) could be caused by a fracturing of the crystallite during transformation, ${ }^{7}$ it is more likely the result of pressure inhomogeneity (an established problem at these pressures ${ }^{8}$ ). Because the crystallites are randomly oriented, however, pressure inhomogeneity should broaden all peaks equally. The ratio of the (001) and (100) domain sizes can thus still be interpreted in terms of overall changes in shape of the nanocrystal. This change in shape must be accompanied by a change in surface structure and the destruction of the low energy $\mathrm{Si} / \mathrm{SiO}_{2}$ interface.

1. S. H. Tolbert and A. P. Alivisatos, Science, 265, 373 (1994); S. H. Tolbert and A. P. Alivisatos, accepted for publication in $J$. Chem. Phys.

2. This is actually somewhat of a simplification. Bulk Si appears to transform from the $\beta$-Sn phase to the closely related Imma structure via a second order transformation. The Imma phase then transforms to a primitive hexagonal structure, again via a second order transformation. A variety of references combine 
to explain this complicated phase behavior: R. H.

Wentorf, Jr. and J. S. Kasper, Science 139, 338

(1963); J. C. Jamieson, Science 139, 762 (1963); J.

S. Kasper and S. M. Richards, Acta. Cryst. 17, 752

(1964); F. P. Bundy, J. Chem. Phys. 41, 3809 (1964);

J. Z. Hu and I. L. Spain, Solid State Com. 51, 263

(1984); H. Olijnyk, S. K. Sikka, and W. B. Holzapfel,

Phys Lett. 103A, 137 (1984); M. I. McMahon and R.

J. Nelmes, Phys. Rev. B 47, 8337 (1993).

3. S. H. Tolbert, A. B. Herhold, L. E. Brus, and A. P. Alivisatos, to be submitted.

4. JCPDS-ICDD powder pattern card \#27-1402, (c) 1989.

5. JCPDS-ICDD powder pattem card \#17-901, (c) 1989.

6. R. E. Hanneman, M. D. Banus, and H. C. Gatos, J. Phys. Chem. Solids 25, 293 (1964); M. T. Yin and M.

L. Cohen, Phys. Rev. B 26, 5668 (1982); R. J. Needs and R. M. Martin, Phys. Rev. B 30, 5390 (1984); K. J. Chang and M. L. Cohen, Phys. Rev. B 31, 7819 (1985); S. P. Lewis and M. L. Cohen, Phys. Rev. B 48, 16144 (1993).

7. In this case, the different (001) and (100) domain sizes could be interpreted in terms of nucleation and growth effects, rather than shape changes.

8. Large deviations in pressure (up to $20 \%$ ) can be observed from ruby fluorescence measurements on $\mathrm{Si}$ nanocrystals in ethylene glycol at pressures near $\mathbf{3 0}$ $\mathrm{GPa}$.

Figure 1:

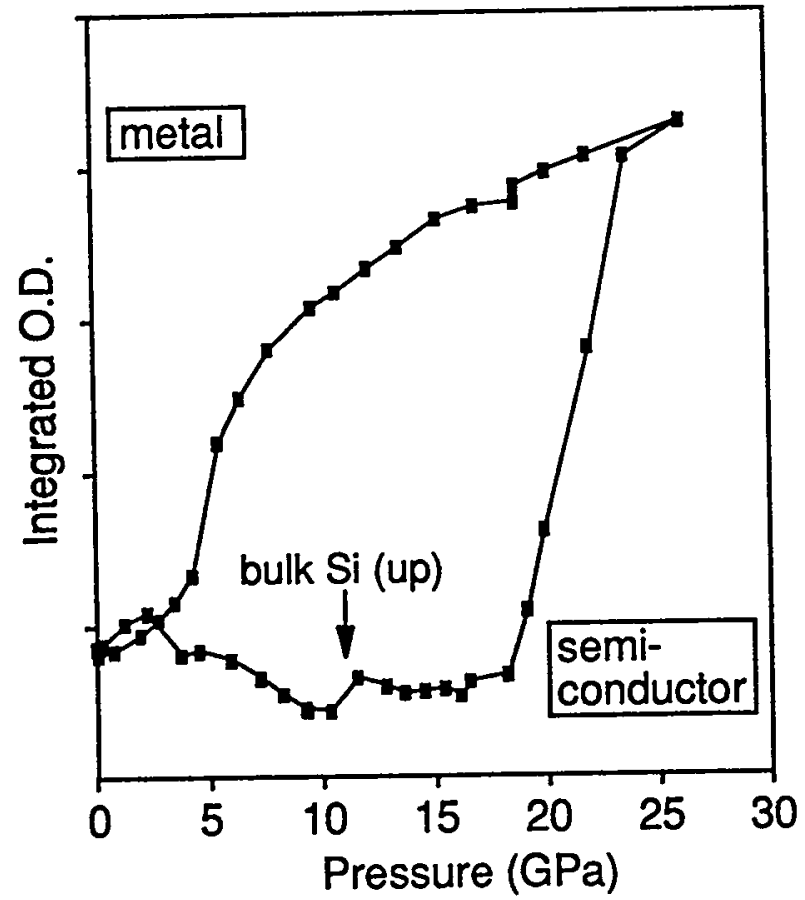

Figure 2:

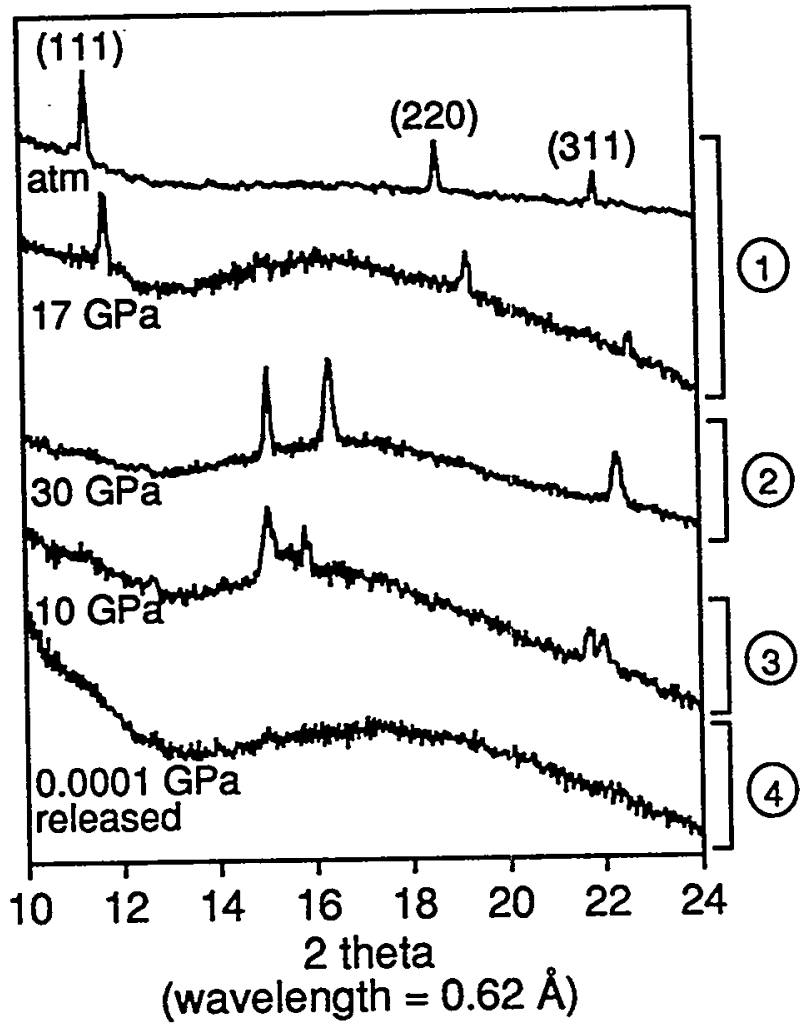

Figure 3:

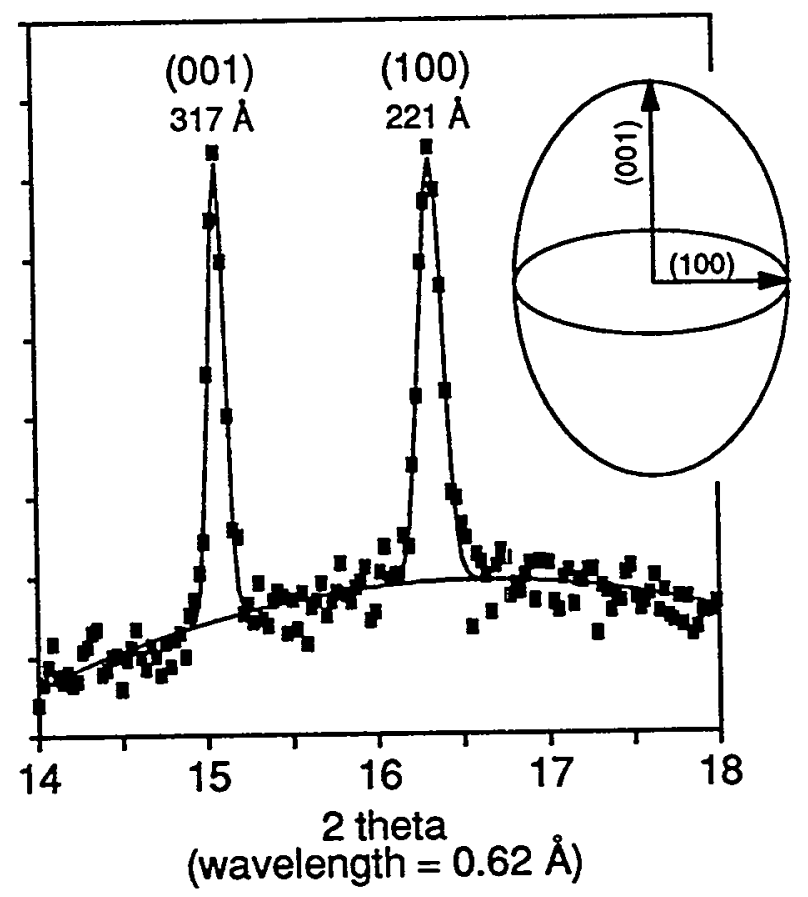




\title{
Synthesis and Characterization of Novel Materials at High Pressures and Temperatures by DAC .X-ray Laser Heating Studies
}

\author{
Choong-Shik Yoo and Jagan Akella \\ Lawrence Livermore National Laboratory, Livermore, California 94551 \\ and \\ Malcolm F. Nicol \\ Department of Chemistry and Biochemistry, UCLA
}

We have developed the DAC X-ray laser-heating technique crucial for the studies of melting, EOS, phase diagram of elements as well as synthesis and characterization of materials in-situ at high pressures to $100 \mathrm{GPa}\left(1 \mathrm{GPa}=10^{4}\right.$ atmosphere) and temperatures to several $1000 \mathrm{~K}$. The system consisted of three components: i) a $\mathrm{Nd}$ :Yag laser-heating system to heat the sample in a DAC (1), ii) a microscope to measure temperature and to view the sample for alignment and observation, and iii) synchrotron $\mathrm{X}$-ray coupled with an image plate detector. The feasibility of the study has already been demonstrated in the studies of iron phase diagram, which we have found a new phase of iron $\left(\varepsilon^{\prime}-\mathrm{Fe}\right)$ at high temperatures of $2000 \mathrm{~K}$ and pressures of $40 \mathrm{GPa}$ and metastability of the existing iron phase $(\gamma-\mathrm{Fe})$. These results would imply a major modification of the iron phase diagram, and provide a crucial constraint for Earth core models (2).

In this year we have applied the technique for synthesis and in-situ characterization of novel materials at high pressures and temperatures. The materials of interests include super-hard materials and metastable energetic phases. We have found the direct reactions of nitrogen with various light elements of $\mathrm{B}, \mathrm{C}$ and $\mathrm{Si}$ at high pressures and temperatures, yielding various forms of technologically important nitride ceramics (see the photograph). Some of these elementary reactions were also found to be highly energetic.

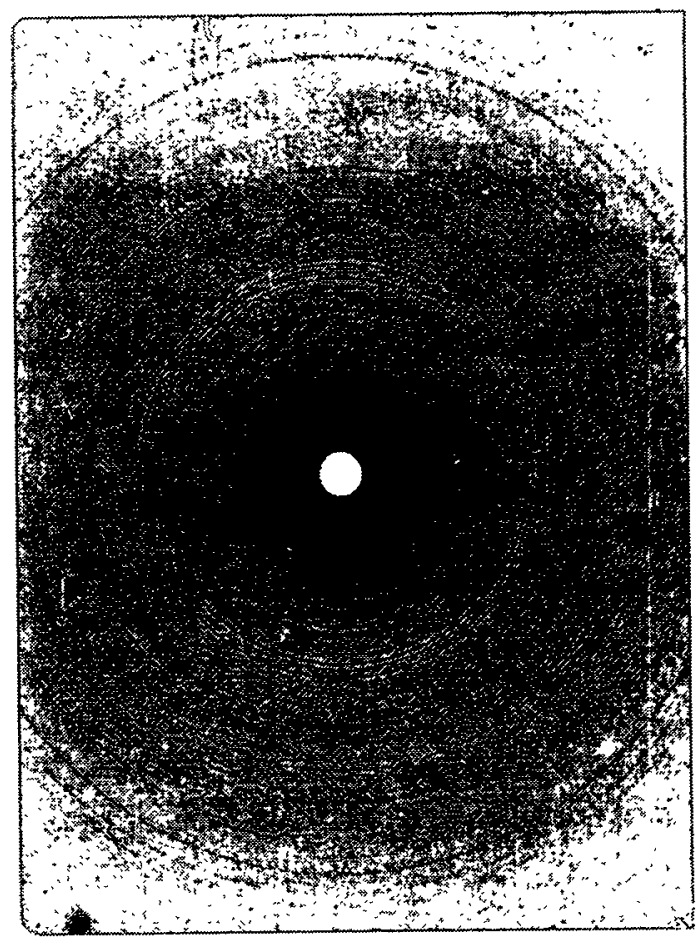

The angle-resolved diffraction pattern of laser heated silicon in nitrogen at $1400 \mathrm{~K}$ and $20 \mathrm{~Kb}$, showing a formation of $\beta$ $\mathrm{Si}_{3} \mathrm{~N}_{4}$. The $\beta-\mathrm{Si}_{3} \mathrm{~N}_{4}$ can be recovered at the ambient condition. The diffraction pattern was obtained using an image plate detector and monochromatic X-ray available at the beamline $10-2$ of the SSRL.

Reference

1. C.S. Yoo, J. Akella, J. Moriarty, Phys. Rev. B 48, 15529 (1993).

2. C.S. Yoo et. al., submitted to Science (1995). 
Proposal 2A14B

\title{
Structural Studies of Type I DNA Topoisomerases.
}

\author{
Christopher Lima, Neal Lue ${ }^{\dagger}$, Amit Sharma, Valerie Tokars, \\ and Alfonso Mondragón \\ Department of Biochemistry, Molecular Biology and Cell Biology \\ Northwestern University \\ Evanston, Il 60208 \\ $\dagger$ Department of Biochemistry and Molecular Biology \\ Harvard University \\ Cambridge, MA 02138
}

\section{Introduction}

DNA topoisomerases are proteins responsible for controlling and maintaining the topological state of DNA the cell. They have been found in all cell types of both eukaryotes and prokaryotes and additionally in some viruses. They are involved in DNA replication, transcription, and genetic recombination $[1,2,3,4]$. Their role in DNA metabolism has made them an important target of novel chemotherapeutic and antibiotic agents $[5,6]$. All topoisomerases work by forming a transient break in DNA through a phosphotyrosine bond. Type I topoisomerases break one DNA strand at a time and then pass another strand through the transient break; type II enzymes break both strands and pass both strands through the break. In this manner, topoisomerases change the linking number in steps of one (type I) or steps of two (type II). Topoisomerases catalyze the following reactions: DNA relaxation and supercoiling, linking of single stranded rings of complementary sequence into duplex rings, knotting and unknotting of double and single stranded rings, and catenation and decatenation of double and single stranded DNA circles. The bond energy of the phosphotyrosine bond is conserved, and hence no external energy source is required for the breakage and rejoining steps.

An understanding of the detailed mechanism of action of DNA topoisomerases is now emerg- ing. The structure of a $67 \mathrm{kDa}$ amino terminal fragment of $E$. coli DNA topoisomerase I [7] has allowed us to suggest a detailed mechanism of action consistent with the existing biochemical, mechanistic, and structural data. We are now in the process of testing this mechanism by studying the interactions of the fragment with short oligonucleotides and also by studying the structure of a $30 \mathrm{kDa}$ fragment that contains the active site tyrosine. The study of this fragment is particularly important for our understanding of the mechanism of action as it plays a crucial role in the reaction. In collaboration with R. DiGate, we are also studying $E$. coli DNA topoisomerase III, a potent catenating decatenating enzyme. We have succeeded in crystallizing the intact protein although the crystals are very small and radiation sensitive.

Recently, we also solved the structure of a 9 $\mathrm{kDa}$ amino terminal fragment of vaccinia virus DNA topoisomerase I [8] which provided the first atomic model of any region of a eukaryoticlike type I topoisomerase. We have solved the structure of a $26 \mathrm{kDa}$ fragment of $S$. cerevisiae DNA topoisomerase I and have refined this structure to $1.9 \AA$ using data collected at SSRL.

\section{Experiments}

We collected data on beamline 7-1 using a rotation/oscillation camera and the MAR phos- 
phor image scanner. Data was collected at a wavelength of $1.07 \AA$ to minimize absorption problems. All crystals were kept at cryogenic temperatures $\left(\approx-170^{\circ} \mathrm{C}\right)$ using a modified Siemens LT I cryocooler. Indexing and integration was done using the program DENZO (Z. Otwinowski, personal communication). Most of the images were processed during data collection to assess the quality of the crystals and optimize the data collection strategy. This proved to be an invaluable aid in collecting very good diffraction data.

We collected data on different protein crystals: a $67 \mathrm{kDa}$ amino terminal fragment of E.coli DNA topoisomerase I soaked with different oligonucleotides, a $30 \mathrm{kDa}$ fragment of $E$. coli DNA topoisomerase I, a $26 \mathrm{kDa}$ fragment of $S$. cerevisiae DNA topoisomerase I, and intact $E$. coli DNA topoisomerase III. In all cases we collected data that was not possible to collect using a conventional X-ray source, either due to the size of the crystals, or radiation sensitivity, or both.

Table I shows the data collection statistics for the $26 \mathrm{kDa}$ fragment of $S$. cerevisiae DNA topoisomerase I. All other data sets are of comparable quality.

\begin{tabular}{|l|l|}
\hline Resolution & $39-1.85 \AA$ \\
\# Reflections & 127,777 \\
\# Unique & 24,425 \\
$R_{\text {sym }}$ & $6.4 \%$ \\
Completeness & $95.1 \%$ \\
\hline
\end{tabular}

Table I. Data collection statistics

\section{Results}

The data collected at SSRL has allowed us to continue our studies of the $67 \mathrm{kDa}$ amino terminal fragment of $E$. coli DNA topoisomerase I in complex with oligonucleotides. We have collected data from crystals soaked with CMP and $\mathrm{dT}_{3}$ to high resolution. We also collected a high resolution data set of the $30 \mathrm{kDa}$ fragment of $E$. coli DNA topoisomerase I. The data is excellent and has allowed us to start Molecular Replacement studies.

We also collected high resolution data on crystals of the $26 \mathrm{kDa}$ fragment of $S$. cerevisiae DNA topoisomerase I. This has allowed us to refine the structure to $1.9 \AA$ resolution. It was impossible to collect data from these crystals beyond $2.8 \AA$ using a conventional source. The overall $\mathrm{R}_{\text {factor }}$ of the structure is now $20.2 \%$ for all data between 5 and $1.9 \AA$. The $\mathrm{R}_{\text {free }}$ for the structure is $28.4 \%$. The stereochemistry of the model is excellent with an r.m.s. deviation from ideality of $0.014 \AA$ for bonds and $1.73^{\circ}$ for angles.

We made considerable progress in our studies of $E$. coli DNA topoisomerase III. The crystals are very small, typically $50 \mu \mathrm{m}$ thick and only $200 \mu \mathrm{m}$ long. The belong to space group $\mathrm{P} 6_{(1,5)} 22$ with cell parameters of $239 \AA$ x $239 \AA$ $x 108 \AA$. We succeeded in collecting one partial native data set. More importantly, we optimized the strategy for cryo-cooling the crystals. This will be extremely important as we continue our studies of this protein.

\section{Acknowledgements}

We are very grateful to M. Soltis, H. Luecke, and P. Phizackerley and the staff at SSRL their help and suggestions. This work is supported by the NIH.

\section{References}

[1] Gellert,M. DNA Topoisomerases. Annu. Rev. Biochem., 50:879-910, 1981.

[2] Wang,J.C. DNA Topoisomerases. Annu. Rev. Biochem., 54:665-697, 1985.

[3] Vosberg,H.-P. DNA Topoisomerases: Enzymes That Control DNA Conformation. Curr. Top. Microbiol. Immunol,, 114:19102,1985 .

[4] Wang,J.C. DNA Topoisomerases: Why so Many? J. Biol. Chem., 266:6659-6662, 1991.

[5] Molecular Biology of DNA Topoisomerases and its Applications to Chemotherapy. In Anhoh.T., Ikeda,H. and Oguro,M., editor, Proceedings of the International Symposium on DNA Topoisomerases in Chemotherapy, 
nagoya, Japan, 1991, Boca Raton, Fla., 1993. CRC Press, Inc.

[6] Liu,L.F. DNA Topoisomerase poisons as antitumor drugs. Ann. Rev. Biochem., 58:351375, 1989.

[7] Lima, C.D., Wang, J.C. and Mondragon, A. Three-dimensional structure of the $67 \mathrm{~K} \mathrm{~N}$ terminal fragment of $\mathrm{E}$. coli DNA topoisomerase I. Nature, 367:138-146, 1994.

[8] Sharma,A., Hanai,R. and Mondragon, A. Crystal structure of the amino terminal fragment of vaccinia virus DNA topoisomerase I at 1.6 A resolution. Structure, 2:767-777, 1994. 


\title{
Evaluation/Data Collection of Cytochrome Reductase Crystals
}

\author{
Edward A. Berry, Li-shar Huang, Vladimir Schulmeister, and Sung-Hou Kim \\ Structural Biology Division \\ Lawrence Berkeley Laboratory \\ University of California \\ Berkeley, CA 94720
}

\begin{abstract}
Summary- The project involves structure determination for an important membrane protein, mitochondrial cytochrome reductase. We have crystallized this protein from beef heart mitochondria in 2 different forms (Berry, E.A. , Huang, L.-s., Earnest, T.N. and Jap, B.K.(1992) J. Mol. Biol. 224, 11611166 and (Berry, Shulmeister, Huang, and Kim (1995) Crystallographica Acta D (in press). In the 1994 run, we have been characterizing new crystals of the protein from different sources, 2 of which diffract markedly better then the beef crystals, and we have developed a method of freezing the crystals without loss of order. These two advances should allow collecting data that will lead to a $3.0 \AA$ structure of the enzyme in the $1995 \mathrm{run}$.

Introduction- Cytochrome reductase (E.C. 1.10.2.2), also known as the cytochrome bc1 complex, ubiquinol:cytochrome c oxidoreductase, or Complex III, is the middle part of the mitochondrial respiratory chain. Reducing equivalents from various substrates get funneled into the ubiquinone pool by NADH and succinate dehydrogenases. Cytochrome reductase oxidizes ubiquinol and reduces cytochrome $c$, using part of the energy released to translocate protons across the mitochondrial inner membrane. Cytochrome $c$ is then oxidized by cytochrome oxidase at the expense of molecular oxygen, with further energy conservation in the proton gradient. Cytochrome reductase is thus vital for aerobic metabolism. Defects in the respiratory chain lead to mitochondrial myopathies such as Kearns-Sayre Syndrome.
\end{abstract}

Cytochrome reductase is a large membrane protein, with 11 subunits (for the beef enzyme) and a total molecular weight of $230 \mathrm{kDa}$. In 1990 we developed a procedure for growing crystals of cytochrome reductase. In the last year we have learned how to make better crystals from other sources, with diffraction approaching $3.0 \AA$, and how to freeze crystals for cryogenic data collection.

Purpose- We had already collected complete data sets on native beef crystals and several potential heavy atom derivatives, but the data was not of sufficient quality to ascertain whether the derivatives were useful. Because of the large size of the protein, we need very accurate data in order for the small changes in intensity due to a heavy atom to be significant. The accuracy was poor because of the low resolution (best diffraction to $3.8 \AA$ ) and because of the rapid radiation damage which forced us to change crystals or translate to an unexposed portion of a crystal every 5-10 frames. In 1994 we decided to work out conditions for crystallization from a number of different sources, in hopes of getting better ordered crystals. We also committed ourselves to finding freezing conditions that would preserve the order of the crystals.

Results- We crystallized cytochrome reductase from heart of pig, rabbit, and chicken. The rabbit crystals were in the same space group as the hexagonal beef crystals, and had similar dimensions (Table I). The pig and chicken crystals were in new space groups. The pig crystals diffracted very poorly. The rabbit crystals were better than the beef, diffracting to $3.5 \AA$, and the chicken crystals diffracted to nearly $3.0 \AA$. Furthermore the unit cell of the chicken crystals was smaller than any other cytochrome reductase crystal, making spot overlap less of a problem.

For freezing crystals we decided to use glycerol and PEG as cryoprotectants, because PEG is the precipitant and glycerol is a frequent additive in our crystallization procedures. About $25 \%$ glycerol was required to freeze mother liquor as a glass. It proved necessary to first equilibrate the crystals at lower glycerol concentration in a low salt buffer before bringing the mother liquor to $25 \%$, otherwise the crystals were disordered even without freezing. Even with very gradual changes of the mother liquor some cracking was observed, especially with larger crystals. Apparently there is some conformational change, at least in the beef crystals, between 15 and $25 \%$ glycerol. The $c$ axis changes from 378 to $352 \AA$ at the higher concentration. Despite this 
Table 1- Parameters of different forms of cytochrome reductase crystals

\begin{tabular}{|c|c|c|c|c|c|c|c|}
\hline $\begin{array}{l}\text { Protein source, } \\
\text { Crystals habit }\end{array}$ & Space Group & Unit Cell & $\frac{\# \mathrm{AU}^{1}}{\text { cell }}$ & $\frac{\text { Mono }^{2}}{\mathrm{AU}}$ & \multicolumn{2}{|c|}{$\begin{array}{l}\text { UC } \begin{array}{r}\text { AU } \\
\left(10^{6} \AA^{3}\right)\end{array} \\
\end{array}$} & $\AA^{3} / D_{a^{4}}$ \\
\hline \multicolumn{8}{|l|}{ beef bcl: } \\
\hline Hexagonal & $\mathrm{P}_{1(5)} 22$ & $21721737890^{\circ} 90^{\circ} 120^{\circ}$ & 12 & 1 & 15.41 & 1.28 & 5.56 \\
\hline frozen- & $\mathrm{P}_{1(5)} 22$ & $21221235290^{\circ} 90^{\circ} 120^{\circ}$ & 12 & 1 & 13.70 & 1.14 & 4.96 \\
\hline Hex rods 5 & $\mathrm{P}_{1(5)} ? ?$ & $13413475190^{\circ} 90^{\circ} 120^{\circ}$ & $6 / 12$ & (2?) & 11.68 & $1.95 / 0.973$ & 4.23 \\
\hline Orthorhombic & $\mathrm{C} 222_{1}$ & $38411817790^{\circ} 90^{\circ} 90^{\circ}$ & 8 & $1(?)$ & 8.02 & 1.00 & 4.35 \\
\hline Babbitbcl: & $\mathrm{P} 6_{1(5)^{22}}$ & $21221235490^{\circ} 90^{\circ} 120^{\circ}$ & 12 & 1 & 13.779 & 1.15 & 4.99 \\
\hline Pork bce: & $\mathbf{P}$ & $\left(15418015490^{\circ} 107^{\circ} 90^{\circ}\right)$ & & & 4.20 & $?$ & $?$ \\
\hline Chicken bc1: & $\mathrm{P}_{212} \mathrm{2}_{1}$ & $17918724490^{\circ} 90^{\circ} 90^{\circ}$ & 4 & $\begin{array}{l}1(?) \\
2(?)\end{array}$ & $\underset{n}{8.167}$ & 2.04 & $8.87(?)$ \\
\hline $\begin{array}{l}\text { frozen- } \\
\text { Other groups: }\end{array}$ & & $16918123990^{\circ} 90^{\circ} 90^{\circ}$ & $"$ & & 7.333 & 1.83 & $\begin{array}{l}4.43 \\
3.98\end{array}$ \\
\hline Stillwater & $\mathrm{P}_{1(3)}{ }^{22}$ & $15915959390^{\circ} 90^{\circ} 90^{\circ}$ & 8 & $2(?)$ & 15.00 & 1.87 & 4.06 \\
\hline Osaka & $\mathrm{P} 21$ & $19617925390^{\circ} 97^{\circ} 90^{\circ}$ & 2 & $4(? ?)$ & 8.81 & 4.40 & 4.78 \\
\hline & $P 6_{1(5)}$ & $13113172090^{\circ} 90^{\circ} 120^{\circ}$ & $6 / 12$ & $(2 ?)$ & 10.70 & 1.783 & 3.88 \\
\hline & & 190190445909090 & 4 & (2) & 16.06 & 4.02 & 4.36 \\
\hline
\end{tabular}

1. \#AU/cell- the number of asymmetric units in the unit cell for this space group

2. Mono/AU- the number of monomers in the asymmetric unit (speculative, except for the beef $\mathrm{P} 6122$ crystals)

3. volume- Volume of the unit cell, asymmetric unit in $\AA^{3},+10^{6}$.

4. Packing parameter, cubic angstroms per dalton of protein, based on assumed number of monomers per asymmetric unit

5. These hexagonal rod crystals appear to be the same as the hexagonal crystals reported by the Osaka group.

Table 2. Comparison of data from $0^{\circ} \mathrm{C}$ beef (left)

\begin{tabular}{|c|c|c|c|c|c|c|}
\hline Beef $\left(0^{\circ} \mathrm{C}\right)$ & \multicolumn{3}{|c|}{ All data } & \multicolumn{3}{|c|}{ Rejecting outliers } \\
\hline $\begin{array}{c}\text { Resolu- } \\
\text { ution }\end{array}$ & $\begin{array}{r}\text { \# indep } \\
\text { reflec- } \\
\text { tions }\end{array}$ & $\begin{array}{l}\text { Com- } \\
\text { plete- } \\
\text { ness }\end{array}$ & $\begin{array}{c}\mathrm{R}- \\
\text { scale }\end{array}$ & $\begin{array}{r}\text { \# indep } \\
\text { reflec- } \\
\text { tions }\end{array}$ & $\begin{array}{l}\text { Com- } \\
\text { plete- } \\
\text { ness }\end{array}$ & $\begin{array}{c}\mathrm{R}- \\
\text { scale }\end{array}$ \\
\hline $30.0-8.6$ & 4560 & $94.7 \%$ & $6.6 \%$ & 4511 & $93.7 \%$ & $5.5 \%$ \\
\hline & 4323 & $94.3 \%$ & $13.2 \%$ & 4135 & $90.2 \%$ & $10.8 \%$ \\
\hline-6.0 & 4155 & $91.5 \%$ & $27.0 \%$ & 3816 & $84.0 \%$ & $17.2 \%$ \\
\hline $6.0-5.4$ & 4032 & $90.0 \%$ & $34.1 \%$ & 3612 & $80.7 \%$ & $19.9 \%$ \\
\hline $5.4-5.0$ & 4037 & $90.0 \%$ & $37.0 \%$ & 3565 & $79.5 \%$ & $20.7 \%$ \\
\hline-4.7 & 3862 & $86.9 \%$ & $37.4 \%$ & 3410 & $76.8 \%$ & \\
\hline & 3950 & $88.8 \%$ & $40.3 \%$ & 3459 & $77.8 \%$ & $21.2 \%$ \\
\hline $30.0-6.8$ & 8883 & $94.5 \%$ & $7.8 \%$ & 8646 & $92.0 \%$ & $6.4 \%$ \\
\hline $30.0-6.0$ & 13038 & $93.5 \%$ & $9.1 \%$ & 12462 & $89.4 \%$ & \\
\hline & 17070 & $92.7 \%$ & $10.1 \%$ & 16074 & $87.3 \%$ & \\
\hline & 21107 & $92.2 \%$ & $11.2 \%$ & 19639 & $85.7 \%$ & \\
\hline & 24969 & $91.3 \%$ & $12.1 \%$ & & $84.3 \%$ & 8.3 \\
\hline & 28919 & $91.0 \%$ & $12.9 \%$ & 26508 & $83.4 \%$ & \\
\hline
\end{tabular}

problem some crystals survive the treatment, and freezing, with only a slight increase in mosaicity. Table 2 compares a dataset recently collected from a single frozen chicken crystal with our best data from beef crystals at $0^{\circ} \mathrm{C}$. The beef data is reasonable at low resolution, but R-scale exceeds $15 \%$ at resolution higher than $6.8 \AA$. With the frozen chicken crystal, R-scale approaches $10 \%$ only above $3.5 \AA$.

Future plans- There is still room for much improvement in the growth of chicken reductase crystals and in the freezing procedure. However we now have frozen crystals diffracting to nearly $3.0 \AA$, and it is time and frozen chicken (right) crystals:

\begin{tabular}{|c|c|c|c|c|c|c|}
\hline Chicken (-1 & $\left.70^{\circ}\right)$ & llldata & & & & liers \\
\hline $\begin{array}{c}\text { Resolu- } \\
\text { ution }\end{array}$ & $\begin{array}{l}\text { \# indep } \\
\text { reflec- } \\
\text { tions }\end{array}$ & $\begin{array}{l}\text { Com- } \\
\text { plete- } \\
\text { ness }\end{array}$ & $\begin{array}{c}\text { R- } \\
\text { scale }\end{array}$ & $\begin{array}{l}\text { \# indep } \\
\text { reflec- } \\
\text { tions }\end{array}$ & $\begin{array}{l}\text { Com- } \\
\text { plete- } \\
\text { ness }\end{array}$ & $\begin{array}{c}\text { R- } \\
\text { scale }\end{array}$ \\
\hline $999.00-6.46$ & 14327 & $93.8 \%$ & $5.9 \%$ & 13150 & $86.1 \%$ & \\
\hline & 12666 & & $6.8 \%$ & & & \\
\hline 4.48 & 12146 & 82. & $6.7 \%$ & 11166 & & 4. \\
\hline & 11102 & 75.6 & 7.4\% & 10230 & & \\
\hline 4.07 & 10047 & $68.6 \%$ & $8.6 \%$ & 9304 & & 5.8 \\
\hline & s & & 9.9 & 8595 & & \\
\hline & 8269 & & 11.7 & 7661 & $52.4 \%$ & 9.1 \\
\hline $3.38-3.23$ & 6215 & $42.6 \%$ & 12.8 & 5809 & & 10.3 \\
\hline $99.00-5.13$ & 26993 & $89.6 \%$ & $6.1 \%$ & 24803 & & 3.1 \\
\hline & & & 6.2 & & & 3. \\
\hline $0-4.07$ & & $84.3 \%$ & 6.3 & 46199 & & \\
\hline & & & 6.5 & & & 3.7 \\
\hline & & $78.3 \%$ & 6.7 & & & 3.9 \\
\hline & & & 6.8 & & & \\
\hline & & $1.2 \%$ & $6.9 \%$ & 77568 & $657 \%$ & \\
\hline
\end{tabular}

to start seriously collecting isomorphous data. We have a list of 4-5 heavy atom reagents, including a synthetic inhibitor analog, that do not disorder the crystals at reasonable concentrations. In 1995 we will collect data sets for putative derivatives from frozen chicken crystals. We will also collect MAD data on native crystals (There are 5 iron atoms in the molecule, 3 hemes and an $\mathrm{Fe}_{2} \mathrm{~S}_{2}$ ironsulfur cluster). It would be umprecedented to actually phase the reflections of such a large protein by $M A D$, but the anomalous data may help locate irons when some isomorphous phases are available. 


\title{
Bacterial Pilin: $2.6 \AA$ X-Ray Crystal Structure
}

\author{
Katrina Forest, Hans Parge, Michael Hickey, \\ John A. Tainer, Elizabeth D. Getzoff \\ Department of Molecular Biology \\ The Scripps Research Institute \\ La Jolla, California 92037
}

Many pathogenic bacteria require specialized cellsurface fibers called pili to mediate recognition and attachment to host cells (Cannon and Sparling, 1984). Pili are composed of primarily a single protein, pilin. We have solved the $2.6 \AA$ structure of pilin from $N$. gonorrhoeae using data collected at SSRL Beamline 7-1 over the past several years. In particular, the phasing of the $\mathrm{x}$-ray reflections was accomplished with a platinum derivative data set collected at $1.07 \AA$ to maximize anomalous signal. Due to the very small crystal size (approx. $.05 \mathrm{X} .05 \mathrm{X} 1$ $\mathrm{mm}$ ) and poor diffraction, this data could not have been collected on a rotating anode $\mathrm{x}$-ray source.

The structure of pilin is a novel $\alpha-\beta$ roll fold with a remarkable $85 \AA$ alpha helix cradled by a 4 stranded $\beta$ sheet. An additional $\beta$ hairpin is formed by a hypervariable sequence region and is packed against the beta sheet by hydrophobic interactions. The structure suggests an assembly mechanism for fiber formation in which the flat conserved face of the globular $\alpha-\beta$ domain packs against the isolated helix tail of another molecule.

This work was supported by an NIH grant to JAT and an NIH postdoctoral fellowship to KTF. A manuscript describing this work is in preparation by the authors listed above.

J. G. Cannon and P. F. Sparling. 1984. Annu. Rev. Microbiol. 38:111-133. 


\title{
CRYSTALLOGRAPHIC ANALYSES OF (MOSTLY) ELECTRON TRANSFER PROTEINS
}

\author{
D.C. Rees, M.K. Chan, M.H.B. Stowell, L. Joshua-Tor \\ Division of Chemistry and Chemical Engineering 147-75CH \\ California Institute of Technology \\ Pasadena, CA 91125
}

\section{ALDEHYDE FERREDOXIN OXIDOREDUCTASE}

Aldehyde ferredoxin oxidoreductase (AOR) from the hyperthermophilic archaeon, Pyrococcus furiosus, is a dimer of identical 605 residue subunits with unusual thermostability. AOR catalyzes the oxidative conversion of aldehydes to carboxylic acids at high temperatures $\left(\mathrm{T}_{\mathrm{opt}} \geq 90^{\circ} \mathrm{C}\right.$ ). The catalytic site of the aldehyde oxidizing reaction is a pterin containing tungsten cofactor, which is analogous to the molybdopterin-based cofactors contained in a broader class of molybdenum and tungsten oxotransferase enzymes, including xanthine oxidase, sulfate oxidase and nitrate reductase - none of which have been crystallographically characterized. To address questions regarding AOR's extreme thermal stability and the structure of the tungsten cofactor site, the structure of AOR has been solved crystallographically and refined to a current resolution of $2.3 \AA$. Initial phases were determined at $3.0 \AA$ resolution using data obtained in part from the rotation camera at the Stanford Synchrotron facility. Diffraction data were collected on the MAR imaging plate at $\lambda=1.08 \AA$ and processed with MOSFLM/CCP4 $(81,847$ total reflections were merged to 24,312 unique reflections ( $91 \%$ complete to $3 \AA$ resolution), with Rmerge $=0.074$ ). In comparison to data collected at $\lambda=1.54 \AA$ resolution, the anomalous scattering from $W$ sites is enhanced, while from the $\mathrm{Fe}$ sites is diminished at $\lambda=1.08 \AA$, thus permitting initial identification of the different metal sites.

The structure of the AOR dimer is illustrated in Figure 1. Located within the dimer are five metal sites; one putative $\mathrm{Fe}$ site at the dimer interface and one tungsten and $\mathrm{Fe}_{4} \mathrm{~S}_{4}$ site located within each subunit. While the role of the mononuclear $\mathrm{Fe}$ site is unclear, the arrangement of the remaining metal centers is consistent with oxidation of substrate occuring at the tungsten center, with transfer of electrons to the $\mathrm{Fe}_{4} \mathrm{~S}_{4}$ cluster, followed by transfer to ferredoxin, the physiological electron acceptor. The tungsten site sits at the center of each subunit and a channel can be identified which leads from the surface of the protein to the tungsten site. As postulated previously by Rajagopalan et. al., the molydopterin ligands do coordinate the tungsten via the dithiolene sulfurs, but unexpectedly, not one, but two molydopterin groups are found, so that the tungsten is coordinated by a total of four sulfur ligands. The remaining two coordination sites are occupied by ligands currently modeled as oxo and glycerol groups.

Although the origins of the thermostability of AOR have not been conclusively established, in comparison to other proteins, AOR exhibits a relatively small solvent exposed surface area, and a relatively large number of both ion-pairs and buried atoms. Further studies to elucidate the mechanism of the oxotransfer reaction and additional analyses to identify features unique to the AOR protein that may contribute to thermostability are presently underway.

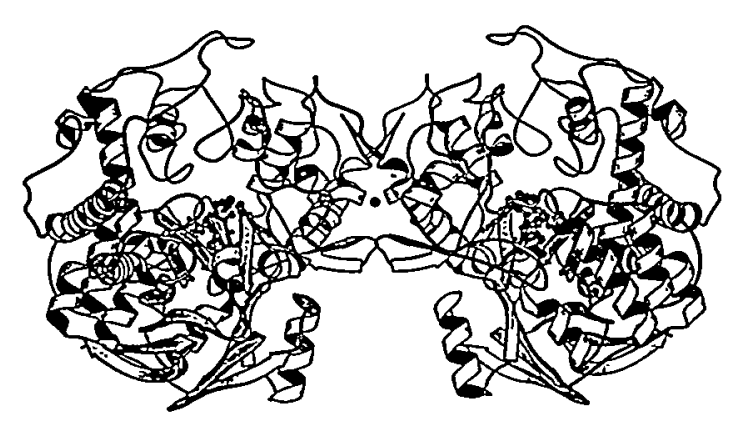

Figure 1. Structure of the AOR dimer 


\section{SUCCINATE-UBIQUNNONE OXIDOREDUCTASE}

$\mathrm{SQR}$ is an integral membrane protein with four subunits and a molecular weight of $\sim 120 \mathrm{kD}$. Redox cofactors associated with SQR include a covalently bound flavin (FAD), $2 \mathrm{Fe}: 2 \mathrm{~S}, 3 \mathrm{Fe}: 4 \mathrm{~S}$ and $4 \mathrm{Fe}: 4 \mathrm{~S}$ clusters, and a b cytochrome. SQR catalyzes the oxidation of succinate to fumarate, coupled to the reduction of ubiquinone to the ubiquinol. The quinone reduction is accompanied by the uptake of protons from one side of the membrane, a process that is analogous to quinone reduction/protonation in the photosynthetic RC. SQR crystals grow in space group R32, with hexagonal constants of $a=173.5 \AA$, $c=345.5 \AA$. We have cryocooled and examined SQR crystals on beam line 7-1 at SSRL. A native data set has been collected on the MAR detector to $3.5 \AA$ resolution and processed with MARXDS (50522 total reduced to 20858 unique reflections (82\% complete), with an Rmerge of 0.15 ). The search for heavy atom derivatives is now in progress.

\section{GAL6}

GAL6 is the first characterized member of a novel class of proteins in eukaryotes which have both DNA-binding and protease activities. The protein is highly conserved from bacteria and yeast to mammals. In yeast, GAL6 is a $52 \mathrm{kD}$ protein under galactose and GALA/GAL80 regulation. It is localized in the nucleus, exists as a hexamer in solution and binds specifically to GAL4 DNA binding sites (UASG). GAL6 is involved in a negative feedback loop in the galactose regulon, decreasing the levels of mRNA transcribed from GAL1 promoters by 3-5 fold. It also appears to delay the onset of induction by galactose. The sequence of GAL6 does not reveal the existence of any of the previously characterized structural DNA binding motifs. The protease activity was, however, first recognized from sequence analysis and then confirmed biochemically. The bacterial homolog of GAL6, PepC, functions as an aminopeptidase. In mammals, although the only function known of this protein is to hydrolyze the anticancer drug bleomycin, it is expressed in all tissues. GAL6 can also function as a bleomycin hydrolase, therefore its relevance in drug design as well. The fact that the sequences of these three proteins are so conserved throughout evolution (around 40\% identity) suggests that this protein has an important cellular function common in all these species.

We have solved the crystal structure to $2.2 \AA$ resolution of this DNA-binding protease from Saccharomyces cerevisiae with initial single isomorphous replacement and anomalous scattering phases calculated using data collected at SSRL. Cryocooled native and mercury derivative data sets were collected to $2.2 \AA$ resolution on the MAR detector, and processed with DENZO (native data set: 202481 total reflections reduced to 31451 unique reflections ( $99.8 \%$ complete) with an Rmerge of 0.074 ; mercury data set: 183105 total reflections reduced to 29482 unique reflections $(94.6 \%$ complete) with an Rmerge of 0.073 ).

The GAL6 hexamer observed in this crystal structure, is a thick ring-like structure with a striking channel through its center (Figure 2). This channel is lined with 60 lysines from the six subunits, giving a significantly positive electrostatic potential at the inner surface. Each subunit is composed of a papainlike core with additional structural and functional modules inserted into loop regions. Each GAL6 subunit has a three-domain structure composed of an oligomerization or the hook domain, a catalytic domain and a helical domain. The resemblance of the core to papain is striking, especially since the sequence similarity is significant only for very short regions around the active site residues. The $\mathrm{C}$ terminal arm of GAL6 extends into the active site cleft, thus blocking the approach to the active site, and perhaps serving a regulatory function.

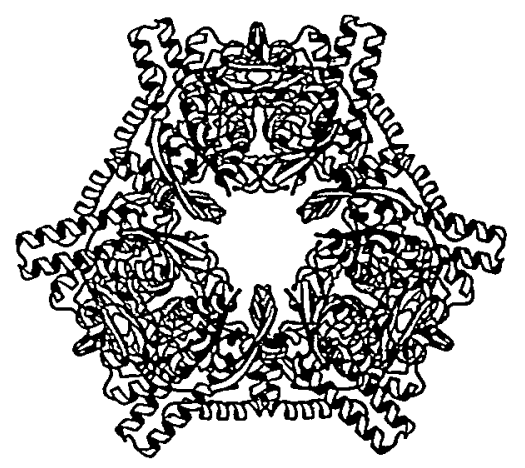

Figure 2. Structure of the GAL6 hexamer.

This work was supported in part by grants from USPHS (GM50775) and the Caltech Chemical Consortium.

\section{REFERENCES ACKNOWLEDGING SSRL SUPPORT}

Chan, M.K., Mukund, S., Kletzin, A., Adams, M.W.W, and Rees, D.C. "The $2.3 \AA$ Resolution Structure of the Tungstopterin Aldehyde Ferredoxin Oxidoreductase from the Hyperthermophilic Archaeon Pyrococcus furiosus", submitted (1994).

Joshua-Tor, L., Xu, H., Johnston, S.A. and Rees, D.C. "The Crystal Structure of GAL6, a DNA Binding Protease", in preparation (1994). 


\title{
X-RAY CRYSTALLOGRAPHIC STUDIES OF THE HYDROXYLASE COMPONENT OF METHANE MONOOXYGENASE FROM METHYLOCOCCUS CAPSULATUS (BATH)
}

\author{
Amy C. Rosenzweig and Stephen J. Lippard \\ Department of Chemistry, Massachusetts Institute of Technology, Cambridge, MA 02139 \\ Christin A. Frederick \\ Department of Biological Chemistry and Molecular Pharmacology, Harvard Medical School and Dana Farber \\ Cancer Institute, 44 Binney St., Boston, MA 02115 \\ Par Nordlund \\ Department of Molecular Biology, Stockholm University, S-10691, Stockholm, Sweden
}

\section{INTRODUCTION}

Methane monooxygenase (MMO) is a complex multicomponent enzyme system which catalyzes the first step in the metabolic pathway of methanotrophic bacteria, the oxidation of methane to methanol (eq. 1). Three distinct proteins comprise the complete system: a (1)

$$
\mathrm{CH}_{4}+\mathrm{NADH}+\mathrm{H}^{+}+\mathrm{O}_{2} \rightarrow \mathrm{CH}_{3} \mathrm{OH}+\mathrm{NAD}^{+}+\mathrm{H}_{2} \mathrm{O}
$$

multi-subunit $\left(\alpha_{2} \beta_{2} \gamma_{2}\right)$ non-heme iron hydroxylase (MW $251 \mathrm{kDa}$ ), which is the site of methane oxidation, an $\mathrm{Fe}_{2} \mathrm{~S}_{2}$, FAD containing reductase (MW 39 kDa) which accepts electrons from NADH, and a regulatory protein (MW $16 \mathrm{kDa}$ ), which has no prosthetic groups. The mechanism by which these three proteins carry out the transformation of methane to methanol is of general interest for several reasons. First, current industrial processes for the conversion of methane to methanol are carried out at high temperature and pressure with a low efficiency. An understanding of the biological oxidation of methane to methanol might enable us to improve these processes and to use the world supply of natural gas more effectively. Second, MMO catalyzes the oxidation of a variety of hydrocarbons in addition to methane. This property of the enzyme has led to the use of methanotrophic bacteria in cleaning up land contaminated by oil spills such as the 1989 Exxon Valdez spill, and in the oxidative removal of hydrocarbon contaminants such as trichloroethylene (TCE) from drinking water. Finally, methane is a greenhouse gas, and methanotrophic bacteria help to control the flux of methane in the atmosphere. Detailed structural characterization of MMO is essential to understanding the reaction mechanism. We are currently studying the hydroxylase component from Methylococcus capsulatus (Bath) by $\mathrm{X}$-ray crystallography.

\section{EXPERIMENTAL}

Crystals of the $M$. capsulatus (Bath) hydroxylase were grown as previously described. ${ }^{1}$ The unit cell dimensions are $62.6 \times 110.1 \times 333.5 \AA$ at 4 ${ }^{\circ} \mathrm{C}$ and $61.7 \times 109.6 \times 330.2 \AA$ at $-170{ }^{\circ} \mathrm{C}$. The lattice is orthorhombic with the observed extinction conditions consistent with the space group $\mathrm{P} 2{ }_{1} 2121$. There is one $\alpha_{2} \beta_{2} \gamma_{2}$ dimer of molecular weight 251 $\mathrm{kDa}$ in the asymmetric unit. Crystals were transported to SSRL in a cooler kept at $4^{\circ} \mathrm{C}$, and cooled to -170 ${ }^{\circ} \mathrm{C}$ in a nitrogen gas stream at beamline 7-1. Data sets were collected by using the Mar Research imaging plate (diamter $300 \mathrm{~mm}$ ) at beamline 7-1. The data were processed with the program DENZO (written by Z. Otwinowski) and merged and scaled with the program SCALEPACK (written by Z. Otwinowski).

\section{RESULTS}

The $2.2 \AA$ structure of the hydroxylase was previously solved by using $2.2 \AA$ native data collected at the Photon Factory and $3.0 \AA$ heavy atom derviative data collected with the $180 \mathrm{~mm}$ diameter Mar Research imaging plate at beamline 7-1. The structure revealed the complete secondary structure of the hydroxylase and the presence of two dinuclear iron active centers. The coordination and surrounding environment of these diiron sites were also revealed, and can now be related to ongoing mechanistic studies of the hydroxylase. ${ }^{2}$ In addition, possible sites of interaction with substrate and with the other methane monooxygenase components were identified. The structure has been fully described elsewhere. 3,4 By using cryocrystallographic techniques and the larger diameter ( 300 $\mathrm{mm}$ ) Mar Research imaging plate, we hoped to extend the resolution of the structure beyond $2.2 \AA$ resolution, thereby clarifying details of the diiron active center. Even with the larger diameter imaging plate, however, adequate resolution of the diffraction spots along the 
$330.2 \AA$ cell dimension prevented us from collecting better than $2.3 \AA$ resolution data, although strong diffraction to $1.8 \AA$ resolution was observed with the frozen crystals. The recent addition of a 2 theta lift at beamline 7-1 should enable us to collect higher resolution data.

\section{CONCLUSIONS}

Crystals of the MMO hydroxylase from $M$. capsulatus (Bath) can be frozen at $-170{ }^{\circ} \mathrm{C}$, which minimizes radiation damage and renders the collection of data beyond $2.2 \AA$ resolution, employing a 2theta lift on the $300 \mathrm{~mm}$ Mar Research imaging plate possible. Future experiments will focus on obtaining high resolution native data as well as on collecting data on crystals in which the diiron center has been reduced to the Fe(II)Fe(II) oxidation state, which is the species that reacts with dioxygen. In addition, we plan to collect data on both oxidized and reduced crystals soaked with various substrates and inhibitors.

\section{REFERENCES}

${ }^{1}$ Rosenzweig, A. C., Frederick, C. A., \& Lippard, S. J. J. Mol. Biol. 227, 283-285 (1992).

${ }^{2}$ Liu, K. E., Wang, D., Huynh, B. H., Edmondson, D., Salifoglou, A. \& Lippard, S. J. J. Am. Chem.

Soc. 116, 7465-7466 (1994).

${ }^{3}$ Rosenzweig, A. C., Frederick, C. A., Lippard, S. J. \& Nordlund, P. Nature 366, 537-543 (1993).

${ }^{4}$ Rosenzweig, A. C. \& Lippard, S. J. Acc. Chem. Res. 27, 229-236 (1994).

\section{ACKNOWLEDGEMENTS}

This work was sponsored by grants from the National Institute of General Medical Sciences (S. J. L.), the Claudia Adams Barr Foundation (C. A. F.), and the Swedish National Research Council (P. N.). 


\title{
Design of Influenza Virus Neuraminidase Inhibitors Based on Crystal Structure
}

\author{
Ming Luo, Center for Macromolecular Crystallography, University of \\ Alabama at Birmingham, Birmingham, AL 35294
}

Influenza virus remains to be a major causative agent for a large number of death among elderly people and young children, and huge economic losses due to illness. Finding a cue will have a general impact both on the basic research of viral pathogenesis of fast evolving infectious agents and clinical treatment of influenza virus infection. Influenza virus is an enveloped virus containing negative strand segmented genomic RNA's. On the viral membrane surface, there are two major antigens, haemagglutinin and neuraminidase. We have been working on the three-dimensional structure of neuraminidase, a tetrameric glycoprotein of 240,000 daltons. The crystal structure of several strains of influenza virus neuraminidase was determined and the structure determination of a neuraminidase-Fab complex is under way. Through the study of neuraminidase complexed with several inhibitors, we were able to identify the active site residues and to have a more clear picture how influenza virus escapes the host immune defense. Based on the structural information, we started to design new inhibitors which should have a potential to be developed as antiviral drugs. The process of inhibitor design involves modeling potential inhibitors using the active site structure and several computer programs; chemical synthesis of the designed inhibitors and testing these inhibitors for their efficacy; and determining the complex structure of new inhibitors and neuraminidase, which could suggest further modifications of the inhibitors. Today, a new series of inhibitors with a good inhibitory effect (best $\mathrm{IC}_{50}>10 \mu \mathrm{M}$ ) has been designed and a patent application has been approved for these compounds. A novel pattern of interactions between NA and inhibitors was identified. This is the first time a noncarbohydrate inhibitor was ever designed to fit the active site of NA. The best compound was shown to inhibit influenza virus replication in cell culture and a mouse model system. The work is now supported by a NIH grant. A cooperative research agreement between UAB and Biocryst Pharmaceuticals, Inc. has been effective since November 1, 1994. 


\section{Structural Study of Calicivirus}

Ming Luo, Center for Macromolecular Crystallography, University of Alabama at Birmingham, Birmingham, AL 35294

Calicivirus is a new class of positive strand RNA virus, including Norwalk virus. The virus can cause acute diarrhea in children and breakouts are found mostly in low income regions. We were able to crystallize the feline calicivirus, a prototype of calicivirus, and its structure determination is under way. The structure of calicivirus is expected to be different from other animal viruses. It has cupshaped depressions on the surface while most of viruses have protrusions. The structure will make an interesting comparison with other virus structures and help us in finding a cue for this newly emerging virus. The crystals were shown to diffract $X$-rays to $3 \AA$ resolution and the space group was determined to be $C 222, a=886 \AA, b=997$
$\AA$ and $c=442 \AA$. Over 250 frames of diffraction data were collected at synchrotron stations and a data set of about $50 \%$ complete to $3.5 \AA$ resolution were processed with acceptabie quality (Rsym=18\%). The particle orientation in the unit cell was determined by rotation function calculations. An envelope was derived from the EM structure at $22 \AA$ resolution provided by Dr. Prasad at Baylor Medical College. A model was built to fit the envelope using the coordinates of a similar virus, TBSV. Using the model, the position of the particles were determined by a R-factor search. A procedure of phase refinement by noncrystallographic averaging will be set to produce the final high resolution structure. 


\section{Low Resolution Data Collection of Yeast RNA Polymerase II \\ Peter R. Davd, David A. Bushnell and Roger D. Kornberg \\ Structutural Biology Dept, Stanford University, Stanford, CA $94305-5400$}

This run was very successful.we were able to successfully screen a variety of freezing and crystallization conditions. Because of this, we were able to extend the diffraction limits of yest RNA polymerase II (Pol II) to better than $3.5 \AA$. This data was gathered on beamline 7.1, using the mar imaging plate area detector under standard wavelength and distance.
The image shown below is from a

$100 \mu \times 125 \mu \times 350 \mu$. crystal.. The arrow at the far right indicates a group of reflections at $3.5 \AA$, as shown in the upper right. The photograph was taken directly from the screen of an image displayed with Wladek Minor's HKLview software.

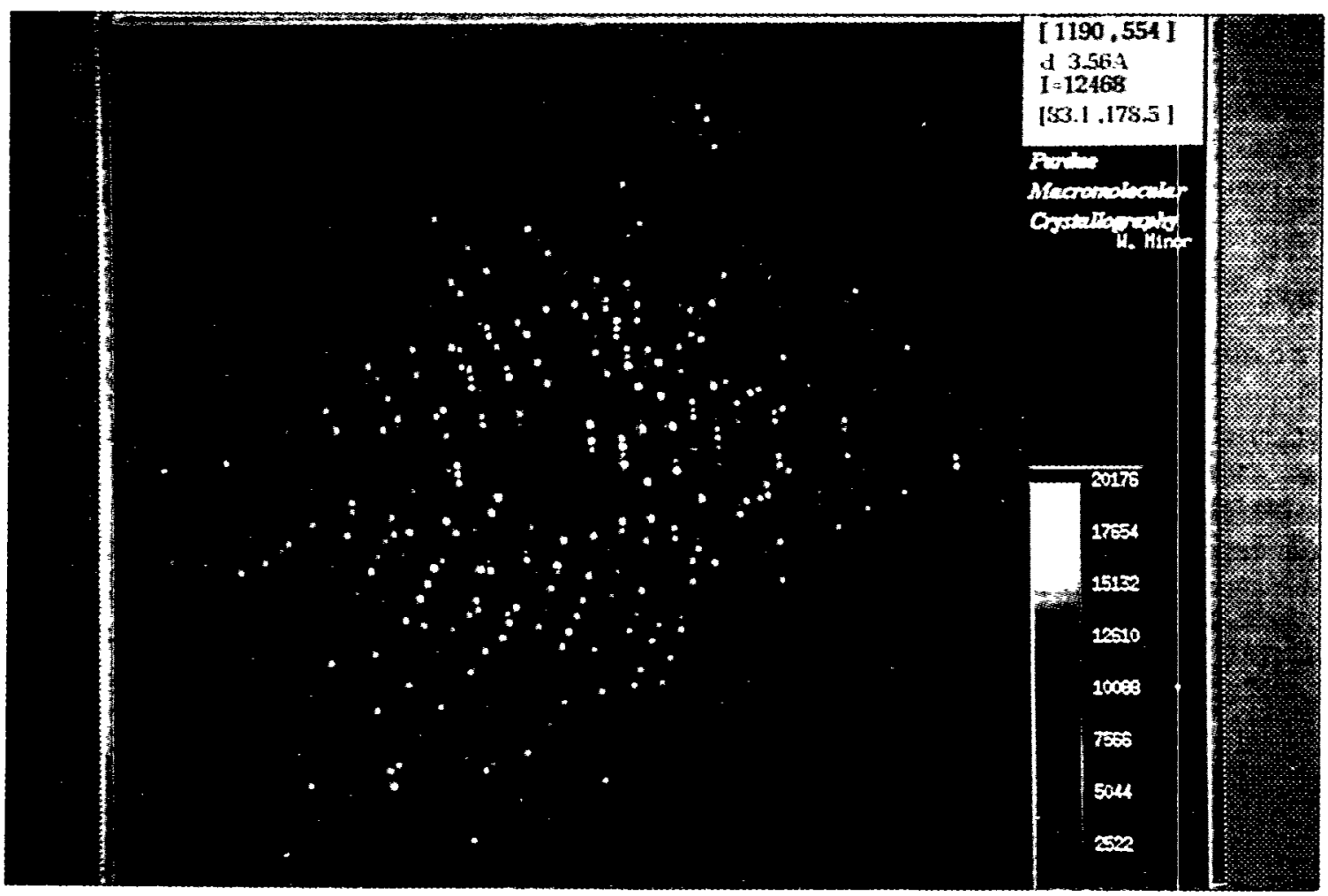




\title{
Heat-labile enterotoxin from $E$. coli (Cholera family toxins)
}

\author{
Ethan A Merritt, Focco van den Akker, Wim G J Hol \\ Department of Biological Structure and Howard Hughes Medical Institution, \\ University of Washington, SM-20, Seattle, WA 98195
}

\section{Introduction}

Cholera toxin is an $\mathrm{AB}_{5}$ hexameric assembly secreted by Vibrio cholerae. As with many other bacterial toxins the catalytic activity resides in the ' $A$ ' fragment, in this case a separate subunit, while receptor binding and delivery of the toxin to the target cell is mediated by a separate ' $B$ ' fragment, in this case a pentamer. The class of $\mathrm{AB}_{5}$ toxins may be subdivided into families based on sequence homology and catalytic activity. The cholera toxin family includes in addition to cholera toxin itself the $E$. coli heat-labile enterotoxins LT and LT-II (Figure 1), and a less well-characterized toxin from Campylo-bacter jejuni. The shiga toxin family comprises a number of toxins from Shigella dysenteriae (SHT) and the 'shiga-like' toxins (SLT, also known as verotoxins) from $E$. coli. The effect of these toxins on human populations ranges from the relatively mild travelers' diarrhea caused by infection with $E$. coli strains producing $\mathrm{LT}$ to the acute and life-threatening diarrhea caused by.$V$. cholerae infection and the equally serious hemolytic uremic syndrome ("hamburger disease') caused by members of the shiga toxin family. Together the $A B_{5}$ toxins are probably .responsible for over a million deaths annually.

\section{Why we are studying these toxins?}

- Questions of basic science (e.g. how does the toxin recognize and bind to the cell surface, how does it then enter the cell, what about the catalytic mechanism)

- Drug design (both the receptor binding site on the B-pentamer and the catalytic site on the A subunit are potential targets for the design of toxin-blocking drugs)

- Vaccine design (Because these toxins stimulate the mucosal immune system there is great interest in using an engineered form of the toxin as a basis for the design of vaccines against a wide range of diseases)

\section{SSRL Data collection}

The toxin project has greatly benefited from access to the high-flux crystallographic station on SSRL beamline 7-1. During 1994 crystallographic data sets were collected from small crystals of several engineered variants of LT-I containing single amino acid

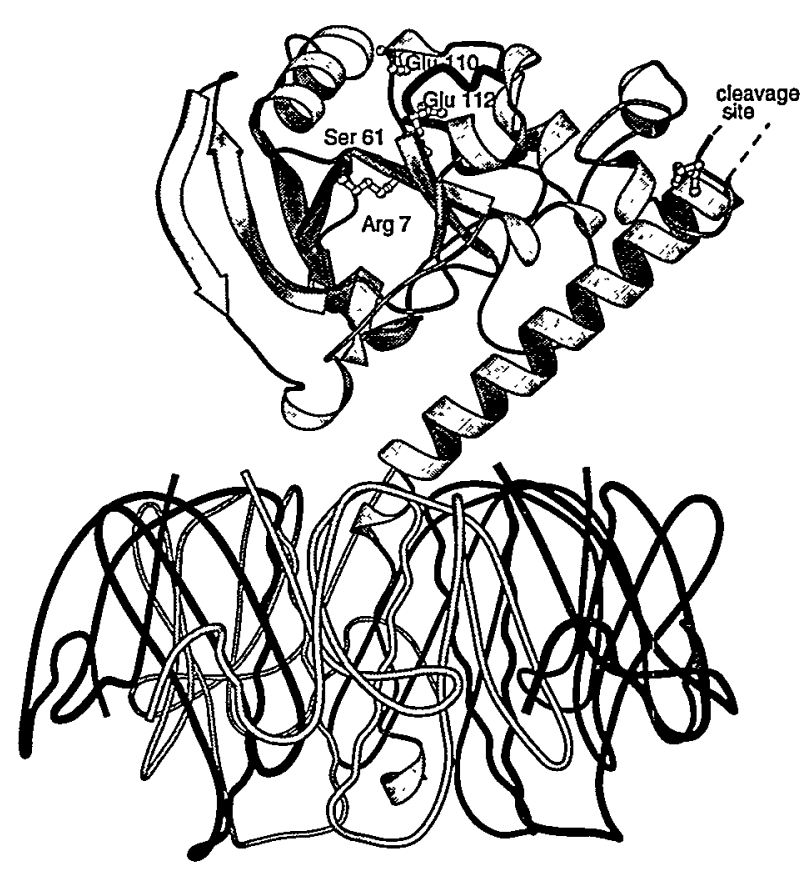

Figure 1: $E$. coli heat-labile enterotoxin (LT), showing the secondary structural elements of the catalytic $A$ subunit and selected residues believed to be involved in catalysis. The $B$ pentamer (lower portion of figure) is responsible for receptor binding. The A subunit of LT-II is homologous to that of LT-I in both sequence and structure; the $B$ subunits of the two toxins share no sequence homology, but may nevertheless exhibit the same secondary structure.

substitutions in the catalytic $A$ subunit, and from crystals of the $\mathrm{AB}_{5}$ holotoxin and of the $\mathrm{B}_{5}$ pentamer of the related toxin LT-IIb.

\section{Results}

The Va197 $\rightarrow$ Lys substitution destroys catalytic activity but does not induce a significant conformational change at the active site. The structure of this mutant has been determined and refined at $2.5 \AA$ resolution using data collected on SSRL beamline 7-1. The final crystallographic residual is $R=0.205$ for data from $15 \AA$ $2.5 \AA$ with $F / \sigma(F)>1.0$. Curiously, residue 97 is an internal residue and does not form part of the accessible surface of the protein in wither the wild type or the mutant structure. The larger Lys sidechain occupies an interior 
cavity, displacing several water molecules seen in the wildtype toxin (Figure 2). The Lysine sidechain also forms a salt bridge to the catalytic residue Glu 112. Either of these two structural differences from the wild type toxin may be responsible for the loss of activity.

Initial model building is in progress for the structures of the LT-IIb holotoxin and $B_{5}$ pentamer using data collected at SSRL. The holotoxin crystallizes in space group $P_{3} 21$ with cell parameters $a=b=105.63 \AA$ $c=171.56 \AA$. Data for the LT-IIb B-pentamer were collected at SSRL to a resolution of $2.0 \AA$ in a triclinic crystal form with cell parameters $a=5103 \AA \mathrm{b}=51.24 \AA$ $c=89.81 \AA, \alpha=105.6^{\circ} \beta=103.3^{\circ} \gamma=96.9^{\circ}$. Preliminary evidence appears to confirm the presence of structural homology between LT and LT-II despite the absence of sequence homology in the B subunits [van den Akker et al, unpublished].

This work is supported by the NIH (AI34501) and by the University of Washington Royalty Research Fund

\section{References}

Hol, WGJ, Sixma, TK, and Merritt, EA: Structure and function of $E$. coli heat labile enterotoxin and cholera toxin B-pentamer. In Bacterial Toxins and Virulence Factors in Disease, edited by Moss, Vaughan, Iglewski, and Tu. New York: M. Dekker (in press).

Merritt, EA, Sarfaty, S, van den Akker, F, L'hoir, C, Martial, JA, and Hol, WGJ: Crystal structure of cholera toxin B-pentamer bound to receptor $\mathrm{G}_{\mathrm{MI}}$ pentasaccharide. Protein Sci 1994, 3: 166-175.

Merritt, EA, Sixma, TK, Kalk, KH, van Zanten, BAM, and Hol, WGJ: Galactose binding site in $E$. coli heat-labile enterotoxin (LT) and cholera toxin (CT). Molec Microbiol 1994, 13: 745-753.

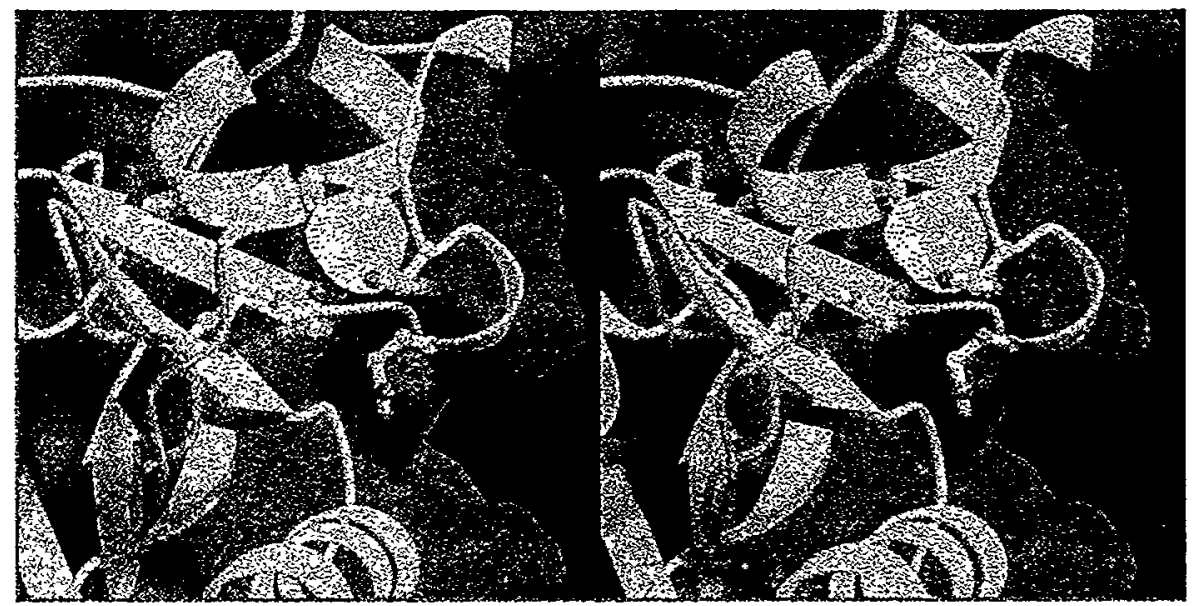

Figure 2: Stereo pair showing he active site of E. coli heat-labile enterotoxin. Extending horizontally across the figure is a crevice in the surface of the $A$ subunit which is believed to constitute the binding site for the substrates of the ADP-ribosylation reaction.. The internal cavity near residue Val 97 in the wild type structure is visible near the center of the figure. The accessible surface depicted is that of the Val97 $\rightarrow$ Lys mutant structure. Note that the sidechain of the mutation site residue Lys 97 does not extend far enough to form part of the protein surface. 


\title{
Crystallographic studies on enzymes of the pyruvate dehydrogenase multienzyme complex (PDC)
}

\author{
Jörg Hendle, Sharmila Mande, Steve Sarfaty \& Wim G. J. Hol
}

Department of Biological Structure, Biomolecular Structure Program and Howard Hughes Medical Institute, SM-20, University of Washington, Seattle, WA 98195, USA

\section{INTRODUCTION}

The giant multi-mega-dalton pyruvate dehydrogenase multienzyme complex (PDC) catalyzes the decarboxylation of pyruvate to acetyl CoA thereby connecting two major biochemical pathways, glycolysis and Krebs' cycle. The PDC is composed of multiple copies of at least three enzymes: the pyruvate decarboxylase-dehydrogenase (E1p), the dihydrolipoamide acetyltransferase (E2p) and the dihydrolipoamide dehydrogenase (E3).

The recently solved structure of the catalytic domain of the dihydrolipoyl transacetylase component (E2pCD) of the PDC from Azotobacter vinelandii and three of its mutants shed light on the mechanism of the E2 component of this multienzyme complex, the binding of substrates and intermediates, and the arrangement of the enzymes and/or subunits in the complex ${ }^{1-3}$.

This report describes the results obtained from diffraction data collected on SSRL beamline 7-1.

\section{Bacillus stearothermophilus E3}

The protein crystallizes in the orthorhombic spacegroup $\mathrm{P} 22_{1} 2_{1}$ ( $\mathrm{a}=64.2 \AA$, $\mathrm{b}=105,7 \AA, c=149.8 \AA$ ) with two independent subunits in the asymmetric unit. Crystals measuring $1.0 \times 0.4 \times 0.4 \mathrm{~mm}^{3}$ diffract to $2.7 \AA$ using the in-house rotating anode/multiwire detector system, whereas similar crystals diffract to $2.3 \AA$ using the MAR image plate on beamline 7-1. The diffraction data are $89 \%$ complete with a $\mathbf{R}_{\text {sym }}$ of $9.8 \%$ using the MOSFLM program package for processing. The structure of $B$. stearothermophilus E3 was determined by molecular replacement using the structure of A. vinelandii E3 as a model ${ }^{4}$.

\section{Bacillus stearothermophilus E1p}

Crystals of the trigonal space group $P 3_{1} 21$ diffract to $2.6 \AA$ on beamline 7-1 compared to $3.2 \AA$ on the in-house system. Structure determination using MIR techniques is in progress.

\section{Enterococcus faecalis E2pCD}

In contrast to the catalytic domain from $A$. vinelandii building the cubic core of the PDC with 24 subunits the protein from $E$. faecalis is assembled in an icosahedral manner ${ }^{5}$ with 60 subunits (total molecular weight $1620 \mathrm{kDa}$ ) related by a 5-fold symmetry among others. The resolution of crystals measuring $1.0 \times 1.0 \times 0.7 \mathrm{~mm}^{3}$ was limited to $7 \AA$ using the in-house system. $3.5 \AA$ data could be collected on beamline 7-1 revealing a very large unit cell with cell constants of $\mathrm{a}=\mathrm{b}=250 \AA$ and $\mathrm{c}=930 \AA$ in space group $\mathrm{P} 3 \mathrm{x} 21$. 


\section{Pseudomonas putida E2bCD}

The catalytic domain of the branchedchain acyltransferase crystallizes in space group I4 $22(a=b=112.7 \AA, c=124.1 \AA)$ with three identical subunits in the asymmetric unit. The crystals diffract to $2.3 \AA$ at beamline 7-1 compared to $3.5 \AA$ at the inhouse system. The structure determination using molecular replacement techniques is in progress.

\section{CONCLUSIONS}

All data sets of components of the 2-oxo acid dehydrogenase multienzyme complexes collected so far on SSRL beamline 7-1 show significantly higher resolution limits compared to the diffraction data collected on the in-house system.

\section{REFERENCES}

1) Mattevi et al. (1993), J. Mol. Biol. 230, 1183-1199.

2) Mattevi et al. (1993), Biochem. 32, 3887-3901.

3) Hendle et al. (1995), Biochem., in press.

4) Mande et al. (1995), in prep.

5) Hackert et al. (1989), Biochem. 28, 6816-6821. 
Proposal 2A61B

\title{
X-ray Crystal Structure Determination of Complexes between Chymotrypsin and Variants of the Inhibitor APPI
}

\author{
Axel J. Scheidig, Abraham M. de Vos and Anthony A. Kossiakoff \\ Deptartment of Protein Engineering, Genentech, Inc., 460 Point San Bruno Boulevard, \\ South San Francisco, CA 94080
}

Chymotrypsin is a serine proteinase with high selectivity to cleave the peptide bond after big hydrophobic amino acid residues. APPI is a member of the Kunitz type inhibitors and inhibits chymotrypsin despite of an arginine at the primary binding site (Arg15). In the context of analysis of protein-protein interactions, we have determined the crystal structure of chymotrypsin complexed with wildtype APPI and with a phage display derived variant of APPI (T11Q, P13R, M17H, S19K) both with an arginine at the primary binding position. Crystals of the $1: 1$ complex are very sensitive toward radiation damage. The initial crystals diffracted the $\mathrm{X}$-ray beam generated with a rotating $\mathrm{Cu}$-anode to a maximum Bragg spacing of $3.2 \AA$ (at room temperature). By the use of cryotechniques the resolution could be improved to 2.5 $\AA$ and in combination with the intense synchrotron beam $(=1.08 \AA)$ at the SSRL to $2.1 \AA$. The statistics for data collection and structure refinement are given in Table 1 . In both structures the binding mode of Arg15 is significantly different compared with the conformation of Arg15 in APPI complexed with trypsin.

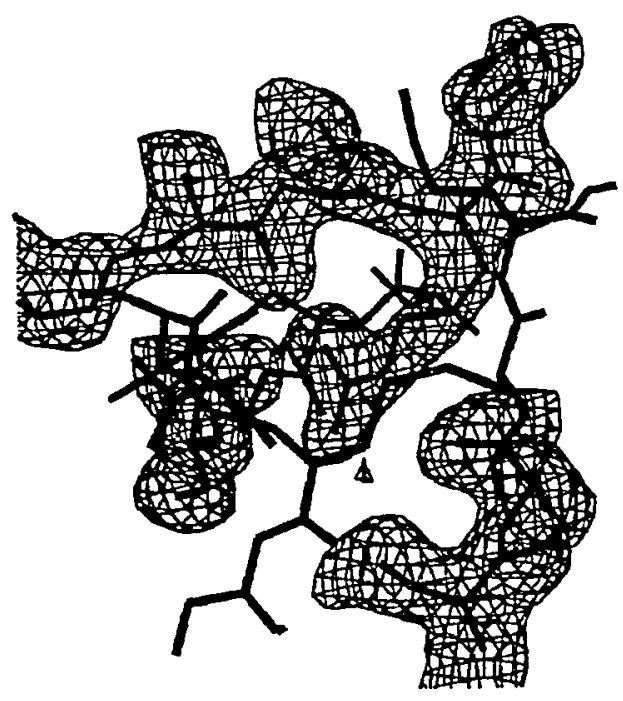

Fig.1: Primary binding pocket of the 1:1 complex between chymotrypsin and the APPI(T11Q, P13R, M17H, S19K) variant.
The high resolution of the data sets allow the analysis of specific water positions in the primary binding pocket. The electron density of the Arg15 bound in the primary binding pocket of chymotrypsin with bound inhibitor is shown in below (Fig. 1). These results complement the data obtained inhouse with other APPI variants, for example one with a histidine at the P1 site (Fig. 2)

Table1: Statistics of data collection and refinement

\begin{tabular}{lll}
\hline $\begin{array}{l}\text { Proteinase } \\
\text { Inhibitor }\end{array}$ & $\begin{array}{l}\text { Chymotrypsin } \\
\text { APPI }\end{array}$ & $\begin{array}{l}\text { Chymotrypsin } \\
\text { APPI(QRRHK) }\end{array}$ \\
\hline detector & MarPlate (30 cm diameter) \\
temperature & $100 \mathrm{~K}$ & \\
spacegroup & $\mathrm{P} 22_{1} 2(2$ molecules/AU) \\
cell axes & $\mathrm{a}=70.5 \AA, \mathrm{b}=181.6 \AA, \mathrm{c}=46.3 \AA$ \\
resolution & $2.1 \AA$ & $2.1 \AA$ \\
completeness & $81.1 \%$ & $97.2 \%$ \\
$\mathrm{R}_{\text {sym }}$ & $3.0 \%$ & $4.7 \%$ \\
$\mathrm{R}_{\text {cryst }}$ & $20.4 \%$ & $21.0 \%$ \\
$\mathrm{R}_{\text {cryst }}$ (free) & $32.6 \%$ & $31.8 \%$ \\
\end{tabular}

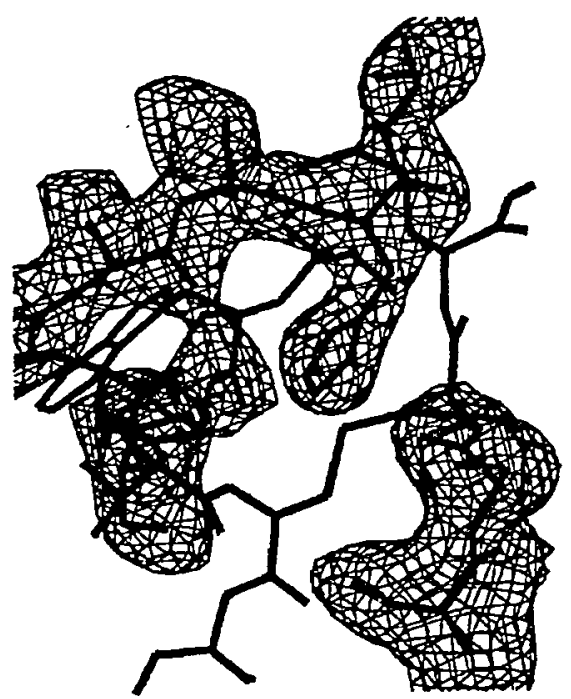

Fig.2: Primary binding pocket of the $1: 1$ complex between chymotrypsin and the APPI(T11R, P13H, R15H, M17A, S19K) variant. 


\section{Structure of the Envelope Glycoprotein from Tick-borne Encephalitis Virus (TBE) \\ Stephen C. Harrison and Félix Rey \\ Howard Hughes Medical Institute and Harvard University}

We have determined the structure of the envelope glycoprotein from TBE. This protein is critical for viral antigenicity and viral infectivity. TBE is a medically important virus in central Europe, and it is closely related to viruses important worldwide (Yellow fever, Dengue, etc.). Understanding the detailed structure of this protein will be valuable for subunit vaccine design, for working out how the virus binds to its receptor, and for elucidating the mechanism by which the virus enters a cell. The only other viral glycoproteins whose structures are known are those from influenza virus. The TBE virus glycoprotein therefore gives significant additional information about the architecture of a class of proteins that includes retroviral envelope antigens such as HIV gp120. The data collected at SSRL, together with data collected at other synchrotron sources, have now yielded an exciting and somewhat surprising structure. The dimeric protein is a very extended molecule that lies on the surface of the viral membrane. The correlation of protein architecture with positions of attentuation mutations and neutralization escape mutations lead us to propose a mechanism for the fusion-activity conformational change.

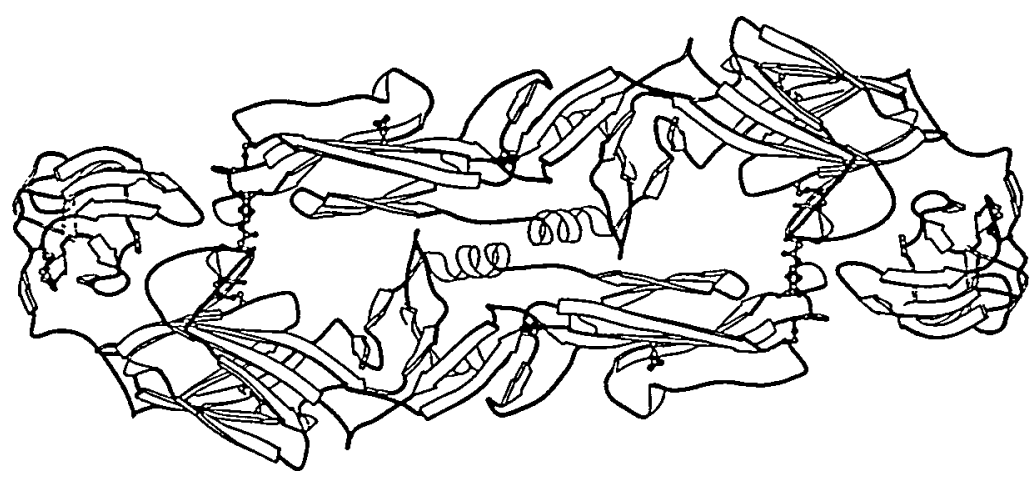

Rey, F.A., Heinz, F. X., Mandl, C., Kunz, C. \& Harrison, Stephen C. "The envelope glycoprotein from tick-borne encephalitis virus at $2 \AA$ resolution." Nature, 375, 291-298 (1995). 


\title{
Three-Dimensional Structure of a Hammerhead RNA
}

\author{
Heinz W. Pley, Kevin M. Flaherty \& David B. McKay \\ Department of Structural Biology \\ Stanford University
}

The hammerhead ribozyme is one of a small number of catalytic RNA motifs that has been identified and characterized in recent years. The consensus sequence required for activity is predicted to have three duplex stems and a conserved "core" of unpaired nucleotides. We have crystallized an RNA-DNA complex that represents a ribozyme (the RNA) bound to an inhibitor (the DNA). The crystals are trigonal, spacegroup $\mathrm{P} 3221, \mathrm{a}=89.7 \AA, \mathrm{c}=185.8 \AA$. On in-house rotating anode sources, the crystals show diffraction to $3.1 \AA$; on beamline 7-1 at SSRL, diffraction can be measured to $2.6 \AA$.

We determined the structure of the hammerhead ribozyme using multiple isomorphous replacement methods. The structure was initially solved to $3.1 \AA$ with in-house native and heavy atom derivative data. $\mathrm{A}$ native dataset was then collected to $2.6 \AA$ resolution at SSRL for refinement of the structure at higher resolution. These data have produced a model of the hammerhead ribozyme, showing that the "core" of the molecule has two structural domains. This work has recently been published:

Pley, H.W., Flaherty, K.M. and McKay, D.B. (1994). Three-dimensional structure of a hammerhead ribozyme. Nature 372, 68-74.

Pley, H.W., Flaherty, K.M. and McKay, D.B. (1994). Model for an RNA tertiary interaction from the structure of an intermolecular complex between a GAAA tetraloop and an RNA helix. Nature 372, $111-$ 113. 


\title{
Crystallographic Studies of Diphtheria Toxin Repressor
}

\author{
Xiayang Qiu and Wim G.J. Hol
}

Department of Biological Structure, Biomolecular Structure Program, and Howard Hughes Medical Institute, SM-20, School of Medicine, University of Washington,Seattle, Washington 98195.

\section{INTRODUCTION}

Iron is essential in virtually all living organisms for a wide variety of cellular processes. In body fluids of humans and animals, free iron ions concentration is extremely low and highly sequestered by proteins such as transferrin and lactoferrin. Therefore, most pathogenic bacteria have developed mechanisms by which they can take up iron from human or animal hosts. One of the most common method used by the bacteria is the synthesis and secretion of siderophores, low molecular weight chelators with high affinity for iron.

When Corynebacterium diphtheriae encounters an environment with a low concentration of iron ions, it initiates the synthesis of siderophores, as well as diphtheria toxin. The diphtheria toxin repressor (DtxR) plays a key role in this Fe(II)-dependent, global regulatory system and is the prototype for a new family of Fe-dependent repressor proteins in gram positive bacteria.

The crystal structure of dimeric DtxR holo-repressor in complex with different transition metals shows that each subunit consists of an $\mathrm{N}$-terminal DNA-binding domain, followed by an interface domain that contains the metal binding sites, and a third, very flexible C-terminal domain. Each DNA-binding domain contains a typical helix-turn-helix motif and has a topology which is very similar to catabolite gene activator protein (CAP). Molecular modeling suggests that bound DNA adopts a bent conformation with helices 3 of DtxR interacting with the major grooves.

There are two metal binding sites per subunit, approximately $10 \mathrm{~A}$ apart from each other. Binding site 1 cause a kink in the long helix connecting the DNA-binding and interface domains, while binding site 2 is positioned at a potential hinge region between the two domains. Residues 98 to 108 appear to be crucial for the functioning of the repressor. This helical segment provides four ligands of the two metal binding-sites plus three residues at the other side of the helix which are at the heart of the dimer interface.

The crystal structure of the DtxR holo-repressor suggests that the divalent cation corepressor controls motions of the DNA binding domain. In this way the metal co-repressor governs the distance between operator recognition elements in the two subunits and, consequently, DNA recognition. 


\section{STRUCTURE DETERMINATION}

The crystal structure of DtxR was determined using multiple isomorphous replacement methods. The native crystals diffract to 1.9 A resolution on a Raxis system in house. The major hurdle in the structure determination is then the introduction of heavy atom derivatives to the protein.

We have tried soaking over 50 heavy atom compounds into the crystals. After nearly 100 data sets were collected in house, a few derivatives were identified. Because they are relatively weak, and share a couple of common sites, combining these derivatives did not provide sufficient phasing to obtain interpretable electron density maps.

Co-crystallization of DtxR in the presence of $15 \mathrm{mM} \mathrm{HgCl} 2$ yielded some small crystals. The crystals looked quite metalic and very promising, yet diffracted only to $5 \mathrm{~A}$ in house. At that time, we had the opportunity to collect data at Stanford Synchrotron Radiation Laboratory (SSRL), and I was able to obtain a good quality 3.0 A data. Two heavy atom sites showed strongly in difference Patterson maps.

Subsequent phasing calculation were done using the new mercury data, and the electron density map improved greatly. The final figure of merit was 0.60 for the reflections from 50.0 to $2.8 \mathrm{~A}$. Density modification including solvent flattening, histogram matching and application of Sayre's equation were carried out, and the resulting electron density map was readily interpretable. The structure is now refined to an R-factor of $23 \%$ at $2.3 \mathrm{~A}$ resolution without adding water molecules.

From our experience on this project, it is quite clear that synchrotron data collection have greatly facilitated crystal structure determination. Even in the cases when high quality native crystals are available, a better derivative data from synchrotron source can also be an essential factor. We appreciate SSRL for their support of beam time, and we would also like to extent of acknowledgments to the staffs at SSRL for their helps during our experiments.

\section{REFERENCE:}

Xiayang Qiu, Christophe L. M. J. Verlinde, Suping Zhang, Michael P. Schmitt, Randall K. Holmes and Wim G. J. Hol (1995) "Three-dimensional structure of the diphtheria toxin repressor in complex with divalent cation co-repressors", Structure 3, Jan. 15. 


\title{
Crystal Structure of a Type II DNA Topoisomerase
}

\author{
James M. Berger, James C. Wang and Stephen C. Harrison \\ Dept. of Molecular and Cellular Biology, Harvard University
}

DNA topoisomerases are enzymes which catalyze the passage of DNA strands through each other. These proteins have been subdivided into two distinct categories based on their catalytic mechanisms, and are referred to as type I and type II enzymes. Type I enzymes alter DNA superhelicity by breaking a single strand of a duplex, passing another single strand through the break and resealing the break. In contrast, type II enzymes work by cleaving both strands of a DNA duplex, transporting another duplex through the break and then religating the duplex. Thus far, all type II enzymes are known to require ATP for catalysis.

We have succeeded in crystallizing the DNA binding/cleavage domain of yeast topoisomerase II. This 800 amino acid fragment gives rise to relatively small crystals with a large unit cell $\left(3,000,000 \AA^{3}\right)$. Because of the relatively poor diffracting power of the crystals, data collection could only be reasonably carried out using synchrotron sources. In addition, because the crystals were extremely radiation sensitive, all data were collected at cryogenic temperatures to slow decay. Despite using high intensity $\mathrm{X}$-ray radiation, a complete data set to $2.7 \AA$ from a single crystal still required approximately 4-6 hours of continuous beam time.

Data were collected at Beam line 7-1 of SSRL over the course of two separate four day time slots. To ensure that only high quality crystals were used, all crystals were frozen and checked for isomorphism at low resolution (4.5 $\AA$ ) on conventional lab X-ray sources prior to their use at SSRL. Both native and heavy atom derivitized crystals were used for data collection.

Data obtained from SSRL have now been processed. Using multiple isomorphous replacement (MIR) methods, we have obtained a solution for the crystallized fragment. Figure 1 shows a representative section of the solvent flattened electron density map with the current model. We are presently in the process of refining the structure to $2.7 \AA$.

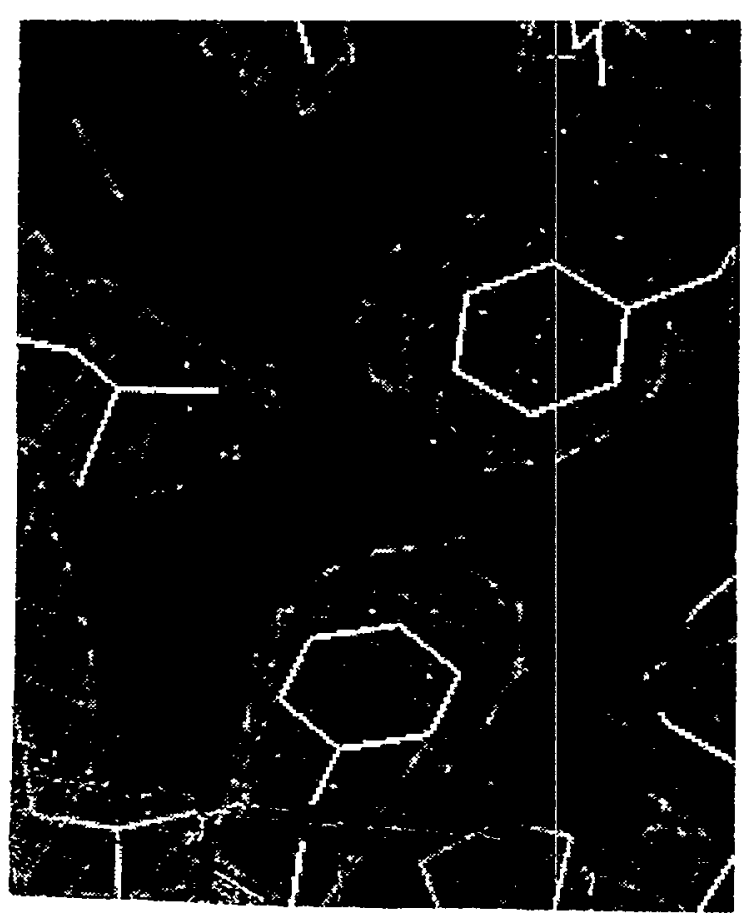

Figure 1. Electron density map using Fobs and solvent flattened phases (no model bias) for a region of the protein core. The current model is indicated by solid lines, the map by wire mesh. Contouring is at $2 \sigma$. 
The Regulatory Subunit of Protein Kinase A.

Ying Su, Nguyen-Huu Xuong, Susan Taylor and Kottayil Varughese. Department of Chemistry and Biochemistry and Department of Biology, University of California, San diego, CA-92093.

The regulatory (R) subunit of cAMP dependent protein kinase is the primary receptor of cAMP in eukaryotic cells. The inactive holoenzyme is a $R_{2} C_{2}$ tetramer. cAMP binding cooperatively to the $R$ subunit causes the complex to dissociate into an $R_{2}(c A M P)_{4}$ dimer and two active catalytic subunits. The $\mathrm{N}$-terminal domain which contains the dimerization region is followed by an inhibitor site and two tandem cAMP binding domains. We crystallized(Su et.al,1993) a monomeric $\Delta 1-91$ mutant of the RI subunit and solved the crystal structure by MIR techniques and refined to an Rfactor of $21.7 \%$ with $2.8 \AA$ data. The diffraction data were collected using a MAR image plate at Stanford Synchrotron Radia- tion Laboratory. Each cAMP binding domain consists of $3 \alpha$ helices and $8 \beta$ strands. The eight $\beta$ strands form two antiparallel $\beta$ sheets each with 4 strands forming a jelly-roll. cAMP binds in the syn conformation in both the domains, where as in the bacterial cAMP binding protein, catabolite gene activating protein, the binding is in the anti conformation. The binding of the cAMP is much tighter in the $R$ subunit due to favorable interactions. One of the most interesting questions being addressed is how cAMP binds to both sites cooperatively.

Su, Y., Taylor, S., Dostmann, W., Xuong, N. H., Varughese, K.I. J. Mol. Biology 230 1091-1093(1993).

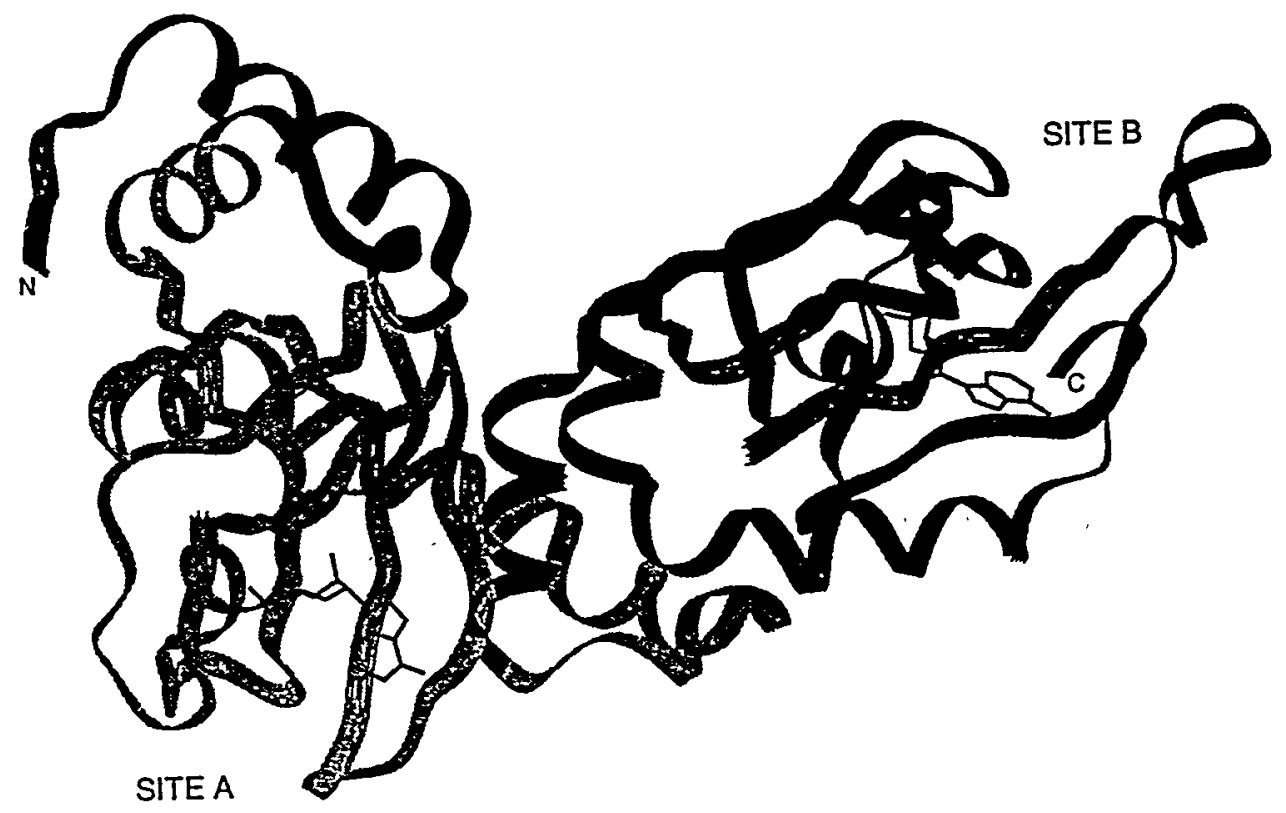

Fig. 1 A Ribbon representation of the structure. 


\title{
Crystallographic Studies of Myosin Subfragment-1
}

\author{
Ivan Rayment \\ Institute for Enzyme Research \\ University of Wisconsin
}

\begin{abstract}
:
The objective of this research is to improve our understanding of the molecular basis of muscle contraction by studying the threedimensional structure of myosin subfragment1 by single crystal x-ray diffraction. Myosin is the major protein in muscle in which it plays both a structural and enzymatic role. The myosin rod forms the backbone of the thick filament whereas the myosin head (subfragment-1, S1) is responsible for the generation of movement though the hydrolysis of ATP and its interaction with actin. The major focus of the present structural studies is to determine the nature of the conformational changes that occur during the contractile cycle and define the nature of the catalytic site. This is being accomplished by determining the structure of the myosin head in the presence of both actin peptides and nucleotide analogues.
\end{abstract}

\section{Progress Report:}

During the most recent data collection session efforts were focused on collecting data for a truncated myosin head that contains all of the components necessary for hydrolysis, actin binding and movement. This minimal motor domain was prepared by mutagensis in Dictyostelium discoideum. Crystals of this fragment diffract to better than $2 \AA$ resolution. Several crystal forms of this protein have been obtained both with and without nucleotide in the active site. At this time the data for this truncated myosin head in the presence of 11 different nucleotide analogs have been recorded. Most of these data sets were recorded at SSRL and are in the process of refinement. So far three publications have arisen from this work. It is expected that at least four more publications will be forthcoming from this data this year. This work has defined the location of the conformational change that occurs during hydrolysis and suggested a structural basis for the catalytic mechanism.

\section{Additional Studies:}

In addition to the data collection for myosin subfragment-1 we collected data for Galactose-1-Phosphate Uridylyltransferase from Escherichia coli at $1.8 \AA$ resolution. This structure has now been solved has been submitted to Biochemistry. 


\title{
ENZYMES CONTROLLING KEY STEPS IN THE SYNTHESIS OF POLYAMINES
}

\author{
Marvin L. Hackert, Marcos Oliveira, Ning Leh Chang, Stephen Ernst, and Andrew Kern \\ Department of Chemistry and Biochemistry, Univ. of Texas, Austin, TX 78712
}

\section{Introduction}

Decarboxylases are involved in a number of physiologically important reactions such as the synthesis of neurotransmitters and polyamines. Polyamines, in turn play a role in the synthesis of DNA, RNAs and proteins. Though some decarboxylases rely on a pyruvoyl moiety for catalysis, a greater number utilize pyridoxal-5'phosphate (PLP). Besides decarboxylation reactions, PLP related cofactors are involved in transamination and various elimination reactions. This versatility arises from the ability of the cofactor to labilize different bonds around the $C^{\alpha}$ carbon of amino acidlike substrates (Dunathan \& Voet, 1974). While a great deal is known about the mode of action of transaminases (Ford et al., 1980; Kamitori et al., 1988) and tryptophan synthase (Hyde et al., 1988), details relating to the mechanism of action of decarboxylases are sparse. Gani (1991) has suggested that the stereochemical course of proton transfer to the cofactor in decarboxylases is similar to that observed for aminotransferases. In particular, it was suggested that the histidine in the canonical sequence for decarboxylases Ser-X-HisLys(Pxl) serves as a proton donor at the $C^{\alpha}$ position. Sandmeier et al.(1994) examined all known sequences of decarboxylases by N-1 profile analysis and reported four, independent groups of decarboxylases.
The structural determination of ODC from L.30a. (Momany et al. 1995) together with sequence alignments indicate that this enzyme together with aspartate amino transferase (Ford et al, 1980) share a common PLP binding domain (Figure 1), while bADC and mODC show considerable differences from ODC, and form a separate group (Momany et al 1995; Sandmeier et al 1984). A similar conclusion has been proposed by Gani (1991) on the basis of stereochemical considerations.

The role of both ornithine decarboxylases (ODCs) and biosynthetic arginine decarboxylase (bADC) is to provide putrescine and agmatine respectively which are starting materials used in the synthesis of polyamines. While ODC has been identified in eukaryotes as catalyzing the first step in the synthesis of polyamines, $A D C$ initiates an alternate pathway used by plants and some human pathogens such as Trypanosoma cruzi. Regulation of ODC activity in the cell occurs at the levels of transcription, translation and post-translation. An antizyme protein has been identified in mammals (Murakami et al., 1992; Li and Coffino, 1993) as well as in $E$. coli (Canellakis et al., 1993) which inhibits enzymatic activity. In eukaryotes the antizyme also targets the enzyme for degradation by proteasomes. We have characterized the activation
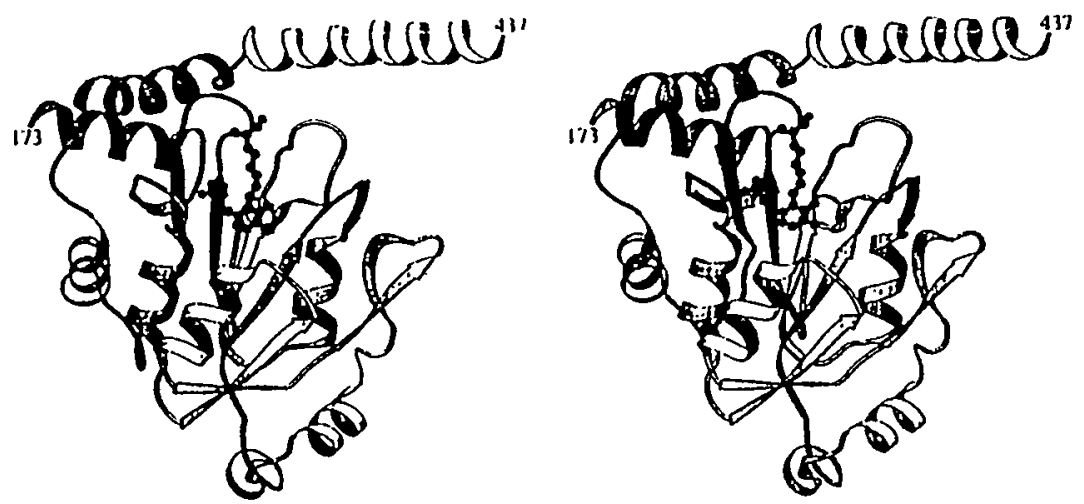

Figure 1. Stereo ribbon representation of the PLP-binding domain of ornithine decarbaxylase from L.30a. The figure was generated using MOLSCRIPT (Kraulis, 1991). 
of ODC $L .30 \mathrm{a}$. by $\mu \mathrm{M}$ concentrations of GTP (Carroll et al., 1995). Rat brain ODC has also been shown to be activated by nucleotides indicating a possible regulatory link between the two enzymes (Kilpelainen \& Hietala, 1994).

In order to determine the mechanism of action of decarboxylases and gain insight into the evolution of the different PLP-dependent enzymes, we have undertaken the structural determination of mouse ODC and bADC. In addition, we have examined the structures inhibitor complexes and of site directed mutants of ODC from Lactobacillus 30 a so that we can identify the residues involved the enzyme reaction as well as identifying different conformational states of the enzyme which may be stabilized by these complexes.

\section{Results}

Crystals of mODC diffract to $2.4 \AA$ resolution at SSRL. The crystals belong to the orthorhombic space group $\mathrm{P}_{2}{ }_{1}{ }_{1} 2$ with cell dimensions of $a=46.4, b=74.4, c=119.0 \AA$. These crystals are radiation sensitive, thus we have only been able to collect data using synchrotron sources such as SSRL. The other decarboxylase believed to be related to the eukaryotic class of decarboxylases is bADC. It crystallizes in the tetragonal space group $\mathrm{P}_{1} 1(3)_{1}{ }_{2}$ with cell dimensions $\mathrm{a}=\mathrm{b}=192.2$, $c=121.1 \AA$ (Rodriquez et al., 1994). The crystals are also radiation sensitive. At SSRL we have been able to extend the resolution of our data from $3.5 \AA$ resolution (using a rotating anode source) to $2.7 \AA$. This is a crucial increment in resolution necessary to improve accuracy of the atomic model for this enzyme. A native bADC data set has been processed using MOSFLM with statistics indicated in Table 1. The structural determination of bADC is being attempted by the identification of potential heavy metal derivatives with data collected using a rotating anode source.

The structures of inhibitor complexes of ODC L.30a. with diaminobutanone (DAB), diaminoheptyne (DAH) and a mutant His $223 \rightarrow$ Ser grown in the presence of ornithine have been determined. The structure of inhibitor complexes of ODC has not been previously possible using conventional sources due to poor resolution and in part due to the time necessary to obtain data using conventional sources. The structure of the inhibitor complex with DAB posed a particularly difficult problem because once it inactivated the enzyme its complex with PLP diffused out of the enzyme in a matter of a few hours. It was only at SSRL with the possibility of collecting rapid oscillation data that it was feasible to observe this inhibitor complex. Crystals of ODC were soaked with DAB in a capillary. After a period of 1 minute the capillary was drained and sealed before being exposed to synchrotron radiation. Using the MARS detector installed at SSRL it was possible to take exposures every three minutes. The rather large time span between exposures occurs because the image plate has to be scanned and erased before the next oscillation image can be recorded. We were able to take five exposures per crystal setting and used 7 crystals for the data set. This data was processed using MOSFLM with statistics shown in Table 1. The electron density map clearly shows the PLP complexed with DAB in the external aldimine form. The stricture of the inhibitor complex with DAH was approached by co-crystallizing ODC with DAH. The data was processed to $3.0 \AA$ resolution (Table 1 ). DAH causes only partial inhibition of ODC (data not shown) and an initial interpretation of the electron density map indicates that it is modifying a residue found in a channel that leads to the active site of ODC. The third data set collected at SSRL involving ODC was that of a site directed mutant His $223 \rightarrow$ Ser. His 223 is a second histidine found in the active site of ODC and is a conserved residue for many decarboxylases. The histidine in the canonical S- $\mathrm{x}-\mathrm{H}-\mathrm{K}$ sequence (His354) interacts with the phosphate of the PLP cofactor. His354 has also been proposed to be the proton donor in the final step of the decarboxylation reaction (Gani , 1991). However, we found that a mutation of His 223 caused a $99.8 \%$ decrease in the activity of the enzyme. This suggested to that His223 and not His354 might be the proton donor. The His $223 \rightarrow$ Ser mutant was grown in the presence of ornithine in order to observe the structure of a bound substrate. Again, with data obtained at SSRL (Table 1), we have been able to verify the His $223 \rightarrow$ Ser mutation at position 223 and our preliminary results indicate that ornithine is bound to the mutant enzyme.

The structures of mODC and $b A D C$ as well as the inhibitor complexes with ODC from L.30a. have the potential of leading to the design of improved anticancer and antiparasitic treatments (ornithine decarboxylase), antihistamines (histidine decarboxylase), control of neurotransmitters (glutamate and dopa decarboxylases), and a host of other reactions that depend on the catalytic cofactor, pyridoxal-5'-phosphate. All three structures of ODC inhibitor complexes and mutant are now been 
refined and a full analysis of the results is forthcoming.

This work was supported from the

NIH(GM30105) and the Foundation for Research.

\section{References:}

Anagnostopoulos, C., Choli, T. and Kyriakidis, D.A. (1992). Bioch. Intl. 27, 991-1000.

Carroll, D.W., Momany, C., Davidson, L., and Hackert, M.L. (1995). Biochemistry, in preparation.

Canellakis E. S., Paterakis A. A., Huang S. C., Panagiotidis C. A., and Kyriakidis D. A. (1993). Proc. Natl. Acad. Sci. USA 90, 7129.7133.

Dunathan, H.C. and Voet, J.G. (1974). Proc. Natl. Acad. Sci. U.S.A 71, 3888-3891.

Ford, G. C., Eichele, G., Jansonius, J. N. (1980). Proc. Natl. Acad. Sci. USA, 77, 2559-2563.

Gani, D. (1991). Phil. Trans. R. Soc. Lond. B332, 131-139.
Gallagher, T., Snell, E.E. and Hackert, M.L. (1989). J. Biol. Chem. 264, 12737-12743.

Guirard, B. M. and Snell, E. E. (1980). J. Biol Chem. 255, 5960-5964.

Kamitori, S., Hirotsu, K., Higuchi, T., Kondo, $\mathrm{K}$., Inoue, K., Kuramitsu,S., Kagamigama, H., Higuchi, Y., Yasuoka, N., Kusunoki, M., and Matsuura, Y. (1988) J. Biochem. Tokyo 104(3), 317-318.

Kilpelainen, P.T. and Hietala, O.A. (1994). Biochem. J. 300, 577-582.

Kraulis, P. (1991). J appl. Crystallogr. 24, 946-950.

Li X., and Coffino P. (1993). Mol. Cell. Biol. 13, 2377-2383.

Momany, C., Ghosh, R., Hackert, M.L. (1995). Protein Science, in press .

Murakami, Y., Matsufiji, S., Kameji, T., Hayashi, S., Igarashji, K., Tamura, T., Tanaka, K., and Ichihara, A. (1992). Nature(London) 360,597-599.

Rodriquez, B.R., Carroll, D.W., Mitchell, D., Momany, C. and Hackert, M.L. (1994). Acta Cryst. D50, 175-177.

Sandmeier, E., Hale, T. I., Christen, P. (1994)." Eur. J. Biochem., 221, 997-1002 .

Table 1. Statistics for data sets collected at SSRL.

\begin{tabular}{lllll}
\hline SAMPLE & $\begin{array}{l}\text { ODC }+ \\
\text { DAH }\end{array}$ & $\begin{array}{l}\text { ODC }+ \\
\text { DAB }\end{array}$ & $\begin{array}{l}\text { H223S }+ \\
\text { ORNTHINE }\end{array}$ & $\begin{array}{l}\text { bADC } \\
\text { NATVE }\end{array}$ \\
\hline RESOLUTION $(\AA)$ & 3.0 & 2.8 & 2.9 & 2.8 \\
PROCESSING PROGRAM & MOSFLM & MOSFLM & MOSFLM & MOSFLM \\
TOTAL REFLECTIONS & 211491 & 454211 & 95306 & 396146 \\
UNIQUE REFLECTIONS & 33916 & 20102 & 37905 & 53264 \\
RSYM & 0.10 & 0.098 & 0.073 & 0.064 \\
RSYM AT HIGHEST RESOLUTION & 0.248 & 0.305 & 0.297 & 0.0221 \\
\% COMPLETE & 79.6 & 38.8 & 81.0 & 94.3
\end{tabular}




\title{
Structure of the Human Protective Protein precursor determined by x-ray crystallography to $2.2 \AA$ resolution.
}

\author{
G. Rudenko ${ }^{\S \mathbb{I}}$, E. Bonten ${ }^{\S}$, A. d'Azzo ${ }^{\S}$, W. Hol ${ }^{\mathbb{I}}$ \\ $\S$ Dept. of Genetics, St. Jude Children's Research Hospital, Memphis TN 38101 \\ II Dept. of Biological Structure, University of Washington, Seattle WA 98195 \\ * Howard Hughes Medical Institute.
}

The human lysosomal storage disease galactosialidosis has been characterized as a combined deficiency of $\beta$-galactosidase and neuraminidase enzymatic activities. Clinical symptoms include neurological-, skeletal- and eye abnormalities, in more serious cases mental retardation and early death. The primary genetic defect in this disease is in fact the deficiency of a third lysosomal enzyme the Human Protective Protein (HPP) (1). HPP, a serine carboxypeptidase by itself, has a ca. $30 \%$ sequence homology to the wheat serine carboxypeptidase, for which the three dimensional structure has recently been solved (2). HPP is thought to be involved in a multi-enzyme complex with $\beta$-galactosidase and neuraminidase, preventing the degradation of the former and providing stabilization/activation of the latter in the harsh acidic environment of the lysosomes. In addition to the protective function, the carboxypeptidase activity is released upon excision of a $2 \mathrm{kDa}$ peptide out of the precursor polypeptide. It is as yet unclear to what extent the loss of carboxypeptidase activity is involved in the clinical phenotype of galactosialidosis.

The precursor form of HPP is currently being produced in the baculo-virus over-expression system. Growing crystals of this glycoprotein turned out to be a formidable task, due to a strong tendency to intergrow and form twinned crystals.

Diffraction was limited to $3.5 \AA$ or worse. In total six crystal forms were found before one was found yielding useful data. These crystals grow in spacegroup $\mathrm{P}_{1} 2_{1} 2$, with cell dimensions $\mathrm{a}=115.04$, $\mathrm{b}=148.11, \mathrm{c}=80.97 \AA$, but with varying morphology. Cryo-cooling proved absolutely essential for data collection, as the crystals die within 5 minutes at $0^{\circ} \mathrm{C}$. The marvelous cryo-cooling facilities at SSRL enabled us to collect data
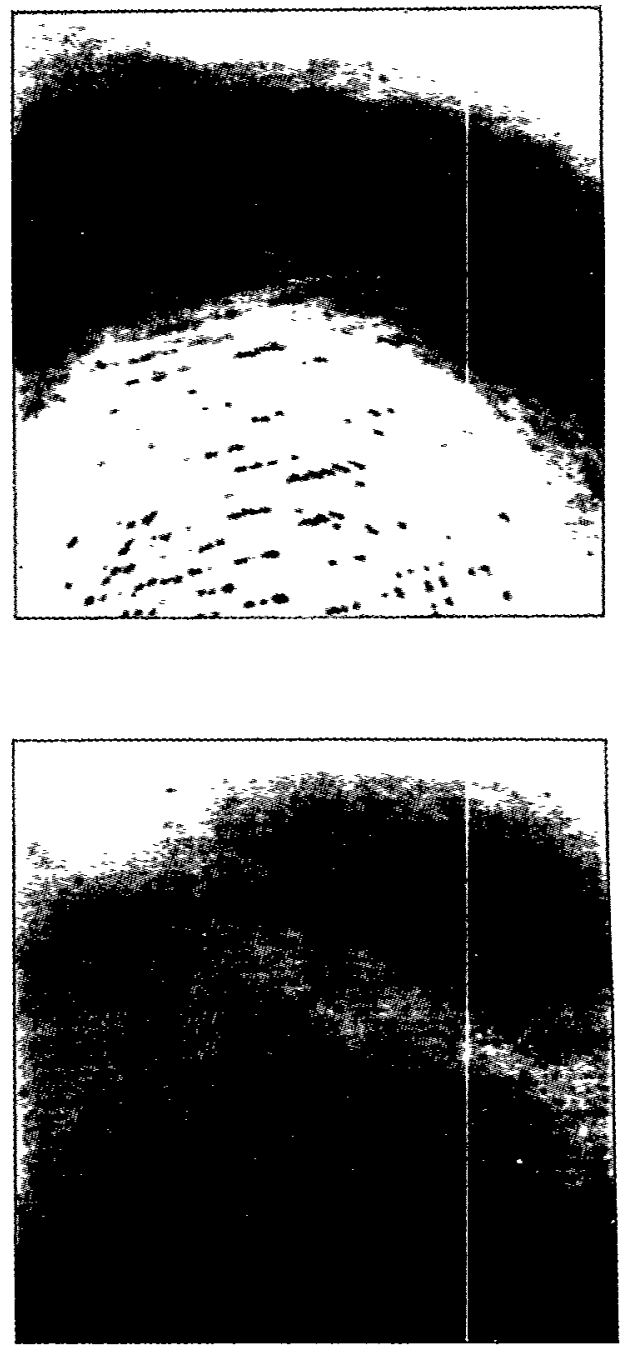

Fig.1: Optimization of cryo-cooling procedure and crystal choice greatly influences the quality of diffraction obtained from the HPP crystals, as apparent from the images above taken from two different crystals. 
extending to $2.0 \AA$ resolution at the beam-line $7-1$. Test experiments at SSRL showed that the cryocooling procedure and choice of crystal morphology was critical for obtaining good diffraction.

\section{See Fig. 1.}

The native data set was collected on the $30 \mathrm{~cm}$ MAR image plate and processed with MOSFLM/CCP4. The final data set contains 83731 unique reflections to $2.0 \AA$, with an $R_{\text {merge }}$ of $5.5 \%$ and $82 \%$ complete.

The structure of the HPP precursor homodimer was solved by molecular replacement. A very partial multi-ala search model was used based on the 3Dstructure of a wheat serine carboxypeptidase monomer (2). The initial model consisted of only 65 $\%$ of the $\mathrm{C}^{\mathrm{a}}$ atoms. Two-fold averaging using the program RAVE (3) was used to bootstrap out the missing 148 residues (out of a total of 452 residues) per monomer. Refinement of the model is in progress. The current $\mathrm{R}$-factor is $24.0 \%$ using data from $10-2.2 \AA$.

The 3D-structure of the precursor HPP allows us to understand why the precursor form of the enzyme is inactive, and to speculate on how removal of the 2 $\mathrm{kDa}$ peptide triggers activation. A number of amino acid substitutions found in defective HPP from different galactosialidosis patients with varying clinical severity have been localized in the 3Dstructure. There appears to be a remarkable correlation between the severity of the mutations for the molecular integrity of HPP and the severity of the disease caused by these mutations. In addition, the structure also provides hints towards the binding sites for $\beta$-galactosidase and neuraminidase, which will form a basis for mutagenesis and biochemical studies to probe the multi-enzyme complex formation in greater detail.

We believe that these studies will aid in the development and engineering of a stable and targetable form of the human protective protein suitable for gene/enzyme therapy.

\section{Acknowledgements:}

M. Soltis and the staff at the SSRL are most gratefully acknowledged for their help and expertise during data collection. This work has been supported by St. Jude Childrens Research Hospital (Memphis TE) and the Dept of Biological Structure, University of Washington (Seattle, WA).
Hoogeveen et al. (1980) Genetic heterogeneity in human neuraminidase deficiency. Nature, Vol. 285 p. 500 - 502.

d' Azzo et al. (1982) Molecular defect in combined $\beta$-galactosidase and neuraminidase deficiencny.

P.N.A.S. Vol. 79 p. 4535 - 4539.

Galjart et al. (1988) Expression of cDNA encoding the human "protective protein" associated with lysosomal $\beta$-galactosidse and neuramindase: homology to yeast proteases. Cell, Vol. 54 p. 755 - 764.

Liao et al. (1992), Refined atomic model of wheat serine carboxypeptidase II at $2.2 \AA$ resolution. Biochemistry, Vol. 31 p. 9796 - 9812.

Atomic coordinates were kindly provided to us by Dr. J. Remington.

Kleywegt, G. and Jones, T.A. (1994)

Convenient single and multiplecrystal real space averaging of macromolecular electron density maps (to be published). 


\title{
A Structural Investigation of Familial Amyotrophic Lateral Sclerosis.
}

\author{
Susan Redford*, Michael Johnson, Robert Hallewell, Elizabeth Getzoff, and John Tainer.
}

The Salk Institute*, 10010 N. Torrey Pines Rd. La Jolla, CA 92037, and

The Scripps Research Institute, 10666 N. Torrey Pines Rd. La Jolla, CA 92037.

Superoxide dismutases (SODs) are key enzymes in the cellular defense against free radical oxidation. SODs catalyze the decomposition of the superoxide radical, a toxic byproduct of aerobic metabolism, to water and hydrogen peroxide. SODs are present in virtually all known cells, and by acting as free radical scavengers have a central role in reducing the damage accompanying, for example, inflammation, viral infection, ischemia, and exposure to ultraviolet light. A tight genetic linkage between the neurodegenerative disease familial amyotrophic lateral sclerosis (FALS) and the gene encoding $\mathrm{Cu}, \mathrm{Zn}$ SOD has been recently reported. To date, 25 point mutations in SOD are known. FALS, also known as Lou Gehrig's disease, is a late onset disease, marked by the degeneration of motor neurons which leads to progressive paralysis. It is a uniformly fatal disease.

Our studies were aimed at elucidating the structural basis of one of the FALS point mutations, Leu 106 to $\mathrm{Val}$ (L106V), on the recombinant human $\mathrm{Cu}, \mathrm{Zn}$ SOD enzyme (HSOD). The crystal structure of the L106V mutant was determined using data collected at SSRL beamline 7-1. The bright synchrotron source allowed data collection to $2.4 \AA$ on $\mathrm{L} 106 \mathrm{~V}$ mutant crystals, which, due to a high solvent content and large unit cell, diffracted to only $3.5 \AA$ resolution with a conventional X-ray source. The crystallographic structure of the wild-type HSOD has been solved to $2.5 \AA$ resolution (Parge et al., $1992^{1}$ ), and is known to be an eight-stranded Greek key $\beta$-barrel. Leu 106 is buried in an hydrophobic environment, and lies in the middle of a cross barrel connection. The sidechain acts as an hydrophobic tether, holding the long Greek key loop in place, and effectively plugging the bottom of the $\beta$-barrel. In the wild-type enzyme, the buried Leu 106 sidechain contacts the sidechains atoms of Ile 113, Ile 112, and Ala 4. All of these residues have been linked by pedigree analysis to FALS, suggesting that this is a critical region of the enzyme. The mutation of L106V results in the loss of these van der Waals contacts, except for a long contact of $4.19 \AA$ between CD1 of Ile 113 and CG1 of Val 106. In the mutant structure, the Val 106 extends back towards Val 29, which pulls it further out of the hydrophobic pocket A gap is formed in the L106V mutant structure, which is not large enough to admit a $1.6 \AA$ water probe, but which results in poorer packing of the hydrophobic sidechains. Together with biochemical data, the crystal structure allows us to hypothesize as to the molecular basis of FALS.

1 Proc. Nath. Acad. Sci, USA, 6109-6113, 1992 


\title{
Human $\mathrm{Cu}, \mathrm{Zn}$ Superoxide Dismutase Turn Mutant and Permutant Structures to Study the Greek Key $\beta$-Barrel Fold
}

\author{
Maria M. Thayer, Hedieh Badkoobehi, Maurice Boissinot, \\ Robert A. Hallewell, John A. Tainer, Elizabeth D. Getzoff \\ Department of Molecular Biology \\ The Scripps Research Institute \\ La Jolla, California 92037
}

It has been suggested that the $\mathrm{N}$-terminal $\beta$ hairpin of the Greek-key $\beta$-barrel of $\mathrm{Cu}, \mathrm{Zn}$ superoxide dismutase is important as a nucleation site to facilitate folding of the enzyme (Getzoff et. al., 1989). In order to investigate the folding and stability of Greek-key $\beta$ barrel proteins, we have made mutants of human $\mathrm{Cu}, \mathrm{Zn}$ superoxide dismutase (HSOD) where all the amino acid residues of the first turn (T1) and/or the second turn (T2) have been changed to Gly. Mutants where one, two or three $\beta$ strands have been moved from the $N$ terminus to the $\mathrm{C}$-terminus of the protein have also been made. All of these mutants have been expressed, purified, and found to retain HSOD activity. Mutations in key structural residues that affect stability of the HSOD $\beta$-barrel can lead to the lethal neurodegenerative disease amyotrophic lateral sclerosis (Deng et. al, 1993, Redford, 1995).

Because of the highly focused synchrotron beam and the cryocrystallography facilities on beam line 7-1 at SSRL, we have been able to overcome the inherent mosaicity of the HSOD crystals and collect X-ray diffraction data to a resolution of $2.1 \AA$ on the T2 mutant. These data are of much higher quality than the $3.0 \AA$ data that we have been able to collect on a conventional source. The crystals have unit cell dimensions of $\mathrm{a}=194.6 \AA, \mathrm{b}=111.5 \AA, \mathrm{c}=144.1 \AA, \beta=94.1^{\circ}$ with C2 crystallographic spacegroup symmetry and 6 fold noncrystallographic symmetry resulting in 6 dimers per asymmetric unit. The data set that was collected at SS$\mathrm{RL}$ is $99.6 \%$ complete to $2.1 \AA$ resolution and has an unweighted $R_{s y m}$ on diffraction intensities of $8 \%$.

The rotation and translation functions using a trimer of dimers without the metal cofactors from the native HSOD structure (Parge et. al. 1992) as a probe revealed all 6 dimers in the asymmetric unit and gave an initial crystallographic $R$ factor of $37 \%$ at $3.0 \AA$ resolution. In the resulting $\mathrm{F}_{o}-\mathrm{F}_{c}$ difference electron density map, there were peaks of positive electron density with a magnitude at least 10 standard deviations above the mean at all 24 of the metal sites, confirming that the correct rotation solution had been found. We are currently in the process of refining the structure. The current R-factor is $27.8 \%$ to $2.1 \AA$ resolution after one cycle of manual fitting, during which only the metal sites were rebuilt, followed by crystallographic refinement with XPLOR.

Deng, H., Hentati, A., Tainer, J.A, Iqbal, Z., Cayabyab, A., Hung, W., Getzoff, E.D., Hu, P., Herzfeldt, B., Roos, R.P., Warner, C., Deng, G., Soriano, E., Celestine, S., Parge, H.E., Ahmed, A., Roses, A.D., Hallewell, R.A., Pericak-Vance, M.A., Siddique, T., (1993) Science 261, 1047-1051.

Getzoff, E.D., Tainer J.A., Stempien, M.M., Graeme, I.B., Hallewell, R.A. (1989) Proteins: Structure Function and Genetics 5, 322-336.

Parge, H.E., Hallewell, R.A., Tainer, J.A., (1992) Biochemistry 89, 6109-6113.

Redford, Susan R. (1995). Ph.D. thesis in Macromolecular and Cellular Structure and Chemistry at The Scripps Research Institute. 


\section{Structure of a $90 \mathrm{kD}$ fragment of yeast topoisomerase II}

James Berger, James C. Wang \& Stephen C. Harrison

Harvard University and Howard Hughes Medical Institute

We have determined the structure of an important fragment of yeast topoisomerase II. This enzyme is essential for untangling DNA during cell division. It is the target for significant antibacterial and anticancer drugs.
The structure suggests how this remarkable enzyme, which divides double-helical DNA and then allows a second DNA duplex to pass through the first, can function as a simple molecular machine.

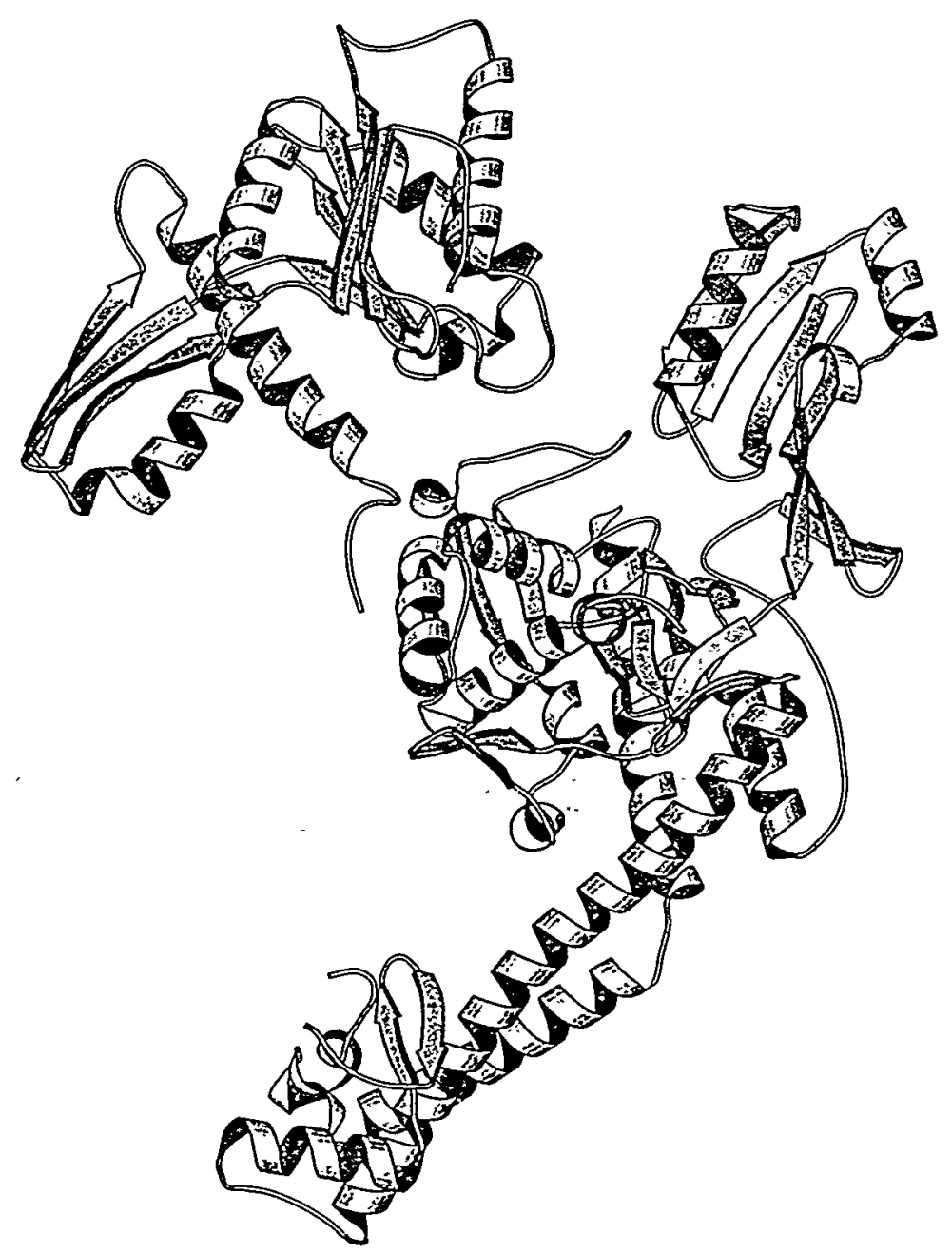




\title{
Structure Determination of Proteins Involved in Signal Transduction
}

\author{
Ursula Schulze-Gahmen, Heather D. Jones ${ }^{\dagger}$, David O. Morgan ${ }^{\dagger}$ and Sung-Hou Kim \\ Department of Chemistry and Lawrence Berkeley Laboratory, University of California, Berkeley, CA 94720 \\ †Department of Physiology, University of California, San Francisco, CA 94143-0444
}

Introdunction. The timing and integration of the eucaryotic cell division and growth cycle is controlled by cyclin-dependent kinases (CDKs), which are highly conserved among eucaryotic species. CDKs are able to phosphorylate many proteins that are involved in cell cycle events, including histones, lamins, and tumor suppressor proteins. In accordance with their central role in the cell cycle, enzyme activity is tightly controlled by multiple mechanisms. Kinase activation requires complex formation with regulatory cyclin proteins, followed by an activating phosphorylation. Our goal is to determine the structure of the complex of CDK2 and a fragment of cyclin A. A comparison of this complex structure with the structure of the inactive apoenzyme ${ }^{1}$ might reveal the mechanism of CDK activation through complex formation with cyclins.

Experimental Methods. The complex of human CDK2 and a fragment of human cyclin A, missing the $\mathrm{N}$-terminal 171 amino acids, were crystallized from $1.0 \mathrm{M} \mathrm{LiSO}_{4}, 0.1 \mathrm{M}$ Tris/HCl $\mathrm{pH}$ 8.4, 3\% PEG 8000, $20 \mathrm{mM}$ DTT, 2 mM EDTA, $0.1 \% \mathrm{n}$-octyl glucoside using microseeding methods. Because the crystals grow as very thin plates $(0.5 \mathrm{x}$ $0.3 \times 0.04 \mathrm{~mm}$ ) which require long exposure times in the $\mathrm{x}$-ray beam, we screened for soaking solutions which would allow flash freezing of the crystals.

Although the crystals are grown from high salt concentrations, they are stable in low salt concentrations. We were able to successfully freeze crystals in $0.15 \mathrm{M} \mathrm{LiSO}_{4}, 0.05 \mathrm{M}$ HEPES $\mathrm{pH} 8.0$, 30-35\% PEG 400.

Data sets at SSRL were collected on beam line 7-1 using a Mar research imaging plate. Since freezing conditions had not been established at that time, data were collected from unfrozen crystals at $4^{\circ} \mathrm{C}$. Each data set was collected as a series of $2.0^{\circ}$ rotations and processed with the program MOSFLM or DENZO. Data were merged and scaled with programs in the CCP4 program suite.

Results. Data reduction from several crystals revealed two different crystal forms grown from the same crystallization conditions. The best data were collected from a monoclinic crystal in spacegroup $\mathrm{P} 21$ with cell dimensions $\mathrm{a}=77.0, \mathrm{~b}=161.34, \mathrm{c}=$ 73.2, $\beta=120.7$. These data are $65 \%$ complete in all resolution shells with an $R_{\text {merge }}$ of 10.6 to $3.4 \AA$. A second crystal form was indexed with unit cell dimensions $\mathrm{a}=73.0, \mathrm{~b}=161.0, \mathrm{c}=140, \alpha, \beta, \gamma$ close to 90.0. At this point, we are not sure yet if these crystals are orthorhombic or a twinned form of the monoclinic crystal form.

Since the $\mathrm{x}$-ray structure of CDK2 is known, we tried to place the molecule into the unit cell of the monoclinic CDK2-cyclin A complex crystals. Although CDK2 constitutes only $50 \%$ of the complex, and the data are only $65 \%$ complete, we obtained relatively strong solutions for the rotation function and translation function in XPLOR. 


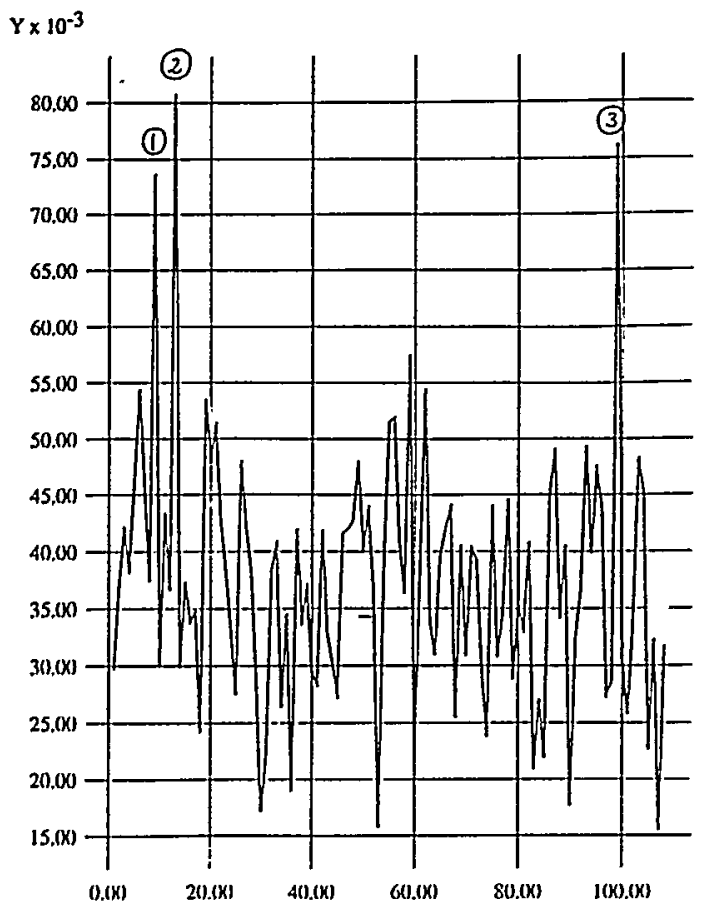

Fig. 1. PC-refinement of results from the rotation function in XPLOR. Peak 1 and 3 are the same orientations. Hence, only two different solutions have strong signals above the background.

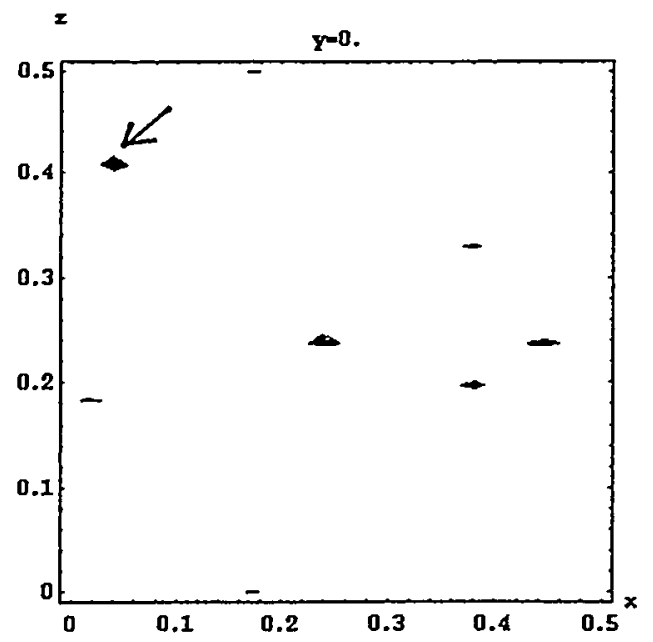

Fig. 2 Results of the translation function for orientation 1 from PC-refinement. The strongest signal, indicated with an arrow, is 1 sigma above the next highest signal.

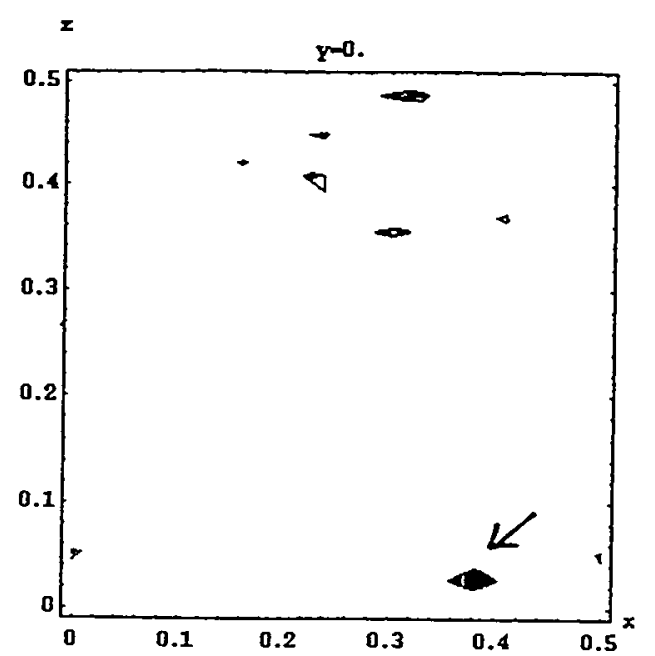

Fig. 3. Translation function for orientation 2 from the PC-refinement. The strongest peak is 1.7 sigma higher than the next highest solution.

sigma

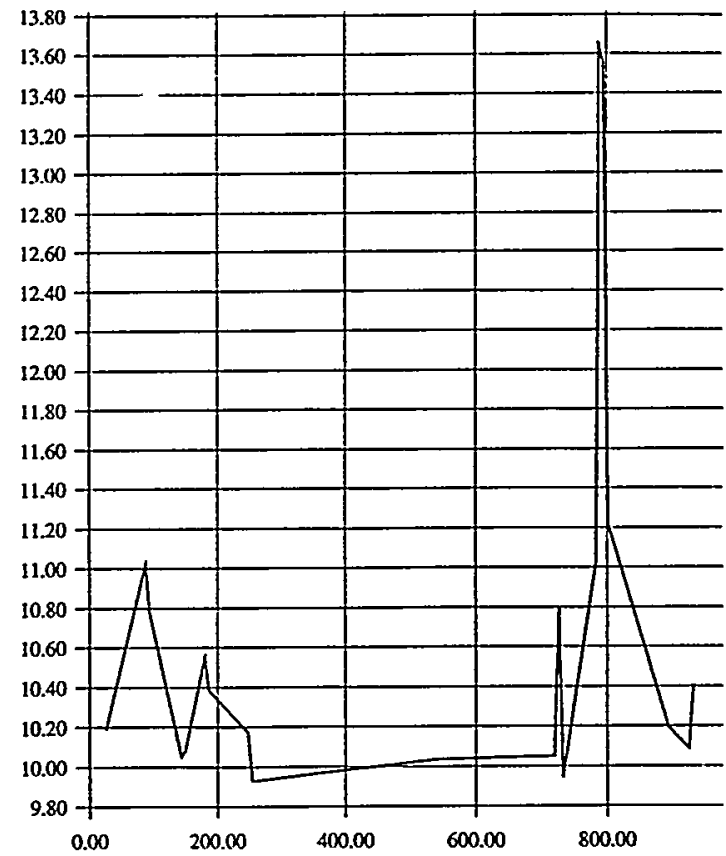

Fig. 4 Results of the combined translation function to determine the relative position in $y$ between molecule 1 and 2 . The solution at $y=0.8$ is 2.5 sigma higher than the next highest solution. 
The molecular packing resulting from the described molecular replacement solution shows no unfavourable contacts. However, with only $50 \%$ of the structure accounted for in the molecular replacement model, electron density maps show no clear density for the unknown structure of cyclin A.

Future experiments. One or two heavy atom derivatives should improve phases sufficiently to be able to locate and trace the cyclin A molecule. Hence, we will be screening for heavy atom derivatives of both crystal forms.In addition, we are exploring the second crystal form, which is predominantly obtained in crystallization experiments. Since we recently found conditions for crystal freezing, we should be able to obtain complete data sets of one crystal at our next synchrotron time.

\section{References.}

1) DeBondt,H.L. et al. (1993) Nature 363:595-602. 


\title{
Synchrotron Radiation Detector Development Program
}

\author{
William K. Warburton, Bradley Hubbard, Yuan Kong and Carl Zhou \\ $\mathrm{X}$-ray Instrumentation Associates \\ 2513 Charleston Road, STE 207, Mountain View, CA 94043-1607
}

The principal activity under this project proposal in 1994 occurred in the area of developing digital signal processing (dsp) electronics to replace the analog electronics which are conventionally used to support energy dispersive solid state detector arrays such as SSRL's pair of Canberra 13 element HPGe detectors. This project is driven by several desires: to reduce costs, to reduce volume, to increase levels of automation, to increase throughput, and to achieve multichannel analysis (MCA) capability on all channels in a detector array. Typical existing electronics consist, on a per channel basis, of a spectroscopy amplifier with pileup rejections (2 wide NIM module), two or three single channel analyzers (SCAs: 4 per 1 wide NIM module), and a CAMAC hex scaler channel per SCA. This ends up filling 3-4 NIM bins plus a few CAMAC slots and creating a tangle of cabling. Tuning the spectrometer entails reconnecting each channel in turn to a MCA in order to set gain and windows and takes a couple of hours to do properly. Once set, channels are rarely checked again to monitor their continued correct performance, the overhead time if much too high. The cost of these electronics runs about $\$ 4,000$ per channel, including NIM bins and cabling.

These systems work fairly well at energies which are high enough so that the detectors can resolve fluorescence $x$-rays from elastically scattered $x$ rays. This limit is somewhere in the $4-6 \mathrm{keV}$ range, depending on what amplifier shaping time can be used. Longer shaping times give higher resolutions but also require lower counting rates to keep pileup losses to an acceptable level. Below this resolution limit the fluorescence and elastic peaks overlap and it is no longer possible to set an SCA window which collects essentially only one or the other. For these energies it would be particularly desirable to have an MCA on each channel so that the areas under the peaks could be determined by deconvolution routines or other correcting algorithms. If this capability could be obtained, then all the well known benefits of single photon counting, which have proven so effective at higher energies, could be applied to such biologically interesting elements as $\mathrm{P}, \mathrm{S}, \mathrm{Cl}, \mathrm{K}$ and $\mathrm{Ca}$.
The approach which we are developing is quite different: first we take the signal straight from the detector's preamplifier and, after some analog signal conditioning, digitize it. The digital signal is then processed by dsp techniques to implement all the usual spectrometer functions, specifically including pulse shaping, peak detection and pileup rejection. This approach has several important advantages. First, because the detected peak is a digital number already, the MCA function is trivial to implement. A memory location with that address is simply incremented by 1 . Moreover, because the number is already available, there is no system deadtime associated with this operation. The total system deadtime becomes as fast as theoretically possible, being set only by the requirements of deadtime inspection. Second, because the digitally shaped peaks can be truly triangular, it is possible to set pileup inspection times very close to the peaking time. This is different from analog shaping, where extended pulse tails require significantly longer inspection times, even for nominally "triangular" shaping. Third, because commercial dsp electronics can be employed, the who processor becomes both far more compact (4 channels in one CAMAC module) and also much cheaper (less than $\$ 2,000 /$ channel, including MCA). Finally, because both the analog functions such as gain and digital functions such as peaking time are all directly under digital control, it becomes quite feasible to completely automate all aspects of setup, data collection, and channel integrity procedures. All this can be accomplished, in a properly designed system without loss of energy resolution.

Our research as SSRL has concentrated on the high count rate aspects of this development and on characterizing changes in resolution with count rate. Some non-beam time was also used to characterize the various energy dispersive spectrometers at SSRL in order to assure that our final electronics could work with them all.

Our first study was to characterize the various preamplifiers' outputs versus countrate. Two issues were important: whether any of the preamplifiers showed any changes in their operating parameters ver- 
sus countrate (for which correction algorithms would have to be devised) and what were the statistics of count arrival (which determine the dynamic range requirements of the analog part of the system). There were two preamplifier categories: resistive feedback (RF) and pulsed optical feedback (POF). The POF amplifiers were found to be very stable and to have characteristics which were essentially count rate independent. The RF preamplifiers, however, produced $x$ ray energies which varied with incoming count rate (ICR). Considerable effort was needed to discover that this resulted from variation in their DC operating point (our system is DC coupled) with rate.- Once discovered, we measured it as a function of rate by establishing the correspondence between count rate and ion chamber current at low rates where our PCbased MCA works linearly, and then using ion chamber readings to infer count rates for measurements up to $500,000 \mathrm{cps}$ ICR. Having determined the amplitude of this effect, we were also able to devise a fairly simple correction for our processing algorithm to correct for it. Figure 1 shows a spectrum from a $\mathrm{Cu}$ target taken using $3 \mu$ s peaking time at $70.4 \mathrm{kcps}$. Only $16 \%$ of the incoming counts are lost to pileup.

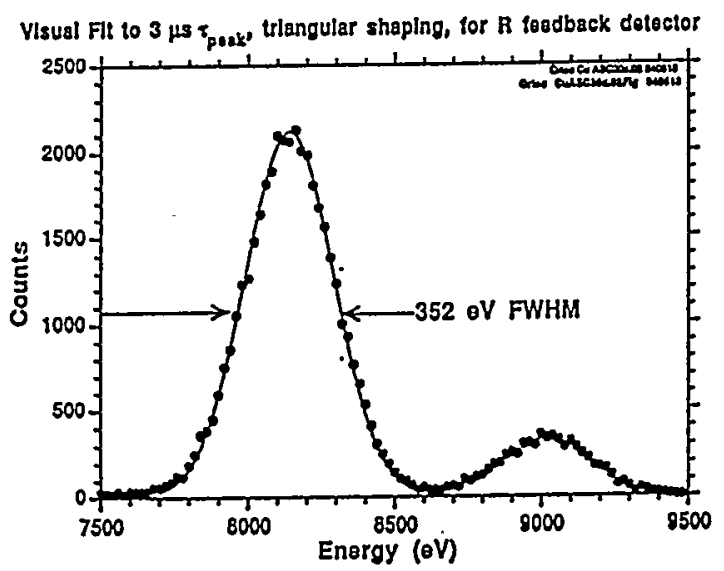

Figure 1: Spectrum taken using software emulation of dsp processing for input data from an RC feedback preamplifier with $3 \mu$ s triangular peaking time.

Figure 2 shows a curve from our study of count statistics. This is the RC preamplifier output (the raw input to our processor) and shows how arrival rates can fluctuate considerably from the average rate. This was taken at $19 \mathrm{kcps}$ ICR, for which the average inter-pulse time is about $50 \mu \mathrm{sec}$. The longest interval is over $240 \mu \mathrm{s}$, the shortest is about $4 \mu \mathrm{s}$. Figure 3 shows how our processor (here emulated in Labview software) takes this data (the dark curve) and converts it into triangular pulses. The figure shows a pile-up event (the second peak, which has
3 pulses) and a not-quite pile-up event (the third peak which has 2 pulses but also two well resolved peaks). The peaking time here is $3.6 \mu \mathrm{s}$, so base widths are $7.2 \mu \mathrm{s}$. These two pulses are separated by about 4.8 $\mu s$ and are easily resolved. With conventional shaping they would be smeared and rejected by pileup inspection circuitry. This illustrates our earlier comments about how precisely triangular digital pulses require shorter pileup inspection times than tailed analog pulses and hence produce higher $\mathrm{OCR}$ values.

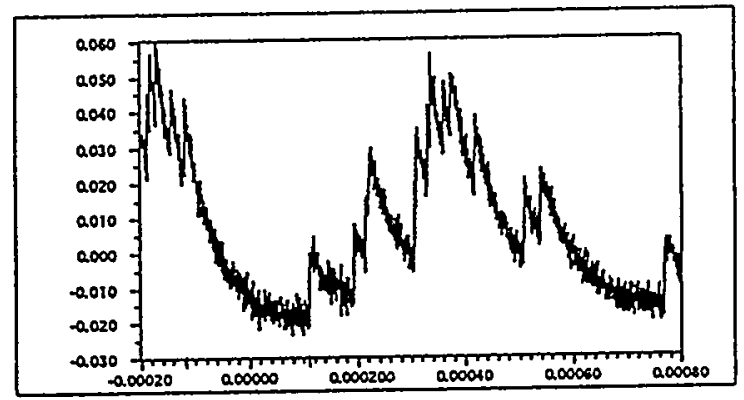

Figure 2: Output of RC feedback preamplifier at 19 $\mathrm{kcps}$ data rate showing fluctuations in $\mathrm{x}$-ray (steps) arrival times.

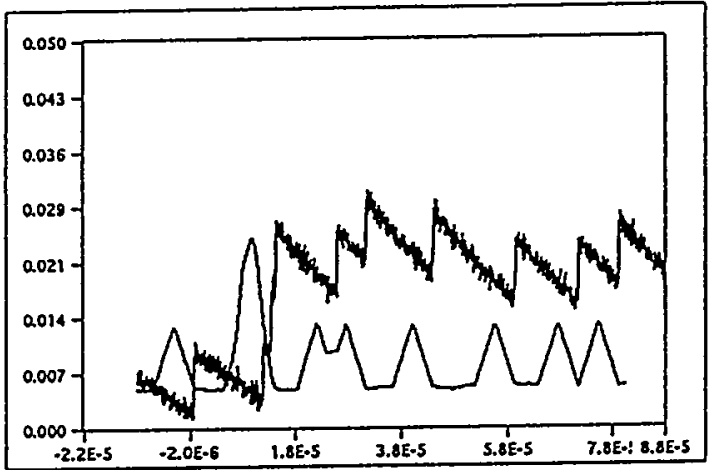

Figure 3: Digital filtering of the RC feedback preamplifier input. The triangular filter peaking time is $3.6 \mu \mathrm{s}$, the triangular pulses' basewidths are thus $7.2 \mu \mathrm{s}$. Both piled-up and almost piled-up pulses can be seen.

Our second and third studies were to verify that increasingly mature versions of our analog signal conditioning (ASC) circuitry were robust at high count rates and did not introduce any degradation into the energy resolution. In the first case (July 1994) we tested a first prototype ASC circuit to assure ourselves that it worked at all data rates and introduced no non-linearity or additional noise. This work was fully successful and no problems were discovered of any kind. Returning in December 1994, we were able to test a full ASC prototype, complete with digital feedback controls for stability. Some signal problems 
were discovered attempting to operate the prototype breadboards at full processing speed, but these were successfully ironed out. Figure 4 shows a spectrum taken (still in emulation mode) through the feedback controlled ASC unit. Using a $3 \mu$ s peaking time, we obtained a resolution of about $235 \mathrm{eV}$ at a ICR of $42.2 \mathrm{kcps}$. These data were taken using our own POF Ge detector, a recent Ortec design for high resolution at faster counting speeds. Using this same detector we were able to collect data at $250 \mathrm{kcps}$ ICR with less than $34 \%$ pileup losses using a $1 \mu$ s peaking time and at $500,000 \mathrm{kcps}$ with less than $26 \%$ pileup losses using an $0.5 \mu$ s peaking time. We were unable to obtain very good energy resolution at these count rates however. An investigation of the problem revealed a hardware dependent design problem which we will be able to correct easily in the next prototype. In principle, the high rate energy resolution should not be too different from values obtained at low rates.

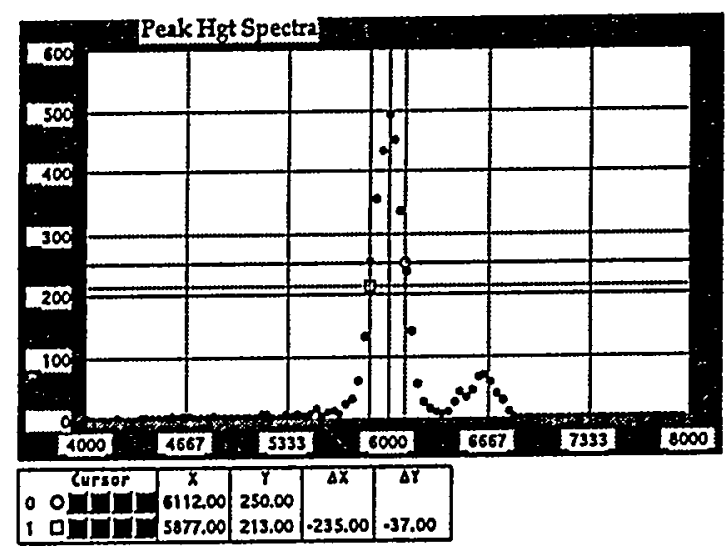

Figure 4: Spectrum taken at $42.2 \mathrm{kcps}$ effective rate by emulation software using a $3 \mu$ s triangular peaking time. The signal from an Ortec POF Ge. detector was passed through the ASC unit under full digital feedback control.

At our next run in March 1995, we expect to be able to test a fully working prototype board with all functions operating digitally on board, particularly the pulse shaping, pileup inspection, and MCA functions. If this pre-production prototype works as expected, we will be testing it for linearity, resolution degradation vs ICR, the sensitivity of its peak detection algorithms to $x$-ray energy, and its capability at extremely high count rates. We have begun working with SSRL staff to assure that this module will integrate smoothly into SSRL's existing EXAFS data collection programs. 


\title{
LIGAND K-EDGE X-RAY ABSORPTION SPECTROSCOPIC STUDIES: METAL- LIGAND COVALENCY IN A SERIES OF TRANSITION METAL COMPLEXES
}

\author{
Susan E. Shadle, ${ }^{1}$ Kendra R. Williams, ${ }^{1}$ Britt Hedman, ${ }^{2}$ Keith O. Hodgson, ${ }^{1,2}$ and Edward I. Solomon ${ }^{1}$ \\ ${ }^{1}$ Department of Chemistry, Stanford University, Stanford, CA 94305 \\ ${ }^{2}$ Stanford Sychrotron Radiation Laboratory, Stanford University, Stanford, CA 94309
}

\section{Introduction}

Analysis of X-ray absorption edge spectra can provide insight into the electronic structure of an absorbing atom. 1,2 While this technique has been used extensively at the $\mathrm{K}$-edge of transition metal centers such as $\mathrm{Cu}, \mathrm{Fe}, \mathrm{Ni}$ and $\mathrm{Mo}^{3-6}$ it has also recently been demonstrated that the absorption edges of ligands such as $\mathrm{S}$ and $\mathrm{Cl}$ can be used to study the electronic structure of inorganic $\mathrm{Cu}^{\mathrm{II}}$ complexes $^{7-9}$ and cupric protein active sites. ${ }^{8}$ The $3 \mathrm{p}$ orbitals of $\mathrm{S}$ and $\mathrm{Cl}$ ligands are directly involved in bonding with transition metal ions. Because the electric dipole-allowed transitions for $\mathrm{K}$-edges are 1s->np, ligand $\mathrm{K}$ edge $\mathrm{X}$-ray absorption spectroscopy provides a direct probe of these ligand-metal bonding interactions.

Recently, the technique of ligand K-edge XAS has been extended to include ligands bound to metal ions other than $\mathrm{Cu}^{\mathrm{II}} .10 \mathrm{As}$ in the $\mathrm{Cu}^{\mathrm{II}}$ systems, the pre-edge feature in other $\mathrm{d}^{\mathrm{n}}$ metal centers corresponds to a transition (or several transitions) from a ligand $1 \mathrm{~s}$ orbital to unoccupied or partially-occupied antibonding orbitals with both metal d- and ligand pcharacter. However, in systems with more than one d-manifold electron or hole, a number of excited states are possible. Further, multiplet effects in the $\mathrm{d}^{\mathrm{n}+1}$ final state can change the observed intensity. The analysis of pre-edge intensity for the determination of covalency in metal d-derived orbitals requires that all contributions to the intensity be taken into account.

The $\mathrm{Cl} \mathrm{K}$-edge XAS pre-edge features for the tetrahedral metal-tetrachloride series $\mathrm{MCl}_{4}{ }^{\mathrm{n}}$ where $\mathrm{M}=\mathrm{Cu}^{\mathrm{II}}, \mathrm{Ni}^{\mathrm{II}}, \mathrm{Co}^{\mathrm{II}}, \mathrm{Fe}^{\mathrm{II}}$, and $\mathrm{Fe}^{\mathrm{III}}$ have been examined and a methodology for the interpretation of pre-edge intensities and energies has been developed for these $\mathrm{d}^{\mathrm{n}}$ hole systems $(\mathrm{n} \geq 1) .10$ This methodology is also being applied to the S K-edge of a metal-tetrathiolate series
$\mathrm{M}(\mathrm{SR}) 4^{\mathrm{n}-}$, where $\mathrm{M}=\mathrm{Ni}^{\mathrm{II}}, \mathrm{Co}^{\mathrm{II}}, \mathrm{Fe}^{\mathrm{II}}, \mathrm{Fe}^{\mathrm{III}}$ and $\mathrm{Mn}$ II.

\section{Experimental}

XAS data were measured at the Stanford Synchrotron Radiation Laboratory using the 54pole wiggler beamline 6-2 in low magnetic field mode ( $5 \mathrm{kG})$ with a Pt-coated focusing mirror and $\mathrm{Si}(111)$ double crystal monochromator, under dedicated conditions $(3.0 \mathrm{GeV}, \sim 50 \mathrm{~mA})$. The monochromator was detuned $\sim 30 \%$ to eliminate higher harmonic components in the $\mathrm{x}$ ray beam. The entire beam path was in $\mathrm{He}$ atmosphere. The data were collected as fluorescence excitation spectra utilizing a $\mathrm{N}_{2}$ filled Lytle detector. Samples were run as very thin powders on mylar tape to avoid selfabsorption. The $\left(\mathrm{Et}_{4} \mathrm{~N}\right)_{2} \mathrm{FeCl}_{4}$ sample was prepared in a dry, anaerobic atmosphere. A $6.35 \mu \mathrm{m}$ polypropylene film window protected the sample from exposure to air.

The intensity of normalized pre-edge features were quantitated by fits to the data using the fitting program EDG_FIT, written by Dr. Graham N. George of the Stanford Synchrotron Radiation Laboratory.

\section{Results}

The $\mathrm{Cl}$ K-edge absorption spectra of the $\sim T_{d}$ complexes $\mathrm{MCl}_{4}{ }^{\mathrm{n}-}\left(\mathrm{M}=\mathrm{Cu}^{\mathrm{II}}, \mathrm{Ni}^{\mathrm{II}}, \mathrm{Co}^{\mathrm{II}}, \mathrm{Fe}^{\mathrm{II}}\right.$, and $\mathrm{Fe}$ III) are shown in Figure 1a'with the pre- . edge feature of these spectra expanded in Figure 1b. Over the divalent series, the energies of the pre-edge features vary in the order $\mathrm{Cu}^{\mathrm{II}}<\mathrm{Ni}^{\mathrm{II}}<$ $\mathrm{Co} \mathrm{II}_{<} \mathrm{Fe}^{\mathrm{II}}$ with the $\mathrm{CuCl}_{4}{ }^{2-}$ pre-edge being the most intense and the $\mathrm{Ni}^{\mathrm{II}}, \mathrm{Co}^{\mathrm{II}}$ and $\mathrm{Fe}{ }^{\mathrm{II}}$ centers having approximately the same intensity within experimental error. The $\mathrm{Fe}^{\mathrm{III}_{\mathrm{Cl}}}$ - pre-edge is at much lower energy and more than 3 times as intense as that of the ferrous complex. 

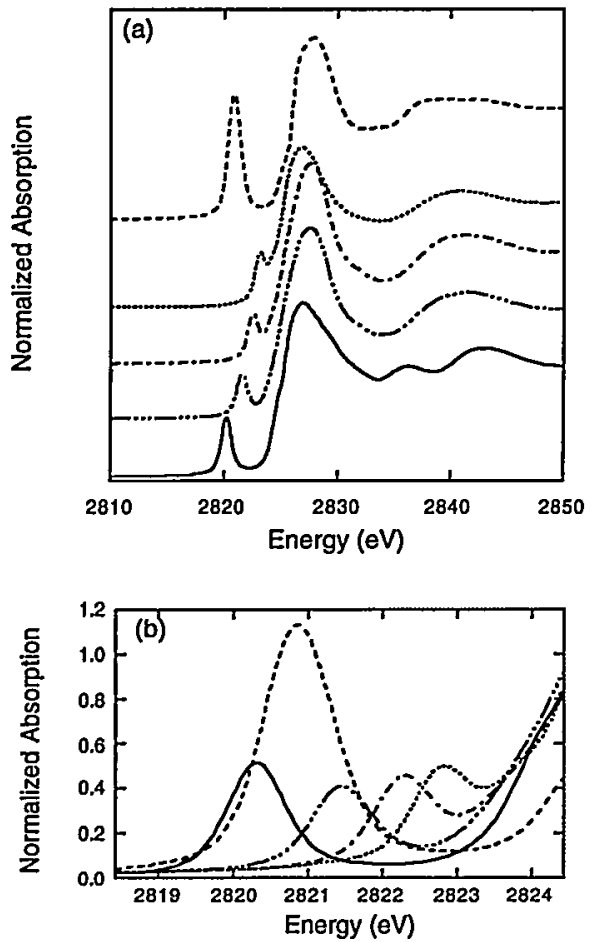

Figure 1. $\mathrm{Cl} \mathrm{K}$-edge XAS spectra of the $T_{d}$ complexes $\mathrm{CuCl}_{4}{ }^{2-(-)}, \mathrm{NiCl}_{4}{ }^{2-(-\cdots)}, \mathrm{CoCl}_{4}{ }^{2-(--)}$,

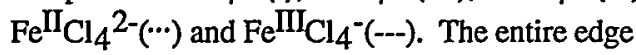
region of each spectrum is shown in (a). Each spectrum exhibits a pre-edge feature, which is shown on an expanded scale in (b).

\section{Analysis}

Pre-edge Energies. There is a combination of factors which contribute to pre-edge energy. First, the relative energy of the $\mathrm{Cl} 1 \mathrm{~s}$ core can change. Also, the d-manifold energy position and the ligand field splitting $(10 D q)$ influence the overall pre-edge energy. Quantitative estimates of the relative $\mathrm{Cl} 1 \mathrm{~s}$ core energy and the ligand field contribution to metal d-derived orbital energy can be obtained from the rising edge inflection points and theoretical energy and intensity ratios, respectively. ${ }^{9}$ By correction of the observed pre-edge energy for these effects, the contribution to the pre-edge energy from energy shifts of the d-manifold can be determined. The d-manifold shifts observed in this $\mathrm{MCl}_{4}{ }^{\mathrm{n}-}$ series are consistent with the periodic trend in the effective nuclear charge ( $Z_{\text {eff }}$ ) on each metal. As the metal $Z_{\text {eff }}$ increases across the first row of the periodic table for the divalent series (from $\mathrm{Fe}^{\mathrm{II}}$ to $\mathrm{Cu}^{\mathrm{I}}$ ), there is a shift in the d-manifold to deeper binding energy. The d-manifold in $\mathrm{Fe}^{\mathrm{III}} \mathrm{Cl}_{4}$ - is also significantly deeper in energy than in $\mathrm{Fe}^{{ }^{I I}} \mathrm{Cl}_{4}$ 2- due to the oxidation state of the iron.

Pre-edge Intensities. Several steps were involved in deriving the relationship between pre-edge intensity and covalency in metal dderived orbitals for metal systems with more than one hole. First, strong field group theoretical predictions of possible one-electron pre-edge transitions were made. The irreducible tensory method was then used to derive dipole strength expressions which describe the intensity of the transitions in the strong field limit. The mixing of excited states in intermediate-strength ligand fields can redistribute pre-edge intensity to higher energy. The degree of this mixing and a total pre-edge multiplet intensity for each spectrum was calculated using ligand field energy splittings and transition intensity ratios for each contributing transition. The total preedge multiplet intensity was used to obtain an experimental estimate of covalency for each complex. This procedure is described in Reference 10.

The calculated covalency (\%) reflected in the dipole strength for the $\mathrm{Cu}^{\mathrm{II}}, \mathrm{Ni}^{\mathrm{II}}, \mathrm{Co}^{\mathrm{II}}, \mathrm{Fe}^{\mathrm{II}}$ and $\mathrm{Fe}^{\mathrm{III}}$ was $30.0,11.8 \pm 0.6,9.0 \pm 0.5,11.9 \pm 1.2$ and $29.8 \pm 1.6$, respectively. Covalencies obtained from SCF-X $\alpha-S W$ calculations of these complexes were within $5 \%$ of the experimental results.

\section{Discussion}

The methodology, briefly described above, allows the ligand XAS pre-edge intensity to be related to metal d-derived orbital covalency in a series of $T_{d}$ metal-tetrachloride systems. The results are understood in terms of the energètics of bonding. The amount of $\mathrm{Cl} 3 \mathrm{p}$ character in each antibonding orbital in the series is determined by the difference in energy between the metal $\mathrm{d}$ orbitals and the $\mathrm{Cl} 3 \mathrm{p}$ orbitals. For increased effective nuclear charge on the metal, the $d$ orbitals are at deeper binding energy and has greatest covalent interaction with ligands.

This ligand $\mathrm{K}$-edge methodology is now being applied to the analysis of S K-edge XAS of $\mathrm{M}(\mathrm{SR}) 4^{\mathrm{n}-}$ complexes. Similar trends are observed in the pre-edge energy and intensities between the metal-tetrachloride and the metaltetrathiolate series. The development of the 
quantitative analysis in $S_{4}$ symmetry, for application to the spectra of the metaltetrathiolates is currently underway. This extension will allow the technique to be applied to the study of $\mathrm{Fe}-\mathrm{S}$ centers.

\section{Acknowledgement}

This research was supported by NSF CHE9217628 (E.I.S.) and CHE-9121576 (K.O.H) and by NIH RR-01209 (K.O.H.). SSRL operations are funded by the Department of Energy, Office of Basic Energy Sciences. The Biotechnology Program is supported by the National Institutes of Health, Biomedical Research Technology Program, National Center for Research Resources. Further support is provided by the Department of Energy, Office of Health and Environmental Research.

We would also like to thank Prof. M. Millar and Prof. S. Koch for graciously providing the $\mathrm{M}(\mathrm{SR}){ }_{4}{ }^{2-}$ complexes.

\section{References}

1. Heald, S.M.; Tranquada, J.M. In Physical Methods of Chemistry; Rossiter, B.W., Hamilton, J.F., Eds.; Wiley: New York, 1990; Vol. 5, pp 189-272.

2. Bianconi, A. In X-ray Absorption; Koningsberger, D.C., Prins, R., Eds.; Wiley: New York, 1988; pp 573-662.

3. Kau, L.S.; Spira-Solomon, D.J.; PennerHahn, J.E.; Hodgson K.O.; Solomon, E.I. J. Am. Chem. Soc. 1987, 109, 6433-6442.

4. Roe, A.L.; Schneider, D.J.; Mayer, R.J.; Pyrz, J.W.; Widom, J.; Que, L., Jr. J. Am. Chem. Soc. 1984, 106, 1676-1681.

5. Eidsness, M.K.; Sullivan, R.J.; Scott, R.A. In Bioinorganic Chemistry of Nickel; Lancaster, J.R., Ed.; VCH: Deerfield Beach, FL, 1988; pp 73-92.

6. Conradson, S.D.; Burgess, B.K.; Newton, W.E.; McDonald, J.W.; Rubinson, J.R.; Gheller, S.F.; Mortenson, L.E.; Adams, M.W.W.; Mascharak, P.K.; Armstrong, W.A.; Holm, R.H.; Hodgson, K.O. J. Am. Chem. Soc. 1985, 107, 7935-7940.

7. Hedman, B.; Hodgson, K.O.; Solomon, E.I. J. Am. Chem. Soc. 1990, 112, 1643-1645.

8. Shadle, S.E.; Penner-Hahn, J.E.; Schugar, H.J.; Hedman, B.; Hodgson, K.O.; Solomon, E.I. J. Am. Chem. Soc. 1993, 115, 767-776.

9. Shadle, S.E.; Hedman, B.; Hodgson, K.O.; Solomon, E.I. Inorg. Chem. 1994, 33, 42354244.
10. Shadle, S.E.; Hedman, B.; Hodgson, K.O.; Solomon, E.I. J. Am. Chem. Soc. 1995, in press. 


\title{
LAUE STUDIES OF Rb. sphaeroides PHOTOSYNTHETIC REACTION CENTER AND OTHER PROTEINS
}

\author{
T. McPhillips ${ }^{1}$, M.H.B. Stowell ${ }^{1}$, M. Mas ${ }^{2}$, M. Soltis ${ }^{3}$, R.P. Phizackerley ${ }^{3}$, D.C. Rees ${ }^{1}$, \\ 1Division of Chemistry, California Institute of Technology, Pasadena, CA 91125 \\ 2Beckman Research Institute, City of Hope, Duarte, CA 91010 \\ ${ }^{3}$ SSRL, Stanford, CA 94309
}

\section{RB. SPHAEROIDES PHOTOSYNTHETIC REACTION CENTER}

Due to the lack of crystals with suitably low mosaicity, we were unable to successfully obtain useful Laue images from the $R b$. sphaeroides photosynthetic reaction center during our Laue time at SSRL during 1994.

\section{PHOSPHOGLYCERATE KINASE}

Experiments have been designed to observe the catalytic intermediates in the turnover of the glycolytic enzyme phosphoglycerate kinase by timeresolved $X$-ray crystallography using both monochromatic and white radiation data collection techniques. The R65Q mutant of yeast PGK has been co-crystallized with both NPE-caged ATP and 3-PG, with the intent of initiating catalysis in the crystal by photolytic removal of the chemical cage attached to the $\gamma$-phosphate of the ATP. Monochromatic diffraction studies of these crystals indicate that this scheme works. Electron density maps calculated from data collected on the pre-flash crystals clearly show caged-ATP bound to the nucleotide binding site of the enzyme. Exposure of the crystals to a UV laser pulse results in a $14 \%$ decrease in unit cell volume over the course of 24 hours, after which time the crystals diffract more strongly than before. Electron density maps calculated from data collected on photolyzed crystals show that ADP has replaced the caged-ATP in the nucleotide binding site on the enzyme. Maps calculated using data collected during the post-flash, cell-transformation period indicate the presence of a long-lived intermediate in the nucleotide binding site. Faster data collection techniques are required, however, to clearly distinguish the apparent intermediate from partial-occupancy caged-ATP.

The Laue method, which allows crystallographic data sets to be collected very rapidly, is being used to more clearly characterize the reaction observed in these crystals. Two Laue diffraction runs were made at SSRL this year using beam line 10-2. Total $\mathrm{X}$-ray exposure times of less than a second were commonly used, with each exposure spread over many seconds using a beam chopper to reduce the effects of radiation damage and crystal heating. Fuji imaging plates were used for all data collection, with each diffraction pattern being recorded on a pack of three imaging plates to allow deconvolution of harmonic overlaps. A Spectra-Physics GCR-12 YAG laser was used to flash the crystals at the third harmonic wavelength of $355 \mathrm{~nm}$. An optics system was used to split the laser pulse into two beams independently directed at the crystal from different directions by fiber-optics.

The general procedure used was to take Laue exposures on the crystals in their native, pre-flashed state, flash the crystals with the laser to remove the cage and initiate reaction, and take further Laue exposures at time-points following the laser flash. Data was collected on a large number of crystals in this way. High quality data was collected from representives of both a monoclinic and an orthorhombic crystal form. Multiple Laue exposures could be taken on a single crystal before significant radiation damage had occurred. For a number of crystals, exposures were taken in several different orientations both before laser-flashing, and at multiple time-points after the laser flash. Several of the data sets have been successfully indexed using LAUEGEN in the CCP4 Laue suite and integration and further analysis of the data is underway.

This work was supported in part by USPHS GM45162. 


\title{
X-RAY ABSORPTION SPECTROSCOPY STUDIES OF MANGANESE REDOX ENZYMES
}

\author{
Pamela J. Riggs-Gelasco, Timothy L. Stemmler, and James E. Penner-Hahn \\ Department of Chemistry, The University of Michgian, Ann Arbor, Michigan 48109-1055
}

Thomas M. Sossong and Dave E. Ash

Deptartment of Biochemistry, Temple University, 3400 N. Broad Street, Philadelphia, Pennsylvania 19140

Rui Mei and Charles F. Yocum

Department of Biology, The University of Michgian, Ann Arbor, Michigan 48109-1048

Demetrios Ghanotakis

Chemistry Department, University of Crete, Iraklion, Crete, Greece

\section{INTRODUCTION}

The goal of this project is to characterize the local structural environments of the Mn sites in Mn redox proteins. The principal focus is on the mononuclear $\mathrm{Mn}$ sites in the $\mathrm{Mn} / \mathrm{Fe}$ superoxide dismutases, the dinuclear $\mathrm{Mn}$ site in Mn catalase and in arginase, and the tetranuclear Mn site in the photosynthetic oxygen complex. Recent results for Mn catalase, arginase, and the oxygen evolving complex are described here.

\section{DINUCLEAR MN SITES}

Catalase catalyzes the disproportionation of $\mathrm{H}_{2} \mathrm{O}_{2}$ to $\mathrm{O}_{2}$ and $\mathrm{H}_{2} \mathrm{O}$. Although most catalases have a heme iron active site, some bacteria produce non-heme, Mn containing catalases. Ariginase catalyzes the hydrolysis of arginine to ornithine and urea. Both Mn catalase and arginase contain dinuclear $\mathrm{Mn}$ active sites that are, at least superficially similar. This simlarity has been strenghtened with the recent discovery that arginase shows slow catalase reactivity.

We have used X-ray absorption spectroscopy to structurally characterize the active site of rat liver arginase and the reduced form of the Lactobacillus plantarum $\mathrm{Mn}$ catalase in an attempt to determine a relationship between structure and catalase activity. We have also used $\mathrm{XAS}$ to monitor structural changes at the $\mathrm{Mn}_{2}$ core of arginase upon addition of ornithine and borate which inhibit the arginase reaction. Initial EXAFS data suggests that active site accessibility and imidizole ligation may be responsible for the differences in the catalase activity between these proteins.

XANES spectra for arginase and for reduced catalase show distinct differences both in edge structure and in $1 s \rightarrow 3 d$ intensity. In contrast, there are no significant differences in either the edge structure or the $1 \mathrm{~s} \rightarrow 3 \mathrm{~d}$ intensity for arginase in the presence or absence of inhibitors (see Figure 1). All of the edge energies are consistent with those seen for Mn(II) models (first inflection point at ca. $6548 \mathrm{eV}$ ). Arginase is thus distinct from catalase, where the $\mathrm{Mn}(\mathrm{II}) / \mathrm{Mn}$ (II) derivative is readily auto-oxidized to a mixture of $\mathrm{Mn}(\mathrm{II}) / \mathrm{Mn}(\mathrm{II})$ and $\mathrm{Mn}(\mathrm{III}) / \mathrm{Mn}(\mathrm{III})$ forms [1].

The $1 \mathrm{~s} \rightarrow 3 \mathrm{~d}$ intensities for arginase are consistent with those found for six coordinate $\mathrm{Mn}$ (II) models. The corresponding intensity for $\mathrm{Mn}$ catalase is ca. $30 \%$ larger than the largest $1 \mathrm{~s} \rightarrow 3 \mathrm{~d}$ intensity observed for a six-coordinate $\mathrm{Mn}$ (II) model. This suggests that at least one of the Mn in Mn catalase must be five-coordiante.

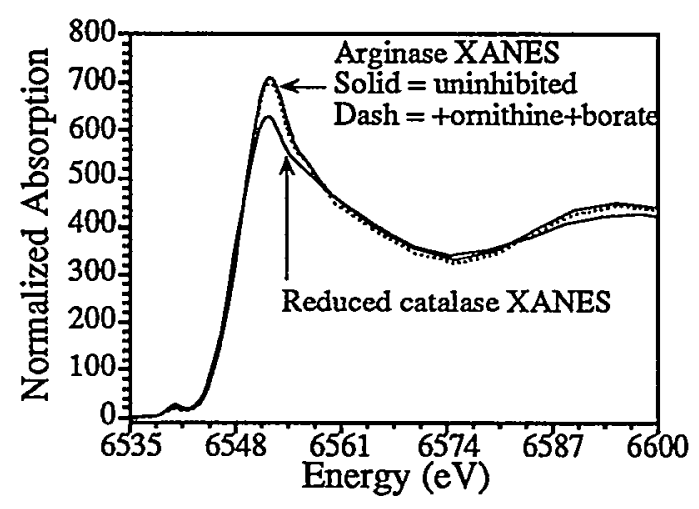

Figure 1 Normalized XANES analysis of the rat liver arginase \pm ornithine/borate and the reduced form of the $\mathrm{Mn}$ catalase.

The Fourier transform of the EXAFS data for arginase with and without the inhibitors ornithine and borate show sharp intense first shell features while that for catalase is substantially broader and damped (see Figure 2). 
Curve fitting analyses show that the nearest neighbor environment for both proteins consists exclusively of low-Z ligands $(N$ and/or 0 ). First shell fitting analysis for uninhibited arginase gives an average first shell distance of $2.17 \AA$ with an apparent coordination number of 4. Attempts to resolve a second shell of Mn-nearest neighbor interactions were not justified, although the existence of a distorted first shell of $\mathrm{Mn}-\mathrm{O} / \mathrm{N}$ ligands is evident from the high disorder and low apparent coordination number obtained. Given the low $1 \mathrm{~s} \rightarrow 3 \mathrm{~d}$ intensity and relatively long Mn-nearest neighbor distance, the average $\mathrm{Mn}$ coordination number must be approximately six.

The first shell distance is unchanged in the presence of ornithine and borate, however the apparent coordination number increases to ca. 5 while the disorder decreases by ca. $50 \%$ relative to the uninhibited sample. The decrease in $\mathrm{Mn}$ nearest neighbor ligand disorder as inhibitor binds to arginase is consistent with EPR evidence which suggests a mixture of species in its native form but only a single species in the inhibited sample. The absence of a structural change at the dinuclear Mn site of arginase upon addition of inhibitors suggests that inhibitor binding site may not take place at the $\mathrm{Mn}_{2}$ core.

First shell fits to reduced catalase show two resolvable Mn-nearest neighbor interactions at $2.10 \AA$ (coordination number of 3.5 ) and $2.27 \AA$ (coordination number of 1.5). Thus catalase, unlike arginase, appears to contain two ordered shells, presumably due to $\mathrm{Mn}-\mathrm{O}$ and $\mathrm{Mn}-\mathrm{N}$. The apparent number of $\mathrm{Mn}$-His interactions, judged either from the first shell fitting or from analysis of the outer shell imidazole scattering, is consistent with approximately 3 His residues per $\mathrm{Mn}_{2}$ core in reduced catalase. In contrast, the outer shell scattering for arginase is substantially weaker (see Fig. 2), suggesting fewer histidines in this case. This is consistent with the somewhat shorter average first shell bond length in arginase.

The EXAFS and XANES data thus suggest two principal differences between the Mn catalase and the arginase active sites. Catalase appears to have a larger number of imidazole ligands and to have a lower coordination number for at least one of the $\mathrm{Mn}$ ions. Either, or both, of these differences may be important in accounting for the tremendous rate difference for the catalase reaction at these two sites.

\section{TETRANUCLEAR MN SITES}

$\mathrm{X}$-ray absorption spectroscopy has been used to structurally characterize the native $\left(S_{1}\right)$ state and chemically altered forms of the tetranuclear Mn cluster responsible for photosynthetic water oxidation in the Oxygen Evolving Complex (OEC) of Photosystem II. EXAFS has been used to characterize three interactions in the OEC: a $1.86 \AA \mathrm{Mn}-\mathrm{O} / \mathrm{N}$ nearest neighbor shell, a $2.72 \AA \mathrm{Mn}-\mathrm{Mn}$ shell, and a $3.3 \AA \mathrm{Mn}-\mathrm{M}$ shell (where $\mathrm{M}=\mathrm{C}, \mathrm{Ca}$, and/or $\mathrm{Mn}$ ).

Effect of reductants on OEC structure. We have shown previously that two different reduced derivatives of the OEC can be prepared by using either small hydrophilic reductants (e.g., $\mathrm{NH}_{2} \mathrm{OH}$ ) or large hydrophobic reductants (e.g., hydroquinone). The former gives a small decrease in edge energy while the latter results in production of ca. $50 \% \mathrm{Mn}$ (II) [3]. We have now extended these studies to include structural characterization of the reduced species. Reduction with $\mathrm{NH}_{2} \mathrm{OH}$ gives no significant change in the average $\mathrm{Mn}$ structure, while hydroquinone reduction causes major structural changes, including disruption of ca. one of two $\operatorname{Mn}(\mu$ O) $2 \mathrm{Mn}$ dimers (data not shown).

Identication of $\mathrm{Mn}-\mathrm{Ca}$ EXAFS. The identity of $\mathrm{X}$ was addressed by substituting $\mathrm{Sr}^{2+}, \mathrm{Dy}^{3+}$ or $\mathrm{La}^{3+}$ for the native $\mathrm{Ca}^{2+}$ cofactor. The substitutions did not result in a detectable change in the $3.3 \AA$ FT peak (see Figure 3 ). On the initial examination, this seems to rule out the possibility of $\mathrm{Mn}-\mathrm{Ca}$ interactions at $3.3 \AA$. 


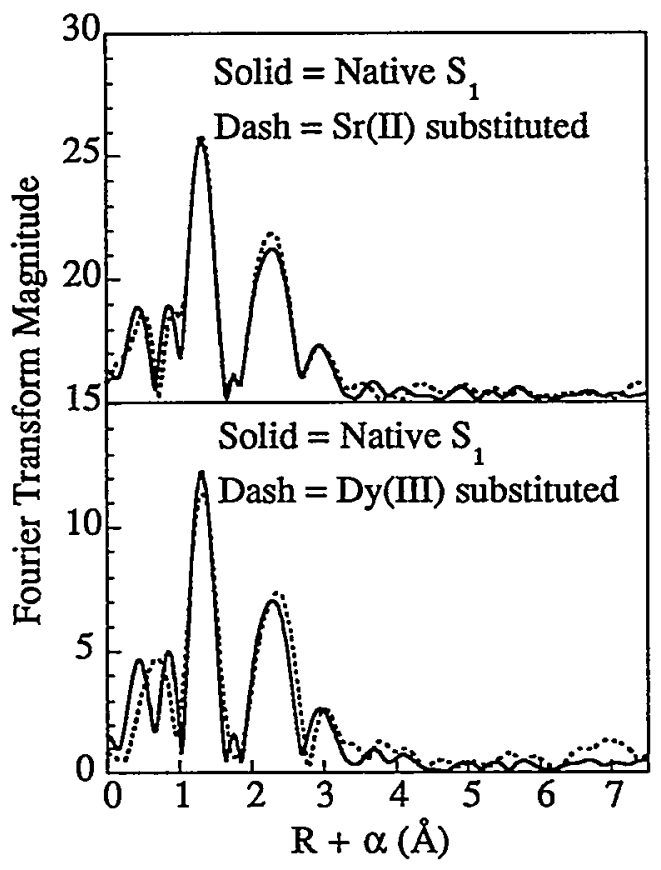

Figure 3. Effect of replacing $\mathrm{Ca}(\mathrm{II})$ with $\mathrm{Sr}$ (II) or Dy(III) on OEC EXAFS.

However, more careful examination of the FTs for simulated (FEFF) $\mathrm{Mn}-\mathrm{M}(\mathrm{M}=\mathrm{Sr}$, Dy, and Mn) interactions (Fig. 4) show that, in reality, only a small enhancement of the $3.3 \AA$ peak is expected, when the data analysis is restricted to that used for the protein $\left(1.5-11.5 \AA^{-1}\right)$. The differences in backscattering amplitude that are expected for these metals are most apparent at $\dot{k} \geq 11.5 \AA^{-1}$, a data range that is currently not accessible for $\mathrm{Mn}$ metalloprotein studies.

Fortunately, the lack of amplitude differences does not preclude identification of the $3.3 \AA$ feature. The difference in EXAFS phase for $\mathrm{M}=\mathrm{Ca}, \mathrm{Sr}$, Dy permit identification of $M$. In particular, attempts to fit an authentic $3.3 \AA \mathrm{Mn}-\mathrm{Mn}$ interaction with $\mathrm{Mn}-\mathrm{Sr}$ scattering give an apparent $\mathrm{Mn}-\mathrm{Sr}$ distance that is $0.1 \AA$ too short. Similarly, attempts to fit a genuine $\mathrm{Mn}-\mathrm{Sr}$ interaction with $\mathrm{Mn}-\mathrm{Mn}$ parameters give an apparent $\mathrm{Mn}-\mathrm{Mn}$ distance that is 0.1 A too long. This change in distance, which reflects the differences in EXAFS phase, allow us to discriminate between the various possibilities for $M$. For the OEC, substitution of $\mathrm{Ca}$ with either Sr or Dy gives no detectable change in the apparent $\mathrm{Mn}-\mathrm{Mn}$ distance. This strongly suggests that there is no EXAFS detectable $\mathrm{Mn}-\mathrm{Ca}$ interaction at $3.3 \AA$ in these samples.

Structural consequences of $\mathrm{Ca}$ replacement. The substitution of $\mathrm{Ca}$ with other cations is known to have pronouced consequences for OEC activity. Only the substitution with Sr results in an active complex. These observations suggest that Ca substiution should have some effect on the OEC structure. Although cation substitutions did not result in a significant change at $3.3 \AA$, the $2.7 \AA$ feature was affected. $\mathrm{Sr}^{2+}$ substitution resulted in a $0.014 \AA$ decrease in $\mathrm{Mn}$-Mn distance. $\mathrm{Dy}^{3+}$ substitution resulted in a $0.011 \AA$ increase in distance and $\mathrm{La}^{3+}$ substitution resulted in a $0.06 \AA$ increase. These differences, while small, are three times larger than the experimental precision in the determination of the $\mathrm{Mn}-\mathrm{Mn}$ distance $(0.004 \AA)$. The changes in the $2.7 \AA$ feature correlate with the Lewis acidity

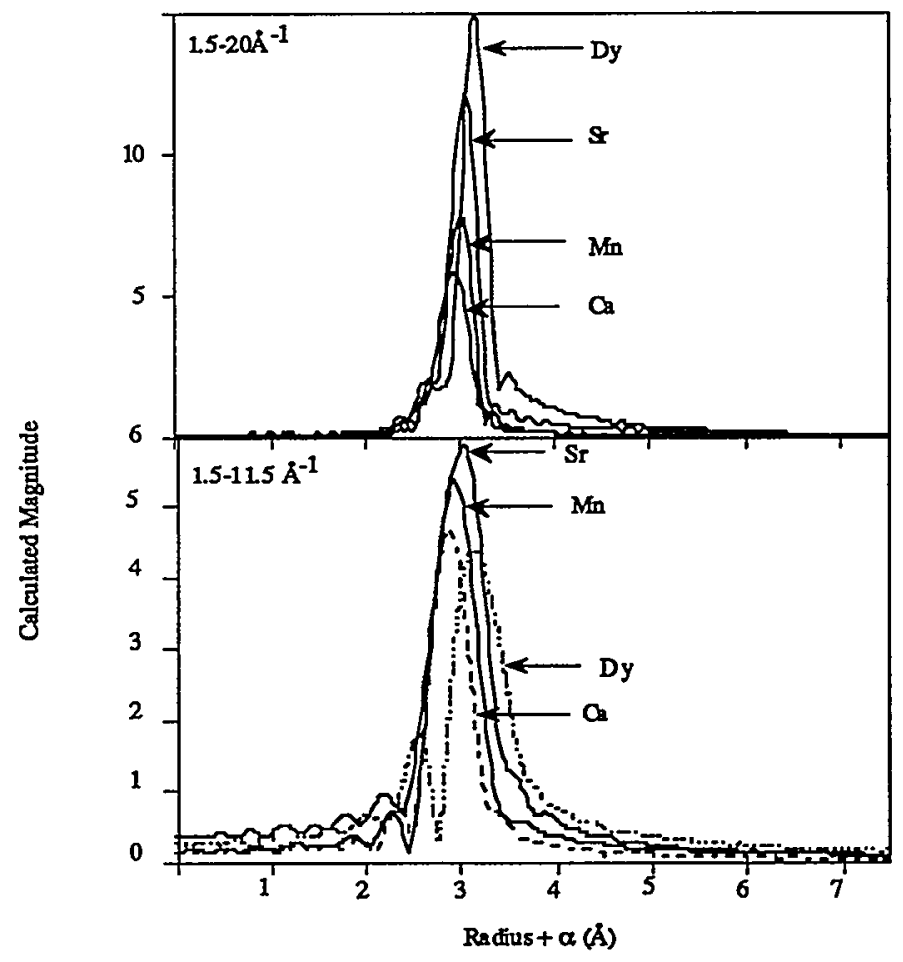

Fig. 4. Calculated EXAFS for $\mathrm{Mn}-\mathrm{M}(\mathrm{M}=\mathrm{Mn}, \mathrm{Sr}, \mathrm{Dy})$ interactions for $k=1.5-20 \AA^{-1}$ (top) and for a more realistic $k$ range or 1.5-11.5 $\AA^{-1}$ (bottom). 
of the cation. One model that explains this correlation is a calcium-bond water that is hydrogen bonded to a single $\mathrm{Mn}_{2}(\mu-\mathrm{O})_{2}$ unit. As the acidity of the cation increases, the affected oxo-bridged dimer becomes more "protonated", accounting for the increase in distance. This model suggests a possible role of calcium is to modulate the protonation state, and consequently the redox potential, of the core $\mathrm{Mn}_{2}(\mu-\mathrm{O})_{2}$ units responsible for water oxidation (see Scheme1).<smiles>[Y19]O[TlH]O[GeH3]</smiles>

Scheme 1: Possible role of calcium in regulation of $\mathrm{Mn}$ cluster potential by hydrogen bonding of a calciumbond water to a single oxo bridge.

\section{REFERENCES}

1. G.S. Waldo and J.E. Penner-Hahn, , Biochemistry , 34 (1995) 1507-1512.

2. S. Khangulov, et al., Biochemistry, 1995, 34, 2015

3. P. Riggs, R.Mei, C. Yocum, and J. PennerHahn,J. Am. Chem. Soc., 114 (1992), 10650 .

\section{ACKNOWLEDGEMENTS}

This work has been supported in part by grants from the NIH (GM-45205 to JEPH; pre-doctoral fellowship to PJRG) and the USDA (NRICGP92-37306-7662 to CFY). Spectra were measured in part at SSRL, which is supported by the US DOE and by the NIH, Biotechnology Resource Program, Division of Research Resources. 


\title{
Small-angle X-ray Scattering Studies of Conformational Changes in E.coli Aspartate Transcarbamoylase
}

\author{
Eric R. Johnson ${ }^{1}$, Hiro Tsuruta ${ }^{2}$, Bin-Bing Zhou ${ }^{1}$, Hiroshi Kihara ${ }^{3}$, and H. K. Schachman ${ }^{1}$ \\ 1) Department of Molecular and Cell Biology, University of California, Berkeley, CA 94720 USA \\ 2) Stanford Synchrotron Radiation Laboratory, Stanford, CA 94309 USA \\ 3) Kansai Medical University, Hirakata, Osaka, Japan
}

\section{Introduction}

E. coli aspartate transcarbamoylase (ATCase) is a hallmark example of an allosteric protein and has been the subject of intensive structural and functional studies because of its intriguing regulatory properties $[1,2]$. ATCase is a relatively large protein (molec. wt. $\sim 300 \mathrm{kDa}$ ), and is comprised of two $100-\mathrm{kDa}$ catalytic (C) trimers and three $34-\mathrm{kDa}$ regulatory $(\mathrm{R})$ dimers. Although a substantial number of $x$-ray crystallography and small-angle scattering studies have been performed on the holoenzyme form of ATCase, structural studies of the isolated $\mathrm{C}$ trimers have been limited. In addition, little is known about the rates of interconversion between the $T$ and $R$ quaternary states of the holoenzyme. We are utilizing small-angle $\mathrm{x}$-ray solution scattering experiments in order to address the following questions: 1) What is the solution structure of the isolated $\mathrm{C}$ trimers of ATCase? 2) What is the relationship between ligand binding and conformational changes in the $C$ trimer? 3) What are the rates of interconversion between the two quaternary states of the ATCase holoenzyme? Both static and time-resolved $x$-ray scattering experiments employing synchrotron radiation have proven to be powerful methods for answering such questions.

\section{Experimental}

The experiments have been performed on SSRL beamline 4-2 or beamline 15-A at the Photon Factory. Protein solutions are irradiated with $1.5 \AA \mathrm{x}$-rays for 530 minutes in plastic cuvettes equipped with 20-50 $\mu$ thickness mica windows. The sample-to-detector distance is typically $2.0-3.0 \mathrm{~m}$, which yields scattering patterns which range from $20-600 \AA$ in Bragg spacing. Some static experiments have been performed at higher protein concentrations and utilize a sample-todetector distance of $<1.0 \mathrm{~m}$ in order to obtain scattering at higher angles. For time resolved experiments, we have used a stopped-flow apparatus similar to that developed previously $[3,4]$.

\section{Results and Discussion}

The radius of gyration $\left(R_{g}\right)$ of the unliganded $C$ trimer extrapolated to zero concentration was $33.5 \AA$, while $\mathrm{R}_{\mathrm{g}}$ decreased to $32.1 \AA$ in the presence of the bisubstrate analog, $\mathrm{N}$-(phosphonacetyl)-L-aspartate (PALA). The $1.4 \AA$ decrease in $\mathrm{R}_{\mathrm{g}}$ is significantly greater than the $0.6 \AA$ decrease expected from the holoenzyme crystal structure. The combination of the substrate carbamoyl phosphate and the substrate analog, succinate, promotes a 1.0-2.0 $\AA$ decrease in $R_{g}$, whereas carbamoyl phosphate alone caused a 1.0 $\AA$ decrease. In contrast, the $C$ trimer exhibits little to no change in $R_{g}$ in the presence of succinate alone. At trimer concentrations of $50-90 \mathrm{mg} / \mathrm{ml}$, scattering in the higher-angle regons $\left(s=(2 \sin \theta) / \lambda<0.056 \AA^{-1}\right)$ could be observed. From comparisons of these scattering patterns with the scattering behavior expected from the crystal structure of the holoenzyme, we conclude that the unliganded $C$ trimer is significantly more expanded in solution than is expected from the crystal model. The conformation of the PALA-liganded trimer, however, appears to be in excellent agreement with the crystal structure of the PALA-liganded holoenzyme. We are currently using the crystal data to model the structure of the isolated unliganded trimers at low resolution.

In another series of experiments we have utilized a stopped-flow mixing apparatus to study the kinetics of the conformational changes in the intact holoenzyme. In this study we have characterized the quaternary structure changes in a variant ATCase holoenzyme in which lysine at position 143 of the regulatory chains has been replaced with alanine (rLys143Ala). Unlike the wild-type enzyme, this mutant exhibits a quaternary structure which is highly dependent on $\mathrm{pH}$. By adjusting the $\mathrm{pH}$ only 0.5 units, the transition between $T$ and $R$ quaternary states can be studied in the absence of ligand binding events. A series of timeresolved $\mathrm{pH}$-jump experiments were performed in which $R_{\mathrm{g}}$ was followed in time steps as small as $\mathbf{5 0}$ msec after the $\mathrm{pH}$ change. In all cases the allosteric transition was complete within the first timeframe of 
data collection which indicates that the sum of the forward and reverse rates of this process must have a time constant which is far less than $50 \mathrm{msec}$.

\section{References}

1) Schachman, H.K. (1993). Curr. Opin. Struc. Biol., 3, 960-967.

2) Lipscomb, W.N. (1992). pp. 103-143 in "Regulation of Proteins by Ligands: Proceedings of the Welch Foundation Conference on Chemical Research," XXXVI, Houston, TX .

3) Nagamura, T. et al. (1985). J. Biochem. Biophys. Methods, 11, 277.

4) Tsuruta, H. et al. (1989). Rev. Sci. Instrum, 60, 2356. 


\title{
Use of White Beam and Small Angle Scattering for the Study of Compact Intermediates
}

\author{
Dan Segel ${ }^{1}$, Hiro Tsuruta ${ }^{2}$, Lingling Chen ${ }^{3}$, David Eliezer ${ }^{1}$, Sebastian Doniach ${ }^{4}$, Keith O. Hodgson ${ }^{2,3}$ \\ Department of Physics ${ }^{1}$, Chemistry ${ }^{3}$, Applied Physics ${ }^{4}$, Stanford University, Stanford, CA 94305 \\ SSRL $^{2}$, Stanford University, Stanford, CA 94309
}

Small angle X-ray scattering (SAXS) studies of compact intermediate states of proteins in equilibrium often require low protein concentration. For stopped flow studies, greater time resolution is needed to study the early kinetics of protein folding. To meet the objectives of static and stopped-flow measurements with beam time limitations, higher flux is needed. Therefore, the use of quasi-white beam has been employed on beam line 4-2.

Compact intermediates have the propensity to aggregate due to substantial exposure of hydrophobic surfaces [1]. A compact intermediate state of apomyoglobin has been observed to aggregate with concentrations $\geq 1 \mathrm{mg} / \mathrm{ml}$ [2]. Concentrations of $\sim 1$ $\mathrm{mg} / \mathrm{ml}$ or less are not reasonable for a monochromatic beam because of the low counting rate. In addition to radius of gyration $\left(R_{g}\right)$ measurements, calculation of the pair distribution function $P(R)$ is a desired result of solution SAXS studies. A software package (GNOM) has been developed by Dmitri Svergun at EMBL to obtain $P(R)$ from the scattering curve. The accuracy of the $P(R)$ fit is proportional to the amount of high angle data. To accurately calculate $P(R)$ of compact intermediates in a reasonable time frame, high beam flux is necessary.

Time-resolved studies of myoglobin refolding using the stopped-flow method and monochromatic data resulted in the time-dependent determination of the radius of gyration $(\mathrm{Rg})$ with a time resolution of $100 \mathrm{~ms}$ for 150 shots [3]. Better time resolution is desired to monitor fast structural events in protein folding such as the formation of compact intermediate states which may have a half life on the order of a few milliseconds or less.

Last June, the first complete SAXS data sets using white light were obtained. The monochromator crystals were removed from the beam's path allowing the raw focused white beam into the hutch; the rest of the experimental setup remained the same as the setup for monochromatic beam. The white beam flux is a few orders of magnitude greater than monochromatic beam flux. With such high flux, we reach the counting limits of the linear gas chamber detector. To attenuate the flux, a $0.5 \mathrm{~mm}$ Al filter was placed in the beam's path. Even with filter in place, the flux remains 30 times greater than the monochromatic beam flux. Also, the filter, along with the focusing mirror, leads to a nearly Gaussian energy profile of the beam (Figure 1).

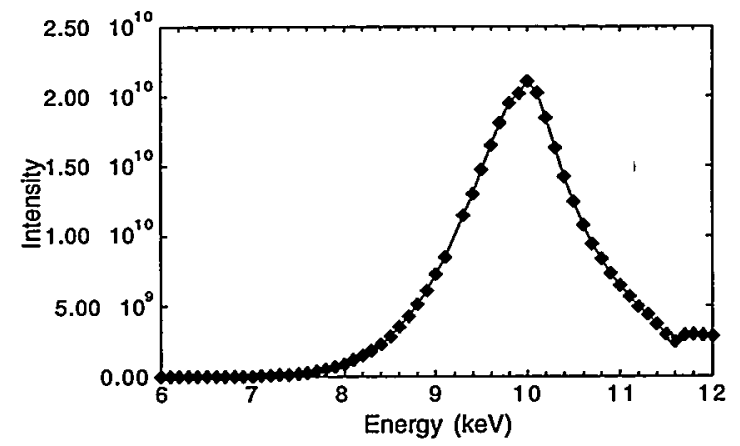

Figure 1. White beam energy profile. Determined by the source characteristics of the SSRL beam, transmission functions of windows/filters in beam's path, and reflection of focusing mirror.

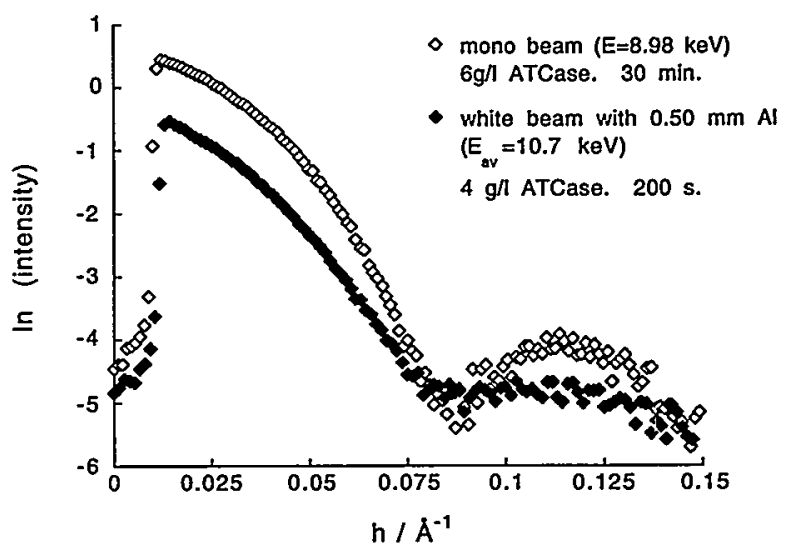

Figure 2. Comparison of ATCase scattering curves. $\mathrm{h}=4 \pi \sin \theta / \lambda$ where $2 \theta$ is the scattering angle.

The white beam scattering curve for aspartate transcarbamoylase (ATCase) exhibits the same features as the monochromatic beam curve, however with somewhat less distinction due to the energy dispersion of the white beam (Figure 2). Exposure time, however, can be significantly shortened with the 
use of the white beam. Taking only the data from the first five seconds of exposure, the radii of gyration for lysozyme (16.1 $\AA$ ) and BSA (33.2 $\AA$ ) show reasonable agreement with the radii of gyration achieved with monochromatic beam (15.1 $\AA$ and $31.2 \AA$, respectively). However, the data from the successive 5 second data frames show an increase in $\mathrm{I}(0)$ (dramatic for lysozyme), which suggests radiation-induced aggregation in the samples. To reduce the radiation effect in future experiments, a sample flow system is being developed. The flow rate would be adjustable; initially we are looking to set the flow rate such that a protein is exposed to the beam for $\sim 1$ second.

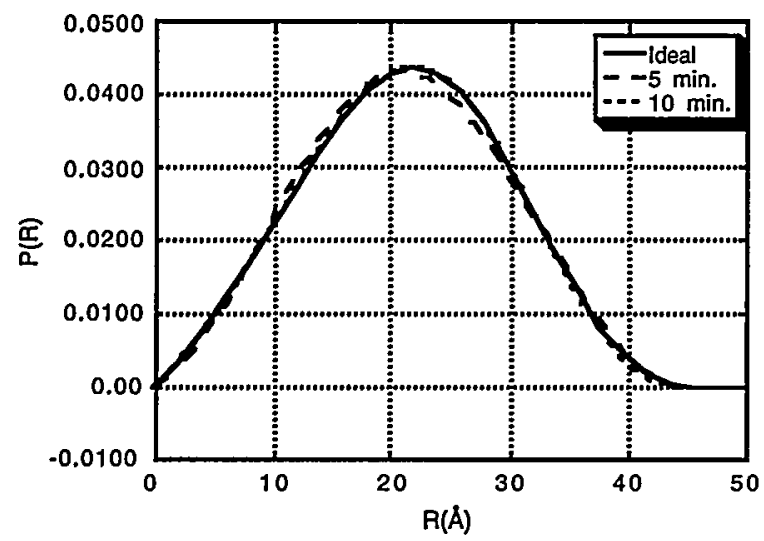

Figure 3. Resultant myoglobin $\mathbf{P ( R )}$ curves from simulated data.

To estimate the amount of exposure time needed with white light, computer simulations were performed on myoglobin. The crystallographic structure (PDB) file was downloaded from Brookhaven. With the coordinates of individual atoms in the PDB file, the Debye formula was used to create a scattering curve. To create a total solution scattering curve, the scattering curve generated from the Debye formula was added to smoothed background data (standard deviations would be added to solution scattering curve) from the June 1994 run. Poisson statistics were then applied to the solution scattering curve for statistical analysis. The resultant scattering curve was then used as an input into the software package GNOM, which calculated $P(R)$. The Guinier plot of the resultant scattering curve was fitted to a straight line with $R_{g}$ (with standard deviation) evaluated from the slope of this curve.

As mentioned, a previous stopped flow study of apomyglobin achieved $100 \mathrm{~ms}$ time resolution for 150 shots in the calculation of $\mathrm{Rg}_{\mathrm{g}}$. Using the white beam with filter and 30 times more flux, we should be able to determine $R_{g}$ to the same accuracy $( \pm 1 \AA$ for the native state) with 50 shots and $10 \mathrm{~ms}$ time resolution, according to the simulation.

Resultant $\mathrm{P}(\mathrm{R})$ calculations from simulations on myoglobin at a concentration of $1 \mathrm{mg} / \mathrm{ml}$ suggest that data taken from exposure time of 10 minutes to white beam should yield a very accurate $P(R)$, with 5 minutes probably acceptable (Figure 3 ). Exposure times of this magnitude are very reasonable for static measurements. Exposure time of 10 minutes to white beam is roughly equivalent to 5 hours of exposure to monochromatic beam.

Simulations suggest in stopped-flow studies that both $R_{g}$ and a very accurate $P(R)$ can be determined with $100 \mathrm{~ms}$ time resolution for 3500 shots using white beam with filter. 1750 shots should yield a suitable $P(R)$. Achievement of such time resolution will lead to better understanding of the kinetics involved in the early process of protein folding.

SSRL is funded by the Department of Energy, Office of Basic Energy Sciences. The Biotechnology Program is supported by the NIH, Biomedical Research Technology Program, Division of Research Resources. Further support is provided by the Department of Energy, Office of Health and Environmental Research.

References:

1.) A.L. Fink. (1995) Annu. Rev. Biophys. Biomol. Struct. 24,495-522.

2.) Y. Goto, L.J. Calciano, \& A.L. Fink. (1990) Proc. Natl. Acad. Sci. USA. 87,573-577.

3.) D. Eliezer, K. Chiba, H. Tsuruta, S. Doniach, K. Hodgson, \& H. Kihara. (1993) Biophys. J. 65,912-917. 


\title{
Photosynthetic Water Oxidation: \\ Structural Insights to the Catalytic Manganese Complex.
}

\author{
Joy C. Andrews ${ }^{a, b}$, Roehl Cinco a,b, Holger Daua,b, Matthew J. Latimer ${ }^{a, b}$, \\ Wenchuan Lianga,b,Theo A. Roelofs ${ }^{a, b}$, Annette Rompel ${ }^{a, b}$, \\ Kenneth Sauer ${ }^{a, b}$, Vittal K. Yachandrab, and Melvin P. Klein ${ }^{b}$. \\ Department of Chemistrya and Structural Biology Division, Lawrence Berkeley Laboratoryb, \\ University of California, Berkeley, CA 94720, USA.
}

\section{Introduction}

The structure and the orientation of the $\mathrm{Mn}$ oxygen-evolving complex (OEC) in photosystem II, which catalyzes the oxidation of water to dioxygen in plants and cyanobacteria, is being studied by $X$-ray absorption spectroscopy. We have proposed a structure consisting of two di- $\mu$-oxo bridged Mn binuclear units, with $\mathrm{Mn}-\mathrm{Mn}$ distance of 2.7 $\AA$, linked by a mono- $\mu$-oxo and carboxylato moieties with a Mn-Mn separation of $3.3 \AA$ [1]. EXAFS studies using oriented PS II samples have shown that the $2.7 \AA \mathrm{Mn}-\mathrm{Mn}$ vectors are at an average angle of $60^{\circ}$ and the $3.3 \AA$ vectors are at an average angle of $43^{\circ}$ (probably an average of $\mathrm{Mn}-\mathrm{Mn}$ and $\mathrm{Mn}-\mathrm{Ca}$ vectors) to the membrane normal [2].

\section{Results and Discussion}

\subsection{Heterogeneity in the Mn-Mn $2.7 \AA$ Vectors}

Guiles et al. [3] described a heterogeneity of the $2.7 \AA \mathrm{Mn}$-Mn distances in the $\mathrm{S}_{3}$ state. DeRose [4] found evidence for a similar effect resulting from fluoride treatment which inhibits oxygen evolution activity. Liang et al [5] have observed a more pronounced heterogeneity (2.73 and $2.85 \AA$ ) in the $\mathrm{Mn}$-Mn distance in the $S_{2}$ state which is characterized by the $g=4.1$ EPR signal.

\subsection{Annealed $\mathrm{NH}_{3} \mathrm{~S}_{2}$-State}

$\mathrm{NH}_{3}$ is a water analog and inhibitor of oxygen evolution. Since it is proposed that $\mathrm{NH}_{3}$ inhibits $\mathrm{O}_{2}$ evolution by binding at the site of water binding, it is important that one understands the structural effects such inhibition produces. Figure 1 shows that the second Fourier peak of the annealed $\mathrm{NH}_{3} \mathrm{~S}_{2}$ state is clearly decreased in comparison with that of the control samples not treated with ammonia. Analysis of the EXAFS spectrum of
$\mathrm{NH}_{3}$-treated PS II samples in the annealed $\mathrm{S}_{2}$ state show that the $2.7 \AA$ distance can be resolved into two components at about 2.72 and $2.87 \AA$.

EXAFS-dichroism measurements indicate that the orientation of the Mn-Mn vector lengthened by ammonia treatment is different from the orientation of the Mn-Mn vectors not lengthened by ammonia. The vector of the binuclear unit which is affected $(2.87 \AA)$ is at an angle of $67^{\circ}$, whereas the second vector $(2.72 \AA)$ is at an angle of $55^{\circ}$ to the membrane normal [6]. The most likely explanation for this difference in orientation is that there are two dissimilar Mn-Mn binuclear units, of which only one is affected by ammonia binding.

The resolution of the Mn-Mn distances in the studies described above indicates the presence of two inequivalent di- $\mu$-oxo bridged Mn binuclear units in the tetranuclear Mn complex and a model based specifically on $\mathrm{NH}_{3}$ studies is shown in Figure 2.

\subsection{XANES Studies of the S-States of Photosystem II}

Oxidation state changes of the Mn complex in the S-states have been studied by XANES. PS II samples were prepared by flash illumination and the S-state composition was characterized by measuring the oscillation in the amplitude of the multiline EPR signal. Second derivatives of the $\mathrm{Mn} \mathrm{K}$-edge spectra of the flash-induced S-states are shown in Figure 3. Distinct changes in shape and an increase in the inflection point energy (measured as the zero crossing of the second derivative) are observed for the $S_{0}$ to $S_{1}$ and $S_{1}$ to $S_{2}$ transition, indicating the oxidation of $\mathrm{Mn}$ during these S-state advances. However, the small increase in the inflection point energy for the $S_{2}$ to $S_{3}$ transition and the similarity of the shape of the second derivative $(6540-6560 \mathrm{eV})$ in the $S_{2}$ and $S_{3}$ states does not provide 
conclusive evidence for $\mathrm{Mn}$ oxidation during the $S_{2}$ to $S_{3}$ transition.

\section{Acknowledgments}

This work was supported by the National Science Foundation Grant DMB91-04104 and by the Director, Division of Energy Biosciences, Office of Basic Energy Sciences, Department of Energy under contract DEAC03-76SF00098. Synchrotron radiation facilities were provided by the Stanford Synchrotron Radiation Laboratory (SSRL) and the National Synchrotron Light Source (NSLS), both supported by DOE. The Biotechnology Laboratory at SSRL and Beam Line X9 at NSLS are supported by the National Center for Research Resources of the National Institutes of Health.

\section{References}

[1] V. K. Yachandra et al. (1993) Science 260, 675.

[2] I. Mukerji et al. (1995) Biochemistry 33, 9712.

[3] R. D. Guiles et al. (1990) Biochemistry 29, 471.

[4] V. J. DeRose et al. (1995) Chem. Phys. 194, 443.

[5] W. Liang et al. (1994) Biochemistry 33, 4923.

[6] H. Dau et al. (1995) Biochemistry 34, 5274.

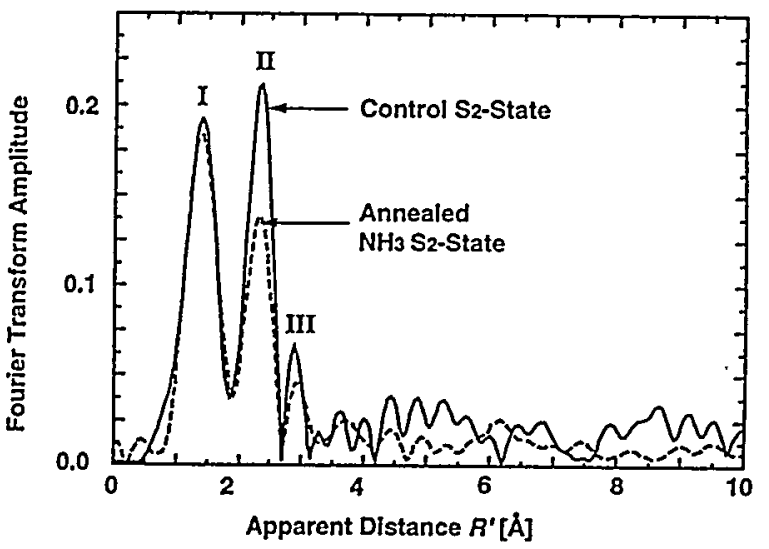

Fig. 1. Fourier transforms of the $k^{3}$-weighted Mn EXAFS data from PS II samples in the control $S_{2}$ state (solid line), and annealed $\mathrm{NH}_{3}$ $\mathrm{S}_{2}$-state (broken line). Peak labeled II is assigned to 2.7-2.8 $\AA \mathrm{Mn}$-Mn distances.

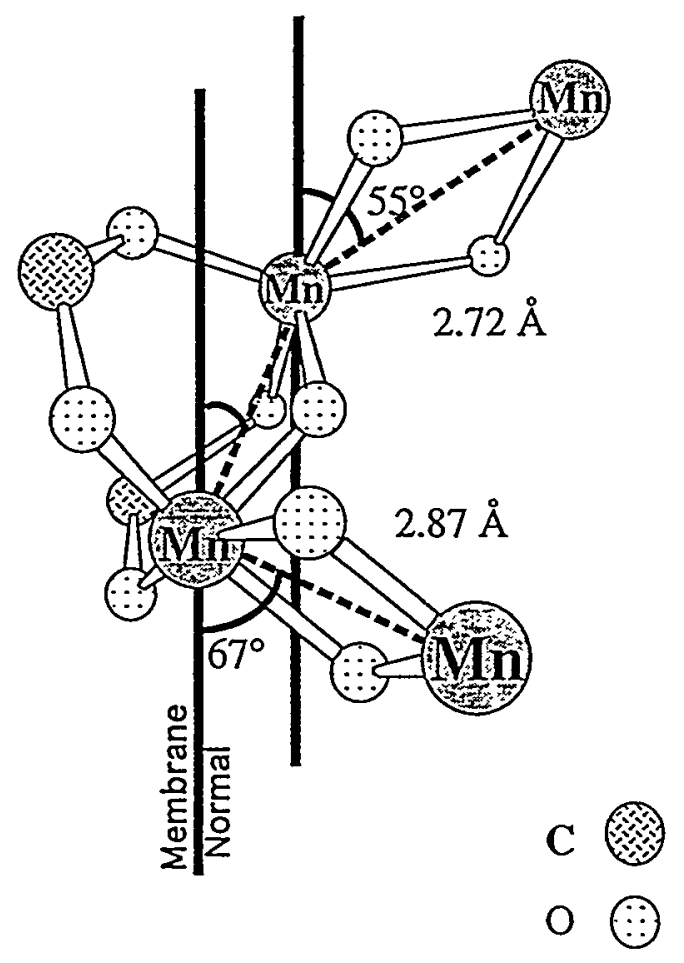

Fig. 2. Proposed model of the PS II Mn complex based on the heterogeneity observed in the $2.7 \AA$ distances and the orientation of the 2.72 and $2.87 \AA \mathrm{Mn}-\mathrm{Mn}$ vectors in annealed $\mathrm{NH}_{3} \mathrm{~S}_{2}$-state samples.

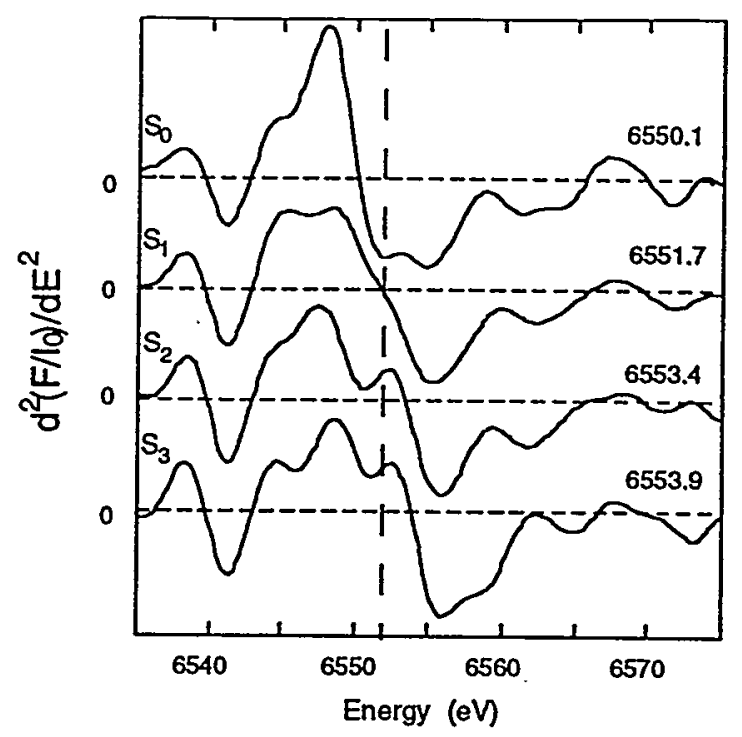

Fig. 3. Second derivatives of $\mathrm{Mn}$ K-edge spectra of PS II samples in the $S_{0}, S_{1}, S_{2}$, and $S_{3}$ states. The inflection point measured as the zero point crossing of the second derivatives is noted on the Figure. 


\title{
Structural Characterization of the Active Site of a Manganese-Enzyme, Prolidase
}

\author{
Paul J. Ellis, ${ }^{1}$ Hans C. Freeman, ${ }^{1}$ Britt Hedman, ${ }^{2}$ Keith O. Hodgson, ${ }^{3}$ \\ Emma M. Proudfoot ${ }^{1}$ and Lianbo Zhang ${ }^{1}$ \\ 1 Department of Inorganic Chemistry, University of Sydney, N.S.W. 2006, Australia \\ ${ }^{2}$ Stanford Synchrotron Radiation Laboratory, Stanford, CA 94309 \\ ${ }^{3}$ Chemistry Department, Stanford University, Stanford, CA 94305
}

The manganese-enzyme prolidase (proline dipeptidase, EC 3.4.13.9) is found in a wide range of microorganisms and mammalian tissues. Its function is to hydrolyse dipeptides in which the Cterminal residue is proline or hydroxyproline. It has been suggested that the proline and hydroxyproline released by the reaction are required for the synthesis of collagen, an essential structural protein. Medical interest in the enzyme stems from the fact that prolidase deficiency in humans is a congenital abnormality whose symptoms include skin lesions, recurrent respiratory tract infections and mental retardation. From a structural point of view it is particularly interesting that a single nucleotide change in the prolidase gene suffices to produce this abnormality. ${ }^{1}$ The occurrence, reactivity, purification and assay of the enzyme have been reviewed by Myara et al. ${ }^{2}$

Prolidase is a dimeric molecule containing one $\mathrm{Mn}$ (II) atom per sub-unit with $\mathrm{M}_{\mathrm{r}} 54,300$. The amino acid sequences of human and $E$. coli prolidase have been determined. ${ }^{3.4}$ Structural similarity with several other aminopeptidases has been inferred from overall similarities among the sequences. ${ }^{5}$ In particular, a number of His, Asp and Glu residues which are putative ligands of two cobalt atoms in methionine aminopeptidase also occur in prolidase. ${ }^{5}$ Replace-ment of one of the conserved Asp residues in prolidase by Asn corresponds to the single nucleotide change that has been mentioned above as being associated with clinical symptoms. A model for the prolidase active site has been proposed on the basis of NMR evidence that the enzyme is specific for the trans configuration of the substrate imide group. ${ }^{6}$ This model has led to the successful design of inhibitors for the enzyme. 7,8 Beyond this, little is known about the active site.
Although manganese-dependent dipeptidases were described by Malmström and collaborators already in the late $1950^{\prime} \mathrm{s}$, metalloproteins containing manganese are - in comparison with $\mathrm{Zn}$-, $\mathrm{Fe}$ - and $\mathrm{Cu}$-proteins - still poorly characterized. The reasons why $\mathrm{Mn}$-proteins are relatively underinvestigated may include the lack of clear correlations between structure and spectroscopic signatures, as well as doubts whether some $\mathrm{Mn}$ potentiated enzymes utilize $\mathrm{Mn}^{2+}$ or $\mathrm{Mg}^{2+}$ in vivo.

We have begun a study of the metal site in prolidase, using EXAFS at the manganese $K$-edge as one of a number of probes of the coordination of the metal. Both the human and $E$. coli prolidase genes $^{3,4}$ have been expressed in $E$. coli (by Dr N E Dixon, Australian National University), and experiments to overproduce the proteins are almost complete. The EXAFS data will be interpreted using new software written at the University of Sydney for the restrained multiple-scattering EXAFS refinement of metal-site geometries.

In order to provide benchmarks for the refinement strategy to be used in the prolidase study, we have recorded EXAFS data at 10K, 173K and 298K for ten manganese(II) and manganese(III) model compounds whose structures are accurately known from X-ray crystal structure analyses. The compounds were selected to include examples of both regular and irregular coordination geometries. Systematic calculations to establish the optimum conditions for the restrained multiple-scattering refinement of the manganese(II) and -(III) sites are in progress.

This work is supported by the Australian Research Council 
1. A. Tanoue, F. Endo, A. Kitano and I. Matsuda, $J$. Clin. Invest., 86, 351 (1990).

2. I. Myara, C. Charpentier \& A. Lemonnier, Life Sci., 34, 1985 (1984).

3. F. Endo, A. Tanoue, H. Nakai, A. Hata, Y. Indo, K. Titani \& I. Matsuda, J. Biol. Chem., 264, 4476 (1989).

4. T. Yoshimoto, H. Tone, T. Honda, K. Osatomi, R. Kobayashi \& D. Tsuru, J. Biochem. 105, 412-416 (1989).
5. J.F. Bazan, L.H. Weaver, S.L. Roderick, R. Huber \& B.W. Matthews, Proc. Natl. Acad. Sci. USA, 91, 2473-2477 (1994).

6. G.F. King, C.R. Middlehurst \& P.W. Kuchel, Biochemistry, 25, 1054 (1986).

7. G.F. King, M.J. Crossley \& P.W. Kuchel, Eur. J. Biochem, 180, 377 (1989).

8. A. Radzicka_\& R. Wolfenden, Biochemistry, 30, 4160- 4164 (1991). 


\title{
EXAFS Determination of a 2.5 å Cu-Cu Interaction at the $\mathrm{Cu}_{\mathrm{A}}$ Center of Oxidized and Reduced Cytochrome Oxidase
}

\section{from Bacillus Subtilis}

\author{
Ninian J. Blackburn and John S. Boswell \\ Dept of Chemistry, Biochemistry and Molecular Biology, Oregon Graduate Institute \\ and \\ Simon de Vries \\ Dept. of Microbiology and Enzymology, Technical University of Delft, Netherlands
}

The $\mathrm{Cu}_{\mathrm{A}}$ center of cytochrome $c$ oxidase has attracted much attention because of its unusual spectroscopy. The emerging consensus is for a dinuclear mixed valence site, (Malmstrom, \& Aasa, 1993) but the nature of the $\mathrm{Cu}-\mathrm{Cu}$ interaction is still hotly debated. Recently we proposed a model based on EXAFS data, involving a short $(2.5 \AA) \mathrm{Cu}-\mathrm{Cu}$ interaction as the origin of a strong second row, or metal scatterer which is present at $\sim 2.5-2.6 \AA$ (Blackburn et al., 1994). In the work described
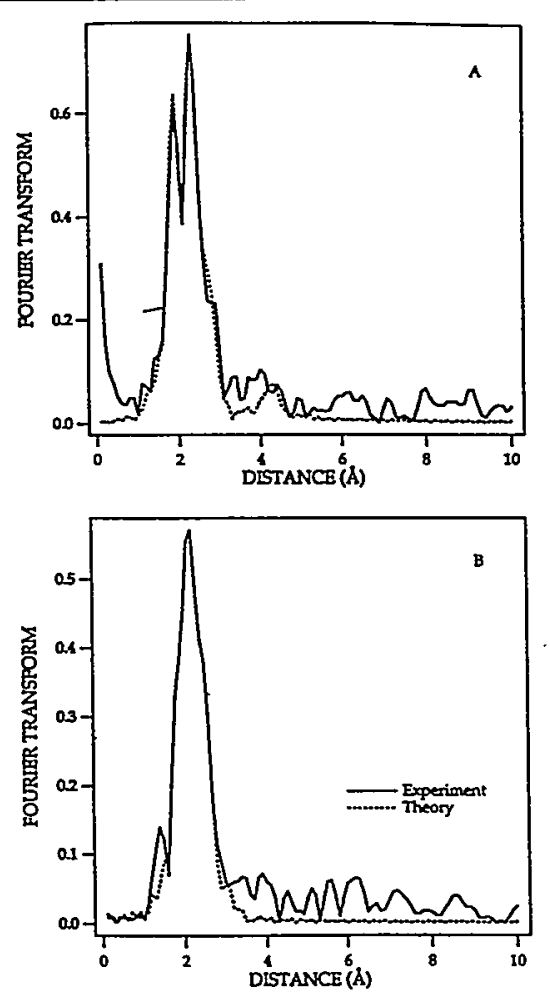

Fig. 1 Fourier Transforms of oxidized $(A)$ and reduced (B) $\mathrm{Cu}_{A}$ here, we have extended our measurements to obtain data to $\mathrm{k}=16 \AA^{-1}$ on both oxidized and reduced forms of the enzyme. The analysis shows that even with this extended data range, it is difficult to distinguish $\mathrm{Cu}-\mathrm{Cu}$ from $\mathrm{Cu}-\mathrm{S}$ at this distance. However, the $\mathrm{Cu}-\mathrm{Cu}$ model gives an excellent fit to both oxidized and reduced datasets. We conclude that the $\mathrm{Cu}-\mathrm{Cu}$ model should be considered a serious candidate for the structure of the $\mathrm{Cu}_{\mathrm{A}}$ center.

The proposed structures and EXAFS derived distances are shown below.
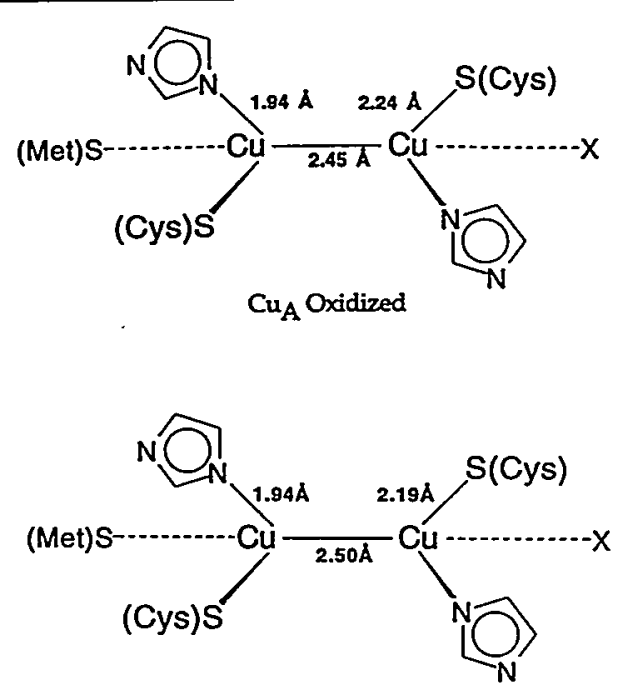

$\mathrm{Cu}_{\mathrm{A}}$ Reduced

Malmstrom, B. G. \& Aasa, R. (1993) Febs Lett 325, 49-52.

Blackburn, N. J., Barr, M. E., Woodruff, W. H., van, d. Oost. J. \& de Vries. S. (1994) Biochemistry 33, 10401-7. 


\title{
EXAFS Studies of the Copper Centers in Peptidylglycine $\alpha$-Hydroxylating Monooxygenase
}

\author{
Ninian J. Blackburn, and John S. Boswell \\ Dept. of Chemistry, Biochemistry and Molecular Biology, Oregon Graduate Institute \\ and \\ Andrew S. Quon, Richard E. Mains, and Betty A. Eipper \\ Dept. of Neuroscience, Johns Hopkins School of Medicine
}

Peptidylglycine $\alpha$-hydroxylating monooxygenase (PHM) catalyzes the final stage in the biosynthesis of amidated peptide hormones. The monooxygenase is a small $35 \mathrm{kD}$ domain of the larger $(75 \mathrm{kD})$ peptide amidating enzyme (PAM). PHM carries out the $\alpha-$ hydroxylation of C-terminal glycine precursor, while a second domain of PAM converts this initial product to the amidated peptide and glyoxalate. It has been known for some time that PHM is very similar to the copper monooxgenase dopamine- $\beta$-monooxygenase $(\mathrm{DBH})$. In this work we have carried out the first XAS studies on PHM, and have demonstrated that the coordination chemistry of the $\mathrm{Cu}$ centers is indeed similar to that of $\mathrm{DBH}$. Previous studies on DBH have shown that the copper centers are inequivalent. $\mathrm{Cu}_{\mathrm{A}}$ is an electron transfer center which shuttles electrons from ascorbate to $\mathrm{Cu}_{\mathrm{B}}$ which is the site of $\mathrm{O}_{2}$ binding and substrate hydroxylation (Reedy and Blackburn, 1994).

EXAFS spectra have been collected on both PHM and PAM. The copper centers of the oxidized forms appear very similar to one another, as well as to the EXAFS of DBH. Simulations have shown that the $\mathrm{Cu}$ (II) is coordinated to 2-3 histidine ligands and 1-2 oxygen donor ligands at an average distance of $1.97 \AA$. In the reduced enzymes, a dramatic change in coordination is apparent. In addition to a shell of 2 histidines at $1.93 \AA$, a strong second row scatterer is evident at $2.25 \AA$ at one of the $\mathrm{Cu}$ centers. Some evidence of Scoordination is seen in the oxidized PHM sample (see figure), Site-directed mutagenesis on the PHM domain indicates that mutation of Met314 to Ile eliminates catalytic activity. On the other hand, all conserved cysteines are involved in disulfide bon formation. For these reasons we believe that one of the $\mathrm{Cu}(\mathrm{I})$ centers of both $\mathrm{PHM}$ and $\mathrm{DBH}\left(\mathrm{Cu}_{\mathrm{B}}\right)$ is coordinated by
2 histidines and a methionine residue. Aspects of this work have recently been published (Quon et al., 1995).
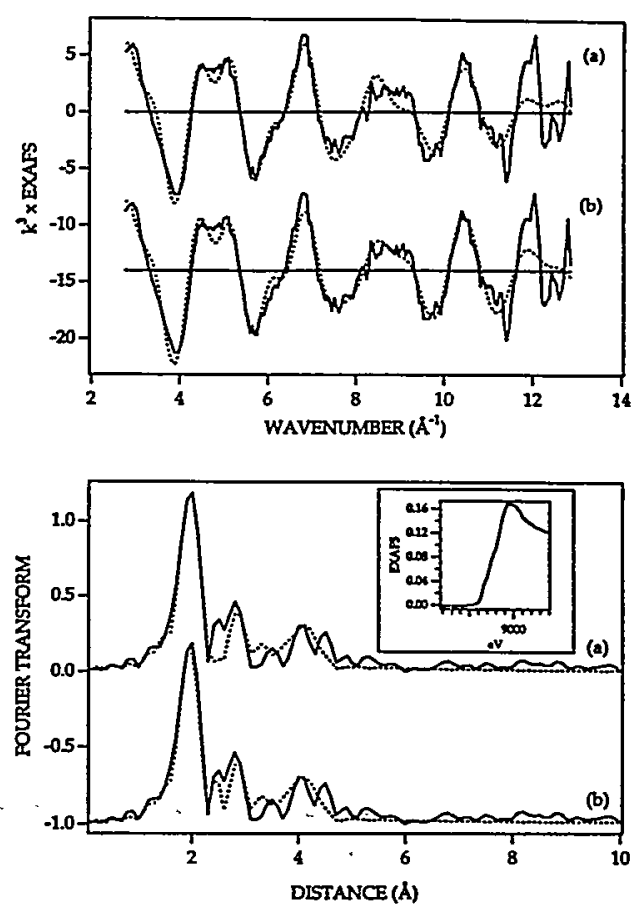

EXAFS and Fts of oxidized PHM (a) no S; (b) 0.5 $S$ per $C u$ at $2.4 \AA$

Reedy, B. J. \& Blackburn, N. J. (1994) J. Am. Chem. Soc. 116, 1924-1931.

Eipper, B. A., Quon, A. S. W., Mains, R. E., Boswell, J. S. \& Blackburn, N. J. (1995) Biochemistry 34, 2857-2865. 


\title{
Structural Investigations on the $\mathrm{Cu}_{\mathrm{B}}$ Center of the Quinol Oxidase from Bacillus Subtilis and an Oxo-bridged Heterobimetallic Fe-Cu Model Compound: Multiple Scattering Determination of the $\mathrm{Cu}-\mathrm{O}(\mathrm{H})$-Fe Angle
}

\author{
Ninian J. Blackburn and John Boswell \\ Dept of Chemistry, Biochemistry and Molecular Biology, Oregon Graduate Institute \\ Marten Wikstrom \\ Bioenergetics Group, Dept of Medical Chemistry, University of Helsinki
}

Kenneth D. Karlin

Dept. of Chemistry, The Johns Hopkins University

The dinuclear $\mathrm{Fe}_{\mathrm{a}}-\mathrm{Cu}_{\mathrm{B}}$ center is the dioxygen binding and reduction site of the super-family of heme-copper oxidases. A combination of spectroscopy and site-directed mutagenesis has provided a structural model for the site, in which the $\mathrm{Fe}$ is 5-coordinate with axial His coordination, and $\mathrm{Cu}_{\mathrm{B}}$ is ligated to $3 \mathrm{His}$ (Hosler et al., 1993). The connectivity between Fe and $\mathrm{Cu}$ is still hotly debated. Some bridging ligand must be present to mediate the observed antiferromagnetic coupling. We have undertaken EXAFS studies of the metal centers in the $\mathrm{Fe}-\mathrm{Cu}$ center of the quinol oxidase from Bacillus subtilis $\left(\mathrm{aa}_{3}-600\right)$, and relevant oxo and hydroxo-bridged model complexes. Since the quinol oxidase lacks the $\mathrm{Cu}_{\mathrm{A}}$ center, interference from the latter can be eliminated from the EXAFS. A previous study had implicated the presence of a $\mathrm{S} / \mathrm{Cl}$ ligand at the $\mathrm{Cu}_{\mathrm{B}}$ center, but $\mathrm{S}$ ligation is inconsistent with sequence and SDM data (Powers et al. 1994). The goal of our studies is to clarify the structure of the $\mathrm{Cu}_{B}$ center, and to probe possible models for the nature of the bridging ligand.

Figure 1 shows a comparison of the background-subtracted $\mathrm{Cu}$ K-EXAFS of three different samples of oxidized $a a_{3}-600$ : (a) the standard preparation, (b) chloride free, and (c) high $\mathrm{pH}(8.8)$ where the magnetic coupling is lost. All three preparations are very similar. Simulations show that the spectra can be simulated by $2-3$ histidine ligands and 1-2 $O$ donor ligands. If the first shell is split ( $3 \mathrm{~N} / 10$ ), the best fit distances are $2.03 \AA$ and $1.92 \AA$ for the His and $O$ shell respectively. ENDOR studies on the high $\mathrm{pH}$ form indicates that the $\mathrm{O}$ ligand carries an exchangeable proton and is thus water or hydroxide. No $\mathrm{Fe}-\mathrm{Cu}$ interaction is seen in the $\mathrm{Cu}$ edge EXAFS data. The high $\mathrm{pH}$ form shows some indication of the presence of substoichiometric coordination of a heavy scatterer such as $\mathrm{Cl}^{-}$, and it is suggested that weak ligation by halides can produce partial occupancy of a coordination position on $\mathrm{Cu}$ (II).

Recently two groups have reported the synthesis and characterization of oxo-bridged heterobimetallic porphyrin $\mathrm{Fe}-\mathrm{Cu}$ complexes which act as good models for the $\mathrm{Fe}-\mathrm{Cu}$

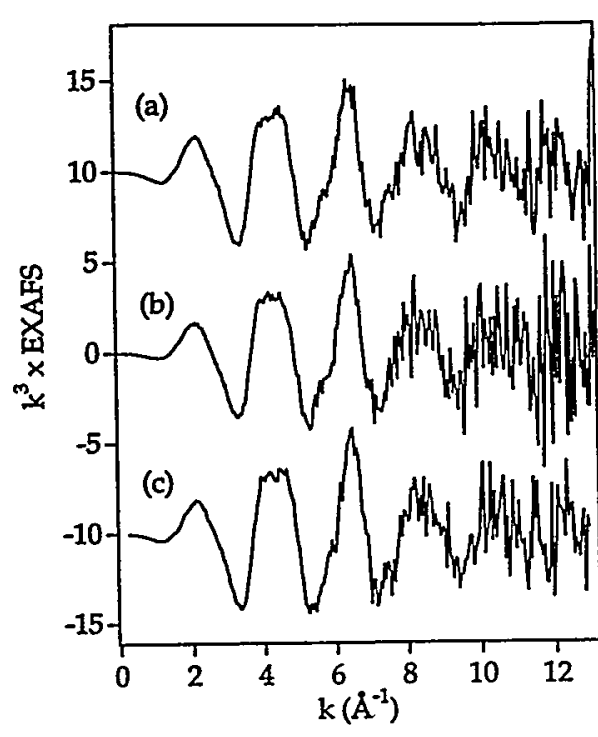

Fig. 1 Comparison of the $C u K$-EXAFS of three preparations of $a a_{3}-600$. (a) standard; (b) $\mathrm{Cl}$-free; (c) high pH 
dinuclear center in the heme-copper oxidases (Karlin et al., 1993; Scott et al., 1994, 1995). The bridging oxo group in these complexes is linear, but undergoes reversible protonation to form a significantly bent $\mathrm{Fe}-\mathrm{OH}-\mathrm{Cu}$ unit. The linear configuration within the $\mathrm{Fe}-\mathrm{O}-\mathrm{Cu}$ unit gives rise to strong multiple scattering interactions. These can be simulated satisfactorily using the program EXCURV (Fig 2(a)). Simulations at both the $\mathrm{Fe}$ and the $\mathrm{Cu}$ edge have allowed us to determine four parameters: three distances: $\mathrm{Cu}-\mathrm{O}, \mathrm{Fe}-\mathrm{O}$, and $\mathrm{Fe}-$ $\mathrm{Cu}$; and one angle $\mathrm{Fe}-\mathrm{O}-\mathrm{Cu}$. Since only three of these parameters are necessary to define the geometry of the $\mathrm{Fe}-\mathrm{O}-\mathrm{Cu}$ unit, there is one additional parameter which can be used to check the validity of the calculations. For the oxo complex we find $\mathrm{Cu}-\mathrm{O}=1.83 \AA, \mathrm{Fe}-\mathrm{O}=1.72 \AA$, $\mathrm{Fe}-\mathrm{Cu}=3.56 \AA$ and $\mathrm{Fe}-\mathrm{O}-\mathrm{Cu}=176^{\circ}$ (simulated), $178^{\circ}$ (calculated). These parameters
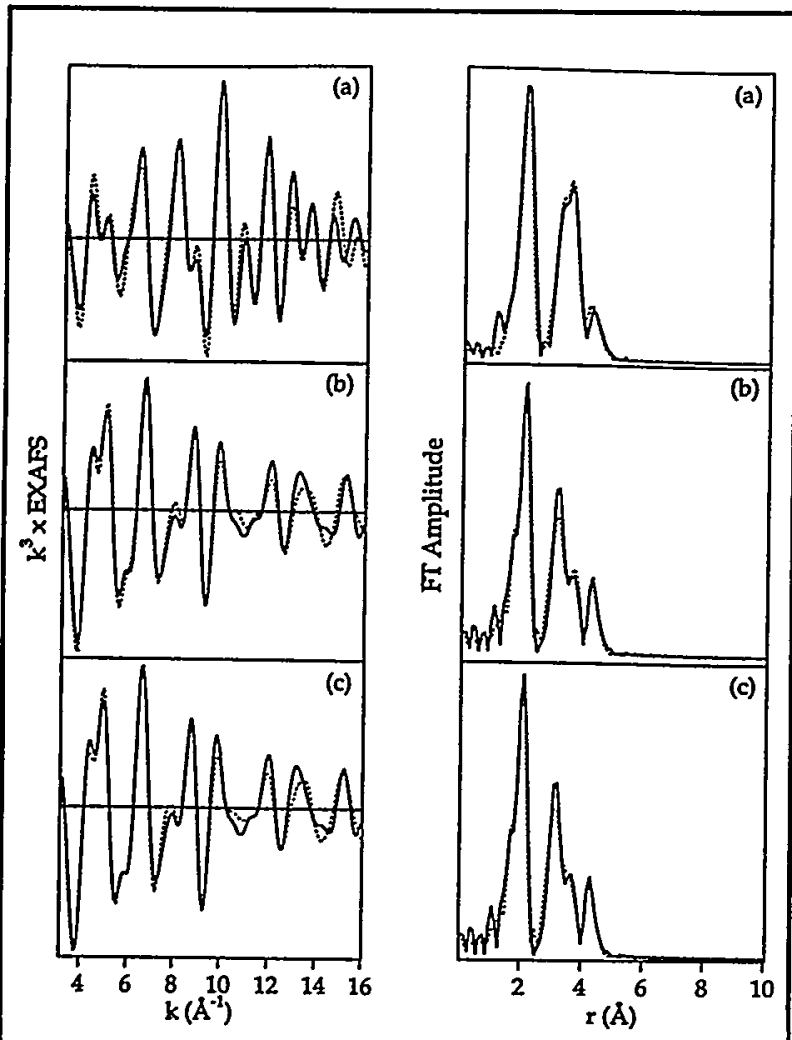

Fig. 2 Fe-EXAFS and Fts of the oxo and hdroxobridged model complexes. (a) oxo MS included, $\mathrm{Fe}$ $\mathrm{Cu} 3.56 \AA$, angle $=174^{\circ}(\mathrm{b})$ hydroxo, no $\mathrm{MS}, \mathrm{Fe}$ $\mathrm{Cu}=3.72 \AA$ (c) hydroxo, $\mathrm{MS}$ included, $\mathrm{Fe}-\mathrm{Cu}=$ 3.66 , angle $=157^{\circ}$. agree closely with crystallography, and demonstrate the power of MS calculations in defining geometry of ligand-bridged heterobimetallic complexes.

Application of these methods to the protonated hydroxo complex has allowed us to define the geometry of the $\mathrm{Fe}-\mathrm{OH}-\mathrm{Cu}$ bridge in the absence of a crystal structure. The Fe-O and Fe$\mathrm{Cu}$ bonds lengthen to $1.87 \AA$ and $3.66 \AA$ respectively, while the $\mathrm{Cu}-\mathrm{O}$ bond lengthens only slightly to $1.89 \AA$. The simulated value of the $\mathrm{Fe}-\mathrm{OH}-\mathrm{Cu}$ angle is $157^{\circ}$, as compared with the value of $155^{\circ}$ calculated from triangulation. The level of agreement is exceptional, and demonstrates the validity of the MS approach to the determination of bridge geometry in ligandbridged heterobimetallic complexes.

Comparison with the protein data indicates that oxo bridging can be ruled out as the origin of the coupling between $\mathrm{Fe}$ and $\mathrm{Cu}$. However, $\mathrm{OH}$ bridging remains an attractive possibility. The similarity between the $\mathrm{Cu}-\mathrm{O}$ bond lengths in protein and model may be coincidental. However if similar $\mathrm{Fe}-\mathrm{O}$ and $\mathrm{Fe}-\mathrm{Cu}$ distances existed in the protein, it would suggest an angle close to $160^{\circ}$, which would likewise be detectable by MS methodologies. Further studies are underway to test these hypotheses.

Hosler, J. P., Ferguson-Miller, S., Calhoun, M. W., Thomas, J. W., Hill, J., Lemieux, L., Ma, J., Georgiou, C., Fetter, J., Shapleigh, J., Tecklenburg, M. M. J., Babcock, G. T. \& Gennis, R. B. (1993) J. Bioenerg. Biomembr. 25, 121-136.

Powers, L., Lauraeus, M., Reddy, K. S., Chance, B. \& Wikstrom, M. (1994) Biochim. Biophys. Acta 1183, 504-512.

Karlin, K. D., Nanthakumar, A., Fox, S., Murthy, N. N., Ravi, N., Huynh, B. H., Orosz, R. D. \& Day, E. P. (1994) J. Am. Chem. Soc. 116, 4753-4763.

Scott, M. J., Lee, S. C. \& Holm, R. H. (1994) Inorg. Chem 33, 4651-62.

Scott, M. J., Zhang, H. H., Lee, S. C., Hedman, B., Hodgson, K. O. \& Holm, R. H. (1995) J. Am. Chem. Soc. $117,568-569$. 


\title{
Solution Small-angle X-ray Scattering Studies of the Heat Shock Cognate Protein
}

\author{
Sigurd M. Wilbanks ${ }^{1}$, Lingling Chen ${ }^{2}$, Hiro Tsuruta ${ }^{4}$, David Eliezer ${ }^{2}$, Sebastian Doniach ${ }^{3}$, \\ Keith O. Hodgson ${ }^{2}$, David B. McKay ${ }^{1}$ \\ ${ }_{1}^{1}$ Department of Structural Biology, Stanford University \\ 2 Department of Chemistry, Stanford University \\ 3 Department of Applied Physics, Stanford University \\ ${ }^{4}$ Stanford Synchrotron Radiation Laboratory
}

The 70 Kilodalton heat shock proteins comprise a family of molecular chaperones that are essential for proper folding of proteins in cells. They bind denatured proteins or unstructured polypeptides, but not their native counterparts. They have an ATPase activity which is coupled to peptide binding and release. The proteins are typically $\sim 650$ amino acids in length. The ATPase activity resides in the first -385 amino acids, while the peptide binding activity is found in residues 385-550.

We have conducted solution small-angle scattering experiments on the bovine heat shock cognate protein (Hsc70) and fragments thereof, including the ATPase fragment (amino acids 1-386), the peptide binding fragment (388-554), full-length protein (1-650), and a "60 kDa" fragment that has approximately 100 amino acids deleted from the carboxy terminus (1-554). From steady-state measurements, we find that $\mathrm{Hsc70}$ is an elongated protein. We find that the ATPase fragment does not show a significant change in $R_{g}$ between its nucleotide-bound and nucleotide-free states. Both fulllength Hsc70 and its $60 \mathrm{kDa}$ fragment show a substantial decrease in $R_{g}$ in the presence of ATP, as compared to in the presence of ADP, AMPPNP, or in the absence of the nucleotide. Kinetic measurements show the ATP-induced decrease to be rapid, relative to the rate of ATP hydrolysis; it occurs in less than a minute, while the time constant for hydrolysis is $\sim 5$ minutes. The transition to the ATP-induced state therefore results from ATP binding, not from ATP hydrolysis. A manuscript from these results is currently in preparation. 


\title{
EXAFS Studies of the Molybdenum-Iron Cluster from Nitrogenase
}

\author{
H. Isaac Liu ${ }^{1}$, Britt Hedman ${ }^{2}$, Keith O. Hodgson ${ }^{1,2}, \mathrm{Li} \mathrm{Ma}^{3}$, Barbara K. Burgess ${ }^{3}$ \\ 1. Department of Chemistry, Stanford University, Stanford, CA 94305 \\ 2. Stanford Synchrotron Radiation Laboratory, Stanford University, Stanford, CA 94309 \\ 3. Department of Molecular Biology and Biochemistry, University of California, Irvine, CA 92727
}

\section{Introduction}

Nitrogenase, which catalyzes the biological reduction of atmosphere $\mathrm{N}_{2}$ to ammonia, is a key enzyme in the nitrogen cycle. It contains an iron protein (Fe protein) and a molybdenum-iron protein. (MoFe protein). The MoFe protein has two types of metal centers, the P-clusters and the iron-molybdenum cofactor (FeMoco) centers, with the latter being the site of substrate binding and reduction.

In addition to studying the FeMoco site of the MoFe protein, since 1977 it has been possible to isolate the FeMo cofactor by extracting it into the solvent $\mathrm{NMF}^{1}$. Although isolated FeMoco cannot fix $\mathrm{N}_{2}$, it can restore activity to inactive FeMo cofactor-deficient forms of the MoFe protein ${ }^{1}$. It has been established by $\mathrm{X}$-ray absorption spectroscopy that isolated FeMoco is similar to, but not identical with, the FeMo cofactor site of the protein ${ }^{2}$. It is also known that FeMoco is responsible for the $S=3 / 2$ EPR signal exhibited by the $\mathrm{MoFe}$ protein in its dithionite reduced state ${ }^{3-5}$. This observation is particularly important because during nitrogenase turnover, the $S=3 / 2$ center is further reduced to an EPR-silent ( $S=$ integer) state ${ }^{6-8}$ which is then capable of substrate reduction.

In 1981 a brief report appeared on the isolation of another metal cluster from the MoFe protein which was designated the molybdenum-iron cluster (MoFe cluster $)^{9}$. This cluster is isolated from MoFe protein into the solvent methyl-ethyl-ketone (MEK) under low pH conditions, rather than into NMF. This species, whose structure is unknown, exhibited an $S=3 / 2$ EPR signal in NMF very similar to that exhibited by isolated FeMoco or the FeMoco site of the MoFe protein. However, this MoFe cluster was not able to activate the FeMoco-deficient forms of the MoFe protein. Thus, the MoFe cluster appeared to be a subset of the FeMoco, containing the magnetic center responsible for the $S=3 / 2$ EPR signal but missing some component that was essential for substrate reduction. In order to gain insight into the relationship between the FeMoco structure, which is known in its protein bound form ${ }^{10}$, and the magnetic center responsible for the $S=3 / 2$ EPR signal we report the characterization of the MoFe cluster using EPR and X-ray absorption spectroscopy ${ }^{11}$.

\section{Experimental}

Iron and molybdenum determinations were carried out as described elsewhere. EPR spectra were obtained using a Bruker ESP $300 \mathrm{~Hz}$ spectrophotometer, interfaced with an Oxford Instruments ESR-9002 liquid helium continuous flow cryostat. Mo K-edge $x$-ray absorption spectra were collected at the Stanford Synchrotron Radiation Laboratory on unfocused multipole wiggler beam line 7-3 under dedicated ring conditions $(3.0 \mathrm{GeV}, 50-100 \mathrm{~mA})$ using a $\mathrm{Si}(220)$ double-crystal monochromator. The spectra were all recorded in fluorescence using a Lyttle detector at sample temperatures of $10 \mathrm{~K}$, maintained using an Oxford Instruments CF1208 liquid He cryostat. Internal energy calibration was performed assigning the first inflection point of Mo foil to $20003.9 \mathrm{eV}$.

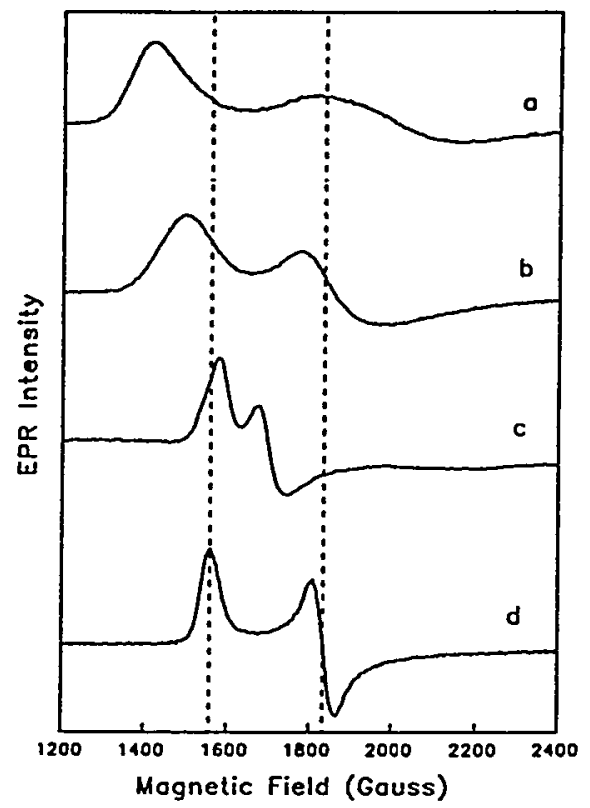

Fig. 1. EPR spectrum recorded at $4 \mathrm{~K}$ for: (a) isolated. FeMo cofactor in NMF, $0.16 \mathrm{mM}$; (b) isolated FeMo cofactor in NMF plus 10 fold excess of benezenethiol, $0.16 \mathrm{mM}$; (c) isolated MoFe cluster in NMF with a 10 fold excess of benezenethiol, $0.1 \mathrm{mM}$ and; (d) MoFe protein in $0.025 \mathrm{M}$ Tris, $\mathrm{pH} 7.4,0.2 \mathrm{mM}$.

\section{Results and Discussion}

As shown in Fig. 1 the $S=3 / 2$ signal exhibited by isolated FeMoco in NMF is very broad. Upon addition 
of benzene thiolate this signal sharpens (Fig. 1b) making it look more like the sharp signal exhibited by the FeMoco site of the MoFe protein (Fig. 1d). This thiol reaction has been studied in detail and the thiol binding site is most likely to be on the Fe atom that is coordinated to Cys 275 of the $\alpha$ subunit of the MoFe protein. It should be noted that the fact that thiol does not sharpen the $\mathrm{MoFe}$ cluster signal does not necessarily mean that MoFe cluster is missing the $\mathrm{Fe}$ atom that coordinated to $\alpha$ Cys 275 , or that thiol does not bind to MoFe cluster. The species that actually binds is thiolate, which is not expected to be present in significant concentration in the pH 4.0 (MEK) or 5.0 (NMF) solutions of MoFe cluster.

Fig. 1 compares the protein and cofactor $S=3 / 2$ EPR signals to that obtained for the MoFe cluster. The results clearly show that the MoFe cluster signal is almost as sharp as the signal exhibited by the MoFe protein, and much sharper than the signals exhibited by FeMoco with or without thiol addition. Thus, the sharpness of the signal is not due to specific interactions with the protein but rather, is an inherent property of the metal cluster. In general, the more sharp a signal the more homogenous the cluster that gives rise to that signal, so it could be that the FeMoco represents a mixture of clusters with varied ligands while the MoFe cluster has a more homogenous ligand set.

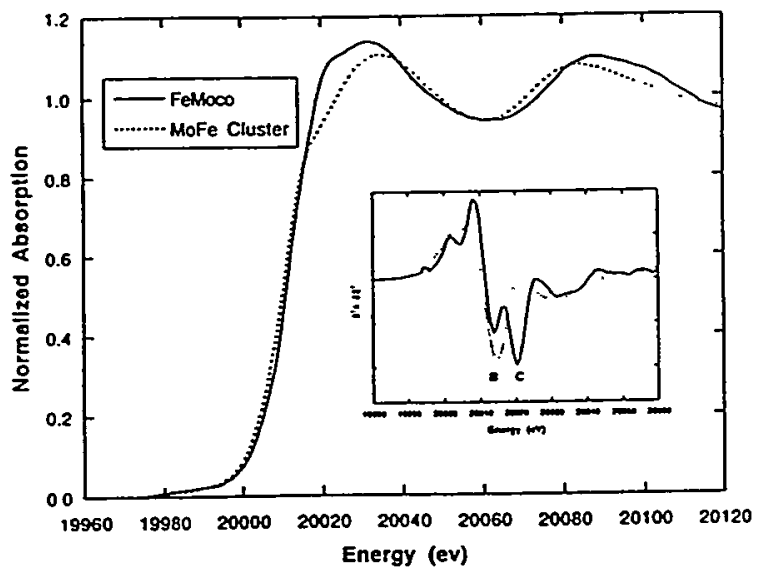

Fig. 2. Comparison of Mo K X-ray absorption edges for isolated FeMo cofactor and MoFe cluster.

Because R-homocitrate is a bidentate ligand to Mo in FeMoco, its removal would require either that the Mo atom adopt a different geometry or, that it be replaced by some anions in solution. To examine the Mo environment in MoFe cluster further we have therefore performed X-ray absorption spectroscopy experiments at the Mo $\mathrm{K}$ edge. Fig. 2 shows a comparison of the
Mo K X-ray absorption edge of FeMoco and MoFe cluster.

In general, the position of this absorption edge is influenced by several factors, including the oxidation state of the Mo, the electron donating character of the ligands and the number of such ligands. As shown in Fig. 2 the position of the absorption edge of the Mo atom in both FeMoco and MoFe cluster are essentially the same. This strongly suggests that the two species have qualitatively similar Mo oxidation states and ligand environments. As also shown in Fig. 2, for the FeMoco there is, however, a difference in intensity in the transition at around $20015 \mathrm{eV}$ which is decrease in the MoFe cluster spectrum.

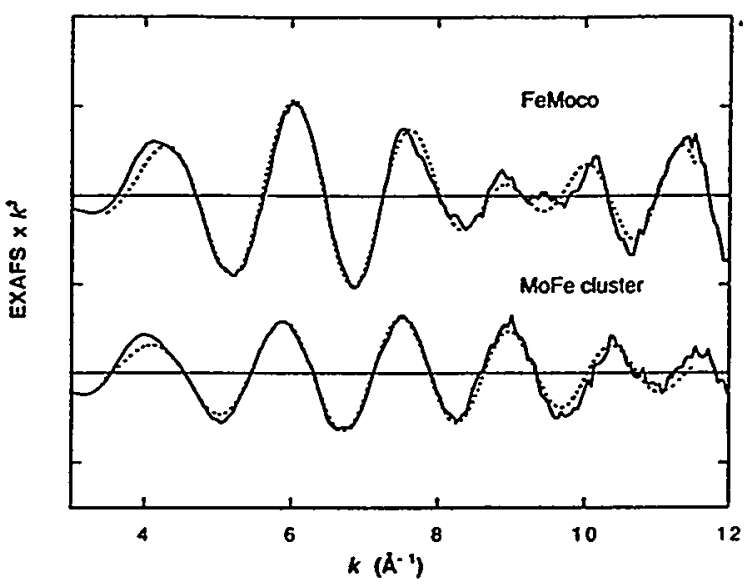

Fig. 3. EXAFS spectra for Mo K-edge data for FeMo cofactor in NMF and MoFe cluster in MEK. The total signal from the best fit which included Mo-O, Mo-S, and Mo-Fe contribution is shown (...) compared with the experimental signal ( $ـ$ )

A comparison of the second derivative for FeMoco and $\mathrm{MoFe}$ cluster edges is even more informative (inset, Fig. 2). Based on our previous X-ray absorption edge studies of synthetic Fe-Mo-S clusters ${ }^{12}$, the features labeled " $B$ " and " $C$ " in this region involve transitions which are indicative of structural as well as electronic properties. Tetrahedral or distorted $\mathrm{C}_{2 \mathrm{v}}$ Mo structure typically have a zero point near $20004 \mathrm{eV}$, which corresponds to the inflection point of a lower energy shoulder, between the preedge and the principal absorption edge discontinuity. Associated with this zero point-is a distinct minimum, around which the shape of the second derivative is relatively symmetric, exhibiting no noticeable structure. As is the case for the FeMoco, this feature is clearly absent in the edge for the MoFe cluster leading to the conclusion that the Mo coordination is definitely not tetrahedral and is likely to 
be pseudo-octahedral. Finally, the data shown in the inset to Fig. 2 also establish that like the FeMoco, the Mo atom in MoFe does not have any double-bonded oxygen or sulfur coordination.

Analysis of the EXAFS region of the spectra provides quantitative information about the number, type and distance of atoms surrounding the Mo atom. Fig. 3 compares the EXAFS and best fits for FeMoco and MoFe cluster. Fourier transforms of the experimental data are shown in Fig. 4. Visual inspection of the data in these two Figures reveals immediately that significant changes to the local environment of the Mo site have occurred. Quantitative EXAFS analysis by curve fitting indicates $3 \mathrm{~S}, 1-2 \mathrm{Fe}$, and 1-2 N/O surround the Mo in the MoFe cluster. This compares with the 3S, 3Fe and 3N/O for FeMoco ${ }^{2}$. Thus, the number of $\mathrm{Fe}$ atoms and the number of $\mathrm{O} / \mathrm{N}$ atoms in the first shell of Mo in MoFe cluster is at least one less than that of FeMoco. The Mo-Fe and Mo-N/O distances are similar to those found in FeMoco, however, the Mo$S$ distance in MoFe cluster is $2.43 \AA$, which is somewhat longer than the $2.36 \AA$ distance in FeMoco.

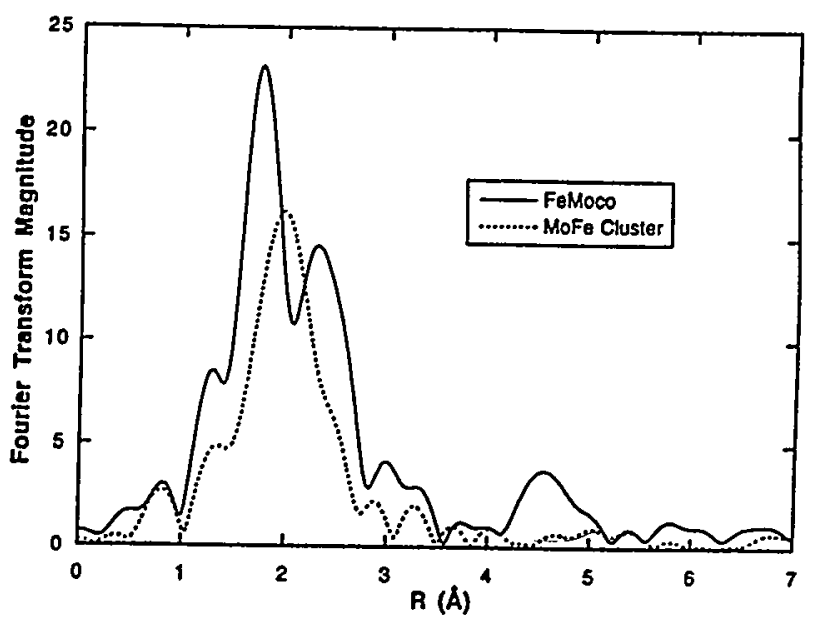

Fig. 4. Comparison of Fourier transforms of the Mo Kedge EXAFS data ( $k$-range of 3.0-12.0 $\AA^{-1}$, no phase correction applied) for isolated FeMo cofactor and the $\mathrm{MoFe}$ cluster. For FeMo cofactor, the lower $\mathrm{R}$ region is dominated by two principal features deriving from the three Mo-S and the three Mo-Fe interactions within the $\mathrm{MoFe}_{3} \mathrm{~S}_{4}$ subcluster.

As shown in Fig. 4 the Fourier transforms of the Mo EXAFS data also give information about the long range order in the cluster. The FeMoco Fourier transform data clearly show a long distance feature which previous analysis has demonstrated arises from the long distance shell of three $\mathrm{Fe}$ atoms in the $\mathrm{Fe}_{4} \mathrm{~S}_{3}$ subcluster that are $5.1 \AA$ from $\mathrm{Mo}^{13}$.This feature is clearly absent in the MoFe cluster data showing a significant perturbation of the long range order of that cluster relative to the FeMoco.

In summary, X-ray absorption spectroscopy experiments show that although the Mo atom in MoFe cluster retains its pseudo-octahedral geometry, its first coordination shell has one less $\mathrm{Fe}$ atom than that of FeMo cofactor and there has been a significant change in the long range order of the cluster.

\section{Acknowledgment}

This research was supported by NSF CHE-91-21576 and NIH RR-01209 (to KOH) and NIH GM-43144 (to BKB). EXAFS data were collected at SSRL, which is supported by the US DOE, Office of Basic Energy Sciences, Divisions of Chemical and Materials Sciences. SSRL is also supported by the NIH, NCRR BRTP program and by the US DOE Office of Health and Environmental Research.

\section{References}

(1) Shah, V. K.; Brill, W. J. Proc. Natl. Acad. Sci. U.S.A. 1977, 74, 3249-3253.

(2) Conradson, S. D.; Burgess, B. K.; Newton, W. E.; Mortenson, L. E.; Hodgson, K. O. J. Am. Chem. Soc. 1987, 109, 7507-7515.

(3) Smith, B.E.; Eady, R. R. Eur. J. Biochem. 1992, 205, 1-15.

(4) Burgess, B. K. Chem. Rev. 1990, 90, 1377-1406.

(5) Newton, W. E. Biological Nitrogen Fixation; Chapman and Hall: New York, 1992, pp 877-930.

(6) Smith, B. E.; Lowe, D. J.; Bray, R. C. Biochem. J. 1973, 135, 331-341.

(7) Mortenson, L. E.; Zumft, W. G.; Palmer, G. Biochim. Biophys. Acta. 1973, 292, 422-435.

(8) Orme-Johnson, W. H.; Hamilton, W. D.; Jones, T. L.; Tso, M.-Y.; Burris, R. H.; Shah, V. K.; Brill, W. J. Proc. Natl. Acad. Sci. USA 1972, 69, 31423145.

(9) Shah, V. K.; Brill, W. J. Proc. Natl. Acad. Sci. USA 1981, 78, 3438-3440.

(10) Kim, J.; Rees, D. C. Nature 1992, 360, 553-560.

(11) Ma, L.; Gavini, N.; Liu, H. I.; Hedman, B.; Hodgson, K. O.; Burgess, B. K. J. Biol. Chem. 1994,

(12) Conradson, S. D.; Burgess, B. K.; Newton, W. E.; Hodgson, K. O.; McDonald, J. W.; Rubinson, J. F.; Gheller, S. F.; Mortenson, L. E.; Adams, M. W. W.; Mascharak, P. K.; Armstrong, W. A.; Holm, R. H. J. Am. Chem. Soc. 1985, 107, 79357940.

(13) Liu, H. I.; Filipponi, A.; Gavini, N.; Burgess, B. K.; Hedman, B.; Di Cicco, A.; Natoli, C. R.; Hodgson, K. O. J. Am. Chem. Soc. 1994, 116, 2418-2423. 


\title{
$\mathrm{K} \beta$ Emission Spectroscopy of Iron and Nickel
}

\author{
Clayton R. Randall, ${ }^{1,2}$.Andrew E. Froeschner, ${ }^{2}$ Melissa M. Grush, ${ }^{2}$ Jeffrey A. Moore, ${ }^{1}$ Xin Wang ${ }^{2}$ \\ and Stephen P. Cramer ${ }^{1,2}$
}

Energy and Environment Division, Lawrence Berkeley Laboratory, Berkeley, CA 94720 and Department of Applied Science, University of California, Davis, CA 95616

$\mathrm{X}$-ray absorption spectroscopy (XAS), in particular EXAFS (Extended X-ray Absorption Fine Structure), has been very useful in determining the coordination chemistry of metal centers in a wide variety of materials. ${ }^{1}$ However, one of the limitations of conventional XAS is that the contributions from all of the species of a given element are averaged together in the spectrum. The signals from different species are usually unresolved, making interpretation of individual site structures difficult or impossible. Since many important metalloenzymes contain two or more atoms of a particular transition element, often in different oxidation states and chemical environments, the ability to characterize individual sites would be an important development.

Small but resolvable shifts occur in the fluorescence emission from metals in different oxidation states or spin states. ${ }^{2}$ For example, it has been found that the $\mathrm{K}_{\mathrm{B}}$ features shift significantly between $\mathrm{Mn}(\mathrm{II})$ and $\mathrm{Mn}(\mathrm{III})$, and somewhat less between $\mathrm{Mn}$ (III) and Mn(IV). ${ }^{3}$ By combining highresolution fluorescence detection with high-resolution absorption measurements, two distinct advantages are gained. First, the measurements can be made selectively for a particular oxidation state or spin state by choosing the appropriate region in an emission spectrum. Second, the absorption spectra that can be obtained are sharper than normal Kedge spectra. With the ability to study elements with particular site symmetries or oxidation states in isolation, site- selective spectroscopy could be a powerful new probe of complex systems.

$$
\text { Recently, a spherically bent Si(440) }
$$
spectrograph was used to record the high-resolution $x$-ray fluorescence spectra of a series of manganese compounds. The $\mathrm{KB} 1,3$ feature (stronger main peak) shifts to lower energy with increasing oxidation state, while the KB' feature (weaker satellite) shifts to higher energy and virtually disappears for low-spin $\mathrm{Mn}$ (III) and all Mn(TV) compounds. ${ }^{4}$ We developed the first spectrograph for $\mathrm{Fe} \mathrm{KB}$ fluorescence emission using a spherically bent $\mathrm{Ge}(620)$ crystal, and recorded spindependent XANES (X-ray Absorption Near-Edge Spectroscopy) spectra. The $1 s \rightarrow 3 d$ transition disappears when the $\mathrm{KB}$ ' emission is monitored. 5

Figure 1 shows the $\mathrm{KB}$ emission spectra of iron-containing compounds in various oxidation states. Iron metal can easily be resolved from ferrous and ferric oxide (top). Unfortunately, the spectra of highspin $\mathrm{Fe}$ (II) in $\mathrm{FeO}$ and high-spin $\mathrm{Fe}$ (III) in $\mathrm{Fe}_{2} \mathrm{O}_{3}$ are very similar, and can not readily be resolved from each other. These results have implications in the study of iron corrosion: it will not be difficult to tell by $\mathrm{x}$-ray emission spectroscpy that a sample is corroded, but will be very difficult to tell the chemical state of the corroded material.

We see similar results for dinuclear synthetic complexes of iron (Figure 1, bottom). Again, it would be difficult to resolve the contributions of the highspin iron sites in the diferrous and diferric complexes to the spectrum of a heterogeneous sample. However, 


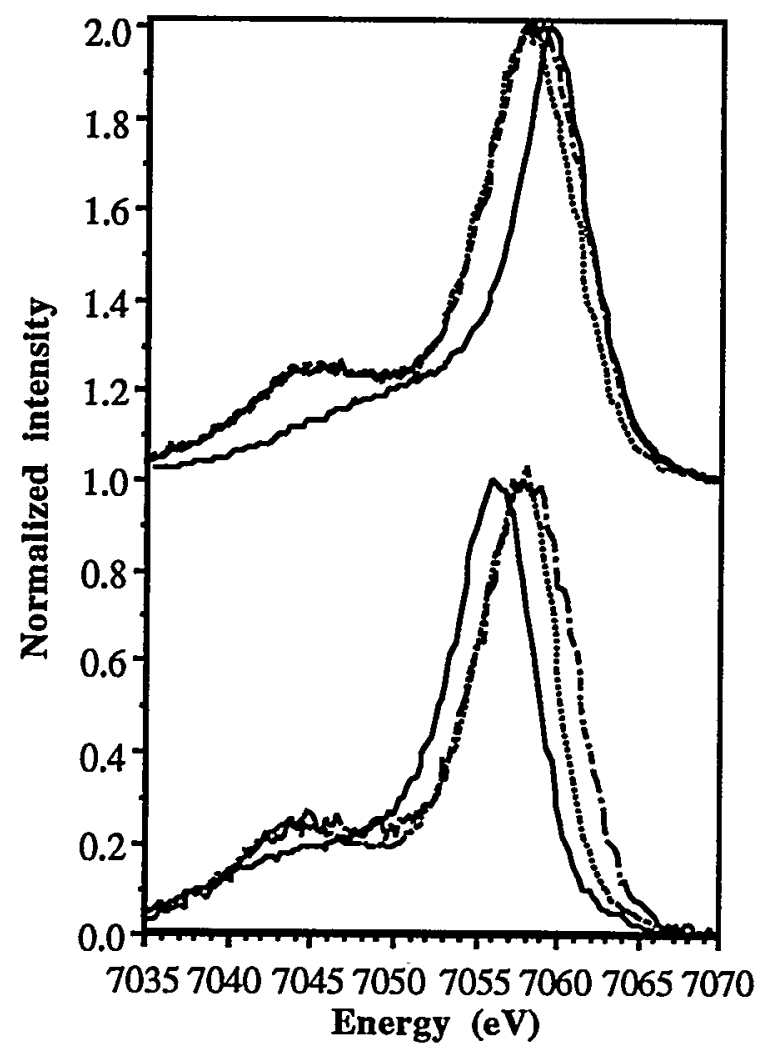

Figure 1. (Top) $\mathrm{K} B$ emission spectra of iron compounds: Fe metal (-), $\mathrm{FeO}(\cdots), \mathrm{Fe}_{2} \mathrm{O}_{3}(--)$ ). (Bottom) $\mathrm{K} B$ emission spectra of dinuclear ironcontaining complexes: $\left[\mathrm{Fe}_{2}(\mathrm{BPMP})(\mathrm{OBz})\right]\left(\mathrm{BPh}_{4}\right)_{2}$ (*.), $\left[\mathrm{Fe}_{2}(\mathrm{~N}-\mathrm{EtHPTB})(\mathrm{OBz})\right]\left(\mathrm{ClO}_{4}\right)_{3}(--)$, $\left[(5-\mathrm{TLA})_{2} \mathrm{Fe}_{2} \mathrm{O}_{2}\right]\left(\mathrm{ClO}_{4}\right)_{3}(-)$.

the $\mathrm{KB}$ emission energy of $\left[(5-\mathrm{TLA})_{2} \mathrm{Fe}_{2} \mathrm{O}_{2}\right]\left(\mathrm{ClO}_{4}\right)_{3}$, a Fe(IV,IV) dimer with an extra electron on the ligands, ${ }^{6}$ is much lower than that of the Fe(II,II) and Fe(III,III) complexes.

This makes it possible for a high-valent iron species in a mixture to be studied in isolation from other low-valent $\left(\mathrm{Fe}^{2+}\right.$ and $\left.\mathrm{Fe}^{3+}\right)$ species. By monitoring the $\mathrm{KB}$ fluorescence emission of the mixture at an energy corresponding mostly to emission from the high-valent species (e.g. at 7054.5 electron volts), one can record site-selective XANES and EXAFS spectra of it. We can then apply the technique of site-selective EXAFS to systems of biological relevance, such as the high-valent iron-containing intermediates of methane monooxygenase.

The hydroxylase component of MMO contains two identical dinuclear non-heme iron sites, and is the locus of substrate hydroxylation. ${ }^{7}$ Lee et al. have studied the reaction kinetics of the reduced $\left(\mathrm{Fe}^{\Pi}{ }_{2}\right) \mathrm{MMO}$ hydroxylase from Methylosinus trichosporium $\mathrm{OB} 3 \mathrm{~b}$ in the presence of component $\mathrm{B} .8$ Among the intermediates observed was one called compound $Q$, a yellow species $\left(\lambda_{\max }=330\right.$ and 430 $\mathrm{nm}$ ) whose rate of formation was not greatly affected by substrates, but whose rate of decay was dependent on both the concentration and type of substrate present. The decay rate also matched the rate of product formation in the active site of MMO hydroxylase, ${ }^{9}$ suggesting that compound $Q$ may be the activated form of the enzyme that leads directly to methane hydroxylation, or its immediate precursor.

Mössbauer spectroscopy of samples prepared by rapid freeze quench techniques showed that in addition to compound $\mathrm{Q}$, two other species were present: unreacted $\mathrm{Fe}^{\mathrm{II}} 2$ hydroxylase, accounting for approximately $30 \%$ of the iron present, and an $\mathrm{Fe} \mathrm{II}_{2}$ species that accounted for approximately $25 \%$ of the iron in the sample. After subtraction of the other two species, only one quadrupole doublet remained, indicating that the two iron atoms of compound $Q$ are in very similar chemical environments. From the isomer shift $(\delta=0.17 \mathrm{~mm} / \mathrm{s})$ and quadrupole splitting $\left(\triangle \mathrm{E}_{\mathrm{Q}}=0.53 \mathrm{~mm} / \mathrm{s}\right)$ of its quadrupole doublet, $\mathrm{Q}$ is very likely at the $\mathrm{Fe}^{\mathrm{IV}} 2$ oxidation level. 8

As an important intermediate in the catalytic cycle of methane monooxygenase and one of very few known high-valent non-heme iron species, it would be very exciting to analyze compound $Q$ by EXAFS spectroscopy, as has been done for the diferrous and 
diferric forms of MMO. 10 Unfortunately, Q can be prepared in at most $45 \%$ yield, making it impractical for sudy by conventional EXAFS methods. However, it may be amenable to study by high-resolution, siteselective EXAFS spectroscopy. By monitoring selectively the Fe(IV) fluorescence emission of the reaction mixture that contains compound $Q$, the other two components of the mixture will be effectively invisible, so that only XAS data from compound Q will be recorded.

Analyzing the EXAFS spectrum of this fascinating new intermediate will help to determine the coordination environment of its dinuclear nonheme iron active site. This will shed some light on how methane monooxygenase accomplishes the energetically difficult process of alkane hydroxylation at ambient temperatures.

The $K \beta$ spectroscopy of nickel has been performed for the last 35 years. ${ }^{11}$ But with the advent of synchrotron light sources, it is now possible to record data of unprecedented quality. We have developed a spherically bent $\mathrm{Ge}(642)$ spectrograph in order to record high-resolution $\mathrm{KB}$ emission spectra of high-spin and low-spin nickel(II) complexes. Our preliminary data, some of which is shown in Figure 2, suggests that it will be possible to resolve high-spin $\mathrm{Ni}$ (II) sites from low-spin $\mathrm{Ni}$ (II) sites, but the reverse will be difficult. Note the difference of approximately

1. X-ray Absorption: Principles, Applications, Techniques of EXAFS, SEXAFS, and XANES, Konigsberger, D. C.; Prins, R., Eds., Wiley: New York, 1988.

2. Ekstig, B.; Källne, E.; Noreland, E.; Manne, R. Phys. Scripta 1970, 2, 38-44.
$1 \mathrm{eV}$ in the $\mathrm{KB}$ emission energies and that the highspin complex has a much larger $\mathrm{KB}^{\prime}$ feature.

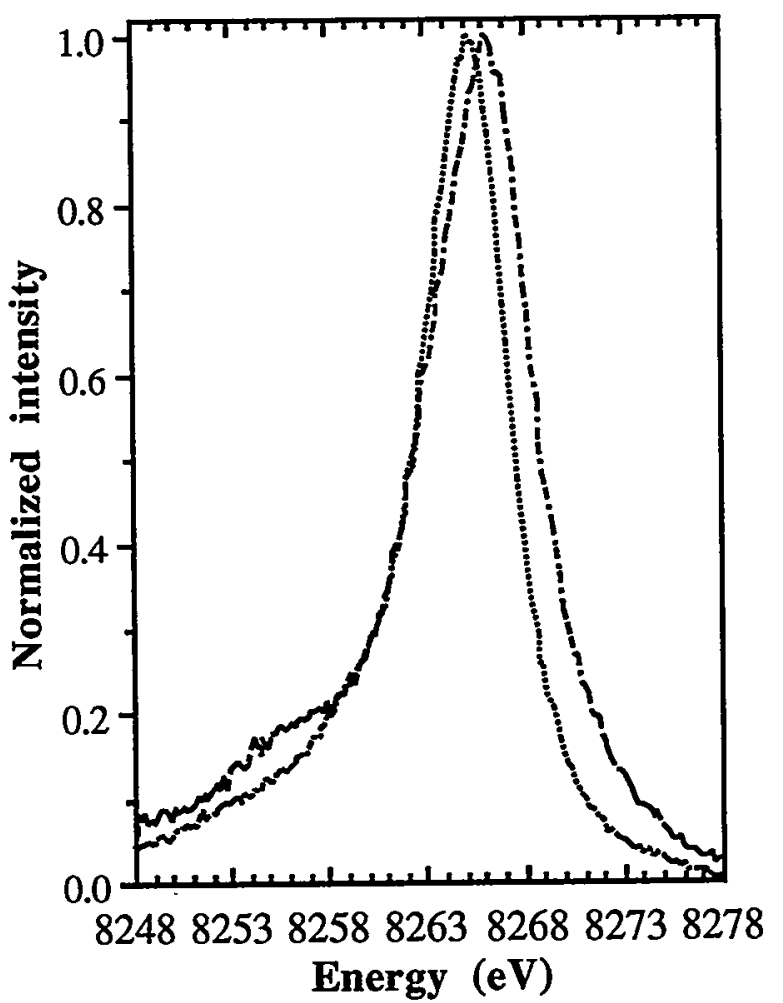

Figure 2. Preliminary $\mathrm{KB}$ emission spectra of highspin trigonal bipyramidal $\mathrm{Ni}(\mathrm{II})$ complex $(-\cdot)$ and lowspin square planar $\mathrm{Ni}(\mathrm{II})$ complex (․).

This work is supported by the Department of Energy, Office of Health and Environmental Research, and the National Institutes of Health (GM-48145).

3. Peng, G.; de Groot, F. M. F.; Hämäläinen, K.; Moore, J. A.; Wang, X.; Grush, M. M.; Hastings, J. B.; Siddons, D. P.; Armstrong, W. H.; Mullins, O. C.; Cramer, S. P. J. Am. Chem. Soc. 1994, 116, 2914-2920.

4. Hämăläinen, K.; Kao, C.-C.; Hastings, J. B.; Siddons, D. P.; Berman, L. E.; Stojanoff, V.; 
Cramer, S. P. Phys. Rev. B 1992, 46, 14274-

14277.

5. Peng, G.; Wang, X.; Randall, C. R.; Moore, J. A.;

Cramer, S. P. Appl. Phys. Lett., 1994, 65, 25272529.

6. Dong, Y.; Fujii, H.; Pan, G.; Randall, C. R.;

Wilkinson, E. C.; Que, L., Jr.; Fox, B. G.;

Kauffmann, K.; Münck, E. J. Am. Chem. Soc., in press.

7. a) Fox, B. G., Froland, W. A., Dege, J. E. and Lipscomb, J. D. J. Biol. Chem., 1989, 264, 1002310033.

b) Froland, W. A., Anderson, K. K., Lee, S.-K., Liu, Y. and Lipscomb, J. D. J. Biol. Chem., 1992, 267, 17588-17597.

8. Lee, S.-K., Nesheim, J. C. and Lipscomb, J. D. J. Biol. Chem., in press.

9. Lee, S.-K., Fox, B. G., Froland, W. A., Lipscomb, J. D. and Münck, E. J. Am. Chem. Soc. 1993, 115, 6450-6451.

10. DeWitt, J. G., Bentsen, J. G., Rosenzweig, A. C., Hedman, B., Green, J., Pilkington, S., Papaefthymiou, G. C., Dalton, H., Hodgson, K. O. and Lippard, S. J. J. Am. Chem. Soc. 1991, 113, 9219-9235.

11. Tsutsumi, K. J. Phys. Soc. Jpn. 1959, 14, 16961706. 


\title{
Site Selective and Spin-Polarized X-ray Fluorescence Spectroscopy
}

\author{
Xin Wang, Clay Randell, Steve Cramer \\ Dept. of Applied Science, UC Davis, Davis, CA 95616
}

$\mathrm{X}$-ray absorption spectroscopy is now a popular tool for elcctronic and geometrical structure determination. A strength of this technique is it's sensitivity to all different species of the same element; which also limit it from interpretation of mixed valence and spin systems. The chemical sensitivity of $\mathrm{K} \beta$ have been known for many years, recent development of intense synchrotron makes $\mathrm{K} \beta$ detection possible for site-selective and spinpolarized $\mathrm{x}$-ray absorptions.

During our last run at SSRL beamline 6-2, we continucd our high resolution-fluorescence spectroscopic study of different Fe compounds, as models to demonstrate the fcasibility of site-selective Fe EXAFS spectroscopy. Using an array of three spherically bent $\mathrm{Ge}(620)$ spectrometers, we recorded $K \beta$ emission spectra of high spin ferric (FcOEPCl), low spin ferric (FeOEPIm2 $\mathrm{Cl}$ ) as models for heme proteins, and $\mathrm{K} 4(\mathrm{Fe}(\mathrm{CN}) 6)$, a pure $\mathrm{Fc}(\mathrm{II})$ low spin compound, Fe2O3, a pure Fe(III) high spin compound with octahedral symmetry, and prussian blue $\mathrm{Fe} 4(\mathrm{Fe}(\mathrm{CN}) 6) 3$, a mixed-valence compound with low spin Fe(II) and high spin Fe(III).

The $\mathrm{K} \beta$ emission spectra for both high spin $\mathrm{Fe}(\mathrm{OEP}) \mathrm{Cl}$ and low spin $\mathrm{Fe}(\mathrm{OEP}) \mathrm{Im}_{2} \mathrm{Cl}$, excited using $7200 \mathrm{cV}$ synchrotron radiation, are shown in Figurc 1 . For the high spin complex, the spectrum is split into two regions. The main pcak (known as $K \beta_{13}$ ) is found at around $7058 \mathrm{eV}$, while the satcllite $\left(\mathrm{K} \beta^{\prime}\right)$ is at $15 \mathrm{eV}$ lower energy side. For the low-spin complex, the centroid of the $K \beta_{1,3}$ pcak is shifted $\sim 0.7 \mathrm{eV}$ to the lower encrgy as comparcd to the high spin complex, and there is also very little $K \beta^{\prime}$ intensity. Similar results were shown previously for $\mathrm{Fe}$, although with lower resolution.

The spin-polarized $X$-ray excitation of high spin $\mathrm{Fe}(\mathrm{OEP}) \mathrm{Cl}$ are shown in Figure 2. The spin-down excitation spectrum was taken by setting the analyzer crystal at $7059 \mathrm{cV}$, the peak centroid of $\mathrm{K} \beta_{1,3}$, while the spin-up spectrum was selected at the emission energy around $7044 \mathrm{eV}$. The spin-down spcctrum does have a airly strong pre-edge pcak around $7112 \mathrm{cV}$ for the five coordinate complex, which is assigned as $1 \mathrm{~s}->3 \mathrm{~d}$

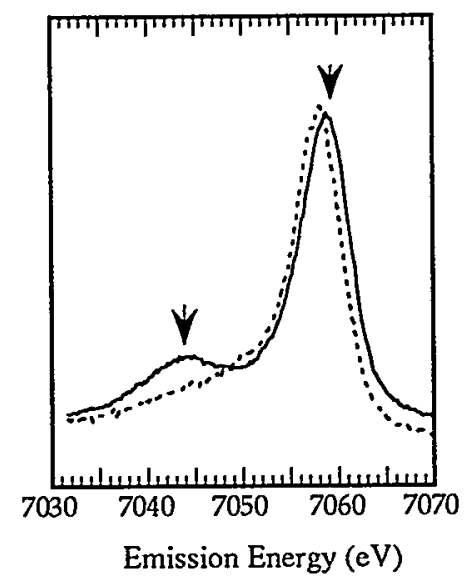

Fig.1 $\mathrm{K} \beta$ emission spectra for both high spin $\mathrm{Fe}(\mathrm{OEP}) \mathrm{Cl}$ (solid) and low spin $\mathrm{Fe}(\mathrm{OEP}) \mathrm{Im}_{2} \mathrm{Cl}$ (dotted).

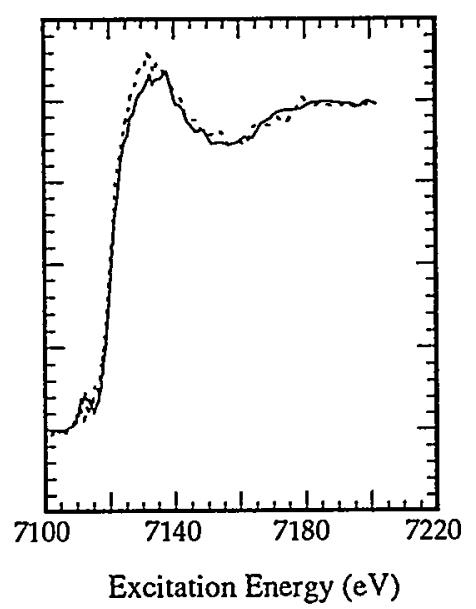

Fig.2 Spin-polarized X-ray excitation of high spin $\mathrm{Fe}(\mathrm{OEP}) \mathrm{Cl}$, monitoring $7059 \mathrm{eV}$ (solid) or 7044 eV(dotted).

transition. This dipole forbidden transition is mainly arise from the iron $4 \mathrm{p}$ atomic orbitals mixing into the predominantly iron 3d orbitals, as well as small part of quadruple transition. Despite the complicated nature of is $>3 \mathrm{~d}$ transitions, the spin selection rule still hold. When there is mainly spin down transition, only spin down $3 \mathrm{~d}$ holes can be reached, which are available for high spin since only the five spin up holes are 
occupied. The broadening of the pre-edge peak could be explained as both $1 \mathrm{~s}->12 \mathrm{~g}$ and $1 \mathrm{~s}->\mathrm{eg}$ transitions are possible. When probing at $7044 \mathrm{eV}$, only spin up transition is monitored, there is no possibility for 1s$>3 \mathrm{~d}$ transitions since all 5 up orbitals are occupied.

The $\mathrm{K} \beta$ emission spectra of prussian blue and two pure compounds are shown in Fig. 3 (solid for prussian blue; dashed line for two pure compounds). The $\mathrm{K} \beta$ peak shifts by $1.2 \mathrm{eV}$ between low spin $\mathrm{Fe}(\mathrm{II})$ and high spin Fe(III). The prussian blue emission spectrum is almost identical an average of the two pure components. By selecting the favorable emission energy for each $\mathrm{Fe}$ site in prussian blue, as indicated by the arrow in Fig. 1 , site sclective XANES were obtained, as shown in Fig. 2. Compared with pure ferric high spin (upper pancl) and ferrous low spin (lower panel) excitation edge spectra, Fe(III) site XANES of prussian blue shows a striking similarity with the pure compound, as well as the Fe(II) site. The calibration between scans was checked with the simultaneous measurement of (NEt4)[FeCl4] by aligning the preedge peak to 7113.0 eV.

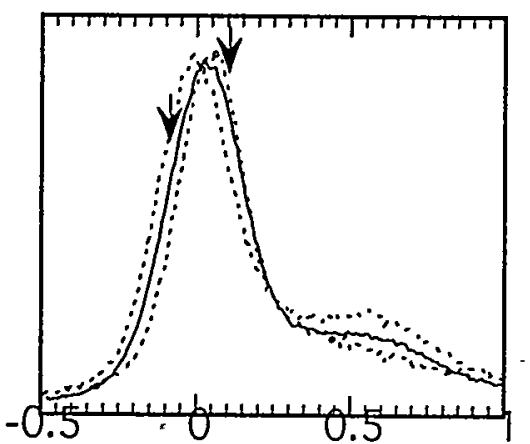

Fig. $3 \mathrm{~K} \beta$ emission spectra of prussian blue(solid), $\mathrm{K} 4 \mathrm{Fe}(\mathrm{CN}) 6$ (dotted) and $\mathrm{Fe} 2 \mathrm{O} 3$ (dotted).

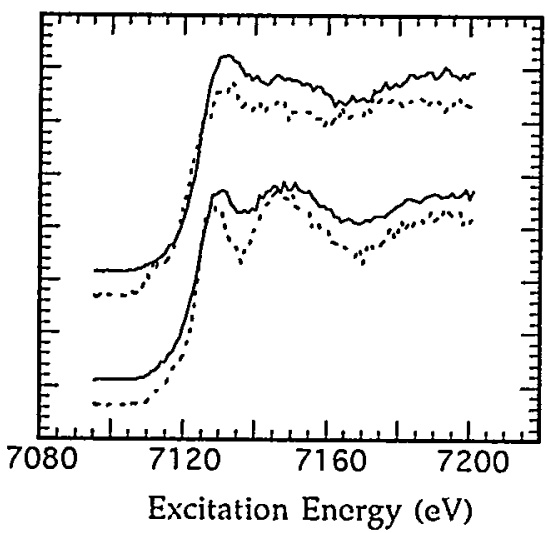

Fig. 4 Site selective XANES of prussian blue (solid), $\mathrm{K} 4 \mathrm{Fc}$ (CN)6 (lower panel, dotted) and $\mathrm{Fc} 2 \mathrm{O} 3$ (upper panel, dotted).

The first site selective EXAFS of an Fe compound are shown in Fig. 5. The data werc smoothed over a $0.2 \AA^{-1}$ interval and a Fourier transform was performed over to
$2-10 \AA^{-1}$ in Fig. 6. From crystal structure and neutron diffraction data, it's known that in prussian blue, the $\mathrm{Fe}(\mathrm{II})-\mathrm{C}$ bond length is $1.92 \AA$, while $\mathrm{Fe}(\mathrm{III})-\mathrm{N}$ is $2.01 \AA$. This bond length difference is qualitatively shown in the Fourier transform. We are currently continuing our data analysis on these spectra. Along with our Mn site selective EXAFS results, this successful demonstration again encourages us to begin application to metalloproteins. With recent improvement on crystal bender and vacuum tubes, we will have even higher efficiency in our spectrometer when we apply this method to some complex proteins like cytochrome oxidase.

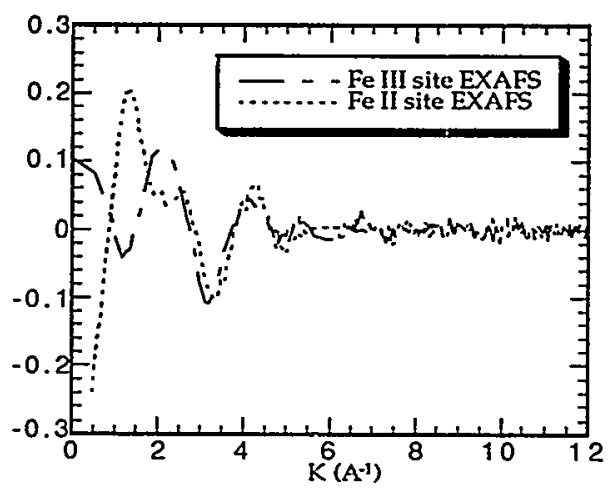

Fig. 5 Site-selective EXAFS of Prussian Blue.

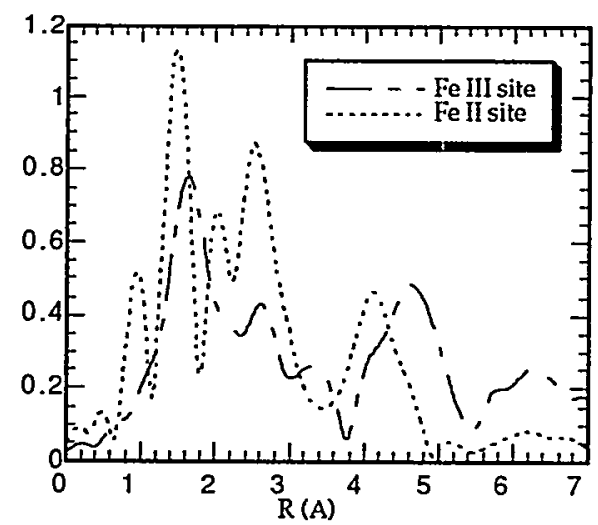

Fig. 6 Fourior transform of site selective EXAFS of Prussian blue.

Other encouraging results came from a rare earth element. We recorded the first Gd L $\beta$ emission and excitation spectra (Fig. 7 and Fig 8) using the same $\mathrm{Ge}(620)$ spectrometer. Since the main line and satellite are spin-polarized, the same as $K \beta$ for transition metals, it is possible to get spin-polarized XANES and EXAFS on this magnetically interesting element, using a higher resolution beamline as an excitation source. . 


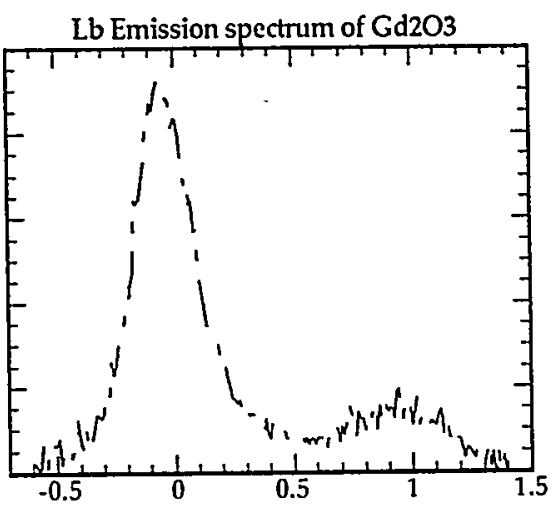

Fig. 7 L $\beta$ Emission spectra of $\mathrm{Gd} 2 \mathrm{O} 3$.

Excitation spectrum of $\mathrm{Gd} 2 \mathrm{O} 3$

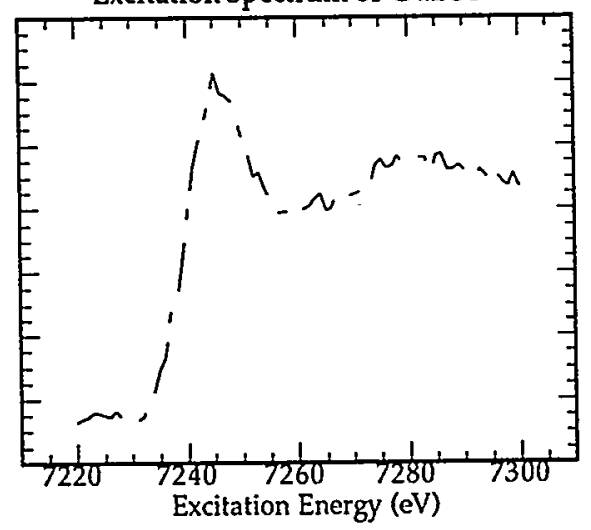

Fig.8 LIII edge absorption of Gd2O3. 


\title{
Determination of Iron-Ligand Bond Lengths in Horse Heart Met-Myoglobin, Deoxy- Myoglobin and Nitrosylmyoglobin using Multiple-Scattering EXAFS
}

\author{
Robert S. Armstrong, ${ }^{1}$ Paul J. Ellis, ${ }^{1}$ Hans C. Freeman, ${ }^{1}$ Britt Hedman, ${ }^{2}$ Keith O. Hodgson, ${ }^{2,3}$ \\ Peter A. Lay, ${ }^{1}$ and Anne M. Rich ${ }^{1}$ \\ ${ }^{1}$ School of Chemistry, University of Sydney, Sydney, NSW 2006, A ustralia. \\ 2 Stanford Synchrotron Radiation Laboratory, Stanford, CA 94309, USA \\ ${ }^{3}$ Department of Chemistry, Stanford University, Stanford, CA 94305, USA
}

Nitric oxide (NO) has recently been found to have diverse biological roles including being a neurotransmitter $[1,2]$. This very unstable molecule is thought to act as a neurotransmitter by binding to hemeproteins. However, little detailed structural information on the binding of NO to hemeproteins is available.

Here we report EXAFS results of experiments on frozen aqueous solutions of myoglobin $(\mathrm{Mb})$ and its NO adducts in both the $\mathrm{Fe}$ (III) and $\mathrm{Fe}$ (II) oxidation states at $10 \mathrm{~K}$.

Horse heart myoglobin was obtained from Sigma, and was further purified by chromatography on a Whatman $\mathrm{CM}-32$ column at $4^{\circ} \mathrm{C}$ using a phosphate buffer solution ( $5 \mathrm{mM} \mathrm{NaH}_{2} \mathrm{PO}_{4}+6.6$ $\mathrm{mM} \mathrm{Na} \mathrm{HPO}_{4}, \mathrm{pH} 7$ ) as an eluent. The myoglobin thus obtained was dialysed against distilled water and then concentrated by ultrafiltration using Amicon ultrafiltration cells. The concentrations of myoglobin were estimated spectroscopically for metmyoglobin in $0.1 \mathrm{M}$ phosphate buffer ( $\mathrm{pH} 6.8$ ) using the following molar absorptivities: $\varepsilon_{280}=3.37 \times 10^{4}$ $\mathrm{M}^{-1} \mathrm{~cm}^{-1} ; \varepsilon_{408}=1.6 \times 10^{5} \mathrm{M}^{-1} \mathrm{~cm}^{-1}$; and $\varepsilon_{505}=1.02$ $x 10^{4} \mathrm{M}^{-1} \mathrm{~cm}^{-1}$ [3]. Solutions were freshly prepared, then syringed into $140-\mu \mathrm{L}$ Lucite EXAFS cells ( $23 \mathrm{x}$ $2 \times 3 \mathrm{~mm}$ ) with $37-\mu \mathrm{m}$ Kapton windows in a nitrogen-filled glove bag. The samples were frozen in liquid nitrogen/hexane. Glycerol (A.R. grade) was used as the glassing agent. The presence of $40 \%$ glycerol in the protein solution was the minimum concentration required to form a homogeneous glass upon freezing. Deoxy-Mb was prepared by reduction of met-Mb with aqueous sodium dithionite. $\mathrm{Mb}(\mathrm{II})$-NO was then prepared by passing $\mathrm{NO}_{(\mathrm{g})}$ over the deoxy-Mb solution. The resulting concentrations were $4.1 \mathrm{mM}, 3.2 \mathrm{mM}$, and $4.0 \mathrm{mM}$ for met-Mb, deoxy-Mb, and $\mathrm{Mb}$ (II)-NO, respectively.

XAS spectra were recorded at the Stanford Synchrotron Radiation Laboratory on the unfocussed beamline 7-3 $(3 \mathrm{GeV}, 50-99 \mathrm{~mA})$. The radiation was monochromatised using a $\mathrm{Si}(220)$ double-crystal monochromator detuned $50 \%$ at $7993 \mathrm{eV}$ to minimise harmonic contamination. An Oxford Instruments continuous-flow liquid helium CF 1208 cryostat was used to maintain a constant temperature of $10 \mathrm{~K}$. The XAS spectra were calibrated using an internal Fe foil standard, assigning the first inflection point to $7111.2 \mathrm{eV}$. Data were collected as fluorescence spectra using a 13-element Ge array detector [4].

The model-fitting calculations were made by means of an in-house program XFIT written by Paul Ellis at the University of Sydney. XFIT incorporates $a b$ initio calculations of the EXAFS using FEFF4.06 (single-scattering) [5] and FEFF6.01 (multiplescattering) [6]. The molecular structures were drawn using the program, MDMovie, written by Dr Jerry $P$. Greenberg, San Diego Supercomputer Center.

The EXAFS structures of met-Mb and deoxy$\mathrm{Mb}$ have been refined using both single-scattering and multiple-scattering analyses (with the aid of published crystallography $[7,8]$ ). The singlescattering analyses provided bond lengths that were consistent with the X-ray data and located 1st shell atoms consisting of pyrrole $N\left(N_{p}\right)$, imidazole $N\left(N_{e}\right)$ and $O$ (in the case of met-Mb) or nitrosyl $N\left(N_{N O}\right)$ in $\mathrm{Mb}(\mathrm{II})-\mathrm{NO}$. The structures were then refined by multiple-scattering, using crystallographically determined coordinates for the porphyrin ligand. The results are contained in Table 1 . The resulting values of $R$ (the difference between the observed and calculated EXAFS) for met-Mb and deoxy-Mb were 14 and $15 \%$, respectively. The best fits obtained from multiple-scattering analyses had significant contributions from all of the porphyrin and imidazole atoms in the coordination environment. The bond distances and angles are consistent with those found for sperm whale $\mathrm{Mb}$ using $\mathrm{X}$-ray crystallography $[7,8]$. The Fe-ligand bond lengths determined from the multiple-scattering analyses probably have a higher precision than those obtained from crystallography.

Table 1. Comparison of Multiple Scattering EXAFS Results with Crystal Structure Data

\begin{tabular}{|c|c|c|l|}
\hline Species & Scatterer & EXAFS $^{\dagger}$ & Crystal $^{\ddagger}$ \\
\hline Met-Mb & $N_{P}(\AA)$ & $2.06_{8}$ & $2.04^{\mathrm{a}}$ \\
& $\mathrm{N}_{\varepsilon}$ & $2.20_{1}$ & 2.13 \\
& $\mathrm{O}$ & $1.98_{4}$ & 2.00 \\
& & & (constrained) \\
\hline Deoxy-Mb & $\mathrm{N}_{\mathrm{P}}(\AA)$ & $2.06_{2}$ & $2.06^{\mathrm{b}}$ \\
& $\mathrm{N}_{\varepsilon}$ & $2.16_{8}$ & 2.1 \\
\hline
\end{tabular}

EXAFS data from horse heart myoglobin;

$¥$ Crystal structure data from sperm whale myoglobin;

${ }^{a}$ Ref. $7 ;{ }^{b}$ Ref. 8 
Multiple-scattering has proven to be very accurate in determining the $\mathrm{Fe}-\mathrm{N}-\mathrm{O}$ angle in $\mathrm{FeNO}$ complexes [9]. The Fe-scatterer coordinates from the deoxy-Mb refinement were used as starting distances in the multiple-scattering analysis of $\mathrm{Mb}(\mathrm{II})-\mathrm{NO}$. Our analysis of the EXAFS data $(\mathrm{R}=$ $16 \%$ ) of $\mathrm{Mb}$ (II)-NO shows the $\mathrm{Fe}(\mathrm{II})-\mathrm{N}-\mathrm{O}$ is bent with a similar angle to that found in simple $\mathrm{Fe}(\mathrm{II})-\mathrm{N}$ $O$ adducts of Fe porphyrin model complexes (Table 2) $[10,11]$. The structure has also shown that the
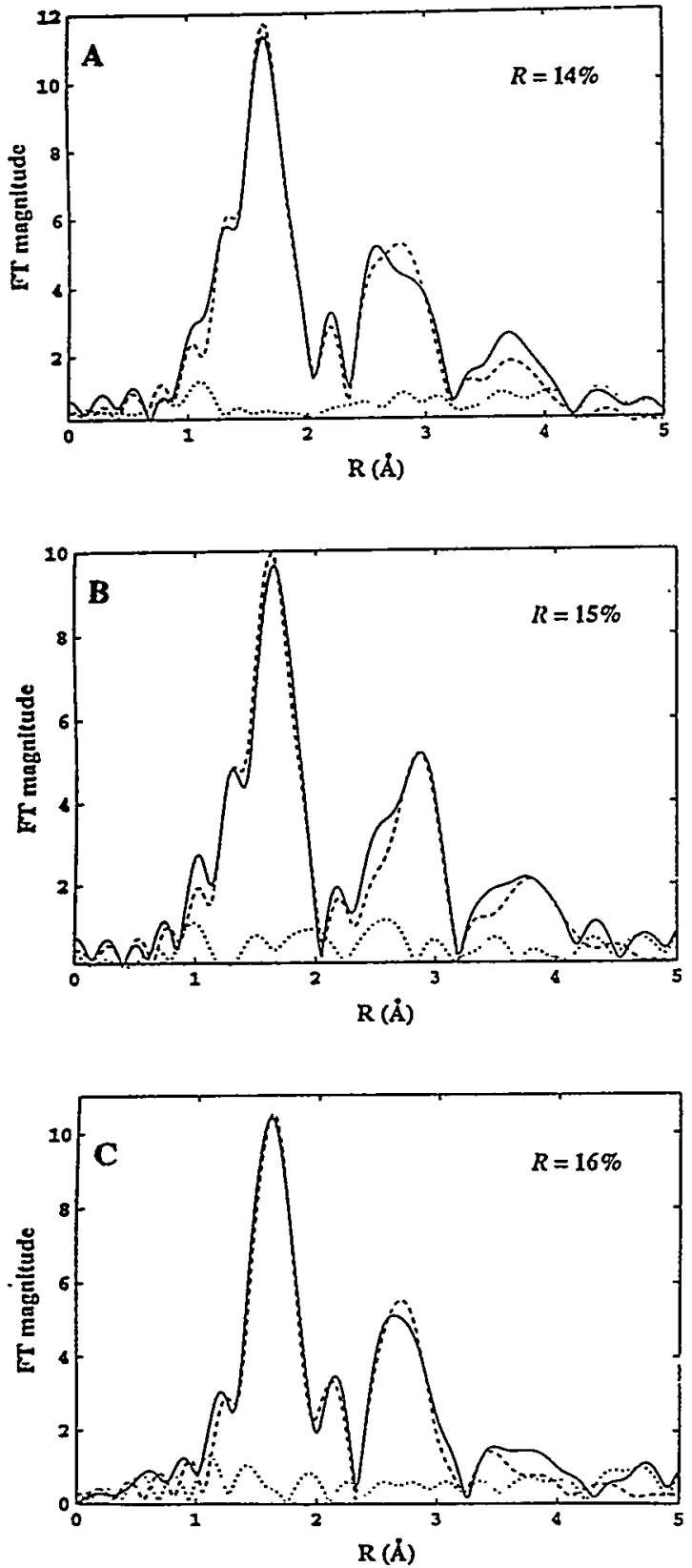

Figure 1. A comparison of the theoretical (- $)$ and experimental $(\longrightarrow$ FT EXAFS of a) met-Mb, b) deoxy-Mb, and c) $\mathrm{Mb}(\mathrm{II})-\mathrm{NO}$, along with the FT of the EXAFS residual (........).
Table 2. Comparison of EXAFS Structure of Mb(II)-NO with X-ray Crystal Structures of Model Compounds

\begin{tabular}{|c|c|c|l|}
\hline Scatterer & $\mathrm{Mb}(\mathrm{II})-\mathrm{NO}$ & $\mathrm{Fe}(\mathrm{TPP}) \mathrm{NO}^{\mathrm{C}}$ & $\begin{array}{l}\text { Fe(TPP)NO } \\
\text {-(1-Melm) }\end{array}$ \\
\hline $\mathrm{N}_{\mathrm{P}}(\AA)$ & $200_{6}$ & $2.001(3)$ & $2.008(15)$ \\
$\mathrm{N}_{\mathrm{c}}$ & $2.52_{2}$ & & $2.180(4)$ \\
$\mathrm{N}_{\mathrm{NO}}$ & $1.75_{2}$ & $1.717(7)$ & $1.743(4)$ \\
$\mathrm{Fe}-\mathrm{N}-\mathrm{O} O$ & 149.6 & $149.2(6)$ & $138(11)$, \\
& & & $142(6)$ \\
\hline
\end{tabular}

'Ref. 10; ${ }^{\mathrm{d}}$ Ref. 11
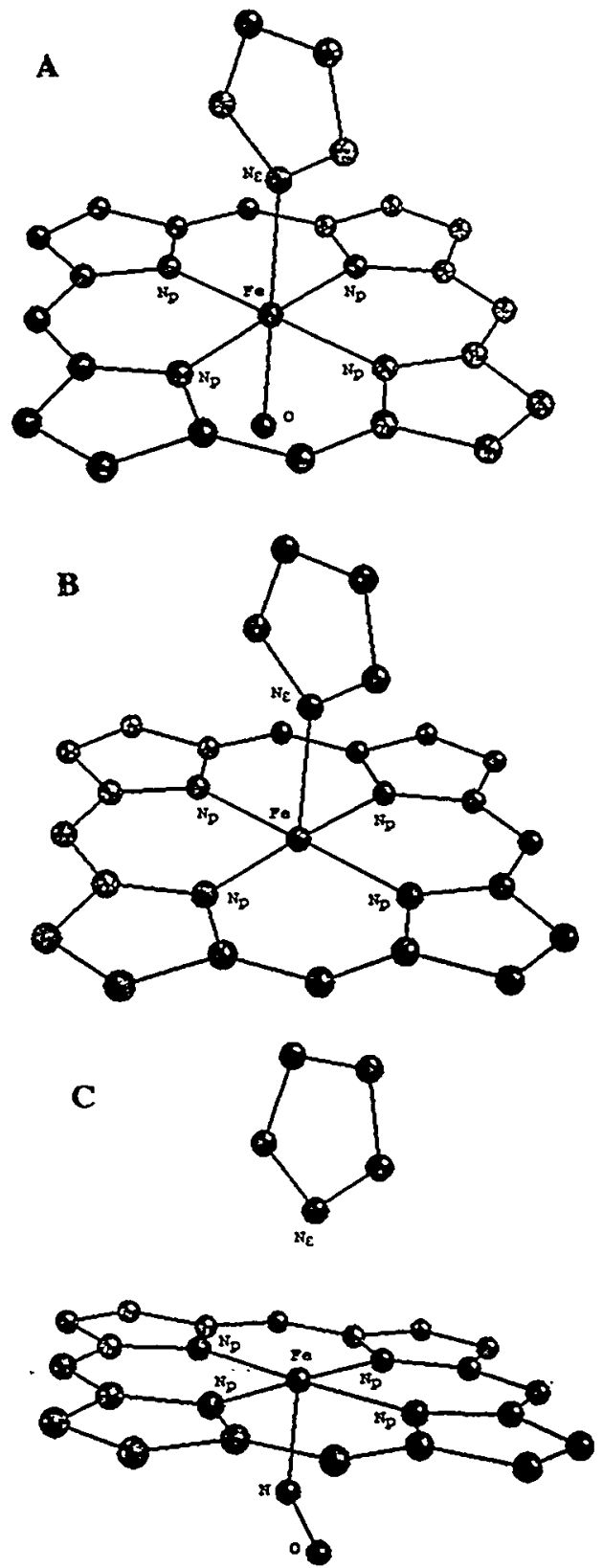

Figure 2. The molecular structures of a) met-Mb, b) deoxy-Mb, and c) $\mathrm{Mb}$ (II)-NO, as determined from the best obtainable EXAFS refinements. 
$\mathrm{Fe}$ (II)-imidazole bond is cleaved (or weakened) on binding of NO. This is important, since in mediating glutamate-stimulated cGMP production, NO may generate an allosteric effect by binding to iron in the heme portion of guanylate cyclase causing a conformational change in the protoheme and possibly untethering it from the protein [12]. The structural information indicates that the best description is consistent with an $\mathrm{Fe}$ (II)-NO electronic structure. Preliminary results with the $\mathrm{Fe}$ (III) adduct indicate a linear $\mathrm{Fe}-\mathrm{N}-\mathrm{O}$ moiety consistent with an $\mathrm{Fe}$ (II)-NO+ electronic structure. These conclusions comply with predictions made from resonance Raman measurements [13].

This work was supported by the Australian Nuclear Science and Technology Organisation. Additional funding was received through an Australian Postgraduate Research Award and the James Kentley Memorial Travelling Scholarship both awarded to Anne Rich. We would like to thank Dr Simon Easterbrook-Smith from the Department of Biochemistry at the University of Sydney for purification of the horse heart myoglobin. We acknowledge the assistance of Robyn Mills from the Sydney Regional Visualisation Laboratory in drawing the molecular structures.

1. A. Verma, D. Hirsch, C. Glatt, G. Ronnett, and S. Snyder, Science, 295, 381 (1993).

2. J. Ribeiro, J. Hazzard, R. Nussenzveig, D. Champagne, and F. Walker, Science, 260 , 539 (1993).

3. Handbook of Biochemistry and Molecular Biology, 3rd Edition, Proteins - Volume II, G. Fasman, Ed., CRC Press, 1976, 477.

4. S. Cramer, O. Tench, M. Yocum, and G. George, Nucl. Instr. Method, A266, 586 (1988).

5. J. Mustre de Leon, J. Rehr, S. Zabinsky, and R. Albers, Phys. Rev. B, 44, 4146 (1991):

6. J. Rehr, R. Albers, and S. Zabinsky, Phys. Rev. Lett, 69, 3397 (1992).

7. T. Takano, J. Mol. Biol., 110, 537 (1977).

8. T. Takano, J. Mol. Biol., 110, 569 (1977).

9. T. Westre, A. Cicco, A. Filipponi, C. Natoli, B. Hedman, E. Solomon, and K. Hodgson, J. Am. Chem. Soc., 116, 6757 (1994).

10. W. Scheidt and M. Frisse, J. Am. Chem. Soc., 97, 17 (1975).

11. W. Scheidt and P. Piciulo, J. Am. Chem. Soc., 98, 1913 (1976).

12. M. Clarke, and J. Gaul, Struct. Bond., 81, 147 (1993).

13. A. M. Rich, Honours Thesis, University of Sydney, 1990. 


\title{
DE NOVO DESIGN OF METALLOPROTEIN METAL SITES
}

\author{
Gregg R. Dieckmann, David L. Tierney, James E. Penner-Hahn, and Vincent L. Pecoraro \\ Department of Chemistry, University of Michigan, Ann Arbor, MI 48109-1055 \\ William F. DeGrado \\ The Dupont-Merck Pharmeceutical Company, PO Box 80328, Wilmington, DE 19880-0328
}

\section{INTRODUCTION}

A fundamental issue in the understanding of metalloprotein structure is the relative importance of the metal's geometric preference vs. the tertiary or quaternary structure inherent in the amino acid sequence of the protein. Examples of both extremes are known. The tertiary structure of plastocyanin generates a distorted tetrahedral binding site for $\mathrm{Cu}$, thus making it efficient in electron transfer by removing the reorganization required in the coordination environment of $\mathrm{Cu}(\mathrm{II})$ upon reduction (1). The zinc finger, at the other extreme, requires $\mathrm{Zn}$ (II) as a template to fold into a functional form. With most metalloproteins falling between these two extremes, deciphering the roles played by protein and metal in determining the final structure is not trivial.

One approach to studying the interplay between metal and protein is the introduction of metal binding sites into de novo designed peptide structures. The use of de novo metallopeptides in the structural and functional modelling of metalloprotein metal sites has many advantages over small molecule models: the peptides are designed to assume well defined tertiary structures in solution, thus providing a molecular scaffolding in which biologically relevant metal binding residues can be introduced; distorted coordination environments, which are difficult to achieve in small, spontaneously assembled complexes, are possible; and these metallopeptides are typically water soluble. This approach, although complementary to one in which metal binding sites are introduced into naturally occuring proteins, has the added versatility that the protein structure is designed, not already defined.

A relatively small number of de novo designed metallopeptides have been reported to date (2). Our approach to metallopeptide design is to introduce a metal binding site into the hydrophobic interior of an $\alpha$-helical coiled coil. This exclusion of solvent or buffer ligation allows us to prepare otherwise difficult to obtain metal coordination geometries and to investigate the ability of our structures to generate potentially charged sites in otherwise hydrophobic environments. Only naturally occuring amino acids or derivatives are utilized in metal ligation. In addition, the metal binding site is designed such that the ligands are donated by all $\alpha$-helices in a non-covalent bundle. With the helices not connected (except through the metal binding site), we can investigate, as desired, the effect on protein stability generated by metal coordination.

The first metal binding site selected to test our approach was $\mathrm{Hg}$ (thiolate)3. The coordination chemistry of $\mathrm{Hg}$ (II), which is dominated by linear, two coordinate thiolate-mercury complexes (3), is few well-characterized mononuclear trigonal thiolate species. The protein structural motif chosen to house our trigonal thiolate binding site was the three $\alpha$-helical bundle. In principle, each helix may provide one thiolate (in the form of Cys) to a trigonal $\mathrm{Hg}$ (II) coordination environment. The preference of $\mathrm{Hg}$ (II) for linear over trigonal coordination thus provides an interesting system in which the relative importance of peptide conformation and metal coordination preference in determining the final metallopeptide structure can bé investigated.

\section{EXPERIMENTAL}

Peptide samples, $4 \mathrm{mM}$ in $\mathrm{Hg}$ with $50 \% \mathrm{v} / \mathrm{v}$ glycerol as glassing agent, were loaded into lucite cuvettes with $6 \mu \mathrm{m}$ kapton windows and flash frozen in liquid nitrogen. X-ray absorption spectra were collected at the Stanford Synchrotron Radiation Laboratory, Beamline VII-3, using a $\mathrm{Si}(220)$ double crystal monochromator that was detuned $50 \%$ for harmonic rejection. Data were measured at $10 \mathrm{~K}$ by fluorescence excitation with a 13 element $\mathrm{Ge}$ detector array, with energy calibration accomplished by simultaneous collection of a $\mathrm{Hg} / \mathrm{Sn}$ amalgam. The inflection point of the amalgam edge was assigned to the $\mathrm{Hg}$ LIII edge at $12284 \mathrm{eV}$. The data were converted to $k$-space using $\mathrm{E}_{0}=12300 \mathrm{eV}$. Windowed (Hg fluorescence) count rates were ca. 3 $\mathrm{kHz}$ at the end of the scan with $15 \mathrm{~s}$ integration times.

Prior to averaging, each individual detector channel was examined for artifacts. Each scan 
consisted of 10-12 useful channels and the resultant EXAFS spectra represent the average of 12 scans per sample. Data reduction followed standard procedures over the energy range $k=1.5$ $14 \AA^{-1}$. The EXAFS data were fit with a nonlinear least-squares algorithm. Amplitude and phase parameters were derived by fitting the EXAFS of $\mathrm{Hg}$ (S-cysteine) 2 for two coordinate $\mathrm{Hg}$, and $\mathrm{Hg}(\mathrm{S} \text {-butyl })_{3}$ for three coordinate $\mathrm{Hg}$. Fits to the peptide data allowed the absorberscatterer bond length, $R_{a b}$, and the Debye-Waller factor, $\sigma$, to vary while holding all other parameters fixed.

\section{RESULTS}

The (S-Cys) 3 coordination environment was engineered into the de novo designed amphiphilic $\alpha$-helical peptide Tri (Figure 1). Tri was based on the sequence of the peptide CoilSer, which has been crystallographically characterized as an up-up-down three-helix bundle. L16C was generated by replacing Leu 16 in Tri with a Cys residue. L16C displays $\mathrm{pH}$ dependent aggregation with 2-helix and 3-helix stoichiometries apparent at $\mathrm{pH} 5.5$ and 8.5 respectively. Each L16C monomer can donate one Cys residue, which is located at an "a" position in the heptad repeat, thus generating a trigonal thiolate metal binding site in the hydrophobic interior of the bundle. The Cys was placed at the center of $\mathrm{L} 16 \mathrm{C}$ so that either possible orientation of the peptides in the bundle (all parallel or up-up-down) should place three Cys in position to bind $\mathrm{Hg}(\mathrm{II})$.
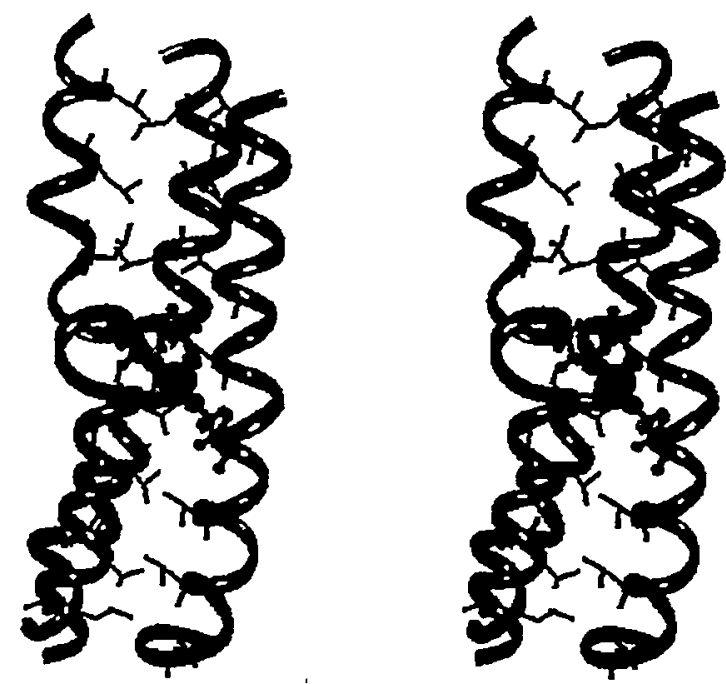

Figure 1: Stereo view of the calculated structure for $\mathrm{Hg}(\mathrm{L} 16 \mathrm{C})_{3}$.

EXAFS data were collected on three samples in order to examine the effect of peptide aggregation tendencies on the $\mathrm{Hg}$ (II) coordination environment. The average bond distances in $\mathrm{Hg}(\mathrm{II})$-thiolate complexes are diagnostic for coordination number, with two coordinate complexes at $2.32 \AA$, three coordinate at $2.43 \AA$ and four coordinate at $2.54 \AA$. The EXAFS spectra are shown in Figure 2. The data for the $1: 2(\mathrm{pH} 8.5)$ and $1: 3(\mathrm{pH}$ 5.5) samples are virtually indistinguishable. However, the 1:3 sample at $\mathrm{pH} 8.5$ shows a rather striking decrease in amplitude. A marked shift to higher frequency is also observed on going from $1: 2 \mathrm{Hg}(\mathrm{II})$ :peptide to $1: 3$ at $\mathrm{pH}$ 8.5. This difference is apparent in the corresponding Fourier transforms (Figure 3). The FTs seem to suggest the presence of two nearly resolved shells.

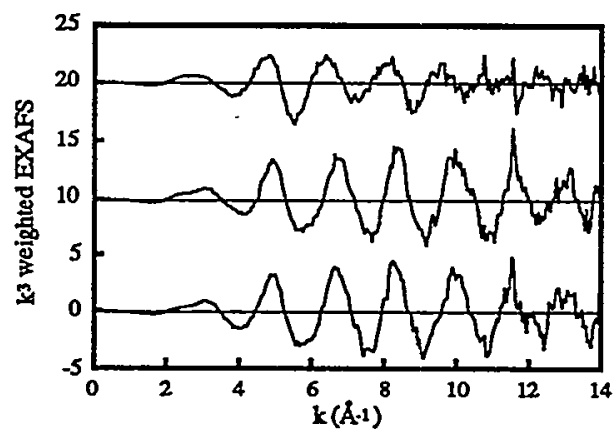

Figure 2 EXAFS data for $\mathrm{Hg}$ peptides. From top: 1:3 Hg:peptide (pH 8.5), 1:2 (pH 8.5), 1:3 (pH 5.5). The curves have been offset for clarity.

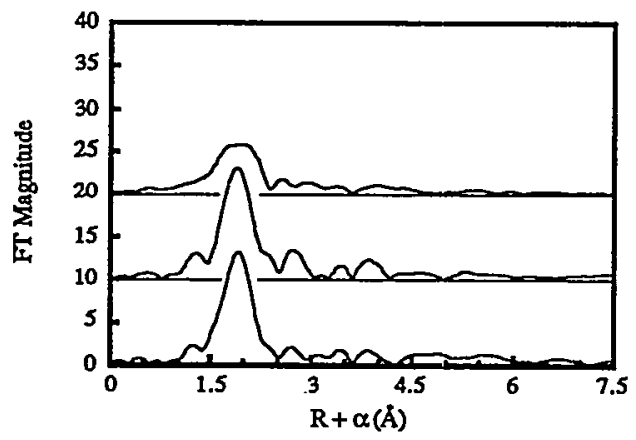

Figure 3 Fourier transforms of $\mathrm{k}^{3}$ weighted EXAFS data. The spectra appear in the same order as in Fig. 2, and also have been offset.

The curve fitting results are summarized in Table 1. Both the 1:2 (pH 8.5) and 1:3 (pH 5.5) data sets give average bond lengths $(2.32 \AA)$ that are consistent with a well ordered, linear twocoordinate mercury (3). The shift in frequency seen for the $1: 3(\mathrm{pH} 8.5)$ sample is reflected in the average $\mathrm{Hg}$-ligand bond length and is 


\begin{tabular}{lcccc} 
Sample & & $\mathrm{R}_{\mathrm{ab}}(\AA)$ & $\sigma^{2}\left(\AA^{2} \times 10^{-3}\right)$ & $\mathrm{F}^{\mathrm{a}}$ \\
\hline $1: 3 \mathrm{Hg}: \mathrm{L16C}$ & $(\mathrm{pH} .5)$ & 2.32 & 0.6 & 0.32 \\
$1: 2$ & $(\mathrm{pH} 8.5)$ & 2.32 & 1.9 & 0.32 \\
$1: 3$ & $(\mathrm{pH} 8.5)$ & 2.41 & 7.7 & 0.24 \\
$\mathrm{a}: \mathrm{F}=\left[\left(\mathrm{k}^{3} \chi_{\text {obs }}\right)^{2}-\left(\mathrm{k}^{3} \chi_{\text {calc }}\right)^{2}\right]^{0.5}$ & &
\end{tabular}

indicative of an increase in $\mathrm{Hg}$ coordination number. The increase of $0.09 \AA$ to $2.41 \AA$ is best described by a change to a three coordinate $\mathrm{Hg}$. The goodness of fit did not increase significantly if nitrogen or oxygen shells were included. It is therefore unlikely that the $\mathrm{Hg}$ (II) is being coordinated by the buffer, $\mathrm{H}_{2} \mathrm{O}$, or a peptide residue other than cysteine. A bond length of $2.41 \AA$ is slightly shorter than that expected for a

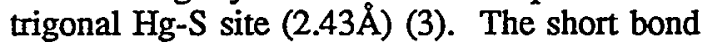
length, in conjunction with the dramatically lower EXAFS amplitude and higher DebyeWaller factor for the 1:3 sample suggest that the $\mathrm{Hg}$ site is distorted from trigonal. Distortion toward a T-shaped site with two short bonds and a long bond would be sufficient to give the observed value of $\sigma^{2}$.

Spectroscopic (4-5) studies have suggested that the selectivity of MerR for $\mathrm{Hg}(\mathrm{II})$ is accomplished by binding one mercuric ion per dimer in a trigonal cysteine environment while other sulfur loving metals adopt a higher coordination number. Previous EXAFS results for HgMerR give an average bond distance of $2.43 \AA$ (4). The difference between $\mathrm{HgMerR}$ and $\mathrm{HgL} 16 \mathrm{C}$ suggest that the $\mathrm{Hg}(\mathrm{II})$ binding site in MerR is more symmetrical than observed for our complex. In future work, we will begin to characterize the metal binding properties of these and similar peptides with other heavy metals, such as Cd(II), $\mathrm{Zn}$ (II) and $\mathrm{Au}(\mathrm{I})$.

\section{REFERENCES}

1. J. M. Guss and H. C. Freeman, J. Mol. Biol. 169, 521 (1983).

2. T. M. Handel, S. A. Williams, W. F. DeGrado, Science 261, 879 (1993); M. R. Ghadiri and M. A. Case, Angew. Chem. Int. Ed. Engl. 32, 1594 (1993); L. Regan and N. D. Clarke, Biochem. 29, 10878 (1990).

3. J. G. Wright, M. J. Natan, F. M. MacDonnell, D. M. Ralston, T. V. O'Halloran, in Progress in Inorganic Chemistry, S. J. Lippard, Ed. (John Wiley \& Sons, Inc., 1990), vol 38.

4. J. G. Wright, H. T. Tsang, J. E. PennerHahn, T. V. O'Halloran, J. Am. Chem. Soc. 112, 2434 (1990).

5. S. P. Watton, J. G. Wright, F. M. MacDonnell, J. W. Bryson, T. V. O'Halloran, J. Am. Chem. Soc. 112, 2824 (1990).

\section{ACKNOWLEDGEMENTS}

This work has been supported in part by grants from the NIH (GM-38049 to JEPH). Spectra were measured in part at SSRL, which is supported by the US DOE and by the NIH, Biotechnology Resource Program, Division of Research Resources. 


\title{
Histone Variants H2A.X and H2B.0 Induce Unfolding of 195bp DNA'from Nucleosomes
}

\author{
Joe M. Gatewood, Brian S. Imai, and Joe Siino \\ Life Sciences Division, Los Alamos National Laboratory \\ and \\ Susan Krueger \\ National Institute of Standards and Technology \\ and \\ Peter Yau \\ Department of Biological Chemistry, University of California,'Davis
}

\begin{abstract}
Although histones are among the most highly conserved protein sequences in nature, families of histone subtypes provide for considerable variation in the packaging of DNA. There are 6 subtypes of human H1, 4 subtypes of $\mathrm{H} 2 \mathrm{~A} ; 3$ subtypes of $\mathrm{H} 2 \mathrm{~B}, 3$ subtypes of $\mathrm{H} 3$ and 1 (possibly 2) H4 subtype(s). Many of these subtypes are cell cycle dependent and package the genome during $S-$ phase of the cell cycle. Other subtypes e.g. $\mathrm{H} 1^{0}, \mathrm{H} 2 \mathrm{~A} . \mathrm{X}$, H2A.Z, H3.3 are cell cycle independent and are thought to be required for the nucleosome packaging of active and potentially active genes of a particular cell type.
\end{abstract}

The chromatin of the human immature sperm consists of nucleohistone and resembles somatic chromatin. During spermiogenesis the histones are replaced by transition proteins and finally by protamines which condense the DNA forming an insoluble complex which is transcriptionally inactive. In humans approximately $15 \%$ of the DNA in the spermatozoan is not condensed by protamine and remains associated with histone. We have previously demonstrated that the DNA packaging in the two chromatin types is sequence-specific (Gatewood et al., 1987). Our protein characterization data indicate that the major histone variants in spermatid chromatin are replaced by protamine leaving the minor hyperacetylated histone variants complexed with a unique subset of the human genome (Gatewood et al., 1990). These unique histone variants and their states of modification have previously been associated with the transcriptionally active state of somatic chromatin. The DNA packaging and histone characterization data were the basis for the hypothesis that the nucleohistone component of human sperm contains the genes programmed for expression in early development (Gatewood, et al., 1990). SAXS experiments conducted during the last year provide support for the hypothesis that histone variant composition is a fundamental control element in gene regulation.

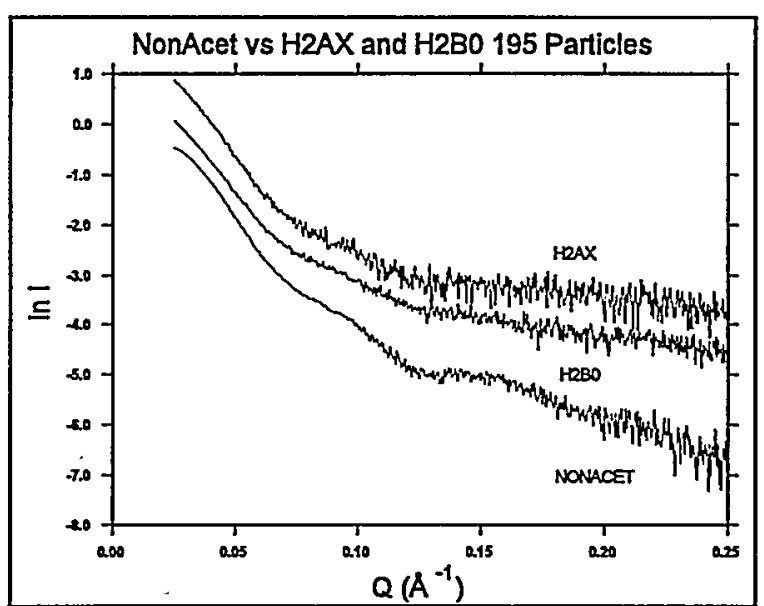

As shown above, the structures of nucleosomes reconstituted with the histone variants found in human sperm are structurally distinct from nucleosomes reconstituted with the major histone variants. The lower scatter profile is of control particles while the two upper profiles are of H2A.X and H2B. 0 containing particles. The difference in scatter profiles is consistant with a partial unwinding of DNA from the histone octamer in the particles containing the histone variants.

Gatewood, J. M., Cook, G. R., Balhorn, R., Bradbury, E. M., and Schmid, C. W., "Sequence-Specific Packaging of DNA in Human Sperm Chromatin," Science, 236, 962964, (1987).

Gatewood, J. M., Cook, G. R., Balhorn, R., Schmid, C. W., and Bradbury, E. M., "Isolation of Four Core Histones from Human Sperm Chromatin Representing a Minor Subset of Somatic Histones," J. Biol. Chem. 265, 20662-20666, (1990). 


\title{
SAXS Measurements on H4 Acetylated and Nonacetylated 195 b.p. Nucleosomes
}

\author{
Brian S. Imai, Joe M. Gatewood, Joseph S. Siino Jr. \\ Life Sciences Division, Los Alamos National Laboratory \\ and \\ Susan Krueger \\ National Institutes of Standards and Technology \\ and \\ Peter M. Yau and E. Morton Bradbury \\ Department of Biological Chemistry, University of California, Davis \\ and \\ Glen McConnell \\ SSRL
}

The nucleosome core particle is the fundamental repeating unit found in eukaryotic chromatin and represents the lowest level of structural organization in the chromosome. In the core particle, 146 b.p. of DNA are wrapped in 1.75 left-handed, superhelical turns around an octamer core of two each of histones $\mathrm{H} 2 \mathrm{~A}$, $\mathrm{H} 2 \mathrm{~B}, \mathrm{H} 3$ and $\mathrm{H} 4$. The overall dimensions of the disc shaped core particle have been determined to be about $55 \AA$ high by $110 \AA$ in diameter ${ }^{1}$.

The reversible acetylation of the N-terminal tails of the core histones has long been associated with transcriptional activity. A potential structural role for acetylation has also been suggested by the finding that nucleosomes introduced onto closed circular DNA molecules introduce fewer supercoils when the histones are highly acetylated compared to nonacetylated histones ${ }^{2}$. This result could be explained by either internal changes in nucleosome structure or structural changes in the DNA beyond the 146 b.p. associated with the core particle region. A small angle neutron scattering study on acetylated core particles ${ }^{1}$ and a footprinting study on reconstituted acetylated nucleosomes ${ }^{3}$ have failed to find a significant difference between acetylated and nonacetylated nucleosomes. This suggests that the regions of interaction of the histone $\mathrm{N}$-terminal tails could be with DNA beyond the 146 b.p. associated with the core particle. In order to test this theory, a SAXS study was performed on BLI-4 on nucleosomes reconstituted onto a 195 b.p. DNA molecule which contained a precise nucleosome positioning sequence ${ }^{4}$ with either fully unacetylated or acetylated $\mathrm{H} 4$ and the remaining histones unacetylated.

The figure shows that while the general features of the acetylated and nonacetylated scattering curves are similar, there is a significant deviation between the two curves at higher Q. This suggests that while the structures of the two particles are similar, the shapes differ significantly. $A P(r)$ analysis was performed on the data which gave a $D_{\max }$ of $140 \AA$ and $160 \AA$ for the nonacetylated and $H 4$ acetylated particles, respectively. Model fitting was done on the $\mathrm{P}(\mathrm{r})$ curves using Biomod, a Monte Carlo based solid modelling and curve generating program developed by $\mathrm{S}$. Henderson (ORNL). This indicated that the best fit models had 2.0 versus 1.83 turns of DNA wrapped around the histone core for the nonacetylated and $\mathrm{H} 4$ acetylated particles, respectively.

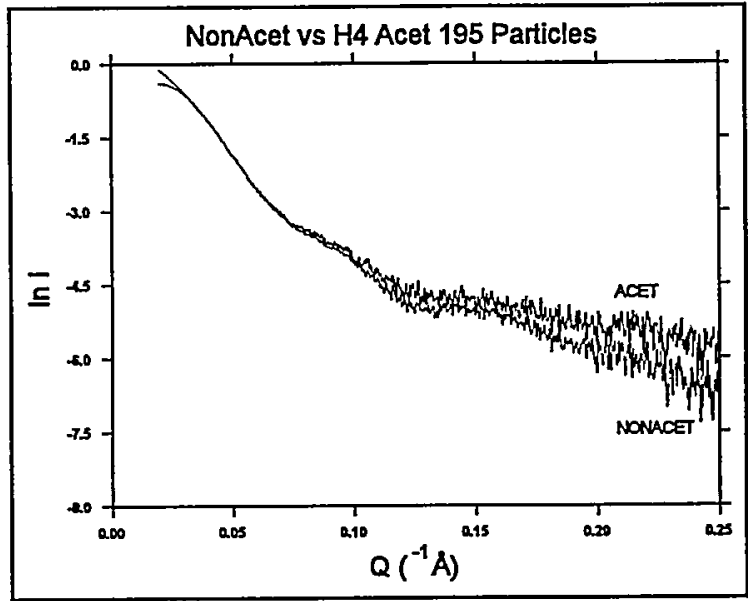

There is clearly a significant difference in the structures of the two resulting best fit models. The nonacetylated histone $\mathrm{N}$-terminal tails hold down 21 b.p. beyond the minimum 146 b.p. of DNA in the core region, while $\mathrm{H} 4$ acetylation reduces this to only 7 b.p. beyond 146 b.p. This data indicates that the role of the histone $\mathrm{N}$-terminal tails is to hold down DNA beyond the 146 b.p. required for the core particle region and that even acetylation of just the $\mathrm{H} 4 \mathrm{~N}$-terminal tails can partially release the extra DNA ends and allow them to extend tangentially out from the nucleosome core. This may be a mechanism by which histone acetylation can modulate chromatin higher order structure.

1. Imai, B. S., Yau, P., Baldwin, J. P., Ibel, K., May, R. 
P. and Bradbury, E. M. (1986) J. Biol. Chem 261, 8784-

8792.

2. Norton, V. G., Imai, B. S., Yau P. and Bradbury E. M. (1989) Cell 57, 449-57.

3. Marvin, K W., Yau, P. and Bradbury, E. M. (1990) J. Biol. Chem. 265, 19839-47.

4. Simpson, R. T., Thoma, F. and Brubaker, J. M. (1985) Cell 42, 799-808. 


\title{
X-ray Solution-Scattering by Myosin Subfragment-one With and Without ATP
}

\author{
Robert Mendelson, Satoru Fujiwara and Deborah Stone \\ Cardiovascular Research Institute and Dept of Biochem. and Biophysics, \\ Univ. of California, San Francisco
}

We have investigated the $\mathrm{X}$-ray scattering by wildtype myosin subfragment-one (S1) as a preliminary to our studies on cloned S1 with site-directed mutations. Surprisingly, we found that earlier studies by Wakabayashi et al. (1992) seem to have overestimated the radius-of-gyration $\left(\mathrm{R}_{\mathrm{g}}\right)$ change induced by adding MgATP to S1. Under the conditions of both experiments about $85 \%$ of $S 1$ population is S1-ADP'P $P_{i}$ (e.g. Bagshaw \& Trentham, 1974). Thus the conformation being sensed is largely that of a state which is fully "cocked" for subsequent force production when the myosin head interacts with actin in contracting muscle.

Typical Guinier plots with and without ATP are shown Figure 1a. The $R_{g}$ change, extrapolated to zero protein concentration (Figure $1 \mathrm{~b}$ ), is about $3 \%$ when corrected for heterogeneity in the S1 population. The $R_{g}$ measured here is in better agreement with that calculated from the complete structure of methylated S1 (Rayment et al. 1993) than is that from the earlier work.

We are currently extending this work using nucleotide analogs.

Bagshaw, C. R. \& Trentham, D. R. (1974). The characterization of myosin product complexes and of product release steps during magnesium ion-dependent adenosine triphosphatase reaction. Biochem. J. 141, 331-349.

Rayment, I., Rypniewski, W. R., Schmidt-Bäse, K., Smith, R., Tomchick, D. R., Benning, M. M., Winkelmann, D. A., Wesenberg, G. \& Holden, H. M. (1993a). Three-dimensional structure of myosin subfragment-1: a molecular motor. Science 261, 50-58.

Wakabayashi, K., Tokunaga, M., Kohno, I., Sugimoto, Y., Hamanaka, T., Takezawa, Y., Wakabayashi, T. \&
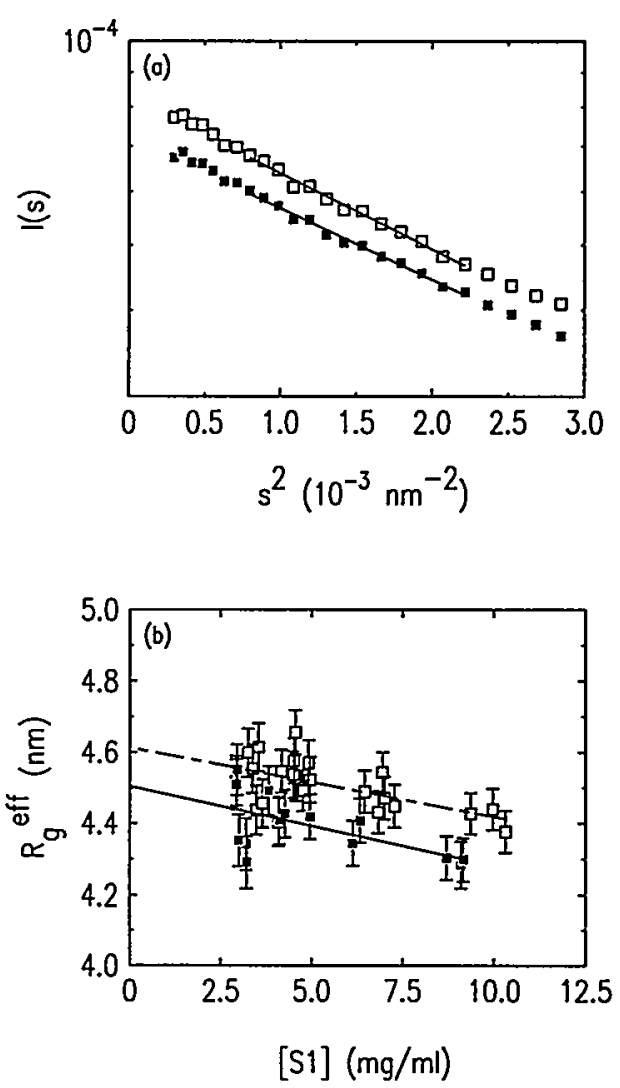

Figure 1 a) Guinier plots of S1 without ( $\square$ ) and with ( $\square$ ) added ATP.. b) Concentration plot of $R_{g}$ with and without added ATP.

Amemiya, Y. (1992). Small-angle synchrotron x-ray scattering reveals distinct shape changes of the myosin head during hydrolysis of ATP. Science 258, 443-447. 


\title{
An X-ray Absorption Spectroscopic study of Pyrococcus furiosus Rubredoxin
}

\author{
Graham N. George ${ }^{1}$, Ingrid J. Pickering ${ }^{1}$, \\ Roger, C. Prince ${ }^{2}$, Michael W. W. Adams ${ }^{3} \&$ Zhi Hao Zhou ${ }^{3}$ \\ 1. Stanford Synchrotron Radiation Laboratory, PO Box 4349 MS 69, Stanford, CA 94309
}

2. Exxon Research and Engineering Co. Route 22 E, Annandale, NJ 08801

3. Center for Metalloenzyme Studies \& Dept. of Biochemistry,

University of Georgia, Athens GA 30602

\section{Introduction}

Rubredoxins are relatively small proteins which contain a single iron atom tetrahedrally coordinated by four cysteinyl sulfur atoms [1] (see Figure 1), and high resolution crystal structures are known for the rubredoxins from Clostridium pasteurianum [2], Desulfovibrio gigas [3] and Pyrococcus furiosus [4]. For the latter rubredoxin, crystal structures for both the oxidized $\left(\mathrm{Fe}^{3+}\right)$ and reduced $\left(\mathrm{Fe}^{2+}\right)$ protein have been determined to 1.8 Å resolution [4].

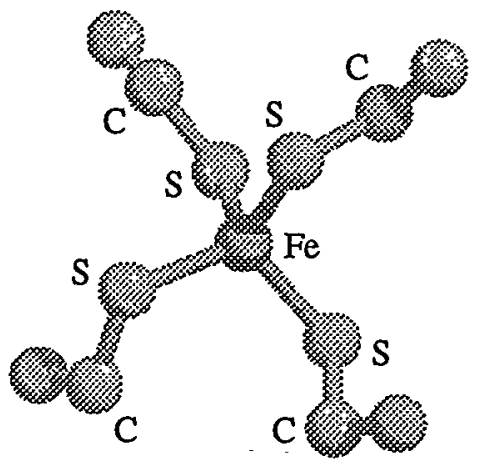

Figure 1: The structure of the iron domain in oxidized Pyrococcus furiosus rubredoxin.

The first reported biological application of $\mathrm{X}$ ray absorption spectroscopy was a study of rubredoxin [5] which corrected errors in the first rubredoxin crystal structure [6] which had reported an anomalously short Fe-S bond of $2.05 \AA$. Later crystal structures $[2,3]$ were in good agreement with the XAS. In the pre- sent work we have used iron K-edge $\mathrm{X}$-ray absorption spectroscopy to probe the structure of the iron site of oxidized and reduced $P$. furiosus nubredoxin.

\section{Experimental}

Data were collected on SSRL BL 7-3 by monitoring the $\mathrm{Fe} \mathrm{K}_{\alpha}$ fluorescence using a 13 element germanium array detector. The EXAFS oscillations were analyzed using theoretical phase and amplitude functions derived from the ab-intio theory code feff $[7,8]$. Curve-fitting, and all other data reduction was performed using the EXAFSPAK program suite.

\section{Results and Discussion}

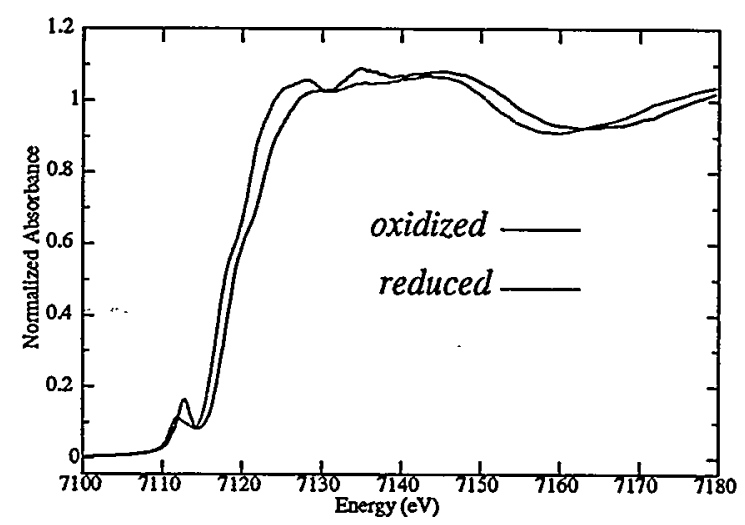

Figure 2: Fe K-edge X-ray absorption near-edge spectra of oxidized and reduced $P$. furiosus rubredoxin.

Figure 2 shows the iron K-edge X-ray absorption near-edge spectrum for both oxidized and reduced rubredoxin. The spectra are typical of high-spin ferrous 
and ferric coordinated by sulfur ligands with approximate tetrahedral geometry.
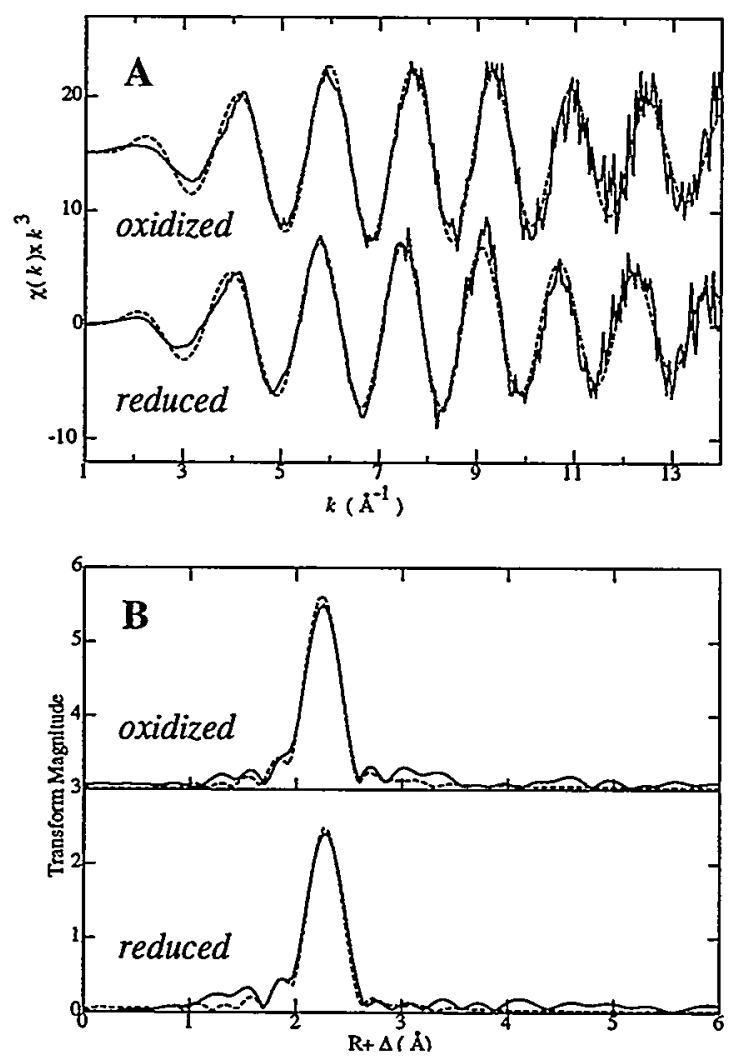

Figure 3: Fe K-edge EXAFS curve fitting results of oxidized and reduced $P$. furiosus rubredoxin. Experimental data is shown using solid lines, and the best fit with broken lines. A shows the EXAFS data, and B the EXAFS Fourier transform phase corrected for $\mathrm{Fe}-\mathrm{S}$ backscattering.

The small features centered at $7111.8 \mathrm{eV}$ (reduced) and $7712.7 \mathrm{eV}$ (oxidized) are the formally dipoleforbidden, quadrupole-allowed $1 \mathrm{~s} \rightarrow 3 \mathrm{~d}$ transitions which gain dipole-allowed intensity in noncentrosymmetric environments by mixing of metal $4 p$ levels. The relatively strong presence of these features here is consistent with tetrahedral coordination. The oxidized and the reduced spectra are in general quite similar with features of the reduced spectrum are shifted by approximately $1 \mathrm{eV}$ to lower energy.
Figure 3 shows the EXAFS curve-fitting results, obtained with the parameters given in Table I.

Table I : EXAFS curve-fitting results.

\begin{tabular}{|l|l|l|l|}
\hline Sample & $R(\AA)$ & $\sigma^{2}\left(\AA^{2}\right)$ & Error* \\
\hline Oxidized & $2.284(6)$ & $0.00306(35)$ & 1.180 \\
\hline Reduced & $2.330(6)$ & $0.00282(31)$ & 1.200 \\
\hline
\end{tabular}

* The fit-error is defined as $\Sigma\left[\chi_{\text {exp }}-\chi_{\text {calc }}\right]^{2} k^{6} /(n$ $m$ ) where $n$ is the number of data points, and $m$ is the number of variables floated in the fit.

For both the oxidized and reduced proteins no outer shell scatterers were observed in the EXAFS, and only a single Fe-S interaction (using a coordination number of four) was required to fit the data. The EXAFS curve-fitting results are in excellent agreement with the crystal structure [4], which gave average $\mathrm{Fe}-\mathrm{S}$ bond lengths of $2.28 \AA$ and $2.32 \AA$, for the oxidized and reduced rubredoxin, respectively. We conclude that there are no significant structural differences between the iron sites of $P$. furiosus rubredoxin in solution and in the crystal. Such differences have been suggested for zinc concanavalain A [9].

\section{References:}

(1) Steifel, E.I.; George, G.N. in Bioinorganic Chemistry (Eds. Bertini, I.; Gray, H.B.; Lippard, S.J.; Valentine J.S.) 1994, pp 365-453.

(2) Watenpaugh, K.D.; Sieker, L.C.; Jensen, L.H. $J$. Mol. Biol. 1980, 138, 615-

(3) Adman, E.T. et. al. J. Mol. Biol. 1977, 112, 113-

(4) Day, M.W.; Hsu, B.T.; Joshua-Tor, L.; Park, J.B.; Zhou, Z.H.; Adams, M.W.W.; Rees, D.C. Protein Science 1992, 1, 1494-1507.

(5) Shulman, R.G. et. al. PNAS USA, 1975, 72, 4003.

(6) Watenpaugh,K.D. et. al. Acta. Cryst. 1973, B29, 943

(7) Rehr, J. J.; Mustre de Leon, J.; Zabinsky, S. I.; Albers, R. C. J. Amer. Chem. Soc. 1991, 113, 51355140.

(8) Mustre de Leon, J.; Rehr, J. J.; Zabinsky, S. I.; Albers, R. C. Phys. Rev. 1991, B44, 4146-4156.

(9) Lin, S.-L.; Stern, E.A.; Kalb, A.J.; Zhang, Y. Biochemistry, 1990, 29, 3599-3603. 


\title{
Constrained EXAFS Refinement of the Core Dimensions of the 3-Iron-Sulfur Ferredoxin from Pyrococcus furiosus
}

\author{
Graham N. George ${ }^{1}$, Ingrid J. Pickering ${ }^{1}$, \\ Roger, C. Prince ${ }^{2}$, Michael W. W. Adams ${ }^{3}$ \& Zhi Hao Zhou ${ }^{3}$
}

1. Stanford Synchrotron Radiation Laboratory, PO Box 4349 MS 69, Stanford, CA 94309

2. Exxon Research and Engineering Co. Route $22 \mathrm{E}$, Annandale, NJ 08801

3. Center for Metalloenzyme Studies \& Dept. of Biochemistry, University of Georgia, Athens GA 30602

\section{Introduction}

Iron-sulfur proteins are a ubiquitous class of proteins which play crucial roles in many biological processes. They most commonly function as agents of electron transfer, and the prototypical members are the ferredoxins. The core dimensions of the iron-sulfur clusters within the proteins are of crucial importance in understanding their physical chemical properties, and the structural nature of the $\mathrm{Fe}_{3} \mathrm{~S}_{4}$ clusters have previously been the focus of considerable debate [see 1 and references therein]. $\mathrm{Fe}_{3} \mathrm{~S}_{4}$ clusters are known to be chemically related to $\mathrm{Fe}_{4} \mathrm{~S}_{4}$ clusters, and are often formed from the latter by loss of a single iron. We report herein a study of the core dimensions of the $\mathrm{Fe}_{3} \mathrm{~S}_{4}$ cluster in the ferredoxin from the hyperthermophilic archæon Pyrococcus furiosus. We have used new constrained EXAFS refinement methods to model the active site using a three-dimensional coordinate model.

The ferredoxin from $P$. furiosus is a monomeric protein of molecular weight 7500 , containing a single iron-sulfur cluster [2], which, in the protein as isolated, is a $\mathrm{Fe}_{4} \mathrm{~S}_{4}$ cluster. The protein is unusual among bacterial ferredoxins in several respects. It has an atypical arrangement of cysteinyl residues for coordinating the $\mathrm{Fe}_{4} \mathrm{~S}_{4}$ cluster; the arrangement of cysteines 11,17 and
56 is analogous to that of other $\mathrm{Fe}_{4} \mathrm{~S}_{4}$ ferredoxins, but an aspartate (asp ${ }^{14}$ ) replaces the cysteinyl residue which would normally complete the cluster ligation. It is very likely that the cluster contains one iron which is coordinated by this aspartate, perhaps together with $\mathrm{H}_{2} \mathrm{O}$ or $\mathrm{OH}^{-}$from solvent water. While a few other bacterial ferredoxins probably contain $\mathrm{Fe}_{4} \mathrm{~S}_{4}$ clusters with similar ligation (e.g. Desulfovibrio africanus ferredoxin III) all of these contain two $\mathrm{Fe}_{4} \mathrm{~S}_{4}$ clusters with the other cluster having the more common $\mathrm{Fe}_{4} \mathrm{~S}_{4}$ coordination of four external cysteines. $P$. furiosus ferredoxin is thus unique in having only a single $\mathrm{Fe}_{4} \mathrm{~S}_{4}$ cluster with one iron possessing non-sulfur coordination. A second unusual feature of the protein is the ease with which the cluster can lose an iron to form a $\mathrm{Fe}_{3} \mathrm{~S}_{4}$ ferredoxin, and it seems highly probable that the labile iron atom is the one with non-sulfur ligation. The resulting $\mathrm{Fe}_{3} \mathrm{~S}_{4}$ ferredoxin can readily be reconstituted with $\mathrm{Fe}^{2+}$ to reform the native $\mathrm{Fe}_{4} \mathrm{~S}_{4}$ cluster, or alternatively with other metals ions to generate a mixed-metal iron sulfur cluster $\mathrm{MFe}_{3} \mathrm{~S}_{4}$, where $\mathrm{M}$ can be cobalt, nickel, zinc, cesium or thallium $[3,4]$. In many ways, $P$. furiosus ferredoxin thus presents an ideal system for study of the biological three-iron clusters and their chemistry [2-4]. In it's redox and spectroscopic properties the $P$. furiosus ferredoxin $\mathrm{Fe}_{3} \mathrm{~S}_{4}$ 
cluster appears to be very similar to other wellcharacterized three-iron clusters $[4,5]$, and there is reason to believe that a common core-structure is shared by these proteins. The cluster $\mathrm{Fe}_{3} \mathrm{~S}_{4}$ can exist in $\left[\mathrm{Fe}_{3} \mathrm{~S}_{4}\right]^{0}$ and $\left[\mathrm{Fe}_{3} \mathrm{~S}_{4}\right]^{-}$oxidation states, for the former (oxidized) redox form, all irons are high-spin ferric, and for the latter (reduced), the additional electron is delocalized between two of the three irons [see 5 and references therein].

\section{Analysis Methods}

Computer software has been developed by two of the authors [G.N.G. and I.J.P.] using the accurate multiple scattering theory feff of John Rehr and coworkers at the University of Washington $[6,7]$. The new software allows refinement of a three-dimensional structural model to the EXAFS data using chemical constraints to restrict the dimensions of the parameter space of the model. We first consider the oxidized three-iron ferredoxin, where there is evidence from Mössbauer spectroscopy and magnetic measurements for an approximately tetrahedral coordination of iron, with all irons being essentially identical and in the high-spin ferric state. The iron K-edge near-edge spectrum (not illustrated) is also typical of tetrahedrally coordinated iron. These spectroscopies indicate that the three-iron cluster in $P$. furiosus ferredoxin is homologous to other well known biological $\mathrm{Fe}_{3} \mathrm{~S}_{4}$ clusters [7]. Additionally, resonance Raman spectroscopy of $P$. furiosus three-iron ferredoxin suggests that the cluster has a $C_{3 v}$ site-symmetry [Adams, M.W.W., Johnson, M.E., personal communication]. Thus, the chemical constraints which can be applied to the oxidized $P$. furiosus three-iron ferredoxin are that the three iron atoms must approach tetrahedral coordination, and a $C_{3 v}$ point-group can be imposed. For the latter, we have only four fractional coordinates to refine.
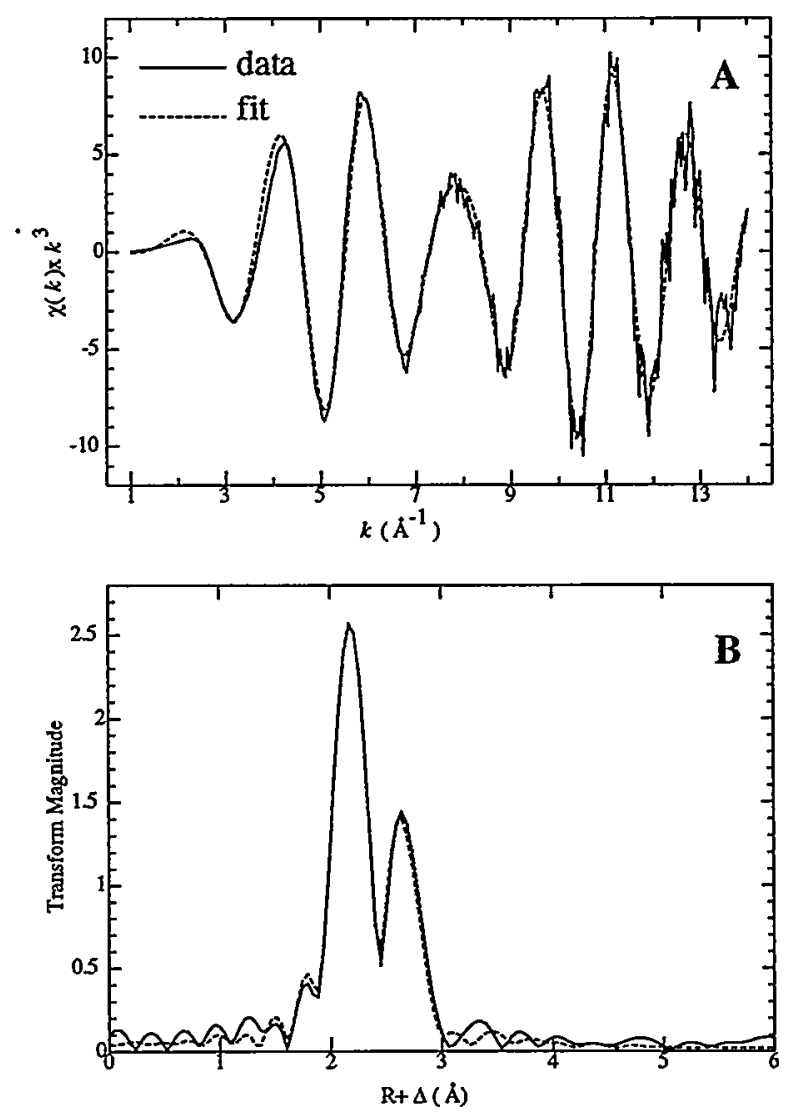

Figure 1 : EXAFS curve-fitting results of the $P$. furiosus oxidized three-iron ferredoxin, obtained using the program DAMP in combination with feff. A shows the EXAFS oscillations and $\mathbf{B}$ shows the Fe-S phasecorrected EXAFS Fourier transform.

The computer program (called DAMP - distance angle minimization program) has an interactive $\mathrm{X}$ Window molecular graphics display, which allows the user to rotate and examine the structure in real-time before, during and after refinement. For the oxidized three-iron ferredoxin, a variety of different starting models were tested, and identical minima were obtained in all cases. No significant difference in the minimum (i.e. the final coordinates obtained) was found when the constraint of $C_{3 v}$ point-group symme- 
try was released. No constraints imposed by the polypeptide backbone were included in the analysis.

Figure 1 shows the iron K-edge EXAFS and Fourier transform together with the best-fit obtained by DAMP, and figure 2 shows the structure determined. The final best fit used six non-degenerate scattering paths (calculated using feff), four single scattering, and two three-leg multiple-scattering paths

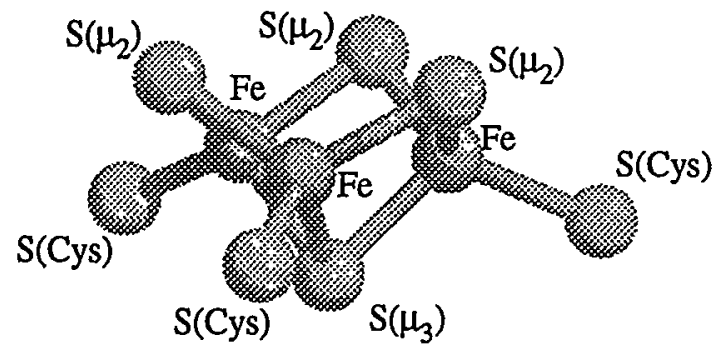

Figure 2 : Results of constrained coordinate refinement of the $\left[\mathrm{Fe}_{3} \mathrm{~S}_{4}\right]^{0}$ core of oxidized $P$. furiosus three-iron ferredoxin.

Final coordinates of the $C_{3 v}$ point-group constrained fit indicated a mean $\mathrm{Fe}-\mathrm{S}$ bond-length of 2.239(3) $\AA$ and a mean Fe-Fe separation of 2.672(6) $\AA$, with bond angles of $110^{\circ}$ for $\left(\mu_{2}\right) \mathrm{S}-\mathrm{Fe}-\left(\mu_{2}\right) \mathrm{S}, 104^{\circ}$ for $\left(\mu_{3}\right) S-F e-\left(\mu_{2}\right) S$, and $113^{\circ}$ for $\left(\mu_{2}\right) S-F e-S(C y s)$. Accuracies (as opposed to the precisions in parentheses) for the $\mathrm{Fe}-\mathrm{S}$ and $\mathrm{Fe}-\mathrm{Fe}$ distances are estimated to be $\pm 0.02 \AA$, while accuracies for the bond angles are more difficult to estimate, as they depend upon the validity of the constraints used in the model.
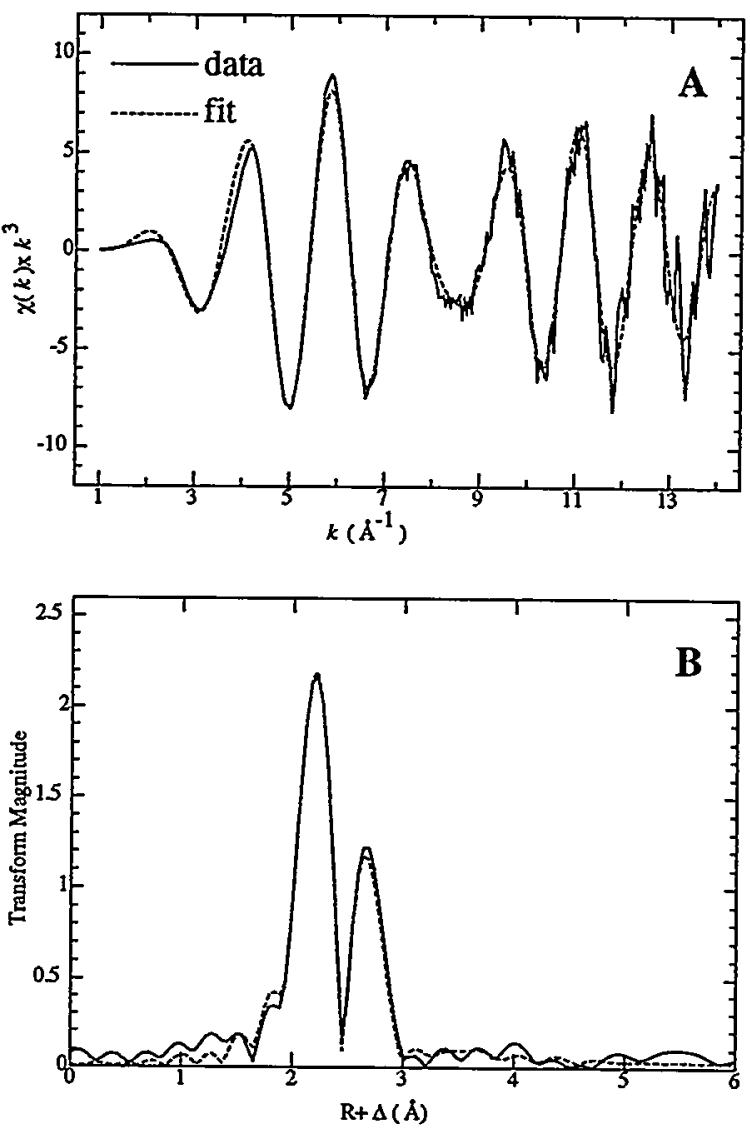

Figure 3 : EXAFS curve-fitting results of the $P$. furiosus reduced three-iron ferredoxin, obtained using the program DAMP in combination with feff. A shows the EXAFS oscillations and $\mathbf{B}$ shows the $\mathrm{Fe}-\mathrm{S}$ phasecorrected EXAFS Fourier transform.

The structure of the related $\left[\mathrm{Fe}_{3} \mathrm{~S}_{4}\right]^{0}$ cluster in Desulfovibrio gigas ferredoxin II has been studied by $\mathrm{X}$-ray crystallography at $1.7 \AA$ resolution [8]. The interatomic mean $\mathrm{Fe}-\mathrm{Fe}$ distance determined by crystallography is larger by $0.08 \AA$ than that determined in the present work. Either this represents a structural difference between the clusters of two proteins, a result of different experimental conditions, or is a reflection of the difficulty of determining exact interatomic distances by crystallography of large molecules. We also note that our estimated bond-lengths are very similar to those estimated from earlier EXAFS of $D$. gigas ferredoxin II [9]. 
We have also examined the EXAFS of the reduced $\left[\mathrm{Fe}_{3} \mathrm{~S}_{4}\right]^{-} P$. furiosus ferredoxin (figure 3). Very similar structural results to the oxidized cluster were obtained, with average $\mathrm{Fe}-\mathrm{S}$ and $\mathrm{Fe}-\mathrm{Fe}$ distances of $2.272(3) \AA$ and $2.668(6) \AA$, respectively. In this case the constraint of $C_{3 v}$ point-group symmetry was not imposed. The major differences between $\left[\mathrm{Fe}_{3} \mathrm{~S}_{4}\right]^{0}$ and $\left[\mathrm{Fe}_{3} \mathrm{~S}_{4}\right]^{-}$clusters is an increase in the Fe-S distance, and slightly larger Debye-Waller factors for both the $\mathrm{Fe}-\mathrm{S}$ and $\mathrm{Fe}-\mathrm{Fe}$ interactions, while no significant difference in the mean $\mathrm{Fe}-\mathrm{Fe}$ distances was observed. Electronically, $\left[\mathrm{Fe}_{3} \mathrm{~S}_{4}\right]^{-}$clusters consists of two strongly coupled irons with an average oxidation state of $\mathrm{Fe}^{2.5+}$ and a single $\mathrm{Fe}^{3+}$ iron [see 5 and references therein]. Thus, it seems likely that the increased Debye-Waller factors for the reduced cluster may be due to differences in individual $\mathrm{Fe}-\mathrm{S}$ and $\mathrm{Fe}-\mathrm{Fe}$ distances. Modelling this with DAMP using the DebyeWallers derived from the oxidized cluster indicates that individual bond lengths may differ by $0.07 \AA \AA$ or by as much as $0.05 \AA$ from the mean distances. Stout has recently reported an X-ray crystallographic study of Azotobacter vinelandii ferredoxin I under a different conditions of $\mathrm{pH}$ and redox state. Comparing the $\left[\mathrm{Fe}_{3} \mathrm{~S}_{4}\right]^{0}$ with the $\left[\mathrm{Fe}_{3} \mathrm{~S}_{4}\right]^{-}$cluster at $\mathrm{pH} 8$, Stout reports a contraction of $\mathrm{Fe}-\mathrm{Fe}$ distances by $0.03,0.06$ and $0.11 \AA$, corresponding to a reduction in the mean $\mathrm{Fe}-\mathrm{Fe}$ distance of $0.07 \AA$. . We find no evidence for such large changes in $\mathrm{Fe}-\mathrm{Fe}$ distances $P$. furiosus three-iron ferredoxin. Either this discrepancy is due to differences in experimental conditions (e.g. temperature) between XAS and crystallography, or a structural difference between the three-iron clusters in the two proteins, or alternatively an error in either the crystallography or XAS analyses. The possibility of a structural difference seems unlikely due to the similarity of the Mössbauer data from the $\mathrm{Fe}_{3} \mathrm{~S}_{4}$ clusters of the two proteins [4].

\section{References:}

(1) George, G.N.; George, S.J. Trends in Biochem. Sci. 1988, 13, 369-370.

(2) Conver, R.C.; Kowal, A.T.; Fu, W.; Park, J.-B.; Aono, S.; Adams, M.W.W.; Johnson, M.K J. Biol. Chem. 1990, 265, 8533-8541.

(3) Conver, R.C.; Park, J.-B.; Adams, M.W.W.; Johnson, M.K J. Amer. Chem. Soc. 1990, 112, 4562 4564 .

(4) Srivastra, K.K.P.; Surerus, K.K..; Conover, R.C.; Johnson, M.K.; Park, J.-B.; Adams, M.W.W.; Münck, E. Inorg. Chem. 1993, 32, 927-936.

(5) Steifel, E.I.; George, G.N. in Bioinorganic Chemistry (Eds. Bertini, I.; Gray, H.B.; Lippard, S.J.; Valentine J.S.) 1994, pp 365-453.

(6) Rehr, J. J.; Mustre de Leon, J.; Zabinsky, S. I.; Albers, R. C. J. Amer. Chem. Soc. 1991, 113, 51355140.

(7) Mustre de Leon, J.; Rehr, J. J.; Zabinsky, S. I.; Albers, R. C. Phys. Rev. 1991, B44, 4146-4156.

(8) Kissinger, C.R.; Adman, E.T.; Sieker, L.C.; Jensen, L.H. J. Amer. Chem. Soc. 1988, 110, 8721-8723.

(9). Antonio, M.R.; Averill, B.A.; Moura, I.; Moura, J. J. G.; Orme-Johnson W.H.; Teo, B.-K.; Xavier, A.V.J. Biol. Chem. 1982, 257, 6646-6649.

(10) Stout, C.D. J. Biol. Chem. 1993, 268, 2592025927. 


\title{
Preliminary X-Ray Crystallographic Analysis of Tritrichomonas foetus Inosine-5'-Monophosphate Dehydrogenase.
}

\author{
Frank G. Whitby ${ }^{1}$, Jorge Huete-Perez ${ }^{1}$, Hartmut Luecke ${ }^{2}$ \& C. C. Wang1 \\ 1UCSF, San Francisco, CA \\ 2SSRL, Stanford, CA
}

Inosine-5'-monophosphate dehydrogenase (IMPDH) catalyzes the NAD-dependent oxidation of inosine monophosphate (IMP) to xanthosine monophosphate (XMP). IMPDH is the key enzyme in the synthesis of guanine nucleotides. Inhibition of IMPDH has been shown to have potentially valuable antiviral, anticancer, immunosuppressive, and antiparasitic effects. Studies of its antiparasitic effects indicate that IMPDH inhibitors inhibit the in vitro growth of the protozoan parasite Tritrichomonas foetus. This parasite infects the urogenital tracts of cattle, leading to sterility and premature abortion. In vitro inhibition of growth by IMPDH inhibitors can be overcome by the addition of guanine or guanosine to the culture medium. The development of specific antiparasitic agents may be helped by the information obtained from a high-resolution three-dimensional structure of IMPDH.

T. foetus IMPDH shares about $35 \%$ sequence identity with human IMPDH. Both human and $T$. foetus enzymes have been shown by gel filtration chromatography to be tetramers of about $240,000 \mathrm{Da}$ at high salt conditions. The native $T$. foetus enzyme has also been shown to aggregate to form a higher molecular weight species of about $340,000 \mathrm{Da}$ at very low salt concentrations. The human enzyme exists as two closely related isoforms of comparable molecular weight and subunit composition. The constitutively produced type I isoform is the dominant form in normal cells, whereas the type II isoform is strongly upregulated in neoplastic and replicating cells. Selective inhibition of the type II isoform offers the opportunity for useful chemotherapies with minimal side effects. Like human IMPDH, $T$. foetus IMPDH uses an unusual sequential, ordered, bi-bi kinetic mechanism in which IMP binds before NAD and NADH is released before XMP. Detail Kcat, substrate specificities? The human enzyme, however, requires $\mathrm{K}^{+}$ for catalytic activity and is inhibited much more strongly by mycophenolic acid (MPA) (type I, $\mathrm{K}_{\mathrm{I}}=37 \mathrm{nM}$; type II, $\mathrm{K}_{\mathrm{I}}=10 \mathrm{nM} ; T$. foetus, $\mathrm{K}_{\mathrm{I}}$ $=7$ to $9 \mu \mathrm{M}$ ) (Carr $e t$ al.). Both human and $T$. foetus IMPDH have been shown to have an active-site cysteine residue that forms a covalent intermediate with IMP. The crystal structure of $T$. foetus IMPDH will help in understanding its relatively weak inhibition by MPA and other strong inhibitors of the human enzyme and will aid in the design of novel inhibitors for control of infection by $T$. foetus. A crystal structure of the $T$. foetus enzyme should also help in modeling the human enzymes. This study aims at solving the high-resolution crystal structure of $T$. foetus IMPDH.

Inosine-5'-monophosphate dehydrogenase (IMPDH) from the protozoan parasite Tritrichomonas foetus has been expressed in $E$. coli and crystallized. Crystals were grown to $0.1 \mathrm{~mm}$ in each dimension in 18 to 72 hours using ammonium sulfate and low molecular weight polyethylene glycols. The crystals belong to the cubic space group P432 with cell edge $157.25 \AA$. The enzyme is a homotetramer with each monomer having a molecular weight of 55,534 Da. There is one monomer per asymmetric unit, based on a volume/mass ratio of $2.7 \AA^{3} / \mathrm{Da}$ and self-rotation analysis. The crystals are adequately stable to allow a complete data set to be collected from a single crystal. Complete native data sets have been collected to $2.3 \AA$ resolution at beam line 7-1 at SSRL. High-quality complete data extending to $3.0 \AA$ resolution has been collected from crystals of four putative derivatives, and the data appear to be isomorphous with that of the native crystals in each case. Efforts to solve the derivatives for use in MIR phasing are underway.

Initial diffraction images collected on a Siemens multiwire area detector, using a 
rotating anode generator, showed very weak diffraction to $3.5 \AA$ resolution from the cubeshaped crystals. Native data were then collected at beam line 7-1 at the Stanford Synchrotron Radiation Laboratory (SSRL) using a MAR imaging plate detector. Diffraction was seen extending to $2.3 \AA$ resolution in all directions on $0.7^{\circ}$ rotation images using a five second exposure. Complete native data sets to $2.3 \AA$ resolution were collected from each of seven crystals by taking rotation images of $0.7^{\circ}$ or $1.0^{\circ}$ for 5 or 10 seconds each. The rotation images were auto-indexed with DENZO, indicating a primitive cubic space group with unit cell edges of $157.25 \AA$. Intensities were integrated using DENZO, and data were scaled and merged using SCALEPACK (denzo and scalepack refs.). Crystals continued to diffract to beyond $3.0 \AA$ resolution after 30 frames had been collected, but an increase in Rsym and change in the frame-to-frame scale factors indicated that the data from later frames were of limited value. Thus, data collection was typically discontinued after 18 to $24^{\circ}$ of rotation for future crystals.

Data were initially indexed in space group P23. A self-rotation function was calculated using the program GLRF. No peaks indicating non-crystallographic symmetry were seen in the self-rotation function at $\mathrm{K}=180^{\circ}$, but strong peaks were seen indicating the crystallographic two-fold operators. The strong peaks in the self-rotation function at $\kappa=90^{\circ}$ coincide with the crystallogrpahic two-folds, indicating that the crystallographic two-folds seen in the self-rotation function at $\mathrm{K}=180^{\circ}$ are actually crystallographic four-folds. Thus, the data were reindexed in space group P432. Re-merging the integrated reflection files in space group P432 had only a minor effect on Rsym, indicating that this is the correct class of cubic space group. Hence the minimum level of symmetry in the crystals was determined to be that of space group P432. Inspection of the reduced data sets revealed no systemmatic absences, ruling out the possibility of higher symmetry space groups that include screw axes. It thus seemed likely that there are six tetrameric molecules per unit cell and that the asymmetric unit contains a monomer. In this case, the crystal volume per mass $\left(V_{M}\right)$ is about $2.7 \AA^{3} / \mathrm{Da}$, giving a solvent content of about $55 \%$ by volume (Matthews). This is in the normal range for protein crystals and indicates that the likelihood of having a dimer in the asymmetric unit is very small. Thus, the asymmetric unit contains a monomer, and the tetramer is generated by the crystallographic operators.

Heavy atom soaks were performed using nine heavy atom compounds that are soluble in the mother liquor. In each case, several crystals were soaked in the heavy atom solution at one or more heavy atom concentrations ( 0.1 to $2 \mathrm{mM}$ ) and for times ranging from one to 48 hours. Apparently isomorphous, high-quality data sets have been collected from crystals soaked in thimerosal, PCMBS, platinum chloride, and mercury cyanide. Derivative data sets were collected at beamline 7-1 at SSRL. Efforts are underway to solve these derivatives for use in MIR phasing.

F. G. Whitby, J. Huete-Perez, H.Luecke, C. C. Wang "Preliminary X-Ray Crystallo-graphic Analysis of Tritrichomonas foetus Inosine-5'Monophosphate Dehydrogenase" Proteins: Struct. Func. Gen., accepted June 1995. 
Proposal 8014B

\title{
The Crystal Structure of Bovine $\gamma D$-Crystallin at $1.65 \AA$ Resolution
}

\author{
David K. Wilson'1, Hartmut Luecke², Florante A. Quiocho ${ }^{1}$ \\ 1HHMI, Baylor College of Medicine, Houston, TX \\ ${ }^{2}$ SSRL, Stanford, CA
}

\begin{abstract}
Crystallins are cytoplasmic proteins abundantly and in most cases selectively expressed in fiber cells of the vertebrate eye lens. Encoded by groups of complex gene families, the crystallins are synthesized in a temporally and spatially precise pattern during lens development (Piatigorsky, 1989). Transparency of the eye lens is attributed to short range ordering of the crystallins (Veretout et al., 1989). While it is not presently understood how the crystallins achieve and maintain this highly ordered array, loss of transparency is known to correlate with separation of lens proteins into distinct liquid phases rich and poor in protein, respectively (Thomson et al., 1987). The crystallins represent the principle protein component linked to phase separation in the lens (Siezen et al., 1985). In the bovine lens, they comprise a group of structurally-related $\sim 20$ kilodalton polypeptides encoded by as many as six related genes. While the structures of their corresponding proteins are over $90 \%$ identical, distinct differences among the crystallins with respect to some biophysical parameters have been noted. For example, phase separation behavior sorts the crystallins into two groups differing in the critical temperature for phase separation: a high $T_{C}$ group and a low $T_{C}$ group.
\end{abstract}

Crystallographic studies of crystallins isolated from calf lenses showed conservation of a compact tertiary structure comprising 2 similar domains each composed of 2 structural motifs likened to a "Greek Key". A structural model for bovine crystallin has been instrumental in guiding structure-function studies of the crystallins, including examination of protein modifications using chemical and biophysical probes (Summers et al., 1984; Najmudin et al., 1993). Since alterations in phase separation behavior probably reflect perturbation of protein-protein interactions occurring between various crystallins, structural models of the pertinent proteins are highly desirable. Bovine crystallin was the first and continues to serve as a prototypical structure with which other crystallins are studied. Structures for bovine $\gamma$ IIIb and $\gamma \mathrm{IVa}$ have been reported (Chirgadze et al., 1991; White et al., 1989). Since recently obtained gene sequencing data indicates that the previously inferred sequence for $\gamma \mathrm{IIIb}$ was incorrect in approximately $10 \%$ of the amino acid assignments (Hay et al., 1994), we have reexamined its structure using high resolution $\mathrm{X}$ ray crystallography.

A wavelength of $1.08 \AA$ was used to collect 598,638 observations to a resolution limit of $1.47 \AA$ at beamline 7-1 at SSRL. These were merged to yield $77,447 \mathrm{~F}_{\text {obs }}$ with an $\mathrm{R}_{\mathrm{MERGE}}$ of 0.087 based on intensity. Removing the weak reflections with $\mathrm{I}<\sim(\mathrm{I})$ left 69,724 remaining. The data used for refinement consisted of 53,136 reflections after the $1.65 \AA$ resolution limit was imposed. The initial phasing for the structure was determined via molecular replacement using the $\gamma \mathrm{B}$ crystallin structure (Najmudin et al.) as the search model.

The $\mathrm{x}$-ray structure of bovine $\gamma \mathrm{D}$ crystallin was solved via molecular replacement and refined to a resolution of $1.65 \AA$ and a crystallographic $R$ factor of 0.215 . This structure was fit using the protein sequence derived from the $\gamma \mathrm{D}$-crystallin gene which was recently associated with the fIIIb protein. The asymmetric unit contains two independent 20 $\mathrm{kDa}$ protein molecules with 173 residues each. These structures are compared with structure of other members of the $\gamma$-crystallin family as well as that of the $\beta 2$-crystallin. Despite evidence that oxidation/crosslinking of cysteines contained in $\gamma$-crystallins are linked to the formation of cataracts, all cysteines are clearly in the reduced state. The packing of the protein within the unit cell is examined and residues involved in a large number of contacts are identified. 


\title{
Very High Resolution Data for Phosphate-Binding Protein
}

\author{
Hartmut Luecke1, Florante A. Quiocho² \\ 1SSRL, Stanford, CA \\ ${ }^{2} \mathrm{HHMI}$, Baylor College of Medicine, Houston, TX
}

The structure of phosphate-binding protein (PBP) at $1.7 \AA$ resolution was solved and published in 1990 (Luecke \& Quiocho, Nature 347, 402-406). The molecule is ellipsoid with overall dimensions of $30 \AA \times 40$ $\AA$ × $70 \AA$. Phosphate-binding protein structurally resembles six other periplasmic binding proteins (specific for L-arabinose, Dgalactose, leucine/isoleucine/valine, leucine, sulfate and maltose). The molecule consists of two domains, each with a central $\beta$-sheet flanked by $\alpha$-helices. The domains are connected by a hinge which is composed of three strands and one helix. The substrate is bound in the cleft between the domains. The general folding pattern is parallel $\alpha / \beta$ with the exception of one antiparallel strand in each $\beta$ sheet as a result of the crossover between the domains. The bound phosphate anion is inaccessible to the bulk solvent. It interacts with the protein through 12 hydrogen bonds, five of which have peptide nitrogen donors. Two of the hydrogen bond partners are charged residues (Asp56 and Arg135). Asp56 accepts the $\mathrm{HPO}_{4}=$ proton through a short $(2.45 \AA)$ hydrogen bond whereas Arg135 donates two hydrogens to the substrate. Asp56 has no formal salt-link partner, and the only other interaction of the carboxyl moiety is through a hydrogen bond from a threonine hydroxyl. The guanidinium moiety of Arg135 is salt-linked to Asp137. The remaining four hydrogen bonds are from two serine and two threonine hydroxyls, respectively. All four hydroxyls in turn also accept a hydrogen bond from NH-groups. There are no solvent molecules within $8 \AA$ of the completely sequestered phosphate anion.

We have now collected a complete very high resolution data set to $1.3 \AA$ for phosphate-binding protein from one crystal at $4^{\circ} \mathrm{C}$ at beam line 7-1 at SSRL. The data set contains 84,564 unique reflections, merged from 256,550 independent observations with an RMERGE of $8.4 \%$. We are in the process of refining the data with the program SHELXL (G. Sheldrick, Göttingen). We hope to be able to resolve the protonation state of the bound inorganic phosphate.

More recently we have successfully flashfrozen PBP crystals to $-170^{\circ} \mathrm{C}$ and observed 5 sigma diffraction spots at $1.05 \AA$ resolution. We plan to collect a complete data set for native PBP crystals as well as crystals of sitedirected mutants in the future. This would be the highest resolution structure of a protein this size. PBP has a 321 amino acid residues with a molecular weight of $34.4 \mathrm{kDa}$. 


\title{
Laue Diffraction Experiments on Phosphoglycerate Kinase
}

\author{
T. McPhillips, M. Stowell and D. Rees (Califomia Institute of Technology) \\ S. M. Soltis and R. P. Phizackerley (Stanford Synchrotron Radiation Laboratory)
}

Introduction. PGK, an important ATP generating enzyme in the glycolytic pathway, catalyzes the conversion of 1,3-bisphospho-D-glycerate and ADP to 3-phospho-D-glycerate and ATP under physiological conditions[1]. PGK is a $45 \mathrm{kD}$ molecular weight protein consisting of two domains connected by a hinge-like region [2,3]. A number of recent spectroscopic studies [4-8] support an earlier proposal[9] that the enzyme undergoes a structural change upon substrate binding. Divalent cations (e.g. $\mathrm{Mg}+2$ or $\mathrm{Mn}+2$ ) are required for catalytic turnover and EPR experiments suggest a cationsubstrate intermediate complex is formed after the substrate binds to the enzyme[4]. The goal of the Laue diffraction experiment is to determine the structure of the purported cation-substrate intermediate complex.

One mutant form of PGK, R65Q, slows the turnover rate from tens of milliseconds to seconds in solution. R65Q crystals diffract to $2.4 \mathrm{E}$ resolution and are characterized by a low inherent mosaicity. The crystal structure, which includes caged ATP bound in the active site was determined from monochromatic $x$-ray diffraction data collected at the California Institute of Technology. The CalTech collaborators were apparently successful in releasing caged ATP in the crystal using a high-powered laser. Unexpectedly, they observed a large continuous change in the unit cell parameters over a period of several hours.

Experiment. A similar experiment was performed at SSRL on BL 10-2. White light Laue diffraction data sets were recorded before, and a few seconds after photolysis from a pulsed Nd:Yag laser $(355 \mathrm{~nm})$. Xray exposure times were typically $\sim 200 \mathrm{msec}$. Several additional data sets were collected over 24 hours. Data were recorded on phosphor imaging plates and scanned using a Fuji BAS2000 imaging plate scanner.

Results. The diffraction spots were somewhat streaky for the smaller crystals, however, the patterns could be indexed. The Laue data was processed using the Daresbury Laboratory suite of programs. We found that the unit cell parameters did not change during the experiment. It is possible that the conditions for the two experiments were different. We now believe there was less caged ATP in solution during the Laue experiments. Further, it may be that the caged ATP bound in the active site, cannot be photolyzed and that active ATP in solution may exchange with it. This would be consistent with the observed slow turnover rate. A microspectrophotometer, under construction at CalTech, will be used in future experiments for monitoring the release of caged ATP in the crystal. We plan to perform additional Laue experiments once larger crystals are obtained and once the photolysis step can be monitored.

1. Blake, C.C.F. and Rice, D.W. Phil. Trans. R. Soc. Lond. A 293, 93-104 (1981).

2. H.C. Watson, N.P.C. Walker, P.J. Shaw, T.N. Bryant, P.L. Wendell, L.A. Fothergill, R.E. Perkins, S.C. Conroy, M.J. Dobson, M.F. Tuite, A.J. Kingsman and S.M. Kingsman E.M.B.O. 1, 1635 (1982).

3. H.C. Watson, P.L. Wendell and R.K. Scopes J. Mol. Biol. 57, 623 (1971).

4. D. Latwesen, Ph.D. Thesis Chap. 4, 85 (1992).

5. G. Haran, E. Haas, B.K. Szpikowska and M.T. Mas P.N.A.S. 89, 11764 (1992).

6. L. Mouwad, M. Desmadril, D. Perahia, J.M. Yon and J.C. Brochon, Biopolymers 30, 1151 (1990).

7. W.J. Fairbrother, H.C. Graham and R.J.P. Williams Eur. J. Biochem. 190, 161 (1990).

8. A.A. Timchenko and S.N. Tsyuryupa Biophys. 27,1065 (1982).

9. R.C. Banks, C.C.F. Blake, P.R. Evans, R. Haser, D.W. Rice, G.W. Hardy, M. Merret and A.W. Phillips Nature 279, 773 (1979). 


\title{
Small-Angle X-Ray Scattering Study on Annexin XII Assembly in Solution
}

\author{
H. Tsuruta, ${ }^{*}$ H. Luecke, ${ }^{*}$ W. S. Mailliard, ${ }^{\#}$ H. T. Haigler" \\ Stanford Synchrotron Radiation Laboratory, ${ }^{*}$ University of California at Irvine ${ }^{\#}$
}

Annexin XII, isolated from Hydra vulgaris, is one of a group of proteins called annexins [1]. Annexins bind to phospholipids in a calcium-dependent manner and are considered to play an important role in intracellular calcium signaling. Annexins are found in a wide variety of organisms and their primary structures are conserved throughout evolution except in the $\mathrm{N}$-terminal region, which seems to possess different functions. Important in vitro functions of annexins include ion channel formation and mediation of membrane fusion. However, there is not enough evidence to determine the molecular mechanism of the annexin interacting with the phospholipid bilayers.

Luecke et al. recently solved the crystallographic structure of annexin XII and suggested a model regarding how the protein might interact with phospholipid bilayers [2]. This model is based on the fact that the protein exists as a $\mathrm{Ca}^{2+}$-dependent hexamer in the crystal. It is crucial to see whether the $\mathrm{Ca}^{2+}$-dependent hexamer formation takes place in solution in order to verify physiological importance of this model. We have thus begun studying the solution structure of annexin XII at different $\mathrm{Ca}^{2+}$ concentrations using small-angle $x$-ray scattering,

All small angle scattering data were collected at the SAS facility on SSRL Beam Line 4-2 with a standard Si(111) two-crystal monochromator or alternatively with the Mo-C multilayer monochromator, which provided considerably higher flux. The concentration of the annexin XII solution ranged from 1 to $6 \mathrm{mg} / \mathrm{ml}$. The solutions also contained $1 \mathrm{mM}$ dithiothreitol in order to prevent radiation-induced aggregation of the protein. A sample solution was loaded in a Kel-F sample cell equipped with $10-25 \mu$ thick mica windows. All measurements were made at $15^{\circ} \mathrm{C}$. The sample-to-detector distance was typically around $2.2 \mathrm{~m}$, and a powder diffraction pattern of cholesterol myristate was used to calibrate the values of momentum transfer $h(h=4 \pi \sin \theta / \lambda$ at a scattering angle $2 \theta$ with the x-ray wavelength $\lambda=1.38 \AA$ ). Individual scattering curves were corrected for a slight distortion of detector response, and corresponding blank scattering curves were subtracted.

Figure 1 shows typical solution scattering curves of annexin XII in the absence or presence of $\mathrm{Ca}^{2+}$. In the presence of $\mathrm{Ca}^{2+}$, there is a drastic change in forward scattering intensity $\mathrm{I}_{(\mathrm{h}->0)}$, which is an indication of $\mathrm{Ca}^{2+}$-induced oligomer formation. Also note that there is a broad scattering peak seen around $\mathrm{h}=0.1 \AA$ in the presence of $\mathrm{Ca}^{2+}$. This might suggest that annexin XII forms an oligomer within which monomers are symmetrically arranged.

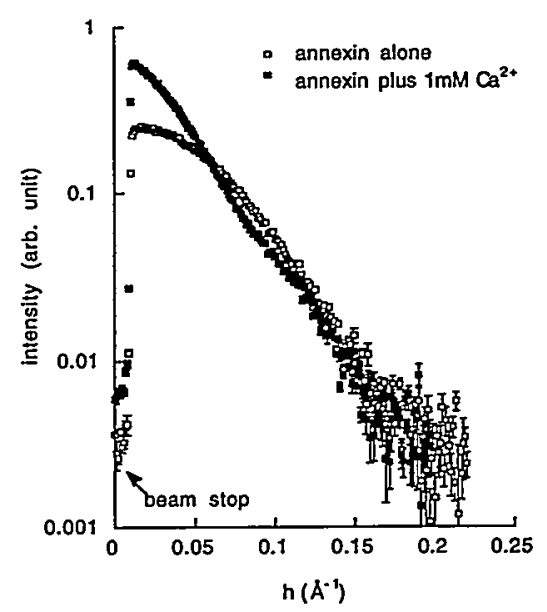

Figure 1. solution scattering curves of annexin in the absence and presence of $\mathrm{Ca}^{2+}$.

The radius of gyration $\left(\mathrm{R}_{\mathrm{g}}\right)$ was evaluated for each scattering curve measured at different $\mathrm{Ca}^{2+}$ concentrations. In the absence of $\mathrm{Ca}^{2+}$, the $\mathrm{R}_{\mathrm{g}}$ slightly depends on protein concentration and ranges from $19.4( \pm 0.4)$ to $22.4( \pm 0.6) \AA$, which are very consistent with the value of $R_{g} 21.6 \AA$ calculated from the annexin monomer in the crystal structure (Table 1). The electron pair distance distribution function $P(r)$ was also calculated from the experimental data obtained without $\mathrm{Ca}^{2+}$. The $\mathrm{P}(\mathrm{r})$ curve looks like that of a monodisperse system, having the largest dimension of the molecule about $70 \AA$ which is in an excellent agreement with that of the monomer in the crystal, though some part of the molecule can be more expanded in solution than in crystal. Also hydrated water surrounding the molecule can make the $\mathrm{Rg}$ slightly larger in solution, and a part of the $P(r)$ curve can be slightly expanded along the $r$ axis. 
Addition of $\mathrm{Ca}^{2+}$ to annexin solutions clearly promotes the formation of oligomeric forms as seen in both $R_{g}$ and $I_{(h->0)}$. This process appears to be reversible, i.e., a further addition of a $\mathrm{Ca}^{2+}$ chelating agent yields essentially monomeric annexin. At a saturating $\mathrm{Ca}^{2+}$ concentration $(1 \mathrm{mM}), \mathrm{R}_{\mathrm{g}}$ depends on annexin concentration: $27.5( \pm 0.5) \AA$ at $3 \mathrm{mg} / \mathrm{ml}$ and $36.2( \pm 0.2) \AA$ at $6 \mathrm{mg} / \mathrm{ml}$. The former value corresponds to that of dimer in crystal and the latter either trimer or hexamer in crystal (Table 1). Analysis of forward scattering intensity $I_{(h->0)}$ supports this possibility, i.e., the largest change in $\mathrm{I}_{(\mathrm{h}}$ $>0$ )/[annexin] is merely a little larger than a factor of two while it would be roughly 3 or 6 in the case of complete trimer or hexamer formation. Thus it is quite likely that annexin XII exists as a mixture of at least two different oligomeric states in solution, depending on its concentration and $\mathrm{Ca}^{2+}$ concentration.

Table 1.

Radii of gyration of oligomers in crystal

\begin{tabular}{ll}
\hline oligomeric state & $R_{\mathrm{g}}(\AA)$ \\
\hline monomer & 21.6 \\
dimer $\dagger$ & 27.2 \\
Ca-dimer $\ddagger$ & 32.2 \\
trimer & 35.1 \\
hexamer & 38.4 \\
\hline$\dagger$ dimer formed side-by-side in trimer \\
$\ddagger$ dimer formed across two trimers, possibly mediated by \\
$\mathrm{Ca}^{2+}$ binding
\end{tabular}

Assuming a mixture of either monomer and trimer, or monomer and hexamer, fraction of monomer forming either trimer $\left(W_{3}\right)$ or hexamer $\left(W_{6}\right)$ was calculated from the values of $R_{g}$ and $I_{(h->0)}$ [3]. Table 2 and 3 summarize $\mathrm{W}_{3}$ and $\mathrm{W}_{6}$, respectively, at different $\mathrm{Ca}^{2+}$ concentrations. Similar values of $\mathrm{R}_{\mathrm{g}}$ were obtained at different $\mathrm{Ca}^{2+}$ concentrations for either assumption. The $\mathrm{Rg}$ values thus obtained for trimer and hexamer are slightly larger than those would be expected for crystal counterparts. It could be due to slightly loose packing of the monomer within the oligomers in solution, compared to that in crystal.

Using the atomic coordinates of the crystal structure, we calculated scattering patterns for different oligomeric states in crystal. None of the scattering curves calculated for the dimer, trimer or hexamer alone fits the experimental scattering curves while a linear combination of the monomer curve and the trimer curve, or a linear combination of the monomer curve and the hexamer curve seems to fit experimental data reasonably well when $\mathrm{W}_{3}$ or $\mathrm{W}_{6}$ is used as a coefficient in the linear combination. Given the fact that the oligomerization is caused by $\mathrm{Ca}^{2+}$ whose binding sites lie between the two trimers in the hexameric structure, hexamer formation is preferentially promoted by $\mathrm{Ca}^{2+}$. This account lends a strong support to the hypothesis that the hexameric structure, as revealed by the crystallographic study, is a physiologically functional form of annexin XII in solution.

Table 2.

Fraction of the monomer forming trimer $\mathrm{W}_{3}$ and evaluated $\mathrm{R}_{\mathrm{g}}$ for trimer

\begin{tabular}{cccc}
\hline [annexin] $(\mathrm{g} / \mathrm{l})$ & {$\left[\mathrm{Ca}^{2+}\right](\mathrm{mM})$} & $\mathrm{W}_{3}$ & $\mathrm{R}_{\mathrm{g}}(\mathrm{A})$ \\
\hline 3.0 & 1.0 & 0.142 & 36.6 \\
4.5 & 1.0 & 0.245 & 37.2 \\
4.5 & 25.0 & 0.558 & 37.1 \\
6.0 & 1.0 & 0.710 & 37.9 \\
\hline
\end{tabular}

Table 3.

Fraction of the monomer forming hexamer $\mathrm{W}_{6}$ and evaluated $R_{g}$ for hexamer

\begin{tabular}{cccc}
\hline$[$ annexin] $(\mathrm{g} / \mathrm{l})$ & {$\left[\mathrm{Ca}^{2+}\right](\mathrm{mM})$} & $\mathrm{W}_{6}$ & $\mathrm{R}_{\mathrm{g}}(\AA)$ \\
\hline 3.0 & 1.0 & 0.057 & 39.5 \\
4.5 & 1.0 & 0.098 & 40.4 \\
4.5 & 25.0 & 0.223 & 40.3 \\
6.0 & 1.0 & 0.284 & 41.2 \\
\hline
\end{tabular}

We hope to establish a solution condition which stabilizes one of the oligomeric states in solution so that detailed structural analysis can be done. Timeresolved scattering experiments using the stoppedflow rapid mixer are also planned to elucidate dynamic aspects of the $\mathrm{Ca}^{2}+$-promoted annexin assembly.

SSRL is funded by the Department of Energy, Office of Basic Energy Sciences. The Biotechnology Program is supported by the NIH, Biomedical Research Technology Program, Division of Research Resources. Further support is provided by the Department of Energy, Office of Health and Environmental Research.

1. M. A. Swairjo \& B. A. Seaton (1994) Annu. Rev. Biophys. Biomol. Struct. 23, 193-213.

2. H. Luecke, B. T. Chang, W. S. Mailliard, D. D. Schlaepfer, \& H. T. Haigler (1995) manuscript in preparation.

3. Z.-X. Wang, H. Tsuruta, Y. Honda, Y. Tachi-iri, K. Wakabayashi, Y. Amemiya, \& H. Kihara (1989) Biophys. Chem. 33, 153-160. 


\title{
Reductive Complexation of Vanadate by Tunichrome: An X-Ray Absorption Spectroscopy Study
}

\author{
Patrick Frank ${ }^{1}$, Daniel Ryan ${ }^{2}$, Kathryn B. Grant ${ }^{2}$, Koji Nakanishi ${ }^{2}$ and Keith O. Hodgson 1,3 \\ 1. Department of Chemistry, Stanford University, Stanford, CA 94305; 2. Department of Chemistry, Columbia University, New \\ York, NY 10027; 3. Stanford Synchrotron Radiation Laboratory, SLAC, Stanford, CA 94309.
}

\section{INTRODUCTION}

Tunichrome is a complex trimer of dopa which occurs in high concentration in the blood cells of marine organisms called tunicates. These organisms exhibit unusual biological chemistry, and have been the subject of increasing study ${ }^{1}$. The structure of tunichrome is shown in Figure 1. Tunichrome has been postulated both as the source of reducing electrons and as a complexing agent for blood cell vanadium, which also occurs in high concentration.<smiles>N[C@@H](Cc1cc(O)c(O)c(O)c1)C(=O)N/C(=C\c1cc(O)c(O)c(O)c1)C(=O)N/C=C/c1cc(O)c(O)c(O)c1</smiles>

Figure 1: The structural formula for tunichrome An-1, as isolated from the blood cells of the tunicate Ascidia ceratodes. Complexation of vanadium would most likely involve the phenolic hydroxyls.

We have shown in previous work that blood cell vanadium is uncomplexed, and exists as the $\mathrm{V}^{3+}$ aquo-ion, most likely in acid $\mathrm{pH}^{2,3}$. Here we describe the use of $\mathrm{x}$-ray absorption spectroscopy (XAS) to probe the reaction of tunichrome with vanadate $\left(\mathrm{VO}_{4}{ }^{3-}\right)$, testing whether this molecule can produce complexed vanadium(III) from vanadate.

\section{MATERIALS AND METHODS}

Tunichrome was isolated as described, and reaction with vanadate and sample preparation was done anaerobically ${ }^{4}$. Data were collected on two independent solid samples on SSRL beamline 7-3 under dedicated operating conditions of $3 \mathrm{GeV}$ and $67 \mathrm{~mA}$ (sample VAn-1,2) or $45 \mathrm{~mA}$ (sample VAn-1) ring current. Wiggler field strength was $18 \mathrm{kG}$. The $\mathrm{X}$-ray beam was energy resolved using a $\mathrm{Si}[220]$ double crystal monochromator. The samples were held at 10K using an Oxford Instruments CF1208 continuous flow helium cryostat. Data were calibrated and normalized as described ${ }^{3}$.

Fits to the data were carried out using the program DATFIT4, which was written by, and obtained from, Dr. Graham George, Stanford Synchrotron Radiation Laboratory, Stanford, CA 94309.

\section{RESULTS}

Two samples were prepared, using two slightly different tunichrome preparations and conditions. Sample VAn-1,2 was prepared using a mixture of tunichrome An-1 and tunichrome An-2. Tunichrome An-2 lacks one external hydroxyl on ring ' $C$ '. The reaction was carried out using $0.88 \mathrm{~mol}$-equivalents of vanadate at $\mathrm{pH} 11$. For the second sample, pure tunichrome An-1 was reacted with $0.8 \mathrm{~mol}$ equivalents of vanadate at $\mathrm{pH} 8.7$, yielding sample VAn-1.

Inspection of the data revealed that the reaction of vanadate with either tunichrome sample produced a complex mixture. Therefore the data were analyzed using a curve-fitting approach. A library of vanadium model complex K-edge XAS spectra was obtained. The vanadium XAS spectra of these models were used to fit the vanadium-tunichrome data The library included the K-edge XAS spectra of vanadium complexes of appropriate oxidation state and ligand array 4 .

In Figure 2 is shown the results of the fit to sample VAn-1. It is clear that vanadate (Figure 2, inset) has been reduced and complexed on reaction with tunichrome. Note the near loss of the vanadate pre-edge feature near $5469 \mathrm{eV}$, in the spectrum of the tunichrome complex. In addition, the $\mathrm{K}$-edge of the 


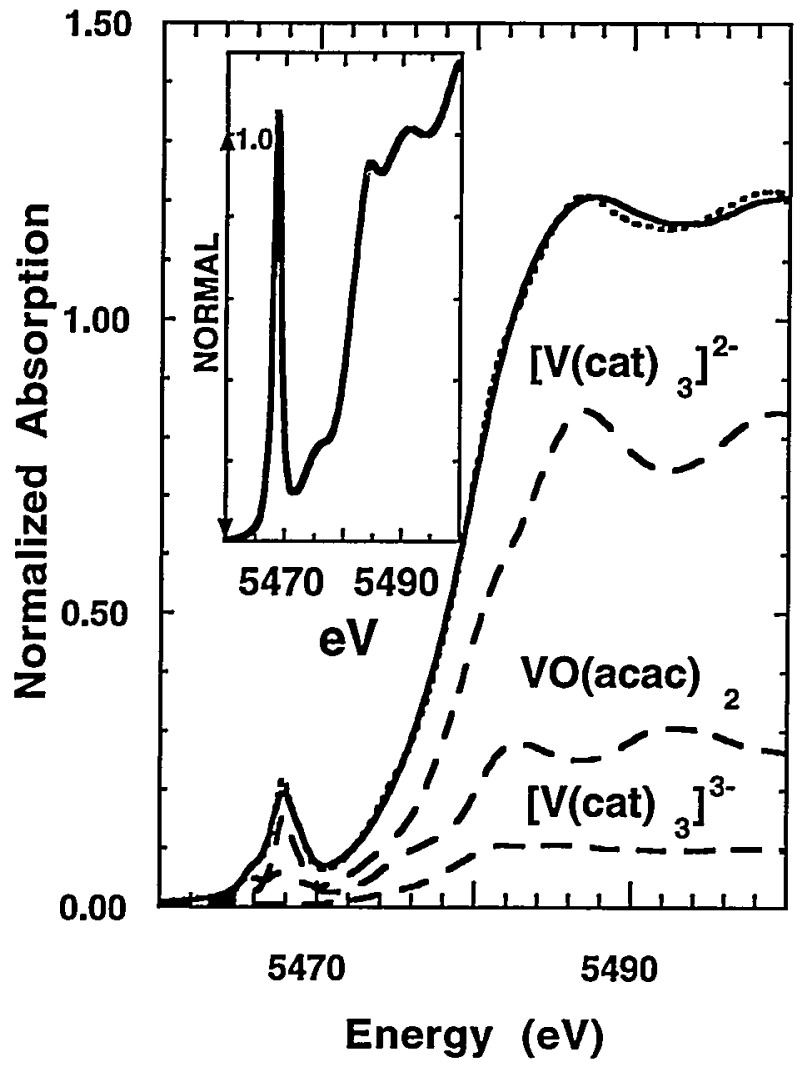

Figure 2. X-ray absorption spectra of: (-) VAn$1 ;(\cdots . \cdot)$ the fit to VAn-1; and (- .-) the three major components of the fit. The identities of the major components are given in the figure. The full component list appears in the Table. Inset: $\mathrm{K}$ edge XAS spectrum of sodium vanadate

product mixture has shifted about $1 \mathrm{eV}$ to lower energy, relative to that of vanadate.

In general, good fits were obtained for both samples. However, since the model complexes cannot reproduce in detail the chemical environment of vanadium in tunichrome, the fits to the data using model complex XAS spectra could not reproduce the details of the shape of the K-edge spectrum of the data. Nevertheless, good fits to the data were obtained. In addition, examination of the second derivative XAS spectra of the data and the fits, indicated that the salient energy positions and multiplicities of the spectral features of the data were reproduced. The results of the fitting experiments for the two data sets are given in the Table.

The major component in each mixture, as implied by the fit, consists of $\mathrm{VIV}_{\text {in a tris-complex }}$ similar to that produced by catechol (1,2-dihydroxybenzene). That is, vanadium(IV) within an octahedral array of phenolic oxygens, and lacking the short oxo ligand. Each mixture also contains about $20 \%$ of, most likely, a bis-chelated vanadyl complex with the vanadium participating in a delocalized oxygen-donating ligand orbital similar to that provided by acetylacetonate. Both reactions produced vanadium in an average oxidation state of IV, demonstrating that the aqueous reductive chemistry of tunichrome cannot produce large amounts of tri-valent vanadium. Therefore, if tunichrome is involved in the biogenic production of vanadium(III), it can only do so either within a nonaqueous regime, or by participation within an enzymatic mechanism, perhaps as a cofactor.

Table

Edge Fit Results

\begin{tabular}{|c|c|c|}
\hline Fit Component & VAn-1 & $\begin{array}{l}\text { VAn- } \\
1,2\end{array}$ \\
\hline$\%\left[\mathrm{~V}^{\mathrm{II}}(\text { catecholate) } 3]^{3-}\right.$ & 9.2 & 1.0 \\
\hline$\%\left[\mathrm{~V}^{\mathrm{IV}}(\text { catecholate) } 3]^{2-}\right.$ & 64.9 & 67.1 \\
\hline$\%\left[\mathrm{~V}^{\mathrm{IV}} \mathrm{O}(\text { catecholate }) 2\right]^{2-}$ & 2.3 & 0.0 \\
\hline$\% \mathrm{~V}^{\mathrm{IV}} \mathrm{O}$ (acetylacetonate) 2 & 23.4 & 19.2 \\
\hline$\% \mathrm{~V}_{\mathrm{O}}($ pivalate) 3 & - & 10.6 \\
\hline$\% \mathrm{HOV}^{\mathrm{V}} \mathrm{O}\left(8 \text {-HOQuin }{ }^{*}\right)_{2}$ & 2.7 & -- \\
\hline $\begin{array}{l}\text { \%Vanadium(III) } \\
\text { \%Vanadium(IV) }\end{array}$ & $\begin{array}{r}9.2 \\
906\end{array}$ & $\begin{array}{r}1.0 \\
863\end{array}$ \\
\hline$\%$ Vanadium(V) & 2.7 & 10.6 \\
\hline $\begin{array}{l}\text { Average Vanadium Oxidation } \\
\text { State }\end{array}$ & $4.04+$ & $4.01+$ \\
\hline
\end{tabular}

* 8-Hydroxyquinolinate

\section{ACKNOWLEDGMENTS}

This work was supported by National Research Service Award GM 14042 (D.R.) and NIH grants GM 08281 (K.G.), GM 10187 (K.N), and RR-01209 (K.O.H) and by NSF CHE-91-21576 (K.O.H). SSRL is supported by the Department of Energy, Office of Basic Energy Sciences, and Office of Health and Environmental Research, and by the National Institutes of Health, National Center for Research Resources

\section{REFERENCES}

1. Metal Ions in Biological Systems, Vol. 31: Vanadium and Its Role for Life, Sigel, H. \& Sigel, A. eds., Marcel Dekker, NY, NY 1995

2. Frank, P., Kustin, K., Robinson, W.E., Linebaugh, L. and Hodgson, K.O. (1995) submitted for publication.

3. Frank, P., Carlson, R.M.K., and Hodgson, K.O. (1986) Inorg. Chem. 25, 470-478.

4. Ryan, D.E., Grant, K.B., Nakanishi, K., Frank, P., and Hodgson, K.O. (1995) submitted for publication. 


\title{
Studies of Lysozyme Folding by Synchrotron Small-Angle X-Ray Scattering
}

\author{
Lingling Chen ${ }^{1}$, Sebastian Doniach ${ }^{2}$ and Keith O. Hodgson ${ }^{1,3}$ \\ 1 Department of Chemistry, Stanford University, Stanford, CA 94305 \\ 2 Department of Applied Physics, Stanford University, Stanford, CA 94305 \\ ${ }^{3}$ Stanford Synchrotron Radiation Laboratory, Stanford, CA 94309
}

\section{Introduction}

Hen egg white lysozyme is one of the best characterized and most studied proteins. It is a small monomeric protein with two domains, termed as $\alpha$ - and $\beta$-domains. The structure is stabilized by four disulfide bridges. There are two proline residues, both with peptide bonds that are trans in the native state(1).

A range of different techniques have been employed in the investigation of folding of lysozyme, and information revealed from these complementary approaches makes it possible to speculate on the nature of the folding pathway of this protein(2). Among knowledge obtained so far, little is known on the shape and the size of the folding intermediates (both equilibrium and transient) of lysozyme. Small-angle $x$-ray scattering (SAXS) provides direct physical information on the size, the shape and the compactness as well as the aggregation state. Further, when combined with the stopped-flow method and with synchrotron sources, SAXS has the potential to probe directly the time-scale of protein folding processes such as compaction, and to detect transient folding intermediates. Here we report initial SAXS studies of lysozyme folding, including static characterization of equilibrium intermediates and time-resolved measurements of the refolding process.

\section{Experimental}

Ten $\mathrm{mg} / \mathrm{ml}$ chicken hen egg-white lysozyme was prepared in $100 \mathrm{mM} \mathrm{NaCl}, 100 \mathrm{mM} \mathrm{Na}$ -citrate, $0-8 \mathrm{M}$ urea at $\mathrm{pH}=2.9$. For native lysozyme at $0 \mathrm{M}$ urea, data were also collected over a range of protein concentrations. This was done to estimate the effect of interparticle interference on the magnitude of $\mathrm{R}_{\mathrm{g}}$ measured at $10 \mathrm{mg} / \mathrm{ml}$. The refolding conditions are described in the legend to Fig. 4.

All samples tested here were allowed to equilibrate for at least 1 hour before SAXS measurement, and were used within 24 hours.

\section{Results And Discussions}

Fig. 1 shows SAXS and CD results of lysozyme unfolding by urea at $\mathrm{pH} 2.9$. The unfolding transition observed by $\mathrm{R}_{\mathrm{g}}$ in SAXS shifts to a lower urea concentration than by the signal at $222 \mathrm{~nm}$ in CD spectroscopy. The shift can be accounted for by different structural information revealed by these two techniques: SAXS probes the global structural compactness, while $C D$ at $222 \mathrm{~nm}$ detects the secondary structure.
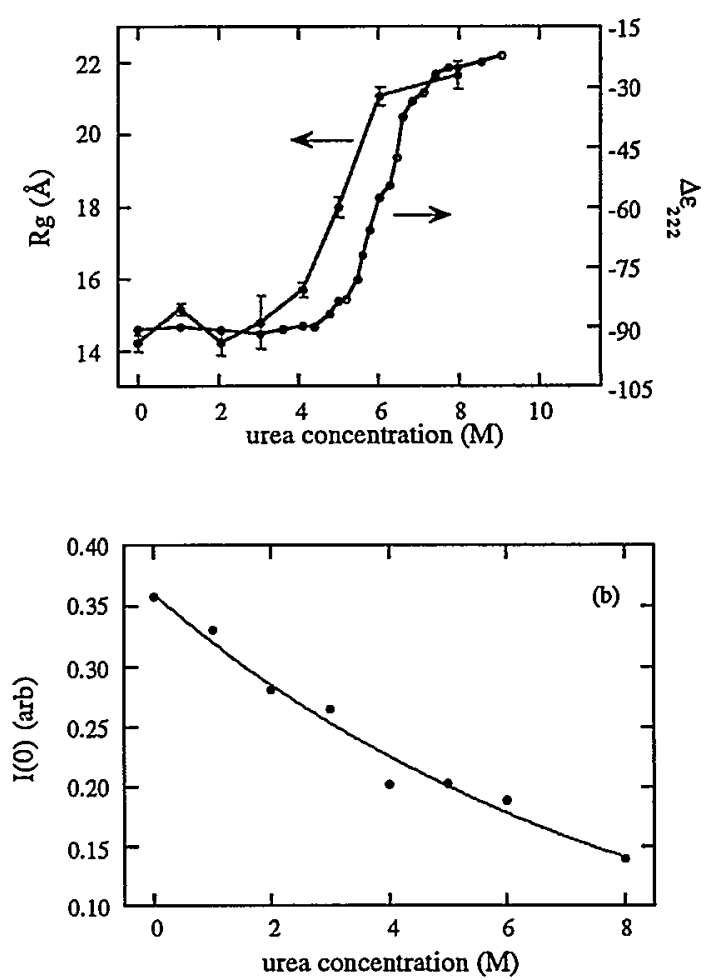

Fig. 1 a) Equilibrium unfolding of lysozyme by urea at $\mathrm{pH}=2.9$ monitored by the radius of gyration $\left(R_{g}\right)(\bullet)$ and by the $C D$ signal at 222 $\mathrm{nm}(\mathrm{O})$. b) The forward scattering intensity $(\mathrm{I}(0))$ as a function of urea concentration.

$\mathrm{R}_{\mathrm{g}}$ of lysozyme in $0 \mathrm{M}$ urea at $10 \mathrm{mg} / \mathrm{ml}$ is measured as $14.2 \AA$, while $R_{g}$ extrapolated to 
zero protein concentration is $15.6 \AA$ (data not shown). Interparticle interference accounts for this $\sim 10 \%$ decrease in $\mathrm{R}_{\mathrm{g}}$. At $8 \mathrm{M}$ urea, $\mathrm{R}_{\mathrm{g}}$ increases to $\sim 22 \AA$. A fully denatured random coil with the same number of amino acid residues could have $R_{g}$ in the range of $36-43 \AA$ (Doniach et al manuscript in preparation), (3). The rather small $R_{\mathrm{g}}$ suggests that lysozyme in 8 $M$ urea is not fully unfolded. After taking into account the change in the electron density due to urea concentration, values of $I(0)$ at different urea concentrations are nearly the same within experimental error. The independence of $\mathrm{I}(0)$ on urea concentration also suggests that sample aggregation is negligible throughout the unfolding process.

A useful SAXS analysis for the folding study is the Kratky plot (4). A well defined peak in the Kratky plot of the native state indicates the presence of a compact globular structure within the folded molecule. The peak diminishes at $8 \mathrm{M}$ urea; however, the Kratky plot is different from that of a fully unfolded chain model (Fig.2). Features in the Kratky plot suggest a picture of random coil loops coexisting with a compact core.

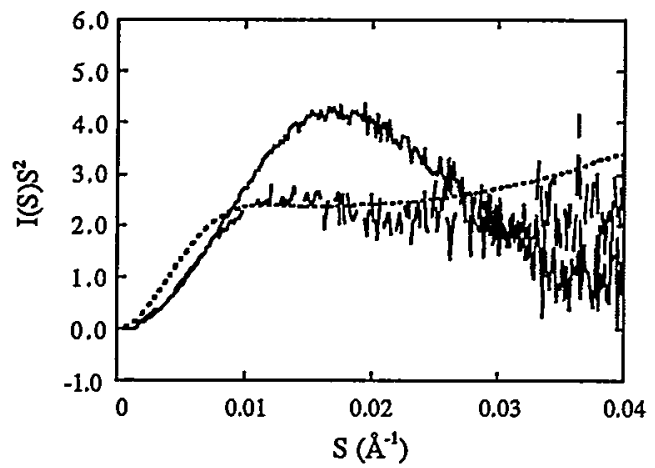

Fig. 2 Kratky plots of lysozyme from SAXS data at $0 \mathrm{M}$ urea (-), at $8 \mathrm{M}$ urea $(-)$, and of a fully unfolded chain (---). A factor of 2.89 is applied to the scattering intensity of the denatured state, which is derived by using 0.423 , 0.343 , and 0.376 as the electron densities of lysozyme, buffer, and $8 \mathrm{M}$ urea in buffer. The model is a chain of 126 links on a cubic lattice whose fully compact structure has a similar $R_{g}$ to that of lysozyme.

The distance distribution $(\mathrm{P}(\mathrm{r})$ ) function for the native state is characteristic of a globular protein, and correlates well with $\mathrm{P}(\mathrm{r})$ computed from the crystal structure (Fig. 3(a)). The shape of the $\mathrm{P}(\mathrm{r})$ function for the denatured state (in 8 M) suggests that the molecules are not completely unfolded. A $\mathrm{P}(\mathrm{r})$ function of a random coil would have a greater density at large distances $(>60 \AA)$, and a larger $D_{\max }(>120 \AA)$ compared with $\sim 80 \AA$ for the sample with $8 \mathrm{M}$ urea (see Fig. 3 (b)).
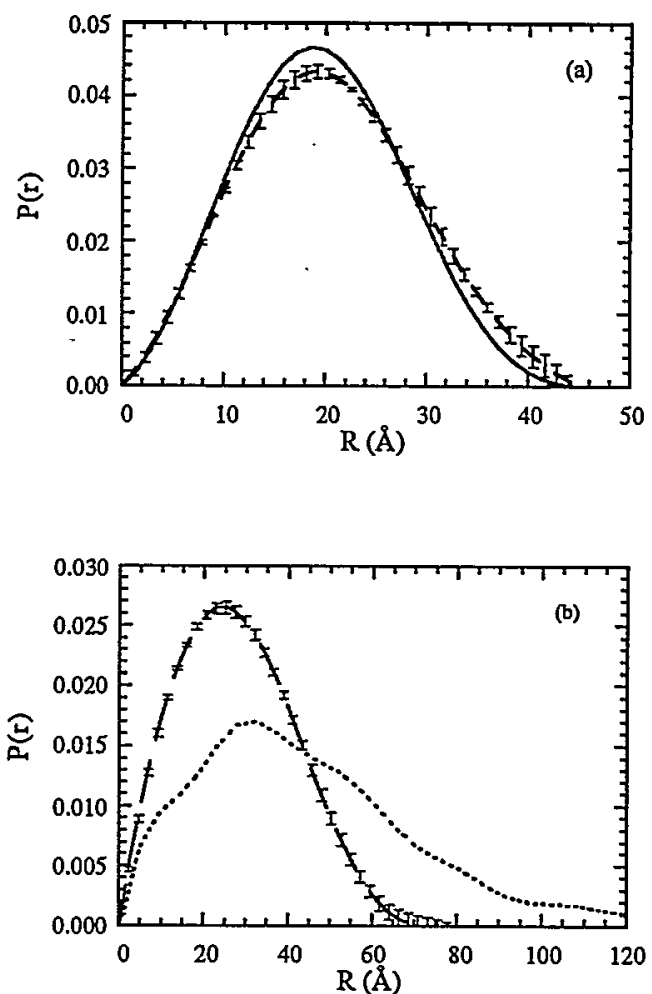

Fig.3 Pair distribution $(\mathrm{P}(\mathrm{r}))$ functions for lysozyme: a) calculated from crystal structure $(-)$, and from SAXS data at $0 \mathrm{M}$ urea $(--) ; \mathrm{b}$ ) from SAXS data at $8 \mathrm{M}$ urea (- ), and a fully unfolded and random-coil-like model (---).

The static SAXS data from $8 \mathrm{M}$ urea sample suggests that lysozyme is not completely unfolded. The non-random coil structure may be attributed to the constraints imposed by the four disulfide bonds, which remain intact in the experiments.

A time course of $\mathrm{R}_{\mathrm{g}}$ in the refolding of lysozyme at $\mathrm{pH}=2.9$ is shown in Fig. 4 . The initial observed $R_{g}$ at $\sim 200 \mathrm{~ms}$ is larger than that of the native state, but smaller than that of the unfolded state. An early compaction event probably occurred within $200 \mathrm{~ms}$. The time course can be fitted with a sum of two exponentials. The time constant of the slower process is several seconds, comparable to the published results $(5,6,7)$. I( 0$)$ during refolding (data not shown) remains nearly constant within experimental errors, indicating that no 
observable dimeric intermediates form, in contrast to that observed in the refolding of myoglobin (8).

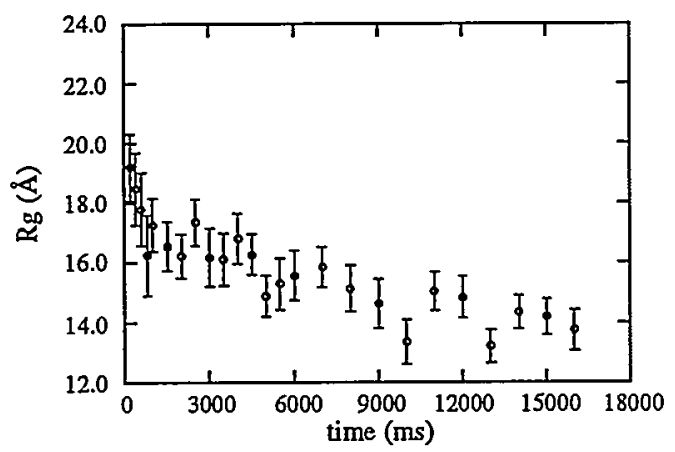

Fig. 4 Refolding kinetics of lysozyme monitored by radii of gyration $\left(R_{g}\right)$. Lysozyme was equilibrated in $8 \mathrm{M}$ urea $\mathrm{pH} 2.9$ at a concentration of $40 \mathrm{mg} / \mathrm{ml}$ for at least 1 hour. The refolding was triggered by a rapid dilution of this denatured sample into a denaturant-free buffer at a ratio of 3 . The final protein concentration was $9.5 \mathrm{mg} / \mathrm{ml}$. Refolding was monitored at various time frames ranging from $20 \mathrm{~ms}$ to $1000 \mathrm{~ms}$, with a total duration time of $16000 \mathrm{~ms}$. Data were accumulated for at least 30 mixing events at a time, and hundreds of separate accumulations were combined. The first $R_{g}$ value shown here is averaged from the first 10 frames of $20 \mathrm{~ms}$.

\section{Acknowledgments}

We would like to acknowledge Dr. Hirotsugu Tsuruta and Dr. David Eliezer for providing assistance in SAXS experiments, and for helpful discussions. We would also like to thank Dr. Dmitri Svergun at EMBL for providing GNOM for indirect transformation, and CRYSOL17 for calculation of $P(r)$ function from crystal structure data. $\mathrm{CD}$ experiments were performed at Dr. Baldwin's laboratory in the Biochemistry Department, Stanford University. This research was supported by NIH Grant RR01209 (to KOH). Data were collected at beam line 4-2 at SSRL which is supported by the U.S. DOE, Office of Basic Energy Sciences, and in part by the NIH NCRR BRTP Program and by the DOE's Office of Health and Environmental Research.

\section{Reference}

1. C. C. F. Blake, D. F. Koenig, G. A. Mair, A. C. T. North, D. C. Phillips and V. R. Sarma, Nature 206, 757-761 (1965).
2. Christopher M. Dobson, Philip A. Evans and Sheena E. Radford, Tibs , 31-37 (1994).

3. Wilmer G. Miller and Carol V. Goebel, Biochemistry 7, 3925-3935 (1968).

4. Mikio Kataoka, Yoshihisa Hagihara, Ken'chi Mihara and Yuji Goto, J. Mol. Biol. , 591596 (1993).

5. Charles Tanford, Kirk C. Aune and Atsushi Ikai, Journal of Molecular Biology 73, 185197 (1973).

6. Shingo Kato, Nobuo Shimamoto and Hiroyasu Utiyama, Biochemistry 21, 38-43 (1982).

7. Kunihiro Kuwajima, Yoshiki Hiraoka, Masamichi Ikeguchi and Shintaro SugaiY, Biochemistry 24, 874-881 (1985).

8. David Eliezer, Kaori Chiba, Hirotsugu Tusuruta, Sebastian Doniach, Keith O. Hodgson and Hiroshi Kihara, Biophysical Journal 65, 912-917 (1993). 


\title{
Vanadium in Blood Cells From Ascidia ceratodes: A Survey Using X-Ray Absorption Spectroscopy
}

\author{
Patrick Frank ${ }^{1}$, Kenneth Kustin ${ }^{2}$, William E. Robinson ${ }^{3}$, Linda Linebaugh ${ }^{2}$, and Keith O. \\ Hodgson 1,4
}

1. Department of Chemistry, Stanford University, Stanford, CA 94305; 2. Department of Chemistry, Brandeis University, Waltham, MA 02254; 3. Environmental Sciences Program, University of Massachusetts-Boston, Boston, MA 02125; 4. Stanford Synchrotron Radiation Laboratory, SLAC, Stanford, CA 94309

\section{INTRODUCTION}

In previous work ${ }^{1,2}$, we have reported on the results of x-ray absorption spectroscopy (XAS) studies on vanadium within the blood cells of the marine Urochordate, Ascidia ceratodes. This organism filters oceanic vanadate, concentrates it within blood cells by a factor of 1-2 million, and reduces the metal from $\mathrm{V}^{5+}$ to air-sensitive $\mathrm{V}^{3+}$. We have found, using XAS, that this metal exists within blood cells as the aquo-ion. EPR studies indicate the endogenous storage regime is most likely acidic, $\mathrm{pH} 1.8$ sulfuric acid, wherein vanadium(III) is resistant to autoxidation ${ }^{3}$.

Here we report on the extension of this work, in order to determine the generality of the result. In addition, we have combined this study with a count of blood cells, in order to correlate the XAS results with blood cell population.

\section{MATERIALS AND METHODS}

Ascidia ceratodes were collected at the Monterey Yacht Harbor, Monterey, CA, by Sea Life Supply Co., Sand City, CA 93955. Blood was obtained, and prepared as described ${ }^{2}$.

XAS data were collected on 8-pole wiggler beamline 7-3 under dedicated operating conditions of $3 \mathrm{GeV}, 50 \mathrm{~mA}$ current, and a wiggler field of $18 \mathrm{kG}$. The $\mathrm{x}$-ray beam was energy resolved using a $\mathrm{Si}[220]$ monochromator, detuned $50 \%$ at $6336 \mathrm{eV}$. Transmission data were collected using $\mathrm{N}_{2}$-filled detectors. Data were calibrated and normalized as described ${ }^{2}$.

\section{RESULTS}

In the Table below, blood cell counts and vanadium contents of the blood cell samples are given. All the samples except VKR4 are comparable in overall count and vanadium concentration. In Figure 1, is shown the vanadium K-edge XAS spectrum of all four samples. The XAS spectrum of sample VKR4, though less intense, has the same general edge shape as that of the others. The close similarity of these XAS spectra with that of V(III) in $\mathrm{pH}$ 1.8 sulfuric acid supports the idea that this ion is the predominant form of intracellular vanadium.

\section{Blood Cell Count and Vanadium Content}

\begin{tabular}{llllll}
\hline $\begin{array}{l}\text { Blood } \\
\text { Cell } \\
\text { Sample }\end{array}$ & $\begin{array}{l}\text { [V]avg } \\
(\mu \mathrm{mol})\end{array}$ & $\begin{array}{l}\text { \%MR } \\
\text { Cells } 1\end{array}$ & $\begin{array}{l}\text { \%SR } \\
\text { Cells }^{2}\end{array}$ & $\begin{array}{l}\text { \%CC } \\
(\mathrm{PV})^{3}\end{array}$ & $\begin{array}{l}\% \\
\text { Other } \\
\text { Cell } \\
\text { Types }\end{array}$ \\
\hline VKR1 & 4.40 & 40.5 & 11.1 & 26.1 & 22.3 \\
VKR2 & 5.97 & 36.1 & 5.3 & 21.1 & 37.5 \\
VKR3 & 4.20 & 39.5 & 6.5 & 29.7 & 24.3 \\
VKR4 & 0.70 & 52.0 & 11.9 & 16.6 & 19.5 \\
\hline
\end{tabular}

1. Morula Cells; 2. Signet Ring Cells; 3. Compartment Cells (predominantly vacuolar), a type of signet ring cell.

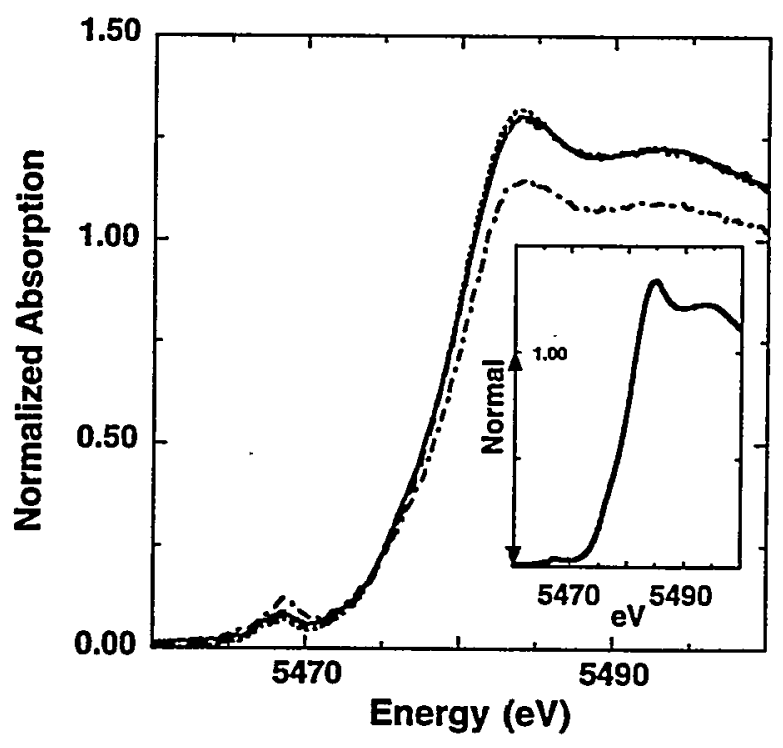

Figure 1: V-K XAS spectra of sample: (-) VKR1; (- - -) VKR2; ( - *) VKR3; (-. -) VKR4. Inset: V-K XAS edge spectrum of $0.1 \mathrm{M}$ V(III) ion in $\mathrm{pH} 1.8$ sulfuric acid solution.

Figure 2 shows the first derivative XAS spectra of the blood cell samples of Figure 1. All display a dispersion-shaped feature near 5475.5 $\mathrm{eV}$. In the inset, is the first derivative of the XAS spectrum of V(III) in $\mathrm{pH} 1.8$ sulfuric acid solution. This spectrum shows the same feature at $5475.5 \mathrm{eV}$ as the blood cell spectra (arrows). 
The $5475.5 \mathrm{eV}$ shoulder has been ascribed to the interaction of vanadium(III) with sulfate ion, and is apparently not present in the XAS edge spectrum of an $\mathrm{HCl}$ solution of V(III) chloride ${ }^{4}$. On cell lysis, the $5475.5 \mathrm{eV}$ shoulder disappears, consistent with dilution of the blood cell contents and the concomitant loss of the vanadium-sulfate interaction.

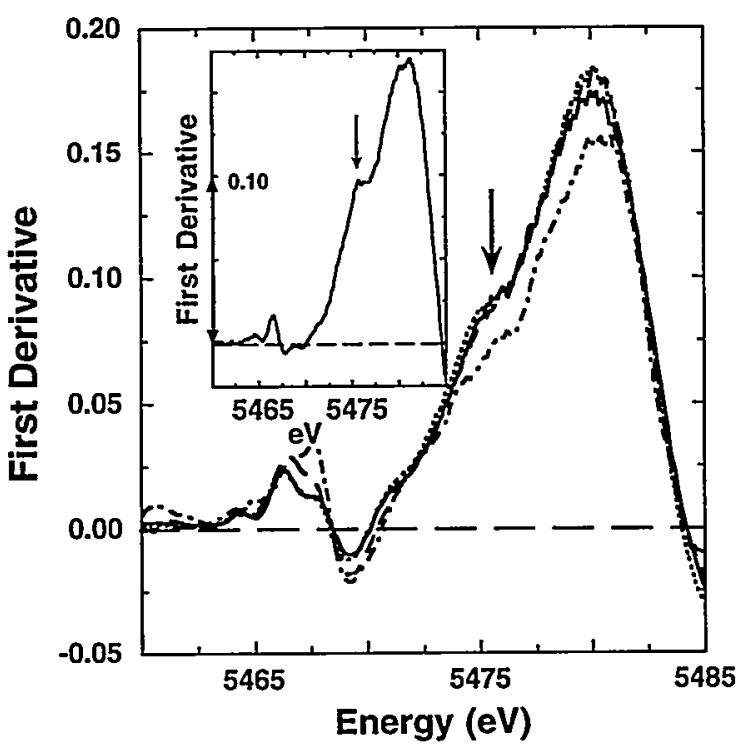

Figure 2: First derivative of the V-K XAS spectra of sample: (-) VKR1; (- - ) VKR2; (‥) VKR3; and (-. -) VKR4. Inset: First deriv-ative of the V-K XAS edge spectrum of $0.1 \mathrm{M} \mathrm{V}^{3+}$ in $\mathrm{pH} 1.8$ sulfuric acid.

Ascidia ceratodes blood cells are known to be rich in sulfate and other sulfur-containing materials. An interaction between endocytic sulfate and vanadium(III) has been imputed previously on the basis of sulfur K-edge XAS investigations of similar blood cells 5 . The data herein, for the first time, show this interaction from the point of view of endogenous V(III).

Figure 3 shows the excellent correspondence of the V-K EXAFS spectrum of sample VKR2 with that of V(III) ion in $9 \mathrm{M}$ sulfuric acid. The latter spectrum is consistent with a ligand sphere of six water molecules at $1.99 \pm 0.02 \AA$ from the vanadium absorber ${ }^{2}$.

Therefore, with this report, a total of at least seven samples of Ascidia ceratodes blood cells, from three independent collections have been examined 1,2 . Each XAS sample represents up to thirty individual animals. All these data strengthen the notion that blood cell vanadium ion is $\geq 90 \%$ trivalent, coordinated by water

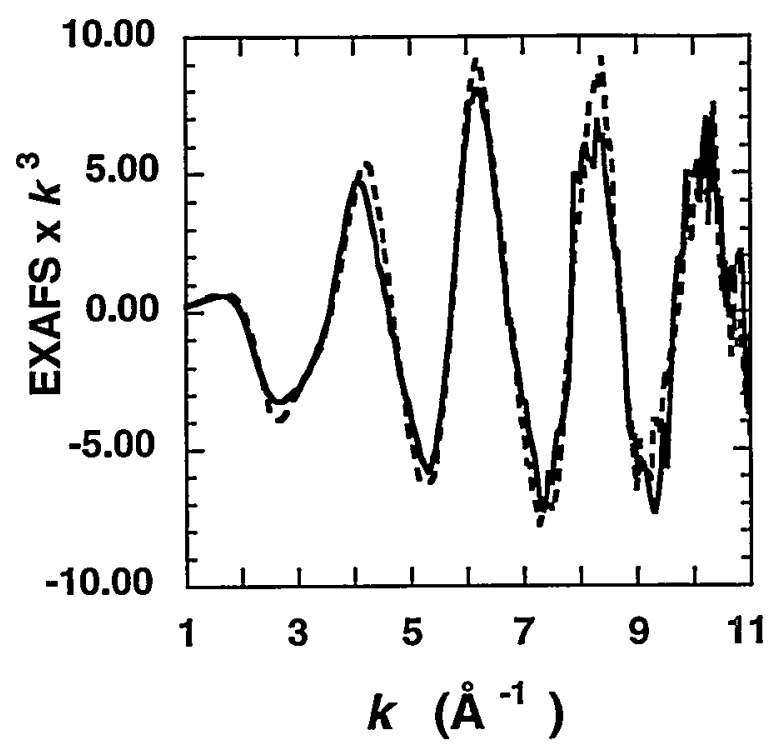

Figure 3. V-K EXAFS spectra of: $(-)$ sample VKR2; ( $\cdots)$ 0.1 M V(III) in $9 \mathrm{M}$ sulfuric acid.

molecules, and able to interact with endogenous dissolved sulfate ion.

In all cases whole blood cell samples were examined. This means that all blood cell types were surveyed for vanadium environment. Thus, these data strongly imply that only less than $10 \%$ of all blood cell vanadium can be other than as described above, no matter the cell type wherein it may reside.

\section{ACKNOWLEDGMENTS}

We thank the researchers in the laboratories of Prof. G. Wallace, University of Massachusetts, for performing the vanadium analyses. This work was supported by MIT Sea Grant G92-RB-33 (to K.K. and W.E.R), by RR-01209 and NSF CHE-91-21576 (to K.O.H.). SSRL is supported by the Department of Energy, Office of Basic Energy Sciences, and Office of Health and Environmental Research, and by the National Institutes of Health, National Center for Research Resources

\section{REFERENCES}

1 Tullius, T.D.; Gillum, W.O.; Carlson, R.M.K.; Hodgson, K.O. (1980) J. Am. Chem. Soc. 102, 5670-5676.

2. Frank, P.; Kustin, K.; Robinson, W.E.; Linebaugh, L.; Hodgson, K.O. (1995) submitted for publication.

3. Frank, P.; Carlson, R.M.K.; Hodgson, K.O. (1986) Inorg. Chem. 25, 470-478.

4. Miyanaga, T.; Watanabe, I.; Ikeda, S. (1990) Bull. Chem. Soc. Jpn 63, 3282-3287.

5. Frank, P.; Hedman, B.; Carlson, R.M.K.; Hodgson, K.O. (1994) Inorg. Chem. 33, 3794-3803. 


\title{
Angle Dependence of Multiple-Scattering Effects in EXAFS As Seen in Cytochrome Oxidase Analogues
}

\author{
Hua H. Zhang1, Michael J. Scott ${ }^{2}$, Sonny C. Lee ${ }^{2}$, Britt Hedman ${ }^{3}$, \\ Richard H. Holm ${ }^{2}$, and Keith O. Hodgson ${ }^{1,3}$ \\ ${ }^{1}$ Department of Chemistry, Stanford University, Stanford, CA 94305 \\ 2 Department of Chemistry, Harvard University, Cambridge, MA 02138 \\ ${ }^{3}$ Stanford Synchrotron Radiation Laboratory, Stanford University, Stanford, CA 94309
}

\section{Introduction}

Cytochrome $c$ oxidase catalyzes the fourelectron reduction of dioxygen to water in the final step of aerobic metabolism. ${ }^{1}$ The enzyme consists of two heme and two copper sites, of which the binuclear heme $a_{3}-\mathrm{Cu}_{\mathrm{B}}$ center is the oxygen binding and reduction site. We have synthesized two molecular bridged complexes to model the binuclear site, $\mathrm{Fe}^{\mathrm{III}}-\mathrm{X}-\mathrm{Cu}$ II with the bridging ligand $\mathrm{X}=\mathrm{O}^{2-}$ or $\mathrm{OH}^{-}$. The two complexes have in common similar structural fragments around each metal, but differ significantly in the bridge angle, $\mathrm{Fe}-\mathrm{X}$ $\mathrm{Cu}$. The hydroxo complex has a bridge angle of $155.6^{\circ}$, whereas the oxo complex has a linear structure. This provides an especially interesting and challenging case for multiple-scattering (MS) EXAFS analysis. ${ }^{2}$ I n particular, there is a significant amplitude enhancement when a backscatterer and an intervening atom form a linear or close-to-linear relationship with the photoabsorber (as in $\mathrm{Fe}-\mathrm{O}-\mathrm{Cu}$ ).

XAS Fe K-edge data of the two complexes have been collected and analyzed using GNXAS MS protocol. $^{3}$ A comparative study has been made to show the angle dependence of MS effects and to address the accuracy of determination of bond distances and angles.

\section{Experimental}

EXAFS transmission samples of the two complexes were run at the Stanford Synchrotron Radiation Laboratory on unfocused beamline 7-3 under dedicated conditions $(3.0 \mathrm{GeV}, 60-100 \mathrm{~mA})$. A Si(220) double-crystal monochromator was used and the radiation was detuned $50 \%$ at $7987 \mathrm{eV}$ to minimize the harmonic contamination. The data were collected at $10 \mathrm{~K}$ using an Oxford Instruments continuous-flow LHe cryostat (model CF1208).

The data analysis was performed using the package GNXAS which has been described in detail in the studies of heterometal Mo-Fe-S clusters ${ }^{4}$ and $\mathrm{Fe}-\mathrm{NO}$ complexes. ${ }^{5}$ EXAFS signals for each of the 2-body and 3-body configurations were calculated based on crystallographic distances and bond angles. These individual signals, including MS contributions, were summed to generate the total theoretical EXAFS spectrum which was then fit to the experimental data by varying structural parameters including distance, angle and Debye-Waller (DW) factor.

\section{Results and Discussion}

The EXAFS Fourier transforms (FT) spectra of the two complexes are presented in Figure 1. The theoretical EXAFS signal matches quite closely the experimental data in both cases. The low-frequency EXAFS is well accounted for by the two single scattering components, $\mathrm{Fe}-\mathrm{O}$ and $\mathrm{Fe}-\mathrm{N}$ (porphyrin) and they give rise to the first peak in the FT at $\sim 1.7 \AA$ (oxo) and at $\sim 1.8$ $\AA$ (hydroxo). The calculated first shell distances show excellent agreement with the crystallographic values, deviating by less than $0.01 \AA$ in both cases (Table I).

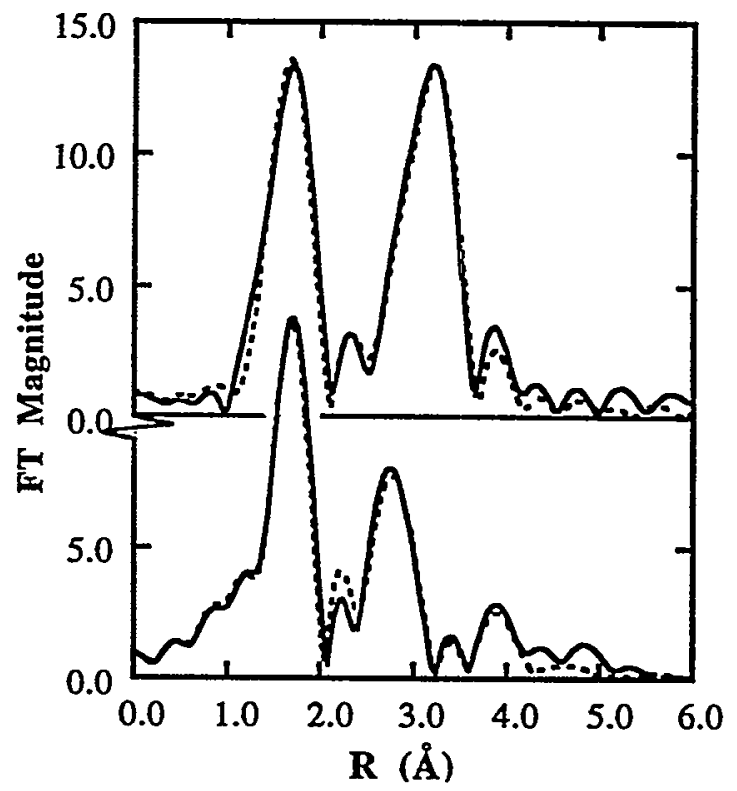

Figure 1. Fourier transforms of the experimental data (-) compared with those of the theoretical signal (...) for oxo (upper) and hydroxo (lower) complexes. The Fourier transforms were performed over the $k$-range of 4.4-14.8 $\AA^{-1}$ and are not phase-shift corrected. 


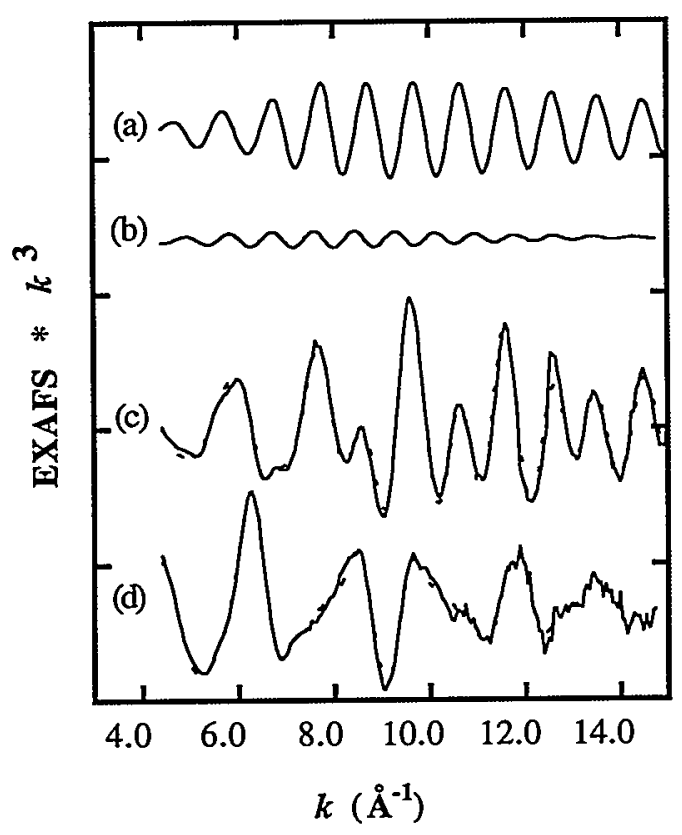

Figure 2. EXAFS signals for $\mathrm{Fe}-\mathrm{X}-\mathrm{Cu}$ contributions for the oxo (a) and for the hydroxo (b), and comparison of the experimental EXAFS data $(-)$ with the total theoretical signals (...) for the oxo (c) and for the hydroxo (d). (The ordinate scale is 10 between successive marks.)

Table I. GNXAS distances and angles for the oxo and the hydroxo complexes, with the crystallographic data shown for comparison.

\begin{tabular}{ccc}
\hline \hline & Oxo & Hydroxo \\
\hline Structural Feature & Cryst. Value & Cryst. Value \\
GNXAS Value & GNXAS Value \\
\hline Fe-O & $1.745 \AA$ & $1.929 \AA$ \\
$(1.75 \pm 0.003) \AA$ & $(1.93 \pm 0.009) \AA$ \\
\hline Fe-N(porphyrin) & $2.110 \AA$ & $2.042 \AA$ \\
$(2.11 \pm 0.002) \AA$ & $(2.04 \pm 0.003) \AA$ \\
\hline \multirow{F}{*}{$\mathrm{Fe} . . \mathrm{Cu}$} & $3.570 \AA$ & $3.804 \AA$ \\
$\mathrm{Fe}-\mathrm{O}-\mathrm{Cu}$ & $(3.57 \pm 0.003) \AA$ & $(3.89 \pm 0.01) \AA$ \\
\hline \hline
\end{tabular}

A dramatic difference is seen in the outer shell region of the EXAFS FTs. In the oxo case, the FT peak at $\sim 3.1 \AA$ is unusually high, having nearly the same magnitude as the first shell peak. This feature compares sharply with the small FT peak at $\sim 3.4 \AA$ in the hydroxo case. This difference is attributed to the different MS contributions from $\mathrm{Fe}-\mathrm{O}-\mathrm{Cu}$ 3-body signals in the two complexes. It is evident from Figure 2 that there is a large amplitude enhancement of this signal for oxo due to its linear configuration. As a result of the strong MS effects, the $\mathrm{Fe}-\mathrm{O}-\mathrm{Cu}$ signal has become the major contributor to the FT peak at $3.1 \AA$. The various fits show that a good fit can be obtained only when the Fe$\mathrm{O}-\mathrm{Cu}$ MS contribution is included. The strong signal makes it possible to accurately determine the structural parameters in the binuclear site for the oxo complex. The calculated distance for $\mathrm{Fe}-\mathrm{Cu}$ is $3.57 \AA$ as compared with the crystallographic distance of $3.570 \AA$ and the angle of $\mathrm{Fe}-\mathrm{O}-\mathrm{Cu}$ is $172^{\circ} \mathrm{vs} .175 .3^{\circ}$ from the crystal structure (Table I).

In contrast, the hydroxo complex has a much weaker Fe-O-Cu signal and contribute less significantly to the total EXAFS signal (Fig. 2(b)). The inclusion of this contribution did, however, improve the fit and the goodness-of-fit value ${ }^{4}$ decreased by $\sim 25 \%$. A comparison of the GNXAS distances and angles with the crystallographic data indicates that the relatively weak $\mathrm{Fe}-\mathrm{O}-\mathrm{Cu}$ signal gives a less accurate determination of the structural parameters. The $\mathrm{Fe}-\mathrm{Cu}$ distance is determined to be $3.89 \AA$ compared to the crystallographic value of $3.804 \AA$. The Fe-O-Cu angle is calculated to be $161.0^{\circ}$, deviating by $4.0^{\circ}$ from the crystallographic value.

\section{Conclusion}

The structural differences of the oxo and the hydroxo complexes give rise to dramatic changes in their EXAFS spectra. GNXAS analysis quantifies the sensitivity of the MS signals to the geometry of the bridging configuration between the metals. As published earlier for Fe-NO angle determination ${ }^{5}$, the technique is relatively sensitive when the angle approaches linearity, but at angles much below about $160^{\circ}$ in these types of complexes (which include significant MS pathways in the porphyrin framework), angle determination becomes less accurate and ultimately unreliable. It is also possible to study the metal-metal assembly from the Cu EXAFS view point where the porphyrin MS effects will be less dominant. This work is currently underway.

\section{Acknowledgments}

This research was supported by grants from the NSF CHE 91-21576 and the NIH RR-01209 (to K.O.H.) and by NSF CHE 92-08387 (to R.H.H.) The Stanford Synchrotron Radiation Laboratory is supported by the Department of Energy, Office of Basic Energy Sciences, Divisions of Chemical and Materials Sciences, and in part by the National Institutes of Health, National Center for Research Resources, Biomedical Research Technology Program, and by DOE's Office of Health and Environmental Research. 


\section{References}

(1) (a) Babcock, G. T.; Wikström, M. Nature 1992, 356, 301. (b) Chan, S.I.; Li, P.M. Biochem. 1990, 29, 1. (c) Malmström, B. G. Chem. Rev. 1990,90, 1247.

(2) The angle dependence of MS effects has been known for some time and, in favorable cases, has been used to obtain angular information (see eg. Teo, B. K. J. Am. Chem. Soc. 1981, 103, 3990 and Co, M. S.; Hendrickson, W. A.; Hodgson, K. O.; Doniach, S. J. Am. Chem. Soc. 1983, 105, 1144).

(3) (a) Filipponi, A.; Di Cicco, A.; Tyson, T. A.; Natoli, C. R. Solid State Commun. 1991, 78, 265. (b) Filipponi, A.; Di Cicco, A. Synch. Rad. News 1992, 6, 13.

(4) Nordlander, E.; Lee, S. C.; Cen, W.; Wu, Z. Y.; Natoli, C. R.; Di Cicco, A.; Filipponi, A.; Hedman, B.; Hodgson, K. O.; Holm, R. H. J. Am. Chem. Soc. 1993, 115, 5549.

(5) Westre, T. E.; Di Cicco, A.; Filipponi, A.; Natoli, C.R.; Hedman, B.; Solomon, E. I.; Hodgson, K.O. J. Am. Chem. Soc. 1994, 116, 6757. 
Proposal 8116B

\title{
DETERMINATION OF THE GEOMETRIC AND ELECTRONIC STRUCTURE OF ACTIVATED BLEOMYCIN USING X-RAY ABSORPTION SPECTROSCOPY
}

\author{
Tami E. Westre, ${ }^{1}$ Kelly E. Loeb, ${ }^{1}$ Jeffrey M. Zaleski, ${ }^{1}$ \\ Britt Hedman, 1,2 Keith O. Hodgson, ${ }^{1,2}$ and Edward I. Solomon 1 \\ 1 Department of Chemistry, Stanford University, Stanford, CA 94305, USA \\ 2 Stanford Synchrotron Radiation Laboratory, Stanford University, SLAC, \\ MS 69, PO Box 4349, Stanford, CA 94309, USA
}

\section{Introduction}

Bleomycin (BLM), a glycopeptide antibiotic produced by strains of Streptomyces verticillus, is currently used in treatment against a variety of carcinomas and lymphomas. ${ }^{1}$ Its therapeutic activity involves selective DNA cleavage at certain GT and GC sites in the presence of metal ions (in particular $\mathrm{Fe}^{2+}$ ) and dioxygen. ${ }^{2,3}$ The coordination environment of the iron is believed to be square pyramidal with the iron ligated to 5 nitrogens from histidine, pyrimidine, a deprotonated peptide function, and primary and secondary amine groups. ${ }^{4}$ Kinetic and spectral studies have demonstrated that the activation mechanism involves high spin $\mathrm{Fe}(\mathrm{II}) \mathrm{BLM}$ reacting with dioxygen and an electron to form activated BLM which is formally at the peroxoferric level. ${ }^{5}$ Activated BLM (and the analogous PMA model complex) ${ }^{6}$ is the first mononuclear nonheme iron oxygen intermediate stable enough for detailed spectroscopic study. DNA degradation by activated BLM involves $\mathrm{C}-\mathrm{H}$ bond cleavage at the C4' of deoxyribose moieties and results in the production of base propenals. ${ }^{3,7}$ This mechanism is similar to the monooxygenation mechanism of cytochrome P-450 and has led researchers to postulate an oxo-ferryl BLM intermediate, ${ }^{3}$ as has been generally considered to be present in P-450 chemistry. Alternatively, spectroscopic model studies $^{6,8 a}$ and mass spectrometric studies ${ }^{8 b}$ have indicated activated BLM to have an iron (III)peroxide site. In this study, X-ray absorption spectroscopy (XAS) has been used to directly probe the oxidation and spin state of the iron in activated BLM and to determine if a short iron-oxo bond is present, which would be characteristic of the oxoferryl species of heme iron. Both the pre-edge and edge regions of the $\mathrm{Fe} \mathrm{K}$-edge spectra indicate that activated BLM is a low spin ferric complex. Bond distances obtained from EXAFS are similar to those in low spin Fe(II)BLM and show no evidence for a short iron-oxo bond. These data are consistent with activated BLM being a peroxy-low spin ferric complex.

\section{Experimental}

Blenoxane (a mixture of $60 \%$ BLM-A $\mathrm{A}_{2}, 30 \%$ BLM$\mathrm{B}_{2}$, and $10 \%$ other BLMs) was obtained as a gift from Bristol-Meyers Squibb and used without further purification. For all XAS samples a $5 \mathrm{mM}$ apo-BLM solution (equal volumes of $300 \mathrm{mM}, \mathrm{pH} 7.0$, HEPES buffer and ethylene glycol) was prepared. Subsequent anaerobic and aerobic additions of $5 \mu \mathrm{L}$ of a Fe( $\left(\mathrm{NH}_{4}\right)_{2}\left(\mathrm{SO}_{4}\right)_{2} \cdot 6 \mathrm{H}_{2} \mathrm{O}$ stock solution yielded $\sim 4$ $\mathrm{mM} \mathrm{Fe}$ (II)BLM and $\mathrm{Fe}$ (III)BLM, respectively. Sample integrity was confirmed by optical absorption and electron paramagnetic resonance (EPR), the latter indicated ferric impurities at less than 5\%. Activated BLM was formed from the reaction of low spin $\mathrm{Fe}(\mathrm{III}) \mathrm{BLM}$ with $\mathrm{H}_{2} \mathrm{O}_{2},{ }^{5}$ because the reaction of $\mathrm{Fe}(\mathrm{II}) \mathrm{BLM}$ with $\mathrm{O}_{2}$ yields a 50:50 mixture of activated BLM and low spin Fe(III)BLM. The intermediate was prepared by rapid addition of 3.8 $\mu \mathrm{L}$ of $\mathrm{H}_{2} \mathrm{O}_{2}$ (100-fold excess) to $80 \mu \mathrm{L} \mathrm{Fe}$ (III)BLM followed by simultaneous freezing in the XAS sample cell and a $3 \mathrm{~mm}$ EPR tube, after a $25 \mathrm{sec}$ incubation. The concentration was determined by EPR spin quantitation resulting in a sample composed of $81(4) \%$ activated BLM, 7(1)\% low spin Fe(III)BLM, 4(1)\% high spin ferric, and $8(1) \% \mathrm{Fe}$ degradation product undetectable by EPR at $77 \mathrm{~K}$. The degradation product of the $\mathrm{Fe}(\mathrm{III}) \mathrm{BLM}-\mathrm{H}_{2} \mathrm{O}_{2}$ reaction was generated by allowing activated BLM to decay for 12 hours producing $8 \%$ high spin ferric with the remaining $92 \% \mathrm{Fe}$ EPR silent at $77 \mathrm{~K}$.

Fe K-edge X-ray absorption spectra were recorded at the Stanford Synchrotron Radiation Laboratory (SSRL). ${ }^{9}$ Standard pre-edge background subtraction and spline fitting techniques were used to normalize the data. ${ }^{10-13}$ EXAFS analysis using empirical phase and amplitude parameters was performed to obtain first shell fits. ${ }^{10-14}$ Contributions to the XAS signal due to low spin Fe(III)BLM and degradation product were subtracted from the total XAS intensity to obtain a $96 \%$ activated BLM edge spectrum. The remaining $4 \%$ corresponds to a high spin ferric impurity for which no reference is available. The $96 \%$ activated BLM edge spectrum was identical in 
shape to that of the $81 \%$ pure sample, however, the edge shifted $0.7 \mathrm{eV}$ to higher energy. The $96 \%$ activated BLM spectrum was used in the edge analysis, whereas the original $81 \%$ activated BLM spectrum was used in the EXAFS analysis.

\section{Results and Discussion}

The Fe-K edge spectra of Fe(II)BLM, Fe(III)BLM, and activated BLM are shown in Figure 1A, where $1 \mathrm{~B}$ shows an expanded view of the $1 \mathrm{~s} \rightarrow 3 \mathrm{~d}$ pre-edge region. The lowest energy peaks arise from the weak $1 \mathrm{~s} \rightarrow 3 \mathrm{~d}$ transition at $\sim 7113 \mathrm{eV}$ followed by the $1 \mathrm{~s}$ $\rightarrow 4 p$ transition at $\sim 7122 \mathrm{eV}$. The spectrum of $\mathrm{Fe}$ (II)BLM has two low-intensity pre-edge peaks at 7111.4 and $7113.6 \mathrm{eV}$. The data for both Fe(III)BLM and activated BLM have similar pre-edge features, each with a maximum at $7112.5 \mathrm{eV}$ with similar intensity, a barely resolvable low energy shoulder for $\mathrm{Fe}$ (III)BLM at $7111 \mathrm{eV}$, and a sloping tail in this lower energy region in activated BLM. The rising edge inflection points occur at $7121.3 \mathrm{eV}$ for $\mathrm{Fe}$ (II)BLM, $7127.0 \mathrm{eV}$ for Fe(III)BLM, and 7127.3 $\mathrm{eV}$ for activated BLM.

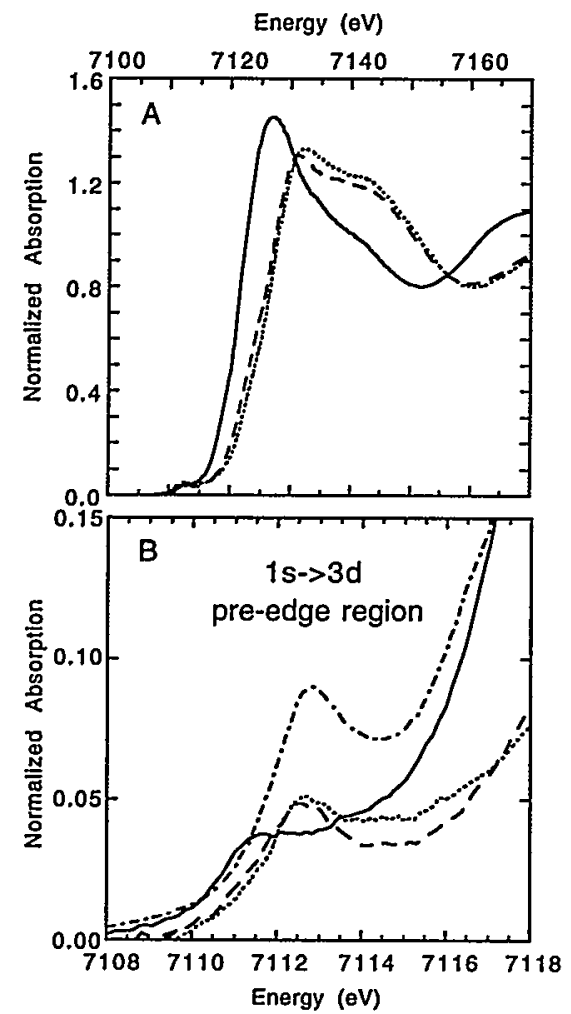

Figure 1. (A) Fe-K XAS edge spectra of $\mathrm{Fe}(\mathrm{II}) \mathrm{BLM}$ $(\longrightarrow), \mathrm{Fe}(\mathrm{III}) \mathrm{BLM}(---)$, and activated BLM (....). (B) Expansion of the $1 \mathrm{~s} \longrightarrow 3 \mathrm{~d}$ pre-edge region of part A with pre-edge data of $\mathrm{Fe}\left(\mathrm{Me}_{3} \mathrm{TACN}\right)(\mathrm{NO})\left(\mathrm{N}_{3}\right)_{2}$ $(-\cdot \cdot)$, which has a Fe-N(O) bond of $1.74 \AA$.
The energy of the edge position (dominated by the $1 s \rightarrow 4 p$ transition) is dependent upon the effective nuclear charge of the absorbing metal atom. This charge is governed by a combination of effects, including the formal metal oxidation state, the number and type of ligating atoms, and the coordination geometry. ${ }^{15-20}$ In this case, the types of ligating atoms and the coordination sphere are similar, thus changes in the edge energy can be correlated to the iron oxidation state. From Figure $1 \mathrm{~A}$, the edge spectrum of activated BLM is very close in energy and similar in shape to that of Fe(III)BLM, in contrast to the $\mathrm{Fe}(\mathrm{II}) \mathrm{BLM}$ spectrum which is $\sim 6$ $\mathrm{eV}$ lower in energy and has a more intense $1 \mathrm{~s} \longrightarrow 4 \mathrm{p}$ feature, indicating that activated BLM contains a ferric metal center. It should be noted that the edge inflection point of horseradish peroxidase compound I, which has a purported $\mathrm{Fe}(\mathrm{IV})=\mathrm{O}$ metal site, was observed to be at $\sim 2 \mathrm{eV}$ higher in energy than that of the resting ferric horseradish peroxidase. ${ }^{21}$

The $1 s \rightarrow 3 d$ pre-edge feature can be used to probe the spin and oxidation states of the iron site in activated BLM, since the final state $\left(1 s^{1} 3 d^{n+1}\right)$ has a different multiplet, splitting for high spin ferrous $\left({ }^{4} \mathrm{~F},{ }^{4} \mathrm{P}\right)$, high spin ferric $\left({ }^{5} \mathrm{D}\right)$, low spin ferrous $\left({ }^{2} \mathrm{E}\right)$, and low spin ferric $\left({ }^{1} \mathrm{~A}_{1},{ }^{3} \mathrm{~T}_{1}\right)$ cases. The number, intensity ratio, and energies of the pre-edge multiplet features are indicative of the specific oxidation and spin states of the iron. The splitting of the pre-edge in $\mathrm{Fe}$ (II)BLM is attributed to the ${ }^{4} \mathrm{~F}$ and ${ }^{4} \mathrm{P}$ free ion splitting of $\sim 2 \mathrm{eV}{ }^{16}$ As observed for $\mathrm{Fe}$ (III)BLM, low spin ferric edges exhibit a pre-edge feature centered at $7112.5 \mathrm{eV}$ with a low energy shoulder at $\sim 7111.0 \mathrm{eV}$, corresponding to the ${ }^{3} \mathrm{~T}_{1}$ and ${ }^{1} \mathrm{~A}_{1}$ transitions, respectively. ${ }^{2}$ The activated $\mathrm{BLM}$ spectrum exhibits the same multiplet splitting, consistent with this complex having a low spin ferric center.

The pre-edge features can also provide information on the geometric structure of the active site. The $1 s \rightarrow 3 d$ pre-edge feature is formally electric dipole forbidden, but gains intensity through an allowed quadrupole transition and through $4 \mathrm{p}$ mixing into the $3 d$ states as a result of the non-centrosymmetric environment of the metal site. When the symmetry of the iron site is lowered, the pre-edge intensity increases due to an increase in the $3 \mathrm{~d}-4 \mathrm{p}$ mixing. 19 Iron-oxo ${ }^{23}$ and iron-nitrosyl ${ }^{24}$ complexes typically have pre-edge features that are a factor of two more intense than their centrosymmetric counterparts, due to their short $\sim 1.8 \AA \mathrm{Fe}-\mathrm{O} / \mathrm{N}$ bond. See, for example, Figure $1 \mathrm{~B}$ where $\mathrm{Fe}\left(\mathrm{Me}_{3} \mathrm{TACN}\right)(\mathrm{NO})\left(\mathrm{N}_{3}\right)_{2},{ }^{25}$ an iron-nitrosyl complex with an $\mathrm{Fe}-\mathrm{N}$ bond length of $1.74 \AA$, is included as a reference. The pre-edge intensity of a 5-coordinate oxo-ferryl porphyrin complex with an Fe-O bond of $1.65 \AA$ is even greater 
than that of $\mathrm{Fe}\left(\mathrm{Me}_{3} \mathrm{TACN}\right)(\mathrm{NO})\left(\mathrm{N}_{3}\right)_{2}$, due to the shorter $\mathrm{Fe}-\mathrm{O}$ bond length. ${ }^{26}$ In contrast, the spectrum of activated BLM has a pre-edge intensity which is typical of a six coordinate low spin ferric complex with no severe distortion around the iron site, eliminating the possibility of a short $\mathrm{Fe}-\mathrm{O}$ bond.

The EXAFS spectra of Fe(II)BLM, Fe(III)BLM, and activated BLM are shown in the Figure $2 \mathrm{~A}$ and the Fourier transforms (FTs), taken over the $k$ range of 3.5-12.5 $\AA^{-1}$, are shown in Figure $2 \mathrm{~B}$. Curve-fitting was performed on filtered first shell contributions over the $k$ range $4-12 \AA^{-1}$ varying bond distances and coordination numbers. The first shell of each sample could not be adequately fit with a single low- $Z$ wave (Fits \#1,3,5 in Table 1). The Fe(II)BLM data were well fit by 2 shells of $N$ atoms (Fit \#2 in Table 1) with an average $\mathrm{Fe}-\mathrm{N}$ distance of $2.16 \AA$. The $\mathrm{Fe}$ (III)BLM and activated BLM data were also fit with two shells of low $Z$ atoms (Fits \#4,6 in Table 1),

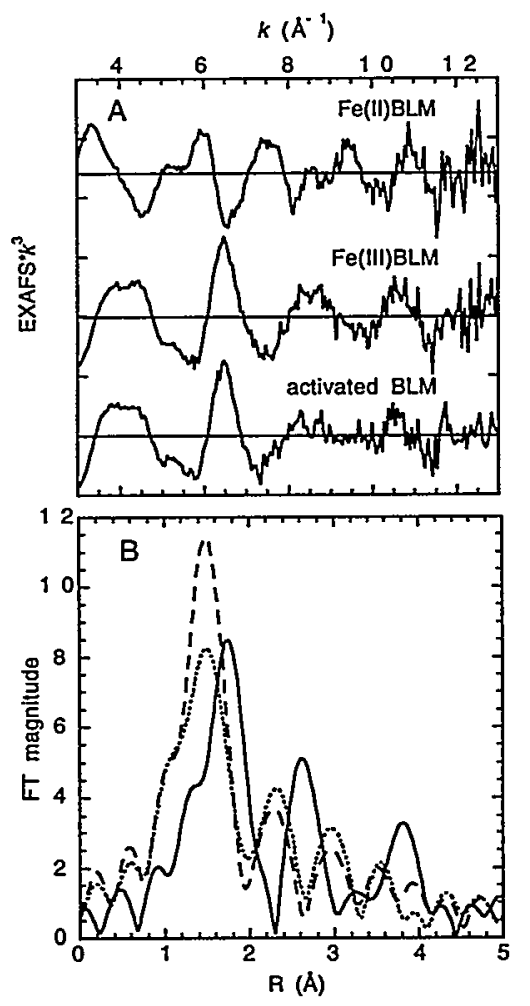

Figure 2. The Fourier transforms of the EXAFS data of Fe(II)BLM (-), Fe(III)BLM (--), and activated BLM (…). The inset contains the EXAFS data $\left({ }^{*} k^{3}\right)$ for Fe(II)BLM, Fe(III)BLM, and activated BLM (the ordinate scale is 5 between major tick marks on an absolute scale from the abscissa with solid horizontal lines indicating the zero point of each plot). but with an average first shell distance $0.2 \AA$ shorter than in Fe(II)BLM (see FTs in Figure 2). The two N shells from the fit to the $\mathrm{Fe}(\mathrm{III}) \mathrm{BLM}$ and activated BLM data have very similar distances with slight changes in the coordination numbers consistent with both being low spin ferric complexes. In contrast, an oxo-ferryl species would have a bond length of $\sim 1.65$ $\AA .21$ However, it was not possible to include an Fe$O$ wave below $1.85 \AA$ in the fit to the activated BLM data, indicating an oxo-ferryl species is clearly not present from the analysis of the EXAFS data.

Table 1. Summary of EXAFS Curve-Fitting Results

\begin{tabular}{ccccccc}
\hline sample & Fit & $\begin{array}{c}\text { FT } \\
\text { window } \\
\text { width }(\AA)\end{array}$ & $\begin{array}{c}\text { ele- } \\
\text { ment }\end{array}$ & $\mathrm{CN}^{a, b}$ & $\begin{array}{c}\text { bond } \\
\text { length } \\
(\AA)\end{array}$ & $\mathrm{F}^{c}$ \\
\hline Fe(II)BLM & 1 & {$[1.0-2.25]$} & $\mathrm{N}$ & 3.0 & 2.16 & 0.41 \\
& 2 & {$[1.0-2.25]$} & $\mathrm{N}$ & 1.4 & 2.08 & 0.29 \\
Fe(III)BLM & 3 & {$[0.75-2.0]$} & $\mathrm{N}$ & 3.3 & 1.94 & 0.73 \\
& 4 & {$[0.75-2.0]$} & $\mathrm{N}$ & 3.4 & 1.90 & 0.31 \\
activated & 5 & {$[0.7-2.0]$} & $\mathrm{N}$ & 2.5 & 1.96 & 0.84 \\
BLM & 6 & {$[0.7-2.0]$} & $\mathrm{N}$ & 2.5 & 1.89 & 0.36 \\
& & & $\mathrm{~N}$ & 3.0 & 2.03 & \\
\hline
\end{tabular}

$a_{\mathrm{CN}}=$ coordination number. ${ }^{b}$ Errors in distances $( \pm 0.02$ $\AA$ ) and coordination numbers $( \pm 25 \%)$ are estimated from the variance between EXAFS fitting results and values from models of crystallographically known structure. 10

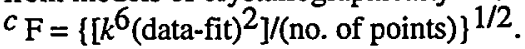

\section{Summary}

Both the energy position and shape of the rising edge of the activated BLM XAS spectrum are very similar to those for $\mathrm{Fe}(\mathrm{MI}) \mathrm{BLM}$, indicating that the iron in activated BLM is ferric. Both Fe(III)BLM and activated BLM exhibit a weak pre-edge feature at $7112.5 \mathrm{eV}$ with a lower energy shoulder indicative of a low spin ferric active site with no severe distortion (i.e. no short iron-oxo bond). The first shell distances obtained from the EXAFS data of activated BLM are within $0.01 \AA$ of the $\mathrm{Fe}(\mathrm{III}) \mathrm{BLM}$ distances with slight changes in the coordination numbers. Thus, the XAS data of activated BLM are not consistent with a $\mathrm{Fe}(\mathrm{TV})=\mathrm{O}$ species, but rather with a low spin ferric peroxide description of the active site. Activated BLM is the first mononuclear non-heme iron oxygen intermediate to be characterized and suggests that such a peroxy-ferric complex may play an important role in $\mathrm{O}_{2}$ activation by this class of enzymes.

\section{Acknowledgments}

This research was supported by grants from the NIH (GM40392, E.I.S.) and NSF (CHE-9121576, K.O.H.). SSRL is supported by the U.S. DOE, Office of Basic Energy Science, Divisions of Chemical and Materials Sciences, and in part by the NIH, 
Biomedical Research Technology Program (RR01209) and the U.S. DOE, Office of Health and Environmental Research. J.M.Z. thanks the Jane Coffin Childs Fund for Medical Research for a postdoctoral fellowship.

\section{References}

(1) Carter, S. K. Bleomycin Chemotherapy; Academic Press: New York, 1985, pp 3-35.

(2) Hecht, S. Acc. Chem. Res. 1986, 19, 383.

(3) Stubbe, J.; Kozarich, J. W. Chem. Rev. 1987, 87, 1107.

(4) Sugiura, Y. Biochem. Biophys. Res. Commun. 1979, 87, 643.

(5) Burger, R. M.; Peisach, J.; Horwitz, S. B. J. Biol. Chem. 1981, 256, 11636.

(6) Guajardo, R. J.; Hudson, S. E.; Brown, S. J.; Mascharak, P. K. J. Am. Chem. Soc. 1993, 7971.

(7) McGall, G. H.; Rabow, L. E.; Ashley, G. W.; Wu, S. H.; Kozarich, J. W.; Stubbe, J. J. Am. Chem. Soc. 1992, 114, 4958.

(8) a. Burger, R. M.; Kent, T. A.; Horwitz, S. B.; Münck, E.; Peisach, J. J. Biol. Chem. 1983, 258,1559 . b. Sam, J. W.; Tang, X. -J.; Peisach, J. J. Am. Chem. Soc. 1994, 116, 5250.

(9) Data were collected on unfocused wiggler beamline 7-3 during dedicated conditions ( 3 $\mathrm{GeV}, 50-100 \mathrm{~mA}$ ) using a $\mathrm{Si}(220)$ doublecrystal monochromator. Data were measured at $10 \mathrm{~K}$ in fluorescence mode with a 13element $\mathrm{Ge}$ detector using an internal $\mathrm{Fe}$ foil calibrant and assigning the first inflection point to $7111.2 \mathrm{eV}$. The spectrometer energy resolution was approximately $1.5 \mathrm{eV}$ with reproducibility of edge position determination of $<0.2 \mathrm{eV}$. Approximately 30 scans were obtained for each BLM sample.

(10) Cramer, S. P.; Hodgson, K. O.; Stiefel, E. I.; Newton, W. E. J. Am. Chem. Soc. 1978, 100, 2748.

(11) Cramer, S. P.; Hodgson, K. O. Prog. Inorg. Chem. 1979, 15, 1.

(12) Scott, R. A. Methods Enzymol. 1985, 117, 414.

(13) Dewitt, J. G.; Bentsen, J. G.; Rosenzweig, A. C.; Hedman, B.; Green, J.; Pilkington, S.; Papaefthymiou, G. C.; Dalton, H.; Hodgson, K. O.; Lippard, S. J. J. Am. Chem. Soc. 1991, $113,9219$.

(14) $\mathrm{Fe}$ (acetylacetonate) 3 and $[\mathrm{Fe}(1,10-$ phenanthroline $\left.)_{3}\right]\left(\mathrm{ClO}_{4}\right)_{3}$ were used to obtain empirical $\mathrm{Fe}-\mathrm{O}$ and $\mathrm{Fe}-\mathrm{N}$ backscattering parameters.

(15) Srivastave, U. C.; Nigam, H. L. Coord. Chem. Rev. 1973, 9, 275.
(16) Shulman, R. G.; Yafet, Y.; Eisenberger, P.; Blumberg, W. E. Proc. Natl. Acad. Sci. USA 1976, 73, 1384.

(17) Cramer, S. P.; Eccles, T. K.; Kutzler, F. W.; Hodgson, K. O. J. Am. Chem. Soc. 1976, 98 , 1287.

(18) Wong, J.; Lytle, F. W.; Messmer, R. P.; Maylotte, D. H. Phys. Rev. B. 1984, 30, 5596.

(19) Roe, A. L.; Schneider, D. J.; Mayer, R. L.; Pyrz, J. W.; Widom, J.; Que, L., Jr. J. Am. Chem. Soc. 1984, 106, 1676.

(20) Kau, L. S.; Spira-Solomon, D. J.; PennerHahn, J. E.; Hodgson, K. O.; Solomon, E. I. J. Am. Chem. Soc. 1987, 109, 6433.

(21) Penner-Hahn, J. E.; McMurry, T. J.; Renner, M.; Latos-Grazynsky, L.; Eble, K. S.; Davis, I. M.; Balch, A. L.; Groves, J. T.; Dawson, J. H.; Hodgson, K. O. J. Biol. Chem. 1983, 258, 12761.

(22) Westre, T. E.; Hedman, B.; Hodgson, K. O.; Solomon, E. I. unpublished data.

(23) Dewitt, J. G. Ph.D. Thesis, Stanford University, 1993.

(24) Zhang, Y.; Pavlosky, M. A.; Brown, C. B.; Westre, T. E.; Hedman, B.; Hodgson, K. O.; Solomon, E. I. J. Am. Chem. Soc. 1992, 114, 9189.

(25) Pohl, K.; Wieghardt, K.; Nuber, B.; Weiss, J. J. Chem. Soc., Dalton Trans. 1987, 187.

(26) Liu, H. I.; Gold, A.; Dawson, J. H.; Hedman, B.; Hodgson, K. O. unpublished data. 
Proposal 9911B

\title{
Electronic and Magnetic Properties of Iron Atoms in Nitrogenase:
}

\section{Fe L-edge Absorption Studies Using Linear and Circular Polarized Light}

\author{
Jie Chen ${ }^{1}$, Corie Y. Ralston ${ }^{1}$, Zhi-Hao Zhou ${ }^{2}$, Michael W.W. Adams ${ }^{2}$, Bin Xia ${ }^{3}$, \\ John L. Markely ${ }^{3}$, Benjamin A. Feinberg ${ }^{4}$, Roland C. Tittsworth ${ }^{5}$, Brian J. Hales ${ }^{5}$, \\ and Stephen P. Cramer 1,6 \\ 1 Department of Applied Science, University of California, Davis \\ 2 Department of Biochemistry, University of Georgia, Athens \\ 3 Department of Biochemistry, University of Wisconsin, Madison \\ ${ }^{4}$ Department of Chemistry, University of Wisconsin, Milwaukee \\ 5 Department of Chemistry, Louisiana State University, Baton Rouge \\ 6 Department of Applied Science, University of California, Davis, \\ and Energy and Environment Division, Lawrence Berkeley Lab
}

The enzyme nitrogenase catalyzes the reduction of dinitrogen to ammonia. The Mo-based nitrogenase requires two proteins: a large $(>200,000) \mathrm{MoFe}$ protein contains 2 types of clusters - the $\mathrm{MoFe}_{7} \mathrm{~S}_{9}$ $\mathrm{M}$-centers and the $\mathrm{Fe}_{8} \mathrm{~S}_{8} \mathrm{P}$-clusters, and a. smaller $(\sim 55,000) \mathrm{Fe}$ protein contains a single $\mathrm{Fe}_{4} \mathrm{~S}_{4}$ cluster. The active site for nitrogen fixation is thought to be in the M-center of the Mo-Fe protein [1]. Resent crystallographic models for $\mathrm{M}$-center can be viewed as fused $\left[\mathrm{MoFe}_{3} \mathrm{~S}_{3}\right]$ and $\left[\mathrm{Fe}_{4} \mathrm{~S}_{3}\right]$ cubane fragments, linked by $3 S^{2-}$ bridges [2]. The P-clusters are seem to be merged $\left[\mathrm{Fe}_{4} \mathrm{~S}_{4}\right]$ cubes, bridged by an $\mathrm{S}-\mathrm{S}$ bond [2]. Despite the structural progress, there are questions about the electronic and magnetic structure need to be answered. For example, what are oxidation states and spin states of Fe and Mo atoms? How different type irons are distributed among these clusters? How spins are coupled within the clusters? Answers to these questions are important for theoretical studies and for understanding catalytic roles of nitrogenase.

We report here the first $X M C D$ results on nitrogenase, together with L-edge absorption studies. We observed strong dichroism effect for both native and thionine-oxidized proteins. The L-edge absorption results suggest that irons in $\mathrm{MoFe}$ proteins are in high-spin states. We found that the ratio of ferrous $\left(\mathrm{Fe}^{2+}\right)$ iron to total $\left(\mathrm{Fe}^{2+}+\mathrm{Fe}^{3+}\right)$ iron in the native protein is $0.65 \pm 0.1$, and it changes to $0.40 \pm 0.1$ in the oxidized form. In addition, $\mathrm{XMCD}$ studies show that the total system net spin is mainly come from ferrous irons in the oxidized state, while both ferrous and ferric irons contribute to the net spin in the resting state.
Nitrogenase solution samples were extracted from appropriate strains of $A$. vinelandii at LSU by methods previously described [3]. Specific activities were $1800 \mathrm{nmol} \mathrm{C}_{2} \mathrm{H}_{2}$ reduced min $^{-1} \mathrm{mg}^{-1}$ protein for both reduced and oxidized proteins. The L-edge experiments were conducted using a $10001 / \mathrm{mm}$ grating monochromator at 10-1 beamline, the XMCD experiments were performed at 8-2 beamline using our 6 Tesla superconducting magnet and liquid $\mathrm{He}$ cryostat. All the spectra were measured in the fluorescence excitation mode using a 13-element $\mathrm{Ge}$ solid state array detector [4].

The L-edge spectroscopy measures the $2 \mathrm{p} \rightarrow 3 \mathrm{~d}$ transitions. Compared to the corresponding K-edge XAS, the spectral resolution is 3-4 fold greater and spectia are much more structured. As L-edge transitions involve the valence (d) orbital, spectra are sensitive to the metal oxidation state, spin state, as well as crystal field symmetry and strength [5]. It is know that both the M-centers and P-clusters in MoFe proteins exhibit a wide range of spin states upon oxidation or reduction (Scheme 1). One limitation of the normal XAS spectroscopy is that it cannot separate these metal centers. A relatively new technique of $\mathrm{x}$-ray magnetic circular dichroism (XMCD) has ability to distinguish these centers. In an XMCD experiment, the absorption of left and right circular polarized light by a magnetized sample is compared. Compared to optical MCD, XMCD is element selective and measures selection rule allowed transitions. As a consequence, big MCD effects (100\%) are expected. Up to $30 \%$ XMCD effects have been observed in the high spin $\mathrm{Fe}$ in $p$. furiosus rubredoxin [6]. XMCD has been used to interpret the 
coupling pattern in mixed-valence and mixed-metal spin-coupled clusters [7]. Comparison with the field direction reveals how the element under study contributes to the bulk magnetic properties. As shown in Scheme 1, the P-clusters are diamagnetic in resting states, and the $\mathrm{M}$-centers are paramagnetic $(S=3 / 2)$, while the $M$-centers become diamagnetic and the P-clusters are paramagnetic in oxidized states $(S=3)$. Thus, Nitrogenase is a good example of how XMCD selectivity for only the paramagnetic centers which allow investigation of complex metalloproteins.

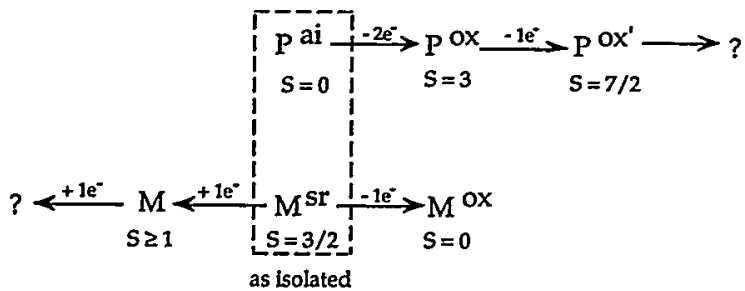

Scheme 1. Magnetic properties of metal clusters in Mo-Fe protein at different redox states.

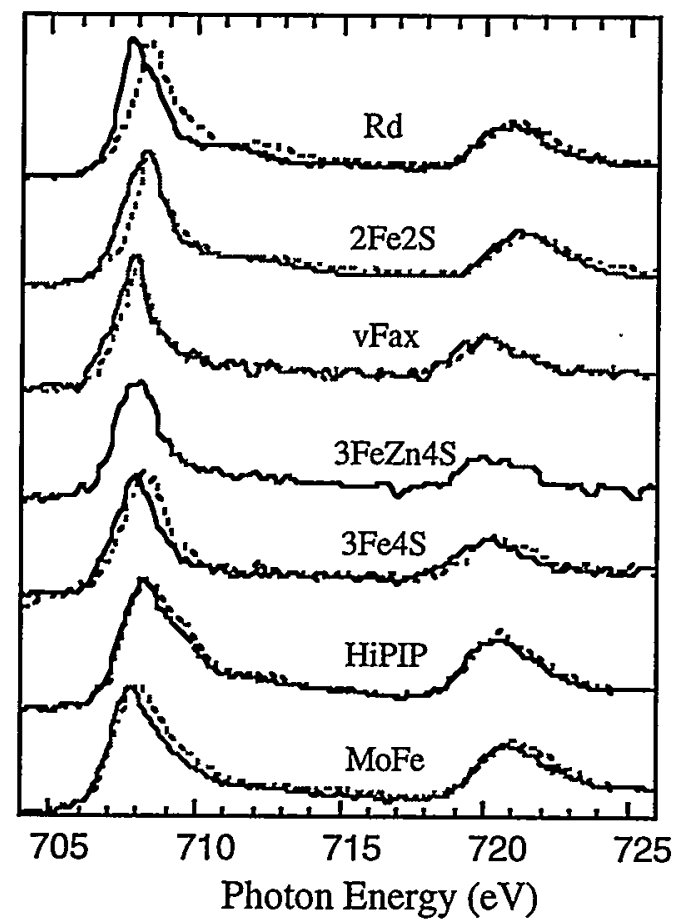

Figure 1. The measured Fe L-edge absorption spectra from reduced (- - ) and (-.-) oxidized Fe-S proteins. Top to bottom: $P$. furiosus rubredoxin, $C$. pasteurianum $2 \mathrm{Fe}, A .7120$ vegetative $2 \mathrm{Fe}, P$. furiosus $3 \mathrm{Fe}$, and $\mathrm{Zn}$ substituted $4 \mathrm{Fe}$ ferredoxins, $C$. vinosum HiPIP, and $A$. vinelandii nitrogenase $\mathrm{MoFe}$-protein.

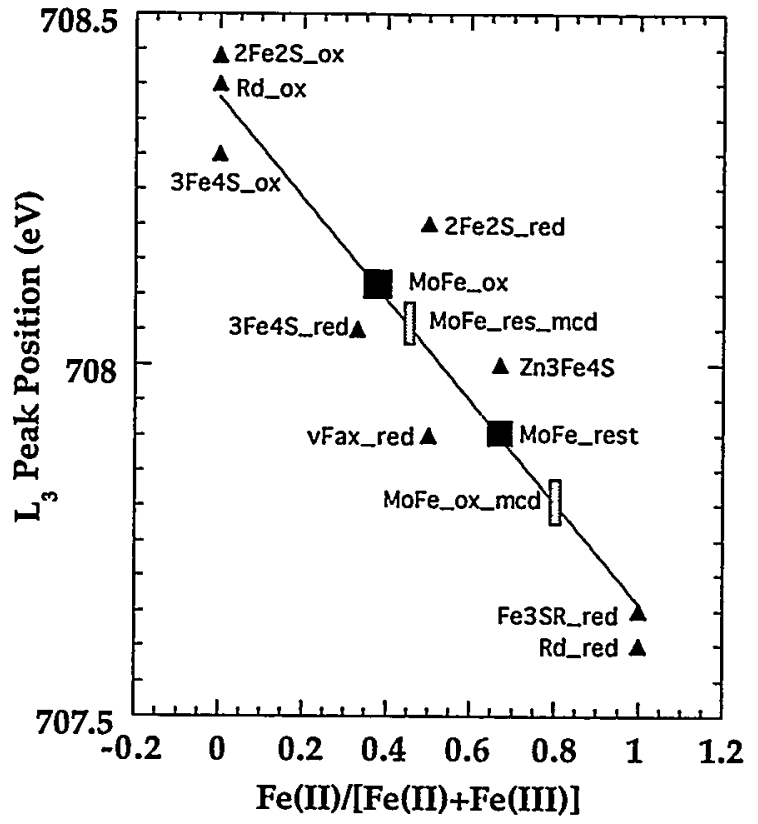

Figure 2. The correlation between the ratio of ferrous $\left(\mathrm{Fe}^{2+}\right)$ iron to total $\left(\mathrm{Fe}^{2+}+\right.$ $\mathrm{Fe}^{3+}$ ) iron and the measured $\mathrm{L}_{3}$ peak position among $\mathrm{Fe}-\mathrm{S}$ proteins. A 3coordinate $\mathrm{Fe}$ model complex (Fe3SR) is also shown for comparison. Note that the whole range of energy spread is less than 1 eV.

To understand the complex systems like nitrogenase, we have examined a series small, well characterized $\mathrm{Fe}-\mathrm{S}$ proteins. These including single iron rubredoxin, $2 \mathrm{Fe}, 3 \mathrm{Fe}$, and $4 \mathrm{Fe}$ ferredoxins, as well as a $\mathrm{Zn}$ substituted $4 \mathrm{Fe}$ ferredoxin, and high potential iron proteins (HiPIP). The Fe L-edge absorption spectra of these samples are shown in Figure 1. Redox shifts are clearly seen for all proteins. The biggest shift is seen in rubredoxins, there is an $800 \mathrm{meV}$ shift upon oxidation. A small but reproducible, $\sim 0.2 \mathrm{eV}$ shift to higher energy is clearly observed in the L-edge spectra of MoFe protein, while extremely small changes are observed at the K-edge. We found that the measured nitrogenase spectra can be simulated by a combination of reduced and oxidized rubredoxin spectra. This result suggests that the $\mathrm{Fe}$ in nitrogenase exists in both $\mathrm{Fe}^{2+}$ and $\mathrm{Fe}^{3}+$ high spin states. The measured $0.2 \mathrm{eV}$ shift corresponds to 7 electron difference (Figure 2) between the resting and thionine-oxidized states, which is in good agreement with the $6 \mathrm{e}^{-}$oxidation process. [8].

As showed in Figure 2, the peak positions in XMCD spectra are reversibly ordered compared to that in L-edge spectra. In particular, the centroid 
position of XMCD peak in oxidized samples corresponds to high ratio $(>0.8)$, which indicates that the system net spin is mainly come from ferrous irons. This agrees well with recently proposed coupling scheme for the oxidized nitrogenase Pclusters [9].

In conclusion, we have studied the electronic structure of $\mathrm{Fe}$ atoms in nitrogenase using both linear and circular polarized lights. The L-edge studies suggest that irons in nitrogenase are in mixed valance and high spin states. The predict redox changes are observed and are in good agreement with predictions. The XMCD results suggest that the system net spin of the oxidized states is mainly come from ferrous irons, while both ferric and ferrous irons contribute to the net spin in resting states.

This work was supported by the NIH through grant GM-44380, the NSF through grants DIR9105323 and DMB-9107312, DOA through grant USDA-94-37305-0701, and by the DOE - Office of Health and Environmental Research. The SSRL is supported by the DOE, Office of Basic Energy Science.

1 W. H. Orme-Johnson, Ann. Rev. Biophys. Biophys. Chem. 14, 419 (1985); R. R. Eady, Adv. Inorg. Chem. 36, 77 (1991).

2 J. Kim, D. C. Rees, Science, 257, 1677 (1992); J. Kim, D. C. Rees, Nature, 360, 553 (1992); M. K. Chan, J. Kim, D. C. Rees, Science, 260, 792 (1993).

3 B. J. Hales, et al, J. Bio. Chem. 261, 15301 (1986).

4 S. P. Cramer et al, Nucl. Inst. Meth., A266, 586 (1988).

5 F. M. F. de Groot, J. Elec. Spectr. Rel. Phenom. 67, 529 (1994).

6 J. van Elp et al, Proc. Natl. Acad. Sci. USA, 90,9664 (1993).

$7 \quad$ P. Rudolf et al, J. Magn. Magn. Mater., 109, 109 (1992).

8 J. Chen et al in Appl. of Synch. Rad. Tech. to Mat. Sci. 63 (1993),

9 J. M. Mouesca et al, Inorg. Chem., 33, 4819 (1994). 


\title{
Angle Resolved Photoemission Spectroscopy (ARPES) Study of MBE-grown $\mathrm{Bi}_{2} \mathrm{Sr}_{2} \mathrm{CaCu}_{2} \mathrm{O}_{8-\delta}$ Thin Films
}

\author{
D.S. Marshall, D.S. Dessau, D.M. King, C.-H. Park, A.Y. Matsuura, Z.-X. Shen, and W.E. Spicer \\ Stanford Electronics Laboratories, Stanford University, Stanford, California 94305 \\ Stanford Synchrotron Radiation Laboratory, Stanford, CA 94309
}

\author{
J. N. Eckstein and I. Bozovic \\ E. L. Ginzton Research Center, Varian Associates, Palo Alto, California 94304
}

\section{INTRODUCTION}

Over the last five years, high-resolution angle-resolved photoemission has emerged as one of the most powerful experimental techniques for studying the electronic properties of high-temperature superconductors. However, virtually all the angle resolved photoemission experiments that demonstrate clear energy dispersion, Fermi level crossings, and superconducting features were performed on bulk crystal samples. 1,2 The difficulty in performing photoemission experiments on thin film samples hinges on two points: (1) only single phase films with a very low defect density and high degree of crystallinity will give good results, and (2) clean surfaces must be prepared for photoemission due to the surface sensitivity of the technique (approximately $15 \AA$ ). The ALLMBE samples used in this experiment were single crystal films without any second phase precipitates. The results reported in this paper were made possible by the development of a technique for mounting and cleaving thin films in ultra high vacuum (UHV). The essence of this technique is to mount the substrate with the film facing outward using electrically conductive epoxy to ground the sample. A toppost with a thermal expansion coefficient matching that of the substrate is then adhered to the face of the sample using epoxy. Once in UHV and cooled to cryogenic temperatures, the top-post is knocked off the sample face by mechanical means leaving behind a freshly cleaved surface.

Most studies of fundamental interest in the area of high temperature superconductivity have been performed on bulk crystals due to the generally higher defect densities and wider superconducting transitions of thin films. However, issues such as the effect of electron doping on the electronic structure in certain materials systems cannot easily be addressed with bulk crystals because the achievable stoichiometries are thermodynamically limited. Using ALL-MBE, metastable materials with a wide stoichiometric range can be synthesized. However, before fundamental issues can be effectively addressed with thin films, comparable results to those of bulk crystals in known systems should be demonstrated. In this letter, we report results of ARPES on $\mathrm{Bi}_{2} \mathrm{Sr}_{2} \mathrm{CaCu}_{2} \mathrm{O}_{8-\delta}$ (2212) thin films grown by ALL-MBE that match those of bulk crystals. We have observed clear features from these films that disperse through the Fermi level. For the first time, the superconducting energy gap has been observed with ARPES in thin films. Both the magnitude of the gap as well as its anisotropy are consistent with the results from bulk crystals. Furthermore, we observe the pile-up and dip in spectral weight associated with the gap. These results open possibilities for future research using thin film samples that take advantage of the ability to grow metastable materials with ALL-MBE.

\section{EXPERIMENT}

The films in this study were grown using ALL-MBE by exposing a heated substrate to shuttered sources of bismuth, strontium, copper, and calcium, all in the presence of ozone. 3 The shuttèr timing was accurately adjusted to deposit $\mathrm{Bi}_{2.1} \mathrm{Sr}_{1.95} \mathrm{Ca}_{0.95} \mathrm{Cu}_{2} \mathrm{O}_{8}$; this composition is typical of the single-phase two-layer $\mathrm{Bi}$ compound. (We will refer to this as 2212 for brevity.) The shuttering sequence was repeated, atomic layer by layer, until approximately $1000 \AA$ of material was deposited. The quality of the samples was quite high, showing single-phase growth and superconducting critical temperatures of $85 \mathrm{~K}$ 
(zero resistance temperature). The superconducting transitions for these thin films were $15 \mathrm{~K}$ wide (10\% to $90 \%$ ) which is typical of 2212 thin films. The samples were mounted in air for photoemission. The surfaces were cleaved in UHV where ARPES measurements were performed using $22.4 \mathrm{eV}$ light from Beam-line 5-3 of the Stanford Synchrotron Radiation Laboratory. Collection of the emitted electrons was accomplished with a hemispherical electron analyzer under conditions that yielded a total experimental resolution of $45 \mathrm{meV}$ (as measured on a gold Fermi edge).

\section{RESULTS}

The photoemission spectra of these film samples were strikingly similar to those of bulk crystals in a number of aspects, including the general shape of the spectra, the normal-state dispersion of bands, the Fermi level crossings, and the symmetry of electronic states with respect to the polarization of the incident light. Figure 1, panel (a) shows a series of spectra from a thin film in the normal (nonsuperconducting) state; and panel (b) shows a similar series from a bulk crystal also in the normal state. Each spectrum of the series is taken at a different collection angle and corresponds to a different point in $\mathrm{k}$-space. Figure 1 shows the dispersion of the uppermost valence bands (within $1 \mathrm{eV}$ of the Fermi level) as they move toward and through the Fermi energy. The dispersions are very similar in both cases.

Figure 2 shows the first Brillouin zone, containing the Fermi surface as mapped by photoemission on bulk crystals ${ }^{2}$ and thin films. The Fermi level crossings of the films agree well with bulk data within the error bars. ${ }^{2}$ The Fermi level crossings were also measured with the sample rotated $90^{\circ}$ so that the Y symmetry point replaced the $X$ symmetry point in the figure. The crossings were identical in both orientations within the error bars shown. These results represent a significant technical step forward, and encourage further studies of ALL-MBE grown thin films such as cation doped materials or metastable materials.

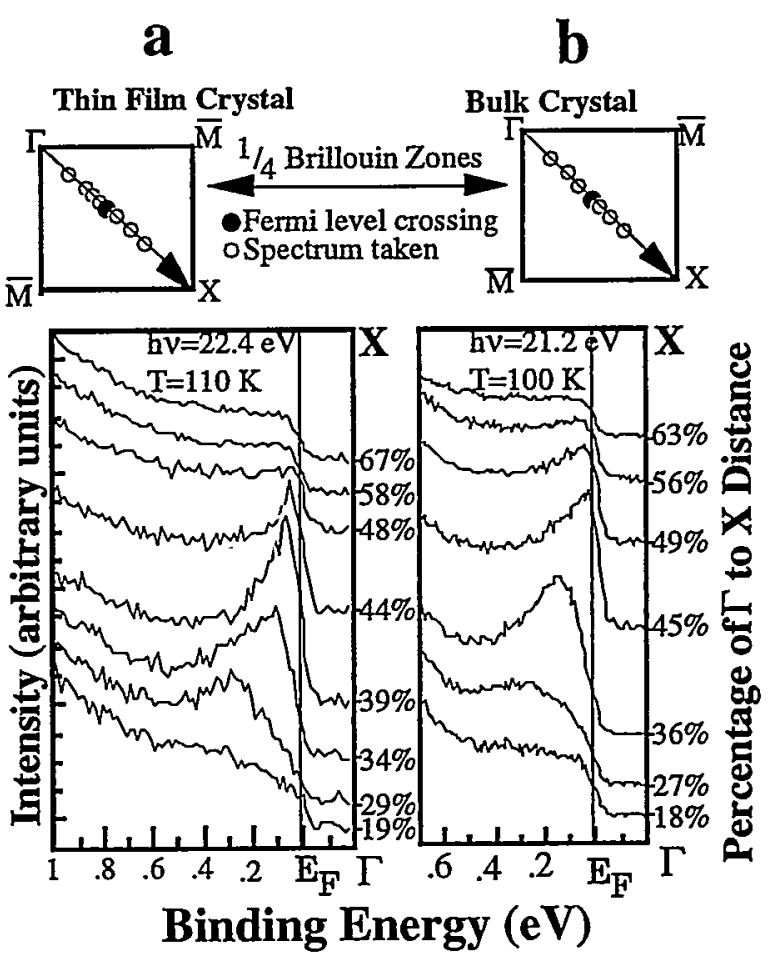

Fig. 1: Spectra of the uppermost valence bands from (a) a thin film crystal and (b) a bulk crystal showing a clearly dispersive feature and a Fermi level crossing near $45 \%$ of the way from the $\Gamma$ to $X$ points of the Brillouin zone. Above each spectrum set is a depiction of $1 / 4$ of the Brillouin zone showing where in $\mathrm{k}$-space the spectra were taken. 


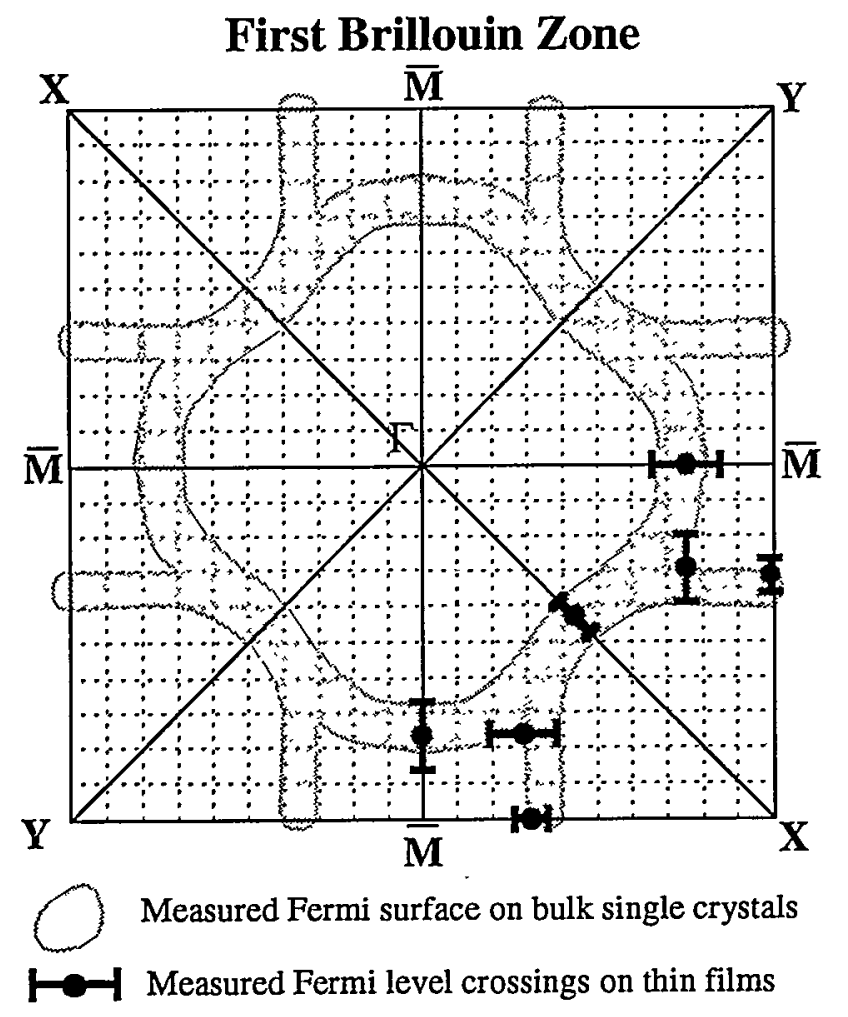

Fig. 2: The first Brillouin zone indicating the Fermi level crossings of thin film samples (with error bars) as well as the Fermi surface of bulk crystals (from ref. 2). Each of the dashed lines represents one tenth of the $\Gamma$ to $\bar{M}$ distance.

The experiments were performed at SSRL which is operated by the DOE's Division of Materials Science. The research at Stanford was the supported by DARPA/Hypress under F33615-90-C-1456 and JSEP under DAAL0391-C-0010, and the research at Varian was supported in part by NRL and ONR under N00014-93-C-2055 and N00014-94-C-2011. DSM would like to thank the Fannie \& John Hertz Foundation for its financial support. ZXS would like to acknowledge the Alfred P. Sloan Foundation for its support.

\section{REFERENCES}

1 C.G. Olson et al, Phys. Rev. B 42, 381 (1990). D.M. King et al, Phys. Rev. Lett. 70, 3159 (1993); ibid. submitted (1994). E.R. Ratner et al, Phys. Rev. B 48, 10482 (1993). R. Liu et al, ibid. 45, 5614 (1992);
46, 11056 (1992). J.G. Tobin et al, ibid. 45, 5563 (1992).

2 D.S. Dessau et al, Phys. Rev. Lett. 71, 2781 (1993).

3 J.N. Eckstein et al, MRS Bulletin 17, (8), 27 (1992); ibid. 19, (9), 44 (1994); Appl. Phys. Lett. 57, 931 (1990); ibid. 57, 1049 (1990).

4 B.O. Wells et al, Phys. Rev. B 46, 11830 (1992). Z.-X. Shen et al, Phys. Rev. Lett. 70, 1553 (1993). G.D. Mahan, D.S. Dessau et al, ibid. 71, 4277 (1993). 


\title{
Development of a Spin-Polarized Inverse Photoemission System
}

\author{
S.L. Friedman ${ }^{1}$ P.J. White ${ }^{1}$ and Z.-X. Shen ${ }^{1,2}$ \\ 1Department of Applied Physics, Stanford University, Stanford, CA 94305 \\ ${ }^{2}$ Stanford Synchrotron Radiation Laboratory, Stanford, CA 94309 \\ J. Hurst \\ IBM Almaden Research Center, 650 Harry Road, San Jose, CA 95120
}

\section{INTRODUCTION}

Magnetic materials have consistently been of interest for their applications in research and industry. Despite substantial research efforts and widespread use, a unified theory of magnetic materials has not emerged. One difficulty has been that conventional analytical techniques provide little information about the factors that contribute to magnetic phenomena. In particular, spectroscopic techniques that map out the electronic structure are rarely sensitive to spin. We are developing a spin polarized inverse photoemission system (SPIPES) to study magnetic materials with emphasis on understanding correlation effects.

Modifying standard techniques in order to obtain spin resolved data is in general difficult. For example, photoemission spectroscopy can be performed using a Mott detector which is capable of measuring the spin polarization of the photoelectrons. The signal, however, is almost 5 orders of magnitude weaker than the signal obtained with non spin-polarized methods. ${ }^{1}$

\section{INVERSE PHOTOEMISSION}

Inverse photoemission spectroscopy (IPES) uses a beam of low energy electrons to directly probe the density of unoccupied states. Since emitted photons are collected, there is no need for an inefficient spin resolving electron detector. Only the electron source need be spin polarized. Fortunately, spin polarized electrons can be generated in an efficient manner. Exciting negative electron affinity (NEA) GaAs photocathodes with circularly polarized light can produce electron beams of $20 \mu \mathrm{A}$ with a polarization of $40 \% .^{2}$ Therefore spin polarized IPES is almost as sensitive as conventional IPES. While photoemission spectroscopy is much more efficient than IPES, the spin polarized versions are equally sensitive.

Developing the SPIPES system has been divided into two components: assembling a conventional momentum resolved inverse photoemission spectroscopy (KRIPES) system and developing a spin polarized electron gun that operates at the appropriate energies. Our conventional momentum resolved inverse photoemission spectroscopy system is based on a band pass photon detector and thermal electron source. With a band pass detector the experiment is conducted in the isochromat mode. The incident electron energy is scanned from a few volts above the sample fermi level to roughly 20 volts above the fermi level. The intensity of $9.8 \mathrm{eV}$ photons measured by the band pass detector is recorded as a function of incident electron energy.

The detector uses a $\mathrm{CaF}_{2}$ window and a $\mathrm{CuBe}$ open mesh electron multiplier. Its response is centered at $9.8 \mathrm{eV}$ with a FWHM of $0.6 \mathrm{eV}$. Resolution can be increased by using a $\mathrm{SrF}_{2}$ window and evaporating $\mathrm{KBr}$ on the photocathode. ${ }^{3}$ We have conducted preliminary tests using a conventional thermal electron source and have begun testing an electron gun designed especially for inverse photoemission that uses an indirectly heated BaO cathode. 4

In addition to characterizing the system, we have begun studies of BiSCCO 2212, a layered high temperature superconductor. To obtain reference spectra, we have conducted studies using a state of the art spectrometer based KRIPES system capable of obtaining $0.2 \mathrm{eV}$ resolution. 5

\section{SPIN POLARIZED ELECTRON SOURCE}

Converting the KRIPES system to a spin polarized system requires replacing the conventional source with a spin polarized one. One of the authors (JH) designed and fabricated a NEA GaAs spin polarized electron gun that was used for spin polarized appearance potential spectroscopy and spin polarized low energy electron microscopy. ${ }^{6}$ Both of these applications, however, required electrons of substantially higher energy than the 5 to $20 \mathrm{eV}$ required for inverse photoemission. Before inverse photoemission can be conducted with this source, we must develop a set of transport optics capable of delivering a well focussed beam at low energy. Currently, though, we are upgrading the cathode. Recent work demonstrates that using strained layer photocathodes spin polarizations of up to $80 \%$ are possible. ${ }^{7}$ Data indicate that we should be able to achieve adequate beam currents while still obtaining $65 \%$ spin polarization. ${ }^{8}$ 


\section{REFERENCES}

${ }^{1}$ J. Kessler, Polarized Electrons. Springer-Verlag, New York (1976)

${ }^{2}$ D.T. Pierce, R.H. Celotta, G.-C. Wang, W.N. Unertl, A. Galejs, C.E. Kuyatt and S.R. Mielczarek, Rev. Sci. Instrum. 51, 478 (1980)

${ }^{3}$ I. Schäfer, W. Drube, M. Schlüter, G. Plagemann and M. Skibowski, Rev. Sci. Instrum. 58, 710 (1987)

${ }^{4}$ N.G. Stoffel and P.D. Johnson, Nuc. Instrum. \& Methods in Phy. Research A. 234, 230 (1985)

${ }^{5}$ P.D. Johnson and S.L. Hulbert, Rev. Sci. Instrum. 61, 2277 (1990)

${ }^{6}$ M.S. Altman, H. Pinkvos, J. Hurst, H. Poppa, G. Marx and E. Bauer, Mat. Res. Soc. Symp. Proc. 232, 125 (1991)

${ }^{7}$ T. Nakanishi, 1992 Lin. Accel. Conf. Proc. (AECL 10728) pp. 268. Ottawa (1992)

${ }^{8} \mathrm{H}$. Tang, SLAC. Private Communication (1994) 


\title{
Low Energy Excit tions and Symmetry of the Order Parameter in High- $T_{c}$ Superconductors
}

\author{
D.S. Dessau, (1) Z.-X. Shen,(1) D.M. King,(1)D.S. Marshall,(1) A.G. Loeser, (1) C.H. Park, (1) A. Matsuura(1), \\ W.E. Spicer $(1)$ and A. Kapitulnik, (2)
}

(1)Department of Applied Physics and Stanford Synchrotron Radiation Laboratory, Stanford University, Stanford, California 94305 (2)Department of Applied Physics, Stanford University, Stanford, California 94305

\section{INTRODUCTION}

High energy resolution angle-resolved photoemission has recently emerged as one of the most direct and powerful probes of the low-energy physics of condensed matter systems. In this report we discuss the results of some studies which delve into some crucial questions about the nature of superconductivity in high temperature superconductors.

Photoemission spectroscopy can probe the superconducting state by measuring the superconducting energy gap. The gap, or minimum excitation energy, is one of the most fundamental quantities of a superconductor in that it is a measure of the interaction strength which binds two electrons to form a Cooper pair. In conventional superconductors, the electrons in a pair are bound by the electron-phonon interaction and have a gap magnitude $2 \Delta / \mathrm{kBT}_{\mathrm{C}}=3.5$, where $\Delta$ is the superconducting gap and $k_{B}$ is Boltzman's constant. As seen from this equation, the gap is isotropic in $\mathbf{k}$-space, although in a few rare cases there may be a gap anisotropy of as much as 20 30\%.

It is widely believed that the conventional electron-phonon interaction is not strong enough to create a superconductor with such a high $\mathrm{T}_{\mathrm{c}}$. However, there is as yet not a consensus on what the mechanism for this pairing must be. Several proposals for such a mechanism require a gap with a very unusual k-space dependence [1-3]. Some gaps even requires nodes (regions where the gap has zero magnitude) at specific locations in $\mathbf{k}$-space and maxima at other specific locations in $\mathrm{k}$-space $[1,2]$. This type of a gap is called d-wave, indicating that the pair has an angular momentum quantum number equal to two, as opposed to the conventional s-wave gap, in which the pairs have zero angular momentum. Most extensively studied of these $d$-wave theories are those that propose a $d_{x} 2$ y2 symmetry gap, where the gap will vary as Icosk $\mathbf{x} \mathrm{a}^{\mathrm{a}} \cos \mathbf{k}_{\mathrm{y}} \mathrm{al}$ $[1,2]$.

\section{EXPERIMENTAL TECHNIQUE}

In a photoemission experiment, electrons are ejected from a sample surface by incident monochromatic photons (hv 21 $\mathrm{eV}$ for this study). These so-called photoelectrons are collected and energy analyzed. When operated in the angleresolved mode, only the photoelectrons emitted in a small solid angle are collected, yielding directional information that determines the electron momentum, or $\mathbf{k}$. Fig. 1 depicts a set of angle-resolved photoemission spectra taken at various emission angles of a 2D material where the interpretation of photoemission data is simplest. Since the binding energy of the photoemission peak gives the energy of the quasiparticles and the emission angle $\theta$ gives $\mathbf{k} / /$ through the relations $\mathbf{k} / /=$ $|\mathbf{k}| \sin \theta$ and $E_{k}=h^{2} k^{2} / 2 m$, the $E$ vs $k / /$ relation can be readily constructed.
Band Structure

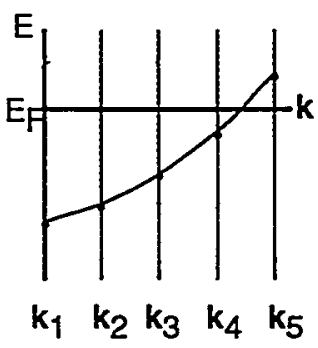

ARPES Spectra

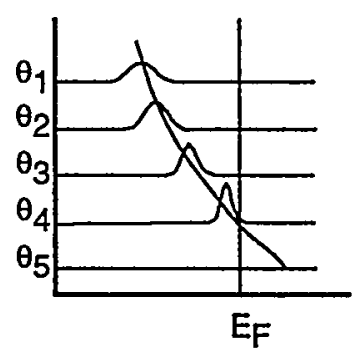

Fig.1 Illustration of angle-resolved photoemission. The crystal momentum $\mathbf{k}$ of the electrons is related to the emission angle $\theta$. The Fermi surface crossing point is determined by the angle corresponding to the peak at the Fermi level.

Our measurements were carried out at SSRL's undulator beam line 5 with the Stanford PRT sidestation (a VSW HA50 system outfitted with a custom multichannel detector). The outstanding performance of this instrumentation has given us the necessary energy resolution at very high fluxes, making this study possible.

\section{RESULTS AND DISCUSSION}

Figure 2 shows the Fermi surface of the high temperature superconductor $\mathrm{Bi} 2 \mathrm{~S}+2 \mathrm{CaCu} 2 \mathrm{O}$ (Bi2212) experimentally determined by us by performing angle-resolved photoemission measurements in the normal state $(T>T c)$. [4] We observed two pieces of Fermi surface, one centered around the $\Gamma$ point 
and one centered around the $X$ or $Y$ points. We interpreted the existence of the two pieces of Fermi surface as an even and odd combination of states from the two conducting $\mathrm{CuO}_{2}$ planes per unit cell. This implies that there must be coupling between the planes in the normal state, an issue of paramount importance for theories of superconductivity based upon spincharge separation. $[3,5,6]$

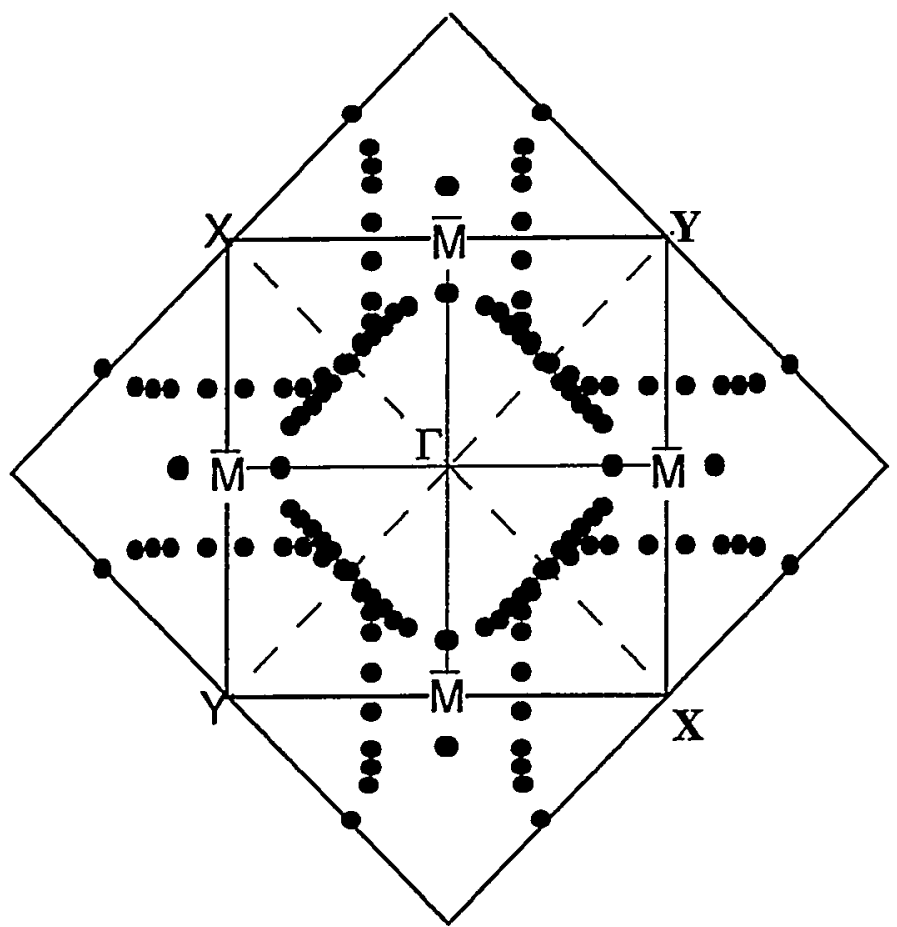

Fig.2 The experimental Fermi surface of Bi2212 as determined by us using angle resolved photoemission.

Fig 3 shows photoemission spectra as a function of temperature for two points on the above measured Fermi surface. [7] For panel $A$ taken near the $M$ point, we see dramatic changes in lineshape as the temperature is lowered through the transition temperature of $\sim 85 \mathrm{~K}$. There is a shifting back of the leading edge corresponding to the opening of the superconducting energy gap, a sharpening of the pole near $40 \mathrm{meV}$ corresponding to increased quasiparticle lifetimes in the superconducting state, and a loss of spectral weight near $80 \mathrm{meV}$. For panel $\mathrm{B}$ taken along the $\Gamma-\mathrm{X}$ direction, there are only minor changes as a function of temperature, indicating a zero or near-zero superconducting gap.

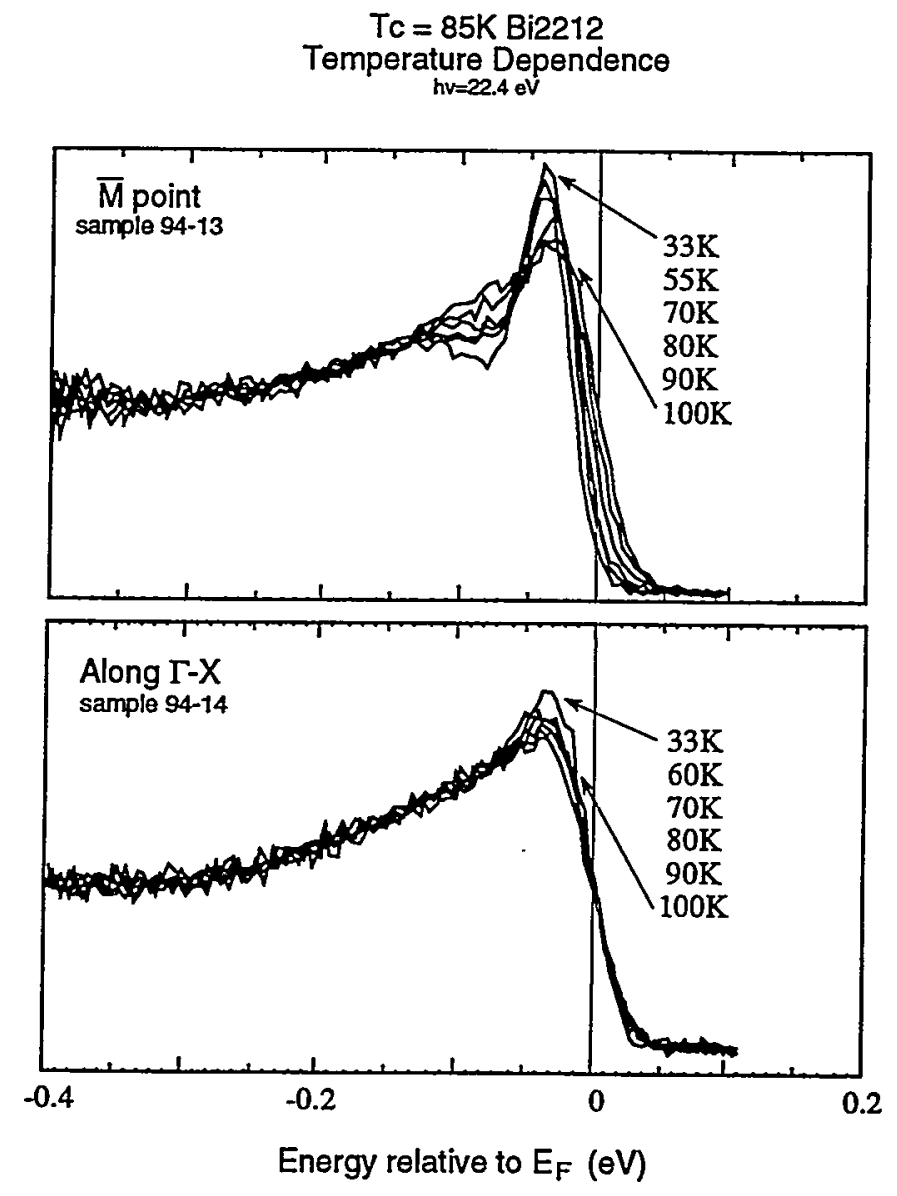

Fig.3 High resolution photoemission spectra from $T \mathrm{~T}=85 \mathrm{~K}$ $\mathrm{Bi} 2 \mathrm{Sr} 2 \mathrm{CaCu} 2 \mathrm{O} 8$ as a function of temperature for two different k-space points on the Fermi surface.

These measurements have been performed on a large number of samples for a variety of $\mathbf{k}$-space points. Fig. 4 plots the measured gap $\Delta(k)$ at low temperature as a function of Icosk $x^{a}$-coskyal for 14 different samples. [8] On this scale, a $d_{x 2-y 2}$ symmetry superconductor would have a gap which is linear with a zero intercept. The figure shows that the data is very consistent with the $d_{x 2}-y 2$ picture and is quite different from the simple s-wave picture, which would not have any variation as a function of $\mathbf{k}$. This finding puts a strong constraint on theoretical models by requiring the superconducting gap to be very anisotropic and to follow a particular functional form in $\mathbf{k}$-space. 


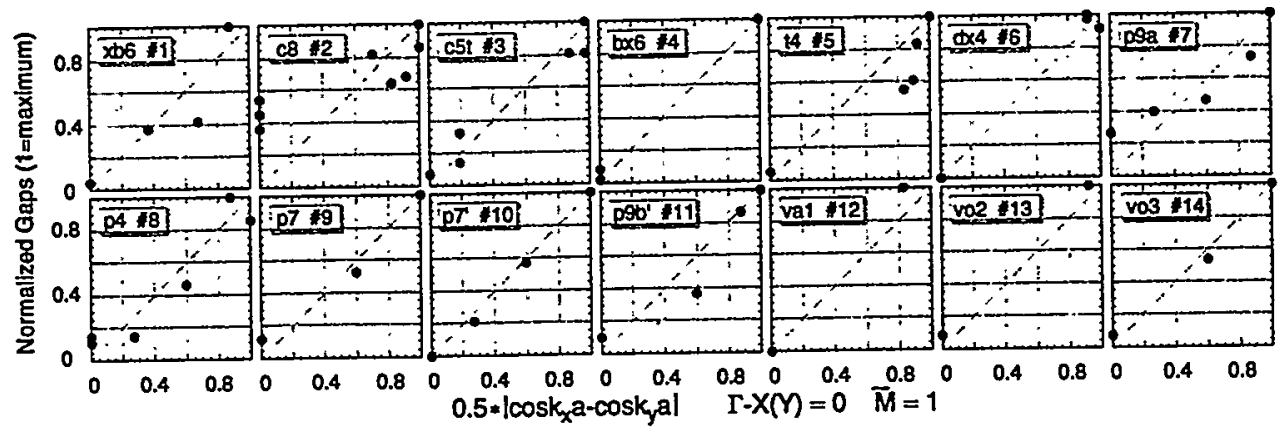

Fig.4 Superconducting gap size of 14 different $\mathrm{Bi} 2212$ samples plotted against the function $1 \cos _{\mathrm{x}} \mathrm{a}$-coskyal. The near-linearity of the data favors a d-wave gap over a simple swave gap.

\section{ACKNOWLEDGMENTS}

This research was supported by the Department of Energy through the Office of Basic Energy Sciences, Division of Chemical Sciences. The office's Division of Materials Science has provided support for this research. Beamline 5 of SSRL was built with DARPA, ONR, AFOSR, AOR, DOE and NSF support. The Stanford work was supported by NSF grants DMR9357507 and DMR9121288, and the NSF grant through the Center of Material Research. D.S.D. acknowledges support from a DOE Distinguished Postdoctoral Research fellowship. Z.X.S. would like to acknowledge the Alfred P. Sloan Foundation for it's support.

\section{REFERENCES}

[1] D.J. Scalapino et al., Phys. Rev. B 34, 8190 (1986); N.E. Bickers et al., Phys. Rev. Lett. 62, 961 (1989)

[2] P. Monthoux et al., Phys. Rev. Lett. 67, 3448 (1991); Phys. Rev. Lett. 69, 961 (1992)

[3] S. Chakravarty et al., Science 261, 337 [1993]

[4] D.S. Dessau et al., Phys. Rev. Lett. 71, 4278 (1993)

[5] P.W. Anderson, Phys. Rev. Lett. 67, 660 (1991)

[6] P.A. Lee et al. [unpublished]

[7] D.S. Dessau et al., (unpublished)

[8] D.S. Dessau et al., Phys. Rev. Lett. 71, 4278 (1993) 


\title{
Photoemission Studies of High- $T_{\mathcal{C}}$ Superconductors
}

\author{
D.M. King, Z.-X. Shen, W.E. Spicer, D.S. Dessau, and B.O. Wells \\ Stanford Synchrotron Radiation Laboratory, Stanford, CA 94309 \\ Stanford Electronics Laboratory, Stanford Universiy, Stanford, CA 94305 \\ Physics Department, Massachusetts Institute of Technology, Cambridge, MA 02139
}

\section{INTRODUCTION}

This report describes recent information we have acquired on the electronic structure from ARPES experiments which was made possible by significant advances in our instrumentation. In the superconductors, interesting features in the electronic structures have been discovered near the Fermi level: apparently band like Fermi surfaces, significantly less quasiparticle dispersion than predicted by band calculations, the presence of an extended saddle point near the Fermi level, and Fermi surfaces with strong nesting features. A critical analysis of experimental data in light of recent advances in many-body theory shows that effects previously thought to be explained by band theory alone (such as the large Fermi surface) may also be accounted for by an idealized many-body approach (1). In addition, there are many elements of the data which can be better described using many-body approaches. Therefore, it appears that it will be necessary to go beyond one-electron theory to understand the electronic structure of the cuprates (1).

\section{EXPERIMENT}

In a photoemission experiment, incident photons excite electrons above the vacuum level so they can be collected and energy analyzed. A typical valence band photoemission spectrum from a single band material consists of a peak and some background. The kinetic and binding energies of the electron are determined by the peak energy position. The crystal momentum is determined by the simple expressions: $|\mathrm{k}|=\left(2 \mathrm{mE} \mathrm{kin}_{\mathrm{n}} / \mathrm{h}^{2}\right)^{1 / 2}$ and $\mathrm{k} / /=|\mathrm{k}| \sin \theta$, where $\theta$ is the angle of the emitted electron with respect to the surface normal. By taking spectra at many angles, $\mathrm{E}$ versus $\mathrm{k}$ relationship can be obtained. For two dimensional materials, which the layered cuprates approximate, $E$ versus $k / /$ is sufficient to determine the band structure throughout the Brillouin zone.

\section{RESULTS}

If one only considers the Fermi surface data from metallic samples, it is tempting to believe that we should model the electronic structure of these superconductors using a one-electron band picture (2). This scenario severs the relationship between the metals and insulators and attributes the separation to either a phase transition (3) or the creation of band-like states near $E_{F}$ with doping (4). There are, however, several reasons to question the use of the delocalized approach to describe the metallic copper oxides. First, photoemission satellite structures are present near the Cu core levels and in the valence band, indicative of the presence of a large $U$ in metallic samples. Second, the band picture predicts a large Fermi surface which should correspond to a high carrier density which varies with doping like $1-x$. This contradicts the results of transport measurements in the low doping regime which reveal a carrier density proportional to $x$, instead of $1-x(5)$.

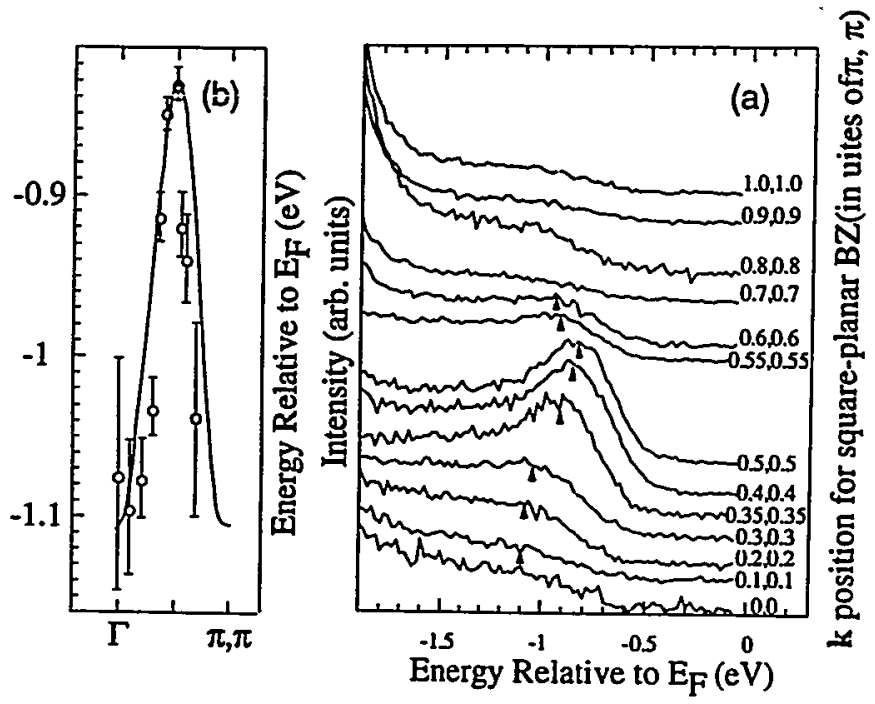

Fig.1. Experimental data from the Mott insulator $\mathrm{Sr}_{2} \mathrm{Cl}_{2} \mathrm{CuO}_{2}$. The inset shows a comparison of the data with a t-J model calculation where $\mathrm{J}$ is determined from Raman experiments.

One consequence of this discrepancy is that while NCCO appears to have a hole like Fermi surface, it is found experimentally to have $n$-type carriers. Third, the experimentally observed band near $\mathrm{EF}_{\mathrm{F}}$ is always much narrower than predicted by LDA calculations everywhere in $k$ space. It is difficult to tell just how much narrower the experimental dispersion is since the peak becomes hard to distinguish at larger binding energies and the top of the band is 
always unoccupied in a metal and therefore cannot be measured by photoemission. Finally, estimates for the ratio of the LDA calculated dispersion to the experimental data vary from a factor of two for some measurements along $(0,0)$ to $(\pi, \pi),(6)$ to a factor of five near the saddle point ( 7$)$.
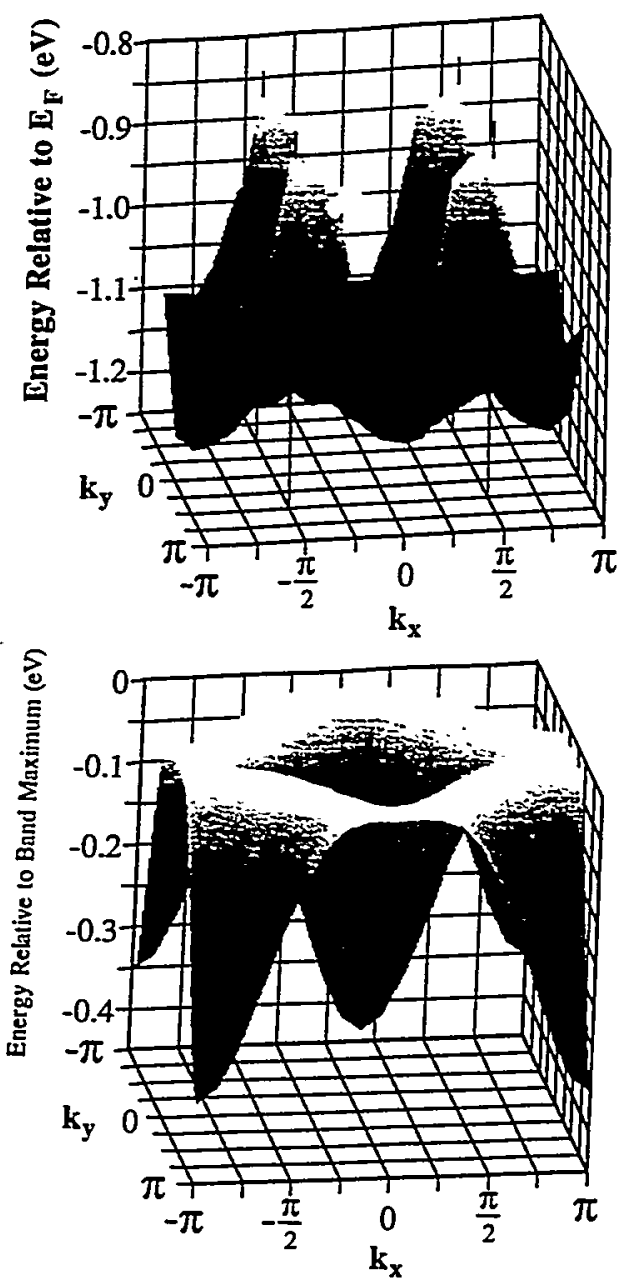

Fig.2. E versus $\mathbf{k}$ relationship of experimental data from $\mathrm{Sr}_{2} \mathrm{Cl}_{2} \mathrm{CuO}_{2}$ (a), and the calculated results from the $\mathrm{t}-\mathrm{J}$ model following a formula presented in reference 11 (b).

To further differentiate the localized and itinerant approaches, it is instructive to study the evolution of the electronic structure as a function of doping. The recent work by Wells et al. (8), which reports the dispersion of the single photohole in the copper oxide insulator $\mathrm{Sr}_{2} \mathrm{CuO}_{2} \mathrm{Cl}_{2}$, pursues this idea. $\mathrm{Sr}_{2} \mathrm{CuO}_{2} \mathrm{Cl}_{2}$ is closely related to the high- $\mathrm{T}_{\mathrm{C}}$ superconductors because it is a layered compound and its electronic properties are dominated by the $\mathrm{CuO}_{2}$ planes. In fact, it has the same structure as the well known high $\mathrm{T}_{C}$ parent compound $\mathrm{La}_{2} \mathrm{CuO}_{4}$ and an almost identical material, $\mathrm{Sr}_{2} \mathrm{CuO}_{2} \mathrm{~F}_{2}+\delta$, which has recently been made superconducting (9). The study of this insulator not only gives information on the phenomenology of the doping process but also provides a test of the localized models in the insulating state where the models are best defined. Fig.1a reproduces data by Wells et al. along the $(0,0)$ to $(\pi, \pi)$ direction (8). A peak disperses towards higher energy, reaches its maximum energy near $(\pi / 2$, $\pi / 2$ ), then loses intensity suddenly and starts to move back to lower energies. The total dispersion is slightly less than 0.3 $\mathrm{eV}$, which gives a rough estimate of the band width. Throughout the Brillouin zone the quasiparticle peak drops in intensity for $\mathrm{k}$ outside a line connecting $(\pi, 0)$ and $(0, \pi)$, the antiferromagnetic Brillouin zone boundary.

Figure 2 shows three dimensional plots that compare the overall shape of the lowest energy bands measured in $\mathrm{Sr}_{2} \mathrm{CuO}_{2} \mathrm{Cl}_{2}$ and that predicted by the $\mathrm{t}-\mathrm{J}$ model as given by Liu and Manousakis or E. Dagotto et al. $(10,11)$. The t-J model is a variation of the Hubbard model with infinite $U$ that includes a t term describing electron hopping and a $\mathrm{J}$ term describing the magnetic interaction (12-14). In this $t-J$ model, the band width is predicted to be 2.2J (10). By using an experimentally determined J from Raman scattering, we are able to compare the experimental data with no free parameters. Experimental data and a theoretically calculated curve along $(0$, $0)$ to $(\pi, \pi)$ is presented in Fig.1b. The results in Fig. 1 and Fig.2 show that both the calculated bandwidth and the prediction that the band reaches its maximum at $(\pi / 2, \pi / 2)$ agree with the data remarkably well. The quantitative agreement between the experiment and many-body theory along the $(0,0)$ to $(\pi, \pi)$ direction is significant because it shows unambiguously that the energy scale of the insulating band is controlled by $\mathrm{J}$. However, the results also show that the $\mathrm{t}-\mathrm{J}$ model cannot describe the overall shape of the band in the insulator and cannot explain the observed spectral weight effect. In addition to the $\mathrm{t}-\mathrm{J}$ model, the Hubbard model has also been used to obtain similar dispersion relations in the intermediate coupling regime (15). In a strong coupling regime, analysis of one hole in an antiferromagnetic background shows that the band reaches a maximum at $(\pi / 2$, $\pi / 2)(16)$.

A simple rigid band picture would predict that doping with a very small number of holes would lower the Fermi level through the band measured in $\mathrm{Sr}_{2} \mathrm{CuO}_{2} \mathrm{Cl}_{2}$ and produce a Fermi surface with pockets centered at $(\pi / 2, \pi / 2)$. These pockets are qualitatively similar to those depicted in Fig.2a. The key issue is understanding how the Fermi surface evolves from small'pockets centered at $(\pi / 2, \pi / 2)$ to a large pocket centered at $(\pi, \pi)$. To illustrate this, Fig.3a and Fig.3b show the experimental $E$ versus $\mathbf{k}$ relations from superconducting samples of $\mathrm{Bi} 2201$ and $\mathrm{NCCO}$. It is very clear that one cannot get the results in Fig. 3 by doping the bands in Fig.2a rigidly either with electrons or holes. 'Although both Bi2201 and $\mathrm{NCCO}$ have one $\mathrm{CuO}_{2}$ plane per unit cell, p- and n- type doping apparently produces very different results. For p-type doping, an important difference in band shape between the insulator and the metal is the saddle points close to the Fermi level near $(\pi, 0)$ that are missing in the insulator. The near degeneracy of the flat band saddle points near $(\pi, 0)$ and the Fermi level may severely affect the shape of the Fermi surface. The development of the flat band saddle points near the Fermi level appears to be the key to the evolution of the Fermi surface. If we consider that the bands in the region outside the 
antiferromagnetic Brillouin zone have very little spectral weight, as noted in Fig. 1a for $\mathbf{k}$ greater than $(\pi / 2, \pi / 2)$, a many-body approach such as the t-J model, shown in Fig.2b, will produce a Fermi surface that closely resembles the observed Fermi surface of Bi2201 if oscillator strength effects are included. The results from n-type NCCO are much harder to reconcile using many-body theories.
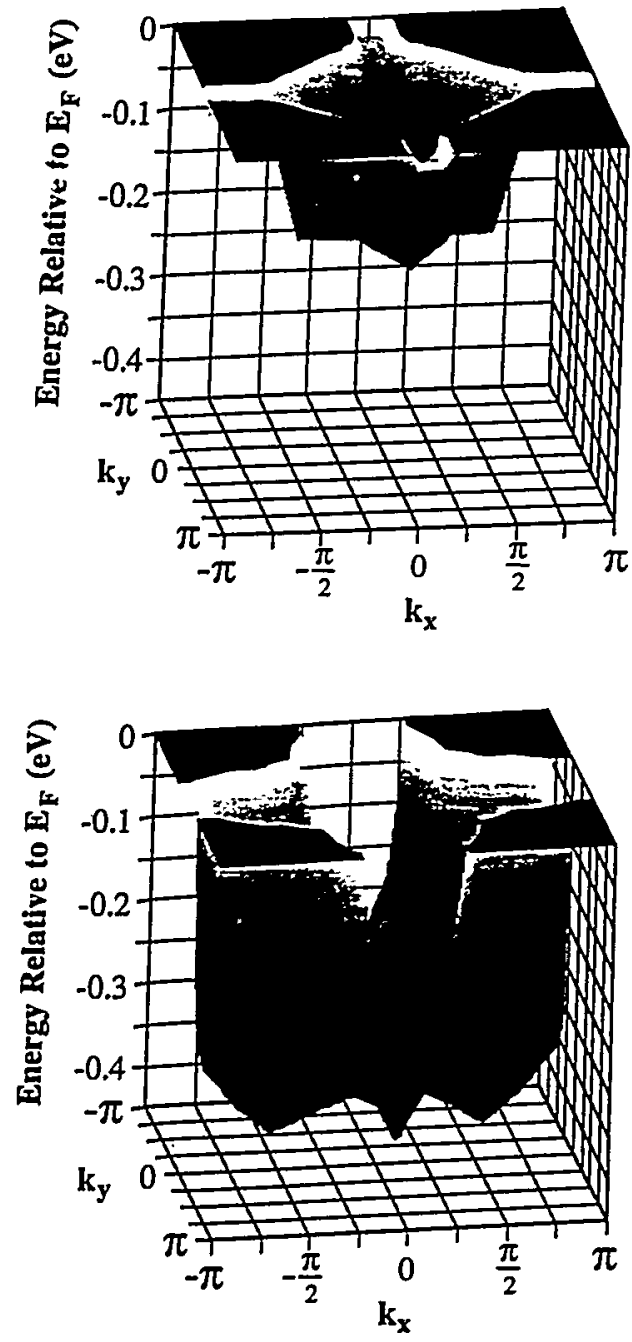

Fig.3. E versus $k$ experimental data from $p$-type Bi2212 (a) and n-type NCCO (b).

The results of Wells et al. have several very important implications. First, it reveals that the lowest energy band in the insulator and the band that crosses $\mathrm{EF}_{\mathrm{F}}$ in the hole doped metals are very similar: the band is separated from the main valence band in the same way, it has similar dispersion, and it has similar intensity modulation as a function of energy. Thus the dispersive band that crosses $\mathrm{EF}_{\mathrm{F}}$ in metallic samples appears not to originate from doping and should thus be explained using the same picture in both doping regimes. Although it is not clear whether many-body theory should give small Fermi surface pockets, the decrease in oscillator strength noted in Fig.1a for $\mathbf{k}$ greater than $(\pi / 2, \pi / 2)$ may make part of the Fermi surface hard to observe by photoemission. We can understand this intensity change in the language of the SDW model, illustrated in Fig.2c (17). The folded back "shadow band" results from the antiferromagnetic order (dashed line) and will have much less oscillator strength and spectral intensity than the original band (dotted line). Therefore, between the mixture of the two bands in the SDW state, one can see part of the band better than the others (dark solid line). This oscillator strength effect is preserved in the intermediate coupling regime (15), and complicates the study of the cross-over. If there are small Fermi surface pockets, centered at $( \pm \pi / 2, \pm \pi / 2)$ in the lightly doped material, then because of the oscillator strength effect only the side of the small Fermi surface, corresponding to the original band, will be clearly seen in a photoemission experiment. This may account for the puzzling experimental data by $\mathrm{Liu}$ et al. from $\mathrm{YBa}_{2} \mathrm{Cu}_{3} \mathrm{O}_{\mathrm{x}}$ which shows that the band crossing behavior along $(0,0)$ to $(\pi, \pi)$ is basically the same as $x$ varies from 6.9 to 6.4 (3). Therefore, the oscillator strength and "shadow band Fermi surface" issues are important for a comprehensive understanding of the photoemission data and provides a heuristic understanding of the subtleties in the evolution of the Fermi surface data as a function of doping. Finally, this work suggests that the electronic structure in the metallic phase may be affected by the short range antiferromagnetic spin fluctuations even though the effects may be very difficult to detect. Recently, Aebi et al. claimed to have observed such an effect (18), but the interpretation of the data is controversial (19).

\section{CONCLUSIONS}

We feel that the overall picture emerging from photoemission leads to the conclusion that a many-body, Hubbard-like approach is appropriate to describe the electronic structure of the cuprate superconductors. However, there are many remaining problems with the many-body theories as they stand. For example, there are no correct predictions of either the overall shape of the lowest energy band in the insulator or the evolution of the saddle point near $(\pi, 0)$ as a function of doping. Furthermore, the $\mathrm{t}-\mathrm{J}$ model, while predicting a correct dispersion and band width from $(0,0)$ to $(\pi, \pi)$, cannot produce the observed oscillator strength change because the insulating gap is set to infinity in the model (8), and the one-band Hubbard model, as it stands now, cannot account for the observed $p$ and n-type asymmetry. There are also serious new attempts to improve the band calculations to better deal with the highly correlated materials. This includes the self-interaction correction, $\mathrm{LDA}+\mathrm{U}$, and other approaches $(20,21)$. These calculations have improved the LDA calculated results in many transition-metal oxides. Unfortunately, by incorporating an ad-hoc inclusion of the Coulomb repulsion $U$ in the potentials, the calculations are no longer from first principles. At this stage, insufficient evidence exists to consistently account for the ARPES data. 
In summary, we continue to be puzzled by the fascinating electronic structure and physical properties but clear progress is being made towards a coherent understanding.

\section{ACKNOWLEDGEMENTS}

We acknowledge the stimulating discussions with R.B. Laughlin, S. Doniach, A. Kapitulnik, D.J. Scalapino, J. Ruvalds, P.W. Anderson and S.C. Zhang. We also acknowledge J.L. Peng, Z.Y. Li, and R.L. Greene for providing the high quality single crystal samples used in most of the work. SSRL is operated by the DOE Office of Basic Energy Science, Division of Chemical Sciences. The Office's Division of Materials Science has provided support for this research. The Stanford work was also supported by NSF grant DMR9121288, DMR9357057, and the NSF grant through the Center of Materials Research. ZXS would like to acknowledge support from the Alfred P. Sloan foundation and DSS would like to acknowledge support from a DOE Distinguished Postdoctoral Research Fellowship.

\section{REFERENCES}

[1] Z.X. Shen et al., Science 267, 343 (1995).

[2] W.E. Pickett et al., Science 255, 46 (1992).

[3] R. Liu et al., Phys. Rev. B 45, 5615 (1992); Phys. Rev.

B 46, 11056 (1992).

[4] T. Takahashi, H. Matsuyama, H. Katayama-Yoshida, Y. Okabe, S. Hosoya, K. Seki, H. Fujimoto, M. Sata, H. Inokuchi, Nature 334, 691 (1988); T. Takahashi in "Strong Correlation and Superconductivity," Eds. H. Fukuyama, S. Maekawa, and A.P. Malozemoff, Springer-Verlag, Berlin, 311 (1989).

[5] N.P. Ong, Z.Z. Wang, J. Clayhold, J.M. Tarascon, L.H. Greene, and W.R. McKinnon, Phys. Rev. B 35, 8807 (1987); N. P. Ong, in Phys. Prop. of the High Temperature Superconductors, vol. 2, ed. by D.M. Ginsberg (World Scient. Singapore 1990).

[6] C.G. Olson, R. Liu, A.-B. Yang, D.W. Lynch, A.J. Arko, R.S. List, B.W. Veal, Y.C. Chang, P.Z. Jiang, A.P.

Paulikas, Science 245, 731 (1989).

[7] D.S. Dessau, Z.-X. Shen, D.M. King, D.S.Marshall, L.W. Lombardo, P.H. Dickinson, J. DiCarlo, C.-H. Park, A.G. Loeser, A. Kapitulnik and W.E. Spicer Phys. Rev. Lett. 71, 2781 (1993).

[8] B.O. Wells, Z.-X. Shen, A. Matsuura, D.M. King, M. Kastmer, O. Jepsen, M. Greven, and R.J. Birgeneau, to be published in Phys. Rev. Lett.

[9] M.Al-Mamouri et al., Nature 369, 382 (1994).

[10] Z. Liu and E. Manousaki, Phys. Rev. B 45, 2425

(1992).

[11] E. Dagotto et al., Phys. Rev. B 41, 9049 (1990); E. Dagotto, Rev. Mod. Phys., July, 1994.

[12] F.C. Zhang and T.M. Rice, Phys. Rev. B 37, 3759 (1988).

[13] H. Eskes, M.B. Meinders, G.A. Sawatzky, Phys. Rev. Lett. 67, 1035 (1991).
[14] R.B. Laughlin, Proc. of VI Physics Summer School, Canberra, Australia, edited by M.P. Das and J. Mahanty (World Sci., Singapore, 1994), p. 294.

[15] N. Bulut, D.J. Scalapino, and S.R. White, Phys. Rev. Lett. 73, 748 (1994).

[16] S.A. Trugman, Phys. Rev. B 37, 1597 (1988).

[17] M. Sigrist and T.M. Rice, Journal of Phys. Soc. of Japan 61, 4283 (1992).

[18] P. Aebi, J. Osterwalder, P. Schwaller, L. Schapbach, M. Shimoda, T. Mochiku and K. Kadowaki, Phys. Rev. Lett. 72, 2757 (1994).

[19] S. Chakravarty, preprint.

[20] V.I. Anisimov et al., Phys. Rev. Lett. 68, 345 (1992).

[21] A. Svane and O. Gunnarsson, Phys. Rev. Lett. 65, 1148

(1990); A. Svane, Phys. Rev. Lett. 68, 1900 (1992). 


\title{
The Interaction of Propylene and Oxygen with $\mathrm{Cu}_{2} \mathrm{O}(111)$ : A Study of Heterogeneous Oxidation Catalysis
}

\author{
Brad Reitz, Pierre Kennepohl, Edward I. Solomon \\ Department of Chemistry, Stanford University \\ Stanford, CA 94305
}

\section{INTRODUCTION}

Research has been performed to understand heterogeneous oxidation catalysis on an atomic level, with particular emphasis being placed on the propylene to acrolein conversion over cuprous oxide. Commercially, a bismuth-molybdate catalyst is uscd $^{1}$, but isolope and other studies indicate $\mathrm{Cu}_{2} \mathrm{O}$ catalyzes this reaction via a similar mechanism ${ }^{2}$ and has well defined surfaces. ${ }^{3}$ Our research focuses on defining the interaction of dioxygen, propylene, and their combination with the $\mathrm{Cu}_{2} \mathrm{O}(111)$ surface and on identifying and isolating novel chemical species formed on the surface. We are interested in identifying the clectronic and geometric structurc of thesc intermediates and in understanding the redox chemistry of the surface and its relevance to hetcrogencous catalysis.

\section{EXPERIMENTAL}

Beamline 3-1 was used to perform valcnce band studics and Beamline 10-1 was used for core level studies.

The surfaces were cleaned in UHV by a series of $\mathrm{Ar}^{+}$ion sputtering and annealing cycles. Research grade oxygen and propylene were used and were introduced through a leak valve into the experimental chamber via a separate UHV line.

\section{RESULTS AND DISCUSSION}

Studics of oxygen exposures on $\mathrm{Cu}_{2} \mathrm{O}(111)$ reveal three different species present at low and room temperature (Figure 1). At low exposures of oxygen $(<100 \mathrm{~L})$ and low temperature (135K), valence band spectra show a two peak pattern (13 and 9.7 $\mathrm{eV}$ binding encrgy). Upon further exposure, a third peak grows in at $5.9 \mathrm{eV}$ binding energy referenced to the vacuum level. Furthermore, upon thermal cycling, the peak at $13 \mathrm{eV}$ decreases in intensity, leaving the two lower binding energy peaks. Preliminary core level studies indicate a broad $\mathrm{O} 1 \mathrm{~s}$ peak at $534 \mathrm{eV}$ binding energy which grows in with exposure and attenuates upon heating (Figure 2). This peak position is consistent with a neutral oxygen species. Gas phase UPS spectra ${ }^{4}$ show molecular oxygen to have a four peak pattern $\left(20.4\left(3 \sigma_{\mathrm{g}}\right), 18.2\left(3 \sigma_{\mathrm{g}}\right)\right.$, $16.6\left(1 \pi_{\mathrm{u}}\right)$, and $12.3 \mathrm{eV}\left(1 \pi_{\mathrm{g}}\right)$ binding energy). The observed peak pattern on $\mathrm{Cu}_{2} \mathrm{O}(111)$ at high exposure and low temperature is consistent with molecular oxygen with overlap of the $3 \sigma_{\mathrm{g}}$ and $1 \pi_{\mathrm{u}}$ orbitals. The higher temperature oxygen intermediate which produces a two peak valence band spectrum $(9.7$ and $5.9 \mathrm{eV})$ is tentatively assigned as atomic oxygen with a possible additional contribution from formation of surface oxide. However, no oxidation to $\mathrm{Cu}^{2+}$ is observed under these conditions as evidenced by the lack of satellite structure in the $\mathrm{Cu} 2 \mathrm{p}$ region, and the fact that no oxygen 1s feature consistent with reduced oxygen species is present. Our research now focuses on using variable energy PES (resonance and cross sections) to identify the origin of the high temperature valence band features, as well as the low temperature, low exposure species.

Studies of propylene chemisorption on $\mathrm{Cu}_{2} \mathrm{O}(111)$ have also been conducted. Valence band data of ambient exposures reveal a complex peak pattern $(20.2,16.8$, 13.6, and $9.6 \mathrm{eV}$ binding energy referenced to the vacuum level) (Figure 3). Carbon 1s 
studies reveal that upon increasing exposure, a broad pcak grows in at $291.6 \mathrm{cV}$ binding encrgy and attenuates upon heating. Oxygen 1s studies reveal a small peak at $532.2 \mathrm{eV}$ (oxide - $529.5 \mathrm{cV}$ ) binding energy which grows in with increasing coverage, but disappcars upon heating, thus indicating the presence of an oxygen containing intermediate (Figure 4). A possible source of this new $O$ is peak would be from deprotonation of propylene to form surface hydroxide. Also, it has been shown that $\mathrm{Cu}_{2} \mathrm{O}$ can catalyze the oxidation of propylene in the absence of oxygen through the incorporation of lattice oxide ${ }^{5}$, so an alternate assignment would be an oxidized propylene intermediate. Current studies focus on identifying the nature of this intermediate and understanding the interaction of propylene with the cuprous oxide surface relative to substrate activation.

\section{SUMMARY}

Three forms of oxygen and at least one propylene intermediate have been observed on $\mathrm{Cu}_{2} \mathrm{O}(111)$. It is now important to define these intermediates, both their chemical nature and their electronic structure, and to relate these to parallel silver/oxygen intermediates to understand elcctrophilic versus nuclcophilic oxidation catalysis.

\section{REFERENCES}

(1) Callahan, J.L.; Grassclli, R.K.; Milberger, E.C.; Strecker, H.A. Ind. Eng. Chem. Prod. Res. Develop., 1970, 9, 134.

(2) Adams, C.R.; Jennings, T.J. J. Catalysis. 1963, 2, 63-68.

(3) Schulz, K.H.; Cox, D.F. Phys. Rev.B. 1991, 43, 3061.

(4) Sicgbahn, K. ESCA Applied to Free Molecules. 1969.

(5) Schulz, K.H.; Cox, D.F. Surface Science 1992, 262, 318-334.

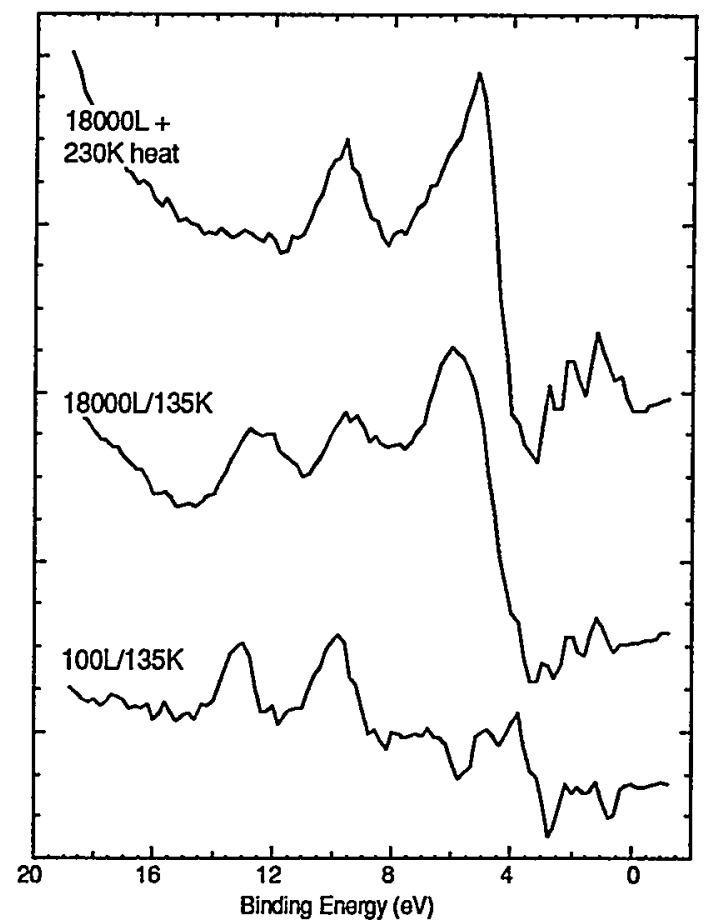

Figure 1: Valence band difference spectra of the different oxygen species observed on the $\mathrm{Cu}_{2} \mathrm{O}(111)$ surface. Taken at BL 3-1 (hv=40eV) and with HeII light (hv=40.8 eV)

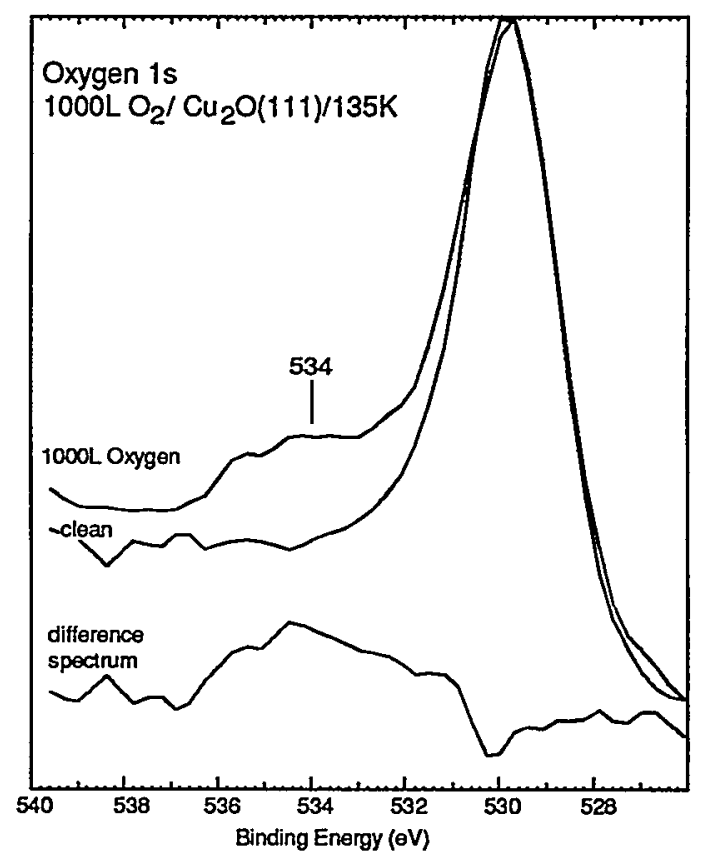

Figure 2: Oxygen 1s spectra of clean and 1000L exposed $\mathrm{Cu}_{2} \mathrm{O}(111)$. Taken at $\mathrm{BL} 10-1 \mathrm{hv}=$ $650 \mathrm{eV}$ ) 


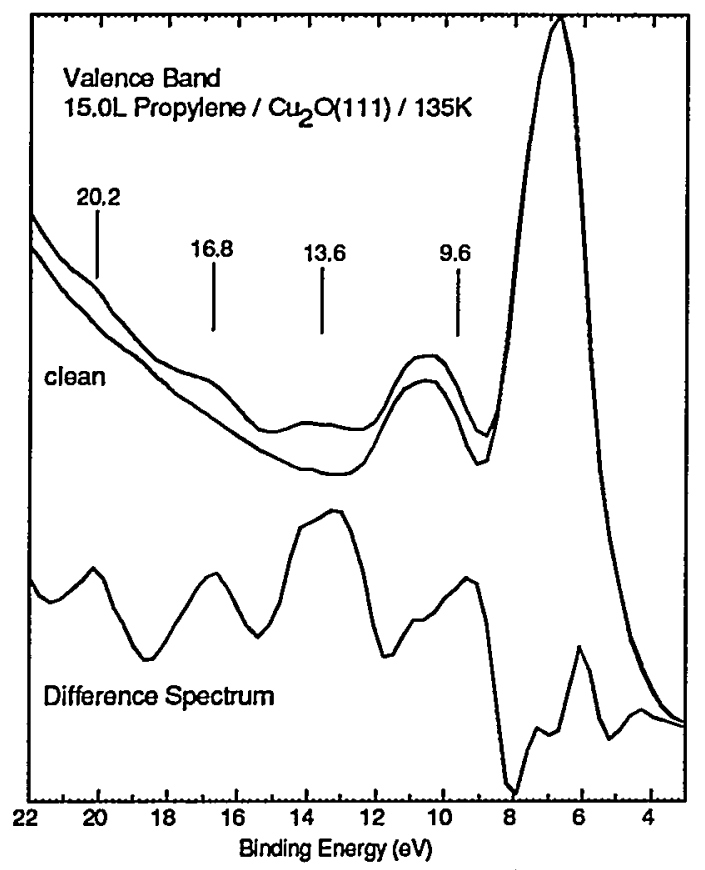

Figurc 3: Valence band spectra of clean and $15 \mathrm{~L}$ propylene exposed $\mathrm{Cu}_{2} \mathrm{O}(111)$. Taken at BL 3-1 $(\mathrm{hv}=40 \mathrm{eV})$.

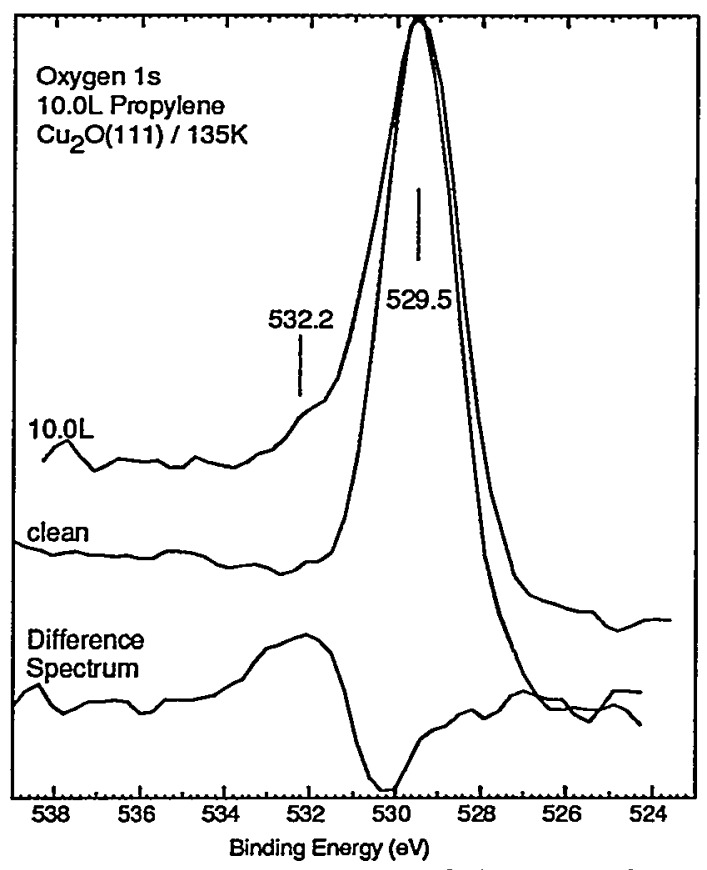

Figure 4: Oxygen 1s spectra of clean and 10L propylene exposed $\mathrm{Cu}_{2} \mathrm{O}(111)$. Taken at $\mathrm{BL} 10$ $1(\mathrm{hv}=650 \mathrm{eV})$. 


\title{
ANGLE RESOLVED PHOTOEMISSION ON QUASI-LOW DIMENSIONAL MATERIALS
}

\author{
G.-H. Gweon ${ }^{1}$, R. Claessen ${ }^{2}$, F. Reinert ${ }^{2}$, W.P. Ellis ${ }^{3}$, J.W. Allen ${ }^{1}$, \\ and Z.-X. Shen ${ }^{4}$ \\ ${ }^{1}$ Department of Physics, University of Michigan, Ann Arbor, MI 48109 \\ ${ }^{2}$ Universität des Saarlandes, Fachrichtung 10.2 - Experimentalphysik, D-66041 \\ Saarbrücken, Germany \\ ${ }^{3}$ Los Alamos National Laboratory, Los Alamos, New Mexico 87545 \\ ${ }^{4}$ Dept. of Applied Physics, Stanford University, Stanford, CA 94305
}

\section{INTRODUCTION}

Our basic interest is to distinguish between Fermi liquid and non-Fermi liquid behavior in angle resolved photoemission spectroscopy (ARPES) of quasi low dimensional materials. The result on $\mathrm{K}_{0.3} \mathrm{MoO}_{3}$, blue bronze, we obtained last year was very interesting ${ }^{1}$ in this respect. So, more experiments were done at the Synchrotron Radiation Center (SRC) during the past year, and more at SSRL during this beamtime period as well. As a result, we made an argument ${ }^{1}$ for the Luttinger liquid $^{2}$ behavior of this material, based on the observation of an ARPES dispersion 5 times bigger than the band calculation and some known transport properties. During this run, we were able to obtain a high resolution ARPES of the blue bronze free of any known photon-induced surface degradation effect ${ }^{3}$, confirming the absence of spectral weight at the Fermi level. We also had intended to look at the temperature and aperture dependence of ARPES of the blue bronze to study the charge density wave (CDW) and the Luttinger liquid ${ }^{2}$ aspects respectively of quasi 1-d metallic system. But for these experiments the sample had to be irradiated too long for the surface to remain undamaged.

This year, we have also extended our study to other inorganic and organic systems. In the former category we have looked at the quasi1d metal $\left(\mathrm{TaSe}_{4}\right)_{2} \mathrm{I}$ and the strongly anisotropic semiconductor $\mathrm{ZrSe}_{3}$ as reference material. The organic systems included (TMTSF) ${ }_{2} \mathrm{ClO}_{4}$ and (fluoranthene) ${ }_{2} \mathrm{PF}_{6}$, both 1-d metals, and the quasi 2-d metal (BEDT-TTF) ${ }_{2} \mathrm{I}_{3}$. Concerning the search for Luttinger liquid behavior the organic ones seem to be more interesting since for many of them the electron-electron correlation is supposed to be important. In contrast, the inorganic metals generally show a CDW transition, which signifies the importance of electron-phonon interaction as well. However, it turns out that the organic samples we studied are very susceptible to the photon beam or do not cleave very well, so that it is very challenging to obtain good ARPES. For the inorganic samples, we were able to obtain good ARPES. The case of $\left(\mathrm{TaSe}_{4}\right)_{2} \mathrm{I}$ is interesting. It shows a nice dispersion along the easy axis of the crystal and still has vanishing spectral weight at the Fermi energy for all the angles, agreeing with the literature ${ }^{4}$. We note that the experimental dispersion is similar in magnitude to the band calculation ${ }^{5}$. This is to be contrasted to the case of blue bronze ${ }^{1}$ for which the band calculation was performed by the same group. In addition, we have observed interesting splittings of the peaks into two components in the CDW phase.

\section{EXPERIMENT}

The angle resolved photoemission spectra were taken at Beamline 5 at SSRL. Samples were cleaved in situ by the top post method. The pressure in the spectrometer was $\sim 5 \times 10^{-11}$ Torr for most of the measurements, while for (TMTSF) ${ }_{2} \mathrm{ClO}_{4}$ it increased to $\sim 2 \times 10^{-10}$ Torr. The temperature of the sample was stabilized by an open cycle He refrigerator and a resistive heater. Spectra were taken from surfaces of freshly evaporated gold to obtain the Fermi level reference energy and instrumental resolution. The combined analyser and monochromator resolution was as good as $\sim 34 \mathrm{meV}$ (FWHM), but for a slightly 


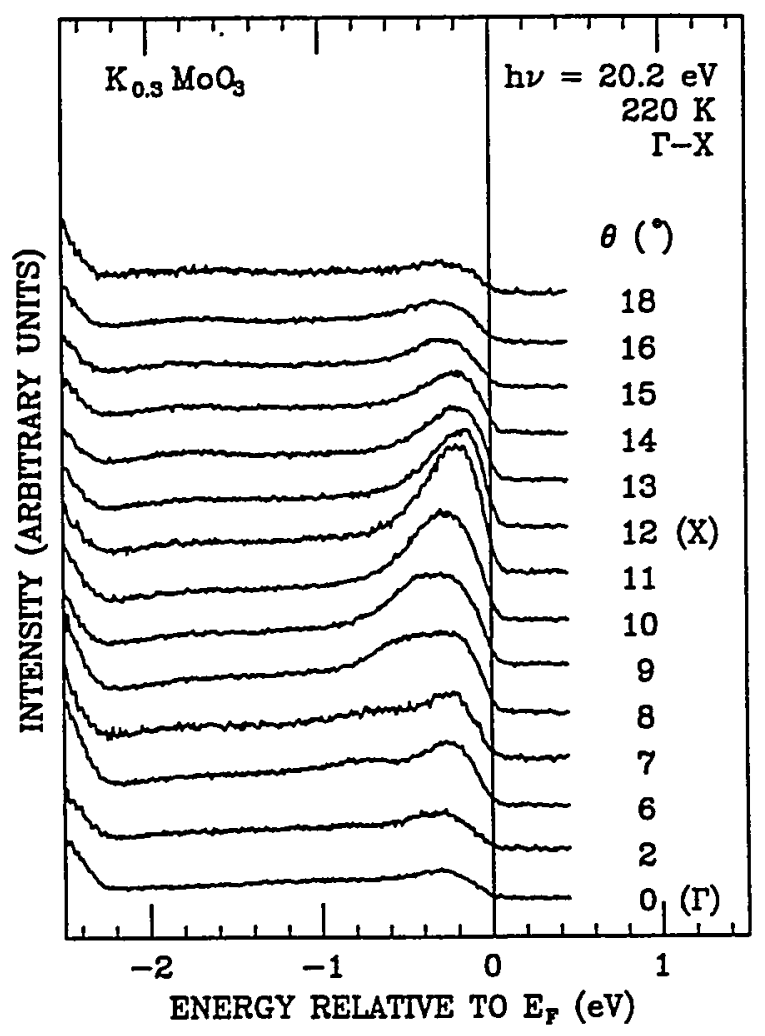

Fig. 1 ARPES along the $b$ axis of $\mathrm{K}_{0.3} \mathrm{MOO}_{3}$.

worse resolution of $\sim 42 \mathrm{meV}$ (FWHM), the detected intensity was much higher, typically thousands of counts per second.

\section{RESULTS}

Fig. 1 shows the ARPES of the blue bronze, $\mathrm{K}_{0.3} \mathrm{MoO}_{3}$, along the easy-conducting axis of the crystal. The data were obtained within 10 hours after the cleave, and with an instrumental resolution of $42 \mathrm{meV}$ (FWHM). Although we obtained the same set of spectra in last year's run $^{1}$ with similar resolution, we also observed then that we had signs of sample damaging, for example the growth of a defect induced structure at binding energy $\sim 2 \mathrm{eV}$. Fig. 1 shows that in this year's run we have eliminated this problem by quick and efficient measurements. The $2 \mathrm{eV}$ structure is hardly visible. Also, one notes that the peak positions are shifted towards lower binding energy. Otherwise the overall spectral features are similar to our previous results ${ }^{1}$. In particular, this high resolution scan confirms that the intensity at the Fermi energy is very low for all the angles, i.e. we cannot observe a Fermi edge. We tried two more experiments on this sample, to observe the CDW gap opening below the transition temperature, and to change the apertures of the electron analyzer in order to observe changes of the power law onset of the spectrum. For the latter we prepared a very small $(1 / 4 \mathrm{~mm} \times 1 / 4 \mathrm{~mm}$ surface) sample. However, both of the experiments required considerable time and beam irradiation, and thus we found that the sample had been damaged before one cycle of the experiment was done. For example, for the temperature dependence we observed a peak-shift of $\sim 50 \mathrm{meV}$ by going from $220 \mathrm{~K}$ to $70 \mathrm{~K}$, but upon increasing the temperature again we could not reproduce the $220 \mathrm{~K}$ data. Instead the data were similar to the $70 \mathrm{~K}$ data.

In Fig. 2 we show ARPES of another inorganic compound, $\left(\mathrm{TaSe}_{4}\right)_{2}$ I. This material is also a CDW material $^{6}$ with a transition temperature of $263 \mathrm{~K}$. As for the blue bronze, angle-integrated high resolution photoemission spectra ${ }^{7}$ show anomalously low weight at the Fermi energy for this material.

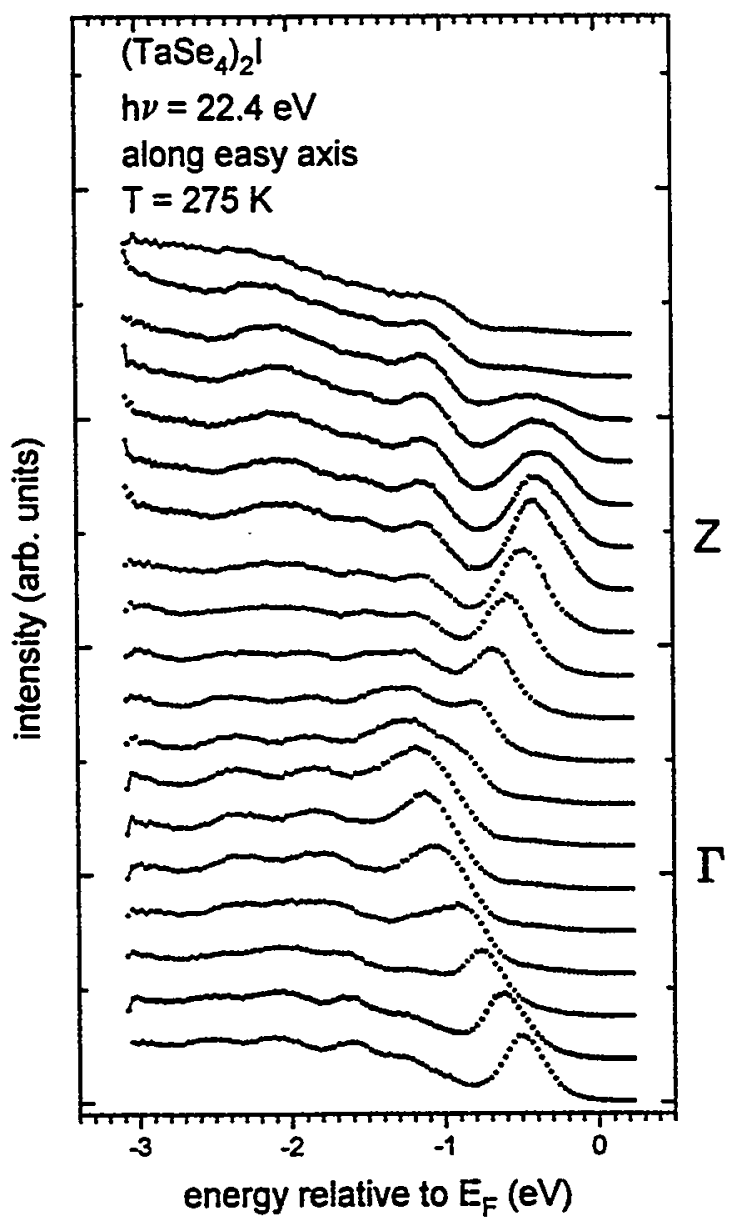

Fig. 2 ARPES along the easy axis of $\left(\mathrm{TaSe}_{4}\right)_{2} \mathrm{I}$. 
We cannot find any weight in ARPES ${ }^{4}$ either. Our ARPES result is similar to that of Ref. [4]. We find that the band calculation reproduces the conduction band ARPES reasonably well. The bandwidth in the band calculation $(0.8 \mathrm{eV})$ is very similar to the experimental finding $(\sim 1.0 \mathrm{eV})$. Notice that this contrasts to the blue bronze case where we found ${ }^{1}$ an anomalous ARPES dispersion which is about 5 times bigger than the band calculation done by the same group.

CDW and Luttinger liquid properties are two main aspects of quasi 1-d (and 2-d as well) electronic system. In this respect, the contrast between $\left(\mathrm{TaSe}_{4}\right)_{2} \mathrm{I}$ and $\mathrm{K}_{0.3} \mathrm{MoO}_{3}$ is interesting. For the blue bronze, we argued ${ }^{1}$ that the Luttinger liquid behavior is observed so that the electronelectron interaction is as important as electronphonon interaction. For $\left(\mathrm{TaSe}_{4}\right)_{2} \mathrm{I}$, we think that electron-phonon interaction is more important than for the blue bronze, for the following two reasons. First, we observed no ARPES anomaly as in the case of the blue bronze. Second, we note that at the CDW transition ${ }^{6}$ the conductivitiy shows a different behavior from that of the blue bronze. While the blue bronze shows a metallic behavior above CDW transtion, the conductivity of $\left(\mathrm{TaSe}_{4}\right)_{2} \mathrm{I}$ just changes slopes at the transition behaving like a gapped system even above the transition ${ }^{6}$. This, being taken as an evidence of a CDW pseudo-gap in $\left(\mathrm{TaSe}_{4}\right)_{2} \mathrm{I}$, also supports our view that electronphonon interaction is more important for this material than for the blue bronze. We add that below the CDW transition we observed a splitting of the peaks near the zone boundary into two components, which may be the two bands in the band calculation. The two bands in the calculation come from bonding and anti-bonding of the $\mathrm{d}_{z^{2}}$ orbitals from two $\mathrm{TaSe}_{4}$ chains in the unit cell. It is not certain yet why the two bands are resolved only in the low temperature phase but it will definitely be closely related to the details of CDW transition.

For the reasons described above it is very desirable to obtain good ARPES data of the organic compounds. For example, (TMTSF) ${ }_{2} \mathrm{ClO}_{4}$, one in the family of materials called Bechgaard salts, is a material for which there is independent evidence of 1-d Luttinger liquid behavior ${ }^{8}$. However, our effort to obtain useful ARPES spectra was hampered by problems encountered in the surface preparation. First, some samples did not cleave very well ((BEDT-TTF $\left.)_{2} \mathrm{I}_{3}\right)$ or after the cleave the sample surfaces looked very rugged and not mirror-like, as expected for a single-crystalline surface. Whether or not this is responsible for the absence of disper-

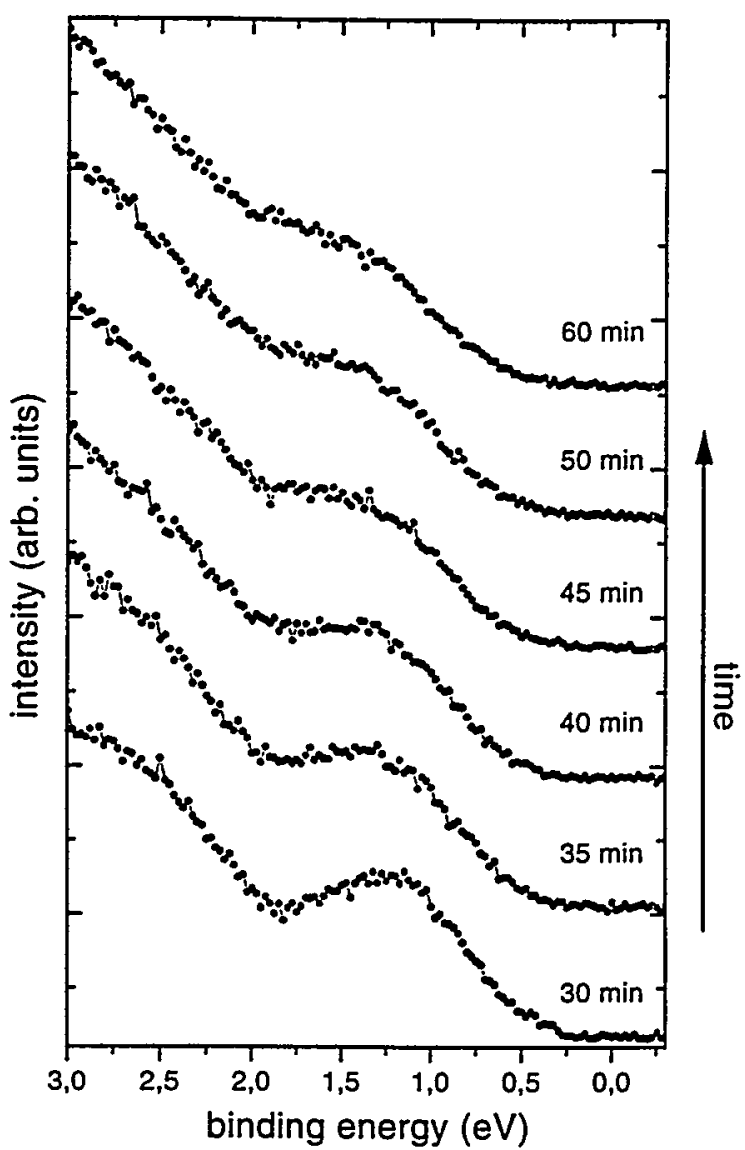

Fig. 3 ARPES spectrum of (TMTSF) $)_{2} \mathrm{ClO}_{4}$ at a fixed angle as a function of time after cleavage.

sive features in the spectra ( (fluoranthene) ${ }_{2} \mathrm{PF}_{6}$, (TMTSF) ${ }_{2} \mathrm{ClO}_{4}$ ) remains to be clarified until we have learned to prepare better surfaces. Second, as in the case of (TMTSF) ${ }_{2} \mathrm{ClO}_{4}$, the spectrum changed rather quickly due to the irradiation with VUV light. We were able to clean the surface repeatedly by scraping and to measure its photon-induced degradation as a function of exposure time. The spectra are shown in Fig. 3. It is nonetheless very exciting that the spectra of (TMTSF) ${ }_{2} \mathrm{ClO}_{4}$ obtained just after the cleavage and just after scraping resemble the angle integrated spectrum of Ref. [8] in that they show no clear Fermi edge. From a double logarithmic plot, the anomalous dimension ${ }^{2}$, assuming that the spectrum that we obtained is an angle-integrated one, is $\alpha \sim 1.2$ or bigger. This is again in good agreement with the estimate of Ref. [8].

In order to compare the spectral onset behavior at the gaps or pseudo-gaps of the quasi-1d metals with CDW and Luttinger liquid behavior to a conventional band-structure gap, we have also per- 
formed ARPES on the strongly anisotropic semiconductor $\mathrm{ZrSe}_{3}$ along the several high-symmetry directions. These data are currently being analysed and complemented by data taken with a discharge light source.

\section{CONCLUSION}

We have made ARPES measurements on various organic and inorganic $1-\mathrm{d}$ or $2-\mathrm{d}$ materials. The work on the organic materials is in the beginning stage due to the difficulty of obtaining a good surface and/or the photon-induced degradation of the surface. For the inorganic 1-d materials, we have obtained a high resolution spectrum for the blue bronze without any photon damaging effect. This shows clearly the absence of the Fermi edge in the ARPES. The overall structure of the dispersions are similar to the previous experiments done at SSRL last year and lower resolution experiments done at SRC in the intervening period. For another quasi-1d metal $\left(\mathrm{TaSe}_{4}\right)_{2} \mathrm{I}$, we have obtained similar ARPES data as in Ref. [4]. In addition, we observed the splitting of the peaks into two components in the CDW phase, a result which requires further research. The dispersions found for $\left(\mathrm{TaSe}_{4}\right)_{2} \mathrm{I}$ are similar in magnitude to the band calculation, which contrasts sharply to the case of the blue bronze. We think that this, together with the pseudo gap observed in the conductivity, indicates that $\left(\mathrm{TaSe}_{4}\right)_{2} \mathrm{I}$ is a system where $\mathrm{CDW}$ mechanism is more dominant over the electron-electron interaction than in the blue bronze.

\section{ACKNOWLEDGEMENTS}

We gratefully acknowledge the assistance of C.H. Park, D.S. Dessau and D. King at the beamline. Work at the University of Michigan was supported by U.S. DoE Grant No. DE-FG02-90ER45416 and NSF' Grant No. DMR-91-08015. Work at the Universität of Saarbrücken was funded by the Deutsche Forschungsgemeinschaft. Work at Stanford University was supported by the Materials Science Division of DoE. Work at LANL was supported by DoE.

\section{REFERENCES}

1. G.-H. Gweon et al, preprint; SSRL activity report (1993), p366.

2. V. Meden and K. Schönhammer, Phys. Rev. B 46, 15753 (1992); J. Voit, Phys. Rev. B 47, 6740 (1993) and references therein.

3. K. Breuer et al, preprint.

4. Y. Hwu et al, Phys. Rev. B 46, 13624 (1992).
5. P. Gressier et al, Inorg. Chem. 23, 1221 (1984).

6. P. Gressier et al, J. Solid State Chem. 51, 141 (1984).

7. Dardel et al, Phys. Rev. Lett. 67, 3144 (1991).

8. Dardel et al, Europhys. Lett. 24, 687 (1993). 
Proposal 2202Vp

\title{
Test of XMCD Sum Rules with Total Electron Yield Measurement
}

\author{
Reiko Nakajima ${ }^{1}$, Joachim Stöhr ${ }^{2}$, Dieter Weller ${ }^{2}$ and Mahesh Samant ${ }^{2}$ \\ 'Department of Materials Science, Stanford University, Stanford, CA 94305 \\ ${ }^{2}$ IBM Research Division, Almaden Research Center, 650 Harry Road, San Jose, CA 95120
}

$\mathrm{X}$-ray magnetic circular dichroism (XMCD) is a promising technique for the quantitative determination of magnetic materials. The usefulness of XMCD for the quantitative determination of magnetic moments critically depends on the practical reliability of theoretical sum rules which establish the link between measured dichroism intensities and the magnetic orbital $^{1}$ and spin $^{2}$ moments. Experimental verification of the XMCD sum rules has recently been provided for bulk $F e$ and $C o$ by $x$-ray transmission measurements. In this paper, we employ electron yield measurements (photocurrent) to quantitatively measure the moments of the 3d magnetic metals, $\mathrm{Fe}, \mathrm{Co}$ and $\mathrm{Ni}$. Since the orbital and spin moments are well known for these metals, the sum rules may be directly tested.

The elemental specificity of XMCD is due to each element's characteristic photoabsorption resonance energies. XMCD utilizes the difference in the absorption of left-and right-handed circularly polarized $x$-rays. Equivalently, measurements may be taken by switching the magnetic field direction while keeping the polarization of the photons constant. For our experiment, the field was switched at each energy point to avoid energy shift problems between the two spectra. An electromagnet provided an external magnetic field parallel to the $x$-ray propagation direction, with a maximum field of 10kOe. The employed electromagnet was capable of achieving $90 \%$ of full field within one second of switching. A diamond window was used to decouple the sample chamber from the UHV storage ring. The pressure in the sample chamber was of the order of $10^{-6}$ torr. A cylindrical ungsten mesh cage was placed around the sample to provide a bias voltage for total electron yield (TEY) measurements.

In practice, electron yield detection offers many advantages over the transmission method for the study of real samples, which often cannot be prepared as free standing films needed for $x$-ray transmission. However, dichroism measurements are often carried out in external magnetic fields which may cause problems for electron yield studies. Another reason for the present study was therefore to test the feasibility of electron yield detection in large external fields.
Figure 1(a) shows sample absorption spectra of $200 \AA$ thick films of $\mathrm{Fe}, \mathrm{Co}$ and $\mathrm{Ni}$ at their $\mathrm{L}_{3,2}$ edges. For each element, the solid and dashed lines represent the absorption spectra for opposite directions of the external magnetic field. Here we can see that the two curves for different field directions trace each other very closely. Above the $L$ edges, the difference in the signals for the two field directions are typically of the order of 0.1 to $0.5 \%$. Shown in Figures 1(b) through (d) are the dichroism spectra for each element. The curves were obtained by taking the difference between the two curves, and then subtracting a linear background fitted to the difference spectra in the regions above and below the $L$ edges.
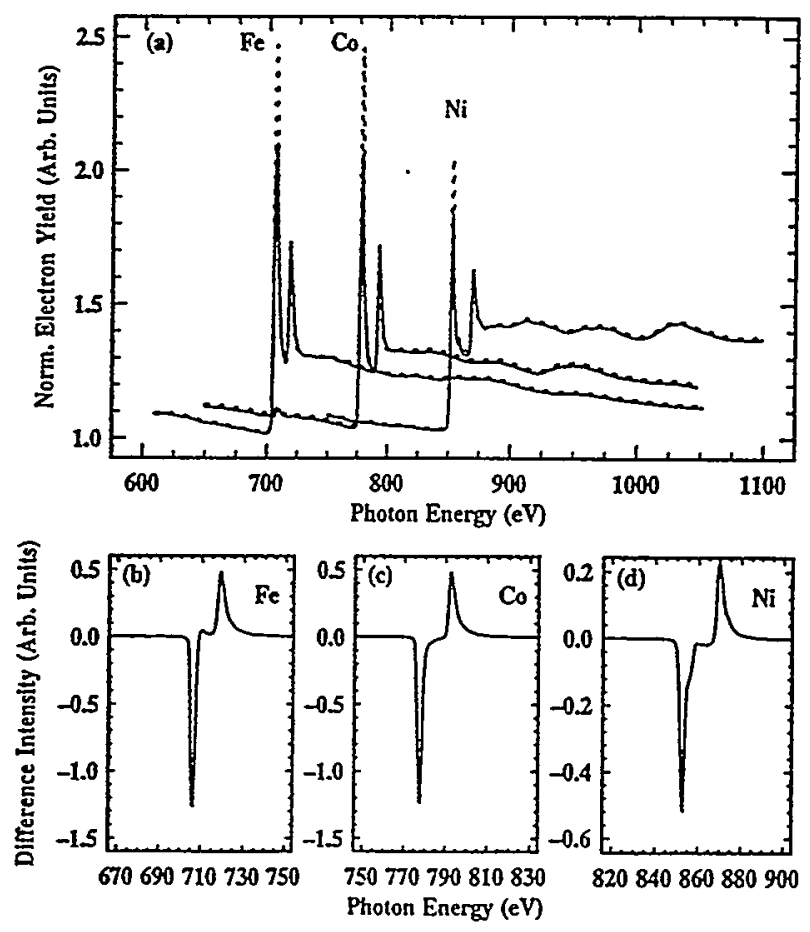

Figures 1: (a) TEY absorption spectra for external magnetic field parallel and antiparallel to $x$-ray wave vector, and dichroism spectra of (b) $\mathrm{Fe}$, (c) $\mathrm{Co}$, and (d) Ni. 
The sum rules for quantitatively determining the orbital and spin magnetic moment are as follows ${ }^{1.2}$. The absorption difference at each resonance peak is defined as

$$
\begin{aligned}
& \Delta \mathrm{A}_{\mathrm{L}_{3}}=\int_{\mathrm{L}_{3}}\left(\mu_{+}-\mu_{-}\right) \mathrm{dE} \\
& \Delta \mathrm{A}_{\mathrm{L}_{2}}=\int_{\mathrm{L}_{2}}\left(\mu_{+}-\mu_{-}\right) \mathrm{dE}
\end{aligned}
$$

where $\mu_{+}$and $\mu_{-}$are the absorption cross-sections with parallel and antiparallel alignments of the photon spin and the magnetic moment, and $\left(\mu_{+}-\mu_{-}\right)$is the dichroism signal.

The simplest test of the sum rules is provided by the following intensity ratio which directly gives the ratio of orbital $\left(\mathrm{m}_{\text {ord }}\right)$ to spin $\left(\mathrm{m}_{\text {spid }}\right)$ moment,

$$
\frac{m_{\text {orb }}}{m_{\text {spin }}}=\frac{2}{3}\left(\frac{\Delta A_{L_{3}}+\Delta A_{L_{2}}}{\Delta A_{L_{3}}-2 \Delta A_{L_{2}}}\right)
$$

Here we have neglected a magnetic dipole term which is typically small in bulk metals ${ }^{3}$.

The actual orbital and spin moments are calculated with the white line intensity, approximated $\mathrm{as}^{3}$

$$
A_{W L}=\int_{L_{2}+L_{3}}\left(\mu_{0}+\mu_{+}+\mu_{-}\right) d E \approx \frac{3}{2} \int_{L_{2}+L_{3}}\left(\mu_{+}+\mu_{-}\right) d E
$$

where $\mu_{0}$ is the absorption cross-section of linearly polarized $x$-rays. The orbital $\left(m_{\alpha b}=-\mu_{B}<L_{z}>\right)$ and spin $\left(m_{\text {spin }}=-2 \mu_{B}\left\langle S_{Z}\right\rangle\right)$ moments are given by:

$$
\left\langle\mathrm{L}_{\mathrm{z}}\right\rangle=2 \mathrm{n}_{\mathrm{h}, 3 \mathrm{~d}} \frac{\Delta \mathrm{A}_{\mathrm{L}_{3}}+\Delta \mathrm{A}_{\mathrm{L}_{2}}}{\mathrm{~A}_{\mathrm{WL}}}
$$

and

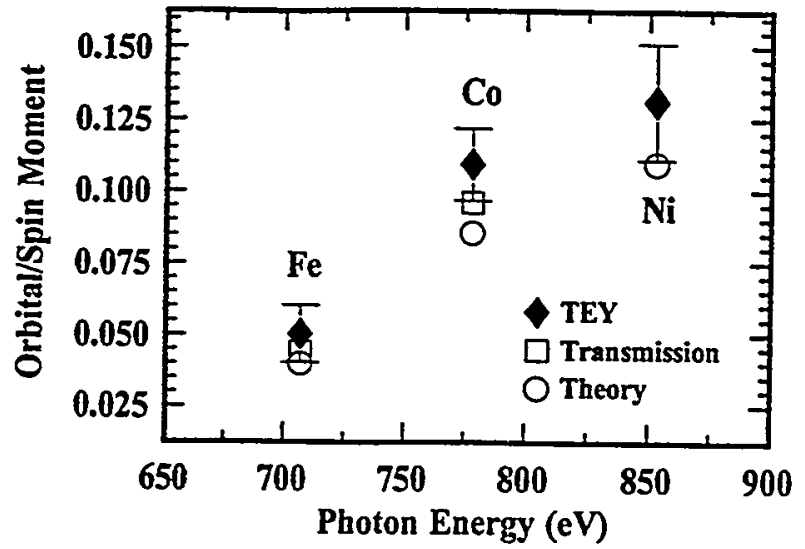

Figure 2: Comparison of the experimental (TEY and transmission ${ }^{3}$ ) and theoretical ${ }^{4}$ values of the orbital to spin moment ratio.

$$
2\left\langle S_{z}\right\rangle+7\left\langle T_{z}\right\rangle=3 n_{h, 3 d} \frac{\Delta A_{L_{3}}-2 \Delta A_{L_{2}}}{A_{W L}}
$$

where $\left\langle\mathrm{L}_{2}\right\rangle$ and $\left\langle\mathrm{S}_{2}\right\rangle$ are the components of the orbital and spin angular moment, $\left\langle\mathrm{T}_{z}\right\rangle$ is the magnetic dipole term, and $n_{b, 3 d}$ is the average number of holes per atom in the $3 \mathrm{~d}$ valence shell.

Figure 2 shows the ratio of the orbital to spin magnetic moments of each element compared to theoretical values ${ }^{4}$ and the values obtained by Chen $e t$ al. in transmission ${ }^{3}$. We chose to display the moment ratios since several uncertain factors are eliminated, such as the degree of polarization of the $x$-rays, the white line intensity, or the average number of holes per atom. The figure shows that the experimental curve follows the theoretical curve, but is higher by about $20 \%$. Within experimental error our results agree with the transmission measurements.

${ }^{1}$ B.T. Thole, P. Carra, F. Sette, and G. van der Laan, Phys. Rev. Lett. 68, 1943 (1992).

${ }^{2}$ P. Carra, B.T. Thole, M. Altarelli, and X. Wang, Phys. Rev. Lett. 70, 694 (1993).

${ }^{3}$ C.T. Chen et al., Phys. Rev. B (submitted).

4Söderlind et al., Phys Rev. B45, 12911 (1992). 
Proposal 2213Vp

\title{
Surface EXAFS and X-ray Standing Wave study of the cleaved $\mathrm{CaO}(100)$ and MgO(100) surfaces.
}

\author{
T. Kendelewicz ${ }^{a}$, P. Liu ${ }^{b}$, W. B. Labiosa ${ }^{b}$ and G. E. Brown, Jr. ${ }^{b}$ \\ aDepartment of Electrical Engineering, and bept. of Geological \& Environmental Sciences, Stanford University, \\ Stanford CA 94305
}

\section{Introduction}

We have applied Surface EXAFS and X-ray Standing Waves (XSW) to study surface relaxation of cleaved $\mathrm{CaO}(100)$ under UHV conditions. Characterization of clean surfaces is a first step for developing a fundamental understanding of surface reactions with adatoms and molecules. The (100) surfaces of $\mathrm{CaO}$ and $\mathrm{MgO}$ arguably have the simplest oxide surface structures, and both can be obtained in atomically clean form by cleavage. Both surfaces are expected to be similar in structure and chemical bonding. $\mathrm{CaO}$ was chosen for a more detail study because of a convenient access to the $\mathrm{Ca} \mathrm{K}$ edge $(\approx 4038 \mathrm{eV})$ and to surfacesensitive Auger electrons (Ca LMM at $290 \mathrm{eV}$ and O $\mathrm{KLL}$ at $\approx 510 \mathrm{eV}$ ). These Auger transitions were used for both EXAFS and XSW detection. The total yield electrons or secondaries are obtained from the same samples and used as bulk standards in EXAFS or bulk background in XSW. Direct comparison of data with different surface sensitivity allows extraction of surface information. Often EXAFS experiments are negatively affected by "Bragg glitches". We use this effect to extract additional information for $\mathrm{CaO}$ and $\mathrm{MgO}$. While EXAFS provides local information on central atom coordination, bond lengths, and angles, the XSW is an ideal probe to determine distances relative to the diffracting Bragg planes of the substrate. For studies of the $\mathrm{CaO}(100)$ and $\mathrm{MgO}(100)$ surfaces, we have used the (200) reflection at $2578 \mathrm{eV}$ and 2945 , respectively.

As $\mathrm{MgO}$ represents a textbook example of an ionic substrate, the $(100)$ surface of this crystal has been studied intensively [1]. The $\mathrm{CaO}(100)$ surface studied in our experiment is expected to behave qualitatively in the same fashion. Despite serious experimental and theoretical efforts, reconstruction of these surfaces is not completely understood. An important observation made in prior studies, which was confirmed in the present study, is the presence of a simple 1x1 LEED pattern, indicating no reconstruction parallel to the $(100)$ surface. Thus only relaxation involving shifts in the vertical positions of the surface atoms, perhaps accompanied by some rumpling (measured by the difference in position of surface anions and cations), is expected. Although surface relaxation may extend into the crystal, effects below the top-most layer are expected to be small. Surface structures of $\mathrm{MgO}$ have been studied with several techniques including LEED and RHEED, which find small surface contraction ( $<3 \%)$ and rumpling ( $<$ $6 \%)[2,3]$. However, the idea of very little surface rearrangement is contradicted by results from other techniques. For example, He scattering indicates a rumpling of 10 to $15 \%$ [4]. Theory predicts small to moderate degrees of relaxation (0 to $11 \%$ ) [5], thus mirror the range of uncertainties of experimental determinations. Until now relatively little was known about the surface relaxation of $\mathrm{CaO}(100)$, although deviation from the truncated bulk is expected to be smaller than that for $\mathrm{MgO}(100)$.

\section{Experimental}

Experiments were performed on UHV cleaved $\mathrm{CaO}(100)$ and $\mathrm{MgO}[100)$ single crystals. Surface EXAFS spectra from $\mathrm{CaO}$ were taken on SSRL beam line 3-3 equipped with a pair of $\mathrm{Ge}(111)$ monochromator crystals. XSW data were taken using Si(111) crystals on the same beam line. Data were collected in the constant final state mode by monitoring the energies of low energy Auger electrons as the energy was swept through the respective Ca K edge or (200) Bragg condition in the back reflection diffraction geometry. A double-pass cylindrical mirror analyzer was used for electron energy discrimination. The energy dependence of the background above the peak was also measured. An electron flood gun was used to eliminate charging. Total yield data were taken from the same samples with the flood gun turned off. The energy dependence of the Bragg reflectivity was measured with an $80 \%$ transmitting metal grid and a channeltron detector upstream of the sample. SEXAFS data were analyzed in the standard fashion using experimental phase and amplitude parameters.

\section{Results and Discussion}

The atomic specificity of the XSW technique allowed us to determine individual displacements of $\mathrm{Ca}(\mathrm{Mg})$ and O surface atoms independently, whereas the local nature of Ca K-SEXAFS permitted us to determine surface Ca$\mathrm{O}$ distances and the coordination number of surface $\mathrm{Ca}$ atoms. An example of the sensitivity of this combined approach is our recent XSW/SEXAFS study of the $\mathrm{CdTe}(110)$ and InSb(110) surfaces [6]. Both of these materials have Auger peaks almost identical to those of $\mathrm{CaO}$ and $\mathrm{MgO}$ and show strong surface relaxations (over $30 \%$ rumpling). In contrast, the $\mathrm{CaO}(100)$ and the $\mathrm{MgO}(100)$ data do not show any surface effects, i.e. the energy dependences of the Auger yields in the vicinity of the (200) Bragg reflection are identical to that of bulksensitive yields for both $\mathrm{O}$ and $\mathrm{Ca}(\mathrm{Mg})$. Thus XSW results suggest immeasurably small relaxation of the $\mathrm{CaO}(100)$ and $\mathrm{MgO}(100)$ surfaces. 
To confirm the above result for $\mathrm{CaO}(100)$, we measured the $\mathrm{Ca} \mathrm{K}$-edge surface EXAFS from the $\mathrm{CaO}(100)$ clean surface by monitoring the $\mathrm{Ca}$ LMM Auger yield and analyzed the data against total yield scans taken from the same sample (used as a phase and amplitude standard). The frequencies of modulations in the surface-sensitive EXAFS scans are identical to that of bulk yield data, but the amplitudes of modulations are measurably reduced, implying similar distances but reduced coordination numbers for the surface relative to the bulk. This observation is further confirmed by detailed analysis of the EXAFS data which yielded a Ca$O$ distance of $2.39 \pm 0.02 \AA$ and $\mathrm{Ca}$ coordination number of $5 \pm 1$. This result agrees with the XSW result which indicates the (100) and bulk atomic positions of $\mathrm{CaO}$ are the same.

This work was performed at SSRL and NSLS which are supported by the US DOE. This work was supported by NSF grant EAR-9305028-001.

\section{References}

[1] V. E. Henrich and P. A. Cox, The Surface Science of Metal Oxides, Cambridge University Press (1994).

[2] C. G. Kinniburgh, J. Phys. C 9 (1976) 2695; T. Urano et al., Surf. Sci. 134 (1983) 109; D. L Blanchard et al., J. Vac. Sci. Technol. A 9 (1991) 1814.

[3] T. Gatoh et al., J. Phys. Soc. Jap. 50 (1981) 2063; P. A. Maxym, Surf. Sci. 149 (1985) 157.

[4] D. R. Jung et al., Phys. Rev. B 39 (1989) 11164.

[5] J. LaFemina and C. Duke, J. Vac. Sci. Technol. A 9 (1991) 1847.

[6] T. Kendelewicz et al., Phys. Rev B, april 15th 1995, in press.; J. C. Woicik et al., Phys. Rev. Lett. 68 (1992) 341, and Phys. Rev. B 46 (1992) 9869. 


\title{
CORRELATION OF MULTIPLE SCATTERING FEATURES IN THE K-EDGE XANES SPECTRA OF Al AND Si TO THE Al-O-Si BOND ANGLE IN ALUMINOSILICATE SODALITES: AN EMPIRICAL STUDY
}

\author{
MICHAEL FRÖBA ${ }^{\ddagger}$, JOE WONG ${ }^{\ddagger}$, P. BEHRENS ${ }^{\# \dagger}$, P. SIEGER ${ }^{\#}$, M. ROWEN", $^{\ddagger}$ \\ T. TANAKA ${ }^{+}, Z$. REK, J. FELSCHE \\ ${ }^{\ddagger}$ Lawrence Livermore National Laboratory, P.O. Box 808, L-369, Livermore, CA 94551, U.S.A. \\ ${ }^{\#}$ Fakultät für Chemie, Universität Konstanz, D-78464 Konstanz, Germany \\ † Institut für Anorganische Chemie, Universität München, D-80333 München, Germany \\ ' Stanford Synchrotron Radiation Laboratory, P.O. Box 4349, Stanford, CA 94309, U.S.A. \\ ${ }^{+}$National Institute for Research in Inorganic Materials, 1-1 Namiki, Tsukuba, Ibaraki 305, Japan
}

\section{INTRODUCTION}

The multiple scattering (MS) features in the XANES contain higher order correlation functions, i.e. bond angles. However, this information cannot be easily extracted because the absorption is influenced by several different MS pathways. Although there now exist some elaborate approaches for the calculations of XANES spectra in the MS region, these have mainly been tested on model compounds with comparatively simple geometric structures. Here we present an empiral study that reveals a correlation between the intensity of MS features at both the $\mathrm{Al} \mathrm{K}$ and $\mathrm{Si} \mathrm{K}$ edges of a series of aluminosilicate sodalites with varying Si-O-Al bond angle [1].

\section{EXPERTMENTAL}

The sodalites have been synthesized under hydrothermal conditions. The phase purity was well as the high degree of crystallinity were determined by powder $x$-ray diffraction [2].

The XAS measurements were performed at the SPEAR ( $3 \mathrm{GeV}$, currents: $100-50 \mathrm{~mA}$ ) storage ring (SSRL) on the JUMBO 3-3 beamline using a $\mathrm{YB}_{66}(400)$ double-crystal monochromator [3-5]. The spectra were recorded in step-scanning mode (counting time: $2 \mathrm{~s}$, step-size: $0.2 \mathrm{eV}$ ) by monitoring the total electron yield with a channeltron. Both edges were each calibrated against the edges of the elements (Al K: $1559 \mathrm{eV}$, Si K: 1839 eV). All spectra were taken at room temperature. A pre-edge fit was applied and the spectra were normalized to an edge jump of 1 .

\section{RESULTS AND DISCUSSION}

The aluminosilicate sodalites selected for this study are host-guest compounds with the general structural formula $\left[\mathrm{Na}_{4} \mathrm{X}\right]_{2}\left[\mathrm{Al}_{3} \mathrm{Si}_{3} \mathrm{O}_{12}\right]_{2}$. The different anions $\mathrm{X}$ of the guest complex $\left[\mathrm{Na}_{4} \mathrm{X}\right]$ are given in Table 1 . In the aluminosilicate framework of these compounds, $\mathrm{Al}$ and $\mathrm{Si}$ are tetrahedrally coordinated by oxygen in which the $\mathrm{Al}-\mathrm{O}$ and $\mathrm{Si}-\mathrm{O}$ bond lengths as well as the $\mathrm{d}_{\mathrm{Na}-\mathrm{O} \text { (framework) }}$ are practically constant and therefore independent of the composition of the guest complex. Nevertheless, the space requirements of this guest complex strongly influence the volume of the cubic unit cell. The sodalite structure adapts the size of its cage via changes in the Al-O-Si bridging angle [6]. Larger guest complexes lead to an increase in this angle and in the lattice constant (Table 1). 


\begin{tabular}{|l|l|l|}
\hline Anion $\mathrm{X}$ & $\Delta \mathrm{Al}-\mathrm{O}-\mathrm{Si}$ & $\mathrm{a}[\AA]$ \\
\hline $\mathrm{Cl}^{-}$ & 138.2 & $8.870[7]$ \\
\hline $\mathrm{NO}_{2}^{-}$ & 140.2 & $8.928[8]$ \\
\hline $\mathrm{B}(\mathrm{OH})_{4}^{-}$ & 144.6 & $9.015[2]$ \\
\hline $\mathrm{SCN}{ }^{-}$ & 146.4 & $9.060[2]$ \\
\hline $1 / 2 \mathrm{CrO}_{4}^{2 \cdot}$ & 148.1 & $9.100[2]$ \\
\hline $1 / 2 \mathrm{MoO}_{4}^{2 \cdot}$ & 149.5 & $9.132[2]$ \\
\hline
\end{tabular}

Table 1. The Al-O-Si bridging angle as a function of the cubic lattice constant for aluminosilicate sodalites $\left[\mathrm{Na}_{4} \mathrm{X}_{2}\left[\mathrm{Al}_{3} \mathrm{Si}_{3} \mathrm{O}_{12}\right]_{2}\right.$ with different anions $\mathrm{X}$.

The normalized $\mathrm{Al} \mathrm{K}$ and Si $\mathrm{K}$ XANES spectra are shown in Fig. 1 and 2, resp. All spectra exhibit strong absorption features $\underline{A}$ (Al K: $1566 \mathrm{eV}$, Si K: $1847 \mathrm{eV}$ ), arising from $1 s \rightarrow 3 p$ bound state transitions and typical for tetrahedral $\mathrm{SiO}_{4}[9]$ and $\mathrm{AlO}_{4}[5,10,11]$ units. The height as well as the energy position for the respective absorption edge are essentially constant.

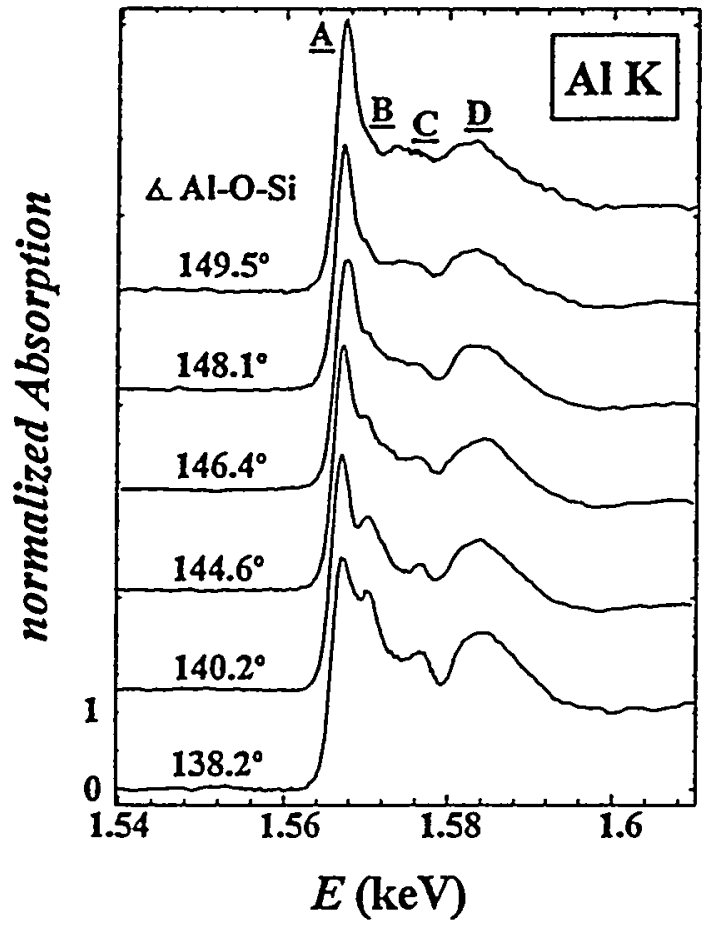

Fig. 1. Al $\mathrm{K}$ XANES spectra of aluminosilicate sodalites with different Al-O-Si bridging angles.

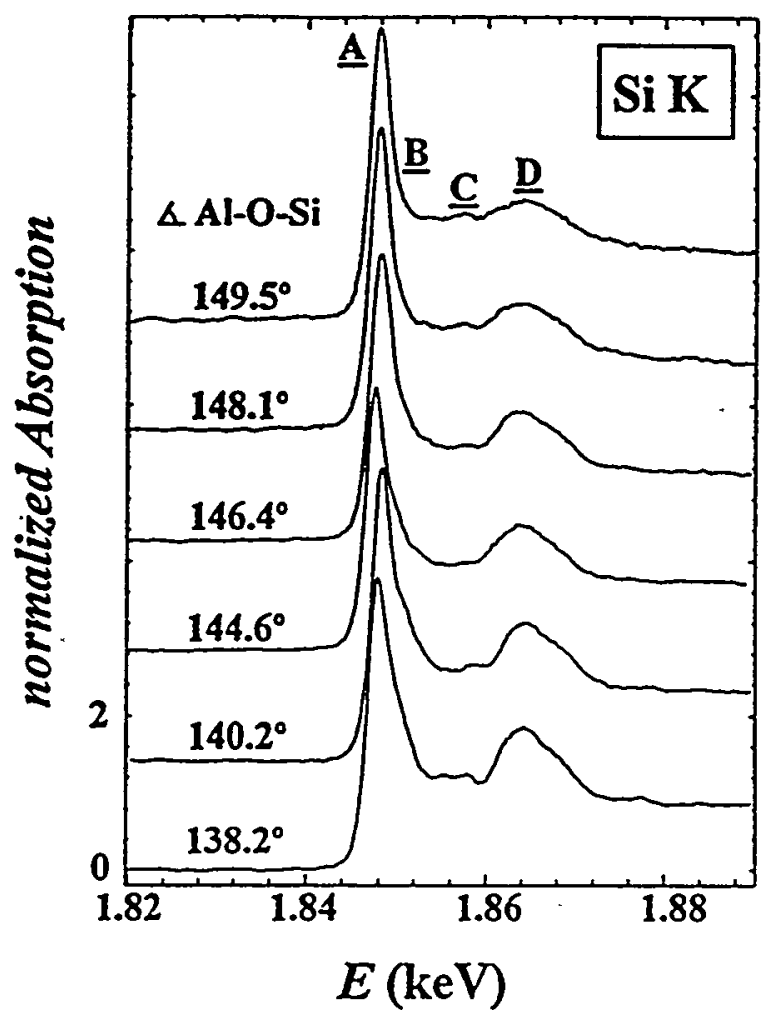

Fig. 2. Si $\mathrm{K}$ XANES spectra of aluminosilicate sodalites with different $\mathrm{Al}-\mathrm{O}-\mathrm{Si}$ bridging angles.

The XANES features at higher energies $(\underline{B}$, $\underline{C}$ and $\underline{D}$ show a decrease in intensity with increasing $\mathrm{Al}-\mathrm{O}-\mathrm{Si}$ bridging angle. $\mathrm{A}$ quantitative determination of this correlation was carried out by approximate the $\mathrm{Al} \mathrm{K}$ and $\mathrm{Si} \mathrm{K}$ XANES with different functions in a least-squares fit and using the integrated intensities of the peaks. In each case 6 symmetric Gaussian functions and one stepfunction (modified arctangent function) were used. The original and the deconvoluted spectra are shown for $\left[\mathrm{Na}_{4} \mathrm{Cl}_{2}\left[\mathrm{Al}_{3} \mathrm{Si}_{3} \mathrm{O}_{12}\right]_{2}\right.$ in Fig. 3. The number of Gaussian functions was determined by using the 2 nd derivative, which yielded 6 negative peaks in this energy range. In particular, we examine the change in intensity as a function of the bridging angle for the 2nd (B), 3rd and 4th (ㅁ) Gaussian function. 


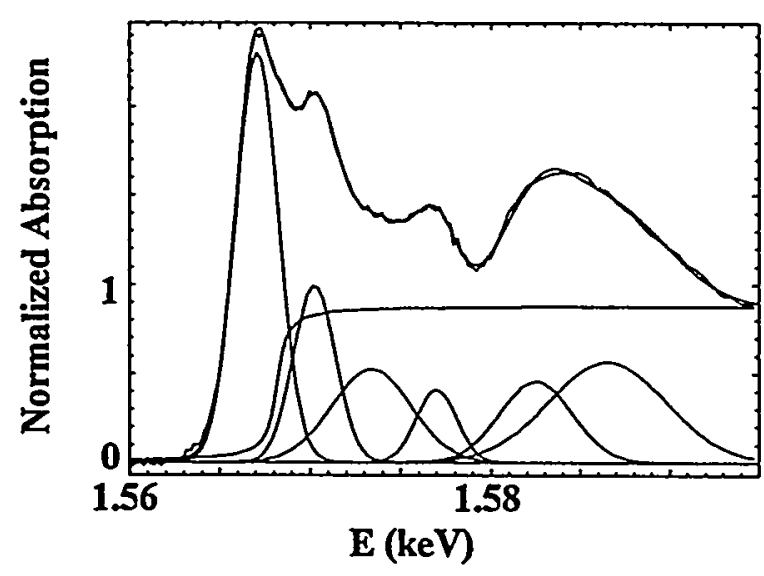

Fig. 3. Deconvolution of the Al $\mathrm{K}$ XANES spectra of the aluminosilicate sodalite $\left[\mathrm{Na}_{4} \mathrm{Cl}_{2}\left[\mathrm{Al}_{3} \mathrm{Si}_{3} \mathrm{O}_{12}\right]_{2}\right.$ using 6 symmetric Gaussian functions and one modified arctangent function.

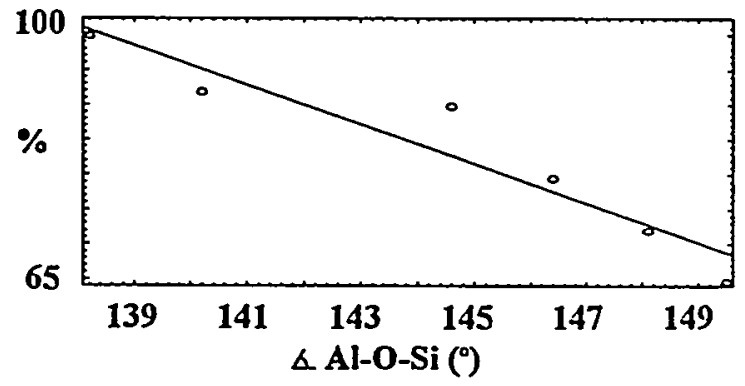

Fig. 4. Sum of the normalized integrated intensity of the 2nd and 3rd Gaussian peak as a function of the Al-O-Si bridging angle in the AI KXANES spectra

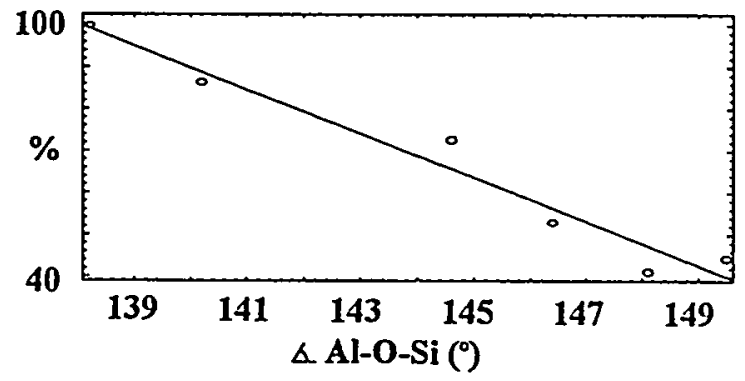

Fig. 5. Normalized integrated intensity of the 4th Gaussian peak as a function of the Al-O-Si bridging angle in the $\mathrm{Al} \mathrm{KXANES} \mathrm{spectra.}$

392
The Fig. 4 and 5 show the results for the Al $\mathrm{K}$ and Fig. 6 and 7 for the Si K spectra. All intensities were normalized to the largest intensity in each plot. In case of Fig. 4 and 6 the normalized sum of the 2nd and 3rd Gaussian function are plotted.

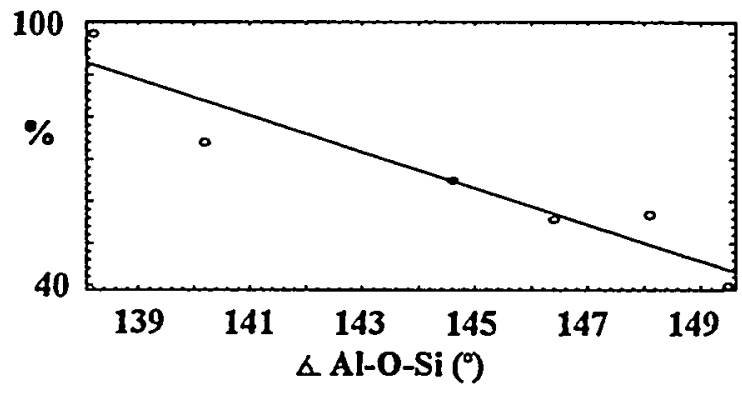

Fig. 6. Sum of the normalized integrated intensity of the 2nd and 3rd Gaussian peak as a function of the Al-O-Si bridging angle in the Si K XANES spectra.

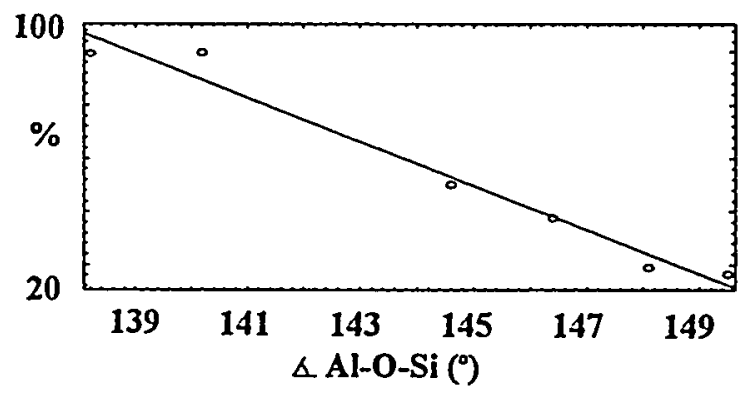

Fig. 7. Normalized integrated intensity of the 4th Gaussian peak as a function of the Al-O-Si bridging angle in the Si K XANES spectra.

The integrated intensities of the considered Gaussian functions show a linear decrease with increasing $\mathrm{Al}-\mathrm{O}-\mathrm{Si}$ angle. A comparison between both edges indicates a larger decrease in case of the Si K XANES spectra.

For further information, in a next step investigations of the geometry of the scattering pathes as a function of different bridging 
pathes as a function of different bridging angles should be carried out. As an indication for backscattering from atoms beyond the shell of nearest neighbors the decrease in the difference in energy position between the 1st and the other Gaussian functions can be seen. For the $\mathrm{Al} \mathrm{K}$ spectra the energy difference between the 1st and the 4th Gaussian function are shown in Fig. 8.

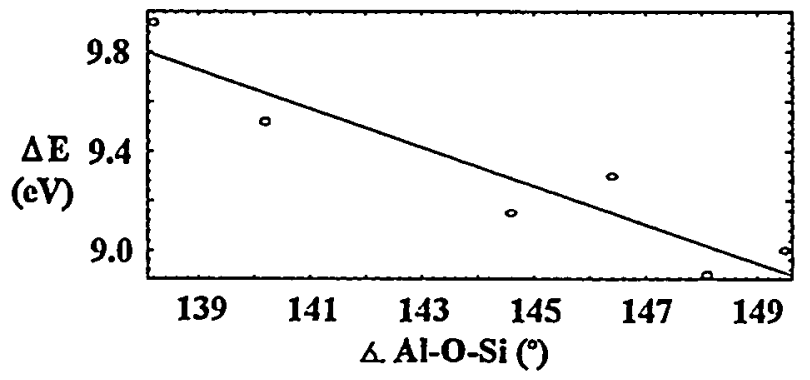

Fig. 8. The energy difference between the 1 st and the 4 th Gaussian function as a function of the Al-O-Si bridging angle in the Al K XANES spectra

Acknowledgements - This work was performed under the auspices of the US Department of Energy (DOE) by the Lawrence Livermore National Laboratory under contract number W-7405-ENG-48. The measurements were carried out at SSRL which is supported by the Chemical Sciences Division of the DOE. M.F. thanks the Alexander von Humboldt Foundation for a Feodor Lynen Research Fellowship. The work in Konstanz was supported by the Deutsche Forschungs-Gemeinschaft (DFG).

\section{REFERENCES}

[1] M. Fröba, Joe Wong, P. Behrens, P. Sieger, M. Rowen, T. Tanaka, Z. Rek, J. Felsche, Physica B (1995), in press.

[2] P. Sieger, $\mathrm{PhD}$ Thesis, University of Konstanz, Germany (1992).
[3] Joe Wong, G. Shimkaveg, W. Goldstein, M. Eckart, T. Tanaka, Z.U. Rek, H. Tompkins, Nucl. Instrum. Meth. A291, 243 (1990).

[4] M. Rowen, Z.U. Rek, Joe Wong, T. Tanaka, G.N. George, I.J. Pickering, G.H. Via, G.E. Brown Jr., Synchrotron Radiation News 6, 25 (1993).

[5] Joe Wong, G.N. George, I.J. Pickering, Z.U. Rek, M. Rowen, T. Tanaka, G.H. Via, B. DeVries, D.E.W. Vaughan, G.E. Brown Jr., Solid State Commun. 92, 559 (1994).

[6] P. Behrens in: Multifunctional Mesoporous Inorganic Solids, eds. M. Hudson and C.A.C. Sequeira, NATO ASI Series C399 (Kluwer, Dordrecht, 1993), p. 73.

[7] J. Löns and H. Schulz, Acta Cryst. 23, 434 (1967).

[8] P. Sieger, M. Wiebcke, J. Felsche, J.-Ch. Buhl, Acta Cryst. C47, 498 (1991).

[9] P. Lagarde, A.M. Flank, 'G. Tourillon, R.C. Liebermann, J.P. Itie, J. Phys. I (France) 2, 1043 (1992).

[10] G.E. Brown Jr., F.D. Dikmen, G.A. Waychunas, SSRL Activity Report, p.146 (1983).

[11] M. Fröba, Joe Wong, M. Rowen, G.E. Brown Jr., T. Tanaka, Z. Rek., Physica B (1995), in press. 


\title{
SiK XANES SPECTROSCOPIC INVESTIGATIONS OF MESOPOROUS M41S SILICA PHASES
}

\author{
MICHAEL FRÖBA ${ }^{\ddagger}$, P. BEHRENS ${ }^{\# \dagger}$, JOE WONG ${ }^{\ddagger}$, CH. HAGGENMÜLLER ${ }^{\sharp}$, G. VAN DE GOOR $^{\#}$, \\ M. ROWEN', T. TANAKA ${ }^{+}$, W. SCHWIEGER ${ }^{\circ}$ \\ ${ }^{\ddagger}$ Lawrence Livermore National Laboratory, P.O. Box 808, L-369, Livermore, CA 94551, U.S.A.

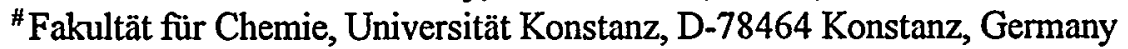 \\ †'Institut für Anorganische Chemie, Universität München, D-80333 München, Germany \\ I Stanford Synchrotron Radiation Laboratory, P.O. Box 4349, Stanford, CA 94309, U.S.A. \\ +National Institute for Research in Inorganic Materials, 1-1 Namiki,Tsukuba, Ibaraki 305, Japan \\ ${ }^{\circ}$ Sektion Chemie, Universität Halle-Wittenberg, D-06108 Halle/Saale, Germany
}

\section{INTRODUCTION}

Recently the discovery of a new family of mesoporous molecular sieves called M41S was reported $^{[1-5]}$. These M41S phases exhibit adjustable pore sizes ranging from 20 to $\approx 60 \AA^{[\sigma]}$ and a narrow pore-size distribution which makes these materials very interesting for size-selective applications. These pores possess a regular arrangement, as shown by $\mathrm{TEM}^{[1-4,7-8]}$. In contrast, the silica walls do not exhibit crystallographic longrange order, as indicated by powder $\mathrm{x}$-ray and electron diffraction as well as IR, Raman and ${ }^{29} \mathrm{Si}$ NMR spectroscopies ${ }^{[1-4,9-11]}$. As a function of the ratio surfactant/silicate, mesoporous silica phases with hexagonal and cubic symmetries can be synthesized ${ }^{[1-5,3]}$. The hexagonal phase is built from parallel, unidimensional channels (MCM-41-type materials) ${ }^{[1-2]}$. In the structure of the cubic Ia $\overline{3} d$ phases ${ }^{[3]}$, an inorganic sheet located on a minimal periodic surface separates the surfactant species into two equal and unconnected volumes. Upon calcination, two mutually interwoven, but otherwise unconnected pore systems are created (MCM-48type materials).

\section{EXPERIMENTAL}

The silica mesostructures were each prepared by hydrothermal treatment of silica solutions containing alkyltrimethylammonium surfactant cations $\left(\mathrm{C}_{\mathrm{n}} \mathrm{TMA}\right)^{+}$. For the hexagonal phase $\underline{1 \mathrm{a}}$ $\mathrm{C}_{12} \mathrm{TMA}^{+}$was used whereas the hexagonal phase $\underline{2 a}$ and the cubic phase 3 a were each synthesized with $\mathrm{C}_{16} \mathrm{TMA}^{+}$. The solutions were heated in a teflon-lined steel autoclave at $110^{\circ} \mathrm{C}$ for two days. Afterwards the solids were recovered by filtration, washed with water until neutral and finally dried overnight at $70^{\circ} \mathrm{C}$. The respective calcined samples $\underline{1 b}-\underline{3 b}$ were prepared by heating $\underline{1 \mathrm{a}}-\underline{3 \mathrm{a}}$ to $550^{\circ} \mathrm{C}$ in an oxygen atmosphere ${ }^{[12]}$.

SiK XANES ( $x$-ray absorption near edge structure) spectra were recorded on the beamline 33 at SSRL with the storage ring SPEAR operating at an electron energy of $3.0 \mathrm{GeV}$ and currents of 95 $\mathrm{mA}$ to $55 \mathrm{~mA}$. Monochromatization was achieved by a double-crystal monochromator using the (400) reflections of two $\mathrm{YB}_{66}$ crystals $^{[13 a]}$. The resolution was $0.8 \mathrm{eV}^{[13 \mathrm{~b}]}$. The spectra were recorded in stepscanning mode (counting time: $4 \mathrm{~s}$, step-size: 0.2 $\mathrm{eV}$ ) by monitoring the total electron yield with a channeltron. A pre-edge fit was applied and the spectra were normalized. The determination of the width of the $1 s \rightarrow 3 p$ bound state transition ("white line") was carried out by calculating the difference in position of the corresponding two zero-points in the 2nd derivative of the spectrum. For comparison, we also measured XANES spectra of the following samples: highly crystalline quartz, coesite and silicalite-1 (designated as MFI), porous silica glasses with surface areas of $402 \mathrm{~m}^{2} / \mathrm{g}$ (glass 1) and $351 \mathrm{~m}^{2} / \mathrm{g}$ (glass 2), resp., fused silica (glass 3), and of synthetic layered sodium silicates ilerite, magadiite and hectorite. 


\section{RESULTS AND DISCUSSION}

The SiK XANES spectra of crystalline and amorphous silicas are shown in Fig. 1, those of layered silicates in Fig. 2 and those of the mesoporous phases in Fig. 3. All spectra exhibit strong absorption features $\underline{A}$, arising from $1 s \rightarrow 3 p$ bound state transitions, which are dipole allowed. The overall shape as well as the energy position ( $1847 \mathrm{eV}$ ) of this white line is characteristic for a tetrahedral arrangement of oxygen around silicon $^{[14]}$, whereas its width is mainly related to the long-range order ${ }^{[15]}$. These widths are listed for all samples in Table 1. A clear differentiation between the crystalline silica phases $(1.45-1.83 \mathrm{eV})$ and the amorphous silicas $(1.94-1.99 \mathrm{eV})$ is possible. The widths of the mesoporous compounds are smaller than those of the glasses, indicative of a higher degree of long-range order in the mesoporous phases than in the glasses, which is in consistence with XRD results.

The broad feature $\underline{C}$ at $\sim 1865 \mathrm{eV}$, which is relatively constant in shape and in energy position for all compounds, is due to scattering processes between the absorbing silicon and its nearest and next-nearest neighbors and thus reflects the shortrange order.

The region $\underline{B}$ between the white line $\underline{A}$ and the broad signal $\mathbf{C}$ mentioned above stands out due to its large variety of different scattering features, which are mainly determined by the medium-range order of the structure. Theoretical multiplescattering calculations using differently sized clusters have shown that the features in this region are related to order extending above $5 \AA$ around the absorbing Si atoms ${ }^{[16]}$. In accordance with this, all crystalline samples possess strong peaks, whereas in the spectra of the glasses only weak signals occur. Considering region $\mathbf{B}$ for the mesoporous phases (Fig. 3), the spectra of the surfactantcontaining samples are totally featureless, whereas the calcined compounds show discernible scattering intensities. In the former case, the absence of any signal may be attribute to the lack of medium-range order in the walls and, possibly, to disorder in the arrangement of the surfactant molecules. The appearance of scattering signals in the case of the calcined samples may be related to an increase in structural order arising from the additional crosslinking taking place during the calcination.

A comparison of the XANES spectra of the mesostructured phases to those of the layered silicates reveals a strong similarity of the surfactant-containing phases 1a-3a to ilerite and of the calcined samples $\underline{1 \mathrm{~b}}-\underline{\mathbf{b}} \mathrm{b}$ to magadiite, resp. This similarity is further confirmed by the ${ }^{29} \mathrm{Si}$ NMR spectra $^{[12,17-18]}$. Therefore, we consider these sheet silicates as potential structural models for the silica walls in the corresponding mesostructures where these layers would be curved in order to form the channel structure. According to a recent structural proposal ${ }^{[17 b]}$, ilerite is a two-layered and magadiite is a three-layered sheet silicate. Our XANES results might indicate that the calcination procedure does not only cause condensation in the walls of the mesostructures but also a rearrangement from a two-layered to a three-layered sheet structure. The silica sheets of the mesoporous phases show a larger disorder than is present in the layered model silicates but, as this study has revealed, are more ordered than "truly" amorphous silicas.

Acknowledgements - This work was performed under the auspices of the U.S. Department of Energy (DOE) by the Lawrence Livermore National Laboratory under contract number W-7405-ENG48. The measurements were carried out at the Stanford Synchrotron Radiation Laboratory (SSRL) which is supported by the Chemical Division of the DOE. M.F. thanks the Alexander von Humboldt Foundation for a Feodor Lynen Research Fellowship. The work in Konstanz and Halle was supported by the Deutsche ForschungsGemeinschaft (DFG).

\section{REFERENCES}

1. C.T. Kresge, M.E. Leonowicz, W.J. Roth, J.C. Vartuli, J.S. Beck, Nature 359, 710 (1992).

2. J.S.Beck, J.C.Vartuli, W.J. Roth, M.E. Leonowicz, C.T. Kresge, K.D. Schmitt, C.T-W. Chu, D.H. Olson, E. W. Sheppard, S.B. McCullen, J.B. Higgins, J.L. Schlenker, J. Am. Chem. Soc. 114, 10834 (1992).

3. A. Monnier, F. Schüth, Q. Huo, D. Kumar, D. Margolese, R.S. Maxwell, G.D. Stucky, M. Krishnamurty, P. Petroff, A. Firouzi, M. Janicke, B.F. Chmelka, Science 261, 1299 (1993). 
4. G.D Stucky, A. Monnier, F. Schüth, Q. Huo, D. Margolese, D. Kumar, M. Krishnamurty, P. Petroff, A. Firouzi, M. Janicke, B.F. Chmelka, Mol. Cryst. Liq. Cryst. 240, 187 (1994).

5. P. Behrens and G.D. Stucky, Angew. Chem. Int. Ed. Engl. 32, 696 (1993).

6. P. Behrens, Adv. Mater. 5, 127 (1993).

7. Q. Huo, D.I. Margolese, U. Ciesla, P. Feng, T.E. Gier, P. Sieger, R. Leon, P.M. Petroff, F. Schüth, G.D. Stucky, Nature 368, 317 (1994).

8. V. Alfredsson, M. Keung, A. Monnier, G.D. Stucky, K.K. Unger, F. Schüth, J. Chem. Soc., Chem. Commun., 921 (1994).

9. C.-Y. Chen, H.-X. Li, M.E. Davis, Microporous Mater. 2, 17 (1993); C.-Y. Chen, S.L. Burkett, H.-X. Li, M.E. Davis, ibid. 2, 27 (1993).

10. W. Kolodziejski, A. Corma, M.-T. Navarro, J. Pérez-Pariente, Solid State Nuclear Magnetic Resonance 2, 253 (1993).

11. D. Akporiaye, E.W. Hansen, R. Schmidt, M. Stöcker, J. Phys. Chem. 98, 1926 (1994).

12. a) M. Fröba, P. Behrens, Joe Wong, G. Engelhardt, Ch. Haggenmüller, G. van de Goor, M. Rowen, T. Tanaka, W. Schwieger, Mater. Res. Soc. Sym. Proc. 371 (1995), in press; b) M. Fröba, G. Engelhardt, $C h$. Haggenmüller, Joe Wong, $M$. Rowen, $T$. Tanaka, W. Schwieger, G. van de Goor, P. Behrens, Adv. Mater., to be submitted (1995).
13. a) Joe Wong, G. Shimkaveg, W. Goldstein, $M$. Eckart, T. Tanaka, Z.U. Rek, H. Tompkins, Nucl. Instrum. Meth. Phys. Res. A 291, 243 (1990); b) M. Rowen, Z.U. Rek, Joe Wong, T. Tanaka, G.N. George, I.L. Pickering, G.H. Via, G.E. Brown Jr., Synchrotron Radiation News 6, 25 (1993).

14. Joe Wong, G.N. George, I.J. Pickering, Z.U. Rek, M. Rowen, T. Tanaka, G.H. Via, B. DeVries, D.E.W. Vaughan, G.E. Brown Jr., Solid State Commun. 92, 559 (1994).

15. P. Lagarde, A.M. Flank, G. Tourillon, R.C. Liebermann, J.P. Itie, J. Phys. I France 2, 1043 (1992).

16. I. Davoli, E. Paris, S. Stizza, M. Fanfoni, A. Gargano, A. Bianconi, F. Seifert, Phys. Chem. Miner. 19, 171 (1992).

17. a) W. Schwieger, D. Heidemann, K.-H. Bergk, Rev. Chem. Mineral. 22, 639 (1985); b) D. Heidemann, W. Schwieger, K.-H. Bergk, Z. Anorg. Allg. Chem. 555, 129 (1987); c) W. Schwieger, K.-H. Bergk, D. Heidemann, G. Lagaly, K. Beneke, Z. Kristallogr. 197, 1 (1991).

18. T.J. Pinnavaia, I.D. Johnson, M. Lipsicas, J. Solid State Chem. 63, 118 (1986).

Table 1: Widths of the white lines $A$ in SiK XANES spectra (in eV).

\begin{tabular}{lllllll}
\hline sample & quartz & coesite & MFI & glass 1 & glass 2 & glass 3 \\
width & 1.49 & 1.45 & 1.71 & 1.94 & 1.94 & 1.99 \\
\hline sample & $\underline{\mathbf{1 a}}$ & $\underline{\mathbf{1 b}}$ & $\underline{\mathbf{2 a}}$ & $\underline{\mathbf{2 b}}$ & $\underline{\mathbf{3 a}}$ & $\underline{\mathbf{3 b}}$ \\
width & 1.85 & 1.69 & 1.83 & 1.80 & 1.70 & 1.70 \\
\hline sample & ilerite & magadiite & hectorite & & & \\
width & 1.80 & 1.83 & 1.79 & & & \\
\hline
\end{tabular}



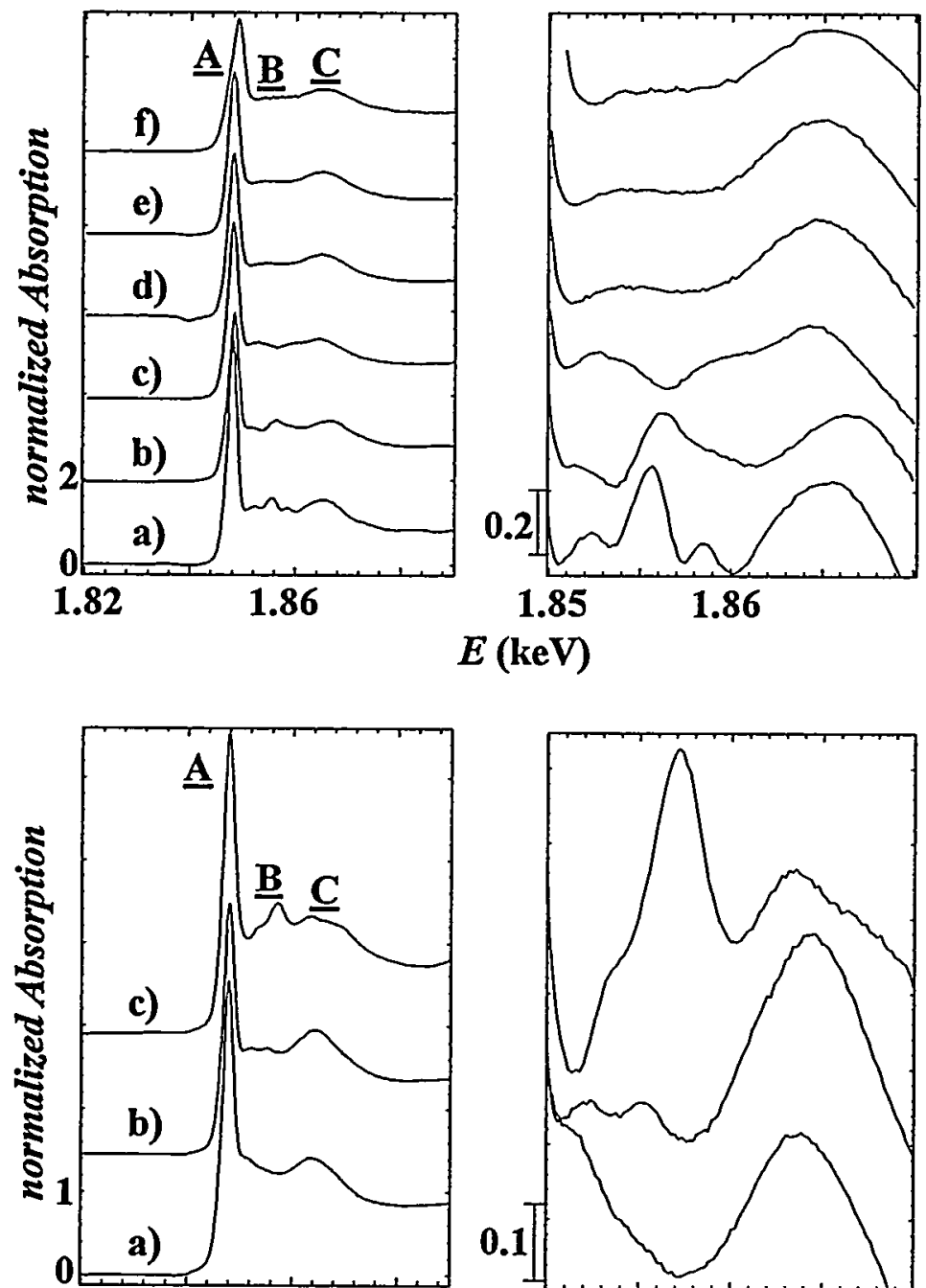

1.82
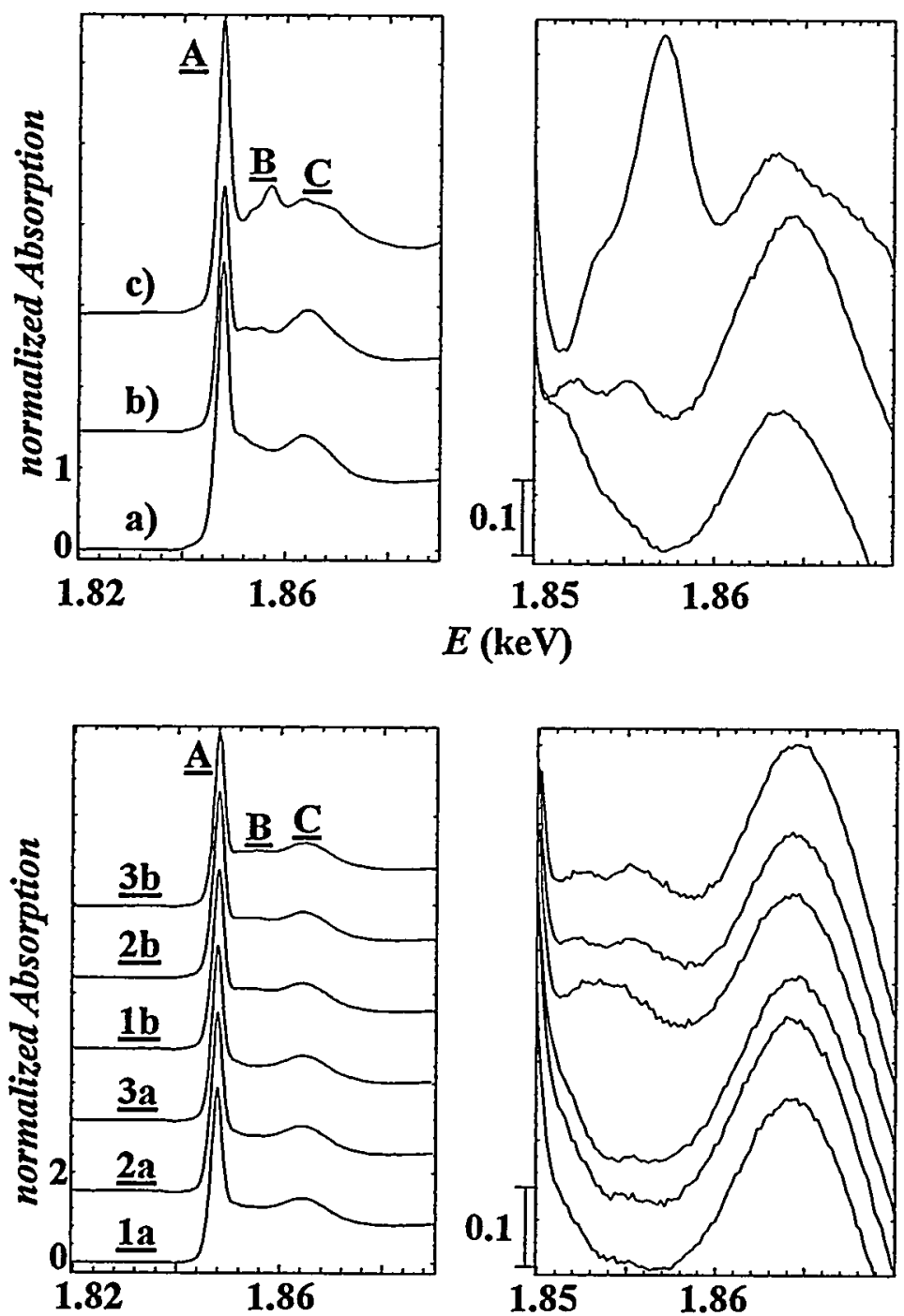

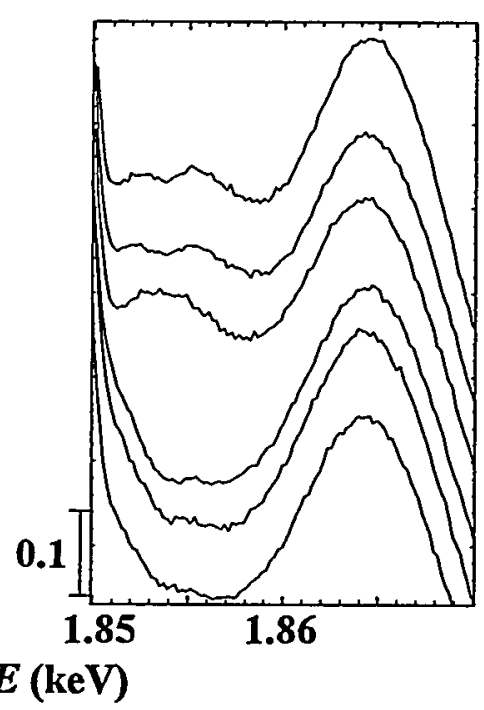

Figure 1: (left) SiK XANES spectra of crystalline and amorphous silica phases. a) $\alpha$-quartz, b) coesite, c) MFI, d) glass 1, e) glass 2 and f) glass 3; (right) zoom into the post-edge region.

Figure 2: (left) SiK XANES spectra of layered silicates: a) ilerite, b) magadiite and c) hectorite; (right) zoom into the post-edge region.

Figure 3: (left) SiK XANES spectra of mesoporous M41S organic-containing silica phases 1a-3a and the calcined compounds $\underline{\mathbf{1 b}} \mathbf{\underline { 3 } \mathbf { b }}$; (right) zoom into the postedge region. 


\title{
Magnetic Circular Dichroism in Soft X-Ray Emission of Fe and Co Excited by Monochromatic Circularly-Polarized X-Rays
}

\author{
L.-C. Duda (1), J. Stöhr (2), M.G. Samant (2), D.C. Mancini (3), N. Wassdahl(1), A. Nilsson (1), P. Kuiper (1), \\ and J. Nordgren (1)
}

(1) Uppsala University, Department of Physics, Box 530, 75121 Uppsala, Sweden

(2) IBM Research Division, Almaden Research Center, 650 Harry Road, San Jose, CA 95120, USA

(3) MAX-LAB, Lund University, Ole Romersvăg 1, 22100 Lund, Sweden

Soft $x$-ray emission (SXE) spectroscopy is particularly useful for the study of the electronic structure of solids because it, as is known for a long time already, gives a rather faithful picture of the partial (i.e. 1-resolved) density of states (DOS) of the occupied part of the valence band [1], e.g. the 3d states of transition metals. The theoretical foundation of this fact is summarized in the initial- and finalstate rules of core-level spectroscopies [2].

Creation of a spin-polarized core hole by excitation with circularly-polarized (CP) synchrotron radiation (SR) adds spin-specificity to the SXE spectra, an effect similar to magnetic circular dichroism (MCD) in X-ray absorption (XA) [3.] This has recently been predicted for Fe by Strange et al. [4] and, using broad-band excitation, was verified by Hague et al. [5]. Spin dependence in radiative excitation and emission processes is due to spin-orbit coupling and spin conservation in dipole transitions [6. The most striking feature of 3d-dichroism spectra (in both absorption and emission) is the sign-reversal between the $L_{2}$ - and $L_{3}$-dichroism which is due to a well-understood atomic property of the $2 p_{1 / 2}$ and $2 \mathrm{p}_{3 / 2}$ sublevels [5]. Magneto-optical sum rules [7] have provided a way to extract the local magnetic spin- and orbital-moments from intensity ratios of the $\mathrm{L}_{3}$ and $\mathrm{L}_{2} \mathrm{x}$-ray absorption edges and have made the $X A-M C D$ technique to an important spectroscopic tool in the study of magnetism. However, XA-MCD supplies a distorted picture of the energy-resolved electronic structure because of the core hole in the final state [2].

We have studied the spin-resolved electronic structure of the $3 \mathrm{~d}$ valence bands of $\mathrm{Fe}, \mathrm{Co}$, and $\mathrm{Ni}$ with the SXE-MCD technique [8]. For this, we measured the magnetization-direction dependent Lemission spectra following narrow-band CPSR for excitation. The SXE-MCD spectra of the $2 \mathrm{p}-3 d$ transition represent to first approximation the spindensity difference of the valence band. The $2 \mathrm{p}-3 \mathrm{~s}$ transition (in the following also called inner transition') takes place between two core levels and should reflect more atomiclike information. Together with spin-resolved 3s-photoemission data [9] it can provide information about the polarization degree of the $2 p$-core-hole immediately before it decays.

We acquired the $x$-ray emission spectra by using a grazing-incidence grating spectrometer with a position-sensitive multichannel-plate detector [10]. The optical axis of the spectrometer was at a right angle to the incidence-photon beam to minimize contributions from elastic scattering. While the sign of the photon-polarization was kept fixed during data taking the magnetic field direction was switched periodically. A typical rate was once in every 30 seconds and ensured equal conditions for both spectra. X-Ray absorption spectra were taken in the sample current mode. The photon beam hit the sample at an angle of $7.5^{\circ}\left(20^{\circ}\right)$ when recording $x$-ray emission (absorption) spectra. 


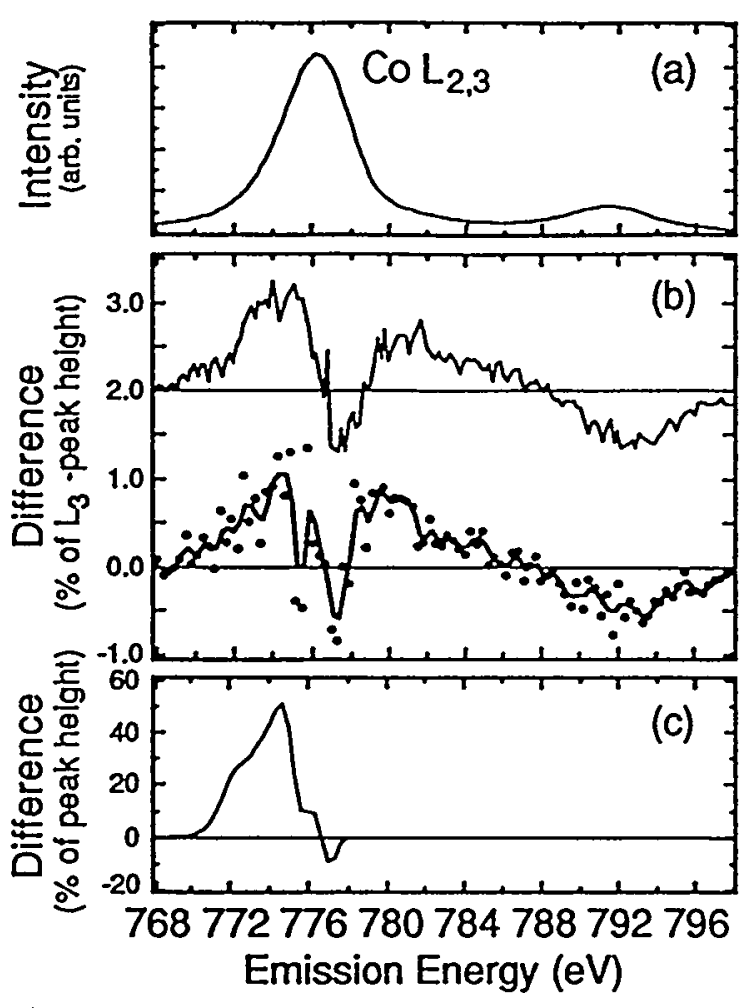

Figure 1: (a) Co $\mathrm{L}_{2,3}$-emission spin-averaged spectrum excited with white light, (b) dichroism spectra recorded with $3 \mathrm{eV}$ (top) and $1 \mathrm{eV}$ (bottom) resolution, (c) spin-density difference broadened by a gaussian of $1 \mathrm{eV}$ FWHM.

Figure la shows a broad-band excited Co L-emission spin-averaged spectrum. Figure $\mathrm{lb}$ is a comparison of two difference spectra (dichroism) recorded with 3 $\mathrm{eV}$ and $1 \mathrm{eV}$ emission-energy resolution, respectively. Though statistics are worse for the higherresolved spectrum we can distinguish an additional structure at $776 \mathrm{eV}$ which is only seen as a shoulder in the low resolution spectrum. This demonstrates the amount of detail this type of spectroscopy is able to reveal. The spin-density difference of the $3 \mathrm{~d}$-band obtained from an LMTO-calculation [11].is shown figure $1 c$ and is in good agreement with the experimental results.

In figure 2 we present magnetic dichroism in $\mathrm{L}_{2,3}$ cmission of Co following energy-selective excitation with $\mathrm{CP}$ x-rays. At least 2 orders of magnitude of incoming flux is lost, compared to zero-order

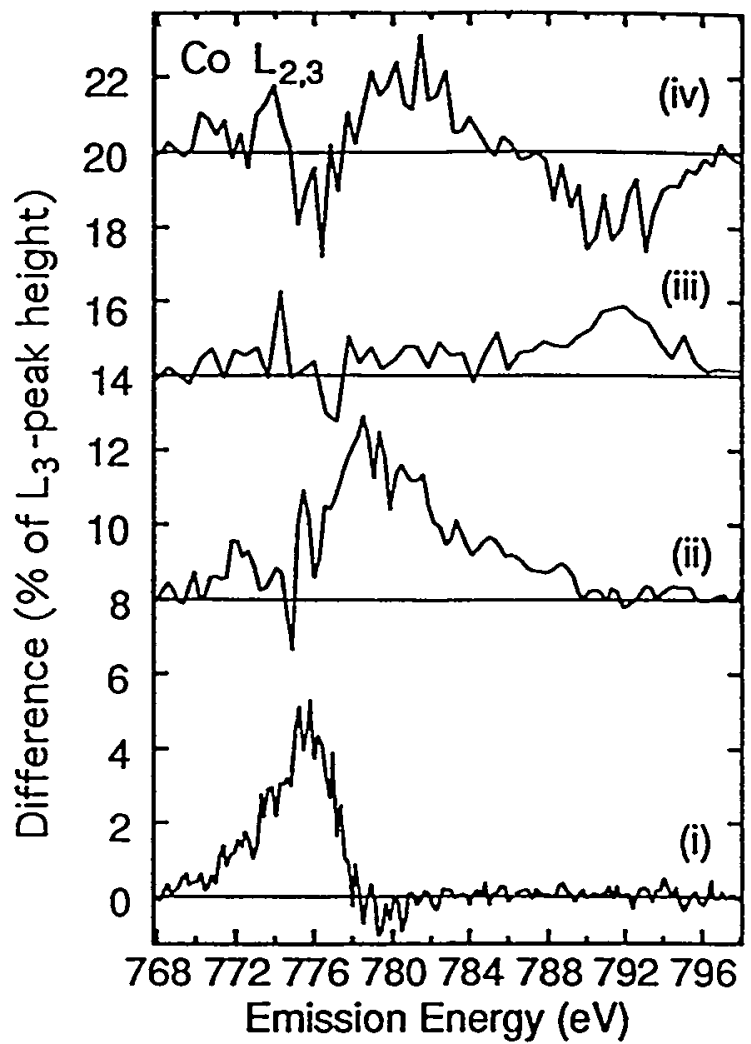

Figure 2: Co $\mathrm{L}_{2,3}$-emission dichroism excited with monochromatized x-rays of $4 \mathrm{eV}$ FWHM: (i) $778 \mathrm{eV}$, (ii) $784 \mathrm{eV}$, (iii) $793 \mathrm{eV}$, (iv) $810 \mathrm{eV}$.

radiation from the monochromator, by going to monochromatic excitation. Therefore, we can present these type of data only with $3 \mathrm{eV}$ resolution in the emission energy.

In the following we point out the important features observed in the dichroism spectra for each energy region seperately. The statements are valid for both figures 2 and 3 .

(i) Excitation at the L3-threshold: The spectral shape of the dichroism spectrum below the excitation energy is consistent with the shape of the white-light excited difference (cf. fig. $1 \mathrm{~b}$ and fig. $3 \mathrm{a}$ for $\mathrm{Co}$ and $\mathrm{Fe}$, resp.). The magnitude of the effect is a few times larger using monochromatic excitation.

(ii) Excitation between $\mathrm{L}_{3}$ and $\mathrm{L}_{2}$; Here the excitation energy is sufficiently high for satellite structures to appear above the main emission line. 


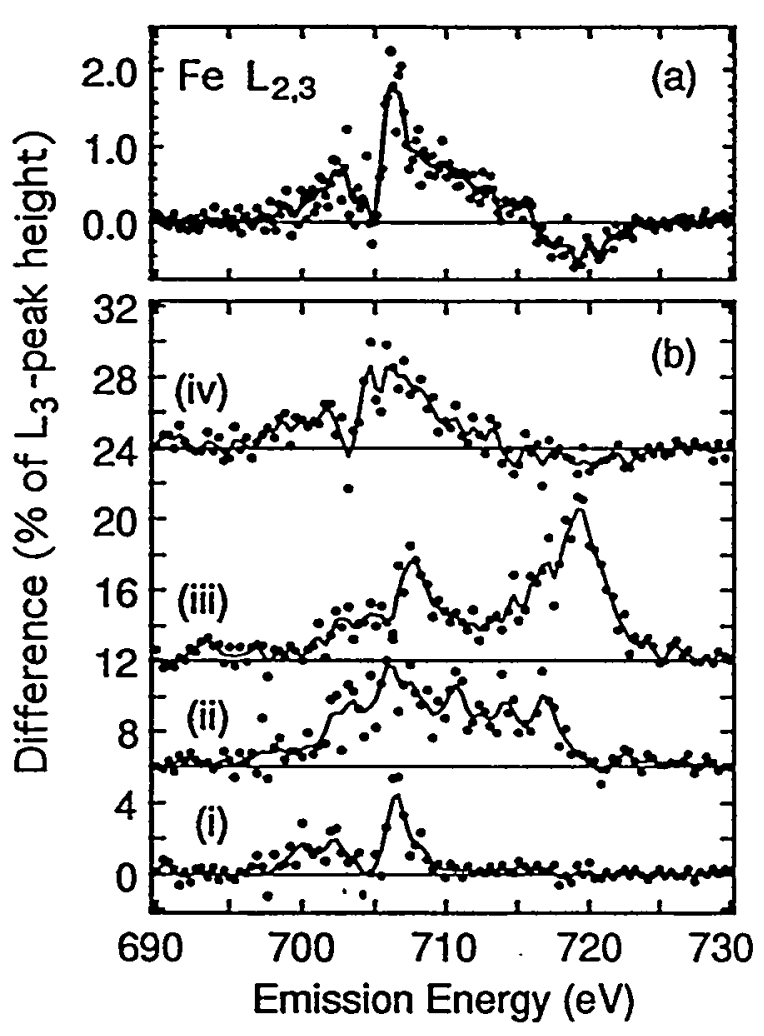

Figure 3: (a) $\mathrm{Fe} \mathrm{L}_{2,3}$ emission dichroism excited with white light and (b) monochromatized $x$-rays of $4 \mathrm{eV}$ FWHM: (i) $708 \mathrm{eV}$, (ii) $715 \mathrm{eV}$, (iii) $722 \mathrm{eV}$, (iv) $735 \mathrm{eV}$.

The statistical accuracy is worse in this spectrum so that the structures of the main line are difficult to compare. Especially in the shake-up satellite region of Fe (709-718 eV)we observe a gigantic SXE-MCD that has a multiple-peak structure (cf. fig. 3b,ii). Here the relative difference exceeds $25 \%$.

(iii) Excitation at the $\mathrm{L}_{2}$-threshold: At this excitation energy $2 p_{1 / 2}$-electrons are excited. The $\mathrm{L}_{2}$-emission line appears and at the same time Coster-Kronig transitions are possible. No clear peaks are discernable in the satellite spectrum which may be due to overlapping of different contributions. The $\mathrm{L}_{2}$ main-peak of the difference spectrum has the same sign as the $\mathrm{L}_{3}$-peak which is due to absorption $\mathrm{MCD}$, i.e. the ratio of the probabilities to excite from

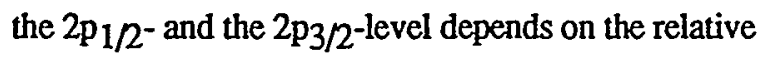
orientation of photon spin and magnetization direction.

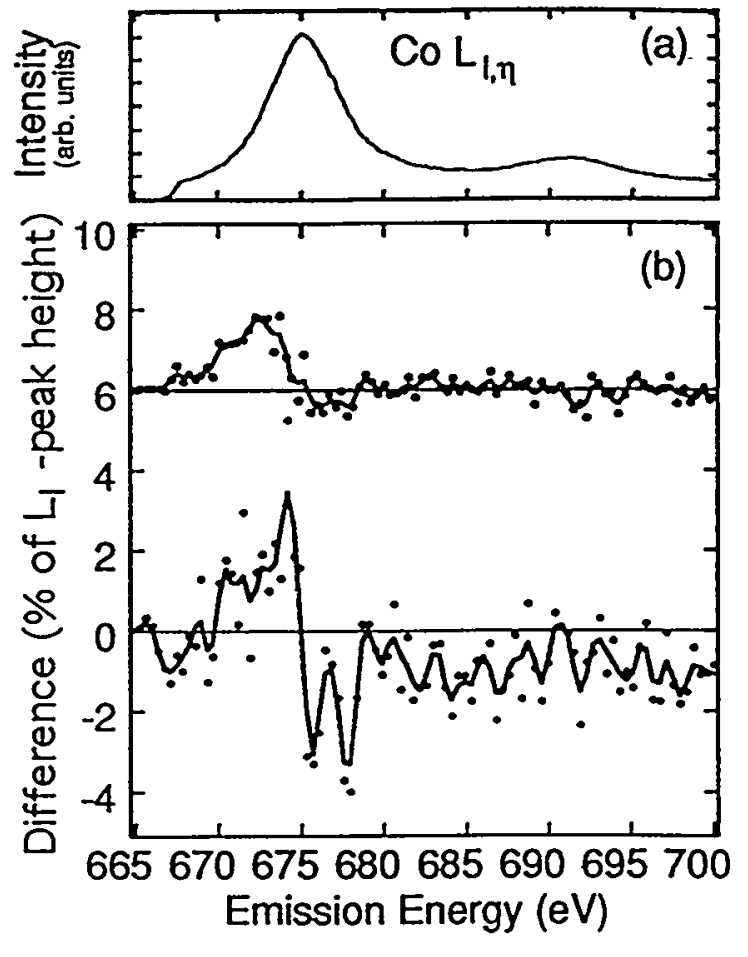

Figure 4: (a) Co $L_{1, \eta} \eta^{-e m i s s i o n ~ s p i n-a v e r a g e d ~}$ spectrum excited with white light and (b) Co $\mathrm{L}_{1, \eta^{-}}$ emission dichroism excited with white light (top) and monochromatized $x$-rays (bottom) of $4 \mathrm{eV}$ FWHM at $778 \mathrm{eV}$.

(iv) Excitation above the $\mathrm{L}_{2}$-threshold: As expected, this spectrum agrees well with the broadband spectrum (cf. fig. $1 \mathrm{~b}$ and fig. $3 \mathrm{a}$ for $\mathrm{Co}$ and $\mathrm{Fe}$, resp.) so that the sign of the $\mathrm{L}_{2}$ - and $\mathrm{L}_{3}$-dichroism features are of opposite sign here as well.

Next, we turn our attention to the Co $\mathrm{L}$ inner transition which is an electronic transition between two (core) shells that both have only small energy dispersion. The overlap of the $2 p$ - and $3 s-$ wavefunctions of the excited atom with the rest of the solid's wave-functions is minimal. Therefore we can expect that the resulting $L_{1, \eta}$-emission spectrum will yield atomiclike information. Spin-resolved $x$-ray photoemission spectroscopy of the Co 3s-shell reveals a doublet structure [8]. The two components are split by some $3.5 \mathrm{eV}$. The higher binding-energy 
component is of purely majority-spin character whereas the lower binding-energy component is of mixed but mainly minority-spin character. The exchange splitting of the $2 \mathrm{p}_{3 / 2}$ states is about $0.2 \mathrm{eV}$ [12] and can be neglected in the discussion on the resulting splitting seen in the $L_{1, \eta}$-emission spectra of the inner transition. Although the $3 \mathrm{~s}$ core-excited final state will distort the energy positions of the $\mathrm{L}_{\mathrm{l}^{-}}$ and $L_{\eta}$-lines to some extent, we still expect the relative energy separation of the centroids of the respective peaks to be almost unaffected.

The experimental results for $\mathrm{Co}_{1, \eta}$ are shown in figure 4. The bump structure at $672 \mathrm{eV}$ in the difference spectrum seen in figure $4 \mathrm{~b}$ is due to an enhancement on the low energy side of the whitelight excited $L_{1}$-peak of one of the spin-dependent spectra (not shown). This is approximately the relative position where-the component of pure majority-spin is observed in [8]. We also see a structure around $677 \mathrm{eV}$ that has a negative sign in the difference spectrum. It is more pronounced in the $L_{3}$-threshold excited dichroism but this could be an artifact that arises in the normalization procedure. More data with better statistical accuracy and higher resolution is needed as well as a means of normalizing in a more strict manner. The magnitude of the difference is just a few percent of the peak height suggesting that spin-flip processes taking place before the decay of the $2 p$ core-hole largely reduce the initial core-hole polarization.

Our results demonstrate the feasability and importance of using energy-selective excitation with CP synchrotron radiation to obtain spin-dependent SXE spectra and the corresponding magnetic dichroism. High-flux sources of $\mathrm{CP} x$-rays (like the elliptical undulator at beam line 5.3) as well as highquality $x$-ray imaging and high-throughput beam lines are badly needed to improve resolution in both excitation and emission.
1. see A. Meisel, G. Leonhardt, R. Szargan, X-Ray Spectra and Chemical Binding, Springer-Verlag, Berlin, 1989, e.g. ch. 6 and references therein

2. U. von Barth and G. Grossmann, Sol. State Comm. 32, 645 (1979); U. von Barth and G. Grossmann, Phys. Rev. B25, 5150 (1982)

3. G. van der Laan et al., Phys. Rev. B34, 6529

(1986); G. Schütz et al., Phys. Rev. Lett. 58, 737

(1987); C.T. Chen et al., Phys. Rev. B42, 7262 (1990)

4. P.M. Strange, B.L. Gyorffy, P.J. Durham, Phys. Rev. Lett. 67, 3590 (1991)

5. C.F. Hague et al., Phys. Rev. B48, 3560 (1993)

6. B.T. Thole, G. van der Laan, G.A. Sawatsky, Phys. Rev. Lett. 55, 1985 (2086); H. Ebert, P.

Strange, B.L. Gyorffy, J. Appl. Phys. 63, 3055 (1988)

7. B.T. Thole et al., Phys. Rev. Lett. 68, 1943 (1992)

8. Duda et al, Phys. Rev. B50, 16758 (1994)

9. D.G. Van Campen, L.E. Klebanoff, Phys. Rev. B49, 2040 (1994)

10. J. Nordgren et al., Rev. Sci. Instrum. 60, 1690 (1989)

11. Per Söderlind, private communication

12. L.E. Klebanoff, D.G. Van Campen, R.J. Pouliot, Phys. Rev. B49, 2047 (1994) 


\title{
Surface Science Study Of Negative Electron Affinity Formation On GaAs With Cs And NF3
}

\author{
R. Cao, H. Tang, and P. Pianetta \\ Stanford Synchrotron Radiation Laboratory \\ Stanford Linear Accelerator Center \\ P.O. Box 4349, Bin 69, Stanford, CA 94309
}

\section{INTRODUCTION}

Negative electron affinity. (NEA) refers to the condition that the vacuum level at the surface of a semiconductor lies below its conduction band minimum in the bulk. Due to the high electron emission efficiency, NEA photocathodes have found wide-ranged applications in both industry and academic research, such as the night vision devices and spin polarized electron source at SLAC. Despite the successful development of NEA photocathode technology, there is no general agreement regarding the mechanism of NEA formation. We strongly believe that a systematic surface science study of such a system is absolutely crucial in order to gain a fundamental understanding of the NEA formation. This stems from fact that the performance of a photocathode is completely determined by the properties of the semiconductor surface and a thin activation layer of 7-10 $[1]$. We have performed a surface science study of NEA formed on GaAs with Cs and NF3. The results have been compared with those from the NEA photocathodes prepared by Cs and $O$. The single valency nature of fluorine makes the system and data interpretation much simpler and will definitely shine light on this issue. It should be pointed out that the performance in terms of quantum yield and life time are comparable for these two types of photocathodes [2].

\section{EXPERIMENTAL}

The experiment was carried out on beam line 81 equipped with a toroidal mirror grating monochromator. The experiment was hosted in a standard ultra-high vacuum (UHV) system equipped with a double pass cylindrical mirror analyzer (CMA) with base pressure better than $1 \times 10^{-10}$ torr scale. High resolution core level and valence band photoelectron spectroscopy was utilized to study the interfacial chemistry, and the quantum yield of the prepared NEA photocathodes were characterized with a calibrated HeNe laser. A commercial available cesium getter made by SEAS was used as the cesium source, and $\mathrm{NF}_{3}$ gas was used as a fluorine carrier. NEA photocathodes were prepared on both cleaved ptype $\mathrm{GaAs}(110)$ surface and thermal cleaned p-type $\operatorname{GaAs}(100)$ surface.

\section{RESULTS AND DISCUSSION}

Figure 1 presents the photoemission spectra of $\mathrm{Ga} 3 \mathrm{~d}$ and $\mathrm{As} 3 \mathrm{~d}$ from the substrate and the $\mathrm{Cs} 5 \mathrm{p}$ and $F 2 p$ from the activation layer at various stage during NEA formation using $\mathrm{Cs}$ and $\mathrm{NF}_{3}$ on a p-type GaAs. For cesiation, cesium is deposited on the 
atomically clean $\mathrm{GaAs}$ surface until the saturation coverage was reached at room temperature. A charge transfer from the adsorbed $\mathrm{Cs}$ to both substrate $\mathrm{Ga}$ and As atoms is evidenced by the presence of shoulders on the low binding energy (high kinetic energy) sides of the substrate core level spectra. This has not been reported before due to lack of energy resolution. The charge transfer leads to strong dipole formation on the substrate surface. This has also been confirmed by an observation of a large reduction of the surface work function. The activation was performed following the so-called yo-yo process with both $\mathrm{Cs}$ and $\mathrm{NF}_{3}$ sources on the one monolayer Cs covered surface, monitored by the photocurrent emitted from the sample [3]. We found that for this system there is no indication of chemical reaction between the substrate and the absorbed fluorine, indicated by absence of the chemically shifted components on the substrate core level spectra, though strong reaction takes place without presence of Cs. This is in sharp contrast to the Cs-O system, where presence of Cs monolayer on the GaAs surface largely enhances the interaction between the substrate and oxygen, leading to strong substrate oxidation. The results also show that an NEA condition can be reached within a wide range of $\mathrm{Cs} / \mathrm{F}$ ratio in the activation layer. The degradation process of a photocathode has been also carefully investigated. Spectroscopically, we have demonstrated that the photocathode degradation is accompanied by a slight reduction of the substrate band bending and accumulation of the contaminants (water is one of the most important one, marked by the arrow in the figure) on the surface.

There have been several models proposed to explain the formation of NEA on GaAs surface, partly based on some experimental findings from the Cs-O prepared photocathodes. However, no general agreement has been reached. The underlying reason for the dispute comes from the complicated chemistry involved in the Cs-O-GaAs system and the fact that device performance is strongly dependent on the detailed preparation process and the vacuum system. The Cs-F-GaAs system shows a much simpler chemistry and should be taken as a model system to investigate the nature of NEA phenomenon. Our study clearly demonstrates that formation of NEA may not be related to the interfacial chemical reaction. We have further proved that in the Cs-F-GaAs system there is no fixed Cs/F ratio in the activation layer, which casts a serious doubt on the validity of the popular cluster model, where formation of the specific Cs-electronegative species cluster is considered to be responsible for enhancement of electron emission from the surface. Finally, our result strongly indicates that accumulation of excess contaminants is the major mechanism for photocathode degradation, contrary to the proposed Cs loss mechanism.

1. D.C. Rodway and M.B. Alleson, J. Phys. D: Appl. Phys. 19, 1353 (1986).

2. F. Ciccacci and G. Chiaia, J. Vac. Sci. Technol. A 9, 2991 (1991).

3. F.C. Tang, M.S. Lubell, K. Rubin, A. Vasilakis, M. Eminyan, and J. Slevin, Rev. Sci. Instrum. 57, 3004 (1986). 


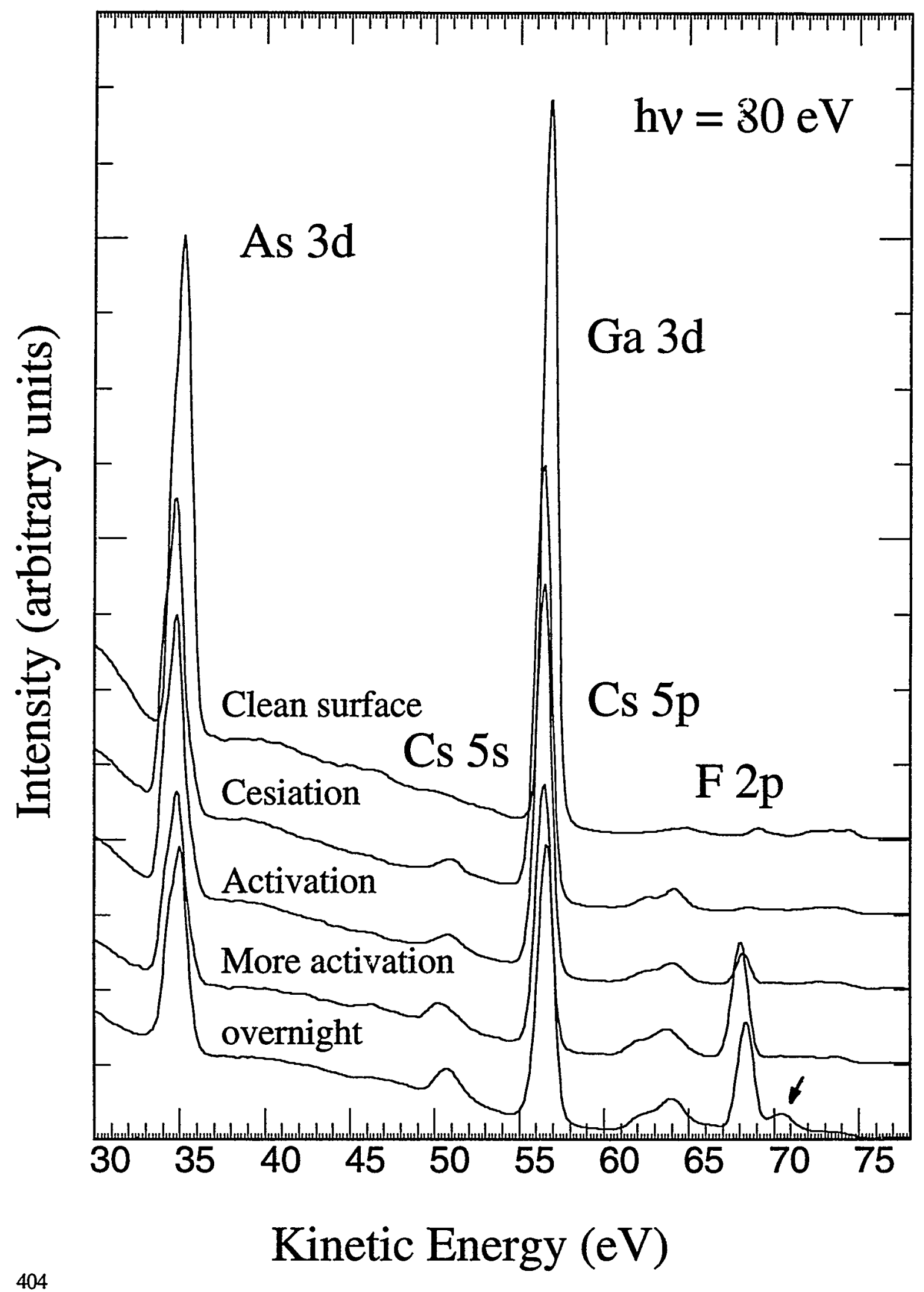




\title{
Direct observation of a narrow band near the gap edge of $\mathrm{FeSi}$
}

\author{
C.-H. Park, Z.-X. Shen, A. G. Loeser D. S. Dessau, \\ Department of Applied Physics, Stanford University, Stanford, \\ California 94305 \\ Stanford Synchrotron Radiation Laboratory, Stanford University, \\ Stanford, California 94309
}

\author{
D. Mandrus, A. Migliori, J. Sarrao, Z. Fisk \\ Los Alamos National Laboratory, Los Alamos, New Mexico 87545
}

\section{INTRODUCTION}

FeSi is a very interesting compound for many reasons. According to band calculations, FeSi is a semiconductor with a small energy gap. In fact, the magnetic susceptibility, $\chi(T)$, and electrical conductivity have a gap-like activated behavior at lower temperatures ${ }^{1}$. However, the band calculation alone can not explain the unusual behavior of $\chi(\mathrm{I})$, nor the recently reported temperature dependence of the optical conductivity data ${ }^{2}$. In particular, the magnetic susceplibility, which can be described with the Curie-Weiss law above $500 \mathrm{~K}$ but drops rapidly below $500 \mathrm{~K}$, has intrigued solid state physicists for a long time.

Recently, motivated by the fact that FeSi exhibits similar properties to some narrow band Kondo insulators ${ }^{3}$, Mandrus et al.$^{4}$ developed a refined version of the narrow band piclure. They found that the thermodynamic properties of FeSi could bc accounted for by a simple model involving two narrow (about $500 \mathrm{~K}$, or $40 \mathrm{meV}$ ) peaks in the DOS at the edges of a narrow gap (about 900K), eliminating the need for the delta-functionlike peaks in the DOS. This is strong circumstantial evidence for a narrow band in $\mathrm{FeSi}$, although the bandwidth is too small to be explained by band calculation alone. Mandrus $\mathrm{et}$ al. suggested that the narrow band is due to a strong renormalization of the band structure in FeSi, implying that this is the first Kondo material to be found which doesn't contain rare earth elements.

In this paper, we report results of our highresolution angle-resolved photoemission data from FeSi. Although our data collected at room temperature in angleintegrated XPS mode is virtually identical to earlier work [9], low-temperature angle-resolved investigation yields some of the most spectacular spectra we have observed in a solid state material. In particular, we observed at low temperature a very sharp and intense peak at the valence band maximum (VBM), which shows a much smaller dispersion than that predicted by band calculations. The peak has a very asymmetric lineshape, which varies dramatically with tcmperalure and emission angle. The data has two implications. First, they are consistent with the picture of I PSi as a highly corrclated matcrial with a very narrow band near the band edge. This constitules the first direct observation of a narrow band at the valence band edge of a highly comelated insulator. Second, the data provides an example of strong temperature dependent photoemission data near the (pinned) Fermi level. We hope to stimulate more work towards understanding temperature dependent photomission data from novel materials.

\section{EXPERIMENT AND ANALYSIS}

The ARPES experiment on FeSi was performed using a VSW system with the undulator beamline 5-3 of SSRL, as described elsewhere ${ }^{5}$. A single crystal of FeSi was transferred into the chamber, and cleaved by a knife edge in-situ, in a vacuum of $5 \mathrm{E}-11$ torr, in order to yield a fresh surface. Even though the cleaved surface does not have a well defined crystal plane, we observed a very sensitive angular dependence near the Fermi level. The samples were oriented so the [100] direction was about 45 degrees from the incident photons. A gold sample was electrically connected so that the Fermi level of the sample could be inferred from a gold spectrum. The combined photon and electron energy resolution was approximately $35 \mathrm{meV}$ at $24 \mathrm{eV}$ photon energy and the angular resolution of the detector was \pm 1 degree. The data was taken in better than $5 \mathrm{E}-11$ torr base pressure. For our angle integrated data, we performed XPS on FeSi in a Surface Science ESCA system. The data was taken at room temperature, with monochromatized $1487 \mathrm{eV}$ photons. The same fracturing method was used on a single crystal, and the sample was cleaved in 1E-7 torr. The overall resolution was about $0.6 \mathrm{eV}$ and the base pressure was about 1E-9 torr when the data was taken.

Figure 1 presents selected spectra of FeSi taken under various conditions. The XPS spectrum (D), gives a rough estimate of the DOS; it is consistent with previously published data ${ }^{6}$, and qualitatively consistent with the band structure calculation. While the spectra $(A)$, (B), and (C), which were taken at $25 \mathrm{~K}$, but at three different analyzer angles, demonstrate the large angular dependence, the difference between $25 \mathrm{~K}$ and $300 \mathrm{~K}$ with the same analyzer angle $(A)$ is also very dramatic. The peak right below the Fermi level in the spectrum (A) at $25 \mathrm{~K}$ is among the sharpest valence band features seen by photoemission. It is also quite asymmetric, and diminishes in the higher temperature spectrum $(A)$ at $300 \mathrm{~K}$. In the spectrum $(B)$, the weight of the sharp peak is reduced dramalically, and double peaks are visible. This implies that there are probably two peaks in the spectra at $(\Lambda)$ also, and the second peak at higher binding energy is buried by the tail of the first peak. In (C), we see the sharp peak diminished even more, and the second is clearly visible. 


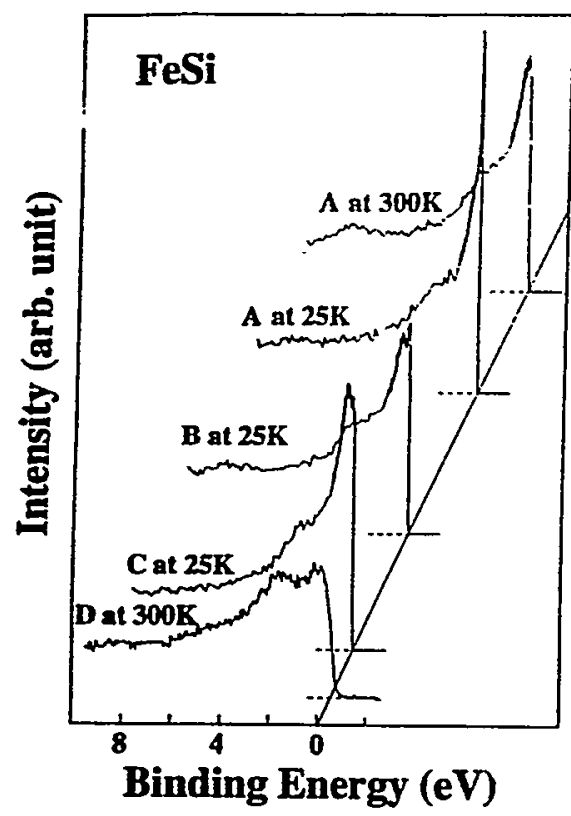

Figure 1. (A) at $300 \mathrm{~K}$ and $(\mathrm{A})$ at $25 \mathrm{~K}$ were taken the same analyzer angle, but at different temperatures. (B) at $25 \mathrm{~K}$ and (C) at $25 \mathrm{~K}$ were taken at different angles from (A). All spectra were taken with $24 \mathrm{eV}$ linearly polarized photons. (D) at $300 \mathrm{~K}$ is XPS at room temperature.

The data in Fig.1 clearly shows that FeSi yields spectacular photoemission spectra: an extremely sharp and intense peak at $\mathrm{E}_{\mathrm{F}}$, with a very asymmetric lineshape and strong temperature and angular dependence. Although one expects photoemission features at the lowest binding energy to be very sharp because the lifetime broadening is small, such features are rarely scen in experiments. To have such a strong and sharp feature right at the VBM is remarkable. The existence of this sharp peak is very reproducible, but the intensity shows some variation from cleave to cleave, presumably due to surface roughness. Even the sharp peak Fig.1 (A) is not the most intense in our data set. An analysis of the rich ARPES data from FeSi will not only help to understand the unusual properties of this material in particular, but also will lead to a better insight of the temperature dependent photoemission spectra from highly correlated materials in general.

For a more detailed look at the peak, we show angular dispersion of the sharp feature obtaincd from a cleave yielding the highest peak intensily, with a magnified energy scale, in Fig. 2. From the bottom to the top curves, the feature is observed dispersing lowards the Fermi level, reaching a maximum at $\theta=23^{\circ}$ and then backing down after $\theta=23^{\circ}$. In the $\theta=23^{\circ}$ spectrum, the highest intensity peak is roughly $25 \mathrm{meV}$ below the Ferni level. The width of the leading edge (10\% 10 $90 \%$ height) is comparable with our $35 \mathrm{meV}$ energy resolution, and thus is mainly limited by the later. In this spectrum, the reference Fermi level lies in this leading edge, more than half way towards the peak. This spectrum is very similar to data from metallic samples such as the high- $T_{c}$ superconductors whose lineshape is highly controversial. 7,8

We believe, however, that the quasiparticle peak is below the Fermi level at $\theta=23^{\circ}$ because it has the highest intensity and energy when compared to neighboring angles; if some of the spectral function weight were above the Fermi level, then the photoemission peak's intensity would be decreased. This critical point behavior is consistent with the fact that FeSi is a semiconductor, placing the VBM at $\theta=23^{\circ}$. The energy separation between the VBM and the pinned Fermi level should be no larger than the $25 \mathrm{meV}$ measured above. Because the peak is very asymmetric and the intensity at higher binding energy could have contributions from secondary electrons, this background makes the peak appear at higher binding energy. Indeed, when we added secondary electrons to the fit, the peak position shifted to $20 \mathrm{meV}$. In addition, it is possible that the peak at $\theta=23^{\circ}$ is only a local maximum, not a global VBM. If we assume that the Fermi level is in the middle of the gap, as for an intrinsic semiconductor, the data suggests that FeSi has a very narrow gap. However, we can not rule out the possibility that the Fermi level of FeSi is pinned very close to the VBM.

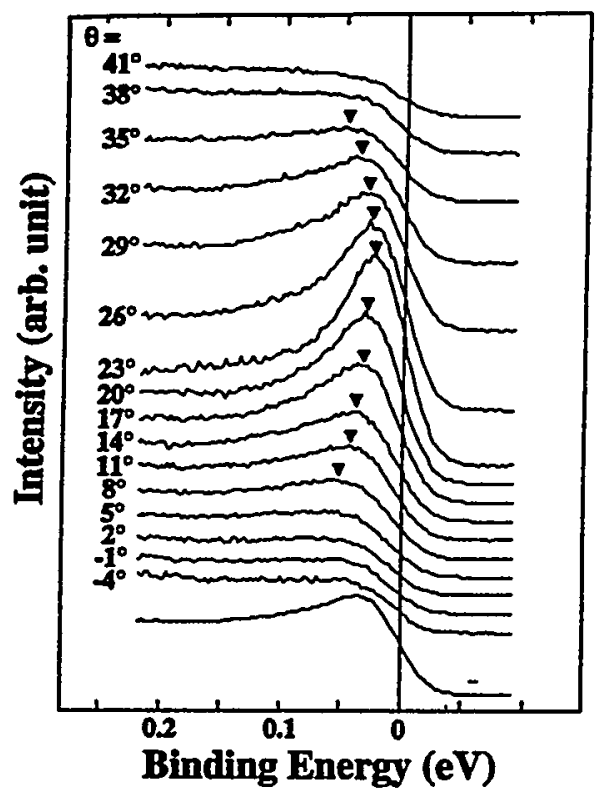

Figure 2. The stack of data shows the angular dispersion of the sharp peak. The triangles roughly indicate peaks, in order to clarify the dispersion. $\mathrm{q}$ is the analyzer angle from which the data were collected. $\theta=0$ when the analyzer is about normal to the [100] plane. As $\theta$ increases, it tilts toward the [110] plane. The bottom curve, the average of all the curve above, is shown to simulate the angle-integrated spectra.

Because FeSi is a three dimensional material, we do not know the exact value of the crystal momentum corresponding to the VBM. However, the peak has been observed very reproducibly at a certain angle between the [100] and [110] directions, as determined from the Laue pattern. We obtained similar results from other cleaved samples. Since the sample surfaces are quite different for each cleave, it is less likely that we see a surface state. Even without the knowledge of specific electron momentum, the data in Fig. 2 still give us a good estimate of the bandwidth near the VBM, because the angular range in Fig. $2\left(45^{\circ}\right)$ is comparable to the width of the first Brillouin zone (about $40^{\circ}$ at the photon energy used). The dispersion seen in the data is $30 \mathrm{meV}$ or less, 
indicating a band width of that magnitude. This number is significantly smaller than the value obtained by the band structure calculations for any band near the Fermi level (about 200 to $500 \mathrm{meV}$ ). Observation of such a narrow band near the Fermi level in FeSi supports the idea that FeSi belongs to the class of highly correlated insulators. It has been shown that a strong renormalization of the band causes a sharp peak at the gap edges. ${ }^{9}$ So far, the narrow band at the gap edge has not been directly observed before in any highly correlated insulator. In essence, Fig. 2 constitutes the first direct observation of a narrow band in this compound.

Next, we consider the very unusual lineshape of the peaks in fig 2 . We first notice that the peak broadens rapidly as it disperses away from EF. The peak broadens more than one would expect from the small change in binding energy as it disperses away from the VBM $(\theta=$ $23^{\circ}$ ). Assuming that the width is directly related to the life-time of the quasi-particle, we speculate that there is some low energy scattering. Such a mechanism would also explain the strong scattering implied by optical experiments, which show a fast disappearance of the gap ${ }^{2}$ As a caution, we note that in the usual picture of peak broadening due to lifetime effects, the weight of the peak above the background should be the same for each $k$ value. To first order, that is not the case here, and so may complicate the above simple interpretation. The second unusual aspect is the asymmetry of the peak. We suggest that this is also due to the correlation effects. The $3 p$ core level photoemission reported elsewhere shows an abnormally large asymmetry of the Doniach-Sunjic lineshape, when compared to the other iron silicides ${ }^{10}$. Knowing that TeSi is not melallic, the asymmetry was attributed to the correlation in the d orbit.

A narrow band yields a peak in the total density of states (DOS), and therefore, assuming similar photoionization cross-sections, onc expects to see a peak in the angle-integrated data. However, in this particular case, we argue that the broadening of the peak as a function of encrgy (as we noted above with Fig.2) makes the angle-integrated spectra look quile different. To demonstrate, the bottom curve in Fig. 2 is the summation of all the spectra above. One can see that the peak is diminished considerably, when only partially angleintegrated. In fach, no strong feature has been observed in the polycrystaline sample ${ }^{11}$, for which the effective angle integration is complete. We have observed a similar effect in other correlated materials, such as the high- $\mathrm{T}_{\mathrm{c}}$ compounds. In this sense, our data is consistent with the previous angle-integrated results. In addition, angleresolved photoemission yeilds more information, which allows us to identify the narrow band and supports the model put forth by Mandrus $\mathrm{ct}$ al.

We tum now to the remarkable temperature dependence in the photoemission data. Fig. 3 shows the temperature dependence of the slarp peak at $\theta=23^{\circ}$. $\Lambda \mathrm{s}$ the temperature increases, the sharp peak reduces rapidly. To compare the spectra at ench temperaturc while compensating for the varying photon flux, we normalized each spectrum with the height of the electron counts near $1 \mathrm{eV}$ binding energy. This procedure only requires a 5 to $10 \%$ rescaling of the spectra as recorded.

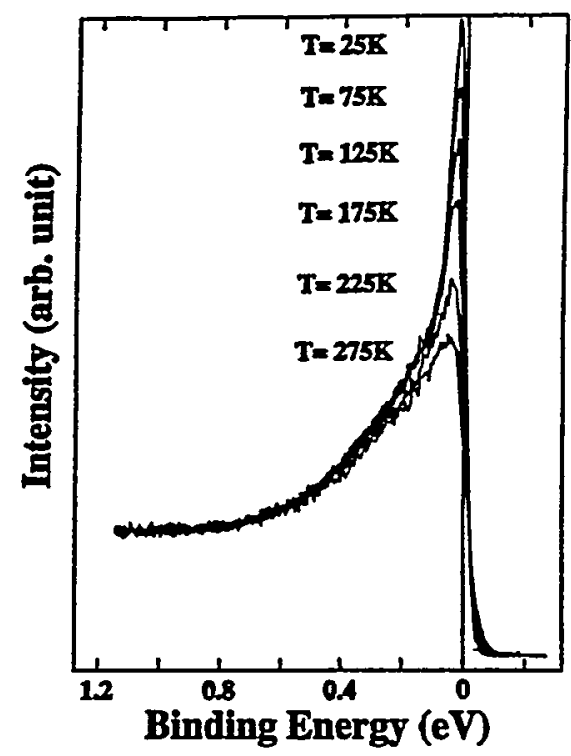

Figure 3. The spectra were collected at an analyzer angle, where the peak is sharpest at $25 \mathrm{~K}(\mathrm{q}=23)$. This angle was measured at $25 \mathrm{~K}$, then $75 \mathrm{~K}, 125 \mathrm{~K}, 175 \mathrm{~K}$, $225 \mathrm{~K}$, and $275 \mathrm{~K}$ as the peak gets smaller.

To the best of our knowledge, the data in Fig.3 constitute the strongest temperature dependence recorded by photoemission. Due to its potential connection to novel phenomena such as the Kondo resonance, metalinsulator transitions and superconductivity, temperature dependent photoemission data near the Fermi level is of great current interest. Not only are issues such as the Kondo resonance highly controversial, but also the temperature dependent data from Cu surfaces are not fully understood. ${ }^{12}$ With the improvement of energy resolution and the proliferation of UHV low temperature capability, it is important to establish a systematic of the temperature dependence of photoemission data. We hope that the data in Fig. 3 will stimulate more interest in this general issue, as well as a better understanding of FeSi itself. In this sense, the data in Fig. 3 provides an extreme example for the temperature dependent photoemission data.

Although the spectra in Fig. 3 line up very well at high binding energy, the total spectral weight is not conserved. The loss, with increasing temperature, of the spectral weight below the Fermi level can not be compensated by the tiny gain above the Fermi level. One way to look at the unconserved spectral weight is to relate this to the fact that FeSi is known to have a narrow indirect gap. The thermally depleted valence band electrons populate the bottom of the conduction band, which is located elsewhere in $\mathrm{K}$-space from the maximum of valence band. In fact, we observed the spectral weight increasing with temperature at a different analyzer angle. Recently, Fu and Doniach proposed a two band model to explain the properties of FeSi. ${ }^{13}$ In this model, two symmetric (Hubbard) bands with moderate on site Coulomb interactions are allowed to hybridize. They solved the problem in infinite dimensions, and compared the results with various experiments. They found that the consideration of the on-site Coulomb interaction leads to a 407 
better description of the susceptibility, and a renormalization of the energy gap. For single particle excitation spectra at low temperature, this model gives a very sharp peak and an incoherent background with a low energy cut-off of the gap energy scale. With increasing temperature, the peak broadens very rapidly and the spectra become a single, very asymmetric feature. They also found that the peak at low temperature broadens very significantly when the energy disperses to a binding energy comparable to the gap. Although the model they are considering is very crude, and the solution is done at infinite dimension, we find many interesting similarities between our data and the model. The lineshape of our data is very asymmetric, which is very similar to data from other highly correlated materials. The qualitatively change of the peak lineshape as a function of temperature and energy position found in our data is also very consistent with this physical picture. In a many body model such as the Hubbard model, a large redistribution of the low energy spectral weight with relatively small temperature change is possible. Within the frame work of the manybody models, the extra spectral weight is either moved to other momentum, different energy, or is cut off by the Fermi function as the peak is broadened.

\section{CONCLUSIONS}

In conclusion, angle-resolved photoemission revealed a very narrow band (about $30 \mathrm{meV}$ ) at the VBM. The spectral feature related to this band is very sharp at low temperatures, and shows a strong and very unusual temperature dependence. This data constitutes the first direct evidence of a very narrow band in FeSi. The significantly renormalized band width, the strong temperature and angular dependence of the data, and the unusual spectral lineshape can all be qualitatively accounted for by a theoretical model with strong electronelectron correlation. This framework of the electronic structure also provides an explanation for the unusual magnetic, thermodynamic properties of FeSi. This finding supports the notion that $\mathrm{FeSi}$ is a highly correlated insulator with a narrow band at the valence band edge. Further, the remarkable data observed in this compound will stimulate more effort to establish a systematic understanding of temperature dependent photoemission data from highly correlated materials.

\section{ACKNOWLEDGMENT}

We would like to acknowledge the stimulating discussions with S. Doniach, C. Fu, and G. Aeppli. SSRL is operated by the DOE office of Basic Energy Science, Division of Chemical Sciences. The office's Division of Material Science provided support for this Research. Beamline 5 of SSRI-was built with DARPA, ONR, AFOSR, AOR, DOE and NSF support.

\section{REFERENCES}

${ }^{1}$ V. Jaccarino et al., Phys. Rev. 160,476 (1967)

2 Z. Schlesinger et al., Phys. Rev. Lett. 71, 1748 (1993)

${ }^{3}$ T. E. Mason et al., Phys. Rev. Lett. 69, 490 (1992)

${ }^{4}$ D. Mandrus et al., to be published in Phys. Rev. B

5D. M. King et al., Phys. Rev. Lett. 70, 3159 (1993)

${ }^{6}$ S. J. Oh et al., Phys. Rev. B 35, 2267 (1987) 408
${ }^{7}$ C. Olson et al., Phys. Rev. B 42, 381 (1990)

${ }^{8} \mathrm{~L}$. Liu Anderson and J. Allen, J. of Phys. \& Chem. of Solids 52, 1473 (1991)

${ }^{9}$ S. Doniach et al., Physica B 199\&200, 450 (1994)

10F. Sirotti et al., Phys. Rev. B 48, 8299 (1993)

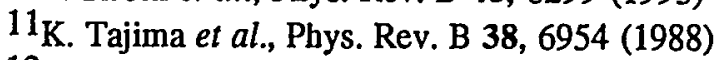

$12_{\mathrm{R}}$. White et al., Phys. Rev. B 35, 1147 (1987)

${ }^{13} \mathrm{C}$. Fu et al., to be published in Phys. Rev. B 


\title{
Imaging of Hydrated Biological Specimens With a New Type of Soft X-ray Microscope
}

\author{
Gregory Hirsch \\ Hirsch Scientific, 365 Talbot Avenue, Suite D8, Pacifica, CA 94044
}

\section{Introduction}

A new type of soft $x$-ray microscope has been undergoing development at SSRL since 1991. Compared to most existing $\mathrm{x}$-ray microscopes, which are fairly elaborate instruments utilizing state-of-theart optics and complex mechanical movement stages, this microscope represents a significant simplification of design, with attendant lowering of costs. The instrument is best suited for examining single biological cells and isolated organelles.

The microscope technique is a variation of the photoelectron-conversion contact method ${ }^{1}$ and the basic principles have been previously described. ${ }^{2}$ The instrument is very similar in geometry to the field emission microscope. As in field emission microscopy, an emitter is positioned in a vacuum chamber, with the tip facing an electron-sensitive area detector but, instead of solid emitters, this instrument uses special cone-like hollow emitters. The specimen to be examined is situated inside the emitter tip. To produce an image, $x$-rays are beamed down the axis of the hollow emitter from the open end, through the specimen, and onto the hemispherical emitter tip membrane. An x-ray contact image is thereby produced on the emitter tip membrane, corresponding to the $\mathrm{x}$-ray absorption of the specimen. The membrane is transparent to soft $\mathrm{x}$-rays and is externally coated with a high-efficiency photoemissive layer. Photoelectrons are liberated into the vacuum, outside the emitter tip, in a pattern corresponding to the contact image of the specimen. A high electric potential placed on the emitter accelerates the photoelectrons radially to the detector. A magnified, high-resolution, real-time image results.

\section{Previous results}

Previous research accomplished at SSRL generated images of various air-dried specimens. Micrographs of fungi and mammalian cells were produced with a resolution down $\approx 1000 \AA .3$ Aqueous samples were introduced into the emitter tips with fine glass micropipettes, and allowed to dry. The emitter interior was exposed to the microscope vacuum environment. Illumination was with broadband radiation, primarily in the water window (the energy range lying between the K-edges of carbon and oxygen, where water is relatively transparent compared to organic material).

Unfortunately, air drying leads to very severe shrinkage and distortion of most biological samples due to the effects of surface tension. The more desirable imaging of specimens in their natural wetstate was the aim of our research in 1994.

\section{Experimental arrangement}

Two different $x$-ray microscopes were used during the 1994 run. The first microscope was the instrument used in previous studies. This microscope is comprised of a small stainless steel chamber, and incorporates a 40-mm-diam Chevron microchannel plate detector (MCP) with phosphor screen output. Images are recorded by photography of the screen with a 35-mm single lens reflex camera using Kodak T-Max 400 film.

During the run, we concurrently constructed a new xray microscope with improved design features. This instrument incorporates a 75-mm-diam MCP. The body of the microscope is constructed from a 6 inch gate valve, with several ports welded on for instrumentation and vacuum connections. This design permits the microscope to be vented for changing emitters, while leaving the expensive and delicate MCP always isolated under vacuum. The arrangement also allows the emitter high-voltage to be raised, while the MCP is protected. Arcing sometimes occurs during this step, which would seriously damage the MCP were the emitter to break while operating the microscope with wet specimens. We find the operation of this new instrument to be very convenient, and the larger screen size allows more of the emitter tip area to be viewed.

Hollow emitters were individually sealed to the back wall of the microscope chamber, with the emitter interior at atmospheric pressure. Thus, the emitter can be thought of as an unusual vacuum window. The sealing surface material was an insulator, which allowed the high negative accelerating-potential to be applied to the emitter. The grounded body of the microscope functions as the anode. 
Beamline 3-4 is a bending magnet line which incorporates a 1.5-degree angle of incidence focusing mirror. This mirror acts as a low-pass filter with a cutoff energy of approximately $3 \mathrm{keV}$. For biological imaging, lower energy $\mathrm{x}$-rays were selected by utilizing mirrors in the beamline monochromator. By selecting the angle of incidence of the beam, and by proper selection of mirror material, it was possible to get fairly well defined and variable cutoff energies in the reflected beam. Sapphire mirrors were used for most of the imaging since they have a very high throughput in the water window and very little throughput above the oxygen edge. A $1500 \AA$ aluminum filter was usually used to remove vacuum ultra-violet radiation.

The $\mathrm{x}$-ray beam was transmitted out of the Beamline 3-4 vacuum system, into a helium atmosphere, via a $1000 \AA$ silicon nitride window. A 25 micron pinhole before the window was used to collimate the beam. The beam then entered the opening of the hollow emitter, where an image of the specimen in the emitter tip at atmospheric pressure was produced. This whole region was enclosed in overlapping Plexiglas cylinders which function both as radiation protection barriers, as well as high voltage protection. The plastic cylinders can be slid apart for specimens changes by sliding the microscope on a mounting table. This motion is interlocked to the SPEAR personnel protection system to prevent radiation exposure.

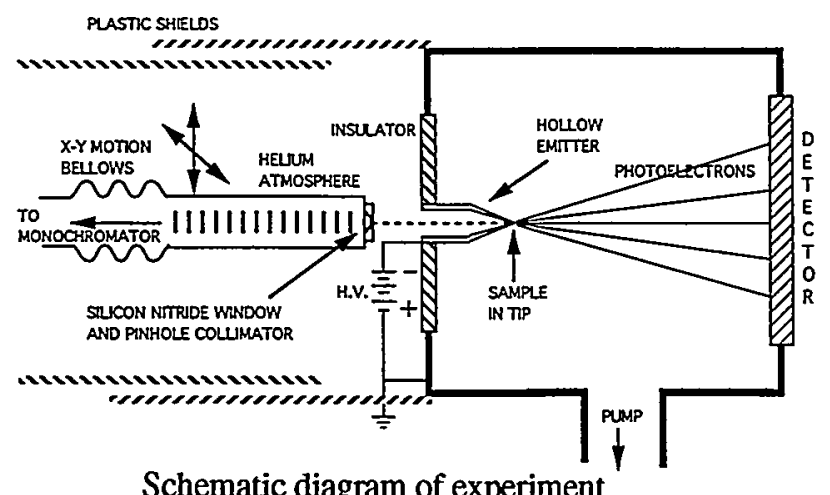

The flange holding the thin-window/pinhole combination was mounted on an $X-Y-Z$ vacuum manipulator. A high voltage electrical break was utilized between this flange and the manipulator, so that the flange could be raised to the same electrical potential as the emitter. This is to prevent electrical breakdown to the window. To form an image, the beam is raster scanned across the entrance of the emitter until an image is observed.

\section{Results}

Images of wet yeast cells in thin water-layers were observed. Several methods for maintaining a water layer of roughly 10 microns were devised. In most of these initial experiments, cells were first air dried in the emitter tips. Water was then added while viewing the tips. A dramatic increase in size could usually be observed as the cells rehydrated and swelled to their normal size. Significant radiation damage could be observed upon extended observation of the wet cells. This was manifest by increased swelling, and eventual disintegration of the whole cell. This was not observed with dry cells. The resolution obtained was somewhat less than seen with dry cells due to the use of lower accelerating voltages. We were very cautious with the the application of the high voltage to prevent any arcing destruction of the emitters.

\section{Significance of results and future plans}

Results obtained in 1994 show that it is feasible to operated the microscope with the emitter interior at atmospheric pressure, and with wet samples. We have, however, found the operation of the microscope with wet specimens to be somewhat problematic. Maintaining a constant and very thin layer of water is possible, but rather difficult. There is also the constant concern that the unexpected rupture of an emitter will cause irreversible damage to the expensive detector.

During the course of this work, we considered the relative merits of examining specimens in the frozenhydrated state. There are several compelling reasons for pursuing this route. The advantages are: image degradation due to radiation damage is essentially eliminated; the specimen can be frozen at a distinct moment in time when an interesting physiological process is happening; the specimen can be carefully placed in the emitter, optimally positioned, and observed by optical microscopy before being frozen and fixed in position; it is not necessary to carefully control the water layer thickness once the specimen is frozen; and image blurring due to specimen motion is eliminated. The disadvantage of observing frozen specimens is the inability to observe dynamic changes in the object. However, due to radiation damage effects, the ability to observe biological activity is rather limited, especially at high resolution. One potential concern is damage to specimens during the freezing process due to icecrystal growth. Fortunately, the hollow-emitter geometry is ideal for achieving very high cooling rates which are known to minimize freezing artifacts. 
Due to the above mentioned difficulties of imaging wet specimens, as well as the advantages of frozen specimens, we have decided to concentrate on using frozen specimens in our 1995 research plans. This work is currently progressing well. Wet specimen experiments may be reinstated in the future

Concurrrent with the research at SSRL, a small laserplasma x-ray source is being constructed. This will allow the instrument to be operated in a small laboratory. This is crucial if this microscope is to become a commercial product. A commercial version of the instrument is envisioned as being a relatively inexpensive microscope that a researcher in the biological sciences can routinely use, generally in conjunction with optical and electron microscopy studies.

\section{Acknowledgments}

The author gratefully acknowledges very helpful discussions and advice from Piero Pianetta and Stephen Rothman. The help of the entire SSRL staff is greatly appreciated. The assistance of Peter Boyd, Brian Burdick and Curtis Troxel was especially valuable. Extensive radiation safety calculations necessary for getting approval of the new microscope system were performed by Michael Rowen. Approval of the experiment was facilitated by Nisy Ipe and Ian Evans. Silicon nitride windows were generously provided by Dino Ciarlo of LLNL. This work was partially supported by NIH SBIR grants 1 R43 RR07941-01 and 9 R44 GM51669-02. Work was done at SSRL, which is operated by the US Department of Energy, Office of Basic Energy Sciences.

\section{References}

1. F. Polack, S. Lowenthal, D. Phalippou, and P. Fournet, in X-ray Microscopy II, edited by D. Sayre, M. Howells, J. Kirz, H. Rarback, (Springer, Berlin, 1988), p. 220

2. G. Hirsch, U.S. Patent 4,829,177 (1989)

3. G. Hirsch, Rev. Sci. Instru., 66(2), 1367 (1995) 
E Versus k Relations And Many Body Effects In The Model Insulating Copper Oxide $\mathrm{Sr}_{2} \mathrm{CuO}_{2} \mathrm{Cl}_{2}$

\author{
B.O. Wells, ${ }^{1}$ Z.-X. Shen, ${ }^{2}$ A. Matsuura, ${ }^{2}$ D.M. King, ${ }^{2}$ M.A. Kastner, ${ }^{1}$ M. Greven, ${ }^{1}$ and R.J. Birgeneau, ${ }^{1}$ \\ ${ }^{1}$ Physics Department, Massachusetts Institute of Technology, Cambridge, MA 02139 \\ ${ }^{2}$ Applied Physics Department, Stanford University, Stanford, CA 94305
}

During the past several years there has been an extensive effort to understand the properties of the layered copper oxides. When undoped, with one hole per copper site, these materials are antiferromagnetic Mott insulators. When doped with sufficient carriers they are superconductors with very high transition temperatures. Traditional one electron based band calculations predict that even the undoped material should be a conductor. It is generally agreed that in the undoped copper oxides, the one-electron assumption breaks down due to the large Coulomb repulsion U between localized electrons. Most of the PES experiments to date have been on the metals and there has been little work on the undoped compounds. ${ }^{1}$ The lack of electronic structure information about the undoped parent compounds has left open several critical questions. One is how the electronic structure evolves with doping, and related to this is whether the doped materials are in a regime where one-electron physics is recaptured. In addition, most of the many body descriptions for the copper oxides rely on developing a model of the undoped material into which carriers are introduced. ${ }^{2}$ However, the accuracy of the underlying model for the undoped state is unknown and should be evaluated before proceeding to the more difficult task of describing the doped material.

We report angle resolved photoemission results on $\mathrm{Sr}_{2} \mathrm{CuO}_{2} \mathrm{Cl}_{2}$, a carrier free layered copper oxide. This is a measurement of the dispersion of a single hole in an antiferromagnetic background, a problem that has been heavily investigated theoretically. We find that the highest occupied band has a similar dispersion and position with respect to the rest of the valence spectrum as the band that crosses $\mathrm{E}_{\mathrm{F}}$ in the metallic copper oxides. This is evidence that hole doping causes the Fermi level to drop into the insulator valence band, although doping apparently leads to significant changes in the shape of the band. The measured band width is close to the value $W=2.25$ predicted by calculations based on the $t-$ $\mathrm{J}$ model. However, the shape of this band is not well described by this model or any other of which we are aware. We find a sudden drop in the spectral weight of this band as a function of wavevector that reflects the antiferromagnetic periodicity. Combining the results for the band width, band shape, and spectral weight variation we arrive at a complete description of the lowest energy quasiparticle spectrum which must be described by any model appropriate for the insulating copper oxides. ${ }^{3}$
Figure la shows a set of ARPES scans taken at different $k$ positions along the direction from the zone center $(0,0)$ to $(\pi, \pi)$. As $\mathbf{k}$ is increased, a peak appears and shifts towards higher energies as $\mathbf{k}$ approaches $(\pi / 2, \pi / 2)$. At larger $\mathbf{k}$ values there is a sudden drop in the intensity of the peak and it appears to move back to lower energies. The $(\pi / 2, \pi / 2)$ point is the global valence band maximum (VBM). The closest that the peak approaches to the Fermi energy is approximately $-0.8 \mathrm{eV}$. We measure a total dispersion of $280 \pm 60 \mathrm{meV}$. For parallel cuts, the peak intensity always drops at $\mathbf{k}$ locations forming a line connecting $(\pi, 0)$ and $(0, \pi)$.
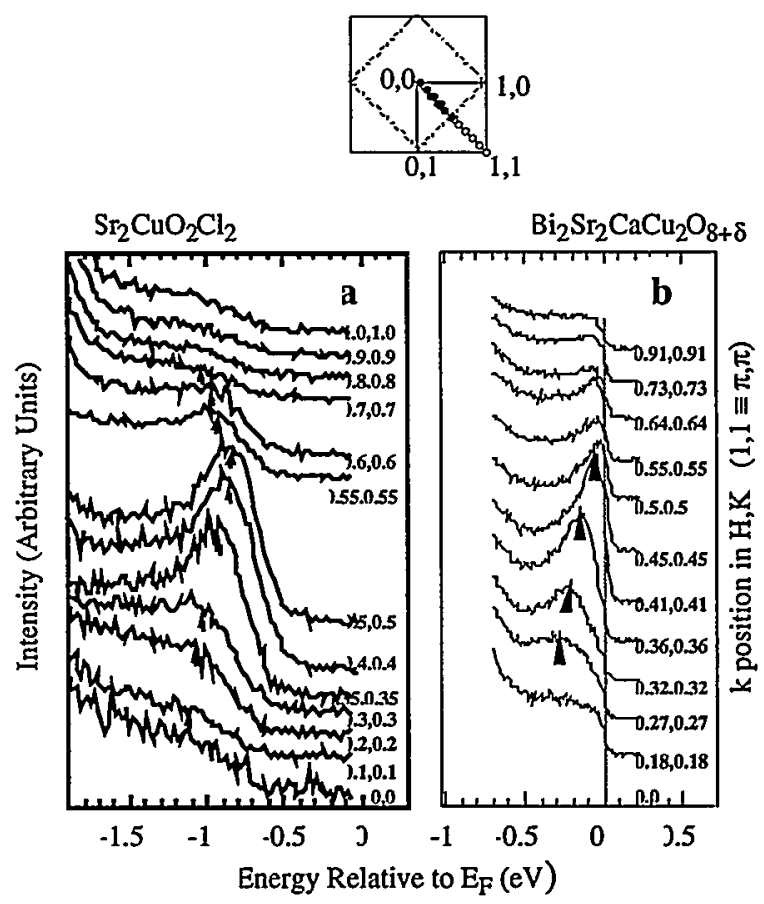

Figure 1. PES data showing the peak dispersion along the $(0,0)$ to $(\pi, \pi)$ direction for insulating $\mathrm{Sr}_{2} \mathrm{CuO}_{2} \mathrm{Cl}_{2}$ (a) and for metallic $\mathrm{Bi}_{2} \mathrm{Sr}_{2} \mathrm{CaCu}_{2} \mathrm{O}_{8+\delta}$ (b). The legend at the top has circles indicating the points in the two dimensional Brillouin zone where the data were taken and is labeled with $(1,1)=(\pi, \pi)$. The dark circles in the legend are on the side of the zone where the peak is strong in the insulator and the open circles where the peak is weak. The size of the circles indicates the $\mathrm{k}$ resolution.

Figure $1 \mathrm{~b}$ shows similar data along the same direction in the square lattice Brillouin zone for the 
metallic copper oxide $\mathrm{Bi}_{2} \mathrm{Sr}_{2} \mathrm{CaCu}_{2} \mathrm{O}_{8+\delta}$ from Dessau et al.. ${ }^{4}$ The peaks in the metal are sharper and disappear after crossing the Fermi level near $(0.45 \pi, 0.45 \pi)$. The spectra are quite similar and suggest that doping has moved the Fermi level into the valence band of the insulator. For comparison, the measured dispersion in $\mathrm{Bi}_{2} \mathrm{Sr}_{2} \mathrm{CaCu}_{2} \mathrm{O}_{8+\delta}$ is $270 \pm 30$ $\mathrm{meV}$ over a $\mathbf{k}$ range from $(0.27 \pi, 0.27 \pi)$ to $(0.45 \pi, 0.45 \pi)$. Over the same $\mathbf{k}$ region we see dispersion of $240 \pm 30 \mathrm{meV}$ in $\mathrm{Sr}_{2} \mathrm{CuO}_{2} \mathrm{Cl}_{2}$, which is the same within the errors.

The peak intensity drops along a line connecting $(\pi, 0)$ to $(0, \pi)$, the boundary of the antiferromagnetic Brillouin zone. The weak part of the band results from a folding of the zone due to antiferromagnetic order. This drop in spectral weight is analogous to that expected for a weak coupling spin density wave model and is also evident in Monte Carlo calculations of the spectral weight function for the Hubbard model at intermediate coupling strengths. ${ }^{5}$ For strong coupling we expect that the original and folded sections of the band would have nearly equal weight. Thus the variation in spectral weight could indicate that $\mathrm{Sr}_{2} \mathrm{CuO}_{2} \mathrm{Cl}_{2}$ is in an intermediate coupling regime.

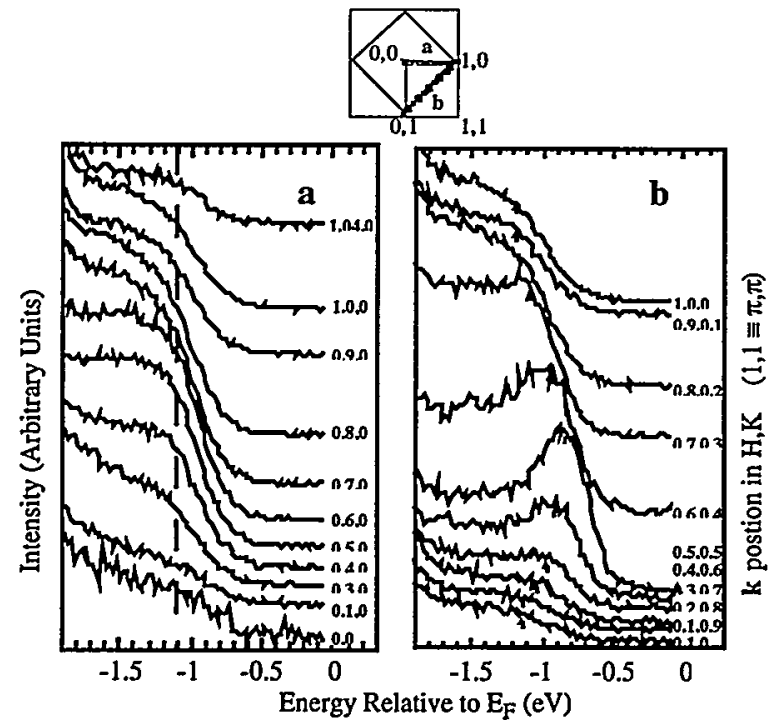

Figure 2. PES data along the $(0,0)$ to $(\pi, 0)$ direction in (a) and along $(0, \pi)-(\pi / 2, \pi / 2)-(\pi, 0)$ in (b). The dashed line in (a) is the maximum energy for any peak in this direction. The legend is the same as in figure 1 .

Figure $2 \mathrm{a}$ shows the data taken from $(0,0)$ to $(\pi, 0)$. There is an overall intensity modulation along this direction but no evidence for band dispersion. Figure $2 b$ shows the peak dispersion along a line from $(0, \pi)$ through $(\pi / 2, \pi / 2)$ to $(\pi, 0)$. From a maximum at $(\pi / 2, \pi / 2)$ the peak disperses to lower energy in either direction to a lowest energy near both $(\pi, 0)$ and $(0, \pi)$. Thus the lack of a dispersing peak in figure $2 \mathrm{a}$ indicates a band that remains at least $1.1 \mathrm{eV}$ below $\mathrm{E}_{\mathrm{F}}$, as indicated by the dashed line. This is in contrast to the hole doped copper oxides which have a flat band close to $\mathrm{EF}_{\mathrm{F}}$ in the vicinity of $(\pi, 0)$, as is discussed further below.

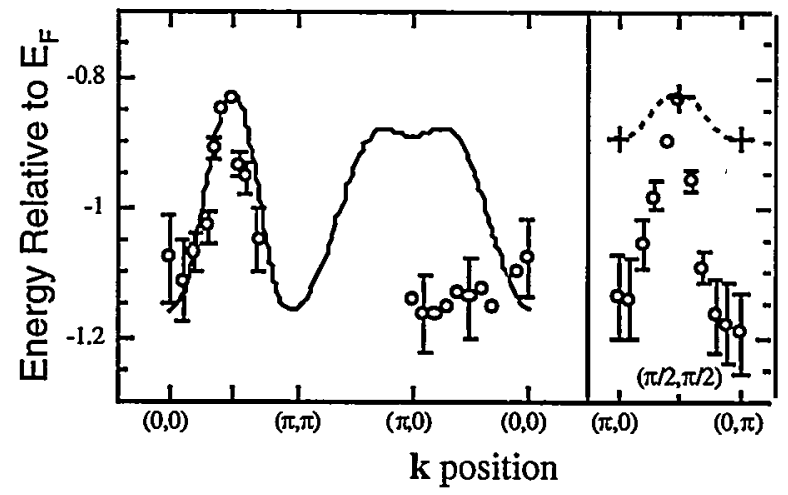

Figure 3. A comparison of the experimentally determined $E$ vs. $k$ relation (circles) with a calculation for the t-J model. All error bars are shown when larger than the symbol size except for the region from $(0,0)$ to $(\pi, 0)$ where for clarity only some representative error bars are shown. The far right panel shows the direction along the antiferromagnetic zone edge from $(0, \pi)$ to $(\pi, 0)$. Here only the points marked by a + are calculated in reference 17 and the dotted line is our interpolation between these points.

In figure 3 the experimentally determined $\mathrm{E}$ vs. $k$ relation is compared with that found for a numerical solution of one hole in a t-J model. ${ }^{6}$ For the region from $(0,0)$ to $(\pi, \pi)$ the $t-J$ model provides a good description of the experimental results. However, near $(\pi, 0)$ the calculation does not describe the data and we see much greater dispersion from $(\pi, 0)$ to $(0, \pi)$ than the calculation predicts. The band width $(\mathrm{W})$ determined by fitting the peaks over the entire region from $(0,0)$ to $(\pi / 2, \pi / 2)$ is $280 \pm 60$ $\mathrm{meV}$. The $\mathrm{t}-\mathrm{J}$ model calculation that gives the curve in figure 3 predicts the quasiparticle bandwidth to be $2.2 \mathrm{~J}$ for a wide range of $t / J$. There are other $t-J$ model calculations that give a comparable band width. Calculations on small clusters of the Hubbard model with intermediate $U$ show a similarly narrow quasiparticle band width. ${ }^{7}$ We find $W / J=2.2 \pm 0.5$, with $\mathrm{J}=125 \pm 6 \mathrm{meV}$ for $\mathrm{Sr}_{2} \mathrm{CuO}_{2} \mathrm{Cl}_{2}{ }^{8}$. This is in contrast to the prediction of one-electron calculations which find a total occupied band width of about $1 \mathrm{eV}$ at half filling, and a total band width of about 3.25 $\mathrm{eV}$. As stated above, the doped samples show similar dispersion to $\mathrm{Sr}_{2} \mathrm{CuO}_{2} \mathrm{Cl}_{2}$, although we cannot be certain of the location of the band maximum in the metals. Still, even for the metals, the LDA calculations predict a band width much too large. 
Our results lead us to speculate about the evolution of the electronic structure with doping. A rigid band doping model would account for the evolution of the data seen from $(0,0)$ to $(\pi, \pi)$, but is not sufficient to account for the changes near $(\pi, 0)$. The overall shape of the band in $\mathrm{Sr}_{2} \mathrm{CuO}_{2} \mathrm{Cl}_{2}$ is that of a peak centered at $(\pi / 2, \pi / 2)$. The effective mass we find at the top of the band is isotropic and equal to $1.5 \pm 0.75$, consistent with transport and optical measurements. ${ }^{10}$ Current models for the insulating state predict that this band should form a ridge extended in the direction from $(\pi, 0)$ to $(0, \pi)$. The photoemission data from the metals are consistent with such a ridge, since the metallic compounds show a band near $E_{F}$ over a wide region of $k$-space near $(\pi, 0)$. This has been described as an extended saddle point and there has been speculation that it is important to the physics of the superconductors. ${ }^{11}$ There are no states near the top of the band in $\mathrm{Sr}_{2} \mathrm{CuO}_{2} \mathrm{Cl}_{2}$ at $(\pi, 0)$. The states forming the extended saddle point must be either created or move up in energy as a result of doping. Such states strongly affect the shape of the Fermi surface since they serve to pin $\mathrm{E}_{\mathrm{F}}$ near the top of this band at the high doping levels of the superconductors. Nevertheless, there is a strong correspondence between the highest occupied band in $\mathrm{Sr}_{2} \mathrm{CuO}_{2} \mathrm{Cl}_{2}$ and the band crossing $\mathrm{E}_{\mathrm{F}}$ in the metallic copper oxides. It appears that while no new band is created by doping, the highest energy band is greatly modified.

There are other experiments which our data may help explain. Liu et al. reported PES data on insulating, but not carrier free, $\mathrm{YBa}_{2} \mathrm{Cu}_{3} \mathrm{O}_{6.35}{ }^{12}$ They did not see any well defined peaks but a strong shoulder that appeared to disperse along the $(0,0)$ to $(\pi, \pi)$ direction, reach a maximum at $\mathrm{EF}$ near $(\pi / 2, \pi / 2)$, and then disappear. This was interpreted as a Fermi level crossing. Since they saw an apparent band at $E_{F}$ only near $(\pi / 2, \pi / 2)$, they interpreted this as evidence for a small pocket Fermi surface even though they did not see the peak reappear at the other side of the pocket. Although no well-defined peak was detected, the $\mathrm{YBa}_{2} \mathrm{Cu}_{3} \mathrm{O}_{6.35}$ data are consistent with our results. The variation in spectral weight makes it impossible to detect a peak for $\mathbf{k}$ greater than $(\pi / 2, \pi / 2)$, thus they would not see the peak reappear. However, without well-defined peaks, this spectral weight drop makes it difficult to distinguish between a small pocket Fermi surface and a model where the small density of holes serves to pin the Fermi level near the VBM. The major change needed to evolve from these data to the large Fermi surfaces apparently seen in the metallic copper oxides is an evolution of the flat band states near $(\pi, 0)$.

In summary, we have measured the $\mathbf{k}$ dependent single particle excitation spectra of the highest energy band in the model insulating copper oxide $\mathrm{Sr}_{2} \mathrm{CuO}_{2} \mathrm{Cl}_{2}$. This band has a strong correspondence to the band crossing $\mathrm{EF}_{\mathrm{F}}$ in the metallic copper oxides. It is well described by a t-J model calculation along $(0,0)$ to $(\pi, \pi)$ but not near $(\pi, 0)$. There is a strong variation in spectral weight as a function of $\mathbf{k}$ which reflects the antiferromagnetic order. Clearly further experimental and theoretical work is necessary to explain the results and explore further the speculations we report in this paper.

We would like to acknowledge helpful conversations with P. W. Anderson, V. Emery, E. Dagotto, R.B. Laughlin, P.A. Lee, and D.J. Scalapino. We thank O. Jepsen for producing an LDA calculation for $\mathrm{Sr}_{2} \mathrm{CuO}_{2} \mathrm{Cl}_{2}$, D.S. Dessau for help with the experimental set up, and P. Blakeslee for characterizing the crystals. SSRL is operated by the DOE Office of Basic Energy Science, Division of Chemical Sciences.The work at Stanford was supported by DOE materials science, NSF grant DMR9357057, and the Stanford Center for Materials Research. The work at M.I.T. was supported by NSF grants number DMR90-22933 and DMR93-15715.

\footnotetext{
${ }^{1}$ For a review of ARPES work the copper oxides see Z.-X. Shen et al., Science 267, 343 (1995) and articles in J. Phys. Chem. Sol. 54, 1073 (1993).

${ }^{2}$ For a review of many body approaches to the high $\mathrm{T}_{\mathrm{c}}$ problem focussed on the $\mathrm{t}-\mathrm{J}$ model see $\mathrm{E}$. Dagotto Rev. Mod. Phys. 66,763 (1994).

${ }^{3}$ For a more complete account of this work see B.O.

Wells et al., Phys. Rev. Lett. 74, 964 (1995).

${ }^{4}$ D.S. Dessau et al., Phys. Rev. Lett. 71, 2781 (1993).

${ }^{5}$ N. Bulut, D.J. Scalapino, and S.R. White, Phys.

Rev. Lett. 73, 748 (1994).

${ }^{6}$ Z. Liu and E. Manousakis, Phys. Rev. B, 45, 2425 (1992).

${ }^{7}$ E. Dagotto et al., Phys. Rev. B 46, 3183 (1992).

${ }^{8}$ M. Greven et al., Phys. Rev. Lett. 72, 1096

(1994). Y. Tokura et al., Phys. Rev. B 41, 11657

(1990).

${ }^{9} \mathrm{O}$. Jepsen supplied us with an unpublished LDA band calculation for $\mathrm{Sr}_{2} \mathrm{CuO}_{2} \mathrm{Cl}_{2}$. For a review of band calculations for the high $\mathrm{T}_{\mathrm{c}}$ materials see W.E. Pickett, Rev. Mod. Phys. 61, 433 (1989).

${ }^{10}$ N.W. Preyer et al. Phys. Rev. B 39, 11563 (1989); J.P. Falck et al., Phys. Rev. B 48, 4043 (1993).

${ }^{11}$ D.S. Dessau et al., Phys. Rev. Lett. 71, 2781

(1993); K. Gofron et al., Phys. Rev. Lett. 73, 3302

(1994), D.M. King et al., ibid 73, 3298 (1994).

${ }^{12}$ R. Liu et al., Phys. Rev. B 46, 11056 (1992).
} 


\title{
Faraday Rotation and Circular Dichroism at the Fe $\mathrm{L}_{2,3}$ Edges
}

\author{
Jeffrey B. Kortright, ${ }^{1}$ Marybeth Rice, ${ }^{1}$ and Roger Carr $^{2}$ \\ ${ }^{1}$ Center for X-ray Optics, Lawrence Berkeley Laboratory, Berkeley, CA 94720 \\ ${ }^{2}$ Stanford Synchrotron Radiation Laboratory, Stanford, CA 94309
}

Using a new, tunable linear polarizer and the newly commissioned elliptically polarizing undulator (EPU) on beamline 5, we measured and compared different manifestations of the magneto-optical response of $\mathrm{Fe}$ at its $\mathrm{L}_{2,3}$ edges. The specific Faraday rotation we observed is larger than that seen in other spectral regions, suggesting that polarization resolved spectroscopies based on optical rotation of linearly polarized soft $x$-rays may be fruitful in studying a variety of materials.

Up to now, magnetic circular dichroism (MCD) has been the primary technique used to study element specific magnetic properties, and has been applied by several groups at the $2 p$ edges of the $3 d$ ferromagnetic elements where the effects are extremely large because of the strong $2 p$ to $3 d$ dipole transitions coupling directly to the $3 \mathrm{~d}$ electrons responsible for magnetism [1-3]. MCD is the differential absorption of circularly polarized light propagating parallel and anti-parallel to the magnetization direction of the sample, and is easily measured in the $x$-ray range using the total electron yield mode. In the visible, magneto-optic effects have been more commonly studied using optical rotation techniques (Faraday, Kerr rotation) which require linear, not circular, polarization and a linear polarizer to measure the rotation. Development of a tunable linear polarizer based on multilayers [4], together with the tunable polarization EPU source, allowed us to make the first measurement of magneto-optical rotation in the soft $x$-ray range, and also to measure $M C D$ from the same sample for comparison.

The new linear polarizer utilizes the polarization dependence of specular reflectance from a multilayer interference structure set to reflect at $45^{\circ}$, the Brewster angle in the x-ray region. Operation at the $\mathrm{Fe} \mathrm{L}_{2}$ and $\mathrm{L}_{3}$ edges $(720$ and $707 \mathrm{eV}$, respectively) required a multilayer with very small period $d=\lambda / 2 \sin \left(45^{\circ}\right) \cong 12.3 \AA$. We used magnetron sputtered multilayers of $W / B_{4} \mathrm{C}$ which have an scomponent reflectance $R_{S}=0.01$ and an extinction ratio $R_{s} / R_{p} \cong 3 \times 10^{4}$ at these energies. The ultrahigh vacuum polarimeter rotates the multilayer about the beam with fixed incidence angle while monitoring
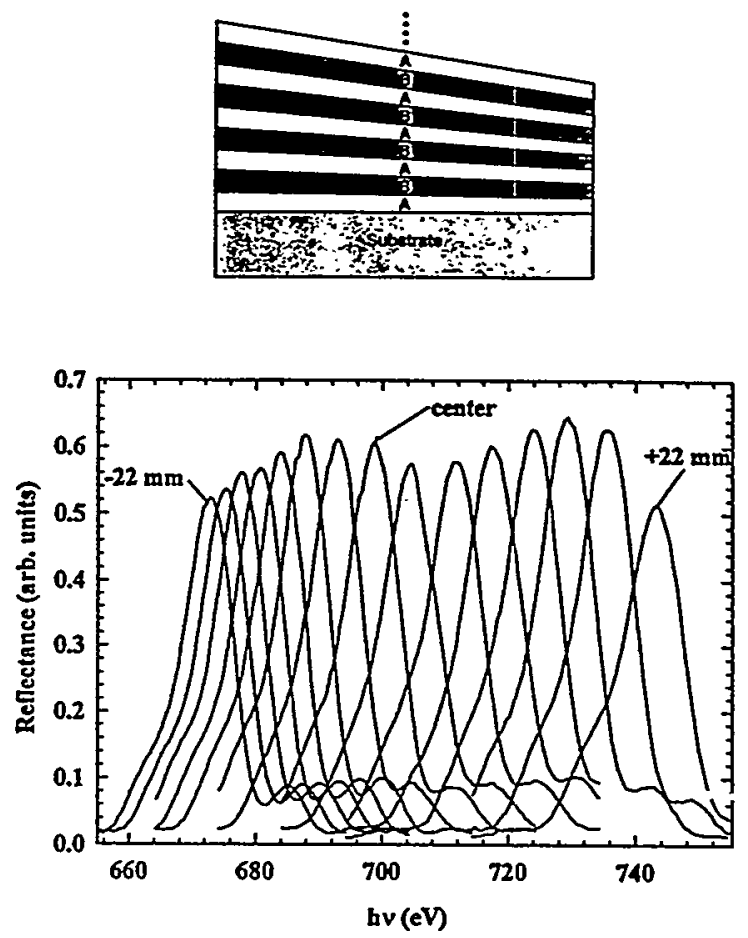

Figure 1. A laterally graded multilayer was used to produce a tunable linear polarizer by translating the multilayer to satisfy the Bragg condition at the desired energy and the $45^{\circ}$ polarizing angle. Measured Bragg spectra taken every $3.1 \mathrm{~mm}$ along the tapered multilayer demonstrate the tunability range available for these measurements.

the reflected intensity with a Si diode. Continuous tunability of the polarizer is achieved by translating the multilayer with a laterally graded period perpendicular to the beam to position the Bragg peak at the desired $\lambda$. Figure 1 shows that a tunability range of $10 \%$ was available for these measurements.

The transmission sample was an $\mathrm{Fe} / \mathrm{Cr}$ multilayer deposited onto a $100 \mathrm{~nm}$ thick silicon nitride membrane. The multilayer nature of this sample is of interest for its magnetic properties, but is not critical for these measurements; any $\mathrm{Fe}$ containing film having a magnetization component along the beam direction will show the effects 


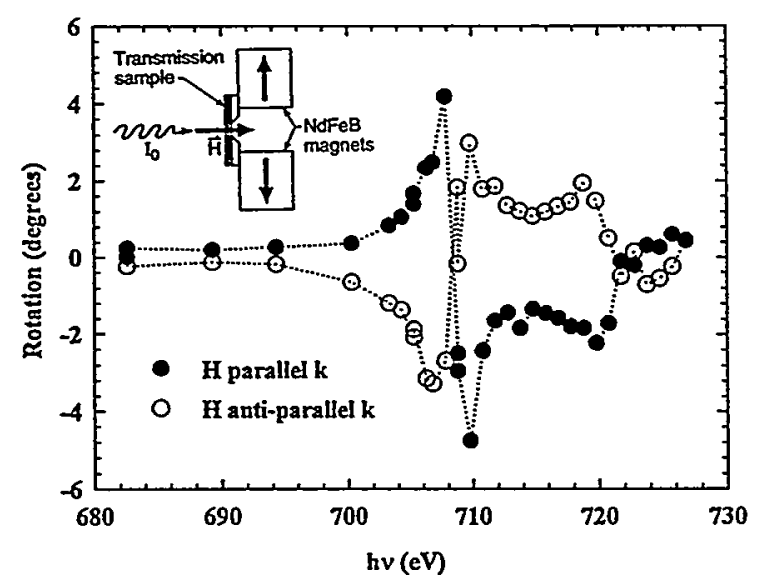

Figure 2. Faraday rotation measured with a $3 \mathrm{kOe}$ applied field parallel and anti-parallel to the beam direction, which is normal to the surface of an $\mathrm{Fe} / \mathrm{Cr}$ multilayer sample supported on a thin membrane. Lines connect data points.

reported here. The multilayer consisted of $2.0 \mathrm{~nm} \mathrm{Fe}$ and $1.9 \mathrm{~nm} \mathrm{Cr}$ magnetron sputter deposited layers repeated for 40 periods with $\mathrm{Cr}$ on top and bottom, mounted with its surface normal parallel to the $\mathrm{x}$-ray beam. A $3 \mathrm{kOe}$ field produced by $\mathrm{NdFeB}$ blocks was applied along the beam direction and sample normal as illustrated in Figure 2. This field strength may not be enough to force the sample's magnetization entirely out of plane, but it does result in a sizable out of plane component as will be demonstrated. The sample and magnet configuration rotated together through $180^{\circ}$ so that the applied field could be aligned parallel and anti-parallel to the beam propagation direction. Comparison measurements of optical rotation and MCD used the same sample and magnetic field configurations, and only changed the polarization of the incident photon beam.

We observe large resonances in the rotation of the plane of horizontal linear polarization on tuning photon energy through the $\mathrm{Fe} \mathrm{L}_{3}$ and $\mathrm{L}_{2}$ edges, as seen in Figure 2. Rotation values were obtained by scanning the polarizer over $\mathrm{a} \pm 40^{\circ}$ range centered about the nominal minimum (with transmission sample removed), and fitting the data to find the deviation of the minimum at each energy. The sense of rotation changes sign with the direction of the applied field, showing large Faraday rotation associated with the $\mathrm{Fe}$ in the sample. Normalizing the maximum $4.8^{\circ}$ rotation by the total $\mathrm{Fe}$ thickness $t_{\mathrm{Fe}}=80 \mathrm{~nm}$ (assuming $\mathrm{Fe}$ at bulk density in distinct layers) yields a Faraday rotation constant of $6.0 \times 10^{4}$ $\mathrm{deg} / \mathrm{mm}$. This is larger than that observed in Fe films in the visible $\left(3.5 \times 10^{4} \mathrm{deg} / \mathrm{mm}\right.$ at $\lambda=546 \mathrm{~nm}$ [5]) and infra-red $\left(5.1 \times 10^{4} \mathrm{deg} / \mathrm{mm}\right.$ at $\left.\lambda=1000 \mathrm{~nm}[6]\right)$
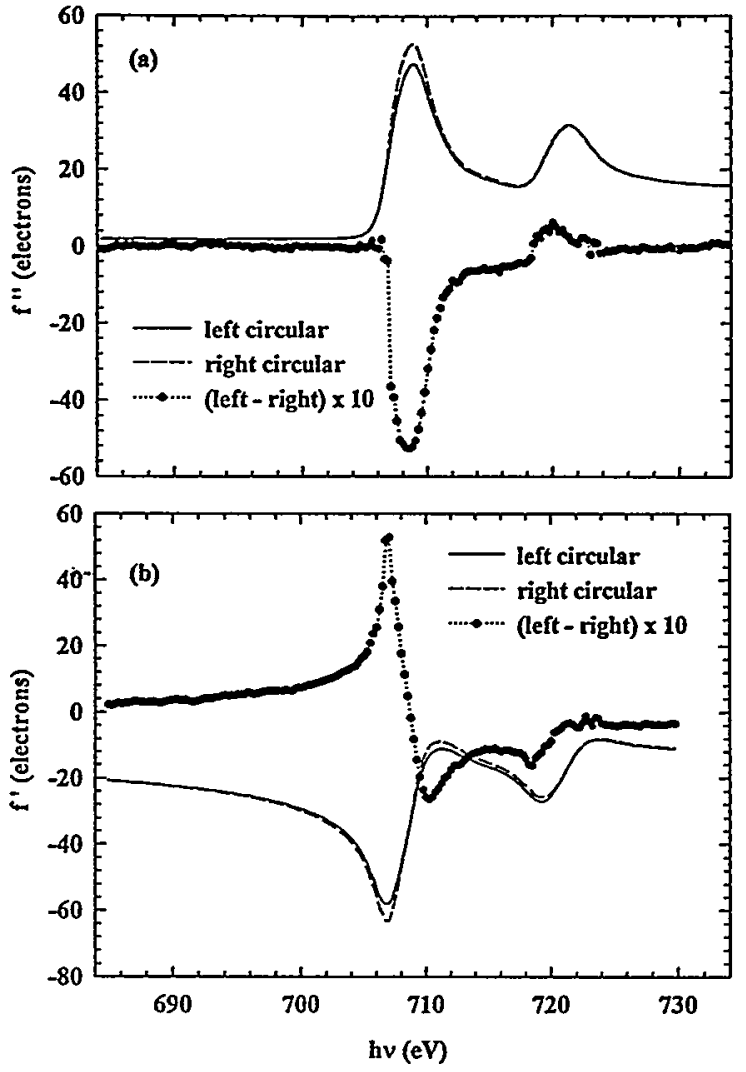

Figure 3. Absorption data (total electron yield) taken with left and right circular polarization and normalized to $f$ " are in (a), as is $f$ " $1-f$ " $r$ that reveals magnetic circular dichroism. The Kramers-Kronig transformation result of the absorption data are shown as $f^{\prime}$ in (b), as is $f^{\prime}{ }_{1}-f^{\prime}{ }^{\prime}$.

spectral regions, and 3 orders of magnitude greater than that observed at the Fe K edge at $7112 \mathrm{eV} \mathrm{[7].}$ Since the magnetization of $\mathrm{Fe}$ may not be saturated along the beam direction, the observed rotation constant is really a lower limit to the specific rotation of $\mathrm{Fe}$ at its $\mathrm{L}_{3}$ edge. This large soft $\mathrm{x}$-ray Faraday rotation, in analogy to magneto-optical effects in the visible region, implies that Kerr rotations (on reflection) should be measurable.

Analysis of MCD absorption spectra using left and right circularly polarized incident beams predicts a Faraday rotation in good agreement with that measured. Absorption data were collected in the total electron yield mode measuring sample current and are shown in Figure 3a, along with the difference that clearly shows the circular dichroism. Absorption data are normalized to $f$ ", the imaginary part of the atomic scattering factor. The real and imaginary parts of the atomic scattering factor, $Z+f$ ' and $f$ ", 

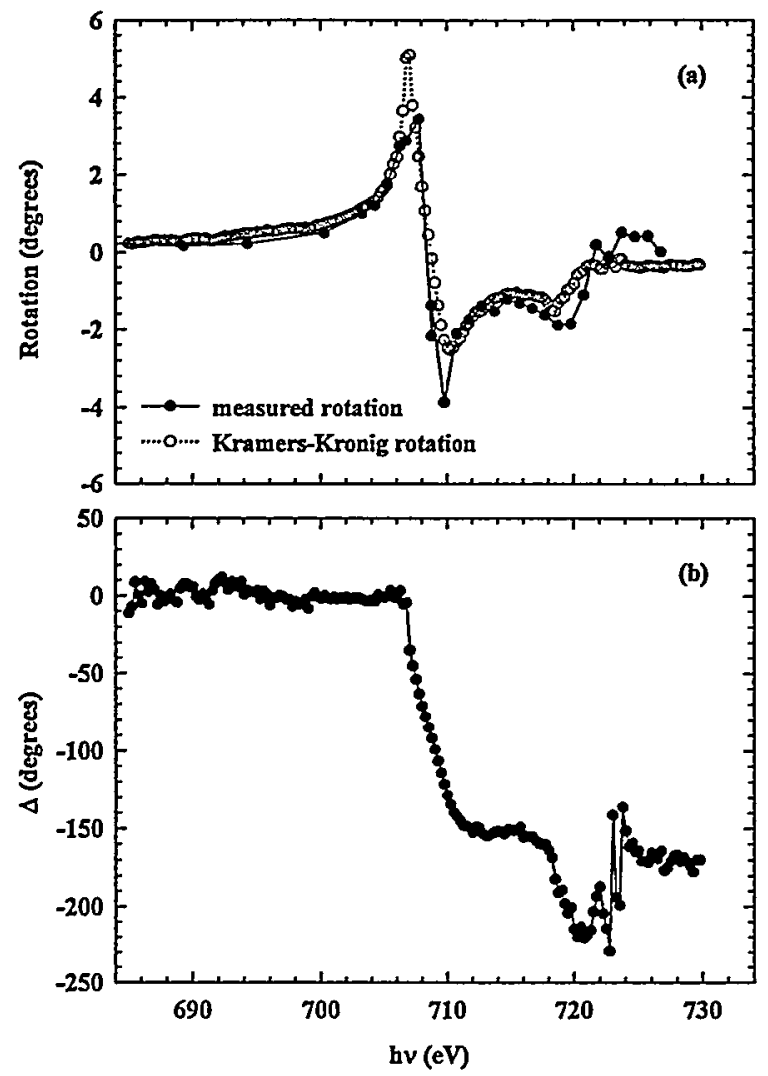

Figure 4. Comparison of the measured rotation of linear polarization (mirror average of two curves in Fig. 2), and that predicted by the absorption data using left and right circular polarization reveals consistency between the two techniques in (a). In (b) is plotted the equivalent phase difference, or retardation, between the horizontal and vertical components describing the transmitted elliptically polarized beam.

are related to the real and imaginary deviations of the refractive index, $\delta$ and $\beta$, from unity by $\delta=\Sigma_{i}\left(Z_{i}+\right.$ $\left.\mathrm{f}_{\mathrm{i}}^{\prime}\right) \mathrm{N}_{\mathrm{i}} \mathrm{r}_{\mathrm{e}} \lambda^{2} / 2 \pi$ and $\beta=\Sigma_{\mathrm{i}} \mathrm{f}_{\mathrm{i}}{ }^{\prime \prime} \mathrm{N}_{\mathrm{i}} \mathrm{r}_{\mathrm{e}} \lambda^{2} / 2 \pi$, where the sum is over atomic species, $N$ is the atomic density and $r_{e}$ the electron radius. Direct measurement of Faraday rotation used linear polarization, which is equivalent to a coherent superposition of equal amounts of left (l) and right (r) circular polarization. KramersKronig transformation of $f$ "1,r yields $f$ ' $_{1, r}$ (Figure 3b), from which $\delta_{\mathrm{I}, \mathrm{r}}$ is obtained. The phase difference between the two components on transmission is $\left(\delta_{\mathfrak{l}}-\delta_{\mathrm{r}}\right) 2 \pi \mathrm{t} \mathrm{Fe}^{/ \lambda}$ radians, and the Faraday rotation angle $\alpha=\left(\delta_{1}-\delta_{r}\right) \pi t_{F e} / \lambda$. We assume that all magneto-optical effects near the $\mathrm{Fe}$ edge are due solely to the $\mathrm{Fe}$ in the sample, and we consider only $\mathrm{Fe}$ in the analysis. Figure $4 \mathrm{a}$ compares this calculated rotation based on MCD data with that measured directly using linear polarization. The agreement is quite good, implying that both measurements are similarly representative of the magnetic properties of the sample along the beam propagation direction.

Both the charge and spin scattering undergo extremely large resonances at the $\mathrm{L}_{2,3}$ edges because of the efficient coupling to the partially filled $3 d$ states. The $f$ " and $f$ ' data in Figure 3 exhibit very large anomalous dispersion of charge scattering, independent of spin effects. $f^{\prime}{ }_{1, r}$ are reduced by more than twice the number of electrons in an $\mathrm{Fe}$ atom. Such large negative resonance results from the strong dipole-allowed matrix elements for transitions from the $2 p_{3 / 2}$ and $2 p_{1 / 2}$ core levels to a high density of empty, localized $3 \mathrm{~d}$ states as expressed by the white line absorption at the edges. The spin-dependent scattering resonates at the same energies, and its resonance $\left(f^{\prime}{ }_{1}-f^{\prime} r\right)$ is large because of the unequal spin density in the $3 \mathrm{~d}$ states. It is because the $L_{2,3}$ resonances couple directly and exclusively to the $3 \mathrm{~d}$ states responsible for magnetism in $\mathrm{Fe}$ that we observe rotation constants larger than in the visible.

Unequal attenuation of the circular components of the initially linearly polarized beam in the rotation measurement induces an ellipticity in the transmitted beam, with ellipticity angle $\omega$ given by $\omega$ $=\left(\beta_{1}-\beta_{\mathrm{r}}\right) \pi \mathrm{t}_{\mathrm{Fe}} / \lambda$ radians. From the data in Figure $3 \mathrm{a}$, the maximum $\omega=5.1^{\circ}$ at the peak of the $\mathrm{L}_{3}$ resonance; $\tan (\omega)$ gives the ratio of the minor to major axes of the ellipse described by the transmitted beam, and the ellipse is rotated by $\alpha$ from the horizontal plane of incident linear polarization. An equivalent description of the elliptically polarized transmitted beam is obtained by resolving it into orthogonal linear components that have a relative phase difference, or retardation, $\Delta$, where $\tan (\Delta)=$ $\tan (2 \omega) / \sin (2 \alpha)[8]$. We find large values of $\Delta$ in the resonance region as shown in Figure $4 b$. This resonant phase retardation is much larger than that expected to be produced by transmission multilayer interference retarders at this energy [4], and may provide useful phase retarding elements, albeit with a very limited energy range.

Optical rotation using linear polarization and MCD using circular polarization are selfconsistent for the sample studied, and we are led to consider the applicability of the different measurements as element-specific magnetic probes. From a classical optics viewpoint, Faraday rotation measures $\left(\delta_{1}-\delta_{r}\right)$ and MCD measures $\left(\beta_{1}-\beta_{r}\right)$ along the propagation direction; the two quantities are 
related by Kramers-Kronig transformation. Thus measurement of one yields the other, assuming $\delta_{1, r}(\lambda)$ and $\beta_{1, r}(\lambda)$ are sufficiently well known away from the energy region of interest so that transformation errors are negligible. Kerr effects only measuring reflected intensity do not separate $\delta$ and $\beta$, but measure a signal influenced by both quantities. MCD measurements in the soft $\mathrm{x}$-ray have required incident light with a large degree of circular polarization, while optical rotation requires only linearly polarized beams. MCD measured using electron yield techniques is limited by electron escape depths to near surface sensitivity. Optical rotation measured in transmission is sensitive to the bulk of thin films, and in reflection has penetration depths ranging from 3 to hundreds of $\mathrm{nm}$ with the incident angle below and above the critical angle, respectively. Optical detection is more compatible than electron detection with measurements in varying magnetic fields.

To summarize, we have measured resonant Faraday rotation on transmission through $\mathrm{Fe}$ containing films in the soft $x$-ray and found rotation constants larger than found in other spectral regions. We have used left and right circular polarization to measure the absorptive part of the magneto-optical response from the same sample, and found the two measurements in good agreement for the sample studied. Soft $\mathrm{x}$-ray optical rotation in both transmission and reflection, using linear incident polarization, thus provides a new and complementary experimental pathway for obtaining element-specific magnetic information from a wide variety of materials. The newly developed optics enabling these measurements are applicable over at least the 50 $900 \mathrm{eV}$ region, covering absorption resonances of numerous elements. The extension of various polarization-resolved spectroscopic techniques into the soft $\mathrm{x}$-ray region is thus possible.

Research was supported by the Director, Office of Basic Energy Sciences, Materials Sciences Division of the U.S. Departement of Energy under contract DE-AC03-76SF00098. M.R is a University of California President's Postdoctoral Fellow.

\section{References}

1. C.T. Chen, F. Sette, and S. Modesti, Phys. Rev. B 42, 7262 (1990).

2. J.G. Tobin, G.D. Waddill, and D.P. Pappas, Phys. Rev. Lett. 68, 3642 (1992).

3. Y. Wu, J. Stohr, B.D. Hermsmeier, M.G. Samant, and D. Weller, Phys. Rev. Lett. 69, 2307 (1992).
4. J.B. Kortright, M. Rice, K. Franck, Rev. Sci. Instrum., accepted for publication.

5. M.J. Freiser, IEEE Transactions on Magnetics Mag-4, 152 (1967).

6. D.O. Smith, J. Appl. Phys. 36, 1120 (1965).

7. D.P. Siddons, M. Hart, Y. Amemiya, and J.B. Hastings, Phys. Rev. Lett. 64, 1967 (1990).

8. D.S. Kliger, J.W. Lewis, and C.E. Randall, "Polarized Light in Optics and Spectroscopy," (Academic Press, Boston) 1990, chapter 5. 


\title{
CHARACTERIZATION OF ALKYL MONOLAYERS ON NH 4 F ETCHED Si(111)
}

\author{
J. Terry, C. Wigren, R. Cao, and P. Pianetta \\ Stanford Synchrotron Kiediation Laboratory, Stanford University, Stanford, CA 94309 \\ M. Linford and C. E. D. Chidsey \\ Dep، rtment of Chemistry, Stanford, CA 94305
}

\begin{abstract}
We present our characterization of a novel passivation layer, alkyl chains on the silicon(111) surface. The alkyl-terminated silicon was investigated using high-resolution photoelectron spectroscopy (PES), scanned-energy photoelectron diffraction (ARPEFS), and near-edge $x$-ray absorption fine structure spectroscopy (NEXAFS). Our Si $2 p$ photoemission data shows significant narrowing of the core level when compared to the core level spectrum of the Si(111)7x7 surface. This narrowing is also observed on the hydrogen terminated surface and is indicative of the loss of surface dangling bonds. This observation suggests that the alkyl chains terminate the Si(111) surface. Our $\mathrm{C}$ 1s photoemission results show two components, one component was attributed to $\mathrm{C}$ atoms bonded to carbon and hydrogen atoms only. We attributed the other component to the carbon atom bonded directly to the silicon surface. We performed normal emission ARPEFS measurements on the $C 1 \mathrm{~s}$ core level over the energy range, $\mathrm{hv}=340 \mathrm{eV}$ to $\mathrm{hv}=600 \mathrm{eV}$ on Beam Line 10-1 at the Stanford Synchrotron Radiation Laboratory. Using a simple Fourier analysis on the data obtained from üic ascond $\mathrm{C} 1 \mathrm{~s}$ component, we observed a total path length difference of 3.56 angstroms. This is consistent with a C-Si bond length of 1.88 angstroms with a silicon backscatter directly underneath the carbon atom. We conclude that the alkyl monolayers are terminating the silicon(111) surface.
\end{abstract}

\section{INTRODUCTION}

Recently, a novel method for producing alkyl monolayers on $\mathrm{H}$-terminated $\mathrm{Si}(111)$ surfaces was developed[1,2,3]. In this method, monolayers are formed by irradiating hydrogen-terminated silicon in the presence of an $\alpha$-olefin, an organic molecule with a terminal carbon-carbon double bond. Because the threshold of the wavelength needed for monolayer formation appears to be near the energy needed to homolytically break the silicon-hydrogen bond, and is far from the absorption maxima of either the olefin or the $\mathrm{Si}-\mathrm{H}$ moiety, the reaction mechanism is likely to involve photon-stimulated desorption of hydrogen, followed by addition of the olefin to the dangling bond. However, direct evidence of a $\mathrm{C}-\mathrm{Si}$ bond had not been observed.

To obtain direct evidence of a $\mathrm{C}-\mathrm{Si}$ bond, we used photoelectron spectroscopy to probe the electronic structure of the monolayers. We compared our observed spectra to those obtained from the decomposition of diethylsilane on $\mathrm{Si}(111)$ and $\mathrm{Si}(100)$ surfaces[4,5].

* Work supported by Department of Energy 
These observations led us to perform scanned energy photoelectron diffraction measurements on the monolayers to determine scattering path length differences along the Si-C bond. We will show that a Si-C bond had formed, that it was normal to the $\operatorname{Si}(111)$ sur-

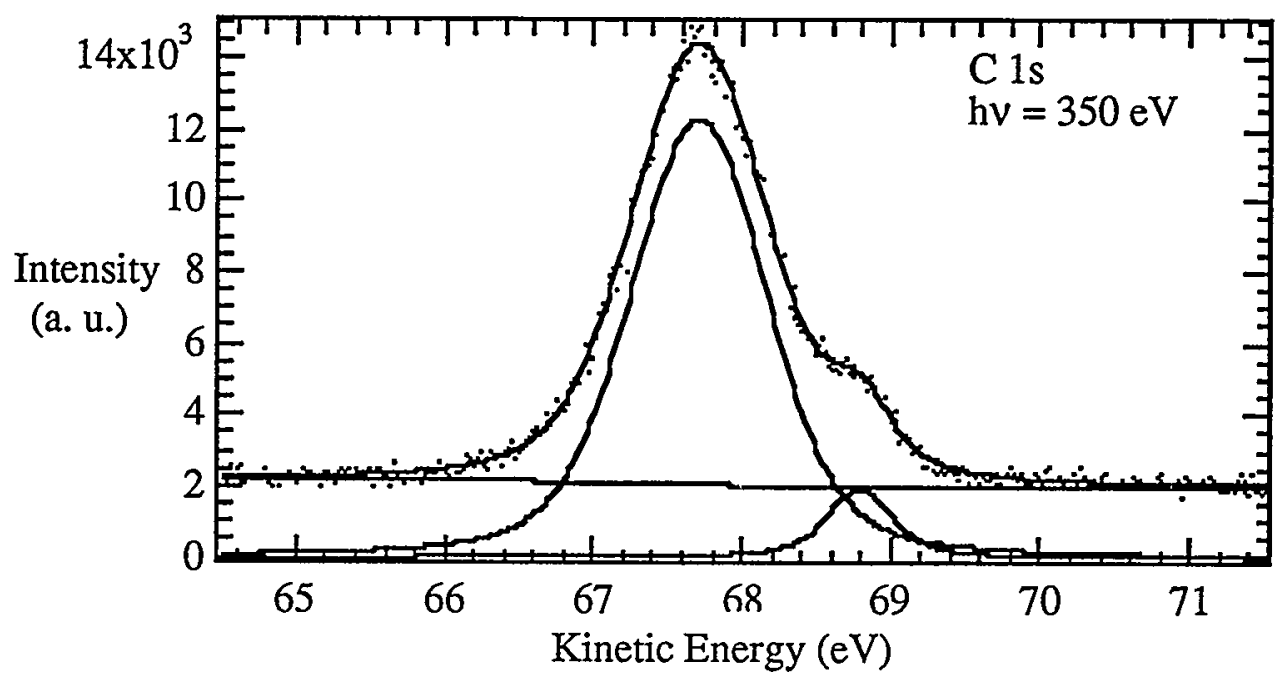

Figure 1: Shows the fitted $C$ 1s spectrum from pentyl-terminated Si(111). Two components are observed. The higher kinetic energy component is attributed to the $\mathrm{C}$ atom bonded to the silicon substrate.

face, and had a

bond length close to the bond length of 1.88 Angstroms found in SiC.

\section{EXPERIMENTAL}

All measurements were performed at Beam Line $10-1$ at the Stanford Synchrotron Radiation Laboratory. Beam Line 10-1 uses a spherical grating monochromator to select VUV photons with energies from $180 \mathrm{eV}$ to $1000 \mathrm{eV}$. We used a Ni refocusing mirror which limited the energy range to $650 \mathrm{eV}$. The scanned energy photoelectron diffraction measurements where taken at photon energies between $300 \mathrm{eV}$ and $650 \mathrm{eV}$.

The analysis chamber consists of a load-lock sample transfer system, a reverse view low energy electron diffraction analyzer, a $100 \mathrm{~mm}$ VSW hemispherical electron energy analyzer, and a sample manipulator with xyz motion and $360^{\circ}$ azimuthal rotation and $180^{\circ}$ polar rotation. The photoemission data was collected at normal emission with total resolution (monochromator and analyzer) of $150 \mathrm{meV}$.

The samples were prepared by exposing 1-pentene and $\mathrm{H} / \mathrm{Si}(111)$ to UV light. They were then brought under argon to the sample load-lock and introduced to the vacuum chamber. No oxidation was observed for samples transported in this manner.

\section{RESULTS AND DISCUSSION}

Figure 1 shows a $C$ 1s spectrum from a pentylterminated surface. Two components are clearly resolved. We assigned the high kinetic energy component to photoelectrons from the carbon atom bonded to the silicon substrate and the low kinetic energy component to the remaining carbon atoms in the chain. This assignment was made because the intensity of the two components varied in predictable ratios as the chain length was increased. This data is not shown here. Further evidence for this assignment is provided by comparing the observed spectra to data from the decomposition of diethylsilane which forms an ethyl-terminated surface[4,5] where a broad spectrum approximately $2 \mathrm{eV}$ wide is observed. The 


\section{INTENSITY of C-Si COMPONENT vs. ELECTRON KINETIC ENERGY}

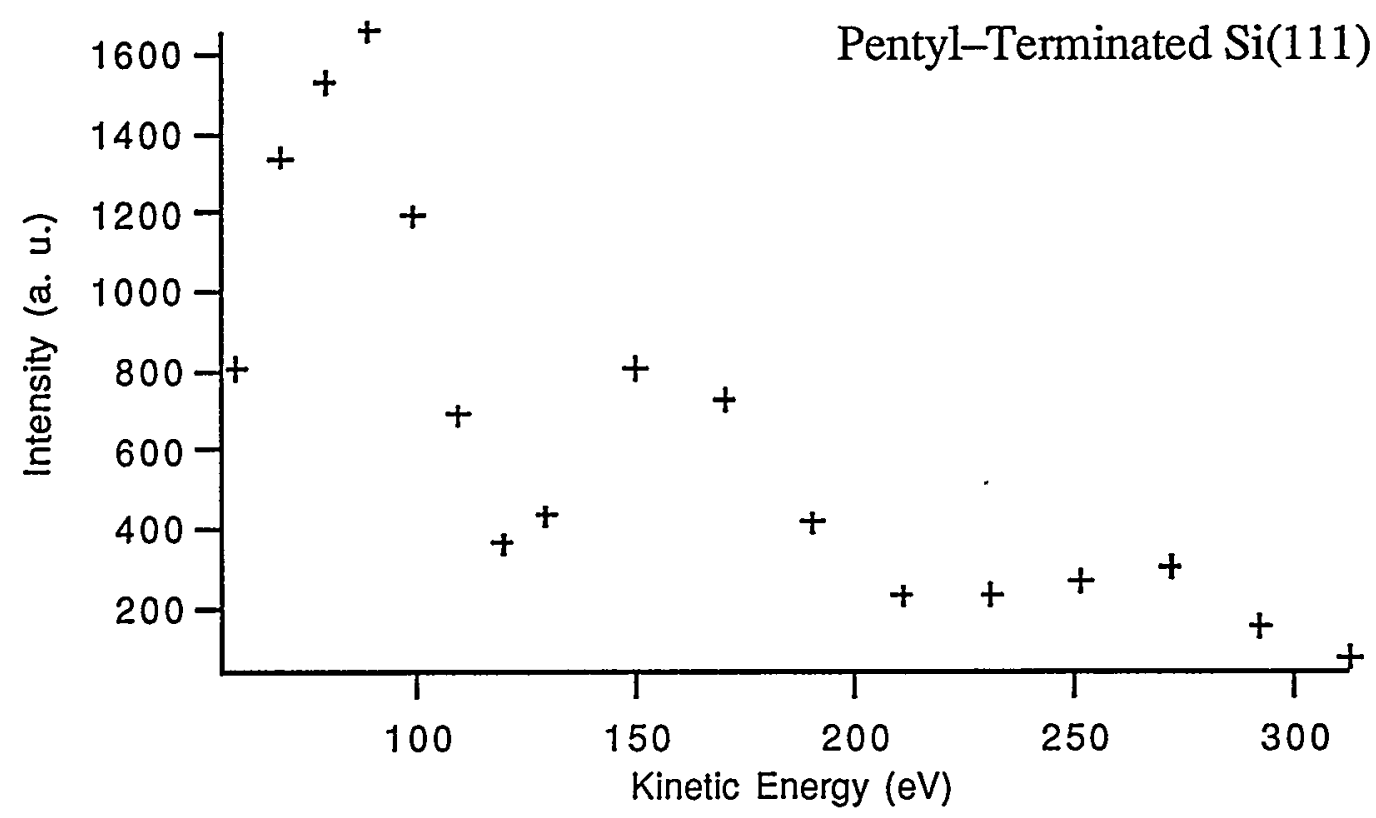

Figure 2: Shows the intensity modulation of the C-Si component of the $\mathrm{C}$ 1s core level spectrum from pentylterminated $\mathrm{Si}(111)$ as the kinetic energy of the photoemitted electron increased (i.e. the photon energy increased).

authors report that upon annealing the spectrum shifts to higher kinetic energy until the position is consistent with that of $\mathrm{Si}-\mathrm{C}$. We speculate that the peak that is $2 \mathrm{eV}$ wide consists of $\mathrm{C}-\mathrm{C}$ and $\mathrm{C}-\mathrm{Si}$ components that were unresolved.

We performed scanned energy photoelectron diffraction measurements on the $\mathrm{C} 1 \mathrm{~s}$ core level at normal emission. The $\mathrm{C}-\mathrm{C}$ component showed a simple decay with increasing energy (data not shown here). However, the $\mathrm{C}-\mathrm{Si}$ component showed strong intensity modulation as the photon energy was varied (Figure 2). The intensity modulation was converted into a function of electron momentum (k) and a zero order emission spectrum, Io, was fit to the data. The resultant functions are shown in Figure 3. The modulations are enhanced by calculating a

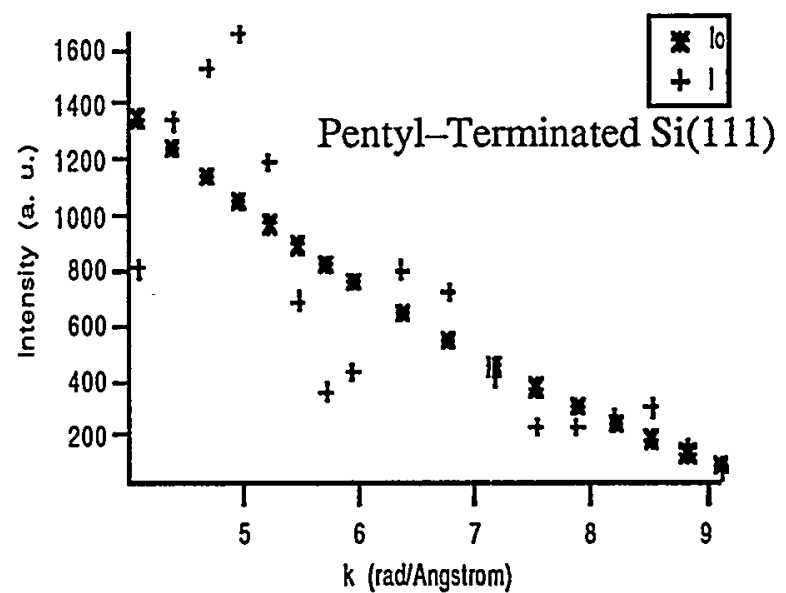

Figure 3: $\mathrm{I}$ is the intensity modulation of Figure 2 as a function of electron momentum (k). Io is a slowly varying function fit to $I$. It represents the intensity in the absence of diffraction.

function $\chi(\mathrm{k})$ where $\chi(\mathrm{k})=(\mathrm{I}-\mathrm{Io}) / \mathrm{Io} . \chi(\mathrm{k})$ is shown in Figure 4. Finally, the total scattering path length difference is obtained by fourier transforming the function $\chi(\mathrm{k})$, (Figure 5). 


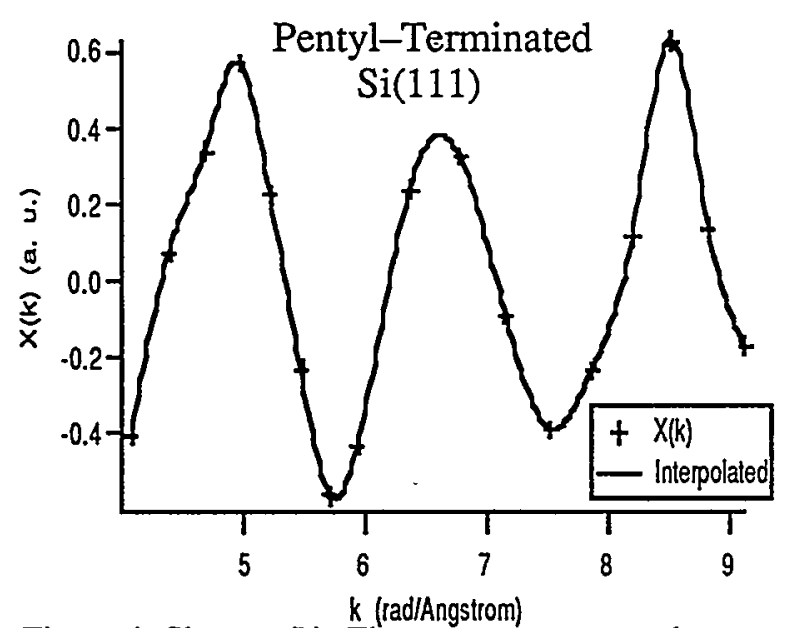

Figure 4: Shows $x(k)$. The crosses represent the actual data points and the solid curve is a cubic spline interpolation of the data points.

The Fourier transform has a peak at $3.47 \mathrm{~A}$. The total scattering path length difference, at normal emission for a Si backscatterer $180^{\circ}$ below the carbon emitter, is equal to $2 \mathrm{Ri}+\varphi \mathrm{i}$. The scattering phase shift, $\varphi \mathrm{i}$, must be determined theoretically[6]. By assuming that the phase shift is $0 \mathrm{~A}$, a first order approximation of the bond length can be obtained from the geometric path length difference. Using $2 \mathrm{Ri}=3.47 \mathrm{~A}$, a value of 1.74 $\mathrm{A}$ is obtained for the $\mathrm{C}-\mathrm{Si}$ bond length. This is in reasonable agreement with the expected value of $1.88 \mathrm{~A}$, from $\mathrm{SiC}$, within this crude approximation.

\section{CONCLUSION}

Using high-resolution photoelectron spectroscopy, we observe two components in the $C$ 1s core level spectra from the alkyl monolayers. Using intensity analysis, we have assigned the high kinetic energy component to emission from the $\mathrm{C}$ atom bonded to the $\mathrm{Si}$ substrate. We performed normal emission scanned energy photoelectron diffraction measurements on the $\mathrm{C} 1 \mathrm{~s}$ core level. We obtained a first order approximation to the bond length of $1.74 \mathrm{~A}$ by assuming the

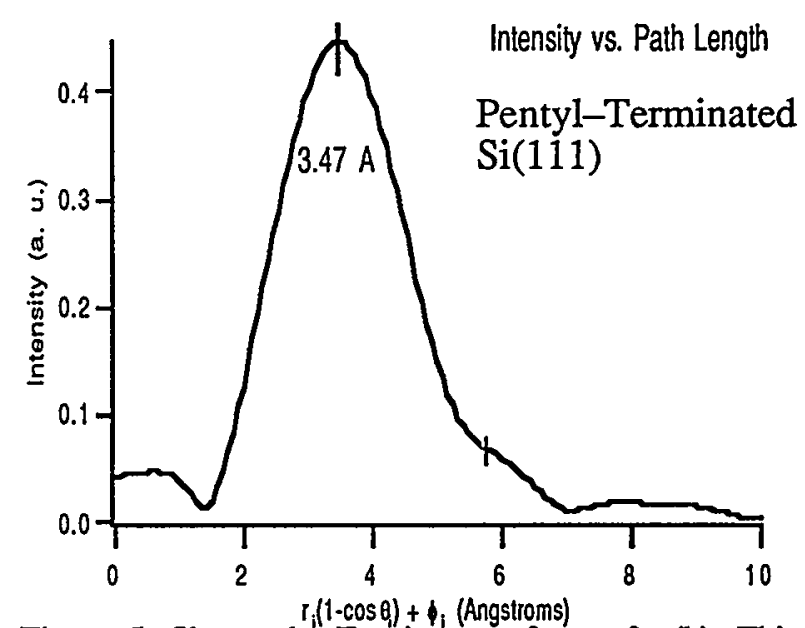

Figure 5: Shows the Fourier transform of $\chi(\mathrm{k})$. This function gives the total scattering path length difference which depends on the distance between the emitter and scatterer and on the phase shift of the scattering atom.

scattering phase shift to be $0 \mathrm{~A}$. Theoretical calculations are in progress to determine the bond length more accurately.

\section{ACKNOWLEDGEMENTS}

SSRL is funded by the Department of Energy, Office of Basic Energy Sciences. Further support is provided by the Department of Energy, Office or Health and Environmental Resèarch.

\section{REFERENCES}

[1]]M. R. Linford, P. Fenter, P. M. Eisenberger, and C. E. D. Chidsey, in prep.

[2]M. R. Linford, and C. E. D. Chidsey, J.Am. Chem. Soc. 115, 12631-12632, (1993).

[3]M. R. Linford, P. Fenter, P. M. Eisenberger, and C. E. D. Chidsey, J. Am. Chem. Soc. 117, 3145-3155, (1995).

[4]D. A. Lapiano-Smith, F. J. Himpsel, and L. J. Terminello, J. Appl. Phys. 74, 5842, (1934).

[5]L. J. Terminello, private communication, April, 1995.

[6]Calculations of the phase shift are presently in progress. 


\title{
Linear and Circular Dichroism in Angle Resolved Fe 3p Photoemission
}

\author{
E. Tamura, ${ }^{1}$ G.D. Waddill, ${ }^{1}$ J. G. Tobin, ${ }^{1}$ and P. A. Sterne ${ }^{1.2}$ \\ 'Department of Chemistry and Materials Science, Lawrence Livermore National Laboratory, Livermore, Califomia 94550 \\ ${ }^{2}$ Department of Physics, University of Califomia, Davis, Califormia 95616 \\ (Received 30 March 1994)
}

\begin{abstract}
Using a recently developed spin-polarized, fully relativistic, multiple scattering approach based on the layer Korringa-Kohn-Rostoker Green function method, we have reproduced the Fe $3 p$ angle resolved soft $x$-ray photoemission spectra and analyzed the associated large magnetic dichroism effects for excitation with both linearly and circularly polarized light. Comparison between theory and experiment yields a spin-orbit splitting of $1.0-1.2 \mathrm{eV}$ and an exchange splitting of $0.9-1.0 \mathrm{eV}$ for $\mathrm{Fe} 3 p$. These values are $50 \%-100 \%$ larger than those hitherto obtained experimentally.
\end{abstract}

Magnetic linear and circular dichroism (MLD, MCD) in core-level photoemission from ferromagnetic materials have been recognized as potentially valuable probes of the magnetic properties of both bulk and surface atoms [1]. Surface sensitivity can be enhanced by grazing-angle emission configurations or by the use of lower photoelectron kinetic energies (below $100 \mathrm{eV}$ ), in which case it is well known from low-energy electron diffraction (LEED) that multiple scattering of photoelectrons by crystal atoms plays an essential role. Previous theories of magnetic effects in core-level photoemission either treat isolated atoms with no multiple scattering effects [2] or include multiple scattering, but neglect the surface completely [3]. So far no theory has been reported in which these multiple scattering effects are taken into account in the propagation of photoelectrons through the surface including both spin-orbit and exchange interactions on an equal footing. 'These two interactions and their interference effects are essential for a complete description of magnetic dichroism. Hence, a full interpretation of magnetic dichroism photoemission requires a fully relativistic and spin-specific multiple scattering approach.

Recently, a large MLD effect was reported in angle resolved soft $\mathrm{x}$-ray photoemission from the $3 p$ state of the $\mathrm{Fe}(001)$ surface [1], in which a striking dependence of photoemission line shape upon the directions of magnetization, light polarization, and electron emission was found. The multiplet structure of the magnetic $\mathrm{Fe} 3 p$ state, which is not well understood [4], is very challenging theoretically in that neither spin-orbit nor exchange interaction can be treated as a perturbation [5].

In this Letter, we demonstrate the importance of appropriate theoretical modeling in the interpretation of magnetic dichroism measurements using angle resolved soft $x$-ray core-level photoemission as a probe of the surface and bulk electronic structures of magnetic materials. Using a recently developed spin-polarized photoemission theory, we show for the first time that these. spectra can be quantitatively explained within a simple single-particle picture taking account of bulk and surface multiple scattering effects in final photoelectron states. We predict numerically three independent spin polarizations, which are induced exclusively by exchange, by spin-orbit, and by their interference effects in the case in which the magnetic moment vector, the electric field vector of $p$-polarized light, and the wave vector of photoelectrons lie simultaneously in a crystallographic symmetry plane. We also present a theoretical analysis of experimental data for $\mathrm{Fe} / \mathrm{Cu}(001)$ [6], revealing a strong emission angle dependence in $\mathrm{MCD}$ spectra.

Neglecting scattering between photoelectrons and their holes in the photoexcitation process [7], the spin density matrix of the photoelectrons can be written in the form

$$
\rho_{\sigma \sigma^{\prime}}=-\frac{1}{\pi} v_{e}\left\langle\psi^{\sigma}\left|\Delta \operatorname{Im} G \Delta^{\dagger}\right| \psi^{\sigma^{\prime}}\right\rangle
$$

where $\left|\psi^{\sigma}\right\rangle$ is the time-reversed LEED state for spin $\sigma, G$ the single hole Green function, and $v_{e}$ the velocity of the photoelectrons. The electron-photon interaction $\Delta$ is well approximated here by the dipole form and the intensity and spin polarization of photoelectrons are obtained from the spin density matrix [8]. Although the photocurrent can be fully described by the single-hole Green function, it is difficult to renormalize the Green function to account for the many-body interaction between the hole and the other crystal electrons [9]. For materials that are well described by a localized picture, it might be easier to treat the many-body effects by explicitly assuming several atomic configurations with a core hole $[10,11]$. This approach has been used to interpret $\mathrm{Ni} 3 p$ corelevel photoemission in terms of multiplet and satellite structures [11]. For delocalized systems like metallic $\mathrm{Fe}$, the problem is more complicated and has been studied only for the free electron system, in which the line spectra are modified with a Doniach-Sunjic (DS) line shape characterized by a singularity parameter [12]. Since a DS line-shape modification does not affect the positions of lines in the multiplet structures, we have not included this type of many-body effect in our calculations.

Many-body effects are included in our calculation in two different ways. First, finite hole lifetime effects are 
included through an imaginary part of the optical potential in the hole Green function, broadening the discrete energy eigenstates into a continuous spectrum. Second, the effective potentials (i.e., self-energy correction) for the $3 p$ holes are expected to be strongly energy (state) dependent and act differently on the majority and minority spins so that the effective spin-orbit and exchange splittings can be modified from the ground-state values in the photoexcitation process. Instead of estimating the effective potentials in a many-electron theory [9], we treat these splittings and a center binding energy of the $3 p$ holes as adjustable parameters and determine them by comparing to the experimental spectra. The magnetic Dirac equation based on density functional theory is accordingly modified in the core-state calculations [13]. The exchange splitting is reduced by renormalizing the difference between the majority- and minority-spin ground-state potentials. For the spin-orbit interaction, we construct a quasirelativistic Dirac equation in which the strength of the spin-orbit coupling can be continuously tuned from the fully relativistic to the scalar relativistic Dirac equation $[13,14]$.

These approximations to the many-body effects allow us to take full advantage of the realistic final state wave function calculated by our fully relativistic multiple scattering computer code based on the layer Korringa-Kohn-Rostoker Green function method [8]. An accurate representation of the final LEED state is very important, since it is known to be very energy sensitive in the low-energy region, and its character can change across the entire $\mathrm{Fe} 3 p$ linewidth, thereby altering the photoemission spectra.

Underlying our spectral analysis is a calculation of the angular-momentum specific density of states (DOS) of the Fe $3 p$ core level. In Fig. 1 , we show the $3 p$ DOS, the imaginary part of the Green function, with both spin-orbit and exchange splittings chosen to be $1 \mathrm{eV}$, compared to the original values of 1.6 and $2.4 \mathrm{eV}$ from band-structure calculations. While the exchange-splitting reduction is commonly observed in photoemission spectra and has been explained theoretically [9], the spin-orbit splitting reduction might be understood as a consequence of the screened interaction between the nucleus and the hole by the other electrons, since the $3 d$ orbitals are more localized than the $3 p$ orbitals in an atomic picture. The hole lifetime is assumed to be $(0.5 \mathrm{eV})^{-1}$ and energy independent. In general, the lifetime is shorter for higher binding-energy states. The exchange splitting can be deduced from the energy difference between the states $\mu=3 / 2$ and $-3 / 2$ of $j .=3 / 2$ (indicated by arrows 3 and 6) since these are pure spin states and their relative energy positions are independent of the spin-orbit interaction. The spin-orbit splitting can be identified by calculating an energy center of the $j$ states, or by setting the exchange splitting to be zero in the hole Green function calculation. Since the total angular momentum $j$ is not a good quantum number for the other four states, they are

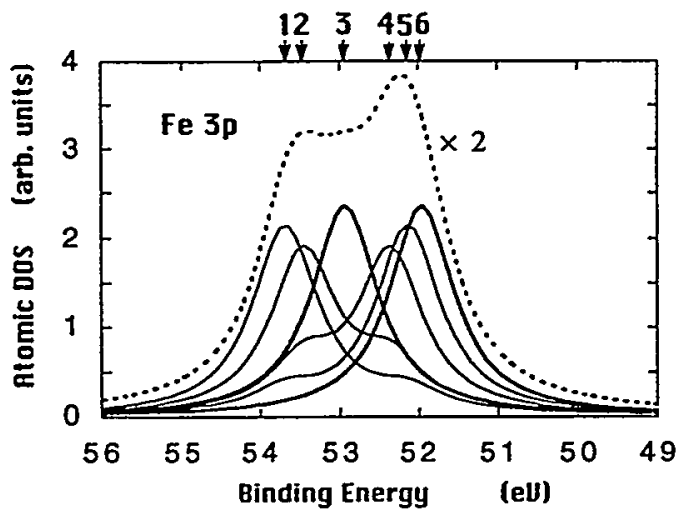

FIG. 1. Angular-momentum projected density of states (DOS) of the Fe $3 p$ state of the bulk atom. To facilitate comparison with experiment, the DOS has been shifted $1 \mathrm{eV}$ to higher binding energy. The thick solid lines correspond to $(j, u)$ of $(3 / 2,3 / 2)$ and $(3 / 2,-3 / 2)$, and the thin lines to $(1 / 2,-1 / 2)$, $(1 / 2,1 / 2),(3 / 2,1 / 2)$, and $(3 / 2,-1 / 2)$, in the order of peak location in decreasing binding energy. The dotted line is to the total DOS. The quantization axis is parallel to the majority-spin direction. The arrows indicate the energies of the eigenstates without lifetime broadening.

strongly hybridized between $j=1 / 2$ and $j=3 / 2$ within the same gyromagnetic quantum number $\mu$.

Using this $3 p$ DOS as a basis, we can analyze the experimental $3 p$ MLD results. The photoemission spectra corresponding to our $\mathrm{Fe} 3 p$ DOS, excited normally by linear polarized light, are compared in Fig. 2 with the experimental data of Ref. [1]. Use of the original bandstructure values for the spin-orbit and exchange splittings does not qualitatively alter the $3 p$ DOS, but the calculated spectra are dramatically affected, and this strong dependence was used to determine the best values for these parameters. Calculation of the final LEED states uses the same bulk muffin-tin potential as the $3 p$ state calculation, but with spin-orbit and exchange splittings taken without modification from the original band-structure calculation. We assume a truncated bulk geometry for the $\mathrm{Fe}(001)$ surface atomic arrangement. Allowing for the background which has not been subtracted from the experimental data, the agreement is excellent for all the spectra with respect to the peak positions, crossover points for the different magnetization directions $\mathbf{M}$, and intensity ratios between $s$ - and $p$-polarized light. The main differences are due to omission of Doniach-Sunjic line shapes and state-dependent lifetime broadenings in the theoretical spectra. Best agreement is obtained with calculations using a spin-orbit splitting of $1.0-1.2 \mathrm{eV}$ and an exchange splitting of $0.9-1.0 \mathrm{eV}$ for the Fe $3 p$ state. A spin-orbit splitting of $1 \mathrm{eV}$ also gives a good fit to the shoulders at $53.4 \mathrm{eV}$ in panel (a) and $53.6 \mathrm{eV}$ in (b).

The peak around $53 \mathrm{eV}$ in both panels is clearly attributed to the transition from the state $\mu=-3 / 2, j=3 / 2$ (cf. Fig. 1). From initial-state-projected calculations in 


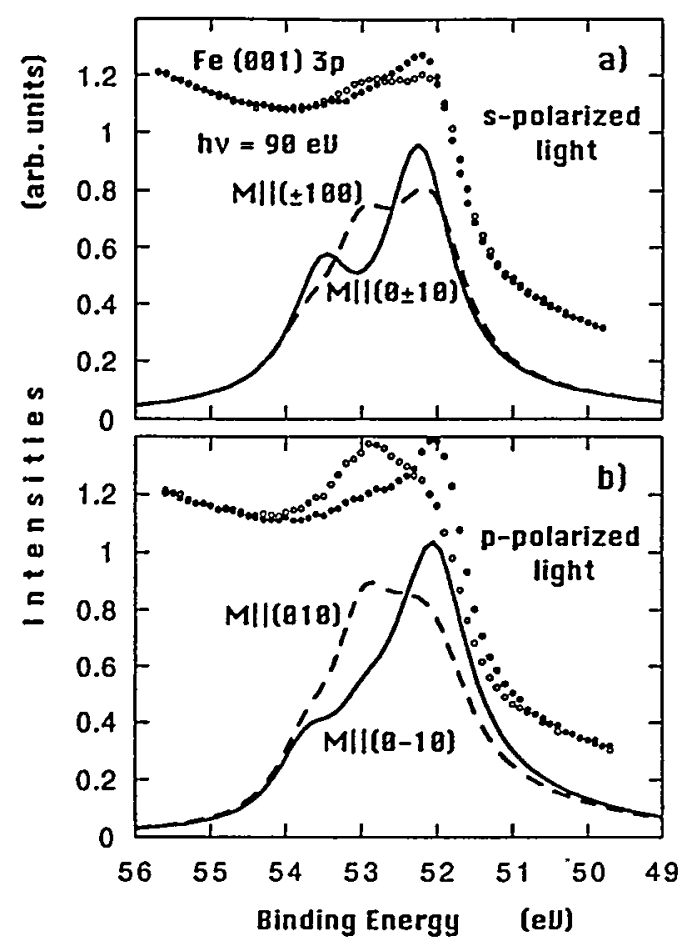

FIG. 2. Experimental and theoretical photoemission spectra emitted normally from the $3 p$ state of the $\mathrm{Fe}(001)$ surface by linearly polarized light of $90 \mathrm{eV}$ incident at polar angle $\theta=74^{\circ}$ parallel to [100]. Magnetic moment vectors lie in the surfaceparallel plane: (a) parallel and antiparallel to [100] (solid circles and solid line) and to [010] (open circles and dashed line) for $s$-polarized light (electric field vector parallel to [010]), and (b) parallel (solid circles and solid line) and antiparallel (open circles and dashed line) to [010] for $p$-polarized light ( $E$ in the plane perpendicular to [010]).

which all transitions are identified, we found that the transitions from the states $\mu= \pm 3 / 2, j=3 / 2$ do not contribute to the spectra when $M$ is parallel to the electric field vector $\mathbf{E}$ of $s$-polarized light [the solid line in (a)]. The spatial part of these initial states is symmetric with respect to the (010) mirror plane (perpendicular to $M$ ), and the final LEED states are also well approximated by a product of a symmetric spatial function and spin functions for normal emission. The matrix element vanishes for $\mathbf{E} \| \mathbf{M}$ which acts as an antisymmetric function in the dipole matrix element calculation. Thus these transitions dominate changes in the spectral line shape with changes in magnetization direction. This was found to be moderately true also for the case of $p$-polarized light, for which spin-resolved intensities (spin polarization) have also been calculated. The theoretical spectra agree well with the experiment by Roth et al. [1] except for the minority-spin peak around $53 \mathrm{eV}$ for $M \|$ [010] (Fig. 3 in Ref. [1]), which is only a shoulder in our calculation (not shown). In this experimental setup, all spin-polarization effects, spin-orbit, exchange, and their interference effects, are induced in the same [010] direc- tion, and so we cannot conclude whether this difference is caused by many-body (multiconfiguration) effects, such as those proposed in $\mathrm{Fe} 3 s$ photoemission [10], or some other effect.

To answer this question, we propose an alternative experimental setup in which $\mathbf{M}$ is chosen in the [100] direction so that the three key vectors, $\mathbf{M}$, the electric field of $p$-polarized light $\mathrm{E}$, and the emission direction are in the same crystallographic symmetry plane. Our theoretical prediction is shown in Fig. 3. When the spinorbit interaction is set equal to zero, the polarizations $P_{y}$ and $P_{z}$ vanish. The existence of $P_{y}$ polarization is already known for nonmagnetic surfaces [15]. Therefore, the intensity asymmetry in the [100] direction [panel (a)] is exclusively due to the exchange interaction, $P_{y}$ is entirely due to the spin-orbit interaction, and $P_{z}$ arises from the interference between these two interactions. Another advantage of this setup is that the absolute values of the spin-polarization and the spin-resolved intensities do not change on reversing the magnetization direction, thereby enabling the elimination of any spurious apparatus asymmetry effects. Our calculation is in very good agreement with the experimental spin-integrated intensity reported in Ref. [1]. It should be kept in mind, however, that the majority-spin spectra are expected to be much broader in shape and the minority-spin spectra somewhat sharper in experiment than the calculated spectra in Fig. 3 , since we use spin-independent hole lifetimes in all calculations.

As a further test of the theory, we have studied the system composed of two monolayers of $\mathrm{fcc} F e$ on $\mathrm{Cu}(001)$,

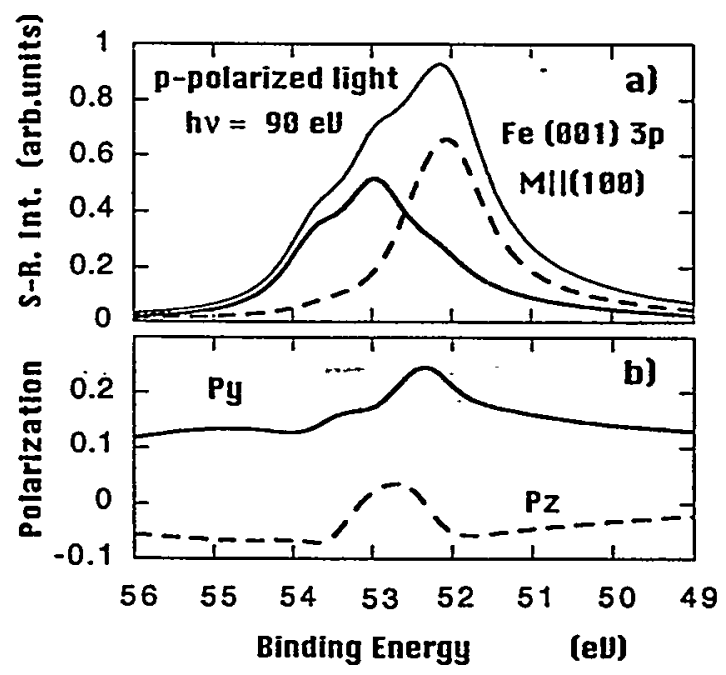

FIG. 3. Theoretical photoemission spectra emitted by $p$ polarized light as Fig. 2(b) but for the magnetic moment vector parallel and antiparallel to [100]: (a) spin-resolved intensities for the majority spin (solid line), the minority spin (dashed line), and the total (thin solid line), and (b) photoelectron spin polarization $P_{y}$ projected to the [010] direction and $P_{z}$ to the surface normal [001] direction. 
both experimentally and theoretically, using magnetic circular dichroism photoemission. The $\mathrm{Fe}$ layers were remanently magnetized along the surface normal and circularly polarized light incident normally. The photoelectrons were collected at a polar emission angle $55^{\circ}$ and an azimuthal angle $-27^{\circ} \pm 5^{\circ}$. Both the helicity and magnetization were independently varied, to consider all four possible configurations.

In our calculations, the geometrical arrangement of the $\mathrm{Fe}$ atoms is assumed to be a perfect continuation of the bulk fcc $\mathrm{Cu}$ crystal. The potentials are obtained from a scalar relativistic linear muffin-tin orbital calculation within the atomic-sphere approximation for the 12-layer superlattice with $5 \mathrm{Cu}, 4 \mathrm{Fe}$, and 3 empty layers. . From our LEED analysis, where a strong diffuse pattem was observed, we concluded that the Fe layers do not grow ideally and a possible clustering takes place under our experimental condition. To allow for this, we neglect the intralayer-multiple scattering for the Fe layers. The self-energy correction for the Fe $3 p$ core-hole state is treated in the same way as in the $\mathrm{Fe}(001)$ surface calculations. The results are presented in Fig. 4 where the theoretical spectra are Gaussian convoluted by the experimental resolution of $0.75 \mathrm{eV}$. The agreement is quite satisfactory, given the simplicity of the model used to describe the quasiordered surface. The main dichroism feature in the energy region $52-53 \mathrm{eV}$, the splitting of $0.25 \mathrm{eV}$, is well reproduced theoretically and can be attributed to the transition from the state $\mu=$ $-3 / 2, j=3 / 2$ as in the case of linearly polarized light. Further calculations were done using different azimuthal angles to find the emission-angle dependence of the splitting; it was found that the splitting varies slowly from 0.0 to $0.4 \mathrm{eV}$ for rotation through $45^{\circ}$. In the past this

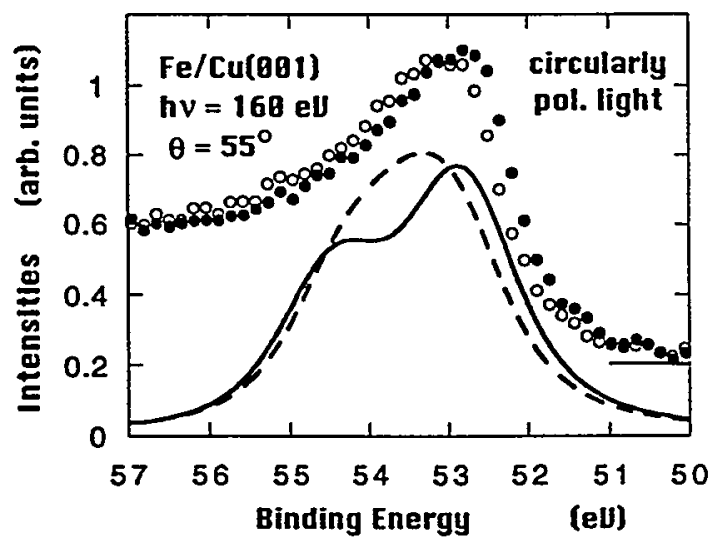

FIG. 4. Experimental and theoretical photoemission spectra emitted from the $\mathrm{Fe} 3 p$ state of the $\mathrm{Fe} / \mathrm{Cu}(001)$ surface by lefthanded circularly polarized (positive helicity) light of $160 \mathrm{eV}$ incident normally. Polar emission angle $\theta=55^{\circ}$ and azimuthal angle $\phi=27^{\circ}\left(25^{\circ}\right)$ for experiment (theory) referred to the [001] direction. The magnetic moment vector is parallel (the solid circles and solid line) or antiparallel (open circles and dashed line) to the light helicity. splitting has often been interpreted as the exchange splitting of $\mathrm{Fe} 3 p$. Unfortunately this is incorrect, although it is certainly introduced by exchange interaction. The splitting simply depends on how strongly the dipoleallowed components are excited in the final states. The specific components are $\mu=-1 / 2, j=1 / 2$ and $5 / 2$ ( $s$ and $d$ waves) for the transition by the positive helicity light and $\mu=-5 / 2, j=5 / 2$ ( $d$ wave) by the negative helicity light from the state $\mu=-3 / 2, j=3 / 2$. The relative strength can be predicted numerically for the offnormal emission into nonsymmetric directions. Based upon our theoretical analysis, in general, the MCD effect is as large as the MLD in some emission angle extent.

We have demonstrated that magnetic dichroism in angle resolved soft $\mathrm{x}$-ray core-level photoemission is a powerful tool for studying the electronic structure of magnetic materials. Interpretation of experimental results is not straightforward, but requires appropriate theoretical modeling to extract unique information. Using our newly developed computer code based on the spin-polarized fully relativistic multiple scattering theory to model linear and circular dichroism spectra we have determined the spin-orbit and exchange splittings of the Fe $3 p$ core level. Our calculations demonstrate that appropriate experimental conditions can further elucidate the role of spin-orbit and exchange interactions in the photoemission process.

The authors gratefully acknowledge A. Gonis for his encouragement. This work was performed under the auspices of the U.S. Department of Energy by Lawrence Livermore National Laboratory under Contract No. W-7405-ENG-48.

[1] Ch. Roth et al., Phys. Rev. Lett. 70, 3479 (1993); Solid State Commun. 86, 647 (1993).

[2] B.T. Thole and G. van der Laan, Phys. Rev. B 49, 9613 (1994).

[3] H. Ebert et al., Phys. Rev. B 44, 4406 (1991).

[4] F. Sorotti et al., Phys. Rev. B 48, 8299 (1993).

[5] H. Ebert, J. Phys. Matter 1, 9111 (1989).

[6] D. P. Pappas et al., J. Appl. Phys. 73, 5926 (1993).

[7] H. Gollisch et al., Solid State Commun. 82, 197 (1992).

[8] E. Tamura, Mater. Res. Soc. Symp. Proc. 253, 347 (1992).

[9] A. Liebsch, Phys. Rev. Lett. 43, 1431 (1979); Phys. Rev. B 23, 5203 (1981).

[10] B.T. Thole and G. van der Laan, Phys. Rev. Lett. 67, 3306 (1991); Phys. Rev. B 44, 12424 (1991).

[11] G. van der Laan et al., Phys. Rev. Lett. 69, 3827 (1992).

[12] S. Doniach and M. Sunjic, J. Phys. C 3, 285 (1970).

[13] E. Tamura (to be published). In contrast to the usual core-state calculation (e.g., see Ref. [5]) we solve the Dirac equation in a Green function formalism on the complex-energy plane.

[14] T. Takeda, Z. Phys. B 32, 43 (1978).

[15] E. Tamura and R. Feder, Europhys. Lett. 16, 695 (1991). 


\title{
Magnetic X-Ray Circular Dichroism in Nickel-Gold Multilayers
}

\author{
Alan F. Jankowski*, G. Dan Waddill** and James G. Tobin* \\ * Lawrence Livermore National Laboratory, Livermore, CA 94551-9900 U.S.A. \\ **University of Missouri, Physics Department, Rolla, MO 65401-0249 U.S.A.
}

Magnetic circular dichroism in $x$-ray absorption is used to investigate the in-plane, remnant magnetization of well-characterized Ni 0.48 A $u_{0.52}$ multilayers. Large superlattice strains are found in this multilayer system for samples with a $2 \mathrm{~nm}$ layer pair spacing. A larger dichroism is found in the Ni $2 p$ absorption edge for a $1.8 \mathrm{~nm}$ than for a $4.4 \mathrm{~nm}$ layer pair sample. The larger dichroism is consistent with a larger magnitude of in-plane strain for the Ni layers and a larger total magnetic anisotropy energy as shown from magnetization curve results.

The observation of magnetic anisotropy in the metallic multilayer systems proves to be of interest for magnetic recording and magneto-optic applications. In general, the magnetic properties of metallic multilayer films are strongly dependent on the relative as well as absolute layer thicknesses. Conventional magnetometry is typically used to investigate magnetization and anisotropy of metallic films. Beyond this application, $\mathrm{x}$-ray absorption spectroscopy (XAS) can be used for measuring magnetic circular dichroism (MCD) providing a sensitive technique for monitoring elemental specific changes in the orientation of sample magnetization. [1] For example, the remnant magnetization of $\mathrm{Fe}$ and $\mathrm{Co}$ are measured as a function of layer thickness for a series of $\mathrm{Fe}_{\mathrm{X}} \mathrm{Co}_{1-\mathrm{X}} / \mathrm{Pt}$ multilayer thin films using MCD. ${ }^{[2-4]}$

The Ni/Au multilayer samples are prepared using sputter deposition. [5-7] The deposition chamber is cryogenically pumped to a base pressure of $6.7 \times 10^{-6}$ $\mathrm{Pa}$. A circular array of planar magnetron sources is situated $20 \mathrm{~cm}$ beneath an oxygen-free copper platen. The purity of the target materials is $0.99995 \mathrm{Ni}$ and $>0.9994 \mathrm{Au}$. The magnetron sources are operated in the $\mathrm{dc}$ mode using an argon working gas pressure of $0.67 \mathrm{~Pa}$ at a flow rate of $20 \mathrm{cc} / \mathrm{min}$. The Si(111) substrates are sequentially rotated over each source and remain at a temperature between 293 and $306 \mathrm{~K}$ during the deposition. The sputter deposition rates of 0.10 to $1.0 \mathrm{~nm} / \mathrm{sec}$ are monitored using calibrated quartz crystals. The layer pair thicknesses (d) are verified using contact profilometry and $x$-ray diffraction for each sample consisting of $\mathrm{N}$ layer pairs. $[5,8-10]$

Microstructural investigation of the Ni/Au multilayers has been initiated using transmission electron microscopy. The films are found to be dense columnar deposits with a (111) textured growth and random inplane orientation. ${ }^{[5,7]}$ High resolution imaging is used to reveal the multilayer lattice structure. Lattice images in cross-section are recorded at the Scherzer defocus condition using a $400 \mathrm{keV}$ electron beam. The $\mathrm{Ni} / \mathrm{Au}$ multilayer samples are strained layered superlattices. ${ }^{[5]}$ Defects in the superlattice are characterized by dislocations in the Ni layers along [-111].

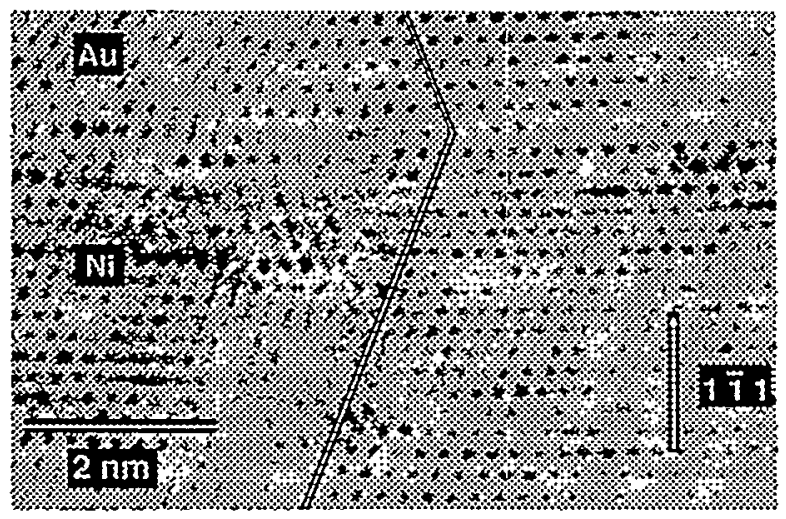

Figure 1 - The lattice image of the $4.4 \mathrm{~nm}$ layer pair $\mathrm{Ni} /$ Au multilayer as viewed in cross-section.

The lattice misfit between the Au and Ni layers is accomodated almost entirely by dislocations within the $\mathrm{Ni}$ layers for the $4.4 \mathrm{~nm}$ repeat periodicity. For the region of the $4.4 \mathrm{~nm} \mathrm{Ni} / \mathrm{Au}$ sample shown in Fig. 1, misfit dislocations along [-111] accomodate $12.5 \%$ of the $13.6 \% \mathrm{Ni}-\mathrm{Au}$ misfit. For the nearly incoherent superlattices as the $4.4 \mathrm{~nm} \mathrm{Ni/Au}$ sample, the $\mathrm{Au}$ layers form twin boundaries on the $\mathrm{Ni}$ layers (as outlined in Fig. 1). Also, the Ni layers contain inplane [1-10] dislocations. However, for the $1.8 \mathrm{~nm}$ sample (Fig. 2), all but 3.2\% of the Ni-Au misfit is accomodated by in-plane strain as divided between the $\mathrm{Ni}$ layer (in tension) and $\mathrm{Au}$ layer (in compression). Selected area diffraction (SAD) patterns of individual columns (imaged in cross-section) are used to compute the in-plane lattice spacings of the Ni layers, hence the [2-20] coherency lattice strains $\left(\varepsilon_{\|}\right)$, as well as the lattice strain along the [111] growth direction $\left(\varepsilon_{\perp}\right)$. Note that these SAD strain values (listed in Table I) reveal a non-Poisson behavior indicating expansion both in-plane and along the growth direction. ${ }^{[5]}$ 


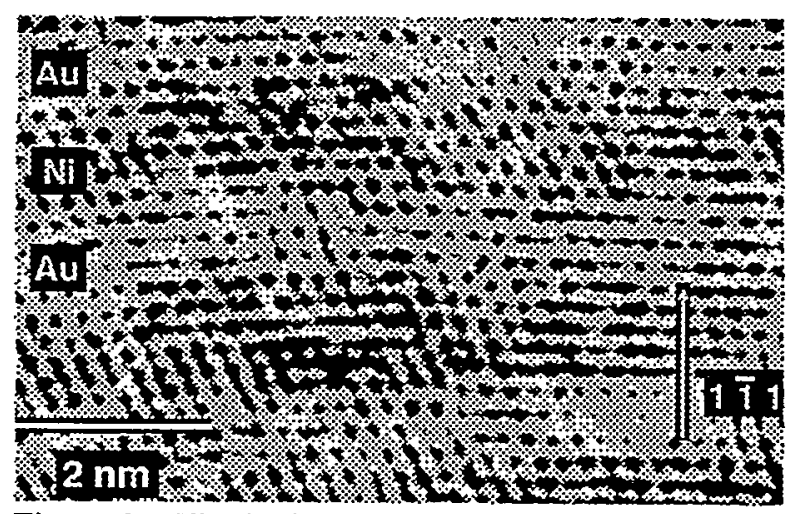

Figure 2 - The lattice image of the $1.8 \mathrm{~nm}$ layer pair $\mathrm{Ni} / \mathrm{Au}$ multilayer as viewed in cross-section.

Table I. Ni/Au Multilayer Parameters

\begin{tabular}{llllllllll}
\hline \hline $\mathrm{d}$ & $\mathrm{N}$ & $\varepsilon_{\|}$ & $\varepsilon_{\perp}$ & $\mu_{\mathrm{sr}} \mathrm{L}$ & $\mu_{\mathrm{sr}} \mathrm{S}$ & $\mu_{\mathrm{sr}}$ & $\mu_{\mathrm{br}}$ & $\mathrm{K}_{\mathrm{u}}$ \\
\hline 1.8 & 193 & .059 & .078 & 0.07 & 0.18 & 0.25 & 0.17 & 3.25 \\
4.4 & 100 & .014 & .020 & 0.03 & 0.02 & 0.04 & 0.00 & 1.70 \\
\hline \hline
\end{tabular}

The magnetic properties of the $\mathrm{Ni} / \mathrm{Au}$ multilayers were studied using vibrating sample magnetometry and a SQUID.[11] The saturation magnetization $\left(M_{S}\right)$ of $\mathrm{Ni}$ was found to decrease inversely with the Ni layer thickness $\left(\mathrm{d}_{\mathrm{Ni}}\right)$ while the Curie temperature $\left(\mathrm{T}_{\mathrm{c}}\right)$ followed a power law behavior. Unlike these dependencies on $\mathrm{d}_{\mathrm{Ni}}$, an abrupt decrease in the magnetic anisotropy was found beyond $2 \mathrm{~nm}$ layer pair spacings. In this study, MCD is used to further investigate the spin and orbital components of magnetic anisotropy of the 1.8 and $4.4 \mathrm{~nm} \mathrm{Ni} / \mathrm{Au}$ samples which characterize the extrema in the magnitude of superlattice strain.

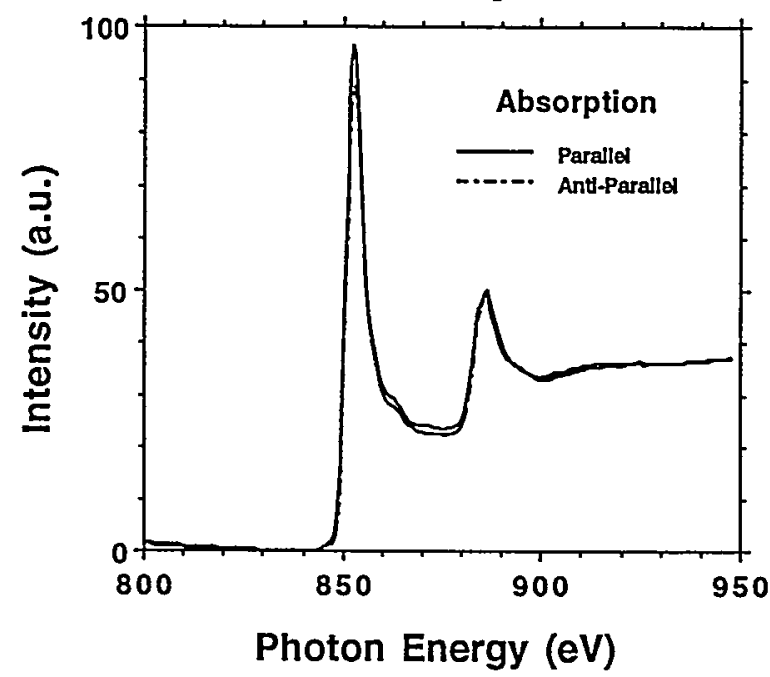

Figure 3 - The polarization dependent XAS curves for the $1.8 \mathrm{~nm} \mathrm{Ni/Au} \mathrm{sample.}$
The x-ray absorption spectroscopy (Fig. 3) and magnetic circular dichroism (Fig. 4) measurements are performed on a spherical grating monochromator with the ability to generate soft $(80-1100 \mathrm{eV}) \mathrm{x}$-rays with a high degree of linear or circular (85-90\%) polarization. ${ }^{[12,13]}$ The Ni/Au films are magnetized in-situ with a pulse coil capable of generating a $3 \mathrm{kOe}$ field. To observe an MCD effect, the in-plane magnetization of the films requires a grazing incidence geometry with alignment of the magnetization and $x$ ray Poynting vectors. In $\mathrm{x}$-ray absorption, $\mathrm{MCD}$ is observed as a circular polarization dependent intensity variation in the LIII and $\mathrm{L}_{\mathrm{II}}$ edges for $3 \mathrm{~d}$ transition metals. The absorption spectra are taken in a total electron yield mode by isolating the sample and measuring the neutralization current. The film is sampled to at least a $20 \mathrm{~nm}$ depth. The polarization dependence requires that the incident $x$-ray helicity (either parallel or anti-parallel to the propagation direction) be aligned or anti-aligned with the sample magnetization. [1,14] MCD is the difference in absorption between these polarized radiation conditions as the photon energy is swept through an $\mathrm{x}$-ray absorption edge. The intensity difference for the LIII and $\mathrm{L}_{\mathrm{II}}$ white lines between the parallel and antiparallel states provides a measure of the magnetic moment as well as its orbital and spin components. $\mathrm{Ni} / \mathrm{Au}$ data is presented without the use of curve smoothing procedures.

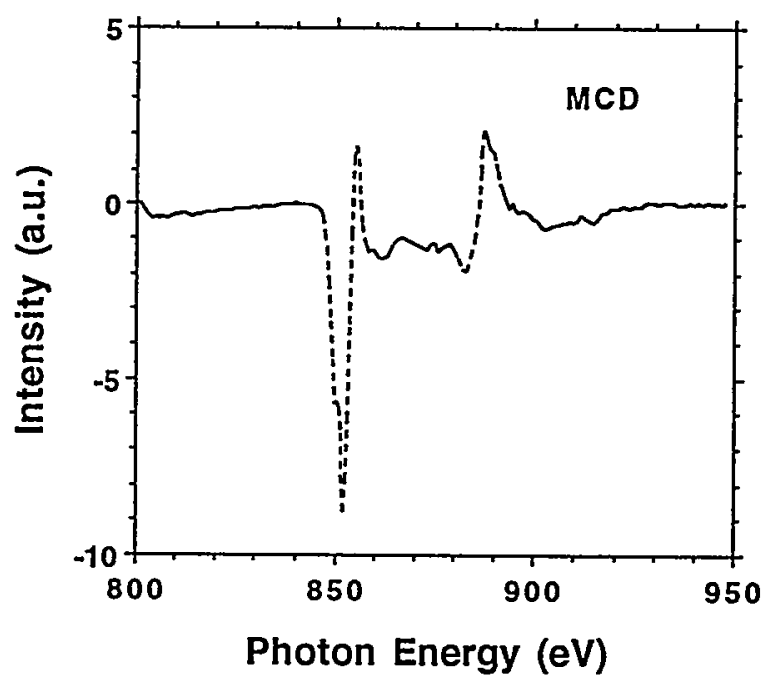

Figure 4 - The MCD curve represents the difference divided by the sum of the XAS curves for the $1.8 \mathrm{~nm}$ $\mathrm{Ni} / \mathrm{Au}$ sample.

The lattice is coupled to the electron spin angular momentum through the spin-orbit interaction. Allowed transitions are determined by the dipole selection rules. In particular, we probe the $2 p$ to $3 d$ transitions. The relative strengths of the $\mathrm{L}_{\mathrm{III}}$ and $\mathrm{L}_{\mathrm{II}}$ absorption edgescontain information about the spindependent density of states near the Fermi level and the 
spin-orbit splitting in the d-bands (Fig. 5). Therefore, element and shell specific information is available about the spin and orbital contributions to the magnetic moments of the material.[1,15-18] Application of the sum-rule (sr) analysis yields values for the spin $\left(\mu_{\mathrm{sr}} \mathrm{S}\right)$ and orbital $\left(\mu_{\mathrm{sr}} \mathrm{L}^{\mathrm{L}}\right)$ components of the total moment $\left(\mu_{\mathrm{sr}}=\mu_{\mathrm{sr}} \mathrm{s}+\mu_{\mathrm{sr}} \mathrm{L}\right)$. The branching-ratio (br) analysis yields a value for the spin moment $\left(\mu_{\mathrm{br}}\right)$ with the apriori assumption of a small orbital moment. For $3 \mathrm{~d}$ elements, the $\mu_{\mathrm{br}}$ is computed with the following relationships. [19]

$$
\begin{aligned}
\mu_{\mathrm{br}}= & \mathrm{C}_{0^{\circ}}\left(\mathrm{BR}^{+}-\mathrm{BR}^{-}\right) /\left(\mathrm{BR}^{+}+\mathrm{BR}^{-}\right) \\
& \mathrm{BR}^{+}=\left(\mathrm{A}^{+}\right) /\left(\mathrm{A}^{+}+\mathrm{B}^{+}\right) \\
& \mathrm{BR}^{-}=\left(\mathrm{A}^{-}\right) /\left(\mathrm{A}^{-}+\mathrm{B}^{-}\right)
\end{aligned}
$$

where $A^{+,-}$is the integrated intensity of the $L_{\text {III }}$ peak above the background intensity and $B^{+,-}$is the integrated intensity of the LII peak above the background intensity for the parallel $(+)$ and antiparallel $(-)$ helicity and magnetization conditions, respectively (Fig. 4).

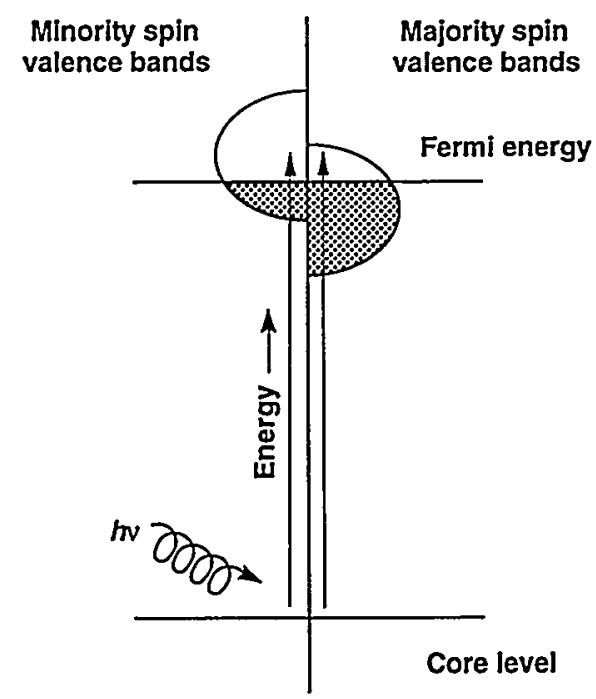

Figure 5 - A schematic of the absorption of a photon and transition of an electron into an exchange split valence band density of states.

For the computation of $\mu_{\mathrm{sr}}$, the following equations apply. [19]

$$
\begin{gathered}
\mu_{\mathrm{Sr}} \mathrm{s} \equiv\left\{\mathrm{C}_{1^{\bullet}}\left[\left(\mathrm{A}^{+} / \mathrm{C}^{+}\right)-\left(\mathrm{A}^{-} / \mathrm{C}^{-}\right)\right] /(\mathrm{SUM})\right\}-3 \cdot \mu_{\mathrm{Sr}} \mathrm{L} \\
\mu_{\mathrm{Sr}}{ }^{\mathrm{L}}=\mathrm{C}_{2^{\bullet}}\left[\left(\mathrm{A}^{+} / \mathrm{C}^{+}\right)-\left(\mathrm{A}^{-} / \mathrm{C}^{-}\right)+\left(\mathrm{B}^{+} / \mathrm{C}^{+}\right)-\left(\mathrm{B}^{-} / \mathrm{C}^{-}\right)\right] /(\mathrm{SUM}) \\
\mathrm{SUM}=\left[\left(\mathrm{A}^{+} / \mathrm{C}^{+}\right)+\left(\mathrm{A}^{-} / \mathrm{C}^{-}\right)+\left(\mathrm{B}^{+} / \mathrm{C}^{+}\right)+\left(\mathrm{B}^{-} / \mathrm{C}^{-}\right)\right]
\end{gathered}
$$

where $\mathrm{C}^{+,-}$is the height of the background curves above the baseline intensity. These analysis procedures applied to the XAS and MCD spectra (Figs. 3 and 4) produce values for the magnetic moments listed in Table I (in units of $\mu_{B} / \mathrm{Ni}$ atom). The analyses may be complicated, however, by the polycrystalline surface. If the sample is not of a single domain, then MCD will average the domains yielding a moment that reflects the average projection of magnetization along the photon propagation direction.

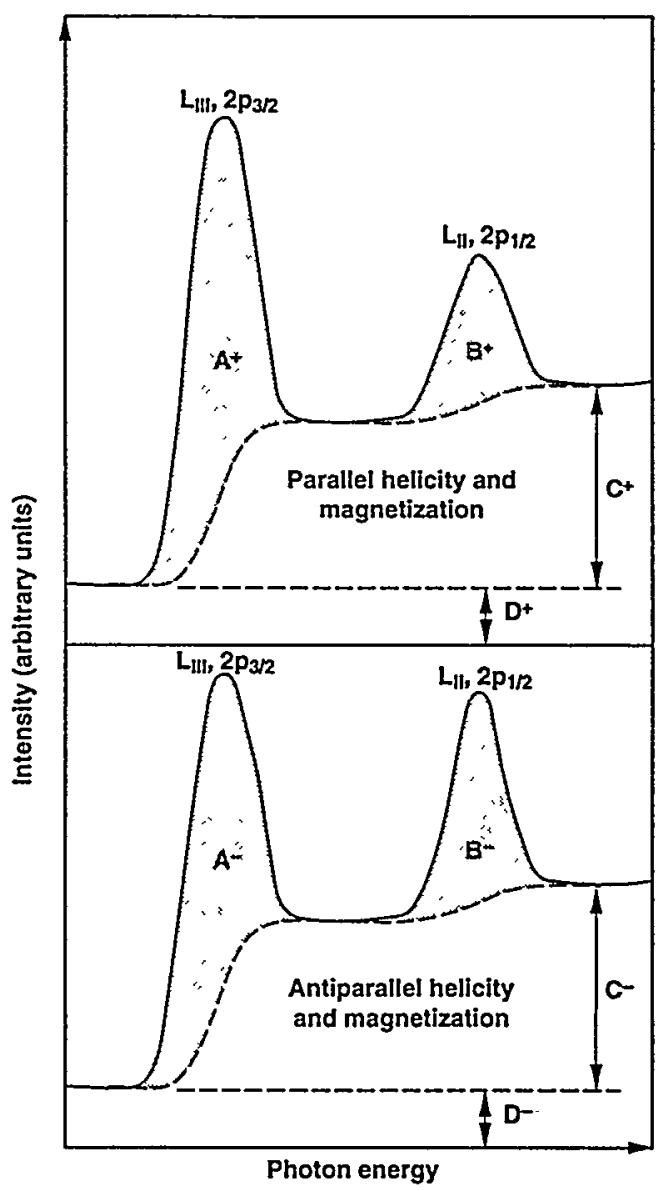

Figure 6 - A schematic of $x$-ray absorption spectra with white line peaks at the $\mathrm{L}_{\mathrm{III}}$ and $\mathrm{L}_{\mathrm{II}}$ edges for the case of ferromagnetic alignment.

The total anisotropy energy $K_{u}$ represents the difference in energy density between the parallel and perpendicular magnetized states. It is equivalent to the difference in area under the magnetization (versus applied field) curves. The total anisotropy energy can be expressed as

$$
\mathrm{K}_{\mathrm{u}}=-\left\{2 \pi \cdot \mathrm{M}_{\mathrm{s}}^{2}+\mathrm{K}_{\mathrm{v}}+2 \cdot \mathrm{K}_{\mathrm{S}} / \mathrm{d}_{\mathrm{Ni}}\right\}
$$

where $\mathrm{K}_{\mathrm{V}}$ and $\mathrm{K}_{\mathrm{S}}$ are the volume and surface anisotropy constants, respectively. ${ }^{[11]}$ The preferred in-plane magnetization for these $\mathrm{Ni} / \mathrm{Au}$ multilayers means that $\mathrm{K}_{\mathbf{u}}$ is always negative. Representative values of $\mathrm{K}_{\mathfrak{u}}$ $\left(10^{6} \mathrm{erg} / \mathrm{cc}\right)$ for the samples examined with XAS for 
MCD are listed in Table I. Whereas the coherent-toincoherent transition for increasing layer pair spacing is observed not to have any noticeable effect on either $M_{S}$ or $T_{C}$, a lattice strain effect is apparent on $K_{u}$.

The magnetic behavior of lattice strained and unstrained $\mathrm{Ni} / \mathrm{Au}$ multilayers have been probed using MCD. The MCD results are consistent with the magnetic anisotropy measurement of these films as previously determined through magnetization curves. ${ }^{[11]}$ A large decrease in the spin component, from 0.18 to $0.02 \mu_{\mathrm{B}} / \mathrm{Ni}$ atom, is found with the sumrule analysis as the in-plane strain of the $\mathrm{Ni}$ layer decreases from $5.9 \%$ to $1.4 \%$ (with an increase in the $\mathrm{Ni}$ layer thickness from 0.91 to $2.1 \mathrm{~nm}$ ). Results for the branching ratio analysis yield nearly equivalent results as for the spin component. The magnitude of decrease (by a factor of 2) in the total anisotropy energy $K_{u}$ is equal to the decrease in the orbital component, from 0.07 to $0.03 \mu_{\mathrm{B}} / \mathrm{Ni}$ atom, which therefore serves as an indicator of elastic strain effects on crystalline lattice. These results confirm a magneto-elastic effect in the magnetization behavior of the $\mathrm{Ni} / \mathrm{Au}$ multilayer system.

This work was performed under the auspices of the United States Department of Energy by Lawrence Livermore National Laboratory under contract W-7405Eng-48.

1. J.G. Tobin, G.D. Waddill and D.P. Pappas, Phys. Rev. Lett. 68, 3642 (1992).

2. A. Jankowski, G.D. Waddill and J. Tobin, Mat. Res. Soc. Symp. Proc. 313, 227 (1993).

3. A.F. Jankowski, G.D. Waddill and J.G. Tobin, J. Vac. Sci. Technol. A 12, 2215 (1994).

4. J. Tobin, A. Jankowski, G. Waddill and P. Sterne, Mat. Res. Soc. Symp. Proc. 343 (1994).

5. A.F. Jankowski, J. Appl. Phys. 71, 1782 (1992).

6. A.F. Jankowski, J. Magn. Magn. Mat. 126, 185 (1993).

7. M.A. Wall and A.F. Jankowski, Thin Solid Films 181, 313 (1989).

8. A.F. Jankowski, Superlatt. Microstruc. 6, 427 (1989).

9. J. Chaudhuri, S. Shah, V. Gondhalekar and A. Jankowski, J. Appl. Phys. 71, 3816 (1992).

10. J. Chaudhuri, S. Alyan and A. Jankowski, Thin Solid Films 219, 63 (1992).

11. J.R. Childress, C.L. Chien and A.F. Jankowski, Phys. Rev. B 45, 2855 (1992).

12. K.G. Tirsell and V. Karpenko, Nucl. Instrum. Meth. A 291, 511 (1990).

13. L.J. Terminello, G.D. Waddill and J.G. Tobin, Nucl. Instrum. Meth. A 319, 271 (1992).
14. J.L. Erskine and E.A. Stern, Phys. Rev. B 12, 5016 (1975).

15. B.T. Thole and G. van der Laan, Phys. Rev. A 38, 1943 (1988).

16. B.T. Thole and G. van der Laan, Phys. Rev. B 42,6670 (1990).

17. B.T. Thole, P. Carra, F. Sette and G. vander Laan, Phys. Rev. Lett. 68, 1943 (1992).

18. P. Carra, B.T. Thole, M. Altarelli and X. Wang, Phys. Rev. Lett. 70, 694 (1993).

19. J.G. Tobin, G.D. Waddill, A.F. Jankowski, P.A. Sterne and D.P. Pappas, submitted for publication. 


\section{ACTIVE PROPOSALS}

SSRL accepts a variety of proposal types. These include 1) full peer-reviewed proposals, b) letters of intent, c) rapid turnaround XAS proposals and d) rotation camera proposals for protein crystallography. Beam time is also assigned by the Participating Research Teams on their beam lines based on application to the PRT. As of December 31, 1994 there were 311 active proposals: 163 peer-reviewed, 12 rapid turnaround XAS, 94 rotation camera proposals, 37 letters of intent and 5 PRTs. The letter suffix appended to the proposal number indicates the Proposal Review Panel to which the proposal is assigned: Materials (M), Biology (B), or Vacuum Ultraviolet (V). The small "p" denotes a program proposal. Peer-reviewed single project proposals remain active for two years after their initial rating, while program proposals can be renewed for an additional two years. The first person listed under each proposal is the proposal spokesperson and the institution listed is that of the spokesperson.

\section{PEER-REVIEWED PROPOSALS}

2128Bp Technetium and Rhenium Imaging Agents and Therapeutic Radiopharmaceuticals

Richard C. Elder

Edward A. Deutsch

(UNIVERSITY OF CINCINNATI)

2137Bp X-ray Absorption Spectroscopic Studies of Nickel-Containing Metalloenzymes

Robert A. Scott

Shengke Wang

Hui Zhang

(UNIVERSITY OF GEORGIA)

2138Mp Local Atomic Structure of High- $\mathbf{T}_{\mathbf{c}}$ Superconductors

James B. Boyce

Corwin H. Booth

Frank G. Bridges

Tord C. Claeson

Guoguang $\mathrm{Li}$

(XEROX)

2139Mp Chemical Vapor Infiltration of Ceramic Matrix Composites

John H. Kinney

Cynthia A. Lundgren

Monte C. Nichols

Thomas L. Starr

Stuart R. Stock

(LAWRENCE LIVERMORE NATIONAL LABORATORY)

2142Bp Anomalous Scattering of X-rays

David H. Templeton

Lieselotte K. Templeton

(LAWRENCE BERKELEY LABORATORY)

2143Mp XAS Study of Cation Chemisorption at Oxide-Water Interfaces

Gordon E. Brown, Jr.

John R. Bargar

Singfoong Cheah

Farrel W. Lytle

George A. Parks

Per K.B. Persson

Steven N. Towle

Ning $\mathrm{Xu}$

(STANFORD UNIVERSTTY) 
2145Vp Photoelectron Spectroscopic Studies of Inorganic Materials as Active Sites in Catalysis Edward I. Solomon

Jeffrey A. Guckert

Paul M. Jones

Jennifer A. May

J. Bradford Reitz

(STANFORD UNIVERSITY)

2146Bp XAS K-edge Studies of Ligands Bound to Open-Shell Metal Ions in Proteins and Model Compounds Keith O. Hodgson

Britt Hedman

Susan E. Shadle

Edward I. Solomon

Kendra R. Williams

(STANFORD SYNCHROTRON RADIATION LABORATORY)

2149Bp Structure $\leftrightarrow$ Function Studies of the Active Sites of Lignin Peroxidase, Peroxidases, and Models

Linda S. Powers

Robert B. Sinclair

(UTAH STATE UNIVERSITY)

2151Bp Structural Characterization of Mercury Metalloproteins Using XAS

James E. Penner-Hahn

Kimber C. Clark

Susan Miller

Thomas V. O'Halloran

Pamela J. Riggs-Gelasco

Timothy L. Stemmler

(UNIVERSITY OF MICHIGAN)

2152Mp XAS Study of Metal Ion Partitioning at Water/Mineral Interfaces

Kim F. Hayes

Lynn E. Katz

James E. Penner-Hahn

(LAWRENCE BERKELEY LABORATORY)

$2153 \mathrm{Mp}$ Composition Fluctuations in Amorphous Films

Michael J. Regan

Arthur I. Bienenstock

(HARVARD UNIVERSITY)

2165Mp Ultra-High Strength-High Temperature Surface Coatings

John C. Bilello

Zofía U. Rek

Jun Tao

Marc A. Vill

Steven M. Yalisove

Zhen Zeng

(UNIVERSITY OF MICHIGAN)

2166Vp Calibration of M.S.S.T.A. Instruments

Maxwell J. Allen

Troy W. Barbee, Jr.

Craig F. DeForest

Richard B. Hoover

Charles C. Kankelborg

Joakim F. Lindblom

Ray H. O'Neal

Arthur B.C. Walker

Thomas D. Willis

(STANFORD UNIVERSITY) 
2169Vp X-ray Standing Wave Studies of Adsorption Geometries at Selected Metal III-V Semiconductor Interfaces

Tom Kendelewicz

Piero A. Pianetta

William E. Spicer

Joseph C. Woicik

(STANFORD UNIVERSITY)

2170Mp X-ray Absorption Spectroscopy of Transformation-Toughened Zirconia Ceramics I-wei Chen

Ping Li

(UNIVERSITY OF MICHIGAN)

2175Mp XAFS Investigation of Selected Topics in Coal and Environmental Science

Gerald P. Huffiman

Frank E. Huggins

Naresh Shah

(UNIVERSITY OF KENTUCKY)

2176Vp Growth, Structure and Electronic Properties of Epitaxially Grown Si, Ge and SiGe Structure Renyu Cao

Piero A. Pianetta

(STANFORD SYNCHROTRON RADIATION LABORATORY)

2178M Mechanism of Formation of Synthetic Clay Catalysts by SAXS and ASAXS

Pappannan Thiyagarajan

Kathleen G. Carrado

Keith O. Hodgson

(ARGONNE NATIONAL LABORATORY)

2179M Distribution of Active Sites on Clay Surfaces by SAXS and ASAXS

Pappannan Thiyagarajan

Kathleen G. Carrado

Keith O. Hodgson

Stephen R. Wasserman

(ARGONNE NATIONAL LABORATORY)

2181Bp X-ray Absorption Spectroscopy Studies of Manganese Redox Enzymes

James E. Penner-Hahn

Martha Ludwig

Rui Mei

Vincent L. Pecoraro

Pamela J. Riggs-Gelasco

Timothy L. Stemmler

Charles F. Yocum

(UNIVERSITY OF MICHIGAN)

2182Mp In Situ X-ray Studies of OMVPE Growth

Paul H. Fuoss

$\therefore$. . Sean M.'Brennan

David W. Kisker

Richard M. Lum

- G. Brian Stephenson

(AT\&T BELL LABORATORIES)

2186Bp XAS Structural Characterization of the Dinuclear Iron Center in Methane Monooxygenase Keith O. Hodgson

Jane G. DeWitt

Britt Hedman

Stephen J. Lippard

Amy C. Rosenzweig

(STANFORD SYNCHROTRON RADIATION LABORATORY) 
Sebastian Doniach

Robert L. Byer

Lingling Chen

David Eliezer

Keith O. Hodgson

Hirotsugu Tsuruta

(STANFORD UNIVERSITY)

2189Mp Speciation of Hazardous Waste by X-ray Absorption Spectroscopy

Farrel W. Lytle

C. Mel Lytle

(THE EXAFS COMPANY)

2190Bp X-ray Absorption Spectroscopy of Manganese Involved in Photosynthetic Oxygen Evolution Melvin P. Klein

Holger Dau

Matthew J. Latimer

Wenchuan Liang

Theo A. Roelofs

Kenneth H. Sauer

Vittal K. Yachandra

Jean-Luc Zimmermann

(LAWRENCE BERKELEY LABORATORY)

2192Bp XAS Structural Characterization of a Manganese-Enzyme, Prolidase

Hans C. Freeman

Paul J. Ellis

Britt Hedman

Keith O. Hodgson

Emma M. Proudfoot

(UNIVERSITY OF SYDNEY)

2193M XAFS Studies of Perovskite Related Structures at High Pressure

Robert L. Ingalls

E. Daryl Crozier

Brian D. Houser

(UNIVERSITY OF WASHINGTON)

2194Mp Determination of the Strain Distribution in Metallic Thin Films and Lines as a Function of Temperature Using GIXS

John C. Bravman

Paul R. Besser

Sean M. Brennan

Ramnath Venkatraman

Richard P. Vinci

(STANFORD UNIVERSITY)

2197Mp Investigation of Heavily-Doped III-V and II-VI Semiconductors Using Fluorescence EXAFS

Kin Man Yu

Edith D. Bourret

Eugene E. Haller

Joseph M. Jaklevic

Carolyn S. Rossington

Wladyslaw Walukiewicz

(LAWRENCE BERKELEY LABORATORY) 
2199Vp High-Photon-Flux Photoemission Studies of Narrow Band Materials

James W. Allen

Ralph Claessen

Zhi-xun Shen

L.H. Tjeng

(UNIVERSITY OF MICHIGAN)

2200Mp Kinetic Studies of OMVPE Growth Using In Situ X-ray Scattering

David W. Kisker

Sean M. Brennan

Paul H. Fuoss

Richard M. Lum

G. Brian Stephenson

(IBM RESEARCH LABORATORY)

2201B The Organization of DNA within Mammalian Sperm Cells

Nicholas V. Hud

Rodney L. Balhorn

Hirotsugu Tsuruta

(LAWRENCE LIVERMORE NATIONAL LABORATORY)

2202Vp Application of Magnetic Circular X-ray Dichroism to Magnetic Multilayers and Imaging of Magnetic Domains

Joachim Stöhr

Brent D. Hermsmeier

Mahesh G. Samant

(IBM RESEARCH LABORATORY)

2203Mp EXAFS Spectroelectrochemistry

Richard C. Elder

William R. Heineman

(UNIVERSITY OF CINCINNATI)

2204M Characterization of Amorphous to Crystalline Phase Transformation in Silica

David F. Blake

Jack Farmer

Nancy Hinman

(NASA-AMES RESEARCH)

2205Mp Uranium Speciation in Soils

Steven D. Conradson

Catherine J. Chisholm-Brause

David W. Morris

(LOS ALAMOS NATIONAL LABORATORY)

2206Mp Structure-Reactivity-Performance Relationships in Mesoscale Platinum-Based Catalysts

Steven D. Conradson

Patrick G. Allen

Shimshon Gottesfeld

Ian D. Raistrick

(LOS ALAMOS NATIONAL LABORATORY)

2207Mp Local Structure in High-Temperature Superconductors

Steven D. Conradson

Jose Mustre De Leon

Ian D. Raistrick

(LOS ALAMOS NATIONALLABORATORY)

2209M Structural Changes Induced by Magnetic Fields in Magnetostrictive Materials

E. Daryl Crozier

Yolande R. Bonin

(SIMON FRASER UNIVERSITY) 
2210Mp Grazing-Incidence XAS Study of Cation Chemisorption at Single-Crystal Oxide

Surfaces

Gordon E. Brown, Jr.

Troy W. Barbee, Jr.

John R. Bargar

Ping Liu

George A. Parks

Per K.B. Persson

Ingrid J. Pickering

Steven N. Towle

Glenn A. Waychunas

(STANFORD UNIVERSITY)

2211Vp Electron Spectroscopy of Gases, Solids and Surfaces

Zahid Hussain

Philip A. Heimann

Zheng-qing Huang

Eric A. Hudson

Tony Huff

Scot A. Kellar

Edward J. Moler

Barry L. Petersen

Tobias N. Reich

David A. Shirley

Pradeep Thalappil

Yu Zheng

(LAWRENCE BERKELEY LABORATTORY)

2212Mp Low Temperature Synthesis of Optical Coatings Using Aqueous Precursor Solutions

Nancy J. Hess

Farrel W. Lytle

(PACIFIC NORTHWEST LABORATORIES)

2213Vp Synchrotron X-ray Spectroscopic Studies of Oxide Surfaces and Oxide-Water Interfaces

Tom Kendelewicz

Gordon E. Brown, Jr.

George A. Parks

Piero A. Pianetta

William E. Spicer

Glenn A. Waychunas

Joseph C. Woicik

(STANFORD UNIVERSITY)

2214M X-ray Absorption Studies of Copper, Lead and Cadmium Complexation by Soil Organic Matter William Bleam

(UNIVERSITY OF WISCONSIN)

2215Mp Improved Fluorescent X-ray Detectors: Pin Diodes and a $4 \pi$ Geometry

Farrel W. Lytle

Charles E. Bouldin

(THE EXAFS COMPANY)

2217Mp Giant Magnetoresistance and Interface Structure in Magnetic Multilayers

Thomas A. Rabedeau

Michael F. Toney

(STANFORD SYNCHROTRON RADIATION LABORATORY)

2218M Solvation of Transition Metal Cations in the Interlayer of Clay Minerals

Stephen R. Wasserman

Kathleen G. Carrado

(ARGONNE NATIONAL LABORATORY) 
2219Mp Electronic Structure and Bonding in Pentavalent Uranium Compounds

Mark R. Antonio

Lynda C. Soderholm

J. Simon Xue

(ARGONNE NATIONAL LABORATORY)

2221M X-ray Absorption Study of $\mathrm{Rb}_{.31} \mathrm{WO}_{3}$ as a Function of Pressure

Brian D. Houser

E. Daryl Crozier

Robert L. Ingalls

(EASTERN WASHINGTON UNIVERSITY)

2222M XAS of Models for Supported Rhodium Catalysts

Craig E. Barnes

(UNIVERSTTY OF TENNESSEE)

2223Mp In Situ Structural Studies of the Initial Stages of Growth of Sputter-Deposited Films

Bruce M. Clemens

Dorota Artymowicz

Sean M. Brennan

Brian J. Daniels

Todd C. Hufnagel

Michael C. Kautzky

Bruce M. Lairson

Richard M. Osgood

Noa More Rensing

Glenn F. Simenson

Junfeng Xu

(STANFORD UNIVERSITY)

2224Mp Diffraction Studies of Kinetics and Structure of Thin Films and Multilayers

Bruce M. Clemens

Dorota Artymowicz

Sean M. Brennan

Brian J. Daniels

Todd C. Hufnagel

Michael C. Kautzky

Bruce M. Lairson

Richard M. Osgood

Noa More Rensing

Glenn F. Simenson

Junfeng $\mathrm{Xu}$

(STANFORD UNIVERSITY)

2225Bp Solution Structure Studies of the Nitrogenase Proteins by Small-Angle X-ray Scattering

Keith O. Hodgson

Barbara K. Burgess

Lingling Chen

Sebastian Doniach

David Eliezer

Narasaiah Gavini

Hirotsugu Tsuruta

(STANFORD SYNCHROTRON RADIATION LABORATORY)

2226Mp X-ray Topographic Study of Defects in Mixed Crystals of $\mathrm{K}_{(\mathrm{X}}\left(\mathrm{D}_{\mathrm{X}} \mathrm{H}_{1-\mathrm{x}}\right)_{2} \mathrm{PO}_{4}$

James J. De Yoreo

Zofia U. Rek

Natalia Zaitseva

(LAWRENCE LIVERMORE NATIONAL LABORATORY) 
2227Bp XAS Studies of Copper-Containing Monooxygenase Enzymes and Relevant Inorganic Model Compounds Ninian J. Blackburn

Kaitlin Grammer

Brian J. Reedy

Frank Rhames (OREGON GRADUATE INSTITUTE)

2228Mp EXAFS Studies of Chemisorption Processes on Ferrihydrite and Quartz Surfaces

Glenn A. Waychunas

Gordon E. Brown, Jr.

James A. Davis

Christopher C. Fuller

Michael Rowen

(STANFORD UNIVERSITY)

2229Mp Mechanics of Advanced Composites Using In Situ X-ray Tomographic Microscopy

Thomas M. Breunig

Ulrich K. Bonse

David L. Haupt

John H. Kinney

Monte C. Nichols

(SANDIA NATIONAL LABORATORIES)

2230M Structure and Interactions in Polymeric Micelles

Glen A. McConnell

Alice P. Gast

Eric K. Lin

(STANFORD UNIVERSITY)

2231B Multiwavelength X-ray Analysis of Gene V Protein Crystals Containing Selenomethionine

Thomas $C$. Terwilliger

Henry D. Bellamy

Yue Guan

Dale H. Leschnitzer

Raymond G. Nanni

R. Paul Phizackerley

Matthew M. Skinner

Andrew H.-J. Wang

Hong Zhang

(LOS ALAMOS NATTONAL LABORATORY)

2232M Anomalous X-ray Scattering of $\mathbf{R b}$ - and $\mathbf{Z r}$-Containing Glasses

Bernhard Himmel

Thomas Gerber

(UNIVERSITÄT ROSTOCK)

2233V New XAFS Spectroscopic Investigations in the 1-2 keV Region

Joe Wong

Gordon E. Brown, Jr.

Michael Fröba

Zofia U. Rek

Michael Rowen

Eiichi Tamura

Grayson H. Via

(LAWRENCE LIVERMORE NATIONAL LABORATORY) 
Edwin L. Alderman

John E. Atwood

George S. Brown

Byron W. Brown

L. Dean Chapman

Nicholas F. Gmur

Helen J. Gordon

William M. Lavender

Stephen N. Oesterie

Edward Rubenstein

William C. Thomlinson

Albert C. Thompson

Charles L. Troxel

Herbert D. Zeman

(DEPARTMENT OF VETERANS AFFAIRS MEDICAL CENTER)

2235M Molecular Routes to Crystalline Complex Metal Oxides with Controlled Stoichiometry Mark J. Hampden-Smith

Leo B. Archer

(UNIVERSITY OF NEW MEXICO)

2237Mp Studies of Actinides by X-ray Absorption Spectroscopy

David K. Shuh

Richard A. Andersen

Jerome J. Bucher

Norman M. Edelstein

Heino Nitsche

(LAWRENCE BERKELEY LABORATORY)

2238Bp EXAFS of Novel Forms of the Nitrogenase Enzyme

Brian J. Hales

Jason H. Christiansen

Stephen P. Cramer

Roland C. Tittsworth

(LOUISIANA STATE UNIVERSITY)

2239Vp Fe XAS L 2,3-edge Studies of Non-Heme Iron Enzymes

Keith O. Hodgson

Britt Hedman

Susan E. Shadle

Edward I. Solomon

Tami E. Westre

Kendra R. Williams

(STANFORD SYNCHROTRON RADIATION LABORATORY)

2240Bp Solution Small-Angle X-ray Scattering Studies of the Heat Shock Cognate Protein David B. McKay

Sebastian Doniach

David Eliezer

Keith O. Hodgson

Hirotsugu Tsuruta

Sigurd M. Wilbanks

(STANFORD UNIVERSITY)

2241Bp XAS Studies of Nitrogenase, Its Cofactor and a Cofactor Cluster

Barbara K. Burgess

Britt Hedman

Keith O. Hodgson

H. Isaac Liu

$\mathrm{Li} \mathrm{Ma}$

(UNIVERSITY OF CALIFORNIA at IRVINE) 
XAS Study of Phase Transitions in $\mathrm{La}_{2} \mathrm{NiO}_{4}$

Juana V. Acrivos

Angelica Alvarado

Joy C. Andrews

Charles M. Burch

Lei Chen

C. Jiang

H. Shen

(CALIFORNIA STATE UNIVERSITY at SAN JOSE)

2243Vp Application of Soft X-ray Emission Magnetic Circular Dichroism to Magnetic Multilayers and Other Magnetic Systems

Joseph E. Nordgren

Laurent C. Duda

Joachim Stöhr

(UPPSALA UNIVERSITET)

$2244 \mathrm{M}$ Depth-Controlled Grazing-Incidence Scattering of $\mathrm{TiO}_{2}$ - and $\mathrm{ZrO}_{2}-\mathrm{SiO}_{2}$ Thin Films

Thomas Gerber

Bernhard Himmel

(UNIVERSITÄT ROSTOCK)

2245Mp Interface Structure and Phase Transitions in MBE-Grown Heterostructures

Christopher A. Lucas

Sean M. Brennan

David Loretto

Gerard Wong

(LAWRENCE BERKELEY LABORATORY)

2246Mp X-ray Absorption Studies of Transition Metal Ion Adsorption on Semiconductor Surfaces Nathan S. Lewis (CALIFORNIA INSTITUTE OF TECHNOLOGY)

2247M Synchrotron Scattering Studies of Biomolecular Materials

Cyrus R. Safinya

Stefan H.J. Idziak

Ilya V. Koltover

Keith E. Kraiser

Heidi E. Warriner

(UNIVERSITY OF CALIFORNIA at SANTA BARBARA)

2248Mp X-ray Diffraction Studies of Laser Heated Molecules in a Diamond Anvil Cell

Choong-shik Yoo

Jagannadh Akella

Malcolm F. Nicol

(LAWRENCE LIVERMORE NATIONAL LABORATORY)

2249Mp X-ray Micro-Tomography with a Diamond Anvil Cell

Samuel T. Weir

Jagannadh Akella

(LAWRENCE LIVERMORE NATIONAL LABORATORY)

2250M A Differential Anomalous Scattering Study of Ionomers

Brian P. Grady

(UNIVERSITY OF OKLAHOMA)

2251M Transmutation and Radiation Effects in Crystalline Waste Forms

Nancy J. Hess

Farrel W. Lytle

(PACIFICNORTHWEST LABORATORIES) 
2252Mp In Situ X-ray Scattering from Single Crystal Electrode Interfaces

Nenad M. Markovic

Hubert A. Gasteiger

Philip N. Ross

(LAWRENCE BERKELEY LABORATORY)

2253Mp Structural Characterization of Organocuprate Complexes in Solution

James E. Penner-Hahn

Terrence M. Barnhart

Hui Huang

Paul Knochel

Gerard van Koten

(UNIVERSITY OF MICHIGAN)

2254M Structural Studies of Manganese-Doped Bismuth Vanadate Yonic Conductors

Allan J. Jacobson

Joseph Di Carlo

Ingrid J. Pickering

Longbao Qiu

(UNIVERSITY OF HOUSTON)

2255M XAS Characterization of Mn in Naturally- and Artifically-Irradiated Single Crystals Glenn A. Waychunas

George R. Rossman

(STANFORD UNIVERSITY)

2256Mp X-ray Absorption Spectroscopy of Sorbed Actinides and Fission Products

Eric A. Hudson

Susan A. Carroll

Carlos A. Colmenares

Louis J. Terminello

Brian E. Viani

George D. Waddill

(LAWRENCE LIVERMORE NATIONAL LABORATORY)

2257Bp High Resolution Mn KB Fluorescence

Stephen P. Cramer

Melissa M. Grush

Jeffrey A. Moore

Gang Peng

Clayton R. Randall

Xin Wang

(UNIVERSITY OF CALIFORNIA at DAVIS)

2258Vp Soft X-ray Absorption Spectroscopy of Mn Proteins

Stephen P. Cramer

Jie Chen

Melissa M. Grush

(UNIVERSITY OF CALIFORNIÄ at DAVIS)

2260Bp XAS Structural Characterization of NO Adducts of Hemes

Peter A. Lay

Robert S. Armstrong

Britt Hedman

Keith O. Hodgson

Anne M. Rich

(UNIVERSITY OF SYDNEY)

2261Mp Sorption and Coprecipitation of Arsenate and Chromate in Alkaline Environments Samuel J. Traina

Satish C.B. Myneni

Glenn A. Waychunas

(OHIO STATE UNIVERSITY) 
2262Bp XAS Structural Characterization of Rationally Designed Metalloproteins

Homme W. Hellinga

Britt Hedman

Keith O. Hodgson

Thane Morgan

(DUKE UNIVERSITY)

2263B X-ray Absorption Spectroscopy of Metal Substituted Iron-Sulfur Clusters in Pyrococcus furiosus

Ferredoxin

Michael W.W. Adams

Graham N. George

Ingrid J. Pickering

Roger C. Prince

(UNIVERSTYY OF GEORGIA)

2264Mp X-ray Diffraction Tomographic Microscopy of Polycrystalline Al-Li 2090

Stuart R. Stock

Zofia U. Rek

(GEORGIA INSTITUTE OF TECHNOLOGY)

2265B Structural Characterization of Metallacrown Complexes

James E. Penner-Hahn

Kelly A. Daly

Brian R. Gibney

Vincent L. Pecoraro

Ann Stemmler

David L. Tiemey

(UNIVERSITY OF MICHIGAN)

2266M Structural Characterization of Mercury on Silicate Glasses

James E. Penner-Hahn

Terrence M. Barnhart

Eric Thaler

(UNIVERSITY OF MICHIGAN)

2267B Structural Characterization of de Novo Designed Peptides

James E. Penner-Hahn

Gregg Dieckmann

William De Grado

Susan Heilman

Vincent L. Pecoraro

Timothy L. Stemmler

David L. Tierney

(UNIVERSITY OF MICHIGAN)

2268Mp Structure-Property Relationships of Scintillator Glasses

David L. Blanchard

Mary Bliss

Richard Craig

Farrel W. Lytle

(PACIFIC NORTHWEST LABORATORIES)

2269Mp XAS Study of Metal Ion Partitioning at Clay-Water Interfaces

Kim F. Hayes

Charalambos Papelis

(LAWRENCE BERKELEY LABORATORY)

2270M The Valence and Local Structure of $\mathrm{Mn}$ in $\mathrm{Al}, \mathrm{Mn}$-oxides Formed at the Birnessite/Solution Interface Scott E. Fendorf

Mark J. Fendorf

(UNIVERSITY OF IDAHO) 
2271Bp Time-Resolved Small-Angle X-ray Scattering Studies of the Refolding of Sperm Whale Apomyoglobin

Patricia A. Jennings

Sebastian Doniach

David Eliezer

Keith O. Hodgson

Hirotsugu Tsuruta

Peter E. Wright

(THE SCRIPPS RESEARCH INSTTTUTE)

2272M In Situ XAFS of Electrode Materials for Advanced Rechargeable Battery Systems

Daniel A. Scherson

Donald A. Tryk

(CASE WESTERN RESERVE UNIVERSITY)

2273Bp Small Angle X-ray Scattering on Reconstituted Chromatins

Joe M. Gatewood

E. Morton Bradbury

Brian S. Imai

Susan Krueger

Peter M. Yau

(LOS ALAMOS NATIONAL LABORATORY)

2274Mp Interface Structure in Giant Magnetoresistive Materials

Steven D. Conradson

Patrick G. Allen

James Brug

Janice H. Nickel

(LOS ALAMOS NATIONAL LABORATORY)

2275Mp Local Structure in High Temperature Superconductors

Steven D. Conradson

Patrick G. Allen

José Mustre De Leon

Janice H. Nickel

(LOS ALAMOS NATIONAL LABORATORY)

2276Mp Improvements in the Processing and Storage of Nuclear Materials

Steven D. Conradson

Patrick G. Allen

Lawrence E. Cox

James McNeese

Kirk D. Veirs

(LOS ALAMOS NATTONAL LABORATORY)

2277B X-ray Scattering by Myosin Subfragment 1

Robert Mendelson

Satoru Fujiwara

James Spudich

(UNIVERSITY OF CALIFORNIA at SAN FRANCISCO)

2278B XANES of Manganese in Photosystem II

Melvin P. Klein

Roehl M. Cinco

Matthew J. Latimer

Wenchuan Liang

Taka-aki Ono

Theo A. Roelofs

Kenneth H. Sauer

Vittal K. Yachandra

(LAWRENCE BERKELEY LABORATORY) 
2279Vp 1s Shake-Up Satellites in Third Period Elements

Adriano Filipponi

Andrea Di Cicco

Britt Hedman

Keith O. Hodgson

(UNIVERSITA DEGLI STUDI DELL'AQUILA)

2280M X-ray Studies of Interfacial Structure of the Multilayers

Xiao Yan

Alfonso Cebollada

Robin Farrow

Yi He

(HONG KONG UNIVERSITY OF SCIENCE)

2281M Thermal Vibrations in Single Crystal $\mathrm{YBa}_{2} \mathrm{Cu}_{3} \mathrm{O}_{7}$ Through $\mathrm{T}_{c}$

Peter Wochner

John R. Arthur

W. Hardy

Simon C. Moss

(BROOKHAVEN NATIONAL LABORATORY)

2282V A XAS Study of the Mg K-edge of Omphacites

Augusto Marcelli

Calegero Natoli

Eleonora Paris

Joe Wong

Ziyu Wu

(UNIVERSITÀ DI ROMA)

2283Mp EXAFS Investigation of Carbon Supported Catalysts

Robert M. Friedman

Jerry Ebner

Farrel W. Lytle

(MONSANTO COMPANY)

2284Bp EXAFS Studies of LTA $_{4}$ Hydrolase and Its Enzyme Inhibitors

Robert M. Friedman

Leslie Askonas

Farrel W. Lytle

(MONSANTO COMPANY)

2285Mp Resonant X-ray Diffraction in Self-Intermetallic Compounds: $\alpha-\mathrm{Mn}$ and $\beta-\mathrm{UH}_{3}$

Andrew C. Lawson

(LOS ALAMOS NATIONAL LABORATORY)

2286Mp Solvation of Metal Ions and Complexes in Solution

Ingmar N. Persson

Magnus K. Sandström

(SWEDISH UNIVERSITY OF AGRICULTURAL SCIENCES)

2287Mp A Study of High-Pressure Transitions to Megabars Using Diamond-Anvil Pressure Cell

Li Chung Ming

A. Jayaraman

Murli H. Manghnani

(UNIVERSITY OF HAWAII)

2288Mp Structure of Cd(S, Se) Nanocrystals Grown in Glass

Timothy $M$. Hayes

Peter D. Persans

(RENSSELAER POLYTECHNIC INSTITUTE) 
MAD Phasing of Selenomethionyl $\mathrm{Ca}^{2+}$-Free Mannose-Binding Protein C William I. Weis

Kenneth Ng

(STANFORD UNIVERSITY)

2290Vp Application of Thin Polymeric Films to X-ray Optics

Stephen F. Powell

(ALCHEMIST TECHNOLOGIES)

2291Mp X-ray Absorption Spectroscopy of Incorporated Neptunium in Mammals

Norman M. Edelstein

Patricia W. Durbin

David K. Shuh

(LAWRENCE BERKELEY LABORATORY)

2292Bp Dinuclear $\mathbf{O}_{2}$-Activation Site of the Cytochrome Oxidase Superfamily

Robert A. Scott

Jun Dong

Robert B. Gennis

Jon Rumbley

Hui Zhang

(UNIVERSITY OF GEORGIA)

2293Mp X-ray Absorption Spectroscopy of Ferroelectric Perovskites

I-wei Chen

Ping $\mathrm{Li}$

James E. Penner-Hahn

Ying Wang

(UNIVERSITY OF MICHIGAN)

2294B XAS Study of Carbonmonoxymyoglobin Crystals and Solutions

Hans C. Freeman

Paul J. Ellis

Britt Hedman

Keith O. Hodgson

Dashuang Shi

(UNIVERSITY OF SYDNEY)

2295Mp X-ray Spectroscopic Determination of Analyte Interactions in Solids for Spectrochemical Analysis

David L. Styris

Richard Gordon

Farrel W. Lytle

(PACIFIC NORTHWEST LABORATORIES)

2296Mp Complexation Chemistry Applicable to Cesium Extraction from Acidic Nuclear Waste Streams

Lynda C. Soderholm

Mark L. Dietz

E.P. Horwitz

Suntharalingam Skanthakumar

Urs Staub

(ARGONNE NATIONAL LABORATORY)

2297Mp Coordination of Lanthanides in Heteropolyanions

Mark R. Antonio

Suntharalingam Skanthakumar

Lynda C. Soderholm

(ARGONNE NATIONAL LABORATORY) 
2298Mp Structure-Catalytic Property Relationships of Supported Metal Clusters

Bruce C. Gates

Oleg Alexeev

Steven E. Deutsch

Rolf Jentoft

Sibudjing Kawi

Bill Weber

Feng-shou Xiao

Ailian Zhao

(UNIVERSITY OF CALIFORNIA at DAVIS)

2299B Structural Determination of the Two NiFe Clusters in CODH from Clostridium thermoaceticum

Corie Y. Ralston

Stephen P. Cramer

Manoj Kumar

Stephen Ragsdale

(UNIVERSITY OF CALIFORNIA at DAVIS)

2300M XAS Study of Phase Transitions in $\mathrm{NbO}_{1.1}$

Juana V. Acrivos

Joy C. Andrews

Charles M. Burch

Lei Chen

E. Mpock

Craig M. Strehlow

S. Yem

(CALIFORNIA STATE UNIVERSITY at SAN JOSE)

2301Bp Bioaccummulation of Heavy Metals by Plants

David E. Salt

Ingrid J. Pickering

Roger C. Prince

(RUTGERS UNIVERSITY)

2302Mp Deep Etch X-ray Lithography: The LIGA Technique

Reid A. Brennen

Charles E. Bryson

Michael H. Hecht

Jill Hruby

Keith H. Jackson

Tom W. Kenny

Piero A. Pianetta

(LAWRENCE BERKELEY LABORATORY)

2303Bp Cu XAS Studies of the Multicopper Oxidases

Edward I. Solomon

Britt Hedman

Keith O. Hodgson

Timothy E. Machonkin

Woonsup Shin

Uma M. Sundaram

Holly Zhang

(STANFORD UNIVERSITY) 
2304Bp Halide XAS Studies of the Photosynthetic Water Oxidation Complex

Melvin P. Klein

Joy C. Andrews

Roehl M. Cinco

Holger Dau

Matthew J. Latimer

Wenchuan Liang

Theo A. Roelofs

Annette F. Rompel

Kenneth H. Sauer

Vittal K. Yachandra

(LAWRENCE BERKELEY LABORATORY)

2305Bp Fe XAS K-edge Studies of Non-Heme Iron Enzymes

Keith O. Hodgson

Britt Hedman

Edward I. Solomon

Tami E. Westre

(STANFORD SYNCHROTRON RADIATION LABORATORY)

2306M XAS Study of Derivatives of $\mathrm{Hg}_{1-\mathrm{x}} \mathrm{Mo}_{\mathrm{x}} \mathrm{Sr}_{2} \mathrm{CuO}_{4},(\mathrm{x}=\mathbf{0 . 2 - 0 . 5 )}$

Charles M. Burch

Juana V. Acrivos

Joy C. Andrews

Lei Chen

Vidula Kirtikar

Kaushal K. Singh

(CALIFORNIA STATE UNIVERSITY at SAN JOSE)

2307Vp Investigation of Nanoscale Magnetic Structure with Photoelectron MXCD

James G. Tobin

Roger G. Carr

George D. Waddill

(LAWRENCE LIVERMORE NATIONAL LABORATORY)

2308Mp Characterization of Catalysts and Petroleum by X-ray Absorption and Scattering Using Synchrotron

Radiation

Guang Zhang

Ronald Medrud

Gary S. Mondo

(CHEVRON RESEARCH \& TECHNOLOGY COMPANY)

2309M Thermal Studies of Ceramics

E. Daryl Crozier

Moyra-Kathleen McManus

(SIMON FRASER UNIVERSITY)

2310V Photoemission Spectra for n-CuInSe 2 and p-CuISe 3 Based Heterostructures

Shalini Menezes

(INTERPHASES RESEARCH)

2311Bp SAXS Studies of Protein Folding Intermediates

Tony L. Fink

Lingling Chen

Sebastian Doniach

Keith O. Hodgson

Daniel J. Segel

Sangita Seshadri

Anupam Talapatra

Hirotsugu Tsuruta

(UNIVERSITY OF CALIFORNIA at SANTA CRUZ) 
2312Mp Microsecond Time-Resolved X-ray Spectroscopy

Stephen P. Cramer

Gang Peng

Hongxin Wang

(UNIVERSITY OF CALIFORNIA at DAVIS)

2313M In Situ Quick XAFS Spectroscopic Studies of DCL Catalysts

Naresh Shah

Gerald P. Huffman

Frank E. Huggins

Fulong Lu

Jianmin Zhao

(UNIVERSITY OF KENTUCKY)

2314Vp Interface Studies of Metal-Matrix Composites

Richard A. Rosenberg

Changyoung Kim

John Noonan

Piero A. Pianetta

Jian Ku Shang

(ARGONNE NATIONAL LABORATORY)

2315B MAD Diffraction Data Collection from Cytochrome Reductase Crystals

Edward A. Berry

Vladimir M. Shulmeister

(LAWRENCE BERKELEY LABORATORY)

2316Mp Grazing Incidence XAS of Sol-Gel Films

Russell F. Howe

Ingrid J. Pickering

(UNIVERSITY OF NEW SOUTH WALES)

2317Mp A XAS Study of Pyrope Garnets

A. Mottana

Gabriele Giuli

Augusto Marcelli

Eleonora Paris

Fritz Seifert

Ziyu Wu

(TERZA UNIVERSITA)

2318Mp Structure-Solubility Studies of Uranium Solids Synthesized Under Simulated Environmental Conditions Patrick G. Allen

Linda N. Marquez

Cynthia E.A. Palmer

(LAWRENCE LIVERMORE NATIONAL LABORATORY)

2319Mp X-ray Absorption Fine Structure Studies of Multilayers of High Temperature Superconductors Frank G. Bridges Corwin H. Booth

James B. Boyce

Tord C. Claeson

Theodore H. Geballe

(UNIVERSITY OF CALIFORNIA at SANTA CRUZ)

2320V Faraday Rotation of Soft X-rays in Magnetic Multilayers

Roger G. Carr

Jeffrey B. Kortright

Marybeth A. Rice

(STANFORD SYNCHROTRON RADIATION LABORATORY) 
2321Bp Synchrotron Radiation Detector Development Program

William $K$. Warburton

Mark E. Daly

Bradley Hubbard

Yuan Kong

Carl Zhou

(X-RAY INSTRUMENTATION ASSOCIATES)

2322B High Resolution X-ray Fluorescence Spectroscopy of Transition Metal Coordination Complexes James E. Penner-Hahn

Kelly A. Daly

(UNIVERSITY OF MICHIGAN)

2323B Structural Characterization of Vanadium Peptide Complexes in Solution

James E. Penner-Hahn

Yvette Boyajian

Charles Corman

Kelly A. Daly

Hui Huang

Molly Meixner

(UNIVERSITY OF MICHIGAN)

2324Bp Solution Scattering from Compact, Denatured Forms of Staphylococcal Nuclease

Eaton E. Lattman

Apostolos G. Gittis

(JOHNS HOPKINS UNIVERSITY)

2325Mp Coordination and Bonding of Metal Ions in Natural Soils and Sediments Using XAS

Peggy A. O'Day

Dennis Bird

Susan A. Carroll

Philip S. Neuhoff

Brian Phillips

Phillip A. Sterne

Glenn A. Waychunas

(ARIZONA STATE UNIVERSITY)

2326Mp X-ray Absorption Spectroscopy of Sorbed Uranium and Thorium

Eric A. Hudson

Carlos A. Colmenares

Dale Perry

Louis J. Terminello

Brian E. Viani

(LAWRENCE LIVERMORE NATTONAL LABORATORY)

2327Mp Determination of Chemical Speciation for TWRS Pre-Treatment R\&D

Steven D. Conradson

David L. Blanchard

Richard Gordon

David L. Styris

(LOS ALAMOS NATIONAL LABORATORY)

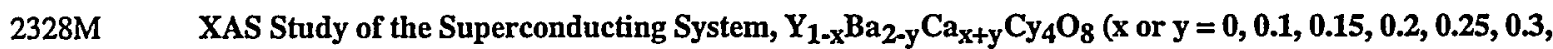
$0.35,0.4$ and 0.5 )

Charles M. Burch

Juana V. Acrivos

Joy C. Andrews

Lei Chen

Kaushal K. Singh

A.P.B. Sinha

(CALIFORNIA STATE UNIVERSITY at SAN JOSE) 
2329Mp Metal Ion Clusters at Oxide-Water Interfaces: Structure and Kinetics of Formation Hillary A. Thompson

John R. Bargar

Gordon E. Brown, Jr.

Singfoong Cheah

Jeffrey P. Fitts

Andrea L. Foster

John D. Ostergren

George A. Parks

Maria L. Peterson

Steven N. Towle

(STANFORD UNIVERSITY)

2330Mp Environmental Geochemistry of Chromium, Selenium and Arsenic

Gordon E. Brown, Jr.

James A. Davis

Jeffrey P. Fitts

Andrea L. Foster

George A. Parks

Maria L. Peterson

Tracy Tingle

Tetsu K. Tokunaga

Glenn A. Waychunas

Art White

(STANFORD UNIVERSITY)

2331Mp High-Resolution EXAFS Investigation of $\mathrm{MnO}_{2}$ Spinel-Based, $\mathrm{Li}^{+}$-Intercalation Battery Electrodes Craig R. Horne

Elton Cairns

Stephen P. Cramer

Melissa M. Grush

(LAWRENCE BERKELEY LABORATORY)

2332Vp Formation and Nature of Negative Electron Affinity on GaAs(100) and GaAs(110) Surfaces

Huan Tang

Renyu Cao

(STANFORD LINEAR ACCELERATOR CENTER)

2333Mp Sorptive Properties of Soil Carbonates for Ni, Se and Mn

Harvey E. Doner

Gordon E. Brown, Jr.

Mavrik Zavarin

(UNIVERSITY OF CALIFORNIA at BERKELEY)

2334Mp Sorption of Plutonium on Geologic Materials and Microbes

Iham Al Mahamid

Anne Happel

Eric A. Hudson

Louis J. Terminello

(LAWRENCE BERKELEY LABORATORY)

2335Mp Development of Soft X-ray Optical Rotation as a Tool for Measuring Magneto-Optical Effects Marybeth A. Rice

Roger G. Carr

Jeffrey B. Kortright

(LAWRENCE BERKELEY LABORATORY)

2336Mp XAFS Studies of High Valent Metal Cations in Silicate Liquids

Gordon E. Brown, Jr.

Francois M. Farges

Georges Calas

(STANFORD UNIVERSITY) 


\section{RAPID TURNAROUND EXAFS PROPOSALS}

1E12M K-edge of S(2472 eV) to Discern the Oxidation State of Sulfur in Soil Organic Matter and Iron Sulfide Oxidation Products

Scott E. Fendorf

Paul A. McDaniel

Matthew J. Morra

(UNIVERSITY OF IDAHO)

1E13M Electronic and Physical Structure of Titanium Fluorides

Ronald G. Cavell

Graham N. George

Ingrid J. Pickering

(UNIVERSITY OF ALBERTA)

1E14M XAS Characterization of Supported Metal Clusters with Nearly Molecular Structures

Bruce C. Gates

Steven E. Deutsch

Sibudjing Kawi

Zhengtian $\mathrm{Xu}$

Ailian Zhao

(UNIVERSITY OF CALIFORNIA at DAVIS)

1E15M XAS of Strontium and Cesium Sorbed on Layer Silicates

Eric A. Hudson

Louis J. Terminello

Brian E. Viani

(LAWRENCE LIVERMORE NATIONAL LABORATORY)

1E16M EXAFS Study of Trace Element Coordination in Natural Samples at Ambient and Cryogenic

Temperatures

Peggy A. O'Day

William L. Bourcier

Susan A. Carroll

Brian L. Phillips

Glenn A. Waychunas

(ARIZONA STATE UNIVERSITY)

1E17M EXAFS and Edges of Zn Model Compounds

Jason H. Christiansen

Stephen P. Cramer

Melissa M. Grush

Corie Y. Ralston

(UNIVERSITY OF CALIFORNIA at DAVIS)

1E18M Di- and Tetranuclear Mo and W Complexes Supported on $\boldsymbol{\gamma}$-Alumina

Gustavo Larsen

Ruben Parra

(UNIVERSITY OF NEBRASKA)

1E19M Probing Vanadium Valence and Coordination in Selected Minerals with K-edge Absorption Spectroscopy Ronald G. Cavell

Graham N. George

Ingrid J. Pickering

(UNIVERSITY OF ALBERTA)

1E20M EXAFS Studies of Electrode Materials for Lithium Batteries

Melissa M. Grush

Elton Cairns

Stephen P. Cramer

Craig R. Horne

(UNIVERSITY OF CALIFORNIA at DAVIS) 
$1 E 21 M$

EXAFS of Ion-Containing Polymers

Brian P. Grady

W. Berlin Genetti

(UNIVERSTTY OF OKLAHOMA)

1E22M XAS Study of Dodecahedral Cation Site in Pyrope

A. Mottana

Fritz Seifert

Gabriele Giuli

Eleonora Paris

Ziyu Wu

Augusto Marcelli

(TERZA UNIVERSITA)

1E23M Investigation of the Metal-Binding Sites on the Cell Walls of Datura innoxia Suspension Culture Cells Jane G. DeWitt

Paul J. Jackson

(LOS ALAMOS NATIONAL LABORATORY)

\section{ROTATION CAMERA PROPOSALS}

2A13B $\quad \alpha_{2}$-Macroglobulin Complexes Suitable for Crystallographic Studies Using Synchrotron Radiation Soren S. Thirup

Gregers Rom Andersen

Trine J. Koch

Jens Nyborg

Anders H.P. Sorensen

(AARHUS UNIVERSITY)

2A14B Structural Studies of Type IDNA Topoisomerases

Alfonso Mondragon

Valerie L. Grum

Christopher D. Lima

Neal F. Lue

Amit P. Sharma

(NORTHWESTERN UNIVERSITY)

2A19B Crystallographic Study of Human Recombinant Factor XII

Vivien Yee

Ethan A. Merritt

Ronald E. Stenkamp

David C. Teller

(UNIVERSTTY OF WASHINGTON)

2A29B High Resolution Structural Studies of Crystalline Pilin

Katrina T. Forest

Elizabeth D. Getzoff

Michael J. Hickey

John A. Tainer

(THE SCRIPPS RESEARCH INSTITUTE)

2A40B Data Collection of Proteasome Crystals

Bing K. Jap

Wolfgang Baumeister

(LAWRENCE BERKELEY LABORATORY)

2A41B Crystallization of a Trimeric DNA-binding Protein in Complex with DNA

Hillary C.M. Nelson

Karen E. Flick

Otis B. Littlefield

(UNIVERSITY OF CALIFORNIA at BERKELEY) 
2A46B X-ray Diffraction Investigation of Cytochrome Reductase Crystals Edward A. Berry

Vladimir M. Shulmeister

(LAWRENCE BERKELEY LABORATORY)

2A47B Crystallographic Analyses of (Mostly) Electron Transfer Proteins

Douglas C. Rees

Michael K. Chan

Leemor Joshua-Tor

Timothy M. McPhillips

Jamie Schlessman

Michael H.B. Stowell

(CALIFORNIA INSTITUTE OF TECHNOLOGY)

2A50B X-ray Crystallographic Studies of Methane Monooxygenase

Stephen J. Lippard

Christin A. Frederick

Pär Nordlund

Amy C. Rosenzweig

(MASSACHUSETTS INSTITUTE OF TECHNOLOGY)

2A51B The Crystal Structure of Colicin Ia: Determination by Cryocrystallography

Michael C. Wiener

Paul G. Foster

Douglas M. Freymann

Veerappa P. Ramalingam

Earl E. Rutenber

(UNIVERSITY OF CALIFORNIA at SAN FRANCISCO)

2A52B Data Collection for Thymidylate Synthase from Pneumocystis carinii Kathleen M. Perry

Diana B. Cherbavaz

Douglas M. Freymann

Michael C. Wiener

(UNIVERSITY OF CALIFORNIA at SAN FRANCISCO)

2A53B Structure of Spherical RNA Viruses, Theiler's Virus and Calicivirus

Ming Luo

Marek Jedrzejas

Kenneth S. Toth

Jun Tsao

Clinton L. White

Carl Zhang

Lan Zhou

(UNIVERSITY OF ALABAMA)

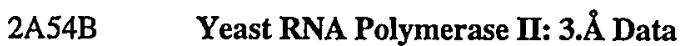

Peter R. David

David A. Bushnell

Kerstin K. Leuther

Gavin D. Meredith

(STANFORD UNIVERSITY)

2A55B Solution of a de Novo Crystal Structure of an Enzyme:Kinase Complex

Diana B. Cherbavaz

Douglas M. Freymann

Veerappa P. Ramalingam

Richard L. Wagner

Michael C. Wiener

(UNIVERSITY OF CALIFORNIA at SAN FRANCISCO) 
2A59B Bacterial Enterotoxins

Ethan A. Merritt

Wilhelmus G.J. Hol

Focco van den Akker

(UNIVERSITY OF WASHINGTON)

2A60B Data Collection of Pyruvate Dehydrogenase (Elp) and a 60mer E2pCD Component of the Pyruvate Dehydrogenase Multienzyme Complex (PDC)

Jörg Hendle

Shekhar C. Mande

Ethan A. Merritt

Xiayang Qiu

Gabrielle Rudenko

(UNIVERSITY OF WASHINGTON)

2A61B Structural Studies of Receptor Signalling: Crystal Structures of Hormone Receptor Complexes

Abraham M. deVos

Charles W. Eigenbrot

Anthony A. Kossiakoff

William S. Somers

(GENENTECH, INC.)

2A62B Structural Studies of Surface Glycoprotein from Tick-Borne Encephalitis Virus and of NFkB

Transcription Factor

Stephen C. Harrison.

Christoph W. Mueller

Felix A. Rey

(HARVARD UNIVERSITY)

Crystal Structure Determination of Botulinum Neurotoxin Type A (BTA)

Cara Marks

Peter R. David

Kenneth E. Goodwill

Reetta Raag

Ray C. Stevens

(UNIVERSITY OF CALIFORNIA at BERKELEY)

2A64B X-ray Data Collection on Amine Oxidase Crystals

Hans C. Freeman

Mitchell Guss

Ian Harvey

Daniel Shi

(UNIVERSITY OF SYDNEY)

2A65B Crystallographic Investigation of Human Cell Cycle Proteins

Michele A. McTigue

Steven Reed

(THE SCRIPPS RESEARCH INSTTTUTE)

2A66B Crystals of $\alpha_{2}$-Macroglobulin, Complement 3 and $3 \mathrm{~b}$ Complexes Suitable for Crystallographic Analysis Using Synchrotron Radiation

Soren S. Thirup

Gregers Rom Andersen

Anders Hanberg

Trine J. Koch

(AARHUS UNIVERSITY)

2A67B X-ray Crystallography of Catalytic Hammerhead RNA

David B. McKay

Kevin M. Flaherty

Heinz W. Pley

(STANFORD UNIVERSITY) 
2A68B X-ray Diffraction of Membrane Protein Complex Crystals - Mitochondrial Cytochrome $b$ - $c_{1}$ Complex Chang-an $Y u$

Johann Deisenhofer

Di Xia

(OKLAHOMA STATE UNIVERSITY)

2A69B Crystallographic Study of Immunodominant Proteins of Mycobacterium leprae and Mycobacterium tuberculosis

Shekhar C. Mande

Wilhelmus G.J. Hol

(UNIVERSITY OF WASHINGTON)

2A70B Structure Determination of Viruses

Michael G. Rossmann

Mavis Agbandje

Michael S. Chapman

Hok-kin Choi

Leodevico L. Ilag

Kyung Hyun Kim

Prasanna Kolatkar

Ladislau Z. Kovari

Robert McKenna

Cory Momany

Andrew $\mathrm{J}$. Prongay

(PURDUE UNIVERSITY)

2A71B X-ray Diffraction Analyses of Proteins of Immunological Interest

Lisa J. Harris

(UNIVERSITY OF CALIFORNIA at RIVERSIDE)

2A72B Crystallographic Studies of Human Topoisomerase I

Xiayang Qiu

James J. Champoux

(UNIVERSITY OF WASHINGTON)

2A73B Crystal Structures of Synthetic Metalloproteins

C.D. Stout

Reza Ghadiri

Enrico A. Stura

(THE SCRIPPS RESEARCH INSTITUTE)

2A74B Structural Studies of Reverse Transcriptase from the Human Immunodeficiency Virus

David W. Rodgers

Steven J. Gamblin

Barbara Harris

Stephen C. Harrison

(HARVARD UNIVERSITY)

2A75B Crystallographic Analysis of the Hormone Binding Domain (HBD) of the Thyroid Hormone Receptor (TR)

Richard L. Wagner

James Apriletti

Linda Brinen

Jenny Buchbinder

Diana B. Cherbavaz

Sarah Gillmor

Kai Lin

Mary E. McGrath

Veerappa P. Ramalingam

Elena P. Sablin

Brian West

Michael C. Wiener

(UNIVERSITY OF CALIFORNIA at SAN FRANCISCO) 
2A76B Intensity Data Collection on the Native Crystals of Human $\beta$-Glucuronidase, and Two Heavy Atom Derivatives

Sanjeev Jain

F. Scott Mathews

William S. Sly

(ST. LOUIS UNIVERSITY)

2A77B Regulatory Subunit of cAMP Dependent Protein Kinase

Kottayil I. Varughese

Susan S. Taylor

$\mathrm{Ng} \mathrm{H}$. Xuong

(UNIVERSITY OF CALIFORNIA at SAN DIEGO)

2A78B High Resolution Structures for the DNA Repair Enzyme Endonuclease III

Maria M. Thayer

John A. Tainer

(THE SCRIPPS RESEARCH INSTTTUTE)

2A79B Structural Studies on Myosin Subfragment-1

Ivan Rayment

Matthew M. Benning

Andrew J. Fisher

Hazel M. Holden

Clyde A. Smith

James B. Thoden

Joseph Wedekind

(UNIVERSITY OF WISCONSIN)

2A80B Structure of Pyruvate Kinase

Ivan Rayment

Todd Larsen

George Reed

(UNIVERSITY OF WISCONSIN)

2A81B

Crystallographic Studies of UDP Galactose 4-Epimerase

Hazel M. Holden

Matthew M. Benning

James B. Thoden

Janeen L. Vanhooke

(UNIVERSTTY OF WISCONSIN)

2A82B Key Enzymes in the Synthesis of Polyamines and Carbon Metabolism

Marvin L. Hackert

Ning Chang

Andrew D. Kern

Marcos A. Oliveira

(UNIVERSITY OF TEXAS)

2A83B Crystallographic Studies on the Human Protective Protein

Gabrielle Rudenko

Alessandra d'Azzo

Wilhelmus G.J. Hol

(UNIVERSITY OFWASHINGTON)

2A84B Crystallographic Analysis of Aldehyde Reductase/NADPH Binary Complex

(ALR1/NADPH)

Ossama A.L. El-Kabbani

Charles E. Bugg

Lawrence J. DeLucas

(UNIVERSITY OF ALABAMA) 
2A85B Crystallographic Structure Determination of the PRO Region of $\alpha$-Lytic Protease Stephen D. Rader (UNIVERSITY OF CALIFORNIA at SAN FRANCISCO)

2A86B Crystallographic Structure Determination of the Heat Shock Protein, HtpG Andrew Shiau (UNIVERSITY OF CALIFORNIA at SAN FRANCISCO)

2A87B Molecular Basis of Amyotrophic Lateral Sclerosis; Crystallographic Studies of Point Mutants of SOD

Susan M. Redford

Elizabeth D. Getzoff

(THE SCRIPPS RESEARCH INSTITUTE)

2A88B Crystallographic Studies of the Anthrax Lethal Toxin

Robert C. Liddington

Jadwiga Bienkowska

Carlo Petosa

(DANA FARBER CANCER INSTITUTE)

2A89B X-ray Crystallographic Studies of Human Antithrombin III Using Synchrotron Radiation with Frozen Crystals

Gloria E. Borgstahl

Teresa Brandt

John Griffin

(THE SCRIPPS RESEARCH INSTTTUTE)

2A90B HSOD Turn Mutant and Permutant Structures to Study the Greek Key $\beta$-Barrel Fold

Maria M. Thayer

Hedieh Badkoobehi

Elizabeth D. Getzoff

John A. Tainer

(THE SCRIPPS RESEARCH INSTTTUTE)

2A91B High Resolution Data Collection from Fab-Antigen

Ian A. Wilson

Jairo H. Arevalo

Matthew Haynes

Robyn L. Stanfield

Rashid Syed

(THE SCRIPPS RESEARCH INSTTTUTE)

2A92B Structure Determination of Proteins Involved in Signal Transduction

Sung-hou Kim

Andrew A. Bohm

Jeroen Brandsen

Li-wei Hung

Kathryn Phillips.

Ursula Schulze-Gahmen . . " .

Xiangwei Weng

(LAWRENCE BERKELEY LABORATORY)

2A93B Crystallographic Studies of Human Thrombin-Inhibitor Complexes

John S. Sack

Pat Bossart-Whitake

Chiey-ying Chang

Howard M. Einspahr

Shari Ohringer

Steven Sheriff

Lydia Tabernero

(BRISTOL-MYERS SQUIBB RESEARCH INSTITUTE) 
2A94B Evaluation/Data Collection of Cytochrome $c$ Oxidoreductase

Bing K. Jap

(LAWRENCE BERKELEY LABORATORY)

2A95B X-ray Diffraction Analysis of Plant Viruses

Nenad M. Ban

(UNIVERSITY OF CALIFORNIA at RIVERSIDE)

2A96B Crystallographic Studies of CHIP28, the Principal Human Water Channel

Michael C. Wiener

Douglas M. Freymann

Veerappa P. Ramalingam

Earl E. Rutenber

A. van Hoek

(UNIVERSITY OF CALIFORNIA at SAN FRANCISCO)

2A97B High Resolution Data Collection on Native and Complexed Sulfite Reductase Hemoprotein Brian R. Crane

Elizabeth D. Getzoff

(THE SCRIPPS RESEARCH INSTITUTE)

2A98B X-ray Diffraction Data Collection on Crystals of Glutathione S-Transferase with Bound Inhibitors Michele A. McTigue

(THE SCRIPPS RESEARCH INSTITUTE)

2A99B Crystallographic Studies on Quinolinate Phosphoribosyltransferase

Narayana Sthanam

(UNIVERSITY OF ALABAMA)

3A01B Structural Studies of NFkB Transcription Factor and a Type II DNA Topoisomerase

Stephen C. Harrison

James M. Berger

Christoph W. Mueller

(HARVARD UNIVERSITY)

3A02B Structure Determination of Proteins Involved in Signal Transduction

Sung-hou Kim

Walter Azevedo

Lan Huang

Li-wei Hung

Ursula Schulze-Gahmen

Xiangwei Weng

(LAWRENCE BERKELEY LABORATORY)

3A03B Measurement of Diffraction Intensities of the "High" Resolution Form of Native and MethylMercuryAcetate Derivative Crystals of the EF-Tu:EF-Ts Complex from E. coli Brian M. Schick

Nenad M. Ban

Lisa J. Harris

Frances Jurnak

(UNIVERSITY OF CALIFORNIA at RIVERSIDE)

3A04B X-ray Crystallographic Studies on Cell Cycle Proteins

Andy S. Arvai

Yves Bourne

Steven Reed

Joanne Stubbe

(THE SCRIPPS RESEARCH INSTITUTE) 
3A05B High Resolution Structures of Heme Containing Proteins

Shankari E. Mylvaganam

Elizabeth D. Getzoff

(THE SCRIPPS RESEARCH INSTTTUTE)

3A06B Structural Basis of C-type Lectin - Carbohydrate Interactions

William I. Weis

Kenneth Ng

(STANFORD UNIVERSITY)

3A07B Antibody Structure and Genetic Engineering

Clifford D. Mol

Andy S. Arvai

Elizabeth D. Getzoff

Michael J. Hickey

John A. Tainer

(THE SCRIPPS RESEARCH INSTTTUTE)

3A08B Structures of DNA Repair Enzymes and Enzyme-DNA Complexes

Clifford D. Mol

Andy S. Arvai

Richard P. Cunningham

H.E. Krokan

(THE SCRIPPS RESEARCH INSTITUTE)

3A09B Determination of the Structure of Ferrous Cytochrome $c$ Peroxidase

Duncan E. McRee

David B. Goodin

Gerard M. Jensen

(THE SCRIPPS RESEARCH INSTITUTE)

3A10B Structure of the N-terminal Domain of a Signal Sequence Recognition Protein

Douglas M. Freymann

Diana B. Cherbavaz

Robert J. Keenan

Veerappa P. Ramalingam

Robert M. Stroud

Michael C. Wiener

(UNIVERSITY OF CALIFORNIA at SAN FRANCISCO)

3A11B Crystallographic Studies of HIV and SIV Proteases in Complex with Non-Peptide Inhibitors Earl E. Rutenber

Charles Craik

(UNIVERSITY OF CALIFORNIA at SAN FRANCISCO)

3A12B Crystallography of the ned Motor Domain

Elena P. Sablin

F. Jon Kull

(UNIVERSITY OF CALIFORNIA at SAN FRANCISCO)

3A13B Analysis of the Kinesin Motor Domain

F. Jon Kull

Elena P. Sablin

(UNIVERSITY OF CALIFORNIA at SAN FRANCISCO) 
3A14B Structural Studies of dUMP Hydroxymethyltransferase from the Bacillius subtilis Phage SP01

Paul G. Foster

Diana B. Cherbavaz

Douglas M. Freymann

Stephen D. Rader

Veerappa P. Ramalingam

Tom Stout

Richard L. Wagner

Michael C. Wiener

(UNIVERSITY OF CALIFORNIA at SAN FRANCISCO)

3A15B X-ray Crystallographic Studies of MurZ Enzyme

Christin A. Frederick

Minfang Gong

Chris Walsh

(DANA FARBER CANCER INSTITUTE)

3A16B Structure of Covalently Modified Glutamine Synthetase

David Eisenberg

Gaston Pfluegl

(UNIVERSITY OF CALIFORNIA at LOS ANGELES)

3A17B Structure of BPI: Human Bactericidal/Permeability-increasing Protein

David Eisenberg

Lesa Beamer

(UNIVERSITY OF CALIFORNIA at LOS ANGELES)

3A18B Crystal Structure of Proteins: HIV-1 Matrix/Lrp/Rad 6/Yuhl/Yugl.Ubal

Christopher P. Hill

Dan Bancroft

Steve Johnston

Felix Vajdos

David Worthylake

(UNIVERSITY OF UTAH)

3A19B Data Collection of Heavy Atom Derivatives of Epstein Barr Virus Nuclear Antigen 1

Aled M. Edwards

Jean Barwell

Alexi Botchkarev

Lori Frappier

Hua Tong

Gerry Wright

(MCMASTER UNIVERSITY)

3A20B X-ray Crystallographic Studies of MurB and MTP Proteins

Howard M. Einspahr

Chiey-ying Chang

Bruce Jacobson

Shari Ohringer

John S. Sack

Steven Sheriff

(BRISTOL-MYERS SQUIBB RESEARCH INSTITUTE)

3A21B Structural Studies of Carbamoyl Phosphate Synthetase from Escherichia coli

Ivan Rayment

Matthew M. Benning

Andrew J. Fisher

Hazel M. Holden

Clyde A. Smith

James B. Thoden

Joseph Wedekind

(UNIVERSITY OF WISCONSIN) 
$\beta$-Complexed with Template-primer and ddCTP

Michael R. Sawaya

Russell F. Doolittle

Samuel H. Wilson

(UNIVERSITY OF CALIFORNIA at SAN DIEGO)

3A23B Crystallography of Ribosomes

Ada Yonath

Heike Bartels

Anat Bashan

William S. Bennett

Harly A.S. Hansen

Jörg Harms

Frank Schlünzen

(WEIZMANN INSTITUTE)

3A24B Crystal Structure of Human Protein Phosphatase 2A

Michael O. Skidmore

Hakon Hope

Joseph Kraut

Michael R. Sawaya

Gernot Walter

(UNIVERSITY OF CALIFORNIA at SAN DIEGO)

3A25B X-ray Diffraction Investigation of Cytochrome Reductase Crystals

Edward A. Berry

Vladimir M. Shulmeister

(LAWRENCE BERKELEY LABORATORY)

3A26B Structural Studies of the Human Transferrin Receptor

Martin Lawrence

(HOWARD HUGHES MEDICAL INSTTTUTE)

3A27B Structure Determination of the N-terminal SH2 Domain from GTPase Activating Protein (GAP) of ras Protein Complexed with Tyrosine-Phosphorylated Peptides

Sung-hou Kim

Li-wei Hung

Kyeong-kyn Kim

(LAWRENCE BERKELEY LABORATORY)

3A28B Structure Determination of Racemic $D-L$ Monellin in a Centrosymmetric Space

Sung-hou Kim

Li-wei Hung

(LAWRENCE BERKELEY LABORATORY)

3A29B Determination of the Structure of the High Affinity IgE Receptor from Mast Cells

Ted Jardetzky

(NORTHWESTERN UNIVERSITY)

3A30B Crystal Structure of Human Fibrinogen Fragment D and Complex

Russell F. Doolittle

Joseph Kraut

(UNIVERSITY OF CALIFORNIA at SAN DIEGO)

3A31B Structural Studies of the KlenTaq DNA Polymerase and Its Complexes with DNA

Sergey Korolev

Wayne Barnes

Gabriel Waksman

(WASHINGTON UNIVERSITY) 
Srinivasan Raghunathan

Timothy M. Lohman

Gabriel Waksman

(WASHINGTON UNIVERSITY)

3A33B Crystallographic Studies of the E. coli Single-Stranded DNA Binding Protein (SSB) in a Complex with Single-Stranded DNA

Srinivasan Raghunathan

Timothy M. Lohman

Gabriel Waksman

(WASHINGTON UNIVERSITY)

Extended Data from Anthranilate Phosphoribosyl Transferase and Cytochrome $c$ Peroxidase from Paracoccus denitrificans

Steven L. Edwards

C. Craig Hyde

Stanley E. Mills

Graham Pettigrew

Charles Yanofsky

(UNIVERSITY OF CALIFORNIA at DAVIS)

3A35B High Resolution Data Collection of Native Diheme Cytochrome $c$ Peroxidase from Nitrosomonas europaea Huiying $\mathrm{Li}$

David M. Arciero

Alan B. Hooper

(UNIVERSITY OF CALIFORNIA at IRVINE)

Structural Studies of Trypanosomal Glycolytic Enzymes

Hidong Kim

Bradley Bernstein

Wilhelmus G.J. Hol

Ethan A. Merritt

(UNIVERSTTY OF WASHINGTON)

3A37B X-ray Diffraction Analyses of Plant Viruses

Mary A. Canady

(UNIVERSITY OF CALIFORNIA at RIVERSIDE)

3A38B Crystallographic Analysis of Insect and Plant Viruses

John E. Johnson

Abhinav Kumar

Tianwei Lin

Padmaja Natarajan

Vijay Reddy

William R. Wikoff

(PURDUE UNIVERSITY)

3A39B Structure of GroEL and Its Complexes with Nucleotides

Paul Curmi

Nicholas Dixon

(UNIVERSITY OF NEW SOUTH WALES) 


\section{LETTERS OF INTENT}

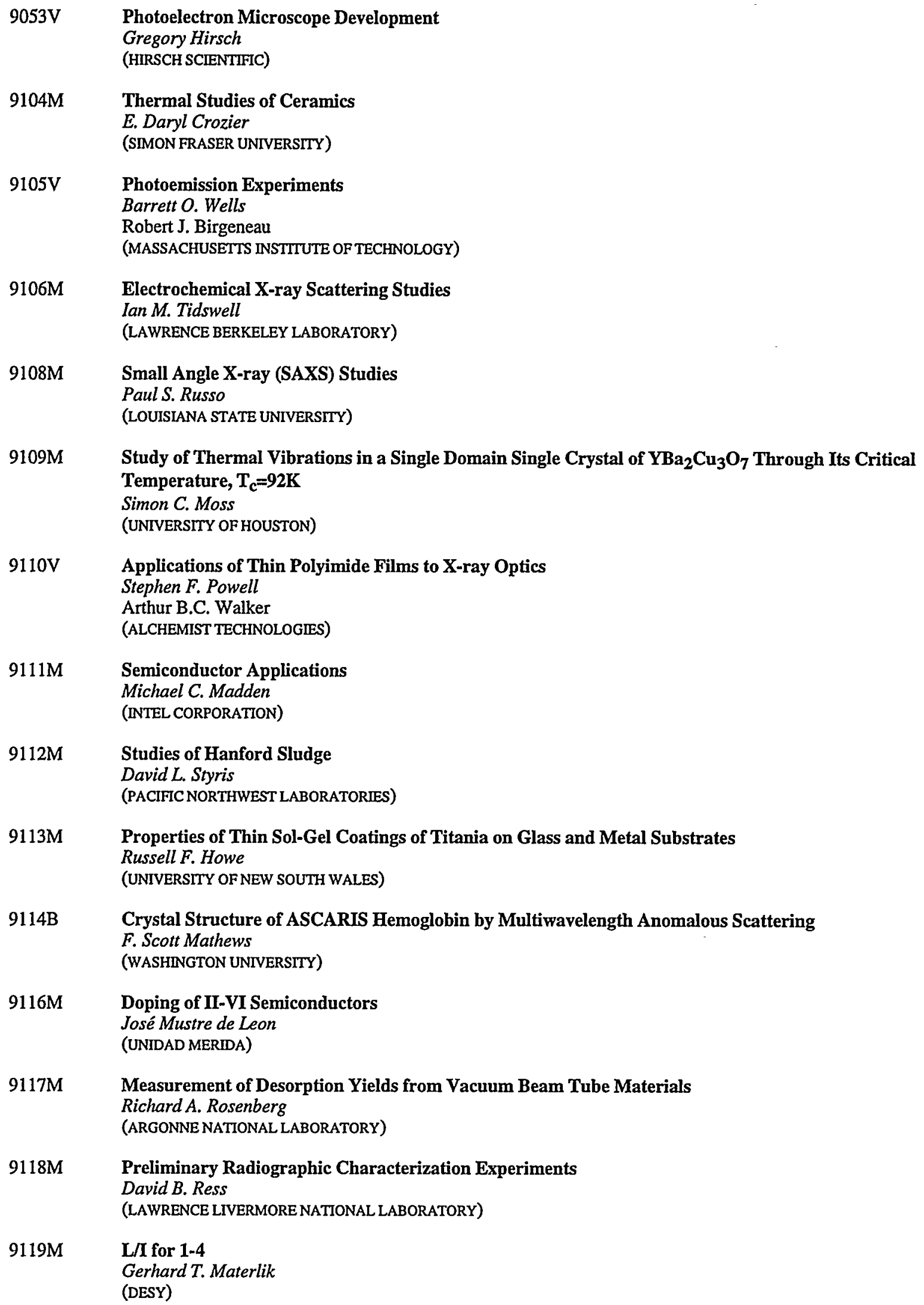

9109M Study of Thermal Vibrations in a Single Domain Single Crystal of $\mathrm{YBa}_{2} \mathrm{Cu}_{3} \mathrm{O}_{7}$ Through Its Critical Temperature, $\mathrm{T}_{\mathrm{c}}=92 \mathrm{~K}$

Simon C. Moss

(UNIVERSITY OF HOUSTON)

9110V Applications of Thin Polyimide Films to X-ray Optics Stephen F. Powell Arthur B.C. Walker (ALCHEMIST TECHNOLOGIES)

9111M Semiconductor Applications Michael C. Madden (INTEL CORPORATION)

9112M Studies of Hanford Sludge

David L. Styris

(PACIFIC NORTHWEST LABORATORIES)

9113M Properties of Thin Sol-Gel Coatings of Titania on Glass and Metal Substrates Russell F. Howe (UNIVERSITY OF NEW SOUTH WALES)

9114B Crystal Structure of ASCARIS Hemoglobin by Multiwavelength Anomalous Scattering F. Scott Mathews (WASHINGTON UNIVERSTTY)

$9116 \mathrm{M} \quad$ Doping of II-VI Semiconductors José Mustre de Leon (UNIDAD MERIDA)

9117M Measurement of Desorption Yields from Vacuum Beam Tube Materials Richard A. Rosenberg (ARGONNE NATIONAL LABORATORY)

9118M Preliminary Radiographic Characterization Experiments David B. Ress (LAWRENCE LIVERMORE NATIONAL LABORATORY)

9119M L/I for 1-4 Gerhard T. Materlik (DESY) 
9120V Calculation of Secondary Enhancement Effects of X-ray Fluorescence Radiation Due to Photoelectrons

Franz A.Weber

Michael K. Mantler

(LAWRENCE LIVERMORE NATIONAL LABORATORY)

9121V Comparison of MCD and Optical Rotation Near the FeL(II) and L(II) Edges

Jeffrey $B$. Kortright

Roger G. Carr

Marybeth A. Rice

(LAWRENCE BERKELEY LABORATORY)

9122B Thermal Unfolding of Staphylococcal Nuclease

Apostolos G. Gittis

(JOHNS HOPKINS UNIVERSITY)

9123B Mosaic Spread Measurements

Howard M. Einspahr

(BRISTOL-MYERS SQUIBB RESEARCH INSTITUTE)

9124M X-ray Crystallographic Studies of MurB and MTP Proteins

Howard M. Einspahr

(BRISTOL-MYERS SQUIBB RESEARCH INSTITUTE)

9125V Investigation of the Nature of Negative Electron Affinity on GaAs

Huan Tang

Renyu Cao

(STANFORD LINEAR ACCELERATOR CENTER)

9126V Investigation of the Bonding of Alkyl Monolayers to Si(111)

Christopher E. Chidsey

Renyu Cao

Jeffrey $\mathrm{H}$. Terry

(STANFORD UNIVERSITY)

9127M TLDS Studies

Nisy E. Ipe

Henry D. Bellamy

R. Paul Phizackerley

(STANFORD LINEAR ACCELERATOR CENTER)

9128V Measuring Absolute Binding Energies of Semiconductor Materials

Terrence Jach

Piero A. Pianetta

(NATIONAL INSTITUTE OF STANDARDS AND TECHNOLOGY)

9129V TXRF Analysis on A1

Robert Helms

(STANFORD UNIVERSITY)

9130B Crystallography of Ribosomes: MAD Phasing

Ada Yonath

(WEIZMANN INSTITUTE)

9131B

Preliminary' White Beam Laue Experiment on Lignin-Degrading Peroxidase

R. Paul Phizackerley

Steven L. Edwards

Michael H. Gold

S. Michael Soltis

(STANFORD SYNCHROTRON RADIATION LABORATORY)

9132B Solving the 3-D Structure of a Catalytic RNA Molecule

Sung-hou Kim

(LAWRENCE BERKELEY LABORATORY) 
Assess Siemens SMART Charged Coupled Device (CCD) Two-dimensional Position Sensitive Area Detector for Applications in Protein Crystallography

James C. Phillips

Henry D. Bellamy

Charles F. Campana

John L. Chambers

Anthony D. Cox

James F. Fait

Hiartmut Luecke

R. Paul Phizackerley

S. Michael Soltis

Robert A. Sparks

(SIEMENS INDUSTRIAL AUTOMATION)

9134B Preliminary White Beam Laue Experiment on Heat Shock Cognate ATPase Fragment

David B. McKay

Kevin M. Flaherty

R. Paul Phizackerley

S. Michael Soltis

Sigurd M. Wilbanks

(STANFORD UNIVERSITY)

9135B Multiple Anomalous Dispersion (MAD) on a Cytochrome C552 from T. thermophilus Duncan E. McRee

(THE SCRIPPS RESEARCH INSTTTUTE)

9136V X-ray Circular Magnetic Dichroism (XCMD)

Piero A. Pianetta

Changyoung Kim

(STANFORD SYNCHROTRON RADIATION LABORATORY)

9137B MAD Feasibility Studies of Mitochondrial Cytochrome Reductase

Edward A. Berry

Henry D. Bellamy

(LAWRENCE BERKELEY LABORATORY)

9138B Mycobacterial Heat Shock Proteins

Shekhar C. Mande

Vivien Yee

(UNIVERSTTY OF WASHINGTON)

9139V Study of Dielectric-semiconductor Interfaces

Zheng Hong $\mathrm{Lu}$

Renyu Cao

(UNIVERSITY OF TORONTO)

9140B X-ray Detector Tests

Stephen P. Cramer

Craig-R: Horne

Brenda J. Weiss

Xin Wang

Clayton R. Randall

(UNIVERSITY OF CALIFORNIA at DAVIS) 
Ercan Adem

Sean M. Brennan

Dennis Erho

Leigh Ann Files

Alice M. Fischer-Colbrie

Alexander Lessmann

Michael C. Madden

Piero A. Pianetta

Jamie Rose

Robert S. Smith

William E. Spicer

Norikatsu Takaura

Anne Testori

Ann Waldhauer

Mary Ann Zaitz

(HEWLETT PACKARD)

\section{PRT PROPOSALS}

9300V IBM-Station 10-1 Joachim Stöhr

Mahesh G. Samant

9400V CENTER FOR MATERIALS RESEARCH (Stanford University) -Station 10-1

Edward I. Solomon

Gordon E. Brown, Jr.

Renyu Cao

Tom Kendelewicz

Robert J. Madix

Piero A. Pianetta

William E. Spicer

Christer Wigren

9500M EXXON RESEARCH AND ENGINEERING - Station 6-2

Grayson H. Via

Richard E. Bare

Stephen D. Cameron

Russell R. Chianelli

Brian D. DeVries

Martin L. Gorbaty

Keng S. Liang

Gary McVicker

George D. Meitzner

Richard S. Polizzotti

Roger C. Prince

Michael Sansone

Paul A. Stevens

David Vaughan

9700V STANFORD UNIVERSITY -Beam Line 5

William E. Spicer

Daniel S. Dessau

Zhi-xun Shen

9900M NATIONAL LABORATORIES/UNIVERSITY OF CALIFORNIA -Beam Lines 8 \& 10

Lloyd L. Chase

Jagannadh Akella

A. Paul Alivisatos

Mehdi Balooch

Trow W. Barbee, Jr.

Frank G. Bridges

Barbara K. Burgess 
Carlos A. Colmenares

Steven D. Conradson

Stephen P. Cramer

Alex V. Hamza

Zahid Hussain

Alan F. Jankowski

Raymond Jeanloz

John H. Kinney

Elise B. Knittle

George H. Kwei

Nancy Lane

Andrew C. Lawson

Sally J. Marsinall

Raymond G. Nanni

Monte C. Nichols

Malcolm F. Nicol

David Rosenfeld

Richard W. Ryon

Cyrus R. Safinya

David K. Shuh

Wigbert J. Siekhaus

Louis J. Terminello

James G. Tobin

Samuel T. Weir

Joe Wong

Choong-shik Yoo 


\section{SSRL EXPERIMENTERS AND PROPOSALS BY INSTITUTION}

As of December 31, 1994 there were 833 experimenters from 147 institutions offically listed on active proposals at SSRL. In addition, well over 150 others (graduate students, etc.) participated in work at the Laboratory in collaboration with these scientists. The 106 US institutions listed on active proposals included 56 universities, 32 corporations, and 18 laboratories

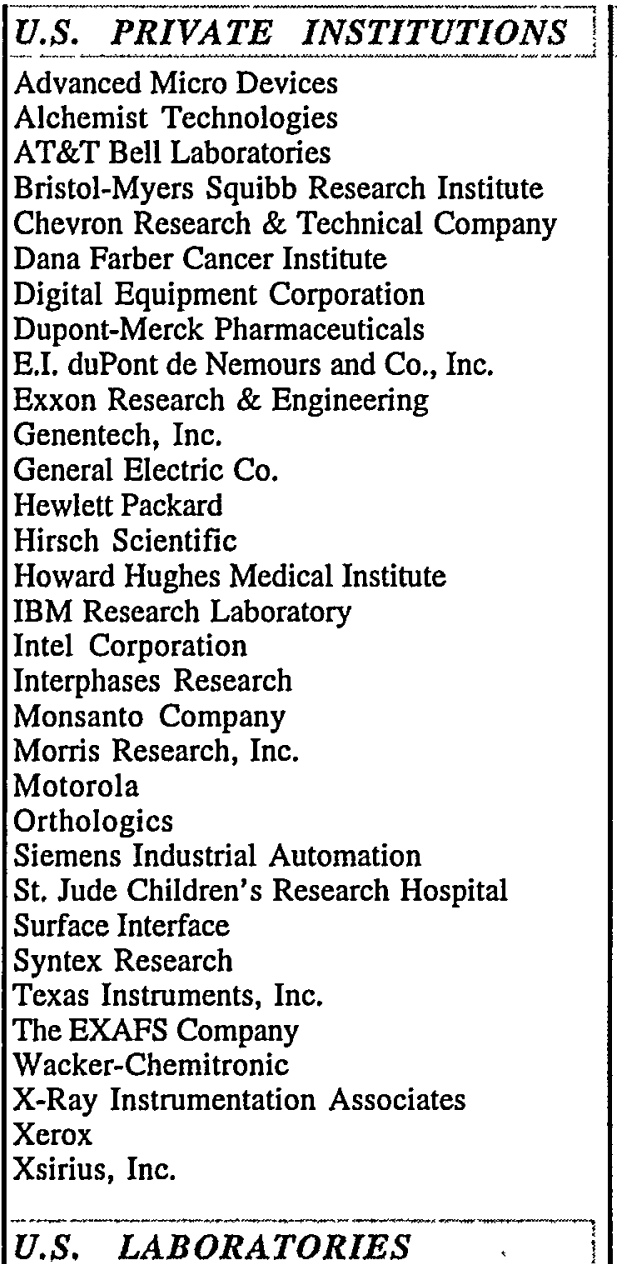

Argonne National Laboratory

Brookhaven National Laboratory

Department of Veterans Affairs Med. Ctr.

Jet Propulsion Laboratory

Lawrence Berkeley Laboratory

Lawrence Livermore National Laboratory

Los Alamos National Laboratory

NASA - Ames Research

NASA - Marshall Space Flight Center

National Institutes of Health

Naval Research Laboratory

National Inst. of Standards \& Technology

Pacific Northwest Laboratories

Sandia National Laboratories

Stanford Linear Accelerator Center

Solar Energy Research Institute

Stanford Synchrotron Radiation Laboratory

US Geological Survey Water Res. Div.

\begin{tabular}{|l|}
\hline U.S. UNIVERSITIES \\
\hline Arizona State University \\
Brigham Young University \\
California Institute of Technology \\
California State University
\end{tabular}

Case Western Reserve University

College of William \& Mary

Duke University

Eastern Washington University

Georgia Institute of Technology

Grand Canyon University

Harvard University

Johns Hopkins University

Louisiana State University

Massachusetts Institute of Technology

North Carolina State University

Northwestern University

Ohio State University

Oklahoma State University

Oregon Graduate Institute

Pennsylvania State University

Purdue University

Rensselaer Polytechnic Institute

Rutgers University

St. Louis University

Stanford University

The State University of New York

The Scripps Research Institute

University of Alabama

University of California

University of Cincinnati

University of Georgia

University of Hawaii

University of Houston

University of Idaho

University of Illinois

University of Kentucky

University of Maine

University of Maryland

University of Michigan

University of Minnesota

University of Missouri

University of Montana

University of Nebraska

University of Nevada

University of New Mexico

University of Oklahoma

University of Oregon

University of Tennessee

University of Texas

University of Utah

University of Virginia

University of Washington
University of Wisconsin

Utah State University

Washington University

Yale University

\section{FOREIGN INSTITUTIONS}

Aarhus University

Chalmers Institute of Technology

CISE S.P.A.

DESY

Forschungszentrum Rossendorf

Gyeong-Sang National University

Hong Kong University of Science

Institute of Physical Chemistry

Kurchatov Institute

Lab Nazionali di Frascati

McMaster University

Max Planck Institut

Moscow State University

National Inst. of Res. in Inorganic Mtls.

Royal Institute of Technology

Siemens AG

Simon Fraser University

Swedish Univ. of Agricultural Sciences

Terza Universita

The Australian National University

TU München

Unidad Merida

Università degli Studi dell'Aquila

Università di Camerino

Università di Roma

Universität Bayreuth

Universität des Saarlandes

Universität Dortmund

Universität Rostock

Université de Paris

University of Alberta

University of British Columbia

University of Edinburgh

University of New South Wales

University of Stockholm

University of Sydney

University of Toronto

University of Trondheim

Uppsala Universitet

Utrecht University

Weizmann Institute 
A note about SSRL proposal numbers: The 1000-2000 numbers are peer-reviewed, rated SSRL proposals, the 9000-9100 numbers are letters of intent. The 9300-9900 are PRT proposals. The 8000 numbers are in-house research experiments. The $2 \mathrm{~A}$ and $3 \mathrm{~A}$ numbers are rotation camera applications and the $1 \mathrm{E}$ numbers are rapid turnaround XAS proposals.

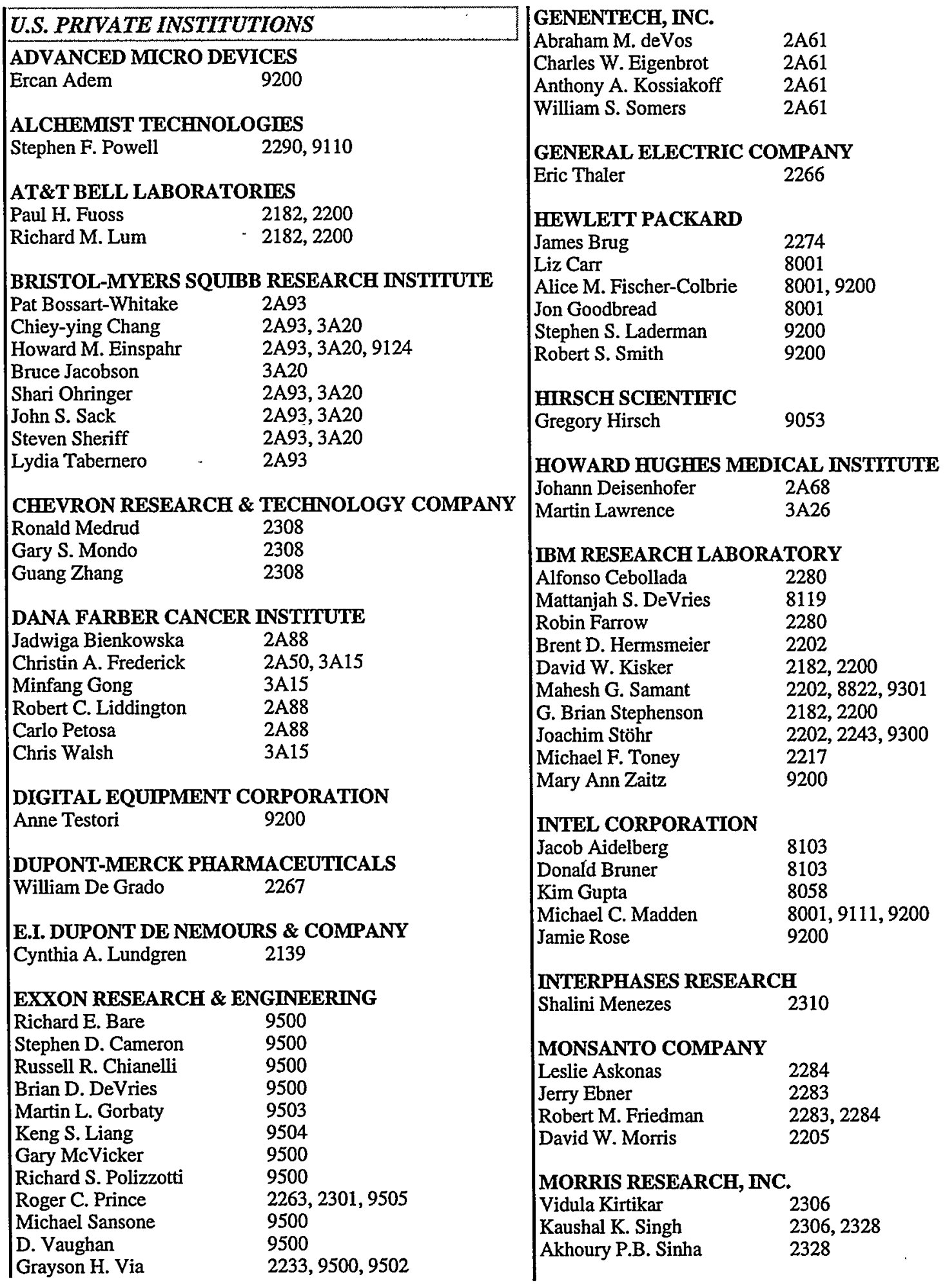




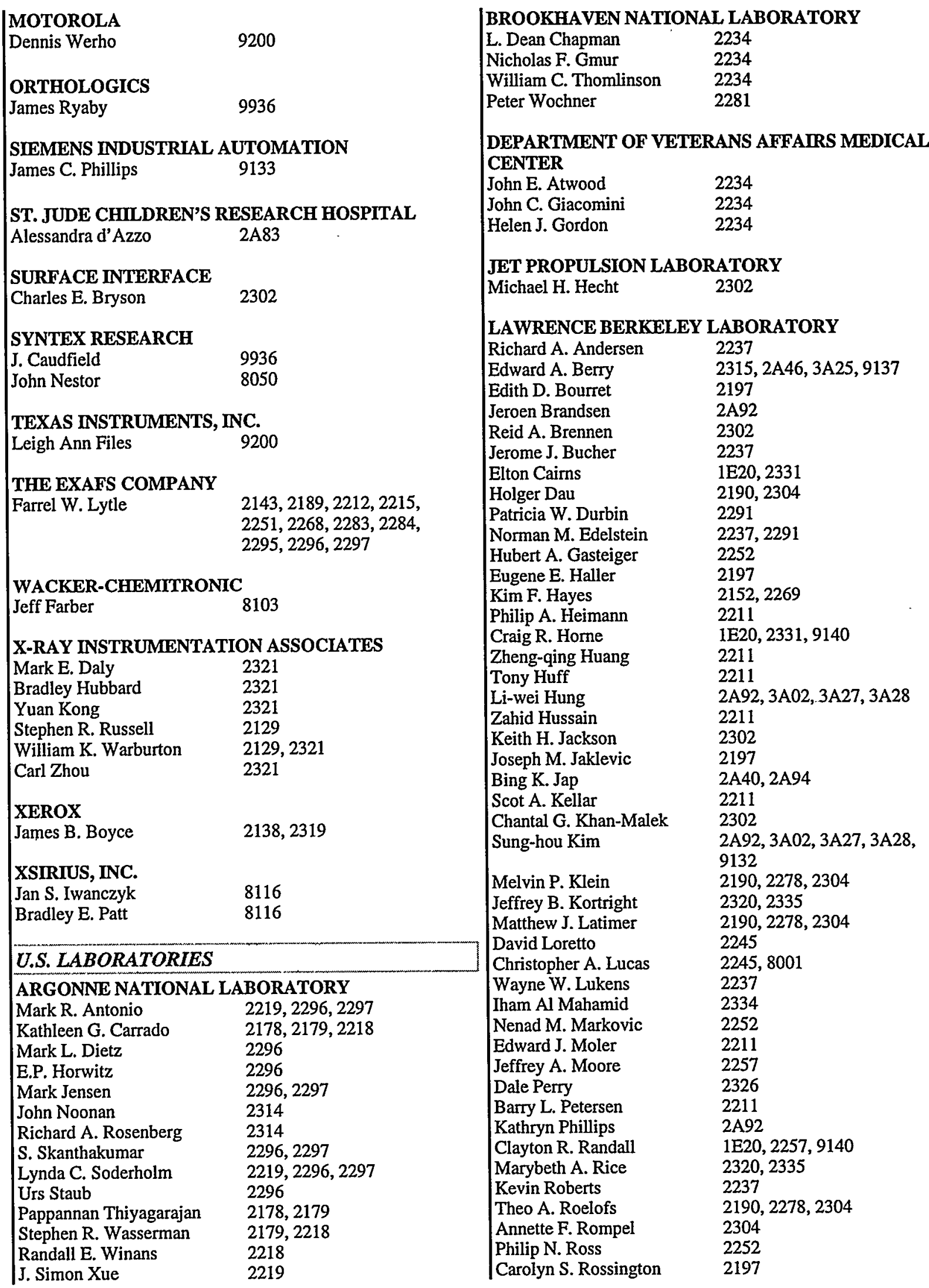




\begin{tabular}{|ll}
\hline LAWRENCE BERKELEY LABORATORY [Cont.] \\
Kenneth H. Sauer & $2190,2278,2304$ \\
David K. Shuh & $2237,2291,9926$ \\
Vladimir M. Shulmeister & 2315,2 A46, 3A25 \\
Geoff Siemering & 2237 \\
David H. Templeton & 2142 \\
Lieselotte K. Templeton & 2142 \\
Pradeep Thalappil & 2211 \\
Albert C. Thompson & 2234 \\
Ian M. Tidswell & 9106 \\
Tetsu K. Tokunaga & 2330 \\
Will Tong & 2237 \\
Wladyslaw Walukiewicz & 2197 \\
Hongxin Wang & 2312 \\
Gerard Wong & 2245 \\
Vittal K. Yachandra & $2190,2278,2304$ \\
Kin Man Yu & 2197 \\
Yu Zheng & 2211 \\
Jean-Luc Zimmermann & 2190
\end{tabular}

\section{LAWRENCE LIVERMORE NATIONAL}

\section{LABORATORY}

Jagannadh Akella 2248, 2249

Patrick G. Allen 2206, 2237, 2274, 2275,

2276,2318

Albert J. Arko $\quad 8119$

Rodney L. Balhorn 2201

Mehdi Balooch 9929

Troy W. Barbee, Jr. $\quad 2166,2210,9903$

William L. Bourcier $1 \mathrm{E} 16$

Susan A. Carroll 1E16, 2256, 2325

Lloyd L. Chase 9900

Carlos A. Colmenares $\quad 2256,2326,9934$

James J. De Yoreo 2226

Alex V. Hamza 9929

Anne Happel 2334

David L. Haupt 2229

Nicholas V. Hud 2201

Eric A. Hudson 1E15, 2211, 2237, 2256,

2326, 2334

Alan F. Jankowski $\quad 9933$

John H. Kinney $\quad 2139,2229,9915,9936$

George H. Kwei 2285, 9930

Linda N. Marquez 2318

Cynthia E.A. Palmer 2318

Brian L. Phillips $1 \mathrm{E} 16$

Richard W. Ryon $\quad 9908$

Wigbert J. Siekhaus $\quad 9929$

Phillip A. Sterne 2325

Eiichi Tamura 2233

Louis J. Terminello 1E15, 2256, 2326, 2334,

James G. Tobin 2307, 9901

Brian E. Viani

Marvin J. Weber

Samuel T. Weir

Joe Wong

Choong-shik Yoo

$1 \mathrm{E} 15,2256,2326$

2268

2249,9931

2233, 2282, 8103, 9902

2248

LOS ALAMOS NATIONAL LABORATORY

Gloria E. Borgstahl 1T02, 2A89

E. Morton Bradbury 2273

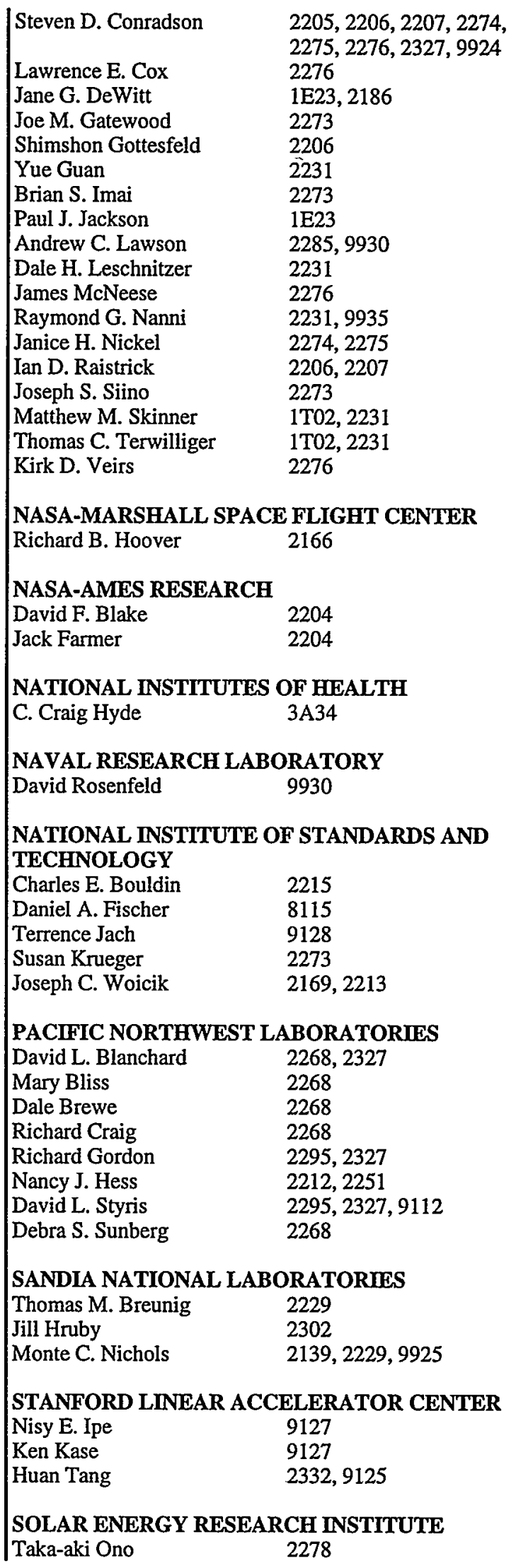




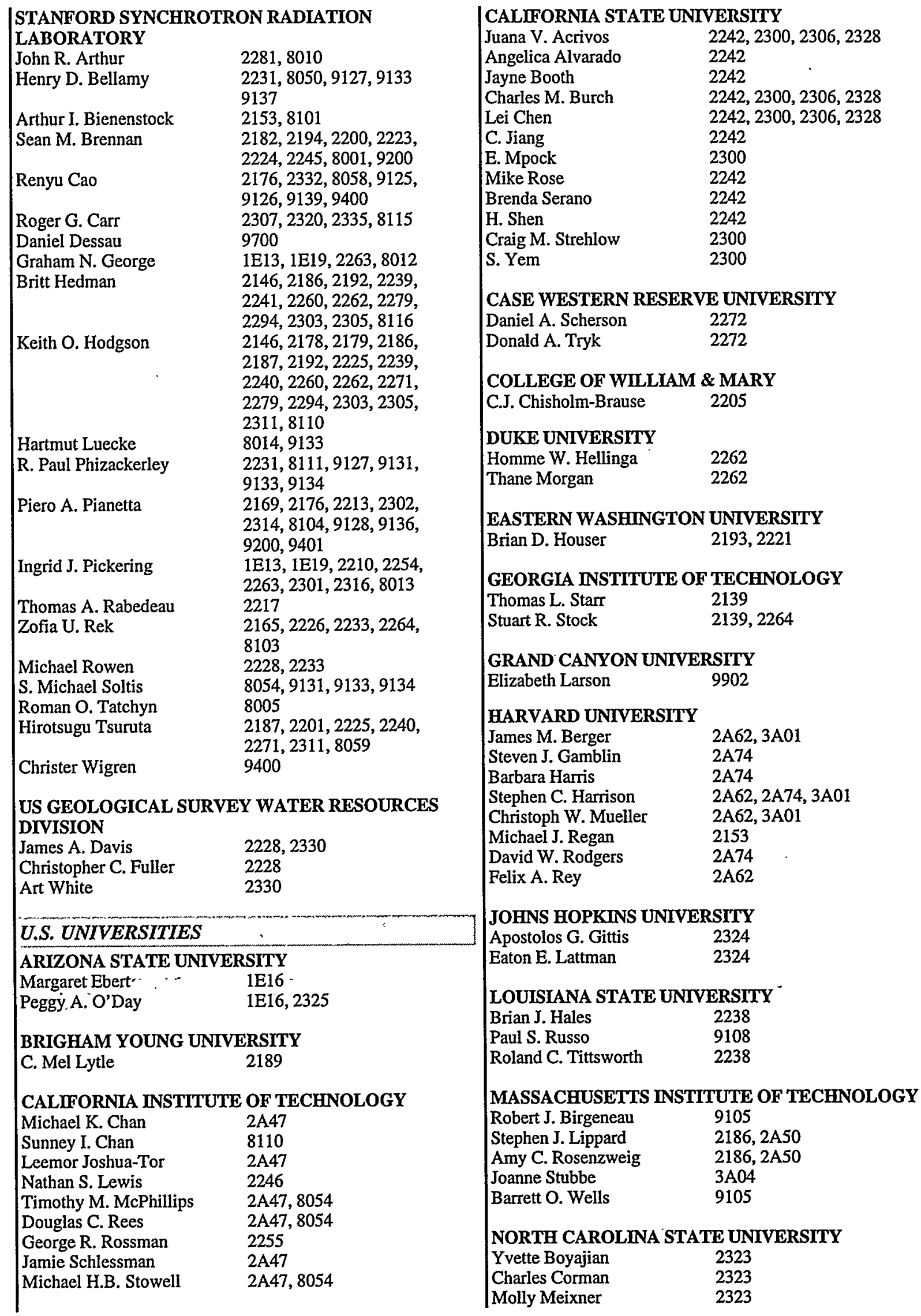




\begin{tabular}{|c|c|}
\hline \\
\hline \multicolumn{2}{|c|}{$\begin{array}{l}\text { NORTHWESTERT UNIVERSITY } \\
\text { Valerie L Grum }\end{array}$} \\
\hline Ted Jardetzky & 3 A29 \\
\hline Christopher D. Lima & $2 \mathrm{~A} 14$ \\
\hline Neal F. Lue & $2 \mathrm{~A} 14$ \\
\hline Alfonso Mondragón & $2 \mathrm{~A} 14$ \\
\hline Thomas V. O'Halloran & 2151 \\
\hline Amit P. Sharma & $2 \mathrm{~A} 14$ \\
\hline \multicolumn{2}{|c|}{ OHIO STATE UNIVERSITY } \\
\hline Satish C.B. Myneni & 2261 \\
\hline Samuel J. Traina & 2261 \\
\hline \multicolumn{2}{|c|}{ OKLAHOMA STATE UNIVERSITY } \\
\hline Chang-an Yu & $2 \mathrm{~A} 68$ \\
\hline \multicolumn{2}{|c|}{ OREGON GRADUATE INSTITUTE } \\
\hline Ninian J. Blackburn & 2227 \\
\hline John S. Boswell & 2227 \\
\hline Michael H. Gold & 9131 \\
\hline Kaitlin Grammer & 2227 \\
\hline Brian J. Reedy & 2227 \\
\hline Frank Rhames & 2227 \\
\hline \multicolumn{2}{|c|}{ PENNSYLVANIA STATE UNIVERSITY } \\
\hline David A. Shirley & 2211 \\
\hline \multicolumn{2}{|c|}{ PURDUE UNIVERSITY } \\
\hline Mavis Agbandje & $2 \mathrm{~A} 70$ \\
\hline Michael S. Chapman & $2 \mathrm{~A} 70$ \\
\hline Hok-Kin Choi & $2 \mathrm{~A} 70$ \\
\hline Leodevico L. Ilag & $2 \mathrm{~A} 70$ \\
\hline John E. Johnson & $3 A 38$ \\
\hline Kyung Hyun Kim & $2 \mathrm{~A} 70$ \\
\hline Prasanna Kolatkar & $2 \mathrm{~A} 70$ \\
\hline Ladislau Z. Kovari & $2 \mathrm{~A} 70$ \\
\hline Abhinav Kumar & $3 A 38$ \\
\hline Tianwei Lin & $3 A 38$ \\
\hline Robert McKenna & $2 \mathrm{~A} 70$ \\
\hline Cory Momany & $2 \mathrm{~A} 70$ \\
\hline Padmaja Natarajan & $3 A 38$ \\
\hline Andrew J. Prongay & $2 \mathrm{~A} 70$ \\
\hline Vijay Reddy & 3 A38 \\
\hline Michael G. Rossmann & $2 \mathrm{~A} 70$ \\
\hline William R. Wikoff & $3 \mathrm{~A} 38$ \\
\hline \multicolumn{2}{|c|}{ RENSSELAER POLYTECHNIC INSTITUTE } \\
\hline Timothy M. Hayes & 2288 \\
\hline Xiaobo Kan & 2288 \\
\hline Peter D. Persans & 2288 \\
\hline \multicolumn{2}{|c|}{ RUTGERS UNIVERSITY } \\
\hline David E. Salt & 2301 \\
\hline \multicolumn{2}{|c|}{ ST. LOUIS UNIVERSITY } \\
\hline Sanjeev Jain & $2 A 76$ \\
\hline William S. Sly & $2 A 76$ \\
\hline \multicolumn{2}{|c|}{ STANFORD UNIVERSITY } \\
\hline Edwin L. Alderman & 2234 \\
\hline Maxwell J. Allen & 2166 \\
\hline Dorota Artymowicz & 2223,2224 \\
\hline John R. Bargar & $2143,2210,2329$ \\
\hline Paul R. Besser & 2194 \\
\hline
\end{tabular}

\begin{tabular}{|c|c|}
\hline Dennis Bird & $1 \mathrm{E} 16,2325$ \\
\hline John C. Bravman & 2194 \\
\hline Byron W. Brown & 2234 \\
\hline Gordon E. Brown, Jr. & $\begin{array}{l}2143,2210,2213,2228, \\
2233,2329,2330,2333, \\
2336,9400\end{array}$ \\
\hline Heather L. Bufford & 2305 \\
\hline David A. Bushnell & $2 A 54$ \\
\hline Robert L. Byer & 2187 \\
\hline Singfoong Cheah & 2143,2329 \\
\hline Lingling Chen & $2187,2225,2311$ \\
\hline Christopher E. Chidsey & 9126 \\
\hline Bruce M. Clemens & 2223,2224 \\
\hline Brian J. Daniels & 2223,2224 \\
\hline Peter R. David & $2 A 54,2 A 63,9085$ \\
\hline Craig F. DeForest & 2166 \\
\hline Sebastian Doniach & $\begin{array}{l}2187,2225,2240,2271, \\
2311\end{array}$ \\
\hline David Eliezer & $2187,2225,2240,2271$ \\
\hline Jeffrey P. Fitts & 2329,2330 \\
\hline Kevin M. Flaherty & $2 A 67,9134$ \\
\hline Andrea L. Foster & 2329,2330 \\
\hline Patrick Frank & 2305 \\
\hline Alice P. Gast & 2230 \\
\hline Theodore H. Geballe & 2319 \\
\hline Jeffrey A. Guckert & 2145 \\
\hline Robert Helms & 9129 \\
\hline Todd C. Hufnagel & 2223,2224 \\
\hline Paul M. Jones & 2145 \\
\hline Charles C. Kankelborg & 2166 \\
\hline Michael C. Kautzky & 2223,2224 \\
\hline Tom Kendelewicz & $\begin{array}{l}2169,2213,9107 \\
9400\end{array}$ \\
\hline Tom W. Kenny & 2302 \\
\hline Changyoung Kim & 2314,9136 \\
\hline Roger D. Komberg & 9085 \\
\hline Bruce M. Lairson & 2223,2224 \\
\hline William M. Lavender & 2234 \\
\hline Kerstin K. Leuther & $2 A 54$ \\
\hline Alexander Lessmann & 9200 \\
\hline Eric K. Lin & 2230 \\
\hline Joakim F. Lindblom & 2166 \\
\hline H. Isaac Liu & 2241 \\
\hline Ping Liu & 2210 \\
\hline Timothy E. Machonkin & 2303 \\
\hline Robert J. Madix & 9400,9402 \\
\hline Jennifer A. May & 2145 \\
\hline Glen A. McConnell & 2230 \\
\hline David B. McKay & $2240,2 A 67,9134$ \\
\hline Gavin D. Meredith & $2 \mathrm{~A} 54$ \\
\hline Kent H. Nakagawa & 2305 \\
\hline Philip S. Neuhoff & $1 \mathrm{E} 16,2325$ \\
\hline Kenneth $\mathrm{Ng}$ & $2289,3 \mathrm{A06}$ \\
\hline Ray H. O'Neal & 2166 \\
\hline Stephen N. Oesterie & 2234 \\
\hline Richard M. Osgood & 2223,2224 \\
\hline John D. Ostergren & 2329 \\
\hline George A. Parks & $\begin{array}{l}2143,2210,2213,2329, \\
2330\end{array}$ \\
\hline Maria L. Peterson & 2329,2330 \\
\hline Heinz W. Pley & $2 \mathrm{~A} 67$ \\
\hline J. Bradford Reitz & 2145 \\
\hline Noa More Rensing & 2223,2224 \\
\hline J. Kurth Reynolds & 2302 \\
\hline
\end{tabular}




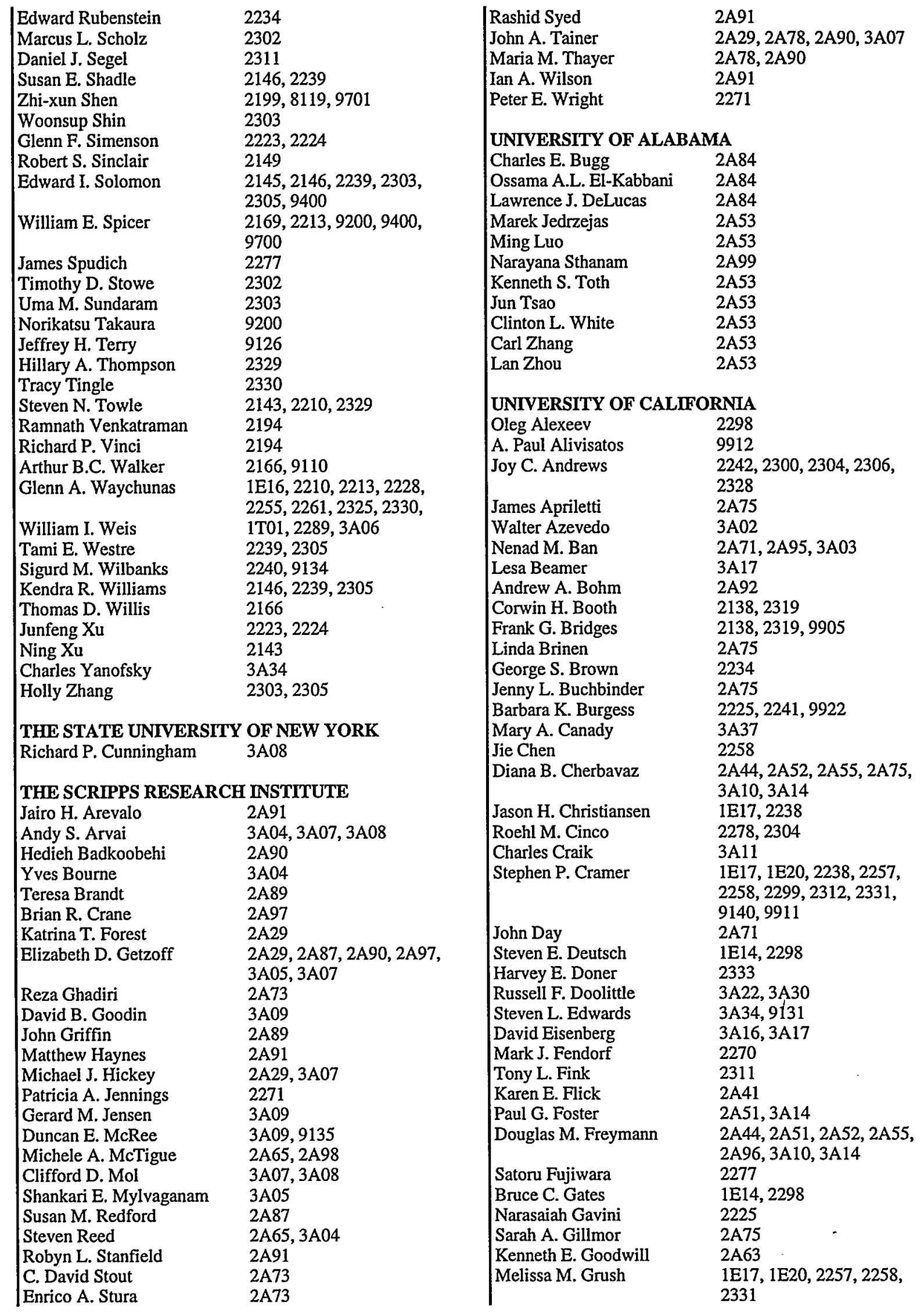




\begin{tabular}{|c|c|}
\hline \multicolumn{2}{|c|}{ UNIVERSITY OF CALIFORNIA [Cont.] } \\
\hline Lisa J. Harris & $2 \mathrm{~A} 71,3 \mathrm{~A} 03$ \\
\hline Hakon Hope & $3 A 24,8054$ \\
\hline Lan Huang & $3 \mathrm{A02}$ \\
\hline Stefan H.J. Idziak & 2247 \\
\hline Raymond Jeanloz & 9937 \\
\hline Rolf Jentoft & 2298 \\
\hline Frances Jurnak & 3A03 \\
\hline Sibudjing Kawi & $1 \mathrm{E} 14,2298$ \\
\hline Robert J. Keenan & $3 \mathrm{~A} 10$ \\
\hline Kyeong-kyn Kim & $3 \mathrm{~A} 27$ \\
\hline Elise B. Knittle & 9923 \\
\hline Ilya V. Koltover & 2247 \\
\hline Keith E. Kraiser & 2247 \\
\hline Joseph Kraut & $3 \mathrm{~A} 24,3 \mathrm{~A} 30$ \\
\hline F. Jon Kull $3 \mathrm{~A} 12,3 \mathrm{~A} 13$ & \\
\hline Steve Larson & $2 \mathrm{~A} 71$ \\
\hline Nancy Lane & 9936 \\
\hline Guoguang $\mathrm{Li}$ & 2138 \\
\hline Huiying $\mathrm{Li} 3 \mathrm{~A} 35$ & \\
\hline Wenchuan Liang & $2190,2278,2304$ \\
\hline Zuzanna Liliental-Weber & 8103 \\
\hline Kai Lin & $2 \mathrm{~A} 75$ \\
\hline Otis B. Littlefield & $2 A 41$ \\
\hline Cara Marks & $2 \mathrm{~A} 63$ \\
\hline Sally J. Marshall & 9927 \\
\hline Mary E. McGrath & $2 A 75$ \\
\hline Robert Mendelson & 2277 \\
\hline Stanley E. Mills & $3 A 34$ \\
\hline Hillary C.M. Nelson & 2A41 \\
\hline Malcolm F. Nicol & 2248,9918 \\
\hline Mike Pannell & 9918 \\
\hline Gang Peng 2257, 2312 & \\
\hline Kathleen M. Perry & $2 A 52$ \\
\hline Gaston Pfluegl & $3 \mathrm{~A} 16$ \\
\hline Brian Phillips & 2325 \\
\hline Reetta Raag & $2 \mathrm{~A} 63$ \\
\hline Stephen D. Rader & $2 \mathrm{~A} 85,3 \mathrm{~A} 14$ \\
\hline Corie Y. Ralston & $1 \mathrm{E} 17,2299$ \\
\hline Veerappa P. Ramalingam & $\begin{array}{l}\text { 2A51, 2A55, 2A75, 2A96, } \\
3 \mathrm{~A} 10,3 \mathrm{~A} 14\end{array}$ \\
\hline Diane Reifschneider & 9918 \\
\hline Earl E. Rutenber & $2 \mathrm{~A} 51,2 \mathrm{~A} 96,3 \mathrm{~A} 11$ \\
\hline Elena P. Sablin & $2 \mathrm{~A} 75,3 \mathrm{~A} 12,3 \mathrm{~A} 13$ \\
\hline Cyrus R. Safinya & 2247,9928 \\
\hline Michael R. Sawaya & $3 \mathrm{~A} 22,3 \mathrm{~A} 24$ \\
\hline Brian M. Schick & $3 \mathrm{A03}$ \\
\hline Ursula Schulze-Gahmen & $2 \mathrm{~A} 92,3 \mathrm{A0} 2$ \\
\hline Sangita Seshadri & 2311 \\
\hline Andrew Shiau & $2 A 86$ \\
\hline Michael O. Skidmore & $3 \mathrm{~A} 24$ \\
\hline Ray C. Stevens & $2 \mathrm{~A} 63$ \\
\hline Tom Stout & $3 \mathrm{~A} 14$ \\
\hline Robert M. Stroud & $2 \mathrm{~A} 44,3 \mathrm{~A} 10$ \\
\hline Anupam Talapatra & 2311 \\
\hline Susan S. Taylor & $2 \mathrm{~A} 77$ \\
\hline A. Van Hoek & $2 A 96$ \\
\hline Kottayil I. Varughese & $2 \mathrm{~A} 77$ \\
\hline Richard L. Wagner & $2 \mathrm{~A} 55,2 \mathrm{~A} 75,3 \mathrm{~A} 14$ \\
\hline Gernot Walter & $3 \mathrm{~A} 24$ \\
\hline Xin Wang & 2257,9140 \\
\hline Heidi E. Warriner & 2247 \\
\hline Bill Weber 2298 & \\
\hline Brenda J. Weiss & 9140 \\
\hline
\end{tabular}

\begin{tabular}{|c|c|}
\hline Xiangwei Weng & $2 \mathrm{~A} 92,3 \mathrm{AO} 2$ \\
\hline Brian West & $2 \mathrm{~A} 75$ \\
\hline Michael C. Wiener & $\begin{array}{l}\text { 2A44, 2A51, 2A52, 2A55, } \\
2 A 75,2 A 96,3 A 10,3 A 14\end{array}$ \\
\hline Feng-shou Xiao & 2298 \\
\hline Zhengtian Xu & $1 \mathrm{E} 14$ \\
\hline Ng H. Xuong & $2 \mathrm{~A} 77$ \\
\hline Peter M. Yau & 2273 \\
\hline Mavrik Zavarin & 2333 \\
\hline Ailian Zhao & $1 \mathrm{E} 14,2298$ \\
\hline \multicolumn{2}{|c|}{ UNIVERSITY OF CINCINNATI } \\
\hline Edward A. Deutsch & 2128 \\
\hline Richard C. Elder & 2128,2203 \\
\hline William R. Heineman & 2203 \\
\hline \multicolumn{2}{|c|}{ UNIVERSITY OF GEORGIA } \\
\hline Michael W.W. Adams & 2263 \\
\hline Jun Dong & 2292 \\
\hline $\mathrm{Li} \mathrm{Ma}$ & 2241 \\
\hline Robert A. Scott & 2137,2292 \\
\hline Shengke Wang & 2137 \\
\hline Hui Zhang & 2137,2292 \\
\hline \multicolumn{2}{|c|}{ UNIVERSITY OF HAWAI } \\
\hline John Balogh & 2287 \\
\hline A. Jayaraman & 2287 \\
\hline Murli H. Manghnani & 2287 \\
\hline Li Chung Ming & 2287 \\
\hline Sean Shieh & 2287 \\
\hline \multicolumn{2}{|c|}{ UNIVERSITY OF HOUSTON } \\
\hline Joseph Di Carlo & 2254 \\
\hline Allan J. Jacobson & 2254 \\
\hline Simon C. Moss & 2281,9109 \\
\hline Longbao Qiu & 2254 \\
\hline \multicolumn{2}{|c|}{ UNIVERSITY OF IDAHO } \\
\hline Scott E. Fendorf & $1 \mathrm{E} 12,2270$ \\
\hline Paul A. McDaniel & $1 \mathrm{E} 12$ \\
\hline Steven L. McGeehan & 2270 \\
\hline Matthew J. Morra & $1 \mathrm{E} 12$ \\
\hline Ron Patterson & 2270 \\
\hline \multicolumn{2}{|c|}{ UNIVERSITY OF ILLINOIS } \\
\hline Robert B. Gennis & 2292 \\
\hline Jon Rumbley & 2292 \\
\hline Jian Ku Shang & 2314 \\
\hline Andrew H.-J. Wang & 2231 \\
\hline Hong Zhang & 2231 \\
\hline \multicolumn{2}{|c|}{ UNIVERSITY OF KENTUCKY } \\
\hline Gerald P. Huffman & 2175,2313 \\
\hline Frank E. Huggins & 2175,2313 \\
\hline Fulong Lu & 2313 \\
\hline Naresh Shah & 2175,2313 \\
\hline Jianmin Zhao & 2175,2313 \\
\hline \multicolumn{2}{|c|}{ UNIVERSITY OF MAINE } \\
\hline Lynn Katz. & 2152 \\
\hline \multicolumn{2}{|c|}{ UNIVERSITY OF MARYLAND } \\
\hline Richard L. Greene & 8119 \\
\hline Jian-liang Peng & 8119 \\
\hline
\end{tabular}




\begin{tabular}{|c|c|}
\hline \multicolumn{2}{|c|}{ UNIVERSITY OF MICHIGAN } \\
\hline \multicolumn{2}{|c|}{$\begin{array}{l}\text { UNIVERSITY OF MICHIGAN } \\
\text { James W. Allen } \\
2199,8119\end{array}$} \\
\hline \multirow{5}{*}{$\begin{array}{l}\text { Terrence M. Barnhart } \\
\text { John C. Bilello } \\
\text { I-wei Chen } \\
\text { Kimber C. Clark } \\
\text { Kelly A. Daly }\end{array}$} & $\begin{array}{l}2151,2170,2253,2265 \\
2266,2267\end{array}$ \\
\hline & 2165 \\
\hline & 2170,2293 \\
\hline & 2151 \\
\hline & $\begin{array}{l}2151,2253,2265,2267, \\
2322,2323\end{array}$ \\
\hline \multirow{4}{*}{$\begin{array}{l}\text { Pamela S. DeMarois } \\
\text { Gregg Dieckmann } \\
\text { Brian R. Gibney } \\
\text { Susan Heilman }\end{array}$} & $2151,2170,2267$ \\
\hline & 2267 \\
\hline & 2265 \\
\hline & 2267 \\
\hline Hui Huang & $2253,2265,2323$ \\
\hline Paul Knochel & 2253 \\
\hline Ping $\mathrm{Li}$ & 2170,2293 \\
\hline Martha Ludwig & 2181 \\
\hline Rui Mei & 2181 \\
\hline Susan Miller & 2151 \\
\hline \multirow{2}{*}{$\begin{array}{l}\text { Vincent L. Pecoraro } \\
\text { James E. Penner-Hahn }\end{array}$} & $2181,2265,2267$ \\
\hline & $\begin{array}{l}2151,2152,2181,2253, \\
2265,2266,2267,2293, \\
2322,2323\end{array}$ \\
\hline \multirow{2}{*}{$\begin{array}{l}\text { Pamela J. Riggs-Gelasco } \\
\text { Timothy L. Stemmler }\end{array}$} & $2151,2170,2181,2267$ \\
\hline & $\begin{array}{l}2151,2170,2181,2253, \\
2265,2267\end{array}$ \\
\hline Jun Tao & 2165 \\
\hline \multirow{2}{*}{ David L. Tierney } & $2151,2170,2265,2267$ \\
\hline & 2199 \\
\hline $\begin{array}{l}\text { L.H. Tjeng } \\
\text { Marc A. Vill }\end{array}$ & 2165 \\
\hline Geoffrey S. Waldo & $1 \mathrm{T02}$ \\
\hline Ying Wang & 2293 \\
\hline Steven M. Yalisove & 2165 \\
\hline \multirow{2}{*}{ Charles F. Yocum } & 2181 \\
\hline & $2151,2170,2267$ \\
\hline Zhen Zeng & 2165 \\
\hline \multicolumn{2}{|c|}{ UNIVERSITY OF MINNESOTA } \\
\hline \multirow{2}{*}{ David M. Arciero } & $3 \mathrm{~A} 35$ \\
\hline & $3 \mathrm{~A} 35$ \\
\hline UNIVERSITY OF MIS & RI \\
\hline George D. Waddill & 2256,2307 \\
\hline UNIVERSITY OF MOI & NA \\
\hline Nancy Hinman & 2204 \\
\hline UNIVERSITY OF NEB & $\mathbf{S K A}$ \\
\hline Manoj Kumar & 2299 \\
\hline Gustavo Larsen & $1 \mathrm{E} 18$ \\
\hline Ruben D. Parra & $1 \mathrm{E} 18$ \\
\hline Stephen Ragsdale & 2299 \\
\hline UNIVERSITY OF NEV & \\
\hline Charalambos Papelis & 2269 \\
\hline UNIVERSITY OF NEV & EXICO \\
\hline Leo B. Archer & 2235 \\
\hline Mark J. Hampden-Smith & 2235 \\
\hline UNIVERSITY OF OKI & OMA \\
\hline W. Berlin Genetti & $1 \mathrm{E} 21,2250$ \\
\hline Brian P. Grady & $1 \mathrm{E} 21,2250$ \\
\hline
\end{tabular}

\begin{tabular}{|c|c|}
\hline \multicolumn{2}{|c|}{ UNIVERSITY OF OREGON } \\
\hline \multicolumn{2}{|c|}{ UNIVERSITY OF TENNESSEE } \\
\hline Craig E. Barnes & 2222 \\
\hline Herbert D. Zeman & 2234 \\
\hline \multicolumn{2}{|c|}{ UNIVERSITY OF TEXAS } \\
\hline Ning Chang & $2 \mathrm{~A} 82$ \\
\hline Marvin L. Hackert & $2 \mathrm{~A} 82$ \\
\hline Andrew D. Kern & $2 \mathrm{~A} 82$ \\
\hline Marcos A. Oliveira & $2 A 82$ \\
\hline Chi-ken Shih & 8119 \\
\hline Samuel H. Wilson & 3 A22 \\
\hline Di Xia & $2 \mathrm{~A} 68$ \\
\hline \multicolumn{2}{|l|}{ UNIVERSITY OF UTAH } \\
\hline Dan Bancroft & $3 \mathrm{~A} 18$ \\
\hline Christopher P. Hill & $3 \mathrm{~A} 18$ \\
\hline Steve Johnston & $3 \mathrm{~A} 18$ \\
\hline Felix Vajdos & 3A18 \\
\hline David Worthylake & $3 \mathrm{~A} 18$ \\
\hline \multicolumn{2}{|c|}{ UNIVERSITY OF VIRGINIA } \\
\hline Yi He & 2280 \\
\hline \multicolumn{2}{|c|}{ UNIVERSITY OF WASHINGTON } \\
\hline Bradley Bernstein & $3 A 36$ \\
\hline James J. Champoux & $2 \mathrm{~A} 72$ \\
\hline Jörg Hendle & $2 \mathrm{~A} 60$ \\
\hline Wilhelmus G.J. Hol & $2 A 59,2 A 69,2 A 83,3 A 36$ \\
\hline Robert L. Ingalls & 2193,2221 \\
\hline Hidong Kim & $3 \mathrm{~A} 36$ \\
\hline Shekhar C. Mande & $2 \mathrm{~A} 60,2 \mathrm{~A} 69,9138$ \\
\hline Ethan A. Merritt & $2 \mathrm{~A} 19,2 \mathrm{~A} 59,2 \mathrm{~A} 60,3 \mathrm{~A} 36$ \\
\hline Xiayang Qiu & $2 \mathrm{~A} 60,2 \mathrm{~A} 72$ \\
\hline Gabrielle Rudenko & $2 A 60,2 A 83$ \\
\hline Ronald E. Stenkamp & $2 \mathrm{~A} 19$ \\
\hline David C. Teller & $2 \mathrm{~A} 19$ \\
\hline Focco van den Akker & $2 A 59$ \\
\hline Vivien Yee & $2 \mathrm{~A} 19,9138$ \\
\hline \multicolumn{2}{|c|}{ UNIVERSITY OF WISCONSIN } \\
\hline Matthew M. Benning & $2 A 79,2 A 81,3 A 21$ \\
\hline William Bleam & 2214 \\
\hline Andrew J. Fisher & $2 \mathrm{~A} 79,3 \mathrm{~A} 21$ \\
\hline Hazel M. Holden & $2 \mathrm{~A} 79,2 \mathrm{~A} 81,3 \mathrm{~A} 21$ \\
\hline Todd Larsen & $2 \mathrm{~A} 80$ \\
\hline Ivan Rayment & $2 \mathrm{~A} 79,2 \mathrm{~A} 80,3 \mathrm{~A} 21$ \\
\hline George Reed & $2 \mathrm{~A} 80$ \\
\hline Clyde A. Smith & $2 \mathrm{~A} 79,3 \mathrm{~A} 21$ \\
\hline James B. Thoden & $2 \mathrm{~A} 79,2 \mathrm{~A} 81,3 \mathrm{~A} 21$ \\
\hline Janeen L. VanHooke & $2 \mathrm{~A} 81$ \\
\hline Joseph Wedekind & $2 \mathrm{~A} 79,3 \mathrm{~A} 21$ \\
\hline \multicolumn{2}{|c|}{ UTAH STATE UNIVERSITY } \\
\hline Linda S. Powers & 2149 \\
\hline \multicolumn{2}{|c|}{ WASEINGTON UNIVERSITY } \\
\hline Wayne Barnes & $3 \mathrm{~A} 31$ \\
\hline Sergey Korolev & $3 \mathrm{~A} 31$ \\
\hline Timothy M. Lohman & $3 \mathrm{~A} 32,3 \mathrm{~A} 33$ \\
\hline F. Scott Mathews & $2 A 76$ \\
\hline Srinivasan Raghunathan & $3 \mathrm{~A} 32,3 \mathrm{~A} 33$ \\
\hline Gabriel Waksman & $3 \mathrm{~A} 31,3 \mathrm{~A} 32,3 \mathrm{~A} 33$ \\
\hline
\end{tabular}


|YALE UNIVERSITY

Vishal Agrawal 1T01

Axel T. Brunger $\quad 1 \mathrm{~T} 01$

Mark Willis 1T01

FOREIGN INSTITUTIONS

AARHUS UNIVERSITY (Denmark)

Anders Hanberg $2 A 66$

Trine J. Koch 2A13, 2 A66

Jens Nyborg 2A13

Gregers Rom Andersen $\quad 2 A 13,2 A 66$

Anders H.P. Sorensen $2 A 13$

Soren S. Thirup 2A13, 2 A66

CHALMERS INSTITUTE OF TECHNOLOGY (Sweden)

Tord C. Claeson 2138, 2319

CISE S.P.A. (Italy)

Fulvio Parmigiani

8119

DESY (Germany)

Gerhard T. Materlik

8001

FORSCHUNGSZENTRUM ROSSENDORF (Germany)

Melissa Denecke

2237

Heino Nitsche

2237

Tobias N. Reich

2211,2237

GYEONG-SANG NATIONAL UNIVERSITY (Korea)

Young-ho Kim 2287

HONG KONG UNIVERSITY OF SCIENCE

(Hong Kong)

Xiao Yan

2280

INSTITUTE OF PHYSICAL CHEMISTRY (Germany)

Michael Fröba

2233

KURCHATOV INSTITUTE (Russia)

Alexander I. Chumakov 8101

Gennadi V. Smimov 8101

LAB NAZIONALI DI FRASCATI (Italy)

Calegero Natoli 2282

Ziyu Wu 1E22, 2282, 2317

MCMASTER UNIVERSITY (Canada)

Jean Barwell 3 A19

Alexi Botchkarev $\quad 3 \mathrm{~A} 19$

Aled M. Edwards $\quad 3 A 19$

Lori Frappier $\quad 3 \mathrm{~A} 19$

Hua Tong 3A19

\section{MAX PLANCK INSTITUT (Germany)}

Heike Bartels 3 A23

Wolfgang Baumeister $\quad 2 \mathrm{~A} 40$

William S. Bennett 3 A23

Olle Gunnarsson $\quad 8119$

Harly A.S. Hansen 3 A23

Jörg Harms 3A23

Ove Jepsen $\quad 8119$

Frank Schlunzen 3 A23
MOSCOW STATE UNIVERSITY (Russia)

Natalia Zaitseva 2226

NATIONAL INSTITUTE OF RESEARCH IN

INORGANIC MATERIALS (Japan)

Takako Tanaka 8103

ROYAL INSTITUTE OF TECHNOLOGY (Sweden)

Magnus K. Sandström 2286

SIEMENS AG (Germany)

Charles F. Campana 9133

John L. Chambers 9133

James F. Fait $\quad 9133$

Manfred R. Schuster $\quad 8001$

Robert A. Sparks 9133

SIMON FRASER UNIVERSITY (Canada)

Yolande R. Bonin 2209

E. Daryl Crozier $\quad 2193,2209,2221,2309$

9104

Moyra-Kathleen McManus 2309

SWEDISH UNIVERSITY OF AGRICULTURAL

SCIENCES (Sweden)

Ingmar N. Persson 2286

Per K.B. Persson $\quad 2143,2210$

TERZA UNIVERSITÀ (Italy)

A. Mottana 1E22, 2317

THE AUSTRALIAN NATIONAL UNIVERSITY

(Australia)

Nicholas Dixon $\quad 3$ A39

TU MÜNCHEN (Germany)

Uwe van Bürck 8101

UNIDAD MERIDA (Mexico)

José Mustre De Leon 2207, 2275, 9116

UNIVERSITÀ DEGLI STUDI DELL'AQUILA (Italy)

Adriano Filipponi 2279

UNIVERSITÀ DI CAMERINO (Italy)

Andrea Di Cicco 2279

Gabriele Giuli $\quad$ 1E22, 2317

Eleonora Paris 1E22, 2282, 2317

UNIVERSITÀ DI ROMA (Italy)

Augusto Marcelli $\quad 1 \mathrm{E} 22,2282,2317$

UNIVERSITÄT BAYREUTH (Germany)

Fritz Seifert 1E22, 2317

UNIVERSITÄT DES SAARLANDES (Germany)

Ralph Claessen 2199

UNIVERSITÄT DORTMUND (Germany)

Ulrich K. Bonse 2229

UNIVERSITÄT ROSTOCK (Germany)

Thomas Gerber 2232, 2244

Bernhard Himmel 2232, 2244

Oliver Stachs 2232 


\begin{tabular}{|c|c|}
\hline \multicolumn{2}{|c|}{ UNIVERSITÉ DE PARIS (France) } \\
\hline Georges Calas & 2336 \\
\hline Francois Farges & 2336 \\
\hline \multicolumn{2}{|c|}{ UNIVERSITY OF ALBERTA (Canada) } \\
\hline Ronald G. Cavell & $1 \mathrm{E} 13,1 \mathrm{E} 19$ \\
\hline \multicolumn{2}{|c|}{ UNIVERSITY OF BRITISH COLUMBIA (Canada) } \\
\hline W. Hardy & 2281 \\
\hline \multicolumn{2}{|c|}{ UNIVERSITY OF EDINBURGH (Scotland) } \\
\hline Graham Pettigrew & $3 \mathrm{~A} 34$ \\
\hline \multicolumn{2}{|c|}{ UNIVERSITY OF NEW SOUTH WALES (Australia) } \\
\hline Paul Curmi & 3 A39 \\
\hline Russell F. Howe & $2316,9.113$ \\
\hline \multicolumn{2}{|c|}{ UNIVERSITY OF STOCKHOLM (Sweden) } \\
\hline Pär Nordlund & $2 \mathrm{~A} 50$ \\
\hline \multicolumn{2}{|c|}{ UNIVERSITY OF SYDNEY (Australia) } \\
\hline Robert S. Armstrong & 2260 \\
\hline Paul J. Ellis & 2192,2294 \\
\hline Hans C. Freeman & $2192,2294,2 \mathrm{~A} 64$ \\
\hline Mitchell Guss & $2 \mathrm{~A} 64$ \\
\hline Ian Harvey & $2 \mathrm{~A} 64$ \\
\hline Peter A. Lay & 2260 \\
\hline Emma M. Proudfoot & 2192 \\
\hline Anne M. Rich & 2260 \\
\hline Daniel Shi & $2294,2 \mathrm{~A} 64$ \\
\hline \multicolumn{2}{|c|}{ UNIVERSITY OF TORONTO (Canada) } \\
\hline Zeng Hong Lu & 9139 \\
\hline \multicolumn{2}{|c|}{ UNIVERSITY OF TRONDHEIM (Germany) } \\
\hline H.E. Krokan & $3 \mathrm{~A} 08$ \\
\hline \multicolumn{2}{|c|}{ UPPSALA UNIVERSITET (Sweden) } \\
\hline Laurent C. Duda & 2243 \\
\hline Joseph E. Nordgren & 2243 \\
\hline \multicolumn{2}{|c|}{ UTTRECHT UNIVERSITÄT (The Netherlands) } \\
\hline Gerard Van Koten & 2253 \\
\hline \multicolumn{2}{|c|}{ WEIZMANN INSTITUTE (Israel) } \\
\hline Anat Bashan & $3 \mathrm{~A} 23$ \\
\hline Ada Yonath & $3 \mathrm{~A} 23,9130$ \\
\hline
\end{tabular}




\section{PUBLICATIONS BASED ON WORK AT SSRL}

\section{JOURNAL ARTICLES}

R. Åkesson, I. Persson, M. Sandström and U. Wahlgren, "Structure and Bonding of Solvated Mercury(II) and Thallium(III) Dihalide and Dicyanide Complexes by XAFS Spectroscopic Measurements and Theoretical Calculations", Inorg. Chem. 33, 3715 (1994)

P.G. Allen, J.J. Bucher, D.L. Clark, N.M. Edelstein, S.A. Ekberg, J.W. Gohdes, E.A. Hudson, N. Kaltsoyannis, W.W. Lukens, M.P. Neu, P.D. Palmer, T. Reich, D.K. Shuh, C.D. Tait and B. Zwick, "Multinuclear NMR, Raman, EXAFS, and X-ray Diffraction Studies of Uranyl Carbonated Complexes in Nearneutral Aqueous Solution. X-ray Structure of $\left[\mathrm{C}\left(\mathrm{NH}_{2}\right)_{3}\left(\mathrm{UO}_{2}\right)_{3}\left(\mathrm{CO}_{3}\right)_{6}\right] * 6.5 \mathrm{H}_{2} \mathrm{O}$ ", submitted to Inorg. Chem.

I. Almahamid, J.J. Bucher, J.C. Bryan, S.B. Clark, N.M. Edelstein, E.A. Hudson, N. Kaltsoyannis, T. Reich, H. Nitsche, W.W. Lukens, P. Torretto, K. Robert and D.K. Shuh, "Electronic and Structural Investigations of Technetium Compounds by XAS", Inorg. Chem., in press

J.C. Andrews, R. Cinco, H. Dau, M.J. Latimer, W. Liang, T.A. Roelofs, A. Rompel, V.K. Yachandra, K. Sauer and M.P. Klein, "Photosynthetic Water Oxidation: Structural Insights to the Catalytic Manganese Complex", Physica $B$, in press

J. Arthur, G. Materlik, R. Tatchyn and H. Winick, "The LCLS: A Fourth Generation Light Source Using the SLAC Linac", Rev. Sci. Instrum. 66, 1987 (199.5)

M.J. Baldwin, T.L. Stemmler, P.J. Riggs-Gelasco, M.L. Kirk, J.E. Penner-Hahn and V.L. Pecoraro, "Structural and Magnetic Effects of Successive Protonations of Oxo Bridges in High Valent Manganese Dimers", J. Am. Chem. Soc., in press

M. Balooch, G.W. Marshall, S.J. Marshall and J.H. Kinney, "AFM of Effects of Conditioning Agents on Dentin", J. Dent. Res. 73, S418 (1994)

A.Q.R. Baron, "Report on the X-ray Efficiency and Time Response of a $1 \mathrm{~cm}^{2}$ Reach Through Avalanche Diode", Nucl. Instrum. Methods A 352, 665 (1995)

A.Q.R. Baron, J. Arthur, S.L. Ruby, A.I. Chumakov, G.V. Smirnov and G.S. Brown, "Angular Dependence of Specular Resonant Nuclear Scattering of X-rays", Phys. Rev. B 50, 10354 (1994)

A.Q.R. Baron and S.L. Ruby, "Time Resolved Detection of X-rays Using Large Area Avalanche Photodiodes", Nucl. Instrum. Methods A 343, 517 (1994)

H.D. Bellamy, R.P. Phizackerley, S.M. Soltis and H. Hope, "An Open-Flow Cryogenic Cooler for Single Crystal Diffraction Experiments", J. Appl. Cryst. 27, 967 (1994)

E.A. Berry, L.-S. Huang, V.M. Shulmeister and S.-H. Kim, "A New Form of Crystals of Bovine Heart. Ubiquinol: Cytochrome $c$ Oxidoreductase-Determination of Space Group and Unit Cell Parameters", Acta Cryst. $D$, in press

P.R. Besser, S. Brennan and J.C. Bravman, "An X-ray Method for Direct Determination of the Strain State and Strain Relaxation in Micron-Scale Passivated Metallization Lines During Thermal Cycling", J. Mater. Res. 9, 13 (1994)

P.R. Besser, J.E. Sanchez, S. Brennan, J.C. Bravman, G. Takaoka and I. Yamada, "Mechanical Behavior of Single Crystal $\mathrm{Al}(111)$ and Bicrystal $\mathrm{Al}(110)$ Films on Silicon Substrates", Mat. Res. Soc. Symp. Proc. 343, 659 (1994)

P.R. Besser, T.N. Marieb and J.C. Bravman, "Measurement and Interpretation of Strain Relaxation in Passivated Al-0.5\% Cu Lines", Mat. Res. Soc. Symp. Proc. 338, 275 (1994) 
J.C. Bilello, S.J. Yalisove and Z.U. Rek, "Texture Evolution in Thin Films and Multilayers Via Synchrotron Topography and GIXS", submitted to $J$. Phys. $D$

R.M. Bionta, K.M. Skulina and J. Weinberg, "Hard X-ray Sputtered-Sliced Phase Zone Plates", Appl. Phys. Lett. 64, 945 (1994)

N.J. Blackburn, M.E. Barr, W.H. Woodruff, J. Van der Oost and S. de Vries, "Metal-metal Bonding in Biology: EXAFS Evidence for a $2.5 \AA$ Copper-Copper Bond in the $\mathrm{Cu}_{\mathrm{a}}$ Center of Cytochrome Oxidase", Biochem. 33, 10401 (1994)

C.H. Booth, F. Bridges, J.B. Boyce, T. Claeson, Z.X. Zhao and P. Cervantes, "Local Disorder in the Oxygen Environment Around Praseodymium in $\mathrm{Y}_{1-\mathrm{x}} \mathrm{Pr}_{\mathrm{x}} \mathrm{Ba}_{2} \mathrm{Cu}_{3} \mathrm{O}_{7}$ from X-ray Absorption Fine Structure", Phys. Rev. B 49, 3432 (1994)

S. Brennan, W. Tompkins, N. Takaura, P. Pianetta, S.S. Laderman, A. Fischer-Colbrie, J.B. Kortright, M.C. Madden and D.C. Wherry, "Wide Band-Pass Approaches to Total Reflection X-ray Fluorescence Using Synchrotron Radiation", Nucl. Instrum. Methods A 347, 417 (1994)

T.M. Breunig, M.C. Nichols, J.S. Gruver, J.H. Kinney and D.L. Haupt, "A Servo-Mechanical Load Frame for In situ, Non-Invasive Imaging of Damage Development”, submitted to Ceram. Soc. Proc.

F. Bridges, C.H. Booth and G.G. Li, "An Iterative Approach to "Atomic Background" Removal in XAFS Data Analysis", submitted to Physica B

C.A. Brown, M.A. Pavlosky, Y. Zhang, T.E. Westre, B. Hedman, K.O. Hodgson and E.I. Solomon, "Spectroscopic and Theoretical Description of the Electronic Structure of $S=3 / 2$ Iron Nitrosyl Complexes and the Relation to $\mathrm{O}_{2}$ Activation by Non-Heme Iron Active Sites", J. Am. Chem. Soc., in press

B.B. Buchanan, J.J. Bucher, D.E. Carlson, N.M. Edelstein, E.A. Hudson, N. Kaltsoyannis, T. Leighton, H. Nitsche, T. Reich, K. Roberts, D.K. Shuh, P. Torretto, J. Woicik, W.-S. Yang, A. Yee and B.C. Yee, "A XANES and EXAFS Investigation of the Speciation of Selenium Following Bacterial Uptake", accepted by Inorg. Chem.

K. Cantwell, "The Stanford Synchrotron Radiation Laboratory - 20 Years of Synchrotron Light", Nucl. Instrum. Methods A 347, 44 (1994)

R. Cao, J. Terry, F. Coffman, P. Pianetta, L. Pan, D. Kania, S. Kapoor and M.A. Kelly, "High Resolution $\mathrm{C}(\mathrm{K})$ Near-edge X-ray Absorption Fine Structure of Graphite and Diamonds", submitted to Phys. Rev.

R. Carr, J.B. Kortright, M. Rice and S. Lidia, "Performance of the Elliptically Polarizing Undulator on SPEAR", Rev. Sci. Instrum. 66, 1862 (1995)

R. Carr, "Exotic Sources of X-rays for Iodine K-edge Angiography", Nucl. Instrum. Methods A 347, 510 (1994)

J. Cerino, T. Rabedeau and W. Bowen, "Photon Beam Position Monitor for SSRL Beamline 9", Rev. Sci. Instrum. 66, 2 (1995)

L. Chen, N. Gavini, H. Tsuruta, D. Eliezer, B.K. Burgess, K.O. Hodgson and S. Doniach, "MgATP-Induced Conformational Changes in the Iron Protein from Azotbacter vinelandii, as Studied by Small-Angle X-ray Scattering", J. Biol. Chem. 269, 3290 (1994)

S. Chiruvolu, H.E. Warriner, E. Naranjo, S.H.J. Idziak, J.O. Radler, J.A. Zasadzinski and C.R. Safinya, "A New Phase of Liposomes: Entangled Tubular Vesicles", submitted to Science

K. Clark, J.E. Penner-Hahn, M. Whittaker and J.W. Whittaker, "Structural Characterization of the Copper Site in Galactose Oxidase Using X-ray Absorption Spectroscopy", Biochem. 33, 12553 (1994) 
J. Cobb and R. Tatchyn, "Tests of Planar Permanent Magnet Multipole Focusing Elements", Nucl. Instrum. Methods A 347, 562 (1994)

F. Coffman, R. Cao, P. Pianetta, S. Kapoor, L.J. Terminello and S. Falabella, "Characterization of Carbon Materials - A Comparison of NEXAFS and Raman Spectroscopy", accepted by Mat. Res. Soc. Symp. Proc.

F. Coffman, R. Cao, P. Pianetta, S. Kapoor, M. Kelly, L.J. Terminello and S. Falabella, "Near-edge X-ray Absorption of Carbon Materials for Determining Bond Hybridization", accepted by Mat. Res. Soc. Symp. Proc.

S.D. Conradson, B.K. Burgess, W.E. Newton, A. Di Cicco, A. Filipponi, Z.Y. Wu, C.R. Natoli, B. Hedman and K.O. Hodgson, "Selenol Binds to Iron in Nitrogenase Iron-Molybdenum Cofactor: An Extended X-ray Absorption Fine Structure Study", Proc. Natl. Acad. Sci. USA 91, 1290 (1994)

W.J. Corbett, M.J. Lee and V. Ziemann, "Model Calibration and Beam Control Systems for Storage Rings", SPIE 2013, 189 (1994)

B.J. Daniels, W.D. Nix and B.M. Clemens, "Effect of Structure, Stress, Strain, and Alloying on the Hardness of Fe(001)/Pt(001) Epitaxial Multilayers", accepted by Mat. Res. Soc. Symp. Proc.

B.J. Daniels and B.M. Clemens, "The Effect of Cr Doping on the Magnetoresistance and Saturation Field of Epitaxial $\mathrm{Fe}_{1-\mathrm{x}} \mathrm{Cr}_{\mathrm{x}}(001)$ Multilayers", accepted by Appl. Phys. Lett.

H. Dau, J.C. Andrews, T.A. Roelofs, M.J. Latimer, W. Liang, V.K. Yachandra, K. Sauer and M.P. Klein, "Structural Consequences of Ammonia Binding to the Manganese Center of the Photosynthetic Oxygen Evolving Complex: An X-ray Absorption Study of Isotropic and Oriented Photosystem II Particles", Biochem., in press

V.J. DeRose, J. Mukerji, M.J. Latimer, V.K. Yachandra, K. Sauer and M.P. Klein, "Comparison of the Manganese Oxygen-Evolving Complex in Photosystem II of Spinach and Synechococcus sp. with Multinuclear Manganese Model Compounds by X-ray Absorption Spectroscopy", J. Am. Chem. Soc. 116, 5239 (1994)

V.J. DeRose, J.J. Latimer, J.-L. Zimmermann, I. Mukerji, V.K. Yachandra, K. Sauer and M.P. Klein, "Fluoride Substitution in the Mn Cluster from Photosystem II: EPR and X-ray Absorption Spectroscopy Studies", Chem. Phys., in press

J.G. DeWitt, A.C. Rosenzweig, B. Hedman, S.J. Lippard and K.O. Hodgson, "X-ray Absorption Spectroscopy Studies of the Diiron Center in Methane Monooxygenase in the Presence of Substrate and the Coupling Protein of the Enzyme System", accepted by Inorg. Chem.

D.S. Dessau, P. Dickinson, S. Doniach, Z.-X. Shen and B.O. Wells, "Comment on Anomalously Large Gap Anisotropy in the a-b Plane of $\mathrm{Bi}_{2} \mathrm{Sr}_{2} \mathrm{CaCu}_{2} \mathrm{O}_{8+\delta}$ ", Phys. Rev. Lett., in press

D.S. Dessau, Z.-X. Shen and B.O. Wells, "Symmetry of the Gap in Bi2212 from Photoemission Spectroscopy", Phys. Rev. Lett. 73, 3044 (1994)

A. Di Cicco, M. De Crescenzi, R. Bernardini and G. Mancini, "Evidence of $K L$ Double-Electron Excitations in X-ray Photoemission Spectra of Solids: The Case of $\mathrm{MgO}$ and $\mathrm{MgF}_{2}$ ", Phys. Rev. B 49, 2226 (1994)

L.-C. Duda, J. Stöhr, D.C. Mancini, A. Nilsson, N. Wassdahl, J. Nordgren and M.G. Samant, "Magnetic Dichroism in $\mathrm{L}_{2,3}$ Emission of Fe, Co and Ni Following Energy-Dependent Excitation with Circularly Polarized X-rays", Phys. Rev. B 50, 16758 (1994)

B.A. Eipper, A. Quon, R.E. Mains, J.S. Boswell and N.J. Blackburn, "The Core of Peptidylglycine $\alpha-$ hydroxylating Monooxygenase. Structural Investigations Using Site Directed Mutagenesis, X-ray Absorption and Electron Paramagnetic Resonance and Comparisons with Dopamine- $\beta$-Monooxygenase", submitted to Biochem. 
P.J. Ellis, H.C. Freeman, M.A. Hitchman, D. Reinen and B. Wagner, "EXAFS Evidence that the $\mathrm{CuCl}_{6}{ }^{4-}$ Ion in (3-Chloroanilinium) $)_{8}\left(\mathrm{CuCl}_{6}\right) \mathrm{Cl}_{4}$ Has an Elongated Rather Than Compressed Tetragonal Geometry", Inorg. Chem. 33, 1249 (1994)

F. Farges, G.E. Brown, Jr. and D. Velde, "X-ray Spectroscopic Study of the Structural Environment of Zr in Two Inosilicates from Cameroon: Implications for Substitution Mechanisms and Zr-partitioning", submitted to Amer. Min.

M.B. Fernández van Raap, M.J. Regan and A. Bienenstock, "Evidence of Phase Separation in Amorphous $\mathrm{Fe}_{\mathrm{x}} \mathrm{Si}_{1-\mathrm{x}}$ Films", submitted to J. Non-Cryst. Sol.

A. Filipponi, M. Passacantando, L. Lozzi, S. Santucci, P. Picozzi and A. Di Cicco, "1s Shake-Up Excitations in $\mathrm{NaF}, \mathrm{NaCl}, \mathrm{NaBr}$, and $\mathrm{Na}_{2} \mathrm{SO}_{4}$ ", Solid State Commun. 91, 55 (1994)

P. Frank, B. Hedman, R.M.K. Carlson and K.O. Hodgson, "Interaction of Vanadium and Sulfate in the Blood Cells of the Tunicate Ascidia ceratodes: Observations Using X-ray Absorption Edge Structure and EPR Spectroscopies", Inorg. Chem. 33, 3794 (1994)

M. Fröba, J. Wong, P. Behrens, P. Sieger, M. Rowen, T. Tanaka, Z. Rek and J. Felsche, "Correlation of Multiple Scattering Features in XANES Spectra of $\mathrm{Al}$ and Si K Edges to the Al-O-Si Bond Angle in Aluminosilicate Sodalites: An Empirical Study", accepted by Physica B

M. Fröba, J. Wong, M. Rowen, G.E. Brown, Jr., T. Tanaka and Z. Rek, "Al and Si K Absorption Edges of $\mathrm{AL}_{2} \mathrm{SiO}_{5}$ Polymorphs Using the New $\mathrm{YB}_{66}$ Soft X-ray Monochromator", accepted by Physica $B$

V. Fülöp, R.P. Phizackerley, S.M. Soltis, I.J. Clifton, S. Wakatsuki, J. Erman, J. Hajdu and S.L. Edwards, "Laue Diffraction Study on the Structure of Cytochrome $c$ peroxidase Compound I", Structure 2, 201 (1994)

P.H. Fuoss, D.W. Kisker, G.B. Stephenson and S. Brennan, "In-situ X-ray Studies of OMVPE Growth", accepted by Mat. Sci. \& Eng. B

P. Ghosh, S.F. Mel and R.M. Stroud, "The Domain Structure of the Ion Channel-Forming Protein Colicin Ia", Nature Structure Biology 1, 597 (1994)

R.J. Goddard, B.P. Grady and S.L. Cooper, "The Room Temperature Annealing Peak in Ionomers: Ionic Crystallites or Water Absorption?", accepted by Macromolecules

B.P. Grady, E.M. O'Connell, C.Z. Yang and S.L. Cooper, "Blends of Metal Acetates and Polyurethanes Containing Pyridine Groups II. SAXS and EXAFS Studies", accepted by J. Polym. Sci.

R.B. Greegor, F.W. Lytle and A. Wu, "Structural Investigation of Bulk and Thin PLZT Using X-ray Absorption Spectroscopy", Thin Solid Films 240, 22 (1994)

A.M. Green, W.E. Spicer, C. Kim, R. Cao and P. Pianetta, " $\mathrm{CaF}_{2}$ Overlayers to Preserve the Ideal Termination of Sb-GaAs(110)", J. Vac. Sci Technol. A 12, 1158 (1994)

J.A. Guckert, M.D. Lowery and E.I. Solomon, "Electronic Structure of the Reduced Blue Copper Active Site: Contributions to Reduction Potentials and Geometry", J. Am. Chem. Soc., in press

G.-H. Gweon, R. Claessen, J.W. Allen, W.P. Ellis, R. Reniert, P.J. Benning, C.G. Olson, Y.X. Zhang, A. Matsuura, Z.-X. Shen and L.F. Schneemeyer, "Anomalous Dispersion in the Single Particle Spectrum of Quasi One-dimensional $\mathrm{K}_{0.3} \mathrm{MoO}_{3}$ ", submitted to Phys. Rev. Lett.

A.V. Hamza, J. Dykes, W.D. Mosley, L. Dinh and M. Balooch, "Reaction and Passivation of Aluminum with $\mathrm{C}_{60}$ ", submitted to Surf. Sci. 
C.J. Harrison, A.A. Bohm and H.C.M. Nelson, "Crystal Structure of the DNA Binding Domain of the Heat Shock Transcription Factor", Science 263, 224 (1994)

G.A. Held, M.G. Samant, J. Stöhr, S.S.P. Parkin, B.D. Hermsmeier, M. van Schilfgaarde and R. Nakajima, "X-ray Magnetic Circular Dichroism Study of the Induced Spin Polarization of $\mathrm{Cu}$ in $\mathrm{Co} / \mathrm{Cu}$ and $\mathrm{Fe} / \mathrm{Cu}$ Multilayers", submitted to Phys. Rev. B

A. Herrera-Gomez, T. Kendelewicz, J.C. Woicik, K.E. Miyano, P. Pianetta, S. Southworth, P.L. Cowan, A. Karlin and W.E. Spicer, "Geometrical Structure of the Bi/GaP(110) Interface: An X-ray Standing Wave Triangulation Study of a Nonideal System", J. Vac. Sci. Technol. A 12, 2473 (1994)

G. Hirsch, "Biological Imaging with a New Type of Soft X-ray Microscope", Rev. Sci. Instrum. 66, 1367 (1995)

F.E. Huggins and G.P. Huffman, "Chlorine in Coal: an XAFS Spectroscopic Investigation", submitted to Fuel

S.H.J. Idziak, C.R. Safinya, R.S. Hill, M. Ruth, H.E. Warriner, K.E. Kraiser, K.S. Liang and J.N. Israelachvili, "The X-ray Surface Forces Apparatus: Structure of a Smectic Liquid Crystal Under Confinement and Flow", Science 264, 1915 (1994)

S.H.J. Idziak, I. Koltover, K.S. Liang, J.N. Israelachvili and C.R. Safinya, "Study of Flow in a Smectic Liquid Crystal in the X-ray Forces Apparatus", accepted by I.J. Thermophy.

M.N. Janakiraman, C.L. White, M. Luo, G. Air and W.G. Laver, "The Structure of B/Lee/40 Influenza Virus Neuraminidase Complexed with Two Neuraminic Acids at $1.8 \AA$ Resolution: Implications for the Catalytic Mechanism", Biochem. 33, 8172 (1994)

A.F. Jankowski, G.D. Waddill and J.G. Tobin, "Dependence of X-ray Absorption Magnetic Circular Dichroism on Layer Periodicity in Iron-Platinum Multilayers", J. Vac. Sci. Technol. A 12, 2215 (1994)

A.F. Jankowski, G.D. Waddill and J.G. Tobin, "Magnetic X-ray Circular Dichroism in Nickel-Gold Multilayers", submitted to Mat. Res. Soc. Symp. Proc.

M.J. Jedrzejas, S. Singh, W. Brouillette, M.G. Air, W.G. Laver and M. Luo, "Structure of Aromatic Inhibitors of Influenza Virus Neuraminidase", Biochem., in press

W.B. Jones, T.E. Elgren, M.M. Morelock, R.C. Elder and D.E. Wilcox, "Technetium Metallothionein: A Spectroscopic and EXAFS Study of ${ }^{99} \mathrm{TcO}^{+3}$ Binding in $\mathrm{Zn}_{7}$-Metallothionein", Inorg. Chem. 33, 5571 (1994)

W.B. Jones, J. Yuan, R. Narayanaswamy, M.A. Young, R.C. Elder, A.E. Bruce and M.R. Bruce, "Solid State EXAFS and Luminescence Studies of Neutral Dinuclear Gold(I) Complexes. Gold(I)-Gold(I) Interactions in the Solid State", Inorg. Chem. 34, 1996 (1995)

L.E. Katz and K.F. Hayes, "Surface Complexation Modeling: I. Strategy for Modeling Monomer Complex Formation at Moderate Surface Coverage", submitted to J. Coll. Inter. Sci.

L.E. Katz and K.F. Hayes, "Surface Complexation Modeling: II. Strategy for Modeling Polymer and Precipitation Reactions at High Surface Coverage", submitted to J. Coll. Inter. Sci.

M. Keenlyside and P. Pianetta, "A Performance and Applications Study of the Photoelectron Spectromicroscope", submitted to J. Elect. Spectrosc.

T. Kendelewicz, J.C. Woicik, K.E. Miyano, S.A. Yoshikawa, P. Pianetta and W.E. Spicer, "Structural Study of Monolayers of Sb on Ge(111) with Different Surface Reconstructions", J. Vac. Sci. Technol. A 12, 1843 (1994) 
S. Kim, D.A. Tryk, M.R. Antonio, R. Carr and D. Scherson, "In situ X-ray Absorption Fine Structure of Foreign Metal Ions in Nickel Hydrous Oxide Electrodes in Alkaline Electrolytes", accepted by J. Phys. Chem.

D.M. King, Z.-X. Shen, D.S. Dessau, D.S. Marshall, C.H. Park, W.E. Spicer, J.L. Peng, Z.Y. Li and R.L. Greene, "Observation of a Saddle Point Singularity in $\mathrm{Bi}_{2} \mathrm{Sr}(0.97)_{2} \mathrm{CuO}_{6+\delta}$ and Its Implications for Normal and Superconducting State Properties", Phys. Rev. Lett. 73, 3298 (1994)

J.H. Kinney, M. Balooch, D.L. Haupt, S.J. Marshall and G.W. Marshall, "Dimensional Changes in Human Coronal Dentin During Demineralization", submitted to J. Dent. Res.

J.H. Kinney, N.E. Lane and D.L. Haupt, "Three-Dimensional, In Vivo Morphometry of Hypoestrogenemic Bone Loss", submitted to J. Bone \& Mineral Res.

J.H. Kinney, G.W. Marshall and S.J. Marshall, "Three-Dimensional Mapping of Mineral Densities in Various Dentin: Theory and Method", submitted to Scanning Microscopy

J.H. Kinney, D.L. Haupt, M.C. Nichols et al., "Three-Dimensional Perspectives Evolving Microstructures", submitted to Nucl. Instrum. Methods

D.W. Kisker, G.B. Stephenson, P.H. Fuoss and S. Brennan, "Characterization of Vapor Phase Growth Using X-ray Techniques", accepted by J. Crys. Growth

J.B. Kortright, M. Rice and R. Carr, "Soft X-ray Faraday Rotation at the Fe $\mathrm{L}_{2,3}$ Edges", Phys. Rev. B 51, 10240 (1995)

A.A. Kossiakoff, W. Somers, M. Ultsch, K. Andow, Y.A. Muller and A.M. deVos, "Comparison of the Intermediate Complexes of Human Growth Hormone and Prolactin Receptors", Protein Science 3, 1697 (1994)

P. Kung, H.-C. Lihn, H. Wiedemann and D. Bocek, “Generation and Measurement of 50-fs(rms) Electron Pulses", Phys. Rev. Lett. 73, 967 (1994)

P.H. Kung, H.-C. Lihn, H. Wiedemann and D. Bocek, "High-intensity Coherent FIR Radiation from Subpicosecond Electron Bunches", SPIE 2118, 191 (1994)

G.H. Kwei, J.M. Lawrence and P.C. Canfield, "Temperature Dependence of the $4 f$ Occupation of $\mathrm{Ce}_{3} \mathrm{Bi}_{4} \mathrm{Pt}_{3}$ ", Phys. Rev. B 49, 14708 (1994)

F.J. Lamelas, P.H. Fuoss, D.W. Kïkker, G.B. Stephenson, P. Imperatori and S. Brennan, "X-ray Scattering Analysis of Surface Structures Produced by Vapor-Phase Epitaxy of GaAs", Phys. Rev. B 49, 1957 (1994)

J. Landro, E. Schmidt, P. Schimmel, D.L. Tierney and J.E. Penner-Hahn, "Thiol Ligation of Two Zinc Atoms to a Class I tRNA Synthetase: Evidence for Unshared Thiols and Role in Amino Acid Binding and Utilization", accepted by Biochem.

M.J. Latimer, A. Rompel, J.H. Underwood, V.K. Yachandra and M.P. Klein, “A Simple In-Hutch Assembly for X-ray Harmonic Suppression", Rev. Sci. Instrum. 66, 1843 (1995)

J.M. Lawrence, G.H. Kwei, P.C. Canfield, J.G. DeWitt and A.C. Lawson, "L(II) X-ray Absorption in Yb Compounds: Temperature Dependence of the Valence", Phys. Rev. B 49, 1627 (1994)

G.G. Li, F. Bridges, J.B. Boyce, T. Claeson, C. Ström, S.-G. Eriksson and S.D. Conradson, "Correlated Local Distortions of the T1O Layers in $\mathrm{T}_{2} \mathrm{Ba}_{2} \mathrm{CuO}_{\mathrm{y}}$ : An X-ray Absorption Study", submitted to Phys. Rev. B

G.G. Li and F. Bridges "XAFS Standards: A Comparison of Experiment and Theory", submitted to Phys. Rev. B 
G.G. Li, F. Bridges and X. Wang, "Monochromator-Induced Glitches in EXAFS Data: II Test of the Model for a Pinhole Sample", Nucl. Instrum. Methods A 340, 420 (1994)

P. Li, Y. Wang and I.-W. Chen, "Local Atomic Structures of $\mathrm{Pb}\left(\mathrm{Zr}_{1 / 3} \mathrm{Nb}_{2 / 3}\right) \mathrm{O}_{3}$ and Related Perovskites. I. An XANES Study of Ionicity/Covalency", accepted by Ferroelectrics

P. Li, Y. Wang and I.-W. Chen, "Local Atomic Structures of $\mathrm{Pb}\left(\mathrm{Zr}_{1 / 3} \mathrm{Nb}_{2 / 3}\right) \mathrm{O}_{3}$ and Related Perovskites II. An EXAFS Study of Cation Distortion", accepted by Ferroelectrics

P. Li, I.-W. Chen and J.E. Penner-Hahn, "Effect of Dopants on Zirconia Stabilization - An X-ray Absorption Study: I. Trivalent Dopants", J. Am. Ceram. Soc. 77, 118 (1994)

P. Li, I.-W. Chen and J.E. Penner-Hahn, "The Effect of Dopants on Zirconia Stabilization - An X-ray Absorption Study. II. Tetravalent Dopants", J. Am. Ceram. Soc. 77, 1281 (1994)

P. Li, I.-W. Chen and J.E. Penner-Hahn, "The Effect of Dopants on Zirconia Stabilization - An X-ray Absorption Study. III. Charge-Compensating Dopants", J. Am. Ceram. Soc. 77, 1289 (1994)

W. Liang, M.J. Latimer, H. Dau, T. Roelofs, V.K. Yachandra, K. Sauer and M.P. Klein, "Correlation Between Structure and Magnetic Spin State of the Mn Cluster in the Oxygen Evolving Complex of Photosystem II in the $S_{2}$ State: Determination by X-ray Absorption Spectroscopy", Biochem. 33, 4923 (1994)

S. Lidia and R. Carr, "Faster Magnet Sorting with a Threshold Acceptance Algorithm", Rev. Sci. Instrum. 66, 1865 (1995)

S. Lidia and R. Carr, "An Elliptically-Polarizing Undulator with Phase Adjustable Energy and Polarization", Nucl. Instrum. Methods A 347, 77 (1994)

C. Lima, J. Wang and A. Mondragon, "Three-Dimensional Structure of the 67K N-Terminal Fragment of $E$. coli DNA Topoisomerase I", Nature 367, 138 (1994)

H.I. Liu, A. Filipponi, N. Gavini, B.K. Burgess, B. Hedman, A. Di Cicco, C.R. Natoli and K.O. Hodgson, "EXAFS Studies of FeMo-cofactor and MoFe Protein: Direct Evidence for the Long Range Mo-Fe-Fe Interaction and Cyanide Binding to the Mo in FeMo-cofactor", J. Am. Chem. Soc. 116, 2418 (1994)

A.G. Loeser, Z.-X. Shen, D.S. Dessau and W.E. Spicer, "Photoemission Studies of the Bi-Sr-Ca-Cu-O Valence Band", J. Elec. Spec. Rel. Phen. 66, 359 (1994)

R.D. Lorentz, A. Bienenstock and T.I. Morrison, "Structural Studies of Amorphous $\mathrm{Fe}_{\mathrm{x}} \mathrm{Ge}_{100-\mathrm{x}}$ Alloys", Phys. Rev. B 49, 3172 (1994)

M. Luo, M.J. Jedrzejas, C. White, S. Singh, W. Brouillette, M.G. Air, W.G. Laver and A. Vasella, "Benzoic Acid Inhibitors of Influenza Virus Neuraminidase", Acta Cryst. D., in press

M. Luo and H.L. Lipton, "The Structure of a Highly Neurovirulent Theiler's Virus (GDVII strain)", submitted to $J$. Virology

C.M. Lytle, F.W. Lytle and B.N. Smith, "Chemical Speciation of Accumulated $\mathrm{Mn}$ and $\mathrm{Fe}$ in a Common Submerged Aquatic, Potamogeton pectinatus L., by X-ray Absorption Spectroscopy", submitted to J. Aquatic Botany

F.W. Lytle, G.L. Bibbins, K.Y. Blohowiak, R.B. Greegor, R.E. Smith and G.D. Tuss, "Investigation of the Structure and Chemistry of a Chromium Conversion Surface Layer on Aluminum", accepted by Corrosion Science

L. Ma, N. Gavini, H.I. Liu, B. Hedman, K.O. Hodgson and B.K. Burgess, "Large Scale Isolation and Characterization of the Molybdenum-Iron Cluster from Nitrogenase”, J. Biol. Chem. 269, 18007 (1994) 
S. Malhotra, Z.U. Rek, L.J. Parfitt, S.M. Yalisove and J.C. Bilello, "Principal Residual Strains as a Function of Depth for Sputter Deposited Mo Thin Films", submitted to Mat. Res. Soc. Symp. Proc.

A. Mäntykenttä-Pramanick and R. Carr, "High Resolution Mammography with Synchrotron Radiation", submitted to Medical Phys.

D.S. Marshall, D.S. Dessau, D.M. King, C.H. Park, A. Matsuura, Z.-X. Shen, W.E. Spicer, J.N. Ecstein and I. Bozovic, "Angle-Resolved Photoemission (ARPES) Study of Bi2212 Thin Films", submitted to Phys. Rev. B

S.J. Marshall, G.W. Marshall, J.H. Kinney and M. Balooch, "Dental Restorative Material-Tooth Interfaces", Scripta Met. et Mater., in press

A.Y. Matsuura, Z.-X. Shen, D.S. Dessau, C.-H. Park, T. Thio, J.W. Bennett and O. Jepsen, "Electronic Structure, Spectral Evolution and the Metal-Insulator Transition in $\mathrm{NiS}_{2-\mathrm{x}} \mathrm{Se}_{\mathrm{x}}$ ", submitted to Phys. Rev. Lett.

M.E. McGrath, R.L. Wagner, J.W. Apriletti, B.L. West, V. Ramalingam, J.D. Baxter and R.J. Fletterick, "Preliminary Crystallographic Studies of the Ligand-binding Domain of the Thyroid Hormone Receptor Complexed with Triiodothyronine", J. Mol. Biol. 237, 236 (1994)

M.A. McTigue, D.R. Williams and J.A. Tainer, "Crystal Structure of a Schistosomal Drug and Vaccine Target: Glutathione-S-Transferase from Schistosoma japonica and Its Complex with the Leading Antischistosomal Drug Praziquantel", J. Mol. Biol., in press

E.A. Merritt, S. Sarfaty, M. Pizza, R. Rappuoli and W.G.J. Hol, "Crystal Structure of E. coli Heat-labile Enterotoxin Mutant Va197 $\rightarrow$ Lys: Mutation of a Buried Residue Causes Loss of Activity but No Conformational Change", Nature Structure Biology, in press

I. Mukerji, J. Andrews, V.J. DeRose, M.J. Latimer, V.K. Yachandra, K. Sauer and M.P. Klein, "Orientation of the Oxygen-Evolving Manganese Complex in a Photosystem II Membrane Preparation: An X-ray Absorption Spectroscopy Study", Biochem., in press

A. Nilsson, P. Bennich, T. Wiell, N. Wassdahl, N. Mårtensson, J. Nordgren, O. Björneholm and J. Stöhr, "Direct Probing of the Adsorbate-Substrate Chemical Bond Using Angle Dependent X-ray Emission Spectroscopy", submitted to Phys. Rev. Lett.

A. Nilsson, J. Stöhr, T. Wiell, M. Aldén, P. Bennich, N. Wassdahl, S.S.P. Parkin, N. Mårtensson, J. Nordgren, B. Johansson and H.L. Skriver, "Determination of the Electronic Density of States at Interfaces: Applications to $\mathrm{Co} / \mathrm{Cu}$ Multilayers", submitted to Phys. Rev. B

P.A. O'Day, J.J. Rehr, S.I. Zabinsky and G.E. Brown, Jr., "Extended X-ray Absorption Fine Structure (EXAFS) Analysis of a Disorder and Multiple-Scattering in Complex Crystalline Solids", J. Am. Chem. Soc. 116, 2938 (1994)

P.A. O'Day, G.E. Brown, Jr. and G.A. Parks, "X-ray Absorption Spectroscopy of Co(II) Multinuclear Surface Complexes and Surface Precipitates on Kaolinites", J. Colloid. Inter. Sci. 165, 269 (1994)

P.A. O'Day, G.A. Parks and G.E. Brown, Jr., "Molecular Structure and Binding Sites of Cobalt(II) Surface Complexes on Kaolinite from X-ray Absorption Spectroscopy", Clays and Clay Minerals 42, 337 (1994)

R.M. Osgood III, B.M. Clemens, R.L. White and S. Brennan, "Strain, Stress, and Magnetic Surface Anisotropy of Epitaxial Fe(110)/Mo(110) Multilayers", accepted by Mat. Res. Soc. Symp. Proc.

C. Papelis and K. Hayes, "Distinguishing Between Interlayer and External Sorption Sites of Clay Minerals Using X-ray Absorption Spectroscopy", submitted to Langmuir

C.H. Park, Z.-X. Shen, A.G. Loeser, D.S. Dessau, D.G. Mandrus, A. Migliori, J. Sarrao and Z.K. Fisk, "Direct Observation of a Narrow Band Near the Gap Edge of FeSi", submitted to Phys. Rev. Lett. 
C.H. Park, Z.-X. Shen, A.G. Loeser, D.S. Dessau, G. Aeppli and Z. Fisk, "Observation of a Very Sharp Spectral Feature at the Band Edge of FeSi”, accepted by Phys. Rev. Lett.

M.A. Pavlosky, Y. Zhang, T.E. Westre, Q.-F. Gan, B. Hedman, K.O. Hodgson and E.I. Solomon, "Near Infrared Circular Dichroism, Magnetic Circular Dichroism and X-ray Absorption Spectral Comparison of the Non-Heme Ferrous Active Sites of Plant and Mammalian 15-Lipoxygenases", accepted by J. Am. Chem. Soc.

A.P. Payne, P.H. Fuoss, D.W. Kisker, G.B. Stephenson and S. Brennan, "GaAs c(4x4) Surface Structure in Organometallic Vapor-phase Epitaxy", Phys. Rev. B 49, 14427 (1994)

C. Pellegrini, J. Rosenzweig, G. Travish, K. Bane, R. Boyce, G. Loew, P. Morton, H.-D. Nuhn, J. Paterson, P. Pianetta, T. Raubenheimer, J. Seeman, R. Tatchyn, V. Vylet, H. Winick, K. Halbach, K.J. Kim, M. Xie, D. Prosnitz, E.T. Scharlemann, R. Bonifacio, L. De Salvo and P. Pierini, "The SLAC Soft X-ray High Power FEL", Nucl. Instrum. Methods A 341, 326 (1994)

G. Peng, F.M.F. deGroot, K. Hämäläinen, J.A. Moore, X. Wang, M.M. Grush, J.B. Hastings, D.P. Siddons, W.H. Armstrong, O.C. Mullins and S.P. Cramer, "High-Resolution Manganese X-ray Fluorescence Spectroscopy. Oxidation-State and Spin-State Sensitivity", J. Am. Chem. Soc. 116, 2914 (1994)

P. Pianetta, N. Takaura, S. Brennan, W. Tompkins, S.S. Laderman, A. Fischer-Colbrie, M. Madden, D.C. Wherry and J.B. Kortright, "Total Reflection X-ray Fluorescence Spectroscopy Using Synchrotron Radiation for Wafer Surface Trace Impurity Analysis", Rev. Sci. Instrum. 66, 1293 (1995)

I.J. Pickering and G.N. George, "Polarized X-ray Absorption Spectroscopy of Cupric Chloride Dihydrate", accepted by Inorg. Chem.

I.J. Pickering, G.E. Brown, Jr. and T.K. Tokunaga, "Quantitative Speciation of Selenium in Soils Using X-ray Absorption Spectroscopy", submitted to Env. Sci. \& Tech.

H.W. Pley, K.M. Flaherty and D.B. McKay, "Model for an RNA Tertiary Interaction from the Structure of an Intermolecular Complex Between a GAAA Tetraloop and an RNA Helix", Nature 372, 111 (1994)

H.W. Pley, K.M. Flaherty and D.B. McKay, "Three-dimensional Structure of a Hammerhead Ribozyme", Nature 372, 68 (1994)

L. Powers, M. Lauraeus, K.S. Reddy, B. Chance and M. Wikström, "Structure of the Binuclear Heme IronCopper Site in the Quinol-oxidizing Cytochrome $\alpha_{3}$ from Bacillus subtilis", Biochim. Biophys. Acta 1183, 504 (1994)

X. Qiu, C.L.M.J. Verlinde, S. Zhang, M.P. Schmitt, R.K. Holmes and W.G.J. Hol, "Three-dimensional Structure of the Diphtheria Toxin Repressor in Complex with Divalent Cation Co-repressors", Structure, in press

C.Y. Ralston, J. Chen, G. Peng, S.J. George, J. van Elp and S.P. Cramer, "Biological X-ray Spectroscopy on 3rd Generation Synchrotron Radiation Sources", Physica B, in press

M.J. Regan and A. Bienenstock, "Characterization of Phase Separation in Amorphous Films Using Modern Small-Angle Scattering Methods", accepted by J. Non-Cryst. Sol.

M.J. Regan and A. Bienenstock, "Phase Separation in Sputtered Amorphous Metal-Germanium Alloys", submitted to Phys. Rev. B

M.J. Regan, M. Rice, M.B. Fernández van Raap and A. Bienenstock, "Anisotropic Phase Separation Through the Metal-Insulator Transition in Amorphous Alloys", Phys. Rev. Lett. 73, 1118 (1994)

J.J. Rehr, C.H. Booth, F. Bridges and S.I. Zabinsky, "X-ray Absorption Fine Structure in Embedded Atoms", Phys. Rev. B 49, 12347 (1994) 
P. Riggs-Gelasco, T.L. Stemmler and J.E. Penner-Hahn, "XAFS of Dinuclear Metal Sites in Proteins and Model Compounds", Coord. Chem. Rev., in press

T.P. Russell, T.E. Karis, Y. Gallot and A.M. Mayes, "A Lower Critical Ordering Transition in a Diblock Copolymer Melt", Nature 368, 729 (1994)

M.G. Samant, J. Stöhr, S.S.P. Parkin, G.A. Held, B.D. Hermsmeier, F. Herman, M. van Schilfgaarde, L.-C. Duda, D.C. Mancini, N. Wassdahl and R. Nakajima, "Induced Spin Polarization in Cu Spacer Layers in $\mathrm{Co} / \mathrm{Cu}$ Multilayers", Phys. Rev. Lett. 72, 1112 (1994)

L. Sangaletti, F. Parmigiani, E. Ratner, Z.-X. Shen, C. Chemelli and O. Jepsen, "Electronic Structure of $\mathrm{K}_{2} \mathrm{NiF}_{4}$ ", Phys. Rev. $B$, in press

M.J. Scott, H.H. Zhang, S.C. Lee, B. Hedman, K.O. Hodgson and R.H. Holm, "Oxygen-Bridged Iron-Copper Assemblies Pertinent to Heme-Copper Oxidases: Synthesis and Structure of an[ $\left.\mathrm{Fe}^{\mathrm{III}}-(\mathrm{OH})-\mathrm{Cu}^{\mathrm{II}}\right]$ Bridge and EXAFS Multiple-Scattering Effects of Linear Oxo and Nonlinear Hydroxo Bridges", J. Am. Chem. Soc., in press

R. Serimaa, O. Serimaa and A. Bienenstock, "Application of Iterative Regularization Technique to Analysis of Anomalous X-ray Scattering Data", accepted by J. Non-Cryst. Sol.

S.E. Shadle, B. Hedman, K.O. Hodgson and E.I. Solomon, "Ligand K-Edge X-ray Absorption Spectroscopy as a Probe of Ligand-Metal Bonding: Charge Donation and Covalency in Copper-Chloride Systems", Inorg.

Chem. 33, 4235 (1994)

S.E. Shadle, B. Hedman, K.O. Hodgson and E.I. Solomon, "Ligand K-edge X-ray Absorption Spectroscopic Studies: Metal-Ligand Covalency in a Series of Late Transition Metal Tetrachlorides", J. Am. Chem. Soc. 117,2259 (1995)

A. Sharma, R. Hanai and A. Mondragon, "Crystal Structure of the Amino Terminal Fragment of Vaccinia Virus DNA Topoisomerase I at $1.6 \AA$ Resolution”, Structure 2, 767 (1994)

Z.-X. Shen, "Fermiology and Superconducting Gap Anisotropy of Cuprate Superconductors", Physica B 197, 632 (1994)

Z.-X. Shen and.D.S. Dessau, "Electronic Structure and Photoemission Studies of Late Transition-Metal Oxides -- Mott Insulators and High-Temperature Superconductors", Phys. Rep., in press

Z.-X. Shen, W.E. Spicer, D.S. Dessau, D.M. King and B.O. Wells, "Photoemission Study of High-T Superconductors ---- From the Electronic Structure to the Superconducting Gap", Science, in press

D.K. Shuh, L.J. Terminello, L.A. Boatner, M.M. Abraham and D. Perry, "Characterization of Ce-Doped LaPO by X-ray Absorption Spectroscopy", Mat. Res. Soc. Symp. Proc. 329, 91 (1994)

D.K. Shuh, N. Kaltsoyannis, J.J. Bucher, N.M. Edelstein, S.B. Clark, H. Nitsche, T. Reich, E.A. Hudson, I. Almahamid, P. Torretto, W.W. Lukens, K. Roberts, B.C. Yee, D.E. Carlson, A. Yee, B.B. Buchanan, T. Leighton, W.-S. Yang and J.C. Bryan, "Environmental Applications of XANES: Speciation of Tc in Cement After Chemical Treatment and Se after Bacterial Uptake", Mat. Res. Soc. Symp. Proc., in press

M.M. Skinner, H. Zhang, D.H. Leschnitzer, Y. Guan, H. Bellamy, R.M. Sweet, C.W. Gray, R.N.H. Konings, A. H.-J. Wang and T.C. Terwilliger, "Structure of the Gene V Protein of Bacteriophage f1 Determined by Multiwavelength X-ray Diffraction on the Selenomethionyl Protein", Proc. Natl. Acad. Sci. USA 91, 2071 (1994)

T.A. Smith, J.G. DeWitt, B. Hedman and K.O. Hodgson, "Sulfur and Chlorine K-edge X-ray Absorption Spectroscopy of Photographic Materials", J. Am. Chem. Soc. 116, 3836 (1994) 
W. Somers, M. Ultsch, A.M. de Vos and A.A. Kossiakoff, "The X-ray Structure of a Growth HormoneProlactin Receptor Complex", Nature 372, 478 (1994)

S.R. Stock, A. Guvenilir, D. Piotrowski and Z.U. Rek, "High Resolution Synchrotron X-ray Diffraction Tomography of Polycrystalline Samples", submitted to Mat. Res. Soc. Symp. Proc.

J. Stöhr, "Determination of Spin-Density and Orbital-Moment Anisotropies in Transition Metals by AngleDependent X-ray Magnetic Circular Dichroism", submitted to Phys. Rev. Lett.

C. Streli, P. Wobrauschek, W. Ladisich, R. Rieder, H. Aiginger, R.W. Ryon, P. Pianetta and P. Pianetta, "Total Reflection X-ray Fluorescence Analysis of Light Elements Using Synchrotron Radiation", Nucl. Instrum. Methods A 345, 399 (1994)

E. Tamura, G.D. Waddill, J.G. Tobin and P.A. Sterne, "Linear and Circular Dichroism in Angle-Resolved Fe 3p Photoemission", Phys. Rev. Lett. 73, 1533 (1994)

T. Tanaka, Y. Ishizawa, J. Wong, Z.U. Rek, M. Rowen, F. Schäfers and B. Müller, "Development of YB 66 Soft X-ray Monochromator for Synchrotron Radiation”, Jpn. J. Appl. Phys. 10, 110 (1994)

D.H. Templeton and L.K. Templeton, "Tetrahedral Anisotropy of X-ray Anomalous Scattering", Phys. Rev. B 49, 14850 (1994)

L.K. Templeton and D.H. Templeton, "Polarized Dispersion for X-rays Scattered by an Aromatic Bromide", J. Sync. Rad. 2, 31 (1995)

L.J. Terminello, A. Chaiken, D.A. Lapiano-Smith, G.L. Doll and T. Sato, "Morphology and Bonding Measured from Boron-Nitride Powders and Films Using Near-Edge X-ray Absorption Fine Structure", J. Vac. Sci. Technol. A 12, 2462 (1994)

J. Terry, R. Cao, C. Wigren and P. Pianetta, "Photoemission Study of $\mathrm{Au}, \mathrm{Ge}$, and $\mathrm{O}_{2}$ Deposition on $\mathrm{NH}_{4} \mathrm{~F}$ Etched Si(111)", J. Vac. Sci. Technol. A 12, 1869 (1994)

J.J.G. Tesmer, T.L. Stemmler, J.E. Penner-Hahn, V.J. Davisson and J.L. Smith, "Preliminary X-ray Analysis of Escherichia coli GMP Synthetase: Determination of Anomalous Scattering Factors for a Cysteinyl Mercury Derivative", Proteins 18, 394 (1994)

J.L. Thorvaldsen, A.K. Sewell, A.M. Tanner, J.M. Peltier, I.J. Pickering, G.N. George and D.R. Winge, "Mixed $\mathrm{Cu}^{+}$and $\mathrm{Zn}^{2+}$ Coordination in the DNA-Binding Domain of the AMT1 Transcription Factor from Candida glabrata", Biochem. 33, 9566 (1994)

I.M. Tidswell, N.M. Markovic and P.N. Ross, "Potential Dependent Structure of Single Crystal Gold Interfaces in Alkaline Electrolyte: An In Situ X-ray Scattering Study", accepted by Surf. Sci.

I.M. Tidswell, N.M. Markovic and P.N. Ross, "Potential Dependent Surface Structure of the Pt(111)/Electrolyte Interface", accepted by $J$. Elec. Chem.

D.L. Tierney, J.A. Fee, M.L. Ludwig and J.E. Penner-Hahn, "X-ray Absorption Spectroscopy of the Iron Site in $E$. coli $\mathrm{Fe}$ (III) Superoxide Dismutase", Biochem., in press

J.G. Tobin, G.D. Waddill, A.F. Jankowski, P.A. Sterne and D.P. Pappas, "Comparison of Branching Ratio and Sum Rule Analyses of Magnetic Circular Dichroism in X-ray Absorption Spectroscopy", submitted to Phys. Rev. $B$

J.G. Tobin and G.D. Waddill, "Resonant Photoemission and Magnetic X-ray Circular Dichroism in the $M$ Shell of Ultrathin Films of Fe", J. Appl. Phys. 75, 6369 (1994) 
J.G. Tobin and G.D. Waddill, "Imaging Surface Atomic Structure with Energy-Dependent Electron Diffraction", Surf. Rev. \& Lett. 1, 213 (1994)

J.G. Tobin, G.D. Waddill, X. Guo and S.Y. Tong, "Spin-Specific Photoelectron Diffraction Using Magnetic X-ray Circular Dichroism", J. Appl. Phys. 76, 10 (1994)

J.G. Tobin, A.F. Jankowski, G.D. Waddill and P.A. Sterne, "Magnetic X-ray Circular Dichroism in Fe Co Pt Multilayers", Mat. Res. Soc. Symp. Proc. 343, 393 (1994)

J.G. Tobin, G.D. Waddill, D.P. Pappas, E. Tamura, P.A. Sterne, X. Guo and S.Y. Tong, "Electron Dichroism Studies of Magnetic Structure Using Circularly-Polarized X-rays", accepted by Spectroscopy

J.G. Tobin, G.D. Waddill, D.P. Pappas, E. Tamura, P.A. Sterne, X. Guo and S.Y. Tong, "Spin-Specific Photoelectron Diffraction, Photoelectron Spectroscopy, and Absorption Using Magnetic X-ray Circular Dichroism", submitted to J. Vac. Sci. Technol.

S.H. Tolbert and A.P. Alivisatos, "Se EXAFS Study of the Elevated Wurtzite to Rock Salt Structural Phase Transition in CdSe Nanocrystals", Nanophase Mat. 471 (1994)

S.H. Tolbert and A.P. Alivisatos, "Size Dependence of a First Order Solid-Solid Phase Transition: The Wurtzite to Rock Salt Transformation in CdSe Nanocrystals", Science 265, 373 (1994)

S.H. Tolbert, A.B. Herhold, C.S. Johnson and A.P. Alivisatos, "A Comparison of Quantum Confinement Effects on the Electronic Absorption Spectra of Direct and Indirect Gap Semiconductor Nanocrystals", submitted to Phys. Rev. Lett.

B.P. Tonner, D. Dunham, J. Zhang, W.L. O'Brien, M. Samant, D. Weller, B.D. Hermsmeier and J. Stöhr, "Imaging Magnetic Domains with the X-ray Dichroism Photoemission Microscope", Nucl. Instrum. Methods A 347, 142 (1994)

K.S. Toth, C.X. Zhang, H.L. Lipton and M. Luo, "The Structure of a Highly Virulent Theiler's Murine Encephalomyelitis Virus (GDVII strain)", submitted to J. Virology

H. Tsuruta, P. Vachette, T. Sano, M. Moody, Y. Amemiya, K. Wakabayashi and H. Kihara, "Kinetics of the Quaternary Structure Change of Aspartate Transcarbamylase Triggered by Succinate, a Competitive Inhibitor", Biochem. 33, 10007 (1994)

M. Valli, P. Persson, I. Persson and M. Sandström, "Extended X-ray Absorption Fine Structure Spectroscopic Study of Acetone Solutions of Zinc and Lead(II) Ethylxanthate", Acta Chem. Scand. 48, 810 (1994)

J. van Elp, G. Peng, B.G. Searle, S. Mitra-Kirtley, Y.-H. Huang, M.K. Johnson, Z.H. Zhou, M.W.W. Adams, M.J. Maroney and S.P. Cramer, "Electronic Structure and Symmetry in Nickel L Edge X-ray Absorption Spectroscopy: Application to a Nickel Protein", J. Am. Chem. Soc. 116, 1918 (1994)

R. Venkatraman, P.R. Besser and J.C. Bravman, "Elastic Strain Gradients and X-ray Line Broadening Effects as a Function of Temperature in Aluminum Thin Films on Silicon", J. Mater. Res. 9, 328 (1994)

R.P. Vinci, E.M. Zielinski and J.C. Bravman, "Effect of Copper Thickness on Crystallographically Orientation-Dependent Stress and Strain", submitted to Mat. Res. Soc. Symp. Proc.

R.P. Vinci, E.M. Zielinski and J.C. Bravman, "Thermal Strain and Stress in Copper Thin Films", submitted to Thin Solid Films

G.D. Waddill, J.G. Tobin, X. Guo and S.Y. Tong, "Magnetic X-ray Circular in Spin Polarized Photoelectron Diffraction", submitted to Mat. Res. Soc. Symp. Proc. 
G.D. Waddill, J.G. Tobin, X. Guo and S.Y. Tong, "Spin-Polarized Photoelectron Diffraction Using CircularlyPolarized X-rays", Phys. Rev. B 50, 6774 (1994)

G.D. Waddill, J.G. Tobin, X. Guo and S.Y. Tong, "A Structural Determination Using Magnetic X-ray Circular Dichroism in Spin-Polarized Photoelectron Diffraction", submitted to Vacuum

S. Wakatsuki, Y. Kimura, W. Stoeckenius, N. Gillis, D. Eliezer, K.O. Hodgson and S. Doniach, "Blue Form of Bacteriorhodopsin and its Order-Disorder Transition During Dehydration", Biochem. Biophys. Acta 1185, 160 (1994)

G.S. Waldo and J.E. Penner-Hahn, "The Mechanism of Mn Catalase Peroxide Disproportionation:

Determination of Mn Oxidation States During Turnover", Biochem., in press

H. Wang, J.C. Woicik, T. Åberg, M.H. Chen, A. Herrera-Gomez, T. Kendelewicz, A. Mäntykenttä, K.E. Miyano, S. Southworth and B. Crasemann, "Threshold $K-L L$ Auger Spectra of P in InP", Phys. Rev. A 50, 1359 (1994)

S. Wang, M.H. Lee, R.P. Hausinger, P.A. Clark, D.E. Wilcox and R.A. Scott, "Structure of the Dinuclear Active Site of Urease. X-ray Absorption Spectroscopic Study of Native and 2-Mercaptoethanol-Inhibited Bacterial and Plant Enzymes", Inorg. Chem. 33, 1589 (1994)

S. Wang, R.A. Scott, L. Lebioda, Z.H. Zhou and J.M. Brewer, "An X-ray Absorption Spectroscopic Study of the Interactions of $\mathrm{Ni}^{2+}$ with Yeast Enolase", accepted by J. Inorg. Biochem.

W.K. Warburton, S.R. Russell, S.A. Kleinfelder and J. Segal, "Direct Observation of Individual SPEAR Electron Bunch Orbit Oscillations at Frequencies Above $30 \mathrm{kHz}$ ", Nucl. Instrum. Methods A 347, 557 (1994)

W.K. Warburton, S.R. Russell, S.A. Kleinfelder and J. Segal, "The 150 nsec Detector Project: Progress with Small Detectors", Nucl. Instrum. Methods A 348, 503 (1994)

W.K. Warburton, S.R. Russell and S.A. Kleinfelder, "The 150 nsec Detector Project: Prototype Preamplifier Results", Nucl. Instrum. Methods A 347, 539 (1994)

H.E. Warriner, S.H.J. Idziak, K.E. Kraiser and C.R. Safinya, "Lamellar Biomembranes Comprised of Lipid and Surfactant-Polymers", Polymer Preprints 35, 641 (1994)

D. Weller, J. Stöhr, R. Nakajima, A. Carl, M.G. Samant, C. Chappert and G. Held, "Microscopic Origin of Magnetic Anisotropy in Au/Co/Au Probed with X-ray Magnetic Circular Dichroism", submitted to Phys. Rev. Lett.

D. Weller, Y. Wu, J. Stöhr, M.G. Samant, B.D. Hermsmeier and C. Chappert, "Orbital Magnetic Moments in Co in Multilayers with Perpendicular Magnetic Anisotropy", Phys. Rev. B 49, 12888 (1994)

B.O. Wells, Z.-X. Shen, A. Matsuura, D.M. King, M.A. Kastner, M. Greven and R.J. Birgeneau, "E Versus k Relations and Many Body Effects in the Model Insulating Copper Oxide $\mathrm{Sr}_{2} \mathrm{CuO}_{2} \mathrm{Cl}_{2}$ ", Phys. Rev. Lett. 74, 964 (1995)

T.E. Westre, A. Di Cicco, A. Filipponi, C.R. Natoli, B. Hedman, E.I. Solomon and K.O. Hodgson, "GNXAS, a Multiple-scattering Approach to EXAFS Analysis: Methodology and Applications to Iron Complexes", J. Am. Chem. Soc. 117, 1566 (1995)

T.E. Westre, A. Di Cicco, A. Filipponi, C.R. Natoli, B. Hedman, E.I. Solomon and K.O. Hodgson, "Determination of the Fe-N-O Angle in $\{\mathrm{FeNO}\}^{7}$ Complexes Using Multiple-Scattering EXAFS Analysis by GNXAS", J. Am. Chem. Soc. 116, 6757 (1994) 
T.E. Westre, K.E. Loeb, J.M. Zaleski, B. Hedman, K.O. Hodgson and E.I. Solomon, "Determination of the Geometric and Electronic Structure of Activated Bleomycin Using X-ray Absorption Spectroscopy", J. Am. Chem. Soc., in press

T.E. Westre, A. Di Cicco, A. Filipponi, C.R. Natoli, B. Hedman, E.I. Solomon and K.O. Hodgson, "Using GNXAS, a Multiple-Scattering EXAFS Analysis Package, for Determination of the Fe-N-O Angle in $\{\mathrm{FeNO}\}^{7}$ Complexes", Physica B 208 \& 209, 137 (1995)

C. White, M.N. Janakiraman, M.J. Jedrzejas, W.G. Laver, J. Vasella, M.G. Air and M. Luo, "A Phosphonate Inhibitor Inhibit Different Strains of Influenza Virus Neuraminidase with Different Efficiencies", J. Mol. Biol., in press

H. Wiedemann, M. Baltay, R. Carr, M. Hernandez and W. Lavender, "A Compact Radiation Source for Digital Subtractive Angiography", Nucl. Instrum. Methods A 347, 515 (1994)

H. Winick, K. Bane, R. Boyce, J. Cobb, G. Loew, P. Morton, H.-D. Nuhn, J. Paterson, P. Pianetta, T. Raubenheimer, J. Seeman, R. Tatchyn, V. Vylet, C. Pellegrini, J. Rosenzweig, G. Travish, D. Rosnitz, E. Scharlemann, K. Halbach, K.-J. Kim, R. Schlueter, M. Xie, R. Bonifacio, L. De Salvo and P. Pierini, "Short Wavelength FELs Using the SLAC Linac", Nucl. Instrum. Methods A 347, 199 (1994)

R.T. Witkowski, K. Clark, D.L. Tierney, J.E. Penner-Hahn, L. Newman, S. Hattman and G. McLendon, "The Zinc Coordination Site of the Bacteriophage Mu Translation Activating Protein, Com", submitted to J. Mol. Biol.

J. Wong, G.N. George, I.J. Pickering, Z.U. Rek, M. Rowen, T. Tanaka, G.H. Via, B. DeVries, D.E.W. Vaughan and G.E. Brown, Jr., "New Opportunities in XAFS Investigation in the 1-2 keV Region", Solid State Commun. 92, 559 (1994)

J. Wong, Z.U. Rek, M. Rowen, T. Tanaka, F. Schäfers, B. Müller, G.N. George, I.J. Pickering, G. Via, B. DeVries, G.E. Brown, Jr. and M. Fröba, "New Opportunities in 1-2 keV Spectroscopy", accepted by Physica B

J. Wong, J. Elmer, P. Waide and E. Larson, "In-Situ X-ray Diffraction of an Arc Weld Showing the Phase Transformation of Ti and Fe as a Function of Position in the Weld", Adv. X-ray Analysis 37, 479 (1994)

J. Wong, M. Fröba and R. Frahm, "A Critical Assessment of the QEXAFS Method", accepted by Physica B

Z. Xu, F.-S. Xiao, S.K. Purnell, O. Alexeev, S. Kawi, S.E. Deutsch and B.C. Gates, "Size-dependent Catalytic Activity of Supported Metal Clusters", Nature 372, 346 (1994)

S.A. Yoshikawa, J. Nogami, C.F. Quate and P. Pianetta, "Behavior of Tellurium on Silicon (100)", Surf. Sci. 321, L183 (1994)

C.A. Yu, D. Xia, J. Deisenhofer and L. Yu, "Crystallization of Mitochondrial Cytochrome $b-c_{1}$ Complex from Gel with or without Reduced Pressure", J. Mol. Biol., in press

K.M. Yu, A.J. Moll, W. Walukiewicz, N. Derhacobian and C. Rossington, "Amphoteric Substitutionality and Lattice Distortion of Ge in InP", Appl. Phys. Lett. 64, 1543 (1994)

- K.M. Yu, A.J. Moll, N. Chan, W. Walukiewicz and P. Becla, "Substitutionality of Ge in Ion Implanted AlSb", submitted to Appl. Phys. Lett.

J. Zhao, Z. Feng, N. Shah, G.P. Huffman and I. Wender, "Role of Molybdenum at the Iron Oxide.Surface", $J$. Catalysis 148, 194 (1994)

J. Zhao, G.P. Huffman and B.H. Davis, "XAFS Study of the State of Platinum in a Sulfated Zirconia Catalyst", Catalysis Letters 24, 385 (1994) 
L. Zhou, Q. Yu and M. Luo, "Characterization of Two Density Populations of Feline calicivirus Particles", J.Virology 205, 530 (1994)

E.M. Zielinski, R.P. Vinci and J.C. Bravman, "Measurement of the Dependence of Stress and Strain on Crystallographic Orientation in $\mathrm{Cu}$ and $\mathrm{Al}$ Thin Films", submitted to Mat. Res. Soc. Symp. Proc.

\section{BOOKS AND CONFERENCE PROCEEDINGS}

F. Bridges, G.G. Li, C.H. Booth, J.B. Boyce, and T. Claeson, "Large Local Distortions Introduced by Defects in $\mathrm{YBa}_{2} \mathrm{Cu}_{3} \mathrm{O}_{7}$ Superconductors: An X-ray-Absorption Study" in Proceedings of the Lattice Defects Conference, Lyon, France (July 1994)

R. Cao, H. Tang and P. Pianetta, "Negative Electron Affinity on $\mathrm{GaAs}$ with $\mathrm{O}_{2}$ and $\mathrm{NF}_{3}$ " in Gordon Research Conference "Excitation at Semiconductor Surfaces-Fundamental Concepts and Application in Semiconductor Processing", Hawaii, Nov. 1994

R. Carr and S., Lidia, "Insertion Device Calculations with Mathematica" in R. Carr et al., Halbach Symposium on Magnet Technology, Berkeley, February 3, 1995

J. Corbett and C. Wermelskirchen, "Controls and Accelerator Modeling" in H. Winick, Synchrotron Radiation Sources: A Primer, World Scientific, Singapore (1994), pp. 215-241

J. Corbett, D. Keeley, R. Hettel, I. Linscott and J. Sebek, "Algorithms for Orbit Control on SPEAR" in Fourth European Particle Accelerator Conference (EPAC'94), London, England, June 27-July 1, 1994

M. Cornacchia, "Lattices" in H. Winick, Synchrotron Radiation Sources: A Primer, World Scientific, Singapore (1994), pp. 30-56

J.H. Dawson, H.I. Liu, M. Sono, S. Kadkhodayan, B. Hedman and K.O. Hodgson, "Direct Evidence of the Electron Releasing Character of the Cysteine Proximal Ligand of Cytochrome P450 from X-ray Absorption Near Edge Spectroscopy" in M.C. Lechner, Cytochrome. Biochemistry, Biophysics and Molecular Biology, John Libby Eurotext, Paris (1994), pp. 441-444

R.C. Elder, W.B. Jones, Z. Zhao, J.G. Dorsey and K. Tepperman, "Myochrisine Solution Structure and Reactivity" in Metal-Based Drugs, Vol. 1 (1994), pp. 363-374

R.C. Elder, L.R. Sharpe, D.H. Igo, R.O. Rigney and W.R. Heineman, "EXAFS Studies of Film Coated Electrodes" in NATO Advanced Science Institutes Series, C: Mathematical and Physical Sciences, Vol. 432 (1994), pp. 335-348

P.H. Fuoss, D.W. Kisker, A.P. Payne, G.B. Stephenson and S. Brennan, "OMVPE Growth: An In Situ X-ray Analysis" in I. Ohdomari et al., Proc. of the First Inter. Symp. on the Control of Semiconductor Interfaces, Elsevier Science, New York (1994), p. 121

J.N. Galayda, Y. Chung and R.O. Hettel, "Orbit Stabilizing and Multibunch Feedback Systems", in H. Winick, Synchrotron Radiation Sources: A Primer, World Scientific, Singapore (1994), pp. 344-375

S.J. George, J. van Elp, J. Chen, S. Mitra-Kirtley, O.C. Mullins and S.P. Cramer, "Soft X-ray Absorption and $X$-ray Magnetic Circular Dichroism in Biology" in B. Chance et al., Synchrotron Radiation in Biosciences, Oxford University Press (1994), pp. 313-334

J.H. Kinney, N.E. Lane, S. Majumdar et al., "Three-Dimensional, in Vivo Microscopy of Trabecular Bone Architecture" in Orthopedic Research Society Symposium, New Orleans, Louisiana, February 1994

M.P. Klein, "Perspectives on Magnetic Resonance and X-ray Spectroscopy in Biochemical Spectroscopy" in K. Sauer, Meth. Enzymol. Vol. 246, Academic Press, Orlando, FL, in press 
M. Luo, "Virus Structure: Atomic Structure" in Encyclopedia of Virology, Academic Press (1994), pp. 15711573

M. Nicol, S.M. Gibson and D. Reifschneider, "Chemistry at Static High Pressure: Some General Principles and Recent Developments" in ARO Workshop on Shock Synthesis of Materials, Atlanta, May 1994

M. Nicol, S.E. Gibson, D. Reifschneider, S.W. Johnson, B.J. Baer and D. Schiferl, "Spectroscopic and X-ray Studies of Materials at High Pressures: Recent Developments" in M. Kamo et al., Advanced Materials '94, Intl. Commun. Spec., Tokyo (1994), pp. 205-210

H.-D. Nuhn, "An Optimized Low Emittance Lattice for SPEAR" in Proceedings of the 4th European Particle Accelerator Conference (EPAC'94), London, England, June 27-July 1, 1994

H.-D. Nuhn, R. Boyce, G. Loew, K. Bane, J. Paterson, R. Miller, T. Raubenheimer, D. Palmer, H. Winick, R. Tatchyn, C. Pellegrini, A.D. Yeremian, G. Travish, J. Rosenzweig, W.M. Fawley, E.T. Scharlemann, K.-J. Kim, R. Schlüter, K. Halbach, M. Xie, R. Bonifacio, A.M. Sessler, P. Pierini and L. De Salvo, "Short Wavelength FELs Using the SLAC Linac" in Proceedings of the 4th European Particle Accelerator Conference (EPAC'94), London, England, June 27-July 1, 1994

L. Powers, R. Sinclair, B. Chance and K.S. Reddy, "The Structure--Function Relationship of the Active Sites in Haemoprotein Catalysis" in B. Chance et al., Synchrotron Radiation in the Biosciences, Oxford University Press (1994), pp. 302-312

P.J. Riggs, R. Mei and J.E. Penner-Hahn, "Structural Characterization of Manganese Redox Enzymes: Results from X-ray Absorption Spectroscopy" in Mechanistic Bioinorganic Chemistry, ACS Advances in Chemistry Series, in press

M.C. Schabel and Z.-X. Shen, "Photoemission Studies of Novel Superconductors" in Proceedings of the International Symposium/Workshop on High-Tc Superconductivity and the $\mathrm{C}_{60}$ Family, Beijing, June, 1994

M.J. Smith, D.E. Ryan, K. Nakanishi, P. Frank and K.O. Hodgson, "Vanadium in Ascidians and the Chemistry of Tunichromes" in H. Sigel and A. Sigel, Metal Ions in Biological Systems: Vanadium and Its Role in Life Vol. 31, Marcel Dekker, New York, in press

E.I. Stiefel and G.N. George, "Ferredoxins, Hydrogenases and Nitrogenases: Metal-Sulfide Proteins" in I. Bertini et al., Bioinorganic Chemistry, University Science Books, Mill Valley (1994), pp. 365-453.

E.I. Stiefel, M.J. Grossman, S.M. Hinton, V. Minak-Berneo, G.N. George, R.C. Prince, R.E. Bare and G.D. Watt, "Bacterioferritin: A Hemoprotein Member of the Ferritin Family" in C. Hershko, Progress in Iron Research, Plenum Press, New York (1994), pp. 157-164

J. Stöhr and Y. Wu, "X-ray Magnetic Circular Dichroism: Basic Concepts and Theory for 3D Transition Metal Atoms" in A.S. Schlachter and F.J. Wuilleumier, New Directions in Research with 3rd Generation Soft X-ray Synchrotron Radiation Sources NATO ASI Series, E Vol. 254, Kluwer Academic, Dordrecht, Netherlands (1994), p. 221

R. Tatchyn, R. Boyce, G. Loew, K. Bane, H.-D. Nuhn, D. Palmer, R. Miller, T. Raubenheimer, J. Paterson, H. Winick, J. Seeman, C. Pellegrini, D. Yeremian, G. Travish, J. Rosenzweig, E.T. Scharlemann, D. Prosnitz, W. Fawley, K. Halbach, S. Caspi, R. Schlüter, M. Xie, K.-J. Kim, P. Pierini, R. Bonifacio and L. De Salvo, "Prospects for High Power Linac Coherent Light Source (LCLS) Development in the $1000 \AA-1 \AA$ Wavelength Range" in Proceedings of the 4th International X-ray Laser Colloquium, Williamsburg, VA, May 16-20, 1994

J.G. Tobin, G.D. Waddill, H. Li and W.Y. Tong, "Imaging of Surface Structure with Energy Dependent Photoelectron Diffraction" in X. Xie et al., The Structure of Surfaces-IV, World Scientific, London (1994) 
G. Travish, W. Fawley, K.-J. Kim, H.-D. Nuhn, C. Pellegrini, H. Winick and M. Xie, "Parameteric Study of an X-ray FEL" in Proceedings of the International Free Electron Laser Conference 1994, Stanford California, August 22-August 26, 1994

H. Tsuruta, T. Sano, P. Vachette, M.F. Moody, I. Narumi, K. Wakabayashi, Y. Amemiya, K. Kimura and H. Kihara, "Time-Resolved X-ray Scattering Study of the Allosteric Transition of $E$. coli Aspartate

Transcarbamylase" in B. Chance et al., Synchrotron Radiation in Biosciences, Oxford University Press (1994), pp. 139-144

D.R. Winge, C.T. Dameron and G.N. George, "The Metallothionein Structural Motif in Gene Expression" in Eichhorn, G.L. and L.G. Marzilli, Adv. Inorg. Biochem. Vol. 10, Prentice Hall, Inc. (1994), pp. 1-48

V.K. Yachandra, V.J. DeRose, M.J. Latimer, I. Mukerji, K. Sauer and M.P. Klein, "A Structural Model for the Photosynthetic Oxygen Evolving Manganese Complex" in B. Chance et al., Synchrotron Radiation in Biosciences, Oxford University Press (1994), pp. 275-283

V.K. Yachandra, "X-ray Absorption Spectroscopy and Applications in Structural Biology in Biochemical Spectroscopy" in K. Sauer, Meth. Enzymol. Vol. 246, Academic Press, Orlando, FL, in press

V.K. Yachandra and M.P. Klein, "X-ray Absorption Spectroscopy" in J. Amesz and A.J. Hoff, Biophysical Techniques in Photosynthesis. Advances in Photosynthesis, Kluwer, Dordrecht, in press

V.K. Yachandra, K. Sauer and M.P. Klein, "Recent Studies on the Mn Complex Involved in Photosynthetic Water Oxidation" in Proceedings of the US-Japan-Binational Conference on Dynamic Aspects of the

Photochemical Reaction Center, Tokyo, Japan, in press

\section{THESES BASED ON WORK AT SSRL}

To date 266 theses from 37 universities have been granted based on work at SSRL. Below are the 29 theses completed in 1994.

A.Q.R. Baron

Stanford University

J. Blixt

Royal Institute of Technology

N. Campobasso

Purdue University

D. Eliezer

Stanford University .

Y. Gea .

The State University of New York at Stony Brook

S.E. Gibson

UC-Los Angeles

B.P. Grady

University of Wisconsin-Madison
"Resonant Nuclear Scattering of Synchrotron Radiation: Detector Development and Specular Scattering from a Thin Layer of ${ }^{57} \mathrm{Fe}$, Advisor-G.S. Brown

"Some Aspects of Thallium(III) Halide and Pseudohalide Coordination in Solution", Advisor - M. Sandström

"Structure Determination and Refinement at $2.2 \AA$ of Nitrogenase MoFeProtein from Clostridium pasteurianum", Advisor - J. Bolin

"Protein:Folding-and Protein Metallocluster Studies Using Synchrotron Small Angle X-ray Scattering", Advisors - S: Doniach/K.O. Hodgson

"Model Compound Synthesis and Physical Studies on Enzymes from Molybdenum-Containing Mesophilic and Tungsten-Containing Hyperthermophilic Organisms", Advisor -C.C.S. Springer

" $o$-Nitroaniline and $p$-Nitroaniline at High Pressures", work partially done at SSRL, Advisor - M. Nicol

"Orientation and Relaxation Behavior in Ionomers", Advisor - S.L. Cooper 
R.J. Goddard

University of Wisconsin-Madison

J.A. Guckert

Stanford University

\author{
A. Herrera-Gomez \\ Stanford University \\ C. $\mathrm{Kim}$ \\ Stanford University
}

G. Li

UC-Santa Cruz

W. Liang

UC-Berkeley

C.D. Lima

Northwestern University

C.M. Lytle

Brigham Young University

M. Najih

University of Kentucky

H.W. Pley

Stanford University

E.M. Proudfoot

University of Sydney

N.M. Rensing

Stanford University

P.J. Riggs-Gelasco

University of Michigan

A.C. Rosenzweig

MIT

S.E. Shadle

Stanford University

K.S. Toth

University of Alabama

M. Valli

Swedish University of

Agricultural Sciences
"Specific Interactions in Polyurethane Cationomers", Advisor - S.L. Cooper

"Electronic Structure Contributions of the Blue Copper Active Site to Reduction Potentials, Geometry, and Electron Transfer Pathways", Advisor - E.I. Solomon

"X-ray Standing Wave Study of the $\mathrm{Bi} / \mathrm{GaAs}$ and $\mathrm{Bi} / \mathrm{GaP}$ Interfaces", Advisor - W.E. Spicer

"Photoelectron Microscopy and Development of an Imaging Band Pass Analyzer for a Magnetic Photoelectron Microscope",

Advisor - P.A. Pianetta

"X-ray Absorption Spectroscopy and Local Structure Studies of Some High $\mathrm{T}_{\mathrm{c}}$ Superconductors", Advisor - F. Bridges

"Structural and Oxidation State Studies of the Manganese Cluster in the Oxygen Evolving Complex of Photosystem II", Advisor - M.P. Klein

"The Three-dimensional Structure of the $67 \mathrm{kDa}$ Amino-Terminal

Fragment of $E$. coli DNA Topoisomerase I.", Advisor-A. Mondragon

"Heavy Metal Accumulation in Great Basin Submersed Aquatic Macrophytes", Advisor - B.N. Smith

"XAFS Spectroscopy of Selected Chromium Compounds and Chromium in Coals and Ashes", Advisor - G.P. Huffman

"Three-dimensional Structures of a Hammerhead Ribozyme and a GAAA Tetraloop-RNA Helix Interaction", Advisor -D. McKay

"The Analysis of the EXAFS of Manganese Complexes", MSc Thesis, Advisor - H.C. Freeman

"The Effect of Structure on the Magnetoresistance of Iron/Chromium Multilayers", Advisor - B. Clemens

"Structural Characterization of the Manganese Cluster of the Oxygen Evolving Complex of Photosystem II Using X-ray Absorption Spectroscopy", Advisor - J.E. Penner-Hahn

"Structural Studies of the Hydroxylase Protein of Methane Monooxygenase from Methylococcus capsulatus (Bath)", Advisor - S.J. Lippard

"Ligand K-Edge X-ray Absorption Spectroscopic Studies of the Electronic Structure of Inorganic Model Complexes and Metalloprotein Active Sites", Advisors - K.O. Hodgson/E.I. Solomon

"Structural Studies of Theiler's Murine Encephalomyelitis Viruses", Advisor - M. Luo

"On the Sorption of Some Soft Ligands on Sulphide Mineral Surfaces", Advisor - I. Persson 
R.P. Vinci

Stanford University

J.E. Whitmore

University of Washington

J. Wu

Stanford University

$X$. Yang

Stanford University

J. Yuan

University of Cincinnati
"Thermal Strains and Stresses in Copper Thin Films and Microelectronic Interconnect Structures", Advisor - J.C. Bravman

"EXAFS Experiments at High Pressure with Small Samples", Advisor - R.L. Ingalls

"A Photoemission Study of the Diamond and the Single Crystal C60", Advisor - I. Lindau

"Photoemission Study of the Epitaxial Growth of Ge on Si(100) Using Surfactants", Advisor - P.A. Pianetta

"EXAFS Study of ReHEDP Anticancer Agents, Scattering Study of $\mathrm{Ni}(\mathrm{acac})$ and $\mathrm{Co}(\mathrm{acac})$ and Molecular Modeling of Metallothioneine and Zinc Finger Proteins", Advisor - R.C. Elder 